\title{
EMBODIED COGNITION OVER THE LIFESPAN AND IN APPLIED SETTINGS
}

EDITED BY: Annalisa Setti and Anna M. Borghi PUBLISHED IN: Frontiers in Psychology
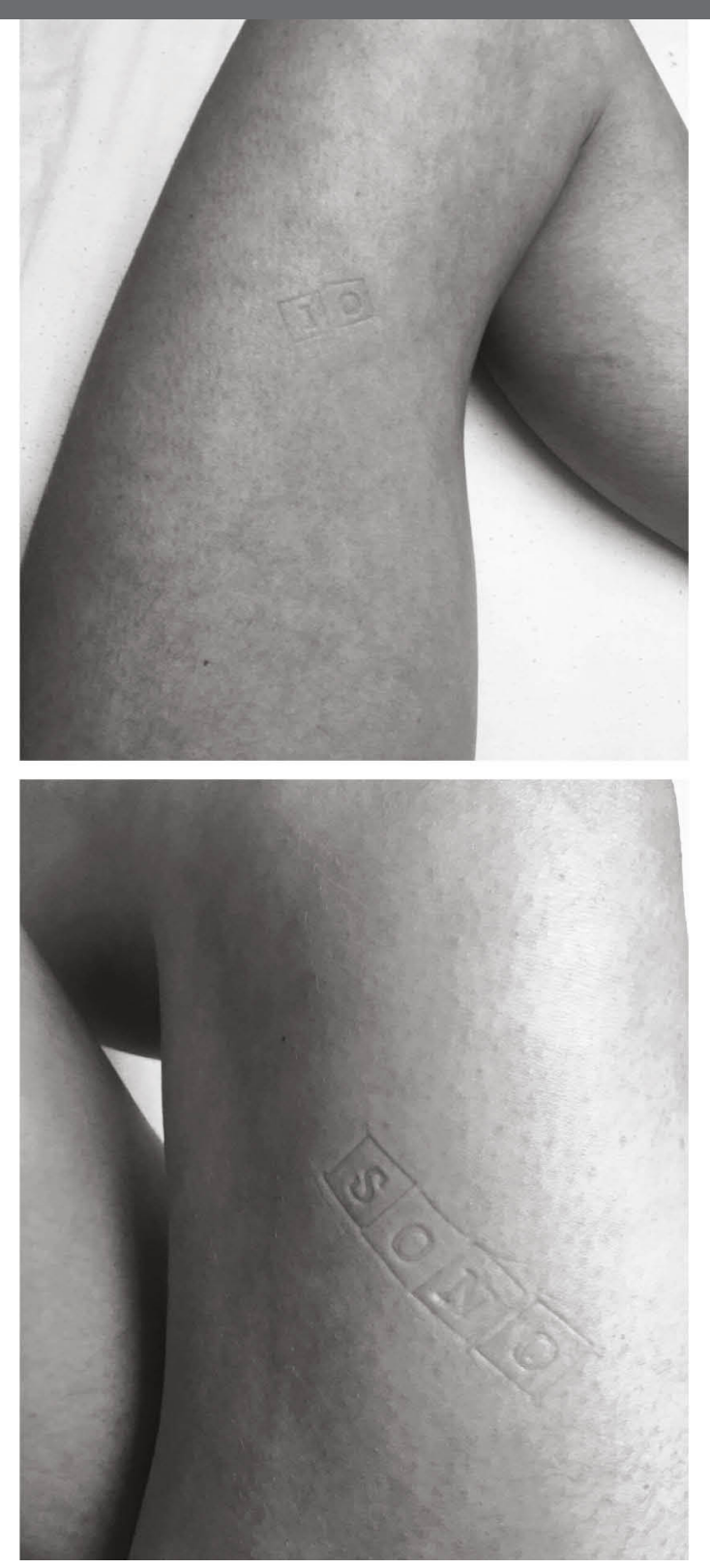


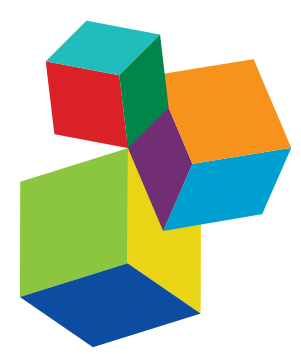

Frontiers Copyright Statement

C Copyright 2007-2018 Frontiers Media SA. All rights reserved.

All content included on this site, such as text, graphics, logos, button

icons, images, video/audio clips, downloads, data compilations and

software, is the property of or is

licensed to Frontiers Media SA

("Frontiers") or its licensees and/or subcontractors. The copyright in the text of individual articles is the property of their respective authors, subject to a license granted to Frontiers.

The compilation of articles constituting this e-book, wherever published, as well as the compilation of all other content on this site, is the exclusive property of Frontiers. For the

conditions for downloading and copying of e-books from Frontiers website, please see the Terms for Website Use. If purchasing Frontiers e-books from other websites or sources, the conditions of the website concerned apply.

Images and graphics not forming part of user-contributed materials may not be downloaded or copied without permission

Individual articles may be downloaded and reproduced in accordance with the principles of the CC-BY

licence subject to any copyright or other notices. They may not be re-sold as an e-book.

As author or other contributor you grant a CC-BY licence to others to reproduce your articles, including any graphics and third-party materials supplied by you, in accordance with the Conditions for Website Use and subject to any copyright notices which you include in connection with your articles and materials.

All copyright, and all rights therein, are protected by national and international copyright laws.

The above represents a summary only.

For the full conditions see the Conditions for Authors and the Conditions for Website Use.

ISSN 1664-8714

ISBN 978-2-88945-492-1

DOI 10.3389/978-2-88945-492-1

\section{About Frontiers}

Frontiers is more than just an open-access publisher of scholarly articles: it is a pioneering approach to the world of academia, radically improving the way scholarly research is managed. The grand vision of Frontiers is a world where all people have an equal opportunity to seek, share and generate knowledge. Frontiers provides immediate and permanent online open access to all its publications, but this alone is not enough to realize our grand goals.

\section{Frontiers Journal Series}

The Frontiers Journal Series is a multi-tier and interdisciplinary set of open-access, online journals, promising a paradigm shift from the current review, selection and dissemination processes in academic publishing. All Frontiers journals are driven by researchers for researchers; therefore, they constitute a service to the scholarly community. At the same time, the Frontiers Journal Series operates on a revolutionary invention, the tiered publishing system, initially addressing specific communities of scholars, and gradually climbing up to broader public understanding, thus serving the interests of the lay society, too.

\section{Dedication to Quality}

Each Frontiers article is a landmark of the highest quality, thanks to genuinely collaborative interactions between authors and review editors, who include some of the world's best academicians. Research must be certified by peers before entering a stream of knowledge that may eventually reach the public - and shape society; therefore, Frontiers only applies the most rigorous and unbiased reviews.

Frontiers revolutionizes research publishing by freely delivering the most outstanding research, evaluated with no bias from both the academic and social point of view. By applying the most advanced information technologies, Frontiers is catapulting scholarly publishing into a new generation.

\section{What are Frontiers Research Topics?}

Frontiers Research Topics are very popular trademarks of the Frontiers Journals Series: they are collections of at least ten articles, all centered on a particular subject. With their unique mix of varied contributions from Original Research to Review Articles, Frontiers Research Topics unify the most influential researchers, the latest key findings and historical advances in a hot research area! Find out more on how to host your own Frontiers Research Topic or contribute to one as an author by contacting the Frontiers Editorial Office: researchtopics@frontiersin.org 


\section{EMBODIED COGNITION OVER THE LIFESPAN AND IN APPLIED SETTINGS}

Topic Editors:

Annalisa Setti, University College Cork, Ireland

Anna M. Borghi, Sapienza Università di Roma, Italy

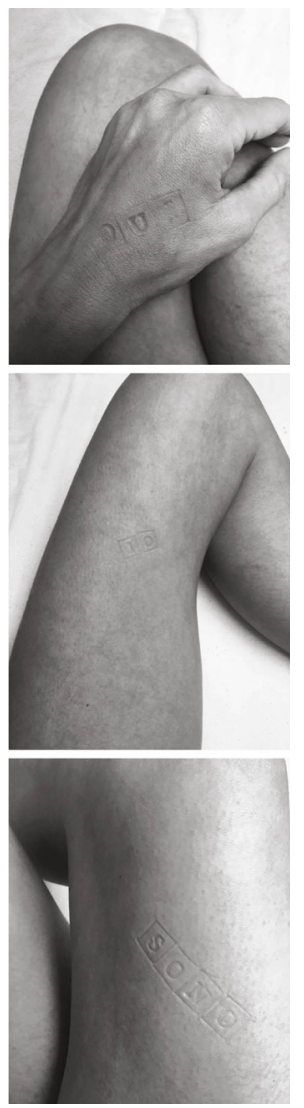

Image: "Io sono qui" (I am here) by Silvia Marchesini, courtesy of the author.

While Embodied Cognition has now been accepted as mainstream in Cognitive Science, the study of its potential contribution to understding child developemnt and ageing, as well as its potential applications, is still in its infancy. This collection of articles explores the contribution of Embodied Cognition to studying the lifespan and potential applied fields. The contributions are theoretical and empirical and offer an important framework for future research and its applications.

Citation: Setti, A., Borghi, A. M., eds. (2018). Embodied Cognition over the Lifespan and in Applied Settings. Lausanne: Frontiers Media. doi: 10.3389/978-2-88945-492-1 


\section{Table of Contents}

06 Editorial: Embodied Cognition Over the Lifespan: Theoretical Issues and Implications for Applied Settings

Annalisa Setti and Anna M. Borghi

\section{PERSPECTIVES}

10 Contribution of Embodiment to Solving the Riddle of Infantile Amnesia Arthur M. Glenberg and Justin Hayes

16 Are Older Adults Less Embodied? A Review of Age Effects Through the Lens of Embodied Cognition

Matthew C. Costello and Emily K. Bloesch

34 Down With Retirement: Implications of Embodied Cognition for Healthy Aging

Bernhard Hommel1 and Armin Kibele

\section{CHILDREN}

\subsection{EC AND LANGUAGE IN CHILDREN}

39 Pacifier Overuse and Conceptual Relations of Abstract and Emotional Concepts

Laura Barca, Claudia Mazzuca and Anna M. Borghi

58 What's on the Inside Counts: A Grounded Account of Concept Acquisition and Development

Serge Thill and Katherine E. Twomey

69 Development of Embodied Word Meanings: Sensorimotor Effects in Children's Lexical Processing

Michelle Inkster, Michele Wellsby, Ellen Lloyd and Penny M. Pexman

77 How Language Is Embodied in Bilinguals and Children With Specific Language Impairment

Ashley M. Adams

\subsection{CHILDREN, BODY, SENSORIMOTOR INFORMATION}

90 Embodied Action Improves Cognition in Children: Evidence from a Study Based on Piagetian Conservation Tasks

Mariana Lozada and Natalia Carro

97 No Interrelation of Motor Planning and Executive Functions Across Young Ages

Kathrin Wunsch, Roland Pfister, Anne Henning, Gisa Aschersleben and Matthias Weigelt

113 Augmenting Instructional Animations With a Body Analogy to Help Children Learn About Physical Systems

Wim T. J. L. Pouw, Tamara van Gog, Rolf A. Zwaan and Fred Paas

124 Stimulus-Response Compatibility Effect in the Near-Far Dimension: A Developmental Study

Aurélien Richez, Gerard Olivier and Yann Coello 
134 Cognitive Mechanisms Underlying Directional and Non-Directional Spatial-Numerical Associations Across the Lifespan

Manuel Ninaus, Korbinian Moeller, Liane Kaufmann, Martin H. Fischer,

Hans-Christoph Nuerk and Guilherme Wood

\subsection{THE ROLE OF ACTION AND TECHNOLOGY IN CHILDREN'S DEVELOPMENT}

147 Hey Teacher, Don't Leave Them Kids Alone: Action Is Better for Memory Than Reading

Mathieu Hainselin, Laurence Picard, Patrick Manolli, Sophie

Vankerkore-Candas and Béatrice Bourdin

156 Commentary: Mobile and Interactive Media Use by Young

Children: The Good, the Bad, and the Unknown

Cedric Galetzka

159 Commentary: Down with Retirement: Implications of Embodied Cognition for Healthy Aging

Alex A. Miklashevsky and Martin H. Fischer

\section{AGEING}

\subsection{LANGUAGE, MEMORY, EXECUTIVE FUNCTIONS}

161 Abstract Concepts and Aging: An Embodied and Grounded Perspective Anna M. Borghi and Annalisa Setti

165 A SEMantic and EPisodic Memory Test (SEMEP) Developed Within the Embodied Cognition Framework: Application to Normal Aging, Alzheimer's Disease and Semantic Dementia

Guillaume T. Vallet, Carol Hudon, Nathalie Bier, Joël Macoir, Rémy Versace and Martine Simard

177 Temporal Information Processing and its Relation to Executive Functions in Elderly Individuals

Kamila Nowak, Anna Dacewicz, Katarzyna Broczek,

Malgorzata Kupisz-Urbanska, Tadeusz Galkowski and Elzbieta Szelag

\subsection{ACTION, PERCEPTION AND COGNITION}

191 Sensorimotor-Conceptual Integration in Free Walking Enhances Divergent Thinking for Young and Older Adults

Chun-Yu Kuo and Yei-Yu Yeh

200 The Age Effects on the Cognitive Processes of Intention-Based and Stimulus-Based Actions: An ERP Study

Ya-Nan Niu, Xinyi Zhu and Juan Li

214 Age-Related Changes in Bimanual Instrument Playing With Rhythmic Cueing

Soo Ji Kim, Sung-Rae Cho and Ga Eul Yoo

\section{EMBODIED COGNITION IN YOUNG ADULTS}

\subsection{LANGUAGE AND EMBODIMENT}

225 The Common Element Effect of Abstract-to-Abstract Mapping in Language Processing

Xuqian Chen, Guixiang Wang and Yuchan Liang 
236 Is Accessing of Words Affected by Affective Valence Only? A Discrete Emotion View on the Emotional Congruency Effect

Xuqian Chen, Bo Liu and Shouwen Lin

\subsection{ACTION, BODY, MOTOR RESONANCE}

246 Social Threat and Motor Resonance: When a Menacing Outgroup Delays Motor Response

Roberta Capellini, Simona Sacchi, Paola Ricciardelli

and Rossana Actis-Grosso

257 Remember Hard But Think Softly: Metaphorical Effects of Hardness/Softness on Cognitive Functions

Jiushu Xie, Zhi Lu, Ruiming Wang and Zhenguang G. Cai

\section{INSTRUMENTS}

266 Virtual Reality as an Embodied Tool to Enhance Episodic Memory in Elderly

Claudia Repetto, Silvia Serino, Manuela Macedonia and Giuseppe Riva

270 Effects of Embodied Learning and Digital Platform on the Retention of Physics Content: Centripetal Force

Mina C. Johnson-Glenberg, Colleen Megowan-Romanowicz,

David A. Birchfield and Caroline Savio-Ramos 


\title{
Editorial: Embodied Cognition Over the Lifespan: Theoretical Issues and Implications for Applied Settings
}

\author{
Annalisa Setti ${ }^{1 *}$ and Anna M. Borghi ${ }^{2}$ \\ ${ }^{1}$ School of Applied Psychology, University College Cork, Cork, Ireland, ${ }^{2}$ Department of Dynamic and Clinical Psychology, \\ Sapienza Università di Roma, Rome, Italy
}

Keywords: emobodied cognition, aging and cognitive function, cognitive development, lifespan, virtual reality, action, abstract

\section{Editorial on the Research Topic}

Embodied Cognition Over the Lifespan: Theoretical Issues and Implications for Applied Settings

\section{INTRODUCTION}

The Special Topic on Embodied Cognition over the Lifespan and in Applied Settings aimed at gathering evidence on the role of EC in development, adulthood, and aging, and to shed light on the applied fields benefiting from this approach. The collection originating from it shows the variety and richness of studies falling under this umbrella. The collection is organized in five sections: the first outlines new perspectives in the potential applications of EC to cognitive development and healthy aging; the second, third, and fourth are dedicated to studies on childhood, older age, and young adults. Each section comprises work on action and language; the fifth section is dedicated to instruments and techniques to utilize within an EC framework, such as Virtual Reality (VR).

OPEN ACCESS

Edited and reviewed by: Lorenza S. Colzato, Leiden University, Netherlands

${ }^{*}$ Correspondence: Annalisa Setti a.setti@ucc.ie

Specialty section: This article was submitted to

Cognition,

a section of the journal

Frontiers in Psychology

Received: 25 February 2018 Accepted: 03 April 2018

Published: 20 April 2018

Citation:

Setti A and Borghi AM (2018) Editorial: Embodied Cognition Over the Lifespan: Theoretical Issues and Implications for Applied Settings.

Front. Psychol. 9:550. doi: 10.3389/fpsyg.2018.00550

\section{PERSPECTIVES}

This section collects proposals of new perspectives and directions of research on embodiment over the lifespan and their implications for social practices. Glenberg and Hayes propose a strongly embodied explanation of two phenomena characterizing childhood-infantile amnesia, consisting in the scarcity of autobiographical memories from early childhood, and childhood amnesia, the quick forgetting of situations until age of seven-and extend the same explanation to cognitive decline with aging. They ascribe a crucial role to the development of the hippocampus, the primary function of which is that of spatial navigation. Before locomotion, they propose that infants have difficulties in associating events with locations, because places are not strongly distinguished by spatial codes, and in memorizing similar events experienced in different places. When locomotion starts, place and grid cells of the hippocampus adapt to the environment and places become stable memory cues. The maturation of locomotion abilities further facilitates long-term episodic memory and lead to the end of infantile amnesia and of childhood amnesia. Within the same framework, the reduction in self-locomotion abilities is at least partially responsible of the cognitive decline with aging.

Costello and Bloesch propose that Embodied Cognition can enhance our understanding of the changes occurring in sensory processing, mental representations, and action perception with aging. They suggest that older adults may rely more on visual inputs than younger adults, possibly due to the degradation of their internal action models, therefore potentially becoming more disembodied. EC can also help understanding the diversity of the aging process across individuals, whereby 
physical and cognitive aging, particularly perceptual and motor skills interplay with cognitive abilities. Importantly, the role the environment emerges as facilitator or barrier to healthy aging.

Hommel and Kibele outline the principles of an embodiedcognition approach to aging, according to which abilities are not a static endowment but rather emerge from fruitful and active exchanges with the environment. The progressive reduction of resources could thus be intended as a modification in the relationship with the environment, that requires reframing. They contend that healthy aging relies on the systematic use of control resources; these resources, even if present, are often not fully exploited. They discuss three phenomena characterizing aging, i.e., the reduction of dopaminergic supply, loneliness, and the loss of body strength, that might lead to the loss and lack of maintenance of cognitive control, leading to a vicious spiral of demotivation and further lack of strategies to contrast cognitive decline. While further research on how use of cognitive control resources affects their maintenance, social practices should promote healthy aging supporting self-empowerment strategies of individuals and reconsidering current policies as retirements laws.

\section{CHILDREN}

\section{EC and Language in Children}

In this section, contributions investigate how language acquisition is influenced by embodied and grounded aspects. Barca et al. show that the extensive use of the pacifier, i.e., of a device actively involving the mouth motor system, influences the acquisition of abstract and emotional concepts, leading to a different pattern of conceptual relations in children who have used it (beyond age 3). The authors interpret their work in the framework of the embodied Words As social Tools (WAT) view (Borghi et al., 2017), according to which abstract concepts activate the linguistic system and the social dimension more than concrete concepts.

Thill and Twomey explore with a computational study the factors affecting Age of Acquisition (AoA) of words; they show that factors traditionally considered in literature such as concreteness, imageability and frequency do not account for the variability of AoA data. AoA can be explained, however, when recurring to "groundability" in sensorimotor and interoceptive experience, i.e., considering concepts as richly embodied.

In the same vein, Inkster et al. study the influence of words' rated body-object interaction (BOI) and imageability in 6-7 years-old and in adults. For the first time they obtain BOI ratings from parents of children (child-BOI), and compare the influence on auditory naming task of high and low imageability words and of high and low child-BOI words. Results showed that children's auditory naming times are influenced by both imageability and child-BOI, testifying the influence of embodiment and groundedness on conceptual representation even in very young children.

Adams provides substantial evidence from the literature that learning a second language should also be grounded in action. In bilingual individuals, both the first and the second language display compatibility effects between semantic content and action. Importantly, children with Specific Language Impairment are known to present motor deficits, therefore they can potentially benefit from the involvement of the body in language learning.

\section{Children, Body, Sensori-Motor Information}

In this section the majority of contributions focuses on the role played by action and movement for cognitive activity in children.

Lozada and Carro used a classical task, the Piagetian conservation task, in 6-7 years-old. They clearly demonstrated the effects of action on cognition: actively manipulating the materials led children to comprehend conservation phenomena better than when they merely observed an adult's demonstration.

In a different perspective, Wunsch et al. tested children aged 3-10 and adults in three motor tasks (e.g., the bar-transport task) and three cognitive tasks on executive functions (e.g., the Tower-of-Hanoi task). They found a general improvement of the performance with age, but also found that, when age was controlled, there was no relationship between motor and cognitive tasks. These results cast doubts on the assumption of a strict interrelationship between action and cognition proposed by embodied and grounded cognition approaches.

Whether the human body and actions can be utilized to support cognitive tasks has also been explored by Pouw et al. The authors looked at the role of utilizing body analogy in explaining physical systems to school age children. They found that instructional animations based on human body were particularly useful for children with lower maths skills for a specific task, while transfer of learning did not benefit from it.

One final study on children focuses on the development of the ability to respond to stimuli in the environment with the appropriate grasp. Richez et al. ask 8-11 years old children to respond to the color of a stimulus presented at different distances from the body. Only over 10 years of age, children responded faster in grasping a proximal switch when they were shown a proximal stimulus, and a distal switch when they were presented with a distal stimulus. The authors argue that this is in line with the view according to which, between 8 and 10 years, children prepare their body to more efficiently act in the environment.

Ninaus et al. investigated the Spatial-Numerical Associations of Response Codes across the lifespan, showing that it increases with age, reflecting the increased experience with the association between numbers magnitude and spatial physical space, which is culturally determined. On the contrary, the interference of flanking numbers on a line bisection task forms a $U$ shape across the lifespan, suggesting that it is grounded in inhibition abilities, which are weaker in children and older adults.

These mixed results, with some positive and some negative findings, highlight that the body plays a complex role in cognitive development, that varies depending on the age, the kind of task and the complexity of the required cognitive skills.

\section{The Role of Action and Technology in Children's Development}

Hainselin et al. test the enactment effect (EE), consisting in better memory for performed actions than for verbally encoded action sentences, in 6-10 years-old children. Overall, action conditions 
(direct action and action observation) were better than nonaction conditions (verbal and listening tasks). Even if younger children memory performance was worse than that of older children, this difference disappeared during learning through action. Their results suggest that episodic memory might not underlie EE in young children, and highlights the importance of action for learning and memory.

Galetzka offers a commentary of a paper of Radesky et al. (2015) according to which technological advances are so fast that research on their impact on children's cognitive processes cannot keep up with them. The author reviews controversial results of research on the effect of interactive technologies as tablets and smartphones. He points out that, while initial results suggested that interacting with tablets was an impoverished experience compared to interacting with objects, when children actively interact with them through touchscreens the typical transfer difficulties characterizing traditional media as television is absent. Hence the advantages of real-life over technology-based learning are highly context-dependent.

Miklashevsky and Fischer adopt the approach outlined by Hommel and Kibele, according to which cognition changes in the dynamical interchange with the environment, to investigate how development changes in the light of the pervasive digitalization and of the extensive use of smartphones and tablets in children. They argue that using oral instead of typed language will likely link linguistic representation to the orofacial rather than to the manual activity, the reduction of hand motor activity in interacting with objects will likely lead to changes in spatial representation and in abstract conceptualization, for example replacing manual with visual metaphors (see the point vs., grasp the idea), and the reduction of finger counting will render individual numbers less accessible; finally, the absence of direct social interaction will reduce the pragmatic value of language, rendering it less linked to action.

\section{AGING}

When looking at cognitive development the importance of an embodied perspective emerges clearly; however very few studies have attempted to implement it in understanding agerelated changes on the other side of the lifespan, in older people.

\section{Language, Memory, Executive Functions}

Borghi and Setti focus on how older people represent abstract concepts and words. They propose an account of conceptual decline in elderly derived from the embodied WAT view (Borghi et al., 2017). The WAT view predicts that in older the decline of abstract concepts should be less marked than that of concrete ones, because the first rely more heavily on language. This contrasts with the view according to which abstract concepts should decline more, because abstract words are typically acquired earlier (AoA) and less frequent than abstract ones. The authors critically analyze current evidence, showing that either the well know advantage in processing of concrete over abstract words (concreteness effect), is reduced with age, or that linguistic-based compensatory strategies are activated. This evidence gives preliminary support to the WAT view.

Vallet et al. start from an embodied view, according to which episodic and semantic memory are grounded in sensorimotor system. They develop and illustrate a memory test, the SEMEP (semantic-episodic) memory test. Results of the test show very distinct pattern of performance across older groups. All older individuals, with or without cognitive deficits, committed more confusion errors than young adults, likely due to a degradation in perception with age. Alzheimer patients showed severe impairments in episodic memory and committed more intrusion errors than the other groups, likely due to difficulties in multimodal integration; finally, semantic dementia patients showed the most severe deficits of semantic memory.

Temporal information processing, the temporal accuracy with which we perceive external stimuli has been linked with efficient cognitive performance; Nowak et al. show the link between perception, assessed by temporal information processing, and executive functions in older adults. They find a correlation between temporal information processing and performance in the Tower of Hanoi.

\section{Action, Perception and Cognition}

As highlighted by both Hommel and Kibele and Costello and Bloesch it is important to understand the role of embodiment and action in aging, potentially shedding a new light on cognitive deficits and cognitive resources in aging.

Creativity is a well-researched topic in young adults, however much less research is devoted to creativity in older. Kuo and Yeh demonstrate that walking enhances divergent thinking. Specifically, older adults can reach the same performance level in a divergent thinking task, when they are free to choose their own walking path as opposed to walking on a fixed path.

Niu et al. in an ERP study, show that a general decline on action preparation occurs with aging, and an age-related impairment on the effect-action retrieval of intention-based actions, possibly due to decline in associative memory.

Kim et al. look at bimanual instrument playing (electronic drum), requiring coordination, in young adults, as well as healthy and Mild Cognitive Impairment older adults. They show that the ability to synchronize with an external cue correlates with cognitive skills; this indicates the close link between action and cognition in aging.

\section{EMBODIED COGNITION IN YOUNG ADULTS}

\section{Language and Embodiment}

According to the conceptual metaphor theory, mappings are formed between abstract and concrete concepts. Chen et al. test the hypothesis that abstract-abstract mappings can be formed focusing on the concept of time and abstract action. Results of two lexical decision tasks revealed robust priming effects when a target verb and its prime (length-changing line or sound beep) lasted the same time (time consistent condition). 
The authors propose that mappings between concepts are influenced by common elements, either linguistic or embodied, that are differently recruited depending on the conceptual difficulty.

Chen et al. look at the role of emotions in words processing. In addition to replicating the effect of priming for words of the same emotional valence, they show that words referring to life-events also activate specific emotions, however it happens at a later stage, as shown by the longer Stimulus Onset Asynchrony compared with priming obtained when valence, as opposed to specific emotions, is manipulated.

\section{Action, Body, Motor Resonance}

Capellini et al. investigate an exquisitely embodied phenomenon, that of motor resonance, i.e., the activation of a simulation while observing the actions of others. Italian participants were presented with videos of an Italian (in-group) or Arabian (outgroup) individuals with a neutral (box of juice) vs. a threatening object (gun) and measured mouse tracker responses. When a threatening object was present, responses were delayed with outgroup members, revealing a higher level of motor resonance with the in-group and the activation of threat-related stereotypes induced by the context.

Xie et al. investigate a debated topic in the embodied literature: metaphorical associations. In two experiments, they manipulated the kind of seat where participants were sitting and recorded performance in a memory task and in a creativity task. The soft seat was associated with better performance in the creativity task, while the hard seat was associated with better performance in the memory task.

\section{REFERENCES}

Borghi, A. M., Binkofski, F., Castelfranchi, C., Cimatti, F., Scorolli, C., and Tummolini, L. (2017). The challenge of abstract concepts. Psychol. Bull. 143, 263-292. doi: 10.1037/bul00 00089

Radesky, J. S., Schumacher, J., and Zuckerman, B. (2015). Mobile and interactive media use by young children: the good, the bad, and the unknown. Pediatrics 135, 1-3. doi: 10.1542/peds.20142251

\section{INSTRUMENTS}

Repetto et al. propose to use VR to improve memory function in elderly. Glenberg and Hayes highlighted the link between locomotion and episodic memory, by means of the alignment of place cells, encoding location, and grid cells updating the viewpoint when self-motion occurs. VR allows the individual to experience a sense of moving in the environment with much limited real movement. Several studies show that episodic memory can be enhanced by using VR exploration, therefore, the authors propose that VR should be exploited to support episodic memory in older adults, who have often reduced mobility.

Johnson-Glenberg et al. are also interested in embodiment through technology. Specifically, they assess the efficacy of different levels of immersiveness and embodiment in supporting learning in college students. They assess both declarative knowledge and generative knowledge immediately after the lesson and at 1 week follow up and found that there was a significant difference after a week, with the more embodied class benefitting more the generative knowledge tests.

\section{CONCLUSION}

The articles collected in this Special Topic trace novel avenues to study Embodied Cognition and its contribution to identifying strengths and weaknesses across the lifespan, and techniques to support the former and prevent the latter.

\section{AUTHOR CONTRIBUTIONS}

All authors listed have made a substantial, direct and intellectual contribution to the work, and approved it for publication.

Conflict of Interest Statement: The authors declare that the research was conducted in the absence of any commercial or financial relationships that could be construed as a potential conflict of interest.

Copyright (c) 2018 Setti and Borghi. This is an open-access article distributed under the terms of the Creative Commons Attribution License (CC BY). The use, distribution or reproduction in other forums is permitted, provided the original author(s) and the copyright owner are credited and that the original publication in this journal is cited, in accordance with accepted academic practice. No use, distribution or reproduction is permitted which does not comply with these terms. 


\section{OPEN ACCESS}

Edited by: Anna M. Borghi,

University of Bologna and Institute of Cognitive Sciences and Technologies, Italy

Reviewed by:

Sascha Topolinski, University of Cologne, Germany Malte Schilling, International Computer Science Institute, USA

*Correspondence: Arthur M. Glenberg glenberg@asu.edu

Specialty section: This article was submitted to Cognition,

a section of the journal Frontiers in Psychology

Received: 21 November 2015 Accepted: 05 January 2016 Published: 25 January 2016

Citation: Glenberg AM and Hayes J (2016) Contribution of Embodiment to Solving the Riddle of Infantile Amnesia. Front. Psychol. 7:10. doi: 10.3389/fpsyg.2016.00010

\section{Contribution of Embodiment to Solving the Riddle of Infantile Amnesia}

\author{
Arthur M. Glenberg ${ }^{1,2 *}$ and Justin Hayes ${ }^{3}$ \\ ${ }^{1}$ Department of Psychology, Arizona State University, Tempe, AZ, USA, ${ }^{2}$ Department of Psychology, University of \\ Wisconsin-Madison, Madison, WI, USA, ${ }^{3}$ Simpson College, Indianola, IA, USA
}

At least since the late nineteenth century, researchers have sought an explanation for infantile amnesia (IA)-the lack of autobiographical memories dating from early childhood-and childhood amnesia (CA), faster forgetting of events up until the age of about seven. Evidence suggests that IA occurs across altricial species, and a number of studies using animal models have converged on the hypothesis that maturation of the hippocampus is an important factor. But why does the hippocampus mature at one time and not another, and how does that maturation relate to memory? Our hypothesis is rooted in theories of embodied cognition, and it provides an explanation both for hippocampal development and the end of IA. Specifically, the onset of locomotion prompts the alignment of hippocampal place cells and grid cells to the environment, which in turn facilitates the ontogeny of long-term episodic memory and the end of IA. That is, because the animal can now reliably discriminate locations, location becomes a stable cue for memories. Furthermore, as the mode of human locomotion shifts from crawling to walking, there is an additional shift in the alignment of the hippocampus that marks the beginning of adult-like episodic memory and the end of CA. Finally, given a reduction in self-locomotion and exploration with aging, the hypothesis suggests a partial explanation for cognitive decline with aging.

Keywords: infantile amnesia, childhood amnesia, aging, memory, locomotion, embodiment

Infantile amnesia (IA) is the well-documented phenomenon describing how most people (including children) explicitly recall little or nothing from the first few years of life. It is not that little is learned. In fact the infant is learning a tremendous amount about bodily control (Adolph, 1997), language (Bruderer et al., 2015), goals (Sommerville et al., 2005), categories (Smith, 2005), causality (Rakison and Krogh, 2010), and more. But there is a curious lack of episodic, autobiographical memory. Even in toddlers and young children up to the age of about seven, fewer episodic memories can be retrieved than would be predicted on the basis of adult rates of forgetting (Bauer, 2015). That is, in addition to IA, there is childhood amnesia (CA; e.g., Newcombe et al., 2011) when childhood experiences are forgotten more quickly than would be predicted based on adult-like memory. We will propose a developmental hypothesis that explains IA and CA using similar mechanisms.

There is no lack of theories to account for IA and CA. Bauer (2015) reviews these theories and explicates her Complementary Processes theory. We take that theory as a starting point, but add what we think is a crucial component based on embodiment, namely, the onset of locomotion. We will argue that it is the onset of locomotion, first crawling and then walking, that provides the conditions for hippocampal place cell and grid cell 
development, and that in turn allows for long-term storage and retrieval of autobiographical memories.

\section{BAUER'S COMPLEMENTARY PROCESSES THEORY}

The first of the complementary processes (or perhaps better, complementary explanations) is that infants lack some of the abilities needed to form episodic memories. For example, Newcombe (e.g., Balcomb et al., 2011; Newcombe et al., 2014) propose that infants cannot bind elements of representations (e.g., time, place, actors, etc.) into events. Howe and Courage (1997) propose that infants lack a self-concept, and hence cannot relate memories to the self. Nelson and Fivush (2004) elaborate on this idea by including, for example, development of processes that control conversation and narrative structure, as necessary for the full expression of autobiographical memory. Whereas these ideas all have some support, we do not view the Howe and Courage and Nelson and Fivush accounts as central to IA and CA for the following reason: not just humans, but all altricial species show a form of IA (Arnold and Spear, 1997). On the assumption that IA across species is not accidental, we need an explanation that does not focus on human-centric concepts such as language and the self.

The second complementary explanation in Bauer's theory is that even the episodic memories that infants and toddlers manage to form are exceptionally vulnerable to forgetting during early years. Bauer, and others, attribute changes in vulnerability to forgetting to maturation of the hippocampus. The hippocampus is a limbic structure located deep within the temporal lobe. It plays a crucial role in binding the spatial and temporal characteristics of long-term episodic memories (e.g., Moscovitch et al., 2006) by coding (or binding) environmental features as an organism moves through time and space. There is little doubt that the hippocampus plays a central role in episodic memory, and there is little doubt that it changes over the course of development. But why does the hippocampus mature at one time and not another, and how does that maturation relate to memory? We propose an embodied answer to these questions.

\section{CONTRIBUTION OF EMBODIMENT}

\section{The Hippocampus and Memory}

The primary purpose of the hippocampus seems to be spatial navigation. Place cells code particular locations (e.g., O'Keefe and Nadel, 1978); grid cells code spatial relations amongst those locations (e.g., Hafting et al., 2005; Castro and Aguiar, 2014); and head direction cells code the current direction of movement (e.g., Taube, 1998). Reciprocal connections between the hippocampus and sensorimotor and emotional cortices, along with Hebbian learning, then produce the ingredients for episodic memory. That is, activity in visual and motor areas (for example) are bound together via the hippocampus which codes the location of the event, resulting in a specific memory of an event at a specific time and place. As the animal changes location, other place and grid cells can be used to bind new memories that occur while at the new location. Thus, the place and grid cells (a) bind together components of events, (b) protect similar events occurring in different locations from catastrophic interference (because of the association with different places), and (c) provide a locationbased retrieval cue, much as with the mnemonic method of loci.

However, consider the following: Why would an animal that cannot engage in self-locomotion need to track location in the environment? The immature altricial animal is completely dependent on caretakers for food, warmth, and transportation. The animal does not explore, and hence there is no need to track novel locations. The animal does not leave the caretaker, so there is no need to track the location of the home environment. Even if the animal were separated from its caretaker, because it does not yet self-locomote, it could not get back home on its own. Thus, there is no need for evolution to have engineered an operating location-tracking mechanism for the animal that cannot self-locomote.

\section{Tuning the Hippocampus to the Environment}

Furthermore, consider how location-tracking mechanisms, such as place cells and grid cells, might become tuned to their environment. One hypothesis is that the tuning comes about through the consistent correlation amongst several cues including optic flow, head direction, and proprioception from self-generated movements. For example, optic flow correlated with proprioception (a) defines for the animal how speed of locomotion translates into change of location, and (b) that correlation comes to represent what is expected of a stable world (Campos et al., 1992; Dahl et al., 2013). To help understand these claims, consider what happens when optic flow and proprioception are decoupled. One way to do that is by walking on a treadmill. While on the treadmill, the brain relearns the correlation so that now proprioception is paired with little flow. One consequence is that when stepping off the treadmill there is a brief period of disorientation during which the visual world appears to move too fast when one begins to walk (Pelah and Barlow, 1996). A second example is when sitting in a car at a red light and the car next to you starts to move, thus generating peripheral optic flow. A typical response is to slam on the brake because the peripheral optic flow is interpreted as self-movement.

Consider now the pre-locomotor infant who is carried from location to location. Because the infant is not constrained to keep her head pointed in the direction of travel, optic flow, head direction, and proprioception are for the most part uncorrelated. Thus, there can be no tuning of the place and grid cells to the local environment. Again, a commonplace experience may help to elucidate the idea. Imagine two people navigating to a new location, for example a driver and a passenger (or two tourists walking together in a new city, where one has a map and the other follows). In this situation, the driver is forced to keep her gaze focused in front, creating optic flow correlated with speed of locomotion, whereas the passenger engages in conversation (while looking at the driver), or looks around at the sights. Thus, for the passenger, there is an inconsistent correlation between 
optic flow, direction of movement, and speed of locomotion (although effects of active versus passive control must also be considered, as in Bakdash et al., 2008). The result is that the driver learns the route, but the passenger does not.

In summary, the pre-locomotor infant does not have the opportunity to tune his hippocampal place and grid cells to the environment. Consequently, the system remains immature and an unreliable contributor to memory. That is, it is difficult for the infant to associate events with a particular place because places are not strongly distinguished by spatial codes. Furthermore, it is difficult to keep track of separate but similar events experienced in different locations (because locations are not strongly differentiated), and this lack of differentiation results in mutual interference and forgetting of the similar events. Note that this hypothesis regarding the pre-locomotor infant corresponds to the first of Bauer's complementary explanations: the infant is unable to form stable episodic memories. However, rather than suggesting a cause particular to humans, such as a poorly developed conception of the self or poor language skills, we offer a mechanism that might be found in every altricial species, namely, a lack of opportunity to tune place and grid cells to the environment, and hence a reduced capability to code the locations of experienced events.

Now consider the situation as the infant begins to crawl. She must keep her head pointed toward her goal, and that generates correlations between optic flow, head direction, and proprioception. We propose that these stable correlations provide the opportunities required to establish a stable system of grid cells linking places. Furthermore, with the advent of crawling, the infant can explore components of her environment (e.g., where the grassy area is relative to the tree; where the kitchen is relative to the TV) and differentiate them in terms of their spatial locations coded through linked grid and place cells. This system then provides the substrate for coding memories of events in distinct locations and keeping track of separate but similar events experienced in different locations. With the establishment of spatial location coding, long-term episodic memory becomes possible. This component of our hypothesis corresponds to the second of Bauer's complementary explanations: vulnerability to forgetting declines over development.

\section{Re-tuning the Hippocampus}

But why is there also CA, that is, continued rapid forgetting even of events experienced by the self-locomoting infant and toddler? One possibility is that it just takes a lot of experience in crawling and exploration to establish a hippocampus with sufficient organization of locations to work with adult-like efficiency. Consider, however, the following: at some point, the infant transitions from crawling to walking. With that transition, there is a new pattern of correlations between head direction, optic flow, and proprioception. The new pattern comes about because the angle between the head and ground (which contributes to optic flow) is changed, the pattern of muscles that produce locomotion and proprioception changes, and the relation between proprioception and optic flow (e.g., speed of movement and speed of flow) changes (Adolph and TamisLeMonda, 2014). This changing pattern of information forces a new organization of grid cells coding the relations between selfmovement and change in location. With that re-organization, the hard-won episodic memories encoded during the crawling period become less accessible, that is, they are forgotten at a rate faster than that of an adult. Furthermore, it is not until walking and associated exploration are well-established that an adultlike hippocampal system can be formed, binding the elements of longer-lasting episodic memories.

Components of this hypothesis are not without precedent; Rovee-Collier (1996) anticipated at least two parts of the hypothesis. First, she noted that what infants learn matches their needs for their current ecological niche. For example, newborns learn appetitive responses, but not defensive ones. She wrote, "... newborns are motorically incapable of escaping or avoiding aversive events signaled by cues in the environment. . .Instead, the newborn's first line of defense is to cry... .Not surprisingly, most of the successful examples of aversive conditioning in infants have been obtained after the age of independent locomotion..." (p. 388). Similarly, we propose that infants are unlikely to develop functioning place and grid cells before the onset of "independent locomotion." Second, she confirms both experimentally and theoretically the relation between selflocomotion and learning spatial relations, “...once infants are able to self-locomote...their definition of context seems to change. Before this time, they know what happens in what place, but they do not know how to get there. Once they can get there without being carried, however, they acquire spatial relations along with their new navigational skills and begin to construct a cognitive map...This is particularly important because once these different places become related to each other, the memories of events that transpired in those places also become associated with each other" (p. 393, emphasis in the original). Nonetheless, Rovee-Collier offers a different explanation for IA and CA.

\section{EVIDENCE SPECIFIC TO THE EMBODIED HYPOTHESIS}

The embodied hypothesis is broadly consistent with the basic facts of IA and CA. Furthermore, by noting the developmental transitions between crawling and walking, the hypothesis provides a simpler explanation of continued weak episodic memory than many other hypotheses. In addition, there are reports that provide evidence consistent with specifics of the hypothesis. What follows is not an exhaustive review of the literature, but a sampling to illustrate some of the highlights.

Herbert et al. (2007) found that 9-months-old infants who had begun crawling were able to successfully perform a deferred imitation task when asked to perform the task in a novel environment with a similar but unique object. Same aged, but immobile infants, were not as successful. As Rovee-Collier (1996) suggests, the mobile infants are better able to associate places with one another and thus retrieve events learned in one environment while located in a different environment.

The literature also documents important changes in cognition that accompany the acquisition of self-locomotion (many are reviewed in Campos et al., 2000). For example, Campos et al. 
(1992) demonstrate how fear of the visual cliff is dependent on self-locomotion. They explain that it is only after the onset of self-locomotion that the relation between optic flow and proprioception comes to define a stable world. Then, when that relation is disrupted by the unusual optic flow near a visual cliff, the infant recognizes a new (and potentially dangerous) type of environment.

Particularly pertinent to our hypothesis are the findings of (Adolph, 1997; Adolph and Tamis-LeMonda, 2014). She documents changes in cognition associated with the transition from crawling to walking. For example, after many weeks of crawling, infants are able to discriminate between slopes that are safe for crawling and those that are not safe. When the infant begins to walk, however, that discrimination is lost, and the toddler must again learn to discriminate between these slopes. Adolph's discussion of this result (e.g., pp. 66-68; 125126 ) is consistent with changes in optic flow and proprioception associated with the transition from crawling to walking.

Bauer et al. (2012) documents how 4-years-old children (compared to 6- and 8-years-old) are not much better than chance in remembering the locations of events. Interestingly, Riggins et al. (2015) uncovered an association between episodic memory and hippocampal volume for 6-years-old, but not for 4-years-old.

Perhaps most important for our hypothesis, the relation between grid cell firing and self-locomotion is now clearly established (at least for rats). Winter et al. (2015) had rats explore an environment while recording from hippocampal grid cells. In the active condition, the rats explored the environment through self-locomotion. In the passive condition, the animal was pulled through the environment in a clear cart. There were several important findings. First, grid cell firing was closely associated with environmental location of the animal in the active condition, but was severely degraded or missing in the passive condition. That is, much like passengers in a car, the rats in the passive condition were not tracking how they got from one location to the next. Second, the firing of head direction cells (that provide the brain with a heading) was not disrupted in the passive condition, but third, the correlation between oscillations in the theta band $(6-10 \mathrm{~Hz})$ and speed of locomotion was disrupted. The second and third findings help to explain the first finding of disorganized grid cell firing in the passive condition. Namely, while in the cart, the animal's head cues the direction of travel. However, the speed of travel, normally associated with movement of and proprioception from the animal's own legs, translated into theta rhythm, is disrupted. Thus, although there is firing in the theta range, it is dissociated from speed of movement (now provided by the cart instead of the animal's legs), and hence there is a disruption in signaling of when grid cell boundaries are crossed.

\section{HOW CAN THIS HYPOTHESIS BE TESTED?}

Some predictions can be tested with animal models. For example, weanling rats can be allowed to explore an open field environment that does or does not contain differentiating features. The animals exposed to the differentiated environment should form a more differentiated grid cell system. Then, episodic memory can be assessed by placing food rewards in specific locations in the radial arm maze where the animal can eat the reward. We predict that rats with differentiated locomotive experience will be less likely to return to the empty locations compared to rats reared in an undifferentiated environment.

Other hypotheses can be tested with human infants (several of these ideas are identical to those in Campos et al., 1992). For example, there should be a correlation between age of onset of crawling (and walking) and performance in episodic memory tasks. A correlation may also be found across cultures that differ in child-rearing practices that encourage or discourage self-locomotion. Similarly, children who have been injured so that self-locomotion is delayed should show a delay in onset of episodic memory.

Experimental tests are also possible. Consider the following test of a counterintuitive prediction (see Table 1). In Phase 1 of the experiment, infants who are still crawling are taught a task (Task 1). Phase 2 occurs when the infant takes her first steps, but while she is still predominately a crawler. In Phase 2, the infant is tested on Task 1 and learns a new task, Task 2. Phase 3 is scheduled so that the interval between Phases 2 and 3 is the same as the interval between Phases 1 and 2. In Phase 3 , the toddler is tested on Task 2 . We predict that the young infant will do relatively well on the test of Task 1 (at Phase 2) because she learned it while having a "crawling" hippocampus and was tested while having a "crawling" hippocampus. However, when the older child is tested on Task 2 (Phase 3), she should do less well because Task 2 was learned while the child had a "crawling" hippocampus, but is tested while she has a "walking" hippocampus.

\section{DISCUSSION}

Although we developed this hypothesis to address IA and CA, it also suggests a novel explanation for some forms of cognitive decline with aging. That is, as the body ages, there may be less self-locomotion and less active exploration. In this case, hippocampal place and grid cell firing may become dissociated from the environment, with a resultant decrease in episodic memory. Prevention of detuning may result from following the common advice to remain active while aging. Furthermore, this extension of the hypothesis suggests an explanation for results reported by Anderson-Hanley et al. (2012). They tested

\section{TABLE 1 | An experimental test of the hypothesis.}

\begin{tabular}{|c|c|c|c|}
\hline & \multicolumn{3}{|c|}{ Phase of experiment } \\
\hline & $\begin{array}{l}\text { (1) Child is } \\
\text { crawling }\end{array}$ & $\begin{array}{l}\text { (2) First } \\
\text { steps }\end{array}$ & $\begin{array}{l}\text { (3) Child is } \\
\text { walking }\end{array}$ \\
\hline $\begin{array}{l}\text { Hypothetical mode of tuning } \\
\text { hippocampus to environment }\end{array}$ & Crawling & $\begin{array}{l}\text { Primarily } \\
\text { crawling }\end{array}$ & Walking \\
\hline Task learned & Task 1 & Task 2 & \\
\hline Task tested & & Task 1 & Task 2 \\
\hline
\end{tabular}


a sample of older adults (average age of 78.8 years) randomly assigned to ride a stationary bicycle (for 3 months) or the same bicycle combined with a virtual reality tour simulating movement through the environment. Riding the stationary bicycle with the virtual tour provided significantly more buffering against mild cognitive impairment than riding the stationary bicycle alone. Furthermore, this buffering effect was associated with a greater increase in BDNF, brain-derived neurotrophic growth factor.

In summary, we have developed a novel explanation for IA and CA. In brief, when an animal cannot self-locomote, there is no opportunity for hippocampal place and grid cells to be tuned to the environment. Consequently, episodic memories (which require memory for place) are difficult to establish. The onset of self-locomotion provides the opportunity for tuning place and grid cells to the environment, and thus the onset of episodic memory. In humans, however, the change in mode of locomotion from crawling to walking rewires the hippocampal cells so that memories created in the crawling phase are less available, thus providing at least a partial explanation for IA and a more extended CA. As the animal continues to explore wider and more diverse areas, a greater number of hippocampal cells can become tuned to the environment, thus establishing a mature basis for coding the locations of remembered events. Unlike some other explanations of IA, this

\section{REFERENCES}

Adolph, K. E. (1997). Learning in the development of infant locomotion. Monogr. Soc. Res. Child Dev. 62, I-VI, 1-158. doi: 10.2307/1166199

Adolph, K. E., and Tamis-LeMonda, C. S. (2014). The costs and benefits of development: the transition from crawling to walking. Child Dev. Perspect. 8, 187-192. doi: 10.1111/cdep.12085

Anderson-Hanley, C., Arciero, P. J., Brickman, A. M., Nimon, J. P., Okuma, N., Westen, S. C., et al. (2012). Exergaming and older adult cognition. Am. J. Prev. Med. 42, 109-119. doi: 10.1016/j.amepre.2011.10.016

Arnold, H. M., and Spear, N. E. (1997). Infantile amnesia: using animal models to understand forgetting. Adv. Study Behav. 26, 251-284. doi: 10.1016/S00653454(08)60381-2

Bakdash, J. Z., Linkenauger, S. A., and Proffitt, D. R. (2008). Comparing decisionmaking vs. control for learning a virtual environment: Backseat drivers learn where they are going. Proc. Hum. Fact. Ergon. Soc. Annu. Meet. 52, 2117-2121. doi: $10.1177 / 154193120805202707$

Balcomb, F., Newcombe, N. S., and Ferrara, K. (2011). Finding where and saying where: developmental relationships between place learning and language in the first year. J. Cogn. Dev. 12, 315-331. doi: 10.1080/15248372.2010.544692

Bauer, P. J. (2015). A complementary processes account of the development of childhood amnesia and a personal past. Psychol. Rev. 122, 204-231. doi: $10.1037 / \mathrm{a} 0038939$

Bauer, P. J., Doydum, A. O., Pathman, T., Larkina, M., Güler, O. E., and Burch, M. (2012). It's all about location, location, location: children's memory for the "where" of personally experienced events. J. Exp. Child Psychol. 113, 510-522. doi: 10.1016/j.jecp.2012.06.007

Bruderer, A. G., Danielson, D. K., Kandhadai, P., and Werker, J. F. (2015). Sensorimotor influences on speech perception in infancy. Proc. Natl. Acad. Sci. U.S.A. 112, 13531-13536. doi: 10.1073/pnas.1508631112

Campos, J. J., Anderson, D. I., Barbu-Roth, M. A., Hubbard, E. M., Hertenstein, M. J., and Witherington, D. (2000). Travel broadens the mind. Infancy 1, 149-219. doi: 10.1207/S15327078IN0102_1

Campos, J. J., Bertenthal, B. I., and Kermoian, R. (1992). Early experience and emotional development: the emergence of wariness of heights. Psychol. Sci. 3, 61-64. doi: 10.1111/j.1467-9280.1992.tb00259.x hypothesis provides an explanation that holds across altricial species.

\section{AUTHOR CONTRIBUTIONS}

AG contributed to developing the hypothesis and writing the manuscript. JH contributed to developing the hypothesis and writing the manuscript.

\section{FUNDING}

AG was partially supported by NSF grants 1020367 and 1324807. Any opinions, findings, and conclusions or recommendations expressed in this material are those of the authors and do not necessarily reflect the views of the funding agencies.

\section{ACKNOWLEDGMENT}

We thank Heather Bimonte-Nelson, William Langston, Lynn Nadel, Nora Newcombe, Dennis Proffitt, and the reviewers for comments on earlier drafts of this paper.

Castro, L., and Aguiar, P. (2014). A feedforward model for the formation of a grid field where spatial information is provided solely from place cells. Biol. Cybern. 108, 133-143. doi: 10.1007/s00422-013-05 81-3

Dahl, A., Campos, J. J., Anderson, D. I., Uchiyama, I., Witherington, D. C., Ueno, M., et al. (2013). The epigenesis of wariness of heights. Psychol. Sci. 24, 1361-1367. doi: 10.1177/0956797613476047

Hafting, T., Fyhn, M., Molden, S., Moser, M.-B., and Moser, E. I. (2005). Microstructure of a spatial map in the entorhinal cortex. Nature 436, 801-806. doi: $10.1038 /$ nature 03721

Herbert, J., Gross, J., and Hayne, H. (2007). Crawling is associated with more flexible memory retrieval by 9-month-old infants. Dev. Sci. 10, 183-189. doi: 10.1111/j.1467-7687.2007.00548.x

Howe, M. L., and Courage, M. L. (1997). The emergence and early development of autobiographical memory. Psychol. Rev. 104, 499-523. doi: 10.1037/0033295X.104.3.499

Moscovitch, M., Nadel, L., Winocur, G., Gilboa, A., and Rosenbaum, R. S. (2006). The cognitive neuroscience of remote episodic, semantic and spatial memory. Curr. Opin. Neurobiol. 16, 179-190. doi: 10.1016/j.conb.2006. 03.013

Nelson, K., and Fivush, R. (2004). The emergence of autobiographical memory: a social cultural developmental theory. Psychol. Rev. 111, 486-511. doi: 10.1037/0033-295X.111.2.486

Newcombe, N. S., Balcomb, F., Ferrara, K., Hansen, M., and Koski, J. (2014). Two rooms, two representations? Episodic-like memory in toddlers and preschoolers. Dev. Sci. 17, 743-756. doi: 10.1111/desc. 12162

Newcombe, N. S., Lloyd, M. E., and Balcomb, F. (2011). "Contextualizing the development of recollection: episodic memory and binding in young children," in Origins and Development of Recollection: Perspectives from Psychology and Neuroscience, eds S. Ghetti and P. J. Bauer (Oxford: Oxford University Press), 73-100.

O'Keefe, J., and Nadel, L. (1978). The Hippocampus as a Cognitive Map, 1st Edn. Oxford: Oxford University Press.

Pelah, A., and Barlow, H. B. (1996). Visual illusion from running. Nature 381, 283. doi: $10.1038 / 381283 \mathrm{a} 0$ 
Rakison, D. H., and Krogh, L. (2010). Does causal action facilitate causal perception in infants younger than 6 months of age? Dev. Sci. 15, 43-53. doi: 10.1111/j.1467-7687.2011.01096.x

Riggins, T., Blankenship, S. L., Mulligan, E., Rice, K., and Redcay, E. (2015). Developmental differences in relations between episodic memory and hippocampal subregion volume during early childhood. Child Dev. 86, 17101718. doi: $10.1111 /$ cdev. 12445

Rovee-Collier, C. (1996). Shifting the focus from what to why. Infant Behav. Dev. 19, 385-400. doi: 10.1016/S0163-6383(96)90001-6

Smith, L. B. (2005). Action alters shape categories. Cogn. Sci. 29, 665-679. doi: 10.1207/s15516709cog0000_13

Sommerville, J. A., Woodward, A. L., and Needham, A. (2005). Action experience alters 3-month-old infants' perception of others' actions. Cognition 96, B1-B11. doi: 10.1016/j.cognition.2004.07.004

Taube, J. S. (1998). Head direction cells and the neurophysiological basis for a sense of direction. Prog. Neurobiol. 55, 225-256. doi: 10.1016/S0301-0082(98)00004-5
Winter, S. S., Mehlman, M. L., Clark, B. J., and Taube, J. S. (2015). Passive transport disrupts grid signals in the parahippocampal cortex. Curr. Biol. 25, 2493-2502. doi: 10.1016/j.cub.2015. 08.034

Conflict of Interest Statement: The authors declare that the research was conducted in the absence of any commercial or financial relationships that could be construed as a potential conflict of interest.

Copyright (c) 2016 Glenberg and Hayes. This is an open-access article distributed under the terms of the Creative Commons Attribution License (CC BY). The use, distribution or reproduction in other forums is permitted, provided the original author(s) or licensor are credited and that the original publication in this journal is cited, in accordance with accepted academic practice. No use, distribution or reproduction is permitted which does not comply with these terms. 


\section{OPEN ACCESS}

Edited by:

Annalisa Setti,

University College Cork, Ireland

Reviewed by:

Martin H. Fischer,

University of Potsdam, Germany

Guillaume T. Vallet,

Laboratoire de Psychologie Sociale et

Cognitive (CNRS), France

${ }^{*}$ Correspondence:

Matthew C. Costello

mcostello@hartford.edu

Specialty section:

This article was submitted to

Cognition,

a section of the journal

Frontiers in Psychology

Received: 30 October 2016 Accepted: 10 February 2017

Published: 27 February 2017

Citation:

Costello MC and Bloesch EK (2017) Are Older Adults Less Embodied? A Review of Age Effects through the Lens of Embodied Cognition.

Front. Psychol. 8:267. doi: 10.3389/fpsyg.2017.00267

\section{Are Older Adults Less Embodied? A Review of Age Effects through the Lens of Embodied Cognition}

\author{
Matthew C. Costello ${ }^{*}$ and Emily K. Bloesch ${ }^{2}$ \\ ${ }^{1}$ Department of Psychology, University of Hartford, West Hartford, CT, USA, ${ }^{2}$ Department of Psychology, Central Michigan \\ University, Mount Pleasant, MI, USA
}

Embodied cognition is a theoretical framework which posits that cognitive function is intimately intertwined with the body and physical actions. Although the field of psychology is increasingly accepting embodied cognition as a viable theory, it has rarely been employed in the gerontological literature. However, embodied cognition would appear to have explanatory power for aging research given that older adults typically manifest concurrent physical and mental changes, and that research has indicated a correlative relationship between such changes. The current paper reviews age-related changes in sensory processing, mental representation, and the actionperception relationship, exploring how each can be understood through the lens of embodied cognition. Compared to younger adults, older adults exhibit across all three domains an increased tendency to favor visual processing over bodily factors, leading to the conclusion that older adults are less embodied than young adults. We explore the significance of this finding in light of existing theoretical models of aging and argue that embodied cognition can benefit gerontological research by identifying further factors that can explain the cause of age-related declines.

Keywords: embodiment, aging, cognition, perception, embodied cognition, older adults, gerontology

\section{INTRODUCTION}

Embodied cognition theory argues that cognitive and perceptual processes are grounded in the organism's sensorimotor capacities. It rests upon a rejection of the classic cognitivist division between mind and body, and holds that the two are intertwined in often unexpected ways (Foglia and Wilson, 2013). Traditional (non-embodied) models of cognition operate through manipulation of symbolic mental representations governed by logical and computational rules. Under this traditional model, the body is not given a privileged position; it is merely the means by which sensory-level inputs are stored and later processed as mental representations. These representations are understood conceptually as independent of the physicality of the body. Embodied cognition rejects this traditional account, and argues that the physicality of the body in action is not merely a vehicle for computational processes, but rather is a co-producer of cognitive processes.

Embodied cognition is generally viewed as a broad theoretical framework for understanding mind-body relations, rather than as a singular theory serving to generate explicit hypotheses. It does not employ any singular experimental methodology, but instead serves as a backdrop to help interpret existing research such as in cognitive, perceptual, and bodily action studies. In this 
regard, embodied cognition theorists generally draw upon traditional 'non-embodied' research, and therefore may be understood as largely consonant with traditional cognitive methodologies (Goldman, 2012) ${ }^{1}$. However, insofar as it rejects some of the central tenets of classical cognitivist theory, embodied cognition retains something of an outsider status in cognitive science. Indeed, for some the ambiguous nature of embodied cognition makes it too difficult to effectively employ (Aizawa, 2015; Mahon, 2015). However, embodied cognition has become increasingly accepted as a viable theoretical option for researchers investigating sensorimotor function and situated cognition (Creem-Regehr and Kunz, 2010; Glenberg et al., 2013; Barsalou, 2016).

Embodied cognition would seem to be a natural fit for gerontology, as aging typically manifests as concurrent physical and cognitive changes (Roberts and Allen, 2016). Physically, older adults suffer from a range of changes to the body and action systems. Older adults experience height decreases, postural changes, decreased bone density, and increased bone brittleness (DiPietro, 2001). The quantity and quality of the muscular system show marked declines with advanced age (Metter et al., 1999), with reductions in muscle mass, strength, and mobility (Visser et al., 2002). These bodily changes are matched by decreases in motor function (cf., Seidler et al., 2010), with older adults exhibiting slower motor responsiveness (Falkenstein et al., 2006), more variable and slower physical movements (Mau-Moeller et al., 2013), and reduced gait speed (Studenski et al., 2011). Mobility is decreased in older adults (Tinetti, 1986), and physical actions are complicated by declines in balance control (Laughton et al., 2003). Accordingly, older adults exhibit decreased walking stability (Lord and Menz, 2000; Lord and Sturnieks, 2005) and increased falls (Kannus et al., 2005).

Cognitively, even healthy older adults exhibit a number of changes, including declines in working memory (Zacks, 1989), selective attention (Verhaeghen and Cerella, 2002), and response inhibition (Kramer et al., 1994). Perhaps the most expected age-related deficit in cognitive processing is in processing speed, with widespread slowdowns in basic cognitive and motor performance (Salthouse, 1996). Cognitive slowdowns for seniors are especially evident in visuospatial processing (Jenkins et al., 2000); relatedly, older adults exhibit declines in visuospatial reasoning and spatial navigation (Moffat, 2009; Klencklen et al., 2012). Interestingly, cognitive slowdowns have been linked to the type of physical activity levels described above (Kraft, 2012), and both cardiovascular health (Hillman et al., 2008) and walking speed (Atkinson et al., 2007) are predictive of cognition in older adults.

In short, there is ample evidence that for older adults, the mind and body are interconnected in a manner that resonates with embodied cognition theory. However, gerontological research has largely avoided the direct application of this framework.

\footnotetext{
${ }^{1}$ At its extreme theoretical variants, embodied cognition theory is both noncomputational and non-representational (cf., Chemero, 2009), although this is not a universal position within the embodied cognition field (Clark, 1997). Given that we will be drawing upon a broad array of research in this review article, we employ a more inclusive variant of embodied cognition in which traditional cognitivist terms such as 'mental representations' are utilized.
}

The goal of this review is to introduce the embodied cognition framework to the field of gerontological research and to argue that it can be used to explain a wide variety of cognitive aging effects. Given that embodied cognition is a broad field of study, the scope of this review article will be limited to addressing how aging affects three subdomains of specific relevance to embodied cognition: sensory processing, mental imagery, and the perception-action link. By way of preview, a central conclusion from this review is a finding common across all three of these subdomains: compared to young adults, older adults exhibit (1) an increased reliance on visual processing (what we will call a visual dominance effect), and (2) a decreased reliance on bodily (kinesthetic, tactile, proprioceptive) factors. At the end of this review, we will discuss the significance of this finding and how it may affect existing models of sensorimotor processing in older adults.

\section{FOUNDATIONS OF EMBODIMENT}

Embodied cognition theorists have argued that sensorimotor factors serve as the groundwork for cognition. For instance, concepts have been characterized as grounded in situated, bodily action and are instantiated by neural reactivations of sensorimotor cortices used in active sensorimotor function (Barsalou, 2008). This embodied view of cognition is generally viewed in contradistinction to traditional cognitivist models in which cognitive processing operates through amodal and abstract mental representations that are conceptually separated from the sensory modalities (Fodor et al., 1975). Because embodied cognition theory argues that higher-level cognition is grounded in sensorimotor processing, it would predict that any changes at this earliest stage will result in corollary changes in more complex cognition. Accordingly, we start our review with age-related sensory changes.

\section{Sensory Perception and Integration}

While the sensory system is subject to age effects across all five senses (Fozard and Gordon-Salant, 2001), our focus will be on declines in visual and tactile ability. Vision and tactition are particularly important senses for embodied cognition, as they provide information about the surrounding environment in which the body is located: vision provides accurate spatial feedback about both near- and far-body space, and tactition provides real-time feedback about the progress and success of physical interactions. Both sensory modalities exhibit decreased sensitivity with advanced age, with documented agerelated declines in vision (Owsley, 2011) and tactile processing (Wickremaratchi and Llewelyn, 2006).

Although age-related unimodal sensory declines are of obvious importance, our perception of the world typically involves more than one sensory modality. Multisensory integration (MSI) is the combination of two or more sensory modalities into a unified percept, a process driven by a neuronal system designed to process multiple sensory inputs simultaneously (Calvert et al., 2004). MSI is particularly important for embodied cognition: in order to have knowledge 
of the body's motor capabilities and the origin of sensory stimuli, there must be seamless integration of both external stimuli (visual and tactile information) and internal representations (motor commands, proprioceptive processing, and vestibular control). Thus, any change in the ability to integrate multiple sensory inputs carries with it changes in the accuracy of embodied representations.

Research indicates that younger and older adults both benefit from multisensory inputs (compared to unisensory inputs) when those inputs provide redundant information, yet this benefit is even greater in the older adults (cf., Freiherr et al., 2013). For instance, Laurienti et al. (2006) had participants discriminate between red and blue stimuli that were presented under both unimodal (either visually as colored circles or auditorily as the verbalized color words) and bimodal (both auditory and visual presentations) conditions. Although all participants evidenced reaction time facilitation in the bimodal compared to unimodal conditions, the gain was significantly greater for older adults. Several subsequent studies have also replicated the finding that older adults gain more than younger adults from bimodal conditions compared to unimodal conditions (Peiffer et al., 2007; Mahoney et al., 2011, 2012). However, when the inputs are unrelated or conflicting, older adults are more negatively affected by the distracting information than young adults (Setti et al., 2011). For example, Poliakoff et al. (2006) tested a crossmodal cuing task that required young and older participants to report the spatial location of vibrotactile stimuli while visual stimuli were presented in either congruent or incongruent locations. Older adults were significantly more distracted by spatially incongruent visual stimuli compared to young participants.

Thus, while older adults can gain from MSI-based facilitation effects, they also exhibit stronger debilitation under MSI-based distraction conditions. What explains these divergent effects? There are likely several contributing factors. First, with deficits in unimodal perception, older adults are likely compensating by relying on the additional information provided by MSI. Consider Mahoney et al. (2014), who found that around 75\% of older adults tested evidenced MSI enhancement (faster performance in visual-somatosensory testing conditions than in either visual or somatosensory alone conditions). However, the remaining 25\% did not show MSI enhancement; rather, their MSI responses were as fast as their unimodal somatosensory responses, which were in turn similar to speeds reported for younger adults in prior literature (e.g., Laurienti et al., 2006; Mahoney et al., 2011). This faster group of elders did not need to draw on multisensory benefit given their command of unimodal processing, whereas the slower older adults drew on the second sensory modality to reinforce the original weaker one.

Second, when vision is one of the modalities being integrated, older adults often appear to prioritize it over audition and touch. Throughout this paper, we will be using the phrase visual dominance to describe this over-reliance on visual processing over other sensory modalities. It is important to point out that visual processing factors dominate over the other sensory modalities for all normally sighted humans, not just older adults (Posner et al., 1976). The findings of this literature review is that visual dominance holds greater sway for older adults when compared to younger adults. Although this is not readily apparent when information from the two modalities provide synchronous information, this bias emerges more clearly during times of conflict (i.e., increased distraction for older adults when visual information conflicts with tactile). This age-related increase in visual capture has been well-explored in crossmodal attention research, indicating that older adult processing is dominated by the visual components under MSI testing conditions (Thompson and Malloy, 2004; Mahoney et al., 2012). A key study in this regard is Diaconescu et al. (2013), who assessed age group differences in brain activity during crossmodal processing using magnetoencephalography (MEG). In the task, younger and older adults made categorization judgments for stimuli presented either unimodally (auditory or visual) or bimodally (auditory + visual). Although both age groups benefited from the addition of visual inputs compared to auditory alone, the benefit was greater for older adults. MEG recordings indicated a probable explanation: multisensory gains in the older adults were associated with increased medial prefrontal and posterior parietal activity, with the latter region implicated in visuo-motor performance. The authors characterized these results as indicative of visual dominance, a compensatory mechanism in which older adults draw more heavily on visual processing brain regions to offset age-related reductions in cortical and subcortical gray matter volume.

Prioritizing visual over somatosensory information may help explain why older adults exhibit balance control issues and increased falls (Kannus et al., 2005). Standing balance is compromised in older adults (Laughton et al., 2003), a finding which has been attributed to their overemphasis of visual processing factors (Horak et al., 1989). When attempting to maintain an upright posture, older adults exhibit increased susceptibility to sway based on visual manipulations (Wade et al., 1995; Prioli et al., 2005; Toledo and Barela, 2014). It is not surprising, then, that walking is also compromised in aging. Efficient walking requires a complex integration of visual, tactile, and vestibular inputs with motor sequencing commands. Yet when walking, older adults tend to focus more on the ground and thus draw more heavily on optic flow information (Anderson et al., 1998), with greater attention to visual factors (Sparrow et al., 2002) which may reflect the decreased processing power of proprioceptive systems (Huitema et al., 2005). Fall-prone older adults appear to rely heavily on visual processing due to interoceptive processing failures (Barrett et al., 2013), and MSI control failures in older adults have been linked to both poor balance (Stapleton et al., 2014) and increased falls (Mahoney et al., 2014).

Yet this visual dominance affecting older adults is neither inevitable nor fixed. Recent research has shown that MSI in older adults is highly plastic, and MSI-based training can result in improved walking and balance performance (Setti et al., 2014). For instance, Merriman et al. (2015) explored whether a balance intervention program could help MSI problems in older adults. They found that older adults benefited from the intervention, with improved balance control when compared to similarly matched peers. Importantly, fall-prone older adults 
showed significant improvement in MSI performance based upon the balance control intervention. Thus, behavioral intervention holds the promise of offsetting the visual dominance exhibited by older adults and reducing the potential for falls.

\section{The Body Schema and Peripersonal Space}

The body schema and peripersonal space are concepts used to explain how humans understand the physical body and the external space immediately surrounding it. While these terms are treated as functionally distinct, they share many similarities and in fact are often used somewhat interchangeably. The body schema is the internalized 'map' of spatial relations for one's own body based on kinesthetic, proprioceptive, and tactile information, which is ultimately used to facilitate successful action; peripersonal space, on the other hand, is the space immediately surrounding the body through which we interact with the surrounding environment. Ambiguity in the exact boundaries of these constructs is understandable: when examining the understanding of physical actions (a body schema question), that movement necessarily takes place in peripersonal space, and the influence of that space is difficult to disentangle from the body schema itself (c.f., Cardinali et al., 2009 for a full review).

Both the body schema and peripersonal space are essential to embodiment theory, for they form the foundation for the intersection of sensory experience and bodily action (Borghi and Cimatti, 2010). Both the body schema and the formation of peripersonal space are mediated through MSI, the result of combining weighted signals from visual, tactile, and proprioceptive domains (Blanke, 2012). Additionally, both allow for multiple action-perception relationships, for the capacity to perform bodily actions requires an awareness of one's own body in space (Reed and Farah, 1995). Accordingly, the body schema and peripersonal space are plastic in nature, and can flexibly adapt to differing physical environments and bodily demands.

It is reasonable to expect that aging will affect the body schema and peripersonal space given that they are based on underlying factors that are vulnerable to aging. First, they represent the holistic integration of multisensory inputs within the body's spatiality (Maravita et al., 2003), a process known to change during aging. Second, body schema mapping and the localization of objects in peripersonal space necessitate accurate proprioception (Walsh et al., 2011; Proske and Gandevia, 2012), which is known to decline with advanced age (Goble et al., 2009). Finally, the body schema is continuously updated by vestibular signals (Lopez et al., 2012), and vestibular control declines with advanced age (Alexander, 1994). Taken together, one would expect profound changes to the body schema and peripersonal space with advanced aging.

Studies examining peripersonal space in young adults have sought to identify perceptual differences between near-body and far-body (extrapersonal) space. One common difference is that in peripersonal space, young adults deploy attention asymmetrically, over-emphasizing the left visual field over the right. This is thought to occur because spatial attention in peripersonal (but not extrapersonal) space is primarily controlled by the right-hemisphere (Fink et al., 2001; Bjoertomt et al., 2002). On tasks such as line bisection, in which participants indicate the center point on a straight horizontal line, it manifests as a slight leftward bias when the line is presented in peripersonal space (Jewell and McCourt, 2000). In extrapersonal space, where the asymmetry does not occur, young adults bisect lines at veridical center or with a slight rightward bias (Varnava et al., 2002; Garza et al., 2008). Older adults, on the other hand, exhibit a rightward bias in peripersonal space (Fujii et al., 1995; Jewell and McCourt, 2000). This occurs even when manual abilities are eliminated and when implicit tasks are used to account for the possibility of strategy differences (Barrett and Craver-Lemley, 2008). This may be occurring because older adults do not have right-hemisphere dominance for coding peripersonal space, requiring activation of the left hemisphere to compensate (Cherry et al., 2005; Chen et al., 2011).

Ghafouri and Lestienne (2000) used a sensorimotor experimental design to evaluate peripersonal space representations in older adults, having participants draw imaginary ellipses in the air in different planes in the space in front of their bodies. The authors argued that representations of 3D space can be revealed by examining goal-directed movements within that space: if space is not represented veridically, the movements should reflect those inaccuracies. The authors compared the abilities of young and older adults to orient ellipses in three different planes by first showing participants a template and asking them to reproduce it. In comparing the plane of motion of each participant's finger, they found that older adults had smaller plane volumes than young adults, indicating a compression in the representation of peripersonal space. Furthermore, by comparing performance across the different planes, they were able to show that the motor component was not responsible for the errors; rather, older adults were representing the spatial coordinates incorrectly.

Although these behavioral tests are in line with peripersonal representations changing with age, not all studies find age differences. Older adults have been found to exhibit leftward biases when drawing or arranging items in peripersonal space, similar to young adults (Barrett and Craver-Lemley, 2008). While these measures were implicit tasks (unlike line bisection), other studies using seemingly implicit dependent measures such as ellipse drawing have shown these differences, suggesting that changes in performance with age are not just due to changing strategies. It is unclear what is causing these divergent results, and further research is needed to clarify why peripersonal space representations sometimes, but not always, show age-related changes.

With regard to the body schema proper, little research has directly explored how it may be affected by aging. Gilpin et al. (2015) recently tested how osteoarthritis affects the body schema of older adults. In their study, arthritis sufferers and non-arthritic controls viewed live video feed of their own hands while their hands were manipulated to be 'stretched' (the imaged hand was digitally elongated while physically pulled), 'shrunk' (digitally contracted while being pushed), or presented without manipulation. Participants were then asked 
to resize digital photographs of their hands to veridical sizes. Analyses indicated two key findings. First, the non-arthritic controls exhibited the expected shrinking and stretching illusion, whereas this manipulation was muted in the arthritis sufferers. Second, arthritic seniors reported smaller hand sizes than nonarthritic controls, suggesting that pain contracted the spatial representation of the hand. Although this study indicates the alteration of the body schema under a common older adult disability, the results point to body schema plasticity based upon age-related pathology (i.e., arthritis) rather than aging itself. However, it indicates that changes in the body and physical ability have the capacity to alter the body schema. Further research is needed to determine how the body and physical changes that accompany healthy aging also alter the body schema.

\section{EMBODIMENT IN MENTAL REPRESENTATIONS}

Having detailed the age-related changes to sensory-level processing, we turn now to how aging alters mental representations based on that sensory processing. The embodied cognition framework regards mental concepts as either heavily interactive with sensorimotor systems or as directly related to sensorimotor function (Glenberg, 2015), whereby concepts are instantiated by reactivations of sensory- and/or actionbased neural pathways (Barsalou, 1999, 2008). As such, mental representations are emblematic of sensorimotor function. This is in contrast with the traditional cognitive science model, wherein perceptual processing of sensory inputs occurs through mental representations that are understood as abstract and amodal symbols for the represented percepts (Fodor et al., 1975; Pylyshyn, 1984). To explore how aging affects the influence of embodiment on mental representations, we will examine age-related differences in mental imagery, motor imagery, and action observation.

\section{Mental Imagery}

Mental imagery is the creation, maintenance, and activation of an internal representation. Although seemingly simple in definition, mental imagery depends upon multiple cognitive subcomponents including sensory processing (the image is typically either visual or auditory in nature), cognitive processing (the image is understood in terms of its physical construction and/or semantic content), and working memory (mental images can be reproduced, maintained, and elaborated upon; Kosslyn, 1995). Mental imagery shares a similar underlying neuroarchitecture with perceptual processing, such that imagining an object elicits similar brain activations as directly perceiving the object (Kosslyn et al., 2001; Wilson-Mendenhall et al., 2013). This close relationship between the perceptual processing and mental imagery suggests that older adult deficits in unisensory and multisensory processing may yield similar deficits in mental representations.

Perhaps the canonical experimental paradigm for mental representation study has been the mental rotation task, first developed by Shepard and Metzler (1971). In such tasks, participants are shown an object on a screen and are required to mentally rotate it, typically responding by selecting the target angle from an array of variously rotated items. Early research on age-related differences in mental rotation found that older adults committed greater errors and yielded slower performance in mental rotation compared to young adults (Cerella et al., 1981; Hertzog and Rypma, 1991), indicative of a generalized decline in mental imagery (Craik and Dirkx, 1992). However, later work found that age effects on mental rotation are not a global failure, but rather are evident primarily under the cognitive strain of difficult rotation angles and increased complexity (McDowd and Craik, 1988; Inui, 1997; Sit and Fisk, 1999). Indeed, recent work from an embodied cognition perspective has found that both older and younger adults have mental representations that are similarly grounded in sensory processing (Vallet et al., 2011, 2013). Age-related deficits in mental rotation, when present, may therefore reflect the increased cognitive demands of spatial processing.

The mental rotation of the body is of particular importance to embodied cognition theory given that it represents the mental manipulation of the internalized body schema (Kaltner et al., 2014). Research with young adults has indicated that such imagery is constrained by body spatiality and bodily actions (Amorim et al., 2006; Steggemann et al., 2011). Importantly, older adult performance in mental rotation worsens when the target shape is a body. Devlin and Wilson (2010) assessed the effect of aging on mental rotations with three different imagery types: an alphanumeric stimuli set ( 5 or $F$ ), a 2D image of a single hand, and 3D whole-body image. Although age effects were evident across all three image types, older adults were significantly worse performing the whole-body rotations, with increasing reaction times and errors when rotating at larger angles. In this case, the whole-body stimuli represented not only a more complex stimuli set, but one intimately linked with the body schema.

While it may not always be clear whether older adult difficulties in whole-body mental rotation are due to task complexity or poor body representations, there is evidence that egocentric perspectives are specifically problematic. First, when the mental rotation requires an egocentric perspective (such as rotating a body image by means of one's own body), older adults show deficits compared to young adults (Jansen and Kaltner, 2014; Kaltner and Jansen, 2016). Second, studies in spatial learning have found that older adults show marked deficits in learning spatial environments when operating through an embodied and first-person perspective (Inagaki et al., 2002; Borella et al., 2015). For instance, Yamamoto and DeGirolamo (2012) had young and older participants encode landmarks in a simulated environment either through a firstperson or third-person perspective. When participants were later asked to reconstruct the spatial layouts, older adults were less accurate than young adults in the first-person, but not the third-person, encoding conditions. Finally, when older adults are required to make mental rotations of hand stimuli, their performance is significantly worse compared to younger adults when the rotations required an egocentric 
(as opposed to allocentric) rotation strategy (De Simone et al., 2013). Taken together, we can interpret these multiple instances of age-related decreases in first-person perspective to indicate that older adults may be downgrading their bodily inputs, resulting in decreases in egocentric (first-person) processing and consequent increases in allocentric (third-person) processing.

\section{Motor Imagery}

A subtype of mental imagery is motor imagery, the mental simulation of an action when there are no overt physical actions being made. Motor imagery is arguably a more complex variant of mental imagery for it includes an implicit action plan (Kosslyn, 1987). Motor imagery is integral to embodied cognition because it combines external representations of the environment and peripersonal space with representations of the body schema to construct simulations. Motor imagery is critical to efficient intentional actions, because it serves as an internal simulation of actions that can moderate overt motoric action (Wolpert, 1997; Wolpert and Kawato, 1998) by working as forwardmodels in the planning of physical actions (Beauchet et al., 2010).

Our understanding of motor imagery has been greatly influenced by Jeannerod, who theorized that covert (mentally simulated) actions are neurologically similar to overt motor actions, such that both elicit comparable cortical activation patterns (Jeannerod, 1994, 2001). Indeed, there has been strong empirical support for this neurological overlap (Decety, 1996; Szameitat et al., 2007; Kraeutner et al., 2014). Motor regions activate when merely viewing action-based objects (Chao and Martin, 2000), and motor imagery elicits sensorimotor brain responses (Hauk et al., 2004). Jeannerod's motor simulation theory is broadly compatible with the embodied cognition framework, and is conceptually akin to action-perception theories that similarly emphasize the close connection between sensation, action, and cognition (Hommel et al., 2001; Proffitt and Linkenauger, 2013).

Research indicates that motor imagery declines are evident in healthy aging (Personnier et al., 2008; Skoura et al., 2008; Saimpont et al., 2009). For instance, Gabbard et al. (2011) asked young and older adults to declare whether a target was within their arm's reach after mentally simulating the action. Older adults overestimated their reach compared to young adults, which may reflect age-related deficits in brain regions critical to motor imagery (Munzert et al., 2009). Declines in motor imagery appear linked to handedness, with older adults exhibiting greater errors when operating with the non-dominant compared to the dominant arm (Skoura et al., 2008; Saimpont et al., 2009).

Perhaps not surprisingly, there are many similarities between the age-related declines in both mental imagery and motor imagery. Like mental imagery, the aging effect on motor imagery is also affected by both the complexity and perspective of the imagery. Under simple motor imagery conditions, older adults perform equivalent to young adults (cf., Saimpont et al., 2013). However, as the motor sequences to be imagined become more challenging, older adult imagery becomes less accurate compared to young adults (Skoura et al., 2005).

One specific form of additional complexity for older adults are first-person motor imagery visualizations. Mulder et al. (2007) had young and older participants report visualization strengths of their own or other's actions. When older adults visualized their own first-person actions (as opposed to a third-person perspective visualization), the vividness in the images decreased. Importantly, this decline in the first-person perspective reflected the declining physical powers of the older adults: imagery vividness scores were correlated with measures of motor capacity and mental and physical speed measures. The effect of aging on motor imagery, therefore, is subtle: relatively preserved in cases of simple motor images without dynamic physical constraints, but degraded under more complex imagined action conditions (Personnier et al., 2008; Saimpont et al., 2013), particularly those involving egocentric perspectives. This result is consistent with theories of aging in which older adults suffer from a diminution of mental resources, resulting in increased difficulties from distraction under complex task requirements (Lustig et al., 2007).

Visual dominance of motor imagery in older adults has also been identified in neuroimaging studies. When using motor imagery, both young and older adults engage a network of neural pathways that include visual imagery and motor pathways as well as frontoparietal, subcortical, and cerebellar areas. However, activation intensity and specificity does differ by age group, suggesting that age effects in motor imagery reflect the changes to these brain networks (Saimpont et al., 2013). During motor imagery, older adult brains respond with larger and more diffuse activation patterns (Léonard and Tremblay, 2007; Nedelko et al., 2010; Sharma and Baron, 2014; Reuter et al., 2015), suggesting degraded or less-specific motor representations leading to compensatory brain recruitment (Zapparoli et al., 2016). When performing overt motor actions, older adults exhibit increased frontal and prefrontal (pre-SMA) activations during more cognitively complex actions, whereas when mentally simulating actions older adults exhibited increased activations in occipito-temporo-parietal areas (Zapparoli et al., 2013). This latter finding suggests that older adults rely more heavily on visual processing while performing motor imagery, consistent with our earlier finding of older adult over-reliance on visual processing in multisensory perception. Thus, behavioral evidence of visual dominance in older adults is matched by neuroimaging results suggesting increased activity in visual processing regions.

\section{Action Observation}

Jeannerod's (2001) motor simulation theory stipulates not only that motor imagery and physical actions share a common neural substrate - it also predicts that there is a similarly shared activation network when observing such actions in others. Indeed, there is mounting evidence indicating that merely observing someone performing an action results in the activation of the sensorimotor pathways responsible for producing that action (Buccino et al., 2004; Lui et al., 2008). This shared cortical activation pattern may offer a twofold advantage: allowing 
superior anticipation of the actions of others and providing information on one's own actions (Jeannerod, 2001; Jeannerod and Anquetil, 2008).

Given the age-related decline in mental and motor imagery, it is not surprising that older adults do not benefit from action observation to the same extent as young adults. Aging decreases the efficiency in learning and then executing novel motor sequences (Coats et al., 2013) with specific losses for action observation (Maryott and Sekuler, 2009). For instance, Maguinness et al. (2013) explored age-related differences in a task in which participants viewed videos of either a hand or a full body lifting weighted boxes and had to indicate the amount of weight being lifted. Older adults were overall less sensitive for weight prediction than young adults, but particularly so when there were fewer visual details on the biomechanics of the lift. Accordingly, the authors argued that older adult performance declines indicate that seniors relied on a more visual-based (rather than felt-based) strategy.

Older adults may rely on visual processing components because their internal action models are degraded (Boisgontier and Nougier, 2013). Diersch et al. (2012) explored this possibility with a task assessing differences between young and older adults in the temporal parameters of action prediction. In the task, participants were shown video clips of everyday action sequences with the scenes interrupted by a black-screen occluder. After the occluder was removed, participants rejoined the original scene, although the timing parameters were manipulated such that the pre- and post-occluder scenes were either temporally continuous or discontinuous with the preceding scene. Participants were tasked with identifying the amount of time that had been altered based on the occluder. Older adults were less accurate than young adults in predicting the time course of another's action, a result that the authors argued indicated a specific age-related decline in action representations. More recently, the same research group found that older adults exhibited increased activity in visual regions when performing action prediction (Diersch et al., 2013), with more diffuse brain activity indicative of less-specific internal models of motor actions (Diersch et al., 2016).

\section{EMBODIMENT IN PERCEPTION AND ACTION}

Our review thus far has focused largely on the bottom-up factors (i.e., physiological systems and sensory processing) and the mental representations that underlie embodied cognition. We have found that aging affects these systems profoundly, with a common finding of visual prioritization over somatosensory processing in older adults. In cases of multisensory perception, older adult deficits in unisensory processing typically result in gains in multisensory inputs, although visual processing is dominant. A similar tendency toward visual dominance occurs in mental imagery (most especially in motor imagery and actionperception), for visual processing factors dominate the motoric representations in older adults. Thus, basic sensorimotor deficits in older adults result in increased reliance on visual processing over other sensory modalities, and this visual dominance alters the nature of action-based mental representations. We now turn to how bodily action alters perceptual experience, a topic that is central to the embodied cognition framework (Glenberg et al., 2013).

\section{Action-Specific Theory of Perception}

A central tenet of embodied cognition is that the mind and the body have reciprocal influence: not only can the mind control and direct the body as traditionally thought, but the body can also direct and influence the mind through its movement, posture, and sensory input. Embodied cognition theorists have long argued that action alters perceptual judgment, and research has confirmed that perceptual judgments of object size and distance correlate with our capacity to act upon the object (Proffitt et al., 2003; Kirsch and Kunde, 2013; Linkenauger et al., 2013). This close link between perception and action is central to both embodied cognition theory (Proffitt, 2006; Glenberg et al., 2013) and ecological theories of perception (Richardson et al., 2008).

Witt (2011) has formalized this general dictum into a specific theory on how action affordances can alter spatial cognition. Her action-specific account of visual perception argues that visual information of an object (in terms of object size or distance) is scaled relative to an observer's effort or ability to effectively interact with that object (Witt et al., 2004). Thus, perceptual appraisals are always scaled as a phenotypic expression of bodily capacity (Proffitt and Linkenauger, 2013) made in relation to action affordances (Kirsch and Kunde, 2013). In line with the action-specific perception theory, physical capacity of the perceiver has been found to be related to perceptual scaling, presumably to maximize efficiency in motor response. Several studies have found that athletes exhibit perceptual recalibrations that correlate with their performance when playing sports such as tennis (Witt and Sugovic, 2010), golf (Witt et al., 2008), softball (Witt and Proffitt, 2005), and football (Witt and Dorsch, 2009).

Given that the older adult body is limited in its physical capacity, it is reasonable to expect age-related changes in perceptual scaling. In an early study by Bhalla and Proffitt $(1999)^{2}$ on the relationship between aging and perceptual capacity, younger and older participants provided slope estimates while looking up from the bottom of variously angled hills. Young adult slope estimates were steeper when placed in physically demanding conditions, a result consistent with the action-specific perception hypothesis. However, older adults provided steeper hill slope estimates than young adults even under typical physical conditions, suggesting that their reduced physiological potential led to perceptual bias. Perceptual scaling tasks have also found that older adults are sensitive to environmental impediments that would complicate walking (Sugovic and Witt, 2013), essentially indicative of increased cautiousness. Similar findings of visuomotor cautiousness have been documented in gait analyses of older adults (Grabiner et al., 2001; Owings and Grabiner, 2004) and shoulder rotation movements when walking through door apertures (Hackney and Cinelli, 2011).

\footnotetext{
${ }^{2}$ Note that this work has been criticized as reflecting participant effects in how participants respond rather than actual measures of perceptual alteration (Firestone, 2013; see Witt et al. (2016) for a reply). Recently, Philbeck and Witt (2015) have offered a more measured action-perception model.
} 
What explains this increasing cautiousness in the actionspecific perception responses of older adults? While the answer is not yet clear, the action-specific account of visual perception would point to the physical limitations of older adults as determinative to perceptual scaling. If true, the number of potential physiological factors affecting the older adult perception-action relationship may be surprisingly broad. Action-specific perception research has generated a dizzying array of physical factors vulnerable to aging that can influence task performance, including strength (Linkenauger et al., 2009), pain levels (Witt et al., 2009), and even respiration capacity (Daviaux et al., 2015). All these factors tie directly to the changing bodies of older adults. Consider the more speculative possibility that the metabolism of older adults might affect their actionperception relationship. It is known that giving participants sugary drinks has been found to decrease judgments of hill steepness (Schnall et al., 2010), presumably due to altered energy levels (although cf., Durgin et al., 2012). Older adults have marked declines in energy intake levels (Vaughan et al., 1991) and metabolism rate decreases (Wilson and Morley, 2003). The cause for this age effect on the regulation of metabolism is diverse, ranging from the delayed absorption of macronutrients and to reduced sensitivity of taste and smell (Roberts and Rosenberg, 2006). This raises the intriguing possibility that such seemingly tangential physiological and/or sensory factors could alter the perceptual judgments of older adults.

\section{Tool Use}

Embodied cognition argues not merely that the body and bodily action is formative toward cognition and perception, but that the mind itself is unconstrained by the physical boundary of the brain and is extended out into the body in action (Noë,, 2009; Clark, 2010). Accordingly, it is logical to assume that the body may not be the only tool for mind-extension. Indeed, embodied cognition theorists have argued that the mind extends itself through a wide array of tools; this goes beyond mere physical objects and includes even metaphorical tools such as cultural artifacts (Malafouris, 2004; Wilson, 2010) and language (Mirolli and Parisi, 2009; Borghi et al., 2013).

The study of how tool use is represented in the brain began largely with primate research using single-cell recordings of neuronal sensitivity. Such work has found that when monkeys use tools, the receptive fields of neurons normally coding the spatial extent of the hand became extended into the more distant space of the tool's reach (cf., Baccarini and Maravita, 2013). An early report indicated that when monkeys were trained to retrieve distant food using a rake, the receptive fields for bimodal (visual and somatosensory) neurons within the postcentral gyrus elongated to include the functional reach of the rake (Iriki et al., 1996). Consequent work has indicated that such toolbased changes to neuronal function are evidence of the plasticity of body representations and the coding of spatial relations (Obayashi et al., 2001; Hihara et al., 2006). Collectively this research indicates that the body schema is instantiated in the brain through multimodal (typically visual and tactile) coding that is subject to alteration based upon tool use (Maravita and Iriki, 2004).
Studies with human participants using tools have found similar results of body schema adaption under both behavioral (Maravita et al., 2002) and neuroimaging (Järveläinen et al., 2004; Peeters et al., 2009) testing conditions. When using tools, peripersonal space becomes extended into extrapersonal space (Berti and Frassinetti, 2000; Farnè and Làdavas, 2000; Maravita et al., 2001; Farnè et al., 2007). Cognitive processes alter in accordance with tool use, as both visual attention (Farnè et al., 2005; Witt and Brockmole, 2012) and memory (Davoli et al., 2012a) become refocused to the space at the functional end of the tool. For instance, participants report the distance of targets to be closer to themselves when wielding a tool directed to the target (Reed et al., 2010). This spatial-contraction effect is evident even when participants merely intend to act upon the target with a tool (Witt et al., 2005) and when the tool offers only remote access (Davoli et al., 2012b).

How might aging affect the ability to incorporate a tool into the body schema? One source of evidence comes from pantomime studies, in which participants are asked to identify or act out a physical action (e.g., "show me how to cut with a knife"). In a manner consistent with our earlier review of action observation research, pantomiming research has found that older adults exhibit general declines in pantomiming human actions (Cavalcante and Caramelli, 2009), especially when the pantomiming involves use of a tool (Mozaz et al., 2002, 2009). When pantomiming tool use, older adults make a characteristic body-part-as-object (BPO) error, wherein body parts rather than simulated actions are used to represent tool use (Ska and Nespoulous, 1987; Peigneux and van der Linden, 1999). For example, when asked to pantomime scissors, older adults are more likely to represent the index and middle fingers as scissor blades than to grip imaginary scissors handles. These agerelated increases in BPO errors may simply reflect the cognitive demands of mimicking a tool-wielded action (Cavalcante and Caramelli, 2009; Maryott and Sekuler, 2009; Mozaz et al., 2009), a pantomime complicated by required mimicry of both the motor intention and detailed hand kinematics. Conversely, older adult deficits in pantomiming may represent a specific deficit, akin to the ideomotor apraxia common to stroke patients (Mizelle and Wheaton, 2010). Yet direct empirical evidence has been minimal.

Costello et al. (2015) recently examined whether aging affects the spatial-contraction effect that occurs during tool use. In their first task, young and older adults were shown target circles at near-body distances (ranging from 44 to $89 \mathrm{~cm}$ away) and asked to estimate their distance after pointing to the target either with their hand or with a reach-extending tool. Only the young adults displayed the expected tool-based spatial-contraction effect, in which the targets appeared closer when the participants were using the tool. In a second task, distance estimates were derived for far distances (ranging from 3.4 to $25.3 \mathrm{~m}$ away) after either pointing with the hand or with a laser pointer, which has been found to produce spatial contraction (Davoli et al., 2012b). Similar to the first experiment, only the young adults displayed the expected spatial-contraction effect when using the laser pointer on the targets. Taken together, the two tasks indicate a generalized phenomenon in which older adults fail to exhibit tool-based plasticity of body representations. 
What might explain older adults' inability to incorporate tools into the body schema? If we assume that tool use is a complex function with multiple cognitive, sensory, and motor components, age effects at lower sensorimotor levels may disrupt the overt performance of using a tool. After all, tool use requires the smooth integration of visual and tactile inputs, and as already discussed, older adults struggle in MSI-based tasks. There is evidence to this effect. Heuer and colleagues have investigated age-related differences in multisensory processing during tool use with a visuo-motor rotation paradigm. In such tasks, participants look at a display screen while moving a mouse cursor to a target, but vision of the hand movements themselves are hidden under an occluder. On some trials, the cursor pathway is diverted from the actual pathway of the hand movement. After the participants complete their reach and return to the home position, the pathway directions of either the cursor or the hand are displayed. Participants are then asked to modify the displayed pathways to the correct location for the remembered target location. Perhaps not surprisingly, under such conditions older adults commit greater errors than young adults (Bock, 2005; Heuer and Hegele, 2008; Heuer et al., 2013). But more importantly, older adults were more heavily influenced by the visual information of the monitor cursor than the tactile information of their own hand movements (Rand and Heuer, 2013; Heuer and Hegele, 2014). Thus, the lack of spatial contraction based upon tool use (Costello et al., 2015) may be due to the aforementioned visual dominance in older adults: because their sensorimotor calibration is more heavily influenced by visual processing, the proprioceptive feedback from the hand using the tool is accordingly diminished.

Secondly, tool use is typically performed with the hand, and there is evidence that the cognitive system of older adults is desensitized to their hands. Young adults appear to automatically orient attention toward their hands both immediately prior to and during a reach (Tipper et al., 1992; Meegan and Tipper, 1998). Older adults, however, do not orient toward the reaching hand; instead, attention remains centered on the space around the trunk (Bloesch et al., 2013). Older adults, in other words, have an attentional reference frame that is less sensitive to their hands, a finding that is consonate with a similar result discussed earlier: when older adults perform mental rotations of hand-based stimuli their errors were greatest for egocentric (as opposed to allocentric) rotations, suggesting that the older adults struggle to extract sensorimotor information of their own hands to assist their mental rotation of the target hand (De Simone et al., 2013). If sensorimotor information about the hands is less available during aging, it would be less likely that representations are flexible enough to easily incorporate tools into the body schema.

One final factor that may also contribute to older adults' ability to understand and use tools is the type of knowledge necessary for this complicated task. Effective tool use relies on both semantic knowledge, information connecting the purpose of a tool to a tobe-acted-upon object, and mechanical knowledge, information about the functional allowances of a tool-based on the properties of both the tool itself and the to-be-acted-upon object (Lesourd et al., 2016). Additionally, tool use depends on reasoning skills that allow for mechanical knowledge to be drawn upon given the tools available and the situational demands (Reynaud et al., 2016). While chronological age is predictive of declines in both types of tool-related knowledge, the relationship is considerably stronger for semantic than mechanical. Further, cognitive functioning mediates age-related declines in semantic, but not mechanical, tasks (Lesourd et al., 2016). Thus, tool use failures in older adults may be partially explained by an inability to access knowledge about a tool's purpose or the type of task a specific tool is used to complete, rather than an inability to access the action affordances of the tool.

\section{ARE OLDER ADULTS LESS EMBODIED?}

This review has explored how the framework of embodied cognition can be applied to the study of aging by detailing research falling under three subdomains pertinent to embodied cognition: sensory perception (with a focus on MSI), mental representation (with a focus on motor imagery), and the effect of action on perceptual judgments. Embodied cognition theory predicts that as the body and sensory systems decline, there will be corollary changes in perception and cognition. Older adulthood is a critical period of physical decline, and therefore embodied cognition is a valuable theoretical prism by which to view aging (Vallet, 2015). As we've described in the prior sections, the aging mind-body relationship is indeed seen consistently across a variety of paradigms measuring different aspects of cognition. The results indicate that aging has profound effect on embodiment, as summarized below.

\section{Embodiment Changes in Older Adults}

While aging is associated with a host of physical changes that produce a variety of effects on embodiment, a consistent finding in the literature is that older adults are influenced largely by visual processing over tactile and kinesthetic processing. This visual dominance effect was evident across all three subdomains we covered. Older adults are more heavily influenced by visual processing components during MSI tasks (Diaconescu et al., 2013), which may explain the increase in falls for older adults (Mahoney et al., 2014; Stapleton et al., 2014). Age-related failures in postural control have been attributed to older adults prioritizing visual inputs over other sensory modalities (Wade et al., 1995; Prioli et al., 2005) due to decreased efficiency in control over body position (Toledo AND Barela, 2014).

Similar instances of visual dominance in older adults have been found when performing tasks involving action observation (Maguinness et al., 2013; Boisgontier et al., 2014), again across a number of domains. Older adults have increased activity in visual processing regions during action prediction (Diersch et al., 2016) and greater reliance on visual over somatosensory signals on visuo-motor tasks (Rand and Heuer, 2013; Rand et al., 2013; Heuer and Hegele, 2014). Similarly, sensorimotor learning in older adults is more strongly directed by visual inputs compared to young adults (Teixeira and Lima, 2009). This over-reliance on visual processing may explain why older adults exhibit declines in spatial cognition: MSI is down-weighting tactile/proprioceptive inputs relative to visual inputs. In essence, due to age-related 
declines in physical capacity, a compensatory mechanism arises that increases the influence of visual processing, resulting in sensorimotor calibrations that are scaled with either distorted tactile/kinematic inputs, or the tactile/kinematic inputs are reduced in their weighted contribution. Older adults, in relying more heavily on a visual signal and less on an internal, bodybased signal, are effectively less embodied than young adults.

An important corollary phenomenon is the age-related change in perspective-taking: older adults struggle when visualizing from their own first-person compared to a third-person perspective, displaying an insensitivity for egocentric awareness. This was primarily evident in motor imagery studies which have found decreases in multiple areas: in first as opposed to third person perspectives (Mulder et al., 2007), in whole-body mental rotation with egocentric requirements (Devlin and Wilson, 2010), and when mentally rotating hand-based stimuli (De Simone et al., 2013). Thus, the older adults are less 'inhabited' within their body and less comfortable when forced to imagine their body in alternative viewpoints. How does this relate to the aforementioned visual dominance effect in older adults? One, first-person perspectives would encompass the body state of the older adult. If older adults have deficiencies in access to their body states, then the visualizations that depend on that perspective would consequently falter. Two, if older adults are less embodied and more objectivist in their perspective, their increased visual processing would force them 'outside' rather than staying in the body. In that sense, mentally seeing the world in the third-person would likely be clearer to older adults compare to an egocentric perspective.

In short, the body's influence on cognition alters in older adults based on their reliance on visual signals over bodily control. Embodied representations rely on externally originating visual and tactile sensory signals as well as internally originating proprioceptive and vestibular sensory signals. These signals will be weighted differently depending on the task and the quality of the signal. For older adults the internal signals are often degraded - proprioception declines with advanced age (Goble et al., 2009), as does vestibular control (Alexander, 1994). Although vision declines with age as well, it can be corrected to normal more easily than these other senses. While it is the case that normally sighted humans, not just older adults, prioritize vision (Posner et al., 1976), when creating embodied representations the visual signal can be balanced with internal signals. If internal signals are less reliable or less available, as they are for older adults, it may be the case that the visual signal becomes most strongly weighted regardless of the task.

This scenario would predict that older adults would be most likely to prioritize visual information because it is the most readily available and often the most spatially and temporally precise. However, in cases where vision is not the most reliable modality, vision should follow perception, not lead it. While that is quite difficult to accomplish in healthy older adults, it is found in some clinical populations. For example, bilateral parietal lobe damage (Balint's Syndrome) can impair the ability to visually localize and attend to stimuli while leaving these abilities relatively intact in the auditory modality. Phan et al. (2000) had both healthy participants and a patient with
Balint's Syndrome localize visual and auditory stimuli that were presented simultaneously. Healthy participants were captured by the visual stimuli, which is expected given the spatial superiority of vision over audition. However, the patient's performance was captured by the auditory stimuli, which is consistent with the prediction that the modality that is most reliable and accurate will be the one whose signal is prioritized. Given the myriad unisensory and physical declines that accompany aging, it is reasonable for vision to be the most reliable; thus, it would make sense for vision to be the default modality.

Note that a similar reliance on visual processing components over somatosensory processing has been identified in older adults suffering from Parkinson's disease (PD) in order to compensate for their physical declines. Imaging studies have found that older adults with PD not only perform worse on motor imagery tasks, but also show increased brain activity in extrastriate visual areas (Helmich et al., 2007). The physical limitations of the patients, in other words, is compensated with additional neural resources drawn from visual processing. At the behavioral level, this manifests in increasing dependence on a visual processing strategy (Poliakoff et al., 2010). Accordingly, motor imagery declines in PD are typically localized to the physical actions most debilitated by PD (Dominey et al., 1995; Helmich et al., 2007). Finally, Conson et al. (2014) found that PD patients had increased difficulty in whole-body mental rotations when the rotation type required a somatosensory 'feel' for the rotated body stimuli in contrast to a more visual grasp. This finding is similar in kind to healthy older adult deficits in first-person perspectives in body schema.

Interestingly, while older adults display visual dominance, infants, and children may display the exact opposite effect a tendency to favor physical factors above visual factors. For instance, Frick et al. (2009) had 5-year-olds, 8-year-olds, 11-yearolds, and young adults ( $M$ age $=37$ years) perform a jigsaw puzzle while working a hand crank. In some trials, the crank rotation matched the expected rotation of the puzzle piece, whereas in other trials the crank rotation was in the opposite direction of the puzzle rotation. When the crank and puzzle rotations matched, performance was improved in the 5- and 8-year-old children, but not the older children or young adults, indicating that motor factors were weighted more heavily early in development. This is consistent with other studies showing that the mental imagery of young children is more heavily influenced by motor processes over visual components (Frick et al., 2005; Funk et al., 2005). Infant research using looking-measure paradigms have similarly found that physical exploration is critical for infants to perceptually discriminate visual scenes (Frick and Möhring, 2013; Möhring and Frick, 2013), suggesting that mental imagery is linked with motor expression from early on. Although there is debate on the age of onset for the visuo-motor matching (cf., Krüger and Krist, 2009; Frick et al., 2013), the evidence points to an increased influence of motor factors on perceptual judgments for infants and children. Thus, from a lifespan perspective, the integration of visual perception and action systems is complex, favoring motor influences early in life, a more leveled integration in young adult period, and diminished motor and increased visual influence in older age. 
The claim of visual dominance in older adults is based on the preponderance of evidence that we have reported in this review, although not surprisingly there are empirical results that run counter to this theme. Recall that we earlier reported an agerelated increase in MSI-based errors during the Sound-Induced Flash Illusion. Recently, McGovern et al. (2014) found that the greatest age effects were found in the fusion illusion (in which two flashes paired with one beep is experienced as one flash) compared to the fission illusion (in which one flash paired with two beeps is reported as two flashes). If the action-perception system of older adults is more heavily driven by visual processing factors, one would have expected performance to be worse in the latter condition. Additionally, results from studies testing the action-specific theory of perception, in which older adults judge hills to be steeper than young adults (Bhalla and Proffitt, 1999) and distances to be greater when walking is difficult (Sugovic and Witt, 2013), appear to run counter to visually dominated perception. If older adults were up-weighting the visual signal, environmental factors that impede physical movements should have less of an impact on perception, not more.

It is difficult to know how to interpret these contrary findings. One possibility could be that differing results are due to the relative demands of different tasks. When judging hill steepness or target distance, explicit judgments are required that may lead to age-related strategy differences, a problem which has been documented in other domains (Geary et al., 1993). These types of perceptual judgments also occur on a much larger environmental scale than tasks assessing peripersonal space, motor imagery, or action execution. Perhaps in these circumstances older adults are able to up-weight internal sensory signals to inform perception. It is important to note here that we are not arguing that older adults do not have access to internal sensory signals. Rather, we are suggesting that the internal signal is discounted relative to vision due to vision's reliability.

\section{An Embodied Cognition Model for Aging}

Our review has indicated that older adults operate with a model of embodied cognition that is differently weighted than younger adults: emphasizing visual processing components and deemphasizing bodily (tactile, kinematic, proprioceptive) factors. Although it is beyond the purview of this review article, a logical continuation would be to formally develop (perhaps via computational modeling) an explicit model of how aging alters the manifold factors operative in sensorimotor-cognitive processing. Developing such a model will be a difficult task given the range of potential age-related causal factors. First, there are a host of 'non-embodied' (as traditionally viewed) cognitive and brain factors that must play a role in how embodiment is altered in aging. As briefly stated in the beginning of this review, older adults exhibit a range of cognitive and brain-based changes. This review has focused almost exclusively on three subdomains pertinent to embodied cognition, but these subdomains are not independent of such factors. Second, social factors are likely operative in the mind-body relationship in aging, although almost no research has been conducted to determine this possibility. This is particularly important considering how negative societal views on aging (i.e., ageism) might alter performance of the older adults. If an older adult believes that he or she is out of shape or cognitively slow, we can expect that participant performance recorded through most behavioral measures will be affected (e.g., Levy, 2003). Third, older adult differences in embodied cognition may reflect cohort differences in strategy. Older adults perform tasks more conservatively, requiring increased evidence prior to making decisions (Ratcliff et al., 2004). It should be noted, however, that these age-related differences in response criterion may themselves simply reflect the age effect in embodied cognition.

We need to be cautious, then, with the concept of an agerelated visual dominance. We do not want to say that in all tasks older adults will favor visual processing. Compensation can take multiple forms depending on the context, and in cases in which the visual inputs are minimized, we would expect that older adults will take advantage of motor factors. Consider Roski et al. (2014), who examined brain activation responses in young and older adults with two task conditions: a motorbased and a visual attention task. In the motor-based task, older adults had decreased activation for motor regions but greater in visual processing regions - an expected result given the visual dominance effect in older adults. However, in the visual attention task there was the opposite effect - activation increased for motor and decreased for visual processing brain regions. In short, older adults were displaying a dedifferentiated brain response in which the distinct functionality of these regions was less defined for older adults, perhaps due to compensatory recruitment. An agerelated visual dominance effect, in other words, may simply be a compensatory draw on any and all available sensory inputs, with visual inputs being the quickest and most salient in most task conditions.

We can best understand this age-related change in sensorimotor control as a change to the internal model of older adults. The internal model is the mental calibration of many inputs, resulting in a controlled and purposeful response (Wolpert et al., 1995). Because older adults are vulnerable to cognitive, perceptual, physiological, and action-based deficits, the calibration of the internal action model is altered, with weaker physiological inputs and consequent over-reliance on visual processing. Several research groups have argued that aging results in degraded internal action models (cf., Cressman et al., 2010; Boisgontier and Nougier, 2013; Lafargue et al., 2013). Degradation in the internal action model may also explain the characteristic dedifferentiated brain response in older adults. If embodied cognitive effects in young adults reflect the efficient integration of sensory, cognitive, and motor systems, the older adult brain is characterized by the failure to recruit specialized neuronal modules and the adoption of a more diffuse cortical response (Mott et al., 2014; Roski et al., 2014). Research indicates that the older adult motor system is affected by dedifferentiation (Bernard and Seidler, 2012; Koppelmans et al., 2015; Reuter et al., 2015). Further, motor performance in older adults is affected by cerebellar changes, leading to 'degraded internal models' (Bernard and Seidler, 2014). Dedifferentiation, which is associated with a lack of specialized neuronal modules, may compromise the ability to calibrate internal and external sensory signals, resulting in inaccurate weighting. Inaccurate or altered 
weighting may explain the age-related behavioral changes in embodiment we've described in the review above.

\section{Future Directions}

As we have highlighted throughout the review, virtually every area of aging would benefit from further research using an embodied cognition perspective. Given that, here we will highlight just a few areas of practical importance. One question that has not yet been explored is the age at which changes in embodiment begin to take place. If age-related changes are based on neural dedifferentiation or compensation, then it would be expected that the changes are gradual and positively correlated with age. Relatedly, given that there are individual differences in the onset and rate of neural change with age, there should be corollary individual differences on tasks that tap into embodied effects. Indeed, there is preliminary support for this, with older adults exhibiting wide variability in the relative impact of the body during visuo-motor performance (Bloesch et al., 2013). Not surprisingly, young adults also show individual differences, which are often overlooked by only examining group differences. By expanding age groups to include middle-aged individuals and testing for individual differences, we can begin to achieve a more nuanced understanding of how and when changes in embodiment occur across the lifespan.

It is clear that older adults have altered MSI, and that this impacts not just sensory processing but also higher-level cognition and physical ability. For example, there is a link between MSI and balance, with training on one factor improving performance on the other (Setti et al., 2014; Merriman et al., 2015). Given that falls are a concern for older adults, anything that can reliably improve balance and decrease fall risk is potentially meaningful. Unfortunately, there is still a paucity of research on this topic. The MSI conditions that have been trained are visual-auditory, but given the link between visualtactile integration and action it may be the case that other types of MSI training would improve balance, and potentially other types of action as well. Visual-tactile integration is also important for successful tool use, which older adults struggle with compared to young adults. While many older adults experience benefits from the use of canes and walkers to assist in mobility, many others report that these tools hinder them and, in fact, increase their risk of falls (Bateni and Maki, 2005). If this struggle is

\section{REFERENCES}

Aizawa, K. (2015). What is this cognition that is supposed to be embodied? Philos. Psychol. 28, 1-21. doi: 10.1080/09515089.2013.875280

Alexander, N. B. (1994). Postural control in older adults. J. Am. Geriatr. Soc. 42, 93-108. doi: 10.1111/j.1532-5415.1994.tb06081.x

Amorim, M. A., Isableu, B., and Jarraya, M. (2006). Embodied spatial transformations: "body analogy" for the mental rotation of objects. J. Exp. Psychol. 135, 327-347. doi: 10.1037/0096-3445.135.3.327

Anderson, P. G., Nienhuis, B., Mulder, T., and Hulstijn, W. (1998). Are older adults more dependent on visual information in regulating self-motion than younger adults? J. Mot. Behav. 30, 104-113. doi: 10.1080/00222899809601328

Atkinson, H. H., Rosano, C., Simonsick, E. M., Williamson, J. D., Davis, C., Ambrosius, W. T., et al. (2007). Cognitive function, gait speed decline, and caused in part by a decreased ability to integrate visual-tactile information about the mobility device and bring it into the body schema, training this type of MSI in older adults may be beneficial.

Embodied cognition claims that there is a causal link between physical ability and cognition, so it should not be surprising that studies have found correlations between older adults' physical capacities and their perceptions (e.g., Sugovic and Witt, 2013). However, many of the studies explicitly examining the relationship between physical declines and the specific cognitive factors assumed to be impacted based on the predictions of EC (including the majority of the studies included in this review) are correlational, leaving unanswered the direction of the relationship or the potential for a third variable. An avenue in which this relationship has been explored experimentally is in fitness training with older adults, which has found that physical interventions such as aerobic exercise have the potential to improve cognitive performance across a variety of tasks, notably processing speed, and visuospatial ability (for recent reviews, see Bherer et al., 2013; Bamidis et al., 2014). Because these are variables that have been implicated in older adult declines on embodied tasks, improving cardiovascular health would perhaps improve embodiment in older adults as well. If this is true, older adults in better physical health should also show greater embodiment than older adults in poor health, a question which can begin to be answered by examining individual differences.

There are many avenues still available for exploration in applying the embodied cognition theoretical framework to understand aging. Indeed, it would appear that we are just scratching the surface of applications to aging, and already we are gaining a richer understanding of age-related declines and the causes of them. The history of gerontological research has focused on isolating and then assessing sensory, cognitive, and physical abilities; it is now more clearly understood that these three systems interact, and that assessing changes means assessing those interactions as well.

\section{AUTHOR CONTRIBUTIONS}

MC compiled original literature review and wrote primary manuscript. EB contributed to both literature review and manuscript preparation.

comorbidities: the health, aging and body composition study. J. Gerontol. 62, 844-850. doi: 10.1093/gerona/62.8.844

Baccarini, M., and Maravita, A. (2013). "Beyond the boundaries of the body: plasticity of the body representation following tool use," in The Hand, an Organ of the Mind: What the Manual Tells the Mental, ed. Z. Radman (Cambridge, MA: MIT Press), 77-99.

Bamidis, P. D., Vivas, A. B., Styliadis, C., Frantzidis, C., Klados, M., Schlee, W., et al. (2014). A review of physical and cognitive interventions in aging. Neurosci. Biobehav. Rev. 44, 206-220. doi: 10.1016/j.neubiorev.2014.03.019

Barrett, A. M., and Craver-Lemley, C. E. (2008). Is it what you see, or how you say it? Spatial bias in young and aged subjects. J. Int. Neuropsychol. Soc. 14, 562-570. doi: $10.1017 /$ S1355617708080764

Barrett, M. M., Doheny, E. P., Setti, A., Maguinness, C., Foran, T. G., Kenny, R. A., et al. (2013). Reduced vision selectively impairs spatial updating in 
fall-prone older adults. Multisens. Res. 26, 69-94. doi: 10.1163/22134808-0000 2412

Barsalou, L. W. (1999). Perceptual symbol systems. Behav. Brain Sci. 22, 577-660. doi: $10.1017 / \mathrm{s} 0140525 \times 99002149$

Barsalou, L. W. (2008). Grounded cognition. Annu. Rev. Psychol. 59, 617-645. doi: 10.1146/annurev.psych.59.103006.093639

Barsalou, L. W. (2016). "Situated conceptualization: theory and application," in Foundations of Embodied Cognition, eds Y. Coello and M. H. Fisher (East Sussex: Psychology Press), 11-37.

Bateni, H., and Maki, B. E. (2005). Assistive devices for balance and mobility: benefits, demands, and adverse consequences. Arch. Phys. Med. Rehabil. 86, 134-145. doi: 10.1016/j.apmr.2004.04.023

Beauchet, O., Annweiler, C., Assal, F., Bridenbaugh, S., Herrmann, F. R., Kressig, R. W., et al. (2010). Imagined timed up \& go test: a new tool to assess higherlevel gait and balance disorders in older adults? J. Neurol. Sci. 294, 102-106. doi: 10.1016/j.jns.2010.03.021

Bernard, J. A., and Seidler, R. D. (2012). Evidence for motor cortex dedifferentiation in older adults. Neurobiol. Aging 33, 1890-1899. doi: 10.1016/j.neurobiolaging. 2011.06.021

Bernard, J. A., and Seidler, R. D. (2014). Moving forward: age effects on the cerebellum underlie cognitive and motor declines. Neurosci. Biobehav. Rev. 42, 193-207. doi: 10.1016/j.neubiorev.2014.02.011

Berti, A., and Frassinetti, F. (2000). When far becomes near: remapping of space by tool use. J. Cogn. Neurosci. 12, 415-420. doi: 10.1162/089892900562237

Bhalla, M., and Proffitt, D. R. (1999). Visual-motor recalibration in geographical slant perception. J. Exp. Psychol. Hum. Percept. Perform. 25, 1076-1096. doi: 10.1037/0096-1523.25.4.1076

Bherer, L., Erickson, K. I., and Liu-Ambrose, T. (2013). A review of the effects of physical activity and exercise on cognitive and brain functions in older adults. J. Aging Res. 2013:657508. doi: 10.1155/2013/657508

Bjoertomt, O., Cowey, A., and Walsh, V. (2002). Spatial neglect in near and far space investigated by repetitive transcranial magnetic stimulation. Brain 125, 2012-2022. doi: 10.1093/brain/awf211

Blanke, O. (2012). Multisensory brain mechanisms of bodily self-consciousness. Nat. Rev. Neurosci. 13, 556-572. doi: 10.1038/nrn3292

Bloesch, E. K., Davoli, C. C., and Abrams, R. A. (2013). Age-related changes in attentional reference frames for peripersonal space. Psychol. Sci. 24, 557-561. doi: $10.1177 / 0956797612457385$

Bock, O. (2005). Components of sensorimotor adaptation in young and elderly subjects. Exp. Brain Res. 160, 259-263. doi: 10.1007/s00221-004-2133-5

Boisgontier, M. P., and Nougier, V. (2013). Ageing of internal models: from a continuous to an intermittent proprioceptive control of movement. Age 35, 1339-1355. doi: 10.1007/s11357-012-9436-4

Boisgontier, M. P., Van Halewyck, F., Corporaal, S. H., Willacker, L., Van Den Bergh, V., Beets, I. A., et al. (2014). Vision of the active limb impairs bimanual motor tracking in young and older adults. Front. Aging Neurosci. 6:320. doi: 10.3389/fnagi.2014.00320

Borella, E., Meneghetti, C., Muffato, V., and De Beni, R. (2015). Map learning and the alignment effect in young and older adults: how do they gain from having a map available while performing pointing tasks? Psychol. Res. 79, 104-119. doi: 10.1007/s00426-014-0543-y

Borghi, A. M., and Cimatti, F. (2010). Embodied cognition and beyond: acting and sensing the body. Neuropsychologia 48, 763-773. doi: 10.1016/j. neuropsychologia.2009.10.029

Borghi, A. M., Scorolli, C., Caligiore, D., Baldassarre, G., and Tummolini, L. (2013). The embodied mind extended: using words as social tools. Front. Psychol. 4:214. doi: 10.3389/fpsyg.2013.00214

Buccino, G., Binkofski, F., and Riggio, L. (2004). The mirror neuron system and action recognition. Brain Lang. 89, 370-376. doi: 10.1016/S0093-934X(03) 00356-0

Calvert, G., Spence, C., and Stein, B. E. (2004). The Handbook of Multisensory Processes. Cambridge, MA: MIT Press.

Cardinali, L., Brozzoli, C., and Farne, A. (2009). Peripersonal space and body schema: two labels for the same concept? Brain Topogr. 21, 252-260. doi: 10.1007/s10548-009-0092-7

Cavalcante, K. R., and Caramelli, P. (2009). Evaluation of the performance of normal elderly in a limb praxis protocol: influence of age, gender, and education. J. Int. Neuropsychol. Soc. 15, 618-622. doi: 10.1017/S13556177090 90663

Cerella, J., Poon, L. W., and Fozard, J. L. (1981). Mental rotation and age reconsidered. J. Gerontol. 36, 620-624. doi: 10.1093/geronj/36.5.620

Chao, L. L., and Martin, A. (2000). Rapid communication representation of manipulable man-made objects in the dorsal stream. Neuroimage 12, 478-484. doi: $10.1006 /$ nimg.2000.0635

Chemero, A. (2009). Radical Embodied Cognitive Science. Cambridge, MA: MIT Press.

Chen, P., Goedert, K. M., Murray, E., Kelly, K., Ahmeti, S., and Barrett, A. M. (2011). Spatial bias and right hemisphere function: sex-specific changes with aging. J. Int. Neuropsychol. Soc. 17, 455-462. doi: 10.1017/S13556177110 $0004 \mathrm{X}$

Cherry, B. J., Adamson, M., Duclos, A., and Hellige, J. B. (2005). Aging and individual variation in interhemispheric collaboration and hemispheric asymmetry. Aging Neuropsychol. Cogn. 12, 316-339.

Clark, A. (2010). "Memento's revenge: the extended mind, extended," in The Extended Mind, ed. R. Menary (Cambridge, MA: MIT Press), 43-66.

Clark, A. (1997). Being There: Putting Brain, Body and World Together Again. Cambridge, MA: MIT Press.

Coats, R. O., Snapp-Childs, W., Wilson, A. D., and Bingham, G. P. (2013). Perceptuo-motor learning rate declines by half from 20s to 70/80s. Exp. Brain Res. 225, 75-84. doi: 10.1007/s00221-012-3349-4

Conson, M., Trojano, L., Vitale, C., Mazzarella, E., Allocca, R., Barone, P., et al. (2014). The role of embodied simulation in mental transformation of wholebody images: evidence from Parkinson's disease. Hum. Mov. Sci. 33, 343-353. doi: 10.1016/j.humov.2013.10.006

Costello, M. C., Bloesch, E. K., Davoli, C. C., Panting, N. D., Abrams, R. A., and Brockmole, J. R. (2015). Spatial representations in older adults are not modified by action: evidence from tool use. Psychol. Aging 30, 656-668. doi: 10.1037/pag0000029

Craik, F. I. M., and Dirkx, E. (1992). Age-related differences in three tests of visual imagery. Psychol. Aging 7, 661-665. doi: 10.1037/0882-7974.7.4.661

Creem-Regehr, S. H., and Kunz, B. R. (2010). Perception and action. Wiley Interdiscip. Rev. Cogn. Sci. 1, 800-810. doi: 10.1002/wcs.82

Cressman, E. K., Salomonczyk, D., and Henriques, D. Y. (2010). Visuomotor adaptation and proprioceptive recalibration in older adults. Exp. Brain Res. 205, 533-544. doi: 10.1007/s00221-010-2392-2

Daviaux, Y., Cremoux, S., Tallet, J., Amarantini, D., Cornu, C., and Deschamps, T. (2015). An enhanced experimental procedure to rationalize on the impairment of perception of action capabilities. Psychol. Res. 80, 1-11. doi: 10.1007/s00426015-0653-1

Davoli, C. C., Brockmole, J. R., and Goujon, A. (2012a). A bias to detail: how hand position modulates visual learning and visual memory. Mem. Cogn. 40, 352-359. doi: 10.3758/s13421-011-0147-3

Davoli, C. C., Brockmole, J. R., and Witt, J. K. (2012b). Compressing perceived distance with remote tool-use: real, imagined, and remembered. J. Exp. Psychol. Hum. Percept. Perform. 38, 80-89. doi: 10.1037/a0024981

De Simone, L., Tomasino, B., Marusic, N., Eleopra, R., and Rumiati, R. I. (2013). The effects of healthy aging on mental imagery as revealed by egocentric and allocentric mental spatial transformations. Acta Psychol. 143, 146-156. doi: 10.1016/j.actpsy.2013.02.014

Decety, J. (1996). Do imagined and executed actions share the same neural substrate? Cogn. Brain Res. 3, 87-93.

Devlin, A. L., and Wilson, P. H. (2010). Adult age differences in the ability to mentally transform object and body stimuli. Neuropsychol. Dev. Cogn. B Aging Neuropsychol. Cogn. 17, 709-729. doi: 10.1080/13825585.2010. 510554

Diaconescu, A. O., Hasher, L., and McIntosh, A. R. (2013). Visual dominance and multisensory integration changes with age. Neuroimage 65, 152-166. doi: 10.1016/j.neuroimage.2012.09.057

Diersch, N., Cross, E. S., Stadler, W., Schütz-Bosbach, S., and Rieger, M. (2012). Representing others' actions: the role of expertise in the aging mind. Psychol. Res. 76, 525-541. doi: 10.1007/s00426-011-0404-x

Diersch, N., Jones, A. L., and Cross, E. S. (2016). The timing and precision of action prediction in the aging brain. Hum. Brain Mapp. 37, 54-66. doi: 10.1002/hbm. 23012 
Diersch, N., Mueller, K., Cross, E. S., Stadler, W., Rieger, M., and Schütz-Bosbach, S. (2013). Action prediction in younger versus older adults: neural correlates of motor familiarity. PLoS ONE 8:e64195. doi: 10.1371/journal.pone.0064195

DiPietro, L. (2001). Physical activity in aging: changes in patterns and their relationship to health and function. J. Gerontol. 56, 13-22. doi: 10.1093/gerona/ 56.suppl_2.13

Dominey, P., Decety, J., Broussolle, E., Chazot, G., and Jeannerod, M. (1995). Motor imagery of a lateralized sequential task is asymmetrically slowed in hemi-Parkinson's patients. Neuropsychologia 33, 727-741. doi: 10.1016/00283932(95)00008-Q

Durgin, F. H., Klein, B., Spiegel, A., Strawser, C. J., and Williams, M. (2012). The social psychology of perception experiments: hills, backpacks, glucose, and the problem of generalizability. J. Exp. Psychol. Hum. Percept. Perform. 38, 1582-1595. doi: 10.1037/a0027805

Falkenstein, M., Yordanova, J., and Kolev, V. (2006). Effects of aging on slowing of motor-response generation. Int. J. Psychophysiol. 59, 22-29. doi: 10.1016/j. ijpsycho.2005.08.004

Farnè, A., Iriki, A., and Làdavas, E. (2005). Shaping multisensory action-space with tools: evidence from patients with cross-modal extinction. Neuropsychologia 43, 238-248. doi: 10.1016/j.neuropsychologia.2004.11.010

Farnè, A., and Làdavas, E. (2000). Dynamic size-change of hand peripersonal space following tool use. Neuroreport 11, 1645-1649. doi: 10.1097/00001756200006050-00010

Farnè, A., Serino, A., and Làdavas, E. (2007). Dynamic size-change of perihand space following tool-use: determinants and spatial characteristics revealed through cross-modal extinction. Cortex 43, 436-443. doi: 10.1016/S00109452(08)70468-4

Fink, G. R., Marshall, J. C., Weiss, P. H., and Zilles, K. (2001). The neural basis of vertical and horizontal line bisection judgments: an fMRI study of normal volunteers. Neuroimage 14, S59-S67. doi: 10.1006/nimg.2001.0819

Firestone, C. (2013). How "Paternalistic" is spatial perception? Why wearing a heavy backpack doesn't-and couldn't-make hills look steeper. Perspect. Psychol. Sci. 8, 455-473. doi: 10.1177/1745691613489835

Fodor, J. D., Fodor, J. A., and Garrett, M. F. (1975). The psychological unreality of semantic representations. Linguist. Inq. 6, 515-531.

Foglia, L., and Wilson, R. A. (2013). Embodied cognition. Wiley Interdiscip. Rev. Cogn. Sci. 4, 319-325. doi: 10.1002/wcs.1226

Fozard, J. L., and Gordon-Salant, S. (2001). "Changes in vision and hearing with aging," in Handbook of the Psychology of Aging, 5th Edn, eds J. E. Birren and K. W. Schaie (San Diego, CA: Academic Press), 241-266.

Freiherr, J., Lundstrom, J. N., Habel, U., and Reetz, K. (2013). Multisensory integration mechanisms during aging. Front. Hum. Neurosci. 7:863. doi: 10. 3389/fnhum.2013.00863

Frick, A., Daum, M. M., Walser, S., and Mast, F. W. (2009). Motor processes in children's mental rotation. J. Cogn. Dev. 10, 18-40. doi: 10.1080/ 15248370902966719

Frick, A., Ferrara, K., and Newcombe, N. S. (2013). Using a touch screen paradigm to assess the development of mental rotation between $3^{1 / 2}$ and $5^{1 / 2}$ years of age. Cogn. Process. 14, 117-127. doi: 10.1007/s10339-012-0534-0

Frick, A., Huber, S., Reips, U.-D., and Krist, H. (2005). Task-specific knowledge of the law of pendulum motion in children and adults. Swiss J. Psychol. 64, 103-114. doi: 10.1024/1421-0185.64.2.103

Frick, A., and Möhring, W. (2013). Mental object rotation and motor development in 8-and 10-month-old infants. J. Exp. Child Psychol. 115, 708-720. doi: 10. 1016/j.jecp.2013.04.001

Fujii, T., Fukatsu, R., Yamadori, A., and Kimura, I. (1995). Effect of age on the line bisection test. J. Clin. Exp. Neuropsychol. 17, 941-944. doi: 10.1080/ 01688639508402443

Funk, M., Brugger, P., and Wilkening, F. (2005). Motor processes in children's imagery: the case of mental rotation of hands. Dev. Sci. 8, 402-408. doi: 10. 1111/j.1467-7687.2005.00428.x

Gabbard, C., Cacola, P., and Cordova, A. (2011). Is there an advanced aging effect on the ability to mentally represent action? Arch. Gerontol. Geriatr. 53, 206-209. doi: 10.1016/j.archger.2010.10.006

Garza, J. P., Eslinger, P. J., and Barratt, A. M. (2008). Perceptual-attentional and motor-intentional bias in near and far space. Brain Cogn. 68, 9-14. doi: 10.1016/ j.bandc.2008.02.006
Geary, D. C., Frensch, P. A., and Wiley, J. G. (1993). Simple and complex mental subtraction: strategy choice and speed-of-processing differences in younger and older adults. Psychol. Aging 8, 242-256. doi: 10.1037/0882-7974.8.2.242

Ghafouri, M., and Lestienne, F. G. (2000). Altered representation of peripersonal space in the elderly human subject: a sensorimotor approach. Neurosci. Lett. 289, 193-196. doi: 10.1016/S0304-3940(00)01280-5

Gilpin, H. R., Moseley, G. L., Stanton, T. R., and Newport, R. (2015). Evidence for distorted mental representation of the hand in osteoarthritis. Rheumatology 54, 678-682. doi: 10.1093/rheumatology/keu367

Glenberg, A. M. (2015). Few believe the world is flat: how embodiment is changing the scientific understanding of cognition. Can. J. Exp. Psychol. 69, 165-171. doi: $10.1037 /$ cep0000056

Glenberg, A. M., Witt, J. K., and Metcalfe, J. (2013). From the revolution to embodiment: 25 years of cognitive psychology. Perspect. Psychol. Sci. 8, 573585. doi: 10.1177/1745691613498098

Goble, D. J., Coxon, J. P., Wenderoth, N., Impe, A. V., and Swinnen, S. P. (2009). Proprioceptive sensibility in the elderly: degeneration, functional consequences and plastic-adaptive processes. Neurosci. Behav. Rev. 33, 271-278. doi: 10.1016/ j.neubiorev.2008.08.012

Goldman, A. I. (2012). A moderate approach to embodied cognitive science. Rev. Philos. Psychol. 3, 71-88. doi: 10.1007/s13164-012-0089-0

Grabiner, P. C., Biswas, S. T., and Grabiner, M. D. (2001). Age-related changes in spatial and temporal gait variables. Arch. Phys. Med. Rehabil. 82, 31-35. doi: 10.1053/apmr.2001.18219

Hackney, A. L., and Cinelli, M. E. (2011). Action strategies of older adults walking through apertures. Gait Posture 33, 733-736. doi: 10.1016/j.gaitpost.2011. 02.019

Hauk, O., Johnsrude, I., and Pulvermüller, F. (2004). Somatotopic representation of action words in human motor and premotor cortex. Neuron 41, 301-317. doi: 10.1016/S0896-6273(03)00838-9

Helmich, R. C., de Lange, F. P., Bloem, B. R., and Toni, I. (2007). Cerebral compensation during motor imagery in Parkinson's disease. Neuropsychologia 45, 2201-2215. doi: 10.1016/j.neuropsychologia.2007.02.024

Hertzog, C., and Rypma, B. (1991). Age differences in components of mentalrotation task performance. Bull. Psychon. Soc. 29, 209-212. doi: 10.3758/ BF03342680

Heuer, H., and Hegele, M. (2008). Adaptation to visuomotor rotations in younger and older adults. Psychol. Aging 23, 190-202. doi: 10.1037/0882-7974.23.1.190

Heuer, H., and Hegele, M. (2014). Age-related variations of visuo-motor adaptation beyond explicit knowledge. Front. Aging Neurosci. 6:152. doi: 10.3389/fnagi. 2014.00152

Heuer, H., Hegele, M., and Rand, M. K. (2013). “Age-related variations in the control of electronic tools," in Age-Differentiated Work Systems, eds C. M. Schlick, E. Frieling, and J. Wegge (Berlin: Springer), 369-390. doi: 10.1007/9783-642-35057-3_16

Hihara, S., Notoya, T., Tanaka, M., Ichinose, S., Ojima, H., Obayashi, S., et al. (2006). Extension of corticocortical afferents into the anterior bank of the intraparietal sulcus by tool-use training in adult monkeys. Neuropsychologia 44, 2636-2646. doi: 10.1016/j.neuropsychologia.2005.11.020

Hillman, C. H., Erickson, K. I., and Kramer, A. F. (2008). Be smart, exercise your heart: exercise effects on brain and cognition. Neuroscience 9, 59-65. doi: $10.1038 / \mathrm{nrn} 2298$

Hommel, B., Musseler, J., Aschersleben, G., and Prinz, W. (2001). The theory of event coding (TEC): a framework for perception and action planning. Behav. Brain Sci. 24, 849-937. doi: 10.1017/S0140525X01000103

Horak, F. B., Shupert, C. L., and Mirka, A. (1989). Components of postural dyscontrol in the elderly: a review. Neurobiol. Aging 10, 727-738. doi: 10.1016/ 0197-4580(89)90010-9

Huitema, R. B., Brouwer, W. H., Mulder, T., Dekker, R., Hof, A. L., and Postema, K. (2005). Effect of ageing on the ability to adapt to a visual distortion during walking. Gait Posture 21, 440-446. doi: 10.1016/j.gaitpost.2004.05.007

Inagaki, H., Meguro, K., Shimada, M., Ishizaki, J., Okuzumi, H., and Yamadori, A. (2002). Discrepancy between mental rotation and perspective-taking abilities in normal aging assessed by Piaget's three-mountain task. J. Clin. Exp. Neuropsychol. 24, 18-25. doi: 10.1076/jcen.24.1.18.969

Inui, N. (1997). Simple reaction times and timing of serial reactions of middle-aged and old men. Percept. Mot. Skills 84, 219-225. doi: 10.2466/pms.1997.84.1.219 
Iriki, A., Tanaka, M., and Iwamura, Y. (1996). Coding of modified body schema during tool use by macaque postcentral neurones. Neuroreport 7, 2325-2330. doi: $10.1097 / 00001756-199610020-00010$

Jansen, P., and Kaltner, S. (2014). Object-based and egocentric mental rotation performance in older adults: the importance of gender differences and motor ability. Aging Neuropsychol. Cogn. 21, 296-316. doi: 10.1080/13825585.2013. 805725

Järveläinen, J., Schuermann, M., and Hari, R. (2004). Activation of the human primary motor cortex during observation of tool use. Neuroimage 23, 187-192. doi: 10.1016/j.neuroimage.2004.06.010

Jeannerod, M. (1994). The representing brain: neural correlates of motor intention and imagery. Behav. Brain Sci. 17, 187-202. doi: 10.1017/S0140525X00034026

Jeannerod, M. (2001). Neural simulation of action: a unifying mechanism for motor cognition. Neuroimage 14, 103-109. doi: 10.1006/nimg.2001.0832

Jeannerod, M., and Anquetil, T. (2008). Putting oneself in the perspective of the other: a framework for self-other differentiation. Soc. Neurosci. 3, 356-367. doi: 10.1080/17470910701563715

Jenkins, L., Myerson, J., Joerding, J. A., and Hale, S. (2000). Converging evidence that visuospatial cognition is more age-sensitive than verbal cognition. Psychol. Aging 15, 157-175. doi: 10.1037/0882-7974.15.1.157

Jewell, G., and McCourt, M. E. (2000). Pseudoneglect: a review and meta-analysis of performance factors in line bisection tasks. Neuropsychologia 38, 93-110. doi: 10.1016/S0028-3932(99)00045-7

Kaltner, S., and Jansen, P. (2016). Developmental changes in mental rotation: a dissociation between object-based and egocentric transformations. Adv. Cogn. Psychol. 12, 67-78. doi: 10.5709/acp-0187-y

Kaltner, S., Riecke, B. E., and Jansen, P. (2014). Embodied mental rotation: a special link between egocentric transformation and the bodily self. Front. Psychol 5:505. doi: 10.3389/fpsyg.2014.00505

Kannus, P., Sievänen, H., Palvanen, M., Järvinen, T., and Parkkari, J. (2005). Prevention of falls and consequent injuries in elderly people. Lancet 366, 1885-1893. doi: 10.1016/S0140-6736(05)67604-0

Kirsch, W., and Kunde, W. (2013). Moving further moves things further away in visual perception: position-based movement planning affects distance judgments. Exp. Brain Res. 226, 431-440. doi: 10.1007/s00221-013$3455-\mathrm{y}$

Klencklen, G., Després, O., and Dufour, A. (2012). What do we know about aging and spatial cognition? Reviews and Perspectives Ageing Res. Rev. 11, 123-135. doi: 10.1016/j.arr.2011.10.001

Koppelmans, V., Hirsiger, S., Mérillat, S., Jäncke, L., and Seidler, R. D. (2015). Cerebellar gray and white matter volume and their relation with age and manual motor performance in healthy older adults. Hum. Brain Mapp. 36, 2352-2363. doi: $10.1002 / \mathrm{hbm} .22775$

Kosslyn, S. M. (1987). Seeing and imagining in the cerebral hemispheres: a computational approach. Psychol. Rev. 94, 148-175. doi: 10.1037/0033-295X. 94.2.148

Kosslyn, S. M. (1995). "Mental imagery," in An Invitation to Cognitive Science: Visual Cognition, 2nd edn, eds S. M. Kosslyn and D. N. Osherson (Cambridge, MA: MIT Press).

Kosslyn, S. M., Ganis, G., and Thompson, W. L. (2001). Neural foundations of imagery. Nat. Rev. Neurosci. 2, 635-642. doi: 10.1038/35090055

Kraeutner, S., Gionfriddo, A., Bardouille, T., and Boe, S. (2014). Motor imagerybased brain activity parallels that of motor execution: evidence from magnetic source imaging of cortical oscillations. Brain Res. 1588, 81-91. doi: 10.1016/j. brainres.2014.09.001

Kraft, E. (2012). Cognitive function, physical activity, and aging: possible biological links and implications for multimodal interventions. Aging Neuropsychol. Cogn. 19, 248-263. doi: 10.1080/13825585.2011.645010

Kramer, A. F., Humphrey, D. G., Larish, J. F., Logan, G. D., and Strayer, D. L. (1994). Aging and inhibition: beyond a unitary view of inhibitory processing in attention. Psychol. Aging 9, 491-512. doi: 10.1037/0882-7974.9. 4.491

Krüger, M., and Krist, H. (2009). Imagery and motor processes-When are they connected? The mental rotation of body parts in development. J. Cogn. Dev. 10, 239-261. doi: 10.1080/15248370903389341

Lafargue, G., Noël, M., and Luyat, M. (2013). In the elderly, failure to update internal models leads to over-optimistic predictions about upcoming actions. PLoS ONE 8:e51218. doi: 10.1371/journal.pone.0051218
Laughton, C. A., Slavin, M., Katdare, K., Nolan, L., Bean, J. F., Kerrigan, D. C., et al. (2003). Aging, muscle activity, and balance control: physiologic changes associated with balance impairment. Gait Posture 18, 101-108. doi: 10.1016/ S0966-6362(02)00200-X

Laurienti, P. J., Burdette, J. H., Maldjian, J. A., and Wallace, M. T. (2006). Enhanced multisensory integration in older adults. Neurobiol. Aging 27, 1155-1163. doi: 10.1016/j.neurobiolaging.2005.05.024

Léonard, G., and Tremblay, F. (2007). Corticomotor facilitation associated with observation, imagery and imitation of hand actions: a comparative study in young and old adults. Exp. Brain Res. 177, 167-175. doi: 10.1007/s00221-0060657-6

Lesourd, M., Baumard, J., Jarry, C., Le Gall, D., and Osiurak, F. (2016). A cognitivebased model of tool use in normal aging. Aging Neuropsychol. Cogn. doi: 10. 1080/13825585.2016.1218822 [Epub ahead of print].

Levy, B. R. (2003). Mind matters: cognitive and physical effects of aging selfstereotypes. J. Gerontol. B Psychol. Sci. Soc. Sci. 58, 203-211. doi: 10.1093/ geronb/58.4.P203

Linkenauger, S. A., Leyrer, M., Bülthoff, H. H., and Mohler, B. J. (2013). Welcome to wonderland: the influence of the size and shape of a virtual hand on the perceived size and shape of virtual objects. PLoS ONE 8:e68594. doi: 10.1371/ journal.pone.0068594

Linkenauger, S. A., Witt, J. K., Stefanucci, J. K., Bakdash, J. Z., and Proffitt, D. R. (2009). The effects of handedness and reachability on perceived distance. J. Exp. Psychol. 35, 1649-1660. doi: 10.1037/a0016875

Lopez, C., Schreyer, H. M., Preuss, N., and Mast, F. W. (2012). Vestibular stimulation modifies the body schema. Neuropsychologia 50, 1830-1837. doi: 10.1016/j.neuropsychologia.2012.04.008

Lord, S. R., and Menz, H. B. (2000). Visual contributions to postural stability in older adults. Gerontology 46, 306-310. doi: 10.1159/000022182

Lord, S. R., and Sturnieks, D. L. (2005). The physiology of falling: assessment and prevention strategies for older people. J. Sci. Med. Sport 8, 35-42. doi: 10.1016/S1440-2440(05)80022-2

Lui, F., Buccino, G., Duzzi, D., Benuzzi, F., Crisi, G., Baraldi, P., et al. (2008). Neural substrates for observing and imagining non-object-directed actions. Soc. Neurosci. 3, 261-275. doi: 10.1080/17470910701458551

Lustig, C., Hasher, L., and Zacks, R. (2007). "Inhibitory deficit theory: recent developments in a "New View," in Inhibition and Cognition, eds D. Gorfein and C. MacLeod (Washington, DC: American Psychological Association), 145-162. doi: 10.1037/11587-008

Maguinness, C., Setti, A., Roudaia, E., and Kenny, R. A. (2013). Does that look heavy to you? Perceived weight judgment in lifting actions in younger and older adults. Front. Hum. Neurosci. 7:795. doi: 10.3389/fnhum.2013.00795

Mahon, B. Z. (2015). The burden of embodied cognition. Can. J. Exp. Psychol. 69, 172-178. doi: $10.1037 /$ cep0000060

Mahoney, J. R., Holtzer, R., and Verghese, J. (2014). Visual-somatosensory integration and balance: evidence for psychophysical integrative differences in aging. Multisens. Res. 27, 17-42. doi: 10.1163/22134808-00002444

Mahoney, J. R., Li, P. C. C., Oh-Park, M., Verghese, J., and Holtzer, R. (2011). Multisensory integration across the senses in young and old adults. Brain Res. 1426, 43-53. doi: 10.1016/j.brainres.2011.09.017

Mahoney, J. R., Verghese, J., Dumas, K., Wang, C., and Holtzer, R. (2012). The effect of multisensory cues on attention in aging. Brain Res. 1472, 63-73. doi: 10.1016/j.brainres.2012.07.014

Malafouris, L. (2004). "The cognitive basis of material engagement: where brain, body and culture conflate," in Rethinking Materiality: The Engagement of Mind with the Material World, eds E. DeMarrais, C. Gosden, and C. Renfrew (Cambridge: McDonald Institute for Archaeological Research), 53-61.

Maravita, A., Husain, M., Clarke, K., and Driver, J. (2001). Reaching with a tool extends visual-tactile interactions into far space: evidence from cross-modal extinction. Neuropsychologia 39, 580-585. doi: 10.1016/S0028-3932(00)00150-0

Maravita, A., and Iriki, A. (2004). Tools for the body (schema). Trends Cogn. Sci. 8, 79-86. doi: 10.1016/j.tics.2003.12.008

Maravita, A., Spence, C., and Driver, J. (2003). Multisensory integration and the body schema: close to hand and within reach. Curr. Biol. 13, R531-R539. doi: 10.1016/S0960-9822(03)00449-4

Maravita, A., Spence, C., Kennett, S., and Driver, J. (2002). Tool-use changes multimodal spatial interactions between vision and touch in normal humans. Cognition 83, B25-B34. doi: 10.1016/S0010-0277(02)00003-3 
Maryott, J., and Sekuler, R. (2009). Age-related changes in imitating sequences of observed movements. Psychol. Aging 24, 476-486. doi: 10.1037/a0015266

Mau-Moeller, A., Behrens, M., Linder, T., Bader, R., and Bruhn, S. (2013). Age-related changes in neuromuscular function of the quadriceps muscle in physically active adults. J. Electromyogr. Kinesiol. 23, 640-648. doi: 10.1016/j. jelekin.2013.01.009

McDowd, J. M., and Craik, F. I. (1988). Effects of aging and task difficulty on divided attention performance. J. Exp. Psychol. 14, 267-280. doi: 10.1037/00961523.14.2.267

McGovern, D. P., Roudaia, E., Stapleton, J., McGinnity, T. M., and Newell, F. N. (2014). The sound-induced flash illusion reveals dissociable age-related effects in multisensory integration. Front. Aging Neurosci. 6:250. doi: 10.3389/fnagi. 2014.00250

Meegan, D. V., and Tipper, S. P. (1998). Reaching into cluttered visual environments: spatial and temporal influences of distracting objects. Q. J. Exp. Psychol. 51, 225-249. doi: 10.1080/02724989843000004

Merriman, N. A., Whyatt, C., Setti, A., Craig, C., and Newell, F. N. (2015). Successful balance training is associated with improved multisensory function in fall-prone older adults. Comput. Human Behav. 45, 192-203. doi: 10.1016/j. chb.2014.12.017

Metter, E. J., Lynch, N., Conwit, R., Lindle, R., Tobin, J., and Hurley, B. (1999). Muscle quality and age: cross-sectional and longitudinal comparisons. J. Gerontol. A Biol. Sci. Med. Sci. 54, B207-B218. doi: 10.1093/gerona/54.5.b207

Mirolli, M., and Parisi, D. (2009). Language as a cognitive tool. Minds Mach. 19, 517-528. doi: 10.1007/s11023-009-9174-2

Mizelle, J. C., and Wheaton, L. A. (2010). The neuroscience of storing and molding tool action concepts: how "plastic" is grounded cognition. Front. Psychol. 1:195. doi: $10.3389 /$ fpsyg.2010.00195

Moffat, S. D. (2009). Aging and spatial navigation: what do we know and where do we go? Neuropsychol. Rev. 19, 478-489. doi: 10.1007/s11065-009-9120-3

Möhring, W., and Frick, A. (2013). Touching up mental rotation: effects of manual experience on 6-month-old infants' mental object rotation. Child Dev. 84, 1554-1565. doi: 10.1111/cdev.12065

Mott, K. K., Alperin, B. R., Holcomb, P. J., and Daffner, K. R. (2014). Age-related decline in differentiated neural responses to rare target versus frequent standard stimuli. Brain Res. 1587, 97-111. doi: 10.1016/j.brainres.2014.08.057

Mozaz, M., Rothi, L. J. G., Anderson, J. M., Crucian, G. P., and Heilman, K. M. (2002). Postural knowledge of transitive pantomimes and intransitive gestures. J. Int. Neuropsychol. Soc. 8, 958-962. doi: 10.1017/\$1355617702870114

Mozaz, M. J., Crucian, G. P., and Heilman, K. M. (2009). Age-related changes in arm-hand postural knowledge. Cogn. Neuropsychol. 26, 675-684. doi: 10.1080/ 02643291003651571

Mulder, T., Hochstenbach, J. B. H., Van Heuvelen, M. J. G., and den Otter, A. R. (2007). Motor imagery: the relation between age and imagery capacity. Hum. Mov. Sci. 26, 203-211. doi: 10.1016/j.humov.2007.01.001

Munzert, J., Lorey, B., and Zentgraf, K. (2009). Cognitive motor processes: the role of motor imagery in the study of motor representations. Brain Res. Rev. 60, 306-326. doi: 10.1016/j.brainresrev.2008.12.024

Nedelko, V., Hassa, T., Hamzei, F., Weiller, C., Binkofski, F., Schoenfeld, M. A., et al. (2010). Age-independent activation in areas of the mirror neuron system during action observation and action imagery: a fMRI study. Restor. Neurol. Neurosci. 28, 737-747. doi: 10.3233/RNN-2010-0542

Noë, A. (2009). Out of Our Heads: Why you are not your Brain, and other Lessons From the Biology of Consciousness. New York, NY: Hill and Wang.

Obayashi, S., Suhara, T., Kawabe, K., Okauchi, T., Maeda, J., Akine, Y., et al. (2001). Functional brain mapping of monkey tool use. Neuroimage 14, 853-861. doi: $10.1006 /$ nimg.2001.0878

Owings, T. M., and Grabiner, M. D. (2004). Variability of step kinematics in young and older adults. Gait Posture 20, 26-29. doi: 10.1016/S0966-6362(03)00088-2

Owsley, C. (2011). Aging and vision. Vis. Res. 51, 1610-1622. doi: 10.1016/j.visres. 2010.10.020

Peeters, R., Simone, L., Nelissen, K., Fabbri-Destro, M., Vanduffel, W., Rizzolatti, G., et al. (2009). The representation of tool use in humans and monkeys: common and uniquely human features. J. Neurosci. 29, 11523-11539. doi: 10.1523/JNEUROSCI.2040-09.2009

Peiffer, A. M., Mozolic, J. L., Hugenschmidt, C. E., and Laurienti, P. J. (2007). Age-related multisensory enhancement in a simple audiovisual detection task. Neuroreport 18, 1077-1081. doi: 10.1097/WNR.0b013e3281e72ae7
Peigneux, P., and van der Linden, M. (1999). Influence of ageing and educational level on the prevalence of body-part-as-objects in normal subjects. J. Clin. Exp. Neuropsychol. 21, 547-552. doi: 10.1076/jcen.21.4.547.881

Personnier, P., Paizis, C., Ballay, Y., and Papaxanthis, C. (2008). Mentally represented motor actions in normal aging II. The influence of the gravityinertial context on the duration of overt and covert arm movements. Behav. Brain Res. 186, 273-283. doi: 10.1016/j.bbr.2007.08.018

Phan, M. L., Schendel, K. L., Recanzone, G. H., and Robertson, L. C. (2000). Auditory and visual spatial localization deficits following bilateral parietal lobe lesions in a patient with Balint's syndrome. J. Cogn. Neurosci. 12, 583-600. doi: $10.1162 / 089892900562354$

Philbeck, J. W., and Witt, J. K. (2015). Action-specific influences on perception and postperceptual processes: present controversies and future directions. Psychol. Bull. 141, 1120-1144. doi: 10.1037/a0039738

Poliakoff, E., Ashworth, S., Lowe, C., and Spence, C. (2006). Vision and touch in ageing: crossmodal selective attention and visuotactile spatial interactions. Neuropsychologia 44, 507-517. doi: 10.1016/j.neuropsychologia.2005.07.004

Poliakoff, E., Galpin, A. J., Dick, J. P. R., and Tipper, S. P. (2010). Does Parkinson's disease affect judgement about another person's action? Exp. Brain Res. 204, 327-331. doi: 10.1007/s00221-009-1976-1

Posner, M. I., Nissen, M. J., and Klein, R. M. (1976). Visual dominance: an information-processing account of its origins and significance. Psychol. Rev. 83, 157-171. doi: 10.1037/0033-295X.83.2.157

Prioli, A. C., Freitas Júnior, P. B., and Barela, J. E. A. (2005). Physical activity and postural control in the elderly: coupling between visual information and body sway. Gerontology 51, 145-148. doi: 10.1159/000083984

Proffitt, D. R. (2006). Embodied perception and the economy of action. Perspect. Psychol. Sci. 1, 110-122. doi: 10.1111/j.1745-6916.2006.00008.x

Proffitt, D. R., and Linkenauger, S. A. (2013). "Perception viewed as a phenotypic expression," in Action Science: Foundations of an Emerging Discipline, eds W. Prinz, M. Beisert, and A. Herwig (Cambridge, MA: MIT Press), 171-227. doi: 10.7551/mitpress/9780262018555.003.0007

Proffitt, D. R., Stefanucci, J., Banton, T., and Epstein, W. (2003). The role of effort in perceiving distance. Psychol. Sci. 14, 106-112. doi: 10.1111/1467-9280.t01-101427

Proske, U., and Gandevia, S. C. (2012). The proprioceptive senses: their roles in signaling body shape, body position and movement, and muscle force. Physiol. Rev. 92, 1651-1697. doi: 10.1152/physrev.00048.2011

Pylyshyn, Z. W. (1984). Computation and Cognition. Cambridge, MA: MIT Press.

Rand, M. K., and Heuer, H. (2013). Implicit and explicit representations of hand position in tool use. PLOS ONE 8:e68471. doi: 10.1371/journal.pone.0068471

Rand, M. K., Wang, L., Müsseler, J., and Heuer, H. (2013). Vision and proprioception in action monitoring by young and older adults. Neurobiol. Aging 34, 1864-1872. doi: 10.1016/j.neurobiolaging.2013.01.021

Ratcliff, R., Thapar, A., Gomez, P., and McKoon, G. (2004). A diffusion model analysis of the effects of aging in the lexical-decision task. Psychol. Aging 19, 278-289. doi: 10.1037/0882-7974.19.2.278

Reed, C. L., Betz, R., Garza, J. P., and Roberts, R. J. (2010). Grab it! Biased attention in functional hand and tool space. Atten. Percept. Psychophys. 72, 236-245. doi: 10.3758/APP.72.1.236

Reed, C. L., and Farah, M. J. (1995). The psychological reality of the body schema: a test with normal participants. J. Exp. Psychol. 21, 334-343. doi: 10.1037/00961523.21.2.334

Reuter, E. M., Behrens, M., and Zschorlich, V. R. (2015). Age-related differences in corticomotor facilitation indicate dedifferentiation in motor planning. Exp. Gerontol. 65, 79-84. doi: 10.1016/j.exger.2015.03.008

Reynaud, E., Lesourd, M., Navarro, J., and Osiurak, F. (2016). On the neurocognitive origins of human tool use: a critical review of neuroimaging data. Neurosci. Biobehav. Rev. 64, 421-437. doi: 10.1016/j.neubiorev.2016. 03.009

Richardson, M. J., Shockley, K., Fajen, B. R., Riley, M. A., and Turvey, M. T. (2008). "Ecological psychology: six principles for an embodied-embedded approach to behavior," in Handbook of Cognitive Science: An Embodied Approach, eds P. Calvo and T. Gomila (Oxford: Elsevier), 161-187. doi: 10.1016/b978-0-08046616-3.00009-8

Roberts, K. L., and Allen, H. A. (2016). Perception and cognition in the ageing brain: a brief review of the short- and long-term links between perceptual and cognitive decline. Front. Aging Neurosci. 8:39. doi: 10.3389/fnagi.2016.00039 
Roberts, S. B., and Rosenberg, I. (2006). Nutrition and aging: changes in the regulation of energy metabolism with aging. Physiol. Rev. 86, 651-667. doi: 10.1152/physrev.00019.2005

Roski, C., Caspers, S., Lux, S., Hoffstaedter, F., Bergs, R., Amunts, K., et al. (2014). Activation shift in elderly subjects across functional systems: an fMRI study. Brain Struct. Funct. 219, 707-718. doi: 10.1007/s00429-013-0530-x

Saimpont, A., Malouin, F., Tousignant, B., and Jackson, P. L. (2013). Motor imagery and aging. J. Mot. Behav. 45, 21-29. doi: 10.1080/00222895.2012.740098

Saimpont, A., Pozzo, T., and Papaxanthis, C. (2009). Aging affects the mental rotation of left and right hands. PLoS ONE 4:e6714. doi: 10.1371/journal.pone. 0006714

Salthouse, T. A. (1996). The processing-speed theory of adult age differences in cognition. Psychol. Rev. 103, 403-428. doi: 10.1037/0033-295X.103.3.403

Schnall, S., Zadra, J. R., and Proffitt, D. R. (2010). Direct evidence for the economy of action: glucose and the perception of geographical slant. Perception 39, 464-482. doi: 10.1068/p6445

Seidler, R. D., Bernard, J. A., Burutolu, T. B., Fling, B. W., Gordon, M. T., Gwin, J. T., et al. (2010). Motor control and aging: links to age-related brain structural, functional, and biochemical effects. Neurosci. Biobehav. Rev. 34, 721-733. doi: 10.1016/j.neubiorev.2009.10.005

Setti, A., Burke, K. E., Kenny, R. A., and Newell, F. N. (2011). Is inefficient multisensory processing associated with falls in older people? Exp. Brain Res. 209, 375-384. doi: 10.1007/s00221-011-2560-Z

Setti, A., Stapleton, J., Leahy, D., Walsh, C., Kenny, R. A., and Newell, F. N. (2014). Improving the efficiency of multisensory integration in older adults: audio-visual temporal discrimination training reduces susceptibility to the sound-induced flash illusion. Neuropsychologia 61, 259-268. doi: 10.1016/j. neuropsychologia.2014.06.027

Sharma, N., and Baron, J. C. (2014). Effects of healthy ageing on activation pattern within the primary motor cortex during movement and motor imagery: an fMRI study. PLoS ONE 9:e88443. doi: 10.1371/journal.pone.0088443

Shepard, R. N., and Metzler, J. (1971). Mental rotation of three-dimensional objects. Science 171, 701-703. doi: 10.1126/science.171.3972.701

Sit, R. A., and Fisk, A. D. (1999). Age-related performance in a multiple-task environment. Hum. Factors 41, 26-34. doi: 10.1518/001872099779577345

Ska, B., and Nespoulous, J. L. (1987). Pantomimes and aging. J. Clin. Exp. Neuropsychol. 9, 754-766. doi: 10.1080/01688638708405214

Skoura, X., Papaxanthis, C., Vinter, A., and Pozzo, T. (2005). Mentally represented motor actions in normal aging I. Age effects on the temporal features of overt and covert execution of actions. Behav. Brain Res. 165, 229-239. doi: 10.1016/j. bbr.2005.07.023

Skoura, X., Personnier, P., Vinter, A., Pozzo, T., and Papaxanthis, C. (2008). Decline in motor prediction in elderly subjects: right versus left arm differences in mentally simulated motor actions. Cortex 44, 1271-1278. doi: 10.1016/j.cortex. 2007.07.008

Sparrow, W. A., Bradshaw, E. J., Lamoureux, E., and Tirosh, O. (2002). Ageing effects on the attention demands of walking. Hum. Mov. Sci. 21, 961-972. doi: 10.1016/S0167-9457(02)00154-9

Stapleton, J., Setti, A., Doheny, E. P., Kenny, R. A., and Newell, F. N. (2014). A standing posture is associated with increased susceptibility to the soundinduced flash illusion in fall-prone older adults. Exp. Brain Res. 232, 423-434. doi: 10.1007/s00221-013-3750-7

Steggemann, Y., Engbert, K., and Weigelt, M. (2011). Selective effects of motor expertise in mental body rotation tasks: comparing object-based and perspective transformations. Brain Cogn. 76, 97-105. doi: 10.1016/j.bandc.2011. 02.013

Studenski, S., Perera, S., Patel, K., Rosano, C., Faulkner, K., Inzitari, M., et al. (2011). Gait speed and survival in older adults. JAMA 305, 50-58. doi: 10.1001/jama. 2010.1923

Sugovic, M., and Witt, J. K. (2013). An older view on distance perception: older adults perceive walkable extents as farther. Exp. Brain Res. 226, 383-391. doi: 10.1007/s00221-013-3447-y

Szameitat, A. J., Shen, S., and Sterr, A. (2007). Motor imagery of complex everyday movements. An fMRI study. Neuroimage 34, 702-713. doi: 10.1016/ j.neuroimage.2006.09.033

Teixeira, L. A., and Lima, S. (2009). Greater specificity of sensorimotor learning in the elderly when acquiring an interceptive task. Curr. Aging Sci. 2, 60-66. doi: $10.2174 / 1874609810902010060$
Thompson, L. A., and Malloy, D. (2004). Attention resources and visible speech encoding in older and younger adults. Exp. Aging Res. 30, 241-252. doi: 10.1080/ 03610730490447877

Tinetti, M. E. (1986). Performance-oriented assessment of mobility problems in elderly patients. J. Am. Geriatr. Soc. 34, 119-126. doi: 10.1111/j.1532-5415.1986. tb05480.x

Tipper, S. P., Lortie, C., and Baylis, G. C. (1992). Selective reaching: evidence for action-centered attention. J. Exp. Psychol. 18, 891-905. doi: 10.1037/0096-1523. 18.4.891

Toledo, D. R., and Barela, J. A. (2014). Age-related differences in postural control: effects of the complexity of visual manipulation and sensorimotor contribution to postural performance. Exp. Brain Res. 232, 493-502. doi: 10.1007/s00221013-3756-1

Vallet, G. T. (2015). Embodied cognition of aging. Front. Psychol. 6:6. doi: 10.3389/ fpsyg. 2015.00463

Vallet, G. T., Simard, M., and Versace, R. (2011). Sensory-dependent knowledge in young and elderly adults: arguments from the cross-modal priming effect. Curr. Aging Sci. 4, 137-149. doi: 10.2174/1874609811104020137

Vallet, G. T., Simard, M., Versace, R., and Mazza, S. (2013). The perceptual nature of audiovisual interactions for semantic knowledge in young and elderly adults. Acta Psychol. 143, 253-260. doi: 10.1016/j.actpsy.2013.04.009

Varnava, A., McCarthy, M., and Beaumont, J. G. (2002). Line bisection in normal adults: direction of attentional bias for near and far space. Neuropsychologia 40 , 1372-1378. doi: 10.1016/S0028-3932(01)00204-4

Vaughan, L., Zurlo, F., and Ravussin, E. (1991). Aging and energy expenditure. Am. J. Clin. Nutr. 53, 821-825.

Verhaeghen, P., and Cerella, J. (2002). Aging, executive control, and attention: a review of meta-analyses. Neurosci. Biobehav. Rev. 26, 849-857. doi: 10.1016/ S0149-7634(02)00071-4

Visser, M., Pluijm, S. M. F., Stel, V. S., Bosscher, R. J., and Deeg, D. J. H. (2002). Physical activity as a determinant of change in mobility performance: the longitudinal aging study Amsterdam. J. Am. Geriatr. Soc. 50, 1774-1781. doi: 10.1046/j.1532-5415.2002.50504.x

Wade, M. G., Lindquist, R., Taylor, J. R., and Treat-Jacobson, D. (1995). Optical flow, spatial orientation, and the control of posture in the elderly. J. Gerontol. B Psychol. Sci. Soc. Sci. 50, 51-54. doi: 10.1093/geronb/50B.1.P51

Walsh, L. D., Moseley, G. L., Taylor, J. L., and Gandevia, S. C. (2011). Proprioceptive signals contribute to the sense of body ownership. J. Physiol. 12, 3009-3021. doi: 10.1113/jphysiol.2011.204941

Wickremaratchi, M. M., and Llewelyn, J. G. (2006). Effects of ageing on touch. Postgrad. Med. J. 82, 301-304. doi: 10.1136/pgmj.2005.039651

Wilson, M. (2010). The re-tooled mind: how culture re-engineers cognition. Soc. Cogn. Affect. Neurosci. 5, 180-187. doi: 10.1093/scan/nsp054

Wilson, M. M. G., and Morley, J. E. (2003). Invited review: aging and energy balance. J. Appl. Physiol. 95, 1728-1736. doi: 10.1152/japplphysiol.00313. 2003

Wilson-Mendenhall, C. D., Simmons, W. K., Martin, A., and Barsalou, L. W. (2013). Contextual processing of abstract concepts reveals neural representations of nonlinguistic semantic content. J. Cogn. Neurosci. 25, $920-$ 935. doi: 10.1162/jocn_a_00361

Witt, J. K. (2011). Action's effect on perception. Curr. Dir. Psychol. Sci. 20, 201-206. doi: $10.1177 / 0963721411408770$

Witt, J. K., and Brockmole, J. R. (2012). Action alters object identification: wielding a gun increases the bias to see guns. J. Exp. Psychol. 38, 1159-1167. doi: 10.1037/ a0027881

Witt, J. K., and Dorsch, T. E. (2009). Kicking to bigger uprights: field goal kicking performance influences perceived size. Perception 38, 1328-1340. doi: 10.1068/ p6325

Witt, J. K., Linkenauger, S. A., Bakdash, J. Z., Augustyn, J. S., Cook, A., and Proffitt, D. R. (2009). The long road of pain: chronic pain increases perceived distance. Exp. Brain Res. 192, 145-148. doi: 10.1007/s00221-0081594-3

Witt, J. K., Linkenauger, S. A., and Wickens, C. D. (2016). Action-specific effects in perception and their potential applications: a reply to commentaries. J. Appl. Res. Mem. Cogn. 1, 88-93. doi: 10.1016/j.jarmac.2016.01.002

Witt, J. K., Linkenauger, S. A., Bakdash, J. Z., and Proffitt, D. R. (2008). Putting to a bigger hole: golf performance relates to perceived size. Psychon. Bull. Rev. 15, 581-585. doi: 10.3758/PBR.15.3.581 
Witt, J. K., Proffitt, D. R., and Epstein, W. (2004). Perceiving distance: a role of effort and intent. Perception 33, 577-590. doi: 10.1068/p5090

Witt, J. K., Proffitt, D. R., and Epstein, W. (2005). Tool use affects perceived distance, but only when you intend to use it. J. Exp. Psychol. 31, 880-888. doi: 10.1037/0096-1523.31.5.880

Witt, J. K., and Proffitt, D. R. (2005). See the ball, hit the ball: apparent ball size is correlated with batting average. Psychol. Sci. 16, 937-938. doi: 10.1111/j.14679280.2005.01640.x

Witt, J. K., and Sugovic, M. (2010). Performance and ease influence perceived speed. Perception 39, 1341-1353. doi: 10.1068/p6699

Wolpert, D. M. (1997). Computational approaches to motor control. Trends Cogn. Sci. 1, 209-216. doi: 10.1016/S1364-6613(97)01070-X

Wolpert, D. M., Ghahramani, Z., and Jordan, M. I. (1995). An internal model for sensorimotor integration. Science 269, 1880-1882. doi: 10.1126/science. 7569931

Wolpert, D. M., and Kawato, M. (1998). Multiple paired forward and inverse models for motor control. Neural Netw. 11, 1317-1329. doi: 10.1016/S08936080(98)00066-5

Yamamoto, N., and DeGirolamo, G. J. (2012). Differential effects of aging on spatial learning through exploratory navigation and map reading. Front. Aging Neurosci. 4:14. doi: 10.3389/fnagi.2012.00014
Zacks, R. T. (1989). Working memory, comprehension, and aging: a review and a new view. Psychol. Learn. Motiv. 22, 193-225.

Zapparoli, L., Invernizzi, P., Gandola, M., Verardi, M., Berlingeri, M., Sberna, M., et al. (2013). Mental images across the adult lifespan: a behavioural and fMRI investigation of motor execution and motor imagery. Exp. Brain Res. 224, 519-540. doi: 10.1007/s00221-012-3331-1

Zapparoli, L., Saetta, G., De Santis, C., Gandola, M., Zerbi, A., Banfi, G., et al. (2016). When I am (almost) 64: the effect of normal ageing on implicit motor imagery in young elderlies. Behav. Brain Res. 303, 137-151. doi: 10.1016/j.bbr. 2016.01.058

Conflict of Interest Statement: The authors declare that the research was conducted in the absence of any commercial or financial relationships that could be construed as a potential conflict of interest.

Copyright $(\odot 2017$ Costello and Bloesch. This is an open-access article distributed under the terms of the Creative Commons Attribution License (CC BY). The use, distribution or reproduction in other forums is permitted, provided the original author(s) or licensor are credited and that the original publication in this journal is cited, in accordance with accepted academic practice. No use, distribution or reproduction is permitted which does not comply with these terms. 


\title{
Down with Retirement: Implications of Embodied Cognition for Healthy Aging
}

\author{
Bernhard Hommel ${ }^{1 *}$ and Armin Kibele ${ }^{2}$ \\ ${ }^{1}$ Cognitive Psychology Unit and Leiden Institute for Brain and Cognition, Leiden University, Leiden, Netherlands, ${ }^{2}$ Institute \\ for Sports and Sport Science, University of Kassel, Kassel, Germany
}

Cognitive and neurocognitive approaches to human healthy aging attribute age-related decline to the biologically caused loss of cognitive-control functions. However, an embodied-cognition approach to aging implies a more interactive view according to which cognitive control emerges from, and relies on a person's active encounters with his or her physical and social environment. We argue that the availability of cognitivecontrol resources does not only rely on biological processes but also on the degree of active maintenance, that is, on the systematic use of the available control resources. Unfortunately, there is evidence that the degree of actual use might systematically underestimate resource availability, which implies that elderly individuals do not fully

OPEN ACCESS

Edited by:

Anna M. Borghi,

University of Bologna, Italy

Reviewed by:

Elisa Di Rosa,

University of Padua, Italy

John George Grundy,

York University, Canada

*Correspondence:

Bernhard Hommel hommel@fsw.leidenuniv.nl

Specialty section: This article was submitted to Cognition,

a section of the journal

Frontiers in Psychology

Received: 25 May 2016

Accepted: 26 July 2016

Published: 09 August 2016

Citation:

Hommel B and Kibele A (2016) Down with Retirement: Implications of Embodied Cognition for Healthy

Aging. Front. Psychol. 7:1184.

doi: 10.3389/fpsyg.2016.01184 exploit their cognitive potential. We discuss evidence for this possibility from three aging-related issues: the reduction of dopaminergic supply, loneliness, and the loss of body strength. All three phenomena point to a downward spiral, in which losses of cognitive-control resources do not only directly impair performance but also more indirectly discourage individuals from making use of them, which in turn suggests underuse and a lack of maintenance-leading to further loss. On the positive side, the possibility of underuse points to not yet fully exploited reservoirs of cognitive control, which calls for more systematic theorizing and experimentation on how cognitive control can be enhanced, as well as for reconsiderations of societal practices that are likely to undermine the active maintenance of control resources - such as retirement laws.

Keywords: aging, aging and longetivity, cognitive enhancement, retirement, loneliness, dopamine, body strength, training

Medical, societal, and economic progress has led to a rather dramatic increase of longevity and population aging in Western societies. Even so-called "healthy aging" often comes with noticeable decline in psychological and physiological functions, however. Particularly problematic is the increasing impairment of cognitive-control functions underlying goal-directed planning, impulse control, working memory, and related processes, which goes hand-in-hand with the loss of the underlying frontal and striatal dopaminergic supply (Umegaki et al., 2008; Cools and D'Esposito, 2011). Myriads of aging studies have concentrated on the targeted processes and the degree of impairment as a function of age(ing). As indicated by the three boxes of Figure 1, this approach is based on the idea that aging reduces the amount and/or quality of available cognitive-control resources, which in turn impairs the quality of the person's actions and the resulting performance 
on control-heavy tasks, be it in everyday life or laboratory challenges designed to assess control abilities.

While this approach seems to make perfect sense, it underestimates the complexity of the interactions between control processes and their application by failing to take the embodiment of cognitive control into consideration. The idea that human cognition is embodied relies on the assumption that cognitive processes and functions should not just be taken as a given, as the common information-processing approach to cognition suggests, but as abilities that emerge from active exchange with one's physical and social environment (Wilson, 2002; Hommel, 2015, 2016). Among other things, this perspective draws attention to the question how people deal with the emergence or disappearance of particular abilities, and what they (could) do to compensate for cognitive and other impairments. Applied to cognitive aging, this suggests to consider natural/biological reductions of cognitive resources not as a given but as a change of the interaction between individual and environment. In other words, cognitive control resources should be considered as both an independent variable (that determines how well control challenges can be met) and a dependent variable (that is affected by dealing with these challenges).

In the present context, we focus on two processes that target cognitive control and that are likely to play a crucial role in cognitive aging. One of these processes is captured by the upper "cognitive effects" route in the figure. If cognitive control is mainly taken as a resource that is reduced in availability as age increases, all that an agent could do to maintain some level of quality of his or her actions and performance is to reduce the amount of required capacity-either by overlearning the action or by choosing less control-demanding actions. From an embodied perspective, however, the connection between cognitive control and action is not uni- but bidirectional. This means that actively exerting cognitive control implies the effective exercise of the underlying processes, which in turn promotes their maintenance. If so, control capacities would not need to be seen as fixed and exclusively regulated by biological factors but, rather, as depending on active use, not much different from a muscle. Even if this may not fully compensate for biological constraints it could very well reduce and dampen their impact.

The other process we focus on is captured by the lower "motivational effects" route in the figure. There is increasing evidence that self-representation and perceived agency relies on active control of one's body and its effects on the environment (Hommel, 2015; Ma and Hommel, 2015). This in turn suggests that perceiving oneself as an agent need not be taken as a given and active agency as a consequence, but rather as emerging from one's interactions with one's environment. With respect to aging, this suggests that the reduced availability of cognitive control resources may not only have direct effects on the quality of actions and the resulting performance, but may also have indirect repercussions for the availability of cognitive control and/or the motivational forces necessary to make active use of them. Abandoning particular actions would lead to perceiving oneself as "someone not performing these kinds of actions" - as a (partial) non-agent that is. If we assume that the motivation to make active use of one's control resources relies on one's self-representation as

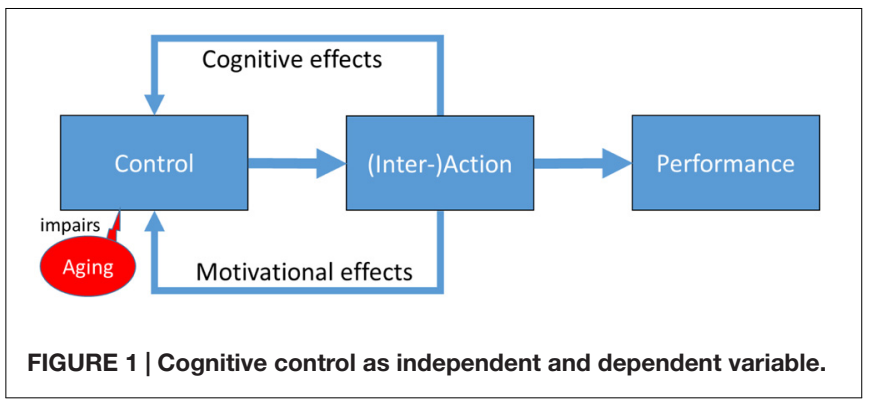

an active agent, this implies that age-related inactivity can lead to the underuse of available control resources. Indeed, there is increasing evidence for substantial interactions between cognitive capacity and motivation in general and in aging in particular (for a comprehensive overview, see Braver et al., 2014), and increasing support for the possibility that substantial amounts of age-related performance deficits actually reflect motivational impairments (e.g., Ennis et al., 2013).

In the following, we will elaborate these considerations, and discuss the (often still preliminary) evidence for the existence of both cognitive and motivational effects on cognitive control, with respect to three important aging-related (and to some degree interrelated) phenomena.

\section{AGING AND DOPAMINERGIC SUPPLY}

Many cognitive processes show some degree of aging-related decline but cognitive-control processes, which orchestrate the more basic processes, are hit particularly hard. While it is difficult to tell cause from effect in this matter, this is likely to do with the particularly strong shrinkage of the frontal lobe, which houses many components of control networks, and the reduction of dopaminergic supply, which is fueling control structures and plays a key role in integrating control relevant cognitive and motivational processes and networks (Braver et al., 2014), with increasing age. Hence, there is ample evidence for aging-related reductions of control-specific resources.

But there is also evidence that engaging in cognitive control might compensate for at least some of these reductions. While tonic dopaminergic activity is assumed to energize exploratory behavior (Niv et al., 2006), engaging in exploratory behavior seems to increase phasic dopaminergic activity (Düzel et al., 2010) - a kind of self-maintaining loop. Unfortunately, the more elderly become aware of reduced cognitive resources, the more they avoid exploring situations that create uncertainty and surprise (de Bruin et al., 2010). This suggests that aging individuals may not fully exploit their potential to refill control resources through actively exposing themselves to as much uncertainty and surprise as possible-and thus reduce cognitive effects of control exercise.

In addition to such cognitive effects, motivational effects might also be involved. It has been argued that the motivation to expose oneself to new situations and environments may rely on the ability to anticipate and build predictive models based on hippocampal episodic memory (Düzel et al., 2010). However, 
extrapolating previous experience to create models of the future requires the availability of such experience, which in turn calls for the very activities that elderly seek to avoid with increasing age. The result is again a downward spiral that prevents elderly from making full use of their remaining cognitive potential.

\section{AGING AND LONELINESS}

Feelings of social isolation and loneliness contribute significantly to the risk of elderly individuals to develop depressive symptoms and mental ill-health (Cacioppo et al., 2006), and reduced social interaction is associated with anxiety and decreases in cognitive functioning (Barnes et al., 2006). This has motivated numerous interventions to increase social interaction in older persons, unfortunately with little success so far (Dickens et al., 2011)-presumably for two reasons. First, aging is associated with reduced mobility and progressive mortality of friends and peers. This objectively provides elderly with fewer opportunities to expose themselves to social encounters and engage in social communication. Social interactions are prime examples for situations with a particularly high degree of uncertainty, which renders them ideal for practicing the very cognitive-control functions that are the most endangered in the aging individual. The natural loss of social networks with increasing age has thus particularly dramatic consequences for the elderly: while maintaining their cognitive-control functions would actually call for more interaction, they get less-an underuse of the cognitive training effect that social interactions provide.

There are also reasons to assume that motivational effects play a role. Fewer opportunities to engage in social interactions lead to a subjective loss of communicative abilities and coping capabilities, and to stronger feelings of insecurity and reduced feelings of safety (Gabriel and Bowling, 2004). This is likely to render social situations less and less rewarding (cf., Düzel et al., 2010), which will in turn make elderly individuals avoid social situations, presumably even more than justified by the actual loss of social skills. In any case, we see the same vicious circle: Objective losses of abilities and opportunities to practice control skills lead to the increased avoidance of situations in which such skills could be practiced and their impairment could be compensated for.

\section{AGING AND BODY STRENGTH}

Age-related loss of body strength has been reported in numerous studies (e.g., Mitchell et al., 2012). This loss is associated with impaired postural control and continuously increasing risk of falling (e.g., Granacher et al., 2008), which both can strongly limit the opportunities to engage in physical and social interactions. Losing body strength can impair performance in various ways. For one, it reduces the quality and accuracy of motor behavior, and by making the translation of action plans into overt behavior less predictable. For another, it increases the control demands of physical and social action, which together with the naturally decreasing control resources increasingly limits the action repertoire. Even actions as wellpracticed as walking can become de-automatized and controldemanding ( $\mathrm{Li}$ and Lindenberger, 2002), and often recruit more extended brain areas as age increases. This in turn puts increasingly high demands on the increasingly impaired cognitive-control processes, as indicated by observations that declines in postural control and risk of falling go hand in hand with decreases in cognitive functioning (e.g., Hsu et al., 2012; Best et al., 2016) and that performance on cognitive-control tasks predicts the risk of falling in the elderly (Buracchio et al., 2011).

As one would expect, strength training has the potential to maintain and enhance body strength in both young and older individuals, and training programs targeting strength, balance, and coordination can improve postural control and prevent falling (e.g., Barry and Carson, 2004; Granacher et al., 2011). However, more interesting for present purposes are findings that body-strength training improves cognitive functioning and conflict resolution (e.g., Liu-Ambrose and Donaldson, 2009; Liu-Ambrose et al., 2010). This suggests that supporting bodily functions to improve physical interactions with one's environment can feed back to cognitive control and help maintaining its functioning-as the cognitive feedback loop of our model suggests.

Though admittedly still somewhat indirect, there is some evidence pointing to the possibility of a motivational feedback loop as well. In younger adults, posture has been demonstrated to affect emotional and motivational behavior and decisionmaking (Neumann et al., 2003), commonly in the sense that approach-associated postures lead to more positive emotions and evaluations than avoidance-associated postures do. More recent studies have revealed that posture also affects self-representation. For instance, at least individuals from Western societies have a greater sense of power when assuming expansive posters, such as standing upright and spreading out their hands or feet (e.g., Park et al., 2013). Importantly, if we consider how posture is affected by aging, it makes sense to expect that decreased posture control is associated with more negative emotions, more pessimistic judgments, and a reduced sense of power. Given that perceived self-efficacy is a key predictor of the likelihood to engage in body-related training at higher age (e.g., Schutzer and Graves, 2004; Costello et al., 2011), it makes sense to assume the existence of a motivational loop from interaction to cognitive control as well: perceiving oneself as being increasingly powerless and inefficient can prevent people from engaging in activities suited to overcome these deficits at least to some degree (cf., McAuley and Blissmer, 2010; Falck et al., 2016).

\section{IMPLICATIONS}

We hope that these examples illustrate the interdependence of cognitive control and its active use during the aging process. Consideration of this mutual interdependence suggests to conceive of cognitive control not as an independent variable that determines the quality of control-demanding performance but, 
rather, as a skill that emerges from and relies on interactions with one's physical and social environment. Accordingly, aging-related losses in control resources may not only reflect natural decline but also the lack of exploiting one's potential to counteract this decline by making use of one's remaining control abilities. Indeed, given that elderly individuals are biased toward affectively positive events (the positivity effect: Reed et al., 2014) and find cognitive effort more costly (Westbrook et al., 2013), it makes sense to assume that they are systematically motivated to under-use their cognitive capacity.

Before discussing the implications of this insight, we note that our arguments are rather conservative. Given that functional and neural descriptions of the same system are theoretically equivalent, finding that functional decline is accompanied by cortical shrinking and dopaminergic dry-out does not necessarily imply that the latter is causing the former, it may just as well be that both functional and biological decline is but the expression of the under-use of one's cognitive potential. For the sake of the argument, we will leave this attractively radical possibility aside, however, and concentrate on our main point: that control needs active maintenance and that elderly may not exploit their full potential to engage therein.

According to a pessimistic interpretation of our reasoning one may be tempted to leave the basic rationale of current aging research intact and simply add the lack of maintenance to the list of dependent measures. According to this view, biological aging leads to both a reduction of cognitive resources and the loss of the ability to efficiently cope with this reduction. But a more optimistic interpretation is also possible. Even if one buys into the assumption that immutable biological factors cause reductions of cognitive-control resources, one could still seek to support the elderly individual's ability to deal with, and at least partially compensate for such reductions. Attempts to do so may raise ethical issues. Note that many, if not all failures to exploit one's potential to practice cognitive control arguably reflect individual preferences: people who feel vulnerable simply do not like to be exposed to situations that they think might reveal these vulnerabilities. Would it be socially responsible and ethically acceptable to encourage elderly with reduced cognitive resources to actively seek cognitively challenging, demanding

\section{REFERENCES}

Barnes, M., Blom, A., Cox, K., and Lessof, C. (2006). The Social Exclusion of Older People: Evidence from the First wave of the English Longitudinal Study of Ageing (Elsa). London: Office of the Deputy Prime Minister.

Barry, B. K., and Carson, R. G. (2004). Transfer of resistance training to enhance rapid coordinated force production by older adults. Exp. Brain Res. 159, 225-238. doi: 10.1007/s00221-004-1950-x

Best, J. R., Liu-Ambrose, T., Boudreau, R. M., Ayonayon, H. N., Satterfield, S., Simonsick, E. M., et al. (2016). An evaluation of the longitudinal, bidirectional associations between gait speed and cognition in older women and men. J. Gerontol. A Biol. Sci. Med. Sci. doi: 10.1093/gerona/glw066 [Epub ahead of print].

Bonsang, E., Adam, S., and Perelman, S. (2012). Does retirement affect cognitive functioning? J. Health Econ. 31, 490-501. doi: 10.1016/j.jhealeco.2012. 03.005

Braver, T. S., Krug, M. K., Chiew, K. S., Koolm, W., Westbrook, J. A., Clement, N. J., et al. (2014). Mechanisms of motivation-cognition interaction: challenges and situations? Even if doing so would reduce the challenge on the long run and turn the downward spiral into an upward spiral, encouraging needy individuals to take this step raises a number of issues that, we do not deny.

And yet, we strongly believe that a more serious consideration of the interdependence between cognitive control and physical and social interactions in theory, experimental practice, and societal reality is badly needed. With respect to research, we need a more systematic investigation and understanding of how the use of cognitive control processes affects their availability and efficiency. With respect to societal conditions, we need to ask whether policy provides sufficient support for self-empowerment of aging individuals. From an embodiment perspective, the probably most effective strike against human cognitive control abilities are retirement laws. In fact, retirement systematically undermines possible efforts to maintain the control abilities under biological challenge by eliminating various (job-related) sources of uncertainty and surprise, cutting substantial parts of the social network, at least for a substantial part of the day, and by possibly taking away opportunities for physical practice (depending on the job). Indeed, there is ample evidence that retirement is specifically associated with a general loss of processing speed (de Grip et al., 2015) and general cognitive functioning (Bonsang et al., 2012). Retirement selectively increases the rate of decline of cognitive abilities, presumably due to a lack of motivation to invest in compensatory activities (Mazzonna and Peracchi, 2012). From our point of view, a responsible societal response to biological aging must not prevent elderly individuals from practicing their cognitive-control skills (as present retirement laws do) but, rather, provide stronger individual and social reinforcement and more opportunities for elderly to engage in control-demanding activities, including physical and social interactions with substantial degrees of uncertainty and surprise.

\section{AUTHOR CONTRIBUTIONS}

All authors listed have made substantial, direct and intellectual contribution to the work and approved it for publication.

opportunities. Cogn. Affect. Behav. Neurosci. 14, 443-472. doi: 10.3758/s13415014-0300-0

Buracchio, T. J., Mattek, N. C., Dodge, H. H., Hayes, T. L., Pavel, M., Howieson, D. B., et al. (2011). Executive function predicts risk of falls in older adults without balance impairment. BMC Geriatr. 11:74. doi: 10.1186/1471-231811-74

Cacioppo, J. T., Hughes, M. E., Waite, L. J., Hawkley, L. C., and Thisted, R. A. (2006). Loneliness as a specific risk factor for depressive symptoms: cross-sectional and longitudinal analyses. Psychol. Aging 21, 140-151. doi: 10.1037/0882-7974.21.1.140

Cools, R., and D'Esposito, M. (2011). Inverted-U-shaped dopamine actions on human working memory and cognitive control. Biol. Psychiatry 69, e113-e125. doi: 10.1016/j.biopsych.2011.03.028

Costello, E., Kafchinski, M., Vrazel, J., and Sullivan, P. (2011). Motivators, barriers, and beliefs regarding physical activity in an older adult population. J. Geriatr. Phys. Ther. 34, 138-147, doi: 10.1519/JPT.0b013e31820e0e71

de Bruin, E. D., Schoene, D., Pichierri, G., and Smith, S. T. (2010). Use of virtual reality technique for the training of motor control in the elderly: 
some theoretical considerations. Z. Gerontol. Geriatr. 43, 229-234. doi: 10.1007/s00391-010-0124-7

de Grip, A., Dupuy, A., Jolles, J., and van Boxtel, M. (2015). Retirement and cognitive development: are the retired really inactive? Econ. Hum. Biol. 19, 157-169. doi: 10.1016/j.ehb.2015.08.004

Dickens, A. P., Richards, S. H., Greaves, C. J., and Campbell, J. L. (2011). Interventions targeting social isolation in older people: a systematic review. BMC Public Health 11:647. doi: 10.1186/1471-2458-11-647

Düzel, E., Bunzeck, N., Guitart-Masip, M., and Düzel, S. (2010). Noveltyrelated motivation of anticipation and exploration by dopamine (NOMAD): implications for healthy aging. Neurosci. Biobehav. Rev. 34, 660-669. doi: 10.1016/j.neubiorev.2009.08.006

Ennis, G. E., Hess, T. M., and Smith, B. T. (2013). The impact of age and motivation on cognitive effort: implications for cognitive engagement in older adulthood. Psychol. Aging 28, 495-504.doi: 10.1037/a0031255

Falck, R. S., Davis, J. C., and Liu-Ambrose, T. (2016). What is the association between sedentary behaviour and cognitive function? A systematic review. $\mathrm{Br}$. J. Sports Med. doi: 10.1136/bjsports-2015-095551 [Epub ahead of print].

Gabriel, Z., and Bowling, A. (2004). Quality of life from the perspectives of older people. Ageing Soc. 24, 675-691. doi: 10.1017/S0144686X03001582

Granacher, U., Mühlbauer, T., Zahner, L., Gollhofer, A., and Kressig, R. (2011). Comparison of traditional and recent approaches in the promotion of balance and strength in older adults. Sports Med. 41, 377-400. doi: 10.2165/11539920000000000-00000

Granacher, U., Zahner, L., and Gollhofer, A. (2008). Strength, power, and postural control in seniors: considerations for functional adaptations and for fall prevention. Eur. J. Sport Sci. 8, 325-340. doi: 10.1080/17461390802478066

Hommel, B. (2015). The theory of event coding (TEC) as embodied-cognition framework. Front. Psychol. 6:1318. doi: 10.3389/fpsyg.2015.01318

Hommel, B. (2016). "Embodied cognition according to TEC," in Foundations of Embodied Cognition: Perceptual and Emotional Embodiment, Vol. 1, eds Y. Coello and M. Fischer (Hove: Psychology Press), 75-92.

Hsu, C. L., Nagamatsu, L. S., Davis, J. C., and Liu-Ambrose, T. (2012). Examining the relationship between specific cognitive processes and falls risk in older adults: a systematic review. Osteoporos. Int. 23, 2409-2424. doi: 10.1007/s00198012-1992-Z

Li, K. Z. H., and Lindenberger, U. (2002). Relations between aging sensory/sensorimotor and cognitive functions. Neurosci. Biobehav. Rev. 26, 777-783. doi: 10.1016/S0149-7634(02)00073-8

Liu-Ambrose, T., and Donaldson, M. G. (2009). Exercise and cognition in older adults: is there a role for resistance training programmes? Br. J. Sports Med. 43, 25-27. doi: 10.1136/bjsm.2008.055616

Liu-Ambrose, T., Nagamatsu, L. S., Graf, P., Beattie, B. L., Ashe, M. C., and Handy, T. C. (2010). Resistance training and executive functions - a 12month randomized controlled trial. Arch. Intern. Med. 170, 170-178. doi: 10.1001/archinternmed.2009.494
Ma, K., and Hommel, B. (2015). The role of agency for perceived ownership in the virtual hand illusion. Conscious. Cogn. 36, 277-288. doi: 10.1016/j.concog.2015.07.008

Mazzonna, F., and Peracchi, F. (2012). Ageing, cognitive abilities and retirement. Eur. Econ. Rev. 56, 691-710. doi: 10.1016/j.euroecorev.2012.03.004

McAuley, E., and Blissmer, B. (2010). Self-efficacy determinants and consequences of physical activity. Exerc. Sport Sci. Rev. 28, 85-88.

Mitchell, W. K., Williams, J., Atherton, P., Larvin, M., Lund, J., and Narici, M. (2012). Sarcopenia, dynapenia, and the impact of advancing age on human skeletal muscle size and strength; a quantitative review. Front. Physiol. 3:260 doi: 10.3389/fphys.2012.00260

Neumann, R., Förster, J., and Strack, F. (2003). "Motor compatibility: the bidirectional link between behavior and evaluation," in The Psychology of Evaluation: Affective Processes in Cognition and Emotion, eds J. Musch and K. C. Klauer (Mahwah, NJ: Lawrence Erlbaum), 7-49.

Niv, Y., Joel, D., and Dayan, P. (2006). A normative perspective on motivation. Trends Cogn. Sci. 10, 375-381. doi: 10.1016/j.tics.2006.06.010

Park, L., Streamer, L., Huang, L., and Galinsky, A. (2013). Stand tall, but don't put your feet up: universal and culturally-specific effects of expansive postures on power. J. Exp. Soc. Psychol. 49, 965-971. doi: 10.1016/j.jesp.2013. 06.001

Reed, A. E., Chan, L., and Mikels, J. A. (2014). Meta-analysis of the age-related positivity effect: age differences in preferences for positive over negative information. Psychol. Aging 29, 1-15. doi: 10.1037/a0035194

Schutzer, K. A., and Graves, B. S. (2004). Barriers and motivations to exercise in older adults. Prev. Med. 39, 1056-1061. doi: 10.1016/j.ypmed.2004. 04.003

Umegaki, H., Roth, G. S., and Ingram, D. K. (2008). Aging of the striatum: mechanisms and interventions. Age 30, 251-261. doi: 10.1007/s11357-0089066-z

Westbrook, A., Kester, D., and Braver, T. S. (2013). What is the subjective cost of cognitive effort? Load, trait, and aging effects revealed by economic preference. PLoS ONE 8:e68210. doi: 10.1371/journal.pone.0068210

Wilson, M. (2002). Six views of embodied cognition. Psychon. Bull. Rev. 9, 625-636. doi: 10.3758/BF03196322

Conflict of Interest Statement: The authors declare that the research was conducted in the absence of any commercial or financial relationships that could be construed as a potential conflict of interest.

Copyright (c) 2016 Hommel and Kibele. This is an open-access article distributed under the terms of the Creative Commons Attribution License (CC BY). The use, distribution or reproduction in other forums is permitted, provided the original author(s) or licensor are credited and that the original publication in this journal is cited, in accordance with accepted academic practice. No use, distribution or reproduction is permitted which does not comply with these terms. 
OPEN ACCESS

Edited by:

Davood Gozli,

University of Macau, China

Reviewed by:

Markus Kiefer,

University of UIm, Germany

Roberta Sellaro,

Leiden University, Netherlands

*Correspondence:

Laura Barca

laurabarcapst@gmail.com

Specialty section:

This article was submitted to

Cognition

a section of the journal

Frontiers in Psychology

Received: 14 November 2016 Accepted: 03 November 2017

Published: 01 December 2017

Citation:

Barca L, Mazzuca C and Borghi AM

(2017) Pacifier Overuse and

Conceptual Relations of Abstract and Emotional Concepts.

Front. Psychol. 8:2014.

doi: 10.3389/fpsyg.2017.02014

\section{Pacifier Overuse and Conceptual Relations of Abstract and Emotional Concepts}

\author{
Laura Barca $^{1 *}$, Claudia Mazzuca ${ }^{2}$ and Anna M. Borghi ${ }^{1,2,3}$ \\ ${ }^{1}$ Institute of Cognitive Science and Technologies, Italian National Research Council (CNR), Rome, Italy, ${ }^{2}$ Department of \\ Philosophy and Communication, University of Bologna, Bologna, Italy, ${ }^{3}$ Department of Dynamic and Clinical Psychology, \\ Sapienza University of Rome, Rome, Italy
}

This study explores the impact of the extensive use of an oral device since infancy (pacifier) on the acquisition of concrete, abstract, and emotional concepts. While recent evidence showed a negative relation between pacifier use and children's emotional competence (Niedenthal et al., 2012), the possible interaction between use of pacifier and processing of emotional and abstract language has not been investigated. According to recent theories, while all concepts are grounded in sensorimotor experience, abstract concepts activate linguistic and social information more than concrete ones. Specifically, the Words As Social Tools (WAT) proposal predicts that the simulation of their meaning leads to an activation of the mouth (Borghi and Binkofski, 2014; Borghi and Zarcone, 2016). Since the pacifier affects facial mimicry forcing mouth muscles into a static position, we hypothesize its possible interference on acquisition/consolidation of abstract emotional and abstract not-emotional concepts, which are mainly conveyed during social and linguistic interactions, than of concrete concepts. Fifty-nine first grade children, with a history of different frequency of pacifier use, provided oral definitions of the meaning of abstract not-emotional, abstract emotional, and concrete words. Main effect of concept type emerged, with higher accuracy in defining concrete and abstract emotional concepts with respect to abstract not-emotional concepts, independently from pacifier use. Accuracy in definitions was not influenced by the use of pacifier, but correspondence and hierarchical clustering analyses suggest that the use of pacifier differently modulates the conceptual relations elicited by abstract emotional and abstract not-emotional. While the majority of the children produced a similar pattern of conceptual relations, analyses on the few (6) children who overused the pacifier (for more than 3 years) showed that they tend to distinguish less clearly between concrete and abstract emotional concepts and between concrete and abstract not-emotional concepts than children who did not use it (5) or used it for short (17). As to the conceptual relations they produced, children who overused the pacifier tended to refer less to their experience and to social and emotional situations, use more exemplifications and functional relations, and less free associations.

Keywords: abstract concepts, pacifier, conceptual relations, facial mimicry, social interaction, embodied cognition 


\section{INTRODUCTION}

\section{Embodied and Grounded Views and Abstract Concepts Representation}

The difficulty in acquiring and processing abstract concepts, such as "freedom" and "phantasy" is widely recognized: they have been named "hard words" (Gleitman et al., 2005; Gentner, 2006)! The way in which we represent abstract concepts has become hotly debated in the last years, also due to the growing interest for them in the context of embodied and grounded (EG) views of cognition (for overviews, see Dove, 2011, 2016; Pecher et al., 2011; Tomasino and Rumiati, 2013; Borghi and Binkofski, 2014; Reilly et al., 2016; for overviews showing the importance of abstract concepts for EG views, see Kiefer and Pulvermüller, 2012; Borghi et al., 2017). According to EG views, language comprehension consists in simulating word meaning re-enacting previous experiences with words' referents (e.g., Barsalou, 2016). While for EG views it is easy to argue that concrete concepts such as "chair" and "cat" are grounded in sensorimotor and emotional systems, it is less straightforward to contend that this is the case for abstract concepts like "justice" and "phantasy." Concrete concepts typically have a single and clearly bounded referent, while abstract ones do not; furthermore, they are generally more complex, they refer more frequently to complex events or situations (Kiefer and Barsalou, 2013), and they are "progressively more detached from physical experience" (Barsalou, 2003; Fernandino et al., 2015) than concrete concepts, even if evidence has shown that they are also grounded in perceptual modalities, particularly in visual ones (Connell and Lynott, 2012). Concrete concepts are typically processed faster and remembered better than abstract ones (concreteness effect, Schwanenflugel et al., 1988, but see counterevidence by Kousta et al., 2011, and no evidence by Barca et al., 2002), and in feature generation tasks they typically elicit more social aspects of situations and more introspective features (Barsalou and Wiemer-Hastings, 2005). As to their neural underpinnings, abstract concept's processing engages more left-lateralized brain areas like the left inferior frontal gyrus and the left middle temporal lobe (see meta-analysis by Wang et al., 2010) and knowledge on abstract concepts is impaired in syndromes such as deep dyslexia and semantic dementia (Shallice and Cooper, 2013). In spite of behavioral and neuropsychological evidence showing differences between concrete and abstract concepts, it is difficult to contend that they are dichotomously organized, since abstractness and concreteness are graded, and different subkinds of concepts exist. We therefore start from the assumption that they are arranged along a continuum spanning from highly concrete to highly abstract concepts. In line with the idea of a continuum, psychological, and neuroscientific studies have recently started to investigate fine-grained distinctions among kinds of abstract concepts, analyzing for example the differences in behavioral effects and neural representation of mental state concepts, social concepts, institutional concepts, mathematic concepts, and emotional concepts (Setti and Caramelli, 2005; Crutch et al., 2013; Ghio et al., 2013, 2016; Roversi et al., 2013). Hence, the category of abstract concepts is highly heterogeneous.

\section{The Peculiarity of Emotional Concepts}

Emotional concepts in particular represent a special case since they have an ambiguous status. From the point of view of an embodied theory, clearly emotional concepts are less difficult to handle with than pure abstract concepts, since when compared with abstract concepts it is much easier to demonstrate that they activate bodily sensations and are grounded in sensorimotor and emotional systems (Borghi and Binkofski, 2014). Empirical research has provided contradictory results. In many studies emotional concepts are considered as a subset of abstract concepts, on the basis of abstractness, concreteness, and imageability ratings provided by participants. Other evidence has instead demonstrated that emotions represent a distinctive kind of concept when compared to both abstract and concrete ones (e.g., Altarriba et al., 1999; Altarriba and Bauer, 2004): they are recalled better than both concrete and abstract words, they are rated differently from both concrete and abstract concepts in concreteness, imageability, and contextual availability, they elicit more different associations, followed by abstract and then by concrete words; finally, independently from their polarity (negative or positive) emotion words are processed faster than other words (Kousta et al., 2009).

As to their development, concrete emotion words are acquired before abstract emotion words. Recent data showed that valenced abstract words are acquired before other abstract words (Kousta et al., 2011, Figure 7, p. 26; Ponari et al., 2017) and that emotional valence facilitates the acquisition of abstract concepts in schoolage children (Ponari et al., 2017). Their early acquisition has been related to the later acquisition of abstract concepts by proponents of the Affective Embodiment Account (AEA). According to the AEA, emotional experience dominates representation of abstract words. Consistently, learning of emotional terms provides a bootstrapping mechanism useful to learn abstract concepts (Kousta et al., 2011; Vigliocco et al., 2013). Since emotional concepts are the first concepts to be acquired that do not possess a concrete referent but rather refer to interoceptive states, they can facilitate the acquisition of abstract concepts, which do not have a concrete referent.

In spite of this hypothesis, to the best of our knowledge, no study directly investigates acquisition and representation of concrete, abstract not-emotional and abstract emotional concepts in children starting from a perspective in which the effects of the bodily involvement on acquisition are analyzed (Pexman, 2017).

The first aim of our paper is to investigate how 7-yearsolds represent concrete, abstract not-emotional, and abstract emotional concepts, in order to verify whether abstract emotional concepts can be assimilated to other abstract concepts or represent instead a distinctive kind of concepts. We wanted to investigate conceptual development in children who had just started a formal linguistic education at school, i.e., first-graders.

We decided to use a word definition task that is typically used to test lexical access, retrieval of stored lexical information, as well as language production in typically developing children and impaired population (Burani et al., 2006; Caramelli et al., 2006). The word definition task would allow us both to verify whether children are able to provide correct definitions of the 
word meanings as well as to analyze and directly compare the network of conceptual relations elicited by the three different kinds of concepts. Stimuli were chosen taking also into account written frequency of texts for first-grade children (Marconi et al., 1993). The selected corpus ensures that children of this age and school class had been exposed to the experimental stimuli. Although we are well aware that abstract concepts are rather heterogeneous and that all abstract concepts might be emotionally connoted, we distinguished purely emotional concepts from other abstract concepts, in order to verify whether abstract emotional concepts can be assimilated to abstract concepts or whether they are represented and processed differently from not-emotional abstract concepts.

The second aim of this work is to investigate whether the representation of the three kinds of concepts in 7-years-old children is differently affected by the use of pacifier in the period of the linguistic burst. The reason why we are interested in the long-term effects of the pacifier use is that, according to some embodied cognition theories and evidence on abstract concepts and on emotional development, the mouth activation plays a crucial role for representing abstract and abstract emotional concepts compared to concrete ones. In the following we first explain why we think that the activation of the mouth is critical for abstract concepts representation and processing, and then we overview some studies on pacifier use and explain why we hypothesize that the acquisition of abstract not-emotional and abstract emotional concepts might be influenced by pacifier use.

\section{Abstract Concepts and Activation of the Mouth}

As to abstract concepts and the activation of the mouth, we will here focus on the WAT (Words As social Tools) view (Borghi and Cimatti, 2009; Borghi and Binkofski, 2014), that underlies how the different acquisition modality of concrete and abstract concepts influences their later representation (see also Wauters et al., 2003). According to the WAT view, both sensorimotor and linguistic-social information concur in representing concrete and abstract concepts, but this information is differently distributed. While the experience with the physical environment has a major weight for the acquisition of concrete concepts, the social, and linguistic input provided by others is crucial for acquiring abstract concepts, since they do not possess a single referent, which can be easily identified through the senses. The first grounded view that highlighted the role not only of sensorimotor but also of linguistic information for characterizing concepts is the LASS (Language and Situated Simulation) view, according to which linguistic representation are more superficial while conceptual content resides in situated simulations (Barsalou et al., 2008). While WAT is strongly inspired by the LASS view, it differs from it for at least two reasons: because it focuses on abstract concepts representation; and because it ascribes more relevance to the linguistic experience as a whole and does not consider language only as a shortcut to access to content, which would be represented only in sensorimotor terms (for a more thorough analysis, see Borghi et al., 2017). In the WAT view language experience plays a crucial role: beyond its communicative role, language influences categorization, supports prediction (Lupyan and Clark, 2015) and, more generally, it can be seen as a tool that widely extends our thought capabilities (Dove, 2014) and affords the realization of a human-specific pedagogical context for efficient learning (Csibra and Gergely, 2009; Pezzulo et al., 2014). In the case of abstract concepts, linguistic labels can thus provide a glue useful to put together category members that can be highly diverse and variable; in addition, language can be a means useful to introspectively reason on abstract concepts and to focus on inner states (Barsalou and Wiemer-Hastings, 2005; Kiefer and Pulvermüller, 2012; Kiefer and Barsalou, 2013). In line with this view, it has been shown that abstract concepts, differently from concrete ones, benefit from rich linguistic contexts (Recchia and Jones, 2012) and that, beyond sensorimotor features, they incorporate more linguistic, social and also interoceptive features than concrete concepts (Thill and Twomey, 2016).

According to the WAT proposal, the major role played by language in the representation of abstract concepts has an embodied counterpart: the activation of the mouth (see Topolinski and Strack, 2009). A number of recent studies seem to support the link between abstract concepts and activation of the mouth. We will briefly review this evidence.

fMRI studies have shown that processing of abstract words engages brain areas dedicated to language processing. A recent meta-analysis (Wang et al., 2010) on abstract concepts processing reports involvement of linguistic production and comprehension areas, in particular the left inferior frontal gyrus (Broca's area) and the left middle temporal lobe. Literature has shown that the LIFG is involved in subvocalizations and in phonological processing and working memory, and it has been hypothesized that abstract words remain longer in working memory in phonological form due to their difficulty (Binder et al., 2005). The activation of these "linguistic" areas is thus compatible with the activation of the mouth. Many behavioral studies have confirmed that abstract word processing implies the activation of the mouth. Experiments on adults in which the acquisition of novel categories and words was mimicked, using novel figures or Lego objects, revealed that new abstract words were responded to faster with the microphone, while new concrete words elicited faster responses with the keyboard (Borghi et al., 2011; Granito et al., 2015). Furthermore, two ratings studies with Italian words derived from two different databases (Barca et al., 2002; Della Rosa et al., 2011) confirmed that abstract words were rated higher on involvement of the mouth, concrete ones of the hand (Granito et al., 2015; Borghi and Zarcone, 2016); higher involvement of the mouth was also found in a rating study with abstract and emotional sentences (Ghio et al., 2013). The significant advantage of abstract categories in the ratings on mouth involvement was true also comparing them with concrete categories involving heterogeneous members, thus it did not depend exclusively on the differences between the category members (Granito et al., 2015). A further study with an implicit definition-word matching task (Borghi and Zarcone, 2016) revealed that the advantage in response times of the hand over the mouth responses was more marked with concrete than with abstract concepts. 
Two possible explanations have been provided, which are not necessarily mutually exclusive. The first is that the activation of the mouth with abstract concepts might depend on the reenactment of their peculiar acquisition modality, which strongly involves linguistic explanations. The second is that, given the higher complexity of abstract words, we might need to re-explain their meaning to ourselves, possibly through a form of inner talk.

\section{Effects of Pacifier Use on Acquisition of Abstract and Abstract Emotional Words}

One way to test whether the mouth involvement is critical during abstract terms acquisition is to investigate the effect of an oral device, as the pacifier, on language development (for further work showing how words referring to emotions, as "to smile," activate the corresponding facial muscle, see Foroni and Semin, 2009). The debate on the use of the pacifier is currently very lively, but mostly confined to its implications for feeding babies (e.g., negative implications for breastfeeding) or for teething and orthodontic problems. Very little is known as to the cognitivelinguistic and emotional implications of its extensive use. Two recent studies link the use of the pacifier with the emotional competence (Niedenthal et al., 2012; Rychlowska et al., 2014). According to the authors, prolonged use of pacifier (duration and frequency of use) would result in an altered facial expression in children and, subsequently, in a reduction in emotional skills (e.g., expressing emotions through facial expressions, and recognizing emotions expressed in faces of others); the effect occurred only in male babies. In light of the numerous studies that show that in adults the mobility of the facial muscles is important in the development of emotional material (e.g., reduced mobility caused by Botox injection affects the ability to process "faces and emotional words", Havas et al., 2010), there may be an interaction between pacifier use and early emotional development of the child, where the use of the pacifier for several hours during the day, and in social contexts, induces a particular motility/location of facial muscles (if the pacifier is used only at night or sleep it may have a minor impact). While recent evidence has shown a negative relation between pacifier use and children's emotional competence (Niedenthal et al., 2012; Rychlowska et al., 2014), the possible interaction between use of pacifier and learning and processing of emotional language has not yet been investigated.

Less is known regarding the relationship between pacifier use and abstract concepts acquisition. As anticipated in the introduction, several studies have shown that the role of linguistic and social input is more relevant for the formation of abstract concepts than of concrete ones, and that this leads to an activation of the mouth (for a review see Borghi et al., 2017). As the representation of abstract concepts not only counts on sensorimotor information but also on linguistic and social information, the overuse of pacifier may interfere with the acquisition of abstract concepts. In other words, because pacifiers occupy the mouth, and because abstract words elicit motor simulations of mouth action, then abstract concepts might develop differently in infants who use pacifiers.

\section{Aims and Hypotheses}

The present study aims to verify whether the extended use of pacifier interferes with the acquisition and consolidation in memory of abstract not-emotional and abstract emotional words meanings compared to that of concrete ones. Specifically, we intend to investigate long-term effects of pacifier overuse using a definition task with 7-year-olds who have never used the pacifier, who have used it for short (until 2 years), until age 2-3 or beyond age 3 .

Based on the aforementioned review, we formulate two hypotheses. The first pertains the distinction between the three conceptual kinds (abstract not-emotional, concrete, and abstract emotional). The second concerns the possible influence of pacifier overuse on the acquisition and consolidation of abstract emotional and not-emotional concepts.

\section{Distinction between Abstract, Concrete, and Abstract Emotional Concepts}

We contrast two possible views. According to the first, emotional concepts can be considered as a subset of abstract concepts (e.g., Kousta et al., 2011). If this is the case, then we should find neither differences in accuracy between abstract emotional and not-emotional concepts nor differences between the network of relations elicited by them; both emotional and abstract concepts should differ from concrete concepts. According to the second view, emotional concepts do not represent a subset of abstract ones but rather differ from both concrete and abstract concepts (Altarriba et al., 1999; Altarriba and Bauer, 2004; Setti and Caramelli, 2005); consistently, abstractness and valence have different neural representation (Skipper and Olson, 2014). If this is the case, then the conceptual relations characterizing emotional concepts should differ from those elicited by both concrete and abstract not-emotional ones.

\section{Effects of Pacifier Use on Conceptual Acquisition}

We predict an influence of the pacifier overuse on the acquisition of both abstract emotional and not-emotional concepts, for which the linguistic and social context of acquisition is particularly important. The use of pacifier would namely render more difficult the formation of a linguistic simulation activating the mouth, and due to its effect on facial expression, it would render social and emotional interactions more difficult. In contrast, the pacifier should not affect the acquisition of concrete concepts, for which the simulation with the mouth would not be necessary and the role of facial expression might be less relevant.

While we predict an influence of the use of pacifier on conceptual development of abstract and emotional concepts, we intend to contrast a stronger and a milder hypothesis. According to the strong hypothesis, pacifier overuse would interfere with the acquisition of abstract and abstract emotional concepts. To test this hypothesis we scored the definitions provided by children distinguishing them in fully correct, partially correct, and incorrect or no response. The strong hypothesis predicts a higher number of incorrect or missing definitions with abstract 
and emotional concepts than with concrete concepts, particularly in children who used the pacifier beyond age 3 .

According to a weak hypothesis, pacifier overuse would influence the organization of conceptual relations elicited by concepts. Accordingly, the three kinds of concepts should not differ in the number of correct definitions but in the pattern of conceptual relations they elicit. To test this hypotheses we coded the definitions provided identifying different kinds of conceptual relations (see section Scoring of the Responses), and we analyzed the pattern of semantic relations characterizing concrete, abstract, and emotional concepts. The weak hypothesis predicts that the pattern of conceptual relations produced by late users of pacifier should be characterized by a less marked difference between concrete and abstract concepts and between concrete and emotional concepts.

\section{EXPERIMENT 1: DEFINITION TASK}

\section{Methods}

\section{Participants}

The sample included 59 children aged 6-7 years (28 male) from a school of Rome. As part of the recruitment procedure, children' parents provided their Informed Consent by means of an enrolling questionnaire requiring information about family composition, socioeconomic status, familiarity with other languages, children cognitive, auditory or language impairments, and pacifier use (if any). Parents also indicated if their child used a pacifier (a) during the day at home, (b) at night, and (c) during the day outside of the home, including school (see also Niedenthal et al., 2012). No a priori selection has been made, that is all children with approved Consent participated in the study.

Data of children who had a language impairment certification were not included in the study.

The demographic characteristics of the sample are reported in the Appendix 2.

Results discussed in the following sections are based on a reduced sample of 46 participants, as in $22 \%$ of cases parents filled the questionnaire but did not provide information about pacifier use (in the Appendix, participants not included are marked by an asterisk).

Children were classified into four subgroups based on parents' responses:

- Never: Children who never used the pacifier (six participants, three males);
- Two: those who used the pacifier up to 2 years of age (17 participants, 10 males);

- Two-Three: those who used the pacifier up to Two-Three years of age (18 participants, nine males);

- Three: those who used the pacifier for 3 years of age and more (five participants, three males) (Table 1).

Overall, the distribution of pacifier use was not homogenous, with most of the children in our sample who have used it (87\%). A large proportion of them used the pacifier for sleeping purposes ( $86 \%$ during the night, $60 \%$ during daytime at home), in few cases they used it also at home (33\%) or at school (27\%), presumably during social interaction but we do not have further information. The school is located in a multiethnic suburb of Rome, thus $26 \%$ of the children had been exposed to other languages, such as English, Singhalese, Portuguese, Spanish, German, Moldovan, Pakistani, Arabic, Albanian, and Romanian. Chi square analyses revealed that the demographic information did not significantly differ between the pacifier groups (Chi-square $=12, d f=9$, $p>0.1$ ).

\section{Materials and Design}

A list of 30 Italian words (10 abstract, 10 concrete, and 10 emotional words) was selected from a larger sample explored in a preliminary study (see Supplementary Materials, Appendix 1). Stimuli characteristics are presented in Table 2. As it can be seen in the appendix, all emotional terms we selected refer to basic emotions (e.g., fear) or to emotional states (e.g., love) or are

TABLE 2 | Psycholinguistic characteristics of the stimuli used for the definition task.

\begin{tabular}{|c|c|c|c|c|c|c|c|c|c|}
\hline & ABS & CONC & EMO & FREQ & IMA & FAM & AoA & MoA & Length \\
\hline $\begin{array}{l}\text { Abstract } \\
\text { words }\end{array}$ & $\begin{array}{l}432 \\
(69)\end{array}$ & $\begin{array}{l}305 \\
(71)\end{array}$ & $\begin{array}{c}3.2 \\
(1.1)\end{array}$ & $\begin{array}{c}89 \\
(104)\end{array}$ & $\begin{array}{c}390 \\
(141)\end{array}$ & $\begin{array}{l}534 \\
(82)\end{array}$ & $\begin{array}{l}326 \\
(70)\end{array}$ & $\begin{array}{c}46 \\
(81)\end{array}$ & $\begin{array}{c}7.2 \\
(0.8)\end{array}$ \\
\hline $\begin{array}{l}\text { Concrete } \\
\text { words }\end{array}$ & $\begin{array}{l}124 \\
(30)\end{array}$ & $\begin{array}{l}676 \\
(27)\end{array}$ & $\begin{array}{l}2.0 \\
(0.6)\end{array}$ & $\begin{array}{c}28 \\
(13)\end{array}$ & $\begin{array}{l}668 \\
(17)\end{array}$ & $\begin{array}{l}562 \\
(75)\end{array}$ & $\begin{array}{l}251 \\
(48)\end{array}$ & $\begin{array}{l}226 \\
(73)\end{array}$ & $\begin{array}{c}7.3 \\
(1.8)\end{array}$ \\
\hline $\begin{array}{l}\text { Emotional } \\
\text { words }\end{array}$ & $\begin{array}{c}410 \\
(128)\end{array}$ & $\begin{array}{c}367 \\
(148)\end{array}$ & $\begin{array}{l}5.7 \\
(0.3)\end{array}$ & $\begin{array}{c}113 \\
(173)\end{array}$ & $\begin{array}{c}445 \\
(117)\end{array}$ & $\begin{array}{l}595 \\
(63)\end{array}$ & $\begin{array}{l}267 \\
(70)\end{array}$ & $\begin{array}{l}369 \\
(96)\end{array}$ & $\begin{array}{c}6.2 \\
(1.6)\end{array}$ \\
\hline
\end{tabular}

ABS, abstractness; CONC, concreteness; EMO, words emotionality; FREQ, written word frequency; IMA, imageability; FAM, familiarity; $A \circ A$, word age of acquisition; MOA, concept mode of acquisition; Length, word length in letters. Mean values and standard deviation are provided (in bracket).

TABLE 1 | Demographic characteristics (percentages) of participants.

\begin{tabular}{|c|c|c|c|c|c|c|c|c|c|c|c|c|c|}
\hline \multirow[t]{2}{*}{ Pacifier } & \multicolumn{2}{|c|}{ Gender } & \multirow{2}{*}{$\frac{\text { Age in months }}{\text { Mean (range) }}$} & \multicolumn{4}{|c|}{ Schooling mother } & \multicolumn{5}{|c|}{ Schooling father } & \multirow{2}{*}{$\begin{array}{l}\text { Exposure to } \\
\text { other languages }\end{array}$} \\
\hline & Male & Female & & $\begin{array}{l}\text { Middle } \\
\text { school }\end{array}$ & $\begin{array}{l}\text { High } \\
\text { school }\end{array}$ & University & NA & $\begin{array}{c}\text { Elementary } \\
\text { school }\end{array}$ & $\begin{array}{l}\text { Middle } \\
\text { school }\end{array}$ & $\begin{array}{l}\text { High } \\
\text { school }\end{array}$ & University & NA & \\
\hline Never & 50 & 50 & 78.3 (73-83) & 17 & 50 & 33 & 0 & 17 & 17 & 50 & 17 & 0 & 50 \\
\hline Two & 59 & 41 & 77.1 (69-83) & 12 & 41 & 41 & 6 & 0 & 12 & 59 & 18 & 12 & 24 \\
\hline Two-Three & 50 & 50 & $77.5(69-83)$ & 22 & 56 & 17 & 6 & 6 & 22 & 61 & 6 & 6 & 22 \\
\hline Three & 60 & 40 & $77.2(72-80)$ & 40 & 20 & 40 & 0 & 0 & 20 & 20 & 60 & 0 & 20 \\
\hline
\end{tabular}


concepts that for children are strongly associated to possession and transmission of emotions (e.g., kiss, heart) (Table 2).

Attention was made in order to control for correlated variables. Nevertheless, abstract words had lower values of Concreteness, Imageability, AOA, Context Availability and MoA than concrete words; concrete words had lower values of Abstractness, Imageability, AOA, Context Availability and MoA than emotional words; and Emotional words had higher values of MoA than abstract words ( $p s<0.05, t$-test computed in Excel). Importantly, the emotional words we selected had abstractness values only slightly lower than abstract concepts and concreteness values much lower than concrete concepts (see Table 2), thus they can be considered, according to the ratings, as subsets of abstract concepts, even if they differed in the acquisition modality, which was mainly linguistic for abstract concepts. Aside from acquisition modality, the main difference between the selected abstract and emotional concepts concerns their valence. To be certain that abstract concepts and emotional concepts differed in emotionality, we performed paired sample $t$-tests (Bonferroni corrected) on the ratings obtained in order to test whether there was a significant difference in emotional ratings between emotional, abstract, and concrete concepts. Even if abstract words were considered as more emotional than concrete ones $\left[t_{(9)}=3.05, p=0.014\right]$, emotional words $(M=5.67)$ were evaluated as significantly more emotional than both concrete words $(M=2.04),\left[t_{(9)}=15.11, p=0.001\right]$, and other abstract words $(M=3.16)\left[t_{(9)}=7.01, p=0.001\right]$, confirming our expectations. Crucially, no abstract word was evaluated higher than any emotional word in emotional valence (see Supplementary Table 1 and Supplementary Figure 1).

\section{Procedure}

Children were enrolled directly at school where data acquisition took place. They were picked up individually from the class and taken to a room specifically dedicated to data collection. They were asked to sit at the table with the experimenter, and a plastic bowl containing pieces of paper was put in front of them. They were asked to pick up a piece of paper one at a time and to provide an oral definition of the word that the experimenter read to them. All the responses were typed online on the computer and were audio recorded. Each session lasted between 15-20 min and, at the end of the session, the child was taken back to the classroom.

\section{Scoring of the Responses}

Definitions were rated using two scoring systems. Level 1 pertained the accuracy of the response and used a three point scale $(2=$ fully correct, $1=$ partially correct, $0=$ not correct or no response, see also Burani et al., 2006).

Level 2 focused on the qualitative analysis of the response, using 11 categories based on the conceptual relations elicited in the response. We assigned one point to each category. Categories Definitions' Features scoring system were:

(1) Perceptual features (referring to perceptual properties of the concept, e.g., "helicopter: something that has a propeller");
(2) Thematic-Spatial (referring to spatial location, e.g., "library: where the books are");

(3) Thematic-Action-Function (referring to the functionality of the concept, e.g., "box: you put something inside");

(4) Emotion (using emotional terms, e.g., "heart: something that is inside us and makes us kind");

(5) Situation (referring to situation and events when the concept might occur, e.g., "shame: when you ashamed to do a play");

(6) Experiential (referring to some experiences, e.g., "brush: when the teacher tells me to paint something and I paint with the brush");

(7) Interaction (referring to social-interactive situation, e.g., "surprise: when someone gives you something and you do not know what it is");

(8) Taxonomic-Superordinate (referring to the a higher level of taxonomy, e.g., "banana: it's a fruit");

(9) Taxonomic-Subordinate (using an example to define the concept, e.g., "agreement: when you get along with a friend");

(10) Norm (referring to social norms, e.g., "helmet: you have to put it on your head when you ride a motorcycle");

(11) Free Association (free association with no conceptual relation with the concept, e.g., "culture: when in the morning you have to go to school and have to wear an apron").

The scoring system we selected was based on previous literature on conceptual development and conceptual representation: in addition to the perceptual/property relations and to the thematic (spatial, action-function, and situation/event) and taxonomic relations (Borghi and Caramelli, 2003; Kalénine et al., 2009; Estes et al., 2011; Mirman et al., 2017), we added free associations, which according to Barsalou and Wiemer-Hastings (2005) should be more typical of abstract concepts, and normative relations, which might characterize abstract concepts of the normative kind (see Roversi et al., 2013). Finally, since we were interested in the role of direct experience and of emotional and social aspects in characterizing abstract concepts we added experiential, emotion (see also $\mathrm{Wu}$ and Barsalou, 2009), and interaction relations.

\section{Reliability Analysis}

Two independent coders (the first two authors of the study) used the two level systems to rate the definitions. A third coder (the last author) intervened in case of disagreement. Inter-judge reliability of coding was calculated by means on inter-rated $t$-test, which showed no significant difference $(t$-value $<1)$.

\section{Data Analysis and Results}

The results were first analyzed considering overall accuracy (total correct definitions). Generalized linear mixed-effects model (GLMM) was used to assess the impact of Concept type and Pacifier use on accuracy data (Baayen, 2008; Bolker et al., 2009). Second, a qualitative analysis of conceptual features was conducted, focusing on conceptual content underlying children's definitions (Borghi and Caramelli, 2003; Caramelli et al., 2006). 
Correspondence analysis (CA) was used to explore relationships among our categorical variables (Concepts type and Pacifier use) and the conceptual relations used in the word definition task. CA is a statistical exploratory technique used to graphically visualize the underlying structure of contingency table. Hierarchical clustering performs an agglomerative hierarchical grouping on results of the CA.

\section{Accuracy of Definitions}

Overall children were accurate in completing the definition task, with $89 \%$ of correct response (44\% of the total were considered fully correct, $45 \%$ of the total partially correct), $10 \%$ of errors and a small percentage of no responses. Correct definitions as a function of Concept types and participants' group are presented in Figure 1.

Generalized linear mixed model (GLMM) fit by maximum likelihood (Laplace Approximation) was used to assess the impact of Concept type and Pacifier use on accuracy data. GLMM was implemented in R Development Core Team (2005) with the lme4 package, with parameter "family = binomial" to account for categorical data (Bates and Maechler, 2009). The model included random intercept for Subjects and Items, and fixed effect of Concept type (Abstract, Concrete, and Emotional) and frequency of Pacifier use (Never, up to 2 years, Two-Three years, more than 3 years). Following the recommendations of Barr et al. (2013), we also included by-subject random slope in the model (that is introducing by-subject adjustments to the intercept as well as by-subject adjustment to the fixed factor Concept).

As the data distribution presents many zeros, we used the Akaike's Information Parameter (AIC) to evaluate the most

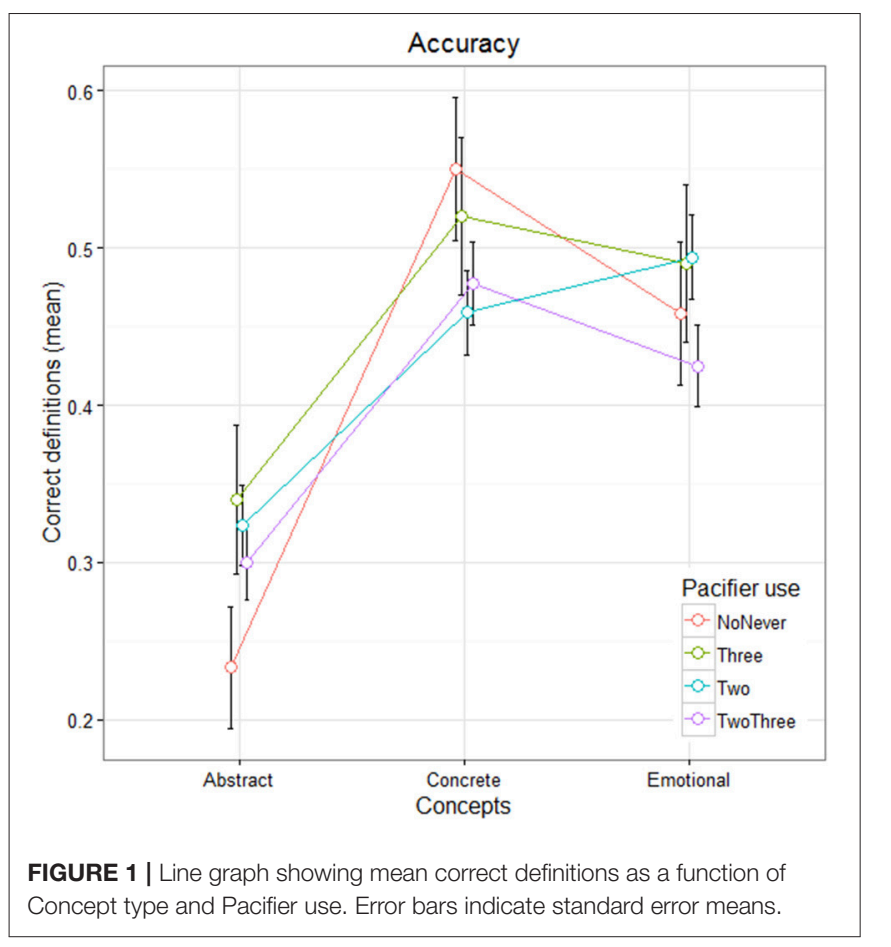

suitable model to data analysis ${ }^{1}$. Models comparison showed that the $\mathrm{LMM}$ is better $(d f=20, \mathrm{AIC}=3,135)$ than the Poisson logit hurdle model (PLH: $\log \mathrm{L}=-1832, d f=24, \mathrm{AIC}=3,712)$ and the zero-inflated negative binomial logit hurdle model (NBLH: $\log \mathrm{L}=-1,832, d f=25, \mathrm{AIC}=3,713)$.

GLMM model showed significant differences between Abstract and Concrete concepts $\left(\beta_{\text {Concrete }}=2.27, z=3.25, p<\right.$ $0.001)$, and between Abstract and Emotional concepts ( $\beta_{\text {Emotional }}$ $=1.6, z=2.6, p<0.001)$. No differences emerged between Concrete and Emotional concepts ( $\beta_{\text {Concrete }: \text { Emotional }}=-0.47, z$ $=-0.89$, ns), nor between different frequencies of pacifier use $(z<1)$. Neither the Concept type per Pacifier interaction was significant, except for the contrast Concrete vs. Pacifier Two $\left(\beta_{\text {Concrete }} \text { : PacifierTwo }=-1.4, z=-2.133, p<0.05\right)^{2}$. Abstract concepts were more difficult to define than both Concrete and Emotional ones and, more interestingly, no differences emerged between Concrete and Emotional concepts, as shown also in Figure 1.

\section{Conceptual Content of Definitions}

Correspondence analysis (CA) was used to explore relationships among our categorical variables (Concepts type and Pacifier use) and the conceptual relations used in the word definition task, which we named "Definitions" Features' (see also Caramelli et al., 2006; Sourial et al., 2010; Ghio et al., 2013). The logic underlying Correspondence analysis is quite similar to that of principal component analysis, but CA applies to categorical data. In CA the frequencies of the conceptual relations give rise to a twodimensional graphical form where they are represented as points in a multidimensional space. The geometrical proximity of the points on the graphs indicates the degree of their association and the similarity of their distribution (Greenacre and Blasius, 1994; Greenacre, 2007). The distances between the points are the weighted distances (Chi-square) between the relative frequencies

\footnotetext{
${ }^{1}$ Due to the high presence of zeros in the distribution, the LMM model may not be appropriate to analyze the data, thus we used the Akaike information criterion for model selection.

The Akaike information criterion (AIC) is a measure of the relative quality of statistical models for a given set of data. Given a collection of models for the data, AIC estimates the quality of each model, relative to each of the other models. Hence, AIC provides a means for model selection. Among the candidate models, we wanted to select the model that minimized the information loss. The smaller the AIC numeric value, the better the fit. AIC has been calculated using the AIC function in R.

${ }^{2}$ We have run a GLMM model including psycholinguistic variables as covariates, that is the fixed-factors of interest and their interaction (Concept Type *Pacifier) and 7 covariates (i.e., Written Frequency, Imageability, Familiarity, Context Availability, Age of Acquisition, Mode of Acquisition, word Length in letters). First, the psycholinguistic variables have been rescaled using the "scale" function. Results are in line with the GLMM model without covariates (significant Abstract-Concrete concepts difference $\left(\beta_{\text {Concrete }}=2.51\right.$, z-value $=2.6, p<0.001)$, and Abstract-Emotional concepts difference $\left(\beta_{\text {Emotional }}=\right.$ $1.36, \mathrm{z}$-value $=2.05, \mathrm{p}<0.05)$. No differences between Concrete - Emotional concepts $\left(\beta_{\text {Concrete }}\right.$ : Emotional $=-0.82$, $\mathrm{z}$-value $\left.=-1.23, \mathrm{~ns}\right)$, nor between different frequencies of pacifier use $(\mathrm{z}$-values $<1)$. Neither the Concept type per Pacifier interaction was significant, except for the contrast Concrete vs. Pacifier Two $\left(\beta_{\text {Concrete }}\right.$ : PacifierTwo $=-1.3, z$-value $\left.=-2.12, p<0.05\right)$. Finally, we have performed models comparison using the ANOVA function, which revealed that adding the covariates is not that informative as the two models are not statistically different (Chi-squared $=6.69$, n.s.)
} 
and not the simple Euclidean distances. Correspondence analysis can be also considered as a method for decomposing the overall Chi-square statistic by identifying a small number of dimensions in which the deviations from the expected values can be represented. In the analyses we conducted we will have two dimensions, the first of which explains the higher amount of inertia. Specifically, we used the "CAinterprTools" package implemented in R statistical environment (Alberti, 2015).

Among the 10 dimensions emerged from the CA analysis, only the first two accounted for most of the inertia, according to the Malinvaud's test (Dimension 1: Eigenvalue $=1.71 \mathrm{e}+05$; Chi-square $=4.96 \mathrm{e}+08, d f=110, p<0.001$; Dimension 2 : Eigenvalue $=3.11 \mathrm{e}+04 ;$ Chi-square $=1.45 \mathrm{e}+08, d f=90$, $p<0.001)$. The scatterplot of the first two CA dimension is in Figure 2.

To make the results more understandable, Figure 3 shows ParetoCharts with the contribution of different variables to the definition of the first two dimensions of the Correspondence Analysis. The charts in Figures 3A,B show the contribution of Definitions' Features to dimension 1 and 2 respectively. Different types of conceptual relationships are contributing to the determination of the two dimensions. In correspondence analysis the first dimension is typically more important than the second. Emotion, Interaction type of definitions have a higher contribution on dimension 1 (with a percentage of explained inertia of 49 and 13.4\%, respectively). Thematic-Spatial,

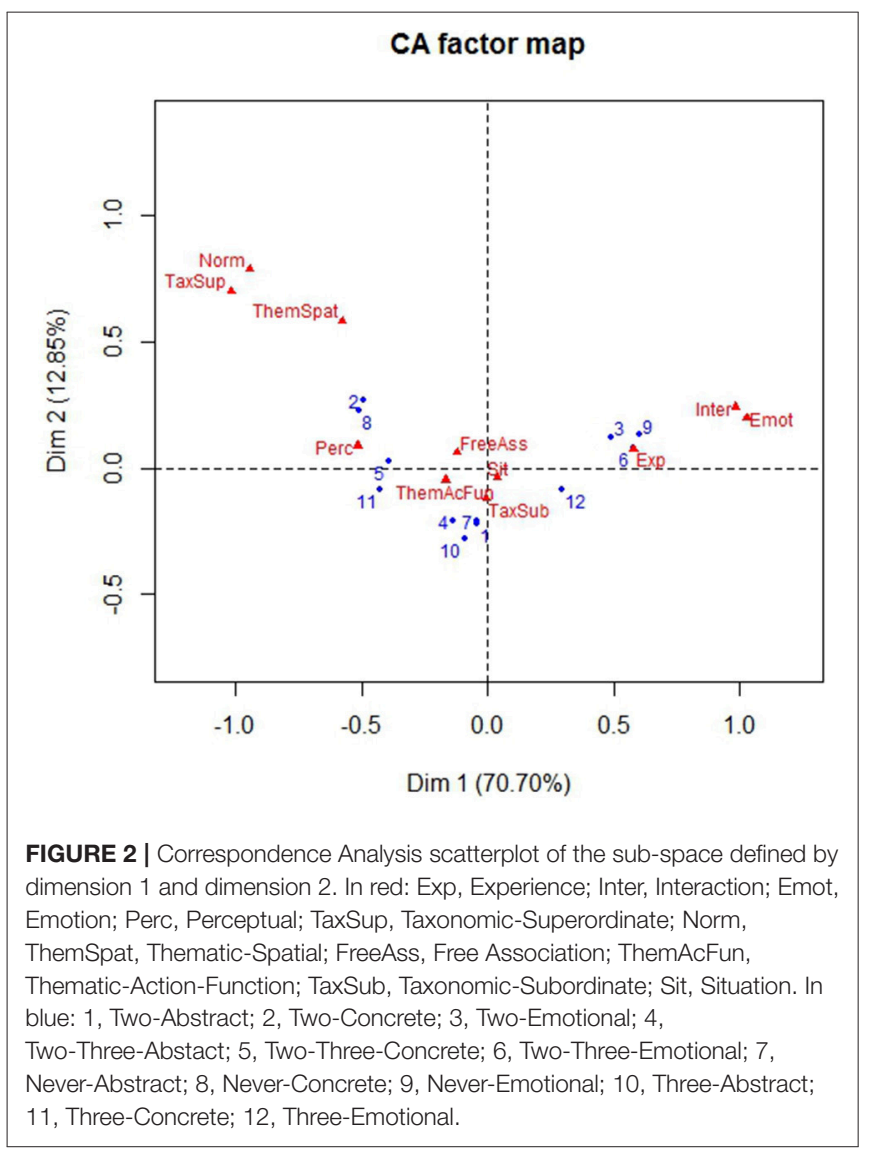

Taxonomic-Superordinate, and Taxonomic-Subordinate conceptual relations have a higher contribution on dimension 2 (with a percentage of explained inertia of 31,25 , and $18 \%$, respectively).

Figures 3C,D shows the contribution of Pacifier by Concepts type to the definition of dimension 1 and 2. The 12 levels of Pacifier by Concepts type combination are differently contributing to the definition of the two dimensions. Never used/Emotional concept, Two-Three years/Emotional, Never used/Concrete, Up to 2 years/Emotional and Up to 2 years/Concrete concepts have a higher contribution on dimension 1 (with a percentage of explained inertia of 21, $17,14,13$ and 14\%, respectively). Up to 2 years/Concrete, 3 years and more/Abstract, Never used/Concrete and Never used/Abstract concept have a higher contribution on dimension 2 (with a percentage of explained inertia of $21,18,16$, and $10 \%$, respectively).

Summarizing, we can see that Dimension 1 (70.70\% of the overall variance) is characterized by the opposition between Emotional and Concrete concepts. Emotional concepts are characterized by the presence of Emotion, Interactive, and Experiential relations in the definitions of all children (i.e., Never Used, Up to 2 years, Two-Three years) apart from late-users of pacifier (3 years and more). Concrete concepts are characterized by the presence of Taxonomic-Superordinate, Perceptual, and Thematic relations (both Thematic-Spatial and Thematic Action-Function) in the definitions of children who Never Used the pacifier or stopped early to use it (Up to 2 years). This suggests that children who used less the pacifier distinguish more markedly between Emotional and Concrete terms, and that definitions of Emotional concepts of late users of pacifiers (3 years and more) are less clearly characterized than those of children who used it less.

On the less relevant Dimension 2 (12.85\% of inertia) Concrete concepts are characterized by children who Never Used the pacifier or stopped early to use it (Up to 2 years) by Taxonomic-Superordinate and Thematic-Spatial relations; they oppose to Abstract concepts characterized in children who Never Used the pacifier and by late users of pacifiers (3 years and more) by Taxonomic-Subordinate/exemplifications relations. Interestingly, the distinction between concrete and abstract concepts is more marked for children who Never Used the pacifier, followed by children who used it until 2 years of age. As to late users of pacifier (3 years and more), similarly to children who did not use pacifiers they produced exemplifications with abstract concepts, but they do not seem to elicit markedly different relations with concrete and abstract concepts.

As to the distinction between concept kinds, we can notice that Emotional concepts oppose to Concrete concepts on Dimension 1, which explains a higher percentage of inertia, while Dimension 2 is characterized by the opposition between Abstract and Concrete concepts. The results therefore indicate that Emotional concepts represent a specific subset of concepts, which however differ more from concrete than from abstract ones.

Hierarchical clustering has been applied over the CA solution depicted in Figure 3, allowing delineating the structure 


\section{A Columns contribution to the Inertia of Dim. 1}

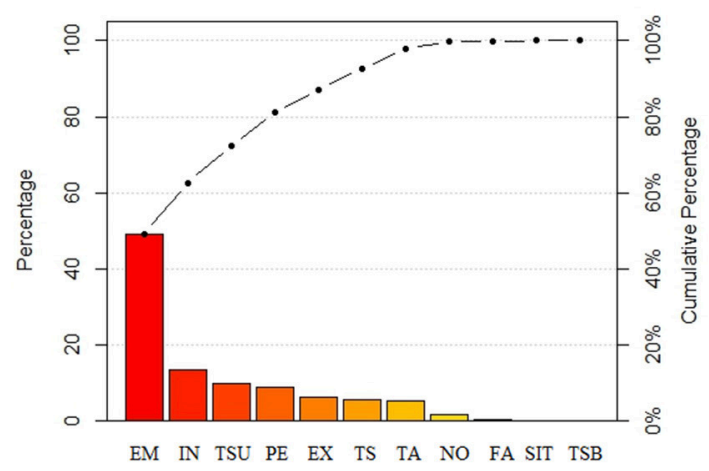

C Rows contribution to the Inertia of Dim. 1

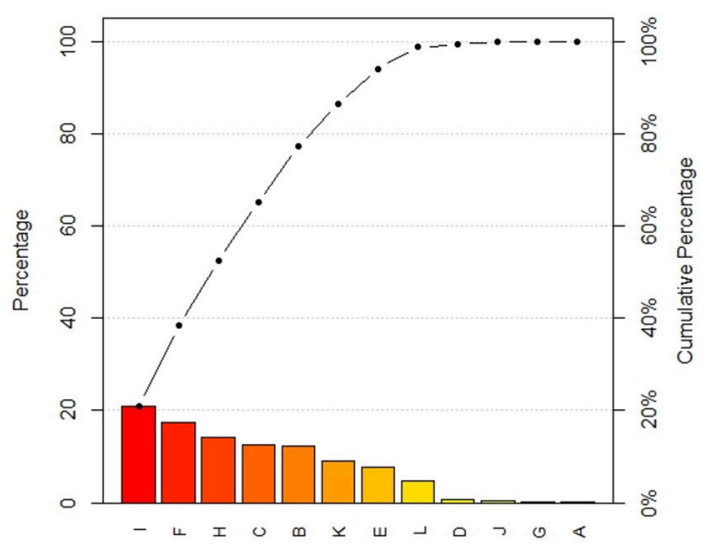

B Columns contribution to the Inertia of Dim. 2

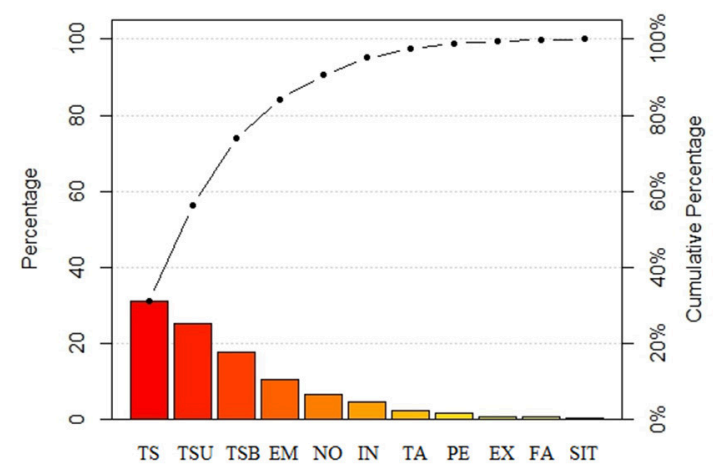

D Rows contribution to the Inertia of Dim. 2

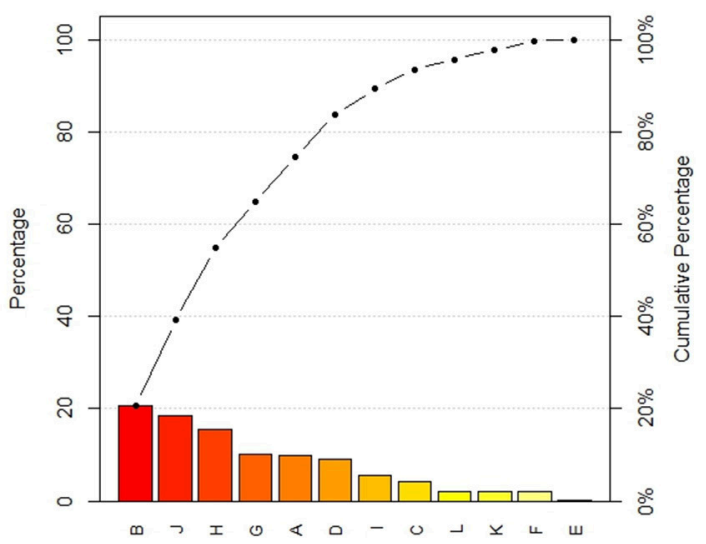

FIGURE 3 | ParetoChart showing which category of Definitions' Features have a higher (in relative terms) contribution to Dimension 1 (A) and Dimension 2 (B). Contribution of Pacifier and Concepts type to the definition of Dimension 1 (C) and Dimension 2 (D). A, TwoAbstract; B, TwoConcrete; C, TwoEmotional; D, Two-ThreeAbstract; E, Two-ThreeConcrete; F, Two-ThreeEmotional; G, NeverAbstract; H, NeverConcrete; I, NeverEmotional; J, ThreeAbstract; K, ThreeConcrete; L, ThreeEmotional.

underlying the dataset by means of "tree" and "clusters" (Husson et al., 2010). The purpose of such analysis is seeking structure in the relations among cases characterized by a number of variables: cases that are similar to each other, in terms of their values for a number of variables, are grouped together forming a cluster.

Hierarchical clustering is performed using an extension of the "CAinterprTools," accomplished via the "FactoMineR" Package (Le et al., 2008). The hierarchy is represented by a dendrogram which is indexed by the gain of within-inertia, with optimal level of division between clusters tree indicated by colored boxes. A barplot of the gained inertia is also returned. Hierarchical clustering of the factor map applied to the Definitions' Features (see Figure 4A) resulted in three clusters: Cluster 1 (black square) with Perceptual, TaxonomicSuperordinate, Norm, and Thematic-Spatial relations; Cluster 2 (red square) with Free Association, Thematic-Action-Function, Taxonomic-Subordinate, and Situation; and Cluster 3 (green square) with Experience, Emotion, and Interaction. Cluster 1 and 3 are opposing on Dimension 1, Cluster 1 and 2 are opposing on Dimension 2. The first dimension accounts for the majority of the inertia of the data, and is determined by the opposition between relational features which typically characterize Concrete concepts in opposition to relations typically associated to Emotional concepts. The second dimension is defined by relational features which typically characterize Concrete concepts, in opposition to relations generally associated to Abstract concepts.

The hierarchical clustering of the factor map confirms that Emotional concepts are clearly different from both Concrete and Abstract concepts, but that the major opposition is that between Concrete and Emotional concepts. This opposition clearly does not depend on the level of abstractness, but seem to be due to the fact that the relations evoked by Emotional concepts pertain emotions and interactive situations, with scarce overlap in particular with the relations elicited by Concrete concepts.

As to Pacifier use by Concepts type (Figure 4B), the clusters are characterized as follows: Cluster 1 (black square) with Concrete concepts and different ages of Pacifier use; Cluster 2 (red square) by Abstract concepts and different ages of Pacifier use; and Cluster 3 (green square) by Emotional concepts and different ages of Pacifier use. Cluster 1 and 3 are opposing on Dimension 1, Cluster 1 and 2 are opposing on Dimension 2. Thus the first dimension, which is accounting for the major part of the 


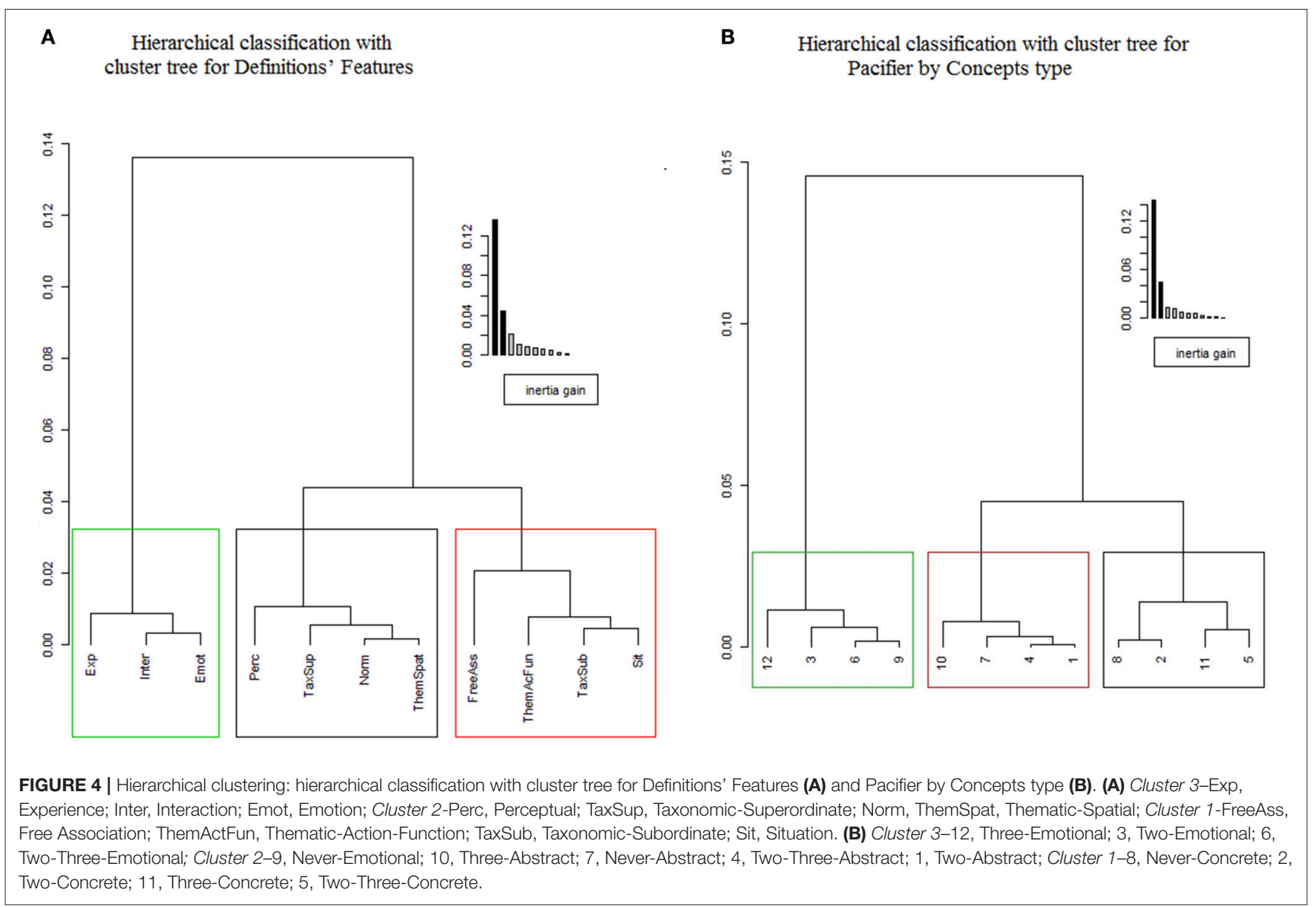

inertia of the data, is determined by the opposition of Concrete and Emotional concepts. The second dimension, which is defined by Concrete and Abstract concepts, is opposing the former to the latter category. When considering the smaller clusters, for both emotion and abstract concepts late users of pacifiers differ from other children. This seems to confirm our hypothesis that the late use of pacifier influences the development of abstract and emotional concepts.

Subsequent Correspondence Analyses performed separately on Concept type and Frequency of Pacifier use provide a more analytical perspective.

\section{Analysis on Concept Type}

Overall, as the percentages show, children extensively uses Thematic-Action-Function and Taxonomic-Subordinate conceptual relations to provide definitions (see Table 3A). This is in line with the predictions of embodied and grounded views, since they seem to situate concepts in action situations and to use exemplifications in order to ground concepts. As to the differences between the concepts, the results of Chi square tests are reported in Table 3B. In line with previous literature, concrete concepts activate more Perceptual properties than Emotional concepts and more Thematic (Thematic-actionfunction and Thematic-spatial) and Taxonomic-Superordinate relations compared to both Emotional and Abstract concepts. Emotional concepts elicit instead more emotional and more interactive relations compared to Concrete and Abstract concepts, and slightly more experiential relations than concrete concepts $(p=0.06)$. As to Abstract concepts, they are not characterized by any specific kind of relation, even if they evoke more emotion relations than concrete concepts (but less than Emotional concepts) and even if, when we look at the percentages, they seem to elicit a high number of free associations (see for consistent results Barsalou and Wiemer-Hastings, 2005).

Correspondence Analysis focused on Concept type and the conceptual relations used in the definition task resulted in two dimensions (Dimension 1-Eigenvalues: 0.19, 85.4\% of inertia; Dimension 2-Eigenvalues: $0.029,14.6 \%$ of inertia). The charts in Figure 5 highlight which type of concepts is defining the first two CA dimensions. The reference line helps in locating which category has an important contribution to the determination of the dimension.

Concrete and Emotional concepts contribute to the definition of the first dimension, while Abstract concepts provide a major contribution to dimension 2, in line with previous analysis. Differently from the previous analysis, however, Concrete and Emotional concepts can be considered as more similar than Abstract concepts (see Figure 5C). 


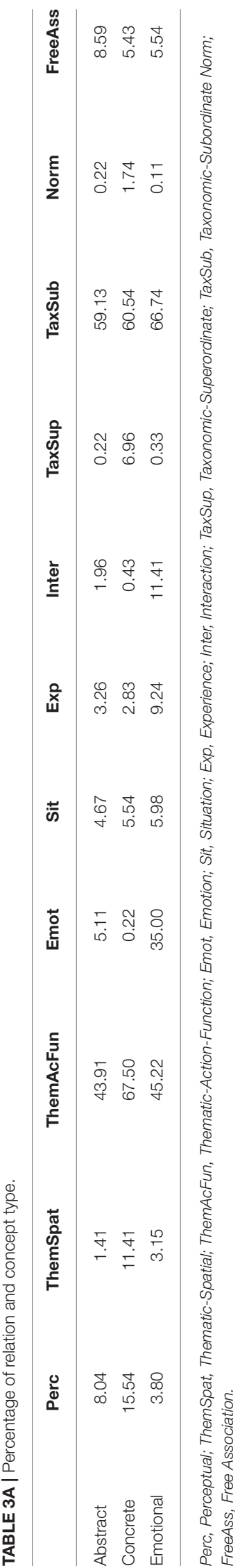

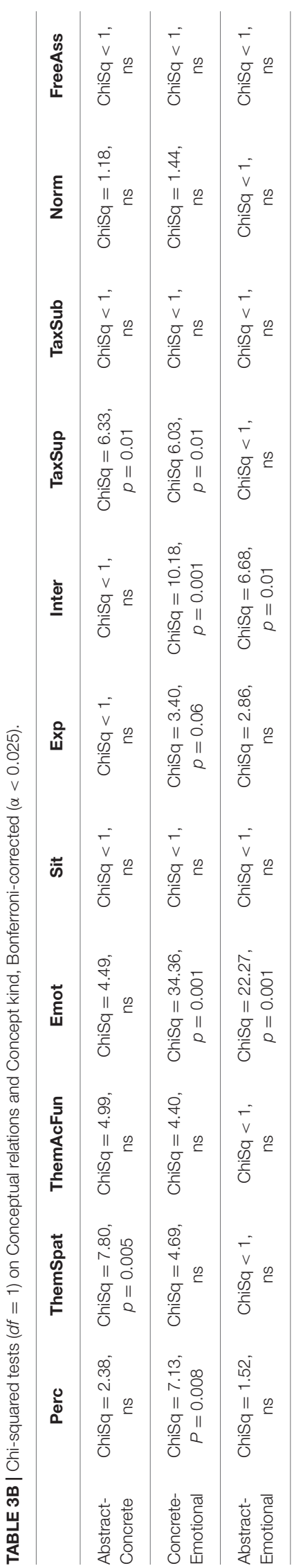

As to the conceptual relations (see Figure 6), Emotional and Interactional type of definitions characterize the first dimension, whereas Thematic-Spatial, Taxonomic-Superordinate, Free Association, Taxonomic-Subordinate and, to a minor extent, Emotional definitions characterize the second dimension.

\section{Analysis on Pacifier Use}

Overall, children extensively use Thematic-Action-Function and Taxonomic-Subordinate relations to provide definitions, independently from the frequency of pacifier use (see Table $4 \mathrm{~A}$ ). However, the frequency of these and other relations is modulated by pacifier use.

If we look at the percentages, we can see that children who Never Used the pacifier produce a higher percentage of perceptual properties as well as of emotion, situation, and experiential relations; furthermore they produce a high percentage of free associations (but slightly lower than children who used it until 2 years). Children who used the pacifiers until age 2 produce more free associations, more interactive properties and more Thematic-spatial relations than other children. The production of children who used the pacifier until 2-3 years is not characterized by a higher percentage of a specific kind of relations than other groups. Compared to other children, late users of pacifier ( 3 years and more), produce more properties related to the interaction with the conceptual referents and their perceptual properties, as the higher percentage of ThematicAction-Function relations and the high percentage of Perceptual relations (but slightly lower than that of children who Never Used the pacifier) testify. Furthermore, they apparently need to ground and exemplify concepts, as the high percentage of Taxonomic-Subordinate indicates.

Overall, the pattern of the relations produced if we look at the percentages suggests that children who did not use pacifier for long produce more relations referring to social and emotional aspects, to experiences and situations, and more free associations, while late users of pacifiers produce mainly exemplifications. If we look at the Chi-squared tests (see Table 4B), we can see however that the only significant differences concern the higher production of free associations of early users of pacifier (Never Used and 2 years) compared to late users (3 years and more). Importantly, free associations are produced more frequently with abstract than with emotional and concrete concepts (see Table 4A).

Correspondence Analysis focused on the frequency of pacifier use and the type of conceptual relations used in the definition task resulted in three dimensions (Dimension 1-Eigenvalues: $0.023,77 \%$ of inertia; Dimension 2-Eigenvalues: 0.004, 14.4\% of inertia; Dimension 3-Eigenvalues: 0.003, 9\% of inertia).

The charts in Figure 7 highlight which frequency of pacifier use is defining the first two CA dimension, accounting for the majority of inertia explained.

The first dimension is mainly characterized by the 3-years group, composed by children who used the pacifier beyond age 3 , and to a minor extent by the 2-years group. The second dimension is defined by the Never and 2 years groups, that is those who did not use the device or used it for a shorter period. 


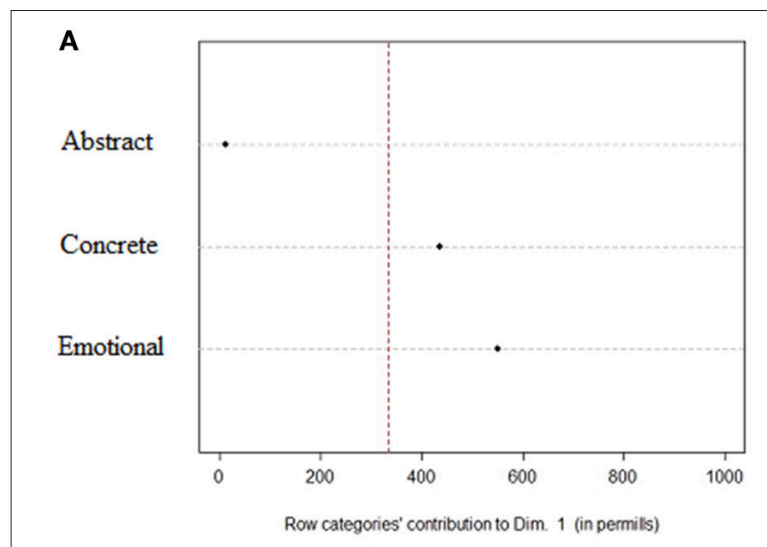

B

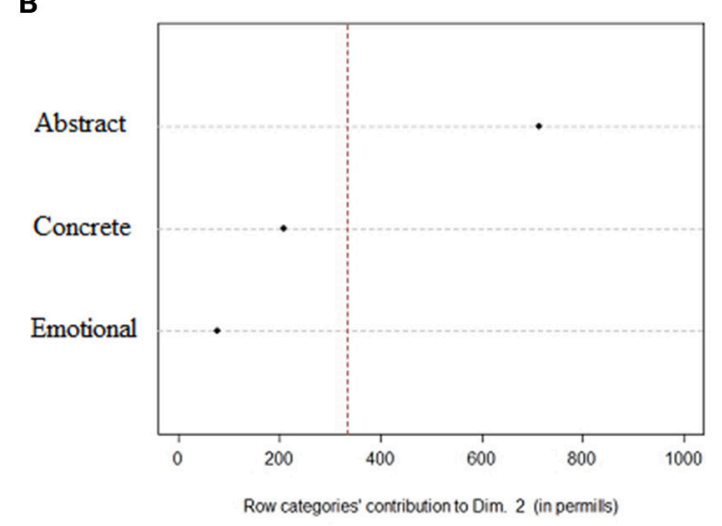

C

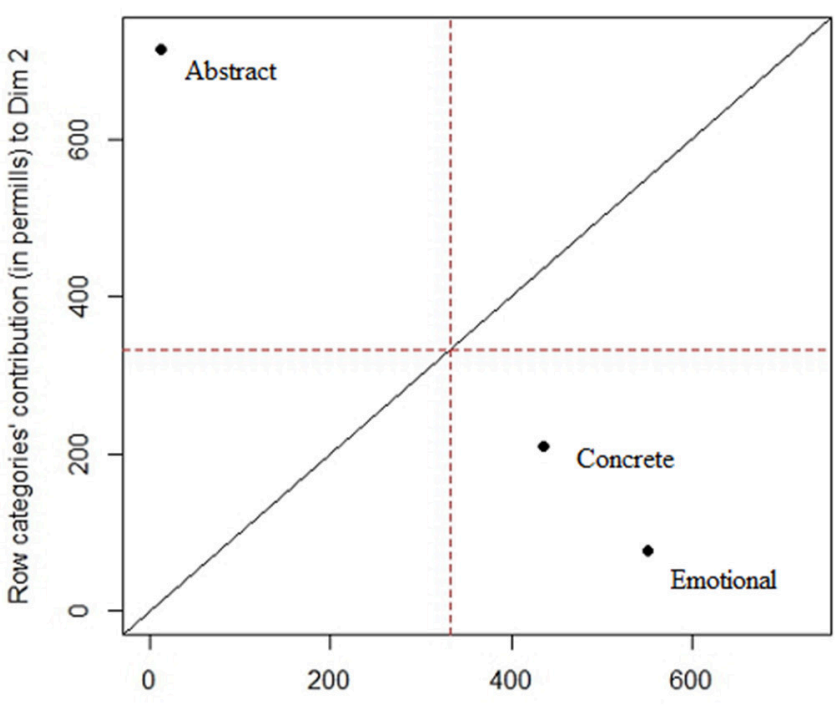

Row categories' contribution (in permills) to Dim 1

FIGURE 5 | Contribution of Concepts type to the definition of dimension 1 (A) and 2 (B); (C) Scatterplot of Concepts type's contribution to dimension 1 and 2.

The two Three-years group does not contributes significantly to the first two dimensions. The analysis thus shows that the use of pacifiers has an impact on the pattern of conceptual relations, as indicated by the distinction between late users of pacifiers and those who never used it or stopped to use it early.

As for the conceptual relations (see Figure 8), Free Association and, to a minor extent Thematic-Spatial relations, define the first dimension, whereas the second dimension is defined by Experiential, Interactional, Emotional, and Taxonomic-Subordinate conceptual relations. Interestingly, the use of Free Association is clearly distinct from use of relations more linked to social and emotional aspects, as well as to examples.

\section{DISCUSSION}

\section{Distinction between Abstract, Concrete, and Emotional Concepts}

Overall, our results clearly support the studies according to which abstract Emotional concepts differ from other, nonemotional Abstract concepts (Altarriba et al., 1999; Altarriba and Bauer, 2004; Setti and Caramelli, 2005) and are not in line with the view according to which all abstract concepts are emotional ones. As anticipated, abstract concepts come in different varieties, and range from social concepts to mental states to mathematical ones (see Borghi and Binkofski, 2014, for a thorough analysis of this). Here we were interested in the relationship between abstract concepts that directly refer to emotions, i.e., emotional concepts, and other kinds of abstract concepts. Even if emotional concepts are abstract by definition, our results show that abstract Emotional concepts represent a very special sub-kind among the sub-kinds of abstract concepts. Our study widely extends previous results showing that in 7-yearolds abstract Emotional concepts differ from Abstract concepts both in accuracy and in the pattern of conceptual relations they elicit.

As to accuracy, linear mixed-effect modeling showed that Abstract and abstract Emotional concepts differed, due to fact that abstract concepts were more difficult to define than Emotional concepts; Emotional and Concrete concepts instead did not differ.

Emotional concepts differed from both Concrete and Abstract concepts also as to the conceptual relations they yielded. In the correspondence analysis in which the conceptual relations elicited by the three kinds of concepts were combined with contribution of pacifier (Figure 2), Emotional concepts opposed on the first and more relevant dimension to Concrete concepts, while in the correspondence analysis on the conceptual relations yielded by the three kinds of concepts without considering the role of pacifier (Figure 5C), abstract Emotional concepts were 


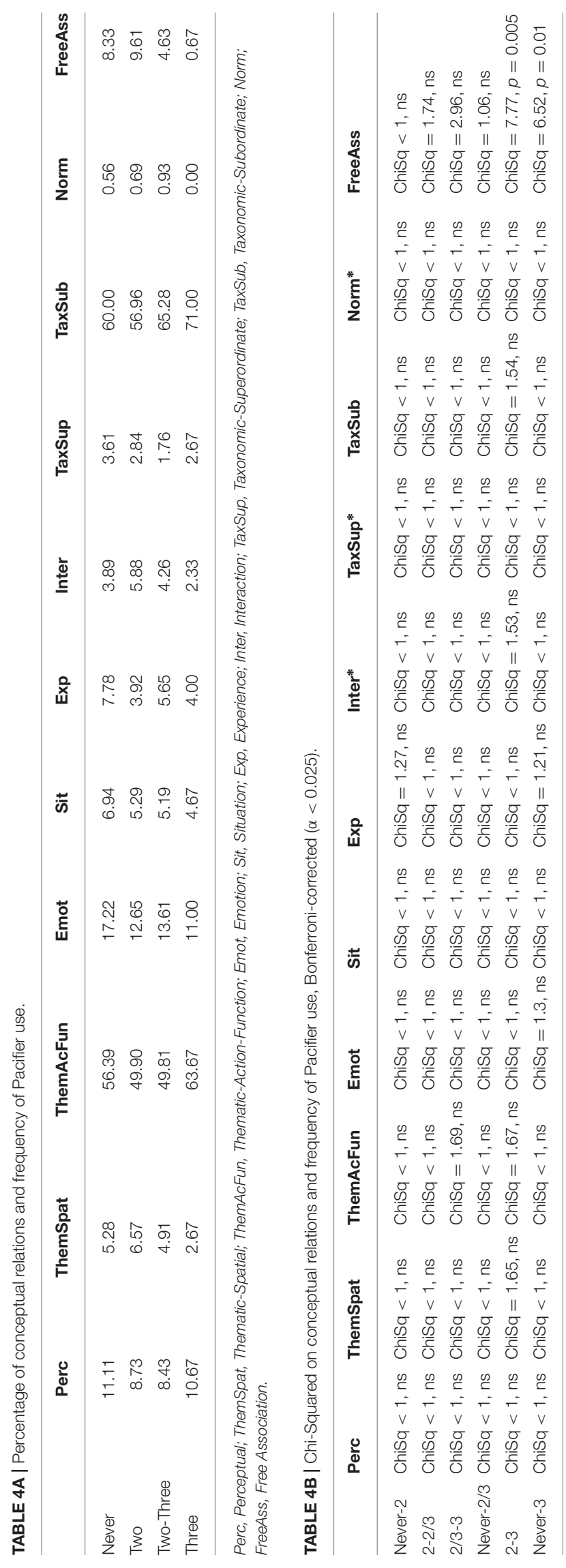

represented on Dimension 1 together with Concrete concepts, while Abstract concepts were represented on Dimension 2.

While our results disconfirm views according to which abstract emotional concepts do not differ from abstract nonemotional concepts, they do not exclude that the presence of emotional features might be relevant in characterizing abstract concepts overall, as proposed by a recent view on abstract concepts (Kousta et al., 2011; Newcombe et al., 2012; Vigliocco et al., 2013; Siakaluk et al., 2014). Indeed, results on correlations in the preliminary study (see Figure S1) suggest that emotionality is correlated, even if slightly, with Abstractness, Age of Acquisition and Modality of Acquisition, and show that Context Availability, Concreteness and Imageability are, even if slightly, negatively correlated with Emotionality. Furthermore, Chi squared tests on the conceptual relations produced revealed that abstract concepts yield more emotion relations than Concrete concepts, even if less than Emotional concepts.

The results we found on abstract Emotional concepts have a number of theoretical implications. First, they highlight the limitations of a view according to which Abstract and Concrete concepts are dichotomously opposed, and favor instead the idea that they are arranged along a continuum (Wiemer-Hastings et al., 2001). Indeed, we found clear processing differences between Abstract concepts, Concrete concepts and abstract Emotional concepts. This is particularly interesting because emotional concepts by definition would be part of abstract concepts, since they do not have a concrete object as referent, and also because in our study the selected Emotional concepts had abstractness and concreteness levels similar to Abstract concepts. Second, they suggest that more studies are needed, aimed at investigating the fine-grained differences and the different typologies of concrete and abstract concepts. The finegrained analysis of differences between kinds of abstract concepts constitutes a new and fruitful research avenue some authors are starting to open (e.g., Crutch et al., 2013; Ghio et al., 2013; Roversi et al., 2013). Third, they indicate that the analysis of conceptual relations elicited might be a promising research avenue to investigate possible differences in the acquisition and development of different kinds of concepts. In spite of the differences we found between Abstract and abstract Emotional concepts, we found one common element: the development of both kinds of concepts is influenced, as predicted, by the use of pacifier.

\section{Effects of Pacifier Use on Conceptual Acquisition}

Our results suggest that the use of pacifier has an effect on the development of abstract and emotional concepts. Importantly, this effect is a long-term one, since we tested the conceptual representation in children who do not use pacifier since years. We did not find support for our strong hypothesis that the use of pacifier would influence the accuracy of the produced definitions. However, we found support to our weak hypothesis, according to which the network of associated relations of emotional and abstract concepts differs depending on how long the pacifier was used. 

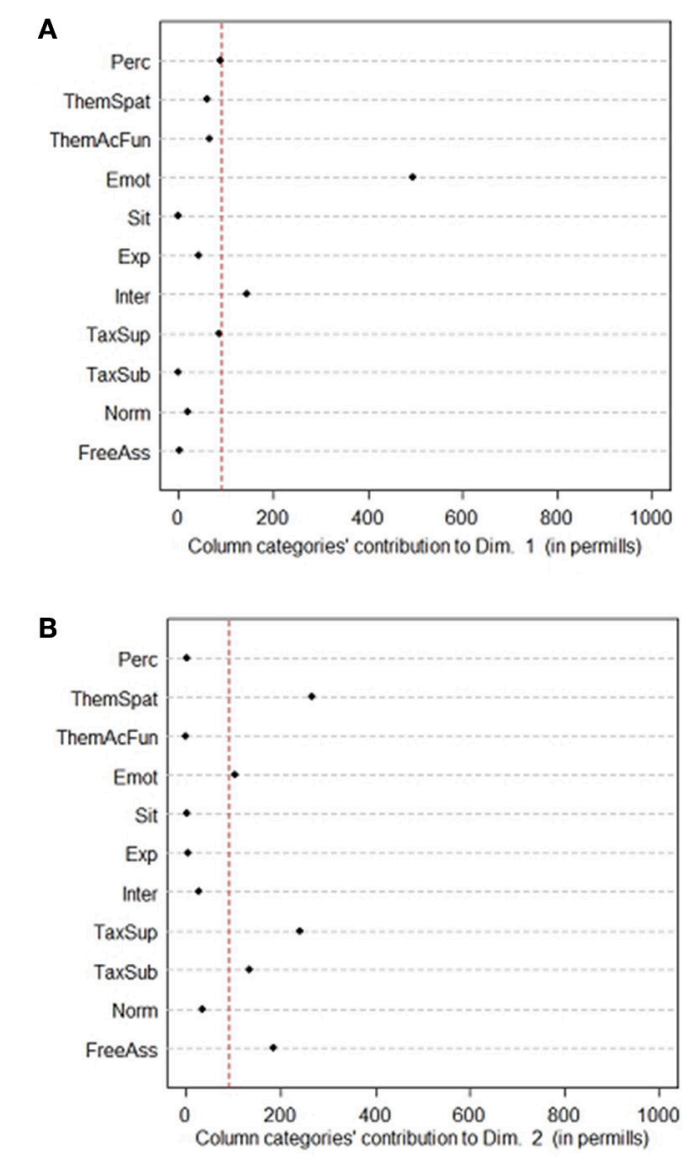

C

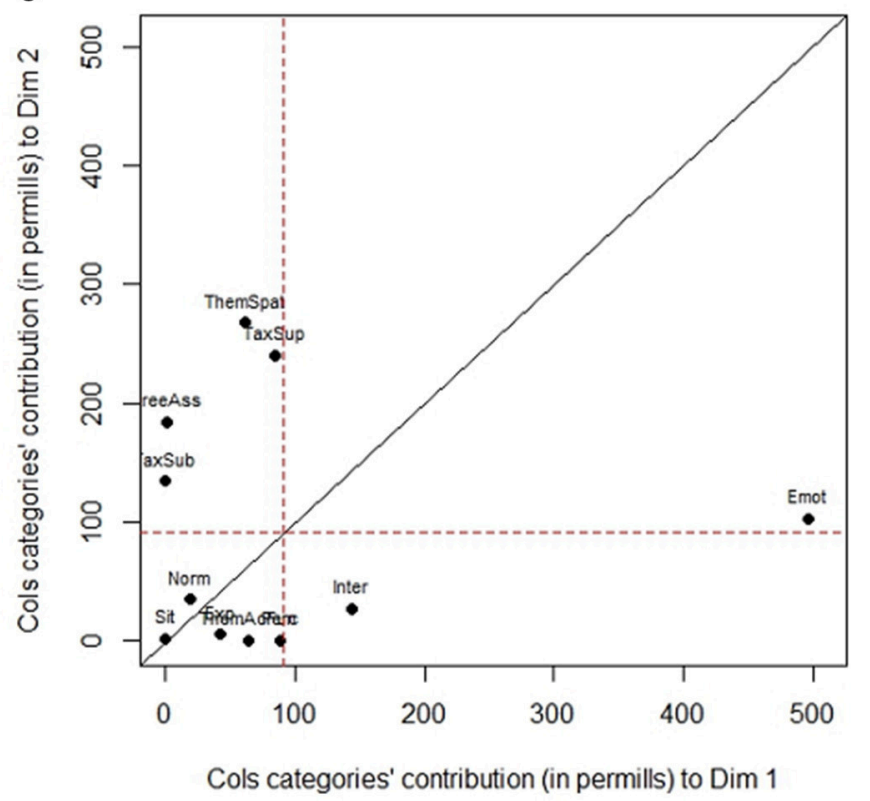

FIGURE 6 | Contribution of the conceptual relations to the definition of dimension 1 (A) and 2 (B); (C) Scatterplot of conceptual relations' contribution to dimension 1 and 2 .

As to the influence of pacifier on abstract Emotional concepts, the correspondence analysis in Figure 2 indicates on Dimension 1 that children who never used or used less the pacifier distinguish more sharply between abstract Emotional and Concrete concepts, and that definitions of abstract Emotional concepts by children who used pacifier beyond age 3 are less clearly characterized than those of children who used it less. The use of pacifier influences also Abstract concepts (see Figure 3, Dimension 2): Children who never used the pacifier and used it until age 2 distinguish more clearly between concrete and abstract concepts, while for late users of pacifier (3 years and more) the distinction between abstract and concrete concepts is unclear. The difference between late users of pacifiers and other children for both emotion and abstract concepts is confirmed by the cluster analysis (Figure 4). If we consider the percentage of conceptual relations produced depending on pacifier use (Table 4), we can notice that the relations produced by late users of pacifiers is confined to exemplifications and thematic-actionfunction relations, with a reduced richness of relations typically associated to emotional and abstract concepts as experiential, interactive, situational, emotional relations as well as spatial relations and free associations. Chi-squared tests revealed that early and late users of pacifiers differed as the first produce more free associations than the others (see Table 4). Finally, the effects of the use of pacifier on the conceptual relations produced is clearly visible in Figure 7C, where 3 years and more significantly contributed to Dimension 1, while children who never used the pacifier or stopped using it early (Never Used, 2 years) contribute to Dimension 2.

Overall, our results indicate that using pacifiers for long leads to a less marked distinction between concrete and abstract emotional concepts and between concrete and abstract concepts; as to conceptual relations, late users of pacifier tend to refer less to their experience, to social and emotional situations, to use more exemplifications and functional relations, and to use less free associations. One possible limitation of our study resides in the sample size: while the overall sample is composed by 59 children, the two samples of children who did not use the pacifier and of children who used it beyond age 3 might appear small. It should be taken into account, however, that the distribution of our sample reflects the distribution of pacifier use in children: the majority of Italian children use the pacifier since the first month of life (Riva et al., 1999) and the majority of them stops to use it before age 3, slightly before starting nursery school. A further limitation is that children with different levels of pacifier's use were not matched for verbal 


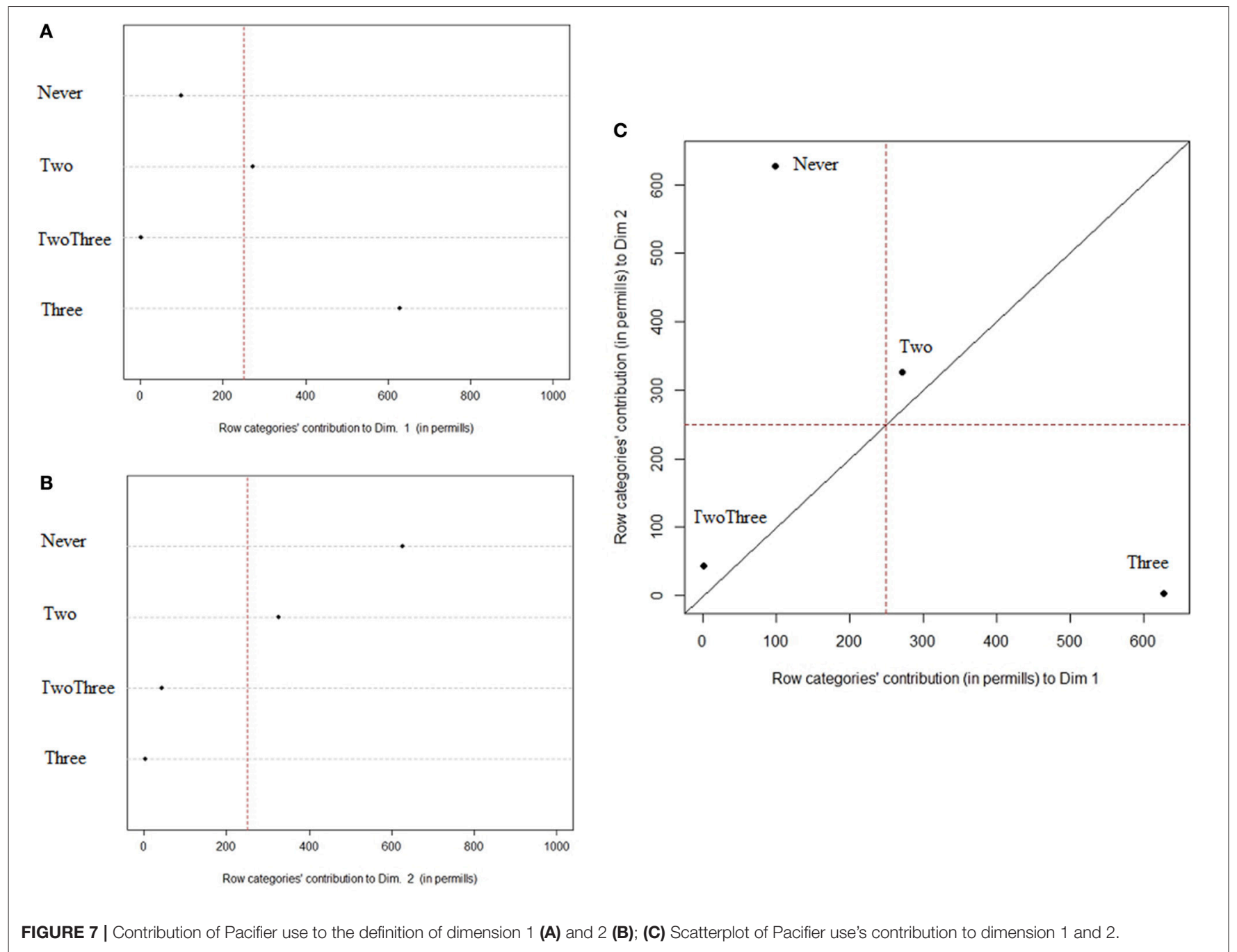

intelligence and vocabulary size, because we intended to have a general picture of the influence of pacifier on acquisition and representation of different kinds of concepts. This might however reduce the strength of our conclusions, hence further work in which these factors are controlled is needed. Importantly, in our sample the differences in use of pacifier cannot be ascribed to demographic characteristics as gender or level of parental instruction, as seen in Table 1. Further studies will be necessary to understand whether children who use less the pacifier differ from children who use it more on the basis of different variables, as for example the level of activity which might render the use of pacifier more/less necessary. Finally, we did not explore the implications of thumb sucking in the present study. Both pacifier use and thumb sucking influence facial mimicry and mouth muscle position, but such behaviors are quite different from each other. While the use of the pacifier is a "passive behavior" for the child as it is induced by parents, thumb sucking is a child volunteer action that is generally despised by parents as it is considered as a "dirty" action. Moreover, from a practical point of view, thumb sucking is more difficult to control especially because we get this information from retrospective questionnaires compiled by parents. Niedenthal et al. (2012) considered both pacifier use and thumb sucking in their study, and found that (differently from pacifier overuse) thumb sucking has no long-term effects on emotional competence, their variable of interest. The implications of both pacifier use and thumb sucking need to be explored in further studies.

The results we found have a number of implications for current theories of abstract and emotional concepts. In general, the influence of the use of pacifier on conceptual development supports the view that abstract concepts are grounded in sensorimotor experience (e.g., Kiefer and Pulvermüller, 2012), and suggests that both linguistic and social and emotional experience might be important for their acquisition and development (Borghi et al., 2017; Pexman, 2017; Ponari et al., 2017).

As to abstract concepts, they confirm the prediction of the WAT theory according to which the use of pacifier, involving the mouth, should interfere with the acquisition of abstract concepts, and influence the pattern of conceptual relations they elicit. Children who did not use the pacifier or used it for short time seem to be more competent in processing abstract concepts 


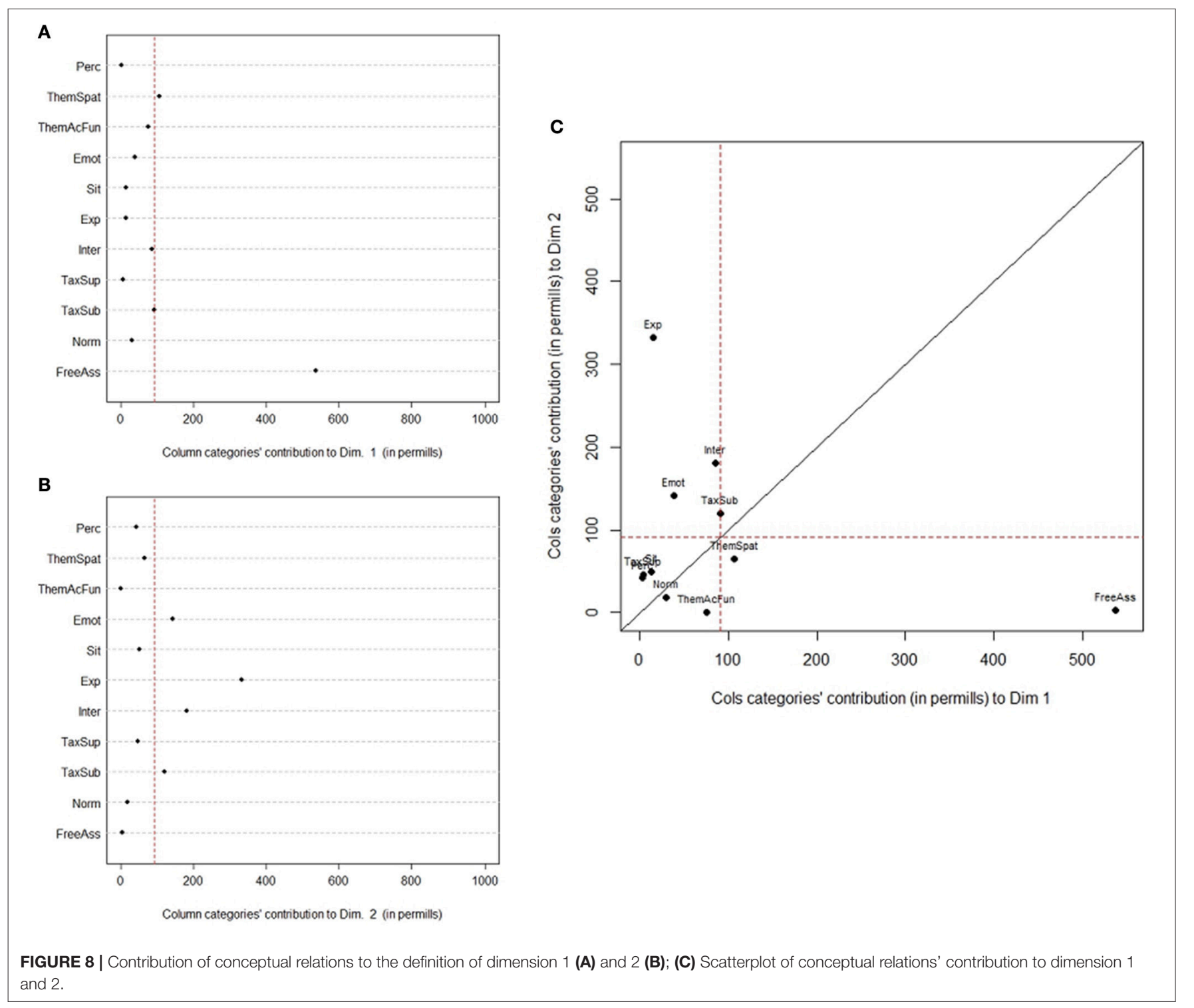

than children who overused the pacifier (for more than 3 years and during social interactions): the pattern of relations the first produce with abstract concepts is richer, and the contrast with the pattern of relations elicited by concrete concepts is more marked and clear in children who did not use the pacifier or used it only for short. An extensive use of pacifier can have impeded them for long time to simulate the word meaning either reenacting the word acquisition experience and/or re-explaining to themselves their meaning through inner talk. This might have influenced the consolidation in memory of abstract concepts meanings. Importantly, the activation of the mouth revealed by the influence of the pacifier is compatible with the views according to which abstract concepts focus attention on internal states (e.g., Barsalou and Wiemer-Hastings, 2005; Kiefer and Barsalou, 2013): introspection could namely occur through inner talk and could involve the recruitment of the mouth motor system (see Borghi and Zarcone, 2016, for developing this issue).
An alternative and not necessarily contrasting explanation ascribes more relevance to the emotional and social dimension: the pacifier might hide more the facial expression, thus it could impede children to fully benefit of the social input necessary for the acquisition of abstract words. The hypothesis that building abstract concepts requires such social input is in keeping with recent evidence on infants (Bergelson and Swingley, 2013) showing that the comprehension of abstract concepts (e.g., "all gone") emerges at around 10 months and becomes more stable at around 14 months. Around 9-10 months, children improve their ability to follow the gaze of others (Beier and Spelke, 2012), while around 14 months they develop forms of joint attention (Carpenter et al., 1998).

Even if current results do not allow us to fully disentangle between the two explanations, we believe that the first is more plausible in light of the current results. If we look at the percentages of relations produced (Table 4), we can see that 
abstract concepts elicit a higher percentage of free associations, rather than of relations involving social and emotional aspects.

Two explanations are possible also in the case of emotional concepts; in this case too the two explanations are not necessarily mutually exclusive. The first relates the difficulty in acquisition/consolidation of Emotional concepts with the reduction of children's emotional competence due the fact that the use of pacifier renders is more difficult to manifest and recognize emotions through mimicry and facial expressions (Niedenthal et al., 2012). The high percentage of emotion and interactive relations produced with Emotional concepts renders this explanation highly plausible.

The second relates the difficulty with Emotional concepts to the fact that the pacifier involves the use of the mouth. Some brain imaging and behavioral studies have shown that emotional concepts activate both the mouth and the hand effectors. Moseley et al. (2012) found with fMRI that processing of abstract emotional words, beyond the limbic regions, engages areas of the precentral cortex activated somatotopically by mouth and hand words. Dreyer et al. (2015) showed that patient CA suffering from lesion in the left supplementary motor area was primarily impaired in abstract-emotional word processing, known to be involved in motor planning independently of a specific effector. At the behavioral level, Ghio et al. (2013) used a rating task in which they asked to what extent actions implied by sentences on emotions, mental states or math concepts referred to the leg, arm or mouth. They found that emotion sentences elicited high ratings for both the hand and the mouth, abstract mental state concepts activated more the mouth and math-related sentences activated more the hand, likely due to the influence of finger counting practice (see also an fRMI follow up by Ghio et al., 2016).

Differently from abstract concepts, for emotional concepts we think that our results render the first explanation more plausible, even if they do not allow to fully disentangle between the two accounts. If we look at the percentages of relations produced (Table 4), we can see that, while abstract concepts elicit a higher percentage of free associations, emotional concepts yield mostly relations involving emotional and interactive aspects.

One further issue remains to be clarified. The influence of pacifier could be occur in different processing phases. The longterm effect we found could be due to the fact that children could not benefit of the linguistic and social input during first encoding in memory of word meanings. Alternatively, it is possible that the effect of pacifier not only influences encoding but is more extended, influencing also consolidation and retrieval. Even if 7-year-olds do not use pacifier, their re-enactment of the word acquisition can be negatively influenced by their previous encoding experience. Further studies are needed to determine in which phase the influence of pacifier occurs.

\section{REFERENCES}

Alberti, G. (2015). CAinterprTools: an $\mathrm{R}$ package to help interpreting correspondence analysis' results. Software $X$ 1-2, 26-31. doi: 10.1016/j.softx.2015.07.001
To summarize, we have demonstrated that in 7-year-olds Concrete, Abstract and Emotional concepts elicit a clearly distinct pattern of conceptual relations. Furthermore, we have shown that the acquisition of the conceptual relations associated to both Abstract and abstract Emotional concepts has an embodied counterpart: it is influenced and modulated by the use of pacifier. Importantly, the influence of pacifier is a long term one. Our results suggest that the influence of pacifier might be due to different mechanisms for the two kinds of concepts-a mechanism ascribing a major role to the linguistic simulation in the case of Abstract concepts, another ascribing a more relevant role to emotional and interactive aspects in the case of abstract Emotional concepts. However, our data do not allow us to conclusively determine which of the two mechanisms is at play; furthermore, the two mechanisms are not necessarily mutually exclusive. Further developmental studies will be needed to investigate more in depth the similarities and differences in the acquisition and representation of different kinds of concepts.

\section{ETHICS STATEMENT}

This study was carried out in accordance with the recommendations of the Ethic Guidelines of the Institute of Cognitive Sciences and Technologies of the Italian National Research Council, with written informed consent from all subjects. All subjects gave written informed consent in accordance with the Declaration of Helsinki. The protocol was approved by the Ethic Committee of the Institute of Cognitive Sciences and Technologies of the Italian National Research Council.

\section{AUTHOR CONTRIBUTIONS}

$\mathrm{LB}$ and $\mathrm{AB}$ contributes to the conception and design of the work; the analysis and interpretation of the data and, with $\mathrm{CM}$, to data acquisition. $\mathrm{LB}$ and $\mathrm{AB}$ contributes to the writing of the manuscript, and CM to revising it.

\section{ACKNOWLEDGMENTS}

We would like to acknowledge Marica Notte for study coordination and data collection. We acknowledge the staff and families of Fausto Cecconi School for participating in the study.

\section{SUPPLEMENTARY MATERIAL}

The Supplementary Material for this article can be found online at: https://www.frontiersin.org/articles/10.3389/fpsyg. 2017.02014/full\#supplementary-material

Altarriba, J., and Bauer, L. M. (2004). The distinctiveness of emotional concepts: a comparison between emotion, abstract, and concrete words. Am. J. Psychol. 117, 389-410. doi: 10.2307/4149007

Altarriba, J., Bauer, L. M., and Benvenuto, C. (1999). Concreteness context availability and imageability ratings and word associations for abstract, 
concrete, and emotion words. Behav. Res. Methods 31, 578-602. doi: 10.3758/BF03200738

Baayen, R. H. (2008). Analyzing Linguistic Data. A Practical Introduction to Statistics using R. Cambridge: Cambridge University Press.

Barca, L., Burani, C., and Arduino, L. S. (2002). Word naming times and psycholinguistic norms for Italian nouns. Behav. Res. Met. Instrum. Comput. 34, 424-434. doi: 10.3758/BF03195471

Barr, D. J., Levy, R., Scheepers, C., and Tily, H. J. (2013). Random effects structure for confirmatory hypothesis testing: keep it maximal. J. Mem. Lang. 68, 255-278. doi: 10.1016/j.jml.2012.11.001

Barsalou, L. W. (2003). Abstraction in perceptual symbol systems. Philos Trans. R. Soc. Lond. B. Biol. Sci. 358, 1177-1187. doi: 10.1098/rstb.2003.1319

Barsalou, L. W. (2016). On staying grounded and avoiding quixotic dead ends. Psychon. Bull. Rev. 23, 1122-1142. doi: 10.3758/s13423-016-1028-3

Barsalou, L. W., and Wiemer-Hastings, K. (2005). "Situating abstract concepts," in Grounding Cognition: the role of Perception and Action in Memory, Language, and Thought, eds D. Pecher and R. Zwaan (New York, NY: Cambridge University Press), 129-163.

Barsalou, L. W., Santos, A., Simmons, W. K., and Wilson, C. D. (2008). "Language and simulation in conceptual processing," in Symbols and Embodiment Debates on Meaning and Cognition, eds M. de Vega, A. Glenberg, and A. Graesser (Oxford: Oxford University Press), 245-284. doi: 10.1093/acprof:oso/9780199217274.003.0013

Beier, J. S., and Spelke, E. S. (2012). Infants' developing understanding of social gaze. Child Dev. 83, 486-496. doi: 10.1111/j.1467-8624.2011.01702.x

Bates, D., and Maechler, M. (2009). Ime4: Linear Mixed-Effects Models using s4 Classes [Computer Software Manual]. Available online at: http://CRAN.Rproject.org/package $=\operatorname{lme} 4$

Bergelson, E., and Swingley, D. (2013). The acquisition of abstract words by young infants. Cognition 127, 391-397. doi: 10.1016/j.cognition.2013.02.011

Binder, J. R., Westbury, C. F., McKiernan, K. A., Possing, E. T., and Medler, D. A. (2005). Distinct brain systems for processing concrete and abstract concepts. J. Cogn. Neurosci. 17, 905-917. doi: 10.1162/0898929054021102

Bolker, B. M., Brooks, M. E., Clark, C. J., Geange, S. W., Poulsen, J. R., Stevens, M. H., et al. (2009). Generalized linear mixed models: a practical guide for ecology and evolution. Trends Ecol. Evol. 24, 127-135. doi: 10.1016/j.tree.2008.10.008

Borghi, A. M., and Binkofski, F. (2014). Words as Social Tools: An Embodied View on Abstract Concepts. Berlin; New York, NY: Springer.

Borghi, A. M., Binkofski, F., Cimatti, F., Scorolli, C., and Tummolini, L. (2017). The challenge of abstract words. Psychol. Bull. 143, 263-292. doi: $10.1037 /$ bul0000089

Borghi, A. M., and Caramelli, N. (2003). Situation bounded conceptual organization in children: from action to spatial relations. Cognit. Dev. 18, 49-60. doi: 10.1016/S0885-2014(02)00161-2

Borghi, A. M., and Cimatti, F. (2009). "Words as tools and the problem of abstract words meanings," in Proceedings of the 31st Annual Conference of the Cognitive Science Society, eds N. Taatgen and H. van Rijn (Amsterdam: Cognitive Science Society), 2304-2309.

Borghi, A. M., Flumini, A., Cimatti, F., Marocco, D., and Scorolli, C. (2011). Manipulating objects and telling words: a study on concrete and abstract words acquisition. Front. Psychol. 2:15. doi: 10.3389/fpsyg.2011.00015

Borghi, A. M., and Zarcone, E. (2016). Grounding abstractness: abstract concepts and the activation of the mouth. Front. Psychol. 7:1498. doi: $10.3389 /$ fpsyg.2016.01498

Burani, C., Bimonte, D., Barca, L., and Vicari, S. (2006). Morphology and lexical comprehension in Williams Syndrome. Brain Lang. 99, 112-113. doi: 10.1016/j.bandl.2006.06.065

Caramelli, N., Borghi, A., and Setti, A. (2006). The identification of definition strategies in children of different ages. Linguist. Comput. 26, 155-178.

Carpenter, M., Nagell, K., Tomasello, M., Butterworth, G., and Moore, C. (1998). Social cognition, joint attention, and communicative competence from 9 to 15 months of age. Monogr. Soc. Res. Child. Dev. 63, 1-174. doi: 10.2307/ 1166214

Connell, L., and Lynott, D. (2012). Strength of perceptual experience predicts word processing performance better than concreteness or imageability. Cognition 125, 452-465. doi: 10.1016/j.cognition.2012.07.010

Crutch, S. J., Troche, J., Reilly, J., and Ridgway, G. R. (2013). Abstract conceptual feature ratings: the role of emotion, magnitude, and other cognitive domains in the organization of abstract conceptual knowledge. Front. Hum. Neurosci. 7:186. doi: 10.3389/fnhum.2013.00186

Csibra, G., and Gergely, G. (2009). Natural pedagogy. Trends Cogn. Sci. 13, 148-153 doi: 10.1016/j.tics.2009.01.005

Della Rosa, P. A., Catricalà, E., Vigliocco, G., and Cappa, S. F. (2011). Beyond the abstract-concrete dichotomy: mode of acquisition, concreteness, imageability, familiarity, age of acquisition, context availability, and abstractness norms for a set of 417 Italian words. Behav. Res. Methods 42, 1042-1048. doi: 10.3758/BRM.42.4.1042

Dove, G. (2011). On the need for embodied and dis-embodied cognition. Front. Psychol. 1:242. doi: 10.3389/fpsyg.2010.00242

Dove, G. (2014). Thinking in words: language as an embodied medium of thought. Topics Cognit. Sci. 6, 371-389. doi: 10.1111/tops.12102

Dove, G. (2016). Three symbol ungrounding problems: abstract concepts and the future of embodied cognition. Psychon. Bull. Rev. 23, 1109-1121. doi: 10.3758/s13423-015-0825-4

Dreyer, F. R., Frey, D., Arana, S., von Saldern, S., Picht, T., Vajkoczy, P., et al. (2015). Is the motor system necessary for processing action and abstract emotion words? Evidence from focal brain lesions. Front. Psychol. 6:1661. doi: 10.3389/fpsyg.2015.01661

Estes, Z., Golonka, S., and Jones, L. L. (2011). Thematic thinking: the apprehension and consequences of thematic relations. Psychol. Learn. Motiv. Adv. Res. Theory 54:249. doi: 10.1016/B978-0-12-385527-5.00008-5

Fernandino, L., Humphries, C. J., Seidenberg, M. S., Gross, W. L., Conant, L. L., and Binder, J. R. (2015). Predicting brain activation patterns associated with individual lexical concepts based on five sensory-motor attributes. Neuropsychology 76, 17-26. doi: 10.1016/j.neuropsychologia.2015.04.009

Foroni, F., and Semin, G. R. (2009). Language that puts you in touch with your bodily feelings the multimodal responsiveness of affective expressions. Psychol. Sci. 20, 974-980. doi: 10.1111/j.1467-9280.2009.02400.x

Gentner, D. (2006). "Why verbs are hard to learn," in Action Meets Words: How Children Learn Verbs, eds K. Hirsch-Pasek and R. Golinkoff (New York, NY: Oxford University Press), 544-564.

Ghio, M., Vaghi, M. M. S., Perani, D., and Tettamanti, M. (2016). Decoding the neural representation of fine-grained conceptual categories. Neuroimage 132, 93-103. doi: 10.1016/j.neuroimage.2016.02.009

Ghio, M., Vaghi, M. M. S., and Tettamanti, M. (2013). Fine-grained semantic categorization across the abstract and concrete domains. PLoS ONE 8:e67090. doi: 10.1371/journal.pone.0067090

Gleitman, L. R., Cassidy, K., Nappa, R., Papafragou, A., and Trueswell, J. C. (2005). Hard words. Lang. Learn. Dev. 1, 23-64. doi: 10.1207/s15473341lld0101_4

Granito, C., Scorolli, C., and Borghi, A. M. (2015). Naming a lego world. The role of language in the acquisition of abstract concepts. PLoS ONE 10:e0114615. doi: 10.1371/journal.pone.0114615

Greenacre, M. (2007). Correspondence Analysis in Practice, 2nd Edn. London: Chapman \& Hall/CRC.

Greenacre, M., and Blasius, J. (1994). Correspondence Analyses in the Social Sciences: Recent Developments and Applications. London: Academic Press.

Havas, D. A., Glenberg, A. M., Gutowski, K. A., Lucarelli, M. J., and Davidson, R. J. (2010).Cosmetic use of botulinum toxin-A affects processing of emotional language. Psychol. Sci. 21, 895-900. doi: 10.1177/0956797610374742

Husson, F., Josse, J., and Pagès, J. (2010). Principal Component Methods Hierarchical Clustering - Partitional Clustering: Why Would we Need to Choose for Visualizing Data? Technical Report, Agrocampus.

Kalénine, S., Peyrin, C., Pichat, C., Segebarth, C., Bonthoux, F., and Baciu, M. (2009). The sensory-motor specificity of taxonomic and thematic conceptual relations: a behavioral and fMRI study. Neuroimage 44, 1152-1162. doi: 10.1016/j.neuroimage.2008.09.043

Kiefer, M., and Barsalou, L. W. (2013). "15 grounding the human conceptual system in perception, action, and internal states," in Action Science: Foundations of an Emerging Discipline, eds W. Prinz, M. Beisert, and A. Herwig (Cambridge: MIT Press), 381-401.

Kiefer, M., and Pulvermüller, F. (2012). Conceptual representations in mind and brain: theoretical developments, current evidence and future directions. Cortex 48, 805-825. doi: 10.1016/j.cortex.2011.04.006

Kousta, S. T., Vigliocco, G., Vinson, D., Andrews, M., and Del Campo, E. (2011). The representation of abstract words: why emotion matters. J. Exp. Psychol. Gen. 140, 14-34. doi: 10.1037/a0021446 
Kousta, S. T., Vinson, D. P., and Vigliocco, G. (2009). Emotion words, regardless of polarity, have a processing advantage over neutral words. Cognition 112, 473-481. doi: 10.1016/j.cognition.2009.06.007

Le, S., Josse, J., and Husson, F. (2008). FactoMineR: an R package for multivariate analysis. J. Stat. Softw. 25, 1-18. doi: 10.18637/jss.v025.i01

Lupyan, G., and Clark, A. (2015). Words and the world predictive coding and the language-perception-cognition interface. Curr. Direct. Psychol. Sci. 24, 279-284. doi: 10.1177/0963721415570732

Marconi, L., Ott, M., Pesenti, E., Ratti, D., and Tavella, M. (1993). Lessico Elementare. Dati Statistici Sullitaliano Letto e Scritto dai Bambini delle Elementari [Elementary Lexicon: Statistical Data on Italian as Written and Read by Elementary School Children]. Bologna: Zanichelli.

Mirman, D., Landrigan, J. F., and Britt, A. E. (2017). Taxonomic and Thematic Semantic Systems. Psychol. Bull. 143, 499-520. doi: 10.1037/bul0000092

Moseley, R., Carota, F., Hauk, O., Mohr, B., and Pulvermüller, F. (2012). A role for the motor system in binding abstract emotional meaning. Cereb. Cortex 22, 1634-1647. doi: 10.1093/cercor/bhr238

Newcombe, P. I., Campbell, C., Siakaluk, P. D., and Pexman, P. M. (2012). Effects of emotional and sensorimotor knowledge in semantic processing of concrete and abstract nouns. Front. Hum. Neurosci. 6:275. doi: 10.3389/fnhum.2012.00275

Niedenthal, P. M., Augustinova, M., Rychlowska, M., Droit-Volet, S., Zinner, L., Knfo, A., et al. (2012). Negative relations between pacifier use and emotional competence. Basic Appl. Soc. Psychol. 34, 387-394. doi: 10.1080/01973533.2012.712019

Pecher, D., Boot, I., and van Dantzig, S. (2011). "Abstract concepts: sensory motor grounding, metaphors, and beyond," in The Psychology of Learning and Motivation, Vol. 54, ed B. Ross (Burlington, NJ: Academic Press), 217-248.

Pexman, P. M. (2017). The role of embodiment in conceptual development. Lang. Cogn. Neurosci. 1-10. doi: 10.1080/23273798.2017.1303522

Pezzulo, G., Barca, L., and D'Ausilio, A. (2014). The sensorimotor and social sides of speech. Behav. Brain Sci. 37, 569-570. doi: 10.1017/S0140525X13004172

Ponari, M., Norbury, C. F., and Vigliocco, G. (2017). Acquisition of abstract concepts is influenced by emotional valence. Dev. Sci. doi: 10.1111/desc.12549. [Epub ahead of print].

R Development Core Team (2005). R: A Language and Environment for Statistical Computing. Vienna: R Foundation for Statistical Computing.

Recchia, G., and Jones, M. N. (2012). The semantic richness of abstract concepts. Front. Hum. Neurosci. 6:315. doi: 10.3389/fnhum.2012.00315

Reilly, J., Peelle, J. E., Garcia, A., and Crutch, S. J. (2016). Linking somatic and symbolic representation in semantic memory: the dynamic multilevel reactivation framework. Psychon. Bull. Rev. 23, 1002-1014. doi: 10.3758/s13423-015-0824-5

Riva, E., Banderali, G., Agostoni, C., Silano, M., Radaelli, G., and Giovannini, M. (1999). Factors associated with initiation and duration of breastfeeding in Italy. Acta Paediatr. 88, 411-415. doi: 10.1111/j.1651-2227.1999.tb01132.x

Roversi, C., Borghi, A. M., and Tummolini, L. (2013). A marriage is an artefact and not a walk that we take together: an experimental study on the categorization of artefacts. Rev. Philos. Psychol. 4, 527-542. doi: 10.1007/s13164-013-0150-7

Rychlowska, M., Korb, S., Brauer, M., Droit-Volet, S., Augustinova, M., Zinner, L., et al. (2014). Pacifiers disrupt adults' responses to infants' emotions. Basic Appl. Soc. Psychol. 36, 299-308. doi: 10.1080/01973533.2014.915217

Schwanenflugel, P. J., Harnishfeger, K. K., and Stowe, R. W. (1988). Context availability and lexical decisions for abstract and concrete words. J. Mem. Lang. 27, 499-520. doi: 10.1016/0749-596X(88)90022-8
Setti, A., and Caramelli, N. (2005). "Different domains in abstract concepts," in Proceedings of the XXVII Annual Conference of the Cognitive Science, eds B. Bara, B. Barsalou, and M. Bucciarelli (Mahwah, NJ: Erlbaum).

Shallice, T., and Cooper, R. P. (2013). Is there a semantic system for abstract words? Front. Hum. Neurosci. 7:175. doi: 10.3389/fnhum.2013. 00175

Siakaluk, P. D., Knol, N., and Pexman, P. M. (2014). Effects of emotional experience for abstract words in the Stroop task. Cognit. Sci. 38, 1698-1717. doi: $10.1111 / \operatorname{cogs} .12137$

Skipper, L., and Olson, I. R. (2014). Semantic memory: distinct neural representations for abstractness and valence. Brain Lang. 130, 1-10. doi: 10.1016/j.bandl.2014.01.001

Sourial, N., Wolfson, C., Zhu, B., Quail, J., Fletcher, J., Karunananthan, S., et al. (2010). Correspondence analysis is a useful tool to uncover the relationships among categorical variables. J. Clin. Epid. 63, 638-646. doi: 10.1016/j.jclinepi.2009.08.008

Thill, S., and Twomey, K. E. (2016). What's on the inside counts: A grounded account of concept acquisition and development. Front. Psychol. 7:402. doi: 10.3389/fpsyg.2016.00402

Tomasino, B., and Rumiati, R. I. (2013). Introducing the special topic "The when and why of sensorimotor processes in conceptual knowledge and abstract concepts." Front. Hum. Neurosci. 7:498. doi: 10.3389/fnhum.2013. 00498

Topolinski, S., and Strack, F. (2009). Motormouth: mere exposure depends on stimulus-specific motor simulations. J. Exp. Psychol. Learn. Mem Cogn. 35:423. doi: $10.1037 / \mathrm{a} 0014504$

Vigliocco, G., Kousta, S., Vinson, D., Andrew, M., and Del Campo, E. (2013). The representation of abstract words: what matters? Reply to Paivio's (2013) comment on Kousta et al. (2011). J. Exp. Psychol. Gen. 142, 288-291. doi: $10.1037 / \mathrm{a} 0028749$

Wang, J., Conder, J. A., Blitzer, D. N., and Shinkareva, S. V. (2010). Neural representation of abstract and concrete concepts: a metaanalysis of neuroimaging studies. Hum. Brain Mapp. 31, 1459-1468. doi: 10.1002/hbm.20950

Wauters, L. N., Tellings, A. E., Van Bon, W. H., and Van Haaften, A. W. (2003). The representation of abstract words: why emotion matters. Appl. Psycholing. 24, 385-406. doi: 10.1017/S0142716403000201

Wiemer-Hastings, K., Krug, J., and Xu, X. (2001). "Imagery, context availability, contextual constraints and abstractness," in Proceedings of the 23rd Annual Meeting of the Cognitive Science Society (Hillsdale, NJ: Erlbaum), 1106-1111.

Wu, L. L., and Barsalou, L. W. (2009). Perceptual simulation in conceptual combination: evidence from property generation. Acta Psychol. 132, 173-189. doi: 10.1016/j.actpsy.2009.02.002

Conflict of Interest Statement: The authors declare that the research was conducted in the absence of any commercial or financial relationships that could be construed as a potential conflict of interest.

Copyright (c) 2017 Barca, Mazzuca and Borghi. This is an open-access article distributed under the terms of the Creative Commons Attribution License (CC BY). The use, distribution or reproduction in other forums is permitted, provided the original author(s) or licensor are credited and that the original publication in this journal is cited, in accordance with accepted academic practice. No use, distribution or reproduction is permitted which does not comply with these terms. 


\section{OPEN ACCESS}

Edited by:

Anna M. Borghi,

University of Bologna \& Institute of Cognitive Sciences and Technologies,

Italy

Reviewed by:

Paul D. Siakaluk,

University of Northern British

Columbia, Canada

Michele Wellsby,

University of Calgary, Canada

*Correspondence:

Serge Thill

serge.thill@his.se

Specialty section: This article was submitted to

Cognition,

a section of the journal

Frontiers in Psychology

Received: 14 January 2016 Accepted: 07 March 2016

Published: 23 March 2016

Citation:

Thill S and Twomey KE (2016) What's on the Inside Counts: A Grounded

Account of Concept Acquisition and

Development. Front. Psychol. 7:402. doi: 10.3389/fpsyg.2016.00402

\section{What's on the Inside Counts: A Grounded Account of Concept Acquisition and Development}

\author{
Serge Thill ${ }^{1 *}$ and Katherine E. Twomey ${ }^{2}$ \\ ${ }^{1}$ Interaction Lab, School of Informatics, University of Skövde, Skövde, Sweden, ${ }^{2}$ Department of Psychology, Lancaster \\ University, Lancaster, UK
}

Understanding the factors which affect the age of acquisition (AoA) of words and concepts is fundamental to understanding cognitive development more broadly. Traditionally, studies of AoA have taken two approaches, either exploring the effect of linguistic variables such as input frequency (e.g., Naigles and Hoff-Ginsberg, 1998) or the semantics of the underlying concept, such as concreteness or imageability (e.g., Bird et al., 2001). Embodied theories of cognition, meanwhile, assume that concepts, even relatively abstract ones, can be grounded in the embodied experience. While the focus of such discussions has been mainly on grounding in external modalities, more recently some have argued for the importance of interoceptive features, or grounding in complex modalities such as social interaction. In this paper, we argue for the integration and extension of these two strands of research. We demonstrate that the psycholinguistic factors traditionally considered to determine AoA are far from sufficient to account for the variability observed in AoA data. Given this gap, we propose groundability as a new conceptual tool that can measure the degree to which concepts are grounded both in external and, critically, internal modalities. We then present a mechanistic theory of conceptual representation that can account for groundability in addition to the existing variables argued to influence concept acquisition in both the developmental and embodied cognition literatures, and discuss its implications for future work in concept and cognitive development.

Keywords: concept grounding, embodiment, developmental linguistics, age of acquisition, SPA

\section{INTRODUCTION}

Within representationalist theories of embodied cognition, the symbol grounding problem has traditionally received much attention. The reason for the focus can be understood from a historical perspective: as Chemero (2009) notes, these theories developed primarily as a reaction to purely computationalist views of cognition. One of the main criticisms leveled at such views was that they assume amodal symbols which are meaningless to the system itself-whatever meaning the symbols might carry was attributed by external observers. How such symbols could acquire meaning that is intrinsic to the system became known as the symbol grounding problem (Harnad, 1990), and the central claim to the solution in embodied terms is that the meaning is acquired through sensorimotor interaction with the world.

\footnotetext{
${ }^{1}$ In contrast, non-representationalist theories of embodied cognition are an evolution of Ecological Psychology and its
} precursors. 
This has led to at least two major research strands. On the more experimental end of the spectrum, much work has focused on detailing the involvement of sensorimotor areas of the brain in, for instance, language processing (see Chersi et al., 2010, for a review). Although such involvement is often taken as evidence for a grounded or embodied understanding of concepts, it is worth pointing out that this is not uncontroversial: Mahon and Caramazza (2008), for instance argue, that the evidence is not sufficient to invalidate disembodied hypotheses.

On the computational end of the spectrum, researchers are interested in creating models of symbol grounding. Eliasmith (2013), for example, details a "semantic pointer architecture," which provides a computational implementation of many aspects of Barsalou's perceptual symbol system (Barsalou, 1999). Other efforts consider robotic implementations of such models (see for instance, Stramandinoli et al., 2012, or for a review, Coradeschi et al. 2013).

A particularly interesting aspect of research across the entire spectrum concerns the putative grounding of abstract conceptsthat is, concepts which do not have a directly perceivable sensorimotor target (see, for instance Dove, 2011; Thill et al., 2014, for recent reviews and discussions). While it is relatively straightforward to propose accounts of sensorimotor grounding of concrete concepts-which do have an observable sensorimotor target in the external world-it is less clear how, if at all, abstract concepts should relate to embodied experience. Mahon and Caramazza (2008) give the example of the concept "beautiful," for which they claim that there is no corresponding consistent sensory or motor information (their emphasis).

An early attempt at explanation is given by the conceptual metaphor theory (Lakoff and Johnson, 1980), which postulates that metaphors and analogical reasoning (e.g., an argument is like war; happiness is up) mediate grounding of abstract concepts in direct sensorimotor experience. However, Dove (2011) points out that the required cognitive mechanisms, such the ability to construct such analogies and metaphors, are not likely to develop until relatively late. He further argues that linguistic representations are dis-embodied (the specific term he coined, and distinct from disembodied) in the sense that they do not acquire semantic content from embodiment, even though they may remain dynamic, multimodal and grounded in linguistic experience. Zwaan (2015) also argues that abstract concepts "acquire a specific sensorimotor instantiation in a discourse context" while being only weakly associated with sensorimotor representations. Similarly, Barsalou et al. (2008) previously proposed the Language And Situated Simulation (LASS) theory, arguing that both linguistic forms and situated simulations are used to represent concepts, including abstract ones.

Other theories imply that the grounding of more abstract concepts can take place in modalities beyond the five senses in the strict sense. The Words As Tools theory (WAT; Borghi and Binkofski, 2014) sees words as social tools, whose use is a "type of experience" (Borghi and Cimatti, 2012, p. 22), which provides a potential way of grounding abstract concepts in a type of social modality. Similarly, Thill et al. (2014) argue that one should not restrict the embodied experience to the "outside" in a theory of concept grounding while Wellsby and Pexman (2014a) note that the focus so far has been more on interaction with the external world and less on "sensing bodies" (their term). This is also true for theories that try to link abstract concepts to embodiment, for instance by grounding them in the sensorimotor representations activated across different linguistic contexts (Barsalou and Wiemer-Hastings, 2005; Zwaan, 2015). As others have noted, the human embodied experience is actually very rich and involves many internal processes (see Stapleton, 2011, 2013, for a thorough review and discussion), including homeostatic and affective mechanisms (e.g., Ziemke and Lowe, 2009; Damasio, 2010) which may directly ground concepts that are considered abstract. As noted by Stapleton (2013), the internal body may $^{2}$ matter to cognition. Of the aspects that comprise this internal body, affect and emotion have received the most attention in discussions of concept grounding so far. Glenberg and Gallese (2012), for instance, propose an account of language acquisition that includes emotional systems as a providing means for grounding in addition to perception and action. Similarly, Kousta et al. (2011) argue that abstract words tend to be more emotionally valenced than concrete ones, and that emotional content might be an important factor in the representation and processing of abstract words in particular. Newcombe et al. (2012) showed a correspondence between emotional experience and speed (and accuracy) of classification of abstract-but not concrete-words, and argue that abstract concepts may be grounded in emotional features that remain stable across different contexts (see also Siakaluk et al., 2014, for a follow-up). The concept of "beautiful," although having no consistent external sensorimotor experience, may thus relate to direct internal experience.

Research into concept grounding tends to focus on adult language and cognition. There are, however, good reasons to approach the topic from a developmental perspective (Kontra et al., 2012). Most immediately, any mechanistic account of concept grounding makes the direct prediction that whatever mechanism is proposed has developed by the time that humans use that concept-recall, for example, Dove's (2011) concern regarding the use of metaphors previously mentioned. Second, bodily and cognitive development may be a crucial component for explanatory accounts of cognitive mechanisms: after all, humans acquire concepts during a period of dramatic change.

Concept grounding depends, by definition, on the sensorimotor experience that is meant to provide this grounding. The importance of this embodied input has been accepted since Piaget's classic work on the sensorimotor roots of cognitive development (Piaget, 1952). More recently, however, new technology has provided striking novel insights into the infant's embodied experience: that is, what infants experience is substantially different from what adults experience. As the body changes-e.g., arms grow longer, walking commences-so too do important characteristics of the body-mediated information available for concept grounding. Studies using head-mounted

\footnotetext{
${ }^{2}$ Stapleton (2013) actually omits the "may," stating that "I argue that recent work in neuroscience and robotics suggests cognitive systems are not merely superficially embodied in the sense that the sensorimotor interactions with the environment are the only interactions relevant to cognitive behavior, but that cognitive systems are 'properly embodied'; the internal body matters to cognition" (pp. 1-2).
} 
eye trackers demonstrate, for example, that the content of the infant's visual field is qualitatively and quantitatively different from that of the adult, because infants' shorter arms lead them to hold objects close to their faces (Smith et al., 2011). The precise nature of the body (e.g., walking vs. crawling, height) is clearly crucial in shaping this experience (Kretch et al., 2014); yet it is also often ignored in the embodied cognitive science literature. For instance, Ziemke (2003) points out that "many discussions/notions of embodied cognition actually pay relatively little attention to the nature and the role of the body involved (if at all)" (p. 1306, emphasis in text) and Borghi et al. (2013) similarly argues that "many versions of the [embodied-grounded] view are too brainbound" (p. 2).

The developmental psychology literature also features a substantial body of work concerned with human concept and word acquisition. This work is highly relevant to the concept grounding discussion. In particular, it illustrates how change over time in the conceptual system reflects change over time in the physical system. For instance, conceptual structure changes radically across development (Quinn and Eimas, 1997; Mandler, 2000): infants as young as 3 months form perceptually-based categories (Quinn et al., 1993), but begin to show evidence of more abstract representations by around 12 months (Mandler and Bauer, 1988), and make conceptuallybased category judgements by 4 years (Keil, 1989). Importantly, early perceptual/conceptual structure and language acquisition are intimately linked. For example, by drawing attention to invariant, category-relevant features, perceptual variability in the objects children see supports category formation and subsequent word learning (e.g., Vlach et al., 2008; Twomey et al., 2014; Goldenberg and Johnson, 2015). Relatedly, English-learning children generalize category labels to new same-shape items, but only if those items are solid rather than non-solid (Samuelson and Horst, 2007). Further, variation in the physical position of the body can disrupt word learning (Samuelson et al., 2011; Morse et al., 2015). Thus, evidence from multiple modalities indicates that the perceptually grounded nature of early concrete concepts interacts with children's ability to learn words. Indeed, the interaction between perceptual grounding and early language has been investigated. For example, in a word naming study which included school-age children, Wellsby and Pexman (2014b) demonstrated that the extent to which the referents of words are easy to physically interact with (as rated by adults) affected 8 - to 9-year old children's written word processing. Specifically, children's naming latencies were shorter for words with high body-object-interaction (BOI) ratings. The authors argued that high-BOI words have richer semantic representations than lowBOI words, leading to greater activation in the semantic system, which in turn facilitates word recognition. Taken together with the adult literature, the developmental embodied cognition approach makes the prediction that the sensorimotor experience associated with a concept should affect how easy it is to acquire that concept.

Recent psycholinguistic studies have focused on the age of acquisition (AoA) of words as a marker of concept learning, and demonstrate that the semantic features of concepts themselves affect the age at which their labels are learned. For example,
McDonough et al. (2011) examined the effect of a word's imageability (the extent to which a word generates a mental image, Paivio et al., 1968) and class (e.g., noun, verb) on AoA. As well as predicting AoA, imageability accounted for variation that word class did not, indicating an independent role of perceptual features in the acquisition of early abstract concepts (for crosslinguistic evidence, see Ma et al., 2009). Closely related to imageability is concreteness, or the extent to which a concept is perceptible (Brysbaert et al., 2014). Bird et al. (2001) showed that imageability and concreteness predicted AoA for children's earlyproduced nouns (see also Barca et al., 2002; Smolík, 2014). In a study in which Dutch adults rated words for emotional valence, arousal, power and AoA, valence was negatively correlated with AoA such that more positive words were acquired earlier (Moors et al., 2013). In addition, linguistic phenomena also affect AoA, including-but not limited to-iconicity (Perry et al., 2015), and in particular, input frequency (Naigles and Hoff-Ginsberg, 1998; Barca et al., 2002; Storkel, 2004; Goodman et al., 2008; Ambridge et al., 2015; Roy et al., 2015). Whether sensorimotor experience predicts AoA, however, remains to be tested.

In the following section we bring together in a single analysis variables that have been shown to affect AoA, specifically, frequency, imageability and valence. Our goal is not to provide an exhaustive account of conceptual and linguistic influences on AoA; indeed, for many of these variables insufficient data are available for a reliable analysis. However, to our knowledge this is the first study to bring together these variables in analysing the reliable measure of AoA provided by the widelyused MacArthur-Bates Communicative Development Inventory vocabulary norms (Fenson et al., 1993). We demonstrate that, when taken together, these variables explain only a minority of the variance, highlighting the importance of identifying and testing new factors. In a second analysis we test our hypothesis that sensorimotor grounding is important to AoA, by adding a measure of body-object interaction. We argue that while existing measures take into account conceptual and linguistic effects on AoA, embodied characteristics of concepts may be an important missing piece of the puzzle.

\section{METHODS}

To explore the effect of conceptual features on AoA we obtained AoA, frequency, imageability and valence ratings from a range of open access sources. Data used in the analyses are provided in Supplementary Materials and Pearson correlations between variables are presented in Table $\mathbf{1}$.

\subsection{Age of Acquisition}

Our goal was to explore the extent to which previously identified variables predict the AoA of words commonly learned by human infants. We took our target words from the MacArthur Bates Communicative Development Inventory (MCDI; Fenson et al., 1993). The MCDI is a well-established, normed and validated list of 680 words that infants and toddlers learn to understand and produce up to 30 months of age, and is widely used in developmental research. We defined AoA as the month in which $50 \%$ or more of 1142 infants in the MCDI sample produced a 
TABLE 1 | Pearson correlations between regression predictors.

\begin{tabular}{lccc}
\hline & BOI & Imageability & Frequency \\
\hline Imageability & $0.44^{\star \star \star}$ & & \\
Frequency & 0.18 & $-0.45^{\star \star \star}$ & \\
Valence & $-0.21^{\star}$ & $0.23^{\star \star \star}$ & $0.22^{\star \star *}$ \\
\hline
\end{tabular}

${ }^{\star} p<0.05,{ }^{* \star *} p<0.001$

given word. AoA in months ranged from 12 (e.g., mommy) to $30+$ (e.g., pretend). AoAs listed as $30+$ months were coded as 31 months for the purposes of the current analysis.

\subsection{Frequency}

Children's language environment has been repeatedly shown to influence their language acquisition (for a review, see Ambridge et al., 2015). We therefore generated our frequency data from real child-directed input, which is representative of the language children hear, rather than relying on corpora of non-childdirected spoken or written speech. CHILDES (MacWhinney, 2000) is a large, open-access online database of transcribed, naturalistic conversations between adults and children. We searched all Northern American corpora for each word in the MCDI, with the exception of some sound effects and routines (e.g., woof, patty cake). Only mothers' utterances were queried, providing an index of children's input. This resulted in frequency ratings for 638 words with frequencies ranging from 0 (cat) to 128124 (you) tokens $(M=2848.82)$.

\subsection{Imageability and Concreteness}

For each MCDI word for which we obtained frequency data we extracted imageability and concreteness ratings from the MRC Psycholinguistic Database (Coltheart, 1981; Wilson, 1988). The database is a large, open-access collection of 26 psycholinguistic variables for up to 150,000 words (although not all words have data for all variables) aggregated from existing studies ${ }^{3}$. Because imageability and concreteness were very highly correlated $(r=$ 0.91, $p<0.0001$ ), in line with Ma et al. (2009) and McDonough et al. (2011), we used imageability as a predictor variable in the following analyses. Imageability scores ranged from 195 (low) to 667 (high; $M=495.58$ ).

\subsection{Valence}

Valence ratings for each word were taken from the 2010 version of the Affective Norms for English Words dataset (ANEW; Bradley and Lang, 2010). This version of ANEW consists of adult ratings of 2476 words for pleasure (i.e., valence), arousal and dominance. Scores ranged from 1.61 (happy) to 8.72 (unhappy; $M=5.92$ ).

\subsection{Body-object Interaction}

To explore our hypothesis that sensorimotor grounding may be important for concept acquisition, we took measures of bodyobject interaction (BOI) from Tillotson et al. (2008) and Bennett et al. (2011), in which adults were asked to rate the extent to which they could easily interact with a named item. Scores ranged

\footnotetext{
${ }^{3}$ Details available at http://www.psych.rl.ac.uk/MRC_Psych_Db_files/mrc2.html.
}

from 1.27 (first; low interactivity) to 6.43 (doll; high interactivity; $M=4.68)$. Specifically, our assumption is that the experience of interacting with concepts that rate highly is more multimodal than that of interacting with low-ranking concepts (if such an experience exists at all), so BOI might serve as a proxy to rank concepts by how much they are defined by an external sensorimotor experience.

\section{RESULTS}

\subsection{The Effect of Conceptual Features on AoA}

To explore the effect of conceptual features on AoA, we first created a conceptual features model. AoA for the 398 words with ratings for every variable was submitted to a linear regression with frequency (log transformed), imageability (mean centred) and valence (mean centred) as fixed effects. Because high frequency function words have little or no semantic content, while rarer nouns have rich semantics, we anticipated that frequency and imageability would interact, so included a frequency-by-imageability interaction term (cf. Roy et al., 2015).

Results are presented in Table 2. The principal result is that the interaction between frequency and imageability predicts AoA, extending the findings of McDonough et al. (2011) and $\mathrm{Ma}$ et al. (2009), who each found correlations between CDI AoA and imageability ratings. As illustrated in Figure 1, although lateacquired words tend to be lower frequency, function words (e.g., an, the, to) have low imageability and are acquired late despite being high frequency. In contrast, high-imageability words for the things infants encounter in their everyday environment (e.g., puppy) are acquired early despite occurring infrequently. In addition to the interaction between imageability and frequency, main effects of these two variables confirmed that as imageability increased, AoA decreased (see also Ma et al., 2009; McDonough et al., 2011), and in line with Roy et al. (2015), as frequency increased, AoA decreased. Interestingly, in contrast with existing studies (e.g., Bird et al., 2001; Moors et al., 2013), valence did not predict AoA; however the adult ratings we used may not capture the effect of a word's valence on young children. More broadly, the differences between our results and existing studies may stem from some important methodological differences: while the majority of work uses adult ratings of word AoA and frequency measures taken from corpora of adult-directed language, we use parental measures of their own children's language and frequencies taken from child-directed speech ( $c f$. McDonough et al., 2011). This contrast highlights the need for child-centric ratings of such predictors, and illustrates the importance of taking seriously the real input to infants when investigating developmental phenomena (Smith et al., 2011).

The goal of this analysis was to illustrate that even well-tested predictors are unable to fully explain AoA. As expected, this model accounted for less than half of the variance (adjusted $R^{2}=$ 0.38 ), leaving substantial scope for the influence of other factors on early concept acquisition. As noted above, our analysis focuses on variables which have repeatedly been shown to influence AoA, and ignores those for which no data are available. Thus, we do not claim that it is an exhaustive model of the factors 
TABLE 2 | Conceptual features model parameters and significance tests $(N=239)$.

\begin{tabular}{lcrcrcc}
\hline & $\boldsymbol{\beta}$ & $\boldsymbol{t}$ & $\boldsymbol{p}$ & $\boldsymbol{F}$ & $\boldsymbol{d f}$ & $\boldsymbol{p}$ \\
\hline Overall model & & & & 37.73 & $(4,234)$ & $<0.0001^{* * *}$ \\
Log frequency & -1.48 & -6.74 & $<0.0001^{* * *}$ & & \\
Imageability & -0.022 & -7.33 & $<0.0001^{* * *}$ & \\
Valence & 0.055 & 0.34 & 0.74 \\
Log frequency $\times$ & -0.0065 & -3.18 & $0.0017^{* *}$ & \\
imageability & & & & \\
\hline$R^{2}$ & 0.39 & & & \\
Adjusted $R^{2}$ & 0.38 & & &
\end{tabular}

${ }^{* *} p<0.01,{ }^{* * *} p<0.001$.

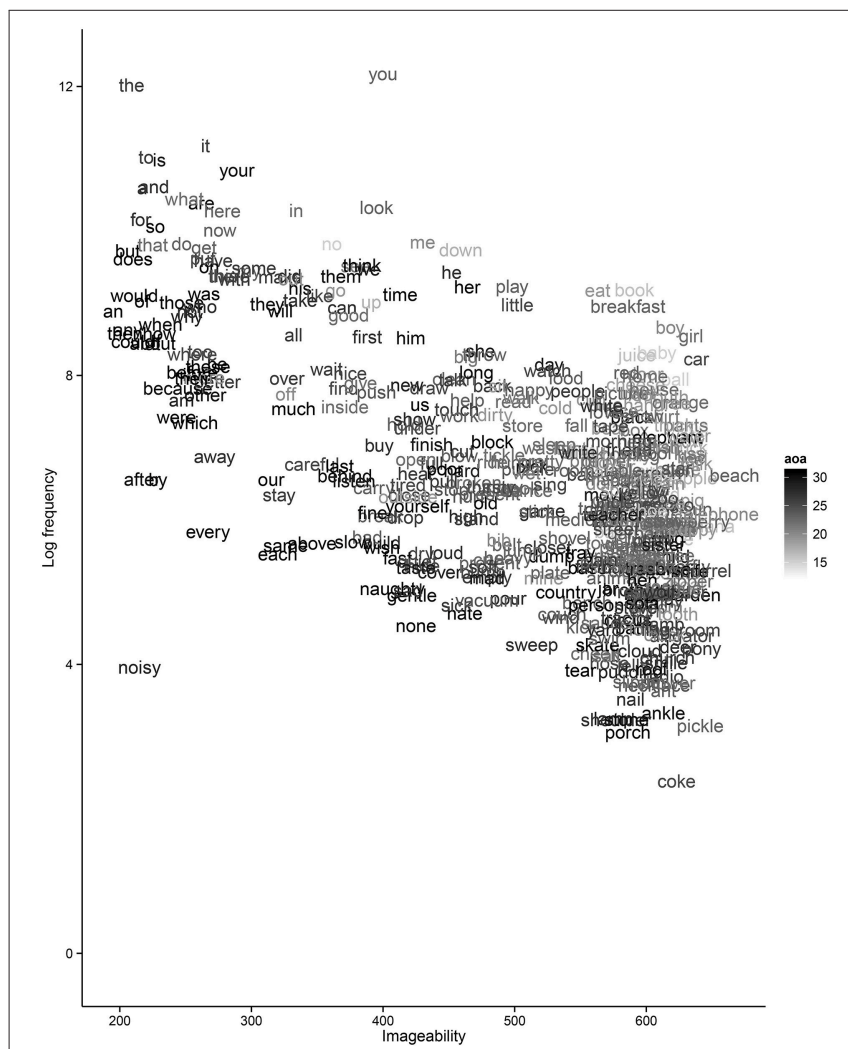

FIGURE 1 | AoA of early concepts plotted by log frequency and imageability. Darker text indicates later AOI.

affecting concept AoA. We do, however, argue that the variance unaccounted for is not simply random variation, but rather the result of linguistic and concept-internal variables not typically included in analyses of AoA. In particular, this leaves open the possibility that embodied aspects of concepts may contribute to the ease with which they are acquired.

\subsection{The Effect of a Sensorimotor Grounding on AoA}

To explore whether the extent of sensorimotor grounding might play a role in concept acquisition (as discussed in Section 2.5), we
TABLE 3 | BOI model parameters and significance tests $(N=151)$.

\begin{tabular}{|c|c|c|c|c|c|c|}
\hline & $\beta$ & $t$ & $p$ & $F$ & $d f$ & $p$ \\
\hline Overall model & & & & 21.32 & $(5,145)$ & $<0.001^{\star * *}$ \\
\hline Log frequency & -0.93 & -1.78 & 0.078 & & & \\
\hline Imageability & -0.013 & -2.22 & $0.028^{*}$ & & & \\
\hline Valence & -0.19 & -0.76 & 0.45 & & & \\
\hline $\begin{array}{l}\text { Body-object } \\
\text { interaction }\end{array}$ & -0.88 & -4.49 & $<0.001^{\star \star \star}$ & & & \\
\hline $\begin{array}{l}\text { Log frequency } x \\
\text { imageability }\end{array}$ & -0.010 & -1.99 & $0.049^{*}$ & & & \\
\hline$R^{2}$ & 0.42 & & & & & \\
\hline Adjusted $R^{2}$ & 0.40 & & & & & \\
\hline
\end{tabular}

${ }^{*} p<0.05,{ }^{\star * \star} p<0.001$

TABLE 4 | Conceptual features model parameters and significance tests fit to dataset used for BOI model $(N=151)$.

\begin{tabular}{lrrrrrr}
\hline & $\boldsymbol{\beta}$ & $\boldsymbol{t}$ & $\boldsymbol{p}$ & $\boldsymbol{F}$ & $\boldsymbol{d f}$ & $\boldsymbol{p}$ \\
\hline Overall model & & & & 19.11 & $(4,146)$ & $<0.001^{* * *}$ \\
Log frequency & -0.59 & -1.07 & 0.28 & & & \\
Imageability & -0.022 & -3.59 & $<0.001^{* * *}$ & & & \\
Valence & 0.037 & 0.27 & 0.14 & & & \\
Log frequency $\times$ & -0.014 & -2.62 & $0.0099^{* *}$ & & \\
imageability & & & & & \\
\hline$R^{2}$ & 0.39 & & & & \\
Adjusted $R^{2}$ & 0.38 & & & & & \\
\hline
\end{tabular}

${ }^{* *} p<0.01,{ }^{* * *} p<0.001$.

added a measure of body-object interaction as a predictor in the conceptual features model to create a BOI model. Because fewer of our target words had ratings for this variable, the final dataset for this analysis consisted of complete ratings for 151 words.

As illustrated in Table 3, when the additional BOI term is included, the frequency-by-imageability interaction and main effect of imageability predict AoA, while the main effect of frequency does not. Critically, in line with our predictions, BOI does predict AoA, such that as words are rated as more difficult to interact with, AoA increases. Importantly, this model also explained a greater proportion of the variance in AoA, with an increase in adjusted R-squared from 0.38 to 0.40 . To compare the fit of our two models, we first refit the conceptual features model to the smaller dataset; this resulted in a similar pattern of results (see Table 4). Including the BOI term resulted in a reduction in AIC from 788.43 to 770.80 . Taken together with the increase in adjusted R-squared, this confirms that the BOI model fits the data better, explaining more variance than the conceptual features model and supporting our claim that the extent to which concepts are grounded in the body affects AoA.

Although including BOI improved the fit of the model, it nonetheless again left a majority of the variance unaccounted for-as expected, given that it did not include linguistic effects 
on AoA, for example iconicity (Perry et al., 2015), ease of pronunciation (Jorm, 1991) and contextual diversity (Hills et al., 2009), and the fact that these ratings came from adults. Thus, it is, for example, possible that using child ratings of BOI could improve the model fit further. What drives concept AoA is far from being fully understood; however the above analyses strongly suggest that grounding in sensorimotor experience could be a critical piece in this puzzle.

\section{WHAT ARE CONCEPTS MADE OF?}

To summarize the results, we first showed that semantic features and linguistic phenomena such as frequency are not sufficient to explain AoA data. Our main hypothesis is that this is because such features do not take into account grounding in a rich or proper sensorimotor experience. We then demonstrated that including predictors related to such a grounding improve on the initial results.

There is clearly much work to be done to validate the hypothesis further. First and foremost, there are currently no major corpora of data that relate to relevant measures other than BOI as used above. Second, the measure of BOI used above takes no account of interoceptive aspects of the sensorimotor experience, which, as noted, are likely to play a part in conceptual structure. How to tap into such interoceptive aspects is not trivial. Although valence ratings may seem like a good starting point (since valence itself is part of the internal sensory experience), they do not provide a measure of how diverse (or multimodal) the internal sensory experience associated with a concept is $^{4}$. Instead, they quantify the strength of one aspect (which is clearly relevant, as argued for instance by Kousta et al., 2011, but not necessarily sufficient since there are other internal modalities as discussed, for example, by Stapleton 2011). Together with the limitations of BOI mentioned before, there is therefore still a need for designing new types of measures that address both internal and external sensorimotor experience more explicitly.

The purpose of the remainder of this paper is therefore to outline a mechanism of concept learning which explicitly takes into account embodied features beyond simple sensorimotor interaction (for instance, interoceptive features) whilst incorporating the variables which have been repeatedly shown to affect AoA, and by extension, conceptual development and structure. In doing so, we will generate testable predictions for future work and lay the groundwork for future research into novel measures that can validate our hypothesis.

To provide this characterization, we cast our discussion in terms of a cognitive architecture since these necessarily formally specify the mechanisms underlying concept use. Specifically, we base our discussion on the semantic pointer architecture (SPA, see Eliasmith, 2013). It would of course be equally possible to formulate these ideas in frameworks other than SPA; the Neural Blackboard Architecture framework (van der Velde and de Kamps, 2006), for example, is also

${ }^{4}$ In PAD space (Mehrabian and Russell, 1974), for example, valence ratings do not relate to the $\mathrm{A}$ or the $\mathrm{D}$. concerned with the creation of combinatorial structures, such as concepts, that might underlie human cognition. For the present purposes, however, we think SPA well-suited: it is inspired by human semantics and syntax in that its "semantic pointers" can be interpreted as perceptually grounded symbols in the sense of Barsalou (1999). SPA can also incorporate mechanisms necessary for concept grounding in terms of a rich sensorimotor experience (see Thill, 2015, for a longer discussion).

The question of when children acquire concepts can therefore be reformulated, for the present purposes, as asking at what age the corresponding semantic pointer forms. In the following, we first give a brief overview of the main computational principles in SPA (we refer the interested reader to Eliasmith, 2013, for a much more thorough discussion, including various demonstrations of cognitive and biological plausibility). We then provide the aforementioned characterization of concepts, which finally allows us to highlight directions for future work.

\subsection{Brief Overview of Semantic Pointers}

Semantic pointers, in SPA, are vectors in a high-dimensional ${ }^{5}$. space. For example, the concept of a robin would thus be described by a vector robin. To specify how such a vector might be obtained, SPA takes inspiration from hierarchical structures in the human brain such as the visual cortex (Felleman and Van Essen, 1991). For example, the retinal image of a robin is successively compressed through the different layers of the hierarchy for object recognition (V1 $\rightarrow \mathrm{V} 2 \rightarrow \mathrm{V} 4 \rightarrow$ IT) into a representation with significantly lower dimensionality than the original retinal input. This resulting representation at the top of the hierarchy would be a semantic pointer robVis encoding the visual appearance of a robin.

Multiple representations can then be bound together to form a new concept. In SPA, the binding operator is circular convolution, denoted by $\circledast$, a vector operation which takes two vectors as an input and returns a vector of the same length as an output. To give an example from Eliasmith (2013), one could construct a semantic pointer for perceptual features of a robin:

$$
\begin{aligned}
\text { robinPercept }= & \text { visual } \circledast \text { robVis }+ \text { auditory } \circledast \text { robAud } \\
& + \text { tactile } \circledast \text { robTact }+\ldots
\end{aligned}
$$

where each element in bold represents a semantic pointer. robin could then be defined as:

$$
\begin{aligned}
\text { robin }= & \text { perceptual } \circledast \text { robinPercept }+ \text { is } A \circledast \text { bird } \\
& + \text { indicates } \circledast \text { spring }+\ldots
\end{aligned}
$$

There are several aspects of semantic pointers that we do not discuss here. It is, for example, possible to "read out" particular components of a semantic pointer (such as what the visual percept RobinVis within the overall concept of Robin is), and to recall the visual image(s) used in forming that particular pointer-a process that can be interpreted as a type of simulation

\footnotetext{
${ }^{5}$ Eliasmith (2013) suggests that 500 dimensions are sufficient for human cognition.
} 
of previous sensorimotor experience as proposed by Barsalou (Barsalou, 1999; Barsalou and Wiemer-Hastings, 2005; Barsalou, 2009). Further discussions of the underlying neural structures, necessary neural mechanisms, and biological plausibility can be found in Eliasmith (2013).

For the present purposes, it is also worth emphasizing that, although it is capable of symbolic manipulation, SPA is not a symbolic account of cognition; the semantic pointers related to any concept are not arbitrary symbols but a compressed combination of perceptual features that make up the concept. As such, the sensorimotor experience of a given concept by an agent plays a fundamental role in forming the concept and shaping computations that use it.

\subsection{Characterization of Richly Grounded Concepts}

In essence, we argue throughout this paper that sensorimotor concept grounding requires a rich perspective of what the term "sensorimotor" actually entails: it is not merely sufficient to consider basic sensorimotor interaction with the external world; internal percepts (including affect, emotional components and other aspects of interoception as discussed in more detail, for example, by Stapleton, 2011) are equally important (Thill et al., 2014; Wellsby and Pexman, 2014a). We therefore postulate that the sensory features of a concept, directly perceived at a given time $t$, can be described as follows:

$$
\begin{aligned}
\mathbf{S}_{\mathbf{t}}^{\mathbf{D}}= & \sum_{i} \sum_{j} \text { Modality }_{\mathbf{i}}^{\text {ext }} \circledast \text { feature }_{\mathbf{j}} \\
& +\sum_{k} \sum_{l} \text { Modality }_{\mathbf{k}}^{\text {int }} \circledast \text { feature }_{\mathbf{~}}
\end{aligned}
$$

where we omit an explicit mention of time on the RHS. Equation (1) simply captures the idea that concepts are multimodal and made up of any number of features from any number of modalities (notably, this number can also be low: constructs are not necessarily complex. In particular, a concept could consist of a single modality, for example the concept "yellow"). What matters is the direct nature of these features; by which we mean that they are not time-dependent. They could for instance relate to a color or the shape of a solid object, as acquired by the visual modality, the smoothness of a surface from a tactile modality, or an affordance elicited by a given object. They could equally relate to direct visceral feelings elicited when experiencing, for example, surprise, pleasure, or to the proprioceptive feeling of an extended arm. Affective mechanisms or emotional components (as highlighted by many, e.g., Kousta et al., 2011; Glenberg and Gallese, 2012; Newcombe et al., 2012) of concepts can be included by representing the different dimensions as internal modalities. For example, in PAD Space (Mehrabian and Russell, 1974), one might posit the following: Pleasure $\circledast$ value $_{\mathbf{p}}+$ Arousal $\circledast$ value $_{\mathbf{a}}+$ Dominance $\circledast$ value $_{\mathrm{d}}$.

Other sensorimotor perceptions, on the other hand, are timedependent: movements are, for example, by definition expressed over time. We sketch such percepts as:

$$
\mathbf{S}^{\mathbf{T}}=f\left(S_{t=1, \ldots, n}^{D}\right)
$$

where the notation again chooses simplicity over being explicit since it is merely meant to be a sketch of a process that would capture temporal aspects of percepts. Here, $f(\cdot)$ is therefore a simply placeholder for a temporal function (see, for example, Pack and Bensmaia, 2015, for a discussion of neural sensitivity to temporal stimuli, and underlying computations, in both the visual and touch modalities).

We argue that Equations ( 1 and 2) provide a reasonable characterization of the sensorimotor experience that may ground concepts and provides a starting point for analysing concept acquisition. To address word acquisition proper, we also need to recognize that verbal labels can be attached to concepts. This gives us the first expression for a concept grounded in rich sensorimotor experience:

$$
\mathrm{C}=\mathrm{S}^{\mathrm{D}}+\mathrm{S}^{\mathrm{T}}+\text { Label } \circledast \text { name }
$$

Next, we note that pointers in SPA can be constructed from other pointers, as in the previous example of the robin. We can introduce a similar idea here by noting that a given concept can be made up by more than just direct sensory features; it can equally include existing concepts:

$$
\mathbf{C}=\mathbf{S}^{\mathbf{D}}+\mathbf{S}^{\mathrm{T}}+\sum_{i} \sum_{j} \text { Includes }_{\mathbf{i}} \circledast \mathbf{C}_{\mathbf{j}}+\text { Label } \circledast \text { name }
$$

where we highlight that other concepts are not merely added by summation (see Eliasmith, 2013); it is rather the compressed vector that is added as a property (that we refer to as Includes here). Equation (4) also captures how some researchers, (particularly those primarily interested in robotic models of concept grounding) believe abstract concepts can be grounded (see Stramandinoli et al., 2012, for an example and Thill et al. 2014, for a larger discussion). In such theories, rather than being grounded in direct sensorimotor features, abstract (or higher order) concepts are instead grounded in other concepts, possibly with no direct sensorimotor component at all, meaning the first two terms on the RHS of Equation (4) would be empty.

In sum, we argue that Equation (4) describes the general form of a grounded concept, can accommodate current views on concepts, can account for abstract concept acquisition, and allows us to incorporate a rich embodied experience without positing a separate mechanism. For example, the modalities that provide features can extend to the social domain, in line with claims that more abstract words go beyond the simple sensorimotor to include a stronger social component (Borghi and Cimatti, 2009, 2012; Borghi and Binkofski, 2014). It is also worth highlighting that the characterization does not require all components to be related to some form of sensorimotor experience (even if rich). The use of Includes allows for the inclusion of purely linguistic features (Kousta et al., 2011), which in turn allows for dis-embodied concepts in the sense of Dove (2011). Indeed, in any of the above, the left-hand term of the $\circledast$ operator in SPA could in principle refer to 
anything and does not necessarily need to be itself something that has a direct sensorimotor grounding (as is clear from the robin example above). This therefore also allows for the construction of metaphors in the sense of Lakoff and Johnson (1980) - as a crude example, one could for instance postulate the following:

$$
\text { Happiness } \approx \text { Modality }^{\text {int }} \circledast \mathbf{U p}
$$

which is meant to express that happiness causes interoceptive feelings that are somewhat akin to the grounded concept of "Up." $\mathbf{U p}$, here is a concept as described by Equation (4).

Finally, it is worth pointing out that this characterization is open to the use of purely amodal symbols, perhaps even in conjunction with grounded ones. Exploring this further would require a theory of how such semantic pointers are formed, but once they are, they could be used at the appropriate places in Equations (1-4) (where one could for instance imagine a dedicated modality for amodal symbols). We do not pursue this here since our main aim is to discuss the grounding of concepts.

\section{DISCUSSION}

Having characterized concepts in terms of the semantic pointer architecture, we now turn to ways in which it can contribute to our understanding of concept acquisition. The first thing to note is that this new account is strongly developmental. As mentioned in the introduction, concepts evolve over timea 5 year old's concept of love is unlikely to be identical to that of a 15-year-old, which in turn is likely to be different from the concept the individual will have at age 35. For any given concept, its characterization in Equation (4) therefore changes over time. In particular, concepts may initially be formed from partial information and additional terms added as the modalities that provide such features develop, or other types of information becomes available, reflecting the rapid development of conceptual structures seen in early childhood (Quinn and Eimas, 1997; Mandler, 2000). The characterization given by Equation (4), for any given concept, is therefore also subject to development. Thus, it is possible to predict a developmental timeline given a hypothesis of necessary constitutents-that is, a concept can only be acquired once its constituent semantic pointers have been acquired. It is worth pointing out that any theory of concept acquisition implicitly makes at least one prediction in this sense: that the proposed cognitive mechanisms exist by the time children begin to acquire the concepts in question. As noted previously for example, Dove (2011) has argued that the ability to form metaphors develops too late to adequately be positioned at the core of abstract concept grounding (although metaphors can contribute to such concepts once available). Similarly, the idea that concepts might be made of contextualized simulations (Barsalou, 1999; Barsalou and Wiemer-Hastings, 2005; Barsalou, 2009) predicts that the necessary mechanisms to develop such simulations develops in a manner consistent with AoA. Conversely, if a developmental timeline for simulation mechanisms is given ${ }^{6}$, it is then possible to sketch how a concept develops from AoA onwards as the simulations it relies on mature.

A historic problem for theories of embodied cognition is how to account for acquisition of concrete and abstract concepts in a single mechanism. For example, while concrete yellow can be directly acquired from the external world, the more abstract lonely requires interoceptive features, while whatever is arguably linguistically mediated. Here, Equation (4) provides a starting point since it can form the basis for a measure of how much of a given concept is grounded in simple, directly perceivable sensorimotor modalities in the sense of Equations ( 1 and 2). In other words, how abstract a concept is is a function of how much of its substance goes beyond simple sensorimotor grounding. This is essentially very similar to the previously mentioned claims from the WAT theory (Borghi and Binkofski, 2014), which argues that more abstract concepts are made up of more social aspects that are not related to an individual's sensorimotor experience. At the same time it extends this to include any source for aspects that are not of a simple external sensorimotor type, including not only more complex sensorimotor experiences related to linguistic usage of the concepts (Barsalou et al., 2008; Dove, 2011; Zwaan, 2015) but also interoceptive (Thill et al., 2014) features.

Because our characterization in Equation (4) incorporates interoceptive features, the conceptual structure it entails is subtly different from that of the commonly and often interchangeably used, adult-rated concreteness or imageability scales (Reilly and Dean, 2007). By trying to provide a way to quantify how much of a concept is grounded in a rich but direct sensorimotor experience, we measure the "groundability" of a concept: the degree to which a concept is directly grounded in embodied processes. Importantly, these embodied processes include internal modalities, including affect and other interoceptive aspects: a concept can thus be directly grounded even if it has no perceivable aspect in the external world. Rather than distinguishing between "concrete" and "abstract" concepts, then, we distinguish between concepts that have a larger or smaller proportion of directly grounded components. Developing a groundability scale, in particular one that can account for development, will be key to empirical tests of this account.

The mechanisms provided by SPA also raise important questions for subsequent work: for example, since SPA uses vectors for the underlying representations, what might the distribution of these vectors be when constructed in a biorealistic fashion, and to what degree does this relate directly to our measure of groundability? Further, a developmental process that enriches concepts over time with newly accessible information from existing or new modalities effectively modifies the direction of the vector in space. This might provide a quantitative measure for the amount of change that the

\footnotetext{
${ }^{6}$ Thill and Svensson (2011) discuss the current lack of such a timeline in more detail and speculate that simulations may co-develop with dreams, with the implication being that the quality of dreams (which do not reach adult-levels of sophistication until the late teens) may serve as an indicator of the sophistication of internal models underlying simulations.
} 
introduction of a new cognitive mechanism can induce in a concept.

Importantly, this approach is also consistent with the developmental literature. Sloutsky (2010), for example, provides such an account of the neural mechanisms underlying concept learning, distinguishing between statistically "dense" and "sparse" categories (the difference being the amount of redundant information that a concept carries). Sloutsky relates these to different learning mechanisms-compression mechanisms for dense, and selection mechansisms for sparse categories. Where abstract concepts (which, in his terms are concepts that have no sensory target, such as "love") are concerned, Sloutsky posits an important role for the executive function, and therefore PFC. Taken together, these insights combine into a developmental hypothesis of category learning: dense categories are easier to learn than sparse because the required compression mechanisms develop earlier while the involvement of the executive function in abstract concepts would predict a late acquisition due to the late maturation of the PFC (for a much more detailed reasoning, see Sloutsky, 2010). The account we have provided here includes these considerations in the precise neural mechanisms that SPA postulates to underlie semantic pointer formation (Eliasmith, 2013), but it also extends them with a more explicit inclusion of embodied mechanisms that have their own developmental timeline. Our account also ties in with Barsalou's idea of situated conceptualization (Barsalou, 2009) and the suggestion that concepts are a "large collection of situational representations" (Barsalou and Wiemer-Hastings, 2005, p. 156) since, as previously noted, SPA can be seen as a computational implementation of Barsalou's (1999) perceptual symbol system. A situated conceptualization could be achieved by decompressing some of the semantic pointers (thus activating simulations of the corresponding sensorimotor experience) that make up a given concept. Conversely a theory of what situated conceptualizations for a given concept need to contain can in turn provide insights into what aspects of (internal and external) sensorimotor experience might make up that concept, thus contributing to insights into the nature of Equation (4) for that concept.

\section{CONCLUSION}

In sum, we have shown how developmental accounts of concept acquisition can include embodied theories of cognition, without being forced to claim that all aspects of all concepts are

\section{REFERENCES}

Ambridge, B., Kidd, E., Rowland, C. F., and Theakston, A. L. (2015). The ubiquity of frequency effects in first language acquisition. J. Child Lang. 42, 239-273. doi: 10.1017/S030500091400049X

Barca, L., Burani, C., and Arduino, L. S. (2002). Word naming times and psycholinguistic norms for italian nouns. Behav. Res. Methods Instr. Comput. 34, 424-434. doi: 10.3758/BF03195471

Barsalou, L. W. (1999). Perceptual symbol systems. Behav. Brain Sci. 22, 577-660. doi: $10.1017 / \mathrm{s} 0140525 \times 99002149$ necessarily grounded in some sensorimotor experience. We have also highlighted the importance of understanding the term "sensorimotor" experience as going beyond sensorimotor interaction with the external world: the inside matters just as much. We refer to the extent to which a concept is richly embodied in this way as its groundability. Using empirical data, we have shown both that the semantic features typically considered in developmental studies are not sufficient to explain variability in AoA and, critically, that including $\mathrm{BOI}$ as a measure which can be related to sensorimotor experience improves the results.

Our account unifies existing theories of embodied cognition in a single mechanism by highlighting how cognitive mechanisms that develop comparatively late can enrich existing concepts. It also makes it clear that concepts which have no components that are available early on can only develop later. It also suggests that additional factors in AoA cover a range of attributes: (a) the complexity of the underlying concepts in terms of how many modalities and features they aggregate, (b) the proportion of directly groundable features, (c) the degree to which such features refer to aspects of the external sensorimotor experience, (d) the development of necessary sophisticated mechanisms, and (e) the ability to communicate about them. Thus, this theoretical account integrates research in embodied cognition and cognitive development, paving, we hope, the way for future empirical tests of the interaction between groundability and concept acquisition.

\section{AUTHOR CONTRIBUTIONS}

All authors listed have made a substantial, direct and intellectual contribution to the work, and approved it for publication.

\section{ACKNOWLEDGMENTS}

KT is a Senior Research Associate in the International Centre for Language and Communicative Development $(\mathrm{LuCiD})$ at Lancaster University, UK. The support of the Economic and Social Research Council [ES/L008955/1] is gratefully acknowledged.

\section{SUPPLEMENTARY MATERIAL}

The Supplementary Material for this article can be found online at: http://journal.frontiersin.org/article/10.3389/fpsyg. 2016.00402

Barsalou, L. W. (2009). Simulation, situated conceptualization, and prediction. Philos. Trans. R. Soc. Lond. B Biol. Sci. 364, 1281-1289. doi: 10.1098/rstb.2008.0319

Barsalou, L. W., Santos, A., Simmons, W. K., and Wilson, C. D. (2008). "Symbols, embodiment, and meaning," in Language and Simulation in Conceptual Processing, eds M. De Vega, A. M. Glenberg, and A. C. Graesser (Oxford: Oxford University Press), 245-283.

Barsalou, L. W., and Wiemer-Hastings, K. (2005). "Situating abstract concepts," in Grounding Cognition, eds D. Pecher and R. A. Zwaan (Cambridge: Cambridge University Press), 129-163. 
Bennett, S. D., Burnett, A. N., Siakaluk, P. D., and Pexman, P. M. (2011). Imageability and body-object interaction ratings for 599 multisyllabic nouns. Behav. Res. Methods 43, 1100-1109. doi: 10.3758/s13428-011-0117-5

Bird, H., Franklin, S., and Howard, D. (2001). Age of acquisition and imageability ratings for a large set of words, including verbs and function words. Behav. Res. Methods Instr. Comput. 33, 73-79. doi: 10.3758/BF03195349

Borghi, A. M., and Binkofski, F. (2014). Words as Social Tools: An Embodied View on Abstract Concepts. New York, NY: Springer-Verlag.

Borghi, A. M., and Cimatti, F. (2009). "Words as tools and the problem of abstract word meanings," in Proceedings of the 31st Annual Conference of the Cognitive Science Society, eds N. Taatgen and H. van Rijn (Austin, TX: The Cognitive Science Society), 2304-2309.

Borghi, A. M., and Cimatti, F. (2012). Words are not just words: the social acquisition of abstract words. RIFL 5, 22-37. doi: 10.4396/20120303

Borghi, A. M., Scorolli, C., Caligiore, D., Baldassarre, G., and Tummolini, L. (2013). The embodied mind extended: Using words as social tools. Front. Psychol. 4:214. doi: 10.3389/fpsyg.2013.00214

Bradley, M. M., and Lang, P. J. (2010). Affective Norms for English Words (Anew): Instruction Manual and Affective Ratings. Technical report, Technical Report C-1, The Center for Research in Psychophysiology, University of Florida.

Brysbaert, M., Warriner, A. B., and Kuperman, V. (2014). Concreteness ratings for 40 thousand generally known english word lemmas. Behav. Res. Methods 46, 904-911. doi: 10.3758/s13428-013-0403-5

Chemero, A. (2009). Radical Embodied Cognitive Science. Cambridge, MA: MIT Press.

Chersi, F., Thill, S., Ziemke, T., and Borghi, A. M. (2010). Sentence processing: linking language to motor chains. Front. Neurorobot. 4:4. doi: 10.3389/fnbot.2010.00004

Coltheart, M. (1981). The mrc psycholinguistic database. Q. J. Exp. Psychol. Sect. A 33, 497-505. doi: 10.1080/146407481084 00805

Coradeschi, S., Loutfi, A., and Wrede, B. (2013). A short review of symbol grounding in robotic and intelligent systems. KI - Künstliche Intelligenz 27, 129-136. doi: 10.1007/s13218-013-0247-2

Damasio, A. (2010). Self Comes to Mind - Constructing the Conscious Brain. New York, NY: Pantheon Books.

Dove, G. (2011). On the need for embodied and dis-embodied cognition. Front. Psychol. 1:242. doi: 10.3389/fpsyg.2010.00242

Eliasmith, C. (2013). How to Build a Brain: A Neural Architecture for Biological Cognition. Oxford: Oxford University Press.

Felleman, D. J., and Van Essen, D. C. (1991). Distributed hierarchical processing in primate visual cortex. Cereb. Cortex 1, 1-47. doi: 10.1093/cercor/1.1.1

Fenson, L., Dale, P. S., Reznick, J. S., Thal, D., Bates, E., amd S Pethick, J. P. H., et al. (1993). The MacArthur Communicative Development Inventories: Users Guide and Technical Manual. Baltimore: Paul H. Brokes Publishing Co.

Glenberg, A. M., and Gallese, V. (2012). Action-based language: a theory of language acquisition, comprehension, and production. Cortex 48, 905-922. doi: 10.1016/j.cortex.2011.04.010

Goldenberg, E. R., and Johnson, S. P. (2015). Category generalization in a new context: the role of visual attention. Infant Behav. Dev. 38, 49-56. doi: 10.1016/j.infbeh.2014.12.001

Goodman, J. C., Dale, P. S., and Li, P. (2008). Does frequency count? parental input and the acquisition of vocabulary. J. Child Lang. 35, 515. doi: 10.1017/S0305000907008641

Harnad, S. (1990). The symbol grounding problem. Phys. D Nonlin. Phenom. 42, 335-346. doi: 10.1016/0167-2789(90)90087-6

Hills, T., Maouene, J., Riordan, B., and Smith, L. B. (2009). “Contextual diversity and the associative structure of adult language in early word learning," in Proceedings of the 31st Annual Conference of the Cognitive Science Society (Austin, TX: The Cognitive Science Society), 2118-2123.

Jorm, A. (1991). The validity of word age-of-acquisition ratings: a longitudinal study of a child's word knowledge. Br. J. Dev. Psychol. 9, 437-444. doi: 10.1111/j.2044-835X.1991.tb00888.x

Keil, F. C. (1989). Concepts, Kinds, and Conceptual Development. Cambridge, MA: MIT Press.

Kontra, C., Goldin-Meadow, S., and Beilock, S. L. (2012). Embodied learning across the life span. Topics Cogn. Sci. 4, 731-739. doi: 10.1111/j.17568765.2012.01221.x
Kousta, S. T., Vigliocco, G., Vinson, D. P., Andrews, M., and Del Campo, E. (2011). The representation of abstract words: Why emotion matters. J. Exp. Psychol. Gen. 140, 14-34. doi: 10.1037/a0021446

Kretch, K. S., Franchak, J. M., and Adolph, K. E. (2014). Crawling and walking infants see the world differently. Child Dev. 85, 1503-1518. doi: 10.1111/cdev.12206

Lakoff, G., and Johnson, M. (1980). Metaphors We Live By. Chicago, IL: University of Chicago Press.

Ma, W., Golinkoff, R. M., Hirsh-Pasek, K., McDonough, C., and Tardif, T. (2009). Imageability predicts the age of acquisition of verbs in chinese children. J. Child Lang. 36, 405-423. doi: 10.1017/S0305000908009008

MacWhinney, B. (2000). The CHILDES Project: The Database, Vol. 2. Mahwah, NJ: Lawrence Erlbaum.

Mahon, B. Z., and Caramazza, A. (2008). A critical look at the embodied cognition hypothesis and a new proposal for grounding conceptual content. J. Physiol. 102, 59-70. doi: 10.1016/j.jphysparis.2008.03.004

Mandler, J. M. (2000). Perceptual and conceptual processes in infancy. J. Cogn. Dev. 1, 3-36. doi: 10.1207/S15327647JCD0101N_2

Mandler, J. M., and Bauer, P. J. (1988). The cradle of categorization: is the basic level basic? Cogn. Dev. 3, 247-264. doi: 10.1016/0885-2014(88)90011-1

McDonough, C., Song, L., Hirsh-Pasek, K., Golinkoff, R. M., and Lannon, R. (2011). An image is worth a thousand words: why nouns tend to dominate verbs in early word learning. Dev. Sci. 14, 181-189. doi: 10.1111/j.14677687.2010.00968.x

Mehrabian, A., and Russell, J. (1974). An Approach to Environmental Psychology. Cambridge, MA: MIT Press.

Moors, A., De Houwer, J., Hermans, D., Wanmaker, S., van Schie, K., Van Harmelen, A.-L., et al. (2013). Norms of valence, arousal, dominance, and age of acquisition for 4,300 dutch words. Behav. Res. Methods 45, 169-177. doi: 10.3758/s13428-012-0243-8

Morse, A. F., Benitez, V. L., Belpaeme, T., Cangelosi, A., and Smith, L. B. (2015). Posture affects how robots and infants map words to objects. PLoS ONE 10:e0116012. doi: 10.1371/journal.pone.0116012

Naigles, L. R., and Hoff-Ginsberg, E. (1998). Why are some verbs learned before other verbs? effects of input frequency and structure on children's early verb use. J. Child Lang. 25, 95-120. doi: 10.1017/S0305000997003358

Newcombe, P. I., Campbell, C., Siakaluk, P. D., and Pexman, P. M. (2012). Effects of emotional and sensorimotor knowledge in semantic processing of concrete and abstract nouns. Front. Hum. Neurosci. 6:275. doi: 10.3389/fnhum.2012.00275

Pack, C. C., and Bensmaia, S. J. (2015). Seeing and feeling motion: canonical computations in vision and touch. PLoS Biol. 13:e1002271. doi: 10.1371/journal.pbio.1002271

Paivio, A., Yuille, J. C., and Madigan, S. A. (1968). Concreteness, imagery, and meaningfulness values for 925 nouns. J. Exp. Psychol. 76, 1-25.

Perry, L. K., Perlman, M., and Lupyan, G. (2015). Iconicity in english and spanish and its relation to lexical category and age of acquisition. PLOS ONE 10:e0137147. doi: 10.1371/journal.pone.0137147

Piaget, J. P. (1952). The Origins of Intelligence in Children. New York, NY: International Universities Press.

Quinn, P. C., and Eimas, P. D. (1997). A reexamination of the perceptual-toconceptual shift in mental representations. Rev. Gen. Psychol. 1, 271. doi: 10.1037/1089-2680.1.3.271

Quinn, P. C., Eimas, P. D., and Rosenkrantz, S. L. (1993). Evidence for representations of perceptually similar natural categories by 3-monthold and 4-month-old infants. Perception 22, 463-463. doi: 10.1068/ p220463

Reilly, J., and Kean, J. (2007). Formal distinctiveness of high- and low-imageability nouns: analyses and theoretical implications. Cogn. Sci. 31, 157-168. doi: $10.1080 / 03640210709336988$

Roy, B. C., Frank, M. C., DeCamp, P., Miller, M., and Roy, D. (2015). Predicting the birth of a spoken word. Proc. Natl. Acad. Sci. U.S.A. 112, 12663-12668. doi: $10.1073 /$ pnas. 1419773112

Samuelson, L. K., and Horst, J. S. (2007). Dynamic noun generalization: momentto-moment interactions shape children's naming biases. Infancy 11, 97-110. doi: 10.1207/s15327078in1101_5

Samuelson, L. K., Smith, L. B., Perry, L. K., and Spencer, J. P. (2011). Grounding word learning in space. PLoS ONE 6:e28095. doi: 10.1371/journal.pone.0028095 
Siakaluk, P. D., Knol, N., and Pexman, P. M. (2014). Effects of emotional experience for abstract words in the stroop task. Cogn. Sci. 38, 1698-1717. doi: $10.1111 /$ cogs. 12137

Sloutsky, V. M. (2010). From perceptual categories to concepts: What develops? Cogn. Sci. 34, 1244-1286. doi: 10.1111/j.1551-6709.2010.01129.x

Smith, L. B., Yu, C., and Pereira, A. F. (2011). Not your mothers view: the dynamics of toddler visual experience. Dev. Sci. 14, 9-17. doi: 10.1111/j.14677687.2009.00947.x

Smolík, F. (2014). Noun imageability facilitates the acquisition of plurals: survival analysis of plural emergence in children. J. Psycholinguist. Res. 43, 335-350. doi: 10.1007/s10936-013-9255-5

Stapleton, M. (2011). Proper Embodiment: The Role of the Body in Affect and Cognition. Ph.D thesis, The University of Edinburgh, UK.

Stapleton, M. (2013). Steps to a "properly embodied" cognitive science. Cogn. Syst. Res. 22-23, 1-11. doi: 10.1016/j.cogsys.2012.05.001

Storkel, H. L. (2004). Do children acquire dense neighborhoods? an investigation of similarity neighborhoods in lexical acquisition. Appl. Psycholinguist. 25, 201-221. doi: 10.1017/S0142716404001109

Stramandinoli, F., Marocco, D., and Cangelosi, A. (2012). The grounding of higher order concepts in action and language: a cognitive robotics model. Neural Netw. 32, 165-173. doi: 10.1016/j.neunet.2012. 02.012

Thill, S. (2015). "Embodied neuro-cognitive integration," in Proceedings of the Workshop on "Neural-Cognitive Integration" (NCI@KI 2015), Vol. 2015-03 of Publication Series of the Institute of Cognitive Science, eds T. R. Besold, and K.-U. Kühnberger, (Osnabrück).

Thill, S., Padó, S., and Ziemke, T. (2014). On the importance of a rich embodiment in the grounding of concepts: perspectives from embodied cognitive science and computational linguistics. Topics Cogn. Sci. 6, 545-558. doi: $10.1111 /$ tops. 12093

Thill, S., and Svensson, H. (2011). "The inception of simulation: a hypothesis for the role of dreams in young children," in Proceedings of the 33rd Annual Conference of the Cognitive Science Society, eds L. Carlson, C. Hoelscher, and T. F. Shipley (Austin, TX: Cognitive Science Society), 231-236.

Tillotson, S. M., Siakaluk, P. D., and Pexman, P. M. (2008). Bodyobject interaction ratings for 1,618 monosyllabic nouns. Behav. Res. Methods 40, 1075-1078. doi: 10.3758/BRM.40.4.1075
Twomey, K. E., Ranson, S. L., and Horst, J. S. (2014). That's more like it: multiple exemplars facilitate word learning. Infant Child Dev. 23, 105-122. doi: 10.1002/icd.1824

van der Velde, F., and de Kamps, M. (2006). Neural blackboard architectures of combinatorial structures in cognition. Behav. Brain Sci. 29, 37-70. doi: $10.1017 / \mathrm{s} 0140525 \mathrm{x} 06009022$

Vlach, H. A., Sandhofer, C. M., and Kornell, N. (2008). The spacing effect in childrens memory and category induction. Cognition 109, 163-167. doi: 10.1016/j.cognition.2008.07.013

Wellsby, M., and Pexman, P. M. (2014a). Developing embodied cognition: Insight from children's concepts and language processing. Front. Psychol. 5:506. doi: 10.3389/fpsyg.2014.00506

Wellsby, M., and Pexman, P. M. (2014b). The influence of bodily experience on children's language processing. Topics Cogn. Sci. 6, 425-441. doi: 10.1111 /tops. 12092

Wilson, M. (1988). Mrc psycholinguistic database: machine-usable dictionary, version 2.00. Behav. Res. Methods Instr. Comput. 20, 6-10. doi: $10.3758 / \mathrm{BF} 03202594$

Ziemke, T. (2003). "What's that thing called embodiment," in Proceedings of the 25th Annual meeting of the Cognitive Science Society (Austin, TX: The Cognitive Science Society), 1305-1310.

Ziemke, T., and Lowe, R. (2009). On the role of emotion in embodied cognitive architectures: from organisms to robots. Cogn. Comput. 1, 104-117. doi: 10.1007/s12559-009-9012-0

Zwaan, R. A. (2015). Situation models, mental simulations, and abstract concepts in discourse comprehension. Psychonom. Bull. Rev. doi: 10.3758/s13423-0150864-x. [Epub ahead of print].

Conflict of Interest Statement: The authors declare that the research was conducted in the absence of any commercial or financial relationships that could be construed as a potential conflict of interest.

Copyright (c) 2016 Thill and Twomey. This is an open-access article distributed under the terms of the Creative Commons Attribution License (CC BY). The use, distribution or reproduction in other forums is permitted, provided the original author(s) or licensor are credited and that the original publication in this journal is cited, in accordance with accepted academic practice. No use, distribution or reproduction is permitted which does not comply with these terms. 


\title{
Development of Embodied Word Meanings: Sensorimotor Effects in Children's Lexical Processing
}

\author{
Michelle Inkster, Michele Wellsby, Ellen Lloyd and Penny M. Pexman* \\ Language Processing Laboratory, Department of Psychology, University of Calgary, Calgary, AB, Canada
}

Previous research showed an effect of words' rated body-object interaction (BOI) in children's visual word naming performance, but only in children 8 years of age or older (Wellsby and Pexman, 2014a). In that study, however, BOI was established using adult ratings. Here we collected ratings from a group of parents for children's $\mathrm{BOI}$ experience (child-BOI). We examined effects of words' child-BOI and also words' imageability on children's responses in an auditory word naming task, which is suited to the lexical processing skills of younger children. We tested a group of 54 children aged 6-7 years and a comparison group of 25 adults. Results showed significant effects of both imageability and child-BOI on children's auditory naming latencies. These results provide evidence that children younger than 8 years of age have richer semantic representations

OPEN ACCESS

Edited by:

Anna M. Borghi,

University of Bologna, Italy

Reviewed by:

Arthur M. Glenberg

Arizona State University, USA Katherine Twomey,

Lancaster University, UK

*Correspondence:

Penny M. Pexman

pexman@ucalgary.ca

Specialty section: This article was submitted to Cognition,

a section of the journa

Frontiers in Psychology

Received: 10 December 2015

Accepted: 18 February 2016

Published: 08 March 2016

Citation:

Inkster M, Wellsby M, Lloyd E and Pexman PM (2016) Development of Embodied Word Meanings:

Sensorimotor Effects in Children's

Lexical Processing.

Front. Psychol. 7:317.

doi: 10.3389/fpsyg.2016.00317 for high imageability and high child-BOI words, consistent with an embodied account of word meaning.

Keywords: sensorimotor, auditory naming, imageability, body-object interaction, language development, semantic processing

\section{INTRODUCTION}

Theories of embodied cognition emphasize the importance of sensorimotor experience for acquiring and representing conceptual knowledge (e.g., Glenberg, 2015). The embodied cognition framework has had a strong influence in recent research on adult language processing. It has received less research attention in the developmental literature and yet, as Wellsby and Pexman (2014b) argued, in order to refine theories of embodied cognition it is important to consider and fully integrate developmental findings. The purpose of the present study was to investigate whether children's previous sensorimotor experience with words' referents influences their lexical processing of those words.

From an embodied cognition perspective, representations of word meaning are, at least in part, grounded in sensorimotor and other bodily systems (for a review see Meteyard et al., 2012). Hence, it should be possible to observe effects of sensorimotor information on lexical processing. Indeed, there is now extensive research examining the effects of imageability in visual word recognition tasks (e.g., Strain et al., 1995; Cortese et al., 1997). Imageability is characterized as a word's ability to arouse mental imagery (visual, sound, etc., Cortese and Fugett, 2004). Typically, word recognition is facilitated for words with referents that can be more easily imaged/sensed.

While imageability primarily measures sensory experience with words' referents, Siakaluk et al. (2008a) have characterized a measure that is more focused on motor experience with words' referents. This is the body-object interaction variable (BOI) and ratings of this dimension capture how easily a human body can interact with a word's referent. Research with adults shows that 
in lexical decision tasks, phonological lexical decision tasks, and semantic categorization tasks, words rated high in BOI (e.g., belt) are processed faster and/or more accurately than words rated low in BOI (e.g., ship; Siakaluk et al., 2008a,b; Tillotson et al., 2008). To be clear, these are all concrete, highly imageable words, and they differ only in rated ease of bodily interaction; word frequency, length, and other factors are matched across high BOI and low BOI word sets. The proposed explanation is that previous motor experience with words' referents (as measured by the BOI variable) facilitates word recognition.

Siakaluk and colleagues have explained BOI effects in adult word recognition in terms of feedback activation in the lexical system. That is, in a fully interactive word recognition system comprised of orthographic, phonological, and semantic units (e.g., Harm and Seidenberg, 2004), it is assumed that words with richer semantic representations provide stronger feedback to the orthographic and phonological units. As a result, orthographic and phonological units are faster to settle into stable patterns of activation for words with relatively richer semantic representations. In most word recognition tasks, activity in the orthographic and phonological units provides the basis for responding (e.g., Hino and Lupker, 1996). If sensorimotor information is part of lexical semantic knowledge, then high BOI words will have richer semantic representations than low BOI words, and phonological and orthographic processing will be facilitated via semantic feedback. Similarly, high imageability words are presumed to have richer semantic representations than low imageability words and thus imageability effects emerge in lexical processing tasks.

While we now understand a great deal about how sensorimotor effects like BOI and imageability influence adult lexical processing, we know much less about when they begin to influence lexical processing in children. In the first study to explore development of BOI effects in children, Wellsby and Pexman (2014a) examined visual word naming performance for high and low BOI words in a group of younger children (aged 6-7 years), a group of older children (aged 8-9 years), and a group of adults. To facilitate comparisons across the groups, Wellsby and Pexman used a composite measure of naming performance that combined naming latency and accuracy information. Results showed a significant facilitatory BOI effect for the older children and the adults but not for the younger children. For children, the size of their BOI effect was related to their age and also to their reading skills; that is, BOI effects were larger for older children and for children with higher scores on a standardized reading test. As such, Wellsby and Pexman concluded that the BOI variable begins to influence children's lexical processing at about 8 years of age. In reasoning about why the effect does not emerge in younger children, Wellsby and Pexman concluded that two explanations were viable: first, younger children may not yet have had sufficient sensorimotor interactions with the words' referents to create richer representations for high BOI items; second, younger children's reading skills may not yet be efficient enough to support semantic feedback. Indeed, consistent with simulations of models of the lexical processing system (e.g., Plaut et al., 1996), it has been suggested that semantic factors become more important as children progress beyond the early stages of reading development (Nation, 2009; Hulme and Snowling, 2013).

There are, however, two issues with the methodology of the Wellsby and Pexman (2014a) study that may have limited detection of BOI effects in the younger children in that study. First, the use of the visual naming task may have limited the effects observed. Wellsby and Pexman reported that the visual naming task was very challenging for the younger children. The younger children made many more naming errors than the older children and also seemed to be sounding out the words, naming them in a step-by-step fashion, which could have complicated the measurement of naming latencies. Visual naming and lexical decision are traditional lexical tasks, yet these have rarely been used with children younger than 8 years of age. This is because in younger children, word reading skills are not advanced enough to support performance in visual naming or lexical decision. Further, in studies using these tasks, there is limited evidence for effects of sensorimotor variables like imageability and concreteness (Coltheart et al., 1988; McFalls et al., 1996). Since naming and lexical decision tasks require that children translate spelling into sound and meaning, performance in those tasks depends heavily on children's reading skills and this could complicate the detection of sensorimotor effects.

A lingering question is thus: would children younger than 8 years of age show sensorimotor effects if given a lexical processing task that was less dependent on reading skills, particularly orthographic skills? To address this question, we chose an alternative task that has been used before to examine semantic effects in adults' lexical processing (Tyler et al., 2000; Wurm et al., 2004). This is the auditory naming task, in which participants hear words and repeat each one aloud as quickly and as accurately as they can. Tyler et al. (2000) compared imageability effects in auditory naming and auditory lexical decision. They found imageability effects in both tasks, even when controlling for word length and familiarity. They concluded that even though the tasks have differences (auditory naming involves articulation while auditory lexical decision involves a decision component), both tasks "tap into the activation of semantic representations" (p. 324). Wurm et al. (2004) noted that unlike visual naming, processing stimuli in auditory naming does not require orthography to phonology translation. As such, the auditory naming task should depend less on orthographic coding skills than do tasks like visual naming and visual lexical decision but should still involve semantic processing. Younger children should be more proficient in an auditory naming task than in a visual naming task as their experience processing spoken language is far greater than their experience processing written language.

An additional issue with the previous examination of BOI effects in children's lexical processing involves the use of adult BOI ratings. While many studies have used adult ratings of semantic variables to assess effects of those variables in children's language processing, this practice is particularly problematic for BOI. The BOI variable is intended to capture fine-grained differences between highly imageable words; that is, it provides an indication of differences in the average person's previous 
experiences physically interacting with the words' referents. Whereas adults rate axe and cane as high BOI, these would likely not be objects with which children have extensive physical experience. While children may have plenty of visual experience with these objects, their physical experience will be different, due to their stature, permitted activities, and other constraints. As such, while adults' ratings of imageability (which primarily assess visual information) are probably applicable to children, adults' BOI ratings of their own bodily experience may not capture variability in children's physical interaction experience. This could have been a particular issue for the younger children in the Wellsby and Pexman study (the 6-7 year olds), who would have had even less experience with some of the objects than the older children, and this issue could have contributed to the null BOI effect observed for that group. There is a need to ensure that the words chosen to assess BOI effects in children's lexical processing are high and low in terms of the average child's BOIs. This was addressed in the present study by asking parents of children in this age range (6-7 years) to provide ratings using a dimension we refer to as child-BOI. We reasoned that parents with children in this age group would be best suited to make ratings of 6-7 year olds' sensorimotor experiences with word referents since these parents have direct knowledge of their children's activities.

In the present study, we thus examined imageability effects (comparing responses to high and low imageability words) and child-BOI effects (comparing responses to high and low child-BOI words, which were a subset of the high imageability words) in the auditory naming task. We tested a group of 6-7 year old children and also a group of adults. Adults were tested primarily as a control group to verify our assumptions about the auditory naming task. That is, although the auditory naming task has been used to examine semantic effects in lexical processing with adults (i.e., Tyler et al., 2000; Wurm et al., 2004), we wanted to assess whether the task was sensitive to the semantic dimensions manipulated here. We included measures of children's reading skills as an additional evaluation of the task. While children's reading skills were correlated with aspects of their visual naming performance in Wellsby and Pexman (2014a), the auditory task used here should be less dependent on children's orthographic skills. Thus we expected that reading skills would not correlated with task performance in the current study.

If BOI effects can be observed before age 8, but were masked in the previous study (Wellsby and Pexman, 2014a) by task difficulty or by items which did not account for adult-child differences in physical interaction experience, then we expected to find a child-BOI effect with 6-7 year olds using the present study's items and auditory naming task. This would be evidence that sensorimotor information is part of children's lexical knowledge, consistent with an embodied framework for word meaning. By this framework, we also expected imageability effects in the auditory naming task. It is also possible, however, that younger children may not have sufficient experience to afford richer semantic representations for high imageability and high childBOI objects and thus we would not expect imageability and child-BOI effects to emerge before age 8 .

\section{MATERIALS AND METHODS}

\section{Participants}

Participants were 54 children aged 6-7 years (32 female, $M$ age $=7 ; 2, S D=0 ; 6$ ) and 25 adults (14 female, $M$ age $=21 ; 2$, $S D=2 ; 4)$. Children were recruited through the University of Calgary Child and Infant Learning and Development (ChILD) database and received a small toy for participating. Adults were undergraduate students in Psychology courses at the University of Calgary who received partial course credit for participating.

\section{Stimuli}

Stimuli for the auditory naming task consisted of 60 high imageability and 60 low imageability monosyllabic words. Imageability was determined based on published norms (Cortese and Fugett, 2004). To ensure that the words were all familiar to 67 year old children and that the high and low imageability word sets differed only in terms of imageability, the two word sets were not significantly different on all of the following characteristics: grade one print frequency (the frequency of a word in academic texts that tend to be part of the Grade 1 curriculum, as indexed by the Educator's Word Frequency Guide, Zeno et al., 1995), children's spoken frequency for the 6-7 year old age group in the Child Language Data Exchange System (CHILDES) database (from the ChildFreq lexical norms described in Bääth, 2010; which include frequency information for all words in CHILDES produced by an English child speaker, providing an estimate of the speech that children typically produce), adults' spoken frequency in child-directed speech (the MacWhinney, 2000, CHILDES sub-corpus extracted by Ping Li, described here: http://childes.talkbank.org/derived/, providing an estimate of the speech that children are typically exposed to), word length, phonological Levenshtein distance (PLD; a measure of words' phonological similarity, Yarkoni et al., 2008), orthographic Levenshtein distance (OLD; a measure of words' orthographic similarity, Yarkoni et al., 2008), and valence (Warriner et al., 2013). Refer to Table 1 for descriptive statistics of the stimuli.

To collect ratings of child-BOI, we presented a separate group of 16 adults (who were all parents to children in the same age range as child participants, but were not parents to the children who participated) with the 60 high imageability words. These adults were instructed to rate each word on a 7 -point scale, and to use a 6-year-old child as their referent. That is, they were asked to rate how much physical interaction experience the average 6-year-old might be likely to have with each thing ( 1 = low, "things a typical child has not touched/held at all"; 7 = high, "things a typical child has touched/held a lot"). Based on these ratings, we selected 12 high child-BOI words (e.g., chair) and 12 low child-BOI words (e.g., knife), again not significantly different on grade one print frequency, child spoken frequency, adult spoken frequency, word length, PLD, OLD, and valence. Descriptive statistics for these items are also provided in Table $\mathbf{1 .}$ There was no significant correlation between child-BOI ratings and imageability ratings for the 60 high imageability words, $r=0.21, p=0.10$. This suggests that child-BOI and imageability are not strongly related constructs. The complete list of stimuli, 
TABLE 1 | Mean characteristics of word stimuli (standard deviations in parentheses).

\begin{tabular}{|c|c|c|c|c|c|c|}
\hline Word characteristic & High imageability & Low imageability & $p$ fordifference test & High child-BOI & Low child-BOI & $p$ for difference test \\
\hline Number of items & 60 & 60 & & 12 & 12 & \\
\hline Imageability & $5.83(0.78)$ & $3.46(0.60)$ & $<0.001$ & $6.40(0.21)$ & $6.34(0.48)$ & 0.38 \\
\hline Child-BOI & & & & $6.21(0.59)$ & $3.83(1.36)$ & $<0.001$ \\
\hline Grade 1 print frequency & $81.87(58.30)$ & $87.77(70.62)$ & 0.62 & $95.08(64.84)$ & $91.50(42.75)$ & 0.87 \\
\hline Child spoken frequency & 87.02 (121.09) & $81.50(145.03)$ & 0.82 & 164.08 (194.95) & $65.58(83.14)$ & 0.13 \\
\hline Adult spoken frequency & $291.72(368.44)$ & $245.93(466.46)$ & 0.55 & $604.42(495.21)$ & 304.25 (341.03) & 0.10 \\
\hline Word length (letters) & $4.28(0.74)$ & $4.30(0.79)$ & 0.91 & $4.17(0.83)$ & $4.58(0.90)$ & 0.25 \\
\hline PLD & $1.29(0.29)$ & $1.23(0.29)$ & 0.27 & $1.23(0.31)$ & $1.31(0.30)$ & 0.53 \\
\hline OLD & $1.46(0.30)$ & $1.47(0.30)$ & 0.90 & $1.46(0.37)$ & $1.58(0.26)$ & 0.35 \\
\hline Valence & $5.23(1.75)$ & $5.30(1.86)$ & 0.83 & $6.46(0.74)$ & $5.65(1.57)$ & 0.12 \\
\hline Sound file length (ms) & 382.67 (53.03) & 395.65 (47.52) & 0.16 & 376.92 (52.32) & $384.50(68.36)$ & 0.76 \\
\hline
\end{tabular}

The high child-BOI low child-BOI words were a subset of the 60 high imageability words. BOI, body-object interaction; PLD, phonological Levenshtein distance; OLD, orthographic Levenshtein distance.

including imageability and child-BOI ratings, can be found in the Appendix.

Word stimuli for the auditory naming task were recorded as sound files by a female speaker who was naïve to the purpose of the study (using Sound Recorder). Since all words were monosyllabic the durations of sound files were quite consistent; to further improve this, however, sound files were edited using the program Praat. The editing ensured that all files included the entire duration of the word and that there were no significant differences between lengths (durations) of the sound files for high imageability and low imageability word sets or for the high child-BOI and low child-BOI word sets (Table 1), while still maintaining perceptibility. Edited files were then played individually to six adults to verify that each word could still be recognized accurately.

\section{Procedure}

Participants were tested in our university laboratory. They sat in front of a computer wearing headphones. A microphone was positioned in front of the child's face, connected to a response box with voice key that measured the onset latency of vocal responses. The child either held the microphone or, if they preferred, the experimenter did so. The experimenter sat beside the participant, and also wore headphones. Sound files were presented one at a time through both the participant's and the experimenter's headphones using E-Prime presentation software (Schneider et al., 2001). Participants were instructed to repeat each word into the microphone immediately after hearing it, and to do so loudly and clearly. The task began with five practice trials, followed by a short break for participants to ask any questions, and then the 120 experimental trials. The words were presented in randomized order to each participant. During the practice and experimental trials, the screen was blank except for a small fixation cross in the center.

Once a word was vocalized, the researcher coded the response as "correct" (participant repeated the correct word), "incorrect" (participant did not repeat the correct word), or "spoiled" (participant hesitated, stuttered, or prematurely triggered the response box) using the computer keyboard. The code of 1, 2, or 3 (respectively) prompted the presentation of the next word; this way, the experiment proceeded at a pace the participant was comfortable with. Participants were also allowed to request a break at any point in order to prevent fatigue effects.

Following the auditory naming task, child participants completed three subtests of the Woodcock Reading Mastery Tests-Revised (WRMT-R; Woodcock, 1997). These represent a subset of the tests administered in the Wellsby and Pexman (2014a) study. These subtests were administered to obtain a measure of participants' reading ability across three different dimensions: their letter identification skills (i.e., what letter is this?), word reading skills (i.e., what word is this?), and orthographic-phonological conversion skills ("word attack," i.e., how does this non-word sound?). Children's mean scores on these subtests (letter identification $M=38.58, S D=3.57$, word reading $M=47.50, S D=21.89$ and word attack $M=19.56$, $S D=10.20)$ were very similar to those obtained for the younger group in the Wellsby and Pexman study.

\section{RESULTS}

We first examined auditory naming accuracy by item to determine whether any items should be excluded from the analysis. The item "full" was removed as it was only named correctly $24.32 \%$ of the time by the child participants. Removing this low imageability item did not compromise the matching illustrated in Table 1. Auditory naming accuracy was high for all other items (97.17\% for children, $98.69 \%$ for adults): too high, in fact, to warrant further analysis of the accuracy data. Before analyzing latency data, we removed latencies for trials with incorrect $(2.83 \%$ for children, $1.18 \%$ for adults) or spoiled responses $(0.81 \%$ for children, $0.13 \%$ for adults). In addition, trials with response latencies faster than $250 \mathrm{~ms}$ or slower than the participant's mean plus 2.5 SD (3.92\% for children, $1.38 \%$ for adults) were removed from the analysis. Since the adults were tested simply to establish that the auditory naming task was sensitive to the kinds of effects tested here, we present the analysis of the adult data first and then, separately, analysis of the child data, including tests for correlations with reading skill variables. 
Imageability effects in response latencies were examined using ANOVAs on the entire dataset [119 items, with imageability (high vs. low) as a fixed factor], whereas child-BOI effects were examined using separate ANOVAs on the subset of the high imageability items for which child-BOI was manipulated [24 items, with child-BOI (high vs. low) as a fixed factor].

\section{Adults}

The analysis of adults' auditory naming latencies showed a significant effect of imageability, $F(1,24)=28.46, p<0.001$, $\eta_{\mathrm{p}}^{2}=0.543$. That is, adults named high imageability words $(M=752.70, S D=77.37)$ faster than low imageability words $(M=768.32, S D=76.27)$. Although it was not our focus for the adult data, we also tested the effect of child-BOI for adults and found that it was not significant, $F(1,24)=3.99$, $p=0.057, \eta_{\mathrm{p}}^{2}=0.142$. Adults did not name high child-BOI words $(M=746.81, S D=76.33)$ significantly faster than low childBOI words $(M=755.43, S D=81.91)$. Given that child-BOI was characterized based on 6-year-olds' physical experience this is not entirely surprising.

\section{Children}

The analysis of children's auditory naming latencies showed a significant effect of imageability, $F(1,53)=57.02, p<0.001$, $\eta_{\mathrm{p}}^{2}=0.518$. That is, children named high imageability words $(M=881.96, S D=123.02)$ faster than low imageability words $(M=906.91, S D=122.12)$. There was also a significant effect of child-BOI for children's naming latencies, $F(1,53)=44.86$, $p<0.001, \eta_{\mathrm{p}}^{2}=0.458$ : Children named high child-BOI words $(M=860.01, S D=126.19)$ faster than low child-BOI words $(M=899.25, S D=132.36)$.

We next examined correlations between children's age, reading skills, imageability effects, and child-BOI effects. These correlations are presented in Table 2. Not surprisingly, there were significant correlations between children's age and scores on each of the reading subtests, since older children also tended to have better reading skills. In addition, children's scores on the reading subtests were correlated. More importantly, children's age was correlated with their child-BOI effect but not their imageability effect, such that older children showed larger BOI effects. There were no significant relationships between the reading skill measures and the imageability or child-BOI effects, and this held regardless of whether age was partialled out of the correlations. There was also no significant relationship between the size of children's imageability and childBOI effects.

In collecting ratings of the child-BOI dimension in the present study, we asked parents to rate "how much experience the average 6-year-old would have likely had physically interacting (using the body: hands, mouth, etc)" with each word's referent. In this way our ratings instructions were somewhat different than those used by Siakaluk and colleagues to collect BOI ratings from adults (e.g., Tillotson et al., 2008). The BOI ratings instructions for adults emphasized ease of interaction. Here, for child-BOI ratings, we emphasized likelihood of interaction in order to highlight the ways in which a child's experience might be different than that of adults. As such, the child-BOI dimension probably measures a slightly different aspect of interaction than does the adult BOI dimension, and frequency of interaction may play a stronger role in child-BOI ratings than in adult-BOI ratings. Further, while the low and high child-BOI word sets do not differ significantly on print frequency or on either of the spoken frequency measures (child spoken frequency, adult spoken frequency), there are large numeric differences between the low and high child-BOI word sets on the spoken frequency dimensions. To evaluate whether the child-BOI effect we observed was incremental to those spoken frequency differences, we conducted linear mixed effects analyses (Baayen et al., 2008) using R. The influence of spoken frequency (either child or adult, in separate models), age, and child-BOI rating were treated as fixed effects, while participants and items were treated as random variables. The dependent measure was auditory naming latency. We began with models including spoken frequency, child-BOI rating and child's age as predictors. However, since child-BOI effects tended to be larger for older children, we also tested the inclusion of an interaction of child-BOI rating and child's age as a predictor. We compared the models with the interaction term to models without the interaction term, and found that including the interaction as a predictor significantly improved model fit: $\chi^{2}(1)=4.43$, $p=0.035$ for the model in Table 3 , with child spoken frequency as a predictor, and $\chi^{2}(1)=4.45, p=0.035$ for the model in Table 4, with adult spoken frequency as a predictor. The resulting model with child spoken frequency as a predictor is presented in Table 3, and the model with adult spoken frequency as a predictor is presented in Table 4. As can be seen, even after including spoken frequency as a predictor, there is evidence that effects of child-BOI may be incremental to the influence of spoken frequency.

\section{TABLE 2 | Correlations among variables.}

\begin{tabular}{|c|c|c|c|c|c|}
\hline Variable & 2 & 3 & 4 & 5 & 6 \\
\hline (2) Imageability effect & - & 0.10 & 0.01 & -0.09 & -0.03 \\
\hline (4) WRMT: letter identification & 0.00 & 0.03 & - & $0.71^{* * *}$ & $0.69^{* * *}$ \\
\hline (5) WRMT: word reading & -0.12 & 0.02 & $0.59^{* * *}$ & - & $0.91^{* * *}$ \\
\hline (6) WRMT: word attack & -0.05 & -0.10 & $0.58^{* * *}$ & $0.88^{* * *}$ & - \\
\hline
\end{tabular}

Raw correlations are presented above the diagonal, and partial correlations (controlling for age) are presented below the diagonal. BOI, body-object interaction; WRMT, Woodcock Reading Mastery Test. ${ }^{* *} p<0.01,{ }^{* * *} p<0.001$. 
TABLE 3 | Summary of regression model predicting child reaction time, with child spoken frequency as a predictor.

\begin{tabular}{|c|c|c|c|c|}
\hline Fixed Effect & Coefficient & $S E$ & $t$ & $p$ \\
\hline Intercept & -286.94 & 273.57 & -1.05 & 0.30 \\
\hline Child spoken frequency & -0.13 & 0.07 & -1.78 & 0.09 \\
\hline Child-BOI rating & 60.93 & 33.60 & 1.81 & 0.07 \\
\hline Age & 14.26 & 3.16 & 4.52 & $<0.001^{* *}$ \\
\hline Child-BOI rating $\times$ Age & -0.81 & 0.38 & -2.11 & $0.04^{*}$ \\
\hline Random effect & \multicolumn{4}{|c|}{$s^{2}$} \\
\hline Subject intercept & \multicolumn{4}{|c|}{11423.33} \\
\hline Item intercept & \multicolumn{4}{|c|}{1886.17} \\
\hline
\end{tabular}

TABLE 4 | Summary of regression model predicting child reaction time, with adult spoken frequency as a predictor.

\begin{tabular}{lrrrc}
\hline Fixed effect & Coefficient & \multicolumn{1}{c}{ SE } & $\boldsymbol{t}$ & $\boldsymbol{p}$ \\
\hline Intercept & 289.47 & 273.71 & 1.06 & 0.29 \\
Adult spoken frequency & 0.04 & 0.03 & 1.56 & 0.13 \\
Child-BOI rating & 62.60 & 33.79 & 1.85 & 0.06 \\
Age & 14.26 & 3.16 & 4.52 & $<0.001^{* * *}$ \\
Child-BOI rating $\times$ Age & 0.81 & 0.38 & 2.11 & $0.04^{*}$ \\
\hline
\end{tabular}

\begin{tabular}{lc}
\hline Random effect & $\boldsymbol{s}^{\mathbf{2}}$ \\
\hline Subject intercept & 11423.33 \\
Item intercept & 1950.11 \\
\hline$N=1184$; log-likelihood $=7472.42 ;$ AlC $=14960.84, * 0.05, * * * p<0.001$
\end{tabular}

\section{DISCUSSION}

The purpose of the present research was to examine whether children's lexical processing is influenced by words' sensorimotor histories (as indexed by imageability and child-BOI) before age 8. Children younger than 8 years of age typically struggle with lexical processing tasks that depend on reading skills. With this in mind, we used an auditory naming task to assess the influence of sensorimotor variables in 6- and 7-year-old children. Even the youngest children in the sample were able to perform this task with high accuracy, suggesting it was well suited to their lexical processing skills. Results showed a significant imageability effect and a significant child-BOI effect in this age group. Thus, there is now evidence that children's lexical processing is influenced by sensorimotor variables before 8 years of age.

Our findings suggest that children do have richer semantic representations for words associated with more sensorimotor information. Thus, sensorimotor information is part of children's lexical knowledge, consistent with an embodied framework for word meaning. Although these representations may not be measurable in orthographically based tasks until age 8 , effects can be observed earlier in a word processing task that does not require orthographic coding. As such, our results provide an important new data point for sensorimotor effects in childhood and are consistent with the assertion that word meaning is embodied throughout the lifespan. This conclusion would be compatible with results showing that during passive listening to verb stimuli (e.g., chase, clap) 4- to 6-year-old children showed activation in the motor cortex (James and Maouene, 2009). Similarly, Maouene et al. (2008) found that the earliest acquired verbs tended to be those associated with the mouth, with verbs associated with hand and arm actions acquired next, and that verbs not associated with any particular body part were acquired later. That is, in the 2nd and 3rd years of life verbs enter children's productive vocabularies at a rapid rate, and the order of acquisition can be predicted, to some degree, by the relationship of the verb to the child's developing motor system.

The present results also showed that while the child-BOI effect in auditory naming was related to age, the imageability effect was not. That is, child-BOI effects tended to increase across the developmental window examined, while imageability effects remained stable. The child-BOI variable is intended to measure physical experiences, and these may be accumulating rapidly in this age group, such that the older children in the sample have sufficiently more physical interaction experiences to effect differences in the underlying semantic representations. In contrast, imageability measures multisensory (visual, sound, etc.) experiences. Since these multisensory experiences do not depend on motor skills to the same degree that child-BOI-related experiences do, they may begin to accumulate earlier and reach a relative plateau during the developmental window assessed here. In future studies, it would be important to test an even younger group of children to determine when imageability effects are first observed in children's lexical processing. Given the high level of accuracy children exhibited for the auditory naming task in the present study, it may be possible to use this task successfully with an even younger group of children in order to test these remaining research questions.

Additional topics for future research include investigating implications for vocabulary acquisition and literacy development: if bodily experiences are an important aspect of children's semantic representations, then providing more of those experiences may facilitate more rapid acquisition of word meaning. In related research, James and Swain (2011) used fMRI to explore differences in the neural correlates of verbs learned through self-generated actions vs. observed actions. Children aged 5-6 years were taught novel verb labels while performing an action on an object or watching an experimenter perform an action on an object. Later, during an fMRI session, children heard the verbs and saw photographs of the objects but did not make a behavioral response. Results showed activity in the motor cortex during passive listening to verbs that had been learned through self-generated actions but not for verbs learned through observed actions. Similarly, children's motor systems were more active when viewing objects experienced through active interaction than when viewing objects experienced through observed interactions. These findings suggest that children's representations of verb meaning involve more motor information if those verbs are learned through direct physical experience with the actions implied. James and Swain (2011) did not test whether these representational differences had 
behavioral consequences, so we don't yet know whether physical experiences facilitate acquisition of word meaning, but this will be an important issue to address in order to more fully understand the applications of this research.

The present results provide new insight about the developing lexicon and suggest a role for sensorimotor experience in the acquisition of word meaning. Sensorimotor effects can be observed in auditory naming in 6-7 year olds, but future research will be required to map out the trajectory of sensorimotor effects in the developing linguistic and conceptual systems.

\section{AUTHOR CONTRIBUTIONS}

MI collected most of the data and wrote the first draft of the manuscript. MW helped design and program the experiment and assisted with analyses. EL assisted with data collection, coding, and writing. PP helped design the experiment, analyze the data, and write the paper.

\section{REFERENCES}

Bääth, R. (2010). ChildFreq: an online tool to explore word frequencies in child language. LUCS Minor 16, 1-6.

Baayen, R. H., Davidson, D. J., and Bates, D. M. (2008). Mixed-effects modeling with crossed random effects for subjects and items. J. Mem. Lang. 59, 390-412. doi: 10.1016/j.jml.2007.12.005

Coltheart, V., Laxon, V. J., and Keating, C. (1988). Effects of word imageability and age of acquisition on children's reading. Br. J. Psychol. 79, 1-12. doi: 10.1111/j.2044-8295.1988.tb02270.x

Cortese, M. J., and Fugett, A. (2004). Imageability ratings for 3,000 monosyllabic words. Behav. Res. Methods Instr. Comput. 36, 384-387. doi: 10.3758/BF03195585

Cortese, M. J., Simpson, G. B., and Woolsey, S. (1997). Effects of association and imageability on phonological mapping. Psychon. Bull. Rev. 4, 226-231. doi: 10.3758/BF03209397

Glenberg, A. (2015). Few believe the world is flat: how embodiment is changing the scientific understanding of cognition. Can. J. Exp. Psychol. 69, 157-163. doi: 10.1037/cep0000056

Harm, M. W., and Seidenberg, M. S. (2004). Computing the meanings of words in reading: cooperative division of labor between visual and phonological processes. Psychol. Rev. 111, 662-720. doi: 10.1037/0033-295X. 111.3.662

Hino, Y., and Lupker, S. J. (1996). Effects of polysemy in lexical decision and naming: an alternative to lexical access accounts. J. Exp. Psychol. 22, 1331-1356.

Hulme, C., and Snowling, M. J. (2013). Learning to read: what we know and what we need to understand better. Child Dev. Perspect. 7, 1-5. doi: 10.1111/cdep.12005

James, K. H., and Maouene, J. (2009). Auditory verb perception recruits motor systems in the developing brain: an fMRI investigation. Dev. Sci. 12, F26-F34. doi: 10.1111/j.1467-7687.2009.00919.x

James, K. H., and Swain, S. N. (2011). Only self-generated actions create sensori-motor systems in the developing brain. Dev. Sci. 14, 673-678. doi: 10.1111/j.1467-7687.2010.01011.x

MacWhinney, B. (2000). The Childes Project, 3rd Edn. Mahwah, NJ: Erlbaum.

Maouene, J., Hidaka, S., and Smith, L. B. (2008). Body parts and early-learned words. Cogn. Sci. 32, 1200-1216. doi: 10.1080/03640210802019997

McFalls, E. L., Schwanenflugel, P. J., and Stahl, S. A. (1996). Influence of word meaning on the acquisition of a reading vocabulary in second-grade children. Read. Writ. 8, 235-250. doi: 10.1007/BF00420277

Meteyard, L., Rodriguez Cuadrado, S., Bahrami, B., and Vigliocco, G. (2012). Coming of age: a review of embodiment and the neuroscience of semantics. Cortex 48, 788-804. doi: 10.1016/j.cortex.2010.11.002

\section{FUNDING}

This work was supported by Insight Grant \# 435-2013-0096 from the Social Sciences and Humanities Research Council (SSHRC) of Canada to PP.

\section{ACKNOWLEDGMENTS}

The authors thank Nicole Ansell and Morgan Teskey for assistance with data collection, and David Sidhu for assistance with data analysis.

\section{SUPPLEMENTARY MATERIAL}

The Supplementary Material for this article can be found online at: http://journal.frontiersin.org/article/10.3389/fpsyg. 2016.00317

Nation, K. (2009). Form-meaning links in the development of visual word recognition. Philos. Trans. R. Soc. B 364, 3665-3674. doi: 10.1098/rstb.2009.0119

Plaut, D. C., McClelland, J. L., Seidenberg, M. S., and Patterson, K. (1996). Understanding normal and impaired word reading: computational principles in quasi-regular domains. Psychol. Rev. 103, 56-115. doi: 10.1037/0033295X.103.1.56

Schneider, W., Eschman, A., and Zuccolotto, A. (2001). E-Prime user's Guide. Pittsburgh, PA: Psychology Software Tools.

Siakaluk, P. D., Pexman, P. M., Aguilera, L., Owen, W. J., and Sears, C. R. (2008a). Evidence for the activation of sensorimotor information during visual word recognition: the body-object interaction effect. Cognition 106, 433-443. doi: 10.1016/j.cognition.2006.12.011

Siakaluk, P. D., Pexman, P. M., Sears, C. R., Wilson, K., Locheed, K., and Owen, W. J. (2008b). The benefits of sensorimotor knowledge: body-object interaction facilitates semantic processing. Cogn. Sci. 32, 591-605. doi: 10.1080/03640210802035399

Strain, E., Patterson, K., and Seidenberg, M. S. (1995). Semantic effects in singleword naming. J. Exp. Psychol. 21, 1140-1154.

Tillotson, S. M., Siakaluk, P. D., and Pexman, P. M. (2008). Body-Object interaction ratings for 1,618 monosyllabic nouns. Behav. Res. Methods 40, 1075-1078. doi: 10.3758/BRM.40.4.1075

Tyler, L. K., Voice, K., and Moss, H. E. (2000). The interaction of meaning and sound in spoken word recognition. Psychon. Bull. Rev. 7, 320-326. doi: 10.3758/BF03212988

Warriner, A. B., Kuperman, V., and Brysbaert, M. (2013). Norms of valence, arousal, and dominance for 13,915 English lemmas. Behav. Res. Methods 45, 1191-1207. doi: 10.3758/s13428-012-0314-x

Wellsby, M., and Pexman, P. M. (2014a). The influence of bodily experience on children's language processing. Top. Cogn. Sci. 6, 425-441. doi: 10.1111 /tops. 12092

Wellsby, M., and Pexman, P. M. (2014b). Developing embodied cognition: insights from children's concepts and language processing. Front. Psychol. 5:506. doi: 10.3389/fpsyg.2014.00506

Woodcock, R. N. (1997). Woodcock Reading Mastery TestsRevised/Normative Update. Circle Pines, MN: American Guidance Service.

Wurm, L. H., Vakoch, D. A., and Seaman, S. R. (2004). Recognition of spoken words: semantic effects in lexical access. Lang. Speech 47, 175-204. doi: 10.1177/00238309040470020401

Yarkoni, T., Balota, D., and Yap, M. (2008). Moving beyond Coltheart's N: a new measure of orthographic similarity. Psychon. Bull. Rev. 15, 971-979. doi: 10.3758/PBR.15.5.971 
Zeno, S. M., Ivens, S. H., Millard, R. T., and Duvvuri, R. (1995). The Educator's Word Frequency Guide. Brewster, NY: Touchstone Applied Science Associates Inc.

Conflict of Interest Statement: The authors declare that the research was conducted in the absence of any commercial or financial relationships that could be construed as a potential conflict of interest.
Copyright (c) 2016 Inkster, Wellsby, Lloyd and Pexman. This is an open-access article distributed under the terms of the Creative Commons Attribution License (CC BY). The use, distribution or reproduction in other forums is permitted, provided the original author(s) or licensor are credited and that the original publication in this journal is cited, in accordance with accepted academic practice. No use, distribution or reproduction is permitted which does not comply with these terms. 
OPEN ACCESS

Edited by:

Annalisa Setti,

University College Cork, Ireland

Reviewed by:

Michael Ziessler

Liverpool Hope University, UK

Maj-Britt Isberner,

University of Kassel, Germany

*Correspondence:

Ashley M. Adams

amadam13@asu.edu

Specialty section:

This article was submitted to

Cognition,

a section of the journal

Frontiers in Psychology

Received: 27 May 2016 Accepted: 02 August 2016 Published: 17 August 2016

Citation:

Adams AM (2016) How Language Is Embodied in Bilinguals and Children with Specific Language Impairment.

Front. Psychol. 7:1209

doi: 10.3389/fpsyg.2016.01209

\section{How Language Is Embodied in Bilinguals and Children with Specific Language Impairment}

\author{
Ashley M. Adams * \\ Bilingual Language and Literacy Laboratory, Speech and Hearing Department, Arizona State University, Tempe, AZ, USA
}

This manuscript explores the role of embodied views of language comprehension and production in bilingualism and specific language impairment. Reconceptualizing popular models of bilingual language processing, the embodied theory is first extended to this area. Issues such as semantic grounding in a second language and potential differences between early and late acquisition of a second language are discussed. Predictions are made about how this theory informs novel ways of thinking about teaching a second language. Secondly, the comorbidity of speech, language, and motor impairments and how embodiment theory informs the discussion of the etiology of these impairments is examined. A hypothesis is presented suggesting that what is often referred to as specific language impairment may not be so specific due to widespread subclinical motor deficits in this population. Predictions are made about how weaknesses and instabilities in speech motor control, even at a subclinical level, may disrupt the neural network that connects acoustic input, articulatory motor plans, and semantics. Finally, I make predictions about how this information informs clinical practice for professionals such as speech language pathologists and occupational and physical therapists. These new hypotheses are placed within the larger framework of the body of work pertaining to semantic grounding, action-based language acquisition, and action-perception links that underlie language learning and conceptual grounding.

\footnotetext{
Keywords: embodied cognition, language disorders, bilingualism, motor cortex, action-based language, conceptual representation, clinical practice
}

The debate about whether human perception of the world can be divorced from motor, sensory, and emotional systems is not new. In fact, as far back as Aristotle, philosophers, and scientists were interested in this question (Barsalou, 1999). In the last several decades, the debate continues especially as it relates to speech perception. Some scientists demonstrate clear effects of the motor system on speech comprehension (e.g., Fadiga et al., 2002; Pulvermüller et al., 2003; Pickering and Garrod, 2006), while others argue against a causal role, demonstrating preservation of comprehension skills despite damage to the parts of the brain responsible for speech motor execution (Hickok, 2009; Rogalsky et al., 2011). There is also contention as to conceptual representation. Some argue for a system of abstract and amodal symbols (Newell and Simon, 1972; Pylyshyn, 1973; Fodor, 1975; Dennett, 1981; Haugeland, 1985) and others claim that conceptual representations are grounded in sensorimotor systems (Glenberg, 2011; Kiefer and Pulvermüller, 2012).

While embodied views of language comprehension have gained momentum in the last 20 years, in order to continue to advance the validity of the embodied approach, the theory should 
demonstrate explanatory power for other kinds of language phenomena such as bilingualism and speech language impairment. The current paper will review the literature on the role of the motor system in language comprehension, and make predictions about how this theory can be extended to bilingualism using popular models of bilingual language processing. Issues such as semantic grounding in a second language and potential differences between early and late acquisition of a second language will be discussed. Next, a review of the literature concerning the comorbidity of speech, language, and motor impairments will demonstrate how embodiment theory informs the discussion of the etiology of these impairments. Finally, based on this discussion, predictions will be made about novel ways to (1) teach a second language and (2) develop interventions for children with speech and language impairments.

\section{EMBODIED THEORIES OF LANGUAGE COMPREHENSION}

The major question that the embodied theory of language comprehension attempts to answer is how meaning is represented when understanding language. An understanding of the history of how the theory emerged is important. The late twentieth century gave rise to many amodal theories of language and reading comprehension (e.g., Kintsch's construction-integration model, 1988) that suggested that language comprehension consisted of manipulation of abstract symbols independent of motor and perceptual systems (Kintsch, 1988). Many of these theories were largely inspired by the invention of the computer, and thus promoted a computational view of the brain's role as a language processor. In fact, the advent of latent semantic analysis (LSA) has demonstrated that computational models do have an impressive ability to identify synonyms and engage in tasks (such as essay scoring) that are believed to depend on meaning, given large amounts of input. According to Kintsch and Mangalath (2011), "semantic models like LSA describe what is stored in long-term memory. Longterm semantic word memory is a decontextualized trace that summarizes all the experiences a person has had with that word. This trace is used to construct meaning in working memory" (p. 365). However, even researchers who work with LSA admit "Discourse comprehension requires not only knowledge of what words mean and how they can be combined, but world knowledge beyond the lexical level-knowledge about causal relations, about the physical and social world, which is not captured by our present techniques" (p. 366).

Herein lies the critical difference between amodal and embodied theories of language comprehension. How is it that we "experience" words and what does world knowledge actually consist of? Certainly there is an articulatory and acoustic experience of production and perception of speech, at least when speaking aloud. Pulvermüller et al. (2005) described action and perception links between articulation and acoustic perception based on the principle of Hebbian learning or "what fires together wires together" (Hebb, 1949; Tsumoto, 1992; Artola and Singer,
1993). Principles of Hebbian learning suggest that saying the correct form of the word (and necessarily also hearing it) while in the process of actually performing the action should strengthen the action-perception links that underlie language and ground concepts (Pulvermüller et al., 2006). As Pulvermüller et al. (2006) note, the fibers connecting the auditory and motor regions of the human brain are significantly more well-developed in humans than in apes, providing an evolutionary neurostructural basis for action-perception links. Research has demonstrated such links between activation of motor and sensory brain areas when listening to syllables and words (Zatorre et al., 1992; Pulvermüller et al., 2003; Wilson et al., 2004), and when producing speech (Paus et al., 1996; Watkins and Paus, 2004). This activation in motor areas occurs almost instantly after activation in auditory areas ( $\sim 20 \mathrm{~ms}$ ), precluding the alternate argument that activation simply spreads to motor areas after meaning has been processed (Pulvermüller et al., 2003).

Several groups of investigators have explored the causal role that motor systems play in speech perception using transcranial magnetic stimulation (TMS; for a review see Möttönen and Watkins, 2012). Watkins et al. (2003) showed increased motor evoked potentials in lip areas of left motor cortex, but not in hand or homologous right hemisphere lip areas, when watching a video of a person speaking (but not when watching random movements of the same person's face). Fadiga et al. (2002) demonstrated that, when listening to speech, motor evoked potentials in the tongue region were greater when words presented required tongue movement to be produced. This type of effect was shown to increase when the quality of the signal was degraded, either auditorily by embedding sound in noise or visually by increasing the speed of the speaker's articulator movements; these results suggest that motor areas may become increasingly important in situations in which the quality of the signal is less than ideal (Wilson, 2009; Murakami et al., 2011).

D'Ausilio et al. (2009) found that stimulating motor areas responsible for articulation of phonemes facilitated recognition of those phonemes. Schomers et al. (2014) found that differentially stimulating tongue and lip areas of the motor cortex delayed comprehension for words in which the articulator being stimulated was necessary for articulation (i.e., slowed comprehension of pool but not tool when the lip area was being stimulated). Taken together, this evidence supports the presence of speech mirror neurons; that is, neurons that respond similarly to both the production and comprehension of speech (Galantucci et al., 2006; Guenther et al., 2006). Therefore, there is a wealth of evidence suggesting that there is a causal role of articulatory motor systems in speech perception, especially under degraded conditions, and action-perception links based on Hebbian learning underlie this connection.

The extent to which words are grounded in articulatory and acoustic expression is only one level of grounding. We also experience concepts using our bodies and our senses, yet based on principles of Hebbian learning one would expect the representation of concepts to overlap with motor plans for the production of the word that represents that concept (Glenberg and Gallese, 2012). Take, for example, the word and concept "garlic." Part of the knowledge of that word is that, in order to 
produce it, you must begin with a voiced velar stop and end with an unvoiced velar stop. Part of the knowledge is what the word sounds like when it is produced. But, perhaps more importantly, part of that knowledge is what garlic tastes like, its pungent smell, and its shape and color. These characteristics of garlic are experienced via our sensory systems, and embodied theory of language comprehension argues that semantic meaning is stored in these same systems.

There is a large amount of evidence to support the claim that semantic information is stored in a distributed fashion in modality-specific sensory and motor areas. González et al. (2006) showed that reading odor-related words such as cinnamon and jasmine produced greater activation of olfactory cortex than control words. Similarly, words related to spatial language differentially activate left inferior parietal cortex (Pulvermüller and Fadiga, 2010), superior temporal cortex is differentially active for words related to sound (Kiefer and Pulvermüller, 2012), motor cortex is somatotopically activated when listening to action verbs (Hauk et al., 2004), and sentences describing motion differentially activate parts of the brain responsible for actual visual motion processing (Rueschemeyer et al., 2010). Glenberg et al. (2008) also showed that performing actions that required hand movement away from the body slowed comprehension of sentences describing both literal and abstract motion toward the body. Lesion studies also provide insight into semantic representation in the sense that damage to the above areas differentially affects the ability to process odor, spatial, and motor words respectively (Bak et al., 2001; Kemmerer, 2006; Boulenger et al., 2008; Kemmerer et al., 2012; Trumpp et al., 2013).

There is also evidence suggesting that emotion word meaning is related to the physical expression of those emotions. To investigate the role of the emotional system in language comprehension, Havas et al. (2010) asked adults to read sentences that contained material classified as happy or sad. During the reading of the sentences, muscle activity was measured. The authors hypothesized that the happy sentences would cause greater activation of the facial muscles responsible for smiling and sad sentences would cause greater activation of the facial muscles responsible for frowning. This is exactly what they found. Greater activity of the zygomaticus muscle was found during reading of the happy sentences and greater activity of the corrugator muscles was found during reading of the sad sentences. This suggests that the reading of sentences containing emotional content is directly linked to the parts of the motor system necessary to express that emotion. To follow this up, Havas et al. (2010) used Botox to temporarily paralyze the corrugator muscles (i.e., the muscles necessary to frown) while participants read sentences that described sad and happy situations. Paralyzing these muscles significantly slowed the reading of the sad sentences, while the reading of the happy sentences was unaffected. The fact that paralysis of the muscles necessary to outwardly express an emotion slowed a reader's comprehension of a sentence meant to evoke that emotion suggests that readers may be using their emotional systems during text comprehension.

Zwaan et al. (2002) investigated the activation of the visual perceptual system during sentence comprehension. They asked undergraduate students to read sentences describing either animals or objects differing in shape based on their location (i.e., the eagle is flying vs. the eagle is in its nest). Immediately following presentation of the sentences, they showed pictures to participants that either corresponded with or conflicted with the shape of the object described in the sentence (i.e., an eagle with its wings spread in flight or an eagle with its wings at its side) and asked if the object or animal was present in the sentence. They found that participants were significantly slower at identifying the objects or animals in the pictures when the shape conflicted with that described in the sentence. The slower reaction times in the conflicting condition suggest that the reader had activated their perceptual system during the comprehension of the written material and had to resolve the conflict before comprehension could occur.

Based on the above evidence, researchers in the embodied area have developed a simulation theory of language comprehension (Rizzolatti et al., 1996; Barsalou, 1999; Zwaan, 2004; Glenberg, 2007; Fischer and Zwaan, 2008; Kiefer and Pulvermüller, 2012). According to simulation theory, language comprehension is accomplished by the use of one's own motor, perceptual and emotional systems to simulate the situations described. Glenberg (2011) asserts that comprehension is closely related to bodily abilities and is revealed by the ability to take appropriate action in response to understanding of language or written material. For example, when one hears a sentence such as "He was doing somersaults down the hallway" one would activate the visual perceptual areas necessary to visualize a hallway and real motion, the motor areas necessary to accomplish the action of somersaulting, and the emotional systems that would respond to the unusual sight of a person attempting to somersault down a hallway.

Glenberg and colleagues developed the Indexical Hypothesis to apply simulation theory to language processing (Glenberg and Robertson, 2000; Kaschak and Glenberg, 2000; Glenberg and Gallese, 2012). The hypothesis includes (a) indexing the linguistic symbols (i.e., letters and words) to one's own experience using a perceptual symbol encoded in memory based on neural patterns generated during previous experience with the objects referenced in the text (Barsalou, 1999); (b) deriving affordances from the objects; (c) integrating the affordances based on the syntax (i.e., determining "who does what to whom?" in the sentence). According to Gibson (1977), an affordance is jointly determined by characteristics of physical objects and characteristics of the body. In brief, an affordance is what one can do with the object.

Glenberg and Gallese (2012) also developed the $A B L$ model of language acquisition based on Wolpert et al. (2003) HMOSAIC more general model of motor control. This model includes a complex set of controllers responsible for generating motor plans, and predictors that make predictions about the sensory consequences of such plans. Central to this model are mirror neuron mechanisms and canonical neurons.

Mirror neurons, first discovered in macaque monkeys by the Parma group in the late 1990's (Gallese et al., 1996; Rizzolatti et al., 1996) and later in humans (Mukamel et al., 2010) are neurons that respond similarly to the execution of an action and to watching that same action performed. Higher-level 
mirror neurons are theorized to encode the goal of the action (Rochat et al., 2010), while lower level mirror neurons encode the sequence of actions necessary to accomplish that goal. As previously discussed, mirror neurons have been implicated in the role of speech motor processes in language comprehension (i.e., covert activation of mirror mechanisms while listening to speech facilitates speech comprehension; Galantucci et al., 2006; Guenther et al., 2006). Canonical neurons fire in response to object affordances (Glenberg and Gallese, 2012); that is, they fire when completing an action such as grasping, but also when they simply see the object to be grasped. However, canonical neurons do not fire during action observation, differentiating them from mirror neurons.

Gallese et al. (1996) go on to describe how these mirror mechanisms in human Broca's area, which controls both the hands and the speech articulators, represents a form of neural exploitation of this area for action control and for speech. Rauscher et al. (1996) found that restricting hand movements during speech slowed lexical retrieval, further supporting the overlap of these two systems. As such, articulation of action words should prime the motor controllers necessary to take those actions. The reverse should also be true in that performing the action should prime the articulators to say the word that names the action.

Based on the framework of the mirror neuron system, the $A B L$ model of language acquisition makes predictions about how children acquire language. Simply put, when young children, at least in Western cultures, are learning language, their more capable language partners tend to label objects in their environment. As such, when children hear words referring to common objects, their speech mirror neurons are activated, and their canonical neurons are activated for the types of actions they can perform with the named object. With repeated exposures, Hebbian learning occurs such that acoustic perceptions, articulatory motor plans, and goal-related motor plans for interacting with the child's environment become linked (Glenberg and Gallese, 2012).

\section{EMBODIED THEORIES APPLIED TO BILINGUALISM}

Taking this evidence base for semantic grounding and theories of language comprehension and acquisition, we will now turn to how these concepts may be applied to bilingualism. But first, why is it important that embodied theory be applied to bilingualism? In most countries in the world, bilingualism is the norm. Some $56 \%$ of individuals in 25 European countries reported being bilingual (European Commission, 2006). In the U.S. alone, one of the countries with a lower percentage of bilingual citizens, there are 60.5 million bilinguals (Ryan, 2013), and these numbers are projected to continue to grow at a rapid pace over the next decade (see Shin and Ortman, 2011 for projected population by 2020). Bilingualism extends across all age groups in all levels of society (Grosjean and Li, 2013). However, there is a large amount of within-group variety among bilinguals, making it important to discuss what "bilingual" exactly means. Is someone who has had one class in a second language a bilingual? Does this term apply only to people who learn two languages from birth? Or before a certain "critical period" closes? These questions are important because second language proficiency varies greatly based on factors such as age of acquisition, patterns of language use, similarities among language pairs, language of education, and social standing of the languages in question (Grosjean and Li, 2013). Such factors will need to be taken into account when making predictions about how embodiment of concepts occurs for bilinguals.

Based on Pulvermüller's research on semantic grounding and Glenberg and Gallese's $A B L$ model, predictions can be made about how this process might occur in early simultaneous bilinguals. Children who acquire two languages from birth learn language in a very similar way to monolingual children. As they interact with their parents, objects are named for them in one or both of their languages depending on language practices in the home. If there are not cultural differences in appropriate ways to interact with an object, children may simply have an additional controller module that contains the speech motor commands for producing a word in two languages. Similarly, to the extent that there are not expected differences in sensorimotor experience of concepts (e.g., apple and manzana), the only additional component to be expected for semantic grounding is a link between the articulatory motor plan for a word in the second language and the meaning of that word as it is grounded in sensorimotor experience. However, children who are bicultural bilinguals may need separate conceptual representations for common objects or actions in their environment. Consider the concept of bread, for example. For a speaker of English in the United States, this would likely evoke the image of a loaf of sliced white or wheat bread that you buy in a grocery store. However, for a French-English bilingual/bicultural child, pain might evoke a different image, one of a baguette that you buy from the boulangerie. De Groot's (1992) distributed conceptual feature model suggests that bilinguals' conceptual representations may have overlapping and non-overlapping features as seen in Figure 1 below.

While De Groot conceptualized these semantic features as abstract and amodal, this same idea could be reconceptualized in terms of semantic grounding. To continue with the bread example, the English and French concept would differ in terms of visual and likely to an extent in gustatory information, also likely in some affordances (i.e., grabbing a piece of sliced bread vs. a baguette would require a different set of motor commands) but possibly not in olfactory information. Therefore, to the extent that sensorimotor information overlaps between the two concepts, one would expect the neural network responsible for semantic grounding to be the same. However, differences in sensorimotor experiences related to the two different concepts would result in neural networks that grounded the concepts in different sensorimotor information.

Jared et al. (2013) tested the hypothesis that there may be differential culture- and language-specific activation of concepts and found that participants were faster to identify culturally biased items (e.g., a traditional Chinese mask) when they read the word mask in Chinese rather than in English. From the 


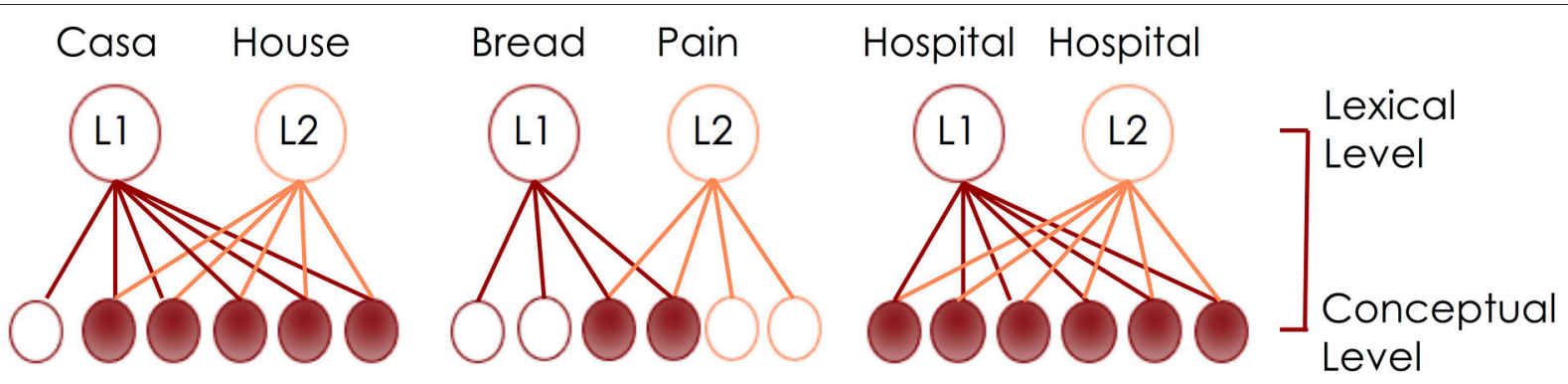

FIGURE 1 | Adaptation of De Groot's (1992) distributed conceptual feature model.

perspective of simulation theory, this advantage in reaction time occurred because hearing the word mask in Chinese activated a specific perceptual memory that was specific to the participant's experience with a mask in their Chinese language environment. The background knowledge that was activated is not composed of arbitrary linguistic information, but instead includes activation of the motor, sensory, and perceptual system. If two languages are learned in different environments at different times, there may be differences in the knowledge that is activated depending on the language of input.

Making these kinds of predictions becomes more complicated when a language learner has acquired a second language later in life, especially if the primary modality in which that language was learned was in a classroom. In this case, a new hypothesis generated from the review of literature concerning embodied language is that late second language learning may be critically different from first language learning in the sense that it may be less likely that vocabulary acquisition happens alongside actual sensorimotor experience (Dudschig et al., 2014). In the case that the learner has a grounded conceptual representation already in place, he or she may simply link the new phonological representation to the previously grounded concept. Kroll and Stewart's (1994) revised hierarchical model, seen below in Figure 2, makes just such a prediction.

When a second language is still emerging, according to this model, the first language mediates L2 access to the conceptual store. As L2 proficiency develops, necessarily through experience with the language, a semantic link begins to strengthen between L2 and the "conceptual store," such that eventually first language mediation may not be necessary if a high enough level of proficiency is achieved. Or, to make a new prediction, one might hypothesize that as new sensorimotor experiences take place in the second language environment, the grounded conceptual representation may gradually shift to incorporate these new experiences. One could conceptualize the arrows in the figure above as neural networks of sensorimotor information that ground concepts. Certainly this is a testable hypothesis and by giving a novice second language learner new experiences with an object, action, or feeling, it should be possible to measure differential activation as it relates to the quantity and quality of sensorimotor experience.

In the case that the learner has no concept in place to attach to a new lexical item, an entirely new, grounded representation must

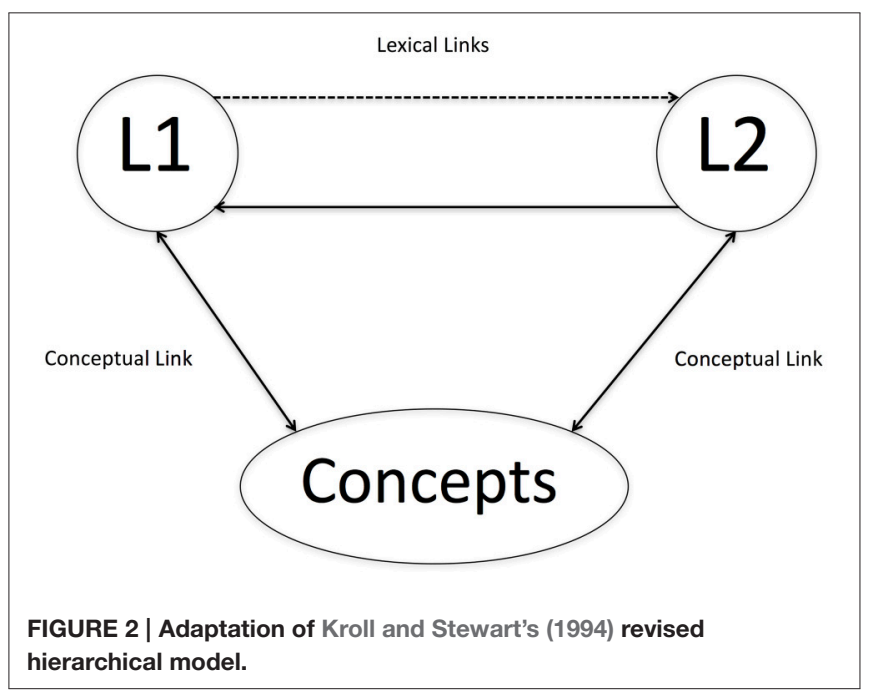

be created. Take for example the distinction of querer and amar in Spanish; the former refers to non-romantic love and the latter to romantic love. This distinction does not exist in English, although certainly the emotions that platonic and romantic loves generate are different. Therefore, the second language learner of Spanish may gradually ground the word amar in feelings of romantic love and the word querer in the feelings of familial love, given enough experience with the language.

An alternative framework in which to consider bilingual language processing within an embodied framework is the Bilingual Interactive Activation Plus Theory (BIA+, Dijkstra and van Heuven, 2002). According to this model, representations are stored in an integrated way, regardless of the language to which they belong. However, there are different resting-level activations connecting lexical items with the semantic system, based on proficiency and word frequency. Therefore, a bilingual who is less proficient in their L2, for example, would require greater activation for the neural network responsible for a given concept to "fire." Words that are less frequent or more unfamiliar to the speaker would have resting-level activations that were more negative, and would therefore require greater activation. One could apply knowledge of both embodied language theories and the BIA+ model to extend current knowledge and conceptualize that embodied neural networks of sensorimotor experience may 
have differential resting-level activations that link the speech motor, acoustic, and semantic information for a given word according to factors such as proficiency, word frequency, and amount of sensorimotor experience with a given object, action, emotion, etc.

In the last 5 years, investigators have started to explore how embodied/simulation processes occur in both languages of a bilingual. Vukovic and Williams (2014) had 24 highly proficient Dutch-English speakers listen to sentences that implied distance (e.g., the microphone across the room vs. the microphone in your hand) in their L2 (English). Critically, target sentences contained an interlingual homophone (a word that sounds the same but has different meanings in the two languages) to test whether simulation of distance occurred in both languages simultaneously. Participants heard a sentence like "on the plate in front of you is a bone." Therefore, participants should be faster to identify a large or close-up picture of a bone than a smaller picture (Winter and Bergen, 2012). However, bone in Dutch means beans. Vukovic and Williams set out to determine if, after hearing the sentence above, participants would be slower to reject a large close-up picture of beans rather than a smaller picture. This would only be true if simulation of distance was taking place in both languages simultaneously. Indeed they found just this effect. The results of this study coincide with previous work suggesting simultaneous activation of both languages in bilingual language processing (Lagrou et al., 2011), and also speak to the automatic process of simulation, even when meaning is only subconsciously processed.

Another study with highly proficient German-Dutch bilinguals used fMRI to study L2 embodiment of action verbs and demonstrated increased activation of the motor and somatosensory regions when listening to simple verbs in their L2 (De Grauwe et al., 2014). Some of the verbs presented were cognate words (similar phonologically and orthographically to German verbs with the same meaning) and the authors found no significant interaction between cognate status and motor activation, suggesting that embodiment of action verbs in L2 is not mediated by first language embodied concepts, at least for highly proficient bilinguals.

The proficiency in the second language may play an important role in how concepts are embodied. In another study with later (and presumably less proficient) second language learners, Dudschig et al. (2014) presented German-English bilinguals with variations of the Stroop task to test for second language (L2) simulation effects. In the first experiment, participants were presented with words that implied a typical location (e.g., bird, shoe, airplane, root) in English, their L2. These words were presented on a screen and were written in one of two colors. Participants were asked to respond to one color with a movement that required hand movement up in space and to the other color with a movement requiring downward motion. The participants were instructed to ignore the meaning of the words. Results showed a significant word-direction and response-direction interaction, such that participants were faster to correctly identify the color by moving their hand upwards when the location implied by the word they read in their L2 was also upwards. Interestingly, similar results were found using emotionally charged words based on previous work by Meier and Robinson (2004) that suggested that positivevalence emotion words are identified faster when they are presented in the upper half vs. the lower half of the screen, and vice versa for negative-valence words. Dudschig et al. (2014) corroborated these results by again finding a worddirection and response-direction interaction for emotion words. These results suggest that semantic grounding in sensorimotor experience occurs in similar ways for both the first and second languages, even when the second language is acquired later in life.

However, another study investigated further the role of proficiency in embodiment of a second language. Bergen et al. (2010) showed adult second language learners two pictures, one of a written verb (e.g., run) and then a drawing of a person doing an action that was either accomplished with the same effector as the verb seen (e.g., kick) or a different effector (e.g., punch). Participants were slower to reject drawings that showed actions accomplished with the same effector implicated by the verb they read, which the authors theorized was due to interference of simulation of the original action. Importantly, accuracy of performance on the task, a proxy for language proficiency, was related to this interference effect; that is, more proficient second language learners had a greater "native-like" interference effect. However, a test of receptive vocabulary was not significantly related to the effect, indicating a complex interplay between language proficiency and the extent to which language can be embodied. Harris et al. (2003) demonstrated that, for late second language learners, taboo and negative words elicited higher levels of skin conductance in the first language relative to the second language, suggesting that embodiment effects may differ between languages of varying proficiency. More work is necessary to determine the extent to which concepts are embodied in more and less proficient speakers of a second language.

A consideration of the totality of this evidence allows for predictions about effective second language teaching and further development of the current theory of embodiment. In very simple terms, second language learning, much like first language learning, should take place in authentic environments and should involve discussion of the immediate perceptual environment, accompanied by interaction with that environment. According to Macedonia (2014), "embodiment is giving language education a cutting edge by authorizing it to consider the body as a learning tool" (p. 4). Several studies have demonstrated that gestures can improve memory for new words and phrases in a second language (Macedonia and Knösche, 2011; Bergmann and Macedonia, 2013; Porter, 2016). Mayer et al. (2015) provided neurological evidence of this improvement. They taught adults foreign language words and their translations and either had participants perform a gesture related to each word, trace a picture related to each word, or simply learn the word verbally (much like what happens in the classroom). They found that the group that received the training with selfperformed gestures recalled more words correctly than the group that learned in the verbal only modality; this behavioral finding was directly related with the amount of activity in 
the left motor cortex and the right biological motion-sensitive superior temporal sulcus (Mayer et al., 2015). The results of this study not only provide strong direct evidence for semantic grounding of words in a second language, but also speak to potential strategies that may be effective for second language teaching.

Atkinson (2010) advocates for a sociocognitive approach to second language teaching that encourages the use of gestures, body positioning, and what he terms "alignment" to improve memory for new words and concepts. A study that followed university students as they learned a second language found that, in contrast to classroom learning, learners' bodies demonstrated a higher level of engagement during a conversational exchange in the second language (Tanous, 2014). This demonstrates that when communication partners are exchanging meaningful information (but not necessarily when they are being taught in a classroom environment) their bodies reflect this language learning experience. As such, teaching of a second language may be reconsidered as guided discovery of the world in a new language environment instead of conveying abstract knowledge in a lecture format (Atkinson, 2010).

In summary, there is a wealth of evidence demonstrating the role of the motor system in language comprehension. The discovery of mirror neurons in human cortex has revived the discussion of the causal role motor neurons play in language processing. Relatedly, sensorimotor experience of the world leads to grounding of semantic information using the brain's sensory, motor, and emotional systems. Simulation theory suggests that concepts are represented using these same systems, as opposed to in some abstract, amodal conceptual store. Emerging research demonstrates that embodiment processes occur in a similar way for highly proficient bilinguals, but may differ in some ways for less proficient second language learners. Several popular models of bilingual language processing allow for predictions about how and why these differences occur. Future research that explores the connections between a well-defined construct of language proficiency and embodiment of concepts in a second language will help to illuminate this issue. Finally, the principles of embodied cognition and simulation theory provide exciting insights into new ways to teach learners a second language. A randomized controlled experiment that explores using the "guided discovery of the environment" method of second language teaching vs. traditional classroom teaching would be one interesting way to test the hypothesis of how embodiment theory applies to second language learning.

\section{EMBODIED THEORIES APPLIED TO SPECIFIC LANGUAGE IMPAIRMENT (SLI)}

Specific language impairments affect $\sim 7-10 \%$ of the population (Tomblin et al., 1997). According to the National Institute on Deafness and Communication Disorders, specific language impairment is "a language disorder that delays the mastery of language skills in children who have no hearing loss or other developmental delays." Decades of research have established that the primary difficulties for children with SLI are in phonological processing (Weismer et al., 2000; Botting and Conti-Ramsden, 2001; Graf-Estes et al., 2007), morphology (Rice and Wexler, 1996; Ullman and Gopnik, 1999), and syntax (Thordardottir and Weismer, 2002; Riches et al., 2010). It is important to note that the focus of this section is not on childhood motor speech disorders such as apraxia of speech, speech sound disorders, or articulation disorders. These subgroups of speech disorders are clearly linked to errors in motor programming and sometimes occur comorbidly with language impairment, but for the scope of the current discussion, the focus will remain on specific language impairment (also popularly referred to as primary language impairment-PLI). This section will extend the previous discussion of language being represented in motor, sensory and emotional areas of the brain to the interesting case of children with SLI who represent what happens when something goes wrong in language development. Perhaps this introduction is a bit misleading since I will spend a good portion of this section suggesting that what is frequently called specific language impairment may not be so specific to the language system after all.

An overview of how cognitive, linguistic, and motor skills develop together in early years and their important crossdomain relationships will set the stage for the discussion of later problems with language impairment. For instance, Angrave and Glenberg (2007) found a linear relationship between the ability to accomplish an action and the ability to say the word related to that action (e.g., drink), with the speech emerging about a year after the action emerged. Thal and Tobias (1992) found that pointing behaviors at 9 months of age were positively correlated with receptive vocabulary skills. Similarly, Acredelo and Goodwyn (1988) found that the rate of expressive vocabulary acquisition is directly related with the earlier ability to label objects using gestures. Standardized language scores at 22 months of age are also predicted by the ability to demonstrate appropriate and functional use of toys during play at 13 months (Ungerer and Sigman, 1984). These studies provide evidence that fits in well with the type of language acquisition model proposed by Glenberg and Gallese (2012) that emphasizes the importance of action-based language.

The relationship between motor and linguistic development is not unidirectional. A series of studies by Green et al. $(2000,2002)$ showed that important changes in lip and jaw movements occur at $\sim 2$ years of age, at the same time as the famed "vocabulary growth spurt" occurs. They found that the production of bilabials changed from being a primarily jawdriven motor act to involving both the jaw and the lips (Green et al., 2000). The authors propose that these changes in motor development are brought about due to increasing demands of the linguistic and cognitive systems. Even in adults, when speech motor stability is measured, researchers have found that stability is directly related to the syntactic complexity of the stimuli (Walsh and Smith, 2002; Kleinow and Smith, 2006), that stability increases with age (Goffman and Smith, 1999; Walsh and Smith, 2002), and that second language learners' speech motor stability increases with second language proficiency (Nip and Blumenfeld, 2015). Finally, Nip et al. (2011) found that speed 
of jaw opening and jaw range of motion were both significantly and positively correlated with the perception and concepts subtest of the Batelle Developmental Inventories, Second Edition (BDI-2, Newborg, 2005) which assesses an infant's active sensorimotor interactions with the immediate environment. As would be predicted by embodiment theory, research in this area demonstrates tight links between speech motor and language abilities (perhaps because these are theorized to be two parts of the same neural network) across the developmental continuum.

Based on the preceding evidence, one might predict that children who have later language impairment would also demonstrate motor deficits. In 2001, Hill published a metaanalysis of previous work done with children with SLI and concluded that this impairment is frequently associated with deficits in both fine and gross motor skills, so much so that it should be considered the rule rather than the exception. While her review included children with a variety of speech and language disorders, only those that involved children with SLI will be reviewed here.

Several studies found that children with SLI demonstrated fine motor deficits using a task that required them to move small pegs from one location to another in a timed fashion (Bishop and Edmundson, 1987; Powell and Bishop, 1992). Children with SLI moved fewer pegs in the time allotted compared to a control group. Children with SLI have also been shown to demonstrate relative difficulty with a finger opposition task, which requires children to touch each of their fingers to their thumb as fast as they can while both accuracy and time are tracked (Johnston et al., 1981; Stark and Tallal, 1981; Katz et al., 1992). Children with SLI were both slower and less accurate at completing this task. Repetitive tapping and threading beads onto a string are other areas in which children with SLI have been shown to be slower than matched controls (Hughes and Sussman, 1983; Powell and Bishop, 1992). In terms of gross motor control, children with SLI primarily demonstrate balance issues as demonstrated by their diminished ability to hop or stand on one foot (Johnston et al., 1981; Stark and Tallal, 1981; Powell and Bishop, 1992).

An alternative methodology that researchers have undertaken to explore the comorbidity of SLI and motor deficits is by comparing children with SLI to children with developmental coordination disorder (DCD). DCD is typically diagnosed using a standardized test of motor abilities, such as the Movement Assessment Battery for Children (Movement ABC, Henderson and Sugden, 1992), and the diagnosis is given to children who fall below the 15th percentile compared to age-matched peers (Hill, 2001). Studies that previously used the Movement ABC found that between 60 and $90 \%$ of children with SLI performed below the 15th percentile on this test (Robinson, 1991; Hill, 1998; Hill et al., 1998; Rintala et al., 1998; but note that the Robinson study involved children with concomitant speech and language disorders). Therefore, the majority of children in these studies qualified for a diagnosis of DCD, suggesting that there is a large overlap between deficits in language and motor abilities.

Hill (1997) compared a group of children with SLI to a group of children with DCD, a group of age-matched controls, and a group of younger controls on their performance on the finger opposition task and the repetitive tapping task. She found that children with SLI's performance on the finger opposition task was statistically different from age-matched controls but was similar to the younger control group and the group with DCD. However, on the repetitive tapping task, no group differences were found (in contrast to Hughes and Sussman, 1983). Powell and Bishop (1992) also compared children with SLI, DCD, and matched controls and found that children with SLI performed similarly to children with DCD on all motor tasks and tasks of visual discrimination, but not on tasks of visuo-spatial processing (SLI > DCD on these subtests). These findings led Hill (2001) to conjecture that co-occurring language and motor deficits may be indicative of a global neurodevelopmental delay or that there may be an "anatomical contiguity of the neural substrates subserving language and motor functions" (p. 165). It is exactly this second hypothesis that embodied theories of language would predict to be correct.

Several more recent studies have continued to investigate this intriguing relationship between language and motor skills. Archibald and Alloway (2008) investigated this relationship from the opposite viewpoint by looking at the language abilities of children diagnosed with DCD and demonstrated that these children, on average, exhibit expressive language difficulties, providing further evidence for overlap between the two skill sets. Flapper and Schoemaker (2013) and Finlay and McPhillips (2013) both demonstrated that $\sim 30 \%$ of children with SLI qualify for a diagnosis of DCD (note that this number is lower than earlier studies reported by Hill, which may be due to a stricter definition of SLI in both of the above studies compared to Hill's earlier studies). Vukovic et al. (2010) further explored how leg coordination, arm coordination and ability to imitate arm and hand gestures related to expressive vocabulary, receptive vocabulary, and articulation. Besides the (now somewhat repetitive) finding that children with SLI performed significantly worse than control children on all tests of motor abilities/coordination, the authors found that imitation of complex arm and hand movements predicted expressive vocabulary in both typically developing and SLI groups while imitation of simple arm and hand movements predicted receptive vocabulary only in the SLI group. Additionally, coordination of arms was highly predictive of expressive and receptive vocabulary in the control group. Zelaznik and Goffman (2010) also report generally poorer performance of children with SLI on motor tasks, but note that, on tasks that required timing precision, there were no group differences, perhaps suggesting a relative strength in this area.

Although articulation and speech sound disorders do not fall under the umbrella of specific language impairment, research has demonstrated that children with SLI still demonstrate subclinical deficits in this area. Findings in this area included that speech rate was overall slower for children with SLI (Andrade et al., 2014) and that articulatory movements were less stable in children with SLI. Higher articulatory variability was correlated with poorer performance on fine motor skills (performed with the hands; DiDonato Brumbach and Goffman, 2014). Additionally, a recent study used gummy bears as bite blocks and had children with SLI and a control group repeat nonwords. They found that 
the bite block was more disruptive for children with SLI when they produced 2 or more syllable-words and theorized that this may be related to difficulties with motor planning. Interestingly, SLI is 1.5-3 times more likely to occur in boys than girls (Tomblin et al., 1997; Broomfield and Dodd, 2004) and Smith and Zelaznik (2004) found that boys (until age 5 years) show a slower maturational course of speech motor development. Evidence that has accumulated over the past two decades continues to point to pervasive deficits in motor skills among children with SLI.

One area of motor ability that may be particularly relevant to theories of embodied cognition is that of hand gestures, since Broca's area is responsible for both articulation and hand movement, and authors have implicated neural exploitation of this area for both speech and action control. Based on this theory and the previous evidence presented here, one would predict that children with SLI would exhibit difficulties in producing communicative gestures. Hill reviewed a group of studies concerning children with SLI and their ability to perform transitive gestures (e.g., show me how you brush your teeth) and intransitive gestures (e.g., show me how you blow a kiss) as well as their ability to imitate novel hand shapes. The overwhelming message of these studies was that children with SLI had difficulty with production and imitation of both types of meaningful gestures and with imitation of novel hand shapes (Dewey et al., 1988; Hill, 1998; Hill et al., 1998).

However, more recent studies call into question these previous findings. Botting et al. (2010) found no differences in the ability of children with SLI to produce representational gestures. In fact, Iverson and Braddock (2011) found that children with SLI were more likely to produce gestures and that the frequency of gestures was negatively correlated with language abilities (i.e., the worse the language skills, the more gestures produced). Evans et al. (2001) found that children with SLI were more likely to use gestures to convey information complementary to the information conveyed in speech, which the authors suggest may be an attempt to externalize some of the processing load to reduce demands on a less stable system of phonological representation. Both sets of studies (the earlier studies reviewed by Hill and the more recent studies reported here) involved children of similar ages, suggesting that differences in task difficulty and individual differences may be the reason such different results emerged. More research into the relationship between motor, linguistic, and gesture abilities are necessary to fully elucidate how these abilities are related, especially since researchers have demonstrated that gesture may help children ground knowledge in sensorimotor experience (Beilock and Goldin-Meadow, 2010).

Based on this evidence that children with SLI exhibit both fine and gross motor subclinical deficits, combined with the evidence from researchers studying the embodied nature of language, informed hypotheses can be formulated to extend the current theoretical approach and describe the difficulties that children with SLI have. If there are weaknesses and instabilities in speech motor control, even at a subclinical level, in children with SLI, then the neural network that connects acoustic input, articulatory motor plans and semantics may be disrupted. Many investigators have theorized that deficits in phonological working memory or instability of phonological representations are at the core of the difficulties that children with SLI experience (Montgomery, 1995; Adams, 1996; Weismer et al., 1999; Evans et al., 2001). However, as embodied theories would predict, at least some part of phonological representation is represented in motor cortex. Therefore, overlapping deficits could be conceptualized to be due to overlapping responsibilities of the motor cortex in both action control and language processing. In fact, researchers have found that the pars triangularis (one part of Broca's area) is significantly smaller in children with SLI and the severity of the language impairment was correlated with the degree of this abnormality (Gauger et al., 1997). This finding provides neurostructural evidence for the critical role of motor control in language pathologies.

According to Glenberg and Gallese's (2012) ABL (Actionbased language) model, based on the HMOSAIC account of motor control, performing an action should prime articulation of the word that labels that action (and vice versa); so one testable hypothesis that may be generated from this theory is whether a child who has difficulty performing an action also has a weaker or nonexistent prime of the related word or sentence. Similarly, if mirror neurons respond similarly when performing an action as when observing an action, it follows that children who have difficulty performing actions may also have difficulty in perceiving them. Such perceptual deficits may lead to further difficulties in acquiring new vocabulary since children may have difficulty learning appropriate ways to interact with their environment (affordances), and therefore have slowed or interrupted development of modules for language and action control. Finally, if language comprehension occurs through simulation of the actions, perceptions, and emotions described, someone who has motor deficits may specifically struggle to understand action verbs, which is a hallmark of SLI, at least in English (Conti-Ramsden and Jones, 1997).

At the very least, the results and theories reported here suggest that motor abilities should be assessed in the process of diagnosis of children with SLI (and language abilities should be assessed when diagnosing DCD). However, there is also the possibility that novel interventions may be developed based on the principles of embodied cognition. Developing language interventions that emphasize that the goal of understanding language is to guide appropriate action may be particularly beneficial and may further the theoretical approach. Therefore, embedding language intervention into activities that require the child to follow a direction and perform the actions required by the direction may prove to have positive results. Activities that require children to get their bodies involved in learning are also recommended (McClelland et al., 2015). Previous research has demonstrated that embodied learning can improve outcomes in areas that range from narrative text comprehension to mathematical equivalence to physics concepts and organic chemistry (Goldin-Meadow et al., 2009; Glenberg, 2011).

Similarly to what was argued about effective second language teaching, interventions designed for children with SLI should require children to interact with their immediate environment. For example, if the activity were to focus on proper use of verbs with the -ing morpheme, children would be asked to perform actions and then describe those actions as they perform 
them. Principles of Hebbian learning allow for development of testable hypotheses such as the idea that saying the correct form of the word (and necessarily also hearing it) while in the process of actually performing the action should strengthen the action-perception links that underlie language and ground concepts. The implications extend beyond speech and language therapy. It may also be possible that more children who are diagnosed with SLI should be referred for services with physical and occupational therapists so as to support simultaneous development of their motor and language skills. However, it should be noted that these systems (motor, perceptual, and linguistic) are not considered independent systems that should be focused on separately; instead it is recommended that speech therapists include action-based activities in their therapy activities and that physical and occupational therapists include language activities in their therapy activities so as to support

\section{REFERENCES}

Acredelo, L., and Goodwyn, S. (1988). Symbolic gesturing in normal infants. Child Dev. 59, 450-466. doi: 10.2307/1130324

Adams, A. M. (1996). Phonological working memory and spoken language development in young children. Q. J. Exp. Psychol. A 49, 216-233. doi: $10.1080 / 713755610$

Andrade, C. R. F. D., Befi-Lopes, D. M., Juste, F. S., Cáceres-Assenço, A. M., and Fortunato-Tavares, T. M. (2014). Aspects of speech fluency in children with specific language impairment. Audiol. Commun. Res. 19, 252-257. doi: 10.1590/S2317-64312014000300008

Angrave, L. C., and Glenberg, A. M. (2007). "Infant gestures predict verb production one year later," in Paper Presented at the Annual Meeting of the American Psychological Association (San Francisco, CA).

Archibald, L. M., and Alloway, T. P. (2008). Comparing language profiles: children with specific language impairment and developmental coordination disorder. Int. J. Lang. Commun. Disord. 43, 165-180. doi: 10.1080/1368282070 1422809

Artola, A., and Singer, W. (1993). Long-term depression of excitatory synaptic transmission and its relationship to long-term potentiation. Trends Neurosci. 16, 480-487. doi: 10.1016/0166-2236(93)90081-V

Atkinson, D. (2010). Extended, embodied cognition and second language acquisition. Appl. Linguist. 31, 599-622. doi: 10.1093/applin/amq009

Bak, T. H., O’Donovan, D. G., Xuereb, J. H., Boniface, S., and Hodges, J. R. (2001). Selective impairment of verb processing associated with pathological changes in Brodmann areas 44 and 45 in the motor neurone disease-dementia-aphasia syndrome. Brain 124, 103-120. doi: 10.1093/brain/124.1.103

Barsalou, L. W. (1999). Perceptual symbol systems. Behav. Brain Sci. 22, 577-660. doi: $10.1017 / \mathrm{s} 0140525 \times 99002149$

Beilock, S. L., and Goldin-Meadow, S. (2010). Gesture changes thought by grounding it in action. Psychol. Sci. 21, 1605-1610. doi: 10.1177/0956797610 385353

Bergen, B., Lau, T. T. C., Narayan, S., Stojanovic, D., and Wheeler, K. (2010). Body part representations in verbal semantics. Mem. Cognit. 38, 969-981. doi: 10.3758/MC.38.7.969

Bergmann, K., and Macedonia, M. (2013). “A virtual agent as vocabulary trainer: iconic gestures help to improve learners' memory performance," in Intelligent Virtual Agents, Lecture Notes in Artificial Intelligence eds R. Aylett, B. Krenn, C. Pelachaud, and H. Shimodair (Berlin: Springer), 139-148.

Bishop, D. V. M., and Edmundson, A. (1987). Specific language impairment as a maturational lag: evidence from longitudinal data on language and motor development. Dev. Med. Child Neurol. 29, 442-459. doi: 10.1111/j.14698749.1987.tb02504.x

Botting, N., and Conti-Ramsden, G. (2001). Non-word repetition and language development in children with specific language impairment (SLI). Int. J. Lang. Commun. Disord. 36, 421-432. doi: 10.1080/13682820110074971 development of neural networks that use motor, perceptual, and linguistic capacities.

\section{AUTHOR CONTRIBUTIONS}

The author confirms being the sole contributor of this work and approved it for publication.

\section{FUNDING}

The author is a doctoral student funded by NSF grant \#1324807 titled EMBRACEing English Language Learners with Technology that focuses on using the theory of embodied cognition to develop educational technology to improve reading comprehension in elementary school children who are learning English as a second language.

Botting, N., Riches, N., Gaynor, M., and Morgan, G. (2010). Gesture production and comprehension in children with specific language impairment. Br. J. Dev. Psychol. 28, 51-69. doi: 10.1348/026151009X482642

Boulenger, V., Mechtouff, L., Thobois, S., Broussolle, E., Jeannerod, M., and Nazir, T. A. (2008). Word processing in Parkinson's disease is impaired for action verbs but not for concrete nouns. Neuropsychologia 46, 743-756. doi: 10.1016/j.neuropsychologia.2007.10.007

Broomfield, J., and Dodd, B. (2004). Children with speech and language disability: caseload characteristics. Int. J. Lang. Commun. Disord. 39, 303-324. doi: $10.1080 / 13682820310001625589$

Conti-Ramsden, G., and Jones, M. (1997). Verb use in specific language imp airment. J. Speech Lang. Hear. Res. 40, 1298-1313. doi: 10.1044/jslhr.400 6.1298

D’Ausilio, A., Pulvermüller, F., Salmas, P., Bufalari, I., Begliomini, C., and Fadiga, L. (2009). The motor somatotopy of speech perception. Curr. Biol. 19, 381-385. doi: 10.1016/j.cub.2009.01.017

De Grauwe, S., Willems, R. M., Rueschemeyer, S. A., Lemhöfer, K., and Schriefers, H. (2014). Embodied language in first- and second-language speakers: neural correlates of processing motor verbs. Neuropsychologia 56, 334-349. doi: 10.1016/j.neuropsychologia.2014.02.003

De Groot, A. M. (1992). Bilingual lexical representation: a closer look at conceptual representations. Adv. Psychol. 94, 389-412.

Dennett, D. (1981). “The nature of images and the introspective trap," in Imagery, ed N. Block (Cambridge, MA: MIT Press), 51-61.

Dewey, D., Roy, E. A., Square-Storer, P. A., and Hayden, D. C. (1988). Limb and oral praxic abilities of children with verbal sequencing deficits. Dev. Med. Child Neurol. 30, 743-751. doi: 10.1111/j.1469-8749.1988.tb14636.x

DiDonato Brumbach, A. C., and Goffman, L. (2014). Interaction of language processing and motor skill in children with specific language impairment. J. Speech Lang. Hear. Res. 57, 158-171. doi: 10.1044/1092-4388 (2013/12-0215)

Dijkstra, T., and van Heuven, W. J. (2002). Modeling bilingual word recognition: past, present and future. Bilingualism 5, 219-224. doi: 10.1017/s136672890 2283017

Dudschig, C., De La Vega, I., and Kaup, B. (2014). Embodiment and secondlanguage: automatic activation of motor responses during processing spatially associated L2 words and emotion L2 words in a vertical Stroop paradigm. Brain Lang. 132, 14-21. doi: 10.1016/j.bandl.2014.02.002

European Commission (2006). Europeans and their Languages, Special Eurobarometer 243-Wave 64.3. Available online at: http://pol.gu. se/digitalAssets/759/759844_Europeans_and_their_Languages_-_EC_2006.pdf (Accessed 10 Jan 2016).

Evans, J. L., Alibali, M. W., and McNeil, N. M. (2001). Divergence of verbal expression and embodied knowledge: evidence from speech and gesture in children with specific language impairment. Lang. Cogn. Process. 16, 309-331. doi: 10.1080/01690960042000049 
Fadiga, L., Craighero, L., Buccino, G., and Rizzolatti, G. (2002). Speech listening specifically modulates the excitability of tongue muscles: a TMS study. Eur. J. Neurosci. 15, 399-402. doi: 10.1046/j.0953-816x.2001.01874.x

Finlay, J. C., and McPhillips, M. (2013). Comorbid motor deficits in a clinical sample of children with specific language impairment. Res. Dev. Disabil. 34, 2533-2542. doi: 10.1016/j.ridd.2013.05.015

Fischer, M. H., and Zwaan, R. A. (2008). Embodied language: a review of the role of the motor system in language comprehension. Q. J. Exp. Psychol. 61, 825-850. doi: $10.1080 / 17470210701623605$

Flapper, B. C., and Schoemaker, M. M. (2013). Developmental coordination disorder in children with specific language impairment: co-morbidity and impact on quality of life. Res. Dev. Disabil. 34, 756-763. doi: 10.1016/j.ridd.2012.10.014

Fodor, J. A. (1975). The Language of Thought. Cambridge: Harvard University Press.

Galantucci, B., Fowler, C. A., and Turvey, M. T. (2006). The motor theory of speech perception reviewed. Psychon. Bull. Rev. 13, 361-377. doi: 10.3758/BF03193857

Gallese, V., Fadiga, L., Fogassi, L., and Rizzolatti, G. (1996). Action recognition in the premotor cortex. Brain 119, 593-609. doi: 10.1093/brain/119.2.593

Gauger, L. M., Lombardino, L. J., and Leonard, C. M. (1997). Brain morphology in children with specific language impairment. J. Speech Lang. Hear. Res. 40, 1272-1284. doi: 10.1044/jslhr.4006.1272

Gibson, J. J. (1977). "The theory of affordances," in Perceiving, Acting, and Knowing: Toward an Ecological Psychology, eds R. Shaw and J. Bransford (Hillsdale, NJ: Erlbaum), 67-82.

Glenberg, A. (2011). How reading comprehension is embodied and why that matters. Int. Electron. J. Elemen. Educ. 4, 5-18. doi: 10.1016/j.tics.2009.06.001

Glenberg, A. M. (2007). "Language and action: creating sensible combinations of ideas," in The Oxford Handbook of Psycholinguistics, ed G. Gaskell (Oxford: Oxford University Press), 361-370.

Glenberg, A. M., and Gallese, V. (2012). Action-based language: a theory of language acquisition, comprehension, and production. Cortex 48, 905-922. doi: 10.1016/j.cortex.2011.04.010

Glenberg, A. M., and Robertson, D. A. (2000). Symbol grounding and meaning: a comparison of high-dimensional and embodied theories of meaning. J. Mem. Lang. 43, 379-401. doi: 10.1006/jmla.2000.2714

Glenberg, A. M., Sato, M., and Cattaneo, L. (2008). Use-induced motor plasticity affects the processing of abstract and concrete language. Curr. Biol. 18, R290R291. doi: 10.1016/j.cub.2008.02.036

Goffman, L., and Smith, A. (1999). Development and phonetic differentiation of speech movement patterns. J. Exp. Psychol. 25:649. doi: 10.1037/00961523.25.3.649

Goldin-Meadow, S., Cook, S. W., and Mitchell, Z. A. (2009). Gesturing gives children new ideas about math. Psychol. Sci. 20, 267-272. doi: 10.1111/j.14679280.2009.02297.x

González, J., Barros-Loscertales, A., Pulvermüller, F., Meseguer, V., Sanjuán, A., Belloch, V., et al. (2006). Reading cinnamon activates olfactory brain regions. Neuroimage 32, 906-912. doi: 10.1016/j.neuroimage.2006.03.037

Graf-Estes, K. G., Evans, J. L., and Else-Quest, N. M. (2007). Differences in the nonword repetition performance of children with and without specific language impairment: a meta-analysis. J. Speech Lang. Hear. Res. 50, 177-195. doi: 10.1044/1092-4388(2007/015)

Green, J. R., Moore, C. A., Higashikawa, M., and Steeve, R. W. (2000). The physiological development of speech motor control: lip and jaw coordination. J. Speech Lang. Hear. Res. 43, 239-255. doi: 10.1044/jslhr.4301.239

Green, J. R., Moore, C. A., and Reilly, K. J. (2002). The sequential development of jaw and lip control for speech. J. Speech Lang. Hear. Res. 45, 66-79. doi: $10.1044 / 1092-4388(2002 / 005)$

Grosjean, F., and Li, P. (2013). The Psycholinguistics of Bilingualism. West Sussex, UK: Wiley-Blackwell.

Guenther, F. H., Ghosh, S. S., and Tourville, J. A. (2006). Neural modeling and imaging of the cortical interactions underlying syllable production. Brain Lang. 96, 280-301. doi: 10.1016/j.bandl.2005.06.001

Harris, C. L., Aycicegi, A., and Gleason, J. B. (2003). Taboo words and reprimands elicit greater autonomic reactivity in a first language than in a second language. Appl. Psycholinguist. 24, 561-579. doi: 10.1017/S0142716403000286

Haugeland, J. (1985). Artificial Intelligence: The Very Idea of it. Cambridge: MIT Press.
Hauk, O., Johnsrude, I., and Pulvermüller, F. (2004). Somatotopic representation of action words in the motor and premotor cortex. Neuron 41, 301-307. doi: $10.1016 /$ S0896-6273(03)00838-9

Havas, D. A., Glenberg, A. M., Gutowski, K. A., Lucarelli, M. J., and Davidson, R. J. (2010). Cosmetic use of botulinum toxin-A affects processing of emotional language. Psychol. Sci. 21, 895-900. doi: 10.1177/0956797610374742

Hebb, D. O. (1949). The Organization of Behavior. A Neuropsychological Theory. New York, NY: John Wiley.

Henderson, S. E., and Sugden, D. A. (1992). Movement Assessment Battery for Children. London: Psychological Corporation.

Hickok, G. (2009). Eight problems for the mirror neuron theory of action understanding in monkeys and humans. J. Cogn. Neurosci. 21, 1229-1243. doi: 10.1162/jocn.2009.21189

Hill, E. L. (1997). An Investigation of the Motor Deficits in Developmental Coordination Disorder and Specific Language Impairment. Ph.D. thesis, University of Cambridge.

Hill, E. L. (1998). A dyspraxic deficit in specific language impairment and developmental coordination disorder? Evidence from hand and arm movements. Dev. Med. Child Neurol. 40, 388-395. doi: 10.1111/j.14698749.1998.tb08214.x

Hill, E. L. (2001). Non-specific nature of specific language impairment: a review of the literature with regard to concomitant motor impairments. Int. J. Lang. Commun. Disord. 36, 149-171. doi: 10.1080/13682820010019874

Hill, E. L., Bishop, D. V. M., and Nimmo-Smith, I. (1998). Representational gestures in developmental co-ordination disorder and specific language impairment: error-types and the reliability of ratings. Hum. Mov. Sci. 17, 655-678. doi: 10.1016/S0167-9457(98)00017-7

Hughes, M.-A., and Sussman, H. M. (1983). An assessment of cerebral dominance in language- disordered children via a time-sharing paradigm. Brain Lang. 19, 48-64. doi: 10.1016/0093-934X(83)90055-X

Iverson, J. M., and Braddock, B. A. (2011). Gesture and motor skill in relation to language in children with language impairment. J. Speech Lang. Hear. Res. 54, 72-86. doi: 10.1044/1092-4388(2010/08-0197)

Jared, D., Poh, R. P. Y., and Paivio, A. (2013). L1 and L2 picture naming in Mandarin-English bilinguals: a test of Bilingual Dual Coding Theory. Bilingualism 16, 383-396. doi: 10.1017/S1366728912000685

Johnston, R. B., Stark, R. E., Mellits, E. D., and Tallal, P. (1981). Neurological status of language- impaired and normal children. Arch. Neurol. 10, 159-163. doi: 10.1002/ana.410100206

Kaschak, M. P., and Glenberg, A. M. (2000). Constructing meaning: the role of affordances and grammatical constructions in sentence comprehension. J. Mem. Lang. 43, 508-529. doi: 10.1006/jmla.2000.2705

Katz, W., Curtiss, S., and Tallal, P. (1992). Rapid automatized naming and gesture by normal and language-impaired children. Brain Lang. 43, 623-641. doi: 10.1016/0093-934X(92)90087-U

Kemmerer, D. (2006). The semantics of space: integrating linguistic typology and cognitive neuroscience. Neuropsychologia 44, 1607-1621. doi: 10.1016/j.neuropsychologia.2006.01.025

Kemmerer, D., Rudrauf, D., Manzel, K., and Tranel, D. (2012). Behavioral patterns and lesion sites associated with impaired processing of lexical and conceptual knowledge of actions. Cortex 48, 826-848. doi: 10.1016/j.cortex.2010.11.001

Kiefer, M., and Pulvermüller, F. (2012). Conceptual representations in mind and brain: theoretical developments, current evidence and future directions. Cortex 48, 805-825. doi: 10.1016/j.cortex.2011.04.006

Kintsch, W. (1988). The role of knowledge in discourse comprehension: a construction-integration model. Psychol. Rev. 95, 163-182. doi: 10.1037/0033295X.95.2.163

Kintsch, W., and Mangalath, P. (2011). The construction of meaning. Top. Cogn. Sci. 3, 346-370. doi: 10.1111/j.1756-8765.2010.01107.x

Kleinow, J., and Smith, A. (2006). Potential interactions among linguistic, autonomic, and motor factors in speech. Dev. Psychobiol. 48, 275-287. doi: $10.1002 / \operatorname{dev} .20141$

Kroll, J. F., and Stewart, E. (1994). Category interference in translation and picture naming: evidence for asymmetric connections between bilingual memory representations. J. Mem. Lang. 33, 149. doi: 10.1006/jmla.1994.1008

Lagrou, E., Hartsuiker, R. J., and Duyck, W. (2011). Knowledge of a second language influences auditory word recognition in the native language. J. Exp. Psychol. Learn. Mem. Cognit. 37, 952. doi: 10.1037/a0023217 
Macedonia, M. (2014). Bringing back the body into the mind: gestures enhance word learning in foreign language. Front. Psychol. 5:1467. doi: 10.3389/fpsyg.2014.01467

Macedonia, M., and Knösche, T. R. (2011). Body in mind: how gestures empower foreign language learning. Mind Brain Educ. 5, 196-211. doi: 10.1111/j.1751228X.2011.01129.x

Mayer, K. M., Yildiz, I. B., Macedonia, M., and von Kriegstein, K. (2015). Visual and motor cortices differentially support the translation of foreign language words. Curr. Biol. 25, 530-535. doi: 10.1016/j.cub.2014.11.068

McClelland, E., Pitt, A., and Stein, J. (2015). Enhanced academic performance using a novel classroom physical activity intervention to increase awareness, attention and self- control: putting embodied cognition into practice. Improv. Schools 18, 83-100. doi: 10.1177/1365480214562125

Meier, B. P., and Robinson, M. D. (2004). Why the sunny side is up: associations between affect and vertical position. Psychol. Sci. 15, 243-247. doi: 10.1111/j.0956-7976.2004.00659.x

Montgomery, J. W. (1995). Examination of phonological working memory in specifically language-impaired children. Appl. Psycholinguist. 16, 355-378.

Möttönen, R., and Watkins, K. (2012). Using TMS to study the role of the articulatory motor system in speech perception. Aphasiology 26, 1103-1118. doi: 10.1080/02687038.2011.619515

Mukamel, R., Ekstrom, A. D., Kaplan, J., Iacoboni, M., and Fried, I. (2010). Singleneuron responses in humans during execution and observation of actions. Curr. Biol. 20, 750-756. doi: 10.1016/j.cub.2010.02.045

Murakami, T., Restle, J., and Ziemann, U. (2011). Observation-execution matching and action inhibition in human primary motor cortex during viewing of speech-related lip movements or listening to speech. Neuropsychologia 49, 2045-2054. doi: 10.1016/j.neuropsychologia.2011.03.034

Newborg, J. (2005). Battelle Developmental Inventory, 2nd Edn. Itasca, IL: Riverside.

Newell, A., and Simon, H. A. (1972). Human Problem Solving. Upper Saddle River, NJ: Prentice-Hall.

Nip, I. S., and Blumenfeld, H. K. (2015). Proficiency and linguistic complexity influence speech motor control and performance in Spanish language learners. J. Speech Lang. Hear. Res. 58, 653-668. doi: 10.1044/2015_jslhr-s-13-0299

Nip, I., Green, J., and Marx, D. (2011). The co-emergence of cognition, language, and speech motor control in early development: a longitudinal correlation study. J. Commun. Disord. 44, 149-160. doi: 10.1016/j.jcomdis.2010.08.002

Paus, T., Perry, D. W., Zatorre, R. J., Worsley, K. J., and Evans, A. C. (1996). Modulation of cerebral blood flow in the human auditory cortex during speech: role of motor-to- sensory discharges. Eur. J. Neurosci. 8, 2236-2246. doi: 10.1111/j.1460-9568.1996.tb01187.x

Pickering, M. J., and Garrod, S. (2006). Do people use language production to make predictions during language comprehension? Trends Cognit. Sci. 11, 105-110. doi: 10.1016/j.tics.2006.12.002

Porter, A. M. (2016). A helping hand with language learning: teaching French vocabulary with gesture. Lang. Learn. J. 44, 236-256. doi: 10.1080/09571736.2012.750681

Powell, R. P., and Bishop, D. V. (1992). Clumsiness and perceptual problems in children with specific language impairment. Dev. Med. Child Neurol. 34, 755-765. doi: 10.1111/j.1469-8749.1992.tb11514.x

Pulvermüller, F., and Fadiga, L. (2010). Active perception: sensorimotor circuits as a cortical basis for language. Nat. Rev. Neurosci. 11, 351-360. doi: $10.1038 / \mathrm{nrn} 2811$

Pulvermüller, F., Hauk, O., Nikulin, V. V., and Ilmoniemi, R. J. (2005). Functional links between motor and language systems. Eur. J. Neurosci. 21, 793-797. doi: 10.1111/j.1460-9568.2005.03900.x

Pulvermüller, F., Huss, M., Kherif, F., Moscoso del Prado Martin, F., Hauk, O., and Shtyrov, Y. (2006). Motor cortex maps articulatory features of speech sounds. Proc. Natl. Acad. Sci. U.S.A. 103, 7865-7870. doi: 10.1073/pnas.0509989103

Pulvermüller, F., Shtyrov, Y., and Ilmoniemi, R. J. (2003). Spatio-temporal patterns of neural language processing: an MEG study using Minimum-Norm Current Estimates. Neuroimage 20, 1020-1025. doi: 10.1016/S1053-8119(03)00356-2

Pylyshyn, Z. W. (1973). What the mind's eye tells the mind's brain: a critique of mental imagery. Psychol. Bull. 80, 1-24. doi: 10.1037/h0034650

Rauscher, F. H., Krauss, R. M., and Chen, Y. (1996). Gesture, speech, and lexical access: the role of lexical movements in speech production. Psychol. Sci. 7, 226-231. doi: 10.1111/j.1467-9280.1996.tb00364.x
Rice, M. L., and Wexler, K. (1996). Toward tense as a clinical marker of specific language impairment in English-speaking children. J. Speech Lang. Hear. Res. 39, 1239-1257. doi: 10.1044/jshr.3906.1239

Riches, N. G., Loucas, T., Baird, G., Charman, T., and Simonoff, E. (2010). Sentence repetition in adolescents with specific language impairments and autism: an investigation of complex syntax. Int. J. Lang. Commun. Disord. 45, 47-60. doi: $10.3109 / 13682820802647676$

Rintala, P., Pienimaki, K., Ahonen, T., and Cantell, M. (1998). Effects of a psychomotor training programme on motor skill development in children with developmental language disorders. Hum. Mov. Sci. 17, 721-737. doi: 10.1016/S0167-9457(98)00021-9

Rizzolatti, G., Fadiga, L., Gallese, V., and Fogassi, L. (1996). Premotor cortex and the recognition of motor actions. Brain Res. Cogn. Brain Res. 3, 131-141. doi: 10.1016/0926-6410(95)00038-0

Robinson, R. J. (1991). Causes and associations of severe and persistent specific speech and language disorders in children. Dev. Med. Child Neurol. 33, 943-962. doi: 10.1111/j.1469-8749.1991.tb14811.x

Rochat, M. J., Caruana, F., Jezzini, A., Escola, L., Intskirveli, I., Grammont, F., et al. (2010). Responses of mirror neurons in area F5 to hand and tool grasping observation. Exp. Brain Res. 204, 605-616. doi: 10.1007/s00221-0102329-9

Rogalsky, C., Love, T., Driscoll, D., Anderson, S. W., and Hickok, G. (2011). Are mirror neurons the basis of speech perception? Evidence from five cases with damage to the purported human mirror system. Neurocase 17, 178-187. doi: $10.1080 / 13554794.2010 .509318$

Rueschemeyer, S., Glenberg, A. M., Kaschak, M., Mueller, K., and Friederici, A. (2010). Top-down and bottom-up contributions to understanding sentences describing objects in motion. Front. Psychol. 1:183. doi: 10.3389/fpsyg.2010.00183

Ryan, C. L. (2013). Language Use in the United States: 2011. US Census Bureau, Washington, DC.

Schomers, M. R., Kirilina, E., Weigand, A., Bajbouj, M., and Pulvermüller, F. (2014). Causal influence of articulatory motor cortex on comprehending single spoken words: TMS evidence. Cereb. Cortex 25, 3894-3902. doi: 10.1093/cercor/bhu274

Shin, H. B., and Ortman, J. (2011). "Language projections: 2010 to 2020," in Presented at the Federal Forecasters Conference (Washington, DC).

Smith, A., and Zelaznik, H. N. (2004). Development of functional synergies for speech motor coordination in childhood and adolescence. Dev. Psychobiol. 45, 22-33. doi: 10.1002/dev.20009

Stark, R. E., and Tallal, P. (1981). "Perceptual and motor deficits in language impaired children," in Central Auditory and Language Disorders in Children, ed R. W. Keith (San Diego, CA: College-Hill Press), 121-144.

Tanous, C. (2014). Embodied Activities and Communicative Strategies: A Case Study on French Informal and Classroom Conversation. Ann Arbor, MI: Proquest LLC.

Thal, D. J., and Tobias, S. (1992). Communicative gestures in children with delayed onset of oral expressive vocabulary. J. Speech Lang. Hear. Res. 35, 1281-1289. doi: 10.1044/jshr.3506.1289

Thordardottir, E. T., and Weismer, S. E. (2002). Verb argument structure weakness in specific language impairment in relation to age and utterance length. Clin. Linguist. Phonet. 16, 233-250. doi: 10.1080/02699200110116462

Tomblin, J. B., Records, N. L., Buckwalter, P., Zhang, X., Smith, E., and O’Brien, M. (1997). Prevalence of specific language impairment in kindergarten children. J. Speech Lang. Hear. Res. 40, 1245-1260. doi: 10.1044/jslhr. 4006.1245

Trumpp, N. M., Traub, F., and Kiefer, M. (2013). Masked priming of conceptual features reveals differential brain activation during unconscious access to conceptual action and sound information. PLOS ONE 8:e65910. doi: 10.1371/journal.pone.0065910

Tsumoto, T. (1992). Long-term potentiation and long-term depression in the neocortex. Progr. Neurobiol. 39, 209-228. doi: 10.1016/0301-0082(92) 90011-3

Ullman, M. T., and Gopnik, M. (1999). Inflectional morphology in a family with inherited specific language impairment. Appl. Psycholinguist. 20, 51-117. doi: $10.1017 /$ S0142716499001034

Ungerer, J. A., and Sigman, M. (1984). The relation of play and sensorimotor behavior to language in the second year. Child Dev. 55, 1448-1455. doi: $10.2307 / 1130014$ 
Vukovic, M., Vukovic, I., and Stojanovik, V. (2010). Investigation of language and motor skills in Serbian speaking children with specific language impairment and in typically developing children. Res. Dev. Disabil. 31, 1633-1644. doi: 10.1016/j.ridd.2010.04.020

Vukovic, N., and Williams, J. N. (2014). Automatic perceptual simulation of first language meanings during second language sentence processing in bilinguals. Acta Psychol. 145, 98-103. doi: 10.1016/j.actpsy.2013.11.002

Walsh, B., and Smith, A. (2002). Articulatory movements in adolescents: evidence for protracted development of speech motor control processes. J. Speech Lang. Hear. Res. 45, 1119-1133. doi: 10.1044/1092-4388(2002/090)

Watkins, K., and Paus, T. (2004). Modulation of motor excitability during speech perception: the role of Broca's area. J. Cogn. Neurosci. 16, 978-987. doi: $10.1162 / 0898929041502616$

Watkins, K. E., Strafella, A. P., and Paus, T. (2003). Seeing and hearing speech excites the motor system involved in speech production. Neuropsychologia 41, 989-994. doi: 10.1016/S0028-3932(02)00316-0

Weismer, S. E., Evans, J., and Hesketh, L. J. (1999). An examination of verbal working memory capacity in children with specific language impairment. J. Speech Lang. Hear. Res. 42, 1249-1260. doi: 10.1044/jslhr. 4205.1249

Weismer, S. E., Tomblin, J. B., Zhang, X., Buckwalter, P., Chynoweth, J. G., and Jones, M. (2000). Nonword repetition performance in school-age children with and without language impairment. J. Speech Lang. Hear. Res. 43, 865-878. doi: 10.1044/jslhr.4304.865

Wilson, S. M. (2009). Speech perception when the motor system is compromised. Trends Cogn. Sci. 13, 329-330. doi: 10.1016/j.tics.2009.06.001

Wilson, S. M., Saygin, A. P., Sereno, M. I., and Iacoboni, M. (2004). Listening to speech activates motor areas involved in speech production. Nat. Neurosci. 7, 701-702. doi: 10.1038/nn1263
Winter, B., and Bergen, B. (2012). Language comprehenders represent object distance both visually and auditorily. Lang. Cogn. 4, 1-16. doi: 10.1515/langcog2012-0001

Wolpert, D. M., Doya, K., and Kawato, M. (2003). A unifying computational framework for motor control and social interaction. Philos. Trans. R. Soc. Lond. B Biol. Sci. 358, 593-602. doi: 10.1098/rstb.2002.1238

Zatorre, R. J., Evans, A. C., Meyer, E., and Gjedde, A. (1992). Lateralization of phonetic and pitch discrimination in speech processing. Science 256, 846-849. doi: 10.1126/science. 1589767

Zelaznik, H. N., and Goffman, L. (2010). Generalized motor abilities and timing behavior in children with specific language impairment. J. Speech Lang. Hear. Res. 53, 383-393. doi: 10.1044/1092-4388(2009/08-0204)

Zwaan, R. A. (2004). "The immersed experiencer: toward an embodied theory of language comprehension," in The Psychology of Learning and Motivation, ed B. H. Ross (New York, NY: Academic Press), 35-62.

Zwaan, R. A., Stanfield, R. A., and Yaxley, R. H. (2002). Language comprehenders mentally represent the shapes of objects. Psychol. Sci. 13, 168-171. doi: $10.1111 / 1467-9280.00430$

Conflict of Interest Statement: The author declares that the research was conducted in the absence of any commercial or financial relationships that could be construed as a potential conflict of interest.

Copyright (c) 2016 Adams. This is an open-access article distributed under the terms of the Creative Commons Attribution License (CC BY). The use, distribution or reproduction in other forums is permitted, provided the original author (s) or licensor are credited and that the original publication in this journal is cited, in accordance with accepted academic practice. No use, distribution or reproduction is permitted which does not comply with these terms. 


\section{OPEN ACCESS}

Edited by:

Anna M. Borghi,

University of Bologna, Italy

Reviewed by:

Tina lachini,

Second University of Naples, Italy

Beata Joanna Grzyb,

Jaume I University, Spain

*Correspondence:

Mariana Lozada

mariana.lozada@gmail.com; lozadam@comahue-conicet.gob.ar

Specialty section:

This article was submitted to Cognition,

a section of the journal

Frontiers in Psychology

Received: 25 November 2015 Accepted: 04 March 2016

Published: 21 March 2016

Citation:

Lozada M and Carro N (2016) Embodied Action Improves Cognition in Children: Evidence from a Study Based on Piagetian Conservation

Tasks. Front. Psychol. 7:393.

doi: 10.3389/fpsyg.2016.00393

\section{Embodied Action Improves Cognition in Children: Evidence from a Study Based on Piagetian Conservation Tasks}

\author{
Mariana Lozada * and Natalia Carro \\ INIBIOMA-CONICET, Universidad Nacional del Comahue, Bariloche, Argentina
}

Converging evidence highlights the relevance of embodied cognition in learning processes. In this study we evaluate whether embodied action (enaction) improves cognitive understanding in children. Using the Piagetian conservation tasks in 6-7 year olds, we analyzed quantity conservation conceptualization in children who were active participants in the transformation process and compared these results to those of children who were mere observers of an adult's demonstration (as traditionally conducted). The investigation was performed with 105 first-graders. Conservation tasks were demonstrated to half the children, while the other half actively carried out the transformation of matter. Our findings showed that active manipulation of the material helped children recognize quantity invariance in a higher proportion than when the demonstration was only observed. That is, their enactive experience enabled them to comprehend conservation phenomena more easily than if they were merely passive observers. The outcome of this research thus emphasizes how active participation benefits cognitive processes in learning contexts, promoting autonomy, and agency during childhood.

Keywords: embodied cognition, enaction, piagetian conservation tasks, agency, children

\section{INTRODUCTION}

Numerous studies have emphasized the importance of embodied action in cognitive processing both in children and adults (e.g., Varela et al., 1991; Barsalou, 1999, 2008; Lakoff and Johnson, 1999; Thompson and Varela, 2001; Sommerville et al., 2005; Johnson and Rohrer, 2007; Glenberg, 2010; Witherington and Heying, 2013). The embodied cognition theory considers cognition as lived, enacted and closely intertwined with dynamic contexts (Varela, 2000; Thompson, 2007). This approach, which prioritizes the role of agency, considers cognition to be a consequence of active manipulation (e.g., Varela, 1999; Di Paolo et al., 2010). Thus, cognition results from sensory-motor experience, where action influences perception and vice versa, indicating that perception and action are inseparable (Prinz, 1990, 1997; Varela et al., 1991; Thelen and Smith, 1994; Jeannerod, 2001; Wilson, 2002; Rizzolatti and Craighero, 2004; Witherington, 2007, 2011; Fischer and Zwaan, 2008; Di Paolo et al., 2010; Di Paolo and De Jaegher, 2012; Anderson et al., 2013). In line with this, it has been stated that conceptual knowledge is embodied, and therefore grounded in sensory-motor systems (Gallese and Lakoff, 2005). To sum up, the embodied approach considers perception, action and cognition as tightly linked, and that previous sensorimotor experiences are seen as the basis of 
knowledge. It is therefore highly likely that first-person action improves conceptual understanding. In this investigation we evaluate how action promotes conceptual understanding.

One precursor of this view was Jean Piaget, who proposed that knowledge is linked to experience and demonstrated the crucial role of recurrent sensorimotor activity in developmental cognitive processes (Piaget, 1952). Piaget's huge contribution to child development showed that cognition is grounded in concrete activity, a theory he developed by studying how children shape their world throughout ontogeny through sensorimotor action (e.g., Piaget, 1952, 1971). Amongst his multiple contributions, Piaget found that understanding of the invariance principle, i.e., the logical concept of quantity conservation, is a developmental process that occurs between 5 and 8 years of age (Piaget, $1965,1971)$. Piaget and other investigators applied a series of tasks in which children observed, then evaluated, whether a certain quantity remained the same when changes in visual appearance were introduced, even though nothing was added or removed (e.g., Piaget, 1971; Inhelder et al., 1974; McGarrigle and Donaldson, 1974; Wilkening, 1979; Alibali and Kita, 2010; Goldin-Meadow and Beilock, 2010; Ping and Goldin-Meadow, 2010). Thus, children's ability to understand conservation despite the apparent transformation of number, matter, or liquid quantity was analyzed. These conservation tasks are ideal for the evaluation of cognitive development, given that they are controllable, replicable and easy to apply in different cultures and socio-economic contexts. In addition, the results are easily measurable and these tests are therefore highly suitable for the assessment of the role of action in children's comprehension. Tasks consisted of showing a child an initial stage (e.g., pouring the same amount of liquid into two identical containers), then a transformation process demonstration (e.g., pouring the same amount of liquid into two different containers, so that quantities appeared to be different), and finally the child was asked whether the amount was the same as before, or different. The transformation processes involved the researcher, for example, flattening a ball of clay, stretching out a row of coins, or transferring a certain amount of liquid into a narrower or wider container.

According to Piaget (1965) and other researchers (e.g., Smedslund, 1968; Siegler, 1981; Alibali and Kita, 2010; GoldinMeadow and Beilock, 2010; Ping and Goldin-Meadow, 2010; Houdé et al., 2011) children under 5-7 years old have difficulty understanding the conservation principle, whereas children older than 7-8 are generally capable of realizing that number, length, volume of liquid, and matter remain unchanged in spite of changes in form. Therefore, for the above situations, three developmental stages are expected to exist within the 5-8 age range: children who consistently recognize the conservation principle (Total Conservers, TC), those who partially recognize this concept (Partial Conservers, PC) and children who do not recognize it in any task (Non Conservers, NC). Children go through a transition stage of being "partial conservers" for liquid quantity, mass, number, and length (Church and GoldinMeadow, 1986). Recognition of weight conservation occurs at 9-10 years of age, and 11-12 year-olds are able to understand volume conservation (Piaget, 1963). In relation to children's increased understanding of the conservation principle, a recent study on number conservation showed that certain neural networks associated with numerical and executive functions were not activated in 5-6 year-old children (non-conservers) whereas in children older than 9-10 (conservers) these networks were activated (Houdé et al., 2011). These authors suggested that the neural contribution of this bilateral parietofrontal network, associated with executive functions such as inhibitory control, plays a crucial role in the acquisition of number-conservation (Borst et al., 2012, 2013; Houdé and Borst, 2014).

Most previous studies on this subject analyzed the conservation principle in a situation where children observed the experimenter's demonstration (e.g., Piaget, 1965, 1971; Inhelder et al., 1974; McGarrigle and Donaldson, 1974; Wilkening, 1979; Goldin-Meadow and Beilock, 2010; Ping and Goldin-Meadow, 2010). These demonstrations precluded the possibility of enaction on the part of participants, who remained "passive," i.e., they were not active agents. Following the enactive theory that action modifies perception, thus promoting further understanding, we considered that it would be of interest to explore whether active manipulation enhances conceptual understanding in children. In the present study we evaluate the role of hands-on experience in cognitive processing, hypothesizing that action will contribute to understanding of the conservation principle, analyzed by means of the well-known Piagetian conservation tasks, but with one major change: the addition of active manipulation. By giving participants the opportunity to handle the materials, we will be able to compare in a precise, concrete way, children's recognition of the conservation concept with and without the incorporation of action. That is, conceptual understanding will be assessed under two conditions: action and observation. Seven conservation tasks were devised using the Piagetian conservation tasks with 6-7 year olds, an age considered to be in the mid-range of this developmental process. We will test the hypothesis that when children are active participants in the transformation process, i.e., the act of transferring liquids, flattening a ball of plasticine, moving coins, or measuring strings with their own hands, conservation understanding is increased. Thus, we hypothesize that children's embodied action will help them to recognize quantity invariance in a higher proportion than children who merely observe the demonstration carried out by an experimenter.

\section{MATERIAL AND METHODS}

\section{Participants}

The study was carried out with 105 first-graders from public and private schools in San Carlos de Bariloche, Argentina (54 girls, 51 boys). The age range of participants was 6-7 years (mean age: 6 years, 8 months \pm 0.6 ).

The participants were all in good health, and there were no significant differences in body mass index or socioeconomic level. Experiments were conducted according to the Helsinki declaration and approved by the Clinical Research Ethics Committee (CEIC) and by the Council of Education of Río Negro Province, Argentina. All procedures were carried out with 
the adequate understanding and written consent of parents and school authorities. In addition, the children gave their verbal consent for participation in the study.

\section{Procedure}

During a normal school day, one child at a time participated in an experimental session of approximately 20-30 min. Two different procedures were followed, corresponding to an observation condition ( $N=47,25$ girls, and 22 boys) and an action condition ( $N=58,29$ girls and 29 boys). In each first grade, approximately half of the children were randomly assigned to each condition.

Children worked individually in a quiet room in the school. Each participant was told that he/she was going to play some games, and sat at a table, facing the researcher. The quantity conservation task materials were laid out on the table. Each session was recorded. We analyzed each answer per task per child, for both groups.

\section{Action and Observation Conditions Action Condition}

In this condition the researcher, with speech and gestures, asked each child to carry out the seven Piagetian conservation tasks by themselves. The tasks were shown in the following order: Two liquid quantity tasks, two mass quantity tasks, a number task and two length tasks. Each task consisted of three stages: Sameness, changing and judgment stages.

1. In the liquid quantity task, the researcher instructed the child to pour the same amount of liquid into two identical containers in order to obtain equal quantities in each one. Each child was asked to make sure that both containers held the same amount of liquid. In the changing stage, the researcher instructed the child to pour the liquid from one of the two identical containers into a narrower one. Finally, in the judgment stage, the researcher asked the child if the quantities were the same or different, and why ("Can you say why you think they are the same/different?").

2. The first stage of Task 1 was repeated but in the changing stage the researcher instructed the child to pour the liquid from one of the two identical containers into a much narrower one (narrower than in the changing stage of 1). In the judgment stage, the questions were repeated as for 1 .

3. In the mass quantity task, the researcher instructed the child to form two identical balls (i.e., with the same amount of plasticine) and each child was asked to make sure the two forms had the same amount of matter. In the changing stage, the researcher instructed the child to roll the plasticine into a thinner, longer shape. In the judgment stage, the questions were repeated as for 1 .

4. The first stage of Task 3 was repeated but in the changing stage the researcher instructed the child to flatten the ball and stretch it; in the judgment stage, the questions were repeated as for 1 .

5. In the number task, the researcher instructed the child to arrange 20 coins in two rows of 10 . Each child was asked to make sure the two rows had the same number of coins. In the changing stage, the researcher instructed the child to spread out the coins in the upper row. These instructions were accompanied with gestures for clarity. In the judgment stage, the questions were repeated as for 1 .

6. In the length task, the researcher instructed the child to lay out two identical strings in a straight line in front of him/her, parallel to each other and to the edge of the table. Each child was asked to make sure the two strings were identical in length. In the changing stage, the researcher asked the child to move the upper string $20-30 \mathrm{~cm}$ to the right of its original location. In the judgment stage, the researcher asked the child if both strings were the same length and asked: "If an ant has to walk along both strings, will it travel the same distance on each? Will it take the same or a different number of steps?" and also asked why.

7. The first stage of Task 6 was repeated but in the changing stage the researcher instructed the child to form a curve with the upper string. These instructions were accompanied with gestures for clarity. Following this, the researcher asked questions as for 6 .

\section{Observation Condition}

In this condition, the child observed demonstrations of the seven Piagetian conservation tasks detailed above, in the same order as in the action condition and consisting of the same three stages, but in this case carried out by the researcher.

\section{Data Analysis \\ Conservation Judgments}

We analyzed children's answers related to each task, i.e., if quantity remained the same or was different after the transformation process. If a child answered that quantity was the same, we considered this a conserver answer for that task. For both observation and action conditions, we analyzed each child's judgment. Children received no feedback on their answers, i.e., the experimenter did not comment on the children's responses.

\section{Comparison of TC, PC, NC in Each Condition}

If a child recognized conservation in all seven tasks, he/she was classified as a total conserver (TC). If a child recognized conservation in at least one task, but not in all, we considered him/her to be a partial conserver (PC), and if a child did not recognize conservation in any of the seven tasks, we considered him/her a non-conserver (NC).

\section{Statistical Analysis Conservation Judgments}

Using a chi square test $(p<0.05)$, we compared the proportion of conserver responses between the action and observation groups for each task category, taking tasks of a similar type together, i.e., liquid, mass, number, and length (Church and Goldin-Meadow, 1986). The conservation response was considered as accurate $($ score $=1)$ and non-conservation as error (score $=0)$.

\section{Comparison of TC, PC, NC}

We compared the proportion of children who recognized conservation in all 7 tasks (TC), those who recognized the conservation principle in at least one task but not all (PC) and those who did not recognize the conservation principle in any 
of the seven tasks (NC) in each group using a chi square test. The relative proportion of TC, PC and NC between groups was conducted by means of the difference test $(p<0.05)$.

\section{RESULTS}

When comparing the proportions of conservation responses for each task category (i.e., liquid, mass, length and number), for the action and observation groups, we found that in the liquid quantity tasks the proportion of conservation answers was significantly higher in the action group than in the observation one $\left(X^{2}=5.82, p=0.0158, d f=1\right)$. That is, the act of transferring liquid by themselves helped the children recognize that liquid quantity was conserved despite the change in the container's shape. Similarly, a higher proportion of conservation answers was found in the action group during the mass tasks $\left(X^{2}=10.61, p=0.0011, d f=1\right)$, i.e., the act of flattening or spreading out plasticine helped them recognize that there was no change in mass despite the transformation in shape. During the number task, the proportion of conservation answers was also higher for the action group $\left(X^{2}=20.08, p=0.000, d f=1\right)$. Furthermore, the opportunity to handle the string during the length tasks led to a greater proportion of conservation answers in the action group than in the observation one $\left(X^{2}=23.21\right.$, $p=0.0000, d f=1$ ) (Figure 1). Thus, in all tasks, a higher proportion of children who were given the opportunity to manipulate the materials showed a greater capacity for reasoning and discernment, which enabled their understanding of the conservation principle. As the study progressed, an overall increase in the proportion of conservation answers was observed in all tasks in both groups.

When comparing the relative proportion of children who recognized conservation in all tasks (TC), in at least one (PC), and in none (NC), we found significant differences between the three categories in the action group $\left(X^{2}=24.24, p=0.0000\right.$, $d f=2$ ). Conversely, in the observation group no significant differences between the relative proportion of children who

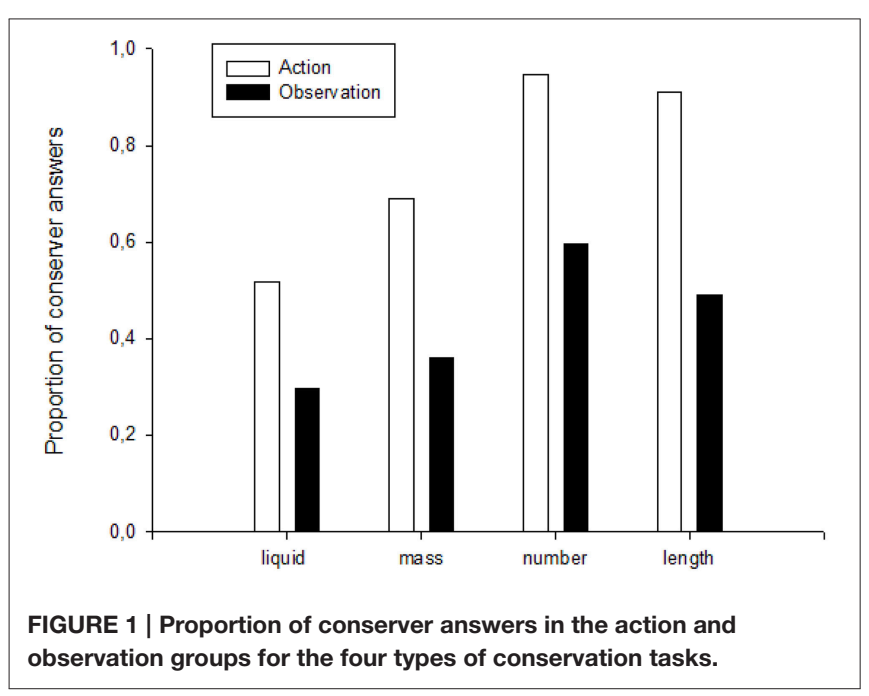

acknowledged conservation in all tasks, in at least one, and in none $\left(X^{2}=2.85, p=0.24, d f=2\right)$. The action group holds a significantly higher proportion $(p<0.028)$ of children who recognized conservation in all tasks $(43.10 \%)$ in comparison with the proportion found in the observation group (25.53\%). In addition, in the action group we found a significantly lower proportion $(p<0.0001)$ of children who did not recognize conservation in any task (3.45\%) compared with the proportion found in the observation group (29.79\%). The extremely low number of non-conservers in the action group shows that when embodied action was allowed, most of the children were aware of conservation in at least one task in a higher proportion than when they were merely observers. The relative proportions of children who understood conservation in at least one task, but not in all, was similar in both groups ( $p=0.21$ ) (Figure 2); this fact could be associated with the high probability of responding correctly to one question out of six for the seven tasks. Therefore, we consider that the relevant finding is the higher proportion of TC found in the action group as well as the lower proportion of NC in this group.

\section{DISCUSSION}

The present study shows how active participation enhances cognitive processing in 6-7 year old children. Using the well-known Piagetian conservation tasks, we discerned that active manipulation, as opposed to mere observation of a demonstration carried out by a researcher, significantly increased understanding of the conservation principle. The fact that conservation performance was higher in the action group than in the observation one suggests that the experience of manipulating objects throughout the transformation processes facilitated recognition of the conservation concept. Thus, the experience of "doing" (i.e., being the agent) seems to favor conceptualization of the fact that liquid quantity, mass, number, and length can remain unaltered despite changes in appearance. Our findings contribute to the understanding of cognition as

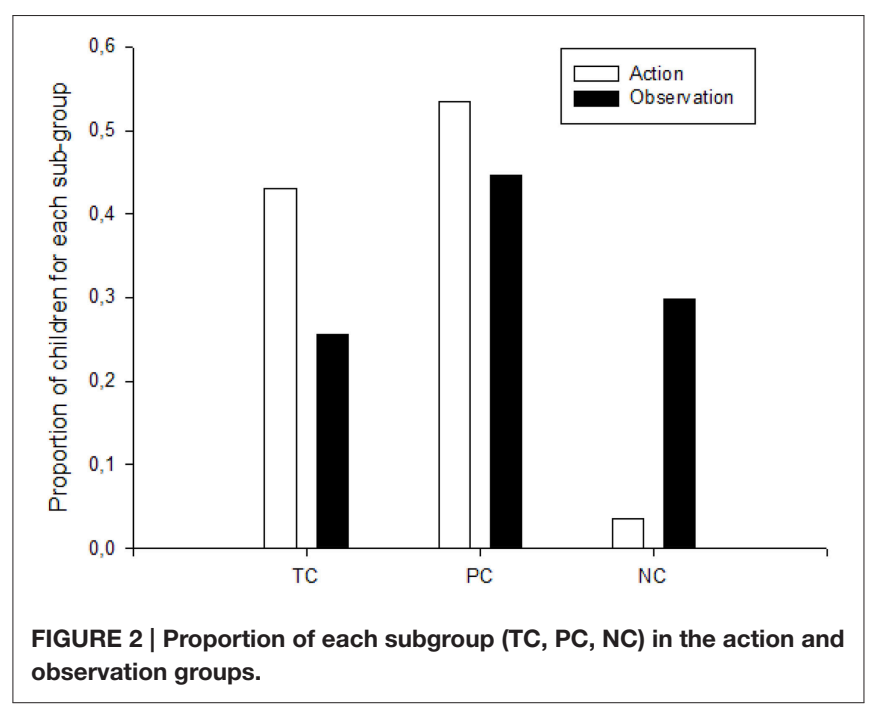


actively embodied, highlighting the impact of action in learning processes. These results suggest that education systems could benefit from the inclusion of embodied experiences in their teaching methods, as suggested in the past (e.g., Montessori, 1914; Dewey, 1920). Our research experimentally confirms the proposal of these well-known educators to incorporate first-hand experience in educational instruction (e.g., Montessori, 1914; Dewey, 1920).

This study aimed to confirm the important role of action in cognition. To this end, we chose to work with Piaget's conservation tasks, as they are well formulated and easily applicable. We sought to evaluate whether active manipulation influences children's cognitive processing, and therefore in our study children had the opportunity to perform the tasks, thus promoting agency and autonomy. The present work contributes to understanding of how children's cognitive abilities can be enhanced by active performance, favoring children's empowerment. Our findings are in line with recent studies that have shown how acting out can improve abstract comprehension of physics and mathematics (e.g., Glenberg et al., 2012; Nemirovsky et al., 2012; Núñez, 2012) and reading comprehension (e.g., Guan et al., 2013), highlighting how embodied experiences promote conceptual comprehension and rational and abstract thinking (Varela et al., 1991).

Our results agree with the embodied cognition theory which asserts that cognitive processes emerge from perception-action patterns, where action guides perception in local situations, which change, in turn, as a consequence of the child's action (e.g., Varela, 1999; Di Paolo et al., 2010). As proposed by the enactive theory, cognitive agents are not passive data collectors who model the world, but active participants who enact a world (Varela et al., 1991; Di Paolo and De Jaegher, 2012). Thus, the world is not something given from outside but something engaged in by doing; then, the world is "brought to life" by concrete handling (Varela, 2000). Along the same lines, a previous study comparing action and observation in conceptual categorization in adults found that action guides object categorization; the authors thus propose that categorization is based on sensorimotor experience (Iachini et al., 2008). In our investigation, the action and observation conditions also implied different sensorimotor experiences, suggesting that increased awareness of the invariance principle could be a result of the children's own activity, where feedback from their action may change their perception of the conservation phenomenon. It is likely that the different kinds of experience, i.e., observing or doing, could have led to different allocation of attention. That is, active manipulation could have helped increase attention to the transformation processes, promoting greater awareness and understanding. It has been proposed that concrete handling helps one focus, and so the present moment can be more salient when it involves concrete action (Varela, 2000). This is in line with previous studies which proposed that lack of attention to relevant quantitative relationships led young children to fail in conservation awareness (e.g., Trabasso and Bower, 1968; Gelman, 1969; Miller and Heller, 1976; Gelman and Baillargeon, 1983).

When analyzing each task performance, the results showed how children in the action group answered as conservers in all tasks in a higher proportion than in the observation group. The task sequence was randomly established and tasks were presented in the same order in both groups, i.e., children underwent the testing in identical conditions and were asked the same questions except for the opportunity to participate actively; the marked difference observed between groups, therefore, must have been due to a different cognitive process occurring while the action group performed the tasks. As the experiment progressed, in both groups a relative increase was observed in children's correct total conservation answers in all tasks except for the length one; this could indicate that generalization or learning transfer has occurred in both conditions. However, our objective was to compare performance under the action and observation conditions; that is, whether the act of "doing" or "observing" generated changes in the understanding of the conservation principle. To this end, the same sequence was followed for each participant and no counterbalancing of task evaluation was carried out. Future studies could further elucidate whether active manipulation of a certain conservation task can improve conservation awareness of a later one, and also evaluate the relative difficulty of each task.

As we worked with children whose age lay in the midrange of the conservation invariance developmental process, it is significant that when comparing the proportion of total conservers, partial conservers, and non-conservers, we found a higher proportion of children in the action group who recognized the conservation principle in all tasks and a lower proportion of children who did not recognize quantity conservation in any task, as hypothesized. We worked with children of 6-7 years old, but previous research has shown that when an easier version of the task is applied, children show the conservation principle earlier in life (Wilkening, 1979); other studies which explored different ways of testing conservation principle allude to potential biases in Piagetian task methodology (e.g., McGarrigle and Donaldson, 1974) and also suggested that conservation conceptualization could occur at earlier ages. Nevertheless, the aim of our study is not to determine at what developmental age the conservation principle is acquired, but rather to compare its conceptualization in two clear-cut circumstances, one of which involved action on the part of children while the other did not.

Other research has demonstrated that children participating in these Piagetian tasks tend to explain the conservation concept with both gestures and speech; these embodied gestures can help manifest conceptual knowledge of conservation (e.g., Evans and Rubin, 1979; Church and Goldin-Meadow, 1986; Alibali and Kita, 2010). It has been found that gestures highlight perceptually present information (Alibali and Kita, 2010) and that gestures not only reflect unspoken thought but can also change thought in children (e.g., Goldin-Meadow, 2009; Goldin-Meadow and Beilock, 2010). These authors suggest that gestures provide a bridge between action and abstract thought. Furthermore, although our findings tie in well with these studies, the analysis of gestures constitutes a different approach to the relevance of action in cognitive processing. In a previous study researchers explored the use of training procedures between the pretesting and post-testing of conservation tasks in partial conservers. Results showed that the training, which involved manipulation, 
slightly improved posttest judgment (Church and GoldinMeadow, 1986). In their investigation, the experimenter played a predominant role as he/she filled the two containers with the same quantity of liquid and then asked the child to transfer it to different containers. This fact could explain why they observed a much lower level of impact due to manipulation. In contrast, our research involved no training stage and the children were active agents of the entire process, from the beginning, without the active participation of an adult.

In our work, the fact that children's active experience during the transformation process was effective in facilitating conservation performance highlights the crucial role of agency. This could have an impact on education, given that, as stated by John Dewey, learning methods are generally external to children's existing capabilities, tending to lie beyond the reach of their experience. As he pointed out, "traditional teaching tends to impose from above and from outside adult standards, subject-matter and methods, and this imposition and external control oppose expression and cultivation of individuality" (Dewey, 1920). Similarly, the renowned educator Maria Montessori founded an integral pedagogy system based on the importance of a child's experience, maintaining that children learn when developing activities through the manipulation of objects (e.g., Montessori, 1914). To sum up, active participation during learning, therefore, connects children's experience more closely with cognitive processes, thus enriching educational practices while enhancing learning abilities As proposed by Jonas (1966), we actively maintain our dynamic identity by doing, i.e., our self-produced identity is based on action, and on perception-action patterns that enable constant interchange with the surroundings. The present study provides new evidence of how cognition emerges from experiential (enactive) processes, which contributes to the understanding of how embodied

\section{REFERENCES}

Alibali, M. W., and Kita, S. (2010). Gesture highlights perceptually present information for speakers. Gesture 10, 3-28. doi: 10.1075/gest.10.1.02ali

Anderson, D. I., Campos, J. J., Witherington, D. C., Dahl, A., Rivera, M., He, M., et al. (2013). The role of locomotion in psychological development. Front. Psychol. 4:440. doi: 10.3389/fpsyg.2013.00440

Barsalou, L. W. (1999). Perceptual symbol systems. Behav. Brain Sci. 22, 577-660. doi: $10.1017 / \mathrm{s} 0140525 \times 99002149$

Barsalou, L. W. (2008). Grounded cognition. Annu. Rev. Psychol. 59, 617-645. doi: 10.1146/annurev.psych.59.103006.093639

Borst, G., Poirel, N., Pineau, A., Cassotti, M., and Houdé, O. (2012). Inhibitory control in number-conservation and class-inclusion tasks: a neo-Piagetian inter- tasks priming study. Cogn. Dev. 27, 283-298. doi: 10.1016/j.cogdev.2012.02.004

Borst, G., Simon, G.,Vidal, J., and Houdé, O. (2013). Inhibitory control and visuospatial reversibility in Piaget's seminal number-conservation task: a high- density ERP study. Front. Hum. Neurosci. 7:920. doi: 10.3389/fnhum.2013.00920

Church, R. B., and Goldin-Meadow, S. (1986). The mismatch between gesture and speech as an index of transitional knowledge. Cognition 23, 43-71. doi: 10.1016/0010-0277(86)90053-3

Dewey, J. (1920). Reconstruction in Philosophy. New York, NY: Henry Holt and Company. agency facilitates conceptual processing such as the quantitative conservation principle. The outcome of this research thus emphasizes how active participation benefits cognitive processes in learning contexts, promoting autonomy and agency during childhood.

\section{AUTHOR CONTRIBUTION}

ML conceived, and designed experiments, analyzed data and contributed to the writing of the manuscript, drafting the work, final approval of the version to be published, and agreement to be accountable for all aspects of the work in ensuring that questions related to the accuracy or integrity of any part of the work are appropriately investigated and resolved. NC conducted experiments, collected data and contributed to the writing of the manuscript, drafting the work, final approval of the version to be published, and agreement to be accountable for all aspects of the work in ensuring that questions related to the accuracy or integrity of any part of the work are appropriately investigated and resolved

\section{FUNDING}

This work was partially supported by Universidad Nacional del Comahue and INIBIOMA-CONICET.

\section{ACKNOWLEDGMENTS}

We are grateful to Audrey Shaw who greatly improved the English version of the manuscript, and Nora Scheuer and Veronica Rapela, whose valuable suggestions also helped improve the manuscript.
Di Paolo, E. A., and De Jaegher, H. (2012). The interactive brain hypothesis. Front. Hum. Neurosci. 6:163. doi: 10.3389/fnhum.2012. 00163

Di Paolo, E. A., Rohde, M., and De Jaegher, H. (2010). "Horizons for the Enactive Mind: Values, Social Interaction, and Play," in Enaction: Towards a New Paradigm for Cognitive Science, eds J. Stewart, O. Gapenne, E. A. Di Paolo (Cambridge, MA: MIT Press), 33-87.

Evans, M. A., and Rubin, R. H. (1979). Hand gestures as a communicative mode in school-aged children. J. Gen. Psychol. 135, 189-196. doi: $10.1080 / 00221325.1979 .10534070$

Fischer, M. H., and Zwaan, R. A. (2008). Embodied language: a review of the role of the motor system in language comprehension. Q. J. Exp. Psychol. 61, 825-850. doi: 10.1080/17470210701623605

Gallese, V., and Lakoff, G. (2005). The brain's concepts: the role of the sensorymotor system in conceptual knowledge. Cogn. Neuropsychol. 22, 455-479. doi: $10.1080 / 02643290442000310$

Gelman, R. (1969). Conservation acquisition: a problem of learning to attend to relevant attributes. J. Exp. Child Psychol. 7, 167-187. doi: 10.1016/00220965(69)90041-1

Gelman, R., and Baillargeon, R. (1983). "A review of some Piagetian concepts," in Cognitive Development, Vol. 3, ed P. H. Musen, (New York, NY: Wiley and Sons), 167-230.

Glenberg, A. (2010). Embodiment as a unifying perspective for psychology. Wiley Interdiscip. Rev. Cogn. Sci. 1, 586-596. doi: 10.1002/wcs.55 
Glenberg, A., Willford, J., Gibson, B., Goldberg, A., and Zhu, X. (2012). Improving reading to improve math. Sci. Stud. Read. 16, 316-340. doi: $10.1080 / 10888438.2011 .564245$

Goldin-Meadow, S. (2009). How gesture promotes learning throughout childhood. Child Dev. Perspect. 3, 106-111. doi: 10.1111/j.1750-8606.2009.00088.x

Goldin-Meadow, S., and Beilock, S. (2010). Action's influence on thought: the case of gesture. Perspect. Psychol. Sci. 5, 664-674. doi: 10.1177/1745691610388764

Guan, C. Q., Meng, W., Yao, R., and Glenberg, A. M. (2013). The motor system contributes to comprehension of abstract language. PLOS ONE 8:e75183. doi: 10.1371/journal.pone.0075183

Houdé, O., and Borst, G. (2014). Measuring inhibitory control in children and adults. Front. Psychol. 5:616. doi: 10.3389/fpsyg.2014.00616

Houdé, O., Pineau, A., Leroux, G., Poirel, N., Perchey, G., Lanoe, C., et al. (2011). Functional magnetic resonance imaging study of Piaget's conservationof-number task in preschool and school-age children. J. Exp. Child Psychol. 110, 332-346. doi: 10.1016/j.jecp.2011.04.008

Iachini, T., Borghi, A. M., and Senese, V. P. (2008). Categorization and sensorimotor interaction with objects. Brain Cogn. 67, 31-43. doi: 10.1016/j.bandc.2007.11.002

Inhelder, B., Sinclair, H., and Bovet, M. (1974). Learning and the Development of Cognition. Cambridge: Harvard University Press.

Jeannerod, M. (2001). Neural simulation of action: a unifying mechanism for motor cognition. Neuroimage 14, S103-S109. doi: 10.1006/nimg.2001.0832

Johnson, M., and Rohrer, T. (2007). "We are live creatures: embodiment, american pragmatism, and the cognitive organism" in Body, Language, and Mind, eds J. Zlatev, T. Ziemke, R. Frank, R. Dirven (Berlin: Mouton de Gruyter), 17-54.

Jonas, H. (1966). The Phenomenon of Life: Towards a Philosophical Biology. Evanston, IL: Northwestern University Press.

Lakoff, G., and Johnson, M. (1999). Philosophy in the Flesh. The Embodied Mind and Its Challenge to Western Thought. New York, NY: Basic Books.

McGarrigle, J., and Donaldson, M. (1974). Conservation accidents. Cognition 3, 341-350. doi: 10.1016/0010-0277(74)90003-1

Miller, P. H., and Heller, K. A. (1976). Facilitation of attention to number and conservation of number. J. Exp. Child Psychol. 22, 454-467. doi: 10.1016/00220965(76)90108-9

Montessori, M. (1914). Dr. Montessori's Own Handbook. New York, NY: Frederick A. Stokes Company.

Nemirovsky, R., Rasmussen, C., Sweeney, G., and Wawro, M. (2012). When the classroom floor becomes the complex plane: addition and multiplication as ways of bodily navigation. J. Learn. Sci. 21, 287-323. doi: $10.1080 / 10508406.2011 .611445$

Núñez, R. (2012). On the science of embodied cognition in the 2010s: research questions, appropriate reductionism, and testable explanations. J. Learn. Sci. 21, 324-336. doi: 10.1080/10508406.2011.614325

Piaget, J. (1952). The Origins of Intelligence in Children. New York, NY: International Universities Press.

Piaget, J. (1963). "Intellectual operations and their development" in The Essential Piaget: An Interpretive Reference and Guide, eds H. E. Gruber and J. J. Vonéche (New York, NY: Basic Books), 342-358.

Piaget, J. (1965). The Child's Conception of Number. New York, NY: W.W. Norton and Company.

Piaget, J. (1971). Biology and Knowledge. Chicago, IL: University of Chicago Press.

Ping, R. M., and Goldin-Meadow, S. (2010). Gesturing saves cognitive resources when talking about nonpresent objects. Cogn. Sci. 34, 602-619. doi: 10.1111/j.1551-6709.2010.01102.x
Prinz, W. (1990). “A common coding approach to perception and action," in Relationships between Perception and Action, eds O. Neumann and W. Prinz (Berlin: Springer-Verlag), 167-201.

Prinz, W. (1997). Perception and action planning. Eur. J. Cogn. Psychol. 9, 129-154. doi: $10.1080 / 713752551$

Rizzolatti, G., and Craighero, L. (2004). The mirror-neuron system. Annu. Rev. Neurosci. 27, 169-192. doi: 10.1146/annurev.neuro.27.070203. 144230

Siegler, R. S. (1981). Developmental sequences within and between concepts. Monogr. Soc. Res. Child Dev. 46, 1-84. doi: 10.2307/1165995

Smedslund, J. (1968). Conservation and resistance to extinction: a comment on Hall and Simpson's article. Merrill Palmer Q. 14, 211-214.

Sommerville, J. A., Woodward, A. L., and Needham, A. (2005). Action experience alters 3-month-old infants' perception of others' actions. Cognition 96, B1-B11. doi: 10.1016/j.cognition.2004.07.004

Thelen, E., and Smith, L. B. (1994). A Dynamic Systems Approach to the Development of Perception and Action. Cambridge: MIT Press.

Thompson, E. (2007). Mind in Life: Biology, Phenomenology and the Sciences of Mind. Cambridge: Harvard University Press.

Thompson, E., and Varela, F. J. (2001). Radical embodiment: neural dynamics and consciousness. Trends Cogn. Sci. 5, 418-425. doi: 10.1016/S13646613(00)01750-2

Trabasso, T., and Bower, G. (1968). Attention in Learning: Theory and Research. New York, NY: John and Wiley.

Varela, F. (2000). El Fenómeno de la Vida. Santiago de Chile: Editorial Dolmen.

Varela, F. J. (1999). Ethical Know-How: Action, Wisdom and Cognition. Stanford: University Press.

Varela, F., Thompson, E., and Rosch, E. (1991). The Embodied Mind: Cognitive Science and Human Experience. Cambridge: MIT Press.

Wilkening, F. (1979). Combining of stimulus dimensions in children's and adults' judgments of area: an information integration analysis. Dev. Psychol. 15, 25-33. doi: 10.1037/0012-1649.15.1.25

Wilson, M. (2002). Six views of embodied cognition. Psychon. Bull. Rev. 9, 625-636. doi: 10.3758/BF03196322

Witherington, D. C. (2007). The dynamic systems approach as metatheory for developmental psychology. Hum. Dev. 50, 127-153. doi: 10.1159/000 100943

Witherington, D. C. (2011). Taking emergence seriously: the centrality of circular causality for dynamic systems approaches to development. Hum. Dev. 54, 66-92. doi: 10.1159/000326814

Witherington, D. C., and Heying, S. (2013). Embodiment and agency: toward a holistic synthesis for developmental science. Adv. Child Dev. Behav. 44, 161-192. doi: 10.1016/B978-0-12-3979476.00006-4

Conflict of Interest Statement: The authors declare that the research was conducted in the absence of any commercial or financial relationships that could be construed as a potential conflict of interest.

Copyright (c) 2016 Lozada and Carro. This is an open-access article distributed under the terms of the Creative Commons Attribution License (CC BY). The use, distribution or reproduction in other forums is permitted, provided the original author(s) or licensor are credited and that the original publication in this journal is cited, in accordance with accepted academic practice. No use, distribution or reproduction is permitted which does not comply with these terms. 
OPEN ACCESS

Edited by:

Anna M. Borghi,

University of Bologna, Italy

Reviewed by:

Julius Verrel,

Max Planck Society, Germany Elena Daprati,

Università di Roma Tor Vergata, Italy

*Correspondence:

Kathrin Wunsch

kathrin.wunsch@sport.uni-freiburg.de

Specialty section:

This article was submitted to

Cognition,

a section of the journal

Frontiers in Psychology

Received: 12 April 2016

Accepted: 23 June 2016

Published: 12 July 2016

Citation:

Wunsch K, Pfister R, Henning A, Aschersleben G and Weigelt M (2016) No Interrelation of Motor Planning and

Executive Functions across Young

Ages. Front. Psychol. 7:1031.

doi: 10.3389/fpsyg.2016.01031

\section{No Interrelation of Motor Planning and Executive Functions across Young Ages}

\author{
Kathrin Wunsch ${ }^{1 *}$, Roland Pfister ${ }^{2}$, Anne Henning ${ }^{3}$, Gisa Aschersleben ${ }^{4}$ and \\ Matthias Weigelt ${ }^{5}$
}

\begin{abstract}
${ }^{1}$ Institute of Sport and Sport Science, University of Freiburg, Freiburg, Germany, ${ }^{2}$ Department of Psychology, University of Würzburg, Würzburg, Germany, ${ }^{3}$ Developmental Psychology, SRH Fachhochschule for Healthcare, Gera, Germany, ${ }^{4}$ Developmental Psychology, Saarland University, Saarbrücken, Germany, ${ }^{5}$ Department Sport and Health, University of Paderborn, Paderborn, Germany
\end{abstract}

The present study examined the developmental trajectories of motor planning and executive functioning in children. To this end, we tested 217 participants with three motor tasks, measuring anticipatory planning abilities (i.e., the bar-transport-task, the sword-rotation-task and the grasp-height-task), and three cognitive tasks, measuring executive functions (i.e., the Tower-of-Hanoi-task, the Mosaic-task, and the D2-attention-endurance-task). Children were aged between 3 and 10 years and were separated into age groups by 1-year bins, resulting in a total of eight groups of children and an additional group of adults. Results suggested (1) a positive developmental trajectory for each of the sub-tests, with better task performance as children get older; (2) that the performance in the separate tasks was not correlated across participants in the different age groups; and (3) that there was no relationship between performance in the motor tasks and in the cognitive tasks used in the present study when controlling for age. These results suggest that both, motor planning and executive functions are rather heterogeneous domains of cognitive functioning with fewer interdependencies than often suggested.

Keywords: anticipatory planning, end-state comfort effect, developmental disorders, child development, motor development

\section{INTRODUCTION}

Anticipatory motor planning accounts for future body postures at the end of goal-directed movements. In their everyday lives, people need to plan many movements in advance. When grasping a cup that is standing upside-down in the cupboard, most people use an uncomfortable thumb-down posture to grasp the cup, then turn it around and end in a comfortable thumbup posture, which makes it possible to pour coffee into the cup. This anticipatory planning performance is a signification of the so called end-state comfort (ESC) effect (Rosenbaum et al., 1990).

The ESC effect was first observed and examined by Rosenbaum et al. (1990), who took this observation into laboratory. They designed the bar-transport-task, in which a horizontally oriented bar with one black and one gray end laid horizontally on two supports. This bar had to be placed on either a red or a blue target disc, placed to the right and to the left of the supports, respectively. Participants could grasp the bar either with an overhand-grip or an underhand-grip, using their right hand. Interestingly, they chose the comfortable overhand-grip only to place the right end 
of the bar on the target (irrespective of bar and target color), whereas they used the initially uncomfortable underhand-grip, when the left end of the bar had to be placed on the target. The flexible selection of the initial grasp type allowed participants to end the object manipulation in a comfortable thumb-up posture (as opposed to an awkward thumb-down posture), even if this meant to tolerate an awkward posture at the beginning of the action.

Since its discovery two decades ago (Rosenbaum et al., 1990), a growing body of research has documented the ESC effect as a robust phenomenon for healthy adults, as well as for different clinical populations (see Rosenbaum et al., 2012, for a review). Moreover, ESC effects also seem to arise in different nonhuman animals, (e.g., Zander et al., 2013). Similar anticipatory planning skills also become evident using other measures than the described bar-transport-task and its conceptual replications (see Figures 1A,B). One example for such additional measures is the grasp-height effect (Figure 1C). Here, anticipatory planning is probed by asking participants to put objects onto shelves of varying height. When placing objects on a high shelf, people grasp the object at its lower end. Conversely, when it has to be placed on a low shelf, they grasp the object at its upper end. Both actions result in a maximally comfortable position. Therefore, the future position of an object, which should be placed onto targets of varying heights, is also anticipated (Cohen and Rosenbaum, 2004).

For the following argument, we subsume all such measures under the umbrella term of ESC effects and, as noted above, these effects have been replicated numerous times (Rosenbaum et al., 2012). This, however, mainly concerns ESC effects for young adults, whereas only few studies investigated ESC effects in children (see Wunsch et al., 2013, for a review). Furthermore, the results of these latter studies were inconsistent with regard to onset of ESC effects during ontogenetic development: Whereas a number of studies suggested the ESC effect to occur already for 3-year olds in some task versions (Jovanovic and Schwarzer, 2011; Knudsen et al., 2012), increasing until the age of about 10 years (Thibaut and Toussaint, 2010; Jongbloed-Pereboom et al., 2013; Wunsch et al., 2015), other studies did not find evidence for anticipatory planning in 2- up to 7-year-old children (Smyth and Mason, 1997; Manoel and Moreira, 2005; Adalbjornsson et al., 2008; Van Swieten et al., 2010). The present study aimed at investigating a potential reason for these disparities in the literature by addressing the relation of ESC performance and executive functions, because differences in ESC performance might mirror differences in executive functioning, as outlined in the following.

The results of studies on motor planning and the ESC effect mentioned above indicate that humans, as well as non-human animals, grasp objects in a way that reflect their intentions. Therefore, studying grasping actions provides a window into internal planning processes. Motor planning, in turn, relies on cognitive control as can be inferred from clinical research. For example, it has been shown that motor deficiencies in people suffering from cerebral palsy do not only relate to movement execution, but also to motor planning (Mutsaarts et al., 2006). It can also be concluded from studies using dual-task paradigms. Here, simultaneously performing object manipulation and memory tasks showed a reduced recall ability, which suggests that planning for grasping objects needs (competes for limited) cognitive resources (e.g., Weigelt et al., 2009; Logan and Fischman, 2011; Spiegel et al., 2012). These findings seem to suggest a close link between anticipatory planning and executive functions (EF).

$\mathrm{EF}$ is an umbrella term that incorporates a collection of interrelated processes underlying purposeful, goal-directed behavior (Gioia et al., 2001). These executive processes are essential for the formation and maintenance of goals and strategies, preparation for action, and verification that plans and actions have been implemented appropriately (Luria, 1973). Results from studies using different EF tasks revealed that they can be explained in terms of three to four underlying factors (Levin et al., 1991; Welsh et al., 1991; Kelly, 2000). Based on these results, Anderson (2002) proposed a model of EF, which describes EF as four distinct domains: (1) attentional control, (2) information processing, (3) cognitive flexibility, and (4) goal setting. These functions are assumed to work in an integrative manner, in order to execute certain tasks, and that they can be conceptualized as an overall control system.

Executive processes further develop with the biological maturation of the frontal cortex throughout childhood and adolescence (Stuss, 1992). This development can be described as a multi-stage process, with different developmental trajectories for different functions (Passler et al., 1985). Therefore, EF play an important role in children's cognitive functioning, behavior, emotional control, and social interaction, but develop at different rates and at different times, differently for children's individual development. Accordingly, differences in grip selection between adults and children have been construed as indicating a deficit in children's planning skills (see Hughes, 1996; Smyth and Mason, 1997), with the presence of ESC as an indicator for "thinking ahead," referring somewhat ambiguously to some kind of planning abilities. This led to an important debate, initially raised by Van Swieten et al. (2010), about whether performance in grip selection tasks is driven by executive planning (i.e., actively planning ahead to solve actions correctly or to avoid mistakes, for example) or motor planning (i.e., planning motor actions in advance in order to solve them correctly or most economically), or both (Stöckel et al., 2012; Scharoun and Bryden, 2013). Van Swieten et al. (2010) argued that the ESC effect cannot fully rely on executive planning, because adults do not consistently select grasps which end in comfortable positions. Thus, if executive planning was the driving force, executive functions would fail on some trials, but not on others, which is unlikely to be the case. Instead, Van Swieten et al. (2010) proposed that grip selection relies predominantly on pure motor planning processes and that the most efficient movement is selected for each grasp. On the other hand, it is commonly assumed that motor skills are very similar to intellectual skills in terms of acquisition and representation (Rosenbaum et al., 2001), which suggests the exact opposite of the aforementioned argument. In this regard, it has been assumed for a long time, that perceptual-motor skills and intellectual skills have closely 


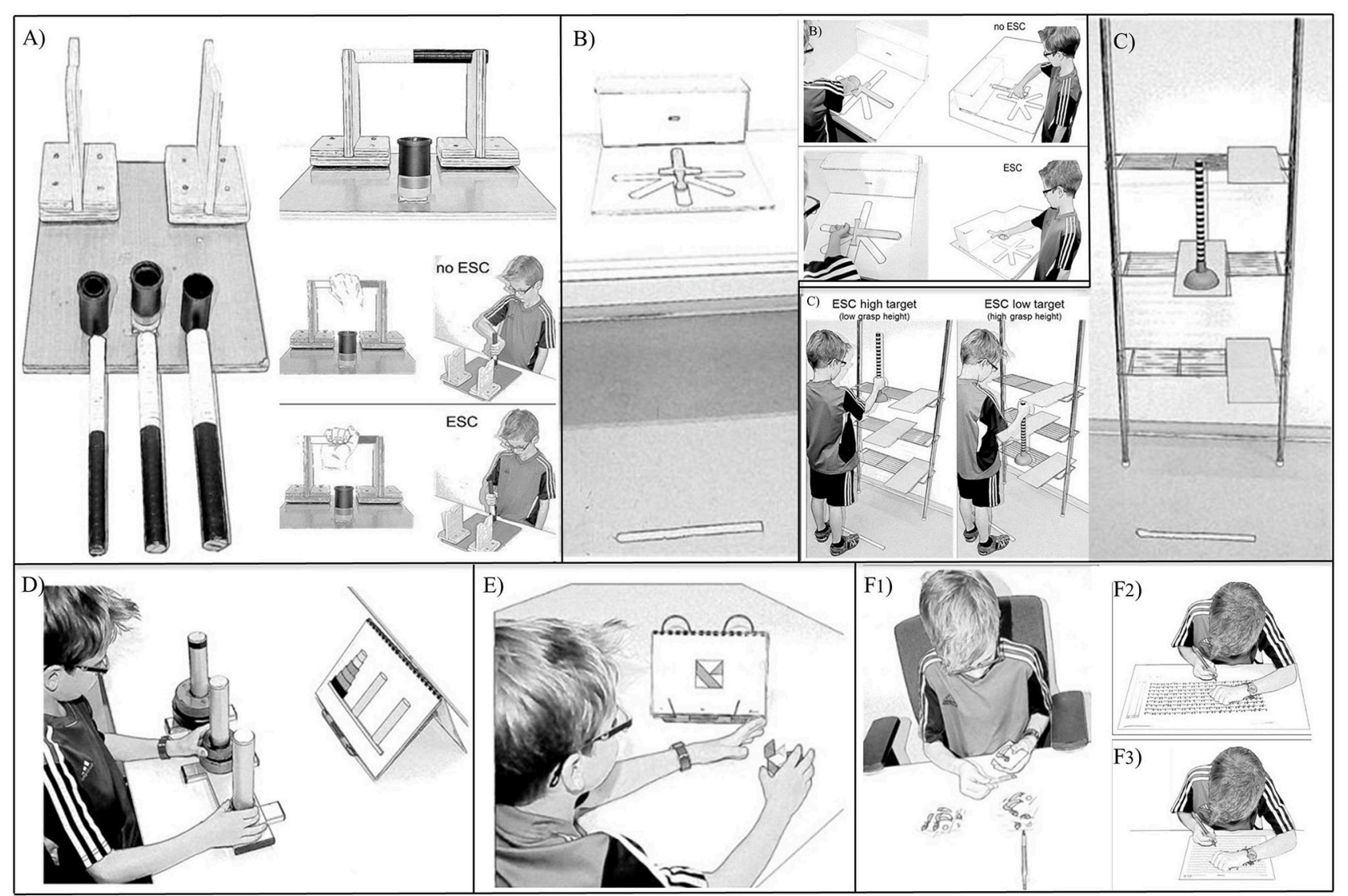

FIGURE 1 | Overview of the tasks used in the study and corresponding action outcomes for the motor tasks. (A) bar-transport-task, (B) sword-rotationtask, (C) grasp-height-task, (D) Tower-of-Hanoi-task, $(\mathbf{E})$ Mosaic-task, $\left(\mathbf{F}_{\mathbf{1}}\right)$ D2-task for preschoolers, $\left(\mathbf{F}_{\mathbf{2}}\right)$ D2-task for school children, $\left(\mathbf{F}_{\mathbf{3}}\right)$ D2-task for adults.

related developmental origins, as already noted by Piaget (1952), who based the development of intelligence upon the emergence of skilled action.

A strong interrelation between perceptual-motor skills and intellectual skills also follows from embodied or grounded accounts of cognition and action (Barsalou, 2008; Borghi and Caruana, 2015). These accounts assume that behavioral decisions and cognitive operations alike are guided by modal simulations. Thus, even high-level functions, such as working memory, are assumed to be based on sensorimotor mechanisms (Borghi, 2005). In this perspective, executive functions would develop during, and because of, sensorimotor interactions with the environment. In contrast to the above-stated suggestion that EFs might resemble the driving force behind ESCrelated motor planning, one could therefore argue that the development of executive functions might be driven by motor interactions with the environment instead. This prediction is not a necessary implication of embodied accounts, however: Once a particular function is developed, it may again influence more basic processes, such as motor planning. In any case, embodied accounts of cognition and action would propose a rather strong coupling of EFs and ESC performance.
Despite these theoretical arguments for a strong coupling of motor and cognitive skills, only few studies investigated this relationship directly. In a study conducted by Jenni et al. (2013), children between 7 and 18 years were tested, using the Zurich Neuromotor Assessment (ZNA) test and other, standardized intelligence tests, only weak correlations could be found between the performance in both, motor and cognitive tasks, which led the authors to suggest that motor and intellectual domains are largely independent. Another recent study conducted by Gonzalez et al. (2014) examined children between 5 and 10 years, using the Behavioral Rating Inventory of Executive Function (BRIEF) and two motor tasks with a focus on grasping. Results revealed significant correlations between the strength of right hand preference for grasping and numerous elements of the BRIEF, showing an interconnectedness of lateralization and EF. Moreover, Jansen (2014) conducted a review study, where she summarized results on studies on the relationship of motor activity and cognitive functions. She concluded that there is a positive effect of motor activity on the development of EF, and that specific physical activity can help to enhance specific cognitive functions in children. Altogether, motor (or better: physical) activity can play an important role in the development of EF and 
therefore, can act as a mediator on the relationship of ESC and $\mathrm{EF}$.

Altogether, the question whether EF development may (at least partly) predict or influence the development of motor planning in terms of the ESC effect is still unanswered (see also Stöckel et al., 2012; Scharoun and Bryden, 2013). The similarity of the developmental trajectories of EF and the ESC effect suggests a potential relationship in their development (see Anderson, 2002, for the projected developmental trajectory of the different EF, and Wunsch et al., 2013, for an overview of the developmental trajectories for the different ESC tasks). Based on the assumption that ESC and EF are related, the present study investigated the possible role of executive functioning on the developmental trajectory of the ESC effect. To this end, we presented eight groups of children and a group of adults with three ESC tasks to assess their motor planning abilities and three EF tasks to measure their cognitive planning abilities. We predicted (1) an increase of task performance in each (ESC) subtest as children get older, (2) substantial inter-correlations between the ESC tasks, as well as, (3) positive correlations in each age group between participants' performance on ESC and EF tasks.

\section{METHODS}

\section{Participants}

Nine age groups with a total of 217 participants were recruited. For a detailed overview of participant's demographics please see Table 1. All children were recruited from local daycare centers, elementary schools or via announcements in a local newspaper in Paderborn, Germany; all adults were students at the University of Paderborn. This study was carried out in accordance with the recommendations of the German Psychological Society (Deutsche Gesellschaft für Psychologie, DGPs). For all groups of children, parents provided their written informed consent for participation and for video recording their child during the experiment. All participants or their parents gave written informed consent in accordance with the Declaration of Helsinki. Participation was voluntary, without any financial compensation. Children received a personal certificate of participation and some sweets.

\section{Tasks and Procedures}

A test battery was designed to assess ESC planning and EF, consisting of three different tasks each: for the measurement of ESC we used (1) the bar-transport-task (Rosenbaum et al., 1990; Weigelt and Schack, 2010), in which participants were asked to grasp a horizontally oriented bar lying on two supports, with one black and one white end, and to insert one of its ends into a target hole in front of the support; (2) the sword-rotation-task (Rosenbaum et al., 1993; Crajé et al., 2010), in which participants had to insert a wooden sword, lying in different orientations in front of them on the table, into a target hole in a box behind it; and (3), for the first time in a child population, the graspheight-task (Cohen and Rosenbaum, 2004; Weigelt et al., 2007), in which participants had to transport a vertically oriented dowel (a toilet plunger) from a chest-high platform either to a higher

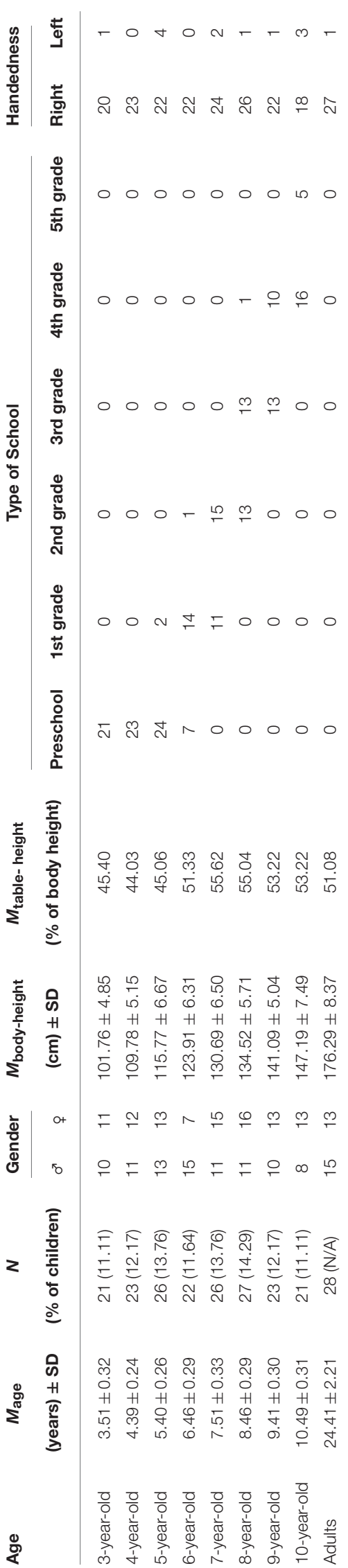


or to a lower platform. The EF tasks were chosen in accordance with the model of Anderson (2002): (1) the Tower-of-Hanoitask (Simon, 1975; Welsh, 1991), which fits into each domain of the model, as feedback utilization, selective attention, planning abilities, strategic organization, and processing speed are needed. Here, participants had to build a tower of discs, as shown by a target position, following several rules with increasing difficulty as trial number increased; (2) the Mosaic-task (Wechsler, 1997, 2002, 2003), which fits into three of the domains, requiring feedback utilization, selective attention, and processing speed. In this task, a given mosaic pattern had to be re-created with a set of building blocks, and (3) the D2-attention-endurance-test (Brickenkamp, 1962), where selective attention, inhibition, and the speed of processing are necessary to achieve good results. Here, "d's" (or ducks in the children's version; Grob et al., 2009, 2013) with a given attribute had to be sketched (or sorted) out.

For each task, the respective materials were placed on a table in front of the participant, except for the grasp-height-task, for which the shelf was placed next to the table. Participants stood in front of the table for the bar-transport-task and for the swordrotation-task, and in front of the shelf for the grasp-height-task. All EF tasks were performed at the same table, but this time with the participants sitting, while seat height was adjusted for each participant. Table height was $55 \mathrm{~cm}$ for kindergarten children and $75 \mathrm{~cm}$ for school children and adults. To adjust for differences in body height, children smaller than $110 \mathrm{~cm}$ stood on a $10 \mathrm{~cm}$ high podium, and children smaller than $120 \mathrm{~cm}$ or $130 \mathrm{~cm}$ stood on a 20 or $10 \mathrm{~cm}$ high podium in order to level the requirements in the ESC tasks. To control for differences in the relative table height compared to body height of participants, mean table heights were computed, indicating the relative table height compared to body height (including the podium height in percent) of participants (please see Table 1). Relative table height was between 44 and $55 \%$ of individual body height, meaning that the apparatus for the ESC tasks was located at body-center $( \pm 5 \%)$. A camera was positioned $150 \mathrm{~cm}$ besides the participant, at a height of $160 \mathrm{~cm}$, and recorded the whole experiment for later coding.

Participants were tested individually by one experimenter. For several preschoolers, a teacher or a parent was also present in order to make the child feel comfortable. Prior to testing, adult participants or children's parents completed a short questionnaire on handedness, on how they completed their way to kindergarten/school/university, and on leisure time activities, sport participation, and spoken languages. Afterwards, children completed a short test to determine handedness as the hand that was used in at least two out of the three tasks (to throw a ball, use a spoon, and write/draw with a pencil). This hand was marked with an ink stamp. In the ESC tasks, children were instructed to always use the "stamp-hand." The ESC tasks were run without familiarization trials. The order of all six tasks was randomized across participants. In the ESC tasks, participants stood in front of the table or the shelf, $10 \mathrm{~cm}$ away from the edges, respectively, or sat in front of the table with the respective apparatus on it for the EF tasks, with materials $10 \mathrm{~cm}$ away from the edge of the table. On average, the entire session lasted between 90 and $100 \mathrm{~min}$. The duration differed according to participant's time needed to complete the different tasks and according to requests for breaks between the subtests. In general, most of the adults were able to complete the testing session in about $75 \mathrm{~min}$, whereas some children needed up to $150 \mathrm{~min}$ to complete the whole session.

\section{Motor Tasks: Assessing ESC Bar-transport-task}

A modified version of the original bar-transport-task (Rosenbaum et al., 1990) was used, which was similar to the one employed by Weigelt and Schack (2010). Different to the original task version by Rosenbaum et al. (1990), there was only one target at midline in front of the apparatus (and not two targets on either side as in the original task). A wooden bar, 20 $\mathrm{cm}$ long, with one black and one white end rested horizontally on two cradles, $15 \mathrm{~cm}$ above the table. A $5 \mathrm{~cm}$ high, black cylindrical container served as the movement target and was placed 10 $\mathrm{cm}$ in front of the support. To keep precision requirements comparable across age groups, the bar's diameter measured $1.5,2$, or $2.5 \mathrm{~cm}$ for preschoolers, school-children and adults, respectively, and the target hole's diameter measured 2, 2.5, or $3 \mathrm{~cm}$ for preschoolers, school-children and adults, respectively (see Figure 1A).

The start orientation of the bar (i.e., black or white end on the right side) was counterbalanced across participants and remained constant throughout the experiment. Participants were instructed to adopt the starting position (i.e., to stand behind the line with their hands facing their legs), to then grasp the bar firmly with their "stamp-hand," and to insert the black or white end of the bar into the target hole, as indicated by the experimenter. After the insertion, they were instructed to return to the starting position. To prevent observational learning, the experimenter used a pincer grip at one end of the bar to reposition the bar back on the two cradles. Participants completed six trials in randomized order, three trials for each end. They could use either an overhand or an underhand grip to grasp the bar. This resulted in either an upright (thumbup) or an inverted (thumb-down) hand position at the end of the movement. In the three uncritical trials, an overhand grip automatically resulted in a comfortable thumb-up position; in the three critical trials, however, an underhand grip was necessary to end in the comfortable thumb-up position and therefore, in ESC. Grip choice was coded from the video. Following recent studies (Adalbjornsson et al., 2008; Weigelt and Schack, 2010; Stöckel et al., 2012), we considered the ESC effect to be present if in a given condition (critical and uncritical trials) at least two out of the three trials ended in the comfortable position, resulting in a dichotomous outcome.

\section{Sword-rotation-task}

A variation of the original handle-rotation-task by Rosenbaum et al. (1993) was used, which was similar to the task versions created by Crajé et al. (2010) and by Jongbloed-Pereboom et al. (2013). A wooden sword $(30 \mathrm{~cm}$ in length, $3.2 \mathrm{~cm}$ in width, and $0.8 \mathrm{~cm}$ in height; handle length $=10 \mathrm{~cm}$ ) was horizontally placed on a platform $(47 \times 47 \mathrm{~cm})$ in front of a target box in one of six start positions (Position $1=0^{\circ}$ (12 o'clock position), Position $2=90^{\circ}$, Position $3=135^{\circ}$, Position $4=180^{\circ}$, Position $5=225^{\circ}$, and Position $6=270^{\circ}$ ). The sword's blade had to be inserted into 
a tight fitting hole in a wooden block ( $47 \mathrm{~cm}$ in length, $16 \mathrm{~cm}$ in width, and $16 \mathrm{~cm}$ in height; hole: $3.5 \times 1 \mathrm{~cm}$; see Figure 1B). The same apparatus was used for all age groups.

Again, participants were instructed to adopt the startingposition and were told that they were a pirate (to adults this task was explained without the cover story) and that they had to insert the sword into the box by firmly grasping the handle in exactly the position it laid on the table, that is, without turning the sword before grasping it. Each session started with Position 1 to make sure participants understood the task. The experimenter retrieved the sword from the box and repositioned it on the platform always by grasping it at the cross guard to avoid observational learning. The task consisted of three blocks of 6 trials (one for each position), resulting in a total of 18 trials. Trial positions were randomized in each block. Participants could choose grips that resulted in either a comfortable end position (with the thumb pointing toward the blade) or an uncomfortable end position (with the thumb pointing away from the blade). Within each block, two trials were critical. Here, grasping the sword in a more uncomfortable hand position (at Positions 2 and 3) resulted in a comfortable end position. Grip choice was coded from the video. We considered the ESC effect to be present if at least 4 out of the 6 critical trials ended in a comfortable position platform (dichotomous outcome).

\section{Grasp-height-task}

This study is the first to examine the grasp-height effect in children. An adaptation of the original grasp-height-task by Cohen and Rosenbaum (2004) was used, which was similar to the version employed by Weigelt et al. (2007). Participants had to transport a standard toilet plunger from a home platform to a lower or higher target platform, and back to the home platform. The stem of the plunger was painted black and white in an alternating order, with a distance of $1 \mathrm{~cm}$ between each stripe. Board heights were individually adjusted to participants' body height by taking the board heights and average height of adult participants reported in Rosenbaum et al. (2006) as reference. For example, for an $87 \mathrm{~cm}$ tall child, shelf heights were 25.4, 43.2, and $61.0 \mathrm{~cm}$ for the low, middle and high shelf, respectively. On each shelf board, a wooden platform was attached in such a way that it protruded $15 \mathrm{~cm}$ from the shelf. The home platform was attached to the horizontal center of the middle shelf board. The two target platforms were attached to the participant's side of handedness, one on the low and the other one on the high shelf board. The toilet plunger stood on the home platform: a circular rubber base (10 $\mathrm{cm}$ in diameter and $5 \mathrm{~cm}$ high) supported the cylindrical wooden shaft $(2.5 \mathrm{~cm}$ in diameter and $33 \mathrm{~cm}$ or $44 \mathrm{~cm}$ in length for child or adult participants, respectively, see Figure 1C).

Participants were told to adopt the starting position (standing behind the line, with hands facing their legs). Child participants were presented a cover-story suggesting that their performance was videotaped in this task in order to program a robot afterwards, which could then perform the same actions as they did. Participants' task was to stand in the start position, to grasp the plunger firmly on the shaft and to transport it to the platform indicated by the experimenter (home-to-target moves). Afterwards, they had to resume the starting position and were then instructed to bring the plunger back to the home platform (target-to-home moves). Participants were told that they needed to closely follow the instructions and move the plunger on the direct way to the named platform, and that home and target platform could differ across trials. A total of six trials had to be completed, with three movements to each of the target platforms. Conditions were blocked, with the start platform for the first three trials counterbalanced across participants. Grasp height was coded from the video by counting the distance from the plunger base to the hand of the participants. Participants showed planning for ESC if they grasped the plunger lower for the hometo-target moves than for the target-to-home moves for the high platform, and higher for the home-to-target moves than for the target-to-home moves for the low platform. ESC was considered to be present if participants showed this pattern in at least two out of the three trials for each platform (dichotomous outcome). Also, mean differences in grasp heights between home-to-target and target-to-home moves in the two conditions were computed and used for all correlation analyses. Ideal ESC performance would result in mean differences of zero, whereas positive or negative numbers indicate insufficient planning. For example, if the plunger was (initially) grasped slightly too high in the home-to-target moves for the upper target, it could then only be placed at the final position in a somewhat awkward body posture (greater stretch). Likewise, if the plunger was (initially) grasped slightly too low in the home-to-target moves for the lower target, it could only be placed while bending the upper body, which is also more uncomfortable.

\section{Cognitive Tasks: Assessing EF}

\section{Tower-of-Hanoi-task}

We used a slightly modified version of the original task (Simon, 1975), which was similar to Welsh (1991), but used only one apparatus as in the original version. It consisted of 3 pegs in a row (height: $23 \mathrm{~cm}$, diameter: $4 \mathrm{~cm}$, distance between pegs: $15 \mathrm{~cm}$ ) that were attached on a bottom plate. On these pegs, up to five discs of varying size and color could be located: black (diameter: $13 \mathrm{~cm}$ ), blue (diameter: $11 \mathrm{~cm}$ ), green (diameter: $9 \mathrm{~cm}$ ), red (diameter: $7 \mathrm{~cm}$ ) and yellow (diameter: $5 \mathrm{~cm}$ ). The target position was indicated by a picture, displayed at a $75^{\circ}$ angle on a music stand, $20 \mathrm{~cm}$ behind the apparatus (see Figure 1D). The peg on the very right was the target peg, and was marked with a black duct tape at the top.

Participants were instructed to build a tower of discs as shown by the target position on the picture, starting from the arrangement presented. Each participant was to solve up to 10 different tower problems, with increasing degree of difficulty. Depending on the number of discs and on the starting arrangement, trials differed in the minimal number of moves necessary to complete the tower. Three test versions were created with difficulty adjusted to age: a version for 3- and 4-year-olds, for 5- and 6-year-olds, and for 7-year-olds and older children and adults. Every test started with a familiarization trial with three discs $(2,3$, or 6 moves according to the test version), in which rules were explained and questions could be clarified. Participants had to follow three rules: (1) move only one disc at a time, (2) a disc may only be in your hand or on a peg, but not on the table 
or somewhere else, and (3) a smaller disc can be placed on top of a bigger disc, but a bigger disc cannot be placed on top of a smaller disc. In test trials, each starting position was initially covered by placing a cardboard in front of the apparatus to assess latency. The task was terminated whenever participants were not able to solve a tower problem in up to twice the minimal number of moves necessary to solve the problem, or if participants did not move any disc for more than $90 \mathrm{~s}$. The single tower problems included 3 or 4 discs in the two easier test versions, and 4 or 5 discs in the most difficult version. Minimal number of moves necessary ranged between 2 and 15 for 3 - and 4-year-olds, between 4 and 15 for 5 - and 6-year-olds, and between 7 and 31 for children aged 7 and older, and adults ${ }^{1}$. Start- and end-positions of the discs were checked from the video and the number of steps to complete the tower was counted. Number of tower problems completed correctly served as dependent measure.

\section{Mosaic-task}

This task is a subtest of the Wechsler Scale of Intelligence ${ }^{2}$. In the Mosaic-task, a given mosaic pattern has to be re-created with a set of building blocks. The target picture or the 3D model was positioned $18 \mathrm{~cm}$ away from the edge of the table, $10 \mathrm{~cm}$ to the side of participants' body midline on the opposite side of handedness. Participants had to arrange up to nine cubes (side length: $2.5 \mathrm{~cm}$ ) of different colors (all red sides, all white sides or red and white sides) (see Figure 1E). The test was administered in accordance with the test manual and age group. Completion time was coded from the video and performance was checked for accuracy. The percentage of scored points was calculated as indicated in the test manual.

\section{D2-attention-endurance-test}

Three versions of this speeded test of selective attention (Brickenkamp, 1962) were used: the analogous subtests in the Intelligence and Development Scales for preschool children (IDS-P; Grob et al., 2013) and for school children (IDS, Grob et al., 2009), and the D2-R for adults (Brickenkamp et al., 2010). Preschool children's task was to sort cardboard cards $(6 \times 6$ $\mathrm{cm}$ ) showing a duck, according to the presence or absence of a distinct characteristic. A pencil, lying $25 \mathrm{~cm}$ away from the child at the side of their handedness indicated where to stack the cards with the given characteristic (see Figure $\mathbf{1 F}_{1}$ ). In the paper-pencil version for school children, participants were presented with a

\footnotetext{
${ }^{1}$ For each age group, problem difficulties were as follows, whereby the first number always indicates the number of discs used, the second number always indicates the number of moves for optimally solving the problem: $3-2,3-3,3-4,3-5,3-6,4-7,4-8$, $4-9,4-10,4-11$, and $4-15$ for 3- and 4-year olds; 3-4, 3-5, 3-6, 4-7, 4-8, 4-9, 4-10, $4-11,4-13,4-15$ for 5 - and 6-year olds; and 4-7, 4-9, 4-10, 4-11, 4-13, 4-15, 5-20, 5-24, 5-27, and 5-31 for children older than 7 years and adults.

${ }^{2}$ The Mosaic-task is a subtest of the HAWIVA ${ }^{\circledR}$-III [Hannover Wechsler Intelligenztest für das Vorschulalter; German translation and adaption of the WPPSI ${ }^{\circledR}$-III (Wechsler Primary and Preschool Scale of Intelligence) of David Wechsler (2002); Ricken et al. (2007); for preschool children], the HAWIK ${ }^{\circledR}$-IV [Hamburg Wechsler Intelligenztest für Kinder; German translation and adaption of the WISC ${ }^{\circledR}$-IV (Wechsler Intelligence Scale for Children) of David Wechsler (2003); Petermann and Petermann (2008); for school children] and the WIE ${ }^{\circledR}$ [Hamburg Wechsler Intelligenztest für Erwachsene; German translation and adaption of the WAIS ${ }^{\circledR}$-III (Wechsler Adult Intelligence Scale) of David Wechsler (1997); Von Aster et al. (2006); for adults] to assess visuospatial and motor skills.
}

DIN A3 sheet of paper showing rows of ducks with or without distinct characteristics. Children had to mark the ducks with the target characteristics (see Figure $\mathbf{1 F}_{2}$ ). In the paper-pencil version for adults, rows of the letters $p$ and $d$ were presented on a DIN A4 sheet of paper, and letters with distinct characteristics had to be marked (see Figure $\mathbf{1 F}_{3}$ ). Videos of preschool children were checked to verify the number of properly sorted cards. Performance was scored in accordance with the respective test manual. According to the test manual, the total number of scored points served as dependent measure.

\section{Data Analysis/Scoring}

Chi-Square tests were used to examine group differences in the ESC tasks due to the dichotomous nature of the dependent variables. For the $\mathrm{EF}$ tasks $^{3}$, comparison across age groups is difficult due to the usage of different task versions and therefore are not reported in the results section. To test for age effects on ESC performance, we further conducted regression analyses on the mean percentage of participants who showed ESC in each age group. Finally, bivariate Pearson's correlations were computed in order to find possible relationships within the ESC tasks and the EF tasks, and between these motor and cognitive tasks. It should be noted that, for the current sample sizes, the individual correlations within each age group come with sufficient power only for large correlations, whereas the assessment across all age groups ensures sufficient power also to detect rather small effect sizes $(1-\beta=0.80$ for $r=0.19$ with two-tailed tests, as determined via the pwr package in R; Champely, 2015). The findings within each group should thus be treated with caution, whereas there is good reason to interpret the overall finding of a null-correlation as evidence for a true null effect (rather than a Type II error).

\section{RESULTS}

\section{The Development of ESC Bar-Transport-Task}

Figure 2 illustrates the mean percentage of participants in each group who showed ESC planning. In the uncritical trials, all participants in all age groups adopted an overhand grasp in at least two out of the three uncritical trials and therefore ended in a comfortable end position. In the critical trials, only $24 \%$ of the 3 -year old children showed sensitivity for ESC planning. This amount increased up to $62 \%$ in the 5 -year-olds (see Table 2). Here, a stagnation of the developmental trajectory can be seen in 5-to-8-year old children, with a mean of $63 \%$ showing ESC planning. Then, the percentage of participants showing

\footnotetext{
${ }^{3}$ We measured different dependent variables for the Mosaic and the Tower of Hanoi tasks, e.g. the total amount of correctly solved items, the total score and the score with bonus-points for fastest resolve in the Mosaic task, and the amount of incorrect moves, the number of correctly solved items or the number of the item where the first mistake was made before doing the first move in the Tower of Hanoi task. Due to their correlations (Mosaic-task: $r=0.229$ for correctly solved items and total score; $r=0.194$ for correctly solved items and bonus-score, and $r=0.950$ for total score and bonus-score; all $p<0.01$; Tower-of-Hanoi-task: $r=$ 0.261 for the amount of incorrect moves and the item with the first mistake, $r=$ 0.149 for the amount of incorrect moves and the number of correctly solved items, and $r=0.218$ for the item with the first mistake and the number of correctly solved items; all $p<0.05$ ), however, we chose the most superordinate variable for further analysis.
} 
ESC increased again up to $95 \%$ in the 10 -year-olds, which is comparable to adult behavior. A chi-square analysis showed these differences in the proportion of children showing ESC in the critical trials to be significant, $\chi_{(7)}^{2}=34.93, p<0.001$.

A regression analysis of the percentage of participants showing ESC across the child groups revealed the developmental trajectory in the critical trials to be statistically significant with an increase of $7.8 \%$ per year, $t_{(6)}=5.622, p=0.001$. The entire regression model including the intercept yielded an adjusted $R^{2}=0.814, F_{(1,7)}=31.608, p<0.001$. Single chi-square fourfield tests revealed that all children groups up to the age of 9 years behaved significantly less often in terms of ESC than adults did; $\chi_{(1)}^{2}=27.93, p<0.001$ for the 3-year-olds; $\chi_{(1)}^{2}=15.71, p<0.001$ for the 4-year-olds; $\chi_{(1)}^{2}=10.12, p<0.01$ for the 5-year-olds; $\chi_{(1)}^{2}$ $=8.98, p<0.01$ for the 6-year-olds; $\chi_{(1)}^{2}=10.12, p<0.01$ for the 7 -year-olds; $\chi_{(1)}^{2}=9.62, p<0.01$ for the 8-year-olds; $\chi_{(1)}^{2}=4.02$, $p<0.05$ for the 9 -year-olds). There was no difference in behavior between 10-year-olds and adults $\left[\chi_{(1)}^{2}=0.04, p>0.05\right]$.

Considering all children, a Kruskal-Wallis Test revealed the differences between the age groups to be statistically significant across all three trials [Trial $1 \chi_{(7)}^{2}=28.62, p<0.01$; Trial 2 $\chi_{(7)}^{2}=27.71, p<0.001$; Trial $\left.3 \chi_{(7)}^{2}=26.76, p<0.001\right]$. This shows the distinct developmental trajectory for ESC planning. Trial repetition data (i.e., whether children change their grip behavior across the trial repetitions and thus, exhibit short-term learning effects over the course of the three critical trials), is depicted in Figure 3.

Concerning these results, there is no systematic pattern detectable, suggesting that short-term learning effects may rather not have occurred across the three trials.

\section{Sword-Rotation-Task}

Figure 4 illustrates the mean percentage of participants in each group who showed ESC planning for both conditions. In the uncritical trials, all participants adopted a grip with the thumb being oriented toward the blade, and thus, ended in a comfortable position when inserting the sword into the box. In the critical trials, however, only $43 \%$ of the 3 - and 4 -year old children showed sensitivity for ESC planning. This amount increased up to $64 \%$ in the 6-year-olds. Again, similar to the bar-transport-task, even if more delayed, a stagnation of the developmental trajectory can be detected in 7-to-10-year old children, with a mean of $75 \%$ of the children showing ESC. All adults showed ESC in the six critical trials (see Table 2). A chi-square analysis showed these effects in the proportion of children showing ESC in the critical trials to be marginally significant, $\chi_{(7)}^{2}=12.89, p=0.075$.

A regression analysis of the percentage of participants showing ESC revealed the developmental trajectory in the critical trials to be statistically significant with an increase of ESC conform grasps of $5.5 \%$ per year, $t_{(6)}=7.156, p<0.001$, adjusted $R^{2}=$ $0.878, F_{(1,7)}=51.209, p<0.001$. Single chi-square four-field tests revealed that all children groups behaved significantly less often in terms of ESC than adults did; $\chi_{(1)}^{2}=21.19, p<0.001$ for the 3 -year-olds; $\chi_{(1)}^{2}=21.24, p<0.001$ for the 4-year-olds; $\chi_{(1)}^{2}=$ $14.88, p<0.001$ for the 5-year-olds; $\chi_{(1)}^{2}=12.12, p<0.001$ for the 6-year-olds; $\chi_{(1)}^{2}=8.66, p<0.01$ for the 7-year-olds; $\chi_{(1)}^{2}=$ 4.24, $p<0.05$ for the 8-year-olds; $\chi_{(1)}^{2}=6.75, p<0.01$ for the 9 -year-olds; and $\chi_{(1)}^{2}=7.42, p<0.01$ for the 10 -year-olds).

Considering all children, a Kruskal-Wallis Test did not consistently reveal the differences between the age groups to be statistically significant across all three trials in Position 2 [Trial 1 $\chi_{(7)}^{2}=7.22, p>0.05$; Trial $2 \chi_{(7)}^{2}=10.47, p>0.05$; Trial $3 \chi_{(7)}^{2}=$ 15.58, $p<0.05$ ] and in Position 3 [Trial $1 \chi_{(7)}^{2}=29.97, p<0.001$; Trial $2 \chi_{(7)}^{2}=11.44, p>0.05$; Trial $3 \chi_{(7)}^{2}=16.95, p<0.05$ ]. We also investigated whether children change their grip behavior across the trial repetitions and thus, exhibit short-term learning effects over the course of the six critical trials. Figure 5 depicts the percentage of children in each age group performing in a manner consistent with second-order motor planning in each of the three critical trials, in the left for Position 2, in the right for Position 3.

A closer examination of trial repetition data did not yield a systematic pattern. It is not the case that children started to plan for ESC with increasing trial number. Therefore, like in the bar-transport-task, short-term learning effects are unlikely to have improved participants' performance in terms of ESC.

\section{Grasp-Height-Task}

Results showed, that the percentage of participants showing ESC in the high target moves increased from $19 \%$ in the 3 -yearolds up to $45 \%$ in the 10 -year-olds, whereas $93 \%$ of the adults showed ESC planning. In the low target moves, the percentage of ESC conform grasps increased from $5 \%$ in the 3 -year-olds up to $26 \%$ in the 9 -year-olds, with 10 -year-olds showing less ESC. Interestingly, this developmental pattern does not follow a linear increase, with older children sometimes performing poorer than younger ones (see Table 2). Fifty percent of all adults showed ESC like planning. Thus, ESC planning seems to be more frequent for bringing the object to high positions than to low positions. But, even 10-year-old children showed only half as many grasp behaviors in terms of ESC as adults do. This hints to a rather late emergence of ESC planning for tasks exploiting a continuous task space.

A chi-square analysis revealed significant differences between the groups in the amount of participants showing ESC averaged across both positions $\left[\chi_{(7)}^{2}=120.59, p<0.001\right]$. A regression analysis of the mean differences in grasp height across the child groups revealed the developmental trajectory to be statistically significant with a slope of $1.81 \mathrm{~cm}$ per year, $t_{(6)}=12.986$, $p<0.001$, adjusted $R^{2}=0.878, F_{(1,7)}=166.011, p<0.001$. Single chi-square four-field tests revealed that all children groups behaved significantly less often in terms of ESC than adults did $\left[\chi_{(1)}^{2}=34.24\right.$ for the 3-year-olds; $\chi_{(1)}^{2}=27.21$ for the 4-year-olds; $\chi_{(1)}^{2}=36.86$ for the 5 -year-olds; $\chi_{(1)}^{2}=30.49$ for the 6-year-olds; $\chi_{(1)}^{2}=27.34$ for the 7-year-olds; $\chi_{(1)}^{2}=20.95$ for the 8-yearolds; $\chi_{(1)}^{2}=15.32$ for the 9-year-olds; and $\chi_{(1)}^{2}=17.70$ for the 10 -year-olds, all $p<0.001]$.

For further analysis, a score was computed for the mean differences in grasp height across both target positions (see Figure 6). First, the mean grasp heights for home-to-target and 


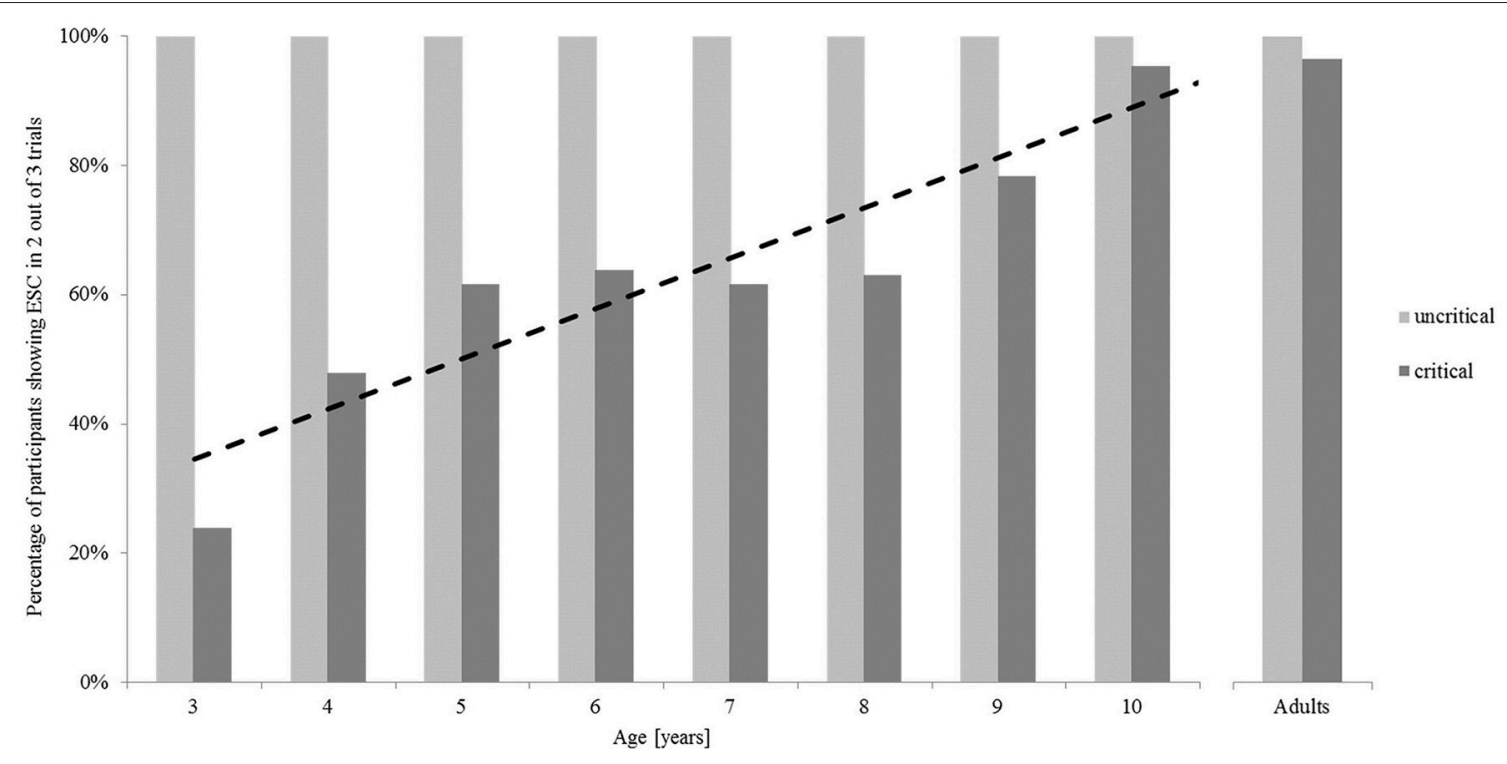

FIGURE 2 | Percentage of the participants in each group showing end-state comfort in at least two out of three trials in the uncritical (initial overhand grip) and critical (initial underhand grip) conditions in the bar-transport-task.

TABLE 2 | Detailed results of all age groups in the six different tasks.

\begin{tabular}{|c|c|c|c|c|c|c|c|c|c|c|}
\hline & \multirow{2}{*}{\multicolumn{2}{|c|}{$\begin{array}{c}\text { Bar-transport- } \\
\text { task } \\
\text { (\% of participants) }\end{array}$}} & \multirow{2}{*}{\multicolumn{2}{|c|}{$\begin{array}{c}\begin{array}{c}\text { Sword-rotation- } \\
\text { task }\end{array} \\
\text { (\% of participants) }\end{array}$}} & \multicolumn{3}{|c|}{$\begin{array}{c}\text { Grasp-height- } \\
\text { task }\end{array}$} & \multirow{3}{*}{$\begin{array}{c}\text { Tower-of-Hanoi- } \\
\text { task } \\
\text { (\% of solved } \\
\text { items) }\end{array}$} & \multirow{3}{*}{$\begin{array}{l}\text { Mosaic- } \\
\text { task } \\
\text { (\% of } \\
\text { points) }\end{array}$} & \multirow{3}{*}{$\begin{array}{c}\text { D2-task } \\
\text { (\% of } \\
\text { points) }\end{array}$} \\
\hline & & & & & \multicolumn{2}{|c|}{ (\% of participants) } & \multirow{2}{*}{$\begin{array}{c}M_{\text {differencce }} \\
\text { in grasp } \\
\text { height }(\mathrm{cm})\end{array}$} & & & \\
\hline & Uncritical & Critical & Uncritical & Critical & HtT-moves & TtH-moves & & & & \\
\hline 3-year-olds & 100.00 & 23.81 & 100.00 & 42.86 & 19.05 & 4.76 & -15.15 & 12.86 & 37.58 & 36.31 \\
\hline 4-year-olds & 100.00 & 47.83 & 100.00 & 43.48 & 34.78 & 4.35 & -14.71 & 23.91 & 48.47 & 47.52 \\
\hline 5-year-olds & 100.00 & 61.54 & 100.00 & 57.69 & 19.23 & 7.69 & -12.92 & 45.77 & 71.15 & 28.12 \\
\hline 6-year-olds & 100.00 & 63.64 & 100.00 & 63.64 & 31.82 & 0.00 & -10.77 & 53.64 & 55.05 & 36.56 \\
\hline 7-year-olds & 100.00 & 61.54 & 100.00 & 73.08 & 38.46 & 3.85 & -8.61 & 41.92 & 51.38 & 45.38 \\
\hline 8-year-olds & 100.00 & 62.96 & 100.00 & 74.07 & 33.33 & 22.22 & -5.35 & 47.78 & 69.00 & 52.54 \\
\hline 9-year-olds & 100.00 & 78.26 & 100.00 & 78.26 & 39.13 & 26.09 & -4.66 & 49.13 & 75.24 & 64.37 \\
\hline 10-year-olds & 100.00 & 95.24 & 100.00 & 76.19 & 45.45 & 13.64 & -3.51 & 56.67 & 85.51 & 71.35 \\
\hline Adults & 100.00 & 96.43 & 100.00 & 100.00 & 92.86 & 50.00 & 4.68 & 66.07 & 67.70 & 43.12 \\
\hline
\end{tabular}

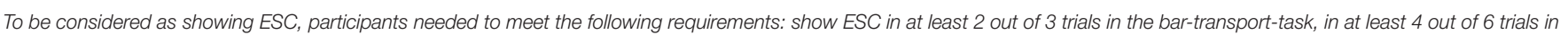
the sword-rotation-task, and in at least 2 out of 3 moves to either platform in the grasp-height-task. HtT, Home-to-Target-moves; TtH, Target-to-Home-moves.

for target-to-home moves were computed for both, high target and low target trials. For both, the differences of the mean grasp heights were computed. The means from these two differences were multiplied by $(-1)$. This resulted in the distribution shown in Figure 6.

Considering all children, a Kruskal-Wallis Test revealed the differences between the age groups to be statistically significant in Trial 1 and Trial 3 for the high target platform [Trial $1 \chi_{(7)}^{2}=$ 17.07, $p<0.05$; Trial $2 \chi_{(7)}^{2}=11.37, p>0.05$; Trial $3 \chi_{(7)}^{2}=$ 22.09, $p<0.01$ ] and in all trials for the low target platform [Trial $1 \chi_{(7)}^{2}=21.78, p<0.01$; Trial $2 \chi_{(7)}^{2}=25.48, p<0.001$; Trial $\left.3 \chi_{(7)}^{2}=28.98, p<0.001\right]$. Again, it was investigated whether participants change their grasp height across the trial repetitions and thus, exhibit short-term learning effects over the course of the six trials. Figure 7 depicts the percentage of children in each age group performing in a manner consistent with second-order motor planning in each of the three trials, in the left graph for the high target platform, in the right graph for the low target platform.

The results show a similar pattern as observed in the other ESC tasks, as there was no systematic pattern of ESC development in grasp height. Therefore, like in the bar-transport-task and in the sword-rotation task, short-term learning effects are unlikely to have improved participants' performance in terms of ESC. 


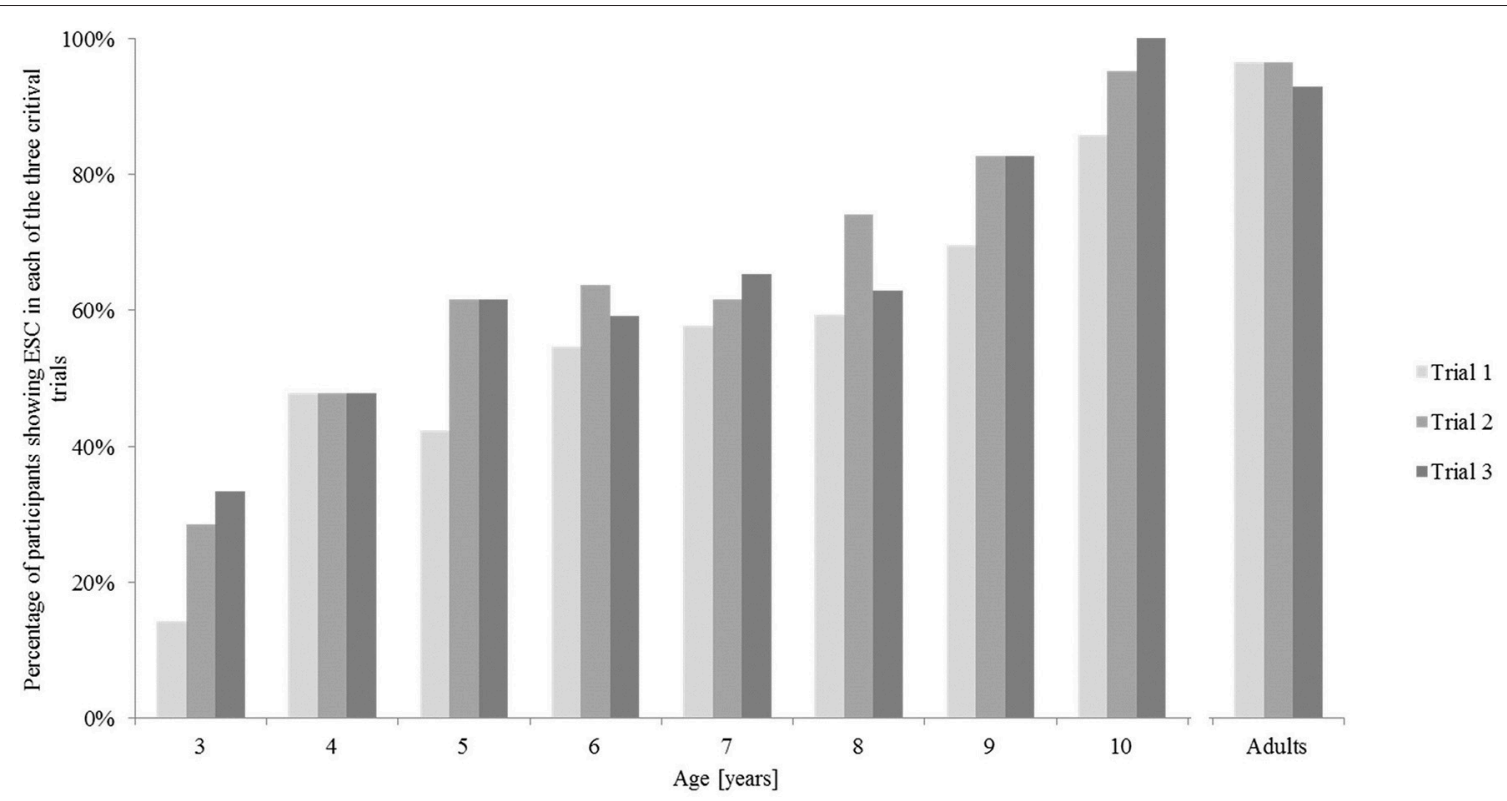

FIGURE 3 | Percentage of participants showing end-state comfort in each of the three critical trials in the bar-transport-task.

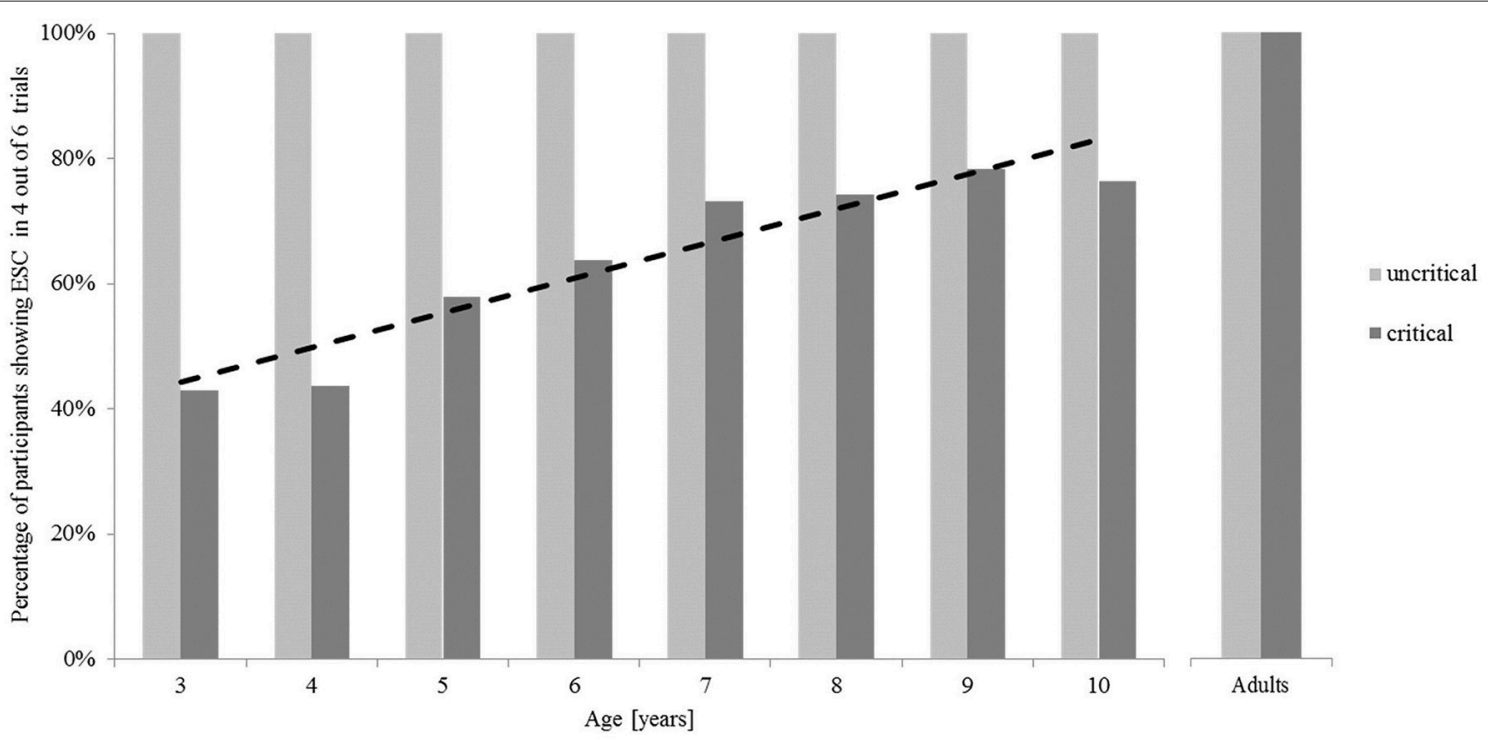

FIGURE 4 | Percentage of participants in each group showing end-state comfort in the sword-rotation task in at least seven out of the 12 uncritical trials and in at least four out of the six critical trials.

In summary, Table 2 provides a detailed overview over the results of all age groups in the six different tasks.

\section{Intercorrelations of the ESC Tasks}

The results above suggested similar developmental trends for ESC performance for the different ESC tasks. Consequently, the question arises whether the different measures of ESC performance are interrelated. For this reason, we computed pairwise correlations between the dichotomous variables for the bar-transport-task and the sword-rotation-task and the continuous variable for grasp height differences (see Table 3) ${ }^{4}$.

${ }^{4}$ Following a suggestion of a reviewer, we verified all bivariate correlations for both ESC tasks with binary outcome measures, i.e., the bar-transport task and the sword rotation task, with the analogous test of the phi coefficient against the $\mathrm{X}^{2}$-distriubtion (with $t_{\text {Pearson }}^{2}=\frac{(n-2) \mathrm{X}^{2}}{n-\mathrm{X}^{2}}$ ). Also, for both tasks, we evaluated the relation to measures of EF via the glmer function of the lme4 package in R. Results mirrored the correlation analyses reported in Tables 3, 4 . 

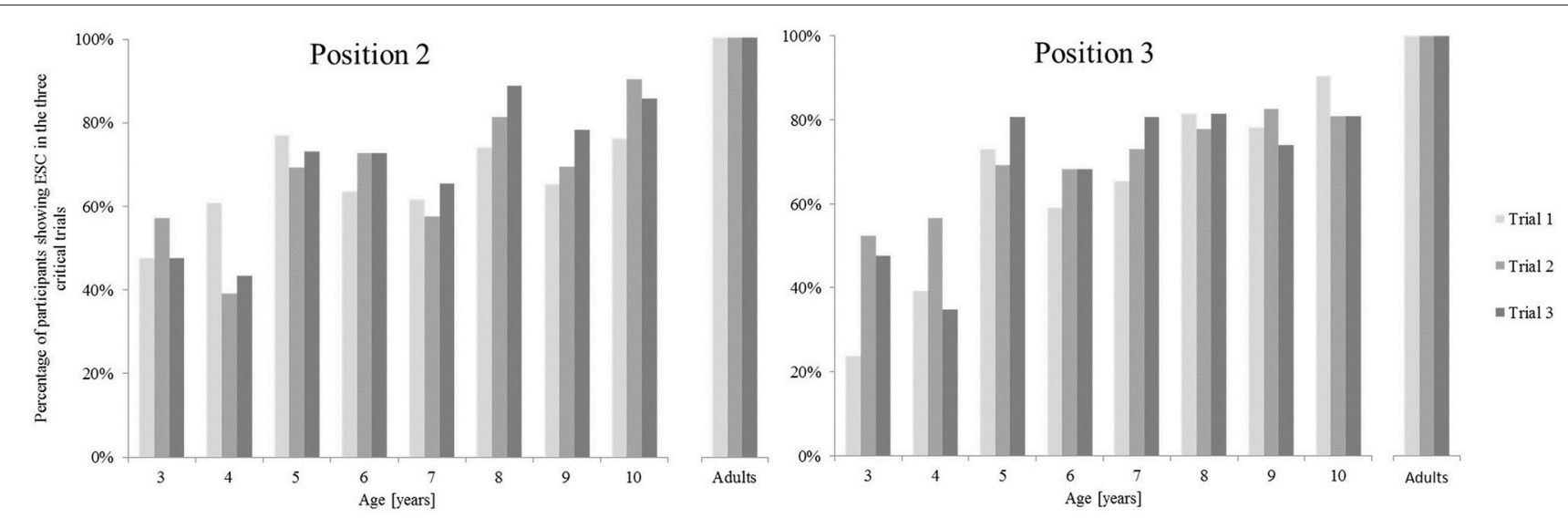

FIGURE 5 | Percentage of the participants showing end-state comfort in each of the three critical trials for Positions 2 and 3, respectively.

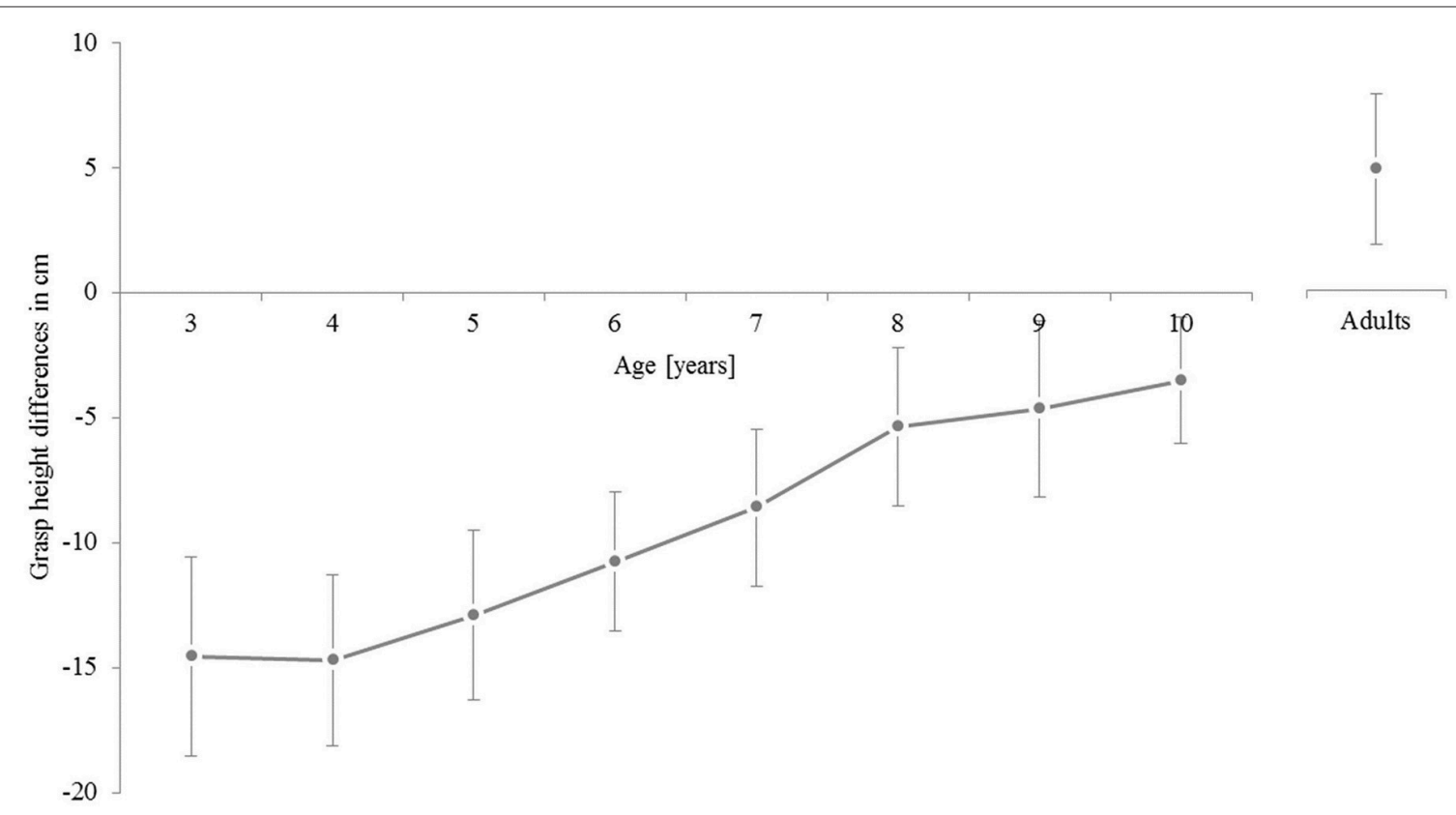

FIGURE 6 | Mean differences in grasp height averaged for the high-target-moves and the low-target-moves. Error bars represent $95 \%$ confidence intervals for the individual means.

Small to medium correlations emerged when considering the entire sample (last row of Table 3), indicating an increase in ESC sensitivity as age increases, even though these correlations are mostly driven by the between-group differences described above. By contrast, there was no sign of intercorrelations of the different ESC measures within the groups (except for the correlation of the bar-transport-task and the sword-rotation-task for the 4-yearolds). To further assess these within-group differences, we Ztransformed each correlation coefficient for each of the non-adult groups, averaged these transformed values and re-transformed the resulting values to correlation coefficients. This procedure yielded mean correlations of $r=0.14$ for the bar-transport-task and the sword-rotation-task, $r=0.07$ for the bar-transport-task and the grasp-height-task, and $r=0.06$ for the sword-rotationtask and the grasp-height-task. Testing the corresponding mean $Z$-values against zero did not yield any significant differences, $p s>0.193$.

\section{The Relationship between ESC and EF}

As in the three ESC tasks, children also improved their performance in the three EF tasks with increasing age. However, as indicated in the Data Analysis, it was not possible to analyze this trajectory due to the usage of different task versions (e.g., a 7-year old child performed numerally "poorer" on the $\mathrm{TOH}$ task, but only because of a switch in task versions from 6 to 7 years, with 7-year-old performing a more difficult version than 


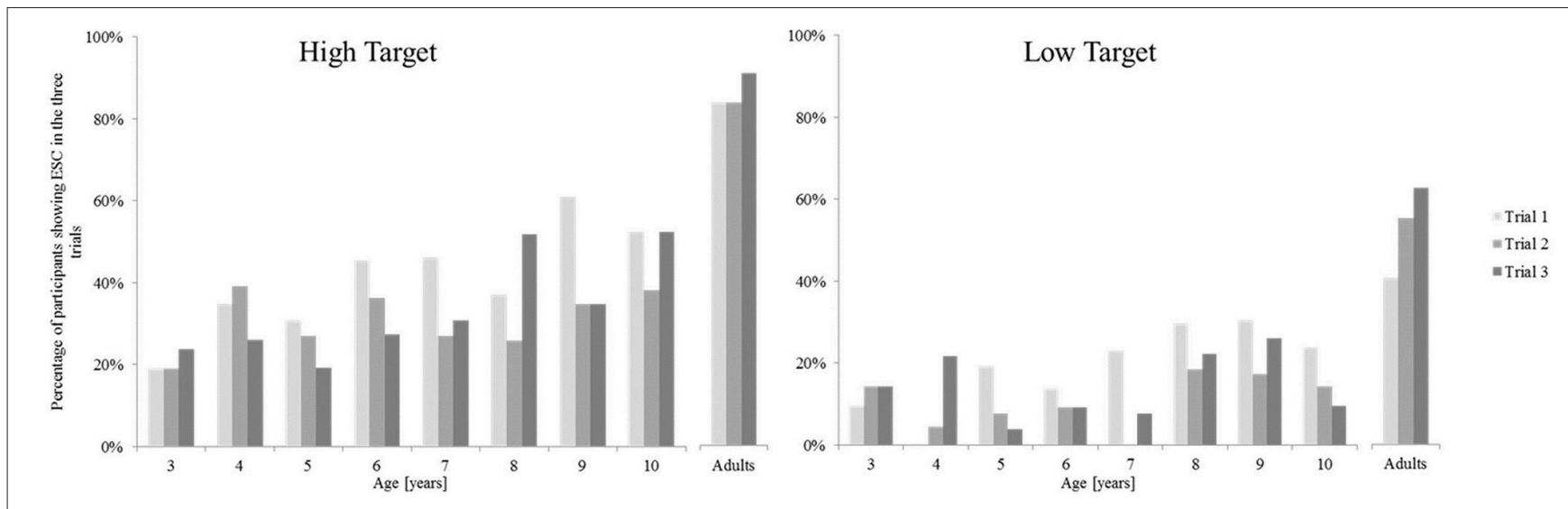

FIGURE 7 | Percentage of participants showing end-state comfort in each of the three trials for the high and the low target platform.

TABLE 3 | Pairwise correlations between the three motor tasks.

\begin{tabular}{lccc}
\hline & & Correlations between the motor tasks \\
\cline { 2 - 4 } Age & $\begin{array}{c}\text { Bar-transport-task and } \\
\text { sword-rotation-task }\end{array}$ & $\begin{array}{c}\text { Bar-transport-task and } \\
\text { grasp-height-task }\end{array}$ & $\begin{array}{c}\text { Sword-rotation-task and } \\
\text { grasp-height-task }\end{array}$ \\
\hline 3-year-olds & -0.258 & -0.144 & -0.155 \\
4-year-olds & $0.565^{* *}$ & 0.263 & -0.113 \\
5-year-olds & 0.283 & 0.210 & 0.126 \\
6-year-olds & 0.214 & 0.247 & 0.261 \\
7-year-olds & 0.055 & 0.079 & 0.236 \\
8-year-olds & 0.071 & 0.071 & -0.020 \\
9-year-olds & 0.233 & -0.115 & -0.326 \\
10-year-olds & -0.125 & -0.058 & 0.386 \\
Adults & $\mathrm{N} / \mathrm{A}$ & 0.367 & $\mathrm{~N} / \mathrm{A}$ \\
Overall & $0.276^{* *}$ & $0.305^{\star *}$ & $0.245^{* *}$ \\
\hline
\end{tabular}

that two correlations for the adult sample could not be computed due to ceiling effects in the sword-rotation-task. ${ }^{* *} p<0.001$.

younger children $)^{5}$. The main purpose of this study was to assess a possible relationship between motor planning and executive functioning. Therefore, Pearson correlations were computed between all motor (dichotomous variables for the bar-transporttask and the sword-rotation-task and continuous variables for the grasp-height-task) and cognitive tasks (see Table 4).

The analyses yielded mostly small and non-significant correlations across the participants of each individual group (for exceptions see Table 4).

In contrast to the ESC tasks, overall correlations across the participants of different groups are not possible for the EF tasks, because we opted to use different versions of the tasks for different age groups. In other words: As cognitive function develops during childhood, it was not possible to use only one test for every single cognitive function. In all tasks, two or three different versions of each test were used to examine executive functions as described in the methods section. As for the intercorrelations of the ESC tasks, however, we computed

\footnotetext{
${ }^{5}$ For further information on EF results please contact the corresponding author.
}

mean correlations across all non-adult groups and tested the resulting mean Z-score against zero. This procedure yielded a significant correlation only between the mosaic task and the bartransport-task, $r=0.218, p=0.018$, whereas the remaining correlations were not significant $p s>0.082$.

\section{DISCUSSION}

The goal of the present study was three-fold: (1) to examine the developmental trajectories of the different (ESC) tasks, (2) to assess possible relationships between all motor tasks used, and (3) to investigate potential relations between the performance in both, the motor and the cognitive task, based on the hypothesis of an association between EFs and ESC planning. To this end, a specific test battery examined the development of motor planning abilities and executive functions in children and adults. To examine motor planning abilities, the bar-transporttask, the sword-rotation-task, and the grasp-height-task were conducted. To test for EF, we used the Tower-of-Hanoi-task, the Mosaic-task, and the D2-attention-endurance-test. This test 
TABLE 4 | Pairwise correlations between the motor and the cognitive tasks.

\begin{tabular}{|c|c|c|c|c|c|c|c|c|c|}
\hline \multirow[b]{3}{*}{ Age } & \multicolumn{9}{|c|}{ Correlations between the cognitive and motor tasks } \\
\hline & \multicolumn{3}{|c|}{ D2-task } & \multicolumn{3}{|c|}{ Mosaic-task } & \multicolumn{3}{|c|}{ Tower of Hanoi } \\
\hline & BTT & SRT & GHT & BTT & SRT & GHT & BTT & SRT & GHT \\
\hline 3-year-old & -0.02 & 0.078 & 0.334 & 0.239 & 0.075 & 0.257 & -0.039 & 0.033 & -0.126 \\
\hline 4-year-old & -0.032 & 0.207 & 0.071 & 0.068 & -0.344 & 0.272 & $0.414^{*}$ & 0.004 & 0.356 \\
\hline 5-year-old & 0.358 & 0.19 & $0.492^{*}$ & 0.279 & 0.278 & $0.406^{\star}$ & 0.112 & -0.201 & -0.06 \\
\hline 6-year-old & 0.064 & 0.129 & -0.046 & 0.059 & 0.165 & -0.112 & 0.072 & 0.072 & 0.053 \\
\hline 7 -year-old & 0.049 & -0.104 & -0.263 & 0.261 & -0.084 & -0.011 & 0.053 & -0.325 & -0.184 \\
\hline 8 -year-old & 0.137 & -0.043 & -0.078 & 0.16 & $0.418^{\star}$ & -0.101 & -0.156 & 0.179 & -0.159 \\
\hline 9-year-old & 0.173 & -0.133 & $0.427^{*}$ & 0.04 & 0.04 & -0.206 & -0.018 & 0.231 & 0.064 \\
\hline 10-year-old & 0.018 & -0.048 & 0.384 & $0.57^{\star}$ & 0.11 & 0.046 & 0.059 & 0.103 & -0.061 \\
\hline Adults & 0.003 & N/A & -0.181 & 0.11 & $\mathrm{~N} / \mathrm{A}$ & 0.124 & 0.038 & $\mathrm{~N} / \mathrm{A}$ & 0.065 \\
\hline
\end{tabular}

BTT, Bar-transport-task; SRT, Sword-rotation-task; GHT, Grasp-height-task. ${ }^{*} p<0.05$.

battery was employed to assess the performance of eight groups of children, aged 3-10 years, and one group of adults.

With regard to the developmental trajectories observed for motor planning abilities (as indicated by the ESC effect), the results support previous studies using the bar-transport-task (Hughes, 1996; Smyth and Mason, 1997; Thibaut and Toussaint, 2010; Weigelt and Schack, 2010; Jovanovic and Schwarzer, 2011; Knudsen et al., 2012; Stöckel et al., 2012) and the sword-rotation-task (Crajé et al., 2010; Jongbloed-Pereboom et al., 2013). Specifically, the likelihood to perform a certain motor action in an ESC-consistent manner (steadily) increased from young kindergarten children to school children and from school children to adults. For the bar-transport task, adult-like performance was reached around the age of 10 years (Thibaut and Toussaint, 2010; Stöckel et al., 2012; see also Knudsen et al., 2012; Stöckel and Hughes, 2016). For the sword-rotation task, this development seems to be somewhat delayed, as even 10-year old children showed the ESC effect less often than adults (see also Jongbloed-Pereboom et al., 2013). In addition, the results of the grasp-height-task suggest that this basic developmental trend across young ages generalizes from a rather dichotomous grip selection (underhand vs. overhand) to grip choices in a continuous task space (here, along the vertical axis of the object, for a different continuous ESC paradigm, see Herbort and Butz, 2012). However, participants appear to display adult-like performance much later in these kinds of tasks, as only about half of the 10-year old children showed the ESC effect in the grasp-height task. Here it should be noted, however, that only $50 \%$ of the adults grasped the plunger in accordance with the ESC effect.

The developmental pattern of motor planning abilities across the different age group, as signified by the ESC effect, did not follow a strictly linear trend. In both, the bar-transport-task and the sword-rotation-task, a stagnation in the developmental trajectory of ESC planning could be observed. These findings are in line with previous studies, and they are commonly explained in terms of the motor re-organization hypothesis (Bard et al., 1990; Thibaut and Toussaint, 2010). According to this hypothesis, motor structures re-organize in children around the age of
8, resulting in a momentary instability of previously acquired abilities. It is likely that different sensory-motor maturation processes, which support the development of cognitive control during early childhood (Piaget and Cook, 1952), also enable the development of ESC planning (Fischer, 1980). Interestingly, 8 -year-old children provided less evidence for anticipatory planning than 6-year-old children in the study of Thibaut and Toussaint (2010), 7-year-old less than 6-year-old children in the study of Jongbloed-Pereboom et al. (2013). The developmental trajectories of the present study can therefore be taken to support the motor re-organization hypothesis, as children aged 7 and 8 years showed a stagnation (or even a decrease in the grasp-height-task) in the development of their motor planning abilities.

As for the second goal of the study, the correlation analyses did not provide much support for intercorrelations between the three motor tasks. Although, correlations emerged when analyzing the data across the entire sample, no such relationship was found within any of the different age groups (with only one exception). The absence of any correlation between the motor tasks is surprising, but is also in line with three other previous studies ${ }^{6}$ testing children in two motor planning tasks within a single experiment (Smyth and Mason, 1997; Knudsen et al., 2012; Stöckel and Hughes, 2016). Please note that in all other studies conducted so far, only a single motor task was used. The current work therefore extends this previous line of research and is the first to investigate the development of motor

\footnotetext{
${ }^{6}$ As the first author of this paper was a co-author in the study by Knudsen et al. (2012), data was checked post-hoc for relations between the two ESC tasks used in either study (the grasp-height-task was excluded due to the continuous nature of the dependent variable). Phi-coefficient analyses revealed similar results: In the study by Knudsen et al. (2012), the bar-transport-tasks and the overturned-glasstask was only correlated in 4 -year-old children $(\phi=0.522, p<0.05)$, In our study, however, we found both tests to be correlated in the 4 - and 5-year-olds ( $\phi=0.476$, $p<0.01$ and $\phi=0.440, p<0.01$ ), but not in the other participant groups. This means, that the two tasks used in our study and the two tasks used in the study by Knudsen et al. (2012) possibly measure different aspects of motor planning. Hence, the developmental trajectories of ESC planning may not be related between these different tasks, possibly due to different task constraints, as already discussed in Wunsch et al. (2013).
} 
planning abilities by using three different tasks in a withinsubjects design. There are several possible reasons why children's performance in the three motor tasks was not correlated. The tasks to measure ESC planning abilities might have been too different to exhibit relationships between their developmental patterns. They differed regarding many details, like the number of required action steps, the precision requirements, children's perception of comfort, the required degree of object rotation, children's familiarity with the task, and/or motivational aspects (for a more detailed discussion of these influencing factors see Wunsch et al., 2013). All of these aspects (or combinations of it) may have prevented intercorrelations between these three tasks to occur. However, it is also possible that the performance in each of these ESC tasks is not based on a common set of motor planning abilities. This notion should certainly be considered in future research.

As for the third goal of the study, we also did not observe any conclusive evidence for interdependencies between the development of ESC performance and EF. Mean correlations within groups of children of similar age were either absent or small. The notion that motor skill development may not be as closely related to the maturation of EF has been previously assumed and put forward by Van Swieten et al. (2010). These authors argued that if adults do not always perform in a manner consistent with ESC, then EF must fail in these cases. However, it is also plausible that EF may sometimes fail under certain circumstances, even in adults, as has been shown, for example, by Blakemore and Choudhury (2006), De Luca et al. (2003) or Salthouse et al. (2003). Alternatively, it might be that the selection of EF tasks may not have been appropriate to examine a relationship to ESC planning. Maybe, the EF tasks chosen for this study (i.e., problem solving, visual perception, and attention) are not appropriate to measure intercorrelations with motor planning abilities. Noteworthy, Stöckel and Hughes (2016) found response planning/problem solving in the Tower of London task to be a significant predictor of anticipatory motor planning performance for a group of 5- to 6-year old children. This suggests that other tests to assess EF than have been used inthe present study should be considered in the future. Moreover, the relatively small number of participants in each age-group is a limitation of the present study. Findings within each group should thus be treated with caution, whereas there is good reason to interpret the overall finding of a null-correlation as evidence for a true null effect (rather than a Type II error).

The observation of largely independent processes underlying EC and ESC also seems to challenge the notion of embodied

\section{REFERENCES}

Adalbjornsson, C. F., Fischman, M. G., and Rudisill, M. E. (2008). The endstate comfort effect in young children. Res. Q. Exerc. Sport 79, 36-41. doi: 10.1080/02701367.2008.10599458

Anderson, P. (2002). Assessment and development of executive function (EF) during childhood. Child Neuropsychol. 8, 71-82. doi: 10.1076/chin.8.2.71.8724

Bard, C., Hay, L., and Fleury, M. (1990). Timing and accuracy of visually directed movements in children: control of direction and amplitude accounts of cognition and action. At the same time, these findings seem to resemble the concept of simulators (rather than simulations), which hold a prominent spot in embodied theorizing (Barsalou et al., 2003). Such simulators are mechanisms that are assumed to provide context-specific simulations and they have been invoked as an embodied alternative to the term of a "concept" (Barsalou, 2003). Our findings therefore suggest that different motor requirements as used in the current bar-transport-task, sword-rotation-task, and grasp-height-task actually rely on rather different simulators to represent the corresponding actions.

\section{CONCLUSION}

In summary, the present study examined children's performance in three object manipulation tasks and compared their performance with three cognitive tasks that measured EF. There was a clear developmental trajectory for all abilities examined here, and this trend occurred in a similar fashion for all motor planning tasks. Contrary to our predictions, however, the findings showed only weak and unreliable intercorrelations between the different motor tasks. In addition, the performance in the cognitive tasks used to test EF did not reliably predict participant's performance in the different ESC tasks. Future research is needed to further assess potential interdependencies between motor skill development and the maturation of cognitive abilities. Specifically, the current findings suggest that motor planning is a rather heterogeneous ability that cannot be captured by one single task.

\section{AUTHOR CONTRIBUTIONS}

All authors made substantial contributions to the conception, analysis and interpretation of results; they drafted and revised the manuscript critically for important intellectual content and approved the final version of this manuscript to be submitted. They all agree to be accountable for all aspects of the work in ensuring that questions related to the accuracy or integrity of any part of the work are appropriately investigated and resolved.

\section{FUNDING}

The article processing charge was funded by the German Research Foundation (DFG) and the Albert Ludwigs University Freiburg in the funding program Open Access Publishing.

Barsalou, L. W. (2003). Situated simulation in the human conceptual system. Lang. Cogn. Process. 18, 513-562. doi: 10.1080/01690960344000026

Barsalou, L. W. (2008). Grounded cognition. Annu. Rev. Psychol. 59, 617-645. doi: 10.1146/annurev.psych.59.103006.093639

Barsalou, L. W., Simmons, W. K., Barbey, A. K., and Wilson, C. D. (2003). Grounding conceptual knowledge in modality-specific systems. Trends Cogn. Sci. 7, 84-91. doi: 10.1016/S1364-6613(02)00029-3 
Blakemore, S.-J., and Choudhury, S. (2006). Development of the adolescent brain: implications for executive function and social cognition. J. Child Psychol. Psychiatry 47, 296-312. doi: 10.1111/j.1469-7610.2006.01611.x

Borghi, A. M. (2005). "Object concepts and action," in Grounding Cognition: The Role of Perception and Action in Memory, Language, and Thinking, eds D. Pecher and R. A. Zwaan (Cambridge: Cambridge University Press), 8-34.

Borghi, A. M., and Caruana, F. (2015). "Embodiment theory," in International Encyclopedia of the Social \& Behavioral Sciences, 2nd Edn., Vol. 7, ed J. D. Wright (Oxford: Elsevier), 420-426. doi: 10.1016/B978-0-08-097086-8.56025-5

Brickenkamp, R. (1962). Test d2 Aufmerksamkeits-Belastungs-Test. Göttingen: Hogrefe.

Brickenkamp, R., Schmidt-Atzert, L., and Liepmann, D. (2010). Test d2 - Revision. Aufmerksamkeits- und Konzentrationstest. Göttingen: Hogrefe.

Champely, S. (2015). pwr: Basic Functions for Power Analysis. R package version 1.1-3. Available online at: http://CRAN.R-project.org/package=pwr

Cohen, R. G., and Rosenbaum, D. (2004). Where grasps are made reveals how grasps are planned: generation and recall of motor plans. Exp. Brain Res. 157, 486-495. doi: 10.1007/s00221-004-1862-9

Crajé, C., Aarts, P., Nijhuis-van der Sanden, M., and Steenbergen, B. (2010). Action planning in typically and atypically developing children (unilateral cerebral palsy). Res. Dev. Disabil. 31, 1039-1046. doi: 10.1016/j.ridd.2010.04.007

De Luca, C., Wood, S. J., Anderson, V., Buchanan, J. A., Proffitt, T., Mahony, K., et al. (2003). Normative data from CABTAB: development of executive function over the lifespan. J. Clin. Exp. Neuropsychol. 25, 242-254. doi: 10.1076/jcen.25.2.242.13639

Fischer, K. W. (1980). A theory of cognitive development: the control and construction of hierarchies of skills. Psychol. Rev. 87, 477-531. doi: 10.1037/0033-295X.87.6.477

Gioia, G. A., Isquith, P. K., and Guy, S. C. (2001). "Assessment of executive function in children with neurological impairments," in Psychological and Developmental Assessment: Children with Disabilities and Chronic Conditions, eds R. J. Simeonsson and S. L. Rosenthal (New York, NY: The Guilford Press), $317-256$.

Gonzalez, C. L. R., Mills, K. J., Genee, I., Li, F., Piquette, N., Rosen, N., et al. (2014). Getting the right grasp on executive function. Front. Psychol. 5:285. doi: 10.3389 /fpsyg.2014.00285

Grob, A., Meyer, C. S., and Hagmann-von Arx, P. (2009). IDS: Intelligence and Development Scales. Intelligenz- und Entwicklungsskalen für Kinder von 5-10 Jahren. Bern: Verlag Hans Huber.

Grob, A., Reimann, G., Gut, J., and Frischknecht, M.-C. (2013). Intelligence and Development Scales - Preschool (IDS-P). Intelligenz- und Entwicklungsskalen für das Vorschulalter. Bern: Verlag Hans Huber.

Herbort, O., and Butz, M. V. (2012). The continuous end-state comfort effect: weighted integration of multiple biases. Psychol. Res. 76, 345-363. doi: 10.1007/s00426-011-0334-7

Hughes, C. (1996). Planning problems in autism at the level of motor control. J. Autism Dev. Disord. 26, 99-107. doi: 10.1007/BF02276237

Jansen, P. (2014). Macht Bewegung unsere Kinder wirklich schlauer? Neue Erkenntnisse zum Zusammenhang von Bewegung und kognitiven Fähigkeiten bei Kindern und Jugendlichen. Sport Orthopäd. Sport Traumatol. 30, 267-273. doi: 10.1016/j.orthtr.2014.02.001

Jenni, O. G., Chaouch, A., Caflisch, J., and Rousson,V. (2013). Correlations between motor and intellectual functions in normally developing children between 7 and 18 years. Dev. Neuropsychol. 38, 98-113. doi: $10.1080 / 87565641.2012 .733785$

Jongbloed-Pereboom, M., Nijhuis-van der Sanden, M. W., Saraber-Schiphorst, N., Crajé, C., and Steenbergen, B. (2013). Anticipatory action planning increases from 3 to 10 years of age in typically developing children. J. Exp. Child Psychol. 114, 295-305. doi: 10.1016/j.jecp.2012.08.008

Jovanovic, B., and Schwarzer, G. (2011). Learning to grasp efficiently: the development of motor planning and the role of observational learning. Vision Res. 51, 945-954. doi: 10.1016/j.visres.2010.12.003

Kelly, T. (2000). The development of executive function in school-aged children. Clin. Neuropsychol. Assessment 1, 38-55.

Knudsen, B., Henning, A., Wunsch, K., Weigelt, M., and Aschersleben, G. (2012). The end-state comfort effect in 3- to 8-year-old children in two object manipulation tasks. Front. Psychol. 3:445. doi: 10.3389/fpsyg.2012.00445
Levin, H. S., Culhane, K. A., Hartmann, J., Evankovich, K., Mattson, A. J., Harward, H., et al. (1991). Developmental changes in performance on tests of purposed frontal lobe functioning. Dev. Neuropsychol. 7, 377-395. doi: $10.1080 / 87565649109540499$

Logan, S. W., and Fischman, M. G. (2011). The relationship between end-state comfort effects and memory performance in serial and free recall. Acta Psychol. 137, 292-299. doi: 10.1016/j.actapsy.2011.03.009

Luria, A. (1973). The Working Brain: An Introduction to Neuropsychology. New York, NY: Basic Books.

Manoel, E. J., and Moreira, C. R. P. (2005). Planning manipulative hand movements: do young children show the end-state comfort effect? J. Hum. Mov. Stud. 49, 93-114.

Mutsaarts, M., Steenbergen, B., and Bekkering, H. (2006). Anticipatory planning deficits and task context effects in hemiparetic cerebral palsy. Exp. Brain Res. 172, 151-162. doi: 10.1007/s00221-005-0327-0

Passler, M., Isaac, W., and Hynd, G. (1985). Neuropsychological development of behavior attributed to frontal lobe functioning in children. Dev. Neuropsychol. 1, 349-370. doi: 10.1080/87565648509540320

Petermann, F., and Petermann, U. (eds.). (2008). Hamburg-Wechsler-Intelligenztest für Kinder IV (HAWIK-IV). Bern: Verlag Hans Huber.

Piaget, J. (1952). The Origins of Intelligence in Children. New York, NY: International Universities Press.

Piaget, J., and Cook, M. (1952). The Origins of Intelligence in Children. New York, NY: W.W. Norton and Co.

Ricken, G., Fritz, A., Schuck, K. D., and Preuß, U. (eds.). (2007). HannoverWechsler-Intelligenztest für das Vorschulalter - III (HAWIVA-III). Bern: Verlag Hans Huber.

Rosenbaum, D. A., Carlson, R. A., and Gilmore, R. O. (2001). Acquisition of intellectual and perceptual-motor skills. Annu. Rev. Psychol. 52, 453-470. doi: 10.1146/annurev.psych.52.1.453

Rosenbaum, D. A., Chapman, K. M., Weigelt, M., Weiß, D. J., and van der Wel, R. (2012). Cognition, action and object manipulation. Psychol. Bull. 138, 924-946. doi: $10.1037 / \mathrm{a} 0027839$

Rosenbaum, D. A., Halloran, E., and Cohen, R. G. (2006). Grasping movement plans. Psychon. Bull. Rev. 13, 918-922. doi: 10.3758/BF03194019

Rosenbaum, D. A., Marchack, F., Barnes, H. J., Vaughan, J., Slotta, J. D., and Jorgensen, M. J. (1990). "Constraints for action selection: overhand versus underhand grips," in Attention and Performance XIII, ed M. Jeannerod (Hillsdale: Erlbaum), 321-342.

Rosenbaum, D. A., Vaughan, J., Jorgensen, M. J., Barnes, H. J., and Stewart, E. (1993). "Plans for object manipulation," in Attention and Performance XIV, eds D. E. Meyer and S. Kornblum (Cambridge: MIT Press), 803-820.

Salthouse, T. A., Atkinson, T. M., and Berish, D. E. (2003). Executive functioning as a potential mediator of age-related cognitive decline in normal adults. J. Exp. Psychol. 132, 566-594. doi: 10.1037/0096-3445.132.4.566

Scharoun, S. M., and Bryden, P. J. (2013). The development of end- and beginningstate comfort in a cup Manipulation Task. Dev. Psychobiol. 56, 407-420. doi: $10.1002 /$ dev. 21108

Simon, H. A. (1975). The functional equivalence of problem solving skills. Cogn. Psychol. 7, 268-288. doi: 10.1016/0010-0285(75)90012-2

Smyth, M. M., and Mason, U. C. (1997). Planning and execution of action in children with or without developmental coordination disorder. J. Child Psychol. Psychiatry 38, 1023-1037. doi: 10.1111/j.1469-7610.1997.tb01619.x

Spiegel, M. A., Koester, D., Weigelt, M., and Schack, T. (2012). The cost of changing an intended action: movement planning, but not execution, interferes with verbal working memory. Neurosci. Lett. 509, 82-86. doi: 10.1016/j.neulet.2011.12.033

Stöckel, T., and Hughes, C. M. L. (2016). The relation between measures of cognitive and motor functioning in 5- to 6-year old children. Psychol. Res. 80, 543-554. doi: 10.1007/s00426-015-0662-0

Stöckel, T., Hughes, C. M. L., and Schack, T. (2012). Representation of grasp postures and anticipatory motor planning in children. Psychol. Res. 76, 768-776. doi: 10.1007/s00426-011-0387-7

Stuss, D. T. (1992). Biological and psychological development of executive functions. Brain Cogn. 20, 8-23. doi: 10.1016/0278-2626(92)90059-U

Thibaut, J. P., and Toussaint, L. (2010). Developing motor planning over ages. J. Exp. Child Psychol. 105, 116-129. doi: 10.1016/j.jecp.2009.10.003 
Van Swieten, L. M., van Bergen, E., Williams, J. H. G., Wilson, A. D., Plumb, M. S., Kent, S. W., et al. (2010). A test of motor (not executive) planning in developmental coordination disorder and autism. J. Exp. Psychol. Hum. Percept. Perform. 36, 493-499. doi: 10.1037/a0017177

Von Aster, M., Neubauer, A., and Horn, R. (eds.). (2006). Wechsler Intelligenztest für Erwachsene (WIE). Frankfurt am Main: Harcourt Test Services.

Wechsler, D. (1997). Wechsler Adult Intelligence Scale, 3rd Edn (WAIS $\left.{ }^{\circledR}-I I I\right)$. San Antonio, TX: Harcourt Assessment Services.

Wechsler, D. (2002). Wechsler Primary and Preschool Scale of Intelligence ${ }^{T M}, 3 r d$ Edn (WPPSI $\left.{ }^{T M}-I I I\right)$. San Antonio, TX: Harcourt Assessment Services.

Wechsler, D. (2003). Wechsler Intelligence Scale for Children, 4th Edn (WISC-IV $\left.{ }^{\circledR}\right)$. San Antonio, TX: Harcourt Assessment Services.

Weigelt, M., Cohen, R. G., and Rosenbaum, D. A. (2007). Returning home: locations rather than movements are recalled in human object manipulation. Exp. Brain Res. 149, 191-198. doi: 10.1007/s00221-006-0780-4

Weigelt, M., Rosenbaum, D. A., Huelshorst, S., and Schack, T. (2009). Moving and memorizing: motor planning modulates the recency effect in serial and free recall. Acta Psychol. 132, 68-79. doi: 10.1016/j.actpsy.2009. 06.005

Weigelt, M., and Schack, T. (2010). The development of end-state comfort planning in preschool children. Exp. Psychol. 57, 476-482. doi: 10.1027/16183169/a000059

Welsh, M. C. (1991). Rule-guided behaviour and self-monitoring on the Tower of Hanoi disk-transfer task. Cogn. Dev. 6, 59-76. doi: 10.1016/08852014(91)90006-Y
Welsh, M. C., Pennington, B. F., and Groisser, D. B. (1991). A normativedevelopmental study of executive function: a window on prefrontal function in children. Dev. Neuropsychol. 7, 131-149. doi: 10.1080/87565649109 540483

Wunsch, K., Henning, A., Aschersleben, G., and Weigelt, M. (2013). A systematic review of the end-state comfort effect in normally developing children and in children with developmental disorders. J. Motor Learn. Dev. 1, 59-76.

Wunsch, K., Weiss, D. J., Schack, T., and Weigelt, M. (2015). Second-order motor planning in children: insights from a cup-manipulation-task. Psychol. Res. 79, 669-677. doi: 10.1007/s00426-014-0596-y

Zander, S. I., Weiss, D. J., and Judge, P. G. (2013). The interface between morphology and action planning: a comparison of two species of New World monkeys. Anim. Behav. 86, 1251-1258. doi: 10.1016/j.anbehav.2013.09.028

Conflict of Interest Statement: The authors declare that the research was conducted in the absence of any commercial or financial relationships that could be construed as a potential conflict of interest.

Copyright (c) 2016 Wunsch, Pfister, Henning, Aschersleben and Weigelt. This is an open-access article distributed under the terms of the Creative Commons Attribution License (CC BY). The use, distribution or reproduction in other forums is permitted, provided the original author(s) or licensor are credited and that the original publication in this journal is cited, in accordance with accepted academic practice. No use, distribution or reproduction is permitted which does not comply with these terms. 
OPEN ACCESS

Edited by:

Annalisa Setti,

University College Cork \& Trinity

College Dublin, Ireland

Reviewed by:

Verónica C. Ramenzoni,

National Scientific and Technical

Research Council, Argentina

Mina Catherine Johnson,

Radboud University, Netherlands

*Correspondence: Wim T. J. L. Pouw pouw@fsw.eur.nl

Specialty section: This article was submitted to Cognition,

a section of the journal

Frontiers in Psychology

Received: 09 December 2015

Accepted: 24 May 2016

Published: 10 June 2016

Citation:

Pouw WTJL, van Gog T, Zwaan RA

and Paas F (2016) Augmenting Instructional Animations with a Body Analogy to Help Children Learn about Physical Systems.

Front. Psychol. 7:860. doi: 10.3389/fpsyg.2016.00860

\section{Augmenting Instructional Animations with a Body Analogy to Help Children Learn about Physical Systems}

\author{
Wim T. J. L. Pouw ${ }^{1 *}$, Tamara van Gog ${ }^{1,2}$, Rolf A. Zwaan ${ }^{1}$ and Fred Paas ${ }^{1,3}$ \\ ${ }^{1}$ Department of Psychology, Education and Child Studies, Faculty of Social Sciences, Erasmus University Rotterdam, \\ Rotterdam, Netherlands, ${ }^{2}$ Department of Education, Utrecht University, Utrecht, Netherlands, ${ }^{3}$ Early Start Research \\ Institute, University of Wollongong, Wollongong, NSW, Australia
}

We investigated whether augmenting instructional animations with a body analogy (BA) would improve 10- to 13-year-old children's learning about class-1 levers. Children with a lower level of general math skill who learned with an instructional animation that provided a BA of the physical system, showed higher accuracy on a lever problemsolving reaction time task than children studying the instructional animation without this BA. Additionally, learning with a BA led to a higher speed-accuracy trade-off during the transfer task for children with a lower math skill, which provided additional evidence that especially this group is likely to be affected by learning with a BA. However, overall accuracy and solving speed on the transfer task was not affected by learning with or without this BA. These results suggest that providing children with a BA during animation study provides a stepping-stone for understanding mechanical principles of a physical system, which may prove useful for instructional designers. Yet, because the BA does not seem effective for all children, nor for all tasks, the degree of effectiveness of body analogies should be studied further. Future research, we conclude, should be more sensitive to the necessary degree of analogous mapping between the body and physical systems, and whether this mapping is effective for reasoning about more complex instantiations of such physical systems.

Keywords: science education, body analogy, embodied learning, digital learning, gestures

\section{INTRODUCTION}

Instructional animations (IA) are increasingly implemented in educational environments (Chandler, 2009). The value of animated over static visualizations for instruction can be intuitively grasped: IA offer the learner direct pick-up of process related information (i.e., information that interacts with time, such as causality and motion), which must be inferred from static visualizations (Spanjers et al., 2010). Surprisingly, empirical results concerning the effectiveness of IA are not as encouraging as these intuitions would predict. For example, in the instructional domain of physical systems (e.g., gears, electrical systems, etc.), although visual presentation benefits learning overall (as opposed to non-graphical instructions), findings regarding the effectiveness of animated versus static visualizations are mixed (Hegarty et al., 2003).

Based on the mixed results Tversky et al. (2002) concluded: "The many failures to find benefits of animation ... calls for deeper inquiry into information processing of animation" (p. 255). This was taken to heart, and later studies have suggested that the main problem with learning from dynamic 
visualizations is that it imposes a high cognitive load on working memory from the learner due to information transience inherent to dynamically changing visualizations (Ayres and Paas, 2007a,b). To be effective, it is argued, the negative effects of transience in IA need to be counteracted, for instance, by means of cueing, or segmentation (Spanjers et al., 2010).

There is one type of task, however, for which IA consistently seem beneficial for learning compared to static visualizations even without measures to counteract transience. Namely, a metaanalysis (Höffler and Leutner, 2007) showed a small effect size of learning gains in animated vs. static visualizations under the condition that the instructional content involves learning bodily routines (e.g., origami, assembly, knot tying). It has been suggested that because human movement is automatically and efficiently processed by the cognitive system (we will return to this in the next section), the transience inherent in IA depicting such tasks may be counteracted (Van Gog et al., 2009).

Indeed, evidence is accumulating that the human cognitive system is distinctively attuned to the body, the body of others, and its possibilities for interactions (e.g., Rizzolatti et al., 1996; Amorim et al., 2006). For example, neuropsychological evidence suggests that perceived human body parts are distinctively processed in particular areas of the brain (extrastriate body area; Peelen and Downing, 2007) as compared to perceived body parts of non-human animals (Peelen and Downing, 2007). Moreover, human bodies are readily mapped onto one's own body schema (Semenza and Goodglass, 1985; Van Gog et al., 2009). For instance, mental rotation of shapes represented as a body is performed faster than mental rotation of inanimate objects (Amorim et al., 2006).

Therefore, in the present paper we investigate whether augmenting IA with a body analogy (BA) improves learning about non-human movement content (originally proposed by De Koning and Tabbers, 2011). Specifically, we investigate whether the effectiveness of IA might be improved by augmenting the learning content (in this study: class 1 lever problems) with a BA. We hypothesize: by meaningfully mapping a physical body on a physical system during instruction, a less cognitively demanding route of knowledge-transfer might be created (as opposed to learning about inanimate objects). "Less demanding," as learners readily map bodily actions on their own body schema. Moreover, learners are very familiar with forces acting on the body, which can be used as an analogy for forces acting on physical systems.

There is evidence already that the body can be mapped on physical systems. For example, when children or adults convey their knowledge about a particular topic they often use gestures that are meaningfully related to the topic's content (e.g., GoldinMeadow and Momeni-Sandhofer, 1999; Garber and GoldinMeadow, 2002; Goldin-Meadow and Alibali, 2002; Hutchins and Nomura, 2011). Importantly, gestures do not simply mirror what is expressed in speech. Rather, gestures can accommodate and complement what is expressed verbally with idiosyncratic information expressed in gesture alone. For instance, in a study by Pine et al. (2004) co-speech gestures that emerged when children explained the workings of a class 1 lever (balance beam) were analyzed (see also Pine and Messer, 2000). To solve lever (e.g., a balance-beam) problems children must attain knowledge about the effects of (I) weights, (II) distance of the weight from the fulcrum, and (III) the positioning of the fulcrum. About one-third of the children (5-9 years) explaining the solution to a balance-beam problem produced gesture-speech mismatches. Children verbally explained the solution to the problem in terms of one property (e.g., I; talking about the weights on the beam), while concurrently expressing another (more advanced property) in gesture (e.g., III; expressing the position of the fulcrum in gesture). Even more remarkably, those children that produced mismatches as compared to those that did not, were more likely to improve on pre- to post-test measures of learning. If knowledge about physical systems develops in sensori-motor modalities as research on gesture suggests, augmenting the learning content with sensori-motor stimuli might improve learning (Höffler and Leutner, 2007; Van Gog et al., 2009; Pouw et al., 2014).

Yet, it seems to be the case that augmenting IA about physical systems with sensori-motor information may be suitable for some but not for others (Zacharia et al., 2012; for an overview, see Pouw et al., 2014). For example, kindergartners' learning about balance beams improved when they were given opportunities to physically interact with a balance beam (class 1 lever), but only when they possessed an incorrect preconception of how a balance beam works (Zacharia et al., 2012). This suggests that especially those with incomplete understanding of a physical system are aided by additional body-analogous information. Therefore, it is important to take into account learners cognitive predispositions when investigating the instructional potency.

\section{Present Study}

In the present study, primary school children learned from IA about a class 1 lever (a seesaw). The workings of levers can be considered as a classic context to test children's conceptual and procedural learning processes about physical systems (KarmiloffSmith and Inhelder, 1974; Dixon and Dohn, 2003; Pine et al., 2004). We designed an IA (duration $6.5 \mathrm{~min}$ ) in which relevant concepts for understanding the working of a seesaw were demonstrated, such as weights, balance, fulcrum, and mechanical advantage. Half of the sample was confronted with a 'BA IA' in which a transparent body was projected onto the seesaw (Figure 1: BA condition) and the other half were given the same IA without this BA (control condition). The body provided an analogy of the concept of mechanical advantage: objects placed further from the fulcrum (analogy: joint) will exert more force than objects placed closer to the fulcrum. Furthermore, if similar weights are put at similar places on the arm they will feel equally heavy (balance) or when they are located at different places, they will not feel equally heavy (disbalance).

Learning performance was assessed through a three choice reaction-time task that assessed accuracy and speed of determining whether a seesaw will pivot to the left or the right, or will balance out, given different configurations of the weights, and the positions of the weight relative to the fulcrum. Additionally, we confronted children with a similar three-choice transfer task that consisted of new concepts, such as interconnecting seesaws, or replacement of the fulcrum.

We hypothesized that the BA condition as compared to the control condition would show better learning overall (i.e., higher 


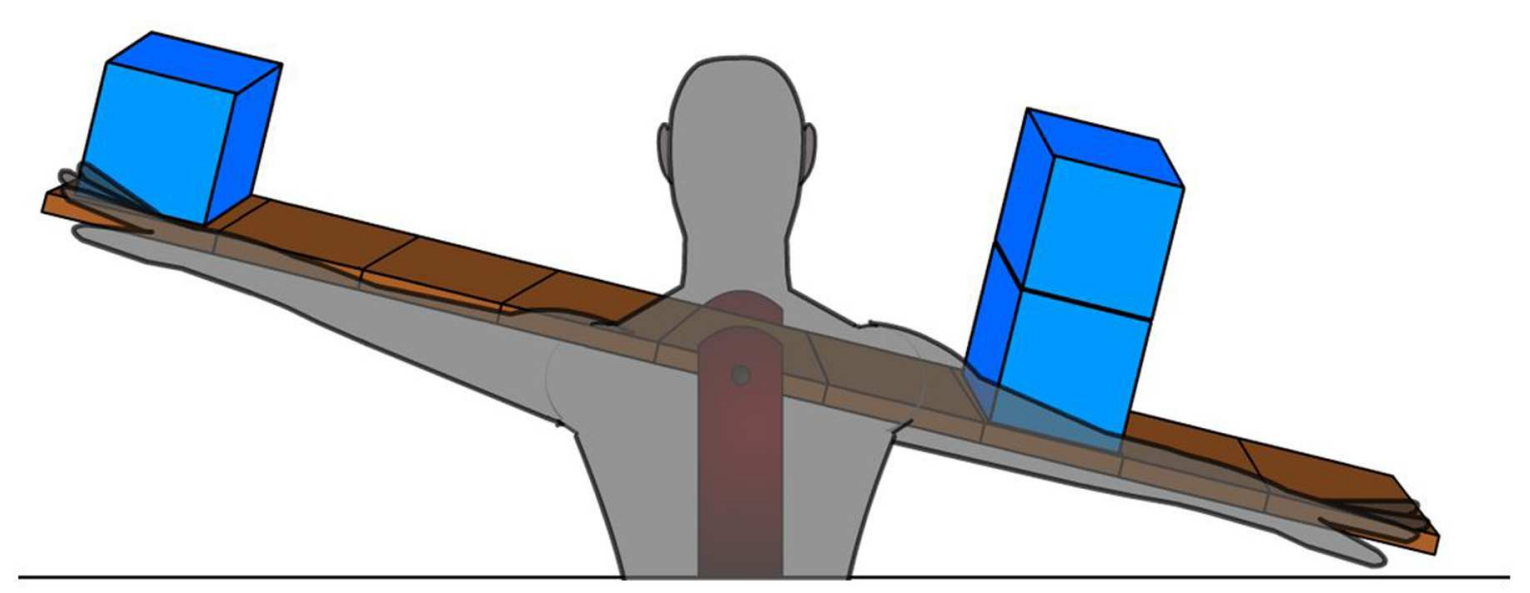

FIGURE 1 | A snapshot of the instructional animation in the BA condition (the seesaw will balance out in this example).

accuracy, faster solving speed on the test tasks). Importantly, to minimize individual cognitive differences between conditions we semi-randomly assigned conditions based on general math scores of the children. We used children's math scores as they are closely related to learning about physical systems, and have been found to strongly correlate with their visuospatial working memory capacity (e.g., Van der Ven et al., 2013), which directly relates to issues of cognitive load associated with IA (Ayres and Paas, 2007a,b). Per exploration we also investigate whether general math skill interacted with the effectiveness of the conditions, as it might be an important cognitive predisposition for learning in the current domain. We also measured subjective experiences of cognitive load, by asking children to rate how much mental effort they invested and how difficult they found the tasks. In addition, we asked them to rate how interesting they found the tasks, which could give an indication of differences in cognitive engagement.

\section{MATERIALS AND METHODS}

\section{Participants and Design}

This study was conducted in accordance with the guidelines of the ethical committee of the institute of psychology at Erasmus University Rotterdam. All children participated based on parental informed consent, where information about the study was provided 2 weeks prior to the experiment and parents were given the opportunity to withdraw their child from participating. A total of 74 Dutch primary school children (three classrooms from two separate schools) were tested (mean age, 12.49, $S D=0.54$; range $10-13 ; 51.4 \%$ female). The two IA-conditions were: control $(N=36,52.8 \%$ female $)$ vs. BA ( $N=38,50 \%$ female). Children were pseudo randomly assigned (see Table 1 for frequencies) to condition by matching for level of general math skill as measured by the national standardized Cito math test or (in one school) an equivalent standardized test that assigns the children to comparable levels of skill as the Cito test does. From highest to lowest, these are: A (highest 25\%), B (next 25\%), C (next 25\%), D (next 15\%), and E (lowest 10\%). This test was taken within the school-semester year in which the experiment took place, and the children's scores were provided by the schools.

\section{Materials}

\section{Instructional Animations}

The IA $^{1}$ were designed in Adobe Flash Professional CS 5.5. The voice-over and textual instructions were programmed in ActionScript $3.0\left(\mathrm{IA}^{\prime} \mathrm{s}^{2}\right)$. The IA consisted of an introduction to the basic concepts of class 1 levers narrated by a female voiceover and explained with a dynamic visualization of a seesaw. In the first part of the IA (3.5 min), basic concepts such as the fulcrum, left and right arm of the seesaw, (dis)balance, weights, and mechanical advantage was introduced. Throughout the instruction no explicit information was provided about formulas related to the constructs. For example, mechanical advantage was explained by showing a balanced seesaw in a mechanical advantage state, with the voiceover instruction informing learners that: "The heavy weight is twice as heavy as the lighter weight, but the seesaw is still in balance! This is

\footnotetext{
${ }^{1}$ The learning effectiveness of the animations was tested with the reaction time task in a pilot-study with adults $(N=78)$ using Amazon's Mechanical Turk. We translated the exact instructional materials designed for Dutch children for the English speaking adult sample. This pilot test showed that the animations were effective for learning (accuracy on the reaction-time task) as compared to no instruction $[t(76)=-2.644, p=0.010$, Cohen's $d=0.602$ (large effect)]. No effects were obtained for solving speed on the RT-task.

${ }^{2}$ http://charlyeielts.nl/bodyanalogy/materials.html
}

TABLE 1 | Number of participants per condition and general math skill.

\begin{tabular}{lcc}
\hline & Control condition & BA condition \\
\hline A & 7 & 8 \\
B & 12 & 12 \\
C & 7 & 8 \\
D & 8 & 7 \\
E & 2 & 3 \\
Total & 36 & 38
\end{tabular}


because the distance of the heavy weight is two times closer to the fulcrum than the lighter weight" (for further instructions ${ }^{2}$ ). The second part of the IA was not narrated and consisted of 24 trials ( $3 \mathrm{~min}$ ) that showed different configurations of weights on varying positions from the fulcrum and its effect on the seesaw (tilt left, right, or balance).

For the BA condition the only difference in the IA as compared to the control condition was that a transparent human body was additionally projected over the seesaw (i.e., no differences in narrated instruction). Importantly, the arms of the projected body moved together with the movement of the seesaw (Figure 1). Only once in the narration (but in both conditions) a reference was made to how it would feel to have weights on one's actual arms. This reference was made after the explanation of mechanical advantage, which showed a seesaw balancing out with unequal amount of weights (Figure 1). This was done to ensure that children in the BA condition would be more likely to see the relevance of the body projected over the seesaw.

\section{Reaction-Time Task}

A three choice reaction-time task was developed (programmed in E-prime) to assess children's accuracy (number of correct responses) and speed (reaction-time) in solving class-1 lever problems. The RT task consisted of 45 trials (and three practice trials) in which children had to judge whether a seesaw would balance, or tilt down to the left or to the right. Each trial showed a seesaw with one or two blocks on either side of the arms of the seesaw on deferring distances from the fulcrum (Figure 2). The number and location of the weight varied for these 45 trials. Children were required to determine which way the seesaw would tilt, or whether it would attain balance, regardless of the current state of the seesaw (i.e., tilted to left/right or balanced). We varied the initial state of the seesaw randomly as to prevent any spurious effects of the initial state of the seesaw on accuracy and speed. Children responded by pressing on a QWERTY keyboard, "P" if the seesaw would tilt to the right, "Q" if it would tilt to the left and SPACE if the seesaw would be in balance.

\section{Transfer Task}

The transfer task, consisting of 15 lever-problems, aimed to assess children's ability and solving speed to further apply the principle of mechanical advantage on new or more complex problems. Twelve problems required children to judge what the end-state would be (tilt left, right, or balance) of a particular seesaw in a set of two interconnected seesaws, in four of those trials the fulcrum was not placed in the center (Figure 3). The last three problems required children to predict how these forces would act on the body (e.g., how heavy a block would feel when placed on the arms, or which seesaw needed to be pushed down the hardest given a number of weights on the seesaws).

\section{Mental Effort, Difficulty, Interest}

We obtained ratings of experienced mental effort, interest, and perceived difficulty of the IA, RT-task, and the transfer task directly after completion. Children answered on a 5-point scale "How hard did you need to think to understand the previous video/task" (mental effort; $1=$ 'not hard,' to $5=$ 'very hard'),
"How interesting did you find this previous video/task" (interest; $1=$ 'not interesting,' to $5=$ 'very interesting') and "How difficult did you find this previous video/task" (difficulty; $1=$ 'not difficult', to $5=$ 'highly difficult').

\section{Demographics}

Information on age, sex, and Cito test score of general math skill of the children were provided by the schools.

\section{Procedure}

Children were tested one or two at a time, in a quiet room at their school. If children were tested at the same time the two experimenters ensured that children did not face each other directly and that there was enough distance between them so that they were not disturbed in any way. Children were seated in front of a laptop and were informed that they would watch an instructional video and perform two tasks to assess what they had learned. They were subsequently asked to put on the headphones so that the experimenter could start the video. Subsequently, children performed the reaction-time task and were instructed to do so "as fast and accurate as possible." Beforehand, children were given three easy practice trials which the experimenter could repeat if needed to ensure they understood the task. Subsequently, children were confronted with the transfer task that was provided in a booklet and they could solve at their own pace (i.e., speed was not emphasized as in the RT-test task). The experimenter used a stopwatch to assess overall solving speed. Immediately after watching the IA, performing the RT, and solving the transfer task, children completed the subjective ratings of effort, interest and difficulty that were printed on a sheet of A4 paper per task. All children received a small present for their participation (handed out in class on the last day of testing).

\section{Data Analyses}

Accuracy and RT-scores for the transfer task and RT-task more than 2 SD from the overall-mean were treated as outliers and were excluded from the analysis (reported in the Results section when applicable).

\section{Reaction-Time Task}

Performance accuracy was measured by summing the correct answers on 45 trials (range: $0-45$ ) and speed was measured by computing the mean reaction time (in $\mathrm{ms}$ ) on correct trials.

\section{Transfer Task}

Performance was measured by summing the correct answers on 15 trials (range: $0-15$ ) as well as overall solving speed in seconds.

\section{RESULTS}

\section{Mental Effort, Difficulty, Interest}

Data are presented in Table 2. T-tests showed no significant differences between conditions in self-reported mental effort, difficulty, or interest, on the IA, RT-task, or transfer task. 


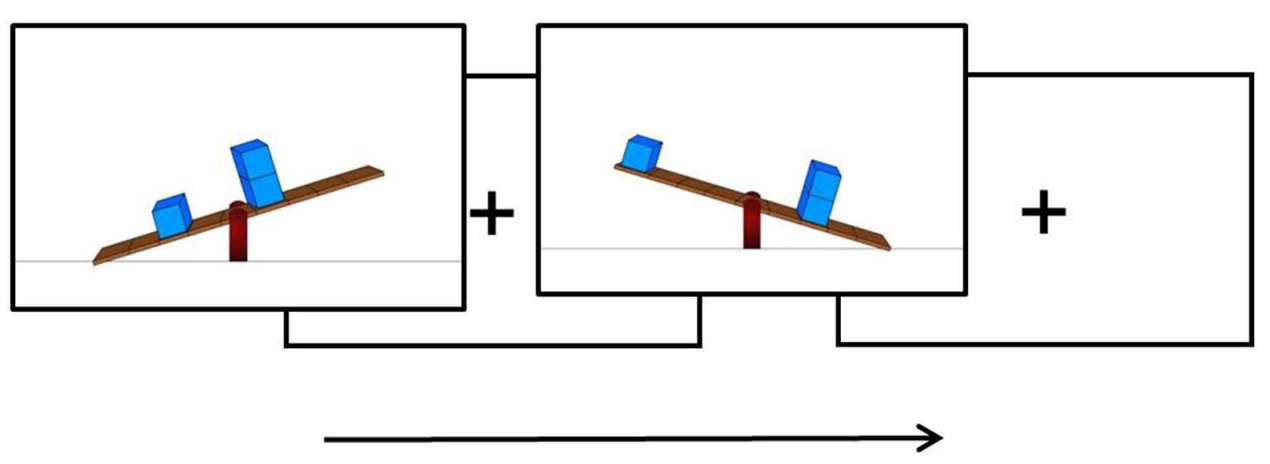

\section{5 trials}

FIGURE 2 | Example of two reaction time trials. Note that trials were given the initial state of the seesaw randomly and the children answered with button presses what the correct state of the seesaw would be (pivot left, balance, pivot right).
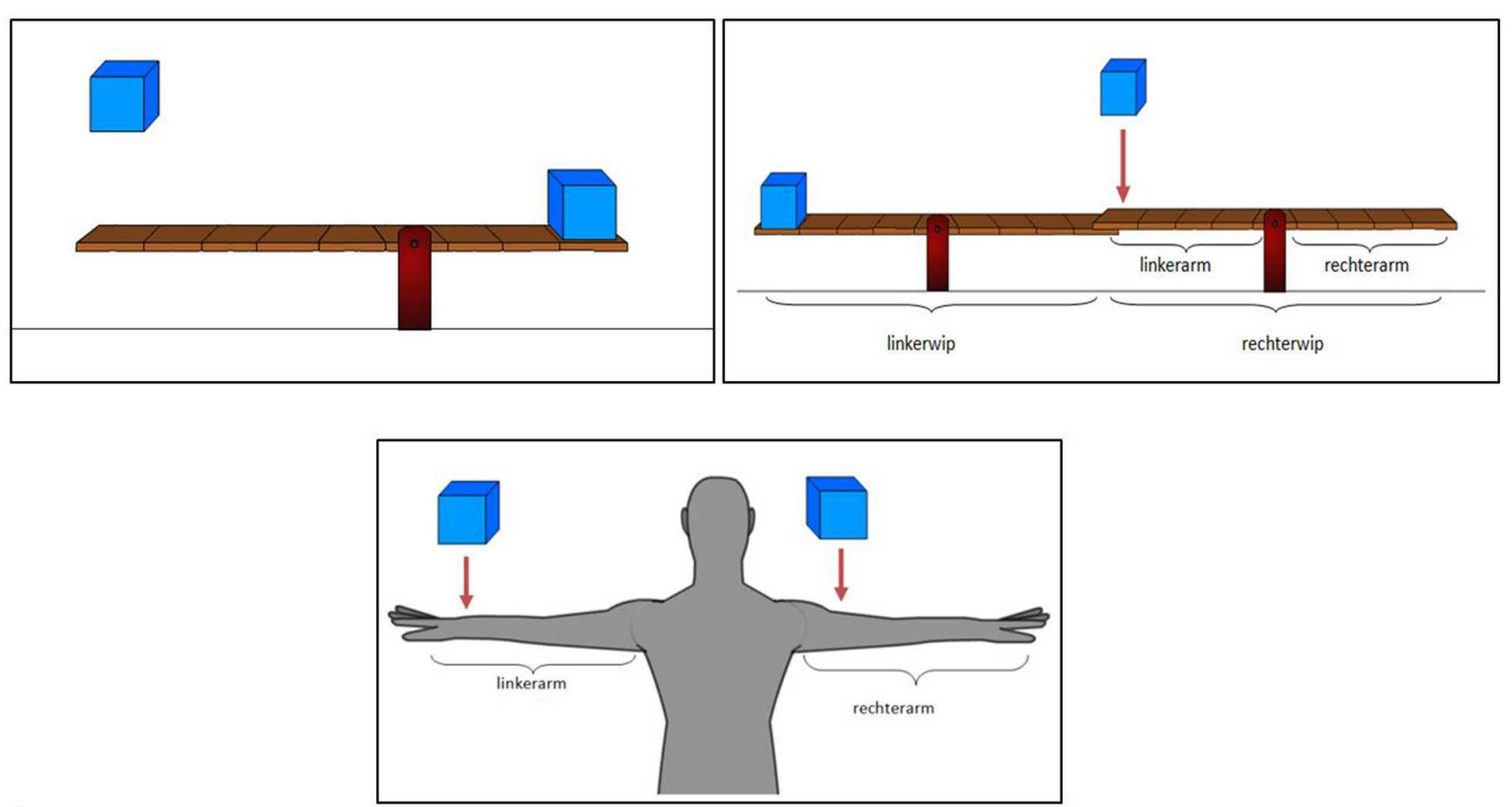

FIGURE 3 | Example of three transfer task problems. In the first example (left above), children were asked to "Judge whether the seesaw pivots to the left, remains in balance, or pivots to the right." This was the same for the second example (right above), but then for the right seesaw [rechterwip]. In the third example children were asked to "which weight will feel the heaviest for this person, or will the weights feel just as heavy?". Note that the terms left arm [linkerarm] and right arm [rechterarm] were added for reference, as learned during the IA.

\section{RT-Task Performance}

\section{Accuracy}

The overall accuracy score on the RT-task was $59.91 \%$ $(M=26.96, S D=5.56)$. Four participants scored $<2$ SDs below the mean (i.e., <15) and were therefore excluded from the analyses (no participants scored $>2 \mathrm{SD}$ ). This resulted in an analysis on data of 70 participants, with $N=34$ in the control condition ( $N=7$ on math skill level $\mathrm{A}, N=11$ on level $\mathrm{B}$, $N=7$ on level C, $N=8$ on level $\mathrm{D}$, and $N=1$ on level E), and $N=36$ in the BA condition ( $N=8$ scoring on math skill level A, $N=12$ on level B, $N=8$ on level C, $N=6$ on level $\mathrm{D}$, and $N=2$ on level E).
We performed a multiple stepwise regression to assess main effects of math skill and condition and its potential interaction. First, we entered math skill (recoded for analysis, $\mathrm{E}=-2, \mathrm{D}=-1, \mathrm{C}=0, \mathrm{~B}=1$, and $\mathrm{A}=2$; higher scores means higher math skill) which was a significant predictor, $F(1,68)=17.256, p<0.001$, explaining $19.1 \%$ of the variance (based on $R^{2}$ adjusted), with higher math skill resulting in higher accuracy, $\beta=0.450, t(68)=4.154, p<0.001$. The effect of condition was assessed by adding condition as a predictor for RT accuracy into a stepwise regression after math skill. Condition was coded as 0 for the control condition and 1 for the BA condition. The overall model 
TABLE 2 | Means and SDs per condition and task-phase for mental effort, interest, and difficulty.

\begin{tabular}{|c|c|c|c|c|c|c|c|}
\hline \multirow[t]{2}{*}{ Condition } & & \multicolumn{2}{|c|}{ Instructional animation } & \multicolumn{2}{|c|}{ Reaction-time task } & \multicolumn{2}{|c|}{ Transfer task } \\
\hline & & $M$ & $S D$ & $M$ & $S D$ & $M$ & $S D$ \\
\hline \multirow[t]{3}{*}{ Control condition } & Mental effort & 1.81 & 1.064 & 2.25 & 1.05 & 2.50 & 1.00 \\
\hline & Interest & 3.56 & 1.319 & 3.58 & 1.36 & 3.69 & 1.33 \\
\hline & Difficulty & 1.92 & 1.13 & 2.58 & 1.16 & 2.64 & 1.10 \\
\hline \multirow[t]{3}{*}{ BA condition } & Mental effort & 1.79 & 0.935 & 2.26 & 1.155 & 2.27 & 1.03 \\
\hline & Interest & 3.45 & 1.350 & 3.74 & 1.178 & 3.26 & 1.35 \\
\hline & Difficulty & 2.11 & 1.23 & 2.37 & 1.08 & 2.71 & 1.04 \\
\hline
\end{tabular}

remained significant, $F(2,67)=9.417, p<0.001$, explaining $19.6 \%$ of the variance in RT-accuracy. Condition was a positive but non-significant predictor for RT-accuracy, $\beta=0.130$, $t(67)=1.208, p=0.231, R_{\text {Partial }}=0.146$. Math skill remained a significant predictor, $\beta=0.429, t(68)=4.136, p<0.001$, $R_{\text {Partial }}=0.451$.

We further assessed whether general math skill moderated the effect of condition by adding an interaction term of condition and math skill into the regression model. This resulted in significant model-fit, $F(3,66)=8.533, p<0.001$, explaining $24.7 \%$ of the variance in RT accuracy. General math skill remained a significant predictor, $\beta=0.704, t(66)=4.645, p<0.001$, $R_{\text {Partial }}=0.496$, and now condition was significantly positively related with RT accuracy, $\beta=0.230, t(66)=2.040, p=0.045$, $R_{\text {Partial }}=0.244$. Furthermore, there was a significant interaction, $\beta=-0.371, t(66)=2.346, p=0.022, R_{\text {Partial }}=-0.277$, indicating that children with lower math skill were more likely to be positively affected by the BA condition (in terms of RT-accuracy) than those with higher math skill (Figure 4).

\section{Speed}

The overall mean reaction time on correct trials was $2791 \mathrm{~ms}$ $(S D=1331)$. Three additional participants were excluded from the analyses as their data fell over 2 SDs above the mean ( $>5453 \mathrm{~ms}$; no participants scored $<2 \mathrm{SD}$ ). This resulted in an analysis (also see Figure 5) on data of 67 participants, with $N=33$ in the control condition ( $N=7$ scoring on math skill level $\mathrm{A}$, $N=10$ on level B, $N=7$ on level C, $N=8$ on level $\mathrm{D}$, and $N=1$ on level $\mathrm{E}$ ), and $N=34$ in the BA condition ( $N=8$ scoring on math skill level A, $N=12$ on level B, $N=6$ on level C, $N=6$ on level $\mathrm{D}$, and $N=2$ on level $\mathrm{E})$. Math skill (recoded $\mathrm{E}=-2$, $\mathrm{D}=-1, \mathrm{C}=0, \mathrm{~B}=1$, and $\mathrm{A}=2$ ) was not a significant predictor, $F(1,65)=0.327, p=0.569, R^{2}$ adjusted $=-0.010$, showing a nonsignificant relation with speed on correct RT trials $\beta=-0.071$, $t(65)=-0.527, p=0.569$. We added condition together with general math skill as a predictor for speed on correct trials into the stepwise regression model. The overall model-fit was non-significant, $F(2,64)=2.878, p=0.064, R^{2}$ adjusted $=0.054$, math skill remained a non-significant predictor, $\beta=-0.083$, $t(64)=-0.695, p=0.490, R_{\text {Partial }}=-0.083$, and condition was a positive significant predictor, with children in the BA condition being slower on correct trials overall, $\beta=0.279, t(64)=2.325$ $p=0.023, R_{\text {Partial }}=0.279$. To assess a possible interaction effect we entered the interaction term of condition and math skill into the regression model, this yielded no significant results, nor a greater fit of the model.

\section{Speed-Accuracy Trade-off}

Additionally, for exploratory purposes we assessed whether there was a speed-accuracy trade-off by calculating an inverse efficiency measure (IES; Townsend and Ashby, 1978; e.g., Setti et al., 2009), IES $=\frac{\text { Reaction-Time }}{\text { Proportion Correct }}$. A higher score entails a more extreme association of speed and accuracy, where slower reaction times are associated with a higher proportion of correct responses or faster reaction time with a higher proportion of incorrect responses.

The overall mean IES was $5709 \mathrm{~ms}(S D=2870)$. Four participants were excluded from this analysis as their scores fell 2 SDs above the mean ( $>11450 \mathrm{~ms}$; no participants scored $<2 S D$ ). The resulting sample consists of 70 participants, with $N=34$ in the control condition ( $N=7$ scoring on math skill level A, $N=12$ on level $\mathrm{B}, N=7$ on level $\mathrm{C}, N=8$ on level $\mathrm{D}$, and $N=0$ on level $\mathrm{E})$, and $N=36$ in the $\mathrm{BA}$ condition $(N=8$ scoring on math skill level A, $N=11$ on level B, $N=7$ on level C, $N=7$ on level $\mathrm{D}$, and $N=3$ on level E).

Math skill (recoded $\mathrm{E}=-2, \mathrm{D}=-1, \mathrm{C}=0, \mathrm{~B}=1$, and $\mathrm{A}=2)$ did not predict IES, $F(1,68)=1.814, R^{2}$ adjusted $=0.013$, $\beta=-0.161, t(68)=-1.347, p=0.183$. Adding condition next to math skill as a predictor of IES resulted in a non-significant overall model-fit, $F(2,67)=1.445, p=0.243, R^{2}$ adjusted $=0.012$; math skill remained non-significant, $\beta=0.124, t(67)=1.037$, $p=0.304, R_{\text {partial }}=-0.155$, and condition was also a nonsignificant predictor of IES, $\beta=-0.154, t(64)=-1.283$ $p=0.204, R_{\text {Partial }}=0.126$. Adding an interaction term of the previous set of predictors (math skill and condition) did not yield a significant fit of the model, $F(3,66)=1.258, p=0.296$, $R^{2}$ adjusted $=0.011$; the interaction term was a non-significant predictor, $\beta=0.181, t(66)=0.942 p=0.350, R_{\text {Partial }}=0.115$, and adding it did not affect results regarding math skill, $\beta=-0.291$, $t(66)=-1.542 p=0.128, R_{\text {Partial }}=-0.186$, or condition, $\beta=0.078, t(66)=0.599 p=0.551, R_{\text {Partial }}=0.074$, in relation to IES.

\section{Transfer Task Performance}

\section{Accuracy}

The overall accuracy on the transfer task was $49.62 \%(M=7.38$, $S D=1.90$. Two participants performed $<2$ SDs below the mean $(<3.58$; no participants scored $>2 \mathrm{SD})$ and were therefore 


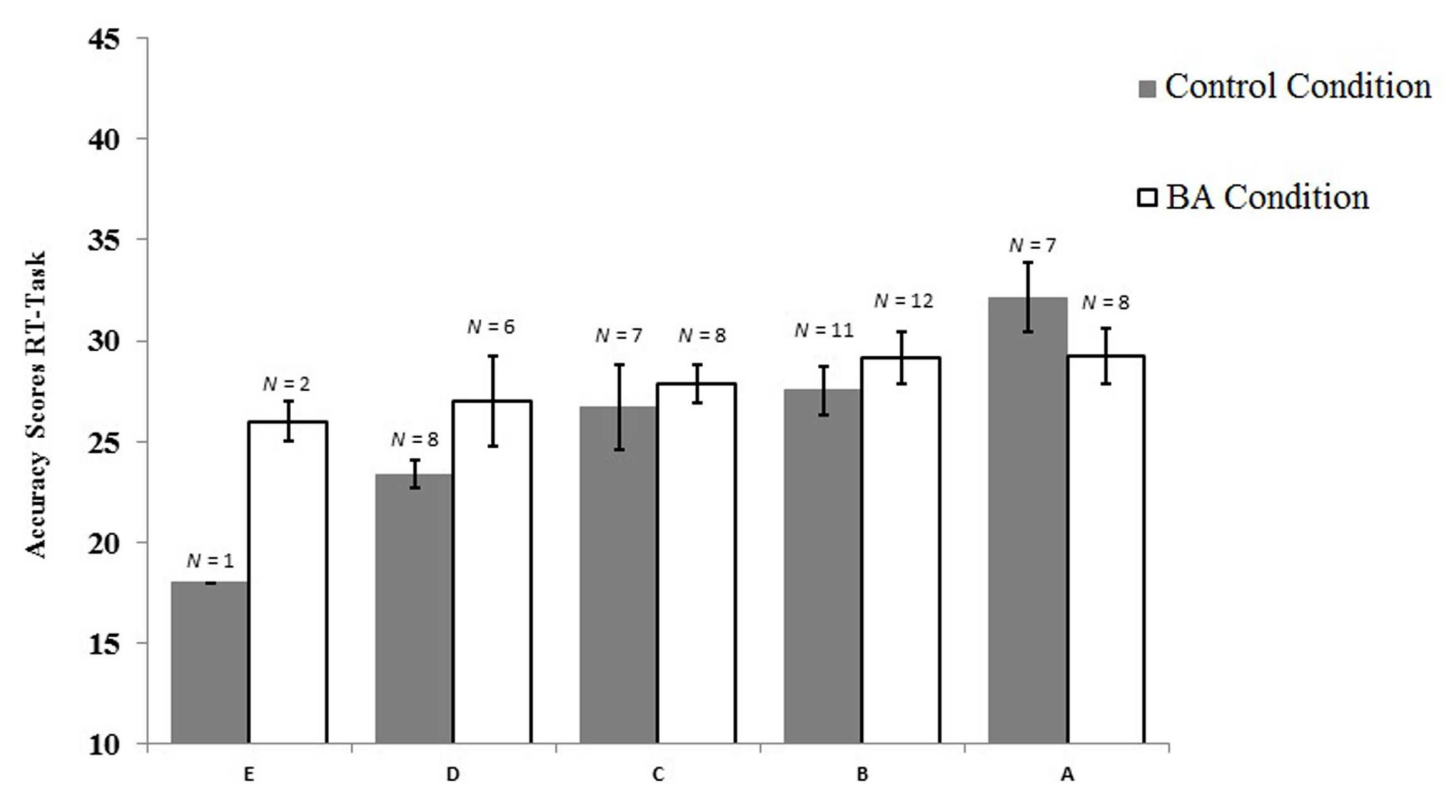

FIGURE 4 | Accuracy scores and standard error per condition and general math skills $(E=$ lower and $A=$ higher general math skill).

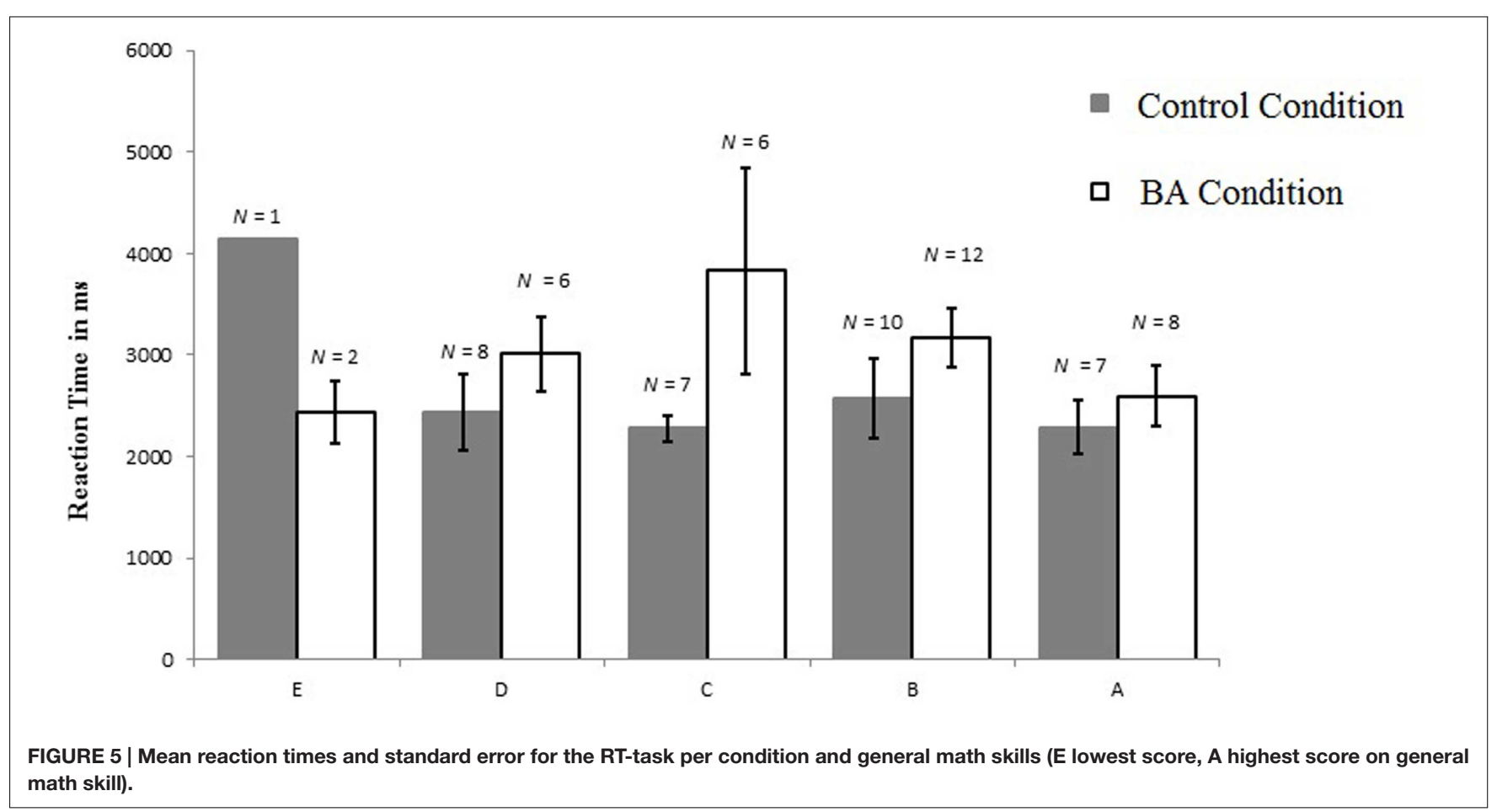

excluded from the analyses. This resulted in an analysis on data of 72 participants, with $N=36$ in the control condition $(N=7$ scoring on math skill level A, $N=12$ on level B, $N=7$ on level C, $N=8$ on level $\mathrm{D}$, and $N=2$ on level E), and $N=36$ in the BA condition ( $N=8$ scoring on math skill level A, $N=12$ on level B, $N=8$ on level C, $N=6$ on level $\mathrm{D}$, and $N=2$ on level E).

A regression analysis showed that math skill (recoded $\mathrm{E}=-2$, $\mathrm{D}=-1, \mathrm{C}=0, \mathrm{~B}=1$, and $\mathrm{A}=2$ ) was a significant predictor of transfer task performance, $F(1,70)=7.320, p=0.009$, $R_{\text {adjusted }}^{2}=0.082$, showing a positive relation with performance $\beta=0.308, t(70)=-0.2 .706, p=0.009$.

We added condition after math skill as a predictor for transfer task performance into the stepwise regression model. The overall model-fit remained significant, $F(1,69)=3.697, p<0.05$, $R^{2}$ adjusted $=0.071$. Math skill remained a significant predictor, $\beta=0.310, t(69)=2.705, p<0.01, R_{\text {Partial }}=0.310$. Condition was 
not a significant predictor, $\beta=-0.403, t(68)=-0.403, p=0.688$, $R_{\text {Partial }}=-0.048$. We further added an interaction term of condition and general math skills into the stepwise regression model, but this resulted in a model with only non-significant predictors $(p>0.246)$.

\section{Speed}

The overall mean solution speed on the transfer task was $308 \mathrm{~s}$ $(S D=77.11)$. Two additional participants were excluded from the analyses as their data fell 2 SD's from the mean (>462 s; no participants scored $<2 \mathrm{SD})$. This resulted in an analysis on data of 70 participants, with $N=34$ in the control condition $(N=6$ scoring on math skill level $\mathrm{A}, N=11$ on level $\mathrm{B}, N=7$ on level C, $N=8$ on level $\mathrm{D}$, and $N=2$ on level $\mathrm{E}$ ), and $N=36$ in the BA condition ( $N=8$ scoring on math skill level A, $N=12$ on level $\mathrm{B}$, $N=8$ on level C, $N=6$ on level $\mathrm{D}$, and $N=2$ on level E). We first assessed whether math skill predicted overall speed on Transfer task in a regression analysis. Math skill was not a significant predictor, $F(1,68)=0.520, p=0.520, R_{\text {adjusted }}^{2}=-0.007$, $\beta=-0.087, t(68)=-0.72, p=0.473$. We added condition next to general math skill as a predictor for speed on transfer task into the stepwise regression model. The overall model-fit was not significant, $F(2,67)=1.699, p=0.395, R_{\text {adjusted }}^{2}=0.020$. Math skill remained a non-significant predictor, $\beta=-0.102$, $t(67)=-0.856, p=0.395, R_{\text {Partial }}=-0.104$, and condition was a non-significant predictor on solving speed on the transfer task, $\beta=0.202, t(67)=1.692, p=0.095, R_{\text {Partial }}=0.202$. We obtained no significant results when entering an interaction term after math skill and condition.

\section{Speed-Accuracy Trade-off}

For exploratory purposes we assessed whether a speed-accuracy trade-off could be detected using the inverse efficiency measure (IES; Townsend and Ashby, 1978), IES $=\frac{\text { Reaction-Time }}{\text { Proportion Correct }}$. As a reminder, a higher score entails a more extreme association of speed and accuracy, where slower reaction times are associated with a higher proportion of correct responses, or faster reaction times with a higher proportion of incorrect responses. Overall mean IES for the transfer task was $674.41 \mathrm{~s}(S D=256.94)$. Three participants' scores fell above 2 SDs above the mean ( $>1188$; no scores $<2 \mathrm{SD}$ ), and were excluded. The resulting sample contained scores of 71 participants, with $N=34$ in the control condition ( $N=7$ scoring on math skill level $\mathrm{A}, N=12$ on level B, $N=6$ on level $\mathrm{C}, N=7$ on level $\mathrm{D}$, and $N=2$ on level E), $N=37$ ( $N=8$ scoring on math skill level $\mathrm{A}, N=12$ on level $\mathrm{B}, N=8$ on level $\mathrm{C}, N=7$ on level $\mathrm{D}$, and $N=2$ on level E).

Interestingly, math skill was predictive for IES, model fit $F(1,69)=5.334, R^{2}$ adjusted $=0.058, \beta=-0.268, t(69)=-2.310$, $p=0.183$, with lower math skill being associated with a lower inverse efficiency score. Adding condition as a predictor next to math skill slightly improved the overall model fit, $F(2,68)=4.234$, $p=0.018, R^{2}$ adjusted $=0.085$, with math skill remaining a significant predictor of IES, $\beta=-0.269, t(68)=-2.356$ $p=0.021, R_{\text {Partial }}=-0.275$, but condition was a nonsignificant predictor of IES, $\beta=0.179, t(68)=1.726 p=0.089$, $R_{\text {Partial }}=0.205$. Subsequently we added the interaction term next to math skill and condition, which substantially improved the overall model fit, $F(3,67)=5.077, p=0.003, R^{2}$ adjusted $=0.149$. In this more predictive moderation model, math skill no longer had a significant main effect on IES, $\beta=0.011, t(67)=0.071$, $p=0.943, R_{\text {Partial }}=0.009$, but condition had a significant main effect on IES, $\beta=0.301, t(67)=2.552, p=0.013, R_{\text {Partial }}=0.298$, which was further qualified by a significant interaction effect, $\beta=-0.406, t(67)=-2.457, p=0.016, R_{\text {Partial }}=-0.289$ (Figure 6). These results support a moderating effect of condition and math skill, such that children in the BA condition were more likely to make less mistakes with slower solving speeds (or more mistakes with faster speeds), especially for those children with a lower math skill score.

\section{DISCUSSION}

We investigated whether children's learning benefited from augmenting an IA about class 1 levers with a BA. It was found that when taking general math skill into account as a moderator, this BA condition was positively affecting lever problem-solving accuracy on the RT-test as compared to the control condition, in which the same instructional animation was shown without the BA. However, this effect was qualified by an interaction, showing that the BA condition improved accuracy on the RT-test for children with a lower level of general math skill, and was absent (if not reversed) for children with a higher math skill. Finally, no evidence was obtained for performance benefits on the transfer task.

As the results are mixed, the question arises whether the BA was "analogous enough" to be informative for learning. Indeed, there are important differences between a seesaw and the BA. Most notably, the BA is imperfect, as the body has two joints with independent moving arms whereas the seesaw has one fulcrum with movement of the arms that are co-dependent. Such (and possibly other) differences might interfere with properly understanding mechanics of seesaws. However, there is some information in the BA that directly corresponds with the mechanics of the seesaw. Namely, there is a one to one correspondence to the difference in weight that would be felt when placing blocks on one's arm with that of the direction of pivot of the seesaw. For example, placing one block on the left arm of the seesaw near the fulcrum and one block on the right arm away from the fulcrum will result in a pivot to the right due to mechanical advantage. This directly corresponds with the relative difference in weight that would be felt when placing one block on the left arm near the fulcrum (joint) and one block on the right arm away from the fulcrum (also due to mechanical advantage). Indeed, in the voiceover of the instructional animations we emphasized to the learner that this was a relevant correspondence.

Therefore, we speculate that the body-analogous information that was present provided a possible means to process the learning content by activating implicit motor knowledge, which provided those children that are least receptive to learning about abstract content (i.e., those with a lower general math skill) 


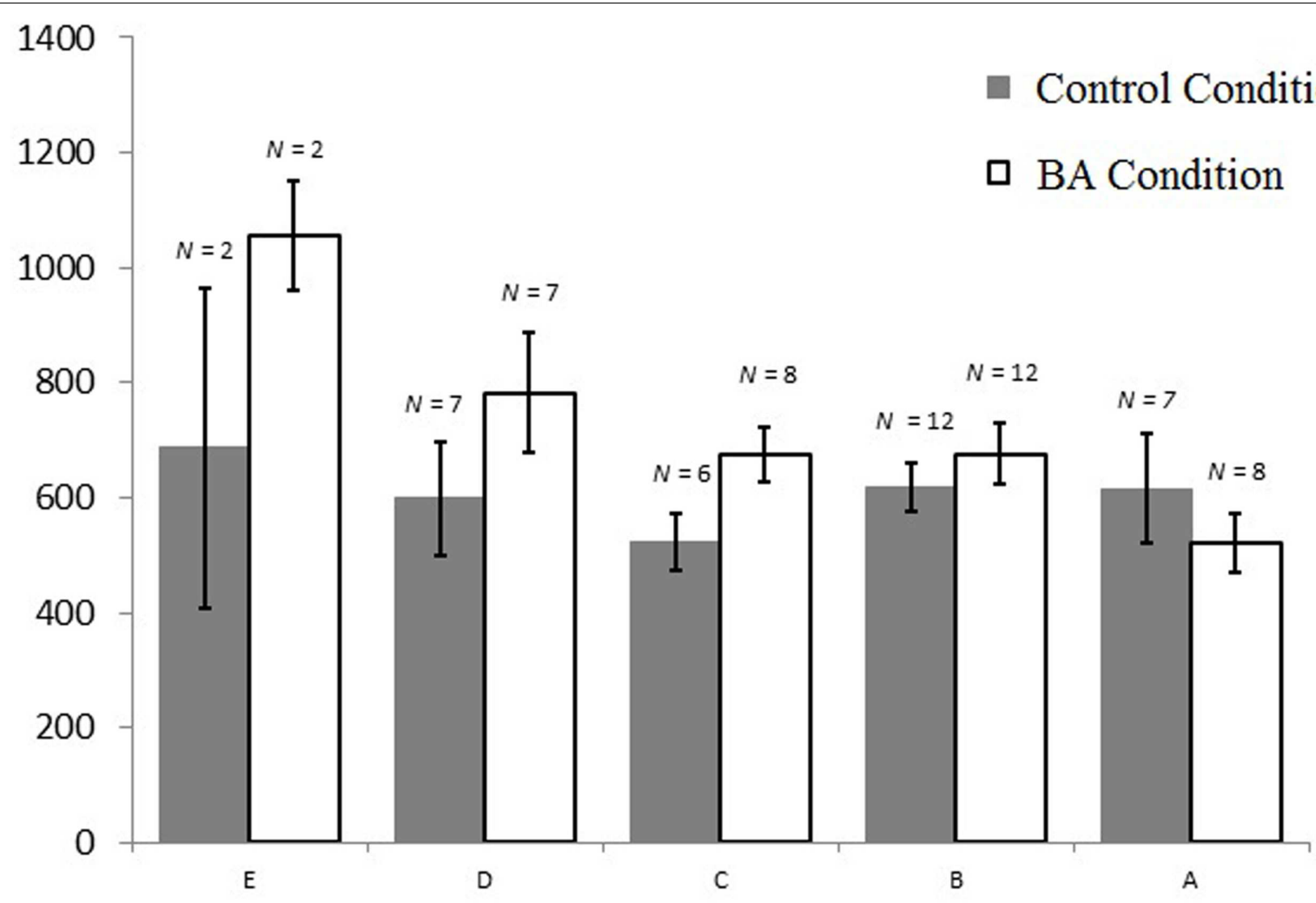

FIGURE 6 | Mean inverse efficiency scores and standard errors for the transfer task per condition and general math skills (E lowest score, A highest score on general math skill).

a way to ground unfamiliar force-dynamics of the seesaw in familiar force-dynamics of the body. In line with observations made by Van Gog et al. (2009), this grounding would be established through mapping of the model's body onto one's own body. Indeed, it seems that when a rule or process is already understood, additional grounding in concrete experiences is unnecessary (Zacharia et al., 2012). We further speculate that in the current case children benefited from the BA condition when performing the reaction time task because they were mentally simulating the force dynamics related to the body (see Van Gog et al., 2009), i.e., mentally re-enacting the learned correspondences of the body and the lever for more accurate problem solving. Yet, we did not find a similar effect of condition on transfer task accuracy as we did in on the reaction timetask. This signals that an efficient strategy on one task does not always readily transfer to another. As shown by Dixon and Dohn (2003), when solving problems with interconnecting balance beams (also included in our transfer task), problem solvers may use more abstract strategies (i.e., alternating strategy; see Dixon and Dohn, 2003 for details) than simply judging each state of each seesaw to judge its effect on the next connected seesaw. Perhaps, judging the forces of a single seesaw like in the RT-task is aided by a BA, whereas this strategy might prove inefficient for solving the transfer problem. On the interconnected seesaw problem, more abstract strategies are more efficient, and discovery of these abstract strategies might even be hampered by a strategy based on a BA. Some evidence speaks to this interpretation as we found that children with lower math scores in the BA condition (as opposed to the control condition) showed higher speed-accuracy tradeoffs, indicating that when these children responded faster their performance was lower (or was higher when they responded more slowly). This might indicate that they attempted to use the $\mathrm{BA}$, but that this was a time-consuming and therefore not necessarily more efficient strategy for solving the transfer task, especially for children with a lower math skill (who would be more likely to use the BA). In sum, future research should be sensitive to the kind of strategy a particular BA solicits, and on which tasks that strategy could be expected to help learning.

Furthermore, in line with findings on the expertise reversal effect (Kalyuga, 2007), the accuracy results show that this mental simulation on the reaction time test was only helpful for children with lower math ability (lower visuospatial working memory capacity) but not helpful, or potentially even detrimental, to those with higher ability (working memory capacity). Perhaps those with a higher ability did not require additional help to induce rules from physical systems so that for them mental simulation during the test task evoked by the BA is superfluous and possibly distracting process. Perhaps this explains why no effects on the transfer test were found, as it was more difficult to use one's 
own body as an analogy on most of the test items that involved multiple balance beams.

It should be noted that the present study provides more of a demonstration than an elaboration of how a BA can affect learning. Indeed, the current design has some shortcomings that prevent such elaboration. For instance, although this task was not one taught in school, the possibility cannot be excluded that some children had more prior knowledge than others; and our current design did not allow for assessing learning gains, as we did not provide children with a pre-test. Furthermore, the current results do not allow us to determine whether higher learning outcomes of children with lower math scores in the BA condition were indeed achieved because cognitive load related to transience was counteracted by more efficient processing due to the BA. There were no differences in mental effort or difficulty ratings between the conditions, but this does not necessarily mean that cognitive load imposed by transience was not reduced. Perhaps the cognitive capacity that was freed-up by reducing the load imposed by transience, was used for processes that were effective for learning, thereby resulting in an similar experience in cognitive load. Future studies might investigate the underlying cognitive mechanisms in more detail, for instance by using continuous and objective cognitive load measures that can be connected to events in the animation such as dual task measures that do not interfere with animation processing (e.g., Park and Brünken, 2014) or EEG measures (e.g., Antonenko et al., 2010).

Future research should further focus on (a) the potential difference in receptivity of children with different individual cognitive capacities for learning with body analogies, (b) the scope of the effectiveness of body analogies on other physical systems (e.g., electrical circuits, gear systems, see for example Johnson-Glenberg et al., 2015), or even more abstract learning domains such as grammatical or language learning (e.g., Lu, 2011), and (c) finally the precise cognitive processes underlying this type of learning (e.g., Brucker et al., 2015).

\section{REFERENCES}

Antonenko, P., Paas, F., Grabner, R., and van Gog, T. (2010). Using electroencephalography to measure cognitive load. Educ. Psychol. Rev. 22, 425-438. doi: 10.1007/s10648-010-9130-y

Amorim, M.-A., Isableu, B., and Jarraya, M. (2006). Embodied spatial transformations: "body analogy" for the mental rotation of objects. J. Exp. Psychol. General 135, 327-347. doi: 10.1037/0096-3445.135.3.327

Ayres, P., and Paas, F. (2007a). Can the cognitive-load approach make instructional animations more effective? Appl. Cogn. Psychol. 21, 811-820. doi: 10.1002/acp.1351

Ayres, P., and Paas, F. (2007b). Making instructional animations more effective: a cognitive load approach. Appl. Cogn. Psychol. 21, 695-700. doi: 10.1002/acp.1343

Brucker, B., Ehlis, A. C., Häußinger, F. B., Fallgatter, A. J., and Gerjets, P. (2015). Watching corresponding gestures facilitates learning with animations by activating human mirror-neurons: an fNIRS study. Learn. Instr. 36, 27-37. doi: 10.1016/j.learninstruc.2014.11.003

Chandler, P. (2009). Dynamic visualisations and hypermedia: beyond the "wow" factor. Comput. Human. Behav. 25, 389-392. doi: 10.1016/j.chb.2008.12.018

De Koning, B. B., and Tabbers, H. K. (2011). Facilitating understanding of movements in dynamic visualizations: an embodied perspective. Educ. Psychol. Rev. 23, 501-521. doi: 10.1007/s10648-011-9173-8

\section{CONCLUSION}

Despite some limitations, our finding that a relatively simple modification of the instructional animation via a BA imbued a positive effect on performance, especially for those with lower general math skill, is a very promising result for future applications in educational practice.

\section{DATA ACCESS}

Data supporting this research report can be retrieved from the Open Science Framework ${ }^{3}$.

\section{AUTHOR CONTRIBUTIONS}

WP designed and collected the data; TvG, RZ, and FP co-designed the experiment. WP written, and TvG, RZ, and FP provided critical revisions on the manuscript.

\section{FUNDING}

This research was funded by the Netherlands Organisation for Scientific Research (NWO-PROO, project number: 411-10-908).

\section{ACKNOWLEDGMENTS}

We would like to thank Gerwin Christiaans for his help in testing the participants and Charly Eielts for developing the instructional animation. We also thank Bonnie Van Huik for her initial contributions to the preparation of the learning material.

\footnotetext{
${ }^{3}$ https://osf.io/4pkjx/
}

Dixon, J. A., and Dohn, M. C. (2003). Redescription disembeds relations: evidence from relational transfer and use in problem solving. Mem. Cogn. 31, 1082-1093. doi: 10.3758/BF03196129

Garber, P. R., and Goldin-Meadow, S. (2002). Gesture offers insight into problemsolving in adults and children. Cogn. Sci. 26, 817-831. doi: 10.1207/s15516 $709 \operatorname{cog} 2606 \_5$

Goldin-Meadow, S., and Alibali, M. W. (2002). "Looking at the hands through time: a microgenetic perspective on learning and instruction," in Microdevelopment: Transition Processes in Development and Learning, eds N. Granott and J. Parziale (Cambridge: Cambridge University Press), 80-105.

Goldin-Meadow, S., and Momeni-Sandhofer, C. (1999). Gestures convey substantive information about a child's thoughts to ordinary listeners. Dev. Sci. 2, 67-74. doi: 10.1111/1467-7687.00056

Hegarty, M., Kriz, S., and Cate, C. (2003). The role of mental animations and external animations in understanding mechanical systems. Cogn. Instr. 21, 325-360. doi: 10.1207/s1532690xci2104_1

Höffler, T. N., and Leutner, D. (2007). Instructional animation versus static pictures: a meta-analysis. Learn. Instr. 17, 722-738. doi: 10.1016/j.learninstruc. 2007.09.013

Hutchins, E., and Nomura, S. (2011). "Collaborative construction of multimodal utterances," in Embodied Interaction: Language and Body in the Material World, eds J. Streeck, C. Goodwin, and C. Lebaron (Cambridge: Academic Press), 29-43. 
Johnson-Glenberg, M. C., Birchfield, D. A., Megowan-Romanowicz, C., and Snow, E. L. (2015). If the gear fits, spin it!: Embodied education and in-game assessments. Int. J. Gaming Comput. Mediat. Simul. 7, 40-65. doi: 10.4018/ IJGCMS.2015100103

Kalyuga, S. (2007). Expertise reversal effect and its implications for learnertailored instruction. Educ. Psychol. Rev. 19, 509-539. doi: 10.1007/s10648-0079054-3

Karmiloff-Smith, A., and Inhelder, B. (1974). If you want to get ahead, get a theory. Cognition 3, 195-212. doi: 10.1016/0010-0277(74)90008-0

Lu, M. T. P. (2011). The Effect of Instructional Embodiment Designs on Chinese Language Learning: the Use of Embodied Animation for Beginning Learners of Chinese Characters. Doctoral dissertation, Columbia University, Columbia.

Park, B., and Brünken, R. (2014). The rhythm method: a new method for measuring cognitive load-An experimental dual-task sudy. Appl. Cogn. Psychol. 29, 232243. doi: 10.1002/acp.3100

Peelen, M. V., and Downing, P. E. (2007). The neural basis of visual body perception. Nat. Rev. Neurosci. 8, 636-648. doi: 10.1038/nrn 2195

Pine, K. J., Lufkin, N., and Messer, D. (2004). More gestures than answers: children learning about balance. Dev. Psychol. 40, 1059-1067 doi: 10.1037/00121649.40.6.1059

Pine, K. J., and Messer, D. J. (2000). The effects of explaining another's actions on children's implicit theories of balance. Cogn. Instr. 18, 37-54. doi: 10.1207/S1532690XCI1801_02

Pouw, W. T. J. L., Van Gog, T., and Paas, F. (2014). An embedded and embodied cognition review of instructional manipulatives. Educ. Psychol. Rev. 26, 51-72. doi: 10.1007/s10648-014-9255-5

Rizzolatti, G., Fadiga, L., Gallese, V., and Fogassi, L. (1996). Premotor cortex and the recognition of motor actions. Cogn. Brain Res. 3, 131-141. doi: 10.1016/0926-6410(95)00038-0

Semenza, C., and Goodglass, H. (1985). Localization of body parts in brain injured subjects. Neuropsychologia 23, 161-175. doi: 10.1016/0028-3932(85) 90101-0
Setti, A., Borghi, A. M., and Tessari, A. (2009). Moving hands, moving entities. Brain Cogn. 70, 253-258. doi: 10.1016/j.bandc.2009.02.012

Spanjers, I. A., Van Gog, T., and Van Merriënboer, J. J. (2010). A theoretical analysis of how segmentation of dynamic visualizations optimizes students' learning. Educ. Psychol. Rev. 22, 411-423. doi: 10.1007/s10648-010-9135-6

Townsend, J. T., and Ashby, F. G. (1978). "Methods of modeling capacity in simple processing systems,". In Cognitive Theory Vol. 3. eds J. Castellan and F. Restle (Hillsdale, N.J.: Erlbaum), 200-239

Tversky, B., Bauer-Morrison, J., and Bétrancourt, M. (2002). Animation: can it facilitate? Int. J. Hum. Comput. Stud. 57, 247-262. doi: 10.1006/ijhc.2002.1017

Van der Ven, S. H. G., Van der Maas, H. L. J., Straatemeier, M., and Jansen, B. R. J. (2013). Visuospatial working memory and mathematical ability at different ages throughout primary school. Learn. Individ. Differ. 27, 182-192. doi: 10.1016/j.lindif.2013.09. 003

Van Gog, T., Paas, F., Marcus, N., Ayres, P., and Sweller, J. (2009). The mirror neuron system and observational learning: implications for the effectiveness of dynamic visualizations. Educ. Psychol. Rev. 21, 21-30. doi: 10.1007/s10648-0089094-3

Zacharia, Z. C., Loizou, E., and Papaevripidou, M. (2012). Is physicality an important aspect of learning through science experimentation among kindergarten students? Early. Child. Res. Q. 27, 447-457. doi: 10.1016/j.ecresq. 2012.02.004

Conflict of Interest Statement: The authors declare that the research was conducted in the absence of any commercial or financial relationships that could be construed as a potential conflict of interest.

Copyright (c) 2016 Pouw, van Gog, Zwaan and Paas. This is an open-access article distributed under the terms of the Creative Commons Attribution License (CC BY). The use, distribution or reproduction in other forums is permitted, provided the original author(s) or licensor are credited and that the original publication in this journal is cited, in accordance with accepted academic practice. No use, distribution or reproduction is permitted which does not comply with these terms. 


\title{
Stimulus-Response Compatibility Effect in the Near-Far Dimension: A Developmental Study
}

\author{
Aurélien Richez ${ }^{1}$, Gerard Olivier ${ }^{2}$ and Yann Coello ${ }^{1 *}$ \\ ${ }^{1}$ CNRS, UMR 9193 - SCALab - Sciences Cognitives et Sciences Affectives, Université de Lille, Lille, France, ${ }^{2}$ Laboratoire \\ Interdisciplinaire Récits Cultures Et Sociétés, University of Nice Sophia Antipolis, Nice, France
}

The present study investigates the developmental aspect of stimulus-response compatibility (SRC) effect in 8-11-years-old children. The task consisted in manually responding to the color of a pawn presented on a chessboard at different distances. Manual responses were provided by reaching a proximal or distal location depending on the color of the stimulus. We found that reaction time was affected by the conflict generated by the response suggested by the location of the stimulus and the response required according to its color. This was not the case for movement time despite we found a higher rate of long duration movements in the incongruent than in the congruent spatial condition. The SRC effect was, however, observed in children older than 10 years

OPEN ACCESS

Edited by:

Anna M. Borghi,

University of Bologna, Italy

Reviewed by:

Costantini Marcello,

University of Chieti-Pescara, Italy Christel Bidet-lldei,

University of Poitiers, France

*Correspondence:

Yann Coello

yann.coello@univ-lille3.fr

Specialty section:

This article was submitted to

Cognition,

a section of the journal

Frontiers in Psychology

Received: 15 June 2016

Accepted: 22 July 2016

Published: 05 August 2016

Citation:

Richez A, Olivier G and Coello Y

(2016) Stimulus-Response Compatibility Effect in the Near-Far

Dimension: A Developmental Study.

Front. Psychol. 7:1169.

doi: 10.3389/fpsyg.2016.01169 old. These findings provide additional evidence for a reorganization of the perceptual system during the period of 8-10 years, integrating progressively multimodal information and preparing more efficiently the body to act in the environment.

Keywords: development, stimulus-response compatibility, reaching, reaction time, movement time

\section{INTRODUCTION}

Since the last century, the role of action in perception and cognition as been widely discussed in philosophy (e.g., Merleau-Ponty, 1945; Hurley, 2002; Noë, 2004), psychology (e.g., James, 1890; Piaget and Inhelder, 1969; Gibson, 1979; Coello and Fischer, 2015) as well as in neurosciences (e.g., Jeannerod, 2006; Rizzolatti and Sinigaglia, 2008). Common to the different theories is the shared idea that perception, action and cognition coevolve through the active exploration of the body and the environment, and contribute through their interaction to knowledge acquisition and retention (Hommel et al., 2001; Chemero, 2003; Barsalou, 2008). In agreement with this view, developmental studies have emphasized the importance of very early interactions with the environment for the ontogenetic development of perceptual (Held and Hein, 1963; Fine et al., 2003) and cognitive abilities (e.g., Wallon, 1945; Piaget and Inhelder, 1969; Thelen and Smith, 1994). On the basis of observations and experimental data, these pioneer researchers have furthermore highlighted the non-linear trajectory of sensorimotor and cognitive developments, characterized by significant quantitative and qualitative changes taking place during the ontogenesis. Within this framework, gradual improvements in perceptual, motor and cognitive abilities through the ontogenetic development were interpreted as relying on structural and functional maturation of the nervous system (Gesell, 1929; Carmichael, 1946; McGraw, 1946), as well as on the multidimensional and dynamical aspect of brain mechanisms relating to behaviors (Greenough et al., 1987; Fischer and Bidell, 1998; Segalowitz, 2002), leading progressively to more efficient processing 
and integration of multimodal information (Choudhury et al., 2007; Gori et al., 2008; Nardini et al., 2010).

Putting development under careful scrutiny has emphasized a particular critical period around the age of 8-10 years, characterized by strong reorganization of motor, perceptual and cognitive activities. Indeed, even if performances are still not equivalent to adults or even adolescents (Choudhury et al., 2007), 8-10 years-old children behave differently than younger children in that they base their perception on multimodal sensory integration (Gori et al., 2008; Nardini et al., 2010), use more efficient predictive mechanisms in their motor performance (Thibaut and Toussaint, 2010) and reveal better flexibility and inhibition capacities in cognitive control (Houdé and Guichart, 2001). Considering the perception of objects' orientation and size for instance, 5-6 years-old children exhibit a clear dominance of one modality upon the other, basing their perception on single (visual or haptic) sensory information. In contrast, 8-10 years children integrate visuo-haptic information in their perceptual estimates (Gori et al., 2008), in accordance with optimal integration statistical models characterizing adults' performances (Ernst and Banks, 2002; see also Nardini et al., 2008, 2010 for similar findings with multidimensional integration of unimodal sensory information). During this period of age, motor control also improves significantly (Assaiante and Amblard, 1995), with more appropriate anticipatory control of posture (Girolami et al., 2010) and object-directed motor actions (Forssberg et al., 1992), as observed in eye-hand coordination (Ego et al., 2016), simple motor tasks such as moving a lever (Bard et al., 1990), or more complex motor tasks such as writing or drawing (Meulenbroek and Van Galen, 1988; Lange-Küttner, 2009). From 8 to 10 years, children show influence of the terminal state of the body during the programming stage of object-directed motor actions (endstate-comfort-effect, Adalbjornsson et al., 2008; Thibaut and Toussaint, 2010) as well as a decrease of movement speed (Bard et al., 1990), suggesting a reorganization of motor planning and guiding strategies based on the refinement of internal models supporting motor skills and predictive behaviors (Thibaut and Toussaint, 2010; Ego et al., 2016). This is also at this age that the capacity to relate sensorimotor and visual information appears a major determinant of perceptual performances, as evidenced, for instance, by the increase accuracy in visually detecting what is reachable with the arm (Richez and Coello, 2015).

As visuo-motor reorganization appears to be spread across a large span of abilities implying the integration of sensorimotor information, we expected developmental changes during the period between 8-10 years to influence the effect of stimulusresponse compatibility (SRC). SRC effect is characterized by a faster and more accurate performance when the location of a visual stimulus is compatible with the location of the motor response provided according to a specific attribute of the stimulus. The classical example of such effect is the Simon effect (Simon and Rudell, 1967; Simon and Small, 1969), which corresponds to the situation where participants must respond to a left or right visual stimulus with one hand (e.g., right) when the stimulus has one color (e.g., red), and with the other hand (e.g., left) when the stimulus has a different color (e.g., green), regardless the location of the visual stimulus. Although the participants are not supposed to take into account the position of the visual stimulus, they usually react faster when the color of the stimulus that appears on one side corresponds to the motor response provided on the same side (congruent trials), compared to the motor response provided on the opposite side (incongruent trials). Following this seminal study, the Simon effect has been used as a valuable tool to study multimodal perception as well as the relation between perception and action (Tucker and Ellis, 1998; Buetti and Kerzel, 2009; Hommel, 2011). Furthermore, Simon's followers have adapted the original paradigm by changing intrinsic and extrinsic features of the stimuli while generally retaining the left-right motor responses (e.g., Hommel, 2011 for a review). To account for SRC effect as a whole, several authors have suggested the existence of two distinct routes from the stimulus to the response, namely a direct and an indirect route (e.g., Kornblum, 1994; Eimer et al., 1995). The first one, the direct route, characterizes an automatic activation of the motor response in relation to the location of the stimulus, and a facilitation effect when both the stimulus and the response features spatially overlap. The second one, the indirect route, links stimulus and response through an arbitrary relationship depending on the experimental instructions. In this respect, Hommel et al. (2001) suggested that the overlap between the perceptual and motor codes within a common and amodal coding system of the various features of an object originates the SRC effect. Accordingly, SRC effect does not depend of the relevant or irrelevant nature of the stimulus-response features in relation to the task, but simply the presence of an overlap between perceptual and motor dimensions (Kornblum, 1994; Hommel, 1997). In agreement with this, SRC effect is not restricted to the right-left dichotomy and several studies have shown that the effect can be extended to the near-far (e.g., Olivier, 2006; Coutte et al., 2015) as well as the up-down (e.g., Cho and Proctor, 2002; Meiran, 2005) spatial dimensions. Furthermore, SRC effect is thought to affect response selection as well as the subsequent programming and execution stages of motor responses (Buetti and Kerzel, 2008, 2009; Coutte et al., 2015).

In order to probe the developmental aspect of the relation between perception and action, we implemented a SRC task in a population of children extending from 8-years to over 11years, a period characterized by major developmental changes related in particular to the integration of multisensory and motor information. In relation to the literature summarized above, we expected to observe the SRC effect with a classical pattern of facilitation for congruent relationship between perception and action, but predominantly in older children when the ability to optimally combine multisensory and motor information arises. In line with Buetti and Kerzel (2008), we expected also an improvement of children performances in terms of lower reaction time (RT) and movement time (MT) in the congruent condition. In our implementation of the SRC paradigm, children were exposed to a chessboard with one black or white pawn presented either at a proximal or distal location, as previously used by Coutte et al. (2015) to evaluate the SRC effect in the near-far dimension. The task was to respond to the color of a chess pawn by responding with the right hand toward either a proximal or distal location, regardless of the proximal-distal position of the 
pawn. We manipulated thus the compatibility of the perceived distance of the stimulus (the irrelevant feature) and the response distance during a simple color discrimination task (the relevant feature).

\section{MATERIALS AND METHODS}

\section{Participants}

A total of 120 children (age range between 8 years 1 month and 12 years 3 months; $M=10$ years, $S D=1$ year) were recruited from French public elementary schools (grades 4-7). The population was then divided into 4 groups, according to participant's age: 8 -years-old $(N=30 ; M=8$ years 8 months, $S D=3$ months), 9-years-old ( $N=30 ; M=9$ years 6 months, $S D=3$ months), 10 -years-old ( $n=30 ; M=10$ years 6 months, $S D=3$ months), 11-years-old $(N=30 ; M=11$ years 6 months, $S D=4$ months).

During the recruitment, consents were obtained from the French National Education Services as well as from the children and their parents. Although practical information was provided to the children, none of them was informed prior to the experimental session about the aim of the study. Full explanations were nonetheless given at the end of the experiment and results were shown to the children. None of the participants had previous record of neurological or psychiatric disorders, or any kind of motor impairment. All participants were right-handed and had a normal (or corrected to normal) vision.

\section{Apparatus and Stimuli}

The experimental apparatus consisted in a computer screen and a response device. The computer screen $\left(22^{\prime \prime}, 100 \mathrm{~Hz}\right)$ was placed vertically on a table in front of the participant at a distance of about $57 \mathrm{~cm}$. The response device was placed on the table between the participant and the computer screen. It consisted of 3 handoperated switches fixed on a $30-\mathrm{cm}$-square wooden panel. The 3 switches were aligned with the participant's mid-body sagittal axis. The closest switch ("starting location switch") was a flat 2$\mathrm{cm}$-square and was situated $1 \mathrm{~cm}$ from the proximal edge of the panel. It could be operated by a simple contact or release using the forefinger. The 2 other switches were cubic sensors $(2 \times 2 \times 2 \mathrm{~cm})$, the first one was placed at $10 \mathrm{~cm}$ and the second one at $25 \mathrm{~cm}$ from the starting location. Both of them needed a precision grip using the thumb and the forefinger to be operated (see Figure 1).

Nine colored pictures $(1280 \times 857$ pixel, resolution $300 \mathrm{dpi})$ representing a square-chessboard $(30 \mathrm{~cm} \times 30 \mathrm{~cm})$ were used as stimuli. The pictures reproduced the visual perspective that a chess player would have when facing an actual chessboard. One picture represented an empty chessboard, the other pictures represented a chessboard with one pawn. The pawn was placed on one of the squares of the 2 central columns. Four possible locations were selected: 2 proximal locations (3rd row) and 2 distal locations (7th row). Half of the pawns were black and the other half was white. Four other pictures were used for the training session. They represented black and white pawns placed at a central location relatively to the other stimuli (5th row). The presence of a black pawn on a white squares, or vice versa, was counterbalanced.

\section{Procedure}

After having been informed about the purpose and conduct of the experiment, the participants were invited to sit in front of the apparatus and to perform a short training session following the same procedure as for the forthcoming experimental session. The actual experiment started when the participants were able to provide motor responses accurately and fluently without the necessity for visual guidance of the moving hand. Before starting the experiment, the participants were allowed to adjust their chair and position to ensure that they could easily perform the proximal and distal manual responses. The proximal response consisted in grasping the nearest switch whereas the distal response consisted in grasping the farthest one. The participants provided their responses with the right hand, the left one remaining in a resting position on the table during the whole experimental session. Half of the participants were instructed to perform a proximal response in the presence of a white stimulus and a distal response in the presence of a black stimulus. The other half received the opposite instruction. At the beginning of each trial, a pictogram was displayed to the participants to indicate that they have to place their hand on the starting position. $500 \mathrm{~ms}$ after having reached the starting position, the photograph of an empty chessboard was displayed. Then, following a constant period of $500 \mathrm{~ms}$, a fixation cross appeared at a location on the chessboard that was presented as corresponding to the location of the starting location for the hand on the response device $(1 \mathrm{~cm}$ from the proximal edge of the chessboard, along the mid-body sagittal plane). After a random period of 500-1500 ms, the fixation cross disappeared and the stimulus (the chessboard with a pawn) was displayed. The participants were instructed to respond as fast and accurately as possible, the stimuli remaining visible until the participants provided their response. An error signal was displayed when the participants anticipated their response (i.e., lift their finger before the apparition of the stimulus) or produced a wrong answer. The whole experimental session was accomplished within one block of trials, including five presentations of the black and white pawns at a proximal and distal location in each column (random order), for a total of 40 trials. All trials with error or anticipation, as well as those with a response time below $200 \mathrm{~ms}$ or above $1000 \mathrm{~ms}$, were repeated at the end of the block of trials. The experimental design corresponded thus to the equation $\mathrm{P}_{15}{ }^{*} \mathrm{I}_{2} * \mathrm{~A}_{4}{ }^{*}<\mathrm{R}_{2} * \mathrm{~S}_{2}>$, with $\mathrm{P}$ for Participants, I for Instruction (white pawn for proximal response or white pawn for distal response), A for Age group (8, 9, 10, 11), R for manual Response (proximal or distal), $\mathrm{S}$ for Stimulus location on the chessboard (proximal or distal).

\section{Data Recording and Analysis}

Both stimulus display and response recording were under the control of Matlab software (R2008b, mathworks). In each trial, the response provided by the participants as well as RT and MT were recorded by the computer. RTs were computed as the time elapsed between the presentation of the stimulus 


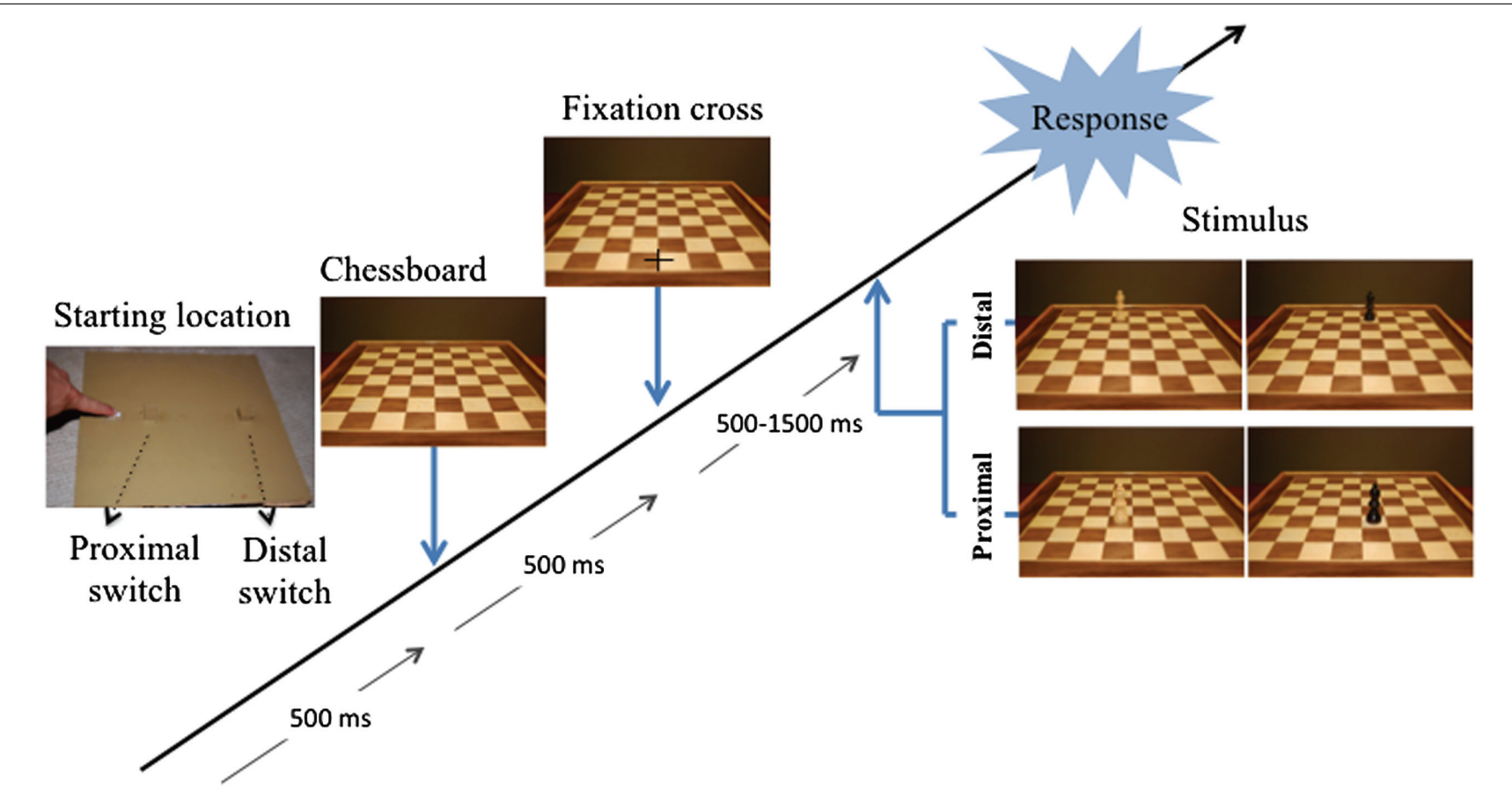

FIGURE 1 | Experimental set up and time sequence of the presentation of the stimuli used in the experiment.

and response initiation (the lift of the switch at the starting position). Movement times were computed as the time elapsed between the release of the starting position switch and the grasping of one of the response switches. In order to precisely catch the temporal dynamics of the individual performances, we analyzed the asymmetry of the distribution of the temporal measures, thought to highlight the variability associated with developmental acquisitions (Immordino-Yang and Fischer, 2007; Rose and Fischer, 2008), by fitting the data with an ex-Gaussian fit procedure (Ratcliff and Murdock, 1976; Lacouture and Cousineau, 2008). The ex-Gaussian distribution is a convolution of a normal and an exponential distribution. The probability density function of the ex-Gaussian fit is given by the following equation:

$$
f(x \mid \mu, \sigma, \tau)=\frac{1}{\tau} \exp \left(\frac{\mu}{\tau}+\frac{\sigma^{2}}{2 \tau^{2}}-\frac{x}{\tau}\right) \Phi\left(\frac{x-\mu-\sigma^{2} / \tau}{\sigma}\right)
$$

where $\Phi$ represents the value of the cumulative density of the Gaussian distribution.

Ex-Gaussian fit procedure was performed using the MATLAB toolbox "DISTRIB" function, in accordance with Lacouture and Cousineau (2008). The ex-Gaussian function applied to empirical RT and MT data provided estimates for three independent parameters: $\mathrm{mu}$, sigma, and tau. $\mathrm{Mu}(\mu)$ represents the mean of the normal component and mainly reflects the average performance. Sigma $(\sigma)$ corresponds to the $S D$ of the normal component and indicates the variability of the performances. Tau $(\tau)$ corresponds to both the mean and the standard deviation of the exponential component and reflects extremes in performance and thus the asymmetry of the function (a greater $\tau$ means a higher amount of long responses compared to short responses, Matzke and Wagenmakers, 2009).

As the instruction factors did not show any significant effect, a $2 \times 2 \times 2$ analysis of Variance (ANOVA) was run on all dependant variables with Target distance (proximal, distal) and Manual response (proximal, distal) as within-subject factors and Age group $(8,9,10,11)$ as between-subjects factor.

\section{RESULTS}

\section{Reaction Time}

The ANOVA performed on the $\mu$ parameter of the RT distribution revealed a significant interaction between Stimulus location and Manual response $[F(1,116)=5.07, p=0.02]$ (see Figure 2). Overall, when children were presented with a proximal stimulus, RT was shorter when they grasped the proximal $(M=426 \mathrm{~ms}, S D=91 \mathrm{~ms})$ than the distal switch ( $M=432 \mathrm{~ms}, S D=79 \mathrm{~ms})$. When the children were presented with a distal stimulus, RT was shorter when they grasped the distal $(M=426 \mathrm{~ms}, S D=85 \mathrm{~ms})$ than the proximal switch $(M=438 \mathrm{~ms}, S D=97 \mathrm{~ms})$. The ANOVA also revealed an interaction between these two factors and Age group $[F(3,116)=3.48, p=0.01]$. Contrasting the data obtained in the different age groups showed that the stimulus location and manual response interaction was not significant for the younger children $[F(1,29)=0.42, p>0.05$ for age group $8 ; F(1,29)=0.55, p>0.05$ for age group 9], emerged as a statistical trend toward significance for the 10 -years-old children 


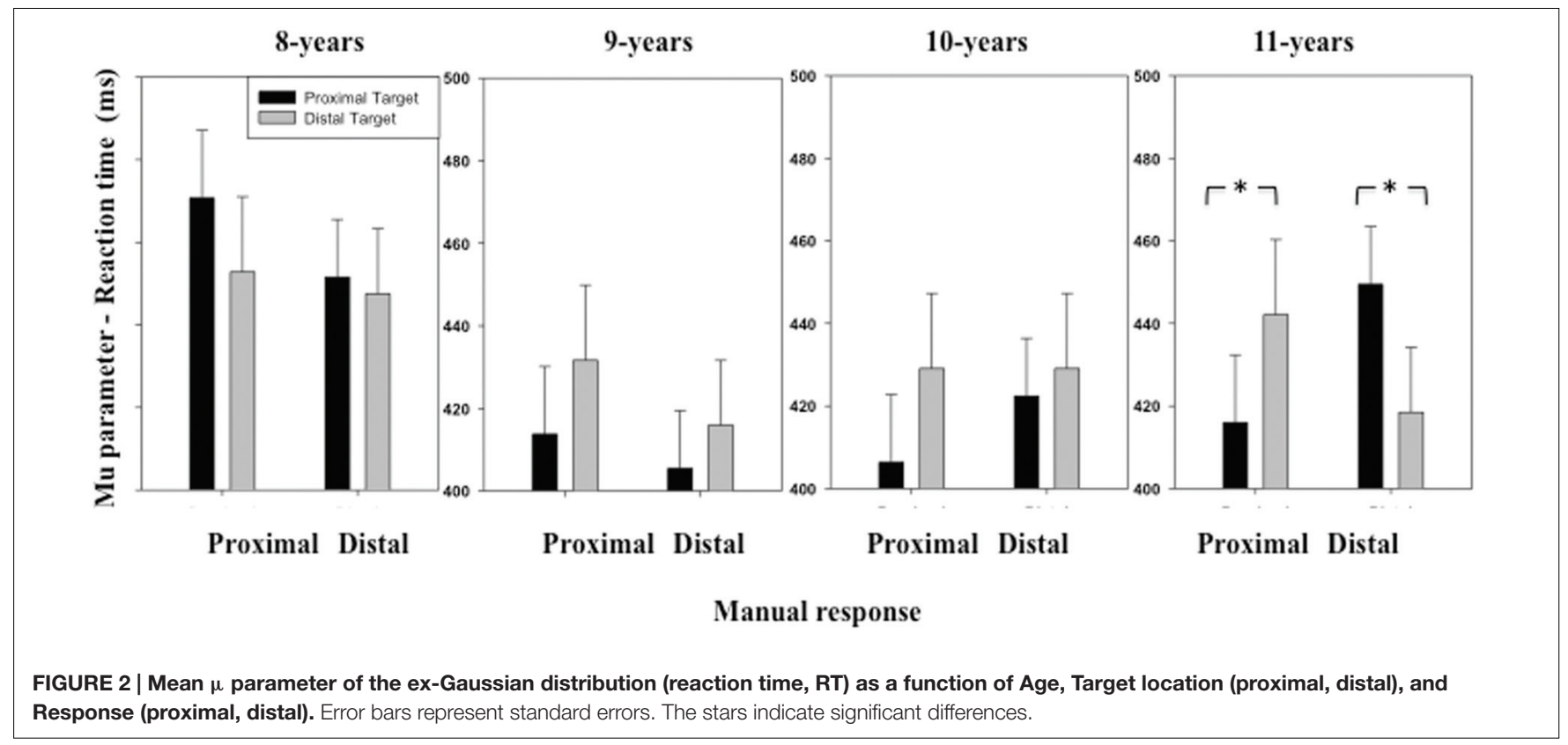

$[F(1,29)=1.98, p=0.16]$, and was significant for the oldest children $[F(1,29)=14.13, p<0.001$ for age group 11]. For the 11-years old children, we observed the classic pattern of shorter RTs for congruent trials than incongruent ones as observed in adults, namely, RTs significantly increased in the incongruent response condition when the target was both at the proximal $[t(29)=2.23, p=0.01]$ or distal $[t(29)=1.70, p=0.04]$ location.

The ANOVA performed on the $\sigma$ and $\tau$ parameters of the exGaussian distribution of RT distribution revealed no significant effect of either the main factors or the interactions between the main factors (see Figures 3 and $\mathbf{4}$ ).

\section{Movement Time}

The ANOVA performed on the $\mu$ parameter of the ex-Gaussian distribution of MT distribution only revealed a main effect of Stimulus location $[F(1,116)=7.34, p<0.01]$ and Manual response $[F(1,116)=246.43, p<0.01]$ (see Figure 5). Overall, MTs were shorter when the stimulus was displayed at a proximal $(M=415 \mathrm{~ms}, S D=108 \mathrm{~ms})$ than a distal location $(M=429 \mathrm{~ms}$, $S D=123 \mathrm{~ms}$ ). MTs were also shorter when the children produced a proximal $(M=376 \mathrm{~ms}, S D=103 \mathrm{~ms})$ than a distal response ( $M=468 \mathrm{~ms}, S D=110 \mathrm{~ms})$. The interactions between these two factors and between the last two factors and Age group were not significant.

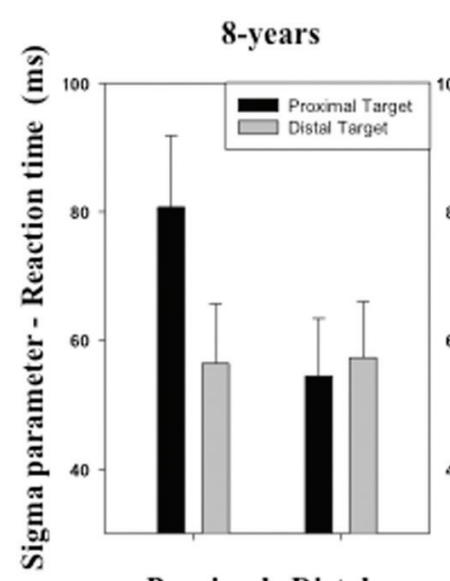

Proximal Distal

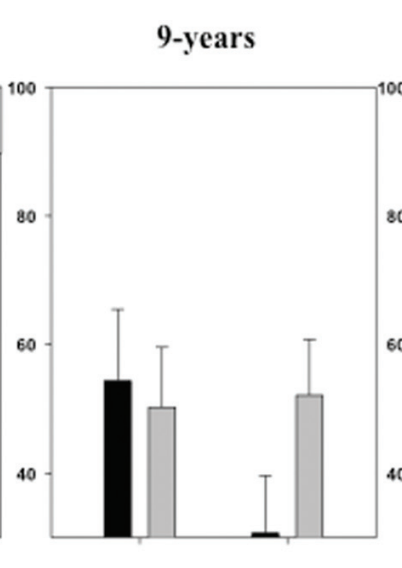

Proximal Distal

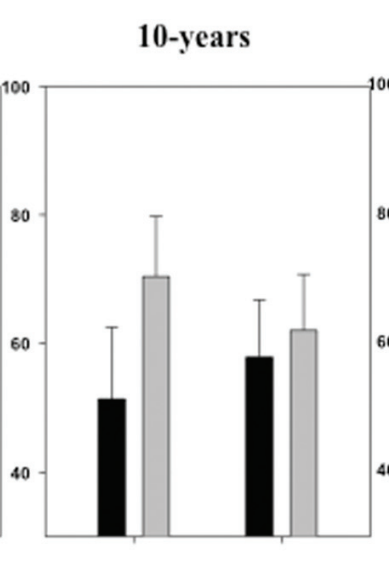

Proximal Distal

\section{1-years}

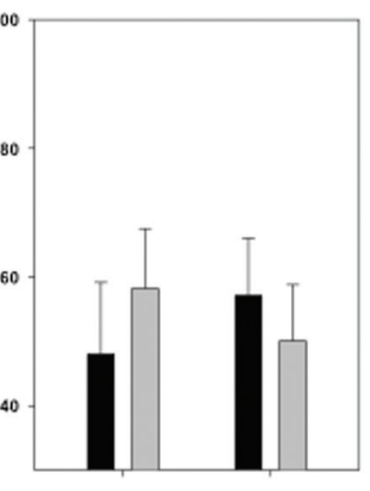

Proximal Distal

Manual response

FIGURE 3 | Mean $\sigma$ parameter of the ex-Gaussian distribution (RT) as a function of Age, Target location (proximal, distal), and Response (proximal, distal). Error bars represent standard errors. 


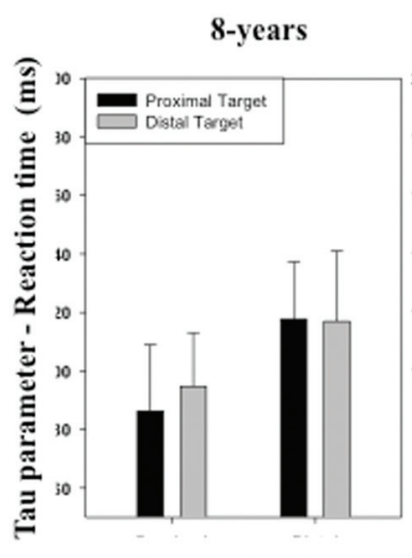

Proximal Distal

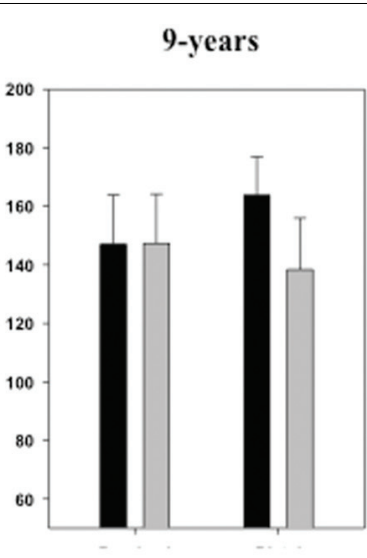

Proximal Distal

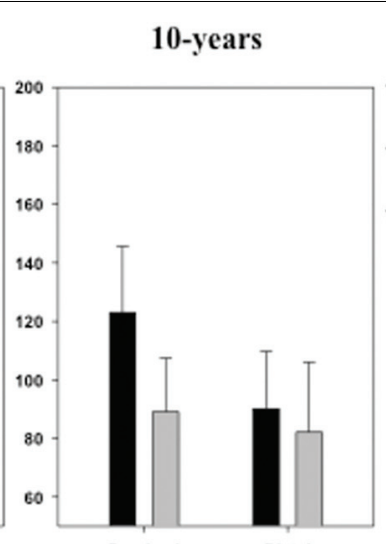

Proximal Distal 11-years

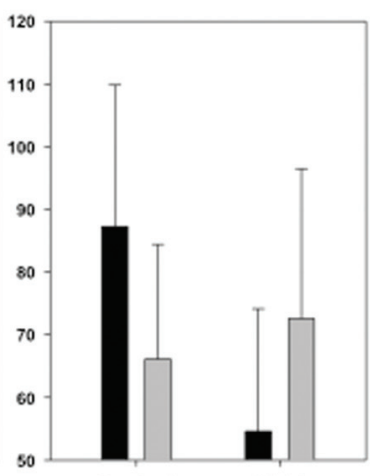

Proximal Distal

Manual response

FIGURE 4 | Mean $\tau$ parameter of the ex-Gaussian distribution (RT) as a function of Age, Target location (proximal, distal), and Response (proximal, distal). Error bars represent standard errors.

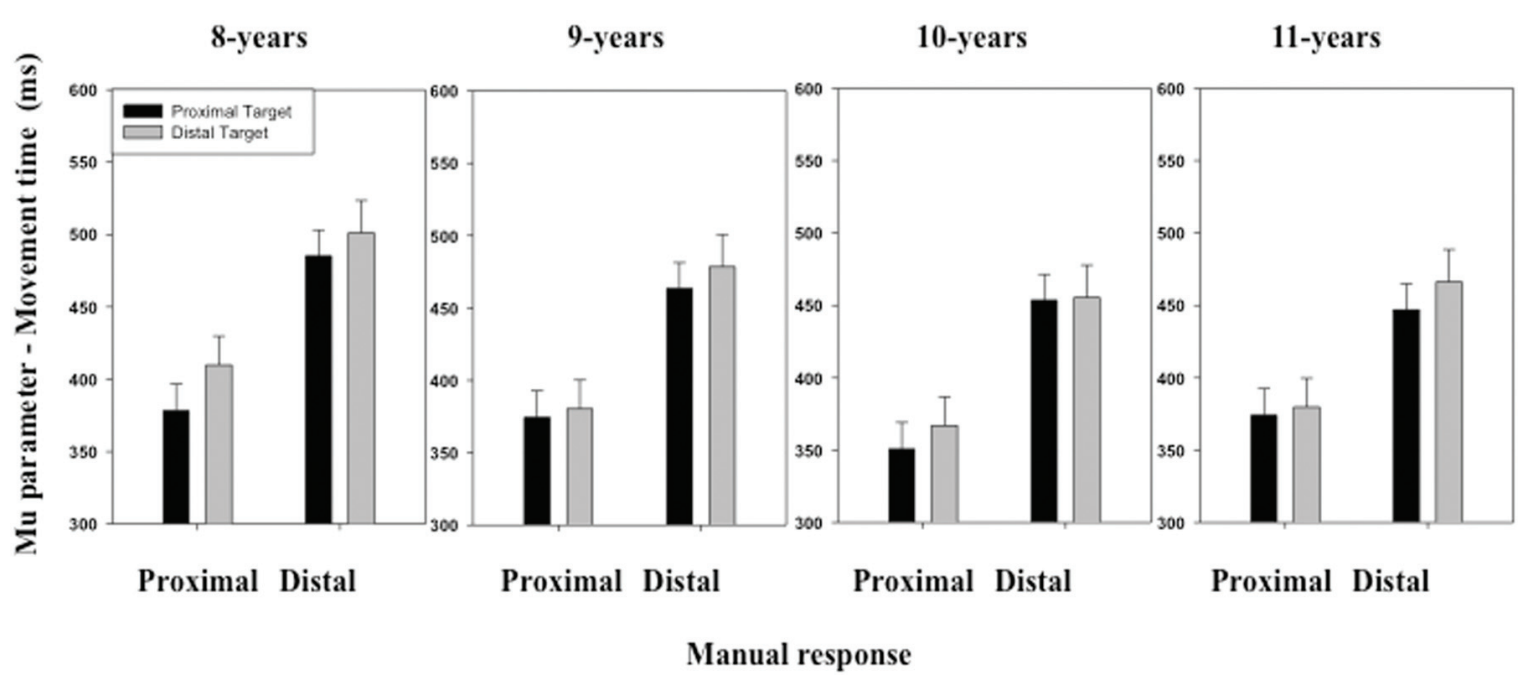

FIGURE 5 | Mean $\mu$ parameter of the ex-Gaussian distribution (movement time, MT) as a function of Age, Target location (proximal, distal), and Response (proximal, distal). Error bars represent standard errors.

The ANOVA performed on the $\sigma$ parameter of the exGaussian distribution of MT distribution revealed no significant effect of either the main factors or the interactions between the main factors (see Figure 6).

The ANOVA performed on the $\tau$ parameter of the exGaussian distribution of MT distribution revealed a significant interaction between Stimulus location and Manual response $[F(1,116)=7.59, p<0.01]$ (see Figure 7). When the participants executed a proximal response, they produced a smaller amount of long responses when the stimulus was displayed at a proximal $(M=129 \mathrm{~ms}, S D=129 \mathrm{~ms})$ than a distal location $(M=145 \mathrm{~ms}$, $S D=120 \mathrm{~ms}$ ). When the participants executed a distal response, they produced a smaller amount of long responses when the stimulus was displayed at a distal $(M=119 \mathrm{~ms}, S D=139 \mathrm{~ms})$ than a proximal location $(M=145 \mathrm{~ms}, S D=102 \mathrm{~ms})$. Although the interaction between these last two factors and Age group was not significant, significant differences between congruent and incongruent conditions were observed for the 11 years children only, when responding to the proximal $[t(29)=1.87, p=0.03]$ or distal $[t(29)=2.70, p<0.01]$ location.

\section{DISCUSSION}

The main objective of the present study was to explore the effect of SRC over the period of age between 8 and 11-years. Overall, the data show that children had better performances for 


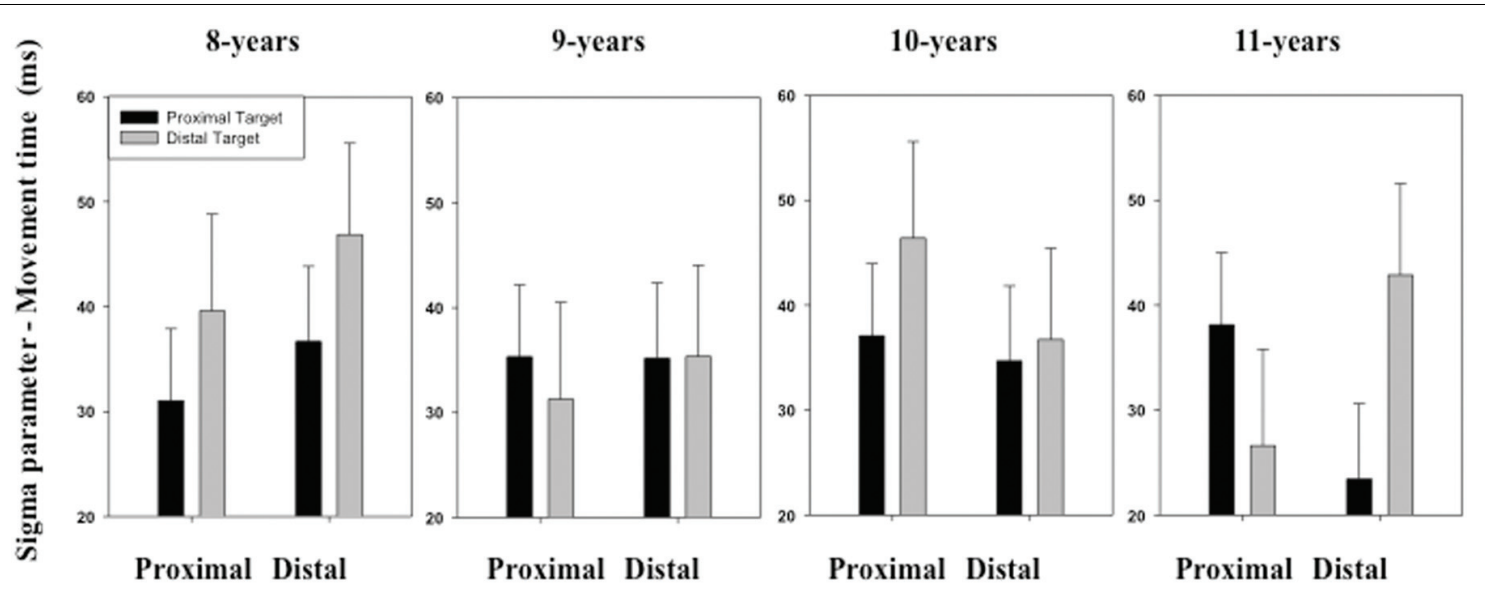

Manual response

FIGURE 6 | Mean $\sigma$ parameter of the ex-Gaussian distribution (MT) as a function of Age, Target location (proximal, distal), and Response (proximal, distal). Error bars represent standard errors.

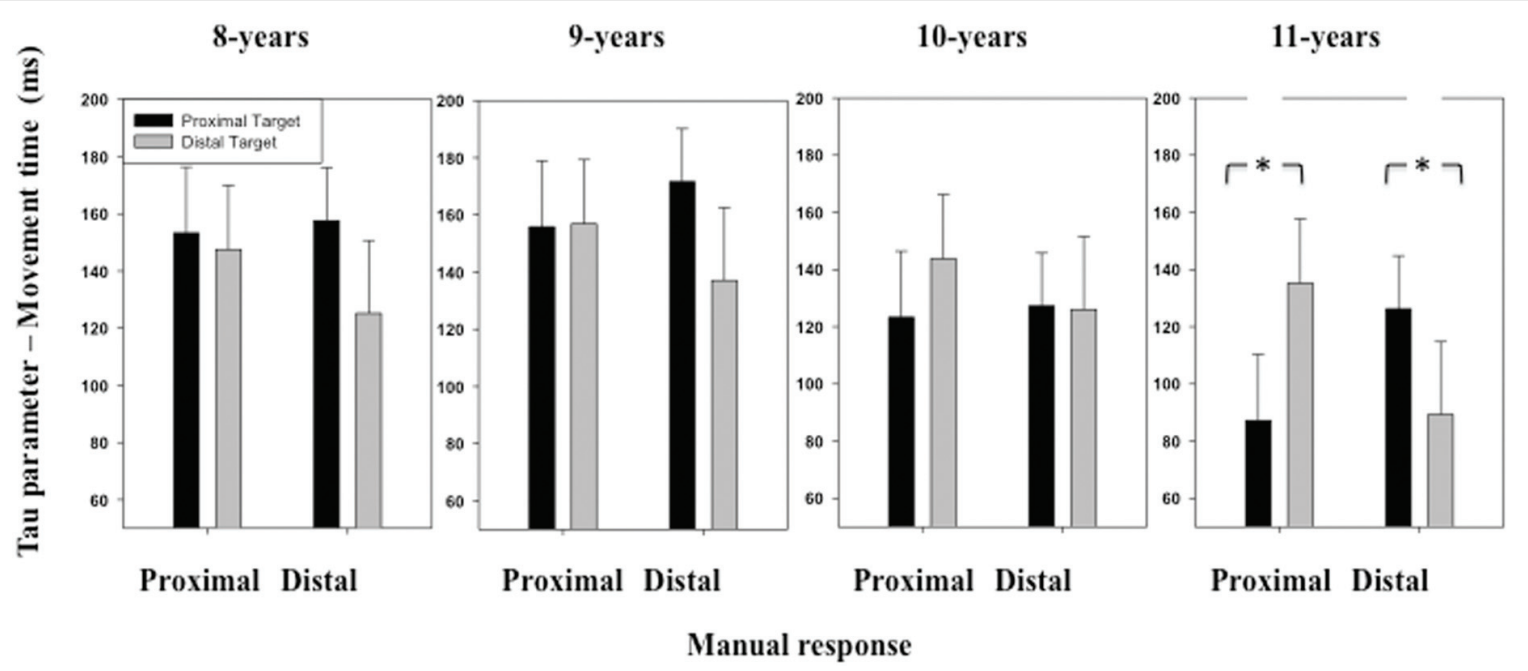

FIGURE 7 | Mean $\tau$ parameter of the ex-Gaussian distribution (MT) as a function of Age, Target location (proximal, distal), and Response (proximal, distal). Error bars represent standard errors. The stars indicate significant differences.

congruent than incongruent trials. In particular, despite the task required to process stimulus color only, RTs were shorter when producing a proximal response in the presence of a proximal stimulus and a distal response in the presence of a distal stimulus, than a proximal response in the presence of a distal stimulus or vice-versa. This effect corresponds to the classical SRC effect and was observed for the $\mu$ parameter (mean performance) but not for the $\sigma$ (performance variability) and $\tau$ (asymmetry in the distribution) parameters of the ex-Gaussian distribution of RT distribution. However, the group analysis revealed that this pattern of results was affected by children age as only the 11years children showed the typical pattern of SRC effect usually observed in adults (Tucker and Ellis, 2001; Olivier, 2006; Buetti and Kerzel, 2008; Coutte et al., 2015). SRC effect seems thus to appear at a specific period of children development, with a shift in the performance occurring between 8 and 11 years, as suggested by the data on RT (we indeed observed a trend toward significant effect for 10-years-old children). The absence of main effect of stimulus location or manual response on RTs suggested that the task difficulty and stimuli visibility were homogeneous across all the conditions and for all age groups. This is confirmed by the lack of effect of congruent-incongruent conditions on the $\sigma$ and $\tau$ parameters of the ex-Gaussian fit procedure of the data relating to RT. Interestingly, 11 years old children produced fewer long-lasting movements in the congruent than the incongruent conditions ( $\tau$ parameter of the ex-Gaussian function for MT). They indeed show a broader amount of long-lasting movements when performing a short movement in the presence of a distal target and when performing a long movement in the presence of a proximal target. This suggests that SRC effect evidenced from 
the age of 11-years in the RT data, also influences movement execution with a higher rate of long-lasting movement when there was no congruence between the location of a visual target and the extent of arm movement.

As a whole, the present findings are thus compatible with the idea of a critical period of qualitative change between the age of 8-10 years, in particular in the way visual information is processed in relation to other sensory systems (Gori et al., 2008) and the motor system (Adalbjornsson et al., 2008; Thibaut and Toussaint, 2010; Ego et al., 2016). The presence of the SRC effect may indeed be interpreted as the effect of predictive mechanisms associated with the perceptual-motor coupling linked to the processing of the visual stimulus (Hommel, 2011; Coutte et al., 2015). According to SRC effect, in the presence of a visual stimulus intrinsic and extrinsic characteristics of that stimulus are automatically processed, as well as the motor response aligned with the distance of the stimulus. Such an automatic motor coding of visual stimulus has been demonstrated in the past using either passive visual observation (Quinlan and Culham, 2007; Proverbio, 2012), visual discrimination task (Tucker and Ellis, 1998), visually triggered motor actions (Costantini et al., 2010) or reachability judgment tasks (Wamain et al., 2015). It has also been found for various spatial dimensions including rightleft (Kornblum, 1994; Hommel, 2011) and up-down dimension (Cho and Proctor, 2002; Meiran, 2005). The pattern of results observed in the 11-years old children is thus compatible with the theory of event coding (Hommel et al., 2001), which argues that stimulus and response coding are not independent but share a common level of processing. The present findings are also compatible with Kornblum's dual route model (Kornblum et al., 1990; Kornblum, 1994), which postulates independent responses selection depending on the stimulus attributes, which could nonetheless overlap in time depending on the one hand on the stimulus features automatically processed and, on the other hand, on the stimulus features intentionally linked to the task requirements. The effect found on RTs measurements may thus reflect the conflict between relevant and irrelevant features influencing response selection: a faster response initiation is indeed observed for the 11-years old in the congruent trials in comparison to incongruent ones.

Surprisingly, no SRC effect was found on MT (mu parameter of the ex-Gaussian function for MT) at all the ages tested in the present experiment. It was indeed reported in previous studies that SRC effect affects both RT and MT (Buetti and Kerzel, 2009; Coutte et al., 2015). According to Buetti and Kerzel (2009), SRC tasks affect the selection of manual responses during both motor preparation and execution stages, such that SRC effect is observed on RT (latency of response initiation), MT (duration of response execution) and movement kinematic parameters (initial direction of the response for instance). When stress is put on movement initiation (following instructions), SRC effect influences MT but not RT. By contrast, response precueing reduces SRC effect on movement parameters, while still preserving SRC effect on RT. As a whole, these results suggested that temporal characteristics of SRC effect reflect the dynamical properties of the planning and execution components of the manual motor responses (Buetti and Kerzel, 2009). The lack of effect of SRC in the present study on mean MT (mu parameter) could thus be attributed to the lack of strict constraint on the organization of the motor response. Because the instructions given to the children did not encourage short RTs, the conflict between target distance and movement amplitude could be resolved before movement initiation, affecting thus essentially RT. Indeed, in order to produce the correct response in the incongruent trials, the automatically activated motor response needed to be inhibited (Kornblum, 1994), at least in the 11 years-old children. According to Buetti and Kerzel (2008), this inhibition may be not dependent on an all-or-none process but may linger in the system depending on processing time and affect motor execution in the case of fast initiated responses, which was not the case in the present study (see also Coutte et al., 2015). However, MT distribution was asymmetric toward long-lasting movements in the incongruent compared to the congruent spatial conditions in the 11 years old children ( $\tau$ parameter of the ex-Gaussian function), suggesting that SRC effect slightly modulated movement execution in the oldest children.

With respect to the developmental trajectory of the SRC effect, the data in the present study suggest then that the two processing routes are not available in younger children, or at least not fully functional. Indeed, the lack of SRC effect on RT before the age of 10 years suggests an absence of automatic motor activation associated with the perception of the irrelevant spatial properties of the stimulus. It seems then that younger children refer to the visual features of the stimulus but fail to integrate multi-modal and motor related information associated with that stimulus. This interpretation is in line with the demonstration by Gori et al. (2008) that it is not until the age of 8 -10-years that multimodal information is integrated in an optimal fashion. Typically, younger children succeed in perceptual tasks by selecting the most appropriate sensory channel (e.g., visual or haptic information) instead of considering more cues and integrate them, as older children do, to comply with the constraints of the task. This interpretation is also supported by the finding that the period between $8-10$-years is characterized by improvements in sensorimotor integration and the development of motor predictive models making possible the response to new or fickle environmental constraints (JansenOsman et al., 2002; Gori et al., 2008; Thibaut and Toussaint, 2010).

To summarize, the present study investigated the developmental aspect of the conflict inherent to the processing of relevant and irrelevant features of a visual stimulus during response selection and execution in a classical SRC task. We found that mainly RT was affected by the incongruence between instruction-dependent and motor related visual information. Furthermore, the effect of the conflict between the different features of the visual stimulus appears only in children older than 10 years. Accordingly, the present results provide additional evidence for a reorganization of the perceptual system integrating multimodal information in a particular period of development and preparing more efficiently the body to act in the environment. Further investigations would be important to evaluate, through different experimental constraints and finer measures of motor responses (e.g., movement kinematics), 
how RTs and MTs are affected in SRC task in childhood. This would provide valuable information on the improvement of the sensory, motor and cognitive mechanisms supporting response selection, planning and execution at major stages of the children development.

\section{AUTHOR CONTRIBUTIONS}

AR organized the experiment, tested the participants, analyzed the data, wrote the paper. GO organized the experiment, wrote the paper. YC organized the experiment, analyzed the data, wrote the paper.

\section{REFERENCES}

Adalbjornsson, C. F., Fischman, M. G., and Rudisill, M. E. (2008). The end state comfort effect in young children. Res. Q. Exerc. Sport 79, 36-41. doi: 10.1080/02701367.2008.10599458

Assaiante, C., and Amblard, B. (1995). An ontogenetic model for the sensorimotor organization of balance control in humans. Hum. Mov. Sci. 14, 13-43. doi: 10.1016/0167-9457(94)00048-J

Bard, C., Hay, L., and Fleury, M. (1990). Timing accuracy of visually directed movement in children: control of direction and amplitude components. J. Exp. Child Psychol. 50, 102-118. doi: 10.1016/0022-0965(90)90034-6

Barsalou, L. W. (2008). Grounded cognition. Annu. Rev. Psychol. 59, 617-645. doi: 10.1146/annurev.psych.59.103006.093639

Buetti, S., and Kerzel, D. (2008). Time course of the Simon effect in pointing movements for horizontal, vertical, and acoustic stimuli: evidence for a common mechanism. Acta Psychol. 129, 420-428. doi: 10.1016/j.actpsy.2008.09.007

Buetti, S., and Kerzel, D. (2009). Conflicts during response selection affect response programming : reaction toward the source of stimulation. J. Exp. Psychol. Hum. Percept. Perform. 35, 816-834. doi: 10.1037/a0011092

Carmichael, L. (1946). “The onset and early development of behavior," in Manual of child psychology, ed. L. Carmichael (New York, NY: Wiley), 43-166.

Chemero, A. (2003). An outline of a theory of affordances. Ecol. Psychol. 15, 181-195. doi: 10.1207/S15326969ECO1502_5

Cho, Y. S., and Proctor, R. W. (2002). Influences of hand posture and hand position on compatibility effects for up-down stimuli mapped to left-right responses: evidence for a hand referent hypothesis. Percept. Psychophys. 64, 1301-1315. doi: 10.3758/BF03194773

Choudhury, S., Charman, T., Bird, V., and Blakemore, S. J. (2007). Development of action representation during adolescence. Neuropsychologia 45, 255-262. doi: 10.1016/j.neuropsychologia.2006.07.010

Coello, Y., and Fischer, M. (2015). The Foundations of Embodied Cognition. Hove: Psychology Press.

Costantini, M., Ambrosini, E., Tieri, G., Sinigaglia, C., and Committeri, G. (2010). Where does an object trigger an action? An investigation about affordances in space. Exp. Brain Res. 207, 95-103. doi: 10.1007/s00221-010-2435-8

Coutte, A., Richez, A., Coello, Y., and Olivier, G. (2015). Concurrent right-left and amplitude spatial congruency effects in a visual discrimination task. Vis. Cogn. 23, 781-795. doi: 10.1080/13506285.2015.1088606

Ego, C., Yüksel, D., Orban de Xivry, J. J., and Lefèvre, P. (2016). Development of internal models and predictive abilities for visual tracking during childhood. J. Neurophysiol. 115, 301-309. doi: 10.1152/jn.00534.2015

Eimer, M., Hommel, B., and Prinz, W. (1995). S-R compatibility and response selection. Acta Psychol. 90, 301-313. doi: 10.1016/0001-6918(95)00022-M

Ernst, M. O., and Banks, M. S. (2002). Humans integrate visual and haptic information in a statistically optimal fashion. Nature 415, 429-433. doi: $10.1038 / 415429 a$

Fine, I., Wade, A. R., Brewer, A. A., May, M. G., Goodman, D. F., Boynton, G., et al. (2003). Long-term deprivation affects visual perception and cortex. Nature $6,1-2$.

\section{FUNDING}

This work was supported by Programme d'Investissements d'Avenir (PIA) and Agence Nationale pour la Recherche (grant ANR-11-EQPX-0023), and European Founds for the Regional Development (Grant FEDER- Presage 41779).

\section{ACKNOWLEDGMENT}

The authors are grateful to the children who participated in the study, to their parents, and the staff and teachers of the French public schools Pierre Mendès France.

Fischer, K. W., and Bidell, T. R. (1998). "Dynamic development of psychological structures in action and thought," in Handbook of Child Psychology: Theoretical Models of Human Development, eds W. Damon and R. M. Lerner (New York, NY: Wiley), 467-561.

Forssberg, H., Kinoshita, H., Eliasson, A. C., Johansson, R. S., Westling, G., and Gordon, A. M. (1992). Development of human precision grip. II. Anticipatory control of isometric forces targeted for object's weight. Exp. Brain Res. 90, 393-398.

Gesell, A. (1929). Maturation and infant behavior pattern. Psychol. Rev. 26, 307319. doi: $10.1037 / \mathrm{h} 0075379$

Gibson, J. J. (1979). The Ecological Approach to Visual Perception. Boston, MA: Houghton Mifflin.

Girolami, G. L., Shiratori, T., and Aruin, A. S. (2010). Anticipatory postural adjustments in children with typical motor development. Exp. Brain Res. 205, 153-165. doi: 10.1007/s00221-010-2347-7

Gori, M., Del Viva, M., Sandini, G., and Burr, D. C. (2008). Young children do not integrate visual and haptic form information. Curr. Biol. 18, 694-698. doi: 10.1016/j.cub.2008.04.036

Greenough, W. T., Black, J. E., and Wallace, C. S. (1987). Experience and brain development. Child Dev. 58, 539-559. doi: 10.2307/1130197

Held, R., and Hein, A. (1963). Movement-produced stimulation in the development of visually guided behavior. J. Comp. Physiol. Psychol. 56, 872-876. doi: $10.1037 / \mathrm{h} 0040546$

Hommel, B. (1997). "Toward an action-concept model of stimulus-response compatibility," in Theoretical Issues in Stimulus-Response Compatibility, eds B. Hommel and W. Prinz (Amsterdam: Elsevier), 281-320.

Hommel, B. (2011). The Simon effect as tool and heuristic. Acta Psychol. 136, 189-202. doi: 10.1016/j.actpsy.2010.04.011

Hommel, B., Müsseler, J., Aschersleben, G., and Prinz, W. (2001). The theory of event coding (TEC): a framework for perception and action planning. Behav. Brain Sci. 24, 849-878. doi: 10.1017/S0140525X0 1000103

Houdé, O., and Guichart, E. (2001). Negative priming effect after inhibition of number/length interference in a Piaget-like task. Dev. Sci. 4, 119-123. doi: 10.1111/1467-7687.00156

Hurley, S. L. (2002). Consciousness in Action. Cambridge, MA: Harvard University Press.

Immordino-Yang, M. H., and Fischer, K. W. (2007). "Dynamic development of hemispheric biases," in Human Behavior, Learning and the Developing BrainTypical Development, eds D. Coch, K. W. Fischer, and G. Dawson (New York, NY: The Guilford Press), 3-29.

James, W. (1890). The Principles of Psychology. NewYork, NY: Dover Publications.

Jansen-Osman, P., Richter, S., Konczak, J., and Kalveram, K. T. (2002). Force adaptation transfers to untrained workspace regions in children: evidence for developing inverse dynamic motor models. Exp. Brain Res. 142, 212-220. doi: 10.1007/s00221-001-0982-8

Jeannerod, M. (2006). Motor Cognition. Oxford: Oxford University Press.

Kornblum, S. (1994). The way irrelevant dimensions are processed depends on what they overlap with: the case of Stroop- and Simon-like stimuli. Psychol. Res. 56, 130-135. doi: 10.1007/BF00419699 
Kornblum, S., Hasbroucq, T., and Osman, A. (1990). Dimensional overlap: cognitive basis of stimulus-response compatibility: a model and taxonomy. Psychol. Rev. 97, 253-270. doi: 10.1037/0033-295X.97. 2.253

Lacouture, Y., and Cousineau, D. (2008). How to use Matlab to fit the ex-Gaussian and other probability functions to a distribution of response times. Tutor. Quant. Methods Psychol. 4, 35-45.

Lange-Küttner, C. (2009). Habitual size and projective size: the logic of spatial systems in children's drawings. Dev. Psychol. 45, 913-927. doi: 10.1037/a0016133

Matzke, D., and Wagenmakers, E. J. (2009). Psychological interpretation of the ex-Gaussian and shifted Wald parameters: a diffusion model analysis. Psychon. Bull. Rev. 16, 798-817. doi: 10.3758/PBR.16. 5.798

McGraw, M. B. (1946). "Maturation of behaviour," in Manual of Child Psychology, ed. L. Carmichael (New York, NY: Wiley), 332-369.

Meiran, N. (2005). Task rule-congruency and Simon-like effects in switching between spatial tasks. Q. J. Exp. Psychol. 58, 1023-1041. doi: 10.1080/02724980443000421

Merleau-Ponty, M. (1945). Phénoménologie de la Perception. Paris: Gallimard.

Meulenbroek, R. G., and Van Galen, G. P. (1988). "The acquisition of skill handwriting: Discontinuous trends in kinematics variables," in Cognition and Action in Skill Behavior, eds A. M. Colley and J. R. Beechs (Amsterdam: Elsevier), 273-281.

Nardini, M., Bedford, R., and Mareschal, D. (2010). Fusion of visual cues is not mandatory in children. Proc. Natl. Acad. Sci. U.S.A. 107, 17041-17046. doi: 10.1073/pnas.1001699107

Nardini, M., Jones, P., Bedford, R., and Braddick, O. (2008). Development of cue integration in human navigation. Curr. Biol. 18, 689-693. doi: 10.1016/j.cub.2008.04.021

Noë, A. (2004). Action in Perception. Cambridge: The MIT press.

Olivier, G. (2006). Visuomotor riming of a manual reaching movement during a perceptual decision task. Brain Res. 1124, 81-85.

Piaget, J., and Inhelder, B. (1969). The Psychology of the Child. New York, NY: Basic Books.

Proverbio, A. M. (2012). Tool perception supresses 10-12 Hz $\mu$ rhythm EEG over the somatosensory area. Biol. Psychol. 91, 1-7. doi: 10.1016/j.biopsycho.2012.04.003

Quinlan, D. J., and Culham, J. C. (2007). fMRI reveals a preference for near viewing in the human parieto-occipital cortex. Neuroimage 36, 167-187. doi: 10.1016/j.neuroimage.2007.02.029

Ratcliff, R., and Murdock, B. B. Jr. (1976). Retrieval process in recognition memory. J. Exp. Psychol. Learn. Mem. Cogn. 16, 163-178.
Richez, A., and Coello, Y. (2015). Representation of peripersonal space in 7- to 14-year old children. Psychol. Française 60, 377-386. doi: 10.1016/j.psfr.2014.10.002

Rizzolatti, G., and Sinigaglia, C. (2008). Mirrors in the Brain: How Our Minds Share Actions, Emotions, and Experience. Oxford: Oxford University Press.

Rose, L. T., and Fischer, K. W. (2008). "Dynamic systems theory," in Chicago Companion to the Child, ed. R. A. Shweder (Chicago, IL: University of Chicago Press).

Segalowitz, S. J. (2002). "The role of neuroscience in historical and contemporary theories of human development," in Human Behavior, Learning, and the Developing Brain, eds D. Coch, K. W. Fischer, and G. Dawson (London: The Guilford Press), 3-29.

Simon, J. R., and Rudell, A. P. (1967). Auditory S-R compatibility: The effect of an irrelevant cue on information processing. J. Appl. Psychol. 51, 300-304. doi: $10.1037 / \mathrm{h} 0020586$

Simon, J. R., and Small, A. M. Jr. (1969). Processing auditory information: interference from an irrelevant cue. J. Appl. Psychol. 53, 433-435. doi: $10.1037 / \mathrm{h} 0028034$

Thelen, E., and Smith, L. B. (1994). A Dynamic Systems Approach to the Development of Cognition and Action. Cambridge, MA: MIT Press-Bradford Books.

Thibaut, J. P., and Toussaint, L. (2010). Developing motor planning over ages. J. Exp. Child Psychol. 105, 116-129. doi: 10.1016/j.jecp.2009.10.003

Tucker, M., and Ellis, R. (1998). On the relation between seen object and components of potential actions. J. Exp. Psychol. Hum. Percept. Perform. 24, $830-846$.

Tucker, M., and Ellis, R. (2001). The potentiation of grasp types during visual object categorization. Vis. Cogn. 8, 769-800. doi: 10.1080/13506280042000144

Wallon, H. (1945). Les Origines de la Pensée Chez L'enfant. Paris: PUF.

Wamain, Y., Gabrielli, F., and Coello, Y. (2015). EEG $\mu$ rhythm in virtual reality reveals that motor coding of visual objects in peripersonal space is task dependent. Cortex 74, 20-30. doi: 10.1016/j.cortex.2015.10.006

Conflict of Interest Statement: The authors declare that the research was conducted in the absence of any commercial or financial relationships that could be construed as a potential conflict of interest.

Copyright (C) 2016 Richez, Olivier and Coello. This is an open-access article distributed under the terms of the Creative Commons Attribution License (CC BY). The use, distribution or reproduction in other forums is permitted, provided the original author(s) or licensor are credited and that the original publication in this journal is cited, in accordance with accepted academic practice. No use, distribution or reproduction is permitted which does not comply with these terms. 


\section{OPEN ACCESS}

Edited by: Annalisa Setti,

University College Cork, Ireland

Reviewed by:

Christine Schiltz,

University of Luxembourg,

Luxembourg

Barbara Treccani,

University of Sassari, Italy

*Correspondence:

Manuel Ninaus

m.ninaus@iwm-tuebingen.de

tThese authors have contributed equally to this work.

Specialty section: This article was submitted to

Cognition,

a section of the journal

Frontiers in Psychology

Received: 31 October 2016 Accepted: 07 August 2017

Published: 23 August 2017

Citation

Ninaus M, Moeller K, Kaufmann L, Fischer $\mathrm{MH}$, Nuerk $\mathrm{H}-\mathrm{C}$ and Wood $\mathrm{G}$

(2017) Cognitive Mechanisms Underlying Directional and Non-directional Spatial-Numerical

Associations across the Lifespan.

Front. Psychol. 8:1421. doi: 10.3389/fpsyg.2017.01421

\section{Cognitive Mechanisms Underlying Directional and Non-directional Spatial-Numerical Associations across the Lifespan}

\author{
Manuel Ninaus ${ }^{1,2,3 *}$, Korbinian Moeller ${ }^{1,2,3+}$, Liane Kaufmann ${ }^{4}$, Martin H. Fischer ${ }^{5}$, \\ Hans-Christoph Nuerk ${ }^{3,6}$ and Guilherme Wood ${ }^{1}$
}

'Leibniz-Institut für Wissensmedien, Tübingen, Germany, ${ }^{2}$ Department of Psychology, University of Graz, Graz, Austria, ${ }^{3}$ LEAD Graduate School and Research Network, Eberhard Karls University of Tübingen, Tübingen, Germany, ${ }^{4}$ Department of Psychiatry and Psychotherapy A, General Hospital, Hall, Austria, ${ }^{5}$ Division of Cognitive Sciences, Department of Psychology, University of Potsdam, Potsdam, Germany, ${ }^{6}$ Department of Psychology, Eberhard Karls University Tübingen, Tübingen, Germany

There is accumulating evidence suggesting an association of numbers with physical space. However, the origin of such spatial-numerical associations (SNAs) is still debated. In the present study we investigated the development of two SNAs in a cross-sectional study involving children, young and middle-aged adults as well as the elderly: (1) the SNARC (spatial-numerical association of response codes) effect, reflecting a directional SNA; and (2) the numerical bisection bias in a line bisection task with numerical flankers. Results revealed a consistent SNARC effect in all age groups that continuously increased with age. In contrast, a numerical bisection bias was only observed for children and elderly participants, implying an U-shaped distribution of this bias across age groups. Additionally, individual SNARC effects and numerical bisection biases did not correlate significantly. We argue that the SNARC effect seems to be influenced by longer-lasting experiences of cultural constraints such as reading and writing direction and may thus reflect embodied representations. Contrarily, the numerical bisection bias may originate from insufficient inhibition of the semantic influence of irrelevant numerical flankers, which should be more pronounced in children and elderly people due to development and decline of cognitive control, respectively. As there is an ongoing debate on the origins of SNAs in general and the SNARC effect in particular, the present results are discussed in light of these differing accounts in an integrative approach. However, taken together, the present pattern of results suggests that different cognitive mechanisms underlie the SNARC effect and the numerical bisection bias.

Keywords: SNARC effect, spatial-numerical bias, line bisection task, cognitive development, aging

\section{INTRODUCTION}

Over the last decades, different effects ascribable to spatial-numerical associations (SNAs) have been described (for reviews see Fischer and Shaki, 2014; Winter et al., 2015). These include, amongst others, spatial biases observed in number magnitude comparison or parity judgment tasks (e.g., Dehaene et al., 1993), line and string bisection tasks with numerical displays 
(Fischer, 2001b; de Hevia et al., 2006), a bias in numerical interval bisection tasks (Priftis et al., 2006; Zorzi et al., 2002) and biased mental arithmetic (e.g., McCrink et al., 2007; Knops et al., 2014; Shaki et al., 2017). They also include number-related effects on pointing and grasping performance (e.g., Fischer, 2003; Andres et al., 2004), on visual detection (e.g., Fischer et al., 2003; Galfano et al., 2006; Ristic et al., 2006; Salillas et al., 2008; Stoianov et al., 2008) and on digit writing (Perrone et al., 2010). Although a lot of research has been devoted to SNAs, no consensus has been reached regarding their origin. In this context, it is of particular interest to establish whether different SNAs draw on the same cognitive underpinning.

In an attempt to investigate this question Cipora et al. (2015) suggested a taxonomy to classify SNAs based on their spatial attributes (extension vs. directionality) and their numerical attributes (cardinality, interval, ordinality, operations). One of the most basic distinctions made by Cipora et al. (2015) is between directional SNAs and non- directional SNAs. An example of directional SNAs are faster responses to small numbers with left-side responses and to larger numbers with right-side responses, known as the "SNARC-effect" (e.g., Dehaene et al., 1993). An example of non-directional SNAs are general biases toward the position of the larger number - left or right as was observed in line bisection with task-irrelevant flanker numbers (e.g., Experiment 2 of Fischer, 2001b). This central theoretical distinction between non-directional vs. directional associations (see also Patro et al., 2014) may also imply distinct origins/ cognitive underpinnings of the respective SNAs. However, this proposal remains to be evaluated empirically.

Recently, Fischer and Brugger (2011, see also Fischer, 2012; Myachykov et al., 2014) argued that three different possible origins of SNAs may be differentiated. According to the authors the mental representation of numerical magnitude information is influenced by (i) general principles in the physical world, such as gravity; (ii) sensory and motor interactions we experience and perform; and (iii) current task constraints on information processing (Fischer and Brugger, 2011). These three hierarchically related levels of influence on numerical representations were referred to as "groundedness," "embodiedness" and "situatedness" of cognition, respectively (Fischer, 2012) - with all three of these jointly determining the strength of SNAs (for a recent review see Winter et al., 2015).

In our view, this hierarchical account of the representation of number magnitude and its implications for SNAs provide a testing bed to investigate the origin of different SNAs. In particular, it allows for rather specific predictions on how certain SNAs may manifest over the lifespan (see Figure 1A; e.g., the increasing influence of embodied number representations via cultural variables over the lifespan). Accordingly, the current study set out to evaluate whether the central theoretical distinction (non-directional vs. directional) of Cipora et al.'s (2015) taxonomy of SNAs may reflect distinct origins of the respective SNAs. Therefore, we selected two SNAs that differ with regard to directional vs. non-directional extension: According to Cipora et al. (2015) the SNARC (spatial-numerical association of response codes) effect is a directional SNA, reflecting that number magnitude is represented on a left-to-right oriented mental number line (MNL) with small numbers on the left and larger numbers represented toward their right side. Accordingly, faster responses are observed for congruent associations (i.e., left-small/right-large) than for incongruent ones (i.e., rightsmall/left-large; for reviews see Fias and Fischer, 2005; Gevers and Lammertyn, 2005; Hubbard et al., 2005; Wood et al., 2008; Fischer and Shaki, 2014).

Other studies found evidence for non-directional SNAs. For example, Fischer (2001b, Experiment 2) designed a version of the line bisection task in which two Arabic numbers presented near the left and right endpoints of horizontal lines served as flankers. When the larger flanker was on the left, a leftward bisection bias was observed, whereas a rightward bisection bias was found when the numerically larger flanker was on the right. Thus, midpoint estimation was distorted by task-irrelevant semantic activity induced by the number symbols (see also de Hevia et al., 2006; Bonato et al., 2008; de Hevia and Spelke, 2009; but see also Gebuis and Gevers, 2011).

Recent evidence also suggested that working memory may be a source of SNAs (Fias et al., 2011; van Dijck and Fias, 2011; Ginsburg et al., 2014). More specifically, van Dijck and Fias (2011) argued that the SNARC effect reflects an association between the ordinal position of an item in working memory and response side. In line with this argument, van Dijck et al. (2009) observed that the SNARC effect disappeared under working memory load. Thus, the SNARC effect may not necessarily reflect overlearned cultural and thus long-term associations between number magnitude and physical space but may, at least partly (cf. Huber et al., 2016), be constructed ad hoc during task execution as well, thus reflecting the situated origin of this SNA.

Examining variations in spatial-numerical performance over the lifespan may give us new insight into the origin(s) of both directional and non-directional SNAs as introduced above (for a similar approach, see Lambrechts et al., 2013). For instance, when the SNARC effect reflects an ad hoc association between the ordinal position of an item in working memory and the response side (van Dijck and Fias, 2011), the effect should be relatively smaller for children as well as the elderly when compared to young and middle-aged adults, due to working memory limitations (see Figure 1C). Specifically, a smaller working memory capacity, in terms of reduced memory spans and thus fewer numbers/ordinal positions maintained in working memory, might lead to smaller SNARC effects. This prediction is due to well-known age-related changes in working memory capacity over the life-span: generally, performance on working memory tasks is much better in adults as compared to children and the elderly (e.g., Hasher and Zacks, 1988; Siegel, 1994; Borella et al., 2008). However, if the SNARC effect instead reflects influences of embodied representations then one may expect a positive association of the SNARC effect with age (as observed in the meta-analyses by Wood et al., 2008) - reflecting an age-related strengthening of SNAs through longer experiences of culturally mediated sensory-motor constraints (for reviews see Fischer and Brugger, 2011; Fischer, 2012; see Figure 1A). In particular, when cognitive capacities become limited in the elderly, embodied cognition effects driven by the reactivation of previously built associations seem to get more pronounced 


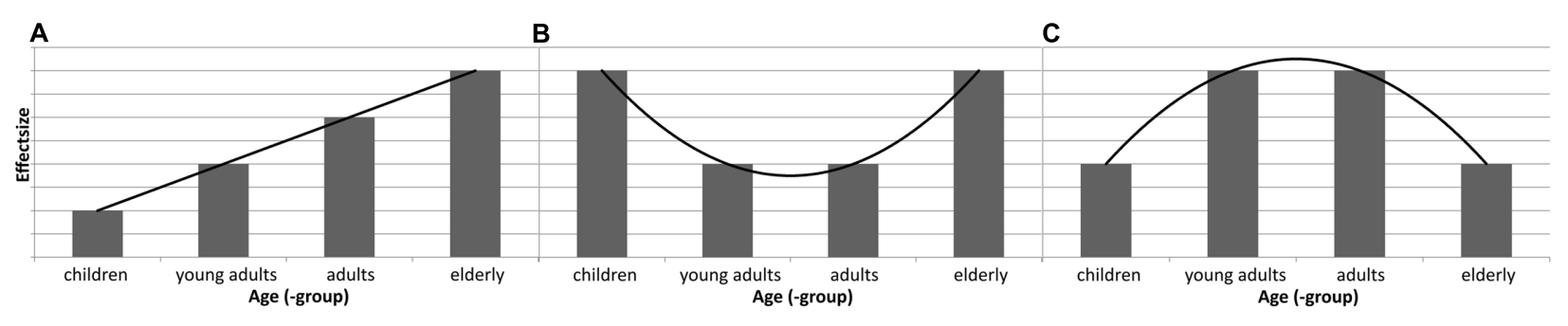

FIGURE 1 | (A) Linear trend of increasing SNAs ( $y$-axis) over the lifespan ( $x$-axis), i.e., children, young adults, middle-aged adults, and elderly; (B) Positive quadratic trend of SNAs over the lifespan; (C) Negative quadratic trend of effect sizes of SNAs over the lifespan.

(e.g., Engelen et al., 2011; Dekker et al., 2014; Loeffler et al., 2016 for an overview). In line with this argument a recent study (Hoffmann et al., 2014) indicated that the SNARC effect was more pronounced in elderly as compared to young adults.

For the case of the non-directional SNA measured with the line bisection task similar competing predictions can be derived: On the one hand, if the bias observed in line bisection is due to embodied mechanisms such as sensory-motor associations of larger magnitudes (e.g., Perrone et al., 2010) one would expect an age-related strengthening; this means a positive association between bisection bias and age (see Figure 1A). On the other hand, working memory might be an important predictor of estimation biases as well. More specifically, inhibitory control, which is seen as a specific part of working memory (for a review see Diamond, 2013) might influence participants' bisection biases as it helps to ignore the task-irrelevant numerical flankers. Hence, weaker inhibitory capacity, as observed in children and elderly participants (e.g., Hasher and Zacks, 1988; Christ et al., 2001), should increase semantically driven bisection biases (see Figure 1B). Interestingly, Hoffmann et al. (2014) also attributed the stronger SNARC effect they observed in their elderly as compared to middle-aged adult participants to reduced inhibitory control over task-irrelevant associations (in this case of numbers and space) in older age (e.g., Hasher and Zacks, 1988). As such, the latter prediction of more pronounced SNAs in children and elderly may also apply to the SNARC effect.

Interestingly, the literature suggests differing developmental trajectories of the SNARC effect and the numerical bisection bias. Consider first the SNARC effect. Depending on the task the SNARC effect can be reliably observed in 7-year-old children (van Galen and Reitsma, 2008, but see Berch et al., 1999, for a SNARC effect from the age of 9 years on only). This suggests that the SNARC effect indeed increases with age. However, one might also speculate that the SNARC effect cannot be measured reliably in children younger than 7 years of age. Nonetheless, Hoffmann et al. (2013) already observed a SNARC effect in a magnitude classification as well as a color judgment task in kindergarten children. Moreover, a parity SNARC effect was already reported for Chinese preschoolers (Yang et al., 2014). Patro and Haman (2012) even observed a SNARC-like effect in 3- to 4-year-olds in a numerosity comparison task. Finally, Bulf and colleagues noticed directional left-to-right mappings in 8-month-old infants (see McCrink and Opfer, 2014 for a review on the development of
SNAs; see Newcombe et al., 2015, for a review on the intertwined development of spatial and numerical competences). Apart from that, and consistent with an age-related increase of the SNARC effect, Wood et al. (2008) found an age-related increase in the SNARC effect size in a meta-analysis. Finally, the SNARC effect is preserved in neurological patients with visuospatial hemineglect even when spatial processing and other SNAs are distorted (Priftis et al., 2006), implying a considerable strength of the effect in this biologically oldest population. In summary, the SNARC effect reflects a robust SNA based on directional spatial representation, which can be found early in life, increases its effect size with age and seems to be preserved in the presence of visuospatial impairments (see Figure 1A).

Consider now the developmental trajectory of bisection biases. On simple line bisection, children between 4 and 12 years showed a clear shift from an initial rightward to a later leftward bias when instructed to bisect lines printed on a sheet of paper (see also Dellatolas et al., 1996). A leftward bisection bias was already found at the age of 7 that decreased slightly up to the age of 12 (Van Vugt et al., 2000). In young adults a systematic leftward bisection bias has been observed (Orr and Nicholls, 2005) whereas a stronger rightward bias was found in elderly participants compared to middle-aged and young adults (Fujii et al., 1995; see also Jewell and McCourt, 2000 for a metaanalysis). So far, there is only one study which compared children and adults regarding a numerical version of the line bisection task (de Hevia and Spelke, 2009). Importantly, de Hevia and Spelke (2009) investigated the numerical bisection bias in young adults as well as 5- and 7-year-old children. While adults presented with a robust bias toward the larger number in both symbolic (i.e., Arabic numbers) and non-symbolic (i.e., dots) flanker conditions, 5- and 7-year-old children only showed this bias for non-symbolic flankers. In sum, these results suggest a visuospatial as well as a (non-symbolic) magnitude bias in children and that these biases increase with age. However, effects of more advanced age on this spatial non-directional representation remain to be studied.

Taken together, the question of whether directional and non-directional SNAs (as for instance the SNARC effect and numerical bisection biases) have a common origin or not remains unanswered so far. On the one side, one may argue that a small set of cognitive processes should account for SNAs observed in different tasks. When several SNAs can be attributed to 
a common set of cognitive mechanisms (e.g., influences of inhibitory control, see above), one would expect that spatialnumerical biases obtained in different tasks should show a non-zero correlation. Moreover, the developmental trajectories of different SNAs should be relatively similar in this case. On the other side, different types of SNAs, e.g., those bound to spatial directions, such as the SNARC effect, or those bound to spatial extensions, such as the numerical bisection bias (see Cipora et al., 2015), may originate from different cognitive processes (e.g., Fischer and Brugger, 2011; Myachykov et al., 2014). In particular, (i) when the SNARC effect is linked to the strength of sensory and motor experiences and thus embodied representations of numbers one would expect a linear relation between the size of the SNARC effect and age (Figure 1A), given that embodied sensory-motor associations underlying the effect would get stronger with age. Moreover, in case the numerical bisection bias originates from the same process, a similar relation between the size of the bias and age should be found (Figure 1A). (ii) However, when the SNARC effect is linked to working memory capacity, one should observe a smaller size of the SNARC effect for both children and elderly than for young adults, because of well-known age-related changes in working memory capacity over the life-span (Figure 1C). In contrast, age related change of working memory capacity should not affect the size of the numerical bisection bias. (iii) Last, in case the SNARC is linked to the strength of inhibitory control abilities, one should observe a larger SNARC effect for both children and elderly adults than for young adults (Figure 1B). Similarly, when the numerical bisection bias originates from the same process, one might expect a larger bisection bias for both children and elderly as compared to adults (Figure 1B). However, as argued above, we do not hypothesize SNARC and bisection bias to originate from the same underlying processes. Instead, the SNARC effect may be driven by sensory and motor experiences whereas numerical line bisection biases may mainly originate from situated aspects of our cognition (e.g., ad hoc effects of inhibitory control). In this case, no significant correlations between these two SNAs should be found and the developmental trajectories of SNAs should be different across the lifespan. In the present study, we tested these predictions on the existence of shared cognitive mechanisms underlying distinct SNAs in a cross-sectional study involving participants between 9 and 86 years of age. In particular, we investigated the developmental trajectories of the SNARC effect and numerical bisection biases as well as the correlations between these SNAs.

\section{MATERIALS AND METHODS}

\section{Participants}

Four groups of participants were included in the present study with an overall $\mathrm{N}$ of 100: 24 children, 25 young adults, 27 middleaged adults and 24 elderly adults. All participants had normal or corrected to normal vision. Participation in the study was voluntary. This study was carried out in accordance with the recommendations of the institutional guidelines of the University of Salzburg and of the Declaration of Helsinki. Informed written consent was obtained from all participants or, in case of children, from their parents or caregivers prior to the study. The protocol was approved by the local ethics committee.

\section{Children}

Twenty-four right-handed third-grade children participated in the study (mean age $=9 \mathrm{y} 1 \mathrm{~m}, S D=0 \mathrm{y} 3 \mathrm{~m}$, range $8 \mathrm{y} 6 \mathrm{~m}$ to $9 y 8 \mathrm{~m})$. Handedness of all participants was assessed with the Edinburgh Handedness Inventory (Oldfield, 1971). Please note that we chose that particular age of our children sample because reliable SNARC effects were previously observed for children of this age or even younger (e.g., Berch et al., 1999, for 9-year-olds; van Galen and Reitsma, 2008, for 7-year-olds; Patro and Haman, 2012, for 4-year-old children using non-symbolic number tasks; Shaki et al., 2012; McCrink et al., 2014, SNARC like effects in counting direction of 3-4 year olds; Bulf et al., 2016, in 8-9month-old infants).

\section{Young Adults}

Twenty-five young adults, all except one being right-handed ${ }^{1}$, were examined (mean age $=21 \mathrm{y} 7 \mathrm{~m} ; S D=1 \mathrm{y} 10 \mathrm{~m}$; range $18 \mathrm{y} 2 \mathrm{~m}$ to $26 \mathrm{y} 5 \mathrm{~m}$ ). All participants completed at least 12 years of general schooling or a specific technical training.

\section{Middle-Aged Adults}

Twenty-five right-handed and two ambidextrous middle-aged adults were examined (mean age $=46 y 0 \mathrm{~m}$; $\mathrm{SD}=4 \mathrm{y} 1 \mathrm{~m}$; range $36 \mathrm{y} 4 \mathrm{~m}$ to $52 \mathrm{y} 0 \mathrm{~m})$.

\section{Elderly}

Twenty-four community-dwelling elderlies, all except one being right-handed, were tested (mean age $=67 \mathrm{y} 0 \mathrm{~m}$; $\mathrm{SD}=6 \mathrm{y} 3 \mathrm{~m}$; range $60 \mathrm{y} 1 \mathrm{~m}$ to $86 \mathrm{y} 5 \mathrm{~m}$ ). According to self-report, all elderly participants were free from major neurological and psychiatric diseases.

\section{Stimuli, Design and Procedure \\ Parity Judgment Task}

Arabic digits 1, 2, 8, and 9 were presented on a CRT monitor in white on a black background. Participants decided whether Arabic digits were odd or even by pressing a right or left response key (i.e., right and left Ctrl keys of a standard keyboard). Arabic digits were shown in Arial font size 50 for a maximum of $2000 \mathrm{~ms}$ and covered a visual angle of $2.5^{\circ}$ vertically and $2^{\circ}$ horizontally from a viewing distance of $50 \mathrm{~cm}$. A fixation cross " + " in the middle of the computer screen was presented in the inter-stimulus interval for $1000 \mathrm{~ms}$ on average (range: 400 $1600 \mathrm{~ms}$ ). Response keys ( 12 by $12 \mathrm{~mm}$ ) were positioned in front of the participant and were $16 \mathrm{~cm}$ apart. Reaction time (RT) was recorded for a maximum of $2000 \mathrm{~ms}$ after stimulus presentation.

The assignment of response keys to even or odd numbers was counterbalanced across participants: After half of the experiment, the parity-to-response-key assignment was reversed (from evenright/odd-left to even-left/odd-right or vice versa). Each stimulus was repeated 40 times per parity-to-response-key assignment,

${ }^{1}$ Results did not change substantially when left-handed and ambidextrous persons were excluded. 
resulting in a total of 320 experimental trials. Additionally, ten practice trials, which were not considered for analyses, preceded the experimental trials in each parity-to-responsekey assignment. In total the experiment took participants approximately $20 \mathrm{~min}$ to complete.

The high number of repetitions of individual stimuli was chosen to ensure sufficient reliability (following the recommendation of Cipora and Nuerk, 2013). To balance the occurrence of individual stimuli, these were presented in 4 blocks of 40 trials for each half. Within each block each stimuli occurred 10 times. Accordingly, split-half reliability was computed by correlating z-transformed SNARC slopes from odd and even blocks across participants for each group separately. Spearman-brown corrected split-half reliability coefficients were: $r_{\text {children }}=0.76, r_{\text {young adults }}=0.93, r_{\text {middle-aged adults }}=0.95$, $r_{\text {elderly }}=0.96$.

\section{Line Bisection Task}

A numerical and a non-numerical version of this task were employed. In the numerical version, participants were asked to precisely bisect 16 horizontal lines; eight lines were printed on each of two A4 sheets of paper and flanked by two Arabic digits. The sheets of paper were positioned in the mid-sagittal plane in front of participants in portrait orientation. Eight lines of $145 \mathrm{~mm}$ length were printed with different horizontal offsets from each other on each of the two pages and with a vertical separation of $28 \mathrm{~mm}$ between them. Numerical distance between flankers was manipulated: digit pairs $1 / 1,2 / 2,8 / 8$ and 9/9 were presented in the symmetrical flankers condition to investigate a possible magnitude-based numerical bias, and digit pairs 1/2, 2/1, 8/9 and $9 / 8$ were presented in the asymmetrical flankers condition to evaluate a possible difference-based numerical bias. Digits were placed close to the start and endpoint of the lines and were printed in boldface 18 point Monaco font.

In the non-numerical version, the only difference to the numerical version of the task was that the flankers were omitted from each line. Importantly, the spatial position of lines on the two sheets in the non-numerical version of the line bisection task was the same as in the numerical version. This allowed for a direct comparison of homolog trials in both numerical and non-numerical tasks. Order of presentation of the numerical and non-numerical task version was counterbalanced between participants. For reasons of consistency, we refer to the homologue trials in the non-numerical tasks (symmetrical and asymmetrical conditions but with no actual flankers) also as if they would exhibit magnitude-based and difference-based spatial bias, respectively, although these tasks were visually identical.

Reliability of the line bisection bias was analyzed separately for the numerical and non-numerical version of the task and for each age group. In particular, split-half reliability scores were computed by correlating performance in two halves of the task matched for the occurrence of asymmetrical and symmetrical flankers (i.e., items $2 / 2,2 / 1,9 / 9,8 / 9$ vs. $1 / 1,1 / 2$, $8 / 8,9 / 8$; corresponding items in the non-numerical version) across participants of the respective age group. Spearman-brown corrected split-half reliabilities were as follows for numerical, $r_{\text {children }}=0.69, r_{\text {young adults }}=0.91, r_{\text {middle-aged adults }}=0.82$, elderly $=0.92$, and non-numerical version of the task, $r_{\text {children }}=0.71, r_{\text {young adults }}=0.94, r_{\text {middle-aged adults }}=0.63$, $r_{\text {elderly }}=0.73$ ).

\section{RESULTS}

\section{Parity Judgment Task}

RT data was trimmed prior to statistical analyses. Correct responses slower than $200 \mathrm{~ms}$ contained in the interval defined by \pm 3 standard deviations from the individual mean RT were kept while responses outside this interval were excluded from analyses. This procedure was repeated iteratively for each individual until no more responses were excluded ( $5 \%$ excluded on average). Because of considerable differences in average RT across groups, which may mask differences between groups, we z-standardized RT using individual means and standard deviations (see Faust et al., 1999 for the statistical rationale). Mean RT as well as the absolute and z-transformed SNARC slopes computed from RT per participant (Lorch and Myers, 1990) served as dependent variables (see Table 1 for descriptive statistics). SNARC slopes describe the best-fitting linear regression of RT difference scores (RT right hand minus RT left hand) on digit magnitudes (see Fias et al., 1996 for a more detailed description of the estimation procedure). Hence, faster right-handed responses to larger numbers result in negative SNARC slopes. Errors were infrequent and will not be analyzed separately (4\% excluded on average).

\section{Mean RT}

Mean RT differed significantly between groups $[F(3,96)=17.77$; $\left.M S E=31.747 ; p<0.05, \eta^{2}=0.36\right]$. Bonferroni-corrected pairwise-comparisons revealed comparable response latencies for children and elderly participants $(p=0.23)$ on the one side and for young adults and middle-aged adults $(p>99)$ on the other side (see also Table 1). Moreover, children $(d=1.67$, $d=1.35)$ and elderly participants $(d=1.46, d=1.03)$ were significantly slower than young adults and middle-aged adults (both comparisons $p<.05$; i.e., children $=$ elderly participants $>$ middle-aged adults $=$ young adults).

\section{SNARC Effects}

Significant SNARC slopes were observed for each age group, with means of $-14,-10,-10$, and $-16 \mathrm{~ms} /$ digit for children, young and middle-aged adults as well as elderly, respectively (all $p<.05$; see Table 1). Due to significant differences in overall RT raw SNARC slopes in ms/digit were not used further in our analyses but z-transformed slopes.

Significant z-transformed SNARC slopes were obtained for each age group [children: $t(23)=-5.44, p<0.001, d=1.11$; young adults: $t(24)=-7.32, p<0.001, d=1.46$; middleaged adults: $t(26)=-5.48, p<0.001, d=1.05$; elderly: $t(23)=-6.42 ; p<0.001, d=1.31$; see Table 1). In order to examine differences between age groups, z-transformed SNARC slopes were submitted to a one-way analysis of variance (ANOVA). A main effect of participant group indicated that standardized SNARC slopes differed significantly between groups 
TABLE 1 | Age, average reaction times as well as the standardized and non-standardized SNARC slopes in the parity decision task.

\begin{tabular}{|c|c|c|c|}
\hline \multicolumn{4}{|l|}{ Children } \\
\hline$n=24$ & RT parity task (ms) & Standardized SNARC slope* & SNARC slope $^{1}$ (ms) \\
\hline Mean & 985 & -0.04 & $-14 a$ \\
\hline$S D$ & 262 & 0.03 & 13 \\
\hline Minimum & 694 & -0.11 & -43 \\
\hline Maximum & 1724 & 0.03 & 6 \\
\hline \multicolumn{4}{|c|}{ Young adults } \\
\hline$n=25$ & RT parity task & Standardized SNARC slope & SNARC slope (ms) \\
\hline Mean & 653 & -0.07 & $-10 b$ \\
\hline SD & 107 & 0.05 & 7 \\
\hline Minimum & 469 & -0.18 & -23 \\
\hline Maximum & 826 & 0.01 & 2 \\
\hline \multicolumn{4}{|l|}{ Adults } \\
\hline$n=27$ & RT parity task & Standardized SNARC slope & SNARC slope (ms) \\
\hline Mean & 715 & -0.07 & $-10 c$ \\
\hline$S D$ & 118 & 0.07 & 10 \\
\hline Minimum & 550 & -0.21 & -32 \\
\hline Maximum & 1087 & 0.06 & 11 \\
\hline \multicolumn{4}{|l|}{ Elderly } \\
\hline$n=24$ & RT parity task & Standardized SNARC slope & SNARC slope (ms) \\
\hline Mean & 876 & -0.08 & $-16 d$ \\
\hline SD & 190 & 0.06 & 12 \\
\hline Minimum & 707 & -0.24 & -45 \\
\hline Maximum & 1458 & 0.04 & 17 \\
\hline
\end{tabular}

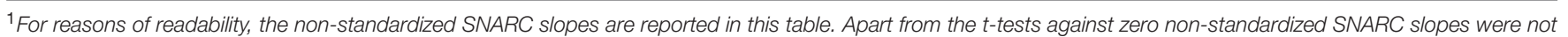

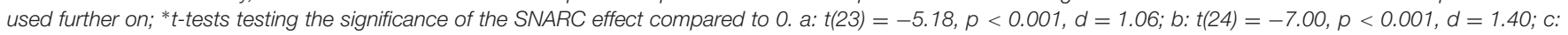
$t(26)=-5.47, p<0.001, d=1.05 ; d: t(23)=-6.44 ; p<0.001, d=1.31$.

$\left[F(3,96)=3.15, M S E=0.003, p<0.05, \eta^{2}=0.09\right]$. Bonferronicorrected pairwise-comparisons revealed a significant difference between children and elderly participants $(p<0.05, d=0.91)$. Other pair-wise comparisons did not reach significance (all $p>0.16$, see also Table 1 ). In each age group, a large proportion of participants presented a negative SNARC slope [22/24 children ( $92 \%), 21 / 25$ young adults ( $84 \%), 23 / 27$ middle-aged adults (85\%) and $22 / 24$ elderly participants (92\%)].

\section{Line Bisection Task}

We measured the constant error to the middle of the lines in millimeters, so that negative values reflected a leftward bias and positive ones a rightward bias. The response of one elderly participant to the symmetrical flanker digit pair $8 / 8$ was replaced by the elderly participants group mean since the response deviated more than three standard deviations from the group mean and probably reflected a momentary lapse of attention.

The biases observed for symmetrical and asymmetrical flankers were analyzed in the numerical version (magnitude-based and difference-based numerical bias) of the line bisection task as well as for their counterparts in the non-numerical version (magnitude-based and difference-based spatial bias). These bias scores were submitted to a $2 \times 4$ ANOVA with the factors flanker magnitude (small vs. large [symmetrical]; large magnitude left vs. large magnitude right [asymmetrical]) and group (children, young adults, middle-aged adults, elderly) in the numerical version of the task.

For the non-numerical version of the task two univariate ANOVAs were conducted with the factor group (children, young adults, middle-aged adults, and elderly), one for the homologue items of the asymmetrical flankers task (difference-based spatial bias; mean of non-numerical items presented at the same position as the items with large magnitude on the left/right) and one for the homologue items of the symmetrical flankers task (magnitude-based spatial bias; mean of non-numerical items for large/small numbers). Moreover, $t$-tests against zero were conducted to evaluate the statistical significance of spatial biases in non-numerical line bisection tasks in the different age groups.

\section{Symmetrical Flankers}

The $t$-tests revealed no significant magnitude-based spatial bias in the non-numerical bisection task (all $p>0.47$ ).

In the ANOVA, no main or interaction effects did reach significance in the numerical version of the line bisection task.

Also, the univariate ANOVA for the non-numerical version of the line bisection task yielded no significant effect $(p=0.93)$.

\section{Asymmetrical Flankers}

Again, the $t$-tests revealed no significant difference-based spatial bias in the non-numerical bisection task (all $p>0.10$ ).

Additionally, the univariate ANOVA for the non-numerical version of the line bisection task did not yield a significant effect 

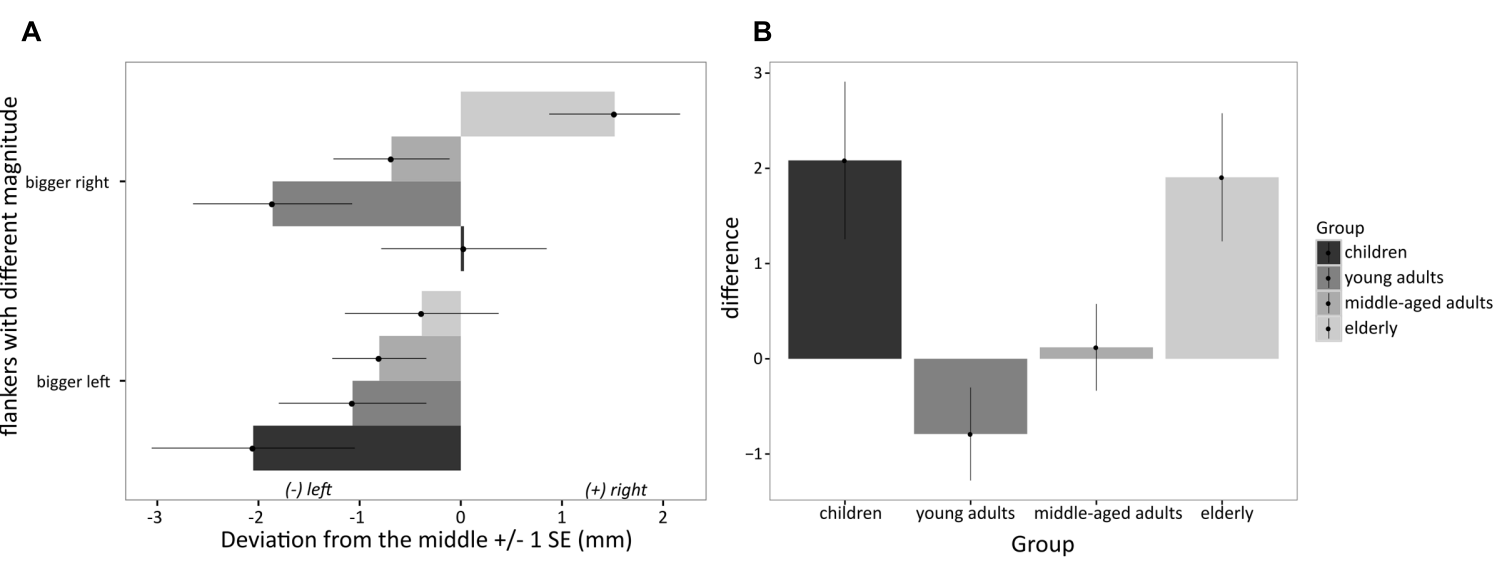

FIGURE 2 | (A) Difference-based numerical bias observed in children, young adults, middle-aged adults and elderly participants for numerical flankers with differing magnitude illustrated for larger number on the left and larger number on the right separately (asymmetrical flankers); positive/negative numbers indicate a rightward/leftward bias, respectively; (B) Difference-based numerical bias observed in the different age groups for numerical flankers with different magnitude illustrated as general bias toward larger numbers independent of side; positive difference values thus indicate a bias toward larger numbers, i.e., the larger the difference the larger is the bias toward the larger number.

$(p=0.62)^{2}$, thus ruling out contributions to the above effects from stimulus order or positioning.

In the numerical version of the task, the ANOVA revealed a significant main effect of side of the larger number $\left[F(1,96)=7.20 ; M S E=4.77 ; p<0.05, \eta_{\mathrm{p}}^{2}=0.07\right.$; see also Figure 2A]: Mean estimations for larger numbers on the left (mean $=-1.07$; negative value indicates bias toward larger number) and larger numbers on the right (mean $=-0.28$; positive value indicates bias toward larger number) differed significantly with regard to the mean position of the estimates on the line. More specifically, mean estimations were located further to the left when the larger number was on the left as compared to the estimations when larger numbers were positioned on the right. Importantly, this main effect was qualified by group $\left[F(3,96)=5.05 ; M S E=4.77 ; p<0.05, \eta_{p}^{2}=0.14\right]$. No other main or interaction effect was significant. In a complementary analysis, planned comparisons were conducted on the difference between the bias obtained when the larger number was on the right vs. on the left. The difference larger number on the right minus larger number on the left was calculated for each person separately and submitted to an univariate ANOVA with the factor group. For this index, positive values indicate a bias toward the larger number (see Figure 2B). A significant effect group was observed $[F(3,96)=5.05 ; M S E=9.54 ; p<0.05$, $\left.\eta^{2}=0.14\right]$. Additionally, the univariate ANOVA revealed a significant positive quadratic trend of the data $[F(3,96)=14.04$; $\left.p<0.05, \eta^{2}=0.13\right]$, indicating a U-shaped relationship between age groups and difference-based numerical bisection bias. This trend reflects a higher bias toward larger numbers in children and elderly as compared to young adults and middle-aged adults (see Figures 1B, 2B). Bonferroni-corrected pairwise-comparisons

${ }^{2}$ Using the mean of all homolog items in the non-numerical version for asymmetrical and symmetrical flanker tasks as dependent variable in one univariate ANOVA and group as factor, we did not observe a significant effect either $(p=0.62)$.

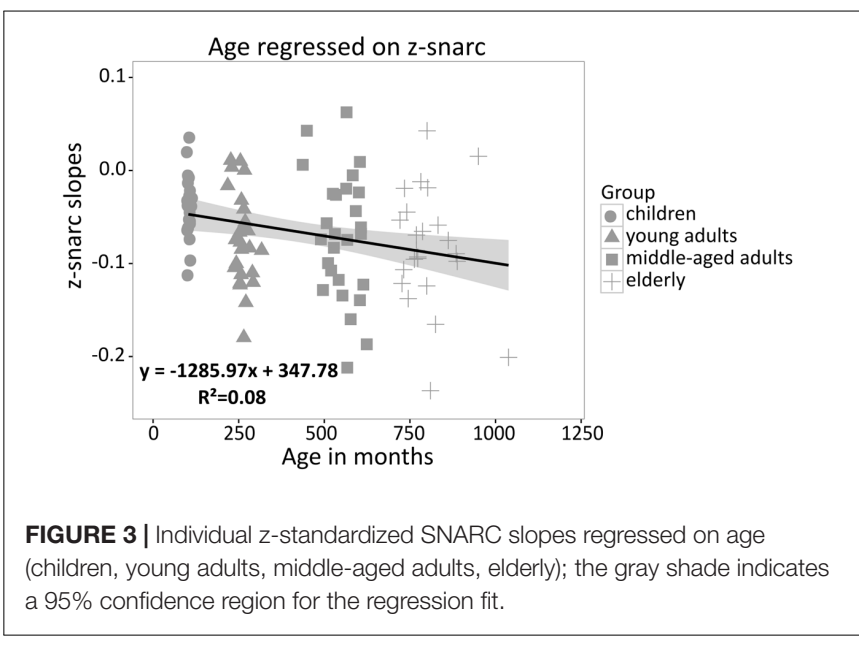

indicated that a larger bisection bias was observed in children $(M=2.08 \mathrm{~mm}, S D=4.05, d=0.86)$ and elderly participants $(M=1.91 \mathrm{~mm}, S D=3.30, d=0.93)$ as compared to young adults $(M=-0.79 \mathrm{~mm}, S D=2.44$; both $p<0.05)$. The bias observed in children, middle-aged adults $(M=0.12 \mathrm{~mm}, S D=2.37)$, and elderly participants did not differ significantly (all $p>0.15$ ), as was that observed for young and middle-aged adults (see Figure 2B).

\section{Age, SNARC and the Bisection Bias}

In order to investigate whether a significant association between the SNARC effect and age was present, individual z-standardized SNARC slopes were regressed on age. The effect of age on the SNARC slope was small but, in line with our expectations and the meta-analytical results reported by Wood et al. (2008), the SNARC slope became significantly more negative with age $\left[R^{2}=8 \%, \beta=-0.27, t(98)=-2.83, p=0.006\right.$; see Figure 3]. 
In order to investigate the existence of a common mechanism underlying SNAs, correlations between age, the SNARC effect and the bisection bias were calculated. Results were very clear: Nonnumerical bisection biases [magnitude-based spatial bias (i.e., mean of homologue conditions for small and large numbers) and difference-based spatial bias (i.e., mean of homologue conditions for larger number on the left and larger number on the right)] correlated moderately with each other but not with the SNARC effect (see Table 2). More importantly, numerical bisection biases [magnitude-based numerical bias (i.e., difference between larger vs. small numbers) and differencebased numerical bias (i.e., difference between larger number on the left vs. on the right)] did not correlate with the SNARC effect either. Finally, we assessed whether the relation between performance in the SNARC and bisection tasks was moderated by age. Thus, we examined the partial correlation between z-SNARC and bisection toward larger number, controlling for individuals age. However, the partial correlation was not significant ( $r=0.08, p=0.39$ ) as well. This indicates that the correlation between directional (SNARC) and non-directional (bisection bias) SNAs may not be moderated by age in the present study. Thus, our results do not corroborate the notion of a common origin of these bisection biases and the SNARC effect.

\section{DISCUSSION}

The aim of this study was to examine whether different SNAs share common or distinct cognitive origins. Based on Cipora et al.'s (2015) distinction between directional and non-directional SNAs, we investigated the SNARC effect in parity judgments (a directional SNA) and the spatial bias in bisecting lines flanked by digits (a non-directional SNA) over the lifespan. Evaluating the developmental trajectories of SNAs should provide meaningful information with respect to the question whether different SNAs share common cognitive underpinnings - as indicated by similar developmental patterns over the lifespan.

Results indicated a reliable SNARC effect in all age groups tested. Moreover, in line with the meta-analytical findings by Wood et al. (2008), we found the SNARC effect to increase with age across the lifespan (see also Hoffmann et al., 2014).
As regards the bisection task, we observed a larger differencebased numerical bias in children and elderly as compared to young adults. However, different from the SNARC effect no linear effect of age on the bisection bias was observed, but a quadratic one. Finally, we did not observe a significant correlation between the SNARC effect and the line bisection bias. This observation, together with a specific effect of age on the SNARC effect, are in line with the view that different cognitive mechanisms seem to underlie the directional and non-directional SNAs, as reflected by the SNARC effect and the bisection bias, respectively. In the following, we will discuss these points in more detail.

\section{The SNARC Effect}

A significant SNARC effect was found in all age groups, and its size increased as a function of age. Importantly, the latter finding cannot be accounted for by differences in processing speed because the increase in the size of the SNARC effect from childhood to old adulthood was found for $\mathrm{z}$-transformed SNARC slopes, thus controlling for differences in absolute response latencies between groups (Faust et al., 1999). In general, these findings confirm the expected age-related increase in the SNARC effect (see Figure 1A) indicated by a meta-analysis (Wood et al., 2008). However, the association between the SNARC effect and age obtained in the current study was lower than the one previously reported in the meta-analysis. Most plausibly, this discrepancy may be due to differences in stimuli, procedures, and populations relative to the 17 studies considered by Wood et al. (2008). The same materials and procedure were, however, used in the present study to examine participants from different age groups; therefore, the observed results corroborate and strengthen the previous conclusion that the SNARC effect increases with age.

Theoretically speaking, the observed increase in the size of the SNARC slopes as a function of age might reflect increasingly automatic associations between number magnitude and physical space across the lifespan. Thereby, the present results are consistent with the role of embodied representations on a SNA bound to spatial directions such as the SNARC effect. In particular, the observed age-related increase of the SNARC effect may be driven by longer-lasting experiences of cultural influences such as reading and writing direction over the lifespan. As such, accumulating sensory-motor associations may let the

TABLE 2 | Correlations between age, SNARC slope and measures of magnitude-based and difference-based bias in the numerical and non-numerical versions of the line bisection task.

\begin{tabular}{|c|c|c|c|c|c|c|}
\hline & Age & SNARC & $\begin{array}{l}\text { Magnitude-based } \\
\text { spatial bias }\end{array}$ & $\begin{array}{l}\text { Magnitude-based } \\
\text { numerical bias }\end{array}$ & $\begin{array}{l}\text { Difference-based } \\
\text { spatial bias }\end{array}$ & $\begin{array}{c}\text { Difference-based } \\
\text { numerical bias }\end{array}$ \\
\hline \multicolumn{7}{|l|}{ age } \\
\hline SNARC & $-0.27^{*}$ & & & & & \\
\hline Magnitude-based spatial bias & 0.08 & -0.05 & & & & \\
\hline Magnitude-based numerical bias & -0.03 & 0.01 & -0.16 & & & \\
\hline Difference-based spatial bias & 0.08 & -0.06 & $0.55^{*}$ & -0.02 & & \\
\hline Difference-based numerical bias & 0.03 & 0.07 & -0.04 & 0.16 & -0.07 & \\
\hline
\end{tabular}

$* p<0.05$. 
SNARC effect grow stronger with age. Nevertheless, one has to acknowledge that the amount of such experiences may well differ across (adult) individuals, thus allowing for differently strong SNAs. However, generally speaking, in children, directional SNAs may not yet be as automatic as in older adult populations. Supporting this view, van Galen and Reitsma (2008) have shown that 7-year-old children only show a SNARC effect in tasks where number magnitude is task-relevant. Only when children were 9 years and older they also showed a reliable SNARC effect in tasks where number magnitude is task-irrelevant - suggesting an increasingly automatic association of number magnitude and physical space. However, Hoffmann et al. (2013) found a SNARClike effect in a color judgment task in children as young as 5 and a half years. This might suggest that the absence of a parity SNARC effect in 7- as opposed to 9-year-old children in the study of van Galen and Reitsma (2008) was not due to a less developed automatic association of number magnitude and physical space at these earlier developmental stages (see also Bulf et al., 2016, who observed directional left-to-right mappings already in 8-monthold infants; and Patro and Haman, 2012, for a SNARC-like effect in 4-year-olds). Importantly, our results are not incompatible with these previous results per se, as we observed a significant SNARC effect in children as well. However, the main focus of our study was on relative differences in SNARC effects between age groups for which we found that SNARC effect sizes increased with age. Moreover, no SNARC effect was observed in illiterate adult populations (Zebian, 2005), emphasizing the role of the spatial experience of reading and writing direction in creating a SNA bound to spatial directions (see McCrink and Opfer, 2014 for a review).

Moreover, the developmental trajectory of the SNARC effect is also meaningful with regard to influences of working memory on the origin of this SNA. In this view, the SNARC effect would reflect an ad hoc association between the ordinal position of an item in working memory and the response side (van Dijck and Fias, 2011). Consequently, the SNARC effect should have been relatively lower for children and elderly persons as compared to young and middle-aged adults (Figure 1C), due to agerelated changes in working memory (i.e., higher working memory capacity in adults as compared to children and the elderly; e.g., Hasher and Zacks, 1988; Siegel, 1994; Borella et al., 2008). This argument is supported by a recent study of randomization behavior across the life span which concluded that cognitive performance peaks at around 25 years of age (Gauvrit et al., 2017).

Alternatively, however, it might be possible that the tendency to code ordinal positions in working memory spatially and the tendency to use strategies involving working memory resources to solve the task increases with increasing age (Figure 1A). According to this view, the working memory account of the SNARC effect might be actually consistent with the finding that the SNARC effect increases with age (i.e., smallest effects for children, intermediate effects for young adults, and largest effects for elderly adults). However, the findings of van Dijck et al. (2009, diminished SNARC effect under working memory load) indicate that working memory resources (in terms of capacity) seem to be necessary to observe a SNARC effect. Accordingly, when working memory capacity declines in elderly, the SNARC effect should decrease rather than increase according to this view. Moreover, it seems rather implausible to assume reliance on increasingly resource-demanding strategies in persons showing an actual decline of cognitive capacities such as working memory due to aging. According to this, elderly people would rely increasingly on strategies requiring cognitive resources that decline, rather than culturally acquired strategies manifested in behavior. In line with this point, it was recently found that effects of embodied cognition driven by the reactivation of previously built associations seem to be stronger in the elderly (e.g., Engelen et al., 2011; Dekker et al., 2014; Loeffler et al., 2016 for an overview). Therefore, we suggest that the observed continuous increase of the SNARC effect over the lifespan is hard to reconcile with such working memory accounts on the SNARC effect. Instead, it corroborates the influence of embodied representations reflecting cultural experiences on long-term associations between number magnitude and physical space. Interestingly, though, the observed change of the size of the SNARC effect across the lifespan might also result from an increase of the tendency to code spatially the numbers from childhood to adulthood joint with a decrease of the ability to inhibit irrelevant information in older age. As such, this account would actually reflect an interplay between mechanisms discussed to influence SNAs on their own earlier. According to this view, the observed trend is consistent with different accounts of the origin of the SNARC effect (e.g. van Dijck et al., 2009, 2012; see also Cheung et al., 2015). These different accounts might even include the polarity correspondence hypothesis, according to which spatial biases result from a binary coding of number magnitudes as being large or small, followed by their association with space that was equally coded into right vs left, respectively (e.g., Proctor and Cho, 2006). Thus, the mastery of spatial language might underlie the observed age-related strengthening of the SNARC effect. In fact, it may by possible that different mechanisms account for developmental changes of, for instance, the SNARC effect.

\section{The Bisection Bias}

In line with previous studies (Fischer, 2001b; Calabria and Rossetti, 2005; de Hevia et al., 2006) we observed no reliable spatial bias for the presence of symmetric flankers. In other words, no magnitude-based numerical bias was observed in the numerical version of the bisection task. Furthermore, no differences between age groups were observed for the magnitudebased numerical bias but rather strong within-group variability. This suggests that the magnitude-based numerical bias is less systematic than other forms of numerical bias and reinforces the view that processing number magnitude alone may not be sufficient for eliciting a numerical bias (Calabria and Rossetti, 2005; de Hevia et al., 2006) or biases for both sides were identical and canceled each other (Fischer, 2006). Moreover, the strong within-group variability observed in this study also reinforces the conclusion that the magnitude-based spatial bias may not be particularly robust. Finally, in line with previous studies, the present results suggest that the familiarity with numbers, which 
presumably increases with age as a result of lifelong handling of numbers (Figure 1A), is not sufficient for producing a stronger magnitude-based numerical bias in elderly participants (relative to children).

On the other hand, a robust difference-based numerical bias was found in the present study following the presentation of asymmetrical flankers. The significant main effect of side on which the larger number was presented indicated an overall numerical bisection bias toward the position of the larger number. These results replicate previous findings by Fischer (2001b) and de Hevia et al. (2006) and are compatible with those reported by Longo and Lourenco (2007). These authors observed a stronger bias in the numerical than in the non-numerical version of the line bisection task. Furthermore, the differencebased numerical bias was particularly stronger in children and elderly participants relative to young adults and middle-aged adults (see Figures 1B, 2B). Interestingly, elderly participants showed a strong absolute rightward bias when the larger number was presented on the right, while children showed only a relative rightward bias when the larger number was presented on the right. A tentative explanation for this finding might be that large numbers on the left are unexpected for children and thus, capture their attention there, because counting usually begins on the left with small numbers (e.g., Shaki et al., 2012). In contrast, for elderly participants all encoding is from left to right (e.g., cultural experiences such as reading and writing direction) and habitually ends on the right, focusing attention there. This is the first time that the development of the difference-based numerical bias was investigated in a sample with an age range from 9 to 86 years. However, we did not observe any agerelated performance differences in the non-numerical version of the line bisection task, a result that is inconsistent with a general leftward bias -/rightward bias in young/old participants observed previously (Jewell and McCourt, 2000). Thus, our findings suggest that the numerical bias can be distinguished from a pure spatial bias observed in the line bisection task.

Finally and importantly, the developmental trajectory of the difference-based numerical bias, a SNA bound to non-directional extensions, was found to differ from that of the SNARC effect, as only the SNARC effect increased with age. These discrepant results suggest that the cognitive origins/mechanisms responsible for these two different types of SNAs, i.e., the non-directional difference-based numerical bias and the directional SNARC effect, seem to differ. This will be discussed in more detail in the next section.

\section{Association between the SNARC Effect, Bisection Bias and Age}

Performance was moderately correlated in the non-numerical versions of the line bisection task. When employing flankers, however, attention orientation is additionally cued by semantic properties of these flankers, such as number magnitude (Fischer, 2001a,b). Therefore, accuracy in the line bisection task should primarily draw on the ability to select relevant spatial information in the presence of cues, which activate an association between space and number magnitude. In the presence of irrelevant and asymmetrical cues, the selection of spatial information should be more difficult and biased by cognitive properties of the cues (Fischer, 2001a; see also Fischer, 2003; Casarotti et al., 2007; Bonato et al., 2008). Therefore, participants with reduced capacity to filter out spatial information should be more sensitive to the effect of numerical cues when bisecting lines. This hypothesis was corroborated by the non-linear association between measures of the difference-based numerical bias and age: The difference-based numerical bias was stronger in children and elderly participants relative to young and middle-aged adults. This can be explained by a general weakness in inhibitory control, as a specific part of working memory (for a review see Diamond, 2013; for age related differences in inhibitory control see also Petersen et al., 2016), that is typically observed in children and elderly participants relative to young adults (Hasher and Zacks, 1988; Christ et al., 2001). As children and elderly participants should be less efficient in inhibiting the magnitude representation of numerical flankers relative to young adults, they may have showed a stronger difference-based numerical bias in the line bisection task. Working memory seems to be a more important factor for the development of the difference-based numerical bias than embodied representations of cultural experiences which result in long-term associations between number magnitude and physical space. However, executive control is involved in two-choice reaction time tasks, such as the parity judgment task, which was used in the current study to measure the SNARC effect, as well. That is, participants have to inhibit the irrelevant information, or the response activated by the irrelevant information (i.e., the spatial information associated with the target number in the case of the SNARC task). Accordingly, and irrespective of the origin of the spatial codes underlying the SNARC effect, we might expect changes in the size of the SNARC effect across the lifespan that follow the development and decline of cognitive-control abilities. This happens, for example, for the Simon effect: the size of the interference effect has been shown to decrease with age during childhood to reach adultlike levels between the sixth and tenth years of life, and then to increase again in older age (e.g., Iani et al., 2014; KuboKawai and Kawai, 2010). However, in order for the interference effect to occur, the interfering information must be available. In fact, consistent evidence shows that there are SNAs in children from very early age on (e.g., de Hevia and Spelke, 2009; Girelli et al., 2009; see de Hevia et al., 2012 for a review on evidence for SNAs in toddlers). As suggested by Wood et al. (2008) there might be more than one cognitive factor which may induce age-related variability in mental associations such as the SNARC effect (i.e. practice with the association and inhibitory abilities).

Nevertheless, our account drawing on a strengthening of embodied representations reflects a more parsimonious explanation of the observed result pattern as it indicates that a single mechanism might be sufficient to account for developmental trajectories. Most importantly, however, the conclusion of two different mechanism underlying the two SNAs investigated in the current study is consistent with the fact that we did not observe a significant correlation between the differencebased numerical bias in line bisection and the SNARC effect. 
The latter would have been expected in case of a shared underlying mechanism incorporating influences of both embodiment and changes of working memory resources. Instead we observed linear and quadratic trends of age on the SNARC effect and the bisection bias, respectively. As such, our results indicate that the SNARC effect may more likely result from the automatic associations between number magnitude and physical space, while working memory contributes to a greater extent to the bisection bias. However, alternative explanations cannot be ruled out completely based on these data.

Taken together, the SNA-specific developmental trajectories are difficult to reconcile with the view of a single set of cognitive mechanisms underlying directional and non-directional SNAs as reflected by the SNARC effect and the numerical bisection bias - be it either embodiment or influences of working memory. However, absence of evidence does not constitute evidence of absence. Therefore, the interpretation of the present results regarding correlations between SNAs remains tentative. While the SNARC effect was associated linearly with age, the differencebased numerical bias presented a quadratic association with age. Regarding the SNARC effect, this indicates an increasing effect of cultural experiences (such as reading and writing direction) and thus embodied influences on long-term associations of number magnitude and physical space. In contrast, the development of the difference-based numerical bias in the numerical version of the line bisection task seems to be associated with changes in working memory between age groups. Even though increasingly automatic associations between number magnitude and physical

\section{REFERENCES}

Andres, M., Davare, M., Pesenti, M., Olivier, E., and Seron, X. (2004). Number magnitude and grip aperture interaction. Neuroreport 15, 2773-2777.

Berch, D. B., Foley, E. J., Hill, R. J., and Ryan, P. M. (1999). Extracting parity and magnitude from Arabic numerals: developmental changes in number processing and mental representation. J. Exp. Child Psychol. 74, 286-308. doi: 10.1006/jecp.1999.2518

Bonato, M., Priftis, K., Marenzi, R., and Zorzi, M. (2008). Modulation of hemispatial neglect by directional and numerical cues in the line bisection task. Neuropsychologia 46, 426-433. doi: 10.1016/j.neuropsychologia.2007.08.019

Borella, E., Carretti, B., and De Beni, R. (2008). Working memory and inhibition across the adult life-span. Acta Psychol. 128, 33-44. doi: 10.1016/j.actpsy.2007. 09.008

Bulf, H., de Hevia, M. D., and Macchi Cassia, V. (2016). Small on the left, large on the right: numbers orient visual attention onto space in preverbal infants. Dev. Sci. 19, 394-401. doi: 10.1111/desc.12315

Calabria, M., and Rossetti, Y. (2005). Interference between number processing and line bisection: a methodology. Neuropsychologia 43, 779-783. doi: 10.1016/j. neuropsychologia.2004.06.027

Casarotti, M., Michielin, M., Zorzi, M., and Umiltà, C. (2007). Temporal order judgment reveals how number magnitude affects visuospatial attention. Cognition 102, 101-117. doi: 10.1016/j.cognition.2006.09.001

Cheung, C., Ayzenberg, V., Diamond, R. F. L., Yousif, S., and Lourenco, S. F. (2015). "Probing the mental number line?: a between - task analysis of spatial - numerical associations," in Proceedings of the 37th Annual Meeting of the Cognitive Science Society, eds D. C. Noelle, R. Dale, A. S. Warlaumont, J. Yoshimi, T. Matlock, C. D. Jennings, et al. (Austin, TX: Cognitive Science Society), 357-362.

Christ, S. E., White, D. A., Mandernach, T., and Keys, B. A. (2001). Inhibitory control across the life span. Dev. Neuropsychol. 20, 653-669. doi: 10.1207/ S15326942DN2003_7 space across the lifespan and age-related changes in working memory seem to be valid explanations for the differential effects of age on the SNARC effect and the bisection bias respectively, alternative interpretations cannot be ruled out completely by the present findings. Therefore, as origins of SNAs are heavily debated, alternative explanations of the present results were discussed in light of these differing accounts in an integrative approach. While the current study contributes interesting results to this ongoing discussion, future studies will be needed to pinpoint the precise cognitive mechanisms of different SNAs. On a broader level, the present results strongly suggest that associations between numbers and space, bound either to spatial directions or non-directional extensions as classified by Cipora et al. (2015), seem to have different origins.

\section{AUTHOR CONTRIBUTIONS}

MN, GW, and KM: analysis and interpretation of data, manuscript writing. GW: subject recruitment and data collection. $\mathrm{LK}, \mathrm{H}-\mathrm{CN}$, and MF: Interpretation of data, manuscript writing. All authors approved the final version of manuscript.

\section{FUNDING}

MN is supported by the Leibniz-Competition Fund (SAW; SAW2016-IWM-3).

Cipora, K., and Nuerk, H.-C. (2013). Is the SNARC effect related to the level of mathematics? No systematic relationship observed despite more power, more repetitions, and more direct assessment of arithmetic skill. Q. J. Exp. Psychol. 66, 1974-1991. doi: 10.1080/17470218.2013.772215

Cipora, K., Patro, K., and Nuerk, H.-C. (2015). Are spatial-numerical associations a cornerstone for arithmetic learning? The lack of genuine correlations suggests no. Mind Brain Educ. 9, 190-206. doi: 10.1111/mbe.12093

de Hevia, M. D., Girelli, L., and Cassia, V. M. (2012). Minds without language represent number through space: origins of the mental number line. Front. Psychol. 3:466. doi: 10.3389/fpsyg.2012.00466

de Hevia, M. D., Girelli, L., and Vallar, G. (2006). Numbers and space: a cognitive illusion? Exp. Brain Res. 168, 254-264. doi: 10.1007/s00221-005-0084-0

de Hevia, M. D., and Spelke, E. S. (2009). Spontaneous mapping of number and space in adults and young children. Cognition 110, 198-207. doi: 10.1016/j. cognition.2008.11.003

Dehaene, S., Bossini, S., and Girau, P. (1993). The mental representation of parity and number magnitude. J. Exp. Psychol. Gen. 122, 371-396. doi: 10.1037/00963445.122.3.371

Dekker, T. M., Mareschal, D., Johnson, M. H., and Sereno, M. I. (2014). Picturing words? Sensorimotor cortex activation for printed words in child and adult readers. Brain Lang. 139, 58-67. doi: 10.1016/j.bandl.2014. 09.009

Dellatolas, G., Coutin, T., and De Agostini, M. (1996). Bisection and perception of horizontal lines in normal children. Cortex 32, 705-715. doi: 10.1016/S00109452(96)80040-2

Diamond, A. (2013). Executive functions. Annu. Rev. Psychol. 64, 135-168. doi: 10.1146/annurev-psych-113011-143750

Engelen, J. A. A., Bouwmeester, S., de Bruin, A. B. H., and Zwaan, R. A. (2011). Perceptual simulation in developing language comprehension. J. Exp. Child Psychol. 110, 659-675. doi: 10.1016/j.jecp.2011.06.009

Faust, M. E., Balota, D. A., Spieler, D. H., and Ferraro, F. R. (1999). Individual differences in information-processing rate and amount: implications for group 
differences in response latency. Psychol. Bull. 125, 777-799. doi: 10.1037/00332909.125.6.777

Fias, W., Brysbaert, M., Geypens, F., and D'Ydewalle, G. (1996). The importance of magnitude information in numerical processing: evidence from the SNARC effect. Math. Cogn. 2, 95-110. doi: 10.1080/135467996387552

Fias, W., and Fischer, M. H. (2005). "Spatial representation of number," in Handbook of Mathematical Cognition, ed. J. I. D. Campbell (New York, NY: Psychology Press), 43-54.

Fias, W., van Dijck, J.-P., and Gevers, W. (2011). "How is number associated with space? The role of working memory," in Space, Time and Number in the Brain, eds S. Dehaene and E. Brannon (Amsterdam: Elsevier), 133-148. doi: 10.1016/B978-0-12-385948-8.00010-4

Fischer, M. H. (2001a). Cognition in the bisection task. Trends Cogn. Sci. 5, 460-462. doi: 10.1016/S1364-6613(00)01790-3

Fischer, M. H. (2001b). Number processing induces spatial performance biases. Neurology 57, 822-826. doi: 10.1212/WNL.57.5.822

Fischer, M. H. (2003). Spatial representations in number processing-evidence from a pointing task. Vis. Cogn. 10, 493-508. doi: 10.1080/13506280244000186

Fischer, M. H. (2006). The future for SNARC could be stark. Cortex 42, 1066-1068. doi: 10.1016/S0010-9452(08)70218-1

Fischer, M. H. (2012). A hierarchical view of grounded, embodied, and situated numerical cognition. Cogn. Process. 13, 161-164. doi: 10.1007/s10339-0120477-5

Fischer, M. H., and Brugger, P. (2011). When digits help digits: spatial-numerical associations point to finger counting as prime example of embodied cognition. Front. Psychol. 2:260. doi: 10.3389/fpsyg.2011.00260

Fischer, M. H., Castel, A. D., Dodd, M. D., and Pratt, J. (2003). Perceiving numbers causes spatial shifts of attention. Nat. Neurosci. 6, 555-556. doi: 10.1038/nn1066

Fischer, M. H., and Shaki, S. (2014). Spatial associations in numerical cognition From single digits to arithmetic. Q. J. Exp. Psychol. 67, 1461-1483. doi: 10.1080/ 17470218.2014.927515

Fujii, T., Fukatsu, R., Yamadori, A., and Kimura, I. (1995). Effect of age on the line bisection test. J. Clin. Exp. Neuropsychol. 17, 941-944. doi: 10.1080/ 01688639508402443

Galfano, G., Rusconi, E., and Umiltà, C. (2006). Number magnitude orients attention, but not against one's will. Psychon. Bull. Rev. 13, 869-874. doi: 10.3758/BF03194011

Gauvrit, N., Zenil, H., Soler-Toscano, F., Delahaye, J.-P., Brugger, P., Schuknecht, B., et al. (2017). Human behavioral complexity peaks at age 25. PLoS Comput. Biol. 13:e1005408. doi: 10.1371/journal.pcbi.1005408

Gebuis, T., and Gevers, W. (2011). Numerosities and space; indeed a cognitive illusion! A reply to de Hevia and Spelke (2009). Cognition 121, 248-252. doi: 10.1016/j.cognition.2010.09.008

Gevers, W., and Lammertyn, J. (2005). The hunt for SNARC. Psychol. Sci. 47, $10-21$.

Ginsburg, V., van Dijck, J.-P., Previtali, P., Fias, W., and Gevers, W. (2014). The impact of verbal working memory on number-space associations. J. Exp. Psychol. Learn. Mem. Cogn. 40, 976-986. doi: 10.1037/a0036378

Girelli, L., Perrone, G., Pisacane, A., and Macchi Cassia, V. (2009). The influence of number and magnitude information on space representation in children. Poster Presented at the Biennal Meeting of the Society for Research in Child Development (SRCD), Denver, CO.

Hasher, L., and Zacks, R. T. (1988). "Working memory, comprehension, and aging: a review and a new view," in The Psychology of Learning and Motivation, ed. G. H. Bower (San Diego, CA: Academic Press), 193-225. doi: 10.1016/S00797421(08)60041-9

Hoffmann, D., Hornung, C., Martin, R., and Schiltz, C. (2013). Developing number-space associations: SNARC effects using a color discrimination task in 5-year-olds. J. Exp. Child Psychol. 116, 775-791. doi: 10.1016/j.jecp.2013.07.013

Hoffmann, D., Pigat, D., and Schiltz, C. (2014). The impact of inhibition capacities and age on number-space associations. Cogn. Process. 15, 329-342. doi: 10.1007/ s10339-014-0601-9

Hubbard, E. M., Piazza, M., Pinel, P., and Dehaene, S. (2005). Interactions between number and space in parietal cortex. Nat. Rev. Neurosci. 6, 435-448. doi: $10.1038 / \mathrm{nrn} 1684$

Huber, S., Klein, E., Moeller, K., and Willmes, K. (2016). Spatial-numerical and ordinal positional associations coexist in parallel. Front. Psychol. 7:438. doi: $10.3389 /$ fpsyg.2016.00438
Iani, C., Stella, G., and Rubichi, S. (2014). Response inhibition and adaptations to response conflict in 6- to 8-year-old children: evidence from the Simon effect. Atten. Percept. Psychophys. 76, 1234-1241. doi: 10.3758/s13414-014-0656-9

Jewell, G., and McCourt, M. E. (2000). Pseudoneglect: a review and meta-analysis of performance factors in line bisection tasks. Neuropsychologia 38, 93-110. doi: 10.1016/S0028-3932(99)00045-7

Knops, A., Dehaene, S., Berteletti, I., and Zorzi, M. (2014). Can approximate mental calculation account for operational momentum in addition and subtraction? Q. J. Exp. Psychol.67, 1541-1556. doi: 10.1080/17470218.2014.890234

Kubo-Kawai, N., and Kawai, N. (2010). Elimination of the enhanced Simon effect for older adults in a three-choice situation: ageing and the Simon effect in a go/no-go Simon task. Q. J. Exp. Psychol. 63, 452-464. doi: 10.1080/ 17470210902990829

Lambrechts, A., Karolis, V., Garcia, S., Obende, J., and Cappelletti, M. (2013). Age does not count: resilience of quantity processing in healthy ageing. Front. Psychol. 4:865. doi: 10.3389/fpsyg.2013.00865

Loeffler, J., Raab, M., and Cañal-Bruland, R. (2016). A lifespan perspective on embodied cognition. Front. Psychol. 7:845. doi: 10.3389/fpsyg.2016.00845

Longo, M. R., and Lourenco, S. F. (2007). Spatial attention and the mental number line: evidence for characteristic biases and compression. Neuropsychologia 45, 1400-1407. doi: 10.1016/j.neuropsychologia.2006.11.002

Lorch, R. F., and Myers, J. L. (1990). Regression analyses of repeated measures data in cognitive research. J. Exp. Psychol. Learn. Mem. Cogn. 16, 149-157. doi: 10.1037/0278-7393.16.1.149

McCrink, K., Dehaene, S., and Dehaene-Lambertz, G. (2007). Moving along the number line: operational momentum in nonsymbolic arithmetic. Percept. Psychophys. 69, 1324-1333. doi: 10.3758/BF03192949

McCrink, K., and Opfer, J. E. (2014). Development of spatial-numerical associations. Curr. Dir. Psychol. Sci. 23, 439-445. doi: 10.1177/ 0963721414549751

McCrink, K., Shaki, S., and Berkowitz, T. (2014). Culturally driven biases in preschoolers' spatial search strategies for ordinal and non-ordinal dimensions. Cogn. Dev. 30, 1-14. doi: 10.1016/j.cogdev.2013.11.002

Myachykov, A., Scheepers, C., Fischer, M. H., and Kessler, K. (2014). TEST: a tropic, embodied, and situated theory of cognition. Top. Cogn. Sci. 6, 442-460. doi: $10.1111 /$ tops. 12024

Newcombe, N. S., Levine, S. C., and Mix, K. S. (2015). Thinking about quantity: the intertwined development of spatial and numerical cognition. Wiley Interdiscip. Rev. Cogn. Sci. 6, 491-505. doi: 10.1002/wcs.1369

Oldfield, R. C. (1971). The assessment and analysis of handedness: the Edinburgh inventory. Neuropsychologia 9, 97-113. doi: 10.1016/0028-3932(71)90067-4

Orr, C. A., and Nicholls, M. E. R. (2005). The nature and contribution of space- and object-based attentional biases to free-viewing perceptual asymmetries. Exp. Brain Res. 162, 384-393. doi: 10.1007/s00221-004-2196-3

Patro, K., and Haman, M. (2012). The spatial-numerical congruity effect in preschoolers. J. Exp. Child Psychol. 111, 534-542. doi: 10.1016/j.jecp.2011 .09 .006

Patro, K., Nuerk, H.-C., Cress, U., and Haman, M. (2014). How number-space relationships are assessed before formal schooling: a taxonomy proposal. Front. Psychol. 5:419. doi: 10.3389/fpsyg.2014.00419

Perrone, G., de Hevia, M. D., Bricolo, E., and Girelli, L. (2010). Numbers can move our hands: a spatial representation effect in digits handwriting. Exp. Brain Res. 205, 479-487. doi: 10.1007/s00221-010-2383-3

Petersen, I. T., Hoyniak, C. P., McQuillan, M. E., Bates, J. E., and Staples, A. D. (2016). Measuring the development of inhibitory control: the challenge of heterotypic continuity. Dev. Rev. 40, 25-71. doi: 10.1016/j.dr.2016.02.001

Priftis, K., Zorzi, M., Meneghello, F., Marenzi, R., and Umiltà, C. (2006). Explicit versus implicit processing of representational space in neglect: dissociations in accessing the mental number line. J. Cogn. Neurosci. 18, 680-688. doi: 10.1162/ jocn.2006.18.4.680

Proctor, R. W., and Cho, Y. S. (2006). Polarity correspondence: a general principle for performance of speeded binary classification tasks. Psychol. Bull. 132, 416-442. doi: 10.1037/0033-2909.132.3.416

Ristic, J., Wright, A., and Kingstone, A. (2006). The number line effect reflects top-down control. Psychon. Bull. Rev. 13, 862-868. doi: 10.3758/BF03194010

Salillas, E., Elyagoubi, R., and Semenza, C. (2008). Sensory and cognitive processes of shifts of spatial attention induced by numbers: an ERP study. Cortex 44, 406-413. doi: 10.1016/j.cortex.2007.08.006 
Shaki, S., Fischer, M. H., and Göbel, S. M. (2012). Direction counts: a comparative study of spatially directional counting biases in cultures with different reading directions. J. Exp. Child Psychol. 112, 275-281. doi: 10.1016/j.jecp.2011. 12.005

Shaki, S., Pinhas, M., and Fischer, M. H. (2017). Heuristics and biases in mental arithmetic: revisiting and reversing operational momentum. Think. Reason. 1-19. doi: 10.1080/13546783.2017.1348987

Siegel, L. S. (1994). Working memory and reading: a life-span perspective. Int. J. Behav. Dev. 17, 109-124. doi: 10.1177/016502549401700107

Stoianov, I., Kramer, P., Umiltà, C., and Zorzi, M. (2008). Visuospatial priming of the mental number line. Cognition 106, 770-779. doi: 10.1016/j.cognition.2007. 04.013

van Dijck, J.-P., and Fias, W. (2011). A working memory account for spatialnumerical associations. Cognition 119, 114-119. doi: 10.1016/j.cognition.2010. 12.013

van Dijck, J.-P., Gevers, W., and Fias, W. (2009). Numbers are associated with different types of spatial information depending on the task. Cognition 113, 248-253. doi: 10.1016/j.cognition.2009.08.005

van Dijck, J.-P., Gevers, W., Lafosse, C., and Fias, W. (2012). The heterogeneous nature of number-space interactions. Front. Hum. Neurosci. 5:182. doi: 10.3389/fnhum.2011.00182

van Galen, M. S., and Reitsma, P. (2008). Developing access to number magnitude: a study of the SNARC effect in 7- to 9-year-olds. J. Exp. Child Psychol. 101, 99-113. doi: 10.1016/j.jecp.2008.05.001

Van Vugt, P., Fransen, I., Creten, W., and Paquier, P. (2000). Line bisection performances of 650 normal children. Neuropsychologia 38, 886-895. doi: 10.1016/S0028-3932(99)00130-X
Winter, B., Matlock, T., Shaki, S., and Fischer, M. H. (2015). Mental number space in three dimensions. Neurosci. Biobehav. Rev. 57, 209-219. doi: 10.1016/j. neubiorev.2015.09.005

Wood, G., Willmes, K., Nuerk, H.-C., and Fischer, M. H. (2008). On the cognitive link between space and number: a meta-analysis of the SNARC effect. Psychol. Sci. Q. 4, 489-525. doi: 10.1027/1618-3169.52.3.187

Yang, T., Chen, C., Zhou, X., Xu, J., Dong, Q., and Chen, C. (2014). Development of spatial representation of numbers: a study of the SNARC effect in Chinese children. J. Exp. Child Psychol. 117, 1-11. doi: 10.1016/j.jecp.2013.08.011

Zebian, S. (2005). Linkages between number concepts, spatial thinking, and directionality of writing: the SNARC effect and the REVERSE SNARC effect in English and Arabic monoliterates, biliterates, and illiterate Arabic speakers. J. Cogn. Cult. 5, 165-190. doi: 10.1163/1568537054068660

Zorzi, M., Priftis, K., and Umiltà, C. (2002). Brain damage: neglect disrupts the mental number line. Nature 417, 138-139. doi: 10.1038/417138a

Conflict of Interest Statement: The authors declare that the research was conducted in the absence of any commercial or financial relationships that could be construed as a potential conflict of interest.

Copyright (c) 2017 Ninaus, Moeller, Kaufmann, Fischer, Nuerk and Wood. This is an open-access article distributed under the terms of the Creative Commons Attribution License (CC BY). The use, distribution or reproduction in other forums is permitted, provided the original author(s) or licensor are credited and that the original publication in this journal is cited, in accordance with accepted academic practice. No use, distribution or reproduction is permitted which does not comply with these terms. 


\section{OPEN ACCESS}

Edited by:

Anna M. Borghi,

Sapienza University of Rome, Italy

Reviewed by:

Mina Catherine Johnson-Glenberg, Arizona State University, USA

Filippo Gomez Paloma

University of Salerno, Italy

*Correspondence:

Mathieu Hainselin

mathieu.hainselin@u-picardie.fr

Specialty section: This article was submitted to Cognition,

a section of the journal

Frontiers in Psychology

Received: 29 October 2016

Accepted: 20 February 2017

Published: 09 March 2017

Citation:

Hainselin M, Picard L, Manolli P, Vankerkore-Candas $S$ and Bourdin $B$ (2017) Hey Teacher, Don't Leave Them Kids Alone: Action Is Better

for Memory than Reading.

Front. Psychol. 8:325

doi: 10.3389/fpsyg.2017.00325

\section{Hey Teacher, Don't Leave Them Kids Alone: Action Is Better for Memory than Reading}

\author{
Mathieu Hainselin ${ }^{1 *}$, Laurence Picard ${ }^{2}$, Patrick Manolli², Sophie Vankerkore-Candas ${ }^{1}$ \\ and Béatrice Bourdin ${ }^{1}$ \\ ${ }^{1}$ CRP-CPO, EA 7273, Université de Picardie Jules Verne, Amiens, France, ${ }^{2}$ Laboratoire de Psychologie, EA 3188, \\ Université Bourgogne Franche-Comté, Besançon, France
}

There is no consensus on how the enactment effect (EE), although it is robust, enhances memory. Researchers are currently investigating the cognitive processes underlying this effect, mostly during adulthood; the link between EE and crucial function identified in adulthood such as episodic memory and binding process remains elusive. Therefore, this study aims to verify the existence of EE in 6-10 years old and assess cognitive functions potentially linked to this effect in order to shed light on the mechanisms underlying the EE during childhood. Thirty-five children (15 second graders and 20 fifth graders) were included in this study. They encoded 24 action phrases from a protocol adapted from Hainselin et al. (2014). Encoding occurred under four conditions: Verbal Task, Listening Task, Experimenter-Performed Task, and SubjectPerformed Task. Memory performance was assessed for free and cued recall, as well as source memory abilities. ANOVAS were conducted to explore age-related effects on the different scores according to encoding conditions. Correlations between EE scores (Subject-Performed Task/Listening Task) and binding memory scores (short-term binding and episodic memory) were run. Both groups benefited from EE. However, in both groups, performance did not significantly differ between Subject-Performed Task and Experimenter-Performed Task. A positive correlation was found between EE and episodic memory score for second graders and a moderate negative correlation was found between EE and binding scores for fifth graders. Our results confirm the existence of $E E$ in 6 and 10 year olds, but they do not support the multimodal theory (Engelkamp, 2001) or the "glue" theory (Kormi-Nouri and Nilsson, 2001). This suggests instead that episodic memory might not underlie EE during early childhood.

Keywords: enactment, memory, action, teaching, education

\section{INTRODUCTION}

Memory for action is one way to assess embodied cognition. Within this field, enactment effect (EE) refers to better memory for performed actions than for verbally encoded action sentences. Even if it is a robust effect in adults, there is still no consensus on the conditions revealing this effect in childhood and on the mechanisms enabled its expression. In fact, we still do not know how it enhances memory, and if the same processes are involved in childhood and adulthood 
(Madan and Singhal, 2012; Hainselin, 2015). The assessment of the EE is generally done through an action phrase memory task (e.g., "put the glass on the table," "move the scissors to the left") when participants perform the actions themselves (SelfPerformed Tasks, SPT), watch the actions performed by the experimenter (Experimenter-Performed Tasks, EPT) or only read the sentence (Verbal Tasks, VT). Most experiments include free and/or cued recall, and recognition.

Different theories attempt to explain EE. The multimodal theory (Engelkamp, 2001) supposes that performing an action involve additional motor components in comparison to observing or reading action sentences. Beyond action planning, visual information and movement control, SPT provide a kinaesthetic feedback during information encoding. This theory is not just about deeper processing but multimodality. Engelkamp (2001) emphasized the superiority of EPT or imagining the action compared to VT was not as large as the SPT superiority over VT, in reference to previous findings of better performance in SPT compared to EPT to support this hypothesis (Engelkamp and Zimmer, 1989; Engelkamp, 1995). In a previous study with transient global amnesia patients, we found an improvement of performance between EPT and VT but no difference between SPT and EPT; so it does not fully support the multimodal theory (Hainselin et al., 2014). Feyereisen (2009) found a similar pattern of result (similar benefit of SPT and EPT to VT) in aging. Another theory, named the "glue" theory, stress the importance the core role of cognitive binding of action and object (KormiNouri and Nilsson, 2001). Accordingly, an active learning episode through enactment enhances episodic integration as it strengthens the link between action verbs and nouns. KormiNouri and Nilsson (2001) claims that the two components (action verbs and noun) are encoded in one memory unit or in two close and binded units. For example, if you scratch the brush, the action (scratch) and the object (the brush), it is engrammed in the same episode. The authors assumed that the interaction between self and object is crucial for action memory performance. Few studies conducted with adults argue for this approach, as they showed that the size of the EE is importantly linked with short-term binding abilities (Mangels and Heinberg, 2006; Hainselin et al., 2014). Generally, binding is defined as an associative process that allows integrating features of episodes into coherent representation. Binding could concern information from different sources, both within and between modalities. For example, Baddeley (2000) assumes that the episodic buffer maintain short-term representations using a multidimensional code that notably serve to integrate information from phonological and visuospatial performance. Adopting such a perspective, Hainselin et al. (2014) tested shortterm binding abilities (a consonant had to be associated with specific locations in a grid) and looked for their links with the size of the EE in adults. They show that binding performances strongly correlated with EE, arguing in favor of the "Glue theory".

In the last years, embodied cognition has been a trending topic, showing how cognition is influenced by sensory-motor processing (Barsalou et al., 2003). For example, participants studying angular momentum by physically tilting a set of wheels had better quiz scores than participants only watching it (Kontra et al., 2015). Surprisingly, few studies have investigated EE in children, despite its potential implication for developmental psychology and education. Describing the encoding conditions that are the most appropriate during childhood is in fact essential, especially as large age-related differences in episodic memory abilities have been observed during childhood and adolescence (e.g., Picard et al., 2017). Globally, performing action has been found to improve memory, in normal children (Blake et al., 1987; Ratner and Hill, 1991; James and Swain, 2011; Badinlou et al., 2015), in children with learning disabilities (Freides and Messina, 1986), in children with attention deficit hyperactivity disorder (Yang et al., 2017) or in developmental pathologies, such as autism (for a review, see Grainger et al., 2014). Memorization of sentences seems in fact facilitated when children perform gestures which are coherent with the meaning of sentences in comparison to verbal learning, that is learning by reading or listening an action sentence. How this pattern varies according to age is, however, a still debated question. Some studies showed in fact that no developmental difference in performance is observed after an action encoding condition (e.g., Cohen and Stewart, 1982) whereas others observed that age-related differences in recall scores remain (e.g., Kormi-Nouri et al., 2008). Contradictory results also concern the effect of the interaction between self and objects. Thus, in agreement with the multimodal theory, some studies observed an advantage of SPTs over EPT in children (e.g., Baker-Ward et al., 1990), whereas others reveal the opposite pattern (Ratner and Hill, 1991) or comparable performance (e.g., Foley and Johnson, 1985). In order to go over the methodological gap of previous studies, an EE paradigm has been recently proposed to children from 8 to 14 years, using the three most common encoding conditions: VT, EPT, and SPT (i.e., Read, Watch, and Perform; Badinlou et al., 2015) and three retrieval phases: free recall, cued recall, and then recognition tests. This research revealed several differences between SPTs and EPTs in their school-aged participants. During free and cued recall, age-related increase in performance was in fact more important for SPTs than for EPTs or VT, which did not differ. Moreover, the developmental effect persisted during recognition for SPT, while it disappeared for EPT (and VT). These results have to be replicated, and completed with another encoding condition, particularly important from an educational perspective: the Listening Task. Listening is probably, with Watching, the most common encoding condition at school and should thus be compared with the three other conditions.

The reasons behind the EE remain also unclear from a developmental perspective. Which are the cognitive processes that underlie this effect and its potential development? Recently, some studies have sought to identify factors that could explain EE variance in childhood, among which verb type, time of testing (immediate or delay), or language proficiency (FrickHorbury, 2002; de Nooijer et al., 2013, 2014). To our knowledge, no study has yet addressed the role of cognitive processes (binding, episodic memory) possibly implicated in the EE from a developmental perspective, although theoretical frameworks 
underlined these cognitive processes' involvement. It is especially important to assess their possible contribution that both shortand long-term binding abilities have been shown to increase largely throughout childhood (Lee et al., 2016; Picard et al., 2017). Thus, this study aimed to (1) verify the existence of $E E$ in 6-11 years old children using four different encoding conditions (VT, LT, EPT, SPT), and (2) assess the link between EE and shortterm binding and episodic memory, in order to improve general knowledge about EE and its mechanisms during childhood.

\section{MATERIALS AND METHODS}

\section{Participants}

Participants were 15 second graders (9 girls and 6 boys) and 20 fifth graders ( 9 girls and 11 boys) from different elementary schools in France. They were all native French speakers. For the first graders group, the mean age was $7.6(S D=1.9$, range: 7.3-7.9 years) and for the fifth graders, the mean age was 10.6 ( $S D=5.7$, range: $9.8-11.3$ years). Experiments were carried out in calm offices in the children's schools. The children's participation was conditioned by their parents', headmasters', and teachers' approval, and their own willingness to take part in the experiment. The children's parents filled in a questionnaire to ensure the absence of a background of neurological or psychiatric medical history, developmental learning disorders and repetition of a grade at school, which constituted exclusion criteria. Participants also had to have normal fluid reasoning performance. To assess it, the Matrix Reasoning subtest of the WISC-IV (Wechsler, 2005) was proposed. Children were presented with a partially filled grid and asked to select one out of four or six items that properly completes the matrix considering a logical criterion. One point was given for each correct answer and testing stop when participants failed to answer correctly to four among the last five items. Standard score had to be in the normal range (4-16); based on this criterion, one boy from the fifth graders group was excluded from the analysis. In the end, we had 19 fifth graders ( 9 girls and 10 boys). This study was carried out in accordance with the recommendations of French law written informed consent from all subjects. All participants gave written informed consent in accordance with the Declaration of Helsinki.

\section{Materials}

Twenty-four poorly integrated action phrases (one action verb + one object noun) were used for the enactment task. Action phrases were created using a list of 24 actions and 24 objects inspired from Hainselin et al. (2014). Action-object pairing was randomly determined. However, associations were controlled so that a possible link exists between the action and the object. Consequently, associations such as "fold the torch in half" were excluded. Actions were chosen to be low associates of the objects with which they were paired and vice versa (i.e., prototypical association such as "read a book" were excluded to avoid answers based on semantic knowledge and to reinforce involvement of binding processes). The enactment task was designed on E-Prime 2.0 and was presented using a 15.4-inch screen ASUS laptop. Participants were seated 50-60 cm away from the computer screen. For the complementary cognitive assessment tasks, verbal memory was assessed with the Narrative Memory subtest (NEPSY-II, Korkman et al., 2012) whereas shortterm binding capacity was assessed with a binding task inspired from Picard et al. (2012) and run with E-Prime 2.0.

\section{Procedure}

Children were tested individually in a separate room in their schools by an experimenter. Each session lasted about an hour. Children first performed the Enactment task after which they completed the Matrix Reasoning subtest (Wechsler, 2005), that also served as interference task. After the Enactment retrieval phases, subjects successively performed the short-term binding task and the Narrative Memory task (Korkman et al., 2012). Tests were administered in the same order for all children. Moreover, children were presented with examples and training for all tasks in order to ensure that all instructions have been fully understood.

\section{Enactment Task}

Participants incidentally encoded 24 poorly integrated action phrases under four conditions: Read out loud (VT), Listen (LT), Watch (EPT), and Perform (Subject-Performed task, SPT). We took poorly integrated (scratch the rub) instead of typical (erase with the rub) action phrases (well integrated) because we wanted to assess the glue theory. With typical action phrases, participants would not had to bind the action and object together as it is already well integrated (Mangels and Heinberg, 2006). In addition, when using typical action phrases, it is very difficult to know if participants genuinely remember the action associated to the object or if they give the most obvious one; for example: erase is generally the first word you can think about after seeing a rubber. Six action phrases were presented for each encoding condition with a fixed order for all subjects. Each action phrase was displayed on the screen for $5 \mathrm{~s}$ during which the subject had to read it silently. Once the action phrase appeared, the object mentioned in the action phrase was physically presented in front of the child. Then the encoding condition appeared above the action phrase and children had to perform the indicated action. For the VT, the participants were instructed to read out loud the phrase; for the LT, they had to listen to the experimenter that read the sentence; for the EPT, they were instructed to watch the experimenter performing the action with the present object; for the SPT, they had to carry out the indicated action with the object in front of them. Once the indicated action has been performed, object was removed and the following action phrase appeared. Action-object pairing and encoding conditions were counterbalanced in two lists to avoid item order bias. Before beginning, children were provided with one example for each condition to ensure that instructions have been fully understood (see Figure 1 for design).

After performing the interference task (Matrix Reasoning subtest, Wechsler, 2005) which lasted around $15 \mathrm{~min}$, children performed three successive memory tests: a free recall, a cued recall, and a recognition test. In the free recall test, they were instructed to recall a maximum number of associations (action + object). If associations cannot be retrieved, they 


\section{Encoding}

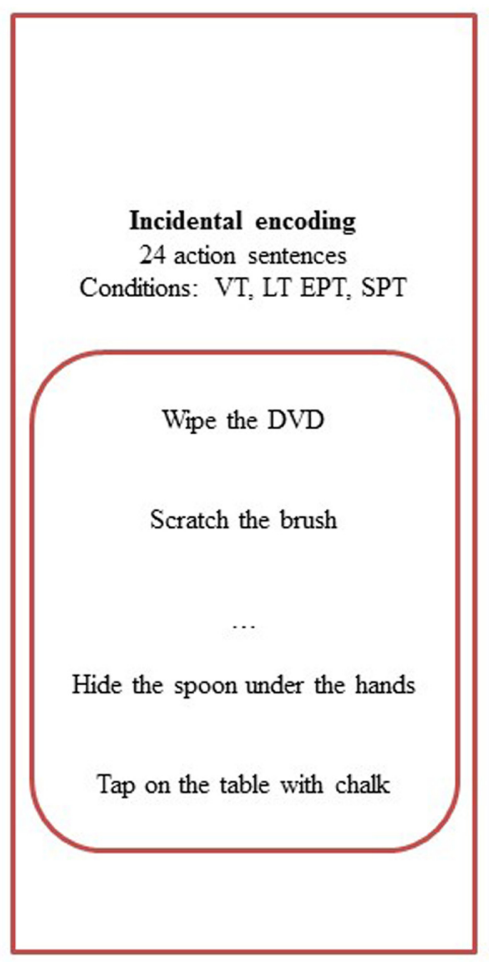

\section{Recall}

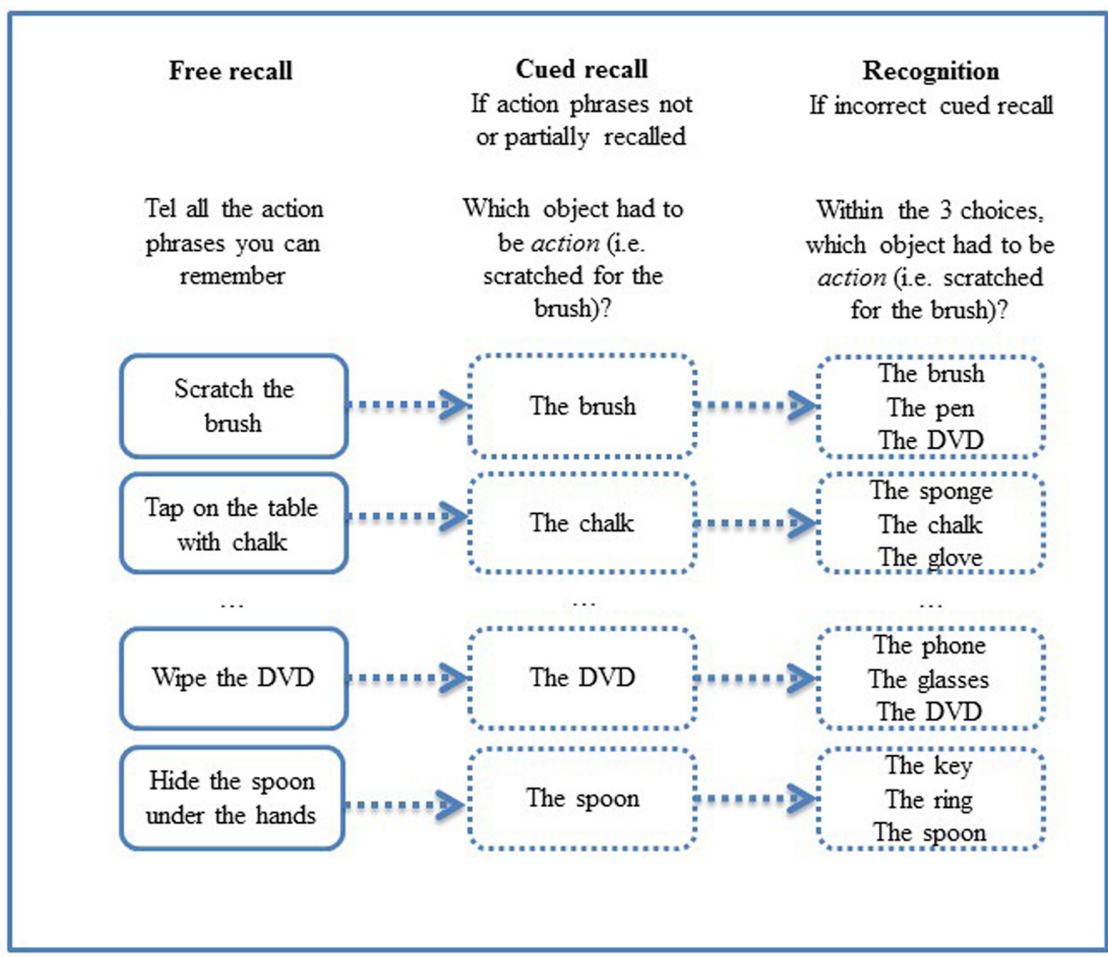

FIGURE 1 | Design for the enactment effect task. After encoding 24 actions, participants have to retrieve actions in free and cued recall. There was a recognition for items incorrectly recalled in the free or cued recall. VT, Verbal Task; LT, Listening Task; EPT, Experimenter-Performed Task; SPT, Self-Performed Task.

were asked to recall action and objects separately. For each action phrase correctly recalled, participants performed a source memory task, as they had to indicate the encoding condition (i.e., Read, Listen, Watch, Perform).

For action phrases that were not or only partially recalled, participants performed a cued recall task: the action was provided as a cue to the object from the encoded action phrase (e.g., what were you required to "fold in half"?). When the cued recall task did not allow retrieving the correct object, a forced choice recognition task was proposed: children had to detect the object associated with the action among a distractor from the same encoding condition as the target and another from a different encoding condition (e.g., "were you required to touch your nose with the modeling clay, with the plastic glove, or with the paintbrush?"). All the proposed items have been previously encoded in order to avoid answers based only on familiarity processes. Position of the cue among the three propositions varied: for one third of the trials the cue object was proposed first, for one third of the trial was proposed in the middle position, and for the last third of the trials it was in the last position.

We marked one point for each correctly retrieved information. Thus, in the free recall task, two points were awarded for each action phrase completely recalled (verb + object), and a single point for each partial recall (i.e., only object or action). The maximum Free Recall score is then 12 for each condition, and
48 for the Total Free Recall score (the sum of the free recall scores for all four conditions). Similarly, one point was awarded for correct answers in cued recall and recognition. As cued retrieval was not proposed for associations correctly remembered during free recall, cued recall scores correspond to the sum of free and cued recall answers. The maximum score was then of 12 for each conditions, and 48 for the Total Cued Recall score. For a better understanding, all scores were converted into percentages. Similarly, the maximum recognition score was of 12 for each condition, and 48 for the Total Recognition score. Source score was calculated as percentage taking correct answer into account.

In order to have an indicator of the size of the EE, the classic improvement index (Perform/Read) was calculated by dividing the Recognition score in the Perform condition by the Recognition score in the Read condition (for similar method, see Hainselin et al., 2014). Given that the youngest participants are only 7 years old in average, reading abilities are not fully automatized. This lack of automaticity could put the youngest' enactment index on a disadvantage. Thus, in an exploratory perspective, we also calculated a second EE index, the Listen improvement index, based on the Listen, instead of the Read condition. It was calculated by dividing the Recognition score in the SPT (Perform) condition by the Recognition score in the VT (Listen) condition. 


\section{Cognitive Assessment Short-term feature-binding}

The task designed to assess the capacity to bind various features during short delay was inspired by the original task created by Prabhakaran et al. (2000) and adapted for children in a non-verbal fashion (e.g., Lorsbach and Reimer, 2005). The task required to memorize during short delay associations between verbal, spatial, and temporal information (for a very close task, see Picard et al., 2012). In the task used here, children had to memorize strings of objects $(2,3$, or 4 objects) that appear one after another in a specific spatial context (the rooms of a house display; Figure 2), in order to recall the three bounded features straight after: verbal feature (name of the object), spatial feature (location), and temporal information (recall had to be in the same order as the presentation). Nine colorized line drawings of common objects were used. All of them were one syllable long, were not complex $($ Mean $=2.5 / 5)$, familiar $($ Mean $=2.5 / 5)$, and very frequently correctly named (Mean $=90.5 \%$ ) according to the norms published by Rossion and Pourtois (2004). Stimuli were displayed using E-Prime 2.0: objects were displayed for $2000 \mathrm{~ms}$ with an inter-stimulus interval of $500 \mathrm{~ms}$. The end of the trial was symbolized by question marks that remained on the screen for $500 \mathrm{~ms}$. The participant then had to orally recall the sequence of encoded objects, pointing to their respective locations, in their order of appearance. The diversity of the representation that have to be associate was strengthen by the use of an oral recall task instead of a recognition one, as it encourage children to maintain object information according to a phonological code rather than a visual ones. The task consisted of two examples and nine trials (three trials for each length), which were presented randomly but in the same order for all participants. One point was awarded for each sequence of items correctly recalled regardless of its length. The maximum score is then equal to nine.

\section{Verbal episodic memory}

The Narrative Memory subtest of the NEPSY-II (Korkman et al., 2012) was used to assess memory for organized verbal material under free and cued recall. The children listened to a brief story and were then asked to recall its main points. In the free recall phase, one point was given for each of the 20 expected details spontaneously recalled (Free Recall score, $\max =20$ ). For each missing detail, a cued recall task was proposed, in the form of a question (e.g., "what was the name of the child?"). One point was given for each additional detail recalled. The Cued Recall score consisted of the sum of the freely recalled details (two point for each detail), and the cued recalled details (one point for each detail; $\max =40$ ). We used the Cued Recall score, taking the Free Recall score into account, in our analysis.

\section{Statistical Methodology}

The statistical analysis of the enactment task scores was carried out using repeated measures ANOVAs, with Group (Second Grader, Fifth Grader) as the between participants factor, and Condition (Read, Listen, Watch, Perform) as the withinparticipants factor, in order to assess the effect of age groups and encoding conditions. The short-term binding and the episodic memory scores were analyzed using a one-way ANOVA, with Group as a between-participants factor. Post hoc Tukey tests were used to carry out paired comparisons. Finally, in order to determine which processes were linked to the EE, Pearson's correlation coefficients were calculated between cognitive scores and the EE (using the classic improvement index Perform/Read and the Listen improvement index Perform/Listen). Analyses were conducted successively for each age group, to detect potential differences in mechanisms associated with age. All analyses were performed with JASP Team (2016).

\section{RESULTS}

\section{Enactment Task}

Descriptive data are presented in Figure 3 and raw data are available in Supplementary Data Sheet 1.

\section{Free Recall}

A main effect of Group was found $[F(1,32)=7.50, p=0.01$, $\left.\eta^{2}=0.19\right]$, such that Fifth Graders recalled more items than Second Graders. An effect of encoding condition was also found $\left[F(3,32)=24.81, p<0.001, \eta^{2}=0.43\right]$, such that the performance in the Watch and Perform conditions were better than that of the Read and Listen conditions $(p<0.001$ for all significant comparisons). The Group $\times$ Condition interaction was not significant $\left[F(3,32)=1.48, p=0.23, \eta^{2}=0.03\right]$. See Supplementary Data Sheet 2 for detailed recall scores.

\section{Cued Recall}

The ANOVA for the cued recall memory performance revealed a very similar pattern: a main effect of Group $[F(1,32)=14.00$, $\left.p<0.001, \eta^{2}=0.30\right]$, with Fifth Graders recalling more items than Second Graders, an effect of encoding Condition $\left[F(3,32)=40.96, p<0.001, \eta^{2}=0.55\right]$, with Watch and Perform items better recalled than that of the Read and Listen

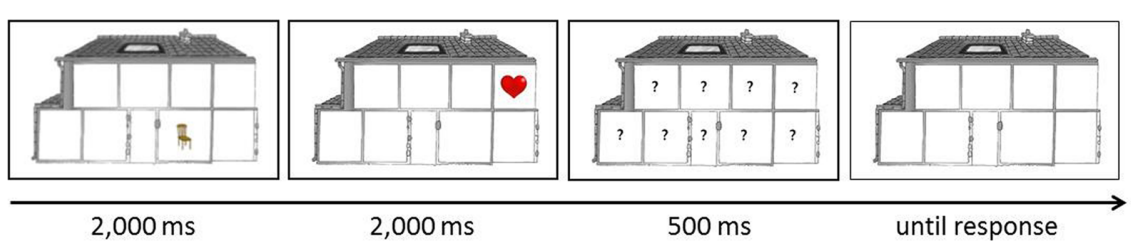

FIGURE 2 | Example of a trial $(N=2)$ taken from the short-term feature-binding task adapted from Picard et al. (2012). 


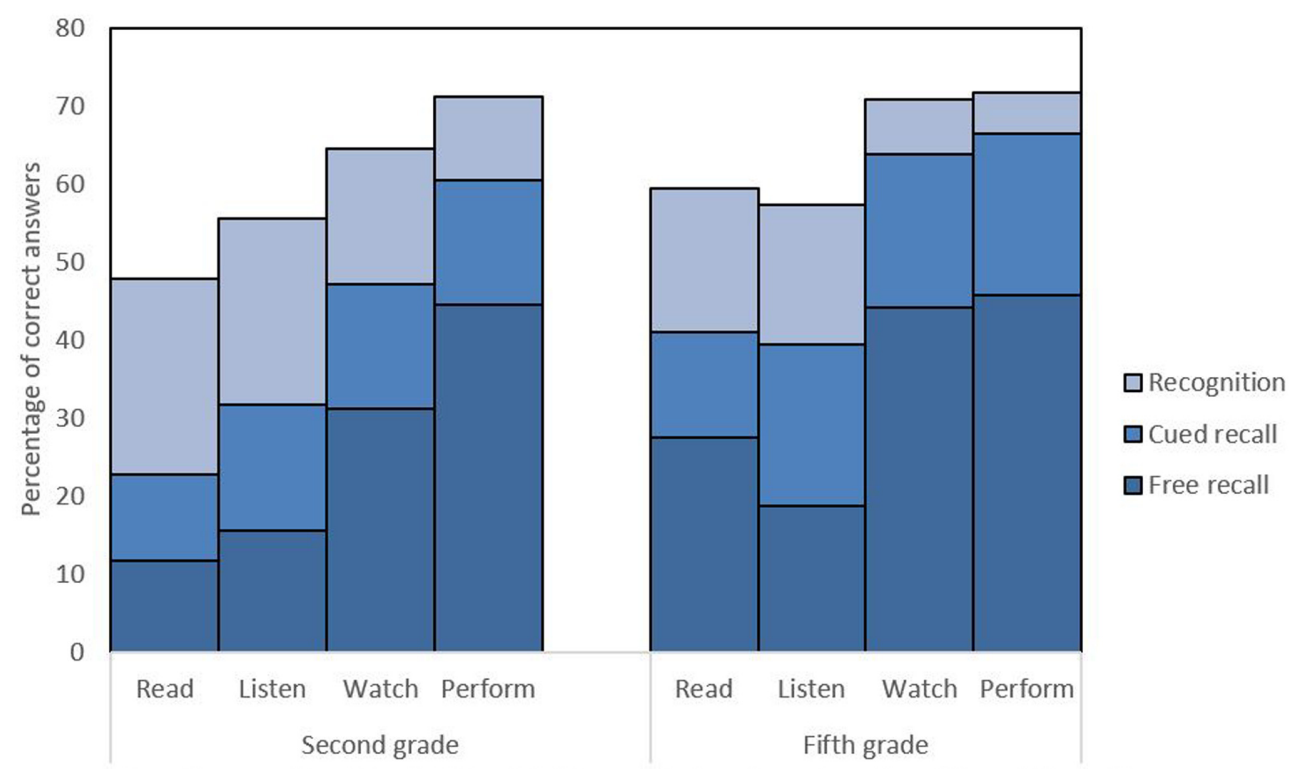

Encoding condition (Read, Listen, Watch, and Perform) as a function of Group (Second grade and Fifth grade)

FIGURE 3 | Percentage of correct answers of action events recalled as a function of type of encoding condition (Read, Listen, Watch, Perform), Group (Second Graders and Fifth Graders), and Retrieval phase (Free Recall, Cued Recall, Recognition).

conditions ( $p<0.001$ for all significant comparisons), and no Group $\times$ Condition interaction $[F(3,32)=1.47, p=0.23$, $\left.\eta^{2}=0.02\right]$.

\section{Recognition}

The ANOVA conducted on the recognition scores showed again a main effect of Group $\left[F(1,32)=6.98, p=0.01, \eta^{2}=0.18\right]$, such that Fifth Graders recalled more items than Second Graders, an effect of Condition $\left[F(3,32)=25.05, p<0.001, \eta^{2}=0.43\right]$, such that the performance in the Watch and Perform conditions were better than that of the Read and Listen conditions, and no Group $\times$ Condition interaction $[F(3,32)=1.82, p=0.15$, $\left.\eta^{2}=0.03\right]$.

\section{Enactment Indexes}

The ANOVAs conducted on the classic enactment index (Perform/Read) did not show a Group effect $[F(1,32)=1.60$, $\left.p=0.22, \eta^{2}=0.05\right]$, while there was a Group effect for the second enactment index (Perform/Listen), $[F(1,32)=11.73, p<0.01$, $\left.\eta^{2}=0.27\right)$.

\section{Source}

The ANOVA conducted on the source score showed a main effect of Group $\left[F(3,32)=4.84, p=0.04, \eta^{2}=0.14\right]$, such that Fifth Graders recalled the encoding condition better than Second Graders, an effect of Condition $[F(3,32)=45.77$, $\left.p<0.001, \eta^{2}=0.58\right]$, such that the performance in the Watch and Perform conditions were better than that of the Read and Listen conditions, and no Group $\times$ Condition interaction $\left[F(3,32)=2.11, p=0.10, \eta^{2}=0.03\right]$.

\section{Cognitive Assessment}

There is a statistical tendency for a difference between the two groups, with better performance for Fifth Graders than Second Graders for Short-Term Binding $[F(1,32)=3.83, p=0.06$, $\left.\eta^{2}=0.11\right]$, and Narrative Recall performance $[F(1,32)=3.87$, $\left.p=0.06, \eta^{2}=0.11\right]$ (see Table 1 ).

\section{Correlation}

For all participants and for Fifth Graders, we found a negative correlation between the Enactment Index and the binding score (respectively, $r=-0.40$ and $r=-0.49$; i.e., the fewer correct answers provided by children in the binding task, the more their memory was enhanced in the Perform condition in comparison with the Read condition). For Second Graders, a positive correlation was found between the Enactment Index and Narrative Recall $(r=0.81)$ scores. There was no significant correlation between the second index (Perform/Listen) and binding or narrative recall scores (see Table 2). See Supplementary Data Sheet 3 for detailed correlation scores.

TABLE 1 | Mean total scores (standard deviation) of the Second and Fifth Graders for the complementary cognitive assessment battery and ANOVAs results.

\begin{tabular}{lccc}
\hline & $\begin{array}{c}\text { Second Graders } \\
(\boldsymbol{n}=\mathbf{1 5})\end{array}$ & $\begin{array}{c}\text { Fifth Graders } \\
(\boldsymbol{n}=\mathbf{1 9})\end{array}$ & $\begin{array}{c}\text { Age effect } \\
\boldsymbol{F ( 1 , 3 2 )}\end{array}$ \\
\hline Binding score & $5.93(1.22)$ & $6.89(1.52)$ & $3.83^{\dagger}$ \\
Narrative Recall & $19.00(5.15)$ & $24.00(8.46)$ & $3.87^{\dagger}$
\end{tabular}

score

$\dagger p=0.06$. 
TABLE 2 | Pearson's correlation between Enactment Indexes and cognitive scores for Second Graders and Fifth Graders.

\begin{tabular}{llcc}
\hline & & $\begin{array}{c}\text { Second Graders } \\
(\boldsymbol{n}=\mathbf{1 5})\end{array}$ & $\begin{array}{c}\text { Fifth Graders } \\
(\boldsymbol{n}=\mathbf{1 9})\end{array}$ \\
\hline $\begin{array}{l}\text { Perform/Read } \\
\text { (classic index) }\end{array}$ & Binding score & -0.10 & $-\mathbf{0 . 4 9 *}$ \\
& $\begin{array}{l}\text { Narrative Recall } \\
\text { score }\end{array}$ & $\mathbf{0 . 8 1 ^ { * * * }}$ & -0.38 \\
\hline $\begin{array}{l}\text { Perform/Listen } \\
\text { (Listen index) }\end{array}$ & Binding score & 0.01 & -0.35 \\
& $\begin{array}{l}\text { Narrative Recall } \\
\text { score }\end{array}$ & 0.42 & -0.13 \\
& & &
\end{tabular}

Significant correlations are in bold.

$* p<0.05$.

${ }^{* * *} p<0.001$

\section{DISCUSSION}

\section{Enactment Theories}

Our results confirmed the presence of an EE in children and the benefit of performing actions for learning purposes. Although we confirmed previous results on the importance of performing actions to enhance memory through the $\mathrm{EE}$, our results are not consistent with the multimodal theory: Watch and Perform conditions performance did not differ for free and cued recalls nor recognition.

Several studies reported that sentences were remembered better if the children mimed the content of the sentences rather than if they were verbally rehearsed (Saltz and Dixon, 1982; Blake et al., 1987; Ratner and Hill, 1991; James and Swain, 2011). Our results showed also that children, whatever their age, exhibited higher recall performance when they had to perform the actions indicated in the sentences than when they have to listen. They also indicate that $\mathrm{EE}$ is observed in the subject-performed condition (differences between Perform and Read) and, inconsistently with Badinlou et al.'s (2015) results, in the experimenter-performed condition (differences between Watch and Read) This might be due to our sample size or the cued recall methodology, for which we only ask for items participants did not recalled freely, contrary to Badinlou et al. (2015) who asked participants to recall every item during cued recall, including those recalled during free recall. With Badinlou et al.'s (2015) methodology, participants with correct answers on free recall could fail to give the correct answer during cued recall, whereas this is not possible with our methodology.

The better performance in memory for action conditions (Watch and Perform) over non-active conditions is also highlighted for source memory (i.e., encoding condition). This adds evidence to the superiority of action conditions, not only for items association, as previously described in the literature, but also for its context. Taken together these results suggest that physical activity, by children themselves, although preferable, is not indispensable to the $\mathrm{EE}$ in younger children. Younger children (Second Graders) recall as much as older children (Fifth Graders) when they learn through action but not when they watch the experimenter performing the actions. Although younger children have lower memory performance than older children, this difference disappears when they learn through action, which is the best encoding condition for both groups. These results are not reflecting a ceiling effect, as our participants have an $80 \%$ success rate for SPT. The importance of actions in cognitive development is known in the education of young children (Rubin et al., 1983; Cohen, 1989), with better learning while acting (Ballantyne and Packer, 2009). While the Listening condition is not always assessed, children exhibited worse memory performance under it for all retrieval conditions, compared to EPT or SPT. Therefore, under this condition, teachers, clinicians, and scientists might under-estimate children's capacities of memorization. In the future, there is a need for more research on memory for action development, with a specific focus on the cognitive processes linked to the $\mathrm{EE}$, such as working memory, which is very important in episodic memory development (Picard et al., 2009; Rhodes et al., 2011; Rajan et al., 2014).

\section{Pedagogical Issues}

It has been shown that action enactment facilitates verbal memory for children. Our results support this finding and research results should encourage teachers to support a more active learning, in particular in young children. Previous publications has shown that watching or performing gestures facilitates acquisition of a variety of tasks, such as mathematical tasks (Cook et al., 2008; Novack et al., 2014) and vocabulary learning (Macedonia and Klimesch, 2014; Mavilidi et al., 2015; Toumpaniari et al., 2015). In particular, foreign language vocabulary learning is easier in preschool children (4-5 years old) when congruent gestures are used in word learning (Mavilidi et al., 2015; Toumpaniari et al., 2015). Gestures can also foster text learning (Cutica et al., 2014).

We did not confirm the glue theory either, as we did not find positive correlation between enactment index score and binding performance (for both enactment indexes). This was the first time that the link between binding and EE was assessed during childhood. As expected, short-term binding performance increases with age, in agreement with previous works (e.g., Gray et al., 2017), confirming that our task was appropriate. When using the classical index (Perform/Read), we did not observe any link with binding for Second Graders and found a negative correlation for the Fifth Graders Group. This unexpected result suggests that the EE might not be supported by the same cognitive processes in childhood as in adulthood. In line with previous work that suggest a need of a different theoretical framework for EE in amnestic patients (Hainselin et al., 2014), it seems that our results cannot be completely explained by the glue theory. In this perspective, research with amnesic children should lighten this actual gray area. Future works should deepened the link between $\mathrm{EE}$ and binding process. As only one specific short-term binding task was used here whereas several types of binding could be identified (see for example Gray et al., 2017). More studies are needed to test if the actual results are also observed with other kind of associative processes. Besides, we tried to use here a 
specific index to avoid any reading ability bias that could lead to differences between age groups. The sensitivity of this new index was not clearly observed here and identification of a new and more appropriate index to reveal EE should pursuit.

The majority of activities in school mostly are based on verbal tasks. Therefore, it is possible that memorization capacity is under-estimated in young children who have a less-developed language capacity or in learning disabled children. In accordance with this conception, a study has shown that children with autism spectrum disorders have better performance when they acted than in another watching condition (Lind and Bowler, 2009). In the same way, learning disabled children, known to have difficulties to memorize verbal information, have memory scores equivalent to the normal control in the motor enactment condition (Freides and Messina, 1986). In our research, children with lower performance can benefit from actions, and future long term research is needed to confirm whether this is an efficient method to improve teaching and reduce inequality between children. In conclusion, we encourage teachers to bring more actions to the classroom, and scientists to evaluate the EE for children with learning disabilities.

\section{REFERENCES}

Baddeley, A. (2000). The episodic buffer: a new component of working memory? Trends Cogn. Sci. 4, 417-423. doi: 10.1016/S1364-6613(00)01538-2

Badinlou, F., Kormi-Nouri, R., Mousavi Nasab, S. M. H., and Knopf, M. (2015). Developmental differences in episodic memory across school ages: evidence from enacted events performed by self and others. Memory 25, 84-94. doi: 10.1080/09658211.2015.1126607

Baker-Ward, L., Hess, T. M., and Flannagan, D. A. (1990). The effects of involvement on children's memory for events. Cogn. Dev. 5, 55-69. doi: 10.1016/0885-2014(90)90012-I

Ballantyne, R., and Packer, J. (2009). Introducing a fifth pedagogy: experiencebased strategies for facilitating learning in natural environments. Environ. Educ. Res. 15, 243-262. doi: 10.1080/13504620802711282

Barsalou, L. W., Kyle Simmons, W., Barbey, A. K., and Wilson, C. D. (2003). Grounding conceptual knowledge in modality-specific systems. Trends Cogn. Sci. 7, 84-91. doi: 10.1016/S1364-6613(02)00029-3

Blake, J., Austin, W., and Lowenstein, J. (1987). The effect of enactment upon sentence imitation in preschool children. J. Psycholinguist. Res. 16, 351-367. doi: 10.1007/BF01069288

Cohen, R. L. (1989). Memory for action events: the power of enactment. Educ. Psychol. Rev. 1, 57-80. doi: 10.1007/BF01326550

Cohen, R. L., and Stewart, M. (1982). How to avoid developmental effects in free recall. Scand. J. Psychol. 23, 9-15. doi: 10.1111/j.1467-9450.1982.tb00408.x

Cook, S. W., Mitchell, Z., and Goldin-Meadow, S. (2008). Gesturing makes learning last. Cognition 106, 1047-1058. doi: 10.1016/j.cognition.2007.04.010

Cutica, I., Ianì, F., and Bucciarelli, M. (2014). Learning from text benefits from enactment. Mem. Cogn. 42, 1026-1037. doi: 10.3758/s13421-014-0417-y

de Nooijer, J. A., van Gog, T., Paas, F., and Zwaan, R. A. (2013). Effects of imitating gestures during encoding or during retrieval of novel verbs on children's test performance. Acta Psychol. 144, 173-179. doi: 10.1016/j.actpsy.2013.05.013

de Nooijer, J. A., van Gog, T., Paas, F., and Zwaan, R. A. (2014). Words in action: using gestures to improve verb learning in primary school children. Gesture 14, 46-69. doi: 10.1075/gest.14.1.03noo

Engelkamp, J. (1995). Visual imagery and enactment of actions in memory. Br. J. Psychol. 86(Pt 2), 227-240. doi: 10.1111/j.2044-8295.1995.tb02558.x

Engelkamp, J. (2001). "Action memory: a system- oriented approach," in Memory for Action: A Distinct form of Episodic Memory?, eds H. D. Zimmer, R. L. Cohen, M. J. Guynn, J. Engelkamp, R. Kormi-Nouri, and M. A. Foley (New York, NY: Oxford University), 46-96.

\section{AUTHOR CONTRIBUTIONS}

MH: Conception and design of the task, analysis and interpretation of the data, writing the manuscript. LP: Conception and design of the task, analysis and interpretation of the data, writing the manuscript. PM: Acquisition of the data, drafting the manuscript. SV-C: Acquisition of the data, drafting the manuscript. BB: Conception and design of the task, analysis and interpretation of the data, writing the manuscript.

\section{ACKNOWLEDGMENT}

Publication fees were supported by the Bonus Qualité Recherche (BQR) of the Université de Picardie Jules Verne (UPJV).

\section{SUPPLEMENTARY MATERIAL}

The Supplementary Material for this article can be found online at: http://journal.frontiersin.org/article/10.3389/fpsyg. 2017.00325/full\#supplementary-material

Engelkamp, J., and Zimmer, H. D. (1989). Memory for action events: a new field of research. Psychol. Res. 51, 153-157. doi: 10.1007/BF00309142

Feyereisen, P. (2009). Enactment effects and integration processes in younger and older adults' memory for actions. Memory 17, 374-385. doi: 10.1080/ 09658210902731851

Foley, M. A., and Johnson, M. K. (1985). Confusions between memories for performed and imagined actions: a developmental comparison. Child Dev. 56, 1145-1155. doi: 10.2307/1130229

Freides, D., and Messina, C. A. (1986). Memory improvement via motor encoding in learning disabled children. J. Learn. Disabil. 19, 113-115. doi: 10.1177/ 002221948601900209

Frick-Horbury, D. (2002). The effects of hand gestures on verbal recall as a function of high- and low-verbal-skill levels. J. Gen. Psychol. 129, 137-147. doi: 10.1080/ 00221300209603134

Grainger, C., Williams, D. M., and Lind, S. E. (2014). Online action monitoring and memory for self-performed actions in autism spectrum disorder. J. Autism Dev. Disord. 44, 1193-1206. doi: 10.1007/s10803-013-1987-4

Gray, S., Green, S., Alt, M., Hogan, T., Kuo, T., Brinkley, S., et al. (2017). The structure of working memory in young children and its relation to intelligence. J. Mem. Lang. 92, 183-201. doi: 10.1016/j.jml.2016.06.004

Hainselin, M. (2015). Memory for action: from cognitive models to clinical evaluation. Socioaffect. Neurosci. Psychol. 5:26091. doi: 10.3402/snp.v5.26091

Hainselin, M., Quinette, P., Juskenaite, A., Desgranges, B., Martinaud, O., de La Sayette, V., et al. (2014). Just do it! How performing an action enhances remembering in transient global amnesia. Cortex 50, 192-199. doi: 10.1016/j. cortex.2013.10.007

James, K. H., and Swain, S. N. (2011). Only self-generated actions create sensorimotor systems in the developing brain. Dev. Sci. 14, 673-678. doi: 10.1111/j. 1467-7687.2010.01011.x

JASP Team. (2016). JASP (Version 0.7.5.5)[Computer Software]. Available at: https: //jasp-stats.org/faq/

Kontra, C., Lyons, D. J., Fischer, S. M., and Beilock, S. L. (2015). Physical experience enhances science learning. Psychol. Sci. 26, 737-749. doi: 10.1177/ 0956797615569355

Korkman, M., Sally, L. K., and Kirk, U. (2012). Bilan Neuropsychologique de l'enfant [NEPSY-II]. Paris: Les Editions de Psychologie Appliquée.

Kormi-Nouri, R., and Nilsson, L.-G. (2001). “The motor component is not crucial!,” in Memory for Action: A Distinct form of Episodic Memory?, eds H. D. Zimmer, R. L. Cohen, M. J. Guynn, J. Engelkamp, R. Kormi-Nouri, and M. A. Foley (New York, NY: Oxford University Press), 97-111. 
Kormi-Nouri, R., Shojaei, R.-S. S., Moniri, S., Gholami, A.-R. R., Moradi, A.R. R., Akbari-Zardkhaneh, S., et al. (2008). The effect of childhood bilingualism on episodic and semantic memory tasks. Scand. J. Psychol. 49, 93-109. doi: 10.1111/j.1467-9450.2008.00633.x

Lee, J. K., Wendelken, C., Bunge, S. A., and Ghetti, S. (2016). A time and place for everything: developmental differences in the building blocks of episodic memory. Child Dev. 87, 194-210. doi: 10.1111/cdev. 12447

Lind, S. E., and Bowler, D. M. (2009). Recognition memory, self-other source memory, and theory-of-mind in children with autism spectrum disorder. J. Autism Dev. Disord. 39, 1231-1239. doi: 10.1007/s10803-0090735-2

Lorsbach, T. C., and Reimer, J. F. (2005). Feature binding in children and young adults. J. Genet. Psychol. 166, 313-328. doi: 10.3200/GNTP.166.3. 313-328

Macedonia, M., and Klimesch, W. (2014). Long-term effects of gestures on memory for foreign language words trained in the classroom. Mind Brain Educ. 8, 74-88. doi: $10.1111 / \mathrm{mbe} .12047$

Madan, C. R., and Singhal, A. (2012). Using actions to enhance memory: effects of enactment, gestures, and exercise on human memory. Front. Psychol. 3:507. doi: $10.3389 /$ fpsyg.2012.00507

Mangels, J. A., and Heinberg, A. (2006). Improved episodic integration through enactment: implications for aging. J. Gen. Psychol. 133, 37-65. doi: 10.3200/ GENP.133.1.37-65

Mavilidi, M.-F., Okely, A. D., Chandler, P., Cliff, D. P., and Paas, F. (2015). Effects of integrated physical exercises and gestures on preschool children's foreign language vocabulary learning. Educ. Psychol. Rev. 27, 413-426. doi: 10.1007/ s10648-015-9337-z

Novack, M. A., Congdon, E. L., Hemani-Lopez, N., and Goldin-Meadow, S. (2014). From action to abstraction: using the hands to learn math. Psychol. Sci. 25, 903-910. doi: 10.1177/0956797613518351

Picard, L., Abram, M., Orriols, E., and Piolino, P. (2017). A new strategy for the neuropsychological assessment of multifaceted episodic memory function in daily life: the use of virtual reality from children to adults. Int. J. Behav. Dev. 41, 211-219. doi: 10.1177/016502541 5616198

Picard, L., Cousin, S., Guillery-Girard, B., Eustache, F., and Piolino, P. (2012). How do the different components of episodic memory develop? Role of executive functions and short-term feature-binding abilities. Child Dev. 83, 1037-1050. doi: 10.1111/j.1467-8624.2012. 01736.x

Picard, L., Eustache, F., and Piolino, P. (2009). De la mémoire épisodique à la mémoire autobiographique: approche développementale. L'Année Psychol. 109, 197-236. doi: 10.4074/S0003503309002012
Prabhakaran, V., Narayanan, K., Zhao, Z., and Gabrieli, J. D. E. (2000). Integration of diverse information in working memory within the frontal lobe. Nat. Neurosci. 3, 85-90. doi: 10.1038/71156

Rajan, V., Cuevas, K., and Bell, M. A. (2014). The contribution of executive function to source memory development in early childhood. J. Cogn. Dev. 15, 304-324. doi: 10.1080/15248372.2013.763809

Ratner, H. H., and Hill, L. (1991). The development of children's action memory: when do actions speak louder than words? Psychol. Res. 53, 195-202. doi: 10.1007/BF00941387

Rhodes, S. M., Murphy, D., and Hancock, P. J. B. (2011). Developmental changes in the engagement of episodic retrieval processes and their relationship with working memory during the period of middle childhood. Br. J. Dev. Psychol. 29, 865-882. doi: 10.1111/j.2044-835X.2010.02014.x

Rossion, B., and Pourtois, G. (2004). Revisiting snodgrass and vanderwart's object pictorial set: the role of surface detail in basic-level object recognition. Perception 33, 217-236. doi: 10.1068/p5117

Rubin, K. H., Fein, G. G., and Vanderberg, B. (1983). “Play," in Handbook of Child Psychology, Socialization, Personality, and Social Developmen, Vol. 4, ed. P. H. Mussen (New York, NY: John Wiley \& Sons), 639-774.

Saltz, E., and Dixon, D. (1982). Let's pretend: the role of motoric imagery in memory for sentences and words. J. Exp. Child Psychol. 34, 77-92. doi: 10.1016/ 0022-0965(82)90032-7

Toumpaniari, K., Loyens, S., Mavilidi, M.-F., and Paas, F. (2015). Preschool children's foreign language vocabulary learning by embodying words through physical activity and gesturing. Educ. Psychol. Rev. 27, 445-456. doi: 10.1007/ s10648-015-9316-4

Wechsler, D. (2005). Echelle d'Intelligence de Wechsler Pour Enfants: WISC-IV. Paris: ECPA, les Editions du Centre de Psychologie Appliquée: Pearson, Cop.

Yang, T., Allen, R. J., Holmes, J., and Chan, R. C. K. (2017). Impaired memory for instructions in children with attention-deficit hyperactivity disorder is improved by action at presentation and recall. Front. Psychol. 8:39. doi: 10.3389/ fpsyg.2017.00039

Conflict of Interest Statement: The authors declare that the research was conducted in the absence of any commercial or financial relationships that could be construed as a potential conflict of interest.

Copyright (C) 2017 Hainselin, Picard, Manolli, Vankerkore-Candas and Bourdin. This is an open-access article distributed under the terms of the Creative Commons Attribution License (CC BY). The use, distribution or reproduction in other forums is permitted, provided the original author(s) or licensor are credited and that the original publication in this journal is cited, in accordance with accepted academic practice. No use, distribution or reproduction is permitted which does not comply with these terms. 


\title{
Commentary: Mobile and Interactive Media Use by Young Children: The Good, the Bad, and the Unknown
}

\author{
Cedric Galetzka* \\ Division of Cognitive Sciences, Psychology Department, University of Potsdam, Potsdam, Germany
}

Keywords: embodied cognition, children's development, touchscreen devices, learning, smartphones

\section{A commentary on}

Mobile and Interactive Media Use by Young Children: The Good, the Bad, and the Unknown by Radesky, J. S., Schumacher, J., and Zuckerman, B. (2015). Pediatrics 135, 1-3. doi: 10.1542/peds. 2014-2251

There seems to be a broad consensus among today's parents that early exposure to digital media is less enriching than real-life experiences (Wooldridge, 2016). While this concern may rightly apply to traditional media such as television, new interactive devices (e.g., smartphones and tablets), on the contrary, are often marketed as supplemental learning tools for children (Kirkorian et al.,

\section{OPEN ACCESS}

Edited by:

Anna M. Borghi,

Sapienza University of Rome, Italy

Reviewed by:

Loïc P. Heurley,

Paris West University Nanterre La

Défense, France

*Correspondence:

Cedric Galetzka

galetzka@uni-potsdam.de

Specialty section:

This article was submitted to

Cognition,

a section of the journal

Frontiers in Psychology

Received: 13 January 2017 Accepted: 13 March 2017 Published: 28 March 2017

Citation:

Galetzka C (2017) Commentary: Mobile and Interactive Media Use by Young Children: The Good, the Bad, and the Unknown.

Front. Psychol. 8:461. doi: 10.3389/fpsyg.2017.00461
2009; Christakis, 2014; Apple, 2016). However, Radesky et al. (2015) recently pointed out that research on the impact of interactive devices on children's cognition cannot keep up with the pace of technological advances. The most recent guidelines on recommended screen time were updated before the first tablets even made their way onto the market (Christakis, 2014). Supplementing Radesky et al. (2015), this commentary aims to clarify the influence of modern touchscreen devices on children's cognitive development from the perspective of embodied cognition.

Embodied cognition highlights that the development of cognitive processes crucially depends on active interactions between one's body and the environment (Barsalou, 1999; Thelen et al., 2001). These sensorimotor interactions are thought to form the basis for many high-level processes such as object recognition and decision-making (Smith, 2005; Rivière and David, 2013). Importantly, children in early developmental stages seem to build up fewer associations from interactions when merely observing an action being executed instead of performing it (Smith, 2005). Modern interactive devices address this concern in that they allow for active bodily interactions via touchscreens (Black et al., 2012; Christakis, 2014).

Radesky et al. (2015) note that research has been sparse on whether children can actually benefit from touchscreen use. However, recent studies indicate that children can transfer acquired knowledge from touchscreen interfaces to physical objects. Wang et al. (2016) compared the effectiveness of teaching children clock reading using an iPad touchscreen app, a toy clock, or a paper drawing. The researchers found that children learned equally well from interactive media and traditional toys, with both conditions outperforming the paper drawing group. Importantly, children were able to transfer learned skills from touchscreens to physical objects. The same has been found in a study teaching children how to solve the Tower of Hanoi problem (Huber et al., 2016). In sum, the typical transfer deficit observed with traditional media seems to be absent when children are actively engaged with devices via touchscreens (Strasburger, 2015; Huber et al., 2016).

However, going beyond Radesky et al. (2015), simple swiping and tapping motions on touchscreens seem impoverished compared to the complex hand movements that facilitate 
exploration of objects (Lederman and Klatzky, 1987; Spitzer, 2013). Confirming this suspicion, Crescenzi et al. (2014) compared children's performance on a drawing task in an iPad vs. paper condition. The researchers point out that touchscreens limit the amount of fingers available for object manipulation. Moreover, qualitative aspects of touch are reduced and important haptic information, such as surface texture, are completely absent. However, the authors note that iPads allow for new types of touch, including more complex touch sequences. Interestingly, a recent study found that touchscreen use in early childhood is correlated with fine motor control (Bedford et al., 2016). Nevertheless, the authors argue that negative effects on more complex motor processes might only become apparent in later stages of development. Therefore, developers of children's applications need to take principles of embodiment, such as allowing for more complex bodily interactions, into account in order to guarantee for a healthy cognitive development (Antle, 2009).

Supporting Radesky et al. (2015), while children are engaged with interactive media, they miss out on other potentially more fruitful activities that foster an understanding around them. However, the limited types of touch of today's smartphones could provide certain beneficial learning effects by replacing traditional forms of media, such as television (Christakis, 2014). Kirkorian et al. (2016) compared the performance of children aged between 24 and 36 months on a word learning task. The children were either instructed to watch a video from a touchscreen or use touch-based gestures during video presentation. The authors observed that especially younger children benefited from contingent touchscreen interactions that accompanied taskrelevant information. Interestingly, this condition appeared to be counterproductive for the oldest children who participated in the experiment. In a follow-up study, Choi and Kirkorian (2016) argued that contingent touch-based interactions mainly facilitate learning in younger children by supporting selective attention mechanism. Future research needs to specify under which conditions contingent touchscreen responses supplement learning.

Moreover, Eisen and Lillard (2016) observed that children consistently prefer real-world objects, such as books, for learning

\section{REFERENCES}

Antle, A. N. (2009). Embodied child computer interaction: why embodiment matters. Interactions 16, 27-30. doi: 10.1145/1487632.1 487639

Apple (2016). Apps for Education. Available online at: http://www.apple.com/ education/products/\#learning-with-ipad (Accessed January 10, 2017).

Barsalou, L. W. (1999). Perceptions of perceptual symbols. Behav. Brain Sci. 22, 637-660.

Bedford, R., de Urabain, I. R. S., Cheung, C. H., Karmiloff-Smith, A., and Smith, T. J. (2016). Toddlers' fine motor milestone achievement is associated with early touchscreen scrolling. Front. Psychol. 7:1108. doi: 10.3389/fpsyg.2016.01108

Black, J. B., Segal, A., Vitale, J., and Fadjo, C. (2012). "Embodied cognition and learning environment design" in Theoretical Foundations of Learning Environments, eds D. Jonassen and S. Land (New York, NY: Routledge), $198-223$. over touchscreen devices. Importantly, children seem to grasp the interactional nature of touchscreens compared to traditional media but fail to conceive them as useful learning tools. The authors reasoned that children discount the learning value of interactive devices due to the circumstances they encounter them. Correspondingly, Radesky et al. (2014) already pointed out that parents mostly let their children use interactive media during routine tasks. Children would benefit from a more systematic approach to learning from touchscreens that takes these aspects into account. Recently, Kucirkova (2016) proposed a framework to bring developers, practitioners, and researchers together to design empirically based applications.

To sum up, the extent to which the advantages of reallife learning might be substitutable by touchscreen devices seems to be heavily context-dependent. Even in the absence of joint engagement, which was shown to greatly benefit learning according to Radesky et al. (2015), interactive media could represent useful supplementary learning tools in educational contexts (Kwok et al., 2016). In that sense, other fruitful approaches could also be to compare the effectiveness of touchscreen devices with other embodied learning approaches such as using active role-play to facilitate reading comprehension (Glenberg et al., 2004; Black et al., 2012). As with any other newly popularized technology, the true potential of smartphones is likely to be discovered along the way (Lovato and Waxman, 2016). Ultimately, insights from embodied cognition contribute to understanding how the touch in touchscreens supports the process of learning.

\section{AUTHOR CONTRIBUTIONS}

The author confirms being the sole contributor of this work and approved it for publication.

\section{ACKNOWLEDGMENTS}

I would like to thank Martin H. Fischer and Alex Miklashevsky for helpful critique and insightful comments.
Choi, K., and Kirkorian, H. L. (2016). Touch or watch to learn? Toddlers' object retrieval using contingent and noncontingent video. Psychol. Sci. 27, 726-736. doi: 10.1177/0956797616636110

Christakis, D. A. (2014). Interactive media use at younger than the age of 2 years: time to rethink the American Academy of Pediatrics guideline? JAMA Pediatr. 168, 399-400. doi: 10.1001/jamapediatrics.2013.5081

Crescenzi, L., Jewitt, C., and Price, S. (2014). The role of touch in preschool children's learning using iPad versus paper interaction. Aust. J. Lang. Literacy $37,86$.

Eisen, S., and Lillard, A. S. (2016). Young children's thinking about touchscreens versus other media in the US. J. Children Media. 1-13. doi: 10.1080/17482798.2016.1254095

Glenberg, A. M., Gutierrez, T., Levin, J. R., Japuntich, S., and Kaschak, M. P. (2004). Activity and imagined activity can enhance young children's reading comprehension. J. Educ. Psychol. 96:424. doi: 10.1037/0022-0663. 96.3.424 
Huber, B., Tarasuik, J., Antoniou, M. N., Garrett, C., Bowe, S. J., Kaufman, J., et al. (2016). Young children's transfer of learning from a touchscreen device. Comput. Human Behav. 56, 56-64. doi: 10.1016/j.chb.2015. 11.010

Kirkorian, H. L., Choi, K., and Pempek, T. A. (2016). Toddlers' word learning from contingent and noncontingent video on touch screens. Child Dev. 87, 405-413. doi: $10.1111 /$ cdev.12508

Kirkorian, H. L., Pempek, T. A., Murphy, L. A., Schmidt, M. E., and Anderson, D. R. (2009). The impact of background television on parentchild interaction. Child Dev. 80, 1350-1359. doi: 10.1111/j.1467-8624.2009. 01337

Kucirkova, N. (2016). iRPD—A framework for guiding design-based research for iPad apps. Br. J. Educ. Technol. 48, 598-610. doi: 10.1111/bjet.12389

Kwok, K., Ghrear, S., Li, V., Haddock, T., Coleman, P., and Birch, S. A. (2016). Children can learn new facts equally well from interactive media versus face to face instruction. Front. Psychol. 7:1603. doi: 10.3389/fpsyg.20 16.01603

Lederman, S. J., and Klatzky, R. L. (1987). Hand movements: a window into haptic object recognition. Cogn. Psychol. 19, 342-368. doi: 10.1016/ 0010-0285(87)90008-9

Lovato, S. B., and Waxman, S. R. (2016). Young children learning from touch screens: taking a wider view. Front. Psychol. 7:1078. doi: 10.3389/fpsyg.2016. 01078

Radesky, J. S., Kistin, C. J., Zuckerman, B., Nitzberg, K., Gross, J., Kaplan-Sanoff, M., et al. (2014). Patterns of mobile device use by caregivers and children during meals in fast food restaurants. Pediatrics 133. doi: 10.1542/peds.2013-3703

Radesky, J. S., Schumacher, J., and Zuckerman, B. (2015). Mobile and interactive media use by young children: the good, the bad, and the unknown. Pediatrics 135, 1-3. doi: 10.1542/peds.2014-2251
Rivière, J., and David, E. (2013). Perceptual-motor constraints on decision making: the case of the manual search behavior for hidden objects in toddlers. J. Exp. Child Psychol. 115, 42-52. doi: 10.1016/j.ecp.2012.11.006

Smith, L. B. (2005). Action alters shape categories. Cogn. Sci. 29, 665-679. doi: 10.1207/s15516709cog0000_13

Spitzer, M. (2013). To swipe or not to swipe?-The question in present-day education. Trends Neurosci. Educ. 3, 95-99. doi: 10.1016/j.tine.2013.09.002

Strasburger, V. (2015). Should babies be watching and using screens? The answer is surprisingly complicated. Acta Paediatr. 104, 967-968. doi: 10.1111/apa.13105

Thelen, E., Schöner, G., Scheier, C., and Smith, L. B. (2001). The dynamics of embodiment: a field theory of infant perseverative reaching. Behav. Brain Sci. 24, 1-34. doi: 10.1017/S0140525X01003910

Wang, F., Xie, H., Wang, Y., Hao, Y., and An, J. (2016). Using touchscreen tablets to help young children learn to tell time. Front. Psychol. 7:1800. doi: 10.3389/fpsyg.2016.01800

Wooldridge, M. B. (2016). An Ecology of Technology: Infants, Toddlers, and Mobile Screen Devices, Doctoral dissertation, University of British Columbia. doi: $10.14288 / 1.0300168$

Conflict of Interest Statement: The author declares that the research was conducted in the absence of any commercial or financial relationships that could be construed as a potential conflict of interest.

Copyright $(\odot 2017$ Galetzka. This is an open-access article distributed under the terms of the Creative Commons Attribution License (CC BY). The use, distribution or reproduction in other forums is permitted, provided the original author (s) or licensor are credited and that the original publication in this journal is cited, in accordance with accepted academic practice. No use, distribution or reproduction is permitted which does not comply with these terms. 


\title{
Commentary: Down with Retirement: Implications of Embodied Cognition for Healthy Aging
}

\author{
Alex A. Miklashevsky ${ }^{1,2 *}$ and Martin H. Fischer ${ }^{2}$ \\ ${ }^{1}$ Laboratory for Cognitive Studies of Language, International Center for Human Development, Tomsk State University, Tomsk, \\ Russia, ${ }^{2}$ Division of Cognitive Sciences, Department of Psychology, University of Potsdam, Potsdam, Germany
}

Keywords: child development, embodied cognition, lifespan, mobile phone, tablet computers, digital technologies

\section{A commentary on}

Down with Retirement: Implications of Embodied Cognition for Healthy Aging

by Hommel, B., and Kibele, A. (2016). Front. Psychol. 7:1184. doi: 10.3389/fpsyg.2016.01184

\section{OPEN ACCESS}

Edited by:

Claudia Repetto,

Università Cattolica del Sacro Cuore,

Italy

Reviewed by:

Daniela Corbetta

University of Tennessee, Knoxville,

USA

Alberto Montebelli,

University of Skövde, Sweden

${ }^{*}$ Correspondence

Alex A. Miklashevsky armanster31@gmail.com

Specialty section:

This article was submitted to

Cognition,

a section of the journal

Frontiers in Psychology

Received: 19 January 2017 Accepted: 31 March 2017

Published: 21 April 2017

Citation:

Miklashevsky AA and Fischer MH

(2017) Commentary: Down with Retirement: Implications of Embodied

Cognition for Healthy Aging.

Front. Psychol. 8:599

doi: 10.3389/fpsyg.2017.00599
Hommel and Kibele (2016) proposed a clear model of understanding age-related cognitive effects in elderly people. They claim that, from an embodied cognition perspective "cognitive processes and functions should not just be taken as a given $\langle\ldots\rangle$, but as abilities that emerge from active exchange with one's physical and social environment" (p. 2). They model this exchange with the environment as a vicious spiral: elderly people tend to reduce interactions with their environment, which in turn reduces their cognitive abilities further.

This insight seems to be productive not only for discussing cognitive aging but also for any investigations of cognitive development from a lifespan perspective. Particularly, it is interesting for studying dramatically changed childhood development in the light of pervasive digitalization. Recent evidence (reviewed by Kucirkova, 2014; Radesky et al., 2015) indicates considerably increased use of smartphones and tablets by children and teenagers. While adults now acquire the skills of typing, working with touch-screens and communicating in social nets after prolonged engagement in and interaction with the physical world, children get engaged in parallel with (or even instead of) it. We argue that this time course difference has consequences for spatial, conceptual, numerical and linguistic development. In particular, cognitive asymmetries will emerge because different proportions of analog and digital sensory experiences will shape cognition differently, eventually creating entirely new cognitive mechanisms underlying seemingly similar mental activities in future adults.

Over $90 \%$ of American teens use the internet daily, sending and receiving on average 30 text messages a day (Lenhart, 2015). This trend of replacing oral with typed language ties linguistic representations to manual instead of orofacial activity. Learning of letters with vs. without typing them results in stronger vs. weaker brain activation in letter processing respectively (James, 2010). Similarly, Chinese children who type extensively using pinyin (a Romanization system for standard Chinese) have reduced Chinese character reading skills (Tan et al., 2013). Both observations show how embodied experiences transform conceptual representations.

Language itself develops other cognitive functions, such as concept formation (Vygotsky, 1986) and our ability to generate extensive representations during thinking. Smartphones and tablets seriously reduce hand motor activity and haptic exploration when compared to traditional object interactions (see also Spitzer, 2013). Such diminished spatial exploration will lead to shallower understanding of spatial relationships represented in language. As spatial schemas underlie much abstract thoughts (e.g., about time or valence; Lakoff and Johnson, 1980), changes in abstract thought are likely. Spatial relationships are culturally fixed through conceptual metaphors acquired by each generation anew. We predict that "digital children" will replace haptic with visual 
metaphors-they will no longer "grasp the idea" but hopefully still "see the point." More generally, as digitally mediated interactions increase, our motor repertoire tends to shrink (even if we play Kinect $^{\mathrm{TM}}$ or search Pokémons ${ }^{\mathrm{TM}}$ ) and we expect to see a drift from motor to visual simulation in language comprehension. For example, when comprehending "he gives you the pizza" we will rely more on a visualization of the implied scene (Stanfield and Zwaan, 2001; for review see Bergen, 2012) rather than engaging our motor system (Glenberg and Kaschak, 2002; for review, see Fischer and Zwaan, 2008).

Finger counting plays an important role in the development of arithmetical skills in children (Domahs et al., 2012). Replacing the sensory and motor activity of natural finger counting with device-supported learning of numbers destroys each number's unique sensory-motor profile, rendering them cognitively less accessible (Sixtus et al., 2017).

There are important differences between real communication and mere observation of communication (e.g., during video watching). Children hardly (verbally) interact with persons on a screen and rarely receive feedback from them. Roseberry et al. (2014) showed that children learn new words from real-life social interaction and communication (also on Skype, i.e., during an interactive process involving perception of the interlocutor) but not from video training alone. Finally, language is a social tool that allows people to achieve goals with the help of others (Borghi and Binkofski, 2014). In virtual scenarios children mostly interact nonverbally. This eliminates the pragmatic value of language, again making it less "woven into action" (Section 7 of Wittgenstein, 1953; as quoted in Pulvermüller, 2012, pp. 425-426).

\section{REFERENCES}

Bergen, B. K. (2012). Louder Than Words: The New Science of How the Mind Makes Meaning. New York, NY: Basic Books.

Borghi, A. M., and Binkofski, F. (2014). Words As social Tools: An Embodied View on Abstract Concepts. Berlin; New York, NY: Springer.

Domahs, F., Kaufmann, L., and Fischer, M. H. (2012). Handy Numbers: Finger Counting and Numerical Cognition. Frontiers Media SA.

Fischer, M. H., and Zwaan, R. A. (2008). Embodied language: a review of the role of the motor system in language comprehension. Q. J. Exp. Psychol. 61, 825-850. doi: 10.1080/17470210701623605

Glenberg, A. M., and Kaschak, M. P. (2002). Grounding language in action. Psychon. Bull. Rev. 9, 558-565. doi: 10.3758/BF03196313

Hommel, B., and Kibele, A. (2016). Down with Retirement: Implications of Embodied Cognition for Healthy Aging. Front. Psychol. 7:1184. doi: 10.3389/fpsyg.2016.01184

James, K. H. (2010). Sensori-motor experience leads to changes in visual processing in the developing brain. Dev. Sci. 13, 279-288. doi: 10.1111/j.14677687.2009.00883.x

Kucirkova, N. (2014). iPads in early education: separating assumptions and evidence. Front. Psychol. 5:715. doi: 10.3389/fpsyg.2014.00715

Lakoff, G., and Johnson, M. (1980). Metaphors We Live By. Chicago, IL: The University of Chicago Press.

Lenhart, A. (2015). Teen, Social Media and Technology Overview 2015. Washington, DC: Pew Research Center.

Pulvermüller, F. (2012). Meaning and the brain: the neurosemantics of referential, interactive, and combinatorial knowledge. J. Neurolinguist. 25, 423-459. doi: 10.1016/j.jneuroling.2011.03.004

Radesky, J. S., Schumacher, J., and Zuckerman, B. (2015). Mobile and interactive media use by young children: the good, the bad, and the unknown. Pediatrics 135, 1-3. doi: 10.1542/peds.2014-2251
Digital technologies became part of our everyday life and children interact with them extensively. We have developed the idea of Hommel and Kibele (2016) that one's cognitive abilities are bi-directionally related with active interactions within one's environment. Recent changes in this environment, such as digitalization, predict potential consequences of these changes for human cognition at different stages of ontogenesis. The same high-level cognitive activities can be performed by means of different underlying cognitive mechanisms in adults and children but due to different early sensorimotor and social experiences. Digitalization of early experiences may abolish or transpose cognitive signatures that hinge on analog experiences in early childhood, such as an extensive haptic repertoire and verbal cooperation. Just as older adults, children already avoid real social and physical interactions, thus creating a cognitive asymmetry that may make them spiral away from the world we wish them to own.

\section{AUTHOR CONTRIBUTIONS}

All authors listed, have made substantial, direct and intellectual contribution to the work, and approved it for publication.

\section{ACKNOWLEDGMENTS}

The authors thank Cedric Galetzka for the valuable discussion on this work. The work by AM was supported by the Ministry of Education and Science of the Russian Federation (the RF Government grant No. 14.Y26.3 1.0014).

Roseberry, S., Hirsh-Pasek, K., and Golinkoff, R. M. (2014). Skype me! Socially contingent interactions help toddlers learn language. Child Dev. 85, 956-970. doi: $10.1111 /$ cdev.12166

Sixtus, E., Fischer, M. H., and Lindemann, O. (2017). Finger posing primes number comprehension. Cogn. Process. doi: 10.1007/s10339-017-0804-y. [Epub ahead of print].

Spitzer, M. (2013). To swipe or not to swipe?-The question in presentday education. Trends Neurosci. Educ. 3, 95-99. doi: 10.1016/j.tine.2013. 09.002

Stanfield, R. A., and Zwaan, R. A. (2001). The effect of implied orientation derived from verbal context on picture recognition. Psychol. Sci. 12, 153-156. doi: 10.1111/1467-9280.00326

Tan, L. H., Xu, M., Chang, C. Q., and Siok, W. T. (2013). China's language input system in the digital age affects children's reading development. Proc. Natl. Acad. Sci. U.S.A. 110, 1119-1123. doi: 10.1073/pnas.12135 86110

Vygotsky, L. S. (1986). Thought and Language. Cambridge, MA; London, UK: The MIT Press.

Wittgenstein, L. (1953). Philosophical Investigations. Oxford: Blackwell Publishers.

Conflict of Interest Statement: The authors declare that the research was conducted in the absence of any commercial or financial relationships that could be construed as a potential conflict of interest.

Copyright (C) 2017 Miklashevsky and Fischer. This is an open-access article distributed under the terms of the Creative Commons Attribution License (CC $B Y)$. The use, distribution or reproduction in other forums is permitted, provided the original author(s) or licensor are credited and that the original publication in this journal is cited, in accordance with accepted academic practice. No use, distribution or reproduction is permitted which does not comply with these terms. 


\title{
Abstract Concepts and Aging: An Embodied and Grounded Perspective
}

\author{
Anna M. Borghi ${ }^{1,2 * t}$ and Annalisa Setti ${ }^{3,4}$ \\ ${ }^{1}$ Institute of Cognitive Sciences and Technologies, Italian National Research Council (CNR), Rome, Italy, ${ }^{2}$ Department of \\ Psychology, University of Bologna, Bologna, Italy, ${ }^{3}$ School of Applied Psychology, University College Cork, Cork, Ireland, \\ ${ }^{4}$ Trinity College Dublin, Dublin, Ireland
}

Keywords: abstract concepts, abstract words, words as tools, aging, elderly, embodied cognition, grounded cognition

OPEN ACCESS

Edited by:

Yann Coello,

University of Lille Nord de France,

France

Reviewed by:

Madeleine Ann Grealy, University of Strathclyde, UK

Tina lachini,

Università degli Studi della Campania

"Luigi Vanvitelli" Caserta, Italy

${ }^{*}$ Correspondence:

Anna M. Borghi

anna.borghi@gmail.com

${ }^{\dagger}$ Present Address:

Anna M. Borghi,

Department of Dynamic and Clinical

Psychology, Sapienza University of

Rome, Rome, Italy

Specialty section:

This article was submitted to

Cognition,

a section of the journal

Frontiers in Psychology

Received: 14 December 2016 Accepted: 08 March 2017

Published: 22 March 2017

Citation:

Borghi AM and Setti A (2017) Abstract

Concepts and Aging: An Embodied

and Grounded Perspective.

Front. Psychol. 8:430.

doi: 10.3389/fpsyg.2017.00430
How do we represent abstract concepts, as "justice" and "phantasy"? This issue has become hotly debated within embodied and grounded cognition views (for reviews: Pecher et al., 2011; Dove, 2016; Borghi et al., 2017). It is in fact unclear how such views can explain how we represent concepts that do not have single concrete referents and are rather detached from sensory experience (Barsalou, 2003; Binder, 2016).

In spite of the increasing interest for this issue, to date evidence on abstract concepts across the lifespan is limited. Assuming that the representation of abstract concepts changes from adulthood to older age, in this paper we discuss how a new embodied and grounded proposal, the Words As social Tools (WAT) view (Borghi and Binkofski, 2014), can explain how abstract concepts are represented by older individuals. More specifically we will advance hypotheses on abstract concepts in aging focusing on WAT, and reinterpret previous findings in light of it. We propose that WAT can account for existing findings and provide a suitable framework to test conceptual knowledge in older adults.

\section{WORDS AS SOCIAL TOOLS (WAT)}

According to WAT all concepts are grounded in perception, action and emotional systems; however, information conveyed through language in a social context is particularly crucial for abstract concepts while sensorimotor information is more crucial for concrete ones. Concrete concepts are typically acquired through sensorimotor interaction with the word referent. To acquire abstract concepts, instead, we benefit more of linguistic and social inputs, since their referents are not clearly defined objects/entities. The importance of language for abstract concepts is due to many reasons. First, labels can help, as a sort of glue, to keep together varied and heterogeneous experiences. Second, explanations offered by others are crucial in order to understand word meaning. Accordingly, abstract concepts are acquired later than concrete ones, they benefit more of linguistically conveyed information and are more influenced by the social context. Consistently, Age of Acquisition (AoA) and modality of acquisition, while being distinct properties of words, are both negatively correlated with concreteness and positively correlated with abstractness (Della Rosa et al., 2010). Third, inner language may help re-explaining to ourselves the meaning of abstract words, typically more complex than concrete ones (Borghi and Zarcone, 2016).

\section{PREDICTION}

In order to advance predictions on abstract concepts in older adults derived from WAT, we refer to recent studies on healthy older, with no claim to be exhaustive.

Even if specific evidence on abstract concepts in healthy aging is lacking, there is evidence that linguistic abilities overall are preserved in elderly, although behavioral performance may be due to activation of different neural networks (Laumann Long and Shaw, 2000; Whiting et al., 2011; Shafto et al., 2012). 
Based on the effect of the word AoA and of the word frequency, which are generally correlated (highly frequency words are typically early acquired words), one could predict higher difficulties in the mastering/use of abstract words in older adults, since these words are typically acquired later. When degradation of the semantic system occurs, frequent words and more imageable words are typically better preserved than less frequent words and less imageable words (Jefferies et al., 2009). However, it is to note that a reverse of the concreteness effect, i.e., the advantage in processing and recall of concrete over abstract concepts, has also been found (see Harciarek and Kertesz, 2011 for a review).

In absence of specific pathologies, since vocabulary is preserved with aging, abstract concepts' decline should be less marked than decline of concrete ones, since, compared to concrete concepts, they were mainly acquired through linguistically conveyed information and their representation relies more heavily on language. Hence, the concreteness effect should be reduced with aging (due to less steep decline of abstract concepts).

\section{EVIDENCE}

Evidence on concreteness effect in healthy aging is scarce.

A reduction of the concreteness effect with normal aging was found by Peters and Daum (2008) who investigated effects of aging on vivid remembering during recognition (recollection). They tested three groups of participants (mean ages 21, 42, and 61 years) in a deep encoding task: participants had to rate words for pleasantness. After an interval participants were required to respond "remember" whether they were certain that they had seen the word, and recollected associations, emotions etc. with it; and to respond "know" when they recognized the word but did not associate any further information with it. Recollection of concrete words declined progressively with age, while recollection of abstract words showed a decline only from the young to the middle age group, with no further decline between middle and older age, revealing the predicted reduction of the concreteness effect in the older group.

Shafto et al. (2012) with a lexical decision paradigm, instead, provided evidence for increased sensitivity to imageability, which is correlated with concreteness, in older adults more than in younger adults. Shafto et al. (2012) manipulated words imageability and phonology (measured by cohort competition) and recorded fMRI. They found preserved lexical abilities (capability to distinguish words from non-words) in older adults, and better performance with high imaginable words in both younger and older adults. Older participants manifested a higher sensitivity to imageability and a lower sensitivity to cohort competition, consistently with evidence showing a reduction of the phonological abilities after the mid 70's.

In a recent fMRI study, Roxbury et al. (2016) also conducted a lexical decision task, in which younger outperformed older adults (mean age 71) in response times and accuracy, and the concreteness effect was preserved with aging. However, the neural underpinnings differed for young and old adults, consistently with the hypothesis that compensatory mechanisms operate in elderly. Interestingly, activity of the left Inferior Frontal Gyrus (IFG), typically associated with phonological processes, increased for abstract words compared with concrete words, only for older adults. Results also showed an increased activity for abstract words in older compared with younger adults in the Left Fusiform Gyrus, an area associated to the retrieval of visual attributes (Binder et al., 2009) (differences were also found in the Angular Gyrus). It is to note that abstract concepts are characterized by visual attributes, while concrete concepts are characterized by different sensory modalities, particularly touch and somatosensation (Connell and Lynott, 2012).

One possibility is that the discrepancies are due to differences in the tasks: the concreteness effect seems to be reduced with age in recollection, but preserved in lexical decision. The difference might be due to the different level of processing the two tasks imply (superficial vs. deep), or to the emphasis placed on speed of processing, which is higher in the lexical decision task. While older adults have well preserved vocabulary, when they need to activate it fast they are disadvantaged due to their general cognitive slowness. However, they may be able to compensate better for words more defined in linguistic form than in perceptual form when the task is not based on speed.

Indeed, during the most superficial auditory lexical decision task (Roxbury et al., 2016) the concreteness effect was preserved at the behavioral level, but the neural underpinnings of the phenomenon differed between the older and the younger group. Specifically, the recruitment of left IFG in older adults for abstract words and pseudo words indicates that some kind of phonological processing takes place. This, according to WAT, could be associated with covert verbalization of the meaning. It is worth noting that phonological processing declines with aging (Burke and Shafto, 2004), however, older adults continue to rely on inner speech (Alderson-Day and Fernyhough, 2015).

As predicted by WAT, the activation of left IFG could reflect the activation of compensatory strategies, which involve linguistic conveyed and linguistic form information, both crucial for abstract concepts representation. The activation of the left IFG would thus play a compensatory role in particular for later acquired abstract concepts. Notice that, according to WAT, abstract concepts do not activate only linguistic information but are also grounded in perception, in particular in the visual modality. In spite of the decline of vision with age, recent evidence has shown that the importance of visual features compared to other sensory modalities is higher for older then for younger adults (Maguinness et al., 2013; Costello and Bloesch, 2017). This activation of the visual modality could be associated with the activation of the Left Fusiform Gyrus (Roxbury et al., 2016).

In sum: according to WAT abstract concepts are grounded in sensorimotor and in linguistic/social experience. Consistently, the loss of abstract words, which are typically later acquired and less frequent than concrete ones, would be compensated reactivating phonological/linguistic and visual features. Based on WAT we propose that abstract and concrete concepts should have a different trajectory of decline. When, with older age, the decline starts to occur, concrete concepts, relying more on sensorimotor 
and episodic knowledge, should decline faster, while abstract concepts should decline slower since they rely also on linguistic knowledge. According to WAT, abstract concepts are grounded in sensorimotor experience similarly to concrete ones, but are more grounded in linguistic experience than concrete concepts. Therefore, we predict that the different trajectory of decline of concrete and abstract concepts is driven by a different weight of the underlying conceptual components (linguistic, visual, motor etc.). Specifically, we predict a less marked decline of abstract compared to concrete concepts, even if the former are typically acquired later. If, instead, the decline of both kinds of concepts was determined mainly by AoA and frequency effects, abstract concepts should decline faster. This inference, however, needs to be tested empirically.

\section{CONCLUSION}

Little is known on how our representation of abstract concepts changes with aging. Some tentative evidence shows that abstract concepts deteriorate less than concrete concepts with age, likely because they rely more on language, and vocabulary and semantic knowledge have been shown to be preserved in older. If they deteriorate, compensatory strategies are activated, recruiting neural networks dedicated to phonological processing and, possibly, to visual processing. This evidence is in line with the WAT proposal, according to which abstract concepts activate both perceptual and linguistic information and it is not in line with the hypothesis that AoA and frequency determine the trajectory of decline of abstract

\section{REFERENCES}

Alderson-Day, B., and Fernyhough, C. (2015). Inner speech: development, cognitive functions, phenomenology, and neurobiology. Psych. Bull. 141, 931-965. doi: 10.1037/bul0000021

Barsalou, L. W. (2003). Abstraction in perceptual symbol systems. Philos. Transact. R. Soc. B Biolog. Sci. 358, 1177-1187. doi: 10.1098/rstb.2003.1319

Binder, J. R. (2016). In defense of abstract conceptual representations. Psychon. Bull. Rev. 23, 1096-1108. doi: 10.3758/s13423-015-0909-1

Binder, J. R., Desai, R. H., Graves, W. W., and Conant, L. L. (2009). Where is the semantic system? A critical review and meta-analysis of 120 functional neuroimaging studies. Cereb. Cortex 19, 2767-2796. doi: 10.1093/cercor/bhp055

Borghi, A. M., and Binkofski, F. (2014). Words as Social Tools: An Embodied View on Abstract Words. New York, NY: Springer.

Borghi, A. M., Binkofski, F., Castelfranchi, C., Cimatti, F., Scorolli, C., and Tummolini, L. (2017). The challenge of abstract words. Psychol. Bull. 143, 263-292. doi: 10.1037/bul0000089

Borghi, A. M., and Zarcone, E. (2016). Grounding abstractness: abstract concepts and the activation of the mouth. Front. Psychol. 7:1498. doi: 10.3389/fpsyg.2016.01498

Burke, D. M., and Shafto, M. A. (2004). Aging and language production. Curr. Dir. Psychol. Sci. 13, 21-24. doi: 10.1111/j.0963-7214.2004.01301006.x

Connell, L., and Lynott, D. (2012). Strength of perceptual experience predicts word processing performance better than concreteness or imageability. Cognition 125, 452-465. doi: 10.1016/j.cognition.2012.07.010

Costello, M. C., and Bloesch, E. K. (2017). Are older adults less embodied? A review of age effects through the lens of embodied cognition. Front. Psychol. 8:267. doi: 10.3389/fpsyg.2017.00267

Della Rosa, P. A., Catricalà, E., Vigliocco, G., and Cappa, S. F. (2010). Beyond the abstract-concrete dichotomy: mode of acquisition, concreteness, imageability, concepts. Further empirical research is however necessary to obtain a clearer framework of abstract words mastering in old age.

\section{AVENUES FOR FUTURE RESEARCH}

Based on WAT, we expect that with non-pathological aging, the definitions of abstract concepts will maintain more richness than concrete ones, with reference to social contexts in which they are acquired and used. Considering the different results on the concreteness effect obtained with different tasks, further studies are needed to determine whether and to what extent the time constraints of speed-based tasks will allow for full access to resources such as inner speech characterizing more abstract than concrete concepts. We also propose that an electrophysiological signature of the relative preservation of abstract concepts could be found with EEG, possibly indicated by a modulation of the N400 component of the ERP related to the concreteness effect (West and Holcomb, 2000), and sentence comprehension and predictability (De Long et al., 2012). Importantly, fMRI studies utilizing speeded and non-speeded tasks with concrete and abstract concepts will allow to elucidate whether the hypothesized compensatory mechanisms in the activation of abstract concepts take place.

\section{AUTHOR CONTRIBUTIONS}

Both authors contributed to the conception and writing of this work.

familiarity, age of acquisition, context availability, and abstractness norms for a set of 417 Italian words. Behav. Res. Methods 42, 1042-1048. doi: 10.3758/BRM.42.4.1042

De Long, K. A., Groppe, D. M., Urbach, T. P., and Kutas, M. (2012). Thinking ahead or not? Natural aging and anticipation during reading Brain Lang. 121, 226-239. doi: 10.1016/j.bandl.2012.02.006

Dove, G. (2016). Three symbol ungrounding problems: abstract concepts and the future of embodied cognition. Psychon. Bull. Rev. 23, 1109-1121. doi: 10.3758/s13423-015-0825-4

Harciarek, M., and Kertesz, A. (2011). Primary progressive aphasias and their contribution to the contemporary knowledge about the brainlanguage relationship. Neuropsychol. Rev. 21, 271. doi: 10.1007/S11065-0119175-9

Jefferies, E., Patterson, K., Jones, R. W., and Lambon Ralph, M. A. (2009). Comprehension of concrete and abstract words in semantic dementia. Neuropsychology 23:492. doi: 10.1037/a0015452

Laumann Long, L., and Shaw, R. J. (2000). Adult age differences in vocabulary acquisition. Educ. Geront. 26, 651-664. doi: 10.1080/03601270050200644

Maguinness, C., Setti, A., Roudaia, E., and Kenny, R. A. (2013). Does that look heavy to you? Front. Hum. Neurosci. 7:795. doi: 10.3389/fnhum.2013. 00795

Pecher, D., Boot, I., and Van Dantzig, S. (2011). Abstract Concepts: Sensory-Motor Grounding, Metaphors, and Beyond. Available online at: http://hdl.handle.net/1765/30616

Peters, J., and Daum, I. (2008). Differential effects of normal aging on recollection of concrete and abstract words. Neuropsychology 22, 255-261. doi: 10.1037/0894-4105.22.2.255

Roxbury, T., McMahon, K., Coulthard, A., and Copland, D. A. (2016). An fMRI study of concreteness effects during spoken word recognition in aging. Preservation or Attenuation? Front. Aging Neurosci. 7:240. doi: 10.3389/fnagi.2015.00240 
Shafto, M., Randall, B., Stamatakis, E. A., Wright, P., and Tyler, L. K. (2012). Age-related neural reorganization during spoken word recognition: the interaction of form and meaning. J. Cognit. Neurosci. 24, 1434-1446. doi: 10.1162/jocn_a_00218

West, C. W., and Holcomb, P. J. (2000). Imaginal, semantic and surface-level processing of concrete and abstract words: an electrophysiological investigation. J. Cogn. Neurosci. 12, 1024-1037. doi: $10.1162 / 08989290051137558$

Whiting, E., Chenery, H. J., and Copland, D. A. (2011). Effect of aging on learning new names and descriptions for objects. Aging Neuropsychol. Cognit. 18, 594-619. doi: 10.1080/13825585.2011.598912
Conflict of Interest Statement: The authors declare that the research was conducted in the absence of any commercial or financial relationships that could be construed as a potential conflict of interest.

Copyright (C) 2017 Borghi and Setti. This is an open-access article distributed under the terms of the Creative Commons Attribution License (CC BY). The use, distribution or reproduction in other forums is permitted, provided the original author(s) or licensor are credited and that the original publication in this journal is cited, in accordance with accepted academic practice. No use, distribution or reproduction is permitted which does not comply with these terms. 


\section{OPEN ACCESS}

Edited by:

Anna M. Borghi,

Sapienza Università di Roma, Italy

Reviewed by:

Arjan Blokland

Maastricht University, Netherlands

Marco Sperduti,

Université Paris Descartes, France

${ }^{*}$ Correspondence:

Guillaume T. Vallet guillaume.vallet@uca.fr

Specialty section:

This article was submitted to

Cognition,

a section of the journal

Frontiers in Psychology

Received: 30 October 2016

Accepted: 18 August 2017

Published: 13 September 2017

Citation:

Vallet GT, Hudon C, Bier N, Macoir J,

Versace $R$ and Simard M (2017) A

SEMantic and EPisodic Memory Test

(SEMEP) Developed within the

Embodied Cognition Framework:

Application to Normal Aging,

Alzheimer's Disease and Semantic

Dementia. Front. Psychol. 8:1493.

doi: 10.3389/fpsyg.2017.01493

\section{A SEMantic and EPisodic Memory Test (SEMEP) Developed within the Embodied Cognition Framework: Application to Normal Aging, Alzheimer's Disease and Semantic Dementia}

\author{
Guillaume T. Vallet ${ }^{1,2,3 *}$, Carol Hudon ${ }^{4,5}$, Nathalie Bier ${ }^{1,6}$, Joël Macoir $^{5,6}$, Rémy Versace $^{7}$ \\ and Martine Simard ${ }^{4,5}$
}

${ }^{1}$ Centre de Recherche de l'IUGM, Université de Montréal, Montreal, QC, Canada, ${ }^{2}$ Département de Psychologie, Université de Montréal, Montreal, QC, Canada, ${ }^{3}$ Laboratoire de Psychologie Sociale et Cognitive, Centre National de la Recherche Scientifique, Université Clermont Auvergne, Clermont-Ferrand, France, ${ }^{4}$ Département de Psychologie, Université Laval, Quebec, QC, Canada, ${ }^{5}$ Centre de Recherche de l'Institut Universitaire en Santé Mentale de Québec, Quebec, QC, Canada, ${ }^{6}$ Département de Réadaptation, Université Laval, Quebec, QC, Canada, ${ }^{7}$ Laboratoire EMC, Université Lyon 2, Lyon, France

Embodiment has highlighted the importance of sensory-motor components in cognition. Perception and memory are thus very tightly bound together, and episodic and semantic memories should rely on the same grounded memory traces. Reduced perception should then directly reduce the ability to encode and retrieve an episodic memory, as in normal aging. Multimodal integration deficits, as in Alzheimer's disease, should lead to more severe episodic memory impairment. The present study introduces a new memory test developed to take into account these assumptions. The SEMEP (SEMantic-Episodic) memory test proposes to assess conjointly semantic and episodic knowledge across multiple tasks: semantic matching, naming, free recall, and recognition. The performance of young adults is compared to healthy elderly adults (HE), patients with Alzheimer's disease (AD), and patients with semantic dementia (SD). The results show specific patterns of performance between the groups. HE commit memory errors only for presented but not to be remembered items. AD patients present the worst episodic memory performance associated with intrusion errors (recall or recognition of items never presented). They were the only group to not benefit from a visual isolation (addition of a yellow background), a method known to increase the distinctiveness of the memory traces. Finally, SD patients suffer from the most severe semantic impairment. To conclude, confusion errors are common across all the elderly groups, whereas AD was the only group to exhibit regular intrusion errors and SD patients to show severe semantic impairment.

Keywords: long-term memory, embodied cognition, aging, Alzheimer's disease, semantic dementia 


\section{INTRODUCTION}

Embodiment has revolutionized how cognition is conceived (Glenberg et al., 2013) to highlight the role of sensory-motor components in cognitive processes (Vallet et al., 2016a). Applied to memory, it was shown repeatedly that semantic knowledge (i.e., knowledge about the world) might also be grounded in sensory-motor features (e.g., Vallet et al., 2010; Casasanto, 2011; Borghi, 2015), as episodic memory (i.e., personal and contextual memories, see Tulving, 1995). Semantic and episodic knowledge may thus share common memory traces, as stated by some memory models (e.g., Minverva II, Hintzman, 1990; Act-In, Versace et al., 2014). The present study proposes a memory test developed to take into account embodiment statements in young adults (YA), healthy elderly adults (HE), patients with Alzheimer's disease $(\mathrm{AD})$ and patients with semantic dementia (SD). These populations exhibit variable levels of perceptual, episodic and semantic memory impairments that allow a differential approach assessing some of the core assumptions of embodiment.

Among the different kinds of memory models, multiple trace models (e.g., Hintzman, 1990) and embodied memory models (e.g., Versace et al., 2014) defines memory as an accumulation of episodic memory traces. All the traces are episodic in nature as all the characteristics of the ongoing event (sensory-motor, emotional, context...) are encoded. Therefore, the distinction between semantic and episodic memory is not in their nature, but rather how knowledge emerges from the activation of a selected (episodic knowledge), or reversely of multiple (semantic knowledge), traces based on the similarity between the target object and each of its traces. A direct consequence of the common traces hypothesis regards the neuropsychological evaluation. Instead of independently assessing semantic and episodic knowledge, the clinician might benefit from a parallel evaluation (see Greenberg and Verfaellie, 2010). This would mainly ensure that the concept to be learned (e.g., a list of words) is known by the patient (e.g., semantic relationship in a matching task), as well as accessible (e.g., lexical access through a naming task) to the patient.

Another consequence of an embodied approach to memory is to consider the role of perception into memory performance because memory traces remain grounded in their sensory components (Borghi, 2015; Brunel et al., 2015). It can be assumed that different levels of perceptual deficits should be associated with different kinds, or levels, of memory impairments. According to the Act-In memory model (Activation-Integration, Versace et al., 2014), the more distinctive a memory trace is, the more likely it can be retrieved (e.g., Brunel et al., 2013). Therefore, if knowledge is grounded in sensory components, a perceptual decline should directly be associated with lower encoding and retrieval performance. This is the case in normal aging in which the sensory and perceptual decline is significantly correlated with the cognitive decline (see Roberts and Allen, 2016, for a recent review). HE are also known to be more prone to memory errors than YA. These errors might be caused by executive (e.g., Meade et al., 2012) and perceptual deficits (e.g., Yeung et al., 2013). It could then be hypothesized that $\mathrm{HE}$ would commit more memory errors than YA, when they have to selectively learn one item among multiple presented items (source memory), and when the lures share common features with the target (especially for perceptual features, Butler et al., 2010).

Interestingly, these memory errors in aging have been associated with an hyper-binding of related or closely presented (in space or time) items (Campbell et al., 2014), which might be explained by their preserved, and perhaps enhanced, higher perceptual integration compared to YA (e.g., Laurienti et al., 2006). Therefore, HE should not falsely recall or recognize items that are not closely related to the targets, on the contrary to patients with $\mathrm{AD}$. Indeed, $\mathrm{AD}$ is characterized by severe episodic memory deficits from encoding to recognition (Fleischman and Gabrieli, 1999). Yet, this population is also associated with a perceptual and sensory decline more severe than that in normal aging. Moreover, their higher perceptual functions, such as multimodal integration, are impaired (Delbeuck et al., 2007) which have been associated by some authors to their memory dysfunctions (e.g., Vallet et al., 2013, 2016b). The disconnection between the different parts of their brain can thus account for the impairment of multimodal integration and memory deficits (Delbeuck et al., 2003). More specifically, the disconnection between the hippocampus and adjacent or distant structures, such as parahippocampal and frontal regions (Rémy et al., 2015), is associated with an episodic memory deficit. These regions are involved in the retrieval of true memories (see Okado and Stark, 2003) which suggests that AD patients are more likely to commit errors for items never presented before, or related to the targets (see MacDuffie et al., 2012). However, classical memory approaches do not predict whether AD patients should commit or not more confusion errors than HE (Waldie and Kwong, 2003; Abe et al., 2011), on the contrary to embodiment in which confusion errors should be similar across these two groups since both groups show relatively similar low-level perceptual decline (see Vallet, 2015).

Furthermore, embodied memory models also assume the multimodal integration occurs during the retrieval of a memory trace, in addition of encoding (e.g., Zimmer et al., 2006), to dynamically bind the components of the trace (e.g., Brunel et al., 2013; Versace et al., 2014). Therefore, the addition of any multimodal components should negatively impair the memory performance of AD patients (Festa et al., 2005), even when this addition is known to increase the distinctiveness of the memory traces in other populations (for a review, see Schmidt, 1991). One can imagine that the disconnection syndrome will reduce the ability of $\mathrm{AD}$ patients to benefit from some perceptual isolation techniques such as adding a colored background. Isolation is a method commonly used to enhance the distinctiveness of a small set of items to be learned by giving them a particularity not shared with the other items, either from an intrinsic characteristic of the stimuli (e.g., Brunel et al., 2010), or from contextual manipulation (Oker et al., 2009).

The present article proposes a SEMantic EPisodic memory test (SEMEP) developed from an embodied cognition perspective adapted from the Pyramid and Palm Tree Test (PPTT, Howard and Patterson, 1992). Our goal is not to prove the different assumptions stated by the embodied cognition theories, but 
rather to illustrate how they could be applied within the clinical context of memory evaluation for differential diagnosis. The main assumptions taken into account are (1) the common memory traces for semantic and episodic knowledge, (2) the sensory-motor nature of the memory traces, and (3) the central role of integration in the emergence of episodic knowledge. It is expected that (1) performances in semantic tasks should directly impact performances in episodic tasks, (2) reduced perceptual ability should decrease episodic memory retrieval and (3) impaired multimodal integration should impair recall and recognition.

The first hypothesis is tested by using the same material in semantic tasks (matching and naming) and in episodic tasks (free recall and recognition), and by including patients with semantic dementia (SD). SD is a rare neurodegenerative disorder characterized by semantic deficits (Hodges and Patterson, 2007). The semantic deficits could be associated with integration failure (e.g., Vallet et al., 2011b; Hoffman et al., 2014). The second and third hypotheses are explored by contrasting populations showing sensory and perceptual declines without and with multisensory integration deficits, respectively in normal aging and in $\mathrm{AD}$. The dynamic integration hypothesis is tested by manipulating visual isolation (Hunt and Lamb, 2001). Onequarter of the items is associated with a distinctive yellow background that should increase memory performance in all groups (YA, HE, SD) except in $\mathrm{AD}$.

In other words, young adults will represent the reference group of the present study. Compared to them, the concomitant decline in perception and cognition (including episodic memory) of the HE would illustrate how reduced perception might impact memory performance (reduced recall and confusion errors). HE will be the control group for $\mathrm{AD}$ and $\mathrm{SD}$ patients. Compared to $\mathrm{HE}, \mathrm{AD}$ shall present significantly worst performances in all episodic memory tasks and shall also commit intrusion errors due to an integration deficit. $\mathrm{AD}$ shall be the only group to not benefit from the perceptual isolation. Finally, SD should exhibit relatively similar performance than $\mathrm{HE}$ on episodic memory tasks, with the exception of free recall tasks (naming deficit), whereas these patients should be the only group with major semantic deficits (matching and naming task).

\section{METHOD}

\section{Participants}

A total of 103 participants were included in the present study (see Table 1). These participants were divided into four groups: 40 young adults, 40 healthy elderly (HE), 20 patients with Alzheimer's disease $(\mathrm{AD})$, and three patients with Semantic Dementia (SD). AD and $\mathrm{SD}$ patients received a diagnosis from a specialist (e.g., a registered neurologist). Diagnoses were confirmed during a consensus meeting between an AD's expert university professor, several neuropsychologists and a neurologist for the $\mathrm{AD}$ patients, with the addition of a speech language pathologist and an occupational therapist for the SD patients.

The AD patients received a diagnosis of probable Alzheimer's disease according to the Diagnostic and Statistical Manual
TABLE 1 | Means (and standard deviations) for the demographic data for the young adults (YA), healthy elderly adults (HE), patients with Alzheimer's disease (AD) and patients with Semantic Dementia (SD).

\begin{tabular}{lcccc}
\hline & $\begin{array}{c}\text { Young } \\
\text { adults } \\
(\boldsymbol{n}=\mathbf{4 0})\end{array}$ & $\begin{array}{c}\text { Healthy } \\
\text { elderly } \\
(\boldsymbol{n}=\mathbf{4 0})\end{array}$ & $\begin{array}{c}\text { Alzheimer's } \\
\text { disease } \\
(\boldsymbol{n}=\mathbf{2 0})\end{array}$ & $\begin{array}{c}\text { Semantic } \\
\text { dementia } \\
(\boldsymbol{n}=\mathbf{3})\end{array}$ \\
\hline Age & $22.9(3.3)$ & $73.85(5.8)$ & $75.95(6.4)$ & $66(12.5)$ \\
Gender (F/M) & $28 / 12$ & $28 / 12$ & $14 / 6$ & $21 / 2$ \\
Education (in years) & $14.9(2)$ & $13.2(4.4)$ & $13.3(4.2)$ & $13.33(1.2)$ \\
\hline
\end{tabular}

of Mental Disorders-Fourth Edition (American Psychiatric Association, 2004) and the National Institute of Neurological and Communicative Disorders and Stroke-Alzheimer's Disease and Related Disorders Association (NINCDS-ADRDA) criteria (McKhann et al., 1984). They were in the early to moderate stages of the disease as defined by a MMSE score between 18 and 27. All SD patients received from a neurologist a diagnosis of probable Semantic Dementia (Neary et al., 1998). They exhibited a significant loss of word meaning and word-finding difficulties. They were in the early to moderate stages of the disease. Despite the number of SD patients seems very small, the disease is rare so that most of the previous publications on SD were done with unique or multiple cases.

AD patients were recruited in Quebec City (Quebec, Canada) in the community, in a community center, or in the pool of patients already followed in our laboratory. SD patients were recruited from a larger project conducted in Quebec City (see Auclair-Ouellet et al., 2016). Participants in the HE group were recruited through public announcements and in two community centers in Quebec City. Participants in YA group were recruited at Laval University and were matched for education and gender with participants in the $\mathrm{HE}$ and $\mathrm{AD}$ groups. In addition, participants in the HE group were also matched for age with the AD patients (see Table 1). Except the SD patients, about the twothird of the participants in each group (YA, HE, AD) took part in a larger study on memory (see Vallet et al., 2013).

Health information was gathered from all participants during an extensive medical history and neuropsychological interview (see Appendix in Supplementary Material for the detailed cognitive profile of each group). In addition, most of the participants also completed the NPI (NeuroPsychiatric Inventory, Cummings et al., 1994) (or completed by a relative in the case of patients with dementia). Participants with a medical history and/or taking medications for conditions with known sensory or neurological effects were excluded, such as schizophrenia, mild to severe head injury, epilepsy, alcohol or other drug abuse, and so on. Participants who reported a diagnosis of depression or anxiety were included only if they were stable on their medication and if they were non-symptomatic at the time of the study. All participants in the study were native French speakers and demonstrated adequate speech, visual and hearing performances.

All participants underwent a neuropsychological screening battery (see Table 1). Nonetheless, the tests completed varied according to the project in which the participant was originally involved. All participants completed a cognitive speed test 
[simple reaction time task (SRT)] and a standard test of general cognitive functioning [Mini-Mental State Examination (Folstein, 1975)]. Except for SD patients, they were all tested on verbal memory [(RL/RI-16 free and cued recall task Van der Linden, 2004)], executive functions [Trail Making Test (TMT, Delis et al., 2001; Lezak et al., 2004); and Stroop test (Godefroy et al., 2010)], and executive-semantic functions [word fluency test (Cardebat et al., 1990)].

\section{Material}

The SEMEP test is based on the visual part of the PPTT in which participants must match semantically related pictures on 52 boards. On each board, three black-and-white line-drawn pictures are displayed as a triangle. On the top, one picture serves as a model (e.g., a pyramid). The two remaining pictures are at the base of the triangle. One picture is the target (e.g., a palm tree) and the other one represents a distractor (e.g., a pine tree).

All the cards of the visual subset of the PPTT were scanned in order to create a numeric version that could be modified. In the encoding/matching phase, 32 of the 52 original cards were selected (cf. Table 2). Among these 32 cards, one-quarter (i.e., 8) were randomly chosen to be visually isolated. The isolation consisted in adding a yellow background to the three items on the board. The non-isolated items remained with the original white background. Illustration of the material used in presented in Figure 1.

A first reason to decrease the number of cards was related to the nature of the material itself as some items were repeated on multiple boards. We thus selected the cards in order to avoid any double presentation of a given item. Furthermore, some associations were demonstrated as being inappropriate for the Quebec French-speaking population. For instance, some items were dramatically poorly processed compared to the others as the windmill-tulip (item 16) or the acorn-pig (item 40) associations (Callahan et al., 2010). The second reason was to decrease the cognitive load associated with the learning phase and to avoid a feeling of failure due to a large number of items to be learned. Finally, the last reason to reduce the number of stimuli regards the need to keep enough items from the original task to be used in the recognition task. The selection was also done to ensure that the correct matching responses were equally presented on each side of the card.

The foils used in the recognition task were selected to have similar visual characteristics than the targets. From the threepicture cards, each item is numerically isolated in order to create new cards with only one item on them. From these pictures, all the targets (items to learn in the encoding phase) were included (32), as well as the same number of foils (32). These foils were selected to be in two conditions: (1) old-foiled: 16 pictures that were presented in the encoding phase, but were not to be learned, (2) new-foiled: 16 pictures that were never presented in the encoding phase. Half of the old-foiled (8) was the distractor and the other half was the model on the original cards. These pictures have been printed and displayed on cards that are easy to manipulate. The order of presentation of the items was randomly defined, but was kept constant for all the participants (cf. Table 3).
TABLE 2 | List of the stimuli included in the encoding/matching phase of the SEMEP.

\begin{tabular}{|c|c|c|c|c|}
\hline & Model & Item 1 & Item 2 & Isolation \\
\hline $\mathrm{P} 1$ & Bottle & mug & glass & \\
\hline $\mathrm{P} 2$ & TV aerial & television & radio & \\
\hline P3 & Fork & ladle & spoon & \\
\hline 1 & glasses & eye & ear & \\
\hline 2 & Hands & gloves & slippers & Isolated \\
\hline 3 & cheese & rabbit & mouse & \\
\hline 4 & thimble & needle & thread & \\
\hline 5 & saddle & goat & horse & \\
\hline 6 & pillow & bed & chair & \\
\hline 7 & trees & onion & apple & \\
\hline 8 & matches & light bulb & candle & Isolated \\
\hline 9 & pyramid & palm tree & pine tree & \\
\hline 10 & bat & owl & woodpecker & \\
\hline 11 & web & bee & spider & \\
\hline 12 & tent & fire & radiator & \\
\hline 13 & soldiers & church & castle & Isolated \\
\hline 14 & caterpillar & butterfly & dragonfly & \\
\hline 15 & nun & convent & house & Isolated \\
\hline 16 & whool & dogs & sheep & \\
\hline 17 & eggs & hen & swan & \\
\hline 18 & puddle & sun & clouds & \\
\hline 19 & fish & cat & dog & \\
\hline 20 & drill & screw & nail & Isolated \\
\hline 21 & stethoscope & tongue & heart & \\
\hline 22 & logs & hammer & saw & Isolated \\
\hline 23 & safety pin & girl & baby & \\
\hline 24 & milk & cow & bull & \\
\hline 25 & razor & chin & noze & Isolated \\
\hline 26 & curtain & door & window & \\
\hline 27 & rocket & star & moon & \\
\hline 28 & mask & clown & mayor & \\
\hline 29 & path & hands & feet & \\
\hline 30 & ink & pencil & pen & \\
\hline 31 & padlock & bicycle & car & Isolated \\
\hline 32 & eskimo & rowing boat & kayak & \\
\hline
\end{tabular}

Eight board were chosen to be isolated by the addition of a yellow background to the pictures in Figure 1. P1, P2, practice items; Bold items, correct matching responses and targets to be learned; Isolated, boards with a yellow background added.

\section{PROCEDURE AND DESIGN}

\section{General Procedure}

This research was approved by the Ethical Committee of the "Centre de recherche Université Laval Robert-Giffard" (project \#228) and all participants signed an informed consent form before the experimental session started. Each participant was tested individually.

All participants completed the whole experimental protocol in one session of $\sim 2 \mathrm{~h}$. Following a short clinical interview, they were submitted to the cognitive battery. They first responded to the MMSE, and then to the RL/RI-16 memory test. During the $15 \mathrm{~min}$ separating the last recall from the 


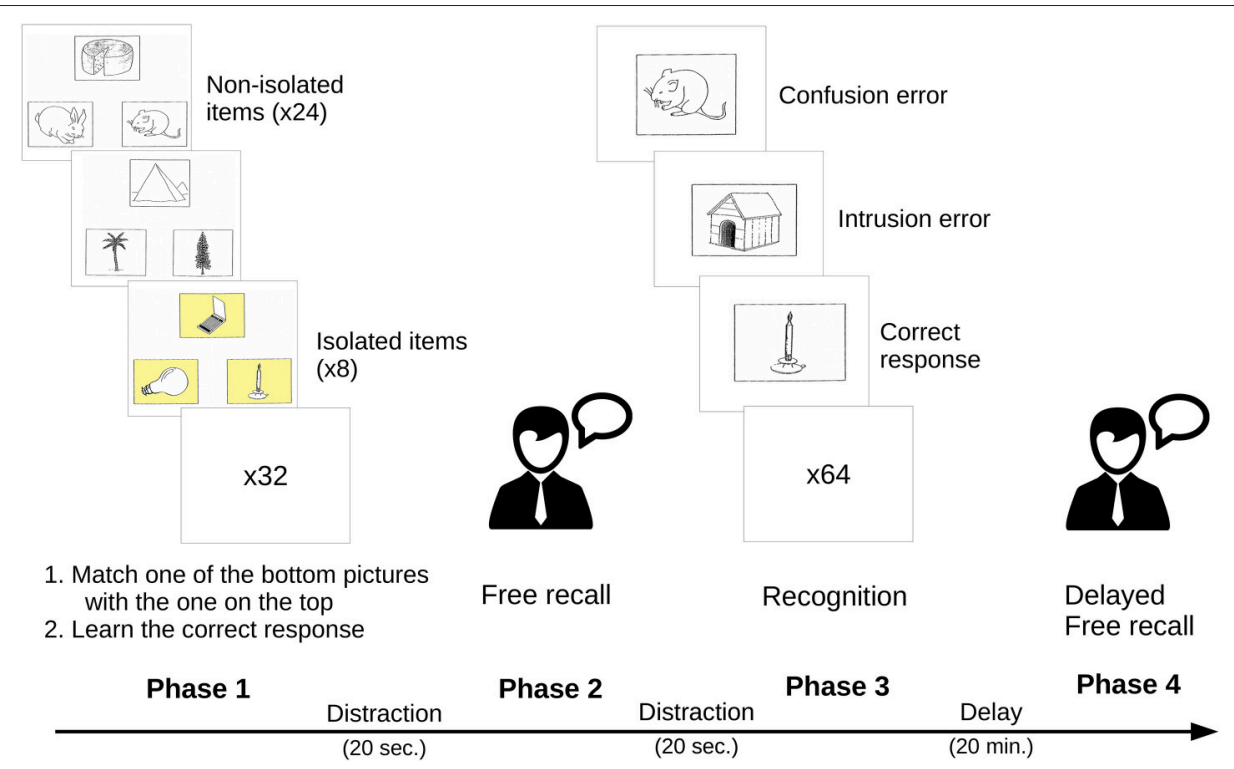

FIGURE 1 | Illustration of the general procedure and of the material used in the SEMEP.

TABLE 3 | List of the stimuli included in the recognition task of the SEMEP.

\begin{tabular}{|c|c|c|c|c|c|c|c|}
\hline & Item & & Item & & Item & & Item \\
\hline 1 & kennel & 17 & armchair & 33 & fire & 49 & heart \\
\hline 2 & candle ${ }^{\star \star}$ & 18 & window & 34 & tulip & 50 & igloo \\
\hline 3 & car & 19 & table & 35 & owl & 51 & feet \\
\hline 4 & spider & 20 & eskimo & 36 & light bulb & 52 & screw $^{\star \star}$ \\
\hline 5 & tent & 21 & bed & 37 & needle & 53 & radiator \\
\hline 6 & blackboard & 22 & ink & 38 & eyes & 54 & lamp \\
\hline 7 & baby & 23 & worm & 39 & bus & 55 & bicycle ${ }^{\star \star}$ \\
\hline 8 & clouds & 24 & glasses & 40 & castle $^{\star \star}$ & 56 & matches \\
\hline 9 & cage & 25 & moon & 41 & hammer & 57 & apple \\
\hline 10 & butterfly & 26 & nose & 42 & gloves** & 58 & pen \\
\hline 11 & battery & 27 & palm tree & 43 & sheep & 59 & ear \\
\hline 12 & windmill & 28 & kayak & 44 & horse & 60 & $\operatorname{chin}^{\star *}$ \\
\hline 13 & couvent $^{\star \star}$ & 29 & razor & 45 & carrot & 61 & cat \\
\hline 14 & pencil & 30 & mouse & 46 & $\operatorname{saw}^{\star *}$ & 62 & cadlock \\
\hline 15 & anchor & 31 & ship & 47 & $\log s$ & 63 & desk \\
\hline 16 & clown & 32 & ring & 48 & cow & 64 & hen \\
\hline
\end{tabular}

Bold items, targets; Italic items, confusion errors; ${ }^{* *}$, isolated items.

delayed recall, they completed different non-verbal tests varying in function of the project (e.g., the simple reaction time, visual perception tasks). This was followed by the SEMEP and between the recognition and delayed free recall tasks (see below), they completed other non-verbal tests such as the TMT test.

\section{SEMEP-Procedure}

A general presentation of the SEMEP and of the different scores computed are provided in Table 4. The experimenter presented the test as a memory test based on pictures. It was highlighted that the participant had to learn a lot of items and it was clearly stated that these items had to be recalled later.

During the encoding phase, the instructions insisted on the fact that two tasks had to be done at the same time: a matching task and a learning task. Participants were told about the matching task that "on each card, you will see three linedrawn pictures organized in a triangle. The picture on the top will be your model. Your first task will be to judge which one of the two pictures, presented at the bottom, best matches the model." The first example was then introduced.

Once the participant had well understood how to proceed, the experimenter presented the learning task: "in addition to the matching task, I will ask you to learn the corresponding name of the correct answer of the matching task. Be careful, your task is to learn and remember only the correct answer. If you happen to recall the other pictures from the card, it will be considered as errors." The two other practice cards were presented by stressing out that it was only a practice, so that the participant did not have to learn these examples.

If the participant had no question about the procedure, the first phase was then summarized: "for each card, you will first tell me which one of the bottom pictures matches the top picture. I will confirm or correct your answer if necessary. Then, I will always confirm which item you will have to learn."

Each board was presented one at a time. The participant did not have any time constraint, but the experimenter tried to keep the presentation of the item relatively constant ( $\sim 8 \mathrm{~s}$ per card). If the participant made a mistake, the experimenter corrected her/him immediately and provided the right answer. Synonyms were accepted and used by the experimenter in the learning instructions. For instance, if the participant said "fortress" instead of "castle," the experimenter then stated: "that's correct, and you will have to learn "fortress." 
In order to avoid recency effect from short-term memory, participants had then to countdown from a random number (e.g., 326) by steps of one during 20 s. Once completed, the experimenter asked the participant to recall as much words as possible that were to be learned. It was highlighted to be careful to give only the correct answer and not the names of other pictures that would be considered as errors. The participant had $2 \mathrm{~min}$ to complete the task. If errors were committed during the recall task, they were not corrected.

A second distractor task was realized before the third phase. The same countdown task was chosen to start from a different number (e.g., 450). The third phase consisted of a yes/no visual recognition task combined with a naming task presenting one card showing one item at a time. For each card, participants were asked to name the depicted object, and then to indicate by a "Yes" or "No" response whether the picture was learned in the learning phase. Once again, it was stressed out that only the correct answers from the matching task had to be considered as a "Yes" response. When an error was made, the experimenter corrected the response.

No mention was made that a delayed free recall task would take place after a 20-min. delay. During this time, the other nonverbal tasks of the general protocol were completed as described in the previous section. At last, the delayed free recall task was completed using the same procedure than that used in the first free recall task.

In the first phase, the correct matching responses were recorded (on 32). In the recall tasks, the experimenter recorded the number of correct responses (items to be remembered, on 32) as well as the number of isolated items correctly recalled (on 8). In the recognition task, the number of correctly named items (on 64) as well as the number of correctly recognized items were noted (on 32) with the addition of the number of isolated items correctly recognized (on 8). In the free recall and recognition tasks, (1) a confusion error was defined as the recall or recognition of an item presented in the encoding phase that was not to be learned; (2) an intrusion error consisted in the recall or recognition of an item never presented to the participant in the encoding phase.

\section{Statistical Analysis}

The data was processed and analyzed using $\mathrm{R}$ version 3.3.1 ( $\mathrm{R}$ Foundation for Statistical Computing). In addition to the raw scores recorded in the SEMEP, the proportion of isolated, confusion errors and intrusion errors were computed for the free recall and delayed free recall tasks. The proportion of isolated item recall was computed with the formula: $\frac{\text { number of isolated items recalled }}{\text { total number of items recalled }}$ and errors rate with the formula: total number of items
number of errors

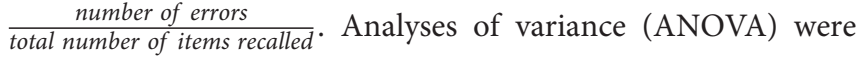
conducted on each dependent variable with the Group (YA vs. $\mathrm{HE}$ vs. $\mathrm{AD}$ ) as a between-subjects variable. $\mathrm{SD}$ patients were excluded from the analyses due to the too limited sample size (only three patients), but they were included in the $\mathrm{Z}$-scores profiling. Z-scores were computed for the mean scores of all the elderly groups using the data from the YA as a reference $\left(\mathrm{z}=\frac{\text { mean }_{\text {score }}-\text { mean }_{Y A}}{s d_{Y A}}\right.$, with mean $\mathrm{YA}$ and $s d_{\mathrm{YA}}$ as the mean and standard deviation values of the young adults). In order to avoid infinite values, the mean and standard deviation values were replaced by the value of 0.1 when they equaled to 0 . Subsequent comparisons were conducted using Tukey post hoc analyses. The common trace hypothesis was tested using a Pearson correlation analysis (bilateral) between the scores of the semantic tasks (matching and naming) and of the episodic tasks (free recall, recognition). An alpha level of 0.05 was used as a significant threshold for all the analyses.

\section{RESULTS}

As shown in Table 5, different patterns of results could be observed on the SEMEP as a function of the comparison underwent. First of all, HE, compared to YA, showed poorer performance on immediate and delayed free recall tasks, whereas recognition (correct scores) appeared preserved. Despite the fact that HE recalled fewer isolated items than YA, their proportion of recall of these items did not differ significantly from that of their younger counterparts. They also did not produce more intrusion errors than YA, but they did commit more confusion errors across the different tasks (recall, recognition and also on the proportions computed).

Secondly, AD patients did exhibit poorer performance than YA in all conditions except for the proportion of isolated items recalled in the immediate recall task. This might be explained by the limited number of items recalled by the AD patients (only 3.5 on average).

Finally, AD patients also showed poorer performances than HE on almost all scores except for the number of confusions. This result has to be moderated by the fact that compared with $\mathrm{HE}, \mathrm{AD}$ patients committed more confusion errors, in proportion, in the two recall tasks.

The common trace hypothesis was also tested using a correlation analysis between the scores of the semantic tasks (matching and naming) and of the episodic tasks (free recall, recognition) as presented in Table 6. The analysis could not be conducted on the intrusion errors in the free recall tasks as there was not enough variance. Corroborating the hypothesis, the semantic scores were significantly associated with most of the episodic scores, excepted for the confusion errors in the recall tasks.

\section{DISCUSSION}

The aim of the present article was to assess a new memory test, the SEMEP, that respect some core assumptions of embodiment in the field of memory, i.e., (1) the common memory traces between semantic and episodic knowledge, (2) the grounding of knowledge into its sensory-motor components, (3) the dynamic integration of knowledge to emerge as episodic memories. Thus, hypotheses associated with these assumptions were tested across four different populations.

It was first hypothesized that HE compared to YA would present reduced recall performances on the SEMEP. As expected, 
TABLE 4 | Summary of the procedure used in the SEMEP with the different scores collected.

\begin{tabular}{|c|c|c|c|}
\hline Phase & Task & Description & Score \\
\hline \multirow[t]{3}{*}{ Phase 1} & Matching task & Match one of the bottom pictures to the top picture & Score /32 \\
\hline & Encoding task & Learn the right answer & \\
\hline & Distraction 1 (20 s.) & Countdown by step of one & \\
\hline \multirow[t]{5}{*}{ Phase 2} & Free recall task & Recall as much as possible words to be learned in Phase 1 & Score /32 \\
\hline & (2 min.) & Intrusion errors (item never presented) & Number of errors \\
\hline & & Confusion errors (item saw, but not to learn) & Number of errors \\
\hline & & Isolation (items recalled that were isolated in Phase 1) & Score /8 \\
\hline & Distraction 2 (20s). & Countdown by step of one & \\
\hline \multirow[t]{6}{*}{ Phase 3} & Naming task & Give the name of the object depicted on the card & Score /64 \\
\hline & Recognition task & Recognize the pictures corresponding to the correct matching in phase 1 & Score /32 \\
\hline & & Intrusion errors (item never presented) & Number of errors \\
\hline & & Confusion errors (item saw, but not to learn) & Number of errors \\
\hline & & Isolation (items recognized that were isolated in Phase 1) & Score $/ 8$ \\
\hline & Delay (20 min.) & & \\
\hline \multirow[t]{4}{*}{ Phase 4} & Delayed free recall task & Recall as much as possible words to be learned in Phase 1 & Score /32 \\
\hline & (2 min.) & Intrusion errors (item never presented) & Number of errors \\
\hline & & Confusion errors (item saw, but not to learn) & Number of errors \\
\hline & & Isolation (item recalled that were isolated in Phase 1) & Score /8 \\
\hline
\end{tabular}

the results showed that $\mathrm{HE}$ recalled statistically fewer items than YA in the immediate and delayed recall tasks. However, their performance was not clinically impaired (z-scores deviation inferior to 1), which might be explained by the visual nature of the material used in the SEMEP. Different studies have indeed shown that $\mathrm{HE}$ benefit from visual material to be learned compared to verbal one (e.g., Smith et al., 2015).

It was also hypothesized that $\mathrm{HE}$ compared to YA would make only more confusion errors. The results supported this hypothesis. HE committed more confusion errors than YA, but did not make more intrusion errors than YA in all conditions of the SEMEP. These findings are supported by the results of previous reports that have demonstrated that $\mathrm{HE}$ are more vulnerable to memory errors, especially to false alarms, than their younger counterparts (see Devitt and Schacter, 2016 for a recent review). However, HE do not falsely recognize stimuli that are not closely related to the target (e.g., Toner et al., 2009). There are different hypotheses in the literature to account for the confusion errors made by HE. The most commonly admitted hypotheses rely on the deficits of executive functions in aging, in line with the alteration of their frontal lobes (e.g., Butler et al., 2004; but see Chan and McDermott, 2007 for a different point of view). For instance, according to the source-monitoring hypothesis, false memories occur when a person is not able to track the source of the stimulus as being old or new (Johnson et al., 1993). Participants need to retrieve specific characteristics from the event that will help them to make a decision about its source. Because these effortful and strategic processes are impaired in aging, HE commit more false alarms than YA (e.g., Meade et al., 2012).
From an embodied perspective, the increase memory errors in HE might be explained by the degradation of perception in aging. It has been known for a long time that the sensory/perceptual decline in healthy aging is associated with the cognitive decline (see Roberts and Allen, 2016 for a recent review). Growing evidence especially shows that memory and perception are very tightly bound (e.g., Graham et al., 2010; Rey et al., 2015; see Appendix B in Supplementary Material for data on a subset of the $\mathrm{HE}$ group), and that memory traces in $\mathrm{HE}$ are grounded in their sensory-motor components, as in YA (e.g., Vallet et al., 2011a, 2013). Therefore, degraded perceptual processing, as in HE, should directly impoverish their memory traces (Humes et al., 2013; Vallet, 2015). The degraded memory traces should, in turn, decrease the distinctiveness of the memory traces, and consequently, it shall be more difficult to distinguish one memory trace from another (see Brunel et al., 2013; Vallet et al., 2016b). In other words, HE should commit more confusion errors as they require more pronounced distinctive features to correctly reject a related lure (Butler et al., 2010).

The distinctiveness heuristic (see Dodson and Schacter, 2002) appears also a useful hypothesis to interpret the results observed for the isolated items in the present study. HE benefited from the visual isolation in a similar fashion than YA (i.e., both groups obtained similar proportions of correct free recall on isolated items; see the bottom of Table 5) (see also Geraci et al., 2009). Isolation is a method commonly used to enhance the distinctiveness of a small set of items to be learned by giving them a particularity not shared with the other items (e.g., Brunel et al., 2010). Despite the altered perception observed in aging, HE shall have preserved, and perhaps enhanced, multisensory integration (Laurienti et al., 2006). This might explain the present result. 


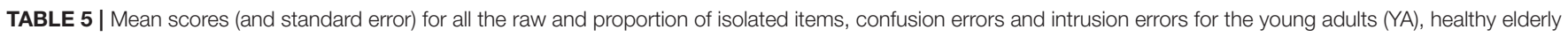
$(\mathrm{HE})$, and patient with Alzheimer's disease (AD).

\begin{tabular}{|c|c|c|c|c|c|c|c|c|}
\hline & YA & HE & AD & SD & YA-HE & YA-AD & HE-AD & $\boldsymbol{F}$ \\
\hline \multicolumn{9}{|l|}{ MATCHING } \\
\hline Correct matching & $31.4(0.24)$ & $30.67(0.24)$ & $29.05(0.34)$ & $26.67(4.16)$ & 0.09 & 0 & 0 & $15.59^{\star \star \star}$ \\
\hline \multicolumn{9}{|l|}{ FREE RECALL } \\
\hline Correct not isolated & $16.33(0.73)$ & $12.67(0.73)$ & $3.5(1.03)$ & $5.67(2.08)$ & 0 & 0 & 0 & $52.19^{\star \star \star}$ \\
\hline Correct isolated & $4.08(0.24)$ & $2.9(0.24)$ & $0.75(0.34)$ & $1.33(1.53)$ & 0 & 0 & 0 & $32.21^{\star \star \star}$ \\
\hline Confusion errors & $0.47(0.14)$ & $1.47(0.14)$ & $1.15(0.2)$ & $0(0)$ & 0 & 0.02 & 0.39 & $12.66^{\star \star \star}$ \\
\hline Intrusions errors & $0(0.11)$ & $0.05(0.11)$ & $1(0.16)$ & $0(0)$ & 0.95 & 0 & 0 & $14.83^{\star \star \star}$ \\
\hline \multicolumn{9}{|c|}{ DELAYED FREE RECALL } \\
\hline Correct not isolated & $20.5(0.8)$ & $15.7(0.8)$ & $5.05(1.14)$ & $8(1)$ & 0 & 0 & 0 & $61.72^{\star \star \star}$ \\
\hline Correct isolated & $5.8(0.24)$ & $4.63(0.24)$ & $0.6(0.34)$ & $2.33(2.08)$ & 0 & 0 & 0 & $78.09^{\star \star \star}$ \\
\hline Confusion errors & $0.4(0.17)$ & $1.52(0.17)$ & $1.5(0.24)$ & $0.33(0.58)$ & 0 & 0 & 1 & $13.37^{\star \star \star}$ \\
\hline Intrusions errors & $0(0.14)$ & $0.05(0.14)$ & $2.1(0.2)$ & $0.33(0.58)$ & 0.97 & 0 & 0 & $41.95^{\star \star \star}$ \\
\hline \multicolumn{9}{|l|}{ RECOGNITION } \\
\hline Correct not isolated & $30.75(0.48)$ & $29.65(0.48)$ & $23.85(0.68)$ & 29.67 (1.53) & 0.24 & 0 & 0 & $36.68^{\star \star \star}$ \\
\hline Correct isolated & $7.77(0.18)$ & $7.17(0.18)$ & $4.85(0.25)$ & $7.33(0.58)$ & 0.05 & 0 & 0 & $46.96^{\star \star \star}$ \\
\hline Confusion errors & $0.22(0.32)$ & $1.65(0.32)$ & $6.2(0.45)$ & $2.33(2.08)$ & 0.01 & 0 & 0 & $60.27^{\star \star \star}$ \\
\hline Intrusions errors & $0(0.38)$ & $0.03(0.38)$ & $5.05(0.53)$ & $0(0)$ & 1 & 0 & 0 & $35.81^{\text {*** }}$ \\
\hline \multicolumn{9}{|l|}{ NAMING } \\
\hline Correct & $63.25(0.46)$ & $62.67(0.46)$ & $57.05(0.65)$ & $52.67(0.58)$ & 0.65 & 0 & 0 & $33.5^{\star \star \star}$ \\
\hline \multicolumn{9}{|l|}{ PROPORTIONS } \\
\hline FR (isolated) & $0.25(0.02)$ & $0.23(0.02)$ & $0.28(0.03)$ & 0.19 (0.19) & 0.84 & 0.78 & 0.52 & 0.62 \\
\hline FR (confusions) & $0.03(0.02)$ & $0.12(0.02)$ & $0.27(0.04)$ & $0(0)$ & 0.02 & 0 & 0 & $16.8^{\star \star \star}$ \\
\hline FR (intrusions) & $0(0.01)$ & $0(0.01)$ & $0.12(0.02)$ & $0(0)$ & 0.97 & 0 & 0 & $19.64^{\star \star \star}$ \\
\hline DFR (isolated) & $0.29(0.02)$ & $0.3(0.02)$ & $0.15(0.02)$ & $0.28(0.25)$ & 0.76 & 0 & 0 & $15.98^{\star \star \star}$ \\
\hline DFR (confusions) & $0.02(0.01)$ & $0.1(0.01)$ & $0.19(0.02)$ & $0.04(0.07)$ & 0 & 0 & 0 & $25.24^{\star \star \star}$ \\
\hline DFR (intrusions) & $0(0.01)$ & $0(0.01)$ & $0.22(0.01)$ & $0.03(0.06)$ & 0.97 & 0 & 0 & $97.97^{\star \star \star}$ \\
\hline
\end{tabular}

$P, p$-values computed for each ANOVA with the group (HE, aMCI, AD) as a between subject variable; ${ }^{* \star *} p<0.001 ; F R$, free recall; DFR, delayed free recall.

TABLE 6 | Correlation analysis between the semantic (matching and naming) scores and the episodic (free recall, recognition, errors) scores of the SEMEP across all groups of participants (young adults, healthy elderly and patients with Alzheimer's disease).

\begin{tabular}{|c|c|c|c|}
\hline Semantic & & Matching & Naming \\
\hline \multicolumn{4}{|l|}{ EPISODIC } \\
\hline \multirow[t]{3}{*}{ FR } & Corr. & $0.29 * *$ & $0.51^{* * *}$ \\
\hline & Corr. Isolated & $0.33^{\star \star \star}$ & $0.45^{\star \star \star}$ \\
\hline & Confusion Err. & -0.15 & 0.07 \\
\hline \multirow[t]{3}{*}{ Delayed FR } & Corr. & $0.26^{\star \star}$ & $0.48^{\star * *}$ \\
\hline & Corr. Isolated & $0.34^{\star * \star}$ & $0.56^{\star \star *}$ \\
\hline & Confusion Err. & -0.17 & -0.18 \\
\hline \multirow[t]{4}{*}{ Recognition } & Corr. & $0.22^{\star}$ & $0.32^{\star \star *}$ \\
\hline & Corr. Isolated & $0.43^{\star \star \star}$ & $0.35^{\star \star \star}$ \\
\hline & Confusion Err. & $-0.37^{\star \star \star}$ & $-0.63^{\star \star \star}$ \\
\hline & Intrusion Err. & $-0.54^{\star \star \star}$ & $-0.59^{\star \star *}$ \\
\hline
\end{tabular}

FR, free recall; Corr., correct; Err., errors; VOSP, visual object and space perception; ${ }^{\star} p<0.05 ;{ }^{* \star} p<0.01 ;{ }^{* \star} p<0.001$; Bold items, significant correlation.

Otherwise, the results of the HE group showed performances relatively similar to those of YA on the semantic and recognition tasks. Numerous studies have found preserved, and sometimes enhanced, semantic memory in aging (e.g., Nyberg et al., 2003; see Park and Gutchess, 2002 for a review). It is also frequent to observe similar, or slightly impaired, recognition performance for HE when the task is not too demanding (Danckert and Craik, 2013; Koen and Yonelinas, 2014).

As expected, AD patients showed severe deficits in episodic memory across the tasks. AD is characterized with severe episodic memory disorders together with a disconnection syndrome (see Delbeuck et al., 2003). It could thus be expected that these patients will show more marked memory impairments, which will expand to memory errors for unrelated content, as well as a deficit to integrate supplementary perceptual information (in the isolation procedure). Nonetheless, AD correctly recalled a relatively similar number of items than SD patients (see Figure 2). This pattern has been previously reported in the literature, especially for the recall of pictures, on the contrary to verbal memory that is more impaired in $\mathrm{AD}$ (Scahill et al., 2005). AD patients also produced a similar amount of confusion errors than $\mathrm{HE}$ in the recall tasks. However, the proportions of confusion errors in the recall tasks and the false alarms in recognition were significantly more important for $\mathrm{AD}$ patients compared to HE.

Interestingly, $\mathrm{AD}$ patients were the only group to commit intrusion errors compared to the other groups. These errors were produced both in the recall and recognition tasks. Despite 


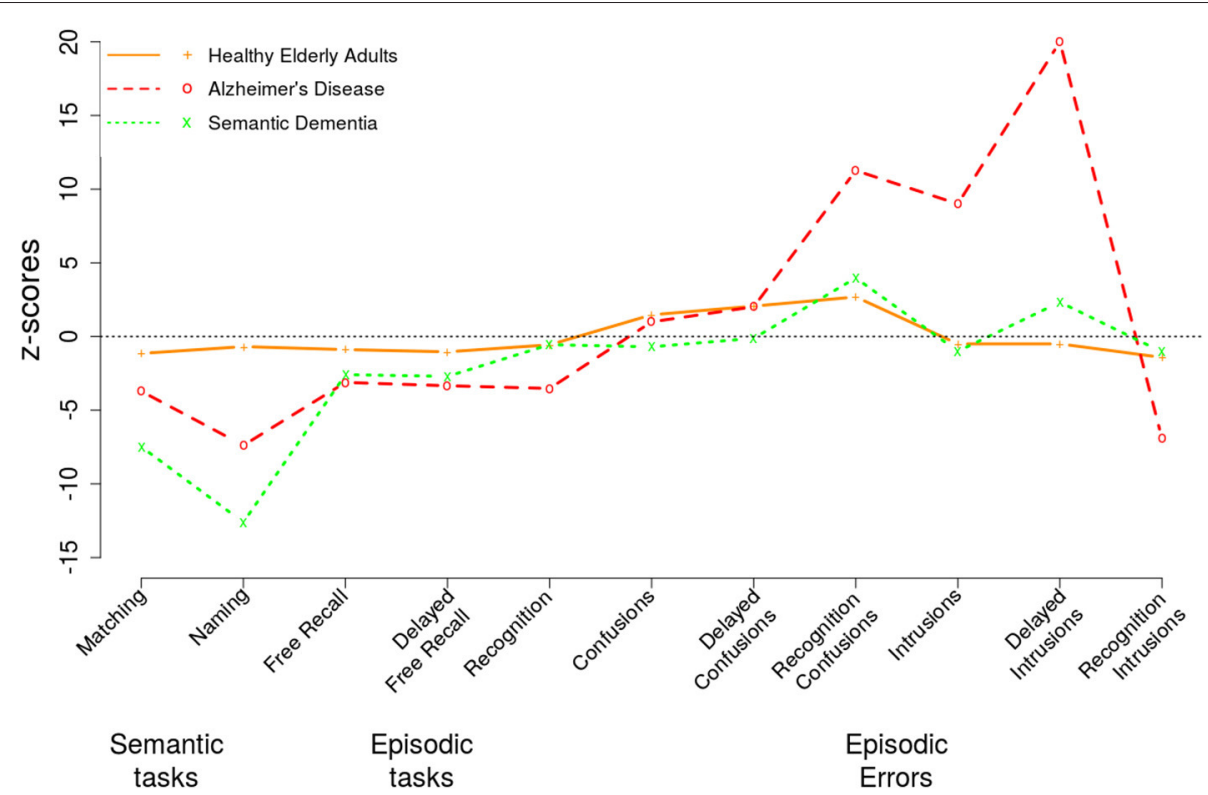

FIGURE 2 | z-scores of healthy elderly adults, patients with Alzheimer's disease and patients with semantic dementia using data from the young adults as reference across the main scores of the SEMEP.

intrusion errors are typically underlied by frontal impairment, such as the one seen in dementia with Lewy bodies (Doubleday and Snowden, 2002), some studies have found a similar pattern of intrusion errors between $\mathrm{AD}$ and fronto-temporal dementia (Pasquier and Grymonprez, 2001). These errors could indicate the weakness of AD's memory traces compared to those of HE (see Vallet et al., 2016b). The overall pattern of performance observed in the present study is yet consistent with other published studies (e.g., Greenaway et al., 2006).

Finally, the isolation procedure let emerges an interesting result. Whereas, all groups of participants appeared to benefit from the increase distinctiveness provided by this procedure, $\mathrm{AD}$ patients did not. All scores for these items, except for the proportions of isolated items recalled in the immediate recall task, were significantly lower in $\mathrm{AD}$ compared to those observed in YA and HE. Moreover, the probability that $\mathrm{AD}$ would recall (in the delayed task) and recognize the isolated items was in fact inferior to chance, which was here at $25 \%^{1}$. The fact that this deleterious effect of isolation was not observed in the immediate free recall might be due to either the very limited number of items recalled then (3.5 in average compared to 5.05 in the delayed recall), or to the accelerate forgetting reported in $\mathrm{AD}$ (e.g., Estévez-González et al., 2003).

This result might be surprising in the traditional multisystem memory approach, but it is expected in embodied cognition theories. Indeed, this deleterious effect is unlikely coming from (1) a specific visuoperceptual decline in $\mathrm{AD}$ patients, as they exhibit preserved visual repetition priming (e.g., Fleischman, 2007); (2) from a specific deficit in the isolation effect as isolation

\footnotetext{
${ }^{1}$ In recognition: $t(19)=3.51, p<0.01$. In delayed free recall: $t(18)=2.34, p<$
} 0.05 . Two-sided $t$-tests. effect appears less efficient in $\mathrm{AD}$, but still beneficial, when it is the font size that is manipulated (Vitali et al., 2006) (3) from an overload of their cognitive resources since the simple addition of visual information, such as a background, is not sufficient to impair cognitive performance in $\mathrm{AD}$ (see Vallet et al., 2013). In the present study, the yellow background appears as a burden which is likely constitutes supplementary information to be bound within the memory trace (see Versace et al., 2014). Indeed, according to the embodied cognition theories, AD show impaired multisensory integration (Delbeuck et al., 2007), which could be related to their disconnection syndrome (Vallet et al., 2013). The disconnection syndrome in AD might explain why these patients present memory deficits in a situation which requires the dynamic interplay between sensory (or multidmodal) components (Festa et al., 2005). This hypothesis remains to be further explored to determine how the integration deficit may play a central role in episodic memory impairment (see also Buschke et al., 2017).

Finally, SD patients showed severe deficits in the semantic memory tasks (matching and naming) as expected in this population Hodges and Patterson, 2007). They also presented reduced free recall performance which appeared to be relatively similar to AD. Nonetheless, this latter finding should be discussed in the light of their naming deficits (Graham et al., 2000). Indeed, SD patients performed well on recognition, comparable to $\mathrm{HE}$, which suggests a relative preservation of episodic memory compared to AD. They also committed a similar amount of confusion and intrusion errors than HE. This suggests that their episodic memory was not as affected by other integration deficits as in $\mathrm{AD}$.

Thus, the difficulties faced by SD on free recall tasks may be due to the difficulty of finding their words rather than 
remembering the words. This hypothesis is supported by the clinical experience during the study; SD patients did try very hard to find the words of items to be recalled, and then abandoned to recall (i.e., name) another item. Of course, these findings should be interpreted with caution as only three patients were included in the present study. A greater number of patients should be included in future studies before drawing any conclusion. Yet, SD patients were included in the present study in order to illustrate the difference of performance pattern between the elderly groups rather than to provide strong evidence in favor of their semantic and episodic patterns of performance.

Taken all together, the data of this study showed very distinctive patterns of performance between the elderly groups, as illustrated with the z-scores in Figure 2. It seems that confusion errors are common across all the elderly groups, with and without cognitive disorders. These errors are supposed to reflect the degradation of the memory traces so that they become less distinctive with aging. According to embodiment theories, this degradation results from the perceptual decline reported in aging. $\mathrm{AD}$ was the only group in the present study that exhibited regular intrusion errors. The intrusion errors could be interpreted as an integration deficit, which is also supported by the detrimental effect of the visual isolation in this group. This difference in the pattern of errors between the elderly and dementia groups emphasizes the need to further consider the type of errors to differentiate clinical population (e.g., Rouleau et al., 2001). Finally, SD patients exhibited the most severe semantic impairment compared to the other groups. They also recalled a few items, as $\mathrm{AD}$ patients, but for a different reason. As all episodic scores were reduced in $\mathrm{AD}$ patients, $\mathrm{SD}$ patients performed similarly to $\mathrm{HE}$ on the recognition task and committed the same type and number of errors as HE.

\section{REFERENCES}

Abe, N., Fujii, T., Nishio, Y., Iizuka, O., Kanno, S., Kikuchi, H., et al. (2011). False item recognition in patients with Alzheimer's disease. Neuropsychologia 49, 1897-1902. doi: 10.1016/j.neuropsychologia.2011.03.015

American Psychiatric Association (2004). DSM-IV-TR Manuel Diagnostique et Statistique des Troubles Mentaux. Paris: Masson.

Auclair-Ouellet, N., Macoir, J., Laforce, R., Bier, N., and Fossard, M. (2016). Regularity and beyond: impaired production and comprehension of inflectional morphology in semantic dementia. Brain Lang. 155-156, 1-11. doi: 10.1016/j.bandl.2016.02.002

Borghi, A. (2015). "An embodied and grounded perspective on concepts," in Epistemology of Ordinary Knowledge, eds M. Bianca and P. Piccari (Cambridge: Scholar), 181-194.

Brunel, L., Goldstone, R. L., Vallet, G. T., Riou, B., and Versace, R. (2013). When seeing a dog activates the bark: multisensory generalization and distinctiveness effects. Exp. Psychol. 60, 100-112. doi: 10.1027/1618-3169/ a000176

Brunel, L., Oker, A., Riou, B., and Versace, R. (2010). Memory and consciousness: trace distinctiveness in memory retrievals. Conscious. Cogn. 19, 926-937. doi: 10.1016/j.concog.2010.08.006

Brunel, L., Vallet, G., Riou, B., Rey, A., and Versace, R. (2015). "Grounded conceptual knowledge emergence from sensorimotor interactions," in Conceptual and Interactive Embodiment: Foundations of Embodied Cognition, eds M. Fisher and Y. Coello (New York, NY: Psychology Press), 108-124.

Buschke, H., Mowrey, W. B., Ramratan, W. S., Zimmerman, M. E., Loewenstein, D. A., Katz, M. J., et al. (2017). Memory binding test distinguishes
To conclude, the SEMEP seems to be an interesting tool to evaluate memory functioning in aging. Beyond the question of embodiment, the test permitted to show specific patterns of results for each group included in the study. In the future, studies may apply the principles used in this test to assess patients with different clinical diagnoses and in different situations to confirm the usefulness of the SEMEP in clinical settings.

\section{AUTHOR CONTRIBUTIONS}

GV designed and conducted the study as well as analyzed the data. RV and MS helped to design the protocol and to analyze the data. CH, JM, and NB helped to conduct the study. All authors were significantly involved in the redaction of the manuscript.

\section{FUNDING}

This research was supported by a grant from the Rhône-Alpes Region in the cluster "Handicap, Aging, Neurosciences."

\section{ACKNOWLEDGMENTS}

The authors wish to thank Benoit Riou and Lionel Brunel for their assistance in this project. The authors also thank Marie Barbier for her help to recruit and evaluate some of the participants included in the study.

\section{SUPPLEMENTARY MATERIAL}

The Supplementary Material for this article can be found online at: http://journal.frontiersin.org/article/10.3389/fpsyg. 2017.01493/full\#supplementary-material

amnestic mild cognitive impairment and dementia from cognitively normal elderly. Arch. Clin. Neuropsychol. 32, 29-39. doi: 10.1093/arclin/acx046

Butler, K. M., McDaniel, M. A., Dornburg, C. C., Price, A. L., and Roediger, H. III. (2004). Age differences in veridical and false recall are not inevitable: the role of frontal lobe function. Psychon. Bull. Rev. 11, 921-925. doi: 10.3758/BF031 96722

Butler, K. M., McDaniel, M. A., McCabe, D. P., and Dornburg, C. C. (2010). The influence of distinctive processing manipulations on older adults' false memory. Neuropsychology 17, 129-159. doi: 10.1080/13825580903029715

Callahan, B., Macoir, J., Hudon, C., Bier, N., Chouinard, N., Cossette-Harvey, M., et al. (2010). Normative data for the pyramids and palm trees test in the Quebec-French population. Arch. Clin. Neuropsychol. 25, 212-217. doi: 10.1093/arclin/acq013

Campbell, K. L., Trelle, A., and Hasher, L. (2014). Hyper-binding across time: age differences in the effect of temporal proximity on paired-associate learning. J. Exp. Psychol. Learn. Mem. Cogn. 40, 293-299. doi: 10.1037/a00 34109

Cardebat, D., Doyon, B., Puel, M., and Goulet, P. (1990). Evocation lexicale formelle et sémantique chez des sujets normaux. Performance et dynamiques de production en fonction du sexe, de l'âge et du niveau culturel. Acta Neurol. Belg. 90, 207-217.

Casasanto, D. (2011). Different bodies, different minds: the body specificity of language and thought. Curr. Dir. Psychol. Sci. 20, 378-383. doi: $10.1177 / 0963721411422058$

Chan, J. C. K., and McDermott, K. B. (2007). The effects of frontal lobe functioning and age on veridical and false recall. Psychon. Bull. Rev. 14, 606-611. doi: 10.3758/BF03196809 
Cummings, J. L., Mega, M., Gray, K., Rosenberg-Thompson, S., Carusi, D., and Gornbein, J. (1994). The neuropsychiatric inventory: comprehensive assessment of psychopathology in dementia. Neurology 44, 2308-2314. doi: 10.1212/WNL.44.12.2308

Danckert, S. L., and Craik, F. I. (2013). Does aging affect recall more than recognition memory? Psychol. Aging 28, 902-909. doi: 10.1037/a00 33263

Delbeuck, X., Collette, F., and Van der Linden, M. (2007). Is Alzheimer's disease a disconnection syndrome? Evidence from a crossmodal audio-visual illusory experiment. Dementia 45, 3315-3323. doi: 10.1016/j.neuropsychologia.2007.05.001

Delbeuck, X., Van der Linden, M., and Collette, F. (2003). Alzheimer's disease as a disconnection syndrome? Neuropsychol. Rev. 13, 79-92. doi: 10.1023/A:1023832305702

Delis, D., Kaplan, E., and Kramer, J. (2001). D-KEFS Executive Function System. San Antonio: The Psychological Corporation, a Harcourt Assessment Company.

Devitt, A. L., and Schacter, D. L. (2016). False memories with age: neural and cognitive underpinnings. Neuropsychologia 91, 346-359. doi: 10.1016/j.neuropsychologia.2016.08.030

Dodson, C. S., and Schacter, D. L. (2002). Aging and strategic retrieval processes: reducing false memories with a distinctiveness heuristic. Psychol. Aging 17, 405-415. doi: 10.1037/0882-7974.17.3.405

Doubleday, E., and Snowden, J. J. S. (2002). Qualitative performance characteristics differentiate dementia with Lewy bodies and Alzheimer's disease. J. Neurol. Neurosurg. Psychiatr. 72, 602-607. doi: 10.1136/jnnp.72.5.602

Estévez-González, A., Kulisevsky, J., Boltes, A., Otermín, P., and García-Sánchez, C. (2003). Rey verbal learning test is a useful tool for differential diagnosis in the preclinical phase of Alzheimer's disease: comparison with mild cognitive impairment and normal. Int. J. Geriatr. Psychiatry 18, 1021-1028. doi: 10.1002 /gps.1010

Festa, E., Insler, R., Salmon, D. P., Paxton, J., Hamilton, J., and Heindel, W. C. (2005). Neocortical disconnectivity disrupts sensory integration in Alzheimer's disease. Neuropsychology 19, 728-738. doi: 10.1037/0894-4105.19.6.728

Fleischman, D. A. (2007). Repetition priming in aging and Alzheimer's disease: an integrative review and future directions. Cortex 43, 889-897. doi: 10.1016/S0010-9452(08)70688-9

Fleischman, D. A., and Gabrieli, J. D. E. (1999). Long-term memory in Alzheimer's disease. Curr. Opin. Neurobiol. 9, 240-244. doi: 10.1016/S0959-4388(99)80034-8

Folstein, M. (1975). "Mini-mental state." A practical method for grading the cognitive state of patients for the clinician. J. Psychiatr. Res. 12, 189-198. doi: 10.1016/0022-3956(75)90026-6

Geraci, L., McDaniel, M. A., Manzano, I., and Roediger, H. L. (2009). The influence of age on memory for distinctive events. Mem. Cognit. 37, 175-180. doi: 10.3758/MC.37.2.175

Glenberg, A. M., Witt, J. K., and Metcalfe, J. (2013). From the revolution to embodiment: 25 years of cognitive psychology. Perspect. Psychol. Sci. 8, 573-585. doi: 10.1177/1745691613498098

Godefroy, O., Azouvi, P., Robert, P., Roussel, M., LeGall, D., and Meulemans, T. (2010). Dysexecutive syndrome: diagnostic criteria and validation study. Ann. Neurol. 68, 855-864. doi: 10.1002/ana.22117

Graham, K. S., Barense, M. D., and Lee, A. C. H. (2010). Going beyond LTM in the MTL: a synthesis of neuropsychological and neuroimaging findings on the role of the medial temporal lobe in memory and perception. Neuropsychologia 48 , 831-853. doi: 10.1016/j.neuropsychologia.2010.01.001

Graham, K. S., Simons, J. S., Pratt, K. H., Patterson, K., and Hodges, J. R. (2000). Insights from semantic dementia on the relationship between episodic and semantic memory. Neuropsychologia 38, 313-324. doi: 10.1016/S0028-3932(99)00073-1

Greenaway, M. C., Lacritz, L. H., Binegar, D., Weiner, M. F., Lipton, A., and Munro Cullum, C. (2006). Patterns of verbal memory performance in mild cognitive impairment, Alzheimer disease, and normal aging. Cogn. Behav. Neurol. 19, 79-84. doi: 10.1097/01.wnn.0000208290.57370.a3

Greenberg, D. L., and Verfaellie, M. (2010). Interdependence of episodic and semantic memory: evidence from neuropsychology. J. Int. Neuropsychol. Soc. 16, 748-753. doi: 10.1017/S1355617710000676
Hintzman, D. L. (1990). Human learning and memory: connections and dissociations. Annu. Rev. Psychol. 41, 109-319. doi: 10.1146/annurev.ps.41.020190.000545

Hodges, J. R., and Patterson, K. (2007). Semantic dementia: a unique clinicopathological syndrome. Lancet Neurol. 6, 1004-1014. doi: 10.1016/S1474-4422(07)70266-1

Hoffman, P., Evans, G. A. L., and Lambon Ralph, M. A. (2014). The anterior temporal lobes are critically involved in acquiring new conceptual knowledge: evidence for impaired feature integration in semantic dementia. Cortex 50 , 19-31. doi: 10.1016/j.cortex.2013.10.006

Howard, D., and Patterson, K. (1992). Pyramids and Palm Trees: a Test of Semantic Access from Pictures and Words. Bury St. Edmunds: Thames Valley Test Company.

Humes, L. E., Busey, T. A., Craig, J., and Kewley-Port, D. (2013). Are age-related changes in cognitive function driven by age-related changes in sensory processing? Atten. Percept. Psychophys. 75, 508-524. doi: 10.3758/s13414-012-0406-9

Hunt, R. R., and Lamb, C. A. (2001). What causes the isolation effect? J. Exp. Psychol. Learn. Mem. Cogn. 27, 1359-1366. doi: 10.1037/0278-7393.27.6.1359

Johnson, M. K., Hashtroudi, S., and Lindsay, D. S. (1993). Source monitoring. Psychol. Bull. 114, 3-28. doi: 10.1037/0033-2909.114.1.3

Koen, J. D., and Yonelinas, A. P. (2014). The effects of healthy aging, amnestic mild cognitive impairment, and Alzheimer's disease on recollection and familiarity: a meta-analytic review. Neuropsychol. Rev. 24, 332-354. doi: 10.1007/s11065-014-9266-5

Laurienti, P. J., Burdette, J. H., Maldjian, J. A., and Wallace, M. T. (2006). Enhanced multisensory integration in older adults. Neurobiol. Aging 27, 1155-1163. doi: 10.1016/j.neurobiolaging.2005.05.024

Lezak, M., Howieson, D., Loring, D., and Fisher, J. (2004). Neuropsychological Assessment 4th Edn. New York, NY: Oxford University Press.

MacDuffie, K. E., Atkins, A. S., Flegal, K. E., Clark, C. M., and ReuterLorenz, P. A. (2012). Memory distortion in Alzheimer's disease: deficient monitoring of short- and long-term memory. Neuropsychology 26, 509-516. doi: $10.1037 / \mathrm{a} 0028684$

McKhann, G., Drachman, D., Folstein, M., Katzman, R., Price, D., and Stadlan, E. M. (1984). Clinical diagnosis of Alzheimer's disease: report of the NINCDS-ADRDA Work Group* under the auspices of department of health and human services task force on Alzheimer's disease. Neurology 34:939. doi: 10.1212/WNL.34.7.939

Meade, M. L., Geraci, L. D., and Roediger, I. I. I., H. (2012). Neuropsychological status in older adults influences susceptibility to false memories. Am. J. Psychol. 125, 449-467. doi: 10.5406/amerjpsyc.125.4.0449

Neary, D., Snowden, J. S., Gustafson, L., Passant, U., Stuss, D., Black, S. E., et al. (1998). Frontotemporal lobar degeneration: a consensus on clinical diagnostic criteria. Neurology 53:1158. doi: 10.1212/WNL.51.6.1546

Nyberg, L., Maitland, S. B., Rönnlund, M., Bäckman, L., Dixon, R. A., Wahlin, A., et al. (2003). Selective adult age differences in an age-invariant multifactor model of declarative memory. Psychol. Aging 18, 149-160. doi: 10.1037/0882-7974.18.1.149

Okado, Y., and Stark, C. (2003). Neural processing associated with true and false memory retrieval. Cogn. Affect. Behav. Neurosci. 3, 323-334. doi: 10.3758/CABN.3.4.323

Oker, A., Versace, R., and Ortiz, L. (2009). Spatial distinctiveness effect in categorisation. Eur. J. Cogn. Psychol. 21, 971-979. doi: 10.1080/09541440802547567

Park, D. C., and Gutchess, A. H. (2002). Aging, cognition, and culture: a neuroscientific perspective. Neurosci. Biobehav. Rev. 26, 859-867. doi: 10.1016/S0149-7634(02)00072-6

Pasquier, F., and Grymonprez, L. (2001). Memory impairment differs in frontotemporal dementia and Alzhemier's disease. Neurocase 7, 161-171. doi: 10.1093/neucas/7.2.161

Rémy, F., Vayssière, N., Saint-Aubert, L., Barbeau, E., and Pariente, J. (2015). White matter disruption at the prodromal stage of Alzheimer's disease: relationships with hippocampal atrophy and episodic memory performance. Neuroimage 7, 482-492. doi: 10.1016/j.nicl.2015.01.014

Rey, A. E., Vallet, G. T., Riou, B., Lesourd, M., and Versace, R. (2015). Memory plays tricks on me: perceptual bias induced by memory 
reactivated size in Ebbinghaus illusion. Acta Psychol. 161, 104-109. doi: 10.1016/j.actpsy.2015.08.011

Roberts, K. L., and Allen, H. A. (2016). Perception and cognition in the ageing brain: a brief review of the short- and long-term links between perceptual and cognitive decline. Front. Aging Neurosci. 8:39. doi: 10.3389/fnagi.2016.00039

Rouleau, I., Imbault, H., Laframboise, M., and Bedard, M. (2001). Pattern of intrusions in verbal recall: Comparison of Alzheimer's disease, Parkinson's disease, and frontal lobe dementia. Brain Cogn. Tennet. 11, 244-249. doi: 10.1016/S0278-2626(01)80076-2

Scahill, V. L., Hodges, J. R., and Graham, K. S. (2005). Can episodic memory tasks differentiate semantic dementia from Alzheimer's disease? Neurocase 11, 441-451. doi: 10.1080/13554790500287734

Schmidt, S. R. (1991). Can we have a distinctive theory of memory? Mem. Cognit. 19, 523-542. doi: 10.3758/BF03197149

Smith, R. E., Hunt, R. R., and Dunlap, K. R. (2015). Why do pictures, but not visual words, reduce older adults' false memories? Psychol. Aging 30, 647-655. doi: $10.1037 /$ pag0000044

Toner, C. K., Pirogovsky, E., Kirwan, C. B., and Gilbert, P. E. (2009). Visual object pattern separation deficits in nondemented older adults. Learn. Mem. 16, 338-342. doi: 10.1101/lm.1315109

Tulving, E. (1995). “Organization of memory: Quo Vadis?” in The Cognitive Neurosciences, ed M. Gazzaniga (Cambridge, MA: MIT Press), 839-847.

Vallet, G. T. (2015). Embodied cognition of aging. Front. Psychol. 6:463. doi: 10.3389/fpsyg.2015.00463

Vallet, G. T., Brunel, L., and Versace, R. (2010). The perceptual nature of the cross-modal priming effect: arguments in favor of a sensory-based conception of memory. Exp. Psychol. 57, 376-382. doi: 10.1027/1618-3169/ a000045

Vallet, G. T., Brunel, L., Riou, B., and Vermeulen, N. (2016a). Editorial: dynamics of sensorimotor interactions in embodied cognition. Front. Psychol. 6:1929. doi: 10.3389/fpsyg.2015.01929

Vallet, G. T., Rouleau, I., Benoit, S., Langlois, R., Barbeau, E. J., and Joubert, S. (2016b). Alzheimer's disease and memory strength: gradual decline of memory traces as a function of their strength. J. Clin. Exp. Neuropsychol. 3395, 1-13. doi: 10.1080/13803395.2016.1147530

Vallet, G. T., Simard, M., and Versace, R. (2011a). Sensory-dependent knowledge in young and elderly adults: arguments from the cross-modal priming effect. Curr. Aging Sci. 4, 137-149. doi: 10.2174/1874609811104020137
Vallet, G. T., Simard, M., Fortin, C., Versace, R., and Mazza, S. (2011b). L'altération des connaissances sémantiques est-elle liée à une altération du traitement perceptif? Étude des atteintes catégories-spécifiques dans la démence sémantique. Geriatr. Psychol. Neuropsychiatr. Vieil. 9, 327-335. doi: 10.1684/pnv.2011.0276

Vallet, G. T., Simard, M., Versace, R., and Mazza, S. (2013). The perceptual nature of audiovisual interactions for semantic knowledge in young and elderly adults. Acta Psychol. 143, 253-260. doi: 10.1016/j.actpsy.2013.04.009

Van der Linden, M. (2004). "L'épreuve de rappel libre/rappel indicé à 16 items (RL/RI-16)," in L'évaluation des Troubles de La Mémoire, eds M. Van der Linden and F. Coyette (Marseille: Solal), 25-47.

Versace, R., Vallet, G. T., Brunel, L., Riou, B., Lesourd, M., and Labeye, E. (2014). ACT-IN: an integrated view of memory mechanisms. J. Cogn. Psychol. 26, 280-306. doi: 10.1080/20445911.2014.892113

Vitali, P., Minati, L., Chiarenza, G., Brugnolo, A., Girtler, N., Nobili, F., et al. (2006). The Von Restorff effect in ageing and Alzheimer's disease. Neurol. Sci. 27, 166-172. doi: 10.1007/s10072-006-0662-3

Waldie, B. D., and Kwong, S. T. (2003). Remembering words never presented: false memory effects in dementia of the Alzheimer type. Neuropsychol. Dev. Cogn. B Aging Neuropsychol. Cogn. 10, 281-297. doi: 10.1076/anec.10.4.281.28969

Yeung, L., Ryan, J., Cowell, R. A., and Barense, M. D. (2013). Recognition memory impairments caused by false recognition of novel objects. J. Exp. Psychol. Gen. 142, 1384-1397. doi: 10.1037/a0034021

Zimmer, H., Mecklinger, A., and Lindenberger, U. (2006). Handbook of Binding and Memory: Perspectives from Cognitive Neuroscience. Oxford: Oxford University Press.

Conflict of Interest Statement: The authors declare that the research was conducted in the absence of any commercial or financial relationships that could be construed as a potential conflict of interest.

Copyright (c) 2017 Vallet, Hudon, Bier, Macoir, Versace and Simard. This is an open-access article distributed under the terms of the Creative Commons Attribution License (CC BY). The use, distribution or reproduction in other forums is permitted, provided the original author(s) or licensor are credited and that the original publication in this journal is cited, in accordance with accepted academic practice. No use, distribution or reproduction is permitted which does not comply with these terms. 
OPEN ACCESS

Edited by:

Annalisa Setti,

University College Cork, Ireland

Reviewed by:

Michael Barnett-Cowan, University of Waterloo, Canada

Richard A. P. Roche

Maynooth University, Ireland

*Correspondence:

Elzbieta Szelag

e.szelag@nencki.gov.pl

Specialty section: This article was submitted to

Cognition,

a section of the journa

Frontiers in Psychology

Received: 30 June 2016 Accepted: 03 October 2016 Published: 19 October 2016

Citation:

Nowak K, Dacewicz A, Broczek K, Kupisz-Urbanska M, Galkowski Tand Szelag E (2016) Temporal Information Processing and its Relation to Executive Functions in Elderly Individuals. Front. Psychol. 7:1599. doi: 10.3389/fpsyg.2016.01599

\section{Temporal Information Processing and its Relation to Executive Functions in Elderly Individuals}

\author{
Kamila Nowak ${ }^{1,2}$, Anna Dacewicz' ${ }^{1}$ Katarzyna Broczek ${ }^{3}$, Malgorzata Kupisz-Urbanska ${ }^{3}$, \\ Tadeusz Galkowski ${ }^{2}$ and Elzbieta Szelag1,2* \\ ${ }^{1}$ Laboratory of Neuropsychology, Department of Neurophysiology, Nencki Institute of Experimental Biology of Polish \\ Academy of Sciences, Warsaw, Poland, ${ }^{2}$ University of Social Sciences and Humanities, Warsaw, Poland, ${ }^{3}$ Department of \\ Geriatrics, Medical University of Warsaw, Warsaw, Poland
}

Normal aging triggers deterioration in cognitive functions. Evidence has shown that these age-related changes concern also executive functions (EF) as well as temporal information processing (TIP) in a millisecond range. A considerable amount of literature data has indicated that each of these two functions sets a frame for our mental activity and may be considered in terms of embodied cognition due to advanced age. The present study addresses the question whether in elderly subjects the efficiency of TIP is related to individual differences in EF. The study involved 53 normal healthy participants aged from 65 to 78 . In these subjects TIP was assessed by sequencing abilities measured with temporal-order threshold (TOT). It is defined as the minimum time gap separating two auditory stimuli presented in rapid succession which is necessary for a subject to report correctly their temporal order, thus the relation 'before-after.' The EF were assessed with regard to the efficiency of the executive planning measured with the Tower of London-Drexel University (TOL ${ }^{\mathrm{DX}}$ ) which has become a well-known EF task. Using Spearman's rank correlations we observed two main results. Firstly, the indices of the TOL ${ }^{\mathrm{DX}}$ indicated a coherent construct reflecting the effectiveness of executive planning in the elderly. Initiation time seemed dissociated from these coherent indices, which suggested a specific strategy of mental planning in the elderly based on on-line planning rather than on preplanning. Secondly, TOT was significantly correlated with the indices of TOL ${ }^{\mathrm{DX}}$. Although some of these correlations were modified by subject's age, the correlation between TOT and the main index of TOL ${ }^{\mathrm{DX}}$ ('Total Move Score') was rather age resistant. These results suggest that normal aging may be characterized by an overlapping of deteriorated TIP and deteriorated EF.

Keywords: normal aging, temporal information processing, executive functions, executive planning, Tower of London, temporal-order judgement

\section{INTRODUCTION}

In recent decades societies worldwide have been facing demographic changes. A growing number of people aged 60 and older in most European countries and in the US has led to a rapid growth of research studies on cognitive functions in the elderly. Aging triggers significant decline in cognitive functioning. Although it is difficult to define exact moment when a mental activity begins to 
deteriorate, a robust body of experimental evidence has documented that cognitive aging is a dynamic process and refers to declined memory, new learning, attention, perception, multisensory integration, language, motor control, speed of processing, and executive functions (EF, e.g., Salthouse, 2009; Szelag et al., 2010; Setti et al., 2011a,b). The results gathered from numerous samples encompassing a wide range of ages indicated that, despite pronounced individual differences, such deterioration increases markedly after 65 years of life. The accumulated data have also indicated age-related deterioration in temporal information processing (TIP, e.g., Fitzgibbons and Gordon-Salant, 1994; Kumar and Sangamanatha, 2011; Turgeon et al., 2016). These literature data have been supported by the results of studies conducted in our laboratory (Kolodziejczyk and Szelag, 2008; Szymaszek et al., 2009). As patterning in time is one of characteristic features of human cognition (e.g., Pöppel, 1997), one may assume that TIP constitutes a neural basis for a mental activity in norm and pathology, including cognitive decline in normal healthy aging (e.g., Teixeira et al., 2013). Given the importance of TIP for our cognitive functions, the present paper focuses on the relationships between deteriorated TIP and deteriorated EF in advanced age. These relationships seem to be of great importance to understand the mechanisms of normal cognitive aging in terms of embodied cognition (Borghi and Cimatti, 2010).

\section{EF in Controlling Mental Activity}

Executive functions are high demand meta-cognitive processes that guide the optimization of goal-oriented behaviors in unfamiliar circumstances (Etnier and Chang, 2009). Despite a long discussion among neuropsychologists about the nature and definition of EF, researchers agree, in general, that these top-down processes make it possible to plan, execute and control intentional actions. In particular, EF include planning of a sequence of processes to complete a goal, inhibition of distracting events and prepotent responses, as well as management of multiple tasks or subprocesses that make up a complex tasks performance. EF serve to control, monitor and adapt our thoughts and behaviors to changing environmental circumstances (see Diamond, 2013 for a review). EF allow to make future plans, play with ideas, as well as to change ineffective behavior, if necessary.

Numerous studies have revealed vulnerability of EF to the effects of age (see Jurado and Rosselli, 2007 for a review), similarly as that of TIP in the millisecond time range (Szymaszek et al., 2009). Starting with the framework proposed by Miyake et al. (2000), it has been emphasized that EF comprise several constructs, including distinct components of inhibition, scheduling, planning, working memory, coordination, and sequencing (Colcombe and Kramer, 2003). Among EF one can distinguish higher-order problem solving, specifically executive planning abilities, which constitute the topic of the present study.

Some researchers have emphasized that planning is one of the main constructs of EF and that it involves several interrelated but dissociable components (Colcombe and Kramer, 2003; Romine and Reynolds, 2005; Hung et al., 2013). They include identification of goal state development of sub-goal representations, anticipation of consequences, determination of requirements and integration of behaviors for achieving subgoals (Sternberg and Ben-Zeev, 2001).

One of the tasks developed to measure this aspect of EF is Tower of London Task (TOL), the most commonly used planning-related neuropsychological tool (Chang et al., 2011). The original paradigm was invented by Shallice (1982). Since then many versions of this test have been applied in the studies of EF, however, the main goal of this task is to mentally plan a series of moves to match the configuration of beads presented by the examiner. The performance involves planning of sequential moves that are executed, monitored and modified in accordance with an overall plan of action, problem constraints and error feedback (Lezak, 2004). The test performance correlates with the outcomes of tasks evaluating other cognitive functions, i.e., attention (Culbertson and Zillmer, 2005), working memory, flexibility, and inhibition control (Welsh et al., 1999; Huizinga et al., 2006; Luciana et al., 2009).

Planning action depends on control and effectiveness of many cognitive functions. Successful solving of TOL problems requires cooperation of working memory, decision-making, inhibitory control, mental flexibility and sustained attention (Lezak, 2004). According to the authors of the TOL version applied here (Tower of London-Drexel University, $\mathrm{TOL}^{\mathrm{DX}}$ ), the measurement is sensitive to executive problem solving and planning, behavioral inhibition and impulsivity control, attentional allocation, cognitive flexibility, abstract/conceptual reasoning, rule-governed behavior and monitoring (Culbertson and Zillmer, 2005). Several studies have revealed that the abilities measured with TOL (or Tower of Hanoi) decreased in patients with dysfunctions resulting from frontal lobe lesions, as well as in normal healthy people in advanced age. This age-related decrease starts at the age of 60 (Zook et al., 2006), 65 (Brennan et al., 1997), or even later, in the eighth decade of life (Davis and Klebe, 2001). Some authors link declined EF with general slowdown of pace of information processing and its contribution to working memory efficiency (Fisk and Warr, 1996).

Age-related changes in EF find its support in the hypothesis of frontal lobe contributions to mental deterioration in aging, assuming that declined cognitive function may be associated with changes in the structure and functionality of frontal lobes (West, 1996). They lead, in consequence, to noticeable decline in various aspects of EF and other non-executive cognitive functions, such as attention, memory, motor control, etc. Structural changes are mostly associated with the reduction in gray and white matter volume of frontal lobes, while functional changes are reflected, e.g., in compensatory higher activity in prefrontal cortex or abnormal functional connectivity in frontal regions (for review see Grady, 2012).

To explain individual differences in age-related changes in EF one may refer to recent neurocognitive models developed to explain deficient or preserved performance in the elderly (Davis et al., 2008; see also Reuter-Lorenz and Park, 2010 and Sala-Llonch, Bartrés-Faz and Junqué, 2015 for a recent summary). For example, PASA model refers to posterioranterior shift of activity in aging and assumes enhanced activity in anterior regions, including prefrontal cortex (e.g., Grady et al., 1994; Cabeza et al., 1997, 2004; Davis et al., 2008). 
On the other hand, HAROLD model (Hemispheric Asymmetry Reduction in Older Adults by Cabeza et al., 1997, 2002; Cabeza and Dennis, 2012) associates compensatory mechanisms with more bilateral activations in prefrontal cortex. However, higher activation in anterior regions in aging does not necessarily have to be accompanied by better performance. This phenomenon was pointed out in CRUNCH model (Compensation-Related Utilization of Neural Circuits Hypothesis; Reuter-Lorenz and Cappell, 2008). Accordingly, the engagement of more resources at lower task demands may result in reduced resources available in more demanding tasks (Reuter-Lorenz and Cappell, 2008; Reuter-Lorenz and Park, 2010). Finally, the STAC model (Scaffolding Theory of Aging and Cognition; Park and ReuterLorenz, 2009) proposes the engagement of alternative neural circuits that allow to maintain a high level of cognitive function in the elderly.

Considering this body of evidence the relations may be anticipated between age-related changes in frontal lobe and tasks that pertain to EF.

\section{TIP in Controlling Mental Activity}

It is commonly accepted that chronological age does not correspond perfectly to mental age, as two people may be of the same age, but differ in their mental capacity (Szelag et al., 2011). A challenging problem is to identify neural processes (or mechanisms) that account for poorer EF in late adulthood. As mentioned at the very beginning, temporal dynamics of information processing creates a neural frame for many cognitive functions, including EF (Pöppel, 1997, 2009; Szelag et al., 2010). Evidence from both various clinical populations and normal sample has indicated that numerous cognitive functions are rooted in the exact temporal template which creates a neural frame for the optimal mental activity. A disordered time frame is often reported in different neurodevelopmental and neurodegenerative deficits, as well as in healthy aging (Fink et al., 2005; see Teixeira et al., 2013 for a review). For example, to create and execute mental activities effectively one has to monitor the passage of time, to react and to change our behavior in time. TIP is embedded in EF such as, for instance, planning, evaluation of previous actions and decision making.

The idea of time being inherent in human cognition is not new. It derived from philosophical ideas (e.g., James, 1890) and has since been investigated in many psychological, psychophysical, and neuroimaging studies (e.g., Pöppel, 1997; Nenadic et al., 2003; Lewandowska et al., 2010; Bedard and Barnett-Cowan, 2016). In general, TIP has been categorized into two major time scales, i.e., milli- and multisecond one. On the basis of various experimental paradigms and subject populations, data has indicated that on these two levels TIP may be influenced by various subject-related factors, among which subject's age seems one of the most important (for the overview see Szelag et al., 2004, 2010, 2011; Bao et al., 2013; Allman et al., 2014; Matthews and Meck, 2014).

The evidence supporting TIP deteriorations resulting from cognitive aging indicates reduced temporal acuity and comes from experiments on duration discrimination, temporal generalization, temporal bisection, time estimation, gap detection, and perception of temporal order (see Allman et al., 2014; Matthews and Meck, 2014; Turgeon et al., 2016 for reviews). The latter paradigm is applied in the present study and concerns the ability to sequence incoming information, considered as a neural basis of the identification of events (Pöppel, 1994, 1997; Szelag et al., 2004). Several studies have revealed that the identification of temporal order of two acoustic stimuli is only possible when they are separated by an interstimulus interval of at least 20-60 ms (Hirsh and Sherrick, 1961, see also Szelag et al., 2004; Wittmann and Fink, 2004 for reviews). Nearly twice as long an interval is required in the elderly, which suggests declined temporal resolution. This supports the notion of temporally discrete information processing within some tens of milliseconds time window (see e.g., Pöppel, 1997; Wittmann, 1999; Szelag et al., 2001) and suggests the existence of an internal timing mechanism that controls our sequential information processing ability (Hirsh and Sherrick, 1961).

The most interesting result was that the sensitive indicator of declined TIP was not the subjects' chronological age, but cognitive competencies (Fink et al., 2005; Teixeira et al., 2013). Seniors beyond 65 years with TIP declined to a lesser extent showed relatively better preserved cognitive status than those with more severely deteriorated TIP (Szelag et al., 2011).

These data may find some support in the processingspeed theory of cognitive aging by Salthouse (1996), which binds cognitive deficits commonly observed in the elderly with a slower pace of information processing. Accordingly, agerelated changes contribute to the decrease in the speed and amount of mental operations that can be processed at a time. Moreover, the reduced speed of processing leads to difficulties in efficient planning, executing and completion of mental processes because of time limitations for these operations in our mental activity.

\section{Study Aims}

The relation 'TIP - EF' has not been investigated comprehensively in the existing literature. The present study, therefore, offers an important new approach addressing the question whether in elderly individuals the differences in millisecond TIP are related to declined EF. Although both these functions (i.e., TIP and EF) were investigated in the previous studies separately (see above), to our knowledge no studies to date have examined the associations between age-related changes in these two domains.

Considering these limitations, the aim of the present study was twofold. Firstly, we attempted to clarify the relationships between particular indices of the EF assessed with the TOL ${ }^{\mathrm{DX}}$ test in subjects beyond 65 years of life. We expected to replicate some previous findings which were focused on much wider range of ages than those tested in our study. Secondly, to understand the relation 'TIP - EF' we verified the correlations between the indices from the TOL ${ }^{\mathrm{DX}}$ and effectiveness of TIP assessed with sequencing abilities in the millisecond time range. 


\section{MATERIALS AND METHODS}

The study was approved by the local Ethical Commission at the University of Social Science and Humanities (permission no 3/II/11-12). All participants gave their written informed consent prior to the study.

\section{Participants}

Fifty-three elderly participants (46 females/7 males), aged 65 to $78(M=69.1 ; S D=3.4)$ took part in the study. They were right-handed (Edinburgh Handedness Inventory, EHI, Oldfield, 1971) and free of any neurological and psychiatric disorders or systemic diseases. The abovementioned criteria were verified during interviews and geriatric examinations performed by professional geriatricians from the Geriatric Clinic of Medical University of Warsaw. The geriatric assessment included physical examination, assessment of functional performance and evaluation of currently taken medications. Individuals with supposedly poor or unstable health conditions or those receiving medications affecting the central nervous system were excluded from the study.

All participants had a normal hearing level (Ansi, 2004) in the frequency range from 250 to $3000 \mathrm{~Hz}$. Frequencies included: 250, $500,750,1000,1500,2000$, and $3000 \mathrm{~Hz}$ and encompassed the sound frequency spectrum presented in our study. An inclusion criterion for all participants was that the difference between these frequencies, as well as between the left and right ear (for a given frequency) did not exceed $30 \mathrm{~dB}$.

Prior to the experiment proper all participants completed Mini-Mental State Examination (MMSE, Folstein et al., 2001) to screen for dementia. An inclusion criterion was the score of at least 27 points on this examination. Additionally, Geriatric Depression Scale (GDS, short form; Shiekh and Yesavage, 1986) was completed to screen for depression. The candidates who scored above 5 points on this scale were excluded from the study.

Verification of all the abovementioned criteria allowed us to expect that the subjects included into our sample were in relatively good health, both physically and mentally. Thus, it may be assumed that they exhibited the level of mental functions characteristic for normal, healthy cognitive aging. The characteristics of the subjects sample are presented in Table 1.

TABLE 1 | The characteristics of the subjects sample.

\begin{tabular}{llccc}
\hline Variable & N & Mean & SD & Range \\
\hline Age (years) & 53 & 69.1 & 3.4 & $65-78$ \\
Sex (female/male) & $46 / 7$ & - & - & - \\
Education (years) & 53 & 15 & 2.7 & $9-21$ \\
GDS (score) & 53 & 2 & 1.7 & $0-5$ \\
MMSE (score) & 53 & 29.2 & 0.8 & $27-30$ \\
EHI (score) & 53 & 89 & 13.1 & $60-100$
\end{tabular}

GDS, Geriatric Depression Scale (short form); MMSE, Mini-Mental State Examination; EHI, Edinburgh Handedness Inventory.

\section{Experimental Procedures}

Two experimental procedures were applied in this study: (1) Tower of London task and (2) Temporal-Order Judgment task. These procedures were completed by the subjects during individual sessions conducted by the experimenter.

\section{Tower of London Task}

Executive functions were assessed with Tower of London-Drexel University task: 2nd Edition $\left(\mathrm{TOL}^{\mathrm{DX}}\right.$, Culbertson and Zillmer, $2005)^{1}$. The test consisted of two identical tower boards, one for the participant and one for the examiner. Each structure contained a board with three pegs and a set of three beads (red, green, and blue). The participant was instructed to replicate configurations of beads presented on the examiner's tower board in as limited number of moves as possible. During the execution of each trial the participant had to follow two rules: (1) it was prohibited to place more beads on a peg than it could accommodate, and (2) one bead could be moved from pegs at the time while other beads had to be kept on pegs. The examples of experimental procedure are displayed in Figure 1.

The task was described in a technical manual (Culbertson and Zillmer, 2005) and consisted of 10 trials of increasing difficulty, which corresponded to the minimum number of moves necessary to replicate the presented configuration. Accordingly, the entire test included two trials with the minimum of four moves, three trials with five moves, three trials with six moves, and two trials with seven moves. Prior to the task proper, the participant completed two practice trials with two moves.

The duration of each trial, including the inspection and execution of a presented target position, was measured by the experimenter. Time limit for each predetermined target position was $1 \mathrm{~min}$. If this limit was exceeded, the trial was classified as time violation (see Table 2). The subject was not informed about any time limitation.

We analyzed seven outcome indices reported by Culbertson and Zillmer (2005). They were grouped into three main categories (Hung et al., 2013): (1) Move Performance ('Total Correct Score,' 'Total Move Score'), (2) Time Efficiency ('Initiation Time,' 'Execution Time,' 'Total Time') and (3) Violation Adherence ('Total Time Violations,' 'Total Rule Violations') which are briefly summarized in Table 2.

\section{Temporal-Order Judgment Task}

To measure the effectiveness of auditory perception of temporal order we used two-element sequences of acoustic stimuli (for detailed description see Szymaszek et al., 2009; Oron et al., 2015). They were paired clicks (rectangular pulses) of $1 \mathrm{~ms}$ duration each. The stimuli were generated by a 16-bit Sound Blaster Extigy Sound Card and delivered at a comfortable listening level via the Sennheiser HD 205 headphones. Paired clicks were presented monaurally, i.e., one click was exposed to the left ear and the other click to the right ear. These clicks were separated by various inter-stimulus intervals (ISIs), which reflected the time

\footnotetext{
${ }^{1}$ This version was applied as it allows to analyze complex subjects' performance on
} the basis of various indices (see below). 


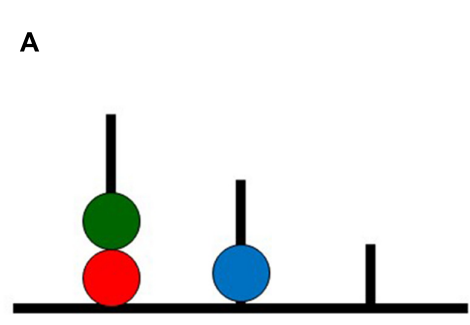

Subject's Tower Board (start position)

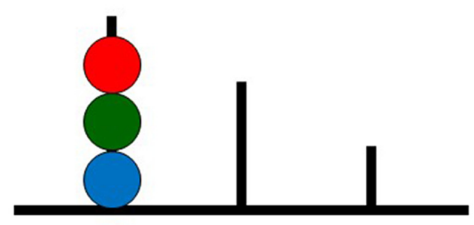

Examiner's Tower Board (presented configuration)

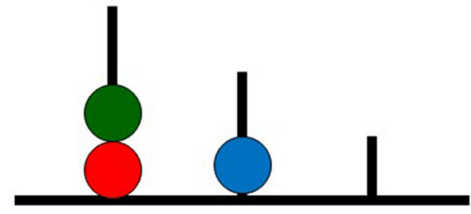

Subject's Tower Board (start position)

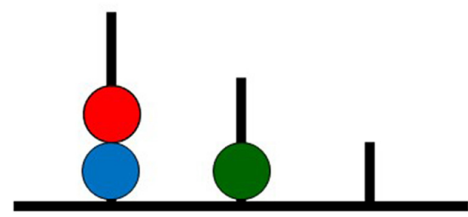

Examiner's Tower Board (presented configuration)

FIGURE 1 | Two examples (A,B) of Tower of London-Drexel University (TOL ${ }^{\mathrm{DX}}$ ) problems with a minimum number of 6 moves necessary to achieve the presented configuration.

TABLE 2 | The TOL ${ }^{\mathrm{DX}}$ outcome measures grouped into three main categories reflecting cognitive processes underlying the test performance.

\begin{tabular}{|c|c|c|c|}
\hline Category & Particular outcome measures & Definition & Associated cognitive processes \\
\hline \multirow[t]{2}{*}{ MOVE PERFORMANCE } & Total Move Score (TMS) & $\begin{array}{l}\text { The number of moves that exceeded the } \\
\text { minimum number of moves necessary to } \\
\text { replicate configurations presented by the } \\
\text { examiner }\end{array}$ & Quality of executive planning \\
\hline & Total Correct Score (TCS) & $\begin{array}{l}\text { the number of trials solved in a minimum } \\
\text { number of moves }\end{array}$ & Working memory capacity and control \\
\hline \multirow[t]{3}{*}{ TIME EFFICIENCY } & Initiation Time (IT) & $\begin{array}{l}\text { The time between the presentation of the } \\
\text { configuration by the experimenter and removing } \\
\text { the first bead from the peg by the participant }\end{array}$ & $\begin{array}{l}\text { Inhibitory response processes and } \\
\text { preparation of planning }\end{array}$ \\
\hline & Execution Time (ET) & $\begin{array}{l}\text { The time needed to solve each trial measured } \\
\text { from the first to the last move by the participant }\end{array}$ & $\begin{array}{l}\text { Speed or pace at which executive } \\
\text { plans are operationalized }\end{array}$ \\
\hline & Total Time (TT) & The sum of the IT and TET & $\begin{array}{l}\text { Overall speed of executive planning and } \\
\text { problem-solving speed }\end{array}$ \\
\hline \multirow[t]{2}{*}{ VIOLATION ADHERENCE } & Total Time Violations (TTV) & $\begin{array}{l}\text { Number of trials in which the } \Pi \text { exceeded } \\
1 \mathrm{~min}\end{array}$ & $\begin{array}{l}\text { Ability to plan and execute problems } \\
\text { solving in a specific temporal period, } \\
\text { cognitive processing control }\end{array}$ \\
\hline & Total Rule Violations (TRV) & The number of violation of required rules & $\begin{array}{l}\text { Ability to govern and control executive } \\
\text { planning according to the applicable } \\
\text { restrictions }\end{array}$ \\
\hline
\end{tabular}

gap between the offset of the first click and the onset of the second click within a pair. The participant reported the temporal order of two clicks presented in rapid sequence by pointing to one of two response cards. Two alternative cards were used: 'right-left' or 'left-right.' Experimental situation is displayed in Figure 2. The values of ISI applied in this study varied from 1 to $200 \mathrm{~ms}$, according to an adaptive maximum-likelihoodbased algorithm (Treutwein, 1997; Szymaszek et al., 2009). First, using up-and-down testing ten paired stimuli were presented with constant decreasing ISIs of $160,120,81,41$ and $1 \mathrm{~ms}$ following by increasing ones of $1,41,81,120$ and $160 \mathrm{~ms}$. On the basis of subject's response correctness, the program estimated the probability of making a correct response to a stimulus having a given ISI according to Treutwein (1997, p. 131). Next, ISI in each trial was adjusted adaptively on the basis of correctness of the subject's previous responses. The algorithm of 


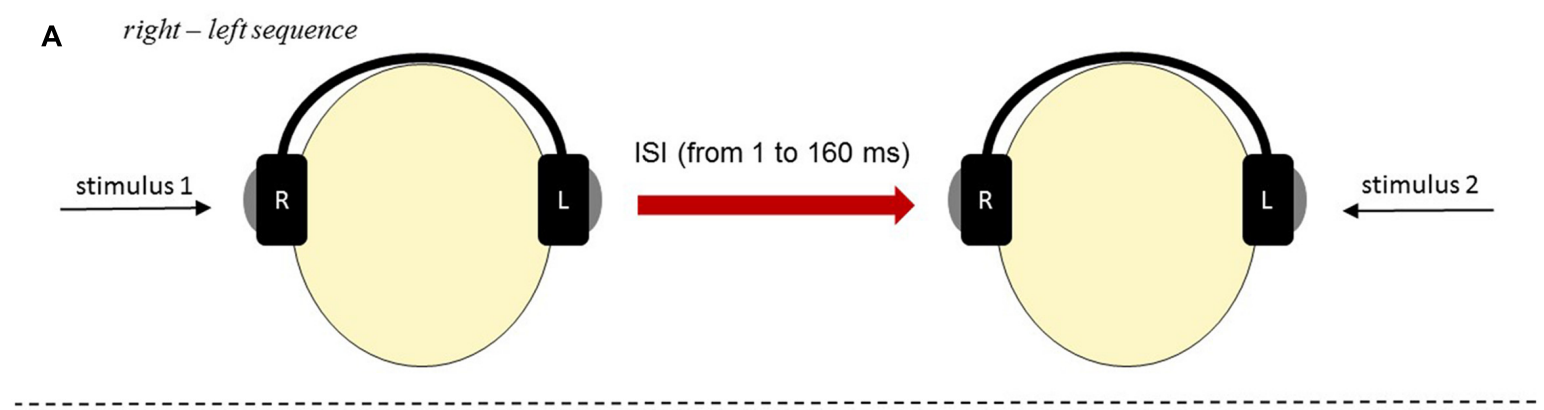

B

left-right sequence

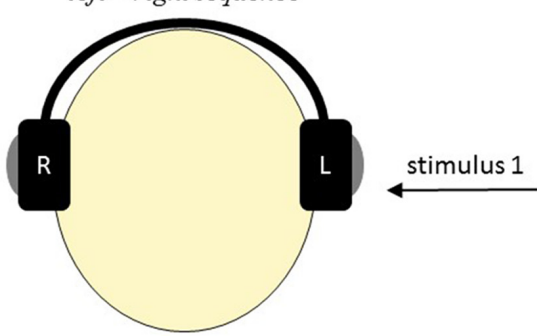

ISI (from 1 to $160 \mathrm{~ms}$ )

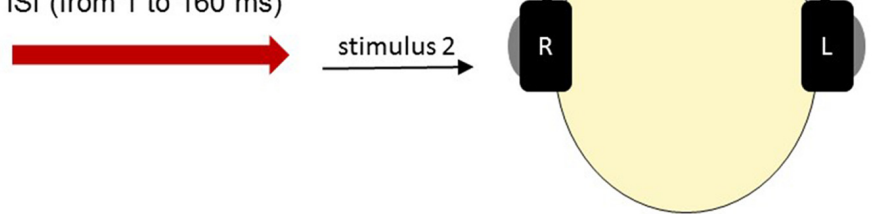

FIGURE 2 | The scheme of stimuli presentation in TOJ Task: (A) 'right-left' sequence and (B) 'left-right' sequence.

the up-and-down method decreased the ISI following a correct response and increased ISI after incorrect responses. ISIs in the consecutive trials were determined by YAAP algorithm based on maximum likelihood parameter estimation. Each session was terminated when a $95 \%$ confidence was reached within \pm 10 steps. The criterion was threshold value within $\pm 10 \mathrm{~ms}$ confidence interval at $75 \%$ probability level. Temporal-order threshold (TOT) reflected effectiveness of TIP and sequencing abilities.

An introductory session was conducted prior to the experiment proper to familiarize the participant with the task. In this session constant, relatively long ISI of $160 \mathrm{~ms}$ was applied in 12 consecutive trials. Once the pre-defined criterion of 10 correct responses was achieved in the last 11 successive trials, the proper experiment started.

\section{Statistical Analyses}

First, Kolmogorov-Smirnov test was applied to verify normal distribution of the data achieved. As the results showed that more than $50 \%$ of the data was not normally distributed, non-parametric Spearman's rank correlations were applied to investigate: (1) the performance of the subjects on $\mathrm{TOL}^{\mathrm{DX}}$ task, and (2) the relationships between the outcome measures obtained on $\mathrm{TOL}^{\mathrm{DX}}$ and TOJ.

\section{RESULTS}

The results obtained in both tasks are summarized in Table 3 . The score of the sample tested are presented with reference to the results obtained in the previous studies (last column) for the comparable age group to that tested presently. Reference data were previously published in (1) normalization study for the $\mathrm{TOL}^{\mathrm{DX}}$ (Culbertson and Zillmer, 2005; Table 4.2, p. 47) and (2) in our previous reports for the TOT (Szymaszek et al., 2009, p. 141).

\section{The Characteristic of Performance on TOL ${ }^{\mathrm{DX}}$ and TOJ Tasks}

As presented in Table 3, mean values of the particular outcome measures of the $\mathrm{TOL}^{\mathrm{DX}}$ task obtained in this study were placed within one standard deviation from the results obtained in the normalization of TOL ${ }^{\mathrm{DX}}$ task for the American-Canadian sample in the similar age group (60- to 80 -years old, $n=39$; Culbertson and Zillmer, 2005; Table 4.2, p. 47).

In the present study, mean TOT value was $92 \mathrm{~ms}( \pm 32 \mathrm{~ms})$. This result is consistent with the data from our previous study where participants aged from 60 to 69 showed the TOT value between 80 and 90 ms (Szymaszek et al., 2009, p. 141). Figure 3 shows typical responses and equation fit from an exemplar participant whose data were close to median responses.

On the basis of this evidence, we may assume that the subjects included into the present study were within the normal limit for both EF (assessed with TOL ${ }^{\mathrm{DX}}$ ) and TIP (assessed with TOJ).

\section{The Relationships Observed in the TOL $^{\text {DX }}$ Task}

In order to better understand the relationship between $\mathrm{EF}$ and timing we analyzed thoroughly how the participants performed in $\mathrm{TOL}^{\mathrm{DX}}$ test and conducted correlational analyses between particular indices of this test. First, we studied the correlations between indices within a given category (Table 2) followed by 
TABLE 3 | Descriptive statistics of results obtained in TOL ${ }^{D X}$ and TOJ tasks.

\begin{tabular}{|c|c|c|c|c|c|}
\hline & Measurement & Mean & $S D$ & Range & Reference data Mean ( \pm SD) \\
\hline & TOL $^{\mathrm{DX}}$ & & & & \\
\hline \multirow[t]{2}{*}{ Move Performance } & TCS & 4.5 & 2 & $1-9$ & $3.3( \pm 1.7)$ \\
\hline & TMS & 33.2 & 17.5 & $1-75$ & $38.8( \pm 15.6)$ \\
\hline \multirow[t]{3}{*}{ Time Efficiency } & $\mathrm{IT}(\mathrm{s})$ & 75 & 40.5 & 29-193 & $72( \pm 64.6)$ \\
\hline & $\mathrm{ET}(\mathrm{s})$ & 304.8 & 112.9 & $111-598$ & $285.3( \pm 119.7)$ \\
\hline & $\Pi(\mathrm{s})$ & 380.1 & 143.4 & $111-799$ & $357.4( \pm 159.5)$ \\
\hline \multirow[t]{3}{*}{ Violation Adherence } & TTV & 1.7 & 1.4 & $0-5$ & $1.0( \pm 1.4)$ \\
\hline & TRV & 0.9 & 1.4 & $0-5$ & $0.7( \pm 1.4)$ \\
\hline & TOT (ms) & 92 & 32 & $32-162$ & 80-90 \\
\hline
\end{tabular}

TCS, Total Correct Score; TMS, Total Move Score; IT, Initiation Time; ET, Execution Time; TT, Total Time; TTV, Total Time Violation; TRV, Total Rule Violation; TOT, Temporal-Order Threshold.

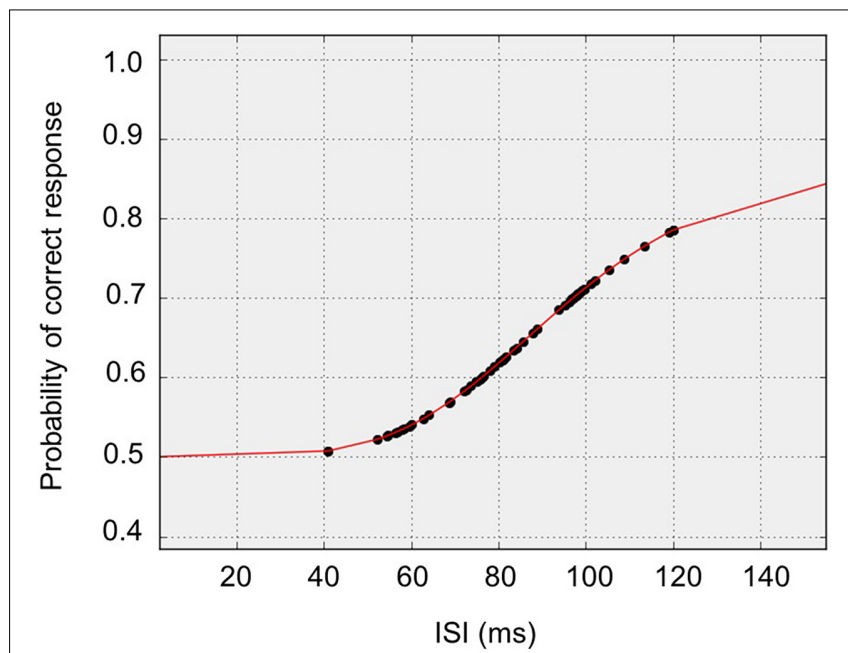

FIGURE 3 | Median subject data: typical responses and equation fit from an exemplar participant whose data was close to median responses.

indices between categories. The results are described below and summarized in Table 4.

The indices from the category Move Performance ('Total Correct Score' and 'Total Move Score') displayed a strong ${ }^{2}$ negative correlation with each other $\left(r_{s}=-0.70 ; p<0.001\right)$. Additionally, 'Total Correct Score' showed significant correlations with all other measures from $\mathrm{TOL}^{\mathrm{DX}}$, such as: a moderate significant relation to 'Execution Time' ( $\left.r_{s}=-0.49 ; p<0.001\right)$, weak but significant correlations with 'Initiation Time' $\left(r_{s}=0.29 ; p<0.04\right)$, 'Total Time' $\left(r_{s}=-0.33 ; p<0.02\right)$, 'Total Rule Violations' $\left(r_{s}=-0.37\right.$; $p<0.01)$ and 'Total Time Violations' $\left(r_{s}=-0.32 ; p<0.05\right)$. On the other hand, 'Total Move Score' strongly correlated with 'Total Time' $\left(r_{s}=0.65 ; p<0.001\right)$, 'Execution Time' $\left(r_{s}=0.76\right.$; $p<0.001)$ and 'Total Time Violations' $\left(r_{s}=0.61 ; p<0.001\right)$. We also found a weak positive correlation between 'Total Move Score' and 'Total Rule Violations' $\left(r_{s}=0.37 ; p<0.01\right)$.

To sum up, a greater number of problems solved correctly was accompanied by fewer redundant moves, shorter total duration

\footnotetext{
${ }^{2}$ Effect sizes according to Cohen (1988).
}

of test performance, shorter initiation time, shorter time of each problem solving, and fewer rule violations. On the other hand, a smaller number of redundant moves was accompanied by shorter duration of test performance, shorter time of each problem solving and fewer time- and rule- violations.

Considering the outcome measures from the category Time Efficiency, we found a very strong positive correlation between 'Total Time' and 'Execution Time' $\left(r_{s}=0.94 ; p<0.001\right)$, as well as a weak positive correlation between 'Total Time' and 'Initiation Time' $\left(r_{s}=0.29 ; p<0.04\right)$. No significant relationship was found between 'Execution Time' and 'Initiation Time.' The correlations between categories indicate that the indices of Time Efficiency correlated with those from the category Violation Adherence. Both 'Execution Time' and 'Total Time' showed strong positive correlations with 'Total Time Violations' (the correlation coefficient was $r_{s}=0.89 ; p<0.001$ for both these indices). Additionally, 'Execution Time' showed a positive, moderate correlation with 'Total Rule Violation' $\left(r_{s}=0.41\right.$; $p<0.002)$. We found also a weak to moderate negative correlation between 'Initiation Time' and 'Total Rule Violations' $\left(r_{s}=-0.33 ; p<0.02\right)$.

To sum up, shorter total duration of test performance (reflected by 'Total Time') was related to both shorter duration of each problem solving (strong relationship) and initiation time (weak but significant relationship). Additionally, longer time spent on solving each test problem was associated with a greater number of time- and rule-violations committed. The longer time spent before beginning of the task solving (reflected by 'Initiation Time'), the fewer rule violations were committed. The particular correlation coefficients and significance levels are given in Table 4 and summarized in Figure 4.

\section{The Relationship between the Performance on TOJ and TOL ${ }^{\mathrm{DX}}$}

The analysis of the relationships between the performance on TOJ and TOL ${ }^{\mathrm{DX}}$ tasks revealed five significant correlations between auditory TOT and particular TOL ${ }^{\mathrm{DX}}$ indices (Table 4). Two moderate correlations between TOT and indices from Move Performance category of TOL ${ }^{\mathrm{DX}}$ were shown, i.e., negative correlation with 'Total Correct Score' $\left(r_{s}=-0.33 ; p<0.015\right)$ and a positive one with 'Total Move Score' $\left(r_{s}=0.46 ; p<0.001\right)$. 
TABLE 4 | Spearman's rho correlations coefficient values (and significance levels) between TOJ and particular outcome measures of TOL ${ }^{\text {DX }}$

\begin{tabular}{|c|c|c|c|c|c|c|c|c|c|}
\hline & & & TCS & TMS & IT & ET & TT & TTV & TRV \\
\hline & Move Performance & TCS & & & & & & & \\
\hline & & TMS & $-0.70 * * *$ & & & & & & \\
\hline \multirow[t]{5}{*}{ TOL $^{\mathrm{DX}}$} & Time Efficiency & $\mathrm{IT}$ & $0.29^{*}$ & -0.23 & & & & & \\
\hline & & ET & $-0.49 * * *$ & $0.76^{* * *}$ & 0.06 & & & & \\
\hline & & $\pi$ & $-0.33^{*}$ & $0.65^{* * *}$ & $0.29 *$ & $0.94 * * *$ & & & \\
\hline & Violation Adherence & TTV & $-0.32^{*}$ & $0.61^{* * *}$ & 0.25 & $0.89 * * *$ & $0.89 * * *$ & & \\
\hline & & $\mathrm{TRV}$ & $-0.37^{* *}$ & $0.37^{* *}$ & $-0.33^{*}$ & $0.41^{* *}$ & 0.25 & $0.28^{*}$ & \\
\hline TOJ & & TOT & $-0.33^{*}$ & $0.46^{* * *}$ & 0.06 & $0.34^{*}$ & $0.28^{*}$ & $0.33^{*}$ & 0.18 \\
\hline TOJ & (age controlled) & TOT & -0.20 & $0.35^{*}$ & 0.04 & 0.19 & 0.14 & 0.18 & 0.03 \\
\hline
\end{tabular}

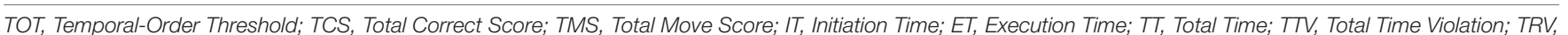

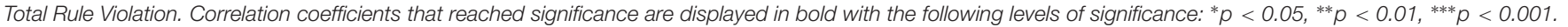
Additional explanations: the correlations between indices within a given category are marked with dark gray fields.

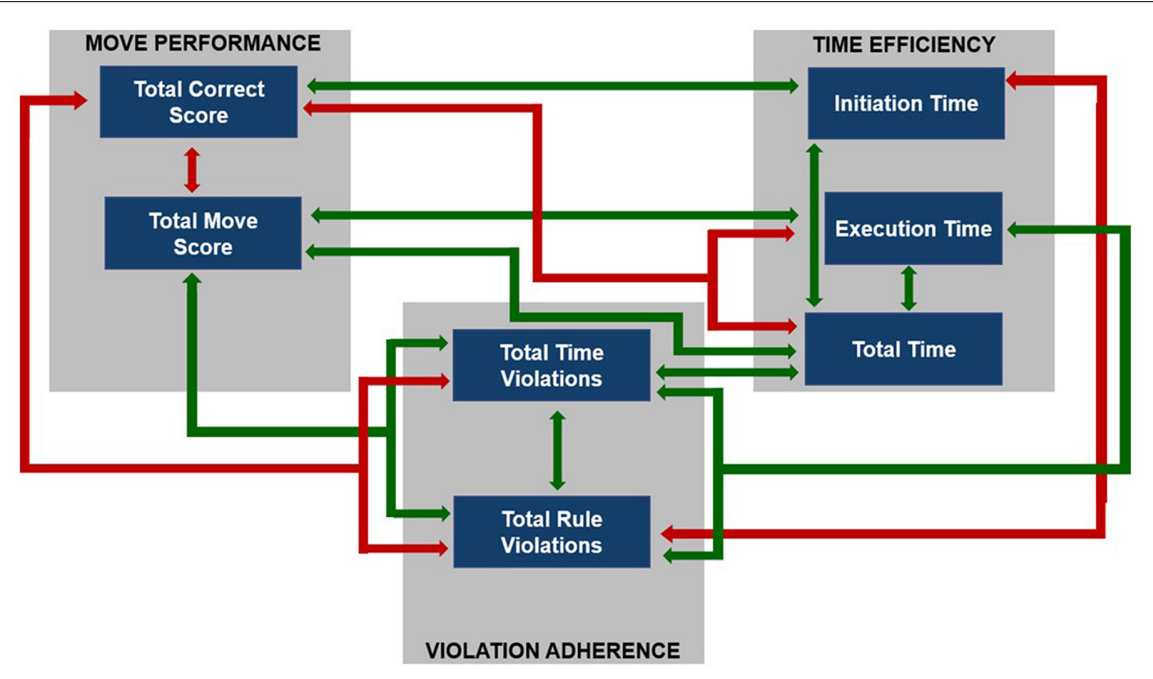

FIGURE 4 | The summary of intercorrelations between TOL ${ }^{\text {DX }}$ outcome measures. Arrows indicate significant correlations: green arrows reflect positive correlations and red arrows -negative ones.

In other words, lower TOT values (better performance) corresponded to a greater number of tasks solved in the minimum number of moves, as well as to a smaller number of redundant moves.

Moreover, we pointed to two weak to moderate positive correlations between TOT and indices from Time Efficiency category of TOL ${ }^{\mathrm{DX}}$, i.e., 'Total Time' $\left(r_{s}=0.28 ; p<0.041\right)$ and 'Execution Time' $\left(r_{s}=0.34 ; p<0.013\right)$. It means that lower TOT values corresponded to shorter both total TOL ${ }^{\mathrm{DX}}$ test performance and each problem solving duration. Furthermore, for the category Violation Adherence we revealed a moderate positive correlation between TOT and 'Total Time Violations' $\left(r_{s}=0.33 ; p<0.017\right)$. Lower TOT (better performance) was accompanied by a smaller number of time violations. These relationship are displayed in the Figures $\mathbf{5}$ and $\mathbf{6}$ and summarized in the Table 4.

To test whether the relationship between TOT and TOL ${ }^{\mathrm{DX}}$ indices may be mediated by age, we used partial correlation analysis (Table 4). Controlling for 'age' partial correlations between TOT and four TOL ${ }^{\mathrm{DX}}$ indices (i.e., 'Total Correct
Score,' 'Execution Time,' 'Total Time' and 'Total Time Violation') turned out non-significant. The relationship between TOT and 'Total Move Score' remained significant but slightly diminished $\left(r_{s}=0.35 ; p<0.05\right)$. These findings suggest that 'Total Move Score' in the lifespan tested here seemed rather resistant to subjects' age, but more depended on TIP resources. The other indices, i.e., 'Total Correct Score,' 'Execution Time,' 'Total Time,' and 'Total Time Violation' were more agedependent.

\section{DISCUSSION}

The study aimed at testing the relationships between particular indices of EF assessed with $\mathrm{TOL}^{\mathrm{DX}}$ test in subjects beyond 65 years of life, as well as to understand the relation 'TIP - EF'.

\section{Summary of Results}

To sum up, the data obtained in both $\mathrm{TOL}^{\mathrm{DX}}$ and TOJ tasks seem to be within a typical range for elderly people. In 
A

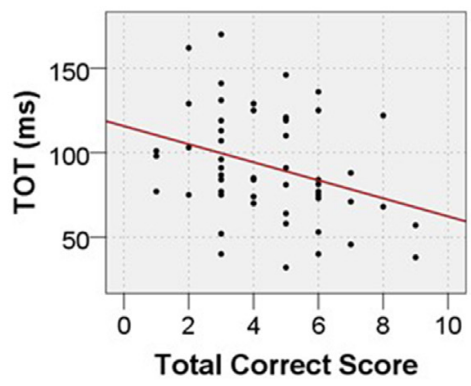

C

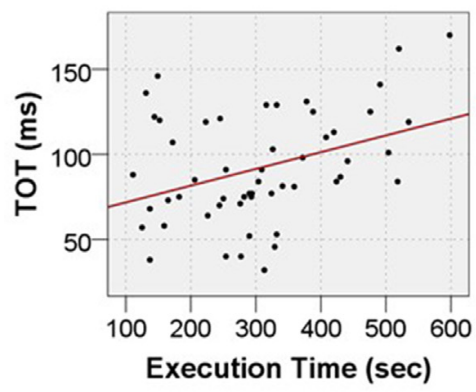

B

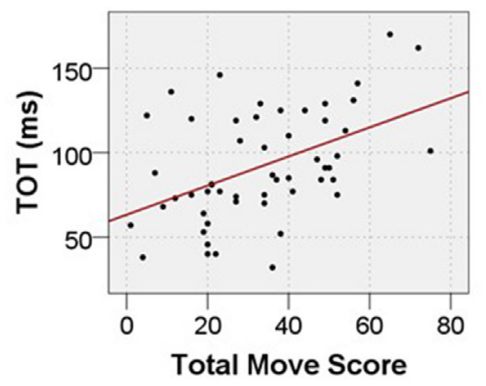

D

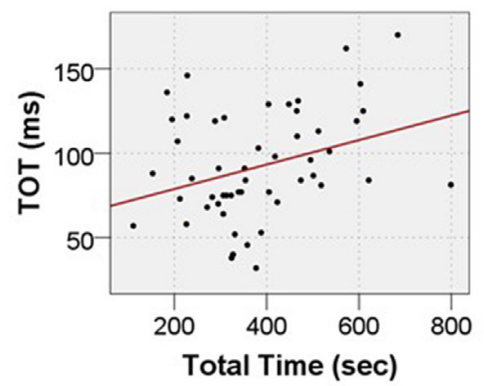

E

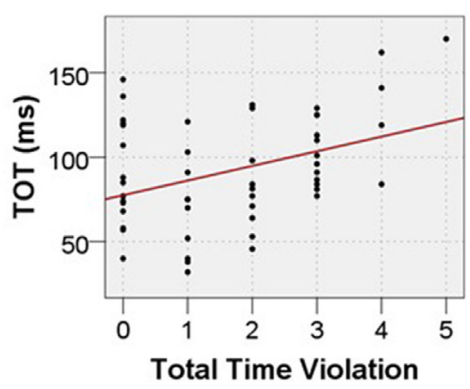

FIGURE 5 | Scatter data illustrating significant correlations between TOT values and outcome measures from TOL ${ }^{\mathrm{DX}}$ test: (A) 'Total Correct Score,' (B) 'Total Move Score,' (C) ‘Execution Time,' (D) ‘Total Time,' (E) ‘Total Time Violations.'

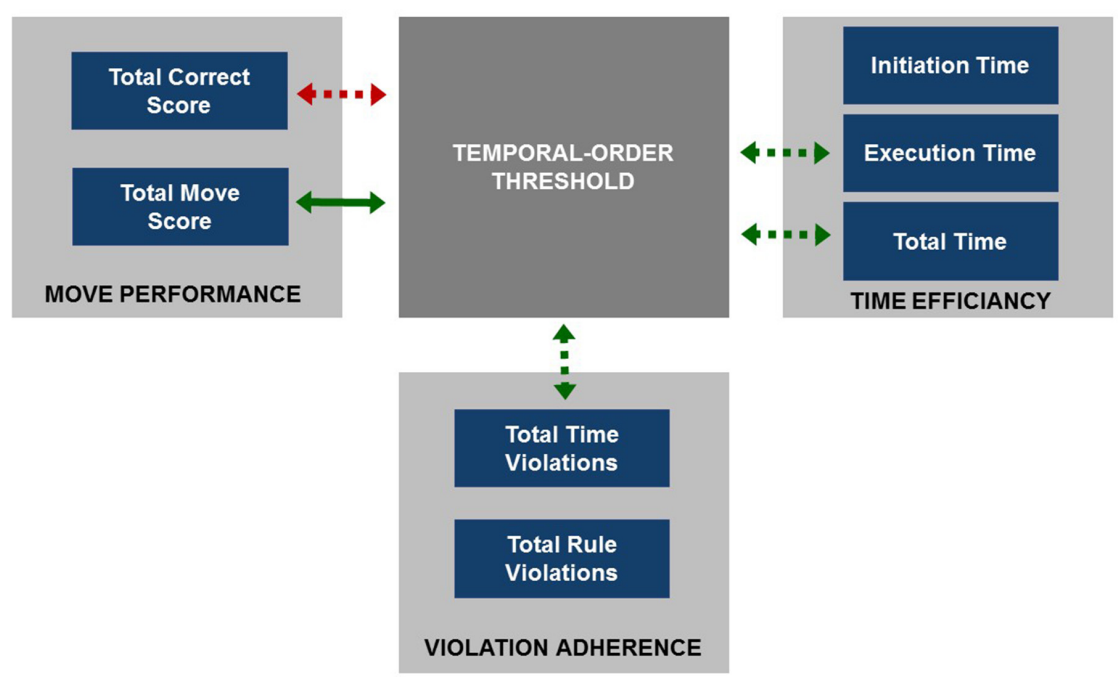

FIGURE 6 | The summary of relationships between TOT (dark gray square) and particular outcome measures of TOL ${ }^{\mathrm{DX}}$ grouped into three categories (light gray squares). Green arrows indicate significant positive correlations and the red one the negative correlation between TOT and TOL ${ }^{\mathrm{DX}}$ outcome measures. The correlation significant after controlling for 'age' is specified with solid arrow whereas those non-significant with dashed ones.

$\mathrm{TOL}^{\mathrm{DX}}$ task the analyses have revealed that the values of the indices obtained are consistent, in general, with the reference data available (Culbertson and Zillmer, 2005) and correlate significantly both within and between categories (Figure 4; Table 4). A smaller number of redundant moves was related to a greater number of correctly solved test problems. Better performance (assessed with two indices from the category Move
Performance) was related to shorter total test duration, shorter time of solving each of the problems, as well as less frequent violations of rules and time limits. Although any relation between initiation time and the number of redundant moves was missing, the subjects who spent more time before starting the task execution solved more test problems correctly (a weak correlation). 
With regard to the relationships between the performance on $\mathrm{TOL}^{\mathrm{DX}}$ and TOJ tasks, we have found that better performance on TOJ (reflected in lower values of auditory TOT) was associated with better performance on TOL ${ }^{\mathrm{DX}}$ (Figures 5 and 6; Table 4) Specifically, subjects characterized by lower TOT made fewer redundant moves on $\mathrm{TOL}^{\mathrm{DX}}$ and solved more problems correctly. These subjects completed the whole TOL ${ }^{\mathrm{DX}}$ test faster and executed each of test problems quicker, which was associated with less frequent time violations. It should be stressed that no significant relations between the performance on TOJ and initiation time, as well as the number of rule violations, was found. After controlling for 'age,' the correlation between TOT and 'Total Move Score' was confirmed, whereas correlations between TOT and other TOL ${ }^{\mathrm{DX}}$ indices were nonsignificant.

\section{Executive Planning in the Elderly}

Before discussing the importance of 'TIP - EF' associations in the elderly, we explain the interrelations between particular indices obtained in $\mathrm{TOL}^{\mathrm{DX}}$ test. Although many existing studies have shown that executive planning measured with different versions of TOL tests decreases with age (e.g., Bugg et al., 2006), to our best knowledge, none of these studies concentrated on complex relations among different performance measures on TOL tests in the elderly. Understanding of the coherence of interrelations between parameters measured with this test may be helpful in our interpretation of the associations between TIP and EF (see below).

The pattern of performance in $\mathrm{TOL}^{\mathrm{DX}}$ test observed in our study was, in general, similar to that reported in the previous literature in younger groups, but we found some important differences. We confirmed the correlations between the indices within the category Move Performance (i.e., 'Total Correct Score' and 'Total Move Score'), as well as proved their associations with those from the category Violation Adherence (Figure 4), indicating the relations between these two categories in the elderly. Such associations seem important, however, were not analyzed thoroughly in published reports in younger adults. The nature of these relations seems reasonable. The greater number of correctly solved problems is obviously accompanied by fewer redundant moves and fewer violations (of both time and rules).

Furthermore, we confirmed previously reported associations in younger adults between indices from categories Move Performance (i.e., 'Total Correct Score,' 'Total Move Score') and Time Efficiency (i.e., 'Execution Time,' 'Total Time'), Figure 4, Table 4. As more moves require more time, both 'Execution Time' and 'Total Time' may be considered as derivatives of motor acts executed during test solving.

These results confirm that all parameters mentioned from these three categories constitute a coherent construct of executive planning and point to some similarities in its specificity in the elderly and in younger groups, tested in previous studies. Beside these similarities, we found some important differences in the strategy of solving the TOL ${ }^{\mathrm{DX}}$ task in the elderly beyond 65 years, as compared to younger groups reported in the literature. These dissimilarities concern the relations of the parameter 'Initiation Time' with the other performance measures.
The previous data obtained in healthy young adults (Hung et al., 2013), college students (Culbertson and Zillmer, 2005, p. 55), or adults aged from 19 to 80 years of life but considered as one subjects pool (Culbertson and Zillmer, 2005) pointed to moderate or even strong interrelations between initiation time and move efficiency (reflected in both 'Total Move Score' and 'Total Correct Score'). In contrast, our results showed no significant correlations between 'Initiation Time' and 'Total Move Score,' accompanied by only a weak correlation with 'Total Correct Score' (Figure 4; Table 4). It seems, thus, that 'Initiation Time' was only weakly associated with move efficiency indices, reflecting quality of executive planning, as well as working memory capacity and control (Table 2).

These results suggest that in the elderly the effectiveness of executive planning cannot be directly attributed to the time period before executing motor acts. The existing literature data on the role of planning on this test in younger adults have shown contradictory results. For example, Unterrainer et al. (2003) indicated that the participants who were instructed to make full mental plans before beginning to execute movements (preplanning) solved significantly more problems than the subjects starting the task immediately with taskrelated movements (on-line). On the contrary, Phillips et al. (2001) showed that the time spent on such preplanning did not necessarily contribute to better performance on TOL task, even if the instruction on taking time to plan actions was explicitly given. They evidenced that better performance in TOL task may not necessarily depend on the time spent on preplanning, but on the ability to plan on-line, i.e., to monitor and plan movements during the execution of particular test problems. Such strategy was also suggested in the earlier work of Gilhooly et al. (1999), who stated that neither young nor elderly participants made a full plan before starting the execution of trials of the TOL task. However, these authors only relied on group comparisons without any correlation analysis between the test indices.

In our opinion, these literature controversies on the planning strategy resulted from uncontrolled contribution of other mental processes incorporated in planning abilities. They involve diminished inhibitory control of actions, mental processing speed and impulsivity of a participant (Culbertson and Zillmer, 2005; Luciana et al., 2009). Many previous studies reported a decline of abovementioned cognitive skills (e.g., Salthouse, 1996; Christ et al., 2001), as well as age-related differences in cognitive styles, i.e., reflection-impulsivity (Coyne et al., 1978).

The hypothesis on the contribution of other mental processes to the planning strategy may be supported by a negative correlation between 'Initiation Time' and 'Total Rule Violation' found in our study (Figure 4; Table 4). It suggested that the participants who spent less time before executing movements committed more rule violations, which reflected difficulties in both postponing of action execution and working under constrains imposed on them by the test instructions.

To sum up, our results indicate that in the elderly, despite a coherent construct of some indices of executive planning, 'Initiation Time' seems rather dissociated from these 
indices and weakly associated with the overall performance on $\mathrm{TOL}^{\mathrm{DX}}$. Therefore, we argue that the elderly may use a different strategy of planning than the younger subjects, i.e., they rely not so much on the preplanning, but rather on the on-line planning during solving particular $\mathrm{TOL}^{\mathrm{DX}}$ test problems.

\section{Associations between TIP and Executive Planning in the Elderly}

Assuming the importance of TIP for our cognitive function (see Introduction), the primary goal of the present study was to test the relation 'TIP - EF' in normal aging in order to understand mental processes characteristic of this period of life. The results reported here confirmed declined temporal acuity in normal healthy elderly (e.g., Bedard and Barnett-Cowan, 2016; Turgeon et al., 2016) and fully matched the data reported previously in our laboratory, when the same experimental procedure as that used in the present study was employed (Szymaszek et al., 2009; Szelag et al., 2011). We also confirmed pronounced heterogeneity in the efficiency of TIP in the subject sample tested here, reflecting individual differences (Matthews and Meck, 2014).

The relevance of the present study is a novel observation that in the elderly such subject-related variability in TIP (assessed with auditory TOT) was associated with the efficiency of executive planning (Figures 5 and 6; Table 4). However, the results of partial correlation analysis (Table 4) indicated that the relation 'TIP - EF' was modified by subject's age in the lifespan tested here (from 65 to 78 years of life, see Table 1). The relation between TIP and 'Total Move Score', considered as the main index of $\mathrm{TOL}^{\mathrm{DX}}$ reflecting the level of executive planning quality (Table 2), was resistant to age-related influences, but strongly associated with TIP. Thus, timing seems to be much more related to executive planning than age in the subjects pool studied here. The persistence of the correlation between TIP and 'Total Move Score' in partial correlations controlling for age supports the hypothesis on an important role of TIP for numerous mental functions, including higher-order metacognitive processes, such as executive planning investigated in our study. It may be assumed, therefore, that the performance on $\mathrm{TOL}^{\mathrm{DX}}$ reflected by 'Total Move Score' - the main index of this test - is rooted in a defined millisecond template, creating a neural base for executive planning abilities. Our results suggest that TIP in a millisecond range is related to these top-down processes engaged in controlling and monitoring our behavior during adaptation to changing environmental conditions. Proper sequencing and maintenance of information provide matrices for building up our sensations, identification of events and ordering them chronologically. Such ability remains the major prerequisite for proper functioning of higher cognitive functions, i.e., EF. It may be suggested, therefore, that the performance on $\mathrm{TOL}^{\mathrm{DX}}$ is rooted in a defined millisecond template, creating a neural base for executive planning abilities.

On the other hand, in partial correlation analysis controlling for age the relation between $\mathrm{TOT}$ and other $\mathrm{TOL}^{\mathrm{DX}}$ indices, i.e., 'Total Correct Score' from the category Move Performance, moreover, 'Executive Time' and 'Total Time' from Time Efficiency, and 'Total Time Violations' from Violation Adherence (Table 2) did not correlate with TIP and were rather age-related (Figure 6; Table 4). Thus, the more advanced age of participants, the poorer performance on these indices was indicated. It may result from declined concomitant functions, like working memory, behavioral control and functioning dynamics, which decline progressively after the age of 65 . The lack of correlations between these indices and TIP (after controlling for age) may also, at least partly, result from the instructions given to participants that did not mention any time limitation. It seems very plausible, therefore, that participants did not perform the task at their maximum tempo. It also seems that the indices from Time Efficiency category, i.e., 'Total Time' and 'Executive Time' may be prone to such physiological factors as slowing of movement in aging, which are not directly related to effectiveness of mental planning but much more to subject's age.

Referring to the specific planning strategy in the elderly (discussed above) based more on on-line planning than on preplanning and reflected in the dissociation of 'Initiation Time' from the other $\mathrm{TOL}^{\mathrm{DX}}$ indices, we should stress that no significant correlation between the effectiveness of TIP and 'Initiation Time' was proven (Figure 6; Table 4). The lack of such associations may indicate that preplanning (reflected in 'Initiation Time,' Table 2) is not so much related to timing, but rather to other processes, among which impulsivity and inhibitory control should be taken into consideration.

The final question concerns potential mechanisms or processes underlying the relationship 'TIP - EF' observed in our study. Despite pronounced individual differences, experimental data have indicated that the perception of succession is controlled by the internal timing mechanism, operating in some tens of millisecond time window implemented probably in neuronal gamma band oscillations with a periodicity of ca. $40 \mathrm{~Hz}$ (VanRullen and Koch, 2003; Oron et al., 2015) Accordingly, one oscillation period has ca. $25 \mathrm{~ms}$ duration. Referring to Pöppel (2009), the relation 'beforeafter' can be properly identified if two stimuli occur at least in two successive oscillatory periods. There is strong evidence that spontaneous (or stimulus triggered) gamma band oscillations, corresponding in duration to TOT, play an important role in human cognition (VanRullen and Koch, 2003).

The relation 'TIP - EF' on some tens of milliseconds level may suggest common neural mechanisms underlying these two mental functions. They create a temporal frame for both our auditory perception of temporal order of incoming events and EF, moreover, for other non-executive cognitive functions, among which language reception was indicated in previous reports (Fink et al., 2006; Oron et al., 2015). It may be hypothesized that the relations 'TIP - EF' cannot be restricted selectively to the auditory modality which was tested here. At this point one may refer to classical reports by Efron (1963) and Swisher and Hirsh (1972), who indicated that deficient TIP in aphasic 
patients was not related selectively to auditory processing but concerned also vision and touch. A similar 'temporal binding window' for audition and vision was also indicated in our previous study (Kanabus et al., 2002). It may suggest the common timing mechanism underlying event ordering which creates a frame for multimodal processing. It may be suspected that this temporal mechanism operates on a very fundamental level, regardless of the specificity of the task, i.e., it seems active in auditory perception of temporal order (reflected in TOT without any motor component) or planning and execution of moves (reflected in solving $\mathrm{TOL}^{\mathrm{DX}}$ problems with a motor component). To understand the potential neural source of these very fundamental processes by means of creating the common neural operational frame on which various mental activities are embedded, one could refer to the neuronal oscillatory activity. Accordingly, expected slower gamma oscillations in advanced age might result in deficient mechanisms operating in some tens of milliseconds time window, less efficient TIP (higher TOT values) and declined executive planning, in which such timing is implemented.

Additional support for strong associations between TIP and EF comes from neuroimaging data. Our previous studies on neuroanatomical representation of TOJ in a millisecond range showed dynamic changes in neural activity depending on task difficulty (Lewandowska et al., 2010). Decreased TOJ difficulty was accompanied by increased activity in bilateral medial frontal gyri, which constitute a part of prefrontal cortex. As mentioned in Introduction, this region is involved in EF. Referring to Bayesian models (Turgeon et al., 2016), such neural network may be related to a central timing mechanism supported by the processes of neurotransmission on the synaptic level, specifically by dopamine-glutamate interactions in cortico-striatal circuits (Turgeon et al., 2016). From this perspective, normal aging is associated with possible reduction in accuracy and precision in dopaminergic functions resulting in age-related decline in TIP and EF. The overlap of neuroanatomical activity in TIP and EF may support a common activation network in which millisecond timing constitutes an essential component of EF governing human cognition.

\section{CONCLUSION}

The results of the present study could shed a new light on our understanding of neural mechanisms underlying human mental activity and allow some generalizations on the taxonomy of functions in neuropsychology.

On the basis of the taxonomy proposed by Pöppel (1994), which was developed in our studies (Szelag et al., 2010, 2011), two classes of cognitive functions may be distinguished. The functions of the first class, i.e., context-related or 'WHAT' functions refer to the mental context of our subjective experience, like conscious percepts, language utterances, new learning material, memory traces, motor acts, etc. The functions of the second class constitute 'HOW' functions. They provide a formal operational basis and control the logistic prerequisite of context-related 'WHAT' functions. Hence, one may assume that logistic-related 'HOW' functions create a neural frame for our mental activity into which particular cognitive non-EF are embedded.

The question arises which functions may be classified into such logistic framework. As TIP provides the crucial component of human cognition (Pöppel, 1994, 1997, 2009; Wittmann, 1999; Allman et al., 2014; Matthews and Meck, 2014), it may be classified as the major example of ' $\mathrm{HOW}$ ' functions. On the basis of a huge amount of literature evidence (see Introduction), EF including executive planning may also be considered as meta-cognitive processes or logistic functions that are responsible for starting, stopping and shifting of the other context-related, non-executive cognitive functions.

Finally, we would like to argue that both TIP and EF create a logistic basis of our mental functioning. The novel outcome of the present study is that the effectiveness of these two logistic functions is intercorrelated, which may support the notion of their common neural substrates. Thus, advanced or deficient executive planning in the elderly corresponds to the effectiveness of TIP in a millisecond range assessed with auditory TOT. The supramodal cooperation between TIP and EF may constitute an example of integrative activity of the brain.

\section{AUTHOR CONTRIBUTIONS}

$\mathrm{KN}$ and $\mathrm{AD}$ : Subject recruitment, acquisition, analysis and interpretation of data, manuscript writing. $\mathrm{KB}$ and $\mathrm{MK}-\mathrm{U}$ : Subject recruitment, geriatric diagnosis of the subjects qualified into the study. TG: Interpretation of data. ES: Acquisition, analysis and interpretation of data, manuscript writing, responsibility for the final version of manuscript.

\section{FUNDING}

The research was supported by National Science Centre (NCN) grant 2015/17/B/HS6/04182.

\section{ACKNOWLEDGMENTS}

The authors would like to thank Anna Bombinska for her technical assistance during the data collection. The computer program for assessment of TOT was obtained from the Human Science Centre, Ludwig-Maximilian University, Bad Tölz. 


\section{REFERENCES}

Allman, M. J., Teki, S., Griffiths, T. D., and Meck, W. H. (2014). Properties of the internal clock: first-and second-order principles of subjective time. Annu. Rev. Psychol. 65, 743-771. doi: 10.1146/annurev-psych-010213-115117

Ansi (2004). ANSI S3.6-2004. American National Standard Specification for Audiometers. New York, NY: American National Standards Institute.

Bao, Y., Szymaszek, A., Wang, X., Oron, A., Pöppel, E., and Szelag, E. (2013). Temporal order perception of auditory stimuli is selectively modified by tonal and non-tonal language environments. Cognition 129, 579-585. doi: 10.1016/j.cognition.2013.08.019

Bedard, G., and Barnett-Cowan, M. (2016). Impaired timing of audiovisual events in the elderly. Exp. Brain Res. 234, 331-340. doi: 10.1007/s00221-015-4 466-7

Borghi, A. M., and Cimatti, F. (2010). Embodied cognition and beyond: acting and sensing the body. Neuropsychologia 48, 763-773. doi: 10.1016/j.neuropsychologia.2009.10.029

Brennan, M., Welsh, M. C., and Fisher, C. B. (1997). Aging and executive function skills: an examination of a community-dwelling older adult population. Percept. Mot. Skill 84, 1187-1197. doi: 10.2466/pms.1997.84.3c.1187

Bugg, J. M., Zook, N. A., DeLosh, E. L., Davalos, D. B., and Davis, H. P. (2006). Age differences in fluid intelligence: contributions of general slowing and frontal decline. Brain Cogn. 62, 9-16. doi: 10.1016/j.bandc.2006.02.006

Cabeza, R., Anderson, N. D., Locantore, J. K., and McIntosh, A. R. (2002). Aging gracefully: compensatory brain activity in high-performing older adults. Neuroimage 17, 1394-1402. doi: 10.1006/nimg.2002.1280

Cabeza, R., and Dennis, N. A. (2012). "Frontal lobes and aging: deterioration and compensation," in Principles of Frontal Lobes Function, 2nd Edn, eds D. T. Stuss, and R. T. Knight (New York, NY: Oxford University Press), 628-652.

Cabeza, R., Grady, C. L., Nyberg, L., McIntosh, A. R., Tulving, E., Kapur, S., et al. (1997). Age-related differences in neural activity during memory encoding and retrieval: a positron emission tomography study. J. Neurosci. 17, 391-400.

Cabeza, R., Nyberg, L., and Park, D. (2004). Cognitive Neuroscience of Aging: Linking Cognitive and Cerebral Aging. New York, NY: Oxford University Press, doi: 10.1093/acprof:oso/9780195156744.001.0001

Chang, Y. K., Tsai, C. L., Hung, T. M., So, E. C., Chen, F. T., and Etnier, J. L. (2011). Effects of acute exercise on executive function: a study with a tower of London task. J. Sport Exerc. Psychol. 33, 847-865. doi: 10.1123/jsep.33.6.847

Christ, S. E., White, D. A., Mandernach, T., and Keys, B. A. (2001). Inhibitory control across the life span. Dev. Neuropsychol. 20, 653-669. doi: 10.1207/S15326942DN2003_7

Cohen, J. (1988). Statistical Power Analysis for Behavioural Sciences. Hillsdale, NJ: Lawrence Eribaum Associates.

Colcombe, S., and Kramer, A. F. (2003). Fitness effects on the cognitive function of older adults: a meta-analytic study. Psychol. Sci. 14, 125-130. doi: 10.1111/14679280.t01-1-01430

Coyne, A. C., Whitbourne, S. K., and Glenwick, D. S. (1978). Adult age differences in reflection-impulsivity. J. Gerontol. 33, 402-407. doi: 10.1093/geronj/3 3.3.402

Culbertson, W. C., and Zillmer, E. A. (2005). Tower of London - Drexel University (TOLDX): Technical Manual, 2nd Edn. Toronto, ON: Multi-Health Systems.

Davis, S. W., Dennis, N. A., Daselaar, S. M., Fleck, M. S., and Cabeza, R. (2008). Que PASA? The posterior-anterior shift in aging. Cereb. Cortex 18, 1201-1209. doi: $10.1093 /$ cercor/bhm155

Davis, P. H., and Klebe, J. K. (2001). A longitudinal study of the performance of the elderly and young on the Tower of Hanoi puzzle and Rey recall. Brain Cogn. 46, 95-99. doi: 10.1006/brcg.2000.1269

Diamond, A. (2013). Executive functions. Annu. Rev. Psychol. 64, 135-168. doi: 10.1146/annurev-psych-113011-143750

Efron, R. (1963). Temporal perception, aphasia and deja vu. Brain 86, 403-424. doi: 10.1093/brain/86.3.403

Etnier, J. L., and Chang, Y. K. (2009). The effect of physical activity on executive function: a brief commentary on definitions, measurement issues and the current state of the literature. J. Sport Exerc. Psychol. 31, 469-483. doi: 10.1123/jsep.31.4.469

Fink, M., Churan, J., and Wittmann, M. (2005). Assessment of auditory temporalorder thresholds-a comparison of different measurement procedures and the influences of age and gender. Restor. Neurol. Neurosci 23, 281-296.
Fink, M., Churan, J., and Wittmann, M. (2006). Temporal processing and context dependency of phoneme discrimination in patients with aphasia. Brain Lang. 98, 1-11. doi: 10.1016/j.bandl.2005.12.00

Fisk, J. E., and Warr, P. (1996). Age and working memory: the role of perceptual speed, the central executive, and the phonological loop. Psychol. Aging 11:316. doi: 10.1037/0882-7974.11.2.316

Fitzgibbons, P. J., and Gordon-Salant, S. (1994). Age effects on measures of auditory duration discrimination. J. Speech. Hear. Res. 37, 662-670. doi: 10.1044/jshr.3703.662

Folstein, M., Folstein, S., and Fanjiang, G. (2001). MMSE Mini-Mental State Examination: Clinical guide. Lutz, FL: Psychological Assessment Resources.

Gilhooly, K. J., Phillips, L. H., Wynn, V., Logie, R. H., and Della Sala, S. (1999). Planning processes and age in the five-disc Tower of London task. Think. Reason. 5, 339-361. doi: 10.1080/135467899393977

Grady, C. (2012). The cognitive neuroscience of ageing. Nat. Rev. Neurosci. 13, 491-505. doi: 10.1038/nrn3256

Grady, C. L., Maisog, J. M., Horwitz, B., Ungerleider, L. G., Mentis, M. J., Salerno, J. A., et al. (1994). Age-related changes in cortical blood flow activation during visual processing of faces and location. J. Neurosci. 14, 1450-1462.

Hirsh, I. J., and Sherrick, C. E. Jr. (1961). Perceived order in different sense modalities. J. Exp. Psychol. 62, 423-432. doi: 10.1037/h0045283

Huizinga, M., Dolan, C. V., and van der Molen, M. W. (2006). Age-related change in executive function: developmental trends and a latent variable analysis. Neuropsychologia 44, 2017-2036. doi: 10.1016/j.neuropsychologia.2006.01.010

Hung, T. M., Tsai, C. L., Chen, F. T., Wang, C. C., and Chang, Y. K. (2013). The immediate and sustained effects of acute exercise on planning aspect of executive function. J. Sport Exerc. Psychol. 14, 728-736. doi: 10.1249/MSS.0b013e3181cbee11

James, W. (1890). Principles of Psychology. New York, NY: Doubleday.

Jurado, M. B., and Rosselli, M. (2007). The elusive nature of executive functions: a review of our current understanding. Neuropsychol. Rev. 17, 213-233. doi: 10.1007/s11065-007-9040-z

Kanabus, M., Szelag, E., Rojek, E., and Pöppel, E. (2002). Temporal order judgement for auditory and visual stimuli. Acta Neurobiol. Exp. 62, 263-270.

Kolodziejczyk, I., and Szelag, E. (2008). Auditory perception of temporal order in centenarians in comparison with young and elderly subjects. Acta Neurobiol. Exp. 68, 373-381.

Kumar, A. U., and Sangamanatha, A. V. (2011). Temporal processing abilities across different age groups. J. Am. Acad. Audiol. 22, 5-12. doi: 10.3766/jaaa.22.1.2

Lewandowska, M., Piatkowska-Janko, E., Bogorodzki, P., Wolak, T., and Szelag, E. (2010). Changes in fMRI BOLD response to increasing and decreasing task difficulty during auditory perception of temporal order. Neurobiol. Learn. Mem. 94, 382-391. doi: 10.1016/j.nlm.2010.08.005

Lezak, M. D. (2004). Neuropsychological Assessment, 4th Edn. New York, NY: Oxford University Press.

Luciana, M., Collins, P. F., Olson, E. A., and Schissel, A. M. (2009). Tower of London performance in healthy adolescents: the development of planning skills and associations with self-reported inattention and impulsivity. Dev. Neropsychol. 34, 461-475. doi: 10.1080/87565640902964540

Matthews, W. J., and Meck, W. H. (2014). Time perception: the bad news and the good. Wiley Interdiscip. Rev. Cogn. Sci. 5, 429-446. doi: 10.1002/wcs.1298

Miyake, A., Friedman, N. P., Emerson, M. J., Witzki, A. H., Howerter, A., and Wager, T. D. (2000). The unity and diversity of executive functions and their contributions to complex "frontal lobe" tasks: a latent variable analysis. Cogn. Psychol. 41, 49-100. doi: 10.1006/cogp.1999.0734

Nenadic, I., Gaser, C., Volz, H. P., Rammsayer, T., Häger, F., and Sauer, H. (2003). Processing of temporal information and the basal ganglia: new evidence from fMRI. Exp. Brain Res. 148, 238-246. doi: 10.1007/s00221-002-1188-4

Oldfield, R. C. (1971). The assessment and analysis of handedness: the Edinburgh inventory. Neuropsychologia 9, 97-113. doi: 10.1016/0028-3932(71)90067-4

Oron, A., Szymaszek, A., and Szelag, E. (2015). Temporal information processing as a basis for auditory comprehension: clinical evidence from aphasic patients. Int. J. Lang. Comm. Dis. 50, 604-615. doi: 10.1111/1460-6984. 12160

Park, D. C., and Reuter-Lorenz, P. (2009). The adaptive brain: aging and neurocognitive scaffolding. Annu. Rev. Psychol. 60, 173-196. doi: 10.1146/annurev.psych.59.103006.093656 
Phillips, L. H., Wynn, V. E., McPherson, S., and Gilhooly, K. J. (2001). Mental planning and the Tower of London task. Q. J. Exp. Psychol. A 54, 579-597. doi: $10.1080 / 02724980042000237$

Pöppel, E. (1994). Temporal mechanisms in perception. Int. Rev. Neurobiol. 37, 185-202. doi: 10.1016/S0074-7742(08)60246-9

Pöppel, E. (1997). A hierarchical model of temporal perception. Trends Cogn. Sci. 1, 56-61. doi: 10.1016/S1364-6613(97)01008-5

Pöppel, E. (2009). Pre-semantically defined temporal windows for cognitive processing. Philos. Trans. R. Soc. B Biol. Sci. 364, 1887-1896. doi: 10.1098/rstb.2009.0015

Reuter-Lorenz, P. A., and Cappell, K. A. (2008). Neurocognitive aging and the compensation hypothesis. Curr. Dir. Psychol. Sci. 17, 177-182. doi: 10.1111/j.1467-8721.2008.00570.x

Reuter-Lorenz, P. A., and Park, D. C. (2010). Human neuroscience and the aging mind: a new look at old problems. J. Gerontol. B Psychol. Sci. Soc. Sci. 65, 405-415. doi: 10.1093/geronb/gbq035

Romine, C. B., and Reynolds, C. R. (2005). A model of the development of frontal lobe functioning: findings from a meta-analysis. Appl. Neuropsychol. 12, 190-201. doi: 10.1207/s15324826an1204_2

Sala-Llonch, R., Bartrés-Faz, D., and Junqué, C. (2015). Reorganization of brain networks in aging: a review of functional connectivity studies. Front. Psychol. 6:663. doi: $10.3389 /$ fpsyg.2015.00663

Salthouse, T. A. (1996). The processing-speed theory of adult age differences in cognition. Psychol. Rev. 103, 403-428. doi: 10.1037/0033-295X.103.3.403

Salthouse, T. A. (2009). When does age-related cognitive decline begin? Neurobiol. Aging 30, 507-514. doi: 10.1016/j.neurobiolaging.2008.09.023

Setti, A., Burke, K. E., Kenny, R. A., and Newell, F. N. (2011a). Is inefficient multisensory processing associated with falls in older people? Exp. Brain Res. 209, 375-384. doi: 10.1007/s00221-011-2560-Z

Setti, A., Finnigan, S., Sobolewski, R., McLaren, L., Robertson, I. H., Reilly, R. B., et al. (2011b). Audiovisual temporal discrimination is less efficient with aging: an event-related potential study. Neuroreport 22, 554-558. doi: 10.1097/WNR.0b013e328348c731

Shallice, T. (1982). Specific impairments of planning. Philos. Trans. R. Soc. Lond. B Biol. Sci. 298, 199-209. doi: 10.1098/rstb.1982.0082

Shiekh, J., and Yesavage, J. (1986). "Geriatric depression scale: recent findings and development of a short version," in Clinical Gerontology: A Guide to Assessment and Intervention, ed. T. L. Brink (New York, NY: The Haworth Press), 165-173.

Sternberg, R. J., and Ben-Zeev, T. (2001). Complex Cognition: The Psychology of Human Thought. New York, NY: Oxford University Press.

Swisher, L., and Hirsh, I. J. (1972). Brain damage and the ordering of two temporally successive stimuli. Neuropsychologia 10, 137-152. doi: 10.1016/0028-3932(72)90053-X

Szelag, E., Dreszer, J., Lewandowska, M., Medrygal, J., Osinski, G., and Szymaszek, A. (2010). "Time and cognition from the aging brain perspective: individual differences," in Personality from Biological, Cognitive, and Social Perspectives, eds T. Maruszewski, M. Fajkowska, and M. W. Eysenck (Nowy Jork, NY: Eliot Warner Publications, Inc.), 87-114.

Szelag, E., Kanabus, M., Kolodziejczyk, I., Kowalska, J., and Szuchnik, J. (2004). Individual differences in temporal information processing in humans. Acta Neurobiol. Exp. 64, 349-366.
Szelag, E., Rymarczyk, K., and Pöppel, E. (2001). Conscious control of movements: increase of temporal precision in voluntarily delayed actions. Acta Neurobiol. Exp. 61, 175-179.

Szelag, E., Szymaszek, A., Aksamit-Ramotowska, A., Fink, M., Ulbrich, P., Wittmann, M., et al. (2011). Temporal processing as a base for language universals: cross-linguistic comparisons on sequencing abilities with some implications for language therapy. Restor. Neurol. Neurosci. 29, 35-45. doi: 10.3233/RNN-2011-0574

Szymaszek, A., Sereda, M., Pöppel, E., and Szelag, E. (2009). Individual differences in the perception of temporal order: the effect of age and cognition. Cogn. Neuropsychol. 26, 135-147. doi: 10.1080/02643290802504742

Teixeira, S., Machado, S., Flávia, P., Velasques, B., Silva, J. G., Sanfim, A. L., et al. (2013). Time perception distortion in neuropsychiatric and neurological disorders. CNS Neurol. Disord. Drug Targets 12, 567-582. doi: $10.2174 / 18715273113129990080$

Treutwein, B. (1997). YAAP: yet another adaptive procedure. Spat. Vis. 11, 129-134.

Turgeon, M., Lustig, C., and Meck, W. H. (2016). Cognitive aging and time perception: roles of bayesian optimization and degeneracy. Front. Aging Neurosci. 8:102. doi: 10.3389/fnagi.2016.00102

Unterrainer, J. M., Rahm, B., Leonhart, R., Ruff, C. C., and Halsband, U. (2003). The Tower of London: the impact of instructions, cueing, and learning on planning abilities. Cogn. Brain Res. 17, 675-683. doi: 10.1016/S0926-6410(03) 00191-5

VanRullen, R., and Koch, C. (2003). Is perception discrete or continuous? Trends Cogn. Sci. 7, 207-213. doi: 10.1016/S1364-6613(03)00095-0

Welsh, M. C., Satterlee-Cartmell, T., and Stine, M. (1999). Towers of Hanoi and London: contribution of working memory and inhibition to performance. Brain Cogn. 41, 231-242. doi: 10.1006/brcg.1999.1123

West, R. L. (1996). An application of prefrontal cortex function theory to cognitive aging. Psychol. Bull. 120:272. doi: 10.1037/0033-2909.120.2.272

Wittmann, M. (1999). Time perception and temporal processing levels of the brain. Chronobiol. Int. 16, 17-32. doi: 10.3109/07420529908998709

Wittmann, M., and Fink, M. (2004). Time and language-critical remarks on diagnosis and training methods of temporal order judgement. Acta Neurobiol. Exp. 64, 341-348.

Zook, N., Welsh, M. C., and Ewing, V. (2006). Performance of healthy, older adults on the Tower of London revised: associations with verbal and nonverbal abilities. Neuropsychol. Dev. Cogn. B Aging Neuropsychol. Cogn. 13, 1-19. doi: $10.1080 / 13825580490904183$

Conflict of Interest Statement: The authors declare that the research was conducted in the absence of any commercial or financial relationships that could be construed as a potential conflict of interest.

Copyright (c) 2016 Nowak, Dacewicz, Broczek, Kupisz-Urbanska, Galkowski and Szelag. This is an open-access article distributed under the terms of the Creative Commons Attribution License (CC BY). The use, distribution or reproduction in other forums is permitted, provided the original author(s) or licensor are credited and that the original publication in this journal is cited, in accordance with accepted academic practice. No use, distribution or reproduction is permitted which does not comply with these terms. 
OPEN ACCESS

Edited by:

Annalisa Setti,

University College Cork, Ireland

Reviewed by:

Andrew Patrick Allen

University College Cork, Ireland

Lorenza S. Colzato,

Leiden University, Netherlands

${ }^{*}$ Correspondence:

Yei-Yu Yeh

yyy@ntu.edu.tw

Specialty section:

This article was submitted to

Cognition

a section of the journal

Frontiers in Psychology

Received: 29 April 2016

Accepted: 29 September 2016

Published: 13 October 2016

Citation:

Kuo C-Y and Yeh Y-Y (2016) Sensorimotor-Conceptual Integration in Free Walking Enhances Divergent Thinking for Young and Older Adults.

Front. Psychol. 7:1580.

doi: 10.3389/fpsyg.2016.01580

\section{Sensorimotor-Conceptual} Integration in Free Walking Enhances Divergent Thinking for Young and Older Adults

\author{
Chun-Yu Kuo ${ }^{1,2}$ and Yei-Yu Yeh ${ }^{2 *}$ \\ ${ }^{1}$ Department of Educational Psychology and Counseling, National Pingtung University, Pingtung, Taiwan, ${ }^{2}$ Department of \\ Psychology, National Taiwan University, Taipei, Taiwan
}

Prior research has shown that free walking can enhance creative thinking. Nevertheless, it remains unclear whether bidirectional body-mind links are essential for the positive effect of free walking on creative thinking. Moreover, it is unknown whether the positive effect can be generalized to older adults. In Experiment 1, we replicated previous findings with two additional groups of young participants. Participants in the rectangularwalking condition walked along a rectangular path while generating unusual uses for chopsticks. Participants in the free-walking group walked freely as they wished, and participants in the free-generation condition generated unconstrained free paths while the participants in the random-experienced condition walked those paths. Only the free-walking group showed better performance in fluency, flexibility, and originality. In Experiment 2, two groups of older adults were randomly assigned to the free-walking and rectangular-walking conditions. The free-walking group showed better performance than the rectangular-walking group. Moreover, older adults in the free-walking group outperformed young adults in the rectangular-walking group in originality and performed comparably in fluency and flexibility. Bidirectional links between proprioceptive-motor kinematics and metaphorical abstract concepts can enhance divergent thinking for both young and older adults.

Keywords: creativity, divergent thinking, cognitive processes, embodied cognition, aging

\section{INTRODUCTION}

Creativity is essential for the advancement of humanity. Creativity is a core activity in arts, sciences, entrepreneurship, and innovation in the workplace. It has been demonstrated that people with higher levels of creativity possess greater occupational self-efficiency (Tierney et al., 1999), healthier psychological functioning (McCracken, 1991; Terr, 1992; Russ, 1998; Kin and Pope, 1999), subjective well-being and successful adaption to daily demands (Reiter-Palmon et al., 1998), and better interpersonal relations (Livingston, 1999). Creativity also contributes to successful aging by encouraging flexible problem-solving skills and promoting self-efficacy in older adults (Fisher and Specht, 1999). In the era of aging and aged societies, empowering older adults with skills geared toward successful aging significantly affects society.

Divergent thinking is a core process of creativity (Guilford, 1967). Divergent thinking entails exploring many alternative and original ideas that differ from the standard responses to a given 
goal. Once a problem is defined, divergent thinking is critical for generating innovative, novel, and useful ideas. Given its importance, numerous methods have been developed to enhance divergent thinking such as brainstorming, structured lectures and exercises, social modeling, behavioral modification, and individualized coaching. Meta-analysis studies showed that programs emphasizing the development of cognitive skills using realistic, domain-specific exercises showed improvements, with effect size $(r)$ ranging from 0.02 to 0.22 (Scott et al., 2004). Adult education programs focusing on divergent thinking strategies showed a large effect $(r=0.35)$ on improving fluency, flexibility, and originality indicators of the Torrance Test of Creative Thinking (Tsai, 2014). The benefit arises from near transfer of applying the trained cognitive strategies to tasks that require divergent thinking.

Three intervention methods that do not share overlapping cognitive processes could produce far transfer on divergent thinking ability. Induction of episodic-specific retrieval processes improves divergent thinking with medium $(r=0.25)$ and large $(r=0.36)$ effects for young adults (Madore et al., 2015). The second approach is to enhance creativity through physical exercises such as running and dancing. Gondola and Tuckman (1985) first demonstrated the benefit of longterm physical exercise on creative performance on Alternative Uses Test (AUT) and Remote Consequences that measured originality. Creativity can be improved by long-term and acute physical exercise (Gondola, 1986); aerobic exercise enhances creativity (Gondola, 1987; Steinberg et al., 1997) and the effect could last for $2 \mathrm{~h}$ (Blanchette et al., 2005). The third method is to enhance divergent thinking through mild bodily movement (Leung et al., 2012; Slepian and Ambady, 2012; Oppezzo and Schwartz, 2014). Fluid movements produced by tracing lines demonstrated a medium-to-large effect size ( $r=0.25,0.38,0.40$, and 0.46 for remote association, category inclusion, originality, and fluency, respectively) compared with rigid movements (Slepian and Ambady, 2012). Walking on a treadmill produced significant improvements compared with sitting (Oppezzo and Schwartz, 2014), using the indicators of appropriate uses $(r=0.33)$ and the number of total ideas $(r=0.44)$ in the AUT (Guilford et al., 1978). Moreover, free walking promoting metaphorical ideas related to divergent thinking enhanced performance in two tasks that required generating novel captions for ambiguous pictures $(r=0.28)$ and generating possible objects from viewing Lego assemblages $(r=0.15)$ compared with walking along a rectangular path (Leung et al., 2012).

Episodic-specificity induction enhances divergent thinking by encouraging recombining ideas across various episodes (Madore et al., 2015). Bodily movements influence divergent thinking by activating metaphorical abstract concepts (Leung et al., 2012; Slepian and Ambady, 2012). The use of a comfortable and selfselected pace in walking on a treadmill (Oppezzo and Schwartz, 2014) may promote a metaphor of unconstrained and free thoughts to enhance divergent thinking. Walking outdoors at one's natural pace (Oppezzo and Schwartz, 2014) may also trigger this abstract concept. The contrast between walking freely inside a rectangle and walking on a rectangular path promotes thinking outside the box (Leung et al., 2012), which entails unconstrained thinking.

If bodily movements encourage creative processes by metaphors (Leung et al., 2012; Slepian and Ambady, 2012), it remains unknown whether bidirectional links between bodily movements and metaphorical abstract concepts are essential for observing the benefits of movements on divergent thinking. According to the simulated sensorimotor metaphor (SSM) theory (Slepian and Ambady, 2014), bidirectional links relating concrete sensorimotor states and metaphorical abstract concepts are critical for a newly learned embodied metaphor to influence sensorimotor judgments. In that study, participants were primed with a novel metaphorical abstract concept that related time (past, present) and weight (heavy) by reading statements. Participants were then requested to estimate the physical weight, popularity, and age of a scientific book that had an old or a new cover. Only the participants who were physically given the book showed the effect of newly learned metaphors, judging the weight according to the primed condition. Participants who simply viewed a photograph of the book did not show the effect. The metaphorical concept must contain the sensorimotor representations (heavy), and the sensorimotor states (feel the weight) must link to the concept for a novel metaphor (e.g., past-heavy) to influence weight judgment. We hypothesize that bidirectional links are also essential for bodily movements to benefit divergent thinking.

The first goal of this study was to verify the hypothesis, adopting the free-walking conditions used in Leung et al.'s (2012) study. We chose walking because walking is easily implemented by people at any age, at any time, with little cost and with no equipment. More importantly, we can verify the hypothesis by examining whether metaphorical abstract (free, unconstrained) concepts or the experienced proprioceptive-motor kinematics (fluid movements) alone can enhance divergent thinking. If either element is effective, it can be beneficial for disabled elders who require assistance to move around. Experiment 1 examined the boundary condition for free walking to enhance divergent thinking. Given the results of Experiment 1, we investigated whether free walking also enhanced divergent thinking for older adults in Experiment 2.

\section{EXPERIMENT 1}

The purpose of this experiment was to investigate whether the bidirectional links between sensorimotor and metaphorical abstract concepts are critical for observing the benefits of free walking on divergent thinking. Leung et al. (2012) showed that participants who walked freely inside a square box performed better on divergent thinking than participants who walked along a rectangular path around the square box. Participants in the rectangular-walking condition did not show better performance compared with participants in the sitting condition. The null effect of the rectangular-walking condition may have arisen from two factors as follows: the rectangular box induced the metaphorical concept of constraining one's thoughts, and 
the experienced proprioceptive-motor kinematics is rigid from walking along lines with straight angles.

To verify the hypothesis, we added the following two conditions: free-generation and random-experienced groups. Participants in the free-generation condition generated free paths for the participants in the random-experienced condition to walk. In the former group, the metaphorical abstract concept was induced without the corresponding sensorimotor experiences. In the latter group, participants walked the random paths without self-initiated abstract concepts in the mind-body connection. Although participants in this group walked predetermined paths as participants in the rectangular-walking condition, these participants walked along random paths comprising many curves. By contrast, participants in the rectangularwalking condition walked along a path comprising angular corners. It has been shown that tracing curved lines enhanced creativity because of the fluid movements compared with tracing lines with rigid angles (Slepian and Ambady, 2012). If bidirectional links between proprioceptive-motor kinematics and metaphorical concepts related to creative processes are critical for producing the benefits of free walking on divergent thinking, we expected that only the participants in the free-walking condition would perform better compared to the rectangular-walking group. The rectangular-walking condition served as the control group because participants in this condition demonstrated a performance comparable to the sitting group (Leung et al., 2012) and participants in this condition also experienced walking movements.

\section{Method \\ Participants}

This study was approved by the Research Ethics Office of the National Taiwan University. Sixty-four undergraduate students (24 males, 40 females, mean at 23.95 years of age with $S D$ of 2.72) volunteered in the experiment for course credit or monetary rewards. The participants were randomly assigned to four different conditions ( $n=16$ in each group). All of the participants had normal or corrected-to-normal vision and were naïve regarding the purpose of the experiment.

\section{Design and Tasks}

We followed the method used in Leung et al.'s (2012) Experiment 2b. The experiment was a single factor between-subjects design, with the four conditions as the sole variable. To evaluate divergent thinking, we adopted an AUT that required the participants to generate as many unusual uses as possible for a common object: chopsticks. To assure that any group differences did not arise from individual characteristics, we also assessed creativity using a Creativity Assessment Packet (CAP; Williams, 1980) questionnaire and basic cognitive functions using a twoalternative forced choice (2AFC) task to measure processing speed and a "hearts and flowers" task (Davidson et al., 2006; Diamond et al., 2007) to assess cognitive flexibility.

\section{CAP instrument}

The original version of CAP is a 50-item questionnaire originally designed by Williams (1980) to measure creativity tendency. We used the Chinese version of CAP (Lin and Wang, 1994). Participants reported how often they believed they had experiences referenced by each item on a 3-point Likert scale from "almost always" to "almost never." Higher averaged total scores purportedly reflect higher tendency in the four dimensions of creativity: adventure, curiosity, imagination, and challenge. The revised CAP had good 4-week test-retest reliability $(r=0.61-0.74)$ and was positively correlated with the Pennsylvania Assessment of Creative Tendency (Rookey, 1973) and Torrance Tests of Creative Thinking (Torrance, 1987). We excluded the items related to experiences in school.

\section{AFC task}

Participants were asked to judge each presented stimulus by pressing the appropriate response key. Each trial began with the presentation of a fixed point for $1,500 \mathrm{~ms}$. A red circle or a green triangle was then presented in the center of the screen. The participants were required to judge which target was presented by pressing the left key of the mouse for one target and the right key for the other. The corresponding key was counterbalanced across participants. The participants were asked to respond to each target as quickly and as accurately as possible.

\section{Hearts and flowers task}

Participants were asked to follow different stimulus-response mapping rules in different blocks. Participants performed the same-side block first. In this block, a black heart was presented on the left or right side of the center fixation and the participants were asked to press the left or right button of a mouse corresponding to the target location (i.e., pressed the left button for a target on the left side). Participants then performed the different-side block. In this block, a black flower was presented on the left or right side of the center fixation and the participants were asked to press the opposite side of the target location (i.e., pressed the left button for a target on the right side). In the third block, a heart or a flower was randomly presented on the left or right side of the center fixation and the participants were required to follow the target-specific rule. They should adopt the same-side rule when a heart was presented and adopt the different-side rule when a flower was shown. The ability to swiftly switch between different rules reflects cognitive flexibility.

\section{Procedure}

Participants entered a room in which a $400 \mathrm{~m} \times 500 \mathrm{~m}$ rectangle was marked with colored tape and a desk was situated in the center of the rectangle. Participants were invited to sit by the desk with the experimenter while listening to the purpose of the study and signing the consent form. Participants were told that the experiment was to investigate how context may influence problem solving and they were asked to read the instructions of the AUT while sitting. Participants in the rectangular-walking and free-walking groups then walked along the rectangular path or freely inside the rectangle, respectively, for $2 \mathrm{~min}$ while contemplating the solutions to the assignment. Participants in the free-generation and random-experienced groups were paired so that one was randomly assigned to generate a free walking path with a laser pointer while the other followed the path. Participants then wrote their AUT responses within $10 \mathrm{~min}$. If participants 
finished the task before the time limit, they were encouraged to continue. Participants then filled the CAP instrument and performed the two cognitive tasks.

\section{Results and Discussion Creativity Assessment Packet (CAP)}

Table 1 shows the CAP score of each group. There were no significant differences among the four groups $[F(3,60)=0.28$, $\left.p=0.840, \eta_{\mathrm{p}}^{2}=0.014\right]$. Participants did not differ in creativity traits.

\section{AFC Task}

Accuracy (see Table 1) was at the ceiling for all groups (99\%), and there was no significant difference among groups $(p>0.80)$. The reaction times (RTs) of correct judgments were analyzed, excluding trials with RTs shorter than $70 \mathrm{~ms}$ or longer than $3000 \mathrm{~ms}$. There was also no significant difference in RTs among groups $(p>0.90)$. Participants did not differ on general processing speed.

\section{Hearts and Flowers Task}

Accuracy was at the ceiling for all three blocks, and there was no significant difference in RTs among groups in each block $(p>0.30)$. The switching cost was calculated by subtracting the RTs of the same-side block and the opposite-side block from the $\mathrm{RTs}$ in the mixed-rules block. There was no difference in the two switching cost measures among groups ( $p s>0.30$ ).

\section{The Alternative Uses Test (AUT)}

Three independent raters who were naive to the study purpose scored participants' responses on the three components of divergent thinking. Fluency was measured by the number of non-repeated responses. Flexibility was evaluated by the number of categories of appropriate uses, which is the most stringent criterion for a use. Originality was defined by novel and appropriate responses compared to normed responses. The intraclass correlation coefficient (ICC) was employed to examine the degree of agreement by three raters on the same item. The ICC was quite high in each component of creativity. The ICC for fluency was 0.997 (95\% confidence interval: 0.996-0.998, $F=1048.60, p<0.001)$, the ICC for flexibility was $0.918(95 \%$ confidence interval: $0.879-0.946, F=34.42, p<0.001$ ), the ICC for originality was 0.942 (95\% confidence interval: $0.914-0.963$, $F=50.12, p<0.001$ ), all of which indicate extremely high inter-rater reliability. Scores were averaged across the three raters.

Figure 1 shows the average scores among the four groups. Scores on the three components of divergent thinking were treated as three dependent measures, and a MANOVA was conducted using the groups (free-walking group, rectangularwalking group, free-generation group, random-experienced group) as a between-subject factor. An alpha level of 0.05 was used for all statistical tests. The results showed significant effects of groups among three components, $F(3,60)=8.44, p<0.001$, $\eta_{\mathrm{p}}^{2}=0.30$ for fluency, $F(3,60)=4.30, p=0.008, \eta_{\mathrm{p}}^{2}=0.18$ for flexibility, $F(3,60)=12.35, p<0.001, \eta_{\mathrm{p}}^{2}=0.38$ for originality. The Scheffe test for the three components of creativity showed that the participants in the free-walking group performed better than the rectangular-walking group ( $p=0.001)$, free-generation group $(p=0.003)$, and random-experienced group $(p=0.002)$ in fluency. Participants in the free-walking group performed better than the rectangular-walking group $(p=0.019)$ and the random-experienced group $(p=0.049)$ in flexibility; the free-walking group did not do better than participants in the free-generation condition $(p=0.16)$. Participants in the freewalking group performed better than the rectangular-walking group $(p<0.001)$, the free-generation group $(p<0.001)$, and the random-experienced group $(p<0.001)$ in originality. Compared with the rectangular-walking group, the effect of free walking on fluency $(r=0.62)$, flexibility $(r=0.52)$ and originality was strong $(r=0.64)$.

The results showed that bidirectional links between sensorimotor and metaphorical abstract concepts are critical for benefitting divergent thinking. Neither proprioceptive-motor kinematics alone in the random-experienced condition nor metaphorical abstract concepts per se in the free-generation condition could produce the benefits. Supporting the SSM model, integration of sensorimotor and conceptual components is critical for effective embodied cognition. More importantly, we observed benefits in all three components of divergent thinking. Thus, the null results of fluency and flexibility observed in Leung et al.'s (2012) experiment with young participants may have resulted from task insensitivity to the changes in these two measures.

One might question that the benefit of free walking arises from social signaling. Because participants were required to walk freely inside the box, they may have been primed to think outside the box without any constraints. By contrast, participants in the rectangular-walking condition were signaled to be rigid in thinking. We argue that social signaling is not a confounding factor for causing the benefits. If social signaling is the reason for observing the benefits, we should also observe benefits in the free-generation condition because these participants were also primed to think outside the box. Another concern is the social elements in pairing participants between the free-generation and random-experienced conditions. Although paired participants did not engage in any interaction, the paring itself may have caused potentially confounding effects. This concern is unlikely to explain the null results compared with the control group unless the pairing counteracts the benefits of metaphorical concepts or the benefits of sensorimotor execution. Social elements may not be a key factor for effective intervention because being more talkative to an experimenter while walking was not what produced benefits to divergent thinking (Oppezzo and Schwartz, 2014). A third possibility for observing the results is that walking provides an opportunity for mind-wandering or incubation to facilitate creative problem solving. Baird et al. (2012) showed that engaging in simple external tasks facilitates creative problem solving via promoting mind wandering. If mind wandering plays an important role, we would expect that participants in the rectangular-walking and free-generation conditions wandered more often that the participants in the random-experienced condition. In the former two conditions, the task was undemanding, following a pre-determined path or generating free paths without walking. In the latter condition, 
TABLE 1 | Mean performance and standard errors (SE, in parentheses) of the creativity assessment packet (CAP), two-alternative forced choice (2AFC task), and hearts and flowers task in Experiment 1.

\begin{tabular}{|c|c|c|c|c|c|}
\hline & CAP & 2AFC Accuracy & 2AFC RT (ms) & Switching cost 1 (ms) & Switching cost 2 (ms) \\
\hline Rectangular-walking group & $57.50(1.45)$ & $0.99(<0.01)$ & $408(18)$ & $240(17)$ & $201(18)$ \\
\hline Free-generation group & $57.75(1.92)$ & $0.99(<0.01)$ & $405(15)$ & $242(29)$ & $222(16)$ \\
\hline Random-experienced group & $57.25(1.87)$ & $0.99(<0.01)$ & $414(17)$ & $245(18)$ & $176(16)$ \\
\hline Free-walking group & $59.31(1.75)$ & $1.00(<0.01)$ & $406(15)$ & $248(20)$ & $201(18)$ \\
\hline
\end{tabular}

Switching cost 1 and cost 2 are the calculated by subtracting the RTs in the same-side block and opposite-side block from the RT in the mixed-block, respectively.

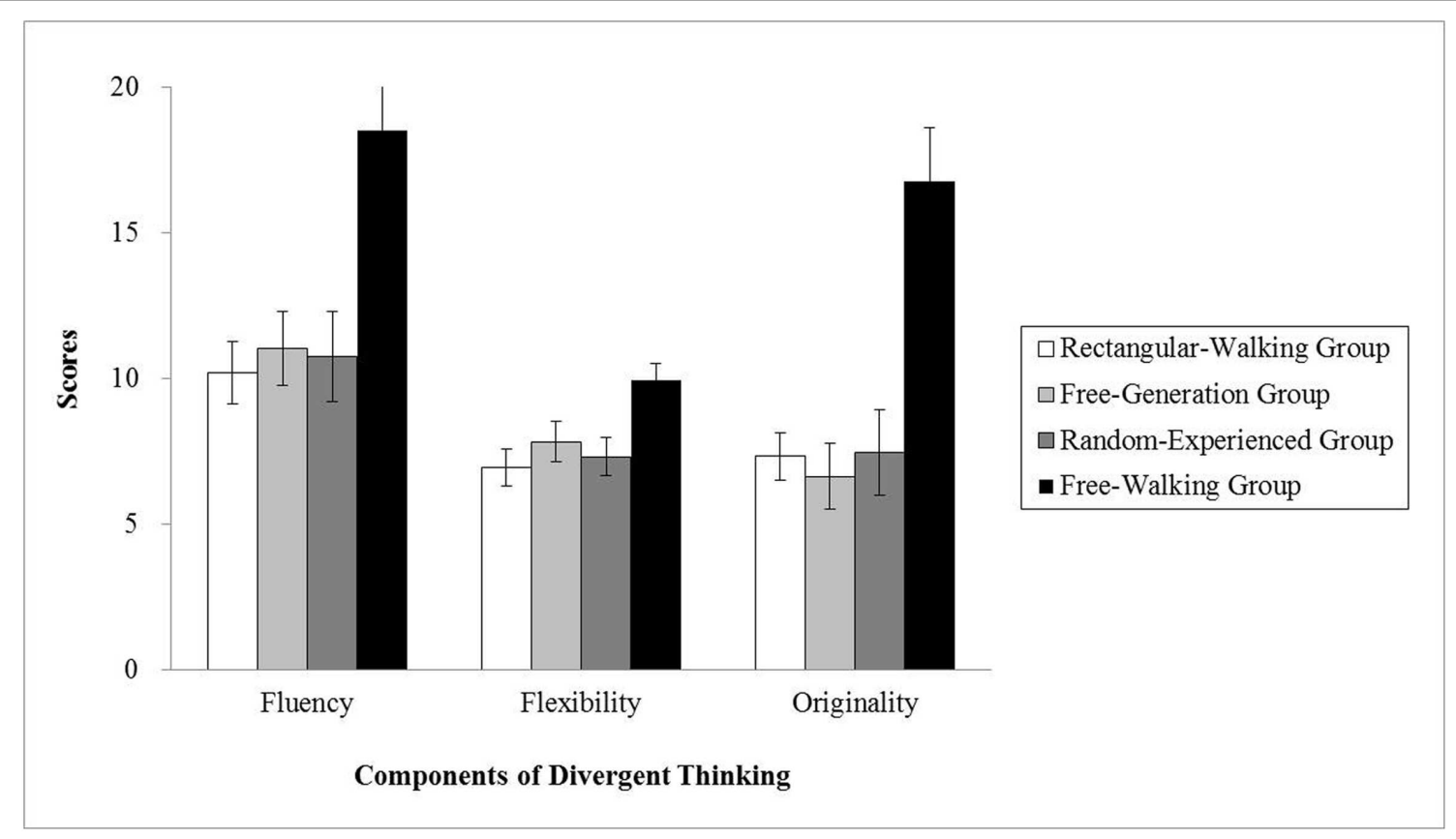

FIGURE 1 | Alternate uses test (AUT) performance in each group in Experiment 1. Error bars represent SES.

participants must pay attention to the paths generated by their partners and followed the paths. The results did not show any differences among these three groups.

Notably, the embodied experiences did not enhance performance on processing speed (2AFC task) and switching ability (the hearts and flowers task). It is likely that the cognitive measures reached the ceiling so that they were insensitive to changes in cognitive functions. This explanation applies only to the measure of processing speed because switch costs in reaction times were large. Moreover, it has been shown that the effect of training on cognitive functions is restricted to the trained tasks or tasks that share similar cognitive processes using similar materials (see Shipstead et al., 2012 for a review). Training is also effective after practicing trained activities for weeks. Training for juggling, for example, emphasizes hand-eye coordination. For such an activity, it could take from 6 weeks (Scholz et al., 2009) to 3 months (Draganski et al., 2004; Boyke et al., 2008) to induce changes in neural activity. For brisk walking to induce changes in neural networks related to cognitive functions, a period of 6 months is necessary (Colcombe et al., 2006). Two minutes of walking are likely insufficient to benefit cognitive functions.

\section{EXPERIMENT 2}

The purpose of this experiment was to investigate whether free walking can also benefit divergent thinking in older adults. It has been shown that scores on divergent thinking were positively related to the complexity in neural networks for older adults (Ueno et al., 2015). Elders with higher scores on divergent thinking showed activated rather than a loss of complexity in neural networks. However, it is unclear whether older adults can benefit from intervention programs that promote divergent thinking. Older adults did not show improvement after 8 weeks of activities (e.g., brainstorming, poetry) that promoted divergent thinking (Flood and Scharer, 2006). By contrast, episodicspecificity induction can enhance divergent thinking for older adults (Madore et al., 2016). One factor may have caused the inconsistent results. Although educational levels were unreported in the former study, approximately $57.9 \%$ of the participants reported "barely get by" as their economic status. By contrast, participants in the episodic-specificity induction study were highly educated $\left(M_{\text {education level }}=15.65, S D=2.17\right)$. Leon et al. (2014) showed that older participants with high education levels 
$\left(M_{\text {education level }}=17.23\right)$ could produce more unique responses on non-timed-constrained tasks of verbal divergent thinking compared to younger participants $\left(M_{\text {education level }}=14.27\right)$. It is possible that only older adults with a higher education may benefit from interventions for divergent thinking ability. We investigated whether older adults with low levels of education can benefit from free walking.

\section{Method}

\section{Participants}

Thirty-two older adults volunteered for the present experiment. These adults were recruited from a community center and received monetary rewards for their participation. None of the participants reported having a diagnosis of Alzheimer disease or a related disorder, history of stroke, head injury, psychiatric illness, or drug abuse problem on the self-report health-screening questionnaire. All participants reported corrected-to-normal vision and they were naïve to the purpose of the experiment.

All participants were over 65 years old, and the mean age was 74.06 years old $(S D=6.22)$. The participants were randomly assigned to two different groups: the rectangularwalking group and the free-walking group ( $n=16$ in each group). Table 2 summarizes the group characteristics. There was no group difference in age, educational level, scores on the MiniMental State Examination (MMSE; Folstein et al., 1975) that assesses global cognitive function, working memory capacity, and reasoning ability (all $p>0.40)$.

\section{Design, Task, and Procedure}

All aspects were the same as those used in Experiment 1, except that only the rectangular-walking and free-walking conditions were conducted. On the AUT, participants spoke the unusual uses while the experimenter recorded the responses.

\section{Results and Discussion Creativity Assessment Packet (CAP)}

There was no significant difference between the two groups $(p>0.20)$ on the CAP measure. The two groups did not differ in creativity traits (see Table 3 ).

\section{AFC Task}

Accuracy was at the ceiling for both groups (99\%), and there was no significant difference between groups $(p>0.90)$. RTs of correct judgments were analyzed, excluding trials with RTs

TABLE 2 | Group means and standard deviations (in parentheses) of age, education in years, global cognitive function measured with Mini-Mental State Examination (MMSE), and working memory performance in Experiment 2.

\begin{tabular}{lcc}
\hline & Rectangular-walking group & Free-walking group \\
\hline Age & $74.75(7.11)$ & $73.38(5.32)$ \\
Education & $5.94(4.60)$ & $5.69(3.00)$ \\
MMSE & $22.94(1.77)$ & $23.63(2.85)$ \\
Forward digit span & $10.75(3.32)$ & $10.88(2.92)$ \\
Backward digit span & $4.69(2.39)$ & $4.19(2.04)$
\end{tabular}

shorter than $70 \mathrm{~ms}$ or longer than $3000 \mathrm{~ms}$. There was also no significant difference in RTs between groups $(p>0.10)$. The two groups did not differ in processing speed (see Table 3 ).

\section{Hearts and Flowers Task}

Accuracy was at the ceiling in all three blocks, and there was no significant difference in RTs between groups in each block (all $p>0.40$ ). The switching cost was calculated by subtracting the RTs in the same-side block and the opposite-side block from the RTs in the mixed-rules block. There was no difference in the two switching cost measures between the two groups ( $p s>0.60)$. The two groups showed comparable performance in cognitive flexibility (see Table 3).

\section{Alternative Uses Test (AUT)}

The ICC was employed to examine the degree of agreement by the three raters on the same item. The ICC was extremely high in each component of creativity. The ICC for fluency was 0.993 (95\% confidence interval: $0.988-0.996, F=444.16$, $p<0.001$ ); for flexibility, 0.937 (95\% confidence interval: $0.890-0.966, F=45.36, p<0.001)$; and for originality, $0.931(95 \%$ confidence interval: $0.880-0.963, F=41.51, p<0.001$ ), which all indicate extremely high inter-rater reliability.

Figure 2 shows the average scores of each group on the three measures of divergent thinking. Scores on the three components of creativity were treated as three dependent measures, and a MANOVA was conducted with the groups (freewalking group and rectangular-walking group) as a betweensubject factor. The results showed a significant effect of groups among three components, $F(1,30)=16.77, p<0.001$, $\eta_{\mathrm{p}}^{2}=0.36$ for fluency, $F(1,30)=12.74, p=0.001, \eta_{\mathrm{p}}^{2}=0.30$ for flexibility, $F(1,30)=15.74, p<0.001, \eta_{p}^{2}=0.34$ for originality. Compared with the rectangular-walking group, the free-walking participants showed better divergent thinking with a large effect size ( $r$ s $=0.59,0.53,0.57$ for fluency, flexibility, and originality, respectively).

\section{Cross-Experiment Comparison}

The following two issues were of interest in this analysis: age effects on divergent thinking and whether free walking can enhance performance for older adults to a degree that is comparable to young adults in the rectangular-walking condition. Scores on the three components of creativity were then treated as three dependent measures, and a MANOVA was conducted with age (young adult compared with older adult) and walking condition (rectangular-walking compared with free-walking) as between-subject factors. The results showed that young adults earned higher scores on all three component measures of divergent thinking as follows: fluency $\left[F(1,60)=17.08, p<0.001, \eta_{\mathrm{p}}^{2}=0.22\right]$, flexibility $\left[F(1,60)=27.04, p<0.001, \eta_{\mathrm{p}}^{2}=0.31\right]$, and originality $\left[F(1,60)=10.56, p=0.002, \eta_{\mathrm{p}}^{2}=0.15\right]$. Participants in the free-walking condition outperformed participants in the rectangular-walking condition for fluency $[F(1,60)=36.95$, $\left.p<0.001, \eta_{\mathrm{p}}^{2}=0.38\right]$, flexibility $\left[F(1,60)=24.53, p<0.001, \eta_{\mathrm{p}}^{2}\right.$ $=0.29]$, and originality $\left[F(1,60)=37.75, p<0.001, \eta_{\mathrm{p}}^{2}=0.39\right]$. 


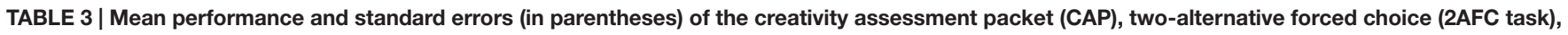
and hearts and flowers task in Experiment 2.

\begin{tabular}{|c|c|c|c|c|c|}
\hline & CAP & 2AFC Accuracy & 2AFC RT (ms) & Switching cost 1 (ms) & Switching cost 2 (ms) \\
\hline Rectangular-walking group & $55.69(1.53)$ & $0.99(0.01)$ & $659(33)$ & $496(53)$ & $331(41)$ \\
\hline Free-walking group & $58.38(1.96)$ & $0.99(0.01)$ & $601(25)$ & $504(40)$ & $365(54)$ \\
\hline
\end{tabular}

Switching cost 1 and cost 2 are the calculated by subtracting RTs in the same-side block and opposite-side block from RT in the mixed-block, respectively.

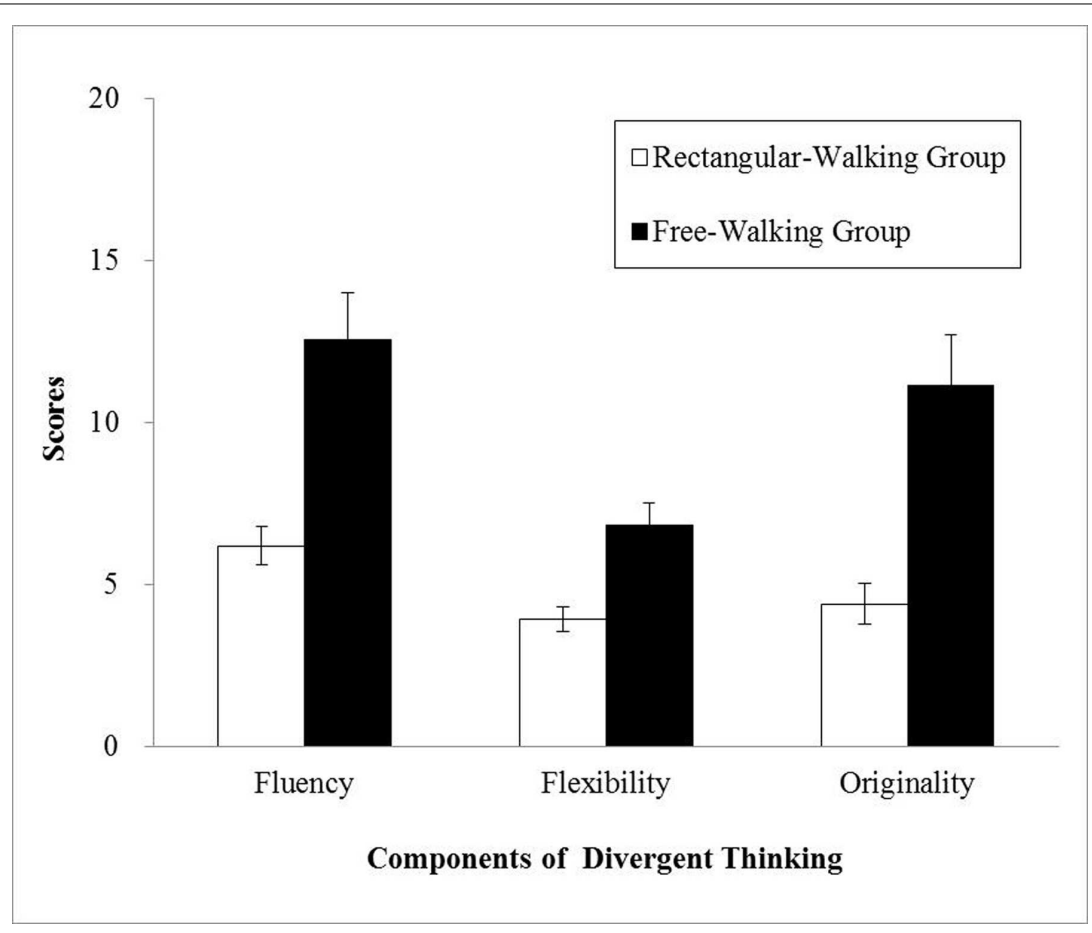

FIGURE 2 | Alternate uses test (AUT) performance of each group in Experiment 2. Error bars represent SEs.

The interaction did not reach significance for all three measures ( $p$ s $>0.30$ ), showing that free walking is beneficial for both younger and older adults. To evaluate whether free walking can improve divergent thinking for older adults compared with young participants in the control condition, we conducted planned comparisons between the older free-walking group and the young rectangular-walking group. The results showed a significant difference in originality $[t(30)=2.15, p=0.04$, $d=0.76, r=0.36]$ although the two groups did not differ on the other two measures $(p s>0.20)$. With free walking, older participants showed comparable or better performance by contrast to young adults who took a fixed walking path.

\section{GENERAL DISCUSSION}

This study had two objectives. First, we investigated the critical element that underlies the benefits of free walking on divergent thinking. Second, we examined whether free walking can benefit divergent thinking for older adults. The results from Experiment 1 showed that bidirectional links between proprioceptive-motor kinematics and metaphorical abstract concepts are critical for observing the benefits of free walking. The results of Experiment 2 showed that older adults can also benefit from free walking when generating unusual uses for a common object. More importantly, older adults in the free walking condition showed a comparable or better performance than young adults in the rectangular-walking control condition.

Supporting the hypothesis based on the SSM theory of embodied cognition, divergent thinking is enhanced only when the participants walked their own unconstrained, free paths. Activation of the concept without the sensorimotor element or vice versa could not produce the benefits to divergent thinking. It is plausible that our manipulation failed to activate the metaphorical concepts in the free-generation condition and to produce fluid movement in the random-experienced condition. Nevertheless, the benefits shown in the free-walking condition are extensive for both younger and older participants. An interesting finding that requires further investigation is the non-significant difference observed in Experiment 1 between the free-walking and free-generation groups on the flexibility measure. Although participants in the free-generation condition did not significantly outperform participants in the rectangularwalking condition, this null result suggests that activation of 
a metaphorical concept may enhance flexibility in divergent thinking.

To our knowledge, this is the first study that demonstrates the benefits of embodied cognition for older adults using the objective measures of divergent thinking. A previous study has shown that 4-week acting interventions may improve word recall and problem solving for older adults (Noice et al., 2004). Embodied cognition in acting includes multiple elements, including the multiple cognitive processes engaged in acting (e.g., mental simulation and deep processing of materials with meanings) in addition to affective and motoric elements (Noice and Noice, 2006). It is unknown which element is essential for acting to improve cognition. The essential element in free walking to benefit divergent thinking is the bidirectional links between conceptual representations and sensorimotor elements. In addition to art programs such as music, theater, dance, and design that promote healthy aging (Hanna et al., 2015), older adults could engage in free walking to improve their divergent thinking ability for creative problem solving in everyday life.

Compared with other art forms such as dance that nurture creative processes by embodiment, free walking can be conducted without any instruction or coaching. Over a short period of time ( $2 \mathrm{~min}$ in this study), this walking exercise improves divergent thinking. More importantly, we identified benefits for both highly educated young adults and less educated older participants. Note that the aging effect on divergent thinking is significant. Given the same condition (rectangular or free walking), older participants performed worse than younger participants. This finding is consistent with previous results (Alspaugh and Birren, 1977; Ruth and Birren, 1985; McCrae et al., 1987; Reese et al., 2001) but conflicts with the findings that age does not impair divergent thinking (Palmiero et al., 2014; Massimiliano, 2015; Madore et al., 2016) or that older adults performed better than younger adults on an AUT (Leon et al., 2014). Two factors may underlie age-related differences in the current study. First, we used a timed AUT. Although participants had 12 min to generate their responses, older adults, slower in processing speed, may not be able to generate as many unusual uses as young adults. Second, older participants were less educated than younger participants. Less educated older adults performed worse than highly educated young adults on divergent thinking under time constraints. However, free walking can enhance divergent thinking for less educated

\section{REFERENCES}

Alspaugh, P., and Birren, J. E. (1977). Variables affecting creative contributions across the adult life span. Hum. Dev. 20, 240-248. doi: 10.1159/000271559

Baird, B., Smallwood, J., Mrazek, M. D., Kam, J. W. Y., Franklin, M. S., and Schooler, J. W. (2012). Inspired by distraction: mind wandering facilitates creative incubation. Psychol. Sci. 23, 1117-1122. doi: 10.1177/0956797612446024

Blanchette, D. M., Ramocki, S. P., O’Del, J. N., and Casey, M. S. (2005). Aerobic exercise and creative potential: immediate and residual effects. Creat. Res. J. 17, 257-264. doi: 10.1207/s15326934crj1702\&3_10

Boyke, J., Driemeyer, J., Gaser, C., Buchel, C., and May, A. (2008). Traininginduced brain structure changes in the elderly. J. Neurosci. 28, 7031-7035. doi: 10.1523/JNEUROSCI.0742-08.2008 older adults. Importantly, older participants in the free-walking condition did not perform worse than younger participants in the rectangular-walking control condition. Older participants in the free-walking condition even outperformed young participants in the control condition on the originality measure. Free paths without angular corners at one's own pace may be the important elements for the metaphors to influence divergent thinking, as walking indoors on a treadmill with a comfortable, self-selected pace or walking outdoors at one's natural gait enhanced divergent thinking in young adults (Oppezzo and Schwartz, 2014). Older adults can take free walks at any time indoors or outdoors to generate novel and useful solutions for the problems they face. When running errands, older adults may adopt various routes rather than taking the same route to reduce their mental fixation. Through bodily movements that activate metaphorical concepts, older adults can improve their divergent thinking. With heightened ability, the loss of complexity in the neural networks may be reduced (Ueno et al., 2015), and self-efficacy may be strengthened for successful aging.

\section{AUTHOR CONTRIBUTIONS}

Y-YY and C-YK developed the study concept and design. Data was collected and analyzed by C-YK. Both authors interpreted the results. C-YK drafted the manuscript under the supervision of Y-YY, and Y-YY provided critical revisions. All authors approved the final version of the manuscript for submission.

\section{FUNDING}

This research was supported by a grant from the Ministry of Science and Technology to Y-YY (NSC 102-2420-H-002-009MY2 \& MOST 104-2410-H-002-056-MY2) and to C-YK (MOST 104-2410-H-153-021-MY3).

\section{ACKNOWLEDGMENT}

We thank Yang-Ming Huang and Cecilia Tien for their assistance on participant recruiting and data collection.

Colcombe, S. J., Erickson, K. I., Scalf, P., Kim, J., Wadhwa, R., McAuley, E., et al. (2006). Aerobic exercise training increases brain volume in aging humans: evidence from a randomized clinical trial. J. Gerontol. A Biol. Sci. Med. Sci. 61B, M1166-M1170. doi: 10.1093/gerona/61.11.1166

Davidson, M. C., Amso, D., Anderson, L. C., and Diamond, A. (2006). Development of cognitive control and executive functions from 4 to 13 years: evidence from manipulations of memory, inhibition, and task switching. Neuropsychologia 44, 2037-2078. doi: 10.1016/j.neuropsychologia.2006.02.006

Diamond, A., Barnett, W. S., Thomas, J., and Munro, S. (2007). Preschool program improves cognitive control. Science 318, 1387-1388. doi: 10.1126/science. 1151148

Draganski, B., Gaser, C., Busch, V., Schuierer, G., Bogdahn, U., and May, A. (2004). Neuroplasticity: changes in grey matter induced by training. Nature 427 , 311-312. doi: $10.1038 / 427311 \mathrm{a}$ 
Fisher, B., and Specht, D. (1999). Successful aging and creativity later in life. J. Aging Stud. 13, 457-472. doi: 10.1016/S0890-4065(99)00021-3

Flood, M., and Scharer, K. (2006). Creativity enhancement: possibilities for successful aging. Issues Ment Health Nurs. 27, 939-959. doi: $10.1080 / 01612840600899832$

Folstein, M. F., Folstein, S. E., and Mchugh, P. R. (1975). Mini-mental state: a practical method for grading the cognitive state of patients for the clinician. J. Psychiatr. Res. 12, 189-198. doi: 10.1016/0022-3956(75)90026-6

Gondola, J. C. (1986). The enhancement of creativity through long and short term exercise programs. J. Soc. Behav. Pers. 1, 77-82.

Gondola, J. C. (1987). The effects of a single bout of aerobic dancing on selected tests of creativity. J. Soc. Behav. Pers. 2, 275-278.

Gondola, J. C., and Tuckman, B. W. (1985). Effects of a systematic program of exercise on selected measures of creativity. Percept. Mot. Skills 60, 53-54. doi: 10.2466/pms.1985.60.1.53

Guilford, J. P. (1967). Creativity: yesterday, today, and tomorrow. J. Creat. Behav. 1, 3-14. doi: 10.1002/j.2162-6057.1967.tb00002.x

Guilford, J. P., Christensen, P. R., Merrifield, P. R., and Wilson, R. C. (1978), Alternate Uses: Manual of Instructions and Interpretations. Beverly Hills: Sheridan Psychological Services.

Hanna, G. P., Noelker, L. S., and Bienvenu, B. (2015). The arts, health, and aging in America: 2005-2015. Gerontologist 55, 271-277. doi: 10.1093/geront/gnu183

Kin, B. J., and Pope, B. (1999). Creativity as a factor in psychological assessment and healthy psychological functioning. J. Pers. Assess. 72, 200-207. doi 10.1207/S15327752JP720204

Leon, S. A., Altmann, L. J. P., Abrams, L., Gonzalez Rothi, L. J., and Heilman, K. M. (2014). Divergent task performance in older adults: declarative memory or creative potential? Creat. Res. J. 26, 21-29. doi: 10.1080/10400419.2014.873657

Leung, A. K. Y., Kim, S., Polman, E., Ong, L. S., Qiu, L., Goncalo, J. A., et al. (2012). Embodied metaphors and creative "acts." Psychol. Sci. 23, 502-509. doi: $10.1177 / 0956797611429801$

Lin, X. T., and Wang, M. R. (1994). Creativity Assessment Packet. Taipei: Psychological Publishing.

Livingston, J. A. (1999). Something old and something new: love, creativity, and the enduring relationship. Bull. Menninger Clin. 63, 40-52.

Madore, K. P., Addis, D. R., and Schacter, D. L. (2015). Creativity and memory: effects of an episodic specificity induction on divergent thinking. Psychol. Sci. 26, 1461-1468. doi: 10.1177/0956797615591863

Madore, K. P., Jing, H. G., and Schacter, D. L. (2016). Divergent creative thinking in young and older adults: extending the effects of an episodic specificity induction. Mem. Cognit. 44, 974-988. doi: 10.3758/s13421-016-0605-Z

Massimiliano, P. (2015). The effects of age on divergent thinking and creative objects production: a cross-sectional study. High Abil. Stud. 26, 93-104. doi: $10.1080 / 13598139.2015 .1029117$

McCracken, J. L. (1991). Creativity and leisure for recovering alcoholics. Alcohol. Treat. Q. 8, 83-89. doi: 10.1300/J020V08N03_08

McCrae, R. R., Arenberg, D., and Costa, P. T. (1987). Declines in divergent thinking with age: cross-sectional, longitudinal, and cross sequential analyses. Psychol. Aging 2, 130-137. doi: 10.1037/0882-7974.2.2.130

Noice, H., and Noice, T. (2006). What studies of actors and acting can tell us about memory and cognitive functioning. Curr. Dir. Psychol. Sci. 15, 14-18. doi: 10.1111/j.0963-7214.2006.00398.x

Noice, H., Noice, T., and Staines, G. (2004). A short-term intervention to enhance cognitive and affective functioning in older adults. J. Aging Health 16, 1-24. doi: $10.1177 / 0898264304265819$

Oppezzo, M., and Schwartz, D. L. (2014). Give your ideas some legs: the positive effect of walking on creative thinking. J. Exp. Psychol. Learn. Mem. Cogn. 40, 1142-1152. doi: $10.1037 / \mathrm{a} 0036577$

Palmiero, M., Di Giacomo, D., and Passafiume, D. (2014). Divergent thinking and age-related changes. Creat Res. J. 26, 456-460. doi: $10.1080 / 10400419.2014 .961786$
Reese, H., Lee, L., Cohen, S., and Puckett, J. M. (2001). Effects of intellectual variables, age, and gender on divergent thinking in adulthood. Int. J. Behav. Dev. 25, 491-500. doi: 10.1080/01650250042000483

Reiter-Palmon, R., Mumford, M. D., and Threlfall, K. U. (1998). Solving everyday problems creatively: the role of problem construction and personality type. Creat. Res. J. 11, 187-198. doi: 10.1207/s15326934crj1103_1

Rookey, T. J. (1973). Pennsylvania Assessment of Creative Tendency. East Stroudsburg, PA: East Stroudsburg State University.

Russ, S. W. (1998). Play, creativity, and adaptive functioning: implications for play interventions. J. Clin. Child Psychol. 27, 469-480. doi: 10.1207/s15374424jccp2704_11

Ruth, J. E., and Birren, J. E. (1985). Creativity in adulthood and old age: relations to intelligence, sex, and mode of testing. Int. J. Behav. Dev. 8, 99-109. doi: $10.1177 / 016502548500800107$

Scholz, J., Klein, M. C., Behrens, T. E. J., and Johansen-Berg, H. (2009). Training induces changes in white-matter architecture. Nat. Neurosci. 12, 1370-1371. doi: $10.1038 / \mathrm{nn} .2412$

Scott, G., Leritz, L. E., and Mumford, M. D. (2004). The effectiveness of creativity training: a quantitative review. Creat. Res. J. 16, 361-388. doi: $10.1080 / 10400410409534549$

Shipstead, Z., Redick, T. S., and Engle, R. W. (2012). Is working memory training effective? Psychol. Bull. 138, 628-654. doi: 10.1037/a0027473

Slepian, M. L., and Ambady, N. (2012). Fluid movement and creativity. J. Exp. Psychol. Gen. 141, 625-629. doi: 10.1037/a0027395

Slepian, M. L., and Ambady, N. (2014). Simulating sensorimotor metaphors: novel metaphors influence sensory judgments. Cognition 130, 309-314. doi: 10.1016/j.cognition.2013.11.006

Steinberg, H., Sykes, E. A., Moss, T., Lowery, S., and LeBoutillier, N. (1997). Exercise enhances creativity independently of mood. Br. J. Sports Med. 31, 240-245. doi: 10.1136/bjsm.31.3.240

Terr, L. C. (1992). Mini-marathon groups: psychological "first aid" following disasters. Bull. Menninger. Clin. 56, 76-86.

Tierney, P., Farmer, S. M., and Graen, G. B. (1999). An examination of leadership and employee creativity: the relevance of traits and relationships. Pers Psychol. 52, 591-620. doi: 10.1111/j.1744-6570.1999.tb00173.x

Torrance, E. P. (1987). Guidelines for Administration and Scoring/Comments on Using the Torrance Tests of Creative Thinking. Bensenville, IL: Scholastic Testing Service, Inc.

Tsai, K. C. (2014). A review of the effectiveness of creative training on adult learners. J. Soc. Sci. Stud. 1, 17-30. doi: 10.5296/jsss.vli1. 4329

Ueno, K., Takahashi, T., Takahashi, K., Mizukami, K., Tanaka, Y., and Wada, Y. (2015). Neurophysiological basis of creativity in healthy elderly people: a multiscale entropy approach. Clin. Neurophysiol. 126, 524-531. doi: 10.1016/j.clinph.2014.06.032

Williams, F. E. (1980). Creativity Assessment Packet. East Aurora, NY: DOK.

Conflict of Interest Statement: The authors declare that the research was conducted in the absence of any commercial or financial relationships that could be construed as a potential conflict of interest.

The reviewer AA and handling Editor declared their shared affiliation, and the handling Editor states that the process nevertheless met the standards of a fair and objective review.

Copyright ( $\odot 2016$ Kuo and Yeh. This is an open-access article distributed under the terms of the Creative Commons Attribution License (CC BY). The use, distribution or reproduction in other forums is permitted, provided the original author(s) or licensor are credited and that the original publication in this journal is cited, in accordance with accepted academic practice. No use, distribution or reproduction is permitted which does not comply with these terms. 


\section{OPEN ACCESS}

Edited by:

Annalisa Setti,

University College Cork, Ireland

Reviewed by:

Edmund Wascher,

Leibniz Research Centre for Working

Environment and Human Factors

(LG), Germany

Jason Chan,

University College Cork, Ireland

${ }^{*}$ Correspondence:

Ya-Nan Niu

niuyn@psych.ac.cn

Juan Li

lijuan@psych.ac.cn

Specialty section:

This article was submitted to

Cognition,

a section of the journal

Frontiers in Psychology

Received: 16 November 2016

Accepted: 02 May 2017

Published: 29 May 2017

Citation:

Niu Y-N, Zhu X and Li J (2017)

The Age Effects on the Cognitive

Processes of Intention-Based and Stimulus-Based Actions: An ERP

Study. Front. Psychol. 8:803.

doi: 10.3389/fpsyg.2017.00803

\section{The Age Effects on the Cognitive Processes of Intention-Based and Stimulus-Based Actions: An ERP Study}

\author{
Ya-Nan Niu ${ }^{1 *}$ Xinyi Zhu ${ }^{1,2}$ and Juan $\mathrm{Li}^{1,2,3 *}$ \\ ${ }^{1}$ Center on Aging Psychology, CAS Key Laboratory of Mental Health, Institute of Psychology, Chinese Academy of Sciences, \\ Beijing, China, ${ }^{2}$ Department of Psychology, University of Chinese Academy of Sciences, Beijing, China, ${ }^{3}$ State Key \\ Laboratory of Brain and Cognitive Science, Institute of Biophysics, Chinese Academy of Sciences, Beijing, China
}

The functional decline in action among older adults is caused not only by physical weakness but also by cognitive decline. In this study, we aimed to compare the cognitive effects of age between intention-based and stimulus-based action modes electrophysiologically. Because age-related declines in cognitive function might proceed distinctly according to specific action modes and processes, four specific cognitive processes, action-effect binding, stimulus-response linkage, action-effect feedback control, and effect-action retrieval, were investigated. We recorded event-related potentials (ERPs) during a modified acquisition-test paradigm in young (mean age $=21$, $S D=2$ ) and old (mean age $=69, S D=5$ ) groups. A temporal bisection task and a movement pre-cuing task were used during the acquisition and test phases, respectively. Using ERP indices including readiness potential (RP), P3, N2 and contingent negative variation (CNV) to identify these four specific processes for the two action modes, we revealed the effects of age on each ERP index. The results showed similar patterns of waveforms but consistently decreasing amplitudes of all four ERP indices in the old age group compared with the young age group, which indicates not only generally declining functions of action preparation in older adults but also age effects specific to the action modes and processes that might otherwise be mixed together under confounding experimental conditions. Particularly, an interference effect indexed by the differences in the amplitudes of CNV between congruent and incongruent tasks was observed in the young age group, which is consistent with previous behavioral reports. However, this effect was absent in the old age group, indicating a specific age-related deficit in the effect-action retrieval process of intentionbased action, which might be caused by an age-related deficit in associative memory. In sum, this study investigated the cognitive processes of two action modes from a developmental perspective and suggests the importance of adding associative memory training to interventions for older adults with the aim of improving intention-based action.

Keywords: age effect, motor cognition, intention-based action, stimulus-based action, bidirectional action-effect association, ERPs, associative memory, executive function 


\section{INTRODUCTION}

The functional decline in action among older adults is caused not only by physical weakness but also by cognitive decline. To date, the effects of healthy aging on cognitive processes of action are not well understood (Onofrj et al., 2001 for a review; Sterr and Dean, 2008; Reuter et al., 2015).

Age-related declines in the prefrontal cortex (PFC) and executive function have been implicated in changes in both the motor preparation and execution phases (Sterr and Dean, 2008; Berchicci et al., 2012; Woods et al., 2015). The possible underlying mechanism involves the compensatory mechanism, overactivation of specific brain areas, and dedifferentiation (see Reuter-Lorenz, 2002 for a review). However, the effect of age on more specific cognitive processes remains unclear. According to the frontal lobe hypothesis (West, 1996, 2000) and reverse theory (Braak et al., 1999), age-related declines in brain areas might proceed distinctly. Thus, the related cognitive processes would be impaired distinctly. Therefore, it is necessary to compare the age effect between different action modes under specific cognitive processes to further understand the effects of age on motor cognition.

Recently, a series of studies investigating motor cognition by comparing intention-based and stimulus-based actions has verified several specific cognitive processes of actions (Elsner and Hommel, 2001; Haggard et al., 2002; Waszak et al., 2005; Keller et al., 2006; Herwig et al., 2007; Herwig, 2015 for a review). Using an acquisition-test paradigm, a bidirectional action-effect association was identified for intention-based action, which included the cognitive processes of actioneffect binding and effect-action retrieval, whereas the cognitive process of stimulus-response linkage was observed for stimulusbased action. Interestingly, the effect-action retrieval process of intention-based action resembled the retrieval of associative memory (Elsner et al., 2002; Melcher et al., 2008, 2013; Pfister et al., 2014), suggesting that associative memory was also involved in the cognitive processes of action. For older adults, it is well known that associative memory is more vulnerable than other types of memory (e.g., Naveh-Benjamin, 2000; see Yonelinas, 2002 for a review; Rhodes et al., 2008). Therefore, in this study, using certain paradigms, we aimed to investigate the age effects on several specific cognitive processes of actions between young and old age groups, with special interest in the associative retrieval of intention-based action.

In previous studies, the acquisition-test paradigm has been primarily used to reveal differences in the cognitive mode between intention-based and stimulus-based actions (e.g., Elsner and Hommel, 2001; Herwig et al., 2007). Participants were divided into two groups and participated in both the acquisition and test phases. During the acquisition phase, the intentionbased group of participants was instructed to make a selfselected keypress (left or right keypress, i.e., action) which was always followed by a certain tone (high or low pitch, i.e., effect), whereas the stimulus-based group were instructed to respond to the stimuli according to prespecified rules, although the response was also followed by certain high- or low-pitch tones. Thus, action-effect binding was acquired by intentionbased acquisition and stimulus-linkage was acquired by stimulusbased acquisition. In the subsequent test phase, participants were asked to perform a rapid keypress for the same tones with a high or low pitch according to either the congruent or incongruent response rule with the acquired linkages. The results showed that the interference effect of reaction time (RT), that is, faster performance on the congruent task compared with incongruent task, was only observed in intention-based action. These results demonstrated a bidirectional action-effect association in intention-based action, that is, the learned action-effect binding during acquisition was then retrieved in reverse (i.e., effect-action retrieval) during the test phase. The RT performance of effectaction retrieval was thus prolonged by the interference from the incongruent task. However, this interference effect was absent in stimulus-based action, suggesting that a mono-directional stimulus-response linkage was formed.

As Elsner et al. (2002) suggested, this effect-action retrieval observed in intention-based actions resembles the process of associative retrieval of memory, and this conclusion has been supported by PET and fMRI studies revealing activation in both the supplementary motor area (SMA) and medial temporal memory system during either action image or execution tasks (Elsner et al., 2002; Melcher et al., 2008, 2013; Pfister et al., 2014). This effect-action retrieval, as well as the age effect on this process, has been seldom investigated using event-related potentials (ERPs); however, several studies have reported the ERP indices of the acquisition phase for intention-based and stimulus-based actions. In these ERP studies (Waszak et al., 2005; Keller et al., 2006), a temporal bisection task was used during the acquisition phase to ensure that the two action modes were measured in a comparable manner when the ERP technique was applied (see Materials and Methods). The results demonstrated that the readiness potential (RP) component, which proceeded slowly and negatively before action execution, reflecting general preparation for voluntary movement (e.g., Shibasaki and Hallett, 2006), was more negative for intention-based acquisition, whereas the P3 component-a positive potential observed approximately $300 \mathrm{~ms}$ after stimulus presentation, reflecting the formation of a link between the stimulus evaluation and response selection (e.g., Petruo et al., 2016) - was more positive under stimulus-based conditions. In this study, we will use the time bisection task during the acquisition phase to compare the age effect on specific cognitive processes of intention-based and stimulusbased actions, with action-effect binding indexed by RP amplitude and stimulus-response linkage indexed by P3 amplitude. In this study, the age effects were predicted to be a decrease in the amplitude of both the RP and P3 components in the old age group because both stimulus processing and motor preparation have been reported to be responsible for the age-related decline of actions (Kolev et al., 2006; Sterr and Dean, 2008; Berchicci et al., 2012; Woods et al., 2015).

In addition to these two cognitive processes during the acquisition phase, another cognitive process, namely feedback control of intention-based action, was also included in this study. When a participant performed an intention-based action to fulfill a certain goal, the action effect was predicted and compared 
with the actual action effect as observed from the external environment using a comparator system (Blakemore et al., 2000 for a review; Hughes and Waszak, 2011; Hughes et al., 2013 for a review). This feedback control mechanism has been reported to be associated with the anterior cingulate cortex (ACC) and function as a performance monitoring and adjustment system (Debener et al., 2005). In a recent ERP study (Hughes and Waszak, 2011), this feedback control process was observed by the $\mathrm{N} 2$ amplitude, which was fronto-centrally distributed within a 150-350 ms time window after the appearance of the action effect, followed immediately by the P3 component. Similarly, in this study, we expected to detect this feedback control process based on the N2 amplitude during the acquisition phase in intentionbased action. The age effect was predicted to be a decrease in the $\mathrm{N} 2$ amplitude in the old age group, especially regarding intention-based action.

In terms of the effect-action retrieval process during the test phase, the contingent negative variation (CNV) amplitude in a movement pre-cuing task was used to reflect the action preparation before the action execution. In the movement pre-cuing task, the upcoming movement was informed in advance by the pre-cue, and the amplitude of the CNV tended to increase with the amount of advance information provided by the pre-cue, either because this information enabled more sufficient preparation for the upcoming action (Leuthold et al., 2004) or because additional resources were available for the subjects to complete the task (Falkenstein et al., 2003). In this study, we assumed that, in intention-based action, the $\mathrm{CNV}$ amplitude would increase in congruent tasks because the congruent response rule facilitates action preparation, in contrast to incongruent tasks, in which the incongruent response rule interferes with action preparation. Therefore, the difference in the CNV amplitude between congruent and incongruent tasks demonstrates the interference effect of RT in intention-based actions in the young age group, as introduced in previous behavioral studies. Such an interference effect of CNV amplitude would not be observed in stimulus-based action. In our previous study (Niu et al., 2016), this interference effect of the CNV amplitude was absent in intention-based action in healthy older adults even after receiving cognitive training. In this study, we predicted that we would observe this interference effect of the $\mathrm{CNV}$ amplitude in the young age group but not in the old age group, which would indicate an age-related impairment on the effect-retrieval process in intention-based action. A correlation analysis was conducted between the cognitive performance and $\mathrm{CNV}$ amplitude in each task to investigate whether associative memory scores were responsible for this interference effect.

In summary, multiple cognitive processes may be involved in intention-based and stimulus-based actions. As suggested by the frontal lobe hypothesis (West, 1996, 2000) and the reverse theory (Braak et al., 1999), age-related declines occur in distinct brain areas, according to the different cognitive functions and processes. Therefore, it is necessary to investigate the age effect under specific cognitive processes, which might be otherwise mixed in confounding experimental conditions. Using the acquisition-test paradigm and time-bisection task, we aimed to investigate the age effects on actions by comparing specific cognitive processes between intention-based and stimulusbased action modes in young and old age groups. Four specific cognitive processes, action-effect binding, stimulusresponse linkage, action-effect feedback control, and effect-action retrieval, were investigated indexed using the RP, P3, N2, and CNV amplitudes, respectively. Compared with stimulus-based actions, intention-based actions have been suggested to be more evolved and play an important role in daily life (Neumann, 1990; see Herwig, 2015 for a review), which was the main aim of this study. In particular, we wanted to investigate the age effect on the effect-action retrieval process in intention-based actions and the potential relationship between action retrieval and associative memory retrieval. We hypothesized that (1) generally declining functions of action preparation in older adults would be revealed by consistently decreasing ERP amplitudes in four cognitive processes of actions; (2) age-related decline would be observed under each specific cognitive process, which might be otherwise mixed and misinterpreted in confounding conditions; (3) particularly, an age effect on effect-action retrieval process of intention-based action would be revealed via the interference effect of the CNV amplitude between congruent and incongruent tasks, which would be observed in the young age group and be absent in the old age group, possibly due to the age-related deficit of associative memory.

\section{MATERIALS AND METHODS}

\section{Participants}

Thirty-six healthy subjects participated in the study, including 18 young subjects (10 women and 8 men) aged 19-24 years (mean age $=21, S D=2)$ and 18 elderly subjects ( 9 women and 9 men) aged 61-74 years (mean age $=69, S D=5$ ). The age groups were matched for years of education (young subjects: 14 years, $S D=1$; elderly: 13 years, $S D=3$ ).

All of the subjects were recruited through advertisements placed in universities and communities near the Institute of Psychology, Chinese Academy of Sciences. The young subjects were all undergraduates that were active in the university. All of the subjects were right-handed, had no history of severe psychiatric or neurological disease, did not use drugs that might adversely affect cognition (i.e., benzodiazepines or antipsychotics), had normal or corrected-to-normal vision and audition, and scored $\leq 16$ on the Center for Epidemiologic Studies Depression Scale (CES-D, Roberts and Vernon, 1983). The Mini Mental State Examination (MMSE, Folstein et al., 1975) was used as a screening test for dementia, and all elderly subjects included in this study scored $\geq 27$ out of 30 possible points. Both the young and elderly subjects were also administered neuropsychological tests, including the Paired Associative Learning Test (PALT, Xu and $\mathrm{Wu}, 1986$ ), a test of associative memory of word pairs, and Trail Making Test A and B (TMT, Reitan, 1955), to test executive function. The test results are shown in Table 1.

All of the subjects were naïve to the purpose of the experiment and were financially reimbursed for their participation. The study was approved by the Ethics Committee of the Institute 
TABLE 1 | Neuropsychological test scores for the two age groups.

\begin{tabular}{|c|c|c|c|}
\hline & $\begin{array}{c}\text { Young }(n=18) \\
\text { Mean }(S D)\end{array}$ & $\begin{array}{l}\text { Old }(n=18) \\
\text { Mean }(S D)\end{array}$ & $P$-values ( $t$-test) \\
\hline MMSE & - & $27.78(1.26)$ & - \\
\hline CESD & $9.39(3.47)$ & $12.17(2.96)$ & 0.014 \\
\hline PALT & $19.89(4.90)$ & 11.78 (8.23) & 0.002 \\
\hline TMT $A^{a}$ & $20.86(6.17)$ & $40.10(17.14)$ & 0.001 \\
\hline TMT B ${ }^{a}$ & 27.55 (6.87) & $62.51(27.85)$ & $<0.001$ \\
\hline
\end{tabular}

PALT, paired associative learning test; TMT, trail making test. ${ }^{a}$ Lower scores indicate better performance.

of Psychology, Chinese Academy of Sciences. Written informed consent was procured from all of the subjects prior to participation.

All subjects participated in both the acquisition and test phases. For the analysis of the RP, N2 and P3 components during the acquisition phase, 18 young subjects and 18 elderly subjects were used. For the analysis of CNV components during the test phase, the subjects in each age group were divided into two subgroups that performed congruent or incongruent tasks. For the young age group, 9 subjects ( 5 women, mean age $=22, S D=1)$ performed congruent tasks, and 9 subjects ( 5 women, mean age $=21, S D=1$ ) performed incongruent tasks. For the old age group, 8 subjects (2 women, mean age $=70, S D=4$ ) performed congruent tasks, and 10 subjects (7 women, mean age $=69, S D=5$ ) performed incongruent tasks. The order of the intention-/stimulus-based acquisition and the congruent/incongruent tasks was counterbalanced for each group.

\section{Apparatus and Stimuli}

The subjects were seated in a relaxed position on a comfortable chair in front of a computer screen placed on a table $75 \mathrm{~cm}$ away from the subjects' eyes in a dimly lit, sound-attenuated and electrically isolated room. The two response keys were located on a keyboard and were separated by a horizontal distance of $45 \mathrm{~mm}$. The fixation site was a white cross ("+") depicted on a black background at a visual angle of $0.8^{\circ} \times 0.8^{\circ}$. The possible visual stimuli were the letters $\mathrm{A}, \mathrm{T}, \mathrm{O}$, and $\mathrm{X}$ presented in white (height: $1.7^{\circ}$ ) against a black background at the center of the computer screen. During the test phase, a red asterisk (“*”) with a visual angle of $0.8^{\circ} \times 0.8^{\circ}$ presented centrally in the screen was used as the imperative signal for a keypress response. At the beginning of each block, an auditory pacing signal composed of sine tones with a frequency of $600 \mathrm{~Hz}$ and a duration of $100 \mathrm{~ms}$ was presented at a comfortable volume. The stimulus presentation and data collection were performed using E-prime (Version 2.0) software in a Windows XP system.

\section{Tasks and Procedures}

The experiment lasted approximately $3 \mathrm{~h}$, including the time required for instruction and practicing, fixing the electroencephalogram (EEG) electrodes to the scalp, and completing the tasks. Each subject completed four experimental sessions, including the acquisition and test phases, for both intention-based and stimulus-based actions. Figure 1A showed the tasks and procedures used in this study. During the acquisition phase, all of the subjects performed intention-based and stimulus-based acquisition with a temporal bisection task to acquire the action-effect and stimulus-response pairs, respectively. During the subsequent test phase, the subjects in each age group were divided into two subgroups to perform a movement pre-cuing task in which the keypress was determined by the letters acquired during acquisition phase. Half of the subjects performed a congruent task in which the keypress was determined by the congruent letter they acquired, whereas the remaining half performed an incongruent task in which the keypress was determined by a letter incongruent with the letter they acquired.

\section{Acquisition Phase}

The temporal bisection task used during the acquisition phase was adapted from Keller et al. (2006). Before the formal experiment, subjects performed practice trials for time bisection per se, which was a pure timing task. This task required them to press either of the two response keys to bisect a 1,600 ms interstimulus interval (ISI) with a fixation cross (“+”) presented at the center of the screen. Each presentation of a 1,600-ms ISI was indicated by a centrally placed number eight (" 8 ") that appeared on the screen for $200 \mathrm{~ms}$. There were 20 trials in each practice block, after which the subjects were informed of their timing performance to indicate whether their responses were relatively fast or slow within $350 \mathrm{~ms}$ of the exact bisection time point. Subjects entered the formal experiment when they reported that they were ready for the task and when their performance was adequate, that is, the $80 \%$ criterion of response accuracy rates was reached.

The subjects were then fixed with EEG electrodes on their scalps before the screen and performed both intention-based and stimulus-based acquisition sessions recorded by EEG. Figure 1A (upper panel) showed the task procedure during acquisition phase. For the intention-based acquisition, the subjects were asked to press the left or right key in a self-selected way to bisect the 1600-ms ISI. Each key press produced an "action effect" in which a letter (i.e., A, T, O, or X) appeared for $200 \mathrm{~ms}$ at the end of each ISI. For example, the self-selection of the left keypress determined the appearance of the letter $A$, and the self-selection of the right keypress determined the appearance of the letter $\mathrm{T}$. The subjects were instructed to attempt to produce a random sequence of letters, as if they were tossing a coin, rather than produce a sequence of a certain order. For the stimulus-based acquisition, the subjects were asked to perform the bisection time task by pressing the left or right key according to the appearance of a letter. For example, the appearance of the letter $\mathrm{O}$ signaled a left keypress, and the appearance of the letter $\mathrm{X}$ signaled the right keypress. These letters were presented in a randomized and counterbalanced sequence. To eliminate carry-over effects, different letters were presented for the same subject across the two actions.

Subjects could take a break between blocks, which contained 40 trials. An auditory pacing signal was presented in the first 


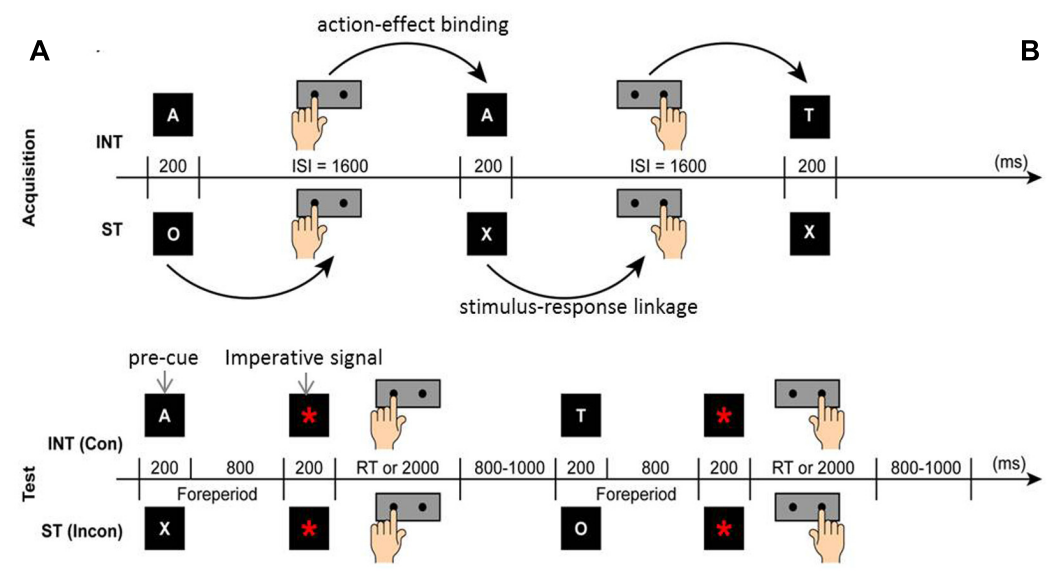

B

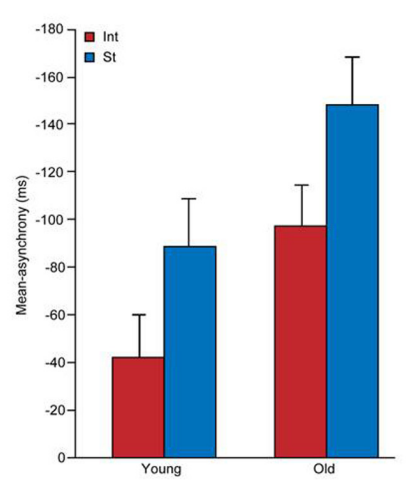

FIGURE 1 | Experiment design (A) and behavioral results (B). (A) Experimental procedure for the acquisition (upper) and test (lower) phases showing the trial sequence of intention-based (INT) and stimulus-based (ST) actions in congruent (Con) and incongruent (Incon) tasks. ISI, Inter-Stimulus Interval (adapted from Niu et al., 2016). (B) Mean asynchrony of temporal bisection for intention-based (red bars) and stimulus-based (blue bars) actions in both age groups during the acquisition phase. The error bars represent the standard errors.

10 trials of each block to indicate the true ISI midpoint and assist subjects with their timing performance. For the remaining 30 trials, the subjects performed the time bisection without the signal. To calculate the correct responses, the time duration of $\pm 350 \mathrm{~ms}$ of the true bisection point of $800 \mathrm{~ms}$ was examined. Responses that fell within this time duration were considered correct. The blocks were repeated until 200 correct responses (excluding auditory signaled trials) were achieved for each action mode. Thus, sufficient trials were ensured for the ERP average under each condition.

\section{Test Phase}

The subjects entered the test phase when the acquisition tasks of both the intention-based and stimulus-based actions were complete. Figure 1A (lower panel) showed the task procedure during test phase. During the test phase, one subgroup performed a congruent task, and the other subgroup performed an incongruent task. Two sessions of the movement pre-cuing task were administered in which the previously acquired two pairs of letters (e.g., A and T, O and X) were used as pre-cues to signal the subsequent keypress. After the presentation of the fixation cross ("+") for $200 \mathrm{~ms}$, the pre-cue (i.e., A, T, O, or X) appeared in the center of the screen for the next $200 \mathrm{~ms}$ to indicate a left or right keypress. For the congruent task, subjects were asked to press the left or right key according to the congruent rule learned during the acquisition phase. For example, subjects who had acquired left keypress $\rightarrow \mathrm{A}$ /right keypress $\rightarrow \mathrm{T}$ were then required to respond to $\mathrm{A}$ with a left keypress and to $\mathrm{T}$ with a right keypress. For the incongruent task, subjects were asked to perform the opposite keypresses. For example, subjects who had acquired $\mathrm{O} \rightarrow$ left keypress $/ \mathrm{X} \rightarrow$ right keypress were then required to respond to $\mathrm{O}$ with a right keypress and to $\mathrm{X}$ with a left keypress.

To ensure sufficient motor preparation before the motor response (Falkenstein et al., 2003; Leuthold et al., 2004), subjects were instructed not to respond immediately to the pre-cue but to withhold their response until the imperative signal (a red asterisk “*”) appeared on the center of the screen $1000 \mathrm{~ms}$ after pre-cue presentation. After the imperative signal appeared for $200 \mathrm{~ms}$, the response period lasted for $2000 \mathrm{~ms}$. Responses that fell beyond this 2200-ms time period were classified as incorrect. The next trial began after the presentation of a black screen for a random duration of 800-1000 ms. Each test session consisted of 200 trials. The experiment ended after the two test sessions were completed.

\section{Behavioral Data Analysis}

The first 20 trials of each block were discarded from the analysis (i.e., the 10 trials in which the pacing signal was presented and the following 10 trials). Data analysis was performed on the correct responses for the remaining trials. Response accuracy during the acquisition task was calculated by measuring the asynchrony (in ms) of each keypress from the true bisection point of $800 \mathrm{~ms}$. In other words, the asynchrony of each keypress was measured by subtracting the actual response time from $800 \mathrm{~ms}$. Asynchronies that fell within $\pm 350 \mathrm{~ms}$ of the true bisection point were considered correct responses and were then averaged according to action modes and age groups. Repeated-measures analysis of variance (ANOVA) considering the action modes (intention-based or stimulus-based action) as a within-subject factor and age group (young or old) as a between-subject factor was performed. Significant interactions were analyzed using simple effects models. The significance level was set at $p=0.05$ for all of the analyses.

\section{Electroencephalogram Recording and Data Analysis}

The Neuroscan EEG system (Neuroscan Inc.) continuously recorded EEG signals from 64 scalp electrodes based on the international 10-20 system, with two electrodes placed on the left and right mastoids. The left mastoid served as an online 
reference. Eye blinks and movements were recorded with two pairs of electrodes as vertical and horizontal electrooculograms (VEOG and HEOG, respectively): one pair placed approximately $1 \mathrm{~cm}$ above and below the left eye, and the other pair placed approximately $1 \mathrm{~cm}$ lateral to the outer canthi of both eyes. The EEG data were amplified with a band-pass filter of $0.05-100 \mathrm{~Hz}$ and were digitized at $500 \mathrm{~Hz}$. Inter-electrode impedances were maintained below $5 \mathrm{k} \Omega$.

Off-line analysis of EEG data was performed, and all data were re-referenced to the average of both mastoids. Ocular artifacts were removed using a regression procedure (Semlitsch et al., 1986). A band-pass filter with a low-pass filter with a cutoff frequency of $30 \mathrm{~Hz}$ was used. Epochs of RP were time-locked to the onset of a keypress response and segmented from $-900 \mathrm{~ms}$ before keypress response to $400 \mathrm{~ms}$ after keypress response, with -900 to $-800 \mathrm{~ms}$ (i.e., the very beginning $100 \mathrm{~ms}$ of RP epoch) serving as the baseline period. Epochs of $700 \mathrm{~ms}$ (including the pre-stimulus baseline time of $100 \mathrm{~ms}$ ) were extracted for N2/P3, and 2,200 ms segments (including the pre-stimulus baseline time of $200 \mathrm{~ms}$ ) were extracted for CNV; both were timelocked to the onset of the stimulus (i.e., A, T, O, or X). Epochs exceeding $\pm 100 \mu \mathrm{V}$ were considered artifacts and were rejected from further analysis for both age groups.

During the acquisition phase, RP was measured as the mean amplitude at electrodes F3, Fz, F4, C3, Cz, C4, P3, Pz, and P4 within a time window of $-400-0 \mathrm{~ms}$ before keypress response because these signals reflect general motor preparation prior to action (Shibasaki and Hallett, 2006). The mean amplitude of N2 was measured within the time window of 300-400 ms over 9 fronto-central sites (F3, Fz, F4, FC3, FCz, FC4, C3, Cz, and C4). The mean amplitude of P3 was measured within 400-500 ms over centro-parietal sites $(\mathrm{C} 3, \mathrm{Cz}, \mathrm{C} 4, \mathrm{CP} 3, \mathrm{CPz}, \mathrm{CP} 4, \mathrm{P} 3, \mathrm{Pz}$, and $\mathrm{P} 4$ ). Finally, during the test phase, the mean $\mathrm{CNV}$ amplitude was measured within 500-1,000 ms, and further analyses were performed at 500-700 ms and 700-1,000 ms, respectively. Repeated-measures ANOVA was performed considering action mode (intention-based and stimulus-based), anterior-posterior scalp location (anterior, medial, and posterior) and scalp laterality (left, middle, and right) as within-subject factors and age group (young and old) as a between-subject factor. Analysis of the congruent and incongruent task subgroups was conducted exclusively for the CNV within each age group, considering the task (congruent and incongruent) as a between-subject factor. Trials classified as incorrect were excluded from ERP analysis. The Greenhouse-Geisser correction was used to adjust for sphericity violations. Post hoc analysis for significant main effects was performed using the Bonferroni method when needed. Significant interactions were analyzed using a simple effects model.

Given the potential relationship between associative memory and bidirectional action-effect binding (Elsner et al., 2002; Melcher et al., 2008, 2013; Pfister et al., 2014), we also computed the correlations (Spearman's rho) separately for the congruent and incongruent subgroups between the neuropsychological test scores and mean $\mathrm{CNV}$ amplitudes (i.e., the average of the $\mathrm{CNV}$ amplitude at the $\mathrm{Cz}, \mathrm{CPz}$ and $\mathrm{Pz}$ electrodes) in each action mode and age group. The alpha level was fixed at $p=0.05$ (two-tailed).

\section{RESULTS}

\section{Behavioral Results}

Significant main effects of age $\left[F_{(1,34)}=5.98, p=0.02\right.$, $\left.\eta_{\mathrm{p}}^{2}=0.149\right]$ and action $\left[F_{(1,34)}=14.99, p<0.001, \eta_{\mathrm{p}}^{2}=0.306\right]$ were revealed in the analysis. The mean asynchrony of temporal bisection, as shown in Figure 1B, was larger (and earlier) for the old age group $(-123 \mathrm{~ms}, S E=17)$ than for the young age group $(-66 \mathrm{~ms}, S E=17)$, which indicates the inaccuracy of motor timing in older adults. Furthermore, the mean asynchrony of temporal bisection for stimulus-based action $(-119 \mathrm{~ms}, S E=14)$ was larger (and earlier) than that for intention-based action $(-70 \mathrm{~ms}, S E=12)$. These results confirmed the temporal attraction effect reported in previous studies (Waszak et al., 2005; Keller et al., 2006). The temporal attraction effect induced a time shift of a response toward its specific stimulus in stimulusbased actions, whereas the action that produced certain effects was shifted closer to its effect in intention-based actions. This effect revealed the mode difference between intention-based and stimulus-based actions, which demonstrates that both modes of action were performed well by the subjects according to the instructions provided to them in this study. The negative mean asynchrony value observed here has been commonly detected in synchronization tasks in previous studies (Aschersleben and Prinz, 1995). There was no significant action $\times$ age group interaction.

\section{ERP Results}

\section{Readiness Potential (-400-0 ms)}

The RP component reflected general movement preparation and the action-effect binding process in intention-based actions (Waszak et al., 2005; Keller et al., 2006). A late RP was used in this study (the $400 \mathrm{~ms}$ before movement onset, as suggested by Shibasaki and Hallett, 2006) because it is considered more sensitive to movement-related parameters than an earlier RP (Keller et al., 2006). Figure 2 (left panel) presents the RP measurements for each condition. There was a significant main effect of age $\left[F_{(1,34)}=16.57, p<0.001, \eta_{\mathrm{p}}^{2}=0.328\right]$ and action $\left[F_{(1,34)}=6.54, p=0.015, \eta_{\mathrm{p}}^{2}=0.161\right]$, indicating that the RP amplitude of the two action conditions was significantly more negative for the young age group than for the old age group, which demonstrates the general age-related decline in the action preparation of both action modes. In addition, the RP amplitude for the intention-based condition was significantly more negative than that for the stimulus-based condition, which replicated the findings of previous studies with regard to the modal differences between the two actions. The interaction between action and age was also significant $\left[F_{(1,34)}=4.27\right.$, $\left.p=0.046, \eta_{\mathrm{p}}^{2}=0.112\right]$. A simple effects analysis revealed that for the old age group, the RP amplitude for the intentionbased condition was significantly more negative than that for the stimulus-based condition $(p=0.002)$, indicating that, although the RP amplitudes were decreased, modal differences between the two actions remained in the old age group. In contrast, these RP amplitudes did not significantly differ for the young age group $(p=0.73)$, which was inconsistent with previous studies. There 

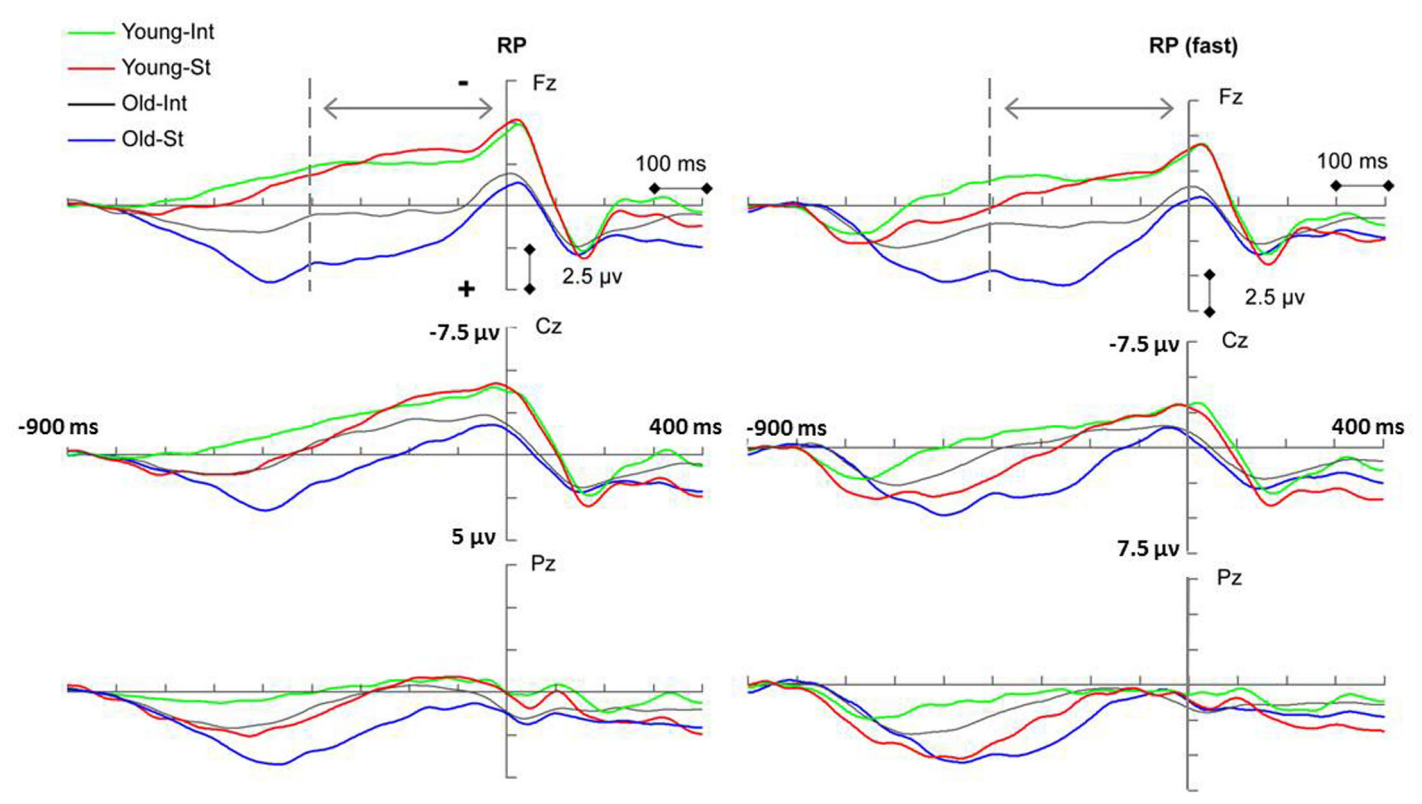

FIGURE 2 | Event-related potentials (ERP) waveforms showing significant main effect of age $(p<0.005)$ and action $(p<0.05)$ of readiness potential (RP) amplitude for both the undivided group (Left penal) and the fast-subgroup (Right penal). INT, intention-based action; ST, stimulus-based action. Keypress response onset occurred at $0 \mathrm{~ms}$.

was no significant interaction between either action or age and scalp location ( $F s<1.33, p>0.27$ for both).

In previous studies, modal differences in RP amplitude were observed among young subjects, specifically when different ISI durations were set between the two stimuli/effects. A 1,200 ms ISI was used in a study by Waszak et al. (2005), and ISIs of both 1,200 ms and 1,600 ms were used in a study by Keller et al. (2006) in which a smaller modal difference was observed for longer ISIs than for shorter ISIs. Therefore, considering the relatively long ISI (i.e., 1,600 ms) used in this study, further analyses were conducted on the young and old age groups whose RTs were shorter than average, classified herein as "fast subgroups." The ERP trials were separated into two subgroups according to the mean RTs. For the "fast subgroup" analysis (see Figure 2, right panel), significant main effects were again found for both age group $\left[F_{(1,34)}=11.12, p=0.002, \eta_{\mathrm{p}}^{2}=0.246\right]$ and action $\left[F_{(1,34)}=30.05, p<0.001, \eta_{\mathrm{p}}^{2}=0.469\right]$. There was also a significant interaction between action and age $\left[F_{(1,34)}=6.82\right.$, $\left.p=0.013, \eta_{\mathrm{p}}^{2}=0.167\right]$. A simple effects analysis revealed a significant difference between the two action conditions for both the young $(p=0.05)$ and old $(p<0.001)$ "fast subgroups." Thus, in this study, modal differences between two actions were observed for the young age group among the "fast subgroup" in which the trials corresponding to shorter than average RTs were pooled together. A possible explanation of the age effect is discussed later in this article.

\section{N2 (300-400 ms)}

The N2 amplitude reflects the feedback control process during the acquisition phase in intention-based actions. For the analysis of the N2 component (Figure 3), there was no significant main effect of age. However, there was a significant main effect of action $\left[F_{(1,34)}=26.93, p<0.001, \eta_{\mathrm{p}}^{2}=0.442\right]:$ the $\mathrm{N} 2$ amplitude evoked by the intention-based condition was significantly more negative than that evoked by the stimulus-based condition, suggesting that compared with the stimulus-based action mode, the higher N2 amplitude was specific to the intention-based action mode. This finding is consistent with previous studies. The feedback control process functioned as a monitor to compare the actual action effect with the anticipated action effect that initiated the intention-based action (Hughes et al., 2013). An enhanced N2 component is observed when the actual action effect does not fit the expectation (Band et al., 2009), whereas an attenuated N2 component is observed when the actual action effect corresponds to the intended expectation (Hughes and Waszak, 2011). In this study, an attenuated N2 component should be detected because the actual action effect corresponded to the intended expectation. However, when comparing the two action modes, the N2 amplitude was more prominent for intention-based actions than stimulus-based actions.

There was also a significant interaction between action and age $\left[F_{(1,34)}=4.12, p=0.05, \eta_{p}^{2}=0.108\right]$. A simple effects analysis showed that the N2 amplitude of the intention-based condition was significantly more negative for the young age group than for the old age group ( $p=0.007)$; however, the N2 amplitude for the stimulus-based condition did not significantly differ between the age groups $(p=0.62)$. This simple effect analysis also revealed an age-related decline in the feedback control process for intentionbased actions, suggesting that this age effect was specific to certain processes and action modes.

Additionally, a significant interaction between action and scalp laterality was observed for the N2 component 


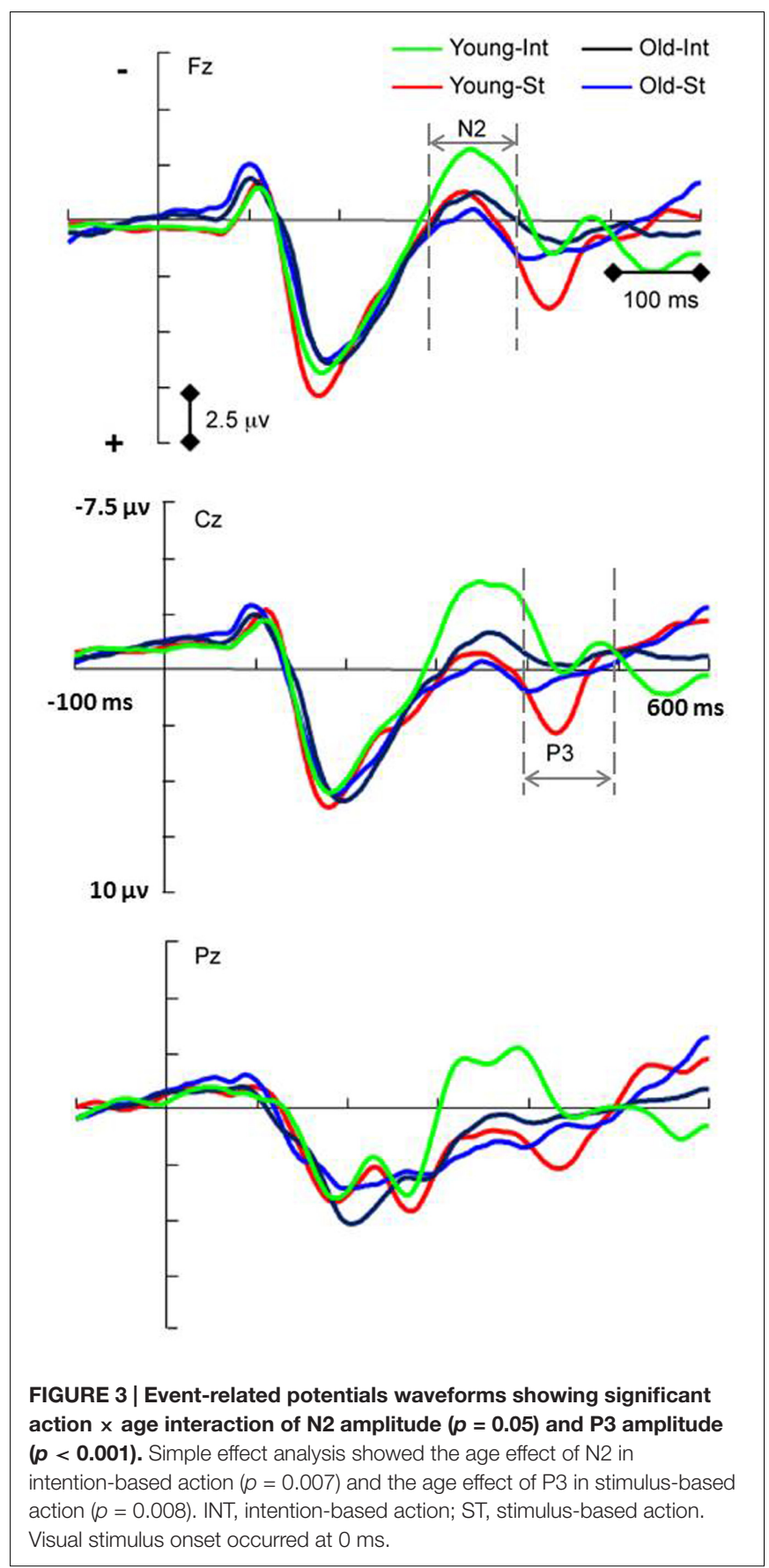

$\left[F_{(2,68)}=5.93, \varepsilon=0.778, p=0.008, \eta_{p}^{2}=0.149\right]$, showing that $\mathrm{N} 2$ amplitude difference between action modes was most pronounced in the middle line of electrodes than in the left or right line of electrodes. This was consistent with the study of Keller et al. (2006) that "the absence of lateralization of sensory areas" might be due to the centrally presented stimuli.

\section{P3 (400-500 ms)}

The P3 component was used in this study to reflect the stimulus-response linkage process during the acquisition phase in stimulus-based actions. There was no significant main effect of age for the P3 component. However, there was a significant main effect of action $\left[F_{(1,34)}=77.55, p<0.001, \eta_{\mathrm{p}}^{2}=0.695\right]$, indicating that the P3 amplitude under the stimulus-based condition was significantly more positive than that under the intention-based condition (Figure 3). These findings replicate the modal differences between two actions reported previously (Waszak et al., 2005; Keller et al., 2006) when age groups were combined. There was also a significant interaction between action and age $\left[F_{(1,34)}=20.99, p<0.001, \eta_{p}^{2}=0.382\right]$. A simple effects analysis of this interaction showed that the P3 amplitude evoked by the stimulus-based condition was significantly more positive for the young age group than for the old age group $(p=0.008)$ but also that the P3 amplitude evoked by the intention-based condition did not differ between age groups $(p=0.141)$. This simple effects analysis revealed an agerelated decline in the stimulus-response linkage process only for stimulus-based actions, suggesting that this age effect was specific to certain processes and action modes. There was also a significant interaction between action and scalp laterality for the P3 component $\left[F_{(2,68)}=8.74, p<0.001, \eta_{\mathrm{p}}^{2}=0.205\right]$, showing that P3 amplitude difference between action modes was most pronounced in the middle line of electrodes than in the left or right line of electrodes, which might be due to the centrally presented stimuli in this study. There were no other significant interactions between action or age and scalp location (all $F<0.93$, all $p>0.35$ ).

\section{CNV (500-1,000 ms, 500-700 ms, or 700-1,000 ms)}

The CNV component was used to reflect the effect-action retrieval process during the test phase in intention-based actions. The CNV measurements for each condition are presented in Figure 4. There was a significant main effect of age $\left[F_{(1,34)}=23.54\right.$, $\left.p<0.001, \eta_{\mathrm{p}}^{2}=0.409\right]$ on the CNV amplitude (500-1,000 ms), which showed that the CNV amplitude of the two action conditions was significantly more negative for the young age group than for the old age group, demonstrating a general agerelated decline in the movement preparation. There was no significant main effect of action or any interaction between age and action for the CNV amplitude.

In this study, the CNV component was recorded for a relatively long time period that included most of the negative deflection for the majority of the motor preparation components because all of the response information was provided by the appearance of a pre-cue. Thus, according to the instructions, the subjects prepared most of their movement response during the time period of the CNV amplitude measurement preceding action initiation. The interference effect of RT between congruent and incongruent tasks has been reported previously (Elsner and Hommel, 2001; Herwig et al., 2007) for intention-based action could be reflected within this period. The CNV amplitude has been linked to the sufficiency of motor preparation or the available resources to complete the task (Falkenstein et al., 2003; Leuthold et al., 2004). In Leuthold et al.'s (2004) study, the CNV amplitude increased with the amount of advanced information concerning movement preparation. Thus, we anticipated that there would be a significant difference in the amplitude of the $\mathrm{CNV}$ between congruent and incongruent tasks in 

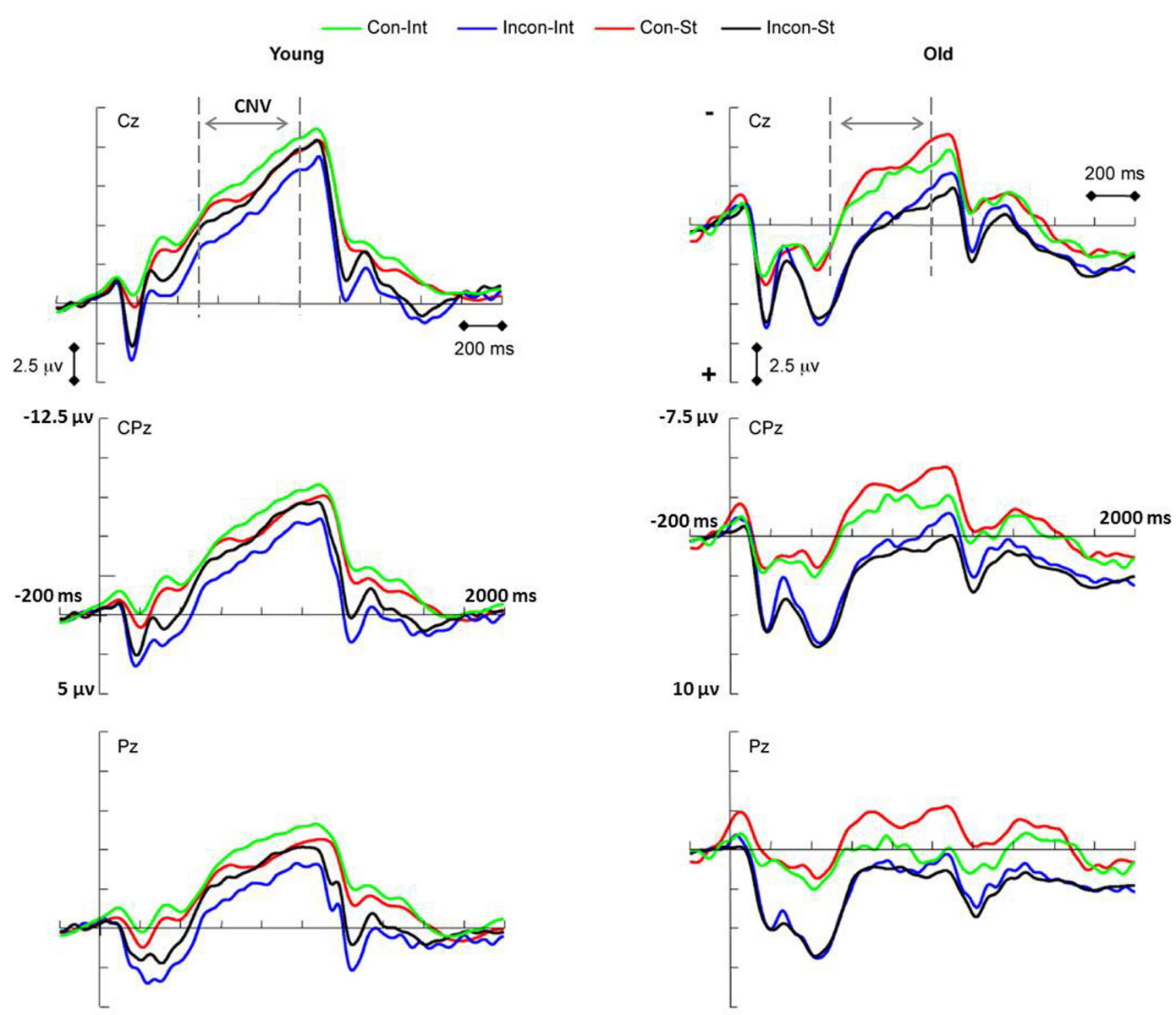

FIGURE 4 | Event-related potential waveforms showing the contingent negative variation (CNV) elicited by congruent (Con) and incongruent (Incon) tasks during the test phase of intention-based (INT) and stimulus-based (ST) actions in young (Left panel) and older (Right panel) adults. A significant main effect of age $(p<0.001)$ was revealed when age groups combined. Left penal: young age group showing significant action $\times$ task interaction $(p=0.001)$. Simple effect analysis showed the interference effect of CNV amplitude in intention-based action $(p=0.045)$. Right penal: old age group showing marginally significant main effect of task $(p=0.051)$. The pre-cuing stimulus onset was set at $0 \mathrm{~ms}$.

intention-based actions in the young age group, specifically that the congruent task would elicit a more negative CNV amplitude than the incongruent task.

To investigate this interference effect of the CNV amplitude, two subgroups stratified by task congruency (congruent and incongruent task subgroups) were created for each age group. For the young age group, a significant interaction between action and task was found $\left[F_{(1,16)}=16.78, p=0.001, \eta_{\mathrm{p}}^{2}=0.512\right]$, although no significant main effect of either action or task was observed. A simple effects analysis revealed the interference effect of $\mathrm{CNV}$ amplitude as expected. The CNV amplitude evoked by the congruent task was significantly more negative than that evoked by the incongruent task under the intentionbased condition $(p=0.045)$. However, under the stimulus-based condition, no significant difference in amplitude was observed between the tasks $(p=0.861)$. Thus, as we predicted, an interference effect of the CNV amplitude in intention-based actions was revealed in the young age group, demonstrating the intact function of the effect-action retrieval process among young adults. Specifically, the results showed that congruent effectaction retrieval might facilitate response preparation, whereas incongruent effect-action retrieval might interfere with response preparation.

However, this effect-action retrieval process might be impaired with age for the deficit of associative memory. For the old age group, there was no significant main effect of action or a task $\times$ action interaction, which demonstrates that the effect-action retrieval process of intention-based actions observed in the young age group was impaired in the old age group. However, there was a marginally significant main effect of task $\left[F_{(1,16)}=4.46, p=0.051, \eta_{\mathrm{p}}^{2}=0.218\right]$, which showed that under both action conditions, the CNV amplitude was more negative for the congruent task than for the incongruent task, suggesting that because of the age-related deficit, other cognitive processes might be involved. A possible explanation for this age effect is discussed later in this article.

Further analysis was conducted on the CNV amplitude within two smaller time windows. Consistent with the results of 
(500-1,000 ms) time window, significant main effect of age was found for $(500-700 \mathrm{~ms})$ time window $\left[F_{(1,34)}=24.40, p<0.001\right.$, $\left.\eta_{\mathrm{p}}^{2}=0.418\right]$ and $(700-1,000 \mathrm{~ms})$ time window $\left[F_{(1,34)}=19.07\right.$, $\left.p<0.001, \eta_{\mathrm{p}}^{2}=0.359\right]$, respectively, showing that the $\mathrm{CNV}$ amplitude was significantly more negative for the young age group than for the old age group. No significance was found for main effect of action or any interaction between age and action. Thus, a general age-related decline was observed continuously during the movement preparation.

The interference effect of CNV amplitude between congruent and incongruent tasks was also investigated within smaller time windows. For the young age group, the results were consistent with those of (500-1,000 ms) time window. Significant interaction between action and task was found within the $500-700 \mathrm{~ms}$ time window $\left[F_{(1,16)}=10.32, p=0.005, \eta_{\mathrm{p}}^{2}=0.392\right]$ and $700-1,000 \mathrm{~ms}$ time window $\left[F_{(1,16)}=18.66, p=0.001\right.$, $\left.\eta_{\mathrm{p}}^{2}=0.538\right]$, respectively, although there was no significant main effect of either action or task. Simple effects analysis revealed that, consistent with the results for the 500-1,000 ms time window, the $\mathrm{CNV}$ amplitude evoked by the congruent task was significantly more negative than that evoked by the incongruent task (500-700 ms: $p=0.028 ; 700-1,000 \mathrm{~ms}: p=0.046)$ for the intention-based condition, whereas no significant difference was observed between tasks under the stimulus-based condition (500-700 ms: $p=0.504 ; 700-1,000 \mathrm{~ms}: p=0.809$ ). Thus, the interference effect of the CNV amplitude observed for the effectaction retrieval process in intention-based actions in the young age group remained consistent during the movement preparation period.

For the old age group, there was no significant main effect of action or action $\times$ task interaction within either the 500-700 ms and 700-1,000 ms time window, indicating that the interference effect of $\mathrm{CNV}$ amplitude observed in young age group was absent in old age group. However, a significant main effect of task condition $\left[F_{(1,16)}=6.74, p=0.02, \eta_{\mathrm{p}}^{2}=0.296\right]$ was found within 500-700 ms time window. This result, which is consistent with the marginal significance observed within the larger time window, indicates that the congruent task evoked a more negative $\mathrm{CNV}$ amplitude in both action modes during a relatively earlier period of movement preparation.

\section{Correlation Results}

In the young age group, associative learning scores, in which higher scores indicate a higher function level of associative memory, were negatively correlated with the CNV amplitudes (Figure 5), which were a negatively trending component, in the congruent task in intention-based action $\left(r_{s}=-0.731, p=0.025\right)$. This result indicated a positive relationship between associative memory performance and motor preparation, as reflected by the CNV amplitude, which supports our assumption that associative memory might be, at least in part, responsible for the effect-action retrieval process in intention-based actions. However, no consistent evidence was observed in the incongruent task for the young age group or old age group. Therefore, further studies and more powerful evidence are needed to draw a cautious conclusion.

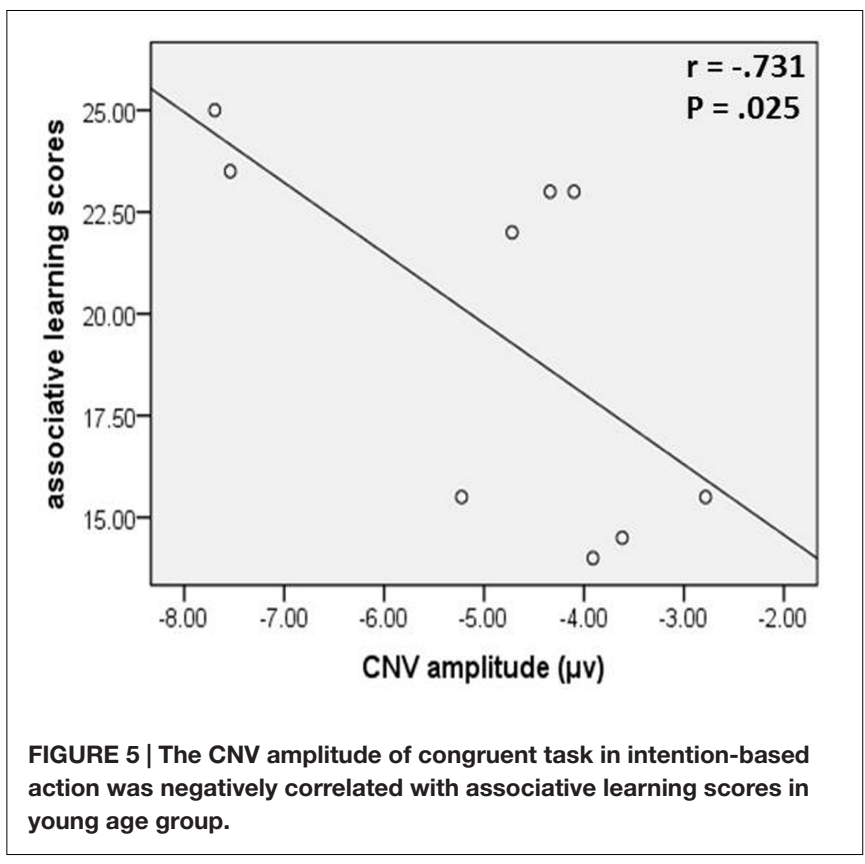

\section{DISCUSSION}

The aim of this study was to use ERP measurements to compare the age effects on four specific cognitive processes, namely actioneffect binding, stimulus-response linkage, feedback control, and effect-action retrieval, between intention-based and stimulusbased actions. The age effects that were specific to these action modes and processes were thus detected. We observed a decrease in ERP amplitudes in the old age group with similar pattern as in the young age group during most specific processes. This result demonstrated a general age-related decline in action preparation. For the effect-action retrieval process in intention-based actions, we revealed an interference effect of the CNV amplitude in the young age group, which was absent in the old age group, indicating an age-related impairment on the effect-action retrieval of intention-based actions. One possible explanation for this finding might be the age-related decline in associative memory.

\section{Behavioral Temporal Attraction}

In the behavioral results, the main effect of age showed larger mean asynchrony in the old age group than in the young age group, indicated age-related decline in motor timing accuracy. This age-related inaccuracy of motor timing might be influenced by both physical and neuromechanistic factors (Aschersleben, 2002). However, the absence of an interaction between action and age suggests that the action mode difference demonstrated by the temporal attraction effect still remained stable during the aging process, or at least in the early phase of aging, as represented by the subjects enrolled in this study.

\section{Action-Effect Binding}

The RP component, a slow negative shift thought to reflect general movement preparation, is used to reflect the process of 
action-effect binding during the acquisition phase in intentionbased actions (Waszak et al., 2005; Keller et al., 2006). The decreased RP amplitude observed for the old age group (compared with the young age group) demonstrated an general age-related decline during action preparation for both action modes. Specifically, when the action mode was considered, the previously reported modal differences in the RP (i.e., higher RP amplitude for intention-based actions than for stimulus-based actions) were observed only in the "fast subgroup" of young age group and with decreased amplitude in old age group, which was absent in young age group when fast and slow responses were combined together. These results suggested an age-related decline on movement preparation.

Given that reaction slowness in older adults has been consistently reported (e.g., Roggeveen et al., 2007; Berchicci et al., 2012; Wolkorte et al., 2014), the relatively long ISI of 1,600 ms utilized in this study seemed suitable for the old age group. However, the young age group may favor a faster response rhythm in the shorter ISI. When a slow response rhythm was demanded (as in this study), young participants might be able to intentionally allocate more resources than older participants within a prolonged period before movement execution due to some underlying mechanism. It has been emphasized that intentional and stimulus-related components might be involved in both action modes to varying extents (Keller et al., 2006; Herwig et al., 2007; Hughes et al., 2011) and that stimulusbased action might become less reflex-like and more intentional when the task becomes more complex or when delayed responses are needed (Herwig et al., 2007). From the grand average RP, we observed a trend in the young age group that the RP amplitude of stimulus-based action (much lower in the early period) increased rapidly in the later period to reach the RP amplitude of intention-based action, and this trend (rather than a reduction in intention-based $\mathrm{RP}$ amplitude) was responsible for the diminishment of the mode difference between the two actions in the young age group. Therefore, we suggest that the rapidly increased RP amplitude for stimulus-based action during the late RP period reflected the use of additional intentional efforts by young adults to hold corresponding responses for prolonged response periods. The absence of such a phenomenon in the old age group might suggest an insufficiency of available resources, which was also reflected by the decreased RP amplitudes for both action modes in the older age group than in the young age group. Interestingly, we previously observed in a training study that the RP amplitude of stimulus-based action (rather than of intentionbased action) in healthy older adults was enhanced after receiving combined cognitive training (Niu et al., 2016), which was similar to the RP amplitudes in the young age group in this study, and might indicate that improved executive function facilitates the sufficiency of available resources. It should be noted, as Herwig et al. (2007) previously revealed, that the "less reflex-likeness" involving a greater intentional effort did not transform the stimulus-based action into an intention-based action. According to the ideomotor theory (Greenwald, 1970; Prinz, 1997; Elsner and Hommel, 2001; Prinz et al., 2009), intention-based actions are acquired/learned specifically through action-effect binding such that an action is performed with the anticipation of fulfilling a certain goal/effect. Therefore, it is necessary to investigate the age effect on separate action modes.

\section{Stimulus-Response Linkage}

For the stimulus-based actions, a monodirectional stimulusresponse linkage was formed during the acquisition phase and was reflected by the P3 components. An age effect was observed for stimulus-based but not intention based conditions, as the P3 amplitudes observed in the old age group were lower than those in the young age group. The P3 amplitude is traditionally considered to reflect attentional allocation during stimulusrelated processing. In the examined action modes, P3 is thought to reflect the attentional process that helped form a linkage between a specific stimulus and its subsequent response (Petruo et al., 2016). Thus, P3 might serve as a marker reflecting completion of stimulus-related processing (Polich and Criado, 2006 for a review) and transformation of stimulus-related information into a corresponding response (Keller et al., 2006). As reviewed by Onofrj et al. (2001), cognitive P3 was most strongly influenced by age effects among all ERPs and was very sensitive to cognitive aging. In this study, the smaller P3 amplitude exhibited by the old age group under stimulus-based action suggested that limited stimulus-related resources were available to perform the action task compared with those available in the young age group. This observation is consistent with previous results showing that age-related declines of stimulus processing contributed to changes in movement with aging (Kolev et al., 2006; Sterr and Dean, 2008; Berchicci et al., 2012; Woods et al., 2015). For example, Berchicci et al. (2012) demonstrated that age-related slowing of stimulus processing correlated with the prolongation of both motor preparation and execution.

\section{Feedback Control}

In this study, the feedback control process, as reflected by the $\mathrm{N} 2$ amplitude, was observed during the acquisition phase in intention-based actions rather than stimulus-based actions. The age effect, reflected by reduced N2 amplitude in the old age group relative to that in the young age group, was also observed for intention-based action only. This feedback control was considered to function as a monitor to compare the actual action effect with the anticipated action effect (Hughes et al., 2013), with a negative feedback mechanism for reinforcement learning (Holroyd and Coles, 2002). Thus, the age effect here suggested that older adults might be inadequately equipped with attentional resources and executive function for action effect monitoring or have impaired reinforcement learning based on negative feedback. Further studies are needed to explore this concept.

An argument might arise that the age effect we observed for the N2 and P3 amplitudes could reflect a general decline in attentional processing rather than a specific decline in pertinent action processing because these two components are related to attention allocation. If this was the case, the age effect should have been observed for both action modes. However, the main effect of age was not significant in this study for either the N2 or P3 component when two actions were combined. Instead, the age effect was revealed under a specific process that was peculiar to 
the action mode. The effect of age on the N2 amplitude reflecting the feedback control process was only observed in intention-based actions, whereas the age effect on the P3 amplitude reflecting the stimulus-response linkage process was only observed in stimulusbased actions. Therefore, as we assumed, we revealed the agerelated declines peculiar to the specific action processes, which might be otherwise confounded under combined action modes or cognitive processes.

We can see that both RP and N2 are components reflecting cognitive processing of intention-based action, and these components occur successively in time. Therefore, the observed age effect on N2 amplitude might be influenced by the age effect on RP amplitude, as both components showed an enhanced amplitude in younger adults compared with that in older adults. However, a time interval (450-1,150 ms) between the execution of motor response and the onset of the following letter (i.e., correct trials) was set in this study, and this interval was longer than the 400-ms time delay suggested by Hughes and Waszak (2011). Based on this rather prolonged time interval, we believe that the N2 component data that we collected did not include an RP component. Moreover, as Onofrj et al. (2001) suggested in their review, age effects were usually reflected by decreased amplitudes of most ERP components. Thus, our findings of decreased amplitude for several ERPs during different processes during the acquisition phase suggest a general decline with age.

\section{Effect-Action Retrieval}

During the test phase, the effect-action retrieval process was investigated using the CNV amplitude, where the age effects can be interpreted from two perspectives. First, the main effect of age resulted in decreased CNV amplitude in the old age group relative to that in the young age group, which demonstrated a general age-related decline in movement preparation. Second, distinct ERP patterns were revealed between the two age groups. The interference effect of CNV amplitude that was unique to intention-based action was absent in the old age group, and a main effect of task showing increased CNV amplitudes for congruent tasks relative to those for incongruent tasks on both action modes was observed within a smaller and earlier time window.

In the young age group, the observed interference effect of the $\mathrm{CNV}$ amplitude was consistent with previous behavioral reports (e.g., Elsner and Hommel, 2001; Herwig et al., 2007), which demonstrated the intact function of the effect-action retrieval process in intention-based actions. However, an absence of this interference effect was revealed in the old age group in this study and in another recent study (Niu et al., 2016) using the same paradigm in healthy older adults, suggesting an age-related impairment of the effect-action retrieval process. The effectaction retrieval in intention-based action has been considered a process of associative memory retrieval (Elsner et al., 2002; Melcher et al., 2008, 2013; Pfister et al., 2014), which was supported in this study by the correlation analysis in the young age group between associative memory performance and the $\mathrm{CNV}$ amplitude in congruent task in intention-based action. Associative memory is more sensitive to aging than other forms of memories (e.g., Naveh-Benjamin, 2000; see Yonelinas, 2002 for a review; Rhodes et al., 2008). Therefore, impaired associative memory might account for the absence of the interference effect of CNV amplitude in the old age group. According to the dualprocess theory of memory, recollection (the retrieval of specific details) and familiarity (the general strength of information) are two main processes that control memory judgment. Recollection is particularly necessary to recognize rearranged pairs from learned pairs (recall-to-reject theory, Rotello and Heit, 2000; Gallo et al., 2004). For older adults, recollection is susceptible to aging, and familiarity may favor the retrieval of associative pairs. For example, older adults tend to recognize rearranged pairs as intact pairs with high scores of false alarm, particularly when the items that formed the pairs were repeatedly learned (Rhodes et al., 2008). In this study, action and effect pairs were repeatedly acquired and rearranged in the incongruent task. Older adults with recollection deficit may fail to recognize the rearrangement of action-effect pairs in the incongruent task and thus be unsusceptible to the interference caused by the incongruence. Therefore, the age-related deficit of recollection might account for the impaired effect-action retrieval in intention-based actions in older adults.

Interestingly, we also observed a main effect of task during the early part of motor preparation; older adults favored congruent tasks rather than incongruent tasks in both action modes, which could not be explained based on the evidence from associative memory. Some recent studies focusing on executive function of inhibition revealed an age effect on processing distractors (see Weeks and Hasher, 2014 for a review; Biss et al., 2013). It has been reported that processing distractors that are congruent with target goals could in fact facilitate the coding and retrieval of target stimuli for older adults. The possible explanations are that older adults are unable to inhibit the processing of irrelevant stimuli in the background and that congruent distractors aid the processing of target stimuli by increasing the depth of processing or implicit rehearsal. If these explanations hold true in the present study, executive function might be responsible for the main effect of task observed in older adults. However, it should be noted that executive function might represent a supplemental or compensative function when associative memory is impaired with age, since executive function has been suggested to play a fundamental and general role in action (see Colcombe and Kramer, 2003 for a review; Berchicci et al., 2012; Niu et al., 2016). The main effect of task occurred only during the early period of motor preparation and then disappeared when the motor response was about to be initiated. Further investigations are needed to understand the potential processes and mechanisms.

Some limitations should be noted in this study. First, the older adults enrolled in this study were less than 74 years of age and had high education levels and MMSE scores. These individuals may exhibit relatively intact cognitive function and reflect the age effect in an early stage. Further studies should include even older adults to evaluate how these age effects vary with the aging process. Second, behavioral performance and sufficient neuropsychological assessments should also be introduced for further validation. Third, during the test phase, a relatively small sample was examined in each subgroup, resulting in a large standard deviation, particularly in the old age group, and 
limiting the statistical power of the test hypothesis. Although the interference effect of CNV amplitude was observed with a small sample in the young age group, conclusions about the age effect must be made with caution. This pilot study provides a new perspective to investigate the age effect by comparing specific cognitive processes between different action modes. Further studies involving large sample sizes and multiple measurements are required to understand the age effects on action.

In summary, this study investigated the cognitive processes of two action modes from a developmental perspective and revealed the age effects between intention-based and stimulusbased actions via four cognitive processes: action-effect binding, stimulus-response linkage, feedback control, and effect-action retrieval. The results revealed not only generally declining functions of action preparation in older adults, as indicated by consistently decreasing ERP amplitudes, but also age effects specific to the action modes and processes, which might otherwise be mixed together under confounding experimental conditions. In particular, an interference effect indexed by the difference in the CNV amplitude between the congruent and incongruent tasks was observed in the young age group, which is consistent with previous behavioral reports. However, this effect was absent in the old age group, indicating an age-related deficit that is particular to the effect-action retrieval process of intention-based actions, which might be due to age-related deficits in associative memory. Further intervention studies

\section{REFERENCES}

Aschersleben, G. (2002). Temporal control of movements in sensorimotor synchronization. Brain Cogn. 48, 66-79. doi: 10.1006/brcg.2001.1304

Aschersleben, G., and Prinz, W. (1995). Synchronizing actions with events: the role of sensory information. Percept. Psychophys. 57, 305-317. doi: 10.3758/ bf03213056

Band, G. P. H., van Steenbergen, H., Ridderinkhof, K. R., Falkenstein, M., and Hommel, B. (2009). Action-effect negativity: irrelevant action effects are monitored like relevant feedback. Biol. Psychol. 82, 211-218. doi: 10.1016/j. biopsycho.2009.06.011

Berchicci, M., Lucci, G., Pesce, C., Spinelli, D., and di Russo, F. (2012). Prefrontal hyperactivity in older people during motor planning. Neuroimage 62, 1750-1760. doi: 10.1016/j.neuroimage.2012.06.031

Biss, R. K., Joan Ngo, K. W., Hasher, L., Campbell, K. L., and Rowe, G. (2013). Distraction can reduce age-related forgetting. Psychol. Sci. 24, 448-455. doi: $10.1177 / 0956797612457386$

Blakemore, S.-J., Wolpert, D., and Frith, C. (2000). Why can't you tickle yourself? Neuroreport 11, R11-R16. doi: 10.1097/00001756-200008030-00002

Braak, E., Griffing, K., Arai, K., Bohl, J., Bratzke, H., and Braak, H. (1999). Neuropathology of Alzheimer's disease: What is new since A. Alzheimer? Eur. Arch. Psychiatry Clin. Neurosci. 249, S14-S22. doi: 10.1007/pl00014168

Colcombe, S., and Kramer, A. F. (2003). Fitness effects on the cognitive function of older adults: a meta-analytic study. Psychol. Sci. 14, 125-130. doi: 10.1111/ 1467-9280.t01-1-01430

Debener, S., Ullsperger, M., Siegel, M., Fiehler, K., von Cramon, D. Y., and Engel, A. K. (2005). Trial-by-trial coupling of concurrent electroencephalogram and functional magnetic resonance imaging identifies the dynamics of performance monitoring. J. Neurosci. 25, 11730-11737. doi: 10.1523/jneurosci.3286-05.2005

Elsner, B., and Hommel, B. (2001). Effect anticipation and action control. J. Exp. Psychol. Hum. Percept. Perform. 27, 229-240. doi: 10.1037//0096-1523.27.1.229

Elsner, B., Hommel, B., Mentschel, C., Drzezga, A., Prinz, W., Conrad, B., et al. (2002). Linking actions and their perceivable consequences in the human brain. Neuroimage 17, 364-372. doi: 10.1006/nimg.2002.1162 targeting the improvement of intention-based action may add sufficient associative memory training to the executive function and physical exercise training.

\section{AUTHOR CONTRIBUTIONS}

Y-NN and JL conceptualized the design of the study. Y-NN researched the data and wrote the draft. XZ researched the data and contributed to the discussion. JL critically reviewed and edited the manuscript.

\section{FUNDING}

This work was supported by the National Science Foundation of China (NSFC, 30900406, 31271108, 31470998, 31671157), the Scientific Foundation of the Institute of Psychology, Chinese Academy of Sciences (Y0CX0601S01), the Pioneer Initiative of the Chinese Academy of Sciences, Feature Institutes Program (TSS-2015-06), Beijing Municipal Science \& Technology Commission No. Z171100000117006, and CAS Key Laboratory of Mental Health, Institute of Psychology, Chinese Academy of Sciences (KLMH2015ZG06). The funders had no role in the study design, data collection and analysis, decision to publish, or preparation of the manuscript.

Falkenstein, M., Hoormann, J., Hohnsbein, J., and Kleinsorge, T. (2003). Shortterm mobilization of processing resources is revealed in the event-related potential. Psychophysiology 40, 914-923. doi: 10.1111/1469-8986.00109

Folstein, M. F., Folstein, S. E., and McHugh, P. R. (1975). Mini-mental state: a practical method for grading the cognitive state of patients for the clinician. J. Psychiatr. Res. 12, 189-198. doi: 10.1016/0022-3956(75)90026-6

Gallo, D. A., Sullivan, A. L., Daffner, K. R., Schacter, D. L., and Budson, A. E. (2004). Associative recognition in Alzheimer's disease: evidence for impaired recall-to-reject. Neuropsychology 18, 556-563. doi: 10.1037/0894-4105.18.3.556

Greenwald, A. G. (1970). Sensory feedback mechanisms in performance control: with special reference to the ideo-motor mechanism. Psychol. Rev. 77, 73-99. doi: $10.1037 /$ h0028689

Haggard, P., Clark, S., and Kalogeras, J. (2002). Voluntary action and conscious awareness. Nat. Neurosci. 5, 382-385. doi: 10.1038/nn827

Herwig, A. (2015). Linking perception and action by structure or process? Toward an integrative perspective. Neurosci. Biobehav. Rev. 52, 105-116. doi: 10.1016/j. neubiorev.2015.02.013

Herwig, A., Prinz, W., and Waszak, F. (2007). Two modes of sensorimotor integration in intention-based and stimulus-based actions. Q. J. Exp. Psychol. 60, 1540-1554. doi: 10.1080/17470210601119134

Holroyd, C. B., and Coles, M. G. H. (2002). The neural basis of human error processing: reinforcement learning, dopamine, and the error-related negativity. Psychol. Rev. 109, 679-709. doi: 10.1037/0033-295x.109.4.679

Hughes, G., Desantis, A., and Waszak, F. (2013). Mechanisms of intentional binding and sensory attenuation: the role of temporal prediction, temporal control, identity prediction, and motor prediction. Psychol. Bull. 139, 133-151. doi: $10.1037 / \mathrm{a} 0028566$

Hughes, G., Schutz-Bosbach, S., and Waszak, F. (2011). One action system or two? Evidence for common central preparatory mechanisms in voluntary and stimulus-driven actions. J. Neurosci. 31, 16692-16699. doi: 10.1523/jneurosci. 2256-11.2011

Hughes, G., and Waszak, F. (2011). ERP correlates of action effect prediction and visual sensory attenuation in voluntary action. Neuroimage 56, 1632-1640. doi: 10.1016/j.neuroimage.2011.02.057 
Keller, P. E., Wascher, E., Prinz, W., Waszak, F., Koch, I., and Rosenbaum, D. A. (2006). Differences between intention-based and stimulus-based actions. J. Psychophysiol. 20, 9-20. doi: 10.1027/0269-8803.20.1.9

Kolev, V., Falkenstein, M., and Yordanova, J. (2006). Motor-response generation as a source of aging-related behavioural slowing in choice-reaction tasks. Neurobiol. Aging 27, 1719-1730. doi: 10.1016/j.neurobiolaging.2005.09.027

Leuthold, H., Sommer, W., and Ulrich, R. (2004). Preparing for action: inferences from CNV and LRP. J. Psychophysiol. 18, 77-88. doi: 10.1027/0269-8803.18. 23.77

Melcher, T., Weidema, M., Eenshuistra, R. M., Hommel, B., and Gruber, O. (2008). The neural substrate of the ideomotor principle: an event-related fMRI analysis. Neuroimage 39, 1274-1288. doi: 10.1016/j.neuroimage.2007.09.049

Melcher, T., Winter, D., Hommel, B., Pfister, R., Dechent, P., and Gruber, O. (2013). The neural substrate of the ideomotor principle revisited: evidence for asymmetries in action-effect learning. Neuroscience 231, 13-27. doi: 10.1016/j. neuroscience.2012.11.035

Naveh-Benjamin, M. (2000). Adult age differences in memory performance: tests of an associative deficit hypothesis. J. Exp. Psychol. Learn. Mem. Cogn. 26, 1170-1187. doi: 10.1037//0278-7393.26.5.1170

Neumann, O. (1990). "Visual attention and action," in Relationships between Perception and Action: Current Approaches, eds O. Neumann, W. Prinz, and P. Bieri (Berlin: Springer-Verlag), 227-267.

Niu, Y.-N., Zhu, X., Li, J., and Fu, J.-N. (2016). The ERP effects of combined cognitive training on intention-based and stimulus-based actions in older Chinese adults. Front. Psychol. 7:1670. doi: 10.3389/fpsyg.2016.01670

Onofrj, M., Thomas, A., Iacono, D., D'Andreamatteo, G., and Paci, C. (2001). Age-related changes of evoked potentials. Neurophysiol. Clin. 31, 83-103. doi: 10.1016/s0987-7053(01)00248-9

Petruo, V. A., Stock, A.-K., Münchau, A., and Beste, C. (2016). A systems neurophysiology approach to voluntary event coding. Neuroimage 135, 324-332. doi: 10.1016/j.neuroimage.2016.05.007

Pfister, R., Melcher, T., Kiesel, A., Dechent, P., and Gruber, O. (2014). Neural correlates of ideomotor effect anticipations. Neuroscience 259, 164-171. doi: 10.1016/j.neuroscience.2013.11.061

Polich, J., and Criado, J. R. (2006). Neuropsychology and neuropharmacology of P3a and P3b. Int. J. Psychophysiol. 60, 172-185. doi: 10.1016/j.ijpsycho.2005. 12.012

Prinz, W. (1997). Perception and action planning. Eur. J. Cogn. Psychol. 9, 129-154. doi: $10.1080 / 713752551$

Prinz, W., Aschersleben, G., and Koch, I. (2009). "Cognition and action," in Oxford Handbook of Human Action, eds E. Morsella, J. A. Bargh, and P. M. Gollwitzer (New York, NY: Oxford University Press), 35-71.

Reitan, R. M. (1955). The relation of the trail making test to organic brain damage. J. Consult. Psychol. 19, 393-394. doi: 10.1037/h0044509

Reuter, E.-M., Behrens, M., and Zschorlich, V. R. (2015). Age-related differences in corticomotor facilitation indicate dedifferentiation in motor planning. Exp. Gerontol. 65, 79-84. doi: 10.1016/j.exger.2015.03.008

Reuter-Lorenz, P. A. (2002). New visions of the aging mind and brain. Trends Cogn. Sci. 6, 394-400. doi: 10.1016/s1364-6613(02)01957-5

Rhodes, M. G., Castel, A. D., and Jacoby, L. L. (2008). Associative recognition of face pairs by younger and older adults: the role of familiarity-based processing. Psychol. Aging 23, 239-249. doi: 10.1037/0882-7974.23.2.239
Roberts, R. E., and Vernon, S. W. (1983). The center for epidemiologic studies depression scale: its use in a community sample. Am. J. Psychiatry 140, 41-46. doi: 10.1176/ajp.140.1.41

Roggeveen, A. B., Prime, D. J., and Ward, L. M. (2007). Lateralized readiness potentials reveal motor slowing in the aging brain. J. Gerontol. B Psychol. Sci. Soc. Sci. 62, 78-84. doi: 10.1093/geronb/62.2.p78

Rotello, M. C., and Heit, E. (2000). Associative recognition: a case of recall-to-reject processing. Mem. Cogn. 28, 907-922. doi: 10.3758/BF03209339

Semlitsch, H. V., Anderer, P., Schuster, P., and Presslich, O. (1986). A solution for reliable and valid reduction of ocular artifacts, applied to the P300 ERP. Psychophysiology 23, 695-703. doi: 10.1111/j.1469-8986.1986.tb00696.x

Shibasaki, H., and Hallett, M. (2006). What is the bereitschaftspotential? Clin. Neurophysiol. 117, 2341-2356. doi: 10.1016/j.clinph.2006.04.025

Sterr, A., and Dean, P. (2008). Neural correlates of movement preparation in healthy ageing. Eur. J. Neurosci. 27, 254-260. doi: 10.1111/j.1460-9568.2007. 05975.x

Waszak, F., Wascher, E., Keller, P., Koch, I., Aschersleben, G., Rosenbaum, D. A., et al. (2005). Intention-based and stimulus-based mechanisms in action selection. Exp. Brain Res. 162, 346-356. doi: 10.1007/s00221-004-2183-8

Weeks, J. C., and Hasher, L. (2014). The disruptive- and beneficial- effects of distraction on older adults' cognitive performance. Front. Psychol. 5:133. doi: 10.3389/fpsyg.2014.00133

West, R. (2000). In defense of the frontal lobe hypothesis of cognitive aging. J. Int. Neuropsychol. Soc. 6, 727-729. doi: 10.1017/s1355617700666109

West, R. L. (1996). An application of prefrontal cortex function theory to cognitive aging. Psychol. Bull. 120, 272-292. doi: 10.1037/0033-2909.120.2.272

Wolkorte, R., Kamphuis, J., and Zijdewind, I. (2014). Increased reaction times and reduced response preparation already starts at middle age. Front. Aging Neurosci. 6:79. doi: 10.3389/fnagi.2014.00079

Woods, D. L., Wyma, J. M., Yund, E. W., Herron, T. J., and Reed, B. (2015). Age-related slowing of response selection and production in a visual choice reaction time task. Front. Hum. Neurosci. 9:193. doi: 10.3389/fnhum.2015. 00193

Xu, S., and Wu, Z. (1986). The construction of "The clinical memory test". Acta Psychol. Sin. 18, 100-108.

Yonelinas, A. P. (2002). The nature of recollection and familiarity: a review of 30 years of research. J. Mem. Lang. 46, 441-517. doi: 10.1006/jmla.2002.2864

Conflict of Interest Statement: The authors declare that the research was conducted in the absence of any commercial or financial relationships that could be construed as a potential conflict of interest.

The reviewer JC and handling Editor declared their shared affiliation, and the handling Editor states that the process nevertheless met the standards of a fair and objective review.

Copyright (c) $2017 \mathrm{Niu}, \mathrm{Zhu}$ and Li. This is an open-access article distributed under the terms of the Creative Commons Attribution License (CC BY). The use, distribution or reproduction in other forums is permitted, provided the original author(s) or licensor are credited and that the original publication in this journal is cited, in accordance with accepted academic practice. No use, distribution or reproduction is permitted which does not comply with these terms. 


\section{OPEN ACCESS}

Edited by: Annalisa Setti,

University College Cork, Ireland

Reviewed by:

Pamela Bryden,

Wilfrid Laurier University, Canada

Bernhard Hommel,

Leiden University, Netherlands

*Correspondence:

Soo Ji Kim

specare@ewha.ac.kr

Ga Eul Yoo

bbird27@hotmail.com

Specialty section:

This article was submitted to

Cognition,

a section of the journal

Frontiers in Psychology

Received: 04 October 2016

Accepted: 28 August 2017

Published: 26 September 2017

Citation:

Kim SJ, Cho S-R and Yoo GE (2017) Age-Related Changes in Bimanual Instrument Playing with Rhythmic Cueing. Front. Psychol. 8:1569. doi: 10.3389/fpsyg.2017.01569

\section{Age-Related Changes in Bimanual Instrument Playing with Rhythmic Cueing}

\author{
Soo Ji Kim ${ }^{1,2 *}$, Sung-Rae Cho ${ }^{3,4,5}$ and Ga Eul Yoo ${ }^{2,6 *}$ \\ ${ }^{1}$ Music Therapy Education, Graduate School of Education, Ewha Womans University, Seoul, South Korea, ${ }^{2}$ Ewha Music \\ Rehabilitation Center, Seoul, South Korea, ${ }^{3}$ Department and Research Institute of Rehabilitation Medicine, Yonsei University \\ College of Medicine, Seoul, South Korea, ${ }^{4}$ Brain Korea 21 PLUS Project for Medical Science, Yonsei University College of \\ Medicine, Seoul, South Korea, ${ }^{5}$ Rehabilitation Institute of Neuromuscular Disease, Yonsei University College of Medicine, \\ Seoul, South Korea, ${ }^{6}$ Department of Music Therapy, Graduate School, Ewha Womans University, Seoul, South Korea
}

Deficits in bimanual coordination of older adults have been demonstrated to significantly limit their functioning in daily life. As a bimanual sensorimotor task, instrument playing has great potential for motor and cognitive training in advanced age. While the process of matching a person's repetitive movements to auditory rhythmic cueing during instrument playing was documented to involve motor and attentional control, investigation into whether the level of cognitive functioning influences the ability to rhythmically coordinate movement to an external beat in older populations is relatively limited. Therefore, the current study aimed to examine how timing accuracy during bimanual instrument playing with rhythmic cueing differed depending on the degree of participants' cognitive aging. Twenty one young adults, 20 healthy older adults, and 17 older adults with mild dementia participated in this study. Each participant tapped an electronic drum in time to the rhythmic cueing provided using both hands simultaneously and in alternation. During bimanual instrument playing with rhythmic cueing, mean and variability of synchronization errors were measured and compared across the groups and the tempo of cueing during each type of tapping task. Correlations of such timing parameters with cognitive measures were also analyzed. The results showed that the group factor resulted in significant differences in the synchronization errors-related parameters. During bimanual tapping tasks, cognitive decline resulted in differences in synchronization errors between younger adults and older adults with mild dimentia. Also, in terms of variability of synchronization errors, younger adults showed significant differences in maintaining timing performance from older adults with and without mild dementia, which may be attributed to decreased processing time for bimanual coordination due to aging. Significant correlations were observed between variability of synchronization errors and performance of cognitive tasks involving executive control and cognitive flexibility when asked for bimanual coordination in response to external timing cues at adjusted tempi. Also, significant correlations with cognitive measures were more prevalent in variability of synchronization errors during alternative tapping compared to simultaneous tapping. The current study supports that bimanual tapping may be predictive of cognitive processing of older adults. Also, tempo and type of 
movement required for instrument playing both involve cognitive and motor loads at different levels, and such variables could be important factors for determining the complexity of the task and the involved task requirements for interventions using instrument playing.

Keywords: older adults, cognitive aging, bimanual coordination, instrument playing, timing accuracy

\section{INTRODUCTION}

Cognitive aging, defined as age-related changes in perceptual and cognitive performance, results in less efficient use of mental resources (Park, 2000). As such, cognitive aging is manifested by slower processing speed, decreased mental capacity during performance of more complex cognitive tasks, and inflexible attentional ability (Han, 2016). Cognitive decline due to dementia interferes with cognitive and motor functioning required for daily living activities at different levels (Giebel et al., 2014). During earlier stages of dementia, affected people demonstrate less executive control, which also impacts motor control, such as a safe gait (van der Wardt et al., 2015). The cognitive difficulties experienced in the early stages of dementia impact one's self-confidence and can lead to anxiety, depression, and withdrawal from activities (Giebel et al., 2014; Han, 2017), which can exacerbate symptoms. As such, there have been increasing calls for interventions targeting the cognitive functioning of older adults with earlier stages of dementia, where the disease has not yet progressed to the point of severe cognitive impairment.

With increasing evidence supporting a correlation between cognitive and motor functions, research has demonstrated that cognitive decline in advanced age also affects motor coordination in association with attentional control and executive function (Temprado et al., 2001; Fujiyama et al., 2013). For example, slower speed or decreased accuracy in performing sequential finger movements in older adults was found to be associated with mental representation of such motor information, which was also supported by increased activation in non-motor areas as well as motor areas in the brain (Caçola et al., 2013). Meanwhile, level of cognitive impairment affects the response time during simple finger tapping in association with working memory capacity (Halliday et al., 2016). With regard to bimanual coordination, which requires the integration of complex neural systems (including motor and perceptual systems; Vaillancourt and Newell, 2002), older adults show decreased accuracy and stability (Lipsitz, 2004). Age-related decline specifically impacts timing processing and control of attentional load, which are viewed as primary factors in task performance of interlimb coordination in the upper extremity (Krampe et al., 2010). In addition, cognitive impairment influences coordination, such that decreased imitation ability of bimanual gestures has been observed in older adults in the early stages of dementia (Nagahama et al., 2015).

Deficits in bimanual coordination have been demonstrated to significantly limit older adults' functioning in daily life (Kilbreath and Heard, 2005). Due to these changes, difficulty in generating appropriate responses for task execution or manipulation of objects is observed in older adults (Tucker et al., 2008).
Accordingly, there is interest in examining how task performance involving bimanual coordination operates and how such motor coordination can be enhanced. When older adults perform a task involving bimanual coordination, the level of performance varies depending on the direction (iso-directional vs. opposite directional) of the involvement of each limb (Meesen et al., 2006) and the speed of movement (Carson et al., 1995; Fujiyama et al., 2013). When the limbs move in opposite directions (Fujiyama et al., 2009) or at a faster speed, the performance level of older adults decreases (Fujiyama et al., 2010). Given these findings concerning the factors that facilitate or limit bimanual coordination of older adults such as the type of movement and tempo, further research is needed to develop evidence-based training to facilitate such ability to control timed movements in older populations (Voelcker-Rehage et al., 2011).

A common task involving bimanual coordination in musicrelated experiences is instrument playing. Instrument playing necessitates movement of the upper limbs and grasping movements, which require interlimb temporal coordination. It involves both symmetric and non-symmetric coordination depending on the type of instrument. In other words, the particular playing methods associated with different instruments facilitate specific temporal timing and movement demands. Given that deficits in bimanual coordination in older populations are attributed to the reduced size of the corpus callosum, which may interfere with interhemisphreic interaction (Sullivan and Pfefferbaum, 2002; Bartzokis et al., 2004; Head et al., 2004; Fling and Seidler, 2012), instrument playing involving upper limb movement using both hands may be promising in recruiting and optimizing neural networks in both the right and left hemispheres of older adults. Engagement in instrument playing in older adults was found to relate to reduced occurrence of dementia within twins (Balbag et al., 2014). Intensive engagement in bimanual synchronized finger movement via piano playing led to more efficient recruitment of motor networks in the brain (Schlaug, 2001) and a higher level of regularity in synchronization to auditory cues and decreased reaction time to such cues (Haslinger et al., 2004). As such, playing instruments with auditory temporal coupling requires motor and cognitive control, which accordingly mediates cognitive stimulation and accordingly predicts preserved cognitive functioning in terms of visual-spatial and executive processing (Hanna-Pladdy and Gajewski, 2012).

As a bimanual sensorimotor task, instrument playing has potential for motor and cognitive training with older adults, with evidence of beneficial effects for cognitive enhancement, gait function and fall risks (Trombetti et al., 2011). Individualized piano instruction that involves multimodal sensorimotor integration and temporal and spatial processing was found 
to effectively facilitate perceptual processing in older adults, which indicates the potential benefits of musical instruction for cognitive aging (Bugos et al., 2007). Intervention with the dual task of handling percussion instruments while walking effectively influences gait parameters and dual task performance, which eventually reduces fall risks. Performance of musical tasks, including rhythm playing, was documented to contribute to delayed cognitive decline in older adults with mild to moderate dementia (Chu et al., 2014).

When performing tasks involving motor control, such as playing a musical instrument, the provision of auditory rhythmic cueing can function as an effective agent for intervening in sensorimotor processing and the timed control of movements (Chen et al., 2006; LaGasse and Knight, 2011). The process of matching a person's repetitive movements to auditory rhythmic cueing was documented to facilitate the precise execution of sequenced motor tasks and increase efficiency in brain activation engaged in such motor control (Witt et al., 2008). Also, the use of repetitive rhythmic cueing presents precise timing information and thereby enhances predictive processing, which leads to decreases in attentional demand and increases in efficiency of motor and attentional control when continuously maintaining rhythmic movements (Ackerley et al., 2011; LaGasse and Knight, 2011; Pecenka et al., 2013). Its application to motor rehabilitation also indicates that rhythmic cueing is an effective agent when intervening with gait of individuals who benefit from external cueing for motor coordination. Previous research demonstrates that older adults in the later stages of dementia could match their gait to external auditory cueing with decreased physical support compared to walking without rhythmic cueing (Clair and O'Konski, 2006). Despite evidence of increased temporal coordination when matched to external cueing in terms of motor and cognitive performance, investigation into whether the synchronization process involving bimanual coordination impacts cognitive and motor functioning in older populations is relatively limited. Therefore, the current study aimed to examine how task performance of bimanual instrument playing with rhythmic cueing differed depending on cognitive aging. The level of performance of bimanual tapping tasks (i.e., synchronization errors) was analyzed and compared depending on the type of tasks (using both hands simultaneously and in alternation) and the tempo of cueing. It also investigated whether there were differences in such performance among young adults and older adults with and without mild dementia. Such results will contribute to better understanding of the factors associated with bimanual coordination when constructing a task for intervening in cognitive aging.

\section{MATERIALS AND METHODS}

\section{Participants}

All procedures and ethical issues related to this study were reviewed and approved by the Institutional Review Board of Ewha Womans University (IRB No. 89-7). Young adults aged 20-40 and older adults aged 65 and over were initially recruited from universities, local community centers, and centers for older adults with dementia: 23 young adults (YA), 22 healthy older adults (OA), and 17 older adults with mild dementia (MD). Eight males and 15 females were recruited for the YA group; 5 males and 17 females were recruited for the OA group; and 6 males and 11 females were recruited for the MD group. An informed consent was obtained from each participant prior to the study. The Korean versions of the Mini-Mental State Examination (MMSE) and Geriatric Depression Scale (GDS) were used to screen for the inclusion criteria for participants. Healthy YA and OA individuals were included if they obtained a score of 24 or higher on the MMSE. Older adults with mild dementia who had been diagnosed with Alzheimer's disease or vascular dementia were recruited and screened for MMSE scores between 19 and 23. Two young adults scored over 14 on the GDS, and in the OA group without diagnosis of dementia, two participants obtained less than 19 on the MMSE. They were excluded from further data analysis. The remaining participants indicated no discernible hearing deficits on a completion of a 10-item questionnaire on hearing loss in various listening conditions. They were also able to follow verbal commands and perform visuospatial tasks, such as writing. In the final analysis, a total of 58 participants were included. Demographic information is displayed in Table $\mathbf{1 .}$

\section{Stimuli}

A 12-inch electronic drum pad (Alesis PercPad, Cumberland, RI, United States) was used with drum sticks. A musical instrument digital interface (MIDI) was used to transfer the signals obtained during drum tapping to the Cubase 5 (Steinberg Media Technologies AG, Hamburg, Germany). The MIDIgenerated signals enabled the collection and analysis of data on the timing of each tapping measured as a unit of seconds. For the provision of rhythmic cueing, a MIDI software-embedded metronome was used.

\section{Measurement}

For cognitive measurements, the Digit Span Test (DST) and Trail Making Test (TMT) were used. The DST (Kaplan et al., 1991; Kang et al., 2002) is used to assess working memory and consists of two subtests: Digit Span Forward (DSF), the test of ability to recall three- to nine-digit numbers in a presented order, and Digit Span Backward (DSB), the test of ability to recall a series of numbers in a reverse order than initially presented (Jahanshahi et al., 2009). The Korean version of TMT (Reitan, 1956; Yi et al., 2007) consists of two subtests (TMT-A and TMT-B) and measures working memory and executive function.

TABLE 1 | Demographic information of participants.

\begin{tabular}{lccc}
\hline Parameter & YA $(\boldsymbol{n}=\mathbf{2 1})$ & OA $(\boldsymbol{n}=\mathbf{2 0})$ & MD $(\boldsymbol{n}=\mathbf{1 7})$ \\
\hline Gender $(\mathrm{M}: \mathrm{F})$ & $8: 13$ & $5: 15$ & $6: 11$ \\
Age, years $(M \pm S D)$ & $28.3 \pm 6.6$ & $75.8 \pm 8.2$ & $77.6 \pm 3.7$ \\
MMSE $(M \pm S D)$ & $29.5 \pm 0.6$ & $27.3 \pm 1.7$ & $21.1 \pm 1.5$ \\
GDS $(M \pm S D)$ & $5.1 \pm 3.4$ & $7.4 \pm 6.2$ & $7.0 \pm 4.1$ \\
Dominant hand (Rt:Lt) & $21: 0$ & $20: 0$ & $17: 0$ \\
\hline
\end{tabular}

MMSE, Mini-Mental State Examination; GDS, Geriatric Depression Scale. 
During the TMT-A, a participant draws a line connecting circled numbers in ascending order from 1 to 15 . The TMT-B requires the participant to draw a line connecting circled numbers and words (i.e., the words for the days of the week) alternatively as in 1, Monday, 2, Tuesday, and so on. While TMT-A and TMT-B both measure visuospatial attention, sequencing, speed of processing and working memory, executive functioning, and cognitive flexibility, the TMT-B also requires attentional control and inhibition and set-shifting task performance (Arbuthnott and Frank, 2000).

For drum tapping tasks, each participant was initially instructed to tap an electronic drum using both hands at their preferred tempo while tapping with both hands simultaneously and in alteration. Then they tapped the drum in time to the rhythmic cueing provided. Such drum tapping tasks were also implemented in two conditions: simultaneous and alternative tapping tasks. When they performed each drum tapping task, they were instructed to maintain the task until they were said to stop. Prior to each trial, participants were presented with a practice trial and after confirming that they understood the task, each trial began. They maintained at least 30 taps for each trial and the duration of its trial was 20-30 s. Regarding the provision of rhythmic cueing, the tempo of cueing was adjusted according to five conditions: each participant's preferred tempo measured during self-paced tempo and adjusted tempo at \pm 10 and $\pm 20 \%$ of the baseline tempo. The order of presenting tasks was randomly determined for each participant prior to the test. The drum tapping tasks provided to each participant are displayed in Table 2.

\section{Procedures}

The current study was individually conducted in a quiet room of a university, a local community center, and a center for older adults with dementia where participants were recruited. In order to minimize the noise in the environment to an equivalent level across settings, isolated places without adjacent rooms where noise could be produced were selected. For each participant who agreed to participate in this study, cognitive measures and drum tapping tasks were administered. During drum tapping tasks, each participant was instructed to tap the drum at their preferred tempo (self-paced tempo) and at a tempo that matched the presented cueing (tapping to rhythmic cueing tasks).

\section{Data Collection and Analysis}

The DST was scored with the number of items that each participant accurately recalled in the presented order (DSF) and the reverse order (DSB). For the TMT, both the TMT-A

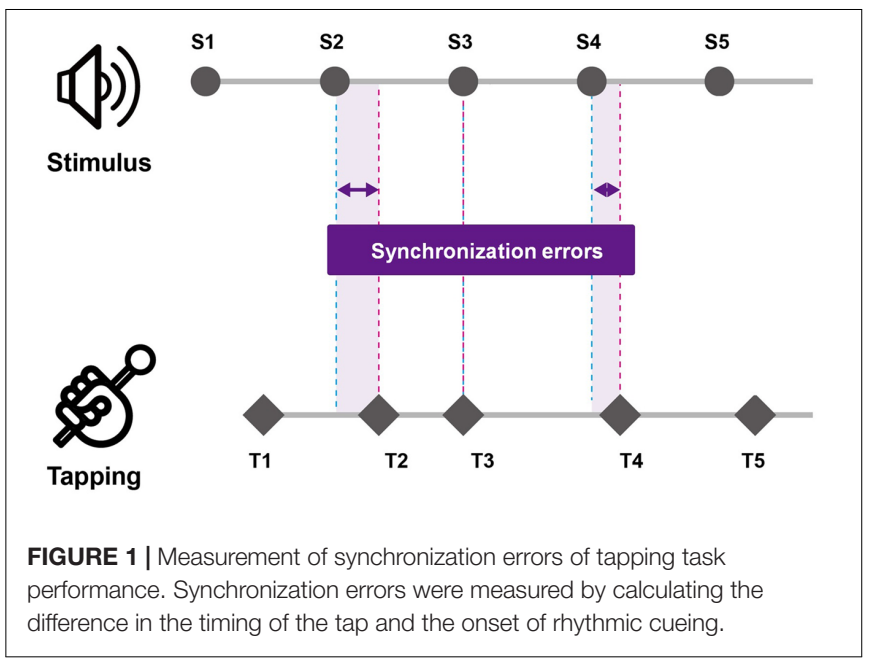

and TMT-B were scored with the time to complete the test. During self-paced tapping, the intervals in the timing of each two consecutive taps were calculated [i.e., inter-tap interval (ITI)] and a total of 29 ITIs for each trial were averaged. Also, in order to measure the regularity of tapping, the variability (standard deviation) of ITI was calculated. And for drum tapping task with rhythmic cueing, the synchronization errors were measured via two parameters: mean synchronization errors and variability of synchronization errors. First, synchronization errors were measured by calculating the difference in the timing of the tap and the onset of rhythmic cueing (see Figure 1). For the mean synchronization errors, the absolute value of each difference in the timing of the tap and the onset of rhythmic cueing was calculated and averaged for each trial. Then variability of synchronization errors was measured with the average coefficient of variation by calculating the standard deviation of the collected time differences divided by the mean inter-stimulus interval of the rhythmic cueing. For each type of tapping task (i.e., simultaneous and alternative tapping), the measures of synchronization errors were analyzed and compared across the age group by conducting a mixed between-within subjects ANOVA with the group as a betweengroup factor and with tempo of the cueing as within-group factor. For post hoc analyses, the Bonferroni correction was used. Furthermore, Spearman's correlations were conducted to examine the relationship between cognitive measures and synchronization errors-related parameters for each playing task condition (i.e., simultaneous and alternative tapping conditions).

TABLE 2 | Drum tapping tasks.

\begin{tabular}{lccc}
\hline Task & Involvement of limbs & Provision of rhythmic cueing & Tempo of rhythmic cueing \\
\hline Self-paced tapping & Simultaneous & $\mathrm{N}$ & $\mathrm{NA}$ \\
& Alternative & $\mathrm{N}$ & $\mathrm{NA}$ \\
Tapping to rhythmic cueing & Simultaneous & $\mathrm{Y}$ & $\mathrm{B}$ \\
& Alternative & $\mathrm{Y}$ & Baseline $/ \pm 10 \% / \pm 20 \%$ \\
& & Baseline $/ \pm 10 \% / \pm 20 \%$ \\
\hline
\end{tabular}

Y, yes; N, no; NA, not applicable; +, faster; -, slower. 
TABLE 3 | Results of cognitive measures for each group.

\begin{tabular}{|c|c|c|c|c|c|}
\hline Parameter & YA $(n=21)$ & OA $(n=20)$ & MD $(n=17)$ & $F(2,55)$ & $P$ \\
\hline \multicolumn{6}{|l|}{ DST } \\
\hline $\mathrm{DSF}(M \pm S D)$ & $8.8 \pm 0.4$ & $5.7 \pm 1.8$ & $4.4 \pm 1.4$ & 57.085 & $<0.001^{* * *}$ \\
\hline \multicolumn{6}{|l|}{ TMT } \\
\hline TMT-A, sec $(M \pm S D)$ & $12.3 \pm 4.0$ & $30.8 \pm 15.7$ & $57.7 \pm 43.0$ & 15.454 & $<0.001^{* * *}$ \\
\hline TMT-B, sec $(M \pm S D)$ & $17.0 \pm 6.8$ & $101.9 \pm 80.6$ & $166.2 \pm 112.0$ & 17.915 & $<0.001^{* * *}$ \\
\hline
\end{tabular}

DST, Digit Span Test; DSF, Digit Span Forward; DSB, Digit Span Backward; TMT, Trail Making Test. *** $p<0.001$.

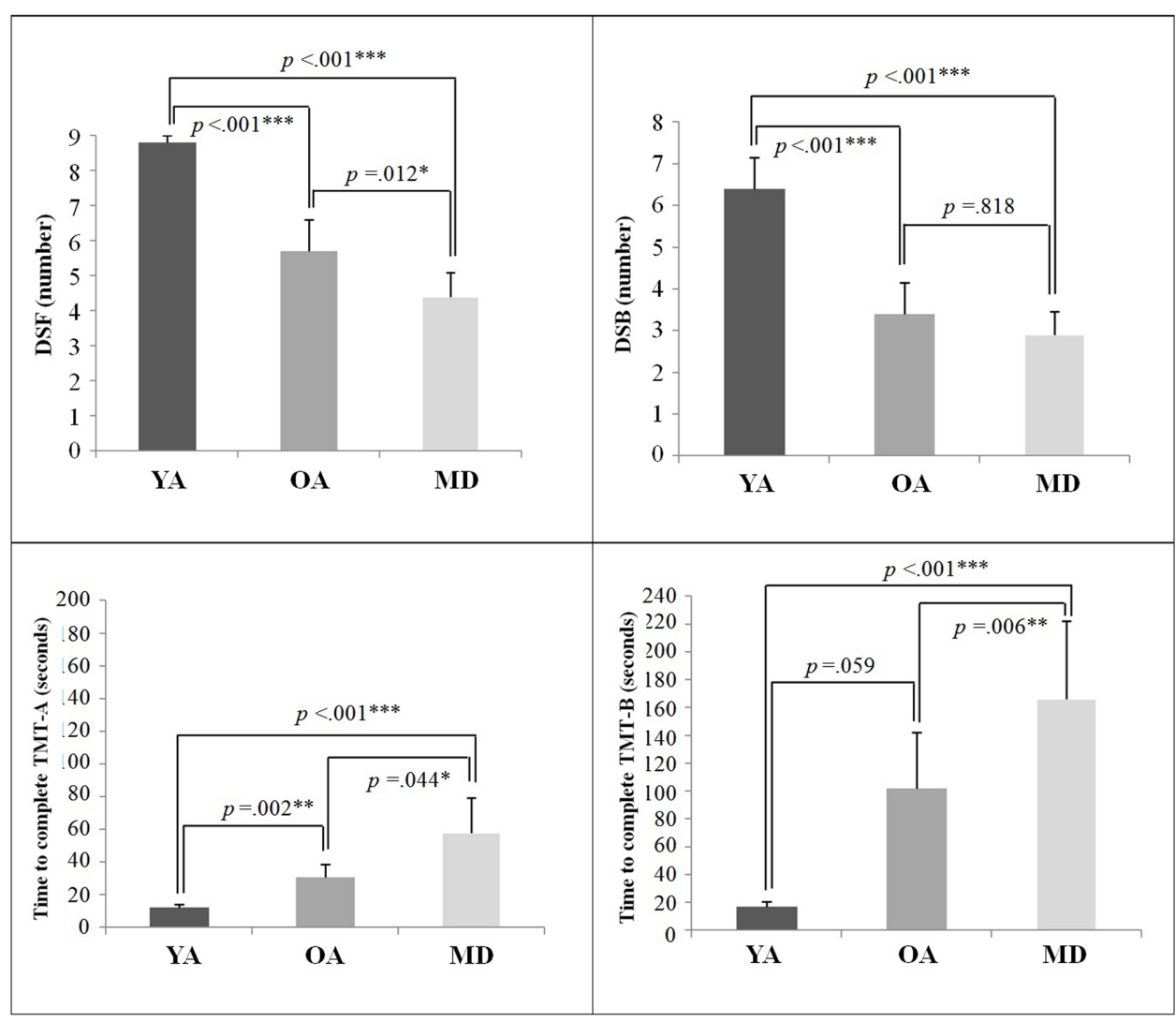

FIGURE 2 | Group comparison of cognitive measures.

\section{RESULTS}

This study investigated whether the performance of bimanual tapping to rhythmic cueing differed depending on the participants' cognitive aging in terms of synchronization errors.

\section{Cognitive Measures of Participants}

The descriptive results of the cognitive measures of the DST and TMT tests are displayed in Table 3. A one-way ANOVA showed that there were significant group differences on all cognitive measures. Post hoc analyses with the Bonferroni correction showed that for DSF and TMT-A measures, the YA group demonstrated significantly greater performance than the OA and MD groups. The OA group also recalled significantly more digits, compared to the MD group. For DSB, while the YA group recalled significantly longer digits than the $\mathrm{OA}$ and $\mathrm{MD}$ groups, the comparison between the $\mathrm{OA}$ and $\mathrm{MD}$ groups did not reach statistical significance. For the TMT-B measure, the YA and OA groups completed the test within significantly less time, compared to the MD group. There was no significant difference in the time to complete the TMT-B between the YA and OA groups. Results on the group comparisons in terms of cognitive measures are displayed in Figure 2.

\section{Self-Paced Bimanual Tapping}

In this study, each participant was instructed to tap the electronic drum with both hands simultaneously and then alternatively 
using one hand and then the other at their preferred tempo. The results of a one-way ANOVA showed that there were significant differences in ITI among the groups during both simultaneous tapping and alternative tapping (see Table 4). Post hoc analyses with the Bonferroni correction showed that the YA group exhibited significantly slower tapping than the OA group during simultaneous $(p=0.005)$ and alternative bimanual tapping $(p=0.005)$. Paired comparisons between the YA and MD groups $(p=0.062$ for simultaneous tapping and $p=1.000$ for alternative tapping) and between the OA and MD groups ( $p=0.092$ for simultaneous tapping and $p=1.000$ for alternative tapping) did not reach statistical significance.

With regard to tapping variability, a one-way ANOVA was conducted to see whether the level of maintaining the regularity of self-paced tapping differed depending on the group. The results showed that there were no significant differences between groups during both simultaneous tapping and alternative tapping (see Table 5).

\section{Mean Synchronization Errors during Bimanual Tapping}

In terms of measures of synchronization errors, in order to investigate the degree of synchronization errors without consideration of the tendency to tap (i.e., the tendency to tap before vs. after the provision of cueing), the absolute values of synchronization errors were calculated and compared across groups. Increases in such value represent increased synchronization errors, indicating lower timing accuracy. Conversely, decreased values indicate higher timing accuracy. For simultaneous tapping, the results of a mixed model repeated measures ANOVA showed that the main effect of tempo was statistically significant, $F(4,220)=3.657$, $p=0.007, \eta^{2}=0.062$, Power $=936$. A post hoc analysis with a Bonferroni correction demonstrated that the $+10 \%$ tempo condition elicit significantly less synchronization errors than the $-20 \%$ tempo condition $(p=0.020)$. The other paired comparisons did not reach statistical significance. The interaction effect between the tempo and group was not significant, $F(8,220)=1.702, p=0.099, \eta^{2}=0.058$, Power $=769$, indicating that changes in synchronization errors depending on the tempo condition were similar across the group. The group significantly affected the mean synchronization errors, $F(2,55)=5.065, p=0.010, \eta^{2}=0.156$, Power $=943$. A post hoc analysis with a Bonferroni correction demonstrated that the YA group showed significantly less synchronization errors than the MD group $(p=0.009)$. The other paired comparisons did not reach statistical significance (see Table 6 and Figure 3).

For alternative tapping, the results of a mixed model repeated measures ANOVA showed that the main effect of tempo

TABLE 4 | Mean ITI (seconds) during self-paced tapping.

\begin{tabular}{|c|c|c|c|c|c|}
\hline \multirow[b]{2}{*}{ Type of tapping } & \multicolumn{3}{|c|}{ Mean ITI $(M \pm S D)$} & \multirow[b]{2}{*}{$F(2,55)$} & \multirow[b]{2}{*}{$p$} \\
\hline & YA $(n=21)$ & OA $(n=20)$ & MD $(n=17)$ & & \\
\hline Alternative & $0.519 \pm 0.223$ & $0.343 \pm 0.147$ & $0.396 \pm 0.107$ & 5.780 & $0.005^{*}$ \\
\hline
\end{tabular}

ITI, inter-tap interval. ** $p<0.01$.

TABLE 5 | Tapping variability during self-paced tapping.

\begin{tabular}{lcccc}
\hline & \multicolumn{3}{c}{ Tapping variability $(\boldsymbol{M} \pm \mathbf{S D})$} & \\
\cline { 2 - 5 } Type of tapping & YA $(\boldsymbol{n}=\mathbf{2 1})$ & OA $(\boldsymbol{n}=\mathbf{2 0})$ & MD $(\boldsymbol{n}=\mathbf{1 7})$ & $\boldsymbol{F ( 2 , 5 5 )}$ \\
\hline Simultaneous & $0.024 \pm 0.012$ & $0.030 \pm 0.028$ & $0.028 \pm 0.020$ & 0.395 \\
Alternative & $0.032 \pm 0.017$ & $0.049 \pm 0.059$ & $0.038 \pm 0.023$ & 0.993
\end{tabular}

TABLE 6 | Mean synchronization errors during bimanual tapping depending on the tempo condition.

\begin{tabular}{|c|c|c|c|c|c|c|}
\hline \multirow[t]{2}{*}{ Tapping task } & \multirow[t]{2}{*}{ Group } & \multicolumn{5}{|c|}{ Synchronization errors, ms $(M \pm S D)$} \\
\hline & & $-20 \%$ & $-10 \%$ & Baseline & $+10 \%$ & $+20 \%$ \\
\hline \multirow[t]{3}{*}{ Simultaneous } & $Y A(n=21)$ & $0.090 \pm 0.077$ & $0.092 \pm 0.072$ & $0.087 \pm 0.075$ & $0.088 \pm 0.072$ & $0.083 \pm 0.067$ \\
\hline & $\mathrm{OA}(n=20)$ & $0.131 \pm 0.070$ & $0.103 \pm 0.063$ & $0.087 \pm 0.055$ & $0.094 \pm 0.062$ & $0.101 \pm 0.061$ \\
\hline & $\operatorname{MD}(n=17)$ & $0.165 \pm 0.078$ & $0.148 \pm 0.073$ & $0.158 \pm 0.088$ & $0.109 \pm 0.047$ & $0.144 \pm 0.060$ \\
\hline \multirow[t]{3}{*}{ Alternative } & $\mathrm{YA}(n=21)$ & $0.080 \pm 0.062$ & $0.087 \pm 0.063$ & $0.087 \pm 0.079$ & $0.068 \pm 0.067$ & $0.074 \pm 0.081$ \\
\hline & $\mathrm{OA}(n=20)$ & $0.099 \pm 0.060$ & $0.099 \pm 0.072$ & $0.086 \pm 0.031$ & $0.070 \pm 0.032$ & $0.084 \pm 0.040$ \\
\hline & $\mathrm{MD}(n=17)$ & $0.169 \pm 0.112$ & $0.149 \pm 0.054$ & $0.131 \pm 0.047$ & $0.147 \pm 0.056$ & $0.181 \pm 0.085$ \\
\hline
\end{tabular}

YA: young adults; OA: older adults; MD: mild dementia. 


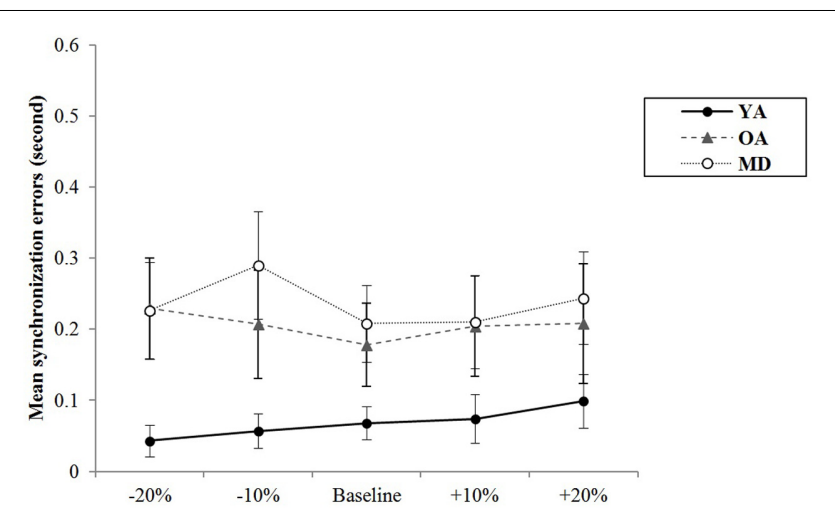

FIGURE 3 | Group comparison of mean synchronization errors during simultaneous bimanual tapping.

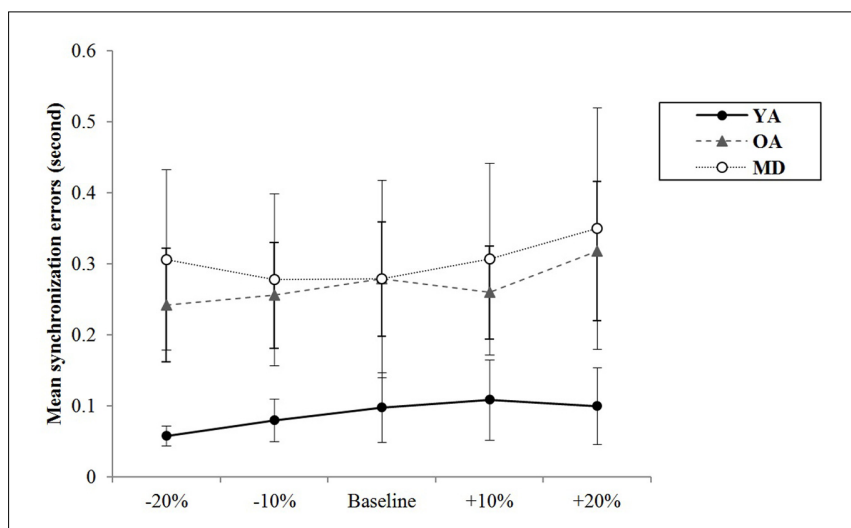

FIGURE 4 | Group comparison of mean synchronization errors during alternative bimanual tapping.

was not statistically significant, $F(4,220)=2.278, p=0.082$, $\eta^{2}=0.040$, Power $=764$, and the interaction effect between tempo and group was also not significant, $F(8,220)=1.688$, $p=0.102, \eta^{2}=0.058$, Power $=761$, indicating that changes in synchronization errors depending on the tempo condition were similar across the groups. The group significantly affects the mean synchronization errors, $F(2,55)=11.503, p<0.001$, $\eta^{2}=0.295$, Power $=0.999$. A post hoc analysis with a Bonferroni correction demonstrated that the YA and OA groups showed significantly less synchronization errors than the MD group ( $p<0.001$ for the YA-MD comparison and $p=0.001$ for the OA-MD comparison). The other paired comparisons did not reach statistical significance (see Table 6 and Figure 4).

\section{Variability of Synchronization Errors during Bimanual Tapping}

For the variability of synchronization errors, the descriptive results of the coefficient of variation during the simultaneous tapping condition are displayed in Table 7. The YA group showed the lowest values for variability of synchronization errors in all tempo conditions. Meanwhile, the MD group showed the highest variability of synchronization errors in all tempo conditions, except the $-20 \%$ tempo condition. The OA group showed the highest variability of synchronization at $-20 \%$ tempo condition and the MD showed it at $-10 \%$ tempo. While the YA group showed the tendency that the variability in maintaining synchronization accuracy slightly increases as the tapping tempo gets faster, the $\mathrm{OA}$ and $\mathrm{MD}$ groups tended to show increased variability of synchronization errors at slower and faster tempi, compared to the preferred tempo.

A mixed model of repeated measures ANOVA was conducted to see whether there were differences in variability of synchronization errors depending on the tempo condition across the groups during simultaneous tapping tasks. There was no significant main effect of the tempo, $F(4,220)=1.994$, $p=0.098, \eta^{2}=0.035$, Power $=0.932$. There was no significant interaction effect between tempo and group, $F(8,220)=1.785$, $p=0.081, \eta^{2}=0.061$, Power $=851$, indicating that the groups showed similar trends in terms of changes in the variability of synchronization errors depending on the tempo condition (see Figure 5). The main effect of group was statistically significant, $F(2,55)=16.979, p<0.001, \eta^{2}=0.382$, Power $=0.999$. Post hoc analyses with the Bonferroni correction demonstrated that the YA group showed significantly less variability in the synchronization errors than the OA $(p<0.001)$ and MD groups $(p<0.001)$. Comparison between the OA and MD groups did not reach statistical significance $(p=0.979)$.

During alternative bimanual tapping, the results for variability of synchronization errors for each group are displayed in Table 7. The YA group showed the least variability of synchronization errors in all tempo conditions. Meanwhile, the MD group showed the highest variability of synchronization errors in all tempo conditions. The YA group showed relatively fewer changes in their variability of synchronization errors in different tempo conditions. The OA group tended to show increased variability of synchronization errors at the slowest tempo. The MD group tended to show increased variability of synchronization errors at adjusted tempi.

The results of a mixed model of repeated measures ANOVA showed that the main effect of the tempo was statistically significant, $F(4,220)=2.693, p=0.039, \eta^{2}=0.047$, Power $=839$. Post hoc analysis with the Bonferroni correction showed while comparison between $+20 \%$ and $-20 \%$ tempo condition $(p=0.123)$ and between $+20 \%$ and $-10 \%$ tempo condition ( $p=0.151)$ elicited the greatest differences, none of the paired comparisons reached statistical significance. There were no significant interaction effects between tempo and group, $F(8,220)=0.704, p=0.688, \eta^{2}=0.025$, Power $=393$, indicating that there were similar trends in terms of changes in synchronization errors depending on the tempo condition across the groups (see Figure 6). The main effect of group was statistically significant, $F(2,55)=29.557, p<0.001$, $\eta^{2}=0.518$, Power $=1.000$. Post hoc analyses with a Bonferroni correction demonstrated that the YA group showed significantly less variability of the synchronization errors than the OA $(p<0.001)$ and MD groups $(p<0.001)$. Comparison between the $\mathrm{OA}$ and $\mathrm{MD}$ group did not reach statistical significance $(p=0.882)$. 
TABLE 7 | Coefficient of variation as measures for variability of synchronization errors during bimanual tapping depending on the tempo condition.

\begin{tabular}{|c|c|c|c|c|c|c|}
\hline \multirow[t]{2}{*}{ Tapping task } & \multirow[t]{2}{*}{ Group } & \multicolumn{5}{|c|}{ Coefficient of variability $(M \pm S D)$} \\
\hline & & $-20 \%$ & $-10 \%$ & Baseline & $+10 \%$ & $+20 \%$ \\
\hline \multirow[t]{3}{*}{ Simultaneous } & $Y A(n=21)$ & $0.043 \pm 0.044$ & $0.057 \pm 0.048$ & $0.068 \pm 0.046$ & $0.074 \pm 0.069$ & $0.099 \pm 0.076$ \\
\hline & $\mathrm{OA}(n=20)$ & $0.229 \pm 0.136$ & $0.207 \pm 0.152$ & $0.178 \pm 0.108$ & $0.204 \pm 0.131$ & $0.208 \pm 0.130$ \\
\hline & $\mathrm{MD}(n=17)$ & $0.226 \pm 0.143$ & $0.290 \pm 0.152$ & $0.208 \pm 0.117$ & $0.210 \pm 0.141$ & $0.244 \pm 0.169$ \\
\hline \multirow[t]{3}{*}{ Alternative } & $\mathrm{YA}(n=21)$ & $0.058 \pm 0.028$ & $0.080 \pm 0.060$ & $0.098 \pm 0.097$ & $0.109 \pm 0.113$ & $0.100 \pm 0.108$ \\
\hline & $\mathrm{OA}(n=20)$ & $0.242 \pm 0.127$ & $0.256 \pm 0.121$ & $0.279 \pm 0.139$ & $0.260 \pm 0.135$ & $0.318 \pm 0.170$ \\
\hline & $\mathrm{MD}(n=17)$ & $0.306 \pm 0.160$ & $0.278 \pm 0.149$ & $0.279 \pm 0.161$ & $0.307 \pm 0.131$ & $0.350 \pm 0.196$ \\
\hline
\end{tabular}

YA: young adults; OA: older adults; MD: mild dementia.

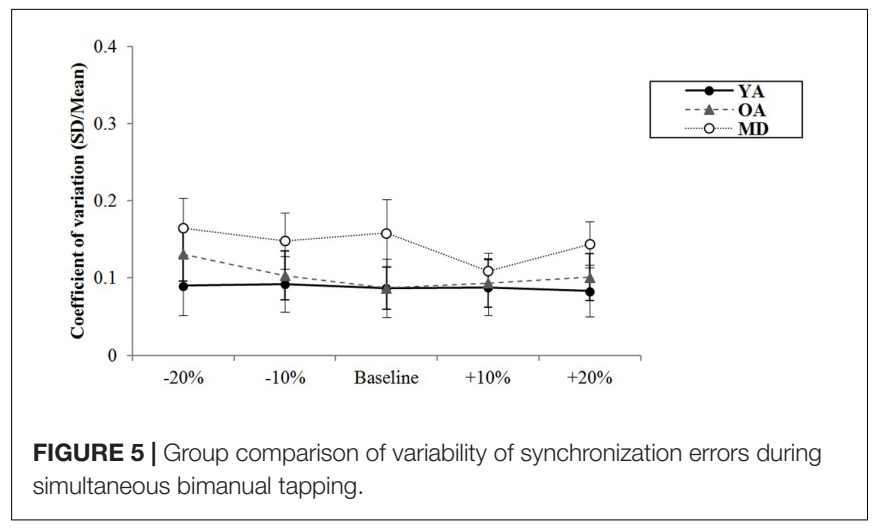

\section{Correlation between Cognitive Measures and Timing Parameters}

Finally, a Spearman's correlation between cognitive measures and variability of synchronization errors during each of the simultaneous and alternative tapping tasks was analyzed. The correlation coefficients are displayed in Table 8 . Correlations between cognitive measures and variability of synchronization errors ranged 0.42 to 80 and all reached statistical significance. The higher correlations over 0.70 , during simultaneous tapping-related parameters indicate a significantly negative correlation between synchronization errors in -20 and $-10 \%$ tempo conditions with the measure

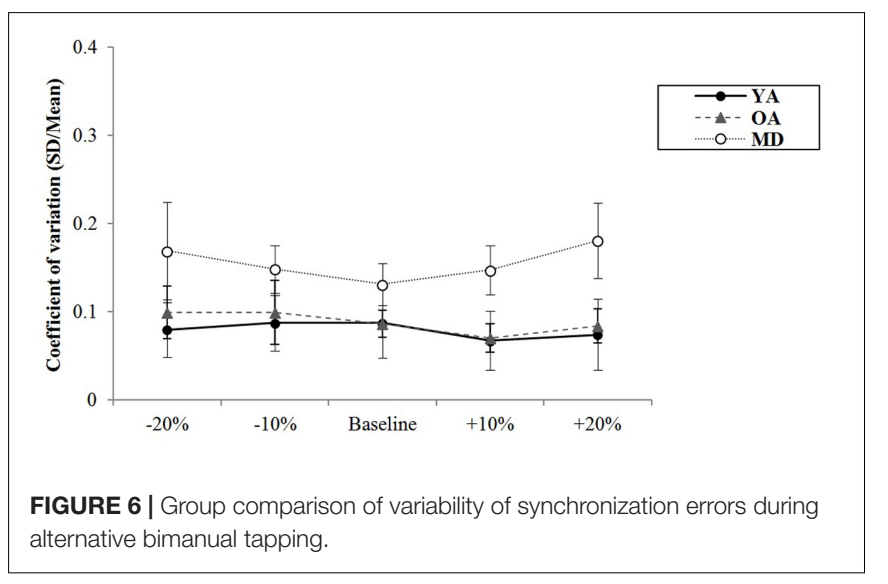

of DSF, indicating that as the difference between timing of tapping and rhythmic cueing became stable (decreased variability of synchronization), the number of digits recalled increased. During alternative tapping at the slowest tempo $(-20 \%$ tempo condition), the variability of synchronization errors was highly correlated with all cognitive measures and such error at $-10 \%$ tempo condition was highly correlated with DSF.

\section{DISCUSSION}

This study investigated whether the measures of synchronization errors during bimanual tapping tasks with rhythmic cueing differed depending on the participants' level of cognitive aging. When asked to tap the drum at a self-paced tempo, the YA group tended to tap the drum significantly slower than the OA and MD groups; meanwhile, the tapping speed was slower for the MD group than the OA group.

Faster tapping speed observed in older adults groups may seem inconsistent with previous research showing significantly slower tapping for older adults than younger adults (Vanneste et al., 2001). However, this study may be associated with other findings showing that reaction time significantly decreased with aging, but that movement time was maintained at a similar level to younger adults (Kauranen and Vanharanta, 1996). In addition, fine motor skills have repeatedly been documented to decrease in older adults, but age-related changes in gross motor skills have not been conclusively demonstrated (VoelckerRehage, 2008). Given that the drum tapping task with drum mallets involves gross motor skills more than a simple finger tapping task, more controlled analysis is needed focusing on the type of involved movement. Furthermore, further studies with increased sample size and both females and males would be needed to corroborate age-related changes in and gender effects on timing measures during bimanual instrument playing. In addition, previous findings were primarily based on participants in Western countries, thereby suggesting that cultural factors, which may influence physical conditions and exposure to and engagement in musical activities, need to be considered in future studies.

The level of tapping variability was not different across the groups, indicating that the participants could maintain their regular tapping. This was consistent during both simultaneous 
TABLE 8 | Correlation between cognitive measures and variability of synchronization errors during bimanual tapping.

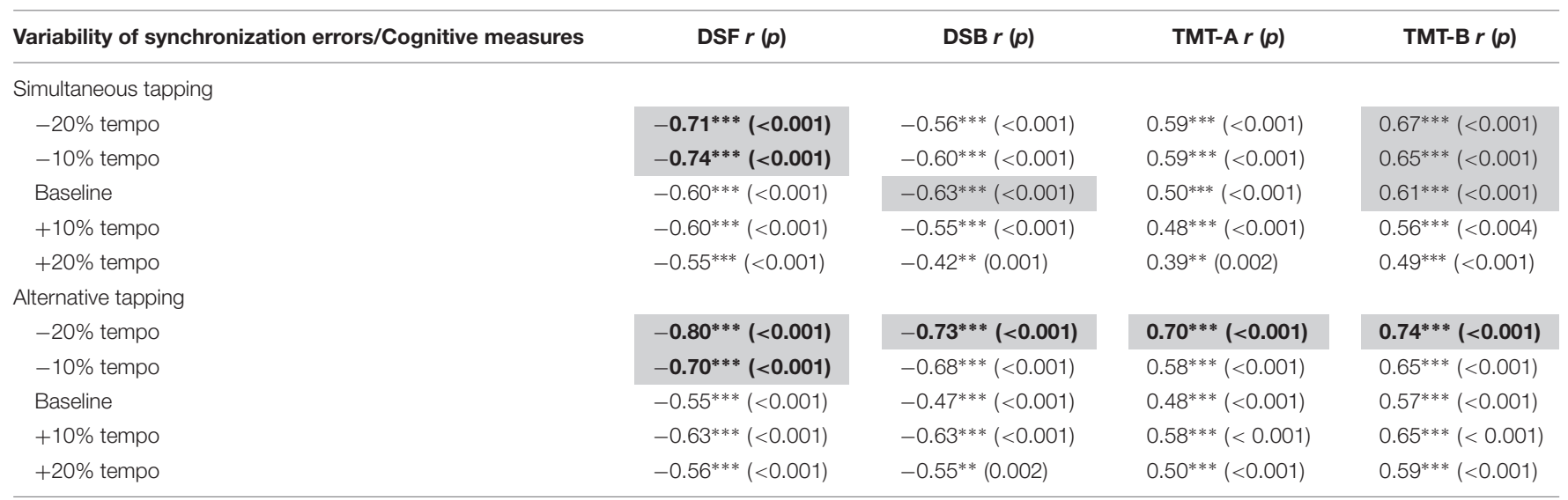

Dark gray and bolded values indicates the case with correlation coefficient over 0.70 and light gray indicates the case with correlation coefficient between 0.60 and 0.70 . ${ }^{* *} p<0.01,{ }^{* * *} p<0.001$.

and alternative tapping. These results indicate that the ability to perceive timing is relatively intact despite cognitive aging.

This finding supports other research demonstrating that internal timing, measured with continuous finger or hand tapping, does not elicit age-related differences (Vanneste et al., 2001; Trugeon et al., 2011). In previous studies, older adults with mild Alzheimer's disease showed no significant differences compared to age-matched older adults and young adults when required to judge the interval of timing and discriminate different intervals (Caselli et al., 2009). These results indicate that the internal timekeeping system operates sufficiently in older adults and older adults with cognitive decline. Although research remains to be done, some brain imaging studies have explained that while decreased volume in the cerebellum and connectivity between motor-related networks negatively affect motor performance of older adults, the compensatory neural system still facilitates the maintenance of sensorimotor functioning of this population, including strengthened connectivity between the motor cortex, putamen, and cerebellum (Seidler et al., 2010). Such findings indicate that the use of external timing cues may be effectively applied to interventions for sensorimotor functioning and motor coordination for daily life activities and tasks.

With regard to synchronization errors, which measure how accurately the participants matched the rhythmic cueing, the group factor (i.e., the level of cognitive aging) resulted in significant differences during both simultaneous and alternative tapping tasks. Interesting to note was while the OA group showed less synchronization errors than the MD group, the comparison between the YA and OA groups did not reach statistical significance. These results indicate that decreases in synchronization errors may be attributed to cognitive impairment, not exclusively to aging. Although older adults could maintain their tapping, the immediacy of adjustment and motor control decreased with cognitive aging. These results also support that decreases in motor response time as reported in the literature result in slower processing speed and efficiency of performance during bimanual coordination (Trapp et al., 2012; Shetty et al., 2014). Despite relatively intact timing perception, the process of transferring perceived information into motor output may be delayed and the efficiency of coordination compromised.

In terms of variability of synchronization errors, the group factor also significantly affected such measures during both simultaneous and alternative tapping tasks. Compared to mean synchronization errors which are indicative of magnitude of asynchrony, the variability of synchronization errors (measured by coefficient of variation) indicates the synchronization precision in terms of how stable timing performance is maintained in relation to the ongoing temporal events. The YA group showed less variability in changes in synchronization errors across the tempo of cueing. Meanwhile, older adults with and without mild dementia showed increased synchronization errors at adjusted tempi. When comparing the two older adults groups, the MD group tended to show greater synchronization errors and increased variability of changes in such errors depending on the tapping task and the tempo or cueing compared to the OA group. Post hoc analyses demonstrated that the YA group showed significantly less variability in synchronization errors than both the OA and MD groups, indicating such group differences may be attributed to aging.

In older adults groups, greater synchronization errors at adjusted tempi than at baseline tempo. This tendency led to significant differences in the variability of synchronization errors depending on the tempo condition. Previous studies demonstrated that older adults tended to have greater difficulty in moving two limbs in opposite directions as required during alternative tapping than in moving two limbs in the same direction as required during simultaneous tapping (Mattay et al., 2002). Such findings indicate that control of two limbs separately in the opposite direction requires inhibitory control, which suppresses conflicting motor output to perform a task (Serrien et al., 2000). The results of this study suggest that decreased processing time for bimanual coordination affects the differences in timing accuracy during simultaneous tapping between younger and older adults regardless of cognitive impairment. Meanwhile, alternative tapping, in which the tempo factor significantly affect timing 
performance, may increase cognitive demands in addition to physical demands.

Furthermore, significantly high correlations between cognitive measures and synchronization errors during bimanual tapping indicate that bimanual coordination in response to external timing cues at adjusted tempi is involved in cognitive processing and cognitive flexibility. Temporal control with bimanual engagement was documented to require working memory and executive control (Bangert et al., 2010). In this study, alternative tapping at slower tempo was associated with a subtest of DST and TMT that require inhibitory control more than simultaneous tapping. It is also noteworthy that significant correlations with cognitive measures were observed in timing measures at adjusted tempi (slower). Such results indicate that the type of involvement of the limbs (simultaneous vs. alternative) and the tempo mediate the processing of external timing cues and motor control based on the perceived input at different levels (Ridderikhoff et al., 2008). Previous studies support that during synchronization tasks, the task to match an individual's tapping to adjusted tempo, that is faster or slower than an individual's preferred tempo, increases dynamic control of attentions by competing with initial expectancy mediated by internal timing and adapting to newly generated expectancy by external cueing (McAuley et al., 2006; Bangert and Balota, 2012).

In sum, the current study supports the notion that bimanual tapping may be predictive of cognitive processing of older adults.

\section{REFERENCES}

Ackerley, S. J., Stinear, C. M., and Byblow, W. D. (2011). Promoting use-dependent plasticity with externally-paced training. Clin. Neurophysiol. 122, 2462-2468. doi: 10.1016/j.clinph.2011.05.011

Arbuthnott, K., and Frank, J. (2000). Trail making test, part B as a measure of executive control: validation using a set-switching paradigm. J. Clin. Exp. Neuropsychol. 22, 518-528.

Balbag, M. A., Pedersen, N. L., and Gatz, M. (2014). Playing a musical instrument as a protective factor against dementia and cognitive impairment: a populationbased twin study. Int. J. Alzheimers Dis. 2014:836748. doi: 10.1155/2014/836748

Bangert, A. S., and Balota, D. A. (2012). Keep up the pace: declines in simple repetitive timing differentiate healthy aging from the earliest stages of Alzheimer's disease. J. Int. Neuropsychol. Soc. 18, 1052-1063. doi: 10.1017/ S1355617712000860

Bangert, A. S., Reuter-Lorenz, P. A., Walsh, C. M., Schachter, A. B., and Seidler, R. D. (2010). Bimanual coordination and aging: neurobehavioral implications. Neuropsychologia 48, 1165-1170.

Bartzokis, G., Sultzer, D., Lu, P. H., Nuechterlein, K. H., Mintz, J., and Cummings, J. L. (2004). Heterogeneous age-related breakdown of white matter structural integrity: implications for cortical disconnection in aging and Alzheimer's disease. Neurobiol. Aging 25, 843-851. doi: 10.1016/j.neurobiolaging.2003. 09.005

Bugos, J. A., Perlstein, W. M., McCare, C. S., Brophy, T. S., and Bedenbaugh, P. H. (2007). Individualized piano instruction enhances executive functioning and working memory in older adults. Aging Ment. Health 11, 464-471. doi: 10.1080/13607860601086504

Caçola, P., Roberson, J., and Gabbard, C. (2013). Aging in movement representations for sequential finger movements: a comparison between young, middle-aged, and older adults. Brain $\operatorname{Cog} n$ 82, 1-5. doi: 10.1016/j.bandc.2013. 02.003

Carson, R. G., Goodman, D., Kelso, J. A. S., and Elliott, D. (1995). Phase-transitions and critical fluctuations in rhythmic coordination of ipsilateral hand and foot. J. Mot. Behav. 27, 211-224. doi: 10.1080/00222895.1995.9941711
It proposes that such instrument playing can be effectively incorporated into the process of assessing and intervening in cognitive and motor functioning of older adults who show limited performance of other types of tasks due to cognitive decline. Furthermore, the current study presents implications for how instrument playing can be used as bimanual coordination tasks for older adults with varying levels of cognitive aging. Results on differences in the timing accuracy depending on the tempo and the type of task indicate that different tapping conditions require cognitive and motor loads at different levels and such variables could be an important factor for determining the complexity of the task and the involved task requirements.

\section{AUTHOR CONTRIBUTIONS}

SJK, S-RC, and GEY contributed to study conception and design, data acquisition and analysis, and manuscript writing.

\section{FUNDING}

This work was supported by the National Research Foundation of Korea Grant funded by the Korean Government (NRF2014S1A5A2A03066008).

Caselli, L., Iaboli, L., and Nichelli, P. (2009). Time estimation in mild Alzheimer's disease patients. Behav. Brain Funct. 5:32. doi: 10.1186/1744-9081-5-32

Chen, J. L., Zatorre, R. J., and Penhune, V. B. (2006). Interactions between auditory and dorsal premotor cortex during synchronization to musical rhythms. Neuroimage 32, 1771-1781. doi: 10.1016/j.neuroimage.2006.04.207

Chu, H., Yang, C., Lin, Y., Ou, K., Lee, T., O’Brien, A. P., et al. (2014). The impact of group music therapy on depression and cognition in elderly persons with dementia: a randomized controlled study. Biol. Res. Nurs. 16, 209-217. doi: 10.1177/1099800413485410

Clair, A. A. C., and O'Konski, M. (2006). The effect of rhythmic auditory stimulation (RAS) on gait characteristics of cadence, velocity, and stride length in persons with late stage dementia. J. Music Ther. 43, 154-163. doi: 10.1093/ jmt/43.2.154

Fling, B. W., and Seidler, R. D. (2012). Fundamental differences in callosal structure, neurophysiologic function, and bimanual control in young and older adults. Cereb. Cortex 22, 2643-2652. doi: 10.1093/cercor/bhr349

Fujiyama, H., Garry, M. I., Levin, O., Swinnen, S. P., and Summers, J. J. (2009). Age-related differences in inhibitory processes during interlimb coordination. Brain Res. 1262, 38-47. doi: 10.1016/j.brainres.2009.01.023

Fujiyama, H., Garry, M. I., Martin, F. H., and Summers, J. J. (2010). An ERP study of age-related differences in the central cost of interlimb coordination. Psychophysiology 47, 501-511. doi: 10.1111/j.1469-8986.2009. 00954.x

Fujiyama, H., Hinder, M. R., Garry, M. I., and Summers, J. J. (2013). Slow and steady is not as easy as it sounds: interlimb coordination at slow speed is associated with elevated attentional demand especially in older adults. Exp. Brain Res. 227, 289-300. doi: 10.1007/s00221-0133511-7

Giebel, C. M., Sutcliffe, C., Stolt, M., Karlson, S., Renom-Guiteras, A., Soto, M., et al. (2014). Deterioration of basic activities of daily living and their impact on quality of life across different cognitive stages of dementia: a European study. Int. Psychogeriatr. 26, 1283-1293. doi: 10.1017/S1041610214000775

Halliday, D. W. R., Stawski, R. S., and MacDonald, S. W. S. (2016). Cognitivelyimpaired-not-demented status moderates the time-varying association 
between finger tapping inconsistency and executive performance. Arch. Clin. Neuropsychol. 2016, 1-7. doi: 10.1093/arclin/acw084

Han, S. A. (2016). Song-induced autobiographical memory of patients with early Alzheimer's Dementia. J. Music Hum. Behav. 13, 49-66. doi: 10.21187/jmhb. 2016.13.2.49

Han, S. J. (2017). The relationship between self-perceived benefits from singing and quality of life in older adults aged over 75 years. J. Music Hum. Behav. 14, 63-84. doi: 10.21187/jmhb.2016.14.1.063

Hanna-Pladdy, B., and Gajewski, B. (2012). Recent and past musical activity predicts cognitive aging variability: direct comparison with general lifestyle activities. Front. Hum. Neurosci. 6:198. doi: 10.3389/fnhum.2012.00198

Haslinger, B., Erhard, P., Altenmüller, E., Hennenlotter, A., Schwaiger, M., von Einsiedel, H. G., et al. (2004). Reduced recruitment of motor association areas during bimanual coordination in concert pianists. Hum. Brain Mapp. 22, 206-215. doi: 10.1002/hbm.20028

Head, D., Buckner, R. L., Shimony, J. S., Williams, L. E., Akbudak, E., Conturo, T. E., et al. (2004). Differential vulnerability of anterior white matter in nondemented aging with minimal acceleration in dementia of the Alzheimer type: evidence from diffusion tensor imaging. Cereb. Cortex 14, 410-423. doi: 10.1093/cercor/bhh003

Jahanshahi, M., Saleem, T. T., Ho, A. K., Fuller, R. R., and Dirnberger, G. (2009). A preliminary investigation of the running digit span as a test of working memory. Behav. Neurol. 20, 17-25. doi: 10.3233/BEN-2008-0212

Kang, Y. W., Chin, J. H., and Na, D. L. (2002). A normative study of the digit span test for the elderly. Korean J. Clin. Psychol. 21, 911-922.

Kaplan, E., Fein, D., Morris, R., and Delis, D. C. (1991). Wechsler Adult Intelligence Scale-Revised as a Neuropsychological Instrument. San Antonio, TX: The Psychological Corporation.

Kauranen, K., and Vanharanta, H. (1996). Influence of aging, gender, and handedness on motor performance of upper and lower extremities. Percept. Mot. Skills 82, 515-525.

Kilbreath, S. L., and Heard, R. C. (2005). Frequency of hand use in healthy older persons. Aust. J. Physiother. 51, 119-122. doi: 10.1016/S0004-9514(05)70040-4

Krampe, R. T., Doumas, M., Lavrysen, A., and Rapp, M. (2010). The costs of taking it slowly: fast and slow movement timing in older age. Psychol. Aging 25, 980-990. doi: 10.1037/a0020090

LaGasse, A. B., and Knight, A. (2011). Rhythm and music in rehabilitation: a critical review of current research. Crit. Rev. Phys. Rehabil. Med. 23, 49-67. doi: 10.1615/CritRevPhysRehabilMed.v23.i1-4.40

Lipsitz, L. A. (2004). Physiological complexity, aging, and the path to frailty. Sci. Aging Knowledge Environ. 2004:pe16. doi: 10.1126/sageke.2004.16.pe16

Mattay, V. S., Fera, F., Tessitore, A., Hariri, A. R., Das, S., Callicott, J. H., et al. (2002). Neurophysiological correlates of age-related changes in human motor function. Neurol 58, 630-635. doi: 10.1212/WNL.58.4.630

McAuley, J. D., Jones, M. R., Holub, S., Johnston, H. M., and Miller, N. S. (2006). The time of our lives: life span development of timing and event tracking. J. Exp. Psychol. 135, 348-367. doi: 10.1037/0096-3445.135.3.348

Meesen, R. L. J., Wenderoth, N., Temprado, J., Summers, J. J., and Swinnen, S. P. (2006). The coalition of constraints during coordination of the ipsilateral and heterolateral limbs. Exp. Brain Res. 174, 367-375. doi: 10.1007/s00221-0060471.1

Nagahama, Y., Okina, T., and Suzuki, N. (2015). Impaired imitation of gestures in mild dementia: comparison of dementia with Lewy bodies, Alzheimer's disease and vascular dementia. J. Neurol. Neurosurg. Psychiatry 86, 1248-1252. doi: 10.1136/jnnp-2014-309436

Park, D. C. (2000). "The basic mechanisms accounting for age-related decline in cognitive function," in Aging and Cognition: A Primer, eds D. C. Park and N. Schwarz (New York, NY: Psychology Press), 3-22.

Pecenka, N., Engel, A., and Keller, P. E. (2013). Neural correlates of auditory temporal predictions during sensorimotor synchronization. Front. Hum. Neurosci. 7:380. doi: 10.3389/fnhum.2013.00380

Reitan, R. M. (1956). Trail Making Test: Manual for Administration, Scoring, and Interpretation. Indianapolis, IN: Indiana University.

Ridderikhoff, A., Peper, C. L. E., and Beek, P. J. (2008). Attentional loads associated with interlimb interactions underlying rhythmic bimanual coordination. Cognition 109, 372-388. doi: 10.1016/j.cognition.2008.10.002
Schlaug, G. (2001). The brain of musicians. Ann. N. Y. Acad. Sci. 930, 281-299. doi: 10.1111/j.1749-6632.2001.tb05739.x

Seidler, R. D., Bernard, J. A., Burutolu, T. B., Fling, B. W., Gordon, M. T., Gwin, J. T., et al. (2010). Motor control and aging: links to age-related brain structural, functional, and biochemical effects. Neurosci. Biobehav. Rev. 34, 721-733. doi: 10.1016/j.neubiorev.2009.10.005

Serrien, D. J., Swinnen, S. P., and Stelmach, G. E. (2000). Age-related deterioration of coordinated interlimb behavior. J. Gerontol. B Psychol. Sci. Soc. Sci. 55, 295-303. doi: 10.1093/geronb/55.5.P295

Shetty, A. K., Shanker, V., and Annamalai, N. (2014). Bimanual coordination: influence of age and gender. J. Clin. Diagn. Res. 8, 15-16. doi: 10.7860/JCDR/ 2014/7333.3994

Sullivan, E. V., and Pfefferbaum, A. (2002). Diffusion tensor imaging and aging. Neurosci. Biobehav. Rev. 30, 749-761. doi: 10.1016/j.neubiorev.2006. 06.002

Temprado, J. J., Zanone, P. G., Monno, A., and Laurent, M. (2001). A dynamical framework to understand performance trade-offs and interference in dual tasks. J. Exp. Psychol. 27, 1303-1313. doi: 10.1037/0096-1523.27.6. 1303

Trapp, S., Lepsien, J., Sehm, B., Villringer, A., and Ragert, P. (2012). Changes of hand switching costs during bimanual sequential learning. PLOS ONE 7:e45857. doi: 10.1371/journal.pone. 0045857

Trombetti, A., Hars, M., Hermann, F. R., Kressig, R. W., Ferrari, S., and Rizzoli, R. (2011). Effect of music-based multitask training on gait, balance, and fall risk in elderly people: a randomized controlled trial. Arch. Intern. Med. 171, 525-533. doi: 10.1001/archinternmed.2010.446

Trugeon, M., Wing, A. M., and Taylor, L. W. (2011). Timing and aging: slowing of fastest regular tapping rate with preserved timing error detection and correction. Psychol. Aging 26, 150-161. doi: 10.1037/a0020606

Tucker, M. G., Kavanagh, J. J., Barrett, R. S., and Morrison, S. (2008). Age-related differences in postural reaction time and coordination during voluntary sway movements. Hum. Mov. Sci. 27, 728-737. doi: 10.1016/j.humov.2008.03.002

Vaillancourt, D. E., and Newell, K. M. (2002). Changing complexity in human behavior and physiology through aging and disease. Neurobiol. Aging 23, 1-11. doi: 10.1016/S0197-4580(01)00247-0

van der Wardt, V., Hood, L. P., Booth, V., Masud, T., and Harwood, R. (2015). The association of specific executive functions and fall risk in people with mild cognitive impairment and early-stage dementia. Dement Geriatr. Cogn. Disord. 40, 178-185. doi: $10.1159 / 000433523$

Vanneste, S., Pouthas, V., and Wearden, J. H. (2001). Temporal control of rhythmic performance: a comparison between young and old adults. Exp. Aging Res. 27, 83-102. doi: 10.1080/03610730125798

Voelcker-Rehage, C. (2008). Motor-skill learning in older adults: a review of studies on age-related differences. Eur. Rev. Aging Phys. Act. 5, 5-16. doi: 10.1007/ s11556-008-0030-9

Voelcker-Rehage, C., Godde, B., and Staudinger, U. M. (2011). Cardiovascular and coordination training differentially improve cognitive performance and neural processing in older adults. Front. Hum. Neurosci. 5:26. doi: 10.3389/fnhum. 2011.00026

Witt, S. T., Laird, A. R., and Meyerand, M. E. (2008). Functional neuroimaging correlates of finger-tapping task variations: an ALE meta-analysis. Neuroimage 42, 343-356. doi: 10.1016/j.neuroimage.2008.04.025

Yi, H., Chin, J., Lee, B. H., Kang, Y., and Na, D. L. (2007). Development and validation of Korean version of trail making test for elderly persons. Dement Neurocogn. Disord. 6, 54-66.

Conflict of Interest Statement: The authors declare that the research was conducted in the absence of any commercial or financial relationships that could be construed as a potential conflict of interest.

Copyright (C) $2017 \mathrm{Kim}$, Cho and Yoo. This is an open-access article distributed under the terms of the Creative Commons Attribution License (CC BY). The use, distribution or reproduction in other forums is permitted, provided the original author(s) or licensor are credited and that the original publication in this journal is cited, in accordance with accepted academic practice. No use, distribution or reproduction is permitted which does not comply with these terms. 


\title{
The Common Element Effect of Abstract-to-Abstract Mapping in Language Processing
}

\author{
Xuqian Chen ${ }^{1 *}$, Guixiang Wang ${ }^{2}$ and Yuchan Liang ${ }^{1}$ \\ ${ }^{1}$ Center for the Study of Applied Psychology, Guangdong Key Laboratory of Mental Health and Cognitive Science, School of \\ Psychology, South China Normal University, Guangzhou, China, ${ }^{2}$ Division of Student Affairs, Psychological Health Center, \\ Guangzhou Academy of Fine Arts, Guangzhou, China
}

Since the 1990s, there has been much discussion about how concepts are learned and processed. Many researchers believe that the experienced bodily states (i.e., embodied experiences) should be an important factor that affects concepts' learning and use, and metaphorical mappings between abstract concepts, such as TIME and POWER, and concrete concepts, such as SPATIAL ORIENTATION, STRUCTURED EXPERIENCEs, etc., suggest the abstract-concrete concepts' connections. In most of the recent literature, we can find common elements (e.g., concrete concepts) shared by different abstract-concrete metaphorical expressions. Therefore, we assumed that mappings might also be found between two abstract concepts that share common

OPEN ACCESS

Edited by:

Anna M. Borghi,

University of Bologna, Italy

Reviewed by:

Claudio Mulatti,

University of Padua, Italy

Katrin Sakreida,

RWTH Aachen University, Germany

*Correspondence: Xuqian Chen

chenxuqian@m.scnu.edu.cn

Specialty section:

This article was submitted to

Cognition,

a section of the journal

Frontiers in Psychology

Received: 22 April 2016 Accepted: 04 October 2016 Published: 24 October 2016

Citation:

Chen X, Wang G and Liang Y (2016)

The Common Element Effect of Abstract-to-Abstract Mapping

in Language Processing.

Front. Psychol. 7:1623.

doi: 10.3389/fpsyg.2016.01623 elements, though they have no symbolic connections. In the present study, two lexical decision tasks were arranged and the priming effect between TIME and ABSTRACT ACTIONs was used as an index to test our hypothesis. Results showed a robust priming effect when a target verb and its prime belonged to the same duration type (TIME consistent condition). These findings suggest that mapping between concepts was affected by common elements. We propose a dynamic model in which mappings between concepts are influenced by common elements, including symbolic or embodied information. What kind of elements (linguistic or embodied) can be used would depend on how difficult it is for a concept to be learned or accessed.

Keywords: common elements, abstract action, time, length, mapping

\section{INTRODUCTION}

What is LOVE? We cannot see it or touch it, but can only feel it or experience it. "An individual's concept of LOVE depends on both his or her own experiences plus the metaphorical concepts for LOVE provided by the culture" (Lakoff and Johnson, 1980, p. 206). Then what kind of information can people get from their experience? Using the concept LOVE as an example, we never really experience what love (i.e., abstract concept) is, but learn what we will do (i.e., concrete concepts and the relevant related experiences, such as KISS, ARGUE, FIGHT, etc.), when we are in LOVE. By doing so, we build up the concept LOVE. As a consequence, researchers are interested in how people think about things they have never experienced, and the notion of embodiment has been offered as a possible solution to this problem. The main idea underlying embodiment is that higher cognitive processes rely heavily on the brain's modality-specific systems and on actual bodily states (Niedenthal et al., 2005). According to the strong embodied approach, researchers believe that 
cognitive processes such as concept learning and concept use involve partial reactivations of the sensory-motor states (i.e., simulation) that occur during experience with the world (Glenberg, 1997; Niedenthal et al., 2009).

In most of the recent literatures, the view of embodiment was discussed based on the idea of conceptual metaphors (Santiago et al., 2011). The embodiment view of abstract concepts has its origins in conceptual metaphor theory, which emerged within cognitive linguistics with a stronger focus on representation than on processing (Lakoff and Johnson, 1980, 1999; Johnson, 1987; Gibbs and O'Brien, 1990). For example, according to what was in our conceptual schema built by experience, we have some metaphorical sayings for LOVE, such as LOVE IS A JOURNEY or LOVE IS WAR. When people use the "A is B" metaphor, A is considered as the target domain while $\mathrm{B}$ is the source domain (Lakoff, 1993). The source domain (e.g., JOURNEY) lays the foundation for the concept, which in its turn forms mappings, or conceptual metaphors. The conceptual metaphor will further provide a whole number of linguistic expressions, or as we might call them linguistic metaphors, that finally deliver the idea to the target domain (e.g., LOVE). In other words, "the human conceptual apparatus may look like the Empire State Building: a rock-solid structure where upper (more abstract) floors are supported by lower (more concrete) floors" (Santiago et al., 2011, p. 43).

However, it is still not clear whether embodied experience was involved in mappings between these abstract and concrete concepts. Some researchers have argued that sensorimotor system activation or experiences during language processing are not as necessary as those mentioned by the strong embodied approach for comprehension (for reviews, see Sakreida et al., 2013). Using fMRI as an index, Sakreida et al. (2013) claimed that concrete word processing relies more on the sensorimotor system, whereas abstract word processing relies more on the linguistic system, though the sensorimotor neural network was found to be engaged in both concrete and abstract language contents in their study. These processing differences between concrete and abstract concepts can be also captured when the processing of phrases is discussed (Scorolli et al., 2012). According to the strong embodied approach and its opposite view, we believe that there might be a possible combination: Reactivations of the sensory-motor states, or so-called sensorymotor states in Sakreida et al.s' (2013) study, are indirectly involved in abstract concepts' learning and use, while the concrete concepts act as mediators.

By analyzing discussions about the metaphorical expressions, we found that metaphorical concepts were always abstract concepts (i.e., difficult to be experienced), whereas nonmetaphorical ones were more concrete (i.e., easy to be experienced). In the conceptual metaphor theory (Lakoff and Johnson, 1980), non-metaphorical concepts include at least (1) spatial orientations (e.g., UP-DOWN, IN-OUT, NEARFAR, and FRONT-BACK), (2) ontological concepts arising in physical experience (e.g., ENTITY, SUBSTANCE, CONTAINER, and PERSON), and (3) structured experiences and activities (e.g., EATING, MOVING, TRANSFERRING OBJECTS FROM PLACE TO PLACE). Evidence in support of this theory came from research on spatial metaphors, such as the POWER and BIG-SMALL metaphor (Freedman, 1979; Lakoff and Johnson, 1980, 1999; Schubert et al., 2009; Yap et al., 2013), POWER and UP-DOWN metaphor (Schubert, 2005; Meier et al., 2007; Zanolie et al., 2012), TIME and UP-DOWN/LEFT-RIGHT metaphor (Boroditsky, 2000; Fuhrman and Boroditsky, 2007; Santiago et al., 2007; Liu and Zhang, 2009a,b; Ouellet et al., 2009), TIME and LONG-SHORT metaphor (Casasanto and Boroditsky, 2008; Zäch and Brugger, 2008; Chen and Zhang, 2011), and EMOTION and UP-DOWN metaphor (Stefanucci and Storbeck, 2009; Lü and Lu, 2013). Evidence also came from metaphors of structured experiences and activities, such as the SOCIAL EMOTION and WARM-COLD metaphor (Williams and Bargh, 2008; IJzerman and Semin, 2010; Bargh and Shalev, 2012; IJzerman et al., 2012) and EMOTION and FACIAL EXPRESSION metaphor (Strack et al., 1988).

As a consequence, learning an abstract concept might be directly facilitated by a metaphorical expression, in which there is embodied mapping between bodily states and the concrete concepts (i.e., "embodied schema" in short in the current study). For example, when people learn the abstract concept RELATIONSHIP and its metaphorical expression such as "We are CLOSE friends," all embodied information about NEARFAR states could be activated and then affect the concept RELATIONSHIP learning. If this argument were true, the abstract-concrete mapping can be found in discussions in a more general way. Here, we further reflect on whether there is also mapping between two abstract concepts that share the same experience information according to their embodied schema, which was built by the relative concrete concepts and their sensorimotor system.

In language processing, especially based on the amodal symbols view (Burgess and Lund, 1997; Landauer and Dumais, 1997), robust priming effects have been widely found in conditions in which primes and targets share the same semantic or other common elements (Plaut, 1995; Bi et al., 1998; Sun and Wang, 2012; Chen et al., 2016). One of the typical examples is the DOCTOR-NURSE word pair, in which both words belong to the same semantic network in our mental lexicon. However, there is still a lack of research on whether similar priming effects can be found between primes and targets sharing common embodied information (e.g., similar experience). It is not surprising that some concepts, such as UP, can be used as the source domain in different metaphorical/embodiment expressions: MORE is UP, CONTROL is UP, GOOD is UP, RATIONAL is UP (Lakoff and Johnson, 1980). Therefore, there is common embodied information among different target domains (e.g., MORE, CONTROL, GOOD, and RATIONAL). By contrast, the same target domain can correspond to different source domains. For example, TIME, as an abstract concept, has several spatial embodied mappings, such as UP-DOWN, LEFT-RIGHT, FRONT-BACK, and LONG-SHORT (Casasanto and Boroditsky, 2008; Zäch and Brugger, 2008; Chen and Zhang, 2011), and some other valuable entailments (Lakoff and Johnson, 1980). There comes the possibility: If the same embodied schema is shared by two abstract concepts in our long-term memory, the 
schema might act as the common element and affect our concept processing.

To investigate this possibility, we chose two concepts-TIME and ABSTRACT ACTIONs - that they do not have directly symbolic connections, and more importantly, that both match the definition proposed by Lakoff and Johnson (1980): These two concepts are understood and structured not merely on their own terms, but also in terms of other concepts. Firstly, the perception of TIME is part of human experience. Based on the experience of the passage of time and anticipated duration, we make everyday decisions, as simple as either waiting for the elevator or taking the stairs (Wittmann and Paulus, 2008). However, "Time is a sort of river of passing events, and strong is its current" (Marcus Aurelius [translated version] Meditations, IV, 43, See Liverence and Scholl, 2012, p. 549). The very expression "the perception of time" invites objection because time is something different from events. When we have "the perception of time," we indeed perceive embodied experiences such as SPATIAL DISTANCEs and other relations between objects (Le Poidevin, 2011). Therefore, we might take stairs to the second floor but wait for the elevator to the 20th. Distances (LONG/SHORT) are different between these two conditions. Secondly, compared with more concrete actions like KISS and STEP that can be seen and understood directly, JUDGE and THINK are more abstract in that they express mental processes, with no reference to a physical object (Scorolli et al., 2012). These actions might have no direct metaphorical mappings, but can be experienced as LONG or SHORT. These experiences are very important for ABSTRACT ACTIONs (Borghi and Cimatti, 2009, 2012), and they are always reflected in our daily expressions, in a somewhat complicated fashion.

For example, in the above elevator/stairs experiences of TIME, distances from the starting point to the goal for specific durations can be easily measured, but it is difficult to find a physically corresponding length for ABSTRACT ACTIONs. Then what could be the common element between TIME and ABSTRACT ACTIONs? In our daily expressions, connections between TIME and LENGTH (an hour is longer than a minute), and between ABSTRACT ACTION and LENGTH (it took me a long time to finish my homework) can be found. Therefore, no matter whether it was an experience of an hour or of fulfilling an action, these can be seen as events with starting and ending points, and the time (duration) for accomplishing these events can be perceived. As a consequence, LENGTH, specifically representing the experiences from the starting point to the ending point of the given event, can be considered as the common element shared by ABSTRACT ACTIONs and TIME. Therefore, concepts involving ABSTRACT ACTIONs (as indicated by the ratings made by participants in the present study) and TIME are suitable for the present research.

Based on the relationships between TIME and LENGTH (i.e., involving metaphorical expressions), and ABSTRACT ACTIONs and LENGTH (i.e., involving no metaphorical expressions but only experiences), it is assumed that there might be an "abstract-to-abstract" mapping with experience as the common element (i.e., “ABSTRACT ACTION-LENGTH-TIME”) during verb processing. In the present study, experience of TIME was manipulated by LENGTH-changing events (i.e., length-changing lines or duration-changing beeps). If this abstract-to-abstract mapping can be quickly activated under the priming task, verb processing should be facilitated by the TIME consistent condition. In other words, the goal of this research was to test whether mappings between two abstract concepts could be connected by their common concrete concept in word processing.

This hypothesis was tested in two experiments. In Experiment 1, abstract words with different DURATION mappings were primed by two kinds of length-changing lines, so that the metaphorical priming effect could be detected under a visual condition. For reasons of experimental rigor, Experiment 2 was arranged to be identical to Experiment 1 except the primes used in Experiment 1 were changed in order to offset any tau effect. The tau effect is a spatial perceptual illusion that reveals that stimulus timing affects the perception of stimulus spacing: The greater the difference between the temporal intervals, the greater must be the spatial difference in the opposite sense in order to offset the tau effect (Helson and King, 1931). There are several explanations for this effect, and one of them is the constant velocity hypothesis (Jones and Huang, 1982), according to which the brain expects spatial intervals that would yield movement with constant velocity. Though we did not use temporal visual intervals, continuous lines might also cause this expectation. Because this so-called tau effect might be involved in Experiment 1, it is not quite clear whether the priming effect, if any, would be caused by the "ABSTRACT ACTION-TIME" mapping or by the "ABSTRACT ACTION-LENGTH (perception of LENGTH caused by the tau effect)" mapping. Thus, in Experiment 2, pure sound beeps with different durations were presented as the primes instead of the length-changing line, so that any influence of the tau effect could be reduced. The common property shared by two kinds of prime in the present study (a line in Experiment 1, a beep in Experiment 2) was that participants could perceive experience from the starting point to the ending point of the prime's presentation.

\section{EXPERIMENT 1}

\section{Method \\ Participants}

Thirty-five right-handed Chinese native speakers took part in the present study $\left(M_{\text {age }}=21.25, S D=2.07 ; 12\right.$ male $)$. Participants gave informed consent before taking part in the experiment.

\section{Materials}

Eighty-five words (49 referring to long-time verbs, and 36 referring to short-time verbs) were selected from the Dictionary of Modern Chinese Language (Institute of Language, 2008), and 60 students who did not take part in the main study completed the following rating questionnaires (20 students for each). Firstly, abstractive degree (abstractness) of candidates was rated on a 7 -point scale $(1=$ concrete action, $7=$ abstract verb). In addition, most of the Chinese words were multi-category words such that verbs and nouns were sometimes difficult to identify. Therefore, all candidate words were also rated according to their 
typical category on a 7-point scale (category rating, $1=$ absolute noun, $7=$ absolute verb), and only words whose means of the abstractive ratings and category ratings were higher than 5 were chosen for use in the experiment. All these chosen candidates were then rated according to their typical perception of duration on a 7 -point scale $(1=$ such an action can be done in a very short time, $7=$ it takes a long time to finish such an action), and verbs with ratings under 2.5 represented short-time verbs whereas verbs with ratings over 5.5 represented long-time verbs. Finally, 46 target words, 23 referring to long-time abstract verbs (occur over a longer period of time, e.g., 缠绵 - lingering, 纠缠 - tangle, etc.) and 23 short-time abstract verbs (occur over a shorter period of time, e.g., 击中 - hit, 崩溃 - breakdown, etc.) were selected for the present study. The two metaphorical durations (long vs. short) of the final selected words did not differ with respect to their familiarity, $t(44)=0.29, p>0.05$, or their category ratings, $t(44)=0.27, p>0.05$, but did differ significantly regarding ratings of their length, $t(44)=39.22, p<0.001$. Moreover, another 46 pseudo words were created as the filler probes so that the number of Yes and No answers could be balanced (details are in Table 1).

In addition, two length-changing lines displayed as primes were created by Macromedia Flash MX 2004. One line was differentiated from the other by its duration. Duration from the starting point to completion of the final line (P-L duration) was $2000 \mathrm{~ms}$ for the long-time prime (10 visual-pictures per second), whereas the P-L duration for the short-time prime was $500 \mathrm{~ms}$ (40 visual-pictures per second). Therefore, both of these lines were visually of the same length when in the final position.

\section{Apparatus and Procedure}

A priming lexical decision task without feedback was used. The experimental software E-Prime presented the stimuli and recorded reaction times. Control files were constructed to display stimuli on a 17-inch IBM (9512-AB1) monitor (screen resolution: 1024 pixels $\times 768$ pixels). Participants were tested individually in a sound-proof room. All priming lines were started from the left ( $X: 25 \% Y: 50 \%)$ of the screen to the right ( $X: 75 \% Y: 50 \%)$. Targets were presented in the center of the monitor. Each trial started with the presentation of a fixation cross for $500 \mathrm{~ms}$. The fixation cross was replaced by a length-changing line (500 or $2000 \mathrm{~ms}$ ), which was followed by the target display for maximum $2000 \mathrm{~ms}$. Participants were asked to judge as quickly and as accurately as possible whether

TABLE 1 | Item characteristics for the final selected targets.

\begin{tabular}{lccccc}
\hline Variables controlled & \multicolumn{2}{c}{ Long-time verbs } & & \multicolumn{2}{c}{ Short-time verbs } \\
\cline { 2 - 3 } \cline { 5 - 6 } & $\boldsymbol{M}$ & $\boldsymbol{S} \boldsymbol{D}$ & & $\boldsymbol{M}$ & $\boldsymbol{S} \boldsymbol{D}$ \\
\hline Abstractness & 5.50 & 0.33 & & 5.52 & 0.32 \\
Familiarity & 5.64 & 0.45 & & 5.68 & 0.47 \\
Category rating & 5.06 & 0.70 & & 5.18 & 0.63 \\
Typical perception of duration*** & 5.83 & 0.32 & & 2.03 & 0.33 \\
\hline
\end{tabular}

*** $p<0.001$. or not the target in each trial was a real word, and to press the corresponding button on a response box. The inter-trial interval was $500 \mathrm{~ms}$ (blank screen) and then the next trial started (see Figure 1). If the participant failed to respond in $2000 \mathrm{~ms}$, the trial was terminated and recorded as an error. Participants received 10 practice trials, for which feedback was provided.

\section{Results and Discussion}

Participants' mean latencies (based on untrimmed correct responses) and error rates are summarized in Table 2, and graphically presented in Figure 2 . Analyses of variance $(2 \times 2$ ANOVAs) were conducted with priming type (duration for the priming lines: $500 \mathrm{~ms}$ vs. $2000 \mathrm{~ms}$ ) and word type (different metaphorical time length for words) as within-subject factors and with latencies (ms) and error rates (\%) as the dependent variables. In addition, as evidence showed that responses to real words and non-words might reflect different mechanisms during word accessing (Yang et al., 2006), latencies and error rates of non-words were submitted to separate $t$-tests.

Results of the analyses on latency showed a significant difference between long-time priming $(M=657 \mathrm{~ms})$ and shorttime priming $(M=615 \mathrm{~ms}), F_{1}(1,34)=28.88, p<0.01$, $\eta^{2}=0.46, F_{2}(1,22)=23.27, p<0.001, \eta^{2}=0.51$, and between long-time concepts $(M=629 \mathrm{~ms})$ and short-time concepts $(M=643 \mathrm{~ms}), F_{1}(1,34)=4.30, p<0.05, \eta^{2}=0.11$, $F_{2}(1,22)=2.27, p>0.10$. Importantly, the interaction between priming type and word type reached significance, $F_{1}(1,34)=16.53, p<0.01, \eta^{2}=0.33, F_{2}(1,22)=4.09, p=0.056$, $\eta^{2}=0.16$. A further simple effect test showed that when primed by the long-time line, long-time concepts produced significantly smaller latency scores than short-time concepts, $F_{1}(1,34)=14.09$, $p<0.01, \eta^{2}=0.29, F_{2}(1,22)=31.10, p<0.001, \eta^{2}=0.59$, whereas when primed by the short-time line, long-time concepts produced significantly larger latency scores than short-time concepts, $F_{1}(1,34)=9.08, p<0.01, \eta^{2}=0.21, F_{2}(1,22)<1$, ns.

Similar analyses were done on error rates and results showed a significant difference between long-time priming and short-time priming, $F_{1}(1,34)=5.56, p<0.05, \eta^{2}=0.14, F_{2}(1,22)=1.82$, $p>0.10$, but the main effect of word type was missing, $F_{1}(1,34)=1.74, p>0.05, F_{2}(1,22)<1$, ns. Importantly, the interaction between priming type and word type also reached significance, $F_{1}(1,34)=13.24, p<0.01, \eta^{2}=0.28$, $F_{2}(1,22)=5.71, p<0.05, \eta^{2}=0.21$. A further simple effect test showed that long-time concepts primed by the long-time line produced significantly higher error rates than short-time concepts primed by the long-time line, $F_{1}(1,34)=11.70, p<0.01$, $\eta^{2}=0.26, F_{2}(1,22)=5.96, p<0.05, \eta^{2}=0.21$; by contrast, longtime concepts primed by the short-time line produced somewhat lower error rates than short-time concepts primed by the shorttime line, $F_{1}(1,34)=3.50, p=0.07, \eta^{2}=0.09, F_{2}(1,22)=1.21$, $p>0.10$.

The interaction between priming type and word type suggests that experience of TIME might act as mediator in the mapping between ABSTRACT ACTIONS and LENGTH. On the one hand, such findings are consistent with the view that TIME 


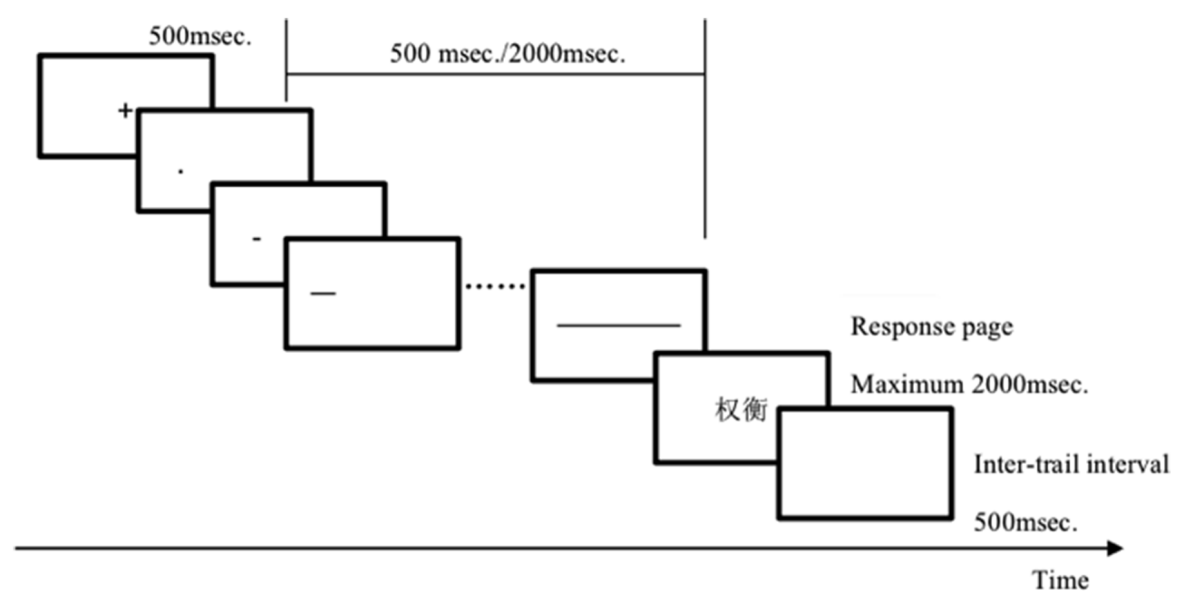

FIGURE 1 | Example trial procedure in Experiment 1. In Experiment 2, pure sound beeps with different durations were displayed instead of the length-changing line.

TABLE 2 | Means of latencies (SE) and error rates (SE) in Experiment 1.

\begin{tabular}{|c|c|c|c|c|c|c|}
\hline & \multicolumn{3}{|c|}{ Long-time priming } & \multicolumn{3}{|c|}{ Short-time priming } \\
\hline & Long-time concepts & Short-time concepts & Non-words & Long-time concepts & Short-time concepts & Non-words \\
\hline Latencies & $631(16)$ & $684(23)$ & $677(21)$ & $628(17)$ & $602(14)$ & $612(19)$ \\
\hline Error rates & $2.91(0.62)$ & $5.46(0.83)$ & $3.34(0.69)$ & $3.94(0.67)$ & $1.51(0.39)$ & $2.27(0.59)$ \\
\hline
\end{tabular}
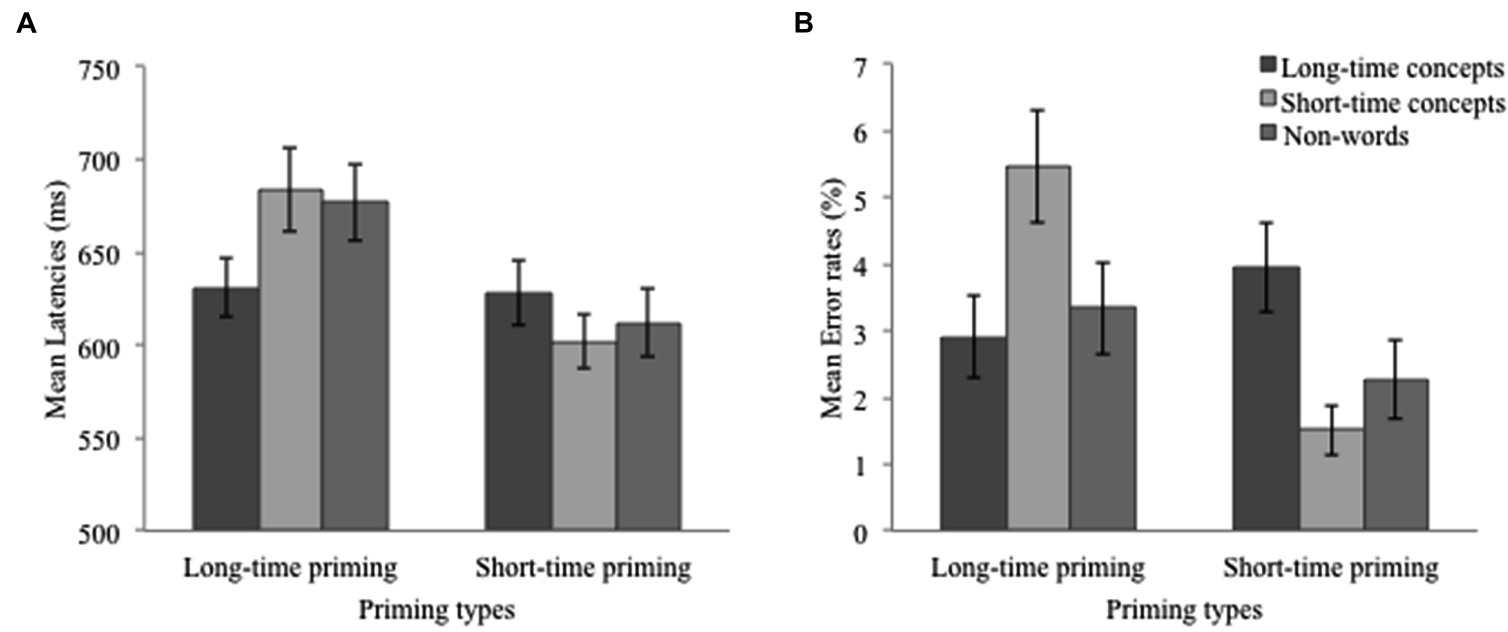

FIGURE 2 | (A) Mean latencies and mean standard errors in Experiment 1. (B) Mean error rates and mean standard errors in Experiment 1.

itself is not a property in the empirical world (Wittmann, 2009). When we talk about time (e.g., "an event lasted a long time," "time flew by"), we use linguistic structures that refer to motion events and to locations and measures in space (Evans, 2004, see Wittmann, 2009). On the other hand, though VERBs used in the present study were somewhat abstractive, they are still relevant to corresponding motion events that people experience as time pressure (e.g., it takes seconds/minutes/hours to finish some ACTIONs) during concept learning (or everyday life). Thus, it was assumed that both TIME and ABSTRACT VERBs can be mapped to the same experience (i.e., experience of duration from starting point to ending point of the length-changing line) and interact with each other in the priming paradigm. The present results were in line with this hypothesis.

In addition, long-term priming trials showed disadvantages, no mater whether the following stimulus was a real word or a non-word. We believed that these findings could be explained 
by processing of internal analog (Moyer, 1973), which will be discussed later again with data of Experiment 2.

\section{EXPERIMENT 2}

The hypothesis of the "ABSTRACT ACTION-LENGTH-TIME" mapping actually referred to an "abstract concept-to-abstract concept" mapping in the present research. However, though Experiment 1 showed that the LENGTH of TIME influences subsequent lexical decisions, the perception of time might become the perception of length of line under visual priming because of the tau effect. In what was probably the first experiment of this type, Benussi (1913, see Sarrazin et al., 2004) presented participants with three successive flashes of light defining two spatial and two temporal interstimulus intervals (ISIs). Distance judgments were found to vary as a function of the duration of the temporal ISIs (Sarrazin et al., 2004). Since then, many studies-involving the visual (Bill and Teft, 1969), auditory (Cohen et al., 1953), or kinesthetic (Helson and King, 1931; Lechelt and Bochert, 1977) modality-have shown that when two constant spatial ISIs are associated with variable temporal ISIs, distance is overestimated or underestimated in accordance with the temporal ISI. Though in Experiment 1 we did not have temporal ISIs, but continuous length-changing lines instead, expectation of movement with constant velocity from the starting point to the end of these lines still existed and there might be a similar tau effect involved in the results of Experiment 1. That is, participants might have perceived the changes of lines (concrete experience), thus affecting the following task. Therefore, instead of visual primes, beeps with different durations were used in Experiment 2 so that the abstractive property of priming could be more carefully manipulated.

\section{Method \\ Participants}

Another 36 right-handed Chinese native speakers, who did not participate in Experiment 1, took part $\left(M_{\text {age }}=21.65, S D=2.32\right.$; 13 male). Participants gave informed consent before taking part in the experiment.

\section{Materials}

Target words were identical to Experiment 1, but the visually length-changing lines were replaced by auditory pure sound ("beep"). A $500 \mathrm{~ms}$ beep was created by Ulead Video Studio 12 for short-time priming while a $2000 \mathrm{~ms}$ beep was used for long-time priming.

\section{Apparatus and Procedure}

Experimental tasks were identical to Experiment 1, except that control files were constructed to play auditory primes through headphones (IRIVER AIRUNKE AE1) to participants, who were tested individually in a sound-proof room.

\section{Results and Discussion}

Participants' mean latencies (based on untrimmed correct responses) and error rates are summarized in Table 3, and graphically presented in Figure 3. Analyses of variance $(2 \times 2$ ANOVAs) were conducted identical to those in Experiment 1.

Results of the analyses on latency showed a significant difference between long-time priming $(M=744 \mathrm{~ms})$ and shorttime priming $(M=673 \mathrm{~ms}), F_{1}(1,35)=46.10, p<0.01$, $\eta^{2}=0.56, F_{2}(1,22)=30.91, p<0.001, \eta^{2}=0.58$, and $a$ marginally significant, but unreliable $17-\mathrm{ms}$, difference between long-time concepts $(M=717 \mathrm{~ms})$ and short-time concepts $(M=700 \mathrm{~ms}), F_{1}(1,35)=3.81, p=0.06, \eta^{2}=0.10, F_{2}(1,22)<1$, ns. Again, the interaction between priming type and word type reached significance, $F_{1}(1,35)=10.17, p<0.01, \eta^{2}=0.23$, $F_{2}(1,22)=7.79, p<0.05, \eta^{2}=0.26$. A further simple effect test showed that long-time concepts primed by the long-time beep produced significantly smaller latency scores than shorttime concepts primed by the long-time beep, $F_{1}(1,35)=24.78$, $p<0.01, \eta^{2}=0.42, F_{2}(1,22)=5.18, p<0.05, \eta^{2}=0.19$, whereas a difference between long-time and short-time concepts primed by the short-time beep was not found, $F_{1}(1,35)=0.30, p>0.05$, $F_{2}(1,22)=4.67, p<0.05, \eta^{2}=1.75$.

Results of error rates showed a significant difference between long-time priming and short-time priming, $F_{1}(1,35)=4.78$, $p<0.05, \eta^{2}=0.12, F_{2}(1,22)=4.52, p<0.05, \eta^{2}=0.17$, but the main effect of word type was again missing, $F_{1}(1,35)=0.01$, $p>0.10, F_{2}(1,22)=2.54, p>0.10$. Importantly, the interaction between priming type and word type reached significance, $F_{1}(1,35)=16.28, p<0.01, \eta^{2}=0.32, F_{2}(1,22)=14.45$, $p<0.01, \eta^{2}=0.40$. A further simple effect test showed that when primed by the long-time BEEP, long-time concepts produced significantly lower error rates than short-time concepts, $F_{1}(1,35)=9.51, p<0.01, \eta^{2}=0.21, F_{2}(1,22)=14.82, p<0.01$, $\eta^{2}=0.40$; however, when primed by the short-time BEEP, long-time concepts produced higher error rates than short-time concepts, $F_{1}(1,35)=7.35, p<0.05, \eta^{2}=0.17, F_{2}(1,22)=3.61$, $p=0.07, \eta^{2}=0.14$.

Under the auditory priming, the interaction between priming type and word type (i.e., difference between RTs of long-time and short-time words under the short-time priming condition) was a bit weakened but still apparent. These findings suggest that the priming effect was still a stable event without the concrete experience (e.g., expectation of movement of constant velocity in Experiment 1). According to the results of Experiments 1 and 2, the hypothesis of the "ABSTRACT ACTION-LENGTH-TIME" mapping was partly proved.

Moreover, similar to the pattern that was found in Experiment 1, long-term priming trials showed disadvantages on both real and non-words. These findings indicated that priming lines (Experiment 1) and beeps (Experiment 2) were actually processed in the current task, and they then affected the following lexical decision task. In one of Moyer's (1973) studies, a size comparison task was designed to manipulate various size differences. Results showed that smaller size differences between target pairs lengthened RTs and increased error rates relative to larger size differences. Moyer believed that participants built "internal analogs" of the given stimuli and did "internal psychophysical (differences) judgment" in the task. The smaller the size differences were, the more difficult the judgment to be made. 
TABLE 3 | Means of latencies (SE) and error rates (SE) in Experiment 2.

\begin{tabular}{|c|c|c|c|c|c|c|}
\hline & \multicolumn{3}{|c|}{ Long-time priming } & \multicolumn{3}{|c|}{ Short-time priming } \\
\hline & Long-time concepts & Short-time concepts & Non-words & Long-time concepts & Short-time concepts & Non-words \\
\hline Latencies & 723 (29) & 765 (29) & $733(28)$ & $677(21)$ & $669(24)$ & $660(20)$ \\
\hline Error rates & $2.54(0.54)$ & $8.21(1.83)$ & $4.35(0.87)$ & $4.59(1.17)$ & $2.05(0.45)$ & $3.27(0.71)$ \\
\hline
\end{tabular}

A

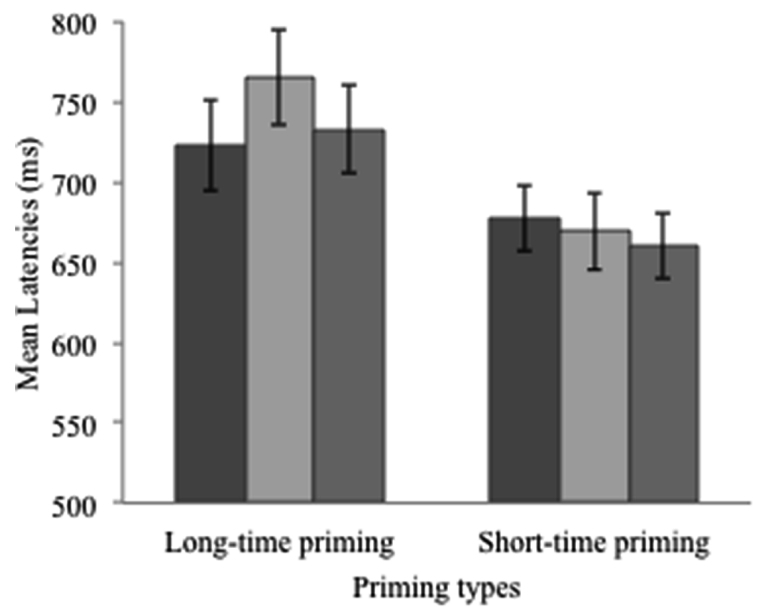

B

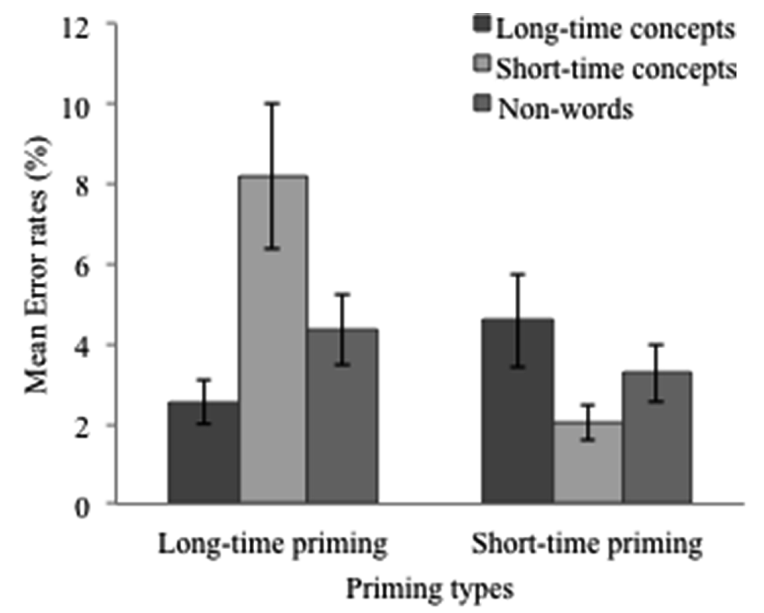

FIGURE 3 | (A) Mean latencies and mean standard errors in Experiment 2. (B) Mean error rates and mean standard errors in Experiment 2.

Similarly, we believed that "internal analogs" of the given lines and beeps were also built during the present priming stage. However, different from research by Moyer (1973), processing of internal analogs in the present study was based on the "size (i.e., length)" of the given stimuli, rather than "size differences" of the stimulus pairs. According to opinion expressed in the literature (Kosslyn et al., 1978, 1979; Kosslyn, 1981), it took a longer time to process larger/longer internal analogs than shorter ones. Therefore, disadvantages were found with long-term priming trials, but not short-term priming trials.

In addition, although responses to real words and nonwords should not be statistically compared (Yang et al., 2006), it was quite clear in both experiments that responses to non-words were slower than those to real words under the consistent condition but quicker than those to real words under the inconsistent condition. These results could be considered as further support for the hypothesis of the "ABSTRACT ACTION-LENGTH-TIME” mapping. That is, participants built the "length" mapping for the words (i.e., ABSTRACT ACTION) while they were building the "length" mapping for the primes (i.e., TIME). In the after-priming stage, processing should begin with accessing the word meaning and end up with the "length" consistency processing (between primes and words). Thus, there might be three kinds of effects in the present study: facilitation (e.g., under the length consistent condition), conflict (e.g., under the length inconsistent condition), or no interaction (e.g., under the non-word condition). Neither facilitation nor confliction could exist between primes and non-words because there were no "length" mappings for these words.

Therefore, the present results might be caused by building different "internal analogs" of the given primes (i.e., significant differences between responses under long- and short-term priming conditions), and also by the different consistencies of "length" mappings between primes and words (i.e., differences among consistent, inconsistent, and non-word conditions). However, these explanations were not convincing enough until further evidence was provided. Thus, the interactions between priming type and word type for the real words will be the main focus in the section "General Discussion."

\section{GENERAL DISCUSSION}

Previous studies on embodied mappings have all focused on two concepts of which at least one was concrete, such as TIME and LENGTH (Casasanto and Boroditsky, 2008; Chen and Zhang, 2011), POWER and SIZE (Freedman, 1979; Lakoff and Johnson, 1980, 1999; Schubert et al., 2009; Yap et al., 2013), POWER and ORIENTATION (Schubert, 2005; Meier et al., 2007; Zanolie et al., 2012). These previous studies have focused on embodied mappings separately, but some so-called common elements (i.e., concrete concepts in more than one mapping) exist in some mappings (e.g., MORE is UP, CONTROL is UP, GOOD is UP, RATIONAL is UP, see Lakoff and Johnson, 1980). Therefore, it has been an open question whether there is mapping between two 
abstract concepts, when these concepts share common elements. This is the first study to test the possibility of this type of mapping. We found a robust priming effect when the primes and targets shared the same experience of length (TIME consistent condition), which was used as an index to test our hypothesis. Based on these findings, we propose a dynamic conceptual model in which mappings between concepts are influenced by common shared elements.

Firstly, our findings extend previous studies on embodiment theory. The most important extension is the idea that abstract concepts should be involved in our embodied schema, at least indirectly. To date, concrete concepts were considered the main part of embodied schema in the literature (Scorolli et al., 2012; Sakreida et al., 2013) and no mappings between two abstract concepts were discussed in relation to this schema. This is because researchers believed that "in general, abstract concepts are defined metaphorically in terms of concepts that are more concrete and more clearly structured on their own terms" (Lakoff and Johnson, 1980, p. 198). However, the embodied schema should be more complex. Findings from neuro-scientific studies on processing linguistic concreteness and abstractness showed that the sensorimotor neural network was engaged in processing abstract concepts but rather less so in processing concrete concepts (Scorolli et al., 2012; Sakreida et al., 2013). According to these findings, the embodied schema might be directly built by concrete concepts and their relative embodied simulations, and then the metaphorical connections between concrete and abstract concepts help build indirect connections between abstract concepts and the embodied simulations. In other words, if we think carefully about the relations among experience, language, and thoughts, we know we should build a more complex conceptual mapping: Those common concrete concepts and their relative embodied experiences help connect two abstract concepts, and the conceptual mappings should be built up not only with the connections between abstract and concrete concepts, but also with connections between abstract concepts.

Actually, this claim is also consistent with the SapirWhorf hypothesis, which pointed toward the possibility that grammatical differences reflect differences in the way that speakers of different languages perceive the world (for reviews, see Kay and Kempton, 1984). That is, the language we speak affects how we think about the world (Kay and Kempton, 1984; Hunt and Agnoli, 1991), and language is a powerful tool in shaping thought, especially habitual thought, about abstract domains (Boroditsky, 2001). Here, the notion of "thought" or "the way that we perceive the world" refers to the non-linguistic cognitive structures (Kay and Kempton, 1984), and, in the present study, precisely refers to the connections between concepts. Therefore, combining the embodiment theory (i.e., experience influences concrete concepts' learning and use) and the Sapir-Whorf hypothesis (i.e., language/daily expression builds connections between concepts), we can infer a pathway for building up the embodied schema between abstract concepts and experiences: Experience + language (or metaphorical mappings between concrete and abstract concepts) thoughts. Using the connection among the concepts of ACTION, TIME, and LENGTH as an example: If LONG-SHORT experiences of events shape expressions such as "an hour is longer than a minute" and "it took me a long time to finish my homework," then these expressions shape our thoughts, i.e., connections between concepts. The process of how people build up these expressions will be discussed again later. According to most of the recent literature, we believe these shaped thoughts (connections between concepts) finally affect the embodiment effects. Specifically in the present work, we argue that connections between two abstract concepts should exist in the conceptual mapping schema.

Next, we draw analogies between the amodal symbols view and embodiment theory according to the present findings, and we propose a dynamic system of word processing. When word processing was discussed in the literature under the amodal symbols view (Burgess and Lund, 1997; Landauer and Dumais, 1997), priming lexical decisions were always found to be affected by common elements between the prime and target in a symbolic system. These symbolic systems include a perceptual representation system (Tulving and Schacter, 1990; Marsolek et al., 1992), phonological system (Lukatela and Turvey, 1991, 1994; Lesch and Pollatsek, 1993; Harm and Seidenberg, 1999), orthographical system (Leck et al., 1995; Xu et al., 1999; Zhang and Weekes, 2009; Zhang et al., 2009; Zou et al., 2012; Chen et al., 2014), and semantic system (Plaut, 1995; Bi et al., 1998; Sun and Wang, 2012). When we learn a word, we also build up a complicated network for it. For example, when we learn the word SOFA, experience, such as seeing a picture of a sofa, or touching a real sofa, might help us to know what a sofa is. More importantly, we also learn SOFA is a kind of furniture and that it is usually put into a building, like chairs, tables, bed, etc., which can be used in our everyday life. Therefore, "a piece of furniture" and "we can use it every day" are common elements for sofa, chair, table, and bed. Some common elements are clear and direct (e.g., phonological and/or orthographical information such as the upper portion of the characters “架” and “驾”, "word" and "work"), while some are more abstractive (e.g., semantic information). All this symbolic information will be encoded in our long-term memory, and affect our word processing.

Though symbolic information discussed under the amodal symbols view was not focused on under the traditional paradigm for discussing embodiment, researchers were actually also looking for some common elements. However, previous research has focused on common elements between two concrete concepts, and between concrete and abstract concepts, and until now there was still a lack of research on common elements between two abstract concepts. According to this point of view, we chose two concepts, TIME and ABSTRACT ACTIONs, which are both abstractive and have similar properties in the present study: Both TIME and ABSTRACT ACTIONs cannot be seen or touched; "experience" of these two concepts must be based on some other concrete concepts; it is never clear how "long" a time it is if it is discussed without specific events. At the 
same time, though people can feel how long an action lasts, this action cannot be physically measured as long or short, either. Importantly, these two concepts have some common elements: They both have long-short expression, but this long or short property cannot be directly measured. Findings in both experiments indicated that priming from the perception of time did influence the following word processing. There was mapping between the perception of TIME and ABSTRACT ACTIONs used in the present study, although they were both abstract. As the experimental manipulation in the present study, information about LENGTH might be the common element between two abstract concepts.

However, how do TIME, ABSTRACT ACTIONs, and LENGTH connect to each other? Metaphor is first of all a phenomenon of pragmatics. For example, when we talk about spatial metaphors, we can find metaphorical expressions such as "we grow close to (or far apart from) people," or "one friend is at the top of the class" (e.g., Lakoff and Johnson, 1980). The experience of "close" and "top" lets us build spatial schemas for abstract concepts such as RELATIONSHIPs and REPUTATION (Boroditsky, 2000; Meier et al., 2007; Schubert et al., 2009). We can easily find a direct connection between TIME and LENGTH in our daily expressions (an hour is longer than a minute), whereas the connection between ABSTRACT ACTION and LENGTH is not so close (it took me a long time to finish my homework). It is not surprising that these expressions differ because "we perceive durations as being filled by particular events" (Liverence and Scholl, 2012). However, experience of ABSTRACT ACTION could be gathered and such abstract concepts could be learned during our lifetime (Chen and Zhu, 2014). In addition, it seems that ABSTRACT ACTIONs should be more closely connected to TIME than to LENGTH. Therefore, we assumed there would be direct mappings between ABSTRACT ACTIONs and TIME. Indeed, this is what we found. More importantly, by manipulating the displayed LENGTH of an event, we showed that TIME affects the accessing of ABSTRACT ACTIONs. During long-time language use, the indirectly embodied connection of ABSTRACT ACTION-TIME is built. This connection affects our word processing.

These elements might not be clear or direct, but they did build the connection between two symbolic "unrelated" concepts. These connections were a function of experience during concept learning. For example, the perception of time is part of human experience; it is essential for everyday behavior and for the survival of the individual organism (Pöppel, 1997; Buhusi and Meck, 2005; Wittmann, 2009). Then how can we "experience" that an hour is longer than a minute (LONG-SHORT metaphor for TIME; see Chen and Zhang, 2011)? At least we can walk a longer distance in an hour than in a minute. Through this experience, the concrete concept of distance becomes the connection between TIME and SPATIAL INFORMATION. Therefore, the key problem was the same under the present experimental tasks: whether there are common elements between two concepts stored in our long-term memory that can be used in accessing words. Therefore, the common element for TIME and ABSTRACT
ACTIONs was the connection to LENGTH, though the strength of connection between TIME and LENGTH, and between ABSTRACT ACTION and LENGTH, might not be the same.

We claim that the "common element" could be the key to combining discussion about word processing under the amodal symbols view and discussion about accessing concepts under embodiment theory. In other words, common elements, either coming from a symbolic system or an embodied system, can facilitate word processing. More importantly, people might flexibly choose whatever is important and when the chosen element should be used. This assumption is one of the plausible explanations for some inconsistent findings under embodiment theory. Some studies have found effects of abstract domain processing on concrete domain processing; based on these results, some researchers have claimed that concrete domains are automatically activated by the situation, and then structure the abstract domains and frame the understanding and reasoning about them (Teuscher et al., 2008; Weger and Pratt, 2008; Ouellet et al., 2010). In contrast, some researchers have argued that the relational structure is stored with the abstract domains, allowing the processing of the abstract domains without having to activate the concrete domains (Santiago et al., 2011). We believe that these different findings are due to what kind of element or information was chosen by participants in the given tasks.

For example, Chinese characters used in the present study hold explicit symbolic information, such as “抵抗resist", in which "f” always exists in characters that relate to actions by hands, such as “抵-against" and “抗-beat back." Thus, symbolic information provided by "f” could be strongly activated in some studies (Zhang et al., 2014a,b; Ma et al., 2015). By contrast, according to the present findings, using common elements was more effective than symbolic information under the present paradigm. Therefore, a dynamic conceptual model should be proposed: Our mental conceptual system was built up by several kinds of information, and we choose the most important or available information under the given task. Thus, the flexible accessing process should be the essence of the dynamic conceptual model.

In addition, we do not argue that mapping between abstract concepts could be as strong as that between an abstract concept and a concrete concept. Compared with the properties of priming in Experiment 2, the length-changing lines used in Experiment 1 were more concrete and their starting point and ending point were more easily captured. As a consequence, length-changing lines produced a larger difference between the consistent and inconsistent priming condition. We believe that besides perceptual information of TIME, length-changing lines might provide concrete information that is more easily activated or accessed, a conjecture that still needs more evidence to prove. Moreover, in the present dynamic system we propose, common embodied information might have less of an effect on concrete-concrete words' mapping. For example, there are several spatial mappings (e.g., UP-DOWN, LEFT-RIGHT, and LONG-SHORT) for TIME, but the experience of TIME, which 
can be seen as a common element among the above mentioned concrete concepts, should not facilitate spatial-spatial (e.g., "UPDOWN"-“LEFT-RIGHT”) concepts' mapping. That is, a concept must first be important to another given concept, and then it becomes the common element.

\section{CONCLUSION}

The more information received, including symbolic or embodied information, the more easily the concept can be accessed.

\section{REFERENCES}

Bargh, J. A., and Shalev, I. (2012). The substitutability of physical and social warmth in daily life. Emotion 12, 154-162. doi: 10.1037/a0023527

Benussi, V. (1913) Psychologie der Zeitaufassung. Heidelberg: Carl Winter's Universitatsbuchhandlung.

Bi, Y., Zhou, X., and Shu, H. (1998). Spread of activation between different lexical representation (in Chinese). Acta Psychol. Sin. 30, 262-268.

Bill, J. C., and Teft, L. W. (1969). Space-time relations: effects of time on perceived visual extent. J. Exp. Psychol. 81, 196-199. doi: 10.1037/h0027425

Borghi, A. M., and Cimatti, F. (2009). "Words as tools and the problem of abstract words meanings," in Proceedings of the 31st Annual Conference of the Cognitive Science Society, eds N. Taatgen and H. van Rijn (Amsterdam: Cognitive Science Society), 2304-2309.

Borghi, A. M., and Cimatti, F. (2012). Words are not just words: the social acquisition of abstract words. RIFL 5, 22-37. doi: 10.4396/20120303

Boroditsky, L. (2000). Metaphoric structuring: understanding time through spatial metaphors. Cognition 75, 1-28. doi: 10.1016/S0010-0277(99)00073-6

Boroditsky, L. (2001). Does language shape thought?: mandarin and english speakers' conceptions of time. Cogn. Psychol. 43, 1-22. doi: 10.1006/cogp.2001.0748

Buhusi, C. V., and Meck, W. H. (2005). What makes us tick? Functional and neural mechanisms of interval timing. Nat. Rev. Neurosci. 6, 755-765. doi: $10.1038 / \mathrm{nrn} 1764$

Burgess, C., and Lund, K. (1997). Representing abstract words and emotional connotation in a high-dimensional memory space. Paper presented at the Proceedings of the Cognitive Science Society, Hillsdale, NJ.

Casasanto, D., and Boroditsky, L. (2008). Time in the mind: using space to think about time. Cognition 106, 579-593. doi: 10.1016/j.cognition.2007.03.004

Chen, L., Zhong, L., Leng, Y., and Mo, L. (2014). The role of the character graphic information in different pinyin processing tasks (in Chinese). Acta Psychol. Sin. $46,1661-1670$.

Chen, X., Liu, B., and Lin, S. (2016). Is accessing of words affected by affective valence only? A discrete emotion view on the emotional congruency effect. Front. Psychol. 7:916. doi: 10.3389/fpsyg.2016.00916

Chen, X., and Zhang, J. (2011). Time metaphor and perceptual representation system: can the information of length be activated when processing the time classifiers? (in Chinese). Acta Psychol. Sin. 43, 863-877.

Chen, Y., and Zhu, L. (2014). Associations of body parts and early-learned mandarin verbs and their effect on AoA of these verbs (in Chinese). Acta Psychol. Sin. 46, 912-921. doi: 10.3724/SP.J.1041.2014.00912

Cohen, J., Hansel, C., and Sylvester, J. (1953). A new phenomenon in time judgement. Nature 172, 901. doi: 10.1038/172901a0

Evans, V. (2004). How we conceptualise time: language, meaning and temporal cognition. Essays Arts Sci. 33, 13-44.

Freedman, D. G. (1979). Human Sociobiology: A Holistic Approach. New York, NY: Free Press.

Fuhrman, O., and Boroditsky, L. (2007). "Mental time-lines follow writing direction," in Proceedings of the 29th Annual Conference of the Cognitive Science Society, eds D. S. McNamara and J. G. Trafton (Austin, TX: Cognitive Science Society)

\section{AUTHOR CONTRIBUTIONS}

XC: Design and drafting the article. GW: analyzed data and explain the results. YL: collection and analysis of data.

\section{ACKNOWLEDGMENT}

This project was supported by the National Natural Science Foundation of China (No. 31400964), and Guangdong Province philosophy social science and the "twelfth-five-year" plan (No. GD12YXL01).

Gibbs, R. W., and O'Brien, J. E. (1990). Idioms and mental imagery: the metaphorical motivation for idiomatic meaning. Cognition 36, 35-68. doi: 10.1016/0010-0277(90)90053-M

Glenberg, A. M. (1997). What memory is for: creating meaning in the service of action. Behav. Brain Sci. 20, 41-50. doi: 10.1017/S0140525X97470012

Harm, M. W., and Seidenberg, M. S. (1999). Phonology, reading acquisition, and dyslexia: insights from connectionist models. Psychol. Rev. 106, 491-528. doi: 10.1037/0033-295X.106.3.491

Helson, H., and King, S. M. (1931). The tau effect: an example of psychological relativity. J. Exp. Psychol. 14, 202. doi: 10.1037/h0071164

Hunt, E., and Agnoli, F. (1991). The whorfian hypothesis: a cognitive psychology perspective. Psychol. Rev. 98, 377-389. doi: 10.1016/j.cogpsych.2008.12.001

IJzerman, H., Gallucci, M., Pouw, W. T. J. L., Weißgerber, S. C., van Doesum, N. J., and Williams, K. D. (2012). Cold-blooded loneliness: social exclusion leads to lower skin temperatures. Acta Psychol. 140, 283-288. doi: 10.1016/j.actpsy.2012.05.002

IJzerman, H., and Semin, G. R. (2010). Temperature perceptions as a ground for social proximity. J. Exp. Soc. Psychol. 46, 867-873. doi: 10.1111/j.14679280.2009.02434.x

Institute of Language (ed.) (2008). Dictionary of Modern Chinese Language, 5th Edn. Beijing: The Commercial Press.

Johnson, M. (1987). The Body in the Mind: The Bodily Basis of Reason and Imagination. Chicago: University of Chicago Press.

Jones, B., and Huang, Y. (1982). Space-time dependencies in psychophysical judgment of extent and duration: algebraic models of the tau and kappa effects. Psychol. Bull. 91, 128-142. doi: 10.1037/0033-2909.91.1.128

Kay, P., and Kempton, W. (1984). What is the sapir-whorf hypothesis? Am. Anthropol. 86, 65-79. doi: 10.1525/aa.1984.86.1.02a00050

Kosslyn, S. M. (1981). The medium and the message in mental imagery: a theory. Psychol. Rev. 88, 46-66. doi: 10.1037/0033-295X.88.1.46

Kosslyn, S. M., Ball, T., and Reiser, B. (1978). Visual images preserve metric spatial information: evidence from studies of image scanning. J. Exp. Psychol. Hum. Percept. Perform. 4, 47-60.

Kosslyn, S. M., Pinker, S., Smith, G., and Shwartz, S. (1979). On the demystification of mental imagery. Behav. Brain Sci. 2, 535-581. doi: 10.1017/S0140525X00 064268

Lakoff, G. (1993). The contemporary theory of metaphor. Metaphor Thought 2, 202-251. doi: 10.1017/CBO9781139173865.013

Lakoff, G., and Johnson, M. (1980). The metaphorical structure of the human conceptual system. Cogn. Sci. 4, 195-208. doi: 10.1207/s15516709cog0402_4

Lakoff, G., and Johnson, M. (1999). Philosophy in the Flesh: The Embodied Mind and its Challenge to Western Thought. New York, NY: Basic books.

Landauer, T. K., and Dumais, S. T. (1997). Solution to Plato's problem: the latent semantic analysis theory of acquisition, induction and representation of knowledge. Psychol. Rev. 104, 211-240. doi: 10.1037/0033-295X. 104.2.211

Le Poidevin, R. (2011). “The experience and perception of time," in The Stanford Encyclopedia of Philosphy, ed. E. N. Zalta (Stanford, CA: Stanford University).

Lechelt, E. C., and Bochert, R. (1977). The interdependence of time and space in somesthesis: the tau effect reexamined. Bull. Psychon. Soc. 10, 191-193. doi: 10.3758/BF03329320 
Leck, K. J., Weekes, B. S., and Chen, M. J. (1995). Visual and phonological pathways to the lexicon: evidence from Chinese readers. Mem. Cogn. 23, 468-476. doi: 10.3758/BF03197248

Lesch, M. F., and Pollatsek, A. (1993). Automatic access of semantic information by phonological codes in visual word recognition. J. Exp. Psychol. Learn. Mem. Cogn. 19, 285-294.

Liu, L., and Zhang, J. (2009a). A study about the psychological mechanism of space-time metaphor (in Chinese). Psychol. Explor. 29, 32-36.

Liu, L., and Zhang, J. (2009b). The effects of spatial metaphoric representation of time on time cognition (in Chinese). Foreign Lang. Teach. Res. 41, 266-271.

Liverence, B. M., and Scholl, B. J. (2012). Discrete events as units of perceived time. J. Exp. Psychol. Hum. Percept. Perform. 38, 549. doi: 10.1037/a0027228

Lü, J., and Lu, Z. (2013). The vertical-spatial metaphor of emotions in text reading (in Chinese). J. Psychol. Sci. 36, 328-334.

Lukatela, G., and Turvey, M. T. (1991). Phonological access of the lexicon: evidence from associative priming with pseudohomophones. J. Exp. Psychol. Hum. Percept. Perform. 17, 166-178.

Lukatela, G., and Turvey, M. T. (1994). Visual lexical access is initially phonological: 1. Evidence from associative priming by words, homophones, and pseudohomophones. J. Exp. Psychol. Gen. 123, 107-128. doi: 10.1037/00963445.123.2.107

Ma, L., Qi, W., and Zhang, J. (2015). The effect of semantic radical, phonological radical and phonology on Chinese words production in picture-word interference paradigm (in Chinese). J. Dali Univ. 14, 79-84.

Marsolek, C. J., Kosslyn, S. M., and Squire, L. R. (1992). Form-specific visual priming in the right cerebral hemisphere. J. Exp. Psychol. Learn. Mem. Cogn. $18,492$.

Meier, B. P., Hauser, D. J., Robinson, M. D., Friesen, C. K., and Schjeldahl, K. (2007). What's "up" with God? Vertical space as a representation of the divine. J. Pers. Soc. Psychol. 93, 699. doi: 10.1037/0022-3514.93.5.699

Moyer, R. S. (1973). Comparing objects in memory: evidence suggesting an internal psychophysics. Percept. Psychophys. 13, 180-184. doi: 10.3758/BF032 14124

Niedenthal, P. M., Barsalou, L. W., Winkielman, P., Krauth-Gruber, S., and Ric, F. (2005). Embodiment in attitudes, social perception, and emotion. Pers. Soc. Psychol. Rev. 9, 184-211. doi: 10.1207/s15327957pspr0903_1

Niedenthal, P. M., Winkielman, P., Mondillon, L., and Vermeulen, N. (2009). Embodiment of emotion concepts. J. Pers. Soc. Psychol. 96, 1120. doi: $10.1037 / \mathrm{a} 0015574$

Ouellet, M., Santiago, J., Funes, M. J., and Lupiánez, J. (2010). Thinking about the future moves attention to the right. J. Exp. Psychol. Hum. Percept. Perform. 36, 17. doi: $10.1037 / \mathrm{a} 0017176$

Ouellet, M., Santiago, J., Sraeli, Z., and Gabay, S. (2009). "Multimodal influences of orthographic directionality on the "Time is Space" conceptual metaphor," in Proceedings of 31th Annual Conference of the Cognitive Science Society, eds N. Taatgen and H. van Rijn (Amsterdam: Cognitive Science Society, Inc.), 1840-1845.

Plaut, D. C. (1995). "Semantic and associative priming in a distributed attractor network," in Proceedings of the 17th Annual Conference of the Cognitive Science Society, (Hillsdale, NJ: Lawrence Erlbaum), 37-42.

Pöppel, E. (1997). A hierarchical model of temporal perception. Trends Cogn. Sci. 1, 56-61. doi: 10.1016/S1364-6613(97)01008-5

Sakreida, K., Scorolli, C., Menz, M. M., Heim, S., Borghi, A. M., and Binkofski, F. (2013). Are abstract action words embodied? An fMRI investigation at the interface between language and motor cognition. Front. Hum. Neurosci. 7:125. doi: 10.3389/fnhum.2013.00125

Santiago, J., Lupiáňez, J., Pérez, E., and Funes, J. M. (2007). Time (also) flies from left to right. Psychon. Bull. Rev. 14, 512-516. doi: 10.3758/BF03194099

Santiago, J., Román, A., and Ouellet, M. (2011). "Flexible foundations of abstract thought: a review and a theory," in Spatial Dimensions of Social Thought, eds A. Maass and T. W. Schubert (Berlin: Mouton de Gruyter), 41-110.

Sarrazin, J. C., Giraudo, M. D., Pailhous, J., and Bootsma, R. J. (2004). Dynamics of balancing space and time in memory: tau and kappa effects revisited. J. Exp. Psychol. Hum. Percept. Perform. 30, 411.

Schubert, T. W. (2005). Your highness: vertical positions as perceptual symbols of power. J. Pers. Soc. Psychol. 89, 1. doi: 10.1037/0022-3514.89.1.1

Schubert, T. W., Waldzus, S., and Giessner, S. R. (2009). Control over the association of power and size. Soc. Cogn. 27, 1-19. doi: 10.1521/soco.2009.27.1.1
Scorolli, C., Jacquet, P. O., Binkofski, F., Nicoletti, R., Tessari, A., and Borghi, A. M. (2012). Abstract and concrete phrases processing differentially modulates cortico-spinal excitability. Brain Res. 1488, 60-71. doi: 10.1016/j.brainres.2012.10.004

Stefanucci, J. K., and Storbeck, J. (2009). Don't look down: emotional arousal elevates height perception. J. Exp. Psychol. Gen. 138, 131. doi: 10.1037/a0014797

Strack, F., Martin, L. L., and Stepper, S. (1988). Inhibiting and facilitating conditions of the human smile: a nonobtrusive test of the facial feedback hypothesis. J. Pers. Soc. Psychol. 54, 768. doi: 10.1037/0022-3514.54.5.768

Sun, H., and Wang, Q. (2012). Interactions of blurredness, frequency, and semantic priming on N400 in Chinese characters: evidence for the IA model of N400 (in Chinese). Acta Psychol. Sin. 44, 745-753. doi: 10.3724/SP.J.1041.2012.00745

Teuscher, U., McQuire, M., Collins, J., and Coulson, S. (2008). Congruity effects in time and space: behavioral and ERP measures. Cogn. Sci. 32, 563-578. doi: 10.1080/03640210802035084

Tulving, E., and Schacter, D. L. (1990). Priming and human memory systems. Science 247, 301-306. doi: 10.1126/science.2296719

Weger, U. W., and Pratt, J. (2008). Time flies like an arrow: space-time compatibility effects suggest the use of a mental time-line. Psychon. Bull. Rev. 15, 426-430. doi: 10.3758/PBR.15.2.426

Williams, L. E., and Bargh, J. A. (2008). Experiencing physical warmth promotes interpersonal warmth. Science 322, 606-607. doi: 10.1126/science.1162548

Wittmann, M. (2009). The inner experience of time. Philos. Trans. R. Soc. B Biol. Sci. 364, 1955-1967. doi: 10.1098/rstb.2009.0003

Wittmann, M., and Paulus, M. P. (2008). Decision making, impulsivity and time perception. Trends Cogn. Sci. 12, 7-12. doi: 10.1016/j.tics.2007.10.004

Xu, Y., Pollatsek, A., and Potter, M. C. (1999). The activation of phonology during silent Chinese word reading. J. Exp. Psychol. Learn. Mem. Cogn. 25, 838-857.

Yang, L., Xiao, Z., Liu, X., Weng, X., and Zhang, X. (2006). Different roles of the left inferior prefrontal cortex in visual Chinese word recognition (in Chinese). Chin. J. Med. Imaging Technol. 22, 833-835.

Yap, A. J., Mason, M. F., and Ames, D. R. (2013). The powerful size others down: the link between power and estimates of others' size. J. Exp. Soc. Psychol. 49, 591-594. doi: 10.1016/j.jesp.2012.10.003

Zäch, P., and Brugger, P. (2008). Subjective time in near and far representational space. Cogn. Behav. Neurol. 21, 8-13. doi: 10.1097/WNN.0b013e31815f237c

Zanolie, K., Dantzig, S. V., Boot, I., Wijnen, J., Schubert, T. W., Giessner, S. R., et al. (2012). Power metaphors: behavioral and ERP evidence that power shifts attention on a vertical dimension. Brain Cogn. 78, 50-58. doi: 10.1016/j.bandc.2011.10.006

Zhang, J., Wang, J., and Chen, X. (2014a). Semantic radicals' research in twenty years: theoretical exploration, experimental evidence and processing model (in Chinese). Adv. Psychol. Sci. 22, 381-399. doi: 10.3724/SP.J.1042.2014. 00381

Zhang, J., Wang, J., and Yin, C. (2014b). The role of phonetic radicals and semantic radicals in phonetics and semantics extraction of phonogram characters: an eye movement study on components perception (in Chinese). Acta Psychol. Sin. 46, 885-900. doi: 10.3724/SP.J.1041.2014.00885

Zhang, Q., Chen, H. C., Weekes, B. S., and Yang, Y. (2009). Independent effects of orthographic and phonological facilitation on spoken word production in Mandarin. Lang. Speech 52, 113-126. doi: 10.1177/0023830908099885

Zhang, Q., and Weekes, B. S. (2009). Orthographic facilitation effects on spoken word production: evidence from Chinese. Lang. Cogn. Process. 24, 1082-1096. doi: 10.1080/01690960802042133

Zou, L., Desroches, A. S., Liu, Y., Xia, Z., and Shu, H. (2012). Orthographic facilitation in Chinese spoken word recognition: an ERP study. Brain Lang. 123, 164-173. doi: 10.1016/j.bandl.2012.09.006

Conflict of Interest Statement: The authors declare that the research was conducted in the absence of any commercial or financial relationships that could be construed as a potential conflict of interest.

Copyright (c) 2016 Chen, Wang and Liang. This is an open-access article distributed under the terms of the Creative Commons Attribution License (CC BY). The use, distribution or reproduction in other forums is permitted, provided the original author(s) or licensor are credited and that the original publication in this journal is cited, in accordance with accepted academic practice. No use, distribution or reproduction is permitted which does not comply with these terms. 


\title{
Is Accessing of Words Affected by Affective Valence Only? A Discrete Emotion View on the Emotional Congruency Effect
}

\author{
Xuqian Chen*, Bo Liu and Shouwen Lin \\ Key Laboratory of Mental Health and Cognitive Science of Guangdong Province, Center for Studies of Psychological \\ Application, School of Psychology, South China Normal University, Guangzhou, China
}

This paper advances the discussion on which emotion information affects word accessing. Emotion information, which is formed as a result of repeated experiences, is primary and necessary in learning and representing word meanings. Previous findings suggested that valence (i.e., positive or negative) denoted by words can be automatically activated and plays a role in many significant cognitive processes. However, there has been a lack of discussion about whether discrete emotion information (i.e., happiness, anger, sadness, and fear) is also involved in these processes. According to the

OPEN ACCESS

Edited by:

Annalisa Setti,

University College Cork and Trinity College Dublin, Ireland

Reviewed by:

Michela Sarlo,

University of Padova, Italy

Luisa Lugli,

University of Bologna, Italy

*Correspondence: Xuqian Chen

cxqpsychology@163.com

Specialty section: This article was submitted to

Cognition,

a section of the journal

Frontiers in Psychology

Received: 25 November 2015

Accepted: 02 June 2016

Published: 17 June 2016

Citation:

Chen X, Liu B and Lin S (2016) Is Accessing of Words Affected by

Affective Valence Only? A Discrete Emotion View on the Emotional

Congruency Effect.

Front. Psychol. 7:916.

doi: 10.3389/fpsyg.2016.00916 hierarchy model, emotions are considered organized within an abstract-to-concrete hierarchy, in which emotion prototypes are organized following affective valence. By controlling different congruencies of emotion relations (i.e., matches or mismatches between valences and prototypes of emotion), the present study showed both an evaluative congruency effect (Experiment 1) and a discrete emotional congruency effect (Experiment 2). These findings indicate that not only affective valences but also discrete emotions can be activated under the present priming lexical decision task. However, the present findings also suggest that discrete emotions might be activated at the later priming stage as compared to valences. The present work provides evidence that information about discrete emotion could be involved in word processing. This might be a result of subjects' embodied experiences.

Keywords: affective valence, discrete emotion, prototypical emotion, congruency effect, embodied cognition

\section{INTRODUCTION}

Relatively enduring beliefs and predispositions toward specific objects or persons are called attitudes (Scherer, 2005). Automatic attitude activation is considered to be a mediating mechanism that plays a role in many significant cognitive processes (Fazio, 2001). In a series of priming studies (Klauer, 1997; Wentura, 1999; Hermans et al., 2001; Herring et al., 2013), significant emotion priming effects were demonstrated using prime-target pairs for which the emotional relation was manipulated, and the influence of affective valence, one of the components of attitudes consisting mostly of differential valence (Scherer, 2005), was most discussed. The evaluation associated with a prime appears to be suppressed when the target's valence is incongruent with primes (Wentura, 1999). To date, researchers reasoned that presentation of an attitude object automatically activates from memory the valence that an individual associates with the object (Fazio, 2001). 
Using the concept of affective valence, emotional priming effects were always discussed in a bipolar fashion: positive and negative. Namely, in most studies, a positive or negative prime stimulus (word or picture) was followed by a positive or negative target stimulus. Results showed that the reaction time was significantly shorter when prime and target shared the same valence (i.e., congruent condition: positive-positive or negativenegative) as compared to trials on which prime and target were of opposite valence (i.e., incongruent condition: positive-negative or negative-positive). Therefore, presentation of "cockroach" as the prime, the attitude toward which is evaluated negatively by an individual, appears to facilitate the activation of the negative evaluation of the target (e.g., disgusting, see Fazio, 2001).

Moreover, Kousta et al. (2009) found an abstractness effect in which words referring to positive or negative emotions are processed faster than neutral words, and words that have more emotional valence trigger a stronger residual latency advantage compared to those that do not, when variables such as imageability (a construct derived from dual coding theory) and rated context availability are held constant (Kousta et al., 2011). According to these findings, Kousta et al. (2011) proposed an embodied abstract semantics hypothesis, in which experiential information is considered as one of the two major types of information during word learning. The other type is languagebased information (Vigliocco et al., 2009).

It seems that most of the above studies were arranged under the dimensional emotion framework. Beginning with Nowlis, most psychologists have described affect as a set of dimensions (see Russell, 1980 as review). In a valence-arousal circumplex model, these affective dimensions are considered to be interrelated in a highly systematic fashion. Interrelationships among affective concepts can be represented by a spatial model, in which valence (pleasantness or hedonic value) and arousal (bodily activation) are the two essential qualities (Russell, 1980; Izard, 1992; Reisenzein, 1994; Reisenzein and Spielhofer, 1994; Posner et al., 2005; Madrid and Patterson, 2014). However, the role of emotion information, one type of experiential information from our experience of our own inner states, is considered to be foundational (i.e., primary and necessary) in learning and representing meanings (Vigliocco et al., 2009). Words that denote emotional states, moods or feelings provide a crucial example of how a word may refer to an entity, even if it is not observable but resides within the organism, so that semantic representations can be developed. For example, a nurse is someone who offers help, and the word nurse should be more positive than words like thief, killer, or robber, etc. However, the nurse could be also a word denoting negative emotion, because a nurse offers injection and piles in our own experience. The fact that a nurse offers injection and piles could be learned as the crucial example of the word nurse. Then what kinds of embodied information about emotion can be acquired? In other words, is it appropriate to discuss emotional priming simply in a bipolar way? This is a question related to the issue of how people label, or make embodied statements about, their affective states.

Some researchers have claimed that this valence-arousal circumplex should be extended to a discrete framework, in which different numbers of basic/discrete emotion labels should be defined. That is, different from the modern dimensional theory (i.e., valence-arousal circumplex model), according to which feelings are described by the valence and arousal dimensional space, discrete emotion theory focuses on the original expressions describing clearly separable states (Scherer, 2005). According to this theory, most of the basic/discrete emotions play an important role in adapting to frequently occurring and prototypically patterned types of significant events in the life of organisms (Barrett, 1998; Scherer, 2005). For example, during emotional experience (i.e., "How do I feel?") and emotion perception (e.g., "Is my baby afraid?" "Is my mother angry?" "Is my cat sad?"), both of which are related to the emotion knowledge that has been learned via prior experience, representations of internal sensations from the body and external sensations from the world become meaningful (Barrett, 2006; Niedenthal, 2007). Experience has also been argued to play an important role in developing concepts (Glenberg, 1997; Barsalou, 1999; Murphy, 2002; Zwaan, 2003; Barsalou et al., 2008), and the embodied simulation account is considered to be viable for understanding the processing and storing of emotional knowledge (Niedenthal et al., 2009). Therefore, the words "happiness," "fear," "sadness," "anger," "surprise," and "interest" function as categorizing labels of emotion concepts that are related to the on-going activity that is realized in the brain (Barrett, 2006, 2009b; Barrett et al., 2009). Therefore, discrete labels, such as joy, anger, sadness and fear, were defined as prototypical emotions in the literature (Shaver et al., 1987; Scherer, 2005).

According to the above argument, one hypothesis can be made: all negatively evaluated emotions should not be the same, and the emotional priming effects should be more meticulously discussed. For example, both the feelings about "defeat" and "war" can activate the emotions of negative valence, but the former feeling should be sadness, whereas the latter one should be fear. As a consequence, it is plausible that both "sadness-war" and "fear-defeat" are emotionally congruent under the dimensional emotion view (positive-negative view), but incongruent under the discrete emotion view ("Sadness-defeat" and "fear-war" are emotionally congruent under both of the dimensional and the discrete emotion views.) Should the emotional priming effect be discussed under a different theoretical framework? We believed that the theoretical framework based only on the emotional valence dimension should be complemented with the more detailed analysis of discrete emotions when discussing emotional priming.

In the current study, emotional congruency effects were discussed under the discrete emotion view. That is, according to the discrete emotion view, though prime and target were both evaluated as words demoting negative emotions, they might be still considered to be incongruent emotions because they belong to different emotion categories. For example, in the current study one focus was anger and fear, which are both labels (or concepts) of negative valence. A key assumption was that when both are followed by a target (event word) denoting negative experience, there would be a significantly shorter reaction time when prime and target shared the same prototypical emotion (i.e., anger as a prime and a target word relating to experience of anger, or fear as a prime and a target word relating to experience of fear) as 
compared to trials on which prime and target are of different prototypical emotion (i.e., fear as a prime and target word relating to experience of anger, or anger as a prime and target word relating to experience of fear). However, this possibility has not yet been discussed. Therefore, the primary purpose of the present experiments was to provide evidence that besides emotional valence, discrete emotion also triggers congruent effects. To fulfill this goal, we arranged two priming lexical decision tasks (LDTs), in one of which (Experiment 1) emotional congruency was manipulated based on emotional valence, whereas in the other of which (Experiment 2) emotional congruency was manipulated based on prototypical emotions.

In addition, we also investigated whether it is the label or the experience/concept of discrete emotion that triggers the congruent effects. On the one hand, emotion labels serve to glue the various instances together into the single category of one emotion, even if they look very different from one another (Barrett, 2009b). In other words, emotion labels facilitate the learning of emotion categories. On the other hand, even trivial events (i.e., "seeing a cockroach") can trigger distinct emotional feelings (Philippot et al., 2003), and the names of these events (i.e., "cockroach") can then trigger the specific emotion (Fazio, 2001), because they might evoke memories of past experiences. However, discrete emotion information evoked by the name of events (i.e., "event words" in short) might not be as clear as those evoked by the label of the emotion. For investigating this statement, either the label of the emotion or event words were manipulated as the prime in the present study.

Though both valence and arousal are important qualities of affect (Barrett, 1998), dimensional labels and discrete labels are believed to be organized at different levels. Shaver et al. (1987) held that interrelated emotion categories are organized within an abstract-to-concrete hierarchy. Namely, the affective words are hierarchically organized first as reflecting their dimensional portrayal (i.e., positive and negative) and then as reflecting their discrete portrayal (e.g., prototypical emotions such as joy, anger, sadness and fear). Moreover, members of these prototypical emotions are stored at subordinate levels (e.g., amusement and pride are subordinate-level emotions of joy; Shaver et al., 1987).

Therefore, information from prototypical emotions might not be as strongly and quickly activated as affective valence. For testing this issue, stimulus onset asynchrony (SOAs; i.e., the interval between the onset of the prime and the onset of the target) was also manipulated in the present study. Researchers have paid great attention to evaluative priming effects under different SOAs, which is considered as a tool to provide insight to the time course of the activation processes underlying automatic affect/attitude activation. In a series of studies (Hermans et al., 1994, 2001; Hu, 2012), automatic evaluative processing has been evaluated under different SOAs (e.g., -150, 0, 50, 100, 150, 200, $300,400,1000 \mathrm{~ms}$, etc.). It has been proposed that the process of automatic stimulus valence occurs at a very early stage in information processing, and researchers believe that the basic process is fast, efficient, and outside awareness (Hermans et al., 2001).

Using the sandwich masks technique, $\mathrm{Hu}$ (2012) reported a converse emotional congruent effect under short SOAs (i.e., 50,
$100 \mathrm{~ms}$ ), and the significant priming effect was reported under $150 \mathrm{~ms}$. A comparison of short and long SOAs showed that affective priming effects were observed at an SOA of $300 \mathrm{~ms}$, but not at a longer SOA of 1000 ms (Hermans et al., 1994; De Houwer et al., 1998); the conclusion was that a SOA of $300 \mathrm{~ms}$ is long enough to activate the associated evaluation so that responding to evaluative congruent targets was facilitated while responding to incongruent targets was inhibited (Hermans et al., 2001). According to the recent literature, especially a report using materials in Chinese (Hu, 2012), SOAs of 50, 150, 200, and $400 \mathrm{~ms}$ were selected.

Before the main study, the most important thing was to select the appropriate prototypical emotions categories and their corresponding event words. Some theories state that the ways people divide the emotional world into discrete categories are different across cultures, and even vary across individuals, and over time (Shaver et al., 1987; Barrett, 2006, 2009a,b). However, at least some researchers have stated that everyone within a culture shares roughly the same representation of emotion concepts (see Barrett, 2006 as review). Therefore, we selected prototypical emotions categories and their corresponding event words based on the culture of Chinese so that the influence from culture can be discreetly avoided. In the culture of Chinese, four essential prototypes (i.e., happiness, anger, sadness, and fear) are mainly discussed (Zhang, 2008). Some prototypical emotions in western culture (e.g., "perhaps" is considered as an emotion mentioned in Shaver et al., 1987 research) are not very distinguished in Chinese. In addition, in different cultures, numbers of positive and negative prototypical emotions are not equal (see samples provided in Barrett, 2006), and apparently, in Chinese there are no positive discrete emotions at the same intensity as negative discrete emotions such as sadness, anger, and fear, with the exception of happiness. More importantly, the influence of affective valence should be reduced when discussing effects of prototypical emotions. Four essential prototypes, happiness, anger, sadness and fear, were selected as materials, but only negative prototypes were used in Experiment 2.

Though we claimed that choosing only negative discrete emotions in Experiment 2 was subject to the goal of the present study, and also to the culture of China, there are still risks for making just conclusions because people might argue that the discrete emotion framework could be incomplete without discussing positive situations. In the literature, there were two hypotheses regarding different effects from positive and negative words: according to a negative-privileged view, negative valence is associated with a general slowdown of the processing of stimuli; but more recently, valence, regardless of polarity, was argued to equally facilitate processing due to the relevance of both negative and positive stimuli (see Kousta et al., 2009 as review). In addition, even according to the privileged hypothesis, negatively valenced stimuli invoke stronger behavioral responses than positive stimuli, even when arousal is held constant (Kousta et al., 2009). Though it was assumed that there should be discrete emotional congruency effects in the present study, congruency effects triggered by discrete emotional words might be not as significant as those triggered by evaluative emotional words. Selecting the more strongly activated emotional words (i.e., 
negative discrete words) should be appropriate for the present investigation.

Therefore, under the manipulations in the present study, the main hypotheses are as follow: (1) emotional congruency effects will be found in both valence priming (Experiment 1) and discrete emotional (Experiment 2) priming conditions; however, (2) this effect might occur later (i.e., in longer SOA conditions) under discrete emotional priming than under valence priming (i.e., in shorter SOA conditions) when SOAs were used as indexes, because the discrete emotional information would not be activated as strongly as valence information in the early stage of word processing.

\section{PRETEST}

The goal of this pretest was to select words denoting different emotions, which would be used in the following two experiments. In Experiment 1, affective words and event words denoting positive and negative emotions were needed, whereas in Experiment 2, the selected words denoted emotions that were negative but belonged to different prototypes of emotions. Therefore, in the present study, happiness was considered to be the positive prototype, while anger, sadness, and fear were considered to be the negative prototypes.

\section{Methods}

\section{Participants}

Ninety university students from the South China Normal University (40 males, 50 females) participated in the experiment voluntarily, and were paid after the task.

\section{Materials and Procedures}

Four affective words, including happiness, sadness, anger, and fear, were selected as the cues, and printed in Chinese on A4 paper. Participants were instructed to freely associate and write down as many as words as possible that they associated with the cue. The part of speech (e.g., noun, adjective, verb, etc.) and relation between cues and word productions (e.g., synonymic, antonymic, etc.) were not restricted.

\section{Results and Analyses}

Ninety questionnaires were delivered but an incomplete one was excluded in the final analyses. Results from the 89 available questionnaires showed 851 associated words were produced for Happiness, 833 for Sadness, 959 for Anger, and 828 for Fear. According to the relation between cues and productions, associate words were categorized into four groups: (1) synonyms, such as happiness-delight, (2) antonymic, such as happinesssorrow, (3) life events, such as sadness-failure, (4) behavior caused by the emotion, such as anger-vent, (5) others which could not be categorized into the previous four groups, such as angerbirds (a name for a popular game). Numbers and frequencies of associated words are shown in Table 1, and a list of the most commonly associated words under each emotional cue is shown in Table 2.
General chi-square test showed that associated frequency of life events was much higher than other associations, $\chi^{2}=53.71$, $d f=3, p<0.001, \eta=0.14$. Further analyses showed that most of the words were associated with the experience of university life, including study, relationships, taking exams, love, family, health, competitions, seeking jobs, ability, and so on, which indicated that there is a strong mapping between affective and event words. More importantly, materials presenting event words could be chosen from associate words produced in the present pretest.

The top 16 associate event words and top 10 synonyms from each emotion group (i.e., happiness, sadness, anger, and fear) were selected. All these candidates were Chinese disyllable words. Because all selected words were relative to university students' experience, but not all of them can be equally paired with word frequencies, familiarity ratings were used instead of word frequencies to control familiarity of materials in the present study. Thirty university students who did not participate in the main study gave five-point familiarity ratings $(1=$ very unfamiliar, $5=$ very familiar) of all these life events and emotion primes. Words with an average rating higher than 3.5 were kept as the final materials (see Appendices A-C).

\section{EXPERIMENT 1}

The goals of Experiment 1 were (1) to replicate the emotional congruent effect in which reaction time was significantly shorter when the prime and target shared the same valence as compared to trials on which prime and target were of opposite valence under four SOAs levels (i.e., 50, 150, 200, $400 \mathrm{~ms}$ ); and (2) to compare such a congruent effect under the affective word priming condition and event word priming condition.

\section{Methods}

\section{Participants}

One hundred students (27 males, 73 females, ages from 18 to 23, $M=21.08$ ) from South China Normal University participated in the present study. They were volunteers and were paid after participation.

\section{Design}

Stimuli in a priming LDT were manipulated in a $2 * 2 * 4$ repeatedmeasures factorial design with evaluative congruency (congruent vs. incongruent) and priming type (affective word vs. event word) as within group factors, SOAs (50/150/200/400 ms) as a between group factor, and latencies and error rates as the dependent variables. Priming (affective word or event word) and probes (event word) sharing the same affective valence were considered as the congruent condition, whereas those representing different affective valence were considered as the incongruent condition. All participants were divided into four groups randomly, 25 students for each group, and fulfilled only one SOA condition.

\section{Materials}

Materials were received from the pretest in which 16 affective words and 32 event words were selected. Affective words, half of which were positive whereas half of which were negative, 
TABLE 1 | Descriptions on the frequencies of associated categories from the associated task $(N=89)$.

\begin{tabular}{|c|c|c|c|c|c|c|}
\hline \multirow[t]{2}{*}{ Affective words } & \multirow[t]{2}{*}{ Answer } & \multicolumn{5}{|c|}{ Categories of associated words } \\
\hline & & Synonyms & Antonym & Life event & Relative behavior & Others \\
\hline \multirow[t]{2}{*}{ Happiness } & Number & 161 & 11 & 531 & 64 & 84 \\
\hline & Frequency (\%) & 18.92 & 1.29 & 62.40 & 7.52 & 9.87 \\
\hline \multirow[t]{2}{*}{ Sadness } & Number & 152 & 2 & 535 & 82 & 62 \\
\hline & Frequency (\%) & 18.24 & 0.24 & 64.22 & 9.84 & 7.44 \\
\hline \multirow[t]{2}{*}{ Anger } & Number & 89 & 1 & 558 & 193 & 118 \\
\hline & Frequency (\%) & 9.28 & 0.10 & 58.19 & 20.12 & 12.30 \\
\hline \multirow[t]{2}{*}{ Fear } & Number & 148 & 2 & 550 & 40 & 88 \\
\hline & Frequency (\%) & 17.87 & 0.24 & 66.42 & 4.83 & 10.63 \\
\hline
\end{tabular}

TABLE 2 | Descriptions on the frequencies of first associated words in Experiment $1(N=89)$.

\begin{tabular}{|c|c|c|c|c|c|c|}
\hline \multirow[t]{2}{*}{ Affective words } & \multirow[t]{2}{*}{ Answer } & \multicolumn{5}{|c|}{ Categories of associated words } \\
\hline & & Synonyms & Antonym & Life event & Relative behavior & Others \\
\hline \multirow[t]{2}{*}{ Happiness } & Number & 36 & 2 & 42 & 7 & 3 \\
\hline & Frequency (\%) & 40.45 & 2.24 & 47.19 & 7.87 & 3.37 \\
\hline \multirow[t]{2}{*}{ Sadness } & Number & 21 & 0 & 47 & 17 & 4 \\
\hline & Frequency (\%) & 23.60 & 0 & 52.81 & 19.10 & 4.49 \\
\hline \multirow[t]{2}{*}{ Anger } & Number & 25 & 1 & 45 & 10 & 8 \\
\hline & Frequency (\%) & 28.09 & 1.12 & 50.56 & 11.24 & 8.99 \\
\hline \multirow[t]{2}{*}{ Fear } & Number & 29 & 0 & 52 & 6 & 1 \\
\hline & Frequency (\%) & 32.58 & 0 & 58.43 & 6.74 & 1.12 \\
\hline
\end{tabular}

were all used as primes. Event words, 16 of which denoted positive emotion 16 of which denoted negative emotion, were used as primes or targets. Therefore, there were eight positive event primes, eight negative event primes, eight positive event targets, and eight negative event targets. In addition, another 16 pseudo words were created as the filler probes so that the number of correct Yes and No responses could be balanced. To establish congruencies in affective valence between primes and probes (affective-event or event-event), four types of prime-probe word pairs were manipulated: (1) affective-event word pairs with congruent evaluation (e.g., positive: 快乐happiness-游戏 play games; negative: 悲伤 sadness-生病 sick), (2) affective-event word pairs with incongruent evaluation (e.g., 高兴 joyfulness-争吵 quarrel; 伤心 feeling bad-录取 being accepted), (3) event-event word pairs with congruent evaluation (e.g., positive: 约会 dating-中奖prizewinning; negative: 失恋 love fails-䯠梦 nightmare), (4) event-event word pairs with incongruent evaluation (e.g., 绝交 break of relations-阅读 reading; 得意 pleased-踥酒 drunk).

\section{Procedure}

A priming LDT without feedback was used. The experimental software E-Prime presented the stimuli and recorded reaction times. Control files were constructed to display on the 17 inch IBM (9512-AB1) monitor (Screen resolution: $1024 \times 768$ Pixels), and participants were tested individually in a sound-proof room. Response errors and reaction times, measured from the onset of words, were automatically recorded by computers for each participant and each item. A trial consisted of the following sequence of events: a fixation point (+) was presented for $500 \mathrm{~ms}$ in the center of the screen, then right after the fixation cross disappeared a priming word was presented for 50,150, 200, or $400 \mathrm{~ms}$ (different SOAs). Probes were presented at the center of the computer screen right after the offset of the priming and remained on the screen for maximum $3000 \mathrm{~ms}$.

Participants were asked to judge as quickly and as accurately as possible whether or not the probe in each trial was a real word, and to press the corresponding button on a response box. The inter-trial interval was $500 \mathrm{~ms}$ (blank screen) and then the next trial started. If the participant failed to respond in $3000 \mathrm{~ms}$, the trial was terminated and the trial was recorded as an error. Participants received 10 practice trials, for which feedback was provided. No feedback was provided during the experiment.

\section{Results}

Participants' mean latencies (based on untrimmed correct responses) and error rates are summarized in Table 3.

Results of the analyses on latency showed a significant difference between congruent and incongruent priming, $F(1,96)=24.60, M S E=250299, p<0.001, \eta^{2}=0.20$, and between affective word priming and event word priming, $F(1,96)=52.89, M S E=544573, p<0.001, \eta^{2}=0.36$. In addition, a main effect of SOAs was found, $F(3,96)=10.08$, $M S E=140450, p<0.001, \eta^{2}=0.24$. Further LSD tests showed that shorter latencies were caused by shorter SOAs $(50,150 \mathrm{~ms})$, rather than by longer ones $(200,400 \mathrm{~ms})$. However, differences between 50 and $150 \mathrm{~ms}$ priming, and between 200 and $400 \mathrm{~ms}$ priming, were not found. The interaction between priming 
TABLE 3 | Means of latencies (ms) and error rates (\%) in Experiment 1 (based on evaluative congruency).

\begin{tabular}{|c|c|c|c|c|c|c|c|c|c|}
\hline \multirow{3}{*}{\multicolumn{2}{|c|}{ Evaluative congruency }} & \multicolumn{8}{|c|}{ SOA } \\
\hline & & \multirow[b]{2}{*}{$50 \mathrm{~ms}$} & \multicolumn{3}{|c|}{ Affective word priming } & \multirow[b]{2}{*}{$50 \mathrm{~ms}$} & \multicolumn{2}{|c|}{ Event word priming } & \multirow[b]{2}{*}{$400 \mathrm{~ms}$} \\
\hline & & & $150 \mathrm{~ms}$ & $200 \mathrm{~ms}$ & $400 \mathrm{~ms}$ & & $150 \mathrm{~ms}$ & $200 \mathrm{~ms}$ & \\
\hline \multirow[t]{2}{*}{ Latencies } & Congruent & $658 \pm 94$ & $652 \pm 70$ & $728 \pm 107$ & $705 \pm 92$ & $595 \pm 69$ & $613 \pm 82$ & $638 \pm 97$ & $618 \pm 58$ \\
\hline & Incongruent & $684 \pm 114$ & $670 \pm 93$ & $809 \pm 128$ & $797 \pm 124$ & $630 \pm 99$ & $652 \pm 61$ & $696 \pm 106$ & $669 \pm 80$ \\
\hline \multirow[t]{2}{*}{ Error rates } & Congruent & $6.2 \pm 7.7$ & $4.0 \pm 5.5$ & $1.8 \pm 2.8$ & $5.8 \pm 10.5$ & $6.2 \pm 8.5$ & $4.2 \pm 4.5$ & $2.6 \pm 4.4$ & $6.0 \pm 7.2$ \\
\hline & Incongruent & $8.8 \pm 9.2$ & $6.4 \pm 6.4$ & $2.2 \pm 3.3$ & $6.3 \pm 9.6$ & $5.8 \pm 7.3$ & $4.8 \pm 3.3$ & $2.2 \pm 2.9$ & $7.0 \pm 10.7$ \\
\hline
\end{tabular}

type and congruency did not reach significance, $F<1$, ns. In addition, the interaction between priming type and SOAs, and the three-way interaction, were missing, $F s<1$, ns.

Results of the analyses on error rates showed that fewer error rates were produced under the $200 \mathrm{~ms}$ priming condition than the other three SOA conditions, $F(3,96)=9.67, M S E=0.046$, $p<0.001, \eta^{2}=0.24$, but a difference among the latter three SOA conditions was not found. However, a main effect of emotional congruency, $F(1,96)=1.22, M S E=0.008, p>0.10$, and priming types, $F(1,96)<1$, ns, were both missing. In addition, the interactions did not reach significance, $F^{\prime}$ s $<1$, ns.

\section{Discussion}

Experiment 1 was mainly focused on whether in a priming LDT event words can elicit a similar emotion priming effect as compared to affective words. As expected, there was a facilitation in priming LDT when the priming and targets were of congruent emotional valence (i.e., positive-positive, or negative-negative), whereas such priming effect was missing when priming and targets were of incongruent emotional valence (i.e., positivenegative, or negative-positive), whether affective or event words were used for priming. These findings are important because they replicate earlier works on the emotional congruency effect (Wentura, 1999; Fazio, 2001). More importantly, Experiment 2 builds on these results in that by controlling congruencies in a discrete emotion level, the discrete emotion view on emotional effect can be tested.

\section{EXPERIMENT 2}

The goal of Experiment 2 was to test the congruent effect with discrete emotions as primes to elicit the emotional congruency effect. Namely, in contrast to the traditional positive-negative categorization, emotions were divided into discrete categories, such as happiness, sadness, anger, and fear. The main hypothesis was that participants would take longer to respond in the priming LDT when prime and target were of different discrete emotion categories (e.g., sadness and fear), although, they were of the same valence (e.g., negative). To avoid an evaluative congruent effect, only one valence (negative) was chosen in the present experiment. That is, of the four basic emotions, only happiness is positive whereas the other three are negative, so materials belonging to the sadness, anger, and fear categories were selected.

\section{Methods}

\section{Participants}

Participants in Experiment 1 also took part in Experiment 2.

\section{Design}

The design was identical to that of Experiment 1 except that emotional congruency was defined based on the discrete emotion. Namely, priming (affective word or event word) and probes (event word) sharing the same discrete emotion (i.e., sadness, fear, anger) were considered as the congruent emotion category group, whereas, those representing different discrete emotions were considered as the incongruent emotion category group.

\section{Materials}

Materials were generated in the pretest. To avoid the effects of positive and negative evaluation, only materials related to sadness, anger, and fear (but not happiness) were used. There were 24 affective words and 48 event words. Affective words, eight of which denoted anger, eight of which denoted sadness, and the rest of which denoted fear, were all used as primes. Event words, 16 of which denoted anger 16 of which denoted sadness, and the rest of which denoted fear, were used as primes or targets. Therefore, there were eight angry-event primes, eight sad-event primes, eight fear-event primes, eight angry-event targets, eight sad-event targets, and eight fear-event targets. To establish congruencies in emotion category between primes and probes (affective-event or eventevent), four types of prime-probe word pairs were manipulated: (1) affective-event word pairs with congruent emotion (e.g., sadness: 伤心 sadness-失去 lose; fear: 焦虑 anxious-论文 writing thesis; anger: 愤怒 rage-背叛 betray), (2) affective-event word pairs with incongruent emotion (e.g., 伤心-论文; 焦虑-背叛; 愤怒失去), (3) event-event word pairs with congruent emotion (e.g., sadness: 失利 defeat-绝交 break off relations; fear: 死亡 death工作 working; anger: 腐败 corruption-露体 naked), (4) event-event word pairs with incongruent emotion (e.g., 失利-工作; 死亡-露体; 腐败绝交).

\section{Procedure}

The procedure was identical to that in Experiment 1.

\section{Results \\ General Analysis}

Participants' mean latencies (based on untrimmed correct responses) and error rates are summarized in Table 4. 
TABLE 4 | Means of latencies (ms) and error rates (\%) in Experiment 2 (based on discrete emotional congruency).

Discrete emotional

congruency
SOA

\begin{tabular}{|c|c|c|c|c|c|c|c|}
\hline $50 \mathrm{~ms}$ & $150 \mathrm{~ms}$ & $200 \mathrm{~ms}$ & $400 \mathrm{~ms}$ & $50 \mathrm{~ms}$ & $150 \mathrm{~ms}$ & $200 \mathrm{~ms}$ & $400 \mathrm{~ms}$ \\
\hline $616 \pm 60$ & $641 \pm 75$ & $708 \pm 97$ & $696 \pm 89$ & $627 \pm 61$ & $631 \pm 87$ & $658 \pm 90$ & $615 \pm 51$ \\
\hline $694 \pm 102$ & $672 \pm 104$ & $751 \pm 106$ & $828 \pm 123$ & $615 \pm 97$ & $640 \pm 62$ & $653 \pm 88$ & $690 \pm 113$ \\
\hline
\end{tabular}

Results of the analyses on latency showed a significant difference between emotional congruent and incongruent priming, $F(1,96)=17.10, M S E=194323, p<0.001$, $\eta^{2}=0.15$, and between affective word priming and event word priming, $F(1,96)=33.35, M S E=360408, p<0.001$, $\eta^{2}=0.26$. In addition, a main effect of SOAs was found, $F(3,96)=7.22, M S E=116134, p<0.001, \eta^{2}=0.18$. Further LSD tests showed that shorter latencies were caused by shorter SOAs $(50,150 \mathrm{~ms})$, rather than by longer ones (200, $400 \mathrm{~ms})$. However, differences between 50 and $150 \mathrm{~ms}$ priming, and between 200 and $400 \mathrm{~ms}$ priming, were not found. The interaction between priming type and congruency reached significance, $F(1,96)=5.94, M S E=72763$, $p<0.05, \eta^{2}=0.06$. Further simple effect analyses were used to test specific comparisons that might explain this interaction. These analyses showed that the congruency effect was generally significant under the affective word priming condition $(p<0.001)$, but not under the event word priming condition $(p>0.10)$. In addition, the interaction between priming type and SOAs was significant, $F(3,96)=3.68, M S E=39788$, $p<0.05, \eta^{2}=0.10$, and the interaction between congruency and SOAs reached significance, as well, $F(3,96)=3.60$, $M S E=40943, p<0.05, \eta^{2}=0.10$. Simple effects analyses on the priming type* SOAs interaction showed significant advantages on emotional words priming occurred only on SOA $=200 \mathrm{~ms}$ $(p<0.05)$, and SOA $=400 \mathrm{~ms}(p<0.05)$; while simple effect analyses on the congruency* SOAs interaction indicated that significant emotional congruency effects occurred when $\mathrm{SOA}=400 \mathrm{~ms}(p<0.05)$ rather than other three SOA conditions ( $p s>0.05)$. However, the three-way interaction was missing, $F<1$, ns.

Results of the analyses on error rates showed that fewer errors were produced under the $200 \mathrm{~ms}$ priming condition than the other three SOA conditions, $F(3,96)=9.43, M S E=0.043$, $p<0.001, \eta^{2}=0.23$, but no difference among the latter three SOA conditions was found. However, a main effect of emotional congruency, $F(1,96)=0.82, M S E=0.005$, $p>0.10$, and of priming types, $F(1,96)=0.35, M S E=0.002$, $p>0.10$, were both missing. In addition, no interaction reached significance [priming type $*$ SOAs: $F(3,96)=1.18$, $M S E=0.006, p>0.10$, emotional congruency $*$ SOAs: $F(3,96)=0.64, M S E=0.004, p>0.10$, priming type $*$ emotional congruency: $F(1,96)=2.21, M S E=0.013, p>0.05$, emotional congruency $*$ priming type $*$ SOAs: $F(3,96)=0.60, M S E=0.003$, $p>0.10]$.

\section{Further Analysis of Congruency Effect Under Event Word Priming Across Experiments}

In the present study, the main focus was emotional congruency effects in event word priming conditions. Therefore, latency data from both experiments under event word priming were also submitted to 2 (Experiments) ${ }^{*} 2$ (Priming types) *4 (SOAs) repeated-measures ANOVAs. Results showed a significant difference between congruent and incongruent priming, $F(1,96)=11.32, M S E=98538, p<0.01, \eta^{2}=0.11$, and among SOAs, $F(3,96)=2.60, M S E=36516, p=0.05$, $\eta^{2}=0.08$. However, the difference between experiments did not reach significance, $F<1$, ns. In addition, the interaction between priming type and experiments reached significance, $F(1,96)=5.72, M S E=20401, p<0.05, \eta^{2}=0.06$, and the three-way interaction was marginally significant, $F(3,96)=2.53$, $M S E=9013, p=0.06, \eta^{2}=0.07$, whereas other interactions were missing [Experiment*SOAs: $F<1$, ns; Congruency*SOAs: $F(3,96)=1.41, M S E=12277, p>0.10]$.

Based on the three-way interaction, further simple effect analyses were conducted to test differences between congruent and incongruent event word priming under different SOA conditions separately (details are in Table 5). Different from emotional congruency effects occurring at the late SOA stage $(\mathrm{SOA}=400 \mathrm{~ms})$ under discrete emotional priming (Experiment 2), a significant priming effect occurred at the early SOA stage (SOA $=50 \mathrm{~ms}$ ) when congruency was defined in terms of positive-negative emotion (Experiment 1).

\section{Discussion}

The novelty of Experiment 2 is that congruency of discrete emotions (i.e., sadness, anger, and fear), all of which were of negative valence, appeared to affect performance in the LDT. However, different from the results of Experiment 1, in which more dimensional emotion information (i.e., positive vs. negative) was manipulated, the congruency effect in Experiment 2 occurred only under the SOA level of $400 \mathrm{~ms}$ and when primes were event words. One of the plausible reasons was that affective information from emotional labels could be more easily activated than that from event words. However, another more possible reason was that life events were often displayed with discrete emotions in everyday expressions. This means that at least a part of the present priming effect under the emotion priming condition, which was larger and more stable than the effect elicited by event priming, was affected by the highly frequent 
TABLE 5 | T-test between congruent and incongruent event priming under different SOAs $(d f=24)$.

\begin{tabular}{|c|c|c|c|c|c|c|c|c|}
\hline & \multicolumn{2}{|c|}{$S O A=50 \mathrm{~ms}$} & \multicolumn{2}{|c|}{$S O A=150 \mathrm{~ms}$} & \multicolumn{2}{|c|}{$S O A=200 \mathrm{~ms}$} & \multicolumn{2}{|c|}{$S O A=400 \mathrm{~ms}$} \\
\hline & $t$ & $p$ & $t$ & $p$ & $t$ & $p$ & $t$ & $p$ \\
\hline EXP1 & 2.312 & $0.03^{*}$ & 1.894 & 0.07 & 2.082 & $0.048 *$ & 2.404 & $0.024 *$ \\
\hline EXP2 & 0.557 & 0.58 & 0.556 & 0.58 & 0.177 & 0.86 & 3.185 & $0.004^{* *}$ \\
\hline
\end{tabular}

${ }^{* * *} p<0.001,{ }^{* *} p<0.01,{ }^{*} p<0.05$.

collocation. Therefore, the priming effect under the event word priming condition should be more pronounced than under the emotion priming condition. The present results indicate that the experiment triggered a discrete emotional congruent effect at a later stage (i.e., $\mathrm{SOA}=400 \mathrm{~ms}$ ) as compared to that triggered by general emotional valence $(\mathrm{SOA}=50 \mathrm{~ms}$ in Experiment 1). This can be considered as evidence that discrete emotion information was activated during the accessing of event words, but this information was much weaker and activated later than in response to words denoted by positive-negative valence.

\section{GENERAL DISCUSSION}

The present study tested whether discrete emotion information can be activated during word processing. In the literature, researchers the emotional dimension was mostly characterized as positive or negative, the emotional congruency effect has yet to be discussed at the discrete emotion level. In addition, we focused on whether discrete emotion can elicit congruency effects as early as the evaluation of emotion. Robust evaluative congruency effects at the SOA levels of 50,200, and $400 \mathrm{~ms}$ (Experiment 1), and the discrete emotional congruency effect at the SOA level of $400 \mathrm{~ms}$ (Experiment 2) under the event word priming condition, indicated that not only general affective valence but also discrete emotions could be activated under the present priming LDT. However, discrete emotions were activated at the later priming stage as compared to general emotional valences. These findings are important because they replicate earlier work on the one hand, but also extend that work by moving from general to discrete emotion. Moreover, the results provide evidence for the hierarchy model of emotion.

It is frequently assumed that people spontaneously evaluate incoming stimuli in terms of bipolar valence, such as pleasant or unpleasant, liked or disliked, good or bad (Klauer, 1997). Altarriba et al. (1999) and Altarriba and Bauer (2004) noted that valence of affective words affects word recognition and retrieval (Altarriba and Bauer, 2004). This effect can be explained by the semantic activation model (Altarriba and Bauer, 2004). However, Kousta et al. (2011) claimed that these findings are consistent with the embodied theory. Research by Kousta et al. (2011) showed that abstract words with affective associations are acquired earlier than are neutral abstract words. Therefore, contrary to the earlier idea that mappings between word and world occur during concrete word learning, Vigliocco et al. (2009) posited a more general explanation. Namely, emotional states, moods or feelings denoted by words represent a mapping from the word to the world, and affect word accessing, no matter which kind (i.e., concrete or abstract) of word it is.

Although, the embodied approaches have been extended to the representation and processing of the valence of experiences of word meanings, it has been far less discussed how a discrete embodied experience could be valid in word accessing. During emotional development, emotions should not be learned only dimensionally as positive, negative, and neutral, but also discretely as happiness, anger, sadness or fear (Shaver et al., 1987). That is, there should be links between particular appraisals and particular emotions (Roseman et al., 1990). As a consequence, it becomes possible that various systematic attempts can be triggered by different emotions to regain control by shifting attention to the threat and physiological arousal in preparation for behavioral responses (Ekman, 2003; Rivers et al., 2007). Therefore, preparation for behavioral responses to "defeat" and "war" are different because experiences of "sadness" and "fear" are different. However, there has been a lack of discussion about whether emotion information or emotional experience affects our word learning and accessing at the discrete emotion level.

Results of Experiment 1 provided evidence that different kinds of words elicited similar emotional congruency effects under the affective valence condition. Based on these results, which can be seen as supporting the argument by Vigliocco et al. (2009), we further investigated a similar effect under the superordinate level in Experiment 2. Results of Experiment 2 indicated that discrete emotion also affects word processing. Different from most of the recent research in which life events were always categorized as positive and negative (Robinson and Kirkeby, 2005; Robinson and Hippel, 2006), Experiment 2 categorized words referring to different discrete emotions belong to the three negative prototypes (namely whether words have sad, angry, or anxious connotations). We found an advantage for the congruent condition in comparison to the incongruent condition, in which congruent emotion facilitation was found when primes and probes belonged to the same prototypical emotion when all materials were of negative valence. In other words, specific prototypical emotion information was accessed in the present priming LDT.

Moreover, the priming effect was found to be robust and stable under affective word priming, no matter whether the emotions were dimensionally or discretely manipulated. This finding might be the evidence that emotion information was more easily activated by clear emotion labels. However, it was not completely clear that such a priming effect elicited by affective words was completely a congruency effect. Affectiveevent word pairs were generated by a word-association test. As a consequence, the priming effects might have been partially 
triggered by the highly frequent collocation between affective words and events in everyday expressions. Discrete affective words and event words in the congruent condition are frequently used in everyday expressions (e.g., I am angry at his defection, or I am happy that you passed the exam). A specific emotional label can semantically activate several candidate events, which can be regarded as preparation for the upcoming LDT, and responses for the congruent trails will be quickened as compared to the incongruent trails. Should the present findings be more consistent with the language-based view (Vigliocco et al., 2009), rather than embodied emotion view?

Therefore, results of event word priming, in which the influence of highly frequent collocation was excluded, are more convincing for the present discussion. According to the SOA effect, under event word priming, an emotional congruency effect was found at the early stage when congruency of affective valence was manipulated (i.e., positive-negative, in Experiment 1), but at the late stage when congruency of discrete emotion was manipuated (i.e., sadness, anger, and fear, in Experiment 2). Apparently, activation of the discrete emotion information was not as fast as that of affective valence.

We believe that the present results are consistent with the hierarchical view of emotion, which is compatible with the bipolar view of the emotional congruency effect. Shaver et al. (1987) proposed a dynamic model of the emotion process implicit in emotion episodes. According to this model, emotions are conceptualized as beginning with an interpretation of events as positive or negative; then, one of the basic emotions, namely one of the prototypical emotions in the present study, is elicited, accompanied by characteristic action tendencies, cognitive biases, and physiological patterns that also arise automatically. This emotion-related information is processed every day, and people can comprehend an event in either a more detailed or more general way. For example, a detailed description such as "a sad woman" is more cognitively vivid and imaginable than a description such as "a woman who is experiencing negative emotion" (Shaver et al., 1987). Therefore, existence of a discrete emotional congruency effect is acceptable.

In addition, according to this dynamic model, experience of emotion could be described under different dimensions: valence (i.e., positive or negative), potency (i.e., weak or strong), and activity (i.e., low or high), all of which are forms of experiential information that would affect word processing. Therefore, experiential information of a word can be processed evaluatively (i.e., positive or negative; Fazio, 2001), and the stronger the feeling is, the faster the process is (i.e., positive/negative vs. neutral; Kousta et al., 2009). More importantly, as mentioned in the three-dimensional solution model (Shaver et al., 1987), anger-related emotions tend to be high in potency, sadnessrelated emotions tend to be low in potency, and fear tends to be intermediate in potency. In other words, results of the present study indicated that the potency can also affect word processing.
We believed that this feeling of potency comes from subjects' everyday experience.

\section{CONCLUSION}

This study provides evidence that emotion information denoted by a word can be considered a prominent feature that affects word accessing, and the results are discussed in terms of the extent to which discrete emotion information is involved in this process. Emotion information as a prominent feature appears beneficial on several levels. Firstly, emotion information is primary and necessary in learning and representing word meanings (Vigliocco et al., 2009), and as a result of repeated experiences, emotions are organized within an abstract-to-concrete hierarchy (Shaver et al., 1987). Therefore, both the bipolar feature (i.e., positive-tonegative valences) and the subordinate feature (i.e., prototypical emotions) affect word accessing. Secondly, activation of emotion information varies under different levels. Valence of emotion denoted by words is fast and strongly activated, and then followed by activation of subordinate emotion information. Thirdly, affective words elicit larger priming effects than event words do. This priming effect might be partially triggered by highly frequent collocation between primes and probes in everyday expressions, which was not tested in the present study.

Taken together, the findings suggest that discrete emotion information produces congruency effects similar to those triggered by general affective valence. In addition, consistent with the hierarchical model of emotion, in which emotion information is organized within an abstract-to-concrete hierarchy, these discrete emotion effects, as compared to dimensional ones, occur at the later stage of word processing.

\section{AUTHOR CONTRIBUTIONS}

XC: idea, design, management, writing; BL: Experiments 1 and 2; SL: pretest.

\section{ACKNOWLEDGMENT}

This project was supported by the National Natural Science Foundation of China (No.31400964), and Guangdong Province philosophy social science and the "twelfth-five-year" plan (No. GD12YXL01).

\section{SUPPLEMENTARY MATERIAL}

The Supplementary Material for this article can be found online at: http://journal.frontiersin.org/article/10.3389/fpsyg. 2016.00916 


\section{REFERENCES}

Altarriba, J., and Bauer, L. M. (2004). The distinctiveness of emotion concepts: a comparison between emotion, abstract, and concrete words. Am. J. Psychol. 117, 389-410. doi: 10.2307/4149007

Altarriba, J., Bauer, L. M., and Benvenuto, C. (1999). Concreteness, context availability, and imageability ratings and word associations for abstract, concrete, and emotion words. Behav. Res. Methods Instrum. Comput. 31, 578602. doi: $10.3758 /$ BF03200738

Barrett, L. F. (1998). Discrete emotions or dimensions? the role of valence focus and arousal focus. Cogn. Emot. 12, 579-599. doi: 10.1080/026999398379574

Barrett, L. F. (2006). Solving the emotion paradox: categorization and the experience of emotion. Pers. Soc. Psychol. Rev. 10, 20-46. doi: 10.1207/s15327957pspr1001_2

Barrett, L. F. (2009a). The future of psychology: connecting mind to brain. Perspect. Psychol. Sci. 4:531. doi: 10.1111/j.1745-6924.2009.01134.x

Barrett, L. F. (2009b). Variety is the spice of life: a psychological construction approach to understanding variability in emotion. Cogn. Emot. 23, 1284-1306. doi: 10.1080/02699930902985894

Barrett, L. F., Gendron, M., and Huang, Y.-M. (2009). Do discrete emotions exist? Philos. Psychol. 22, 427-437. doi: 10.1080/09515080903153634

Barsalou, L. W. (1999). Perceptions of perceptual symbols. Behav. Brain Sci. 22, 637-660. doi: 10.1017/S0140525X99532147

Barsalou, L. W., Santos, A., Simmons, W. K., Wilson, C. D., De Vega, M., Glenberg, A., et al. (2008). "Language and simulation in conceptual processing," in Symbols, Embodiment, and Meaning, eds M. De Vega, A. M. Glenberg, and A. C. Graesser (Oxford: Oxford University Press), 245-283. doi: 10.1093/acprof:oso/9780199217274.003.0013

De Houwer, J., Hermans, D., and Eelen, P. (1998). Affective and identity priming with episodically associated stimuli. Cogn. Emot. 12, 145-169. doi: $10.1080 / 026999398379691$

Ekman, P. (2003). Emotions Revealed: Recognizing Faces and Feelings to Improve Communication and Emotional Life. New York, NY: Times Books: Henry Holt and Co.

Fazio, R. H. (2001). On the automatic activation of associated evaluations: an overview. Cogn. Emot. 15, 115-141. doi: 10.1080/02699930125908

Glenberg, A. M. (1997). What memory is for: creating meaning in the service of action. Behav. Brain Sci. 20, 41-50. doi: 10.1017/S0140525X97470012

Hermans, D., De Houwer, J., and Eelen, P. (1994). The affective priming effect: automatic activation of evaluative information in memory. Cogn. Emot. 8, 515-533. doi: 10.1080/02699939408408957

Hermans, D., De Houwer, J., and Eelen, P. (2001). A time course analysis of the affective priming effect. Cogn. Emot. 15, 143-165. doi: 10.1080/0269993004200033

Herring, D. R., White, K. R., Jabeen, L. N., Hinojos, M., Terrazas, G., Reyes, S. M., et al. (2013). On the automatic activation of attitudes: a quarter century of evaluative priming research. Psychol. Bull. 139:1062. doi: 10.1037/a0031309

$\mathrm{Hu}$, T. (2012). Relationships between word-frequencies of target words and emotional priming (in Chinese). J. Nanjing Xiaozhuang Univ. 2:111.

Izard, C. E. (1992). Basic emotions, relations among emotions, and emotioncognition relations. Psychol. Rev. 99, 561-565.

Klauer, K. C. (1997). Affective priming. Eur. Rev. Soc. Psychol. 8, 67-103. doi: $10.1080 / 14792779643000083$

Kousta, S.-T., Vigliocco, G., Vinson, D. P., Andrews, M., and Del Campo, E. (2011). The representation of abstract words: why emotion matters. J. Exp. Psychol. Gen. 140:14. doi: 10.1037/a0021446

Kousta, S.-T., Vinson, D. P., and Vigliocco, G. (2009). Emotion words, regardless of polarity, have a processing advantage over neutral words. Cognition 112, 473-481. doi: 10.1016/j.cognition.2009.06.007

Madrid, H. P., and Patterson, M. G. (2014). Measuring affect at work based on the valence and arousal circumplex model. Span. J. Psychol. 17:E50. doi: $10.1017 /$ sjp. 2014.54
Murphy, G. L. (2002). The Big Book of Concepts. Cambridge, MA: MIT press.

Niedenthal, P. M. (2007). Embodying emotion. Science 316, 1002-1005. doi: $10.1126 /$ science. 1136930

Niedenthal, P. M., Winkielman, P., Mondillon, L., and Vermeulen, N. (2009). Embodiment of emotion concepts. J. Pers. Soc. Psychol. 96:1120. doi: $10.1037 / \mathrm{a} 0015574$

Philippot, P., Schaefer, A., and Herbette, G. (2003). Consequences of specific processing of emotional information: impact of general versus specific autobiographical memory priming on emotion elicitation. Emotion 3:270. doi: 10.1037/1528-3542.3.3.270

Posner, J., Russell, J. A., and Peterson, B. S. (2005). The circumplex model of affect: an integrative approach to affective neuroscience, cognitive development, and psychopathology. Dev. Psychopathol. 17, 715-734. doi: 10.1017/S0954579405050340

Reisenzein, R. (1994). Pleasure-arousal theory and the intensity of emotions. J. Pers. Soc. Psychol. 67:525. doi: 10.1037/0022-3514.67.3.525

Reisenzein, R., and Spielhofer, C. (1994). Subjectively salient dimensions of emotional appraisal. Motiv. Emot. 18, 31-77. doi: 10.1007/BF022 52474

Rivers, S. E., Brackett, M. A., Katulak, N. A., and Salovey, P. (2007). Regulating anger and sadness: an exploration of discrete emotions in emotion regulation. J. Happiness Stud. 8, 393-427. doi: 10.1007/s10902-0069017-2

Robinson, M. D., and Hippel, W. V. (2006). Rose-colored priming effects: life satisfaction and affective priming. J. Posit. Psychol. 1, 187-197. doi: 10.1080/17439760600885705

Robinson, M. D., and Kirkeby, B. S. (2005). Happiness as a belief system: individual differences and priming in emotion judgments. Personal. Soc. Psychol. Bull. 31, 1134-1144. doi: 10.1177/0146167204274081

Roseman, I. J., Spindel, M. S., and Jose, P. E. (1990). Appraisals of emotion-eliciting events: testing a theory of discrete emotions. J. Pers. Soc. Psychol. 59, 899-915. doi: 10.1037/0022-3514.59.5.899

Russell, J. A. (1980). A circumplex model of affect. J. Pers. Soc. Psychol. 39:1161. doi: $10.1037 / \mathrm{h} 0077714$

Scherer, K. R. (2005). What are emotions? And how can they be measured? Soc. Sci. Inform. 44, 695-729. doi: 10.1177/05390184050 58216

Shaver, P., Schwartz, J., Kirson, D., and O'connor, C. (1987). Emotion knowledge: further exploration of a prototype approach. J. Pers. Soc. Psychol. 52:1061. doi: 10.1037/0022-3514.52.6.1061

Vigliocco, G., Meteyard, L., Andrews, M., and Kousta, S. (2009). Toward a theory of semantic representation. Lang. Cogn. 1, 219-248. doi: 10.1515/LANGCOG.2009.011

Wentura, D. (1999). Activation and inhibition of affective information: evidence for negative priming in the evaluation task. Cogn. Emot. 13, 65-91. doi: $10.1080 / 026999399379375$

Zhang, J. (2008). General Psychology. Guangdong: Guangdong Higher Education Press.

Zwaan, R. A. (2003). The immersed experiencer: toward an embodied theory of language comprehension. Psychol. Learn. Motiv. 44, 35-62. doi: 10.1016/S00797421(03)44002-4

Conflict of Interest Statement: The authors declare that the research was conducted in the absence of any commercial or financial relationships that could be construed as a potential conflict of interest.

Copyright (c) 2016 Chen, Liu and Lin. This is an open-access article distributed under the terms of the Creative Commons Attribution License (CC BY). The use, distribution or reproduction in other forums is permitted, provided the original author(s) or licensor are credited and that the original publication in this journal is cited, in accordance with accepted academic practice. No use, distribution or reproduction is permitted which does not comply with these terms. 


\section{OPEN ACCESS}

Edited by:

Anna M. Borghi,

University of Bologna, Italy

Reviewed by:

Starla Weaver,

Kessler Foundation, USA

Filomena Anelli,

University of Bologna, Italy

${ }^{*}$ Correspondence:

Roberta Capellin

r.capellini@campus.unimib.it

Specialty section:

This article was submitted to

Cognition,

a section of the journa

Frontiers in Psychology

Received: 15 June 2016 Accepted: 14 October 2016 Published: 01 November 2016

Citation:

Capellini $R$, Sacchi S, Ricciardelli $P$ and Actis-Grosso R (2016) Social Threat and Motor Resonance: When a Menacing Outgroup Delays Motor Response. Front. Psychol. 7:1697. doi: 10.3389/fpsyg.2016.01697

\section{Social Threat and Motor Resonance: When a Menacing Outgroup Delays Motor Response}

\author{
Roberta Capellini ${ }^{1 *}$, Simona Sacchi ${ }^{1}$, Paola Ricciardelli1,2 and Rossana Actis-Grosso ${ }^{1,2}$ \\ ${ }^{1}$ Department of Psychology, University of Milano-Bicocca, Milan, Italy, ${ }^{2}$ Milan Center for Neuroscience, Milan, Italy
}

Motor resonance (MR) involves the activation of matching motor representations while observing others' actions. Recent research has shown that such a phenomenon is likely to be influenced by higher order variables such as social factors (e.g., ethnic group membership). The present study investigates whether and how the perception of a social threat elicited by an outgroup member and by contextual cues can modulate motor responses while an individual observes others' movements. In an experimental study based on an action observation paradigm, we asked participants to provide answers through computer mouse movements (MouseTracker). We manipulated the agents' group membership (ingroup vs. outgroup) and the social valence of the objects present in a context (neutral vs. threatening) to elicit social menace through contextual cues. Response times and computer mouse trajectories were recorded. The results show a higher level of MR (i.e., participants started to respond earlier and were faster at responding) when observing an action performed by the ingroup members rather than by the outgroup members only when threatening objects are present in a given context. Participants seem to resonate better with their ingroup; conversely, the outgroup member movements tend to delay motor responses. Therefore, we extend prior research going beyond the general ingroup bias effect on MR and showing that the interaction between membership and contextual cues is likely to elicit threat-related stereotypes. Practical implications of these findings are discussed.

Keywords: social threat, motor resonance, action observation, intergroup relations, MouseTracker

\section{INTRODUCTION}

We tend to move with other people around us. For instance, when we see other people dancing or clapping their hands, we spontaneously synchronize our actions with those of our interaction partners (Cappella, 1997; Chartrand and Bargh, 1999). Furthermore, we resonate with others by mentally simulating and mimicking their gestures, postures, facial expressions, and emotions (Decety and Jackson, 2004). The present research focuses on the relationship between others' action observations and motor resonance (MR) and on the effects of group membership on this phenomenon. More specifically, the present experimental study explores whether and how the perception of social threat elicited by an outgroup member and by contextual cues is likely to modulate motor responses when facing an agent's action. 
Several studies involving the use of different neuroscience techniques ranging from fMRI to TMS (see for instance the body of research on motor neuron systems: Rizzolatti et al., 2001; Rizzolatti and Craighero, 2004; Rizzolatti and Sinigaglia, 2010) and behavioral measures consistently show that observing someone else performing an action elicits a motor activation similar to an activation that occurs when one performing the observed action personally (Fadiga et al., 1995, 2005; Dijksterhuis and Bargh, 2001; Sebanz et al., 2003; Iacoboni, 2005; Fourkas et al., 2006; Press et al., 2011).

Thus, this phenomenon of MR implies one's capacity to embody a representation of others' actions, and it seems to contribute to several complex and crucial social skills, such as one's understanding of others' actions, intentions and emotions (Hurley, 2008; Iacoboni, 2009) and the facilitation of interpersonal coordination and cooperation (Knoblich and Sebanz, 2006). For this reason, this type of ability is fundamental to our success as individuals and as a species and confers significant adaptive social advantages (e.g., Frith, 2007).

Although $\mathrm{MR}$ is an uncontrolled and automatic process (Gallese et al., 1996; Rizzolatti and Craighero, 2004; Wilson and Knoblich, 2005), over the last decade several studies have focused on the possibility that biological, individual and social factors may modulate such an effect.

In this regard, neuroscience evidence (fMRI) suggests that specific motor brain regions (i.e., right rostral parietal foci) are active only when observing biological movements (Perani et al., 2001; see also Kilner et al., 2003) and actions performed by conspecifics (Buccino et al., 2004). Moreover, previous research has shown that tendencies to simulate observed actions (Fadiga et al., 1995, 2005; Urgesi et al., 2006; Aglioti et al., 2008) or sensorimotor states of other individuals (Avenanti et al., 2005; Minio-Paluello et al., 2009) can be affected by individual differences such as gender (Cheng et al., 2008) or high-level personality traits such as empathy (Avenanti et al., 2009).

Among social factors, the actor and perceiver's group membership seems to play a central role. For instance, MolnarSzakacs et al. (2007) found that witnessing actions performed by an individual of one's cultural and ethnic ingroup increases corticospinal excitability to a greater extent than observing actions performed by an outgroup member (see also Liew et al., 2011). In line with this, other recent research has suggested a 'group bias' in MR (Gutsell and Inzlicht, 2010, 2013; for exceptions see Désy and Théoret, 2007; Losin et al., 2012). Moreover, such an effect has been proven to be stronger for those presenting high levels of racial prejudice. Starting from the assumption that an ingroup can be conceived of as an extended self (Aron et al., 1992; Brewer and Gardner, 1996), these results are in line with prior findings showing that action observation related regions are more active in response to stimuli associated with the self than with others (Uddin et al., 2006; Kaplan et al., 2008) and when facing agents physically similar to oneself (Molnar-Szakacs et al., 2007).

Although these studies started to explore the influence of social factors on $\mathrm{MR}$, this line of research leaves open questions on the role of social threats in such a process.
Social threat has been shown to be crucial to social perception. Indeed, research on impression formation suggests that when evaluating others we are primarily interested in defining whether others could represent an advantage or a threat (Wojciszke et al., 1998; Wojciszke, 2005; Fiske et al., 2007; Cuddy et al., 2008). In addition, perceptions of threat have emerged as an important predictor of global group attitude (Stephan et al., 1999; Stephan and Stephan, 2000; Riek et al., 2006; Pettigrew, 2008; Pettigrew and Tropp, 2008) in early stages of the impression formation process (Todorov et al., 2009). However, not every outgroup is stereotypically associated with social menace in every condition. Specific social categories (e.g., Blacks, Latinos and more recently Arabians) are stereotypically associated with aggression and threat (e.g., Payne, 2001; Mange et al., 2012) and are more likely to elicit aggressive responses in a social perceiver.

Recent literature has explored the role of threatening contextual cues on attention and social perception, showing that evolutionary relevant threatening stimuli are effective at capturing attentional resources (Öhman et al., 2001; Fox et al., 2002) thus causing interference with goal-directed activity (Williams et al., 1988, 1996). People are sensitive to dangerous objects (Anelli et al., 2012a,b, 2013), and the dangerousness of everyday graspable objects can influence one's surrounding context and the boundaries of peripersonal space (Coello et al., 2012). Furthermore, as revealed by previous studies, contexts systematically influence social categorizations (Freeman et al., 2013), may modify the interpretation of what a facial expression represents (Righart and De Gelder, 2008) and may affect person perception. Hence, contextual cues may influence the impression of a social target. Threatening contextual cues, for instance, can weaken or strengthen race-based stereotypes of aggressiveness and menace (Trawalter et al., 2008): a threatening context is likely to activate negative stereotypes associated with specific social categories.

Building on this body of work, the present study aimed to explore whether and how MR triggered by observations of others' arm movements toward an object can be modulated by social variables such as ethnic group membership. Specifically, we predicted an increased MR when participants observe an action performed by an ingroup member rather than by an outgroup member in line with prior studies (Gutsell and Inzlicht, 2010). In going beyond prior research showing that MR is modulated by social categorization, we investigated whether the perceived threat posed by a social target is likely to modulate a social perceiver's MR response. More specifically, we expected that social threat elicited by a specific outgroup (i.e., stereotypically aggressive) in a specific context (i.e., threatening contextual cues) is likely to amplify the pattern. This hypothesis is in line with previous studies on the effect of morality on MR (Liuzza et al., 2015), which shows that the phenomenon is significantly reduced when observing immoral actions (namely actions related to social threats; Brambilla et al., 2013) in individuals presenting high levels of harm avoidance.

To investigate these hypotheses, we carried out an experimental study where using an action observation paradigm 
we asked participants to provide responses through computer mouse movements. More specifically, participants observed a movie clip showing an actor moving his arm toward an object; then on the screen, a square appeared in a congruent or incongruent position relative to the direction of the actor's movement. Participants were asked to indicate the square position by performing a computer mouse movement toward one of two labels denoting the position (i.e., left or right). We manipulated the group membership of the actors (ingroup vs. outgroup) and the social valence of objects present in the context (neutral vs. threatening) to elicit social menace through contextual cues. MR has been assessed through the use of an action observation paradigm implemented by MouseTracker software (Freeman and Ambady, 2010), a tool that measures behavioral responses by recording computer mouse trajectories and that provides multiple informative dependent variables as detailed in the results section [e.g., initial response times, total response times, the maximum deviation (MD) point of the trajectory and the area under the curve (AUC)].

Moreover, our paradigm, which orthogonally manipulates directions of an actor's arm movement and participant's response direction, allowed us to distinguish the effect of MR from effects elicited by social attention. Indeed, whether the underlying process was social attention, we would expect to find task facilitation when target-stimuli appeared in a position congruent with the direction of the actor's movement when compared to incongruent positions. Otherwise, if we measured MR, we would expect to find task facilitation when actors move the same arm as the one used by participants to provide their answers. If the congruence between response directions and actors' arm movements does not have an effect, the hypothesis on social attention may be discarded.

\section{MATERIALS AND METHODS}

\section{Participants}

The initial sample comprised 82 participants who volunteered to participate in the study in exchange for course credit. Seventy-nine participants were Italian citizens. Three non-Italian participants (1 Ukrainian, 1 Peruvian, and 1 Italo-Argentine) were excluded. Thus, the final sample included 79 participants $\left(M_{\text {age }}=23.43, S D_{\text {age }}=3.76\right.$, range $18-44$ years, 40 males, 39 females). Sixty-seven participants were right-handed, 10 were left-handed, and 2 were ambidextrous according to self-reports; all of them were naive as to the purpose of the experiment. An a priori power analysis for within-subject ANOVA (medium effect size $=0.20$; power $=0.95$ ) suggested minimum $N=46$. We advertised the study and enrolled all individuals who had responded and volunteered to participate.

\section{Ethical Statements}

All participants provided written informed consent before participating in the study. The study was conducted in accordance with the ethical standards outlined in the 1964 Declaration of Helsinki and with the standard ethical procedures recommended by the Italian Association of Psychology (AIP).
The study was specifically approved by the local Ethics Committee of Milano-Bicocca University.

\section{Materials and Procedure}

The experiment was carried out in a dimly lit room. Participants were asked to complete a questionnaire. On the cover page of the questionnaire, participants provided their demographic data. Participants were then presented with a 7 -item national identification scale (e.g., "I identify with Italians"; Cameron, 2004), a 9-item Modern Prejudice Scale for Islamic people (e.g., "For Italians it's normal to have a relationship with an Islamic person"; McConahay, 1986), and a 10-item Motivation to Respond Without Prejudice Scale (e.g., "Being non-prejudiced toward Islamic people is important to my self-concept"; Plant and Devine, 1998). Participants answered these questions on a 7-point scale ranging from 1 (not at all) to 7 (very much).

The second part of the experiment was run on an Intel ${ }^{\circledR}$ Pentium ${ }^{\circledR}$ G630@2.70 GHz personal computer interfaced with a 22-in LCD computer monitor (Asus ${ }^{\circledR}$ VW226; Resolution: 1680 pixels $\times 1050$ pixels; Refresh rate: $59 \mathrm{~Hz}$ ) equipped with the MouseTracker software program (Freeman and Ambady, 2010). After signing the consent form, participants were comfortably seated in a chair positioned approximately $60 \mathrm{~cm}$ away from the monitor from which they received instructions and were presented with photos and brief descriptions of the actors they were going to watch during the experiment (name, age, and nationality). Hence, the ingroup (Gabriele, 26 years old, Italian) and outgroup targets (Haashim, 27, Iraqi) were introduced.

The experiment was then conducted. Each trial began with the computer screen showing a small box labeled "Start" at the lower center of the screen and two response boxes labeled "Left" and "Right" on the upper left and upper right corners of the screen, respectively. After $500 \mathrm{~ms}$, a random video (WMV format; 25 frames/s; 640 pixels $\times 480$ pixels; $1.296 \mathrm{kbps}$; Duration $=1.388 \mathrm{~ms}$ ) was shown at the center of the screen.

As is shown in Figure 1, videos presented the front view of an actor performing an arm movement toward one out of two objects located on a table on his left and right, at a distance of $55 \mathrm{~cm}$ from his torso and $67.5 \mathrm{~cm}$ apart from each other. In all of the videos, the actor looked straight ahead and did not move any body parts other than his arm. The actors used their right hand to move to the right (the participant's left) and left hand to move to the left (the participant's right). At the end of each movie clip, a blue square appeared to the left or right of the screen. Participants were required to ignore the direction of the actor's arm movement and to indicate with a computer mouse movement the position (left or right) in which the square appeared. They were asked to do this as quickly and accurately as possible by moving their computer mouse cursor from the "Start" button to the chosen response box at the top. Responses were allowed only after the square appeared. A blank screen of $500 \mathrm{~ms}$ was inserted between each response box click and the following trial.

We manipulated within participants the actor's ethnic membership (ingroup vs. outgroup) by presenting an Italian and an Arabian target, which is an outgroup stereotypically related to aggression or threat as suggested by recent research (Oswald, 2005; Mange et al., 2012). Moreover, the object valence 


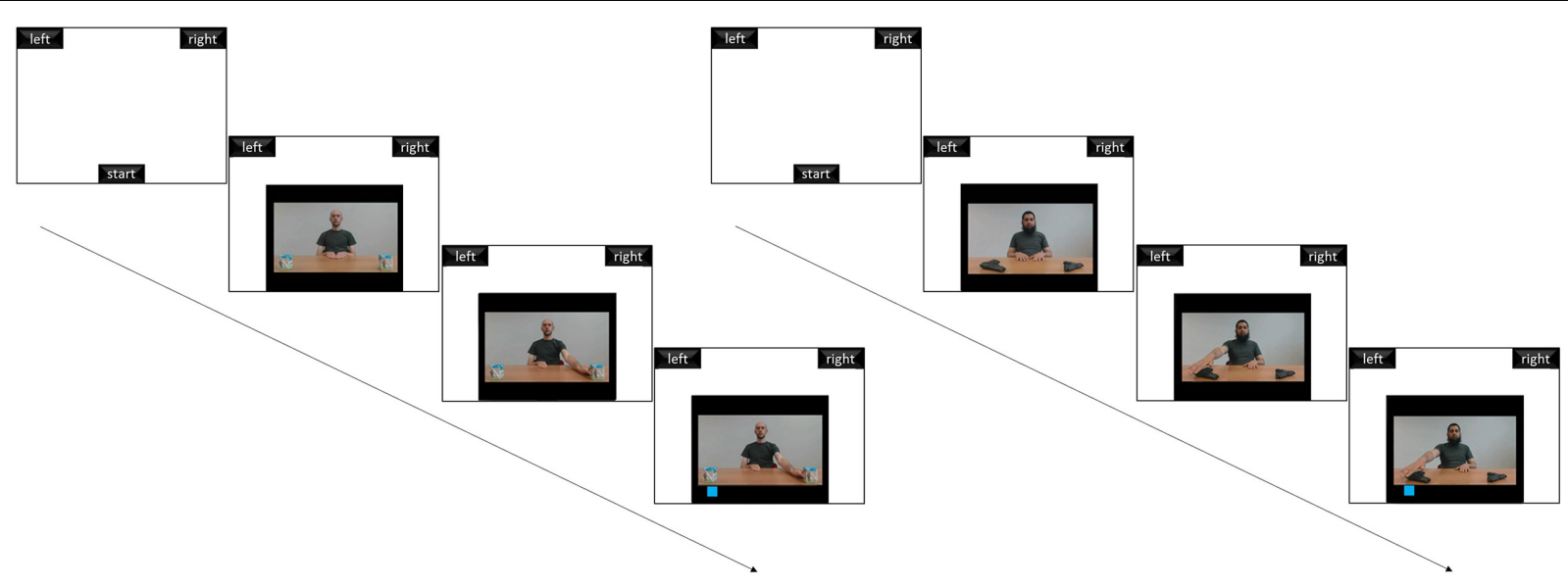

FIGURE 1 | An example of the experimental procedure.

(neutral vs. threatening) was manipulated by showing box of juice as a neutral object or a gun as a menacing object. In a first pretest, 57 Italian participants (26 males; age range: 18-65; $\left.M_{\text {age }}=33.20, S D_{\text {age }}=10.75\right)$ were presented with a picture of Haashim and a picture of Gabriele, and they were asked to evaluate how much they perceived the targets (the order was properly balanced) as threatening ("[...] is a threatening person") and frightening ("[...] is a frightening person") on a 5-point scale (ranging from $1=$ not at all to $5=$ extremely; $\alpha=0.76$ ). In line with prior studies (Mange et al., 2012), the results showed that participants perceived the Arabian outgroup member to be more menacing $(M=2.26, S D=1.02)$ than the Italian ingroup member $(M=1.96, S D=0.87), t(56)=2.12, p=0.04$.

In a second pretest, 14 Italian participants (six males; age range: $21-32 ; M_{\text {age }}=26.07, S D_{\text {age }}=3.95$ ) were presented with a picture of a gun and a picture of a box of juice and they were asked to evaluate how much they perceived the object (the order was properly balanced) as threatening ("[...] is a threatening object") and frightening ("[...] is a frightening object"), $\alpha=0.86$, and graspable ("[...] is a graspable object") on a 5-point scale (ranging from $1=$ not at all to $5=$ extremely). Then, they were asked to rate their overall impression on a 7-point scale range (ranging from $-3=$ extremely negative to $+3=$ extremely positive). The results showed that participants perceived the gun to be more menacing $(M=4.50, S D=0.71)$ than the box of juice $(M=1.14, S D=0.36), t(13)=18.17, p<0.000$, and more negative $(M=2.50, S D=1.7)$ than juices $(M=4.93, S D=1.21)$, $t(13)=4.57, p<0.000$. Moreover, guns were perceived to be as graspable as the boxes of juice, $p=0.90$.

We thus showed a total of 16 different videos as a result of combinations of these four variables (membership: ingroup vs. outgroup; actor's movement direction: left vs. right; object valence: neutral vs. threatening; square position: left vs. right). Each video was presented randomly six times throughout the experiment, resulting in a total of 96 trials with four additional trials presented at the beginning of the session as training trials, producing a total of 100 trials. Trials were split into two blocks: half of the participants were presented with a first block showing actors moving toward neutral objects (e.g., box of juice) followed by a second block with actors moving toward threatening objects (e.g., gun); the other half was presented with a first block showing actors moving toward threatening objects followed by a second block with actors moving toward neutral objects.

Hence, the experimental design consisted of a 2 (block order: neutral first vs. threatening first) $\times 2$ (membership: ingroup vs. outgroup) $\times 2$ (object valence: neutral vs. threatening $) \times 2$ (actor's movement direction: left vs. right) $\times 2$ (square position: left vs. right) design, with the first factor manipulated between subjects and the latter factors manipulated within subjects. It is important to note that 'left' and 'right' always refer to the participants' point of view (the position on the screen); thus, for instance an actor's movement to left means an actor's movement performed with his right hand to his right side. Moreover, as we excluded errors from the data, square positioning corresponds to the direction of a participant's response. Participants were randomly assigned to one of the two experimental groups. As provided by the MouseTracker software, the initial times (IT), response times (RT), AUC, and MD measures were recorded.

\section{RESULTS}

\section{Preliminary Analysis}

Regarding the mouse-tracking data, we conducted the analyses on four different indices provided by MouseTracker that represented our crucial dependent variables. Indices are the following: initial response times (IT), that represents the starting point in which participants begin the mouse trajectory in order to indicate their responses; total $\mathrm{RT}$, which represents the total amount of time taken by participants to perform their responses, $\mathrm{MD}$, which is a common index for assessing response competition, and AUC, which represents another index for evaluate response competition in terms of larger positive AUC values that indicate greater response competition.

Seventy-seven of the 79 participants performed the task using the mouse with their right hand. Since it was not feasible 
to conduct statistical analyses on two cases, participants who performed the task using their left hand were excluded from the sample. Then, training trials have been eliminated from the analysis. Moreover, trials in which participants did not provide a correct response (e.g., answer 'left' when the square compared on the right or vice versa) were discarded (15 of 7,968 recorded trials, corresponding to $0.19 \%$ ). Next, we removed trials in which the dependent variables indices (IT, RT, AUC, and MD) were greater or lower than $\pm 2.5 \mathrm{SD}$. With this procedure 485 trails were removed (485 of 7,968 recorded trials, corresponding to 6.09\%). Finally, we excluded three outlier data points with standardized values greater than \pm 3 from the IT, RT, MD, and AUC averages. Thus, final analysis was conducted on a sample of 74 subjects.

Regarding the three explicit scale analysis, after reversing the items negatively phrased in the questionnaire and testing the scales reliability (national identification: Cronbach's $\alpha=0.72$; prejudice: Cronbach's $\alpha=0.79$; motivation to avoid prejudice: Cronbach's $\alpha=0.85$ ), we computed the average scores for each measure.

Then, to control for possible effects of block order and square position, a 2 (block order: neutral first vs. threatening first) $\times 2$ (membership: ingroup vs. outgroup) $\times 2$ (object valence: neutral vs. threatening) $\times 2$ (actor's movement direction: left vs. right) $\times 2$ (square position: left vs. right) ANOVA was computed, using the first variable as between-participants factor and the other variables as within-participants factors.

The ANOVA carried out on the crucial dependent variables (IT, Total RT, MD, and AUC) did not reveal any interaction effect with block order $\left(p_{\mathrm{s}}>0.05\right)$.

Moreover, the five- and four way interaction with square position (corresponding to the participant's response direction) was non-significant $\left(p_{\mathrm{s}}>0.05\right)$. This result was crucial in order to exclude a possible influence of social attention (namely the congruence between agent's movement direction and the position of the target stimulus to which participants were called to respond). Indeed, if the underlying process were due to social attention, we would expect to find task facilitation when square appeared in a position that was congruent with respect to the direction of the actor's arm movement (e.g., actor's movement toward left - square on the left) and, on the other hand, to find a greater response delay in incongruent positions (e.g., actor's movement toward right - square on the left).

Also participants' handedness, when introduced as factor in the aforementioned analysis, proved to be ineffective $\left(p_{\mathrm{s}}>0.05\right)$.

Therefore, we collapsed data across these factors and the following analyses do not consider these variables.

\section{Initial Times}

After the preliminary analyses a 2 (membership: ingroup vs. outgroup) $\times 2$ (object valence: neutral vs. threatening) $\times 2$ (actor's movement direction: left vs. right) within participants ANOVA on the IT was computed.

In line with the hypothesis, the analysis yielded a significant main effect of membership, $F(1,73)=17.41, p<0.001, \eta_{p}^{2}=0.19$; indeed, participants started their response with mouse earlier when observing an ingroup actor arm's movement $(M=78.99$, $S D=3.08)$ than when observing an outgroup actor $(M=85.89$,
$S D=3.31$ ). This result can be interpreted as a higher level of motor activation when perceiving ingroup members' acts.

As displayed in Figure 2, the ANOVA yielded a significant three-way interaction between membership, actor's movement direction, and object valence, $F(1,73)=21.89, p<0.001$, $\eta_{\mathrm{p}}^{2}=0.23$. As showed by post hoc analyses (LSD tests), when the objects presented in the context were threatening (i.e., guns), and the actor was an ingroup member, participants were faster while perceiving the agent moving to left (it is worthy to note that this condition would imply MR since the actor in front view was executing the movement with his right hand, that was the same hand participants were using to perform the task; $M=73.95$, $S D=3.49)$ than to the right $(M=83.80, S D=3.57), p<0.001$. On the other side, with threatening object but in outgroup condition, participants were even slower while perceiving the agent moving to the left $(M=93.04, S D=4.27)$ than to the right $(M=77.70, S D=3.76), p<0.001$. These results can be interpreted as a higher MR with the ingroup member; in stark contrast, the outgroup member's movement seems to delay motor response. Interestingly, in the other block, when the objects were neutral, in ingroup condition, there was no difference in IT between trials directed to the left $(M=78.98, S D=3.42)$ and to the right $(M=79.24, S D=3.93), p=0.94$. Analogously, in outgroup condition, no difference arose in IT between trials directed to the left $(M=86.17, S D=3.80)$ and to the right $(M=86.64, S D=3.93), p=0.86$. Thus, these results seem to suggest that the crucial interaction between actor's movement direction and membership arouses only when cues in the social context elicit threat.

Moreover, post hoc analyses (LSD tests) showed also significant differences in IT between neutral and threatening objects when participants observed an outgroup member; in particular, they were faster when the actor was moving to the left toward the box of juice than when he was moving to the left toward the gun, $p=0.05$. Conversely, they were slower when the outgroup member was moving to the right toward the box of juice than when he was moving to the right toward the gun, $p=0.03$. Interestingly, no differences arose in IT between neutral and threatening objects in the ingroup condition, neither when the actor was moving to the left, $p=0.14$, nor to the right, $p=0.16$.

Moreover, the ANOVA revealed a significant two-way interaction between membership and actor's movement direction, $F(1,73)=17.83, p<0.001, \eta_{p}^{2}=0.20$, which may be justified by the three-way interaction. The analysis did not yield any other significant effect, $p_{\mathrm{s}}>0.36$.

\section{Total Response Times}

Then a 2 (membership: ingroup vs. outgroup) $\times 2$ (object valence: neutral vs. threatening) $\times 2$ (actor's movement direction: left vs. right) ANOVA on total RT was carried out.

As displayed in Figure 3, the ANOVA yielded a significant three-way interaction between membership, actor's movement direction, and object valence, $F(1,73)=5.84, p=0.02, \eta_{\mathrm{p}}^{2}=0.07$.

As showed by post hoc analyses (LSD tests), when the objects presented in the context were threatening and the actor was an outgroup member, participants were slower when observing an agent moving to the left $(M=785.73, S D=19.19)$ than to the 


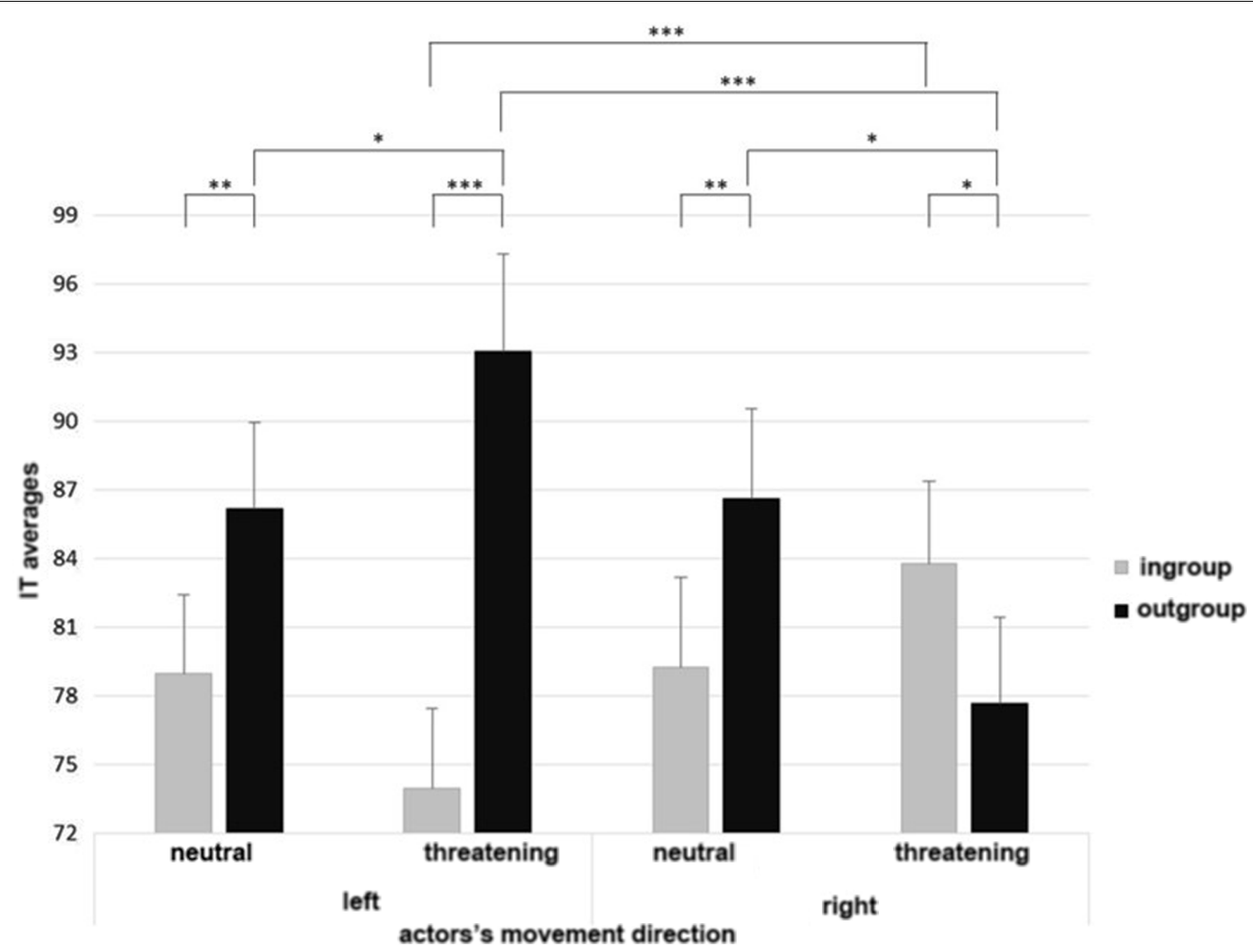

FIGURE 2 | Three-way interactions between membership, actor's movement direction, and object valence [Initial Times (IT)]. Asterisks highlight significantly different means comparisons $\left({ }^{*} p<0.05,{ }^{* *} p<0.01,{ }^{* * *} p<0.001\right)$. Error bars represent standard errors.

right $(M=763.64, S D=18.59), p=0.03$. On the other side, in ingroup condition with menacing objects, no differences in RT were revealed between trials directed to the left $(M=759.09$, $S D=16.30)$ and to the right $(M=761.97, S D=18.84), p=0.75$.

Interestingly, in the other block, when the objects were neutral and in outgroup condition, participants were faster when the agent was moving to the left $(M=756.75, S D=18.72)$ than to the right $(M=772.49, S D=18.62), p=0.08$. In ingroup condition with neutral objects, no differences in RT were revealed between trials directed to the left $(M=763.71, S D=18.15)$ and to the right $(M=763.11, S D=19.36), p=0.97$.

Post hoc analyses (LSD tests) showed also significant differences in IT between neutral and threatening objects when the agent was an outgroup member; in particular, when he was moving to the left, participants were slower in the threatening condition (i.e., with guns) than in the neutral one (i.e., with box of juice), $p=0.04$. There were no differences between neutral and threatening objects when the outgroup member was moving to the right, $p=0.59$. No differences in RT arose between neutral and threatening objects in ingroup condition, neither when the agent was moving to the left, $p=0.70$, nor to the right, $p=0.93$. Furthermore, the ANOVA revealed a significant twoway interaction between object valence and actor's movement direction, $F(1,73)=3.94, p=0.05, \eta_{\mathrm{p}}^{2}=0.05$, which may be justified by the three-way interaction. In sum the results partially confirmed data on IT. We should consider that the present index represents the total time used by participants for providing their response, thus participants could use the entire time of the trajectory to adjust their answer, until they clicked the response box.

No other effects were found, $p_{\mathrm{s}}>0.17$.

\section{Maximum Deviation and Area Under the Curve}

We also computed a 2 (membership: ingroup vs. outgroup) $\times 2$ (object valence: neutral vs. threatening) $\times 2$ (actor's movement direction: left vs. right) ANOVA on two other indices used to measure the response competition: MD and AUC. As shown, using MD versus AUC for the same data does not substantially change the results (Freeman et al., 2008). No effects were found neither on MD nor on AUC, $p_{\mathrm{s}}>0.08$.

These results revealed that participants did not experience response competition in providing their mouse responses. The fact that we found effects on initial and RT and not on the trajectories can be ascribed to the type of task. As shown, we asked participants to indicate the position (left/right) in which the stimulus appeared, that is a simple and quite effortless task. The easiness of this kind of task could have led participants to provide their responses without uncertainties. 


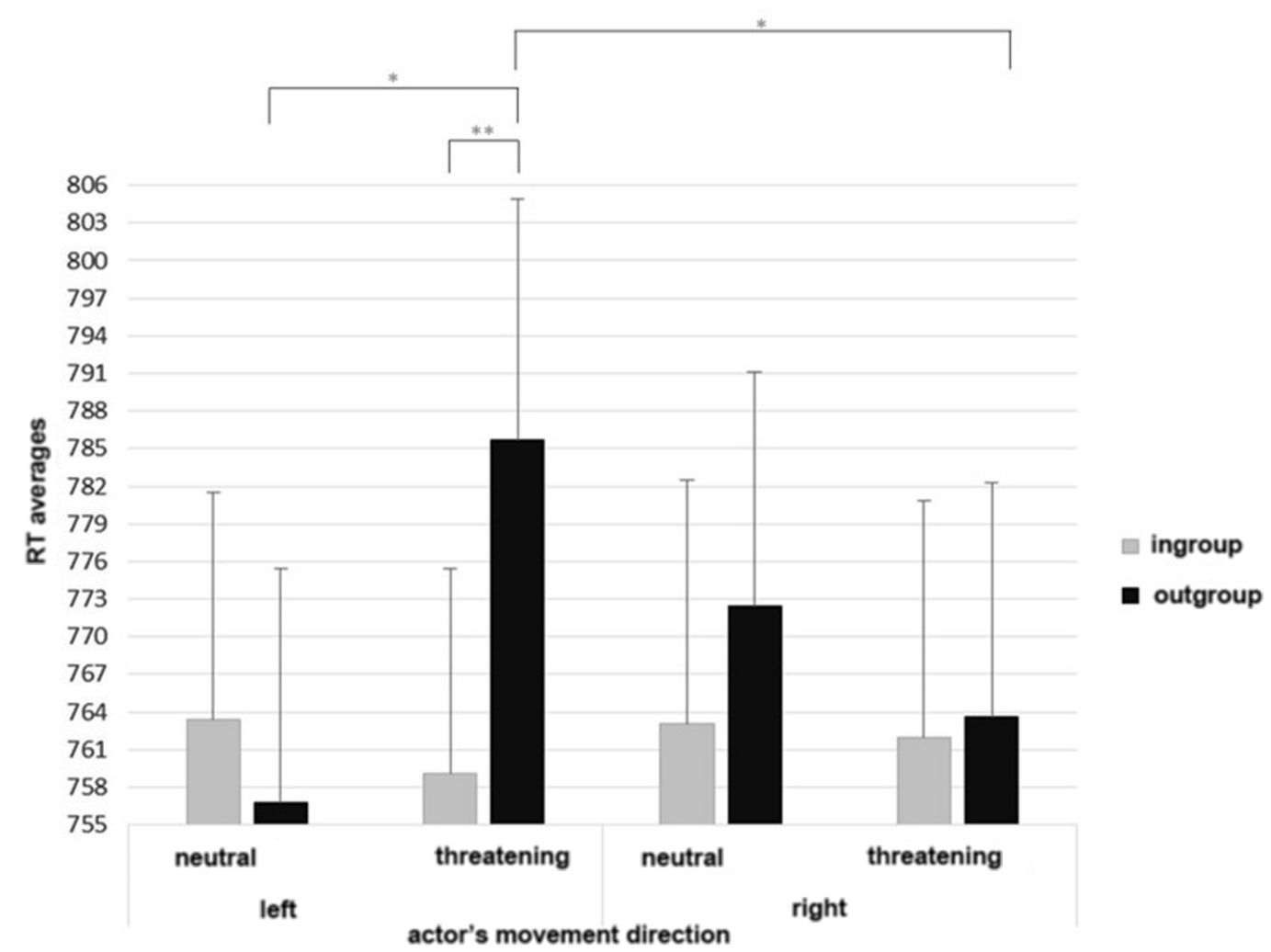

FIGURE 3 | Three-way interactions between membership, actor's movement direction, and object valence (Total Response Times). Asterisks highlight significantly different means comparisons $\left({ }^{*} p<0.05,{ }^{* *} p<0.01,{ }^{* * *} p<0.001\right)$. Error bars represent standard errors.

\section{Explicit Scales}

Finally, in order to investigate whether participants' level of identification with the national group (i.e., Italians), level of prejudice and level of motivation to avoid prejudice were likely to moderate the effects, moderated moderation models were explored using PROCESS macro (Hayes, 2013; model 3, 5000 bootstrap resampling) with actor's movement direction (left vs. right; left defined the condition in which participants provided their responses using the same hand as the one used by the actor in the videos) as an independent variable, membership (ingroup vs. outgroup) as a moderator, the explicit measures as moderator of the moderator, and IT and total RT as dependent variables. The explicit measures used were: identification with the ingroup $(M=4.16, S D=0.91)$, prejudice toward Islamic people $(M=3.20, S D=0.87)$, and motivation to avoid prejudice $(M=3.88, S D=1.06)$. None of these models revealed significant interaction, $p_{\mathrm{s}}>0.35$. Thus, these measures of identification and prejudice did not moderate our effects on MR revealed by previous analyses.

In line with our hypothesis, the results suggest that people are prone to a higher level of MR when observing an action performed by the ingroup members rather than by the outgroup members. Hence, participants seem to resonate better with their ingroup; conversely, outgroup members' movements tend to delay motor responses. Moreover, the perceived social threat elicited by socially menacing cues interferes with this effect; when participants observed an outgroup member moving toward a weapon, they were slower at providing responses. Thus, our results seem to suggest that the crucial interaction between actor's movement direction and membership occurs only when cues in a social context elicit threat.

\section{DISCUSSION}

A robust line of research has widely suggested that observing another person's action activates corresponding motor representations in the observer (Iacoboni et al., 1999; Rizzolatti et al., 2001; Rizzolatti and Craighero, 2004); moreover, prior studies have shown that MR may be influenced by characteristics of an action made and of the performer of that action (MolnarSzakacs et al., 2007; Cheng et al., 2008). The present study investigated whether and how perceptions of social threat elicited by an outgroup member and by social cues can modulate motor responses when a person observes others' actions.

Our findings suggest that MR during action observation is likely to be modulated by ethnic group membership. Indeed, consistent with our hypothesis and in line with the existing literature (Molnar-Szakacs et al., 2007; Avenanti et al., 2010; Gutsell and Inzlicht, 2010, 2013), participants tended to resonate better with their ingroup; in fact, when the ingroup actor 
executed a movement with the same hand as the one used by participants to provide their responses, motor facilitation was found. Conversely, when the outgroup member performed an action using the same hand as participants, a delay in response was found.

These results can be interpreted in the light of the existing literature on group membership and social interactions. Indeed, people empathize more with the ingroup members than the outgroup members (Dovidio and Gaertner, 2010; Trawalter et al., 2012; Gutsell and Inzlicht, 2013) and tend to perceive the ingroup members more favorably and as more similar to themselves (Hewstone et al., 2002). Studies on spontaneous synchrony, mimicry and motor coordination consistently show that individuals are less likely to synchronize their movements with those whom they harbor negative feelings for (Miles et al., 2010). Moreover, this effect may be partially due to familiarity: ingroup members are usually the ones with whom we most often interact (Fiske, 1992). Therefore, moving with others or resonating with their movements could be considered an early embodied form of relation with our conspecifics that could be affected by social perception and cultural inter-individual differences (Sacheli et al., 2015).

However, the present research complements and extends this emerging line of research, going beyond a mere ingroup bias effect and exploring the role of social threat. Our main finding suggests that the perception of social threat, elicited by an outgroup member stereotypically associated with social aggression (Oswald, 2005) and by contextual cues, is likely to interfere with motor response provided when facing an agent's action. In fact, when participants faced a stereotypically aggressive outgroup member moving toward a weapon, they were slower to initiate motor responses. Interestingly, the interaction effect between membership and movement direction disappeared when contextual cues were neutral and unlikely to elicit social threat.

It is worthy to note two additional nuances of our results. First, in neutral conditions, the MR effect disappeared when participants viewed an ingroup member. One possibility for why we did not find MR in this condition is because in this specific context (i.e., when threat is not salient), an individual may be likely to focus on the task and to disregard social stimuli. By contrast, when social threat is elicited, an individual is more heavily influenced by the presence and movements of social targets that can become potentially menacing. As highlighted by the literature of threat and attention, evolutionary relevant threatening stimuli are effective at ensnaring attentional resources (Öhman et al., 2001; Fox et al., 2002), thus interfering with goal-directed activity (Williams et al., 1988, 1996).

Second, no difference was found between neutral and threatening conditions when participants faced the ingroup members: RT differed between neutral and threatening conditions only when participants observed an outgroup agent's movements. This pattern could suggest that threatening objects are likely to activate negative stereotypes associated with specific social categories (e.g., Arabians). As revealed by a prior study, threat cues might weaken or strengthen racebased stereotypes of aggressiveness and menace (Trawalter et al., 2008): hence, only in particular conditions (e.g., in our experiment when participants were presented with guns), negative stereotypes associated with racial minorities may be active and likely to garner attention. As argued by Trawalter et al. (2008), information gleaned from bottom-up (e.g., contextual cues) and top-down processing (e.g., stereotypic expectancies) may have interactive effects on social perception. From these findings, future studies could further explore effects of group membership by presenting participants with control outgroups not stereotypically associated with threat or aggression. In this way, it would be possible to control whether effects that emerge are to be ascribed to the presence of a generic outgroup or to a particular and specific menacing outgroup.

This result seems to be at odds with previous research (Gutsell and Inzlicht, 2013) showing that when outgroup behavior is negative and threatening, individuals begin to process them as ingroup members, thus reducing ingroup bias effects on MR. However, the results are in line with functional perspectives on person perception (e.g., Todorov et al., 2009), suggesting that when an outgroup behavior is threatening, people can allocate cognitive resources to the threatening individual. This form of cognitive resource allocation does not necessarily apply to active movements. In fact, the delay found in response to the Arabian outgroup member as he moved toward a gun can be interpreted as a freeze reaction to a harmfully perceived event as suggested by several studies that show that spontaneous body responses to social threat cues elicit freeze-like behaviors in humans (Roelofs et al., 2010). Moreover, our findings are in line with previous studies on the effects of morality on MR (Liuzza et al., 2015) showing a decrease in MR when observing immoral actions, in particular in individuals presenting high levels of harm avoidance. As an extension of this work, it will be interesting to investigate the relation between MR and visual attention. Indeed, future works could explore whether motor freezing arising as a reaction to a menacing outgroup could be associated with greater visual attention to this agent; participants, for instance, could be attracted to a menacing target while at the same time being frozen in their motor reactions. For this reason, it will be useful to integrate the measure of RT and computer mouse trajectories provided through MouseTracker with the analysis of eye movements using an eye tracker.

Furthermore, an added value of this work lies in the experimental methodology we adopted; on one hand, we used an action observation paradigm implemented using MouseTracker software (Freeman and Ambady, 2010), which is a tool that measures behavioral responses by recording computer mouse trajectories and that provides multiple informative dependent variables (e.g., initial RT, total RT, MD point of the trajectory, and AUC). In this way, it is possible to monitor and implicitly investigate the entire motor response to understand at what level of the process the effects interfere. Thanks to this experimental tool, it was possible to highlight that the effects of our variables are particularly precocious, as they interfered with the task early on in the process (IT).

Moreover, the paradigm we used allows to discriminate between two competing underlying processes. Through our action observation paradigm, we presented participants with 
congruent (i.e., actor's movement toward the left - square on the left) or incongruent (i.e., actor's movement toward the right square on the left) stimuli. Several studies on social attention that have adopted a modified version of the Posner paradigm (Posner, 1978, 1980; Frischen et al., 2007) and the Social Simon Paradigm (Tsai and Brass, 2007; Dolk et al., 2011) manipulated the congruence of stimuli in the same way. In our study, the orthogonal manipulation of the square's position (corresponding with participant response directions) alongside membership and actor's movement directions allowed us to disentangle effects of MR from effects elicited by social attention. Indeed, if the underlying process were due to social attention, we would expect to find task facilitation when stimuli (i.e., square) appeared in a position congruent to the direction of the actor's movement. With incongruent positions, we would expect to find greater response competition and thus an increase in the difficulty in making a decision. Otherwise, if we measured MR, we would expect to find task facilitation with an actor moving the same arm as the one used by participants to provide their answers. The motor facilitation result found when the actors moved the same arm as the one used by participants to provide their answers supports the hypothesis on MR. Moreover, as the square's position did not interact with our crucial effects, the hypothesis on social attention can be discarded.

Moreover, we showed that our findings are not affected by levels of prejudice; both people with a high level of prejudice toward Arabian people and those with low prejudice were influenced when performing the task. From a social psychology perspective, the results are compatible with stereotype activation rather than with prejudice effects. Stereotypes are fixed and over-generalized beliefs about people that are based on their membership to a particular social category (for a review, see Hilton and von Hippel, 1996). Previous research has widely demonstrated that trait concepts and stereotypes, which are generally shared within a community and stable over time, become automatically active in the presence of a relevant behavior or stereotyped-group features. Given their pervasiveness in cultural contexts, stereotypes can have detrimental effects on social perception and elicit stereotype-consistent behavioral responses independent from the individual's attitudes and personal values (i.e., prejudices) toward that social group (Devine, 1989; Devine and Elliot, 1995). From

\section{REFERENCES}

Aglioti, S. M., Cesari, P., Romani, M., and Urgesi, C. (2008). Action anticipation and motor resonance in elite basketball players. Nat. Neurosci. 11, 1109-1116. doi: 10.1038/nn.2182

Anelli, F., Borghi, A. M., and Nicoletti, R. (2012a). Grasping the pain: motor resonance with dangerous affordances. Conscious. Cogn. 21, 1627-1639. doi: 10.1016/j.concog.2012.09.001

Anelli, F., Nicoletti, R., Kalkan, S., Sahin, E., and Borghi, A. M. (2012b). "Human and robotics hands grasping danger," in Proceeding of the 2012 International Joint Conference on Neural Networks (IJCNN) (Piscataway, NJ: IEEE), 1-8. doi: 10.1109/IJCNN.2012.6252590

Anelli, F., Ranzini, M., Nicoletti, R., and Borghi, A. M. (2013). Perceiving object dangerousness: an escape from pain? Exp. Brain Res. 228, 457-466. doi: 10.1007/s00221-013-3577-2 our results and in consideration of the fact that most people internalize stereotypes in the course of normal socialization, future studies could also investigate the role of individual characteristics (e.g., age) in the development of stereotypes. From a lifespan perspective, it will be interesting to explore the development of adaptive human skills (Knoblich and Sebanz, 2006) to resonate with co-specifics during different stages of human life and particularly in relation to ingroup bias and social threat perception.

Finally, from an intergroup point of view, the effect of social threat on MR could have important implications; such an effect may affect the first stage of social perception and may alter communication and interactions with outgroup individuals and the quality of intergroup relations. Moving together and coordinating with other movements can bridge the gap between the self and others and create a sense of social connection. Therefore, investigating the effects of facilitating and hindering factors of MR could help understanding the challenges associated with intergroup interactions. To conclude, the present study provides avenues for further studies on the role of variables likely to reduce intergroup threats and to promote more cooperative relations between members of different social groups.

\section{AUTHOR CONTRIBUTIONS}

RC drafted the first version of the manuscript. RC and SS run the study and conducted data analyses. RC, SS, PR and RA-G conceived the study idea and the experimental paradigm and provided comments on the first version of the manuscript.

\section{FUNDING}

This work was supported by a grant (Fondo di Ateneo) from the University of Milano-Bicocca to SS, PR, and RA-G.

\section{ACKNOWLEDGMENT}

We would like to thank Licia Anna De Salve for her help in collecting data.

Aron, A., Aron, E. N., and Smollan, D. (1992). Inclusion of other in the self scale and the structure of interpersonal closeness. J. Pers. Soc. Psychol. 63, 596-612. doi: 10.1037/0022-3514.63.4.596

Avenanti, A., Bueti, D., Galati, G., and Aglioti, S. M. (2005). Transcranial magnetic stimulation highlights the sensorimotor side of empathy for pain. Nat. Neurosci. 8, 955-960. doi: 10.1038/nn1481

Avenanti, A., Minio-Paluello, I., Sforza, A., and Aglioti, S. M. (2009). Freezing or escaping? Opposite modulations of empathic reactivity to the pain of others. Cortex 45, 1072-1077. doi: 10.1016/j.cortex.2008. 10.004

Avenanti, A., Sirigu, A., and Aglioti, S. M. (2010). Racial bias reduces empathic sensorimotor resonance with other-race pain. Curr. Biol. 20, 1018-1022. doi: 10.1016/j.cub.2010.03.071

Brambilla, M., Sacchi, S., Pagliaro, S., and Ellemers, N. (2013). Morality and intergroup relations: threats to safety and group image predict the desire to 
interact with outgroup and ingroup members. J. Exp. Soc. Psychol. 49, 811-821. doi: 10.1016/j.jesp.2013.04.005

Brewer, M. B., and Gardner, W. (1996). Who is this "We"? Levels of collective identity and self representations. J. Pers. Soc. Psychol. 71, 83-93. doi: 10.1037/0022-3514.71.1.83

Buccino, G., Vogt, S., Ritzl, A., Fink, G. R., Zilles, K., Freund, H. J., et al. (2004). Neural circuits underlying imitation learning of hand actions: an event-related fMRI study. Neuron 42, 323-334. doi: 10.1016/S0896-6273(04) 00181-3

Cameron, J. E. (2004). A three-factor model of social identity. Self Identity 3, 239-262. doi: 10.1080/13576500444000047

Cappella, J. N. (1997). Behavioral and judged coordination in adult informal social interactions: vocal and kinesic indicators. J. Pers. Soc. Psychol. 72, 119-131. doi: 10.1037/0022-3514.72.1.119

Chartrand, T. L., and Bargh, J. A. (1999). The chameleon effect: the perceptionbehavior link and social interaction. J. Pers. Soc. Psychol. 76, 893-910. doi: 10.1037/0022-3514.76.6.893

Cheng, Y., Lee, P. L., Yang, C. Y., Lin, C. P., Hung, D., and Decety, J. (2008). Gender differences in the mu rhythm of the human mirror-neuron system. PLoS ONE 3:e2113. doi: 10.1371/journal.pone.0002113

Coello, Y., Bourgeois, J., and Iachini, T. (2012). Embodied perception of reachable space: how do we manage threatening objects? Cogn. Process. 13, 131-135. doi: 10.1007/s10339-012-0470-z

Cuddy, A. J., Fiske, S. T., and Glick, P. (2008). Warmth and competence as universal dimensions of social perception: the stereotype content model and the BIAS map. Adv. Exp. Soc. Psychol. 40, 61-149. doi: 10.1016/S0065-2601(07) 00002-0

Decety, J., and Jackson, P. L. (2004). The functional architecture of human empathy. Behav. Cogn. Neurosci. Rev. 3, 71-100. doi: 10.1177/ 1534582304267187

Désy, M. C., and Théoret, H. (2007). Modulation of motor cortex excitability by physical similarity with an observed hand action. PLOS ONE 2:e971. doi: 10.1371/journal.pone.0000971

Devine, P. G. (1989). Stereotypes and prejudice: their automatic and controlled components. J. Pers. Soc. Psychol. 56, 5-18. doi: 10.1037/0022-3514.56.1.5

Devine, P. G., and Elliot, A. J. (1995). Are racial stereotypes really fading? The Princeton trilogy revisited. Pers. Soc. Psychol. Bull. 21, 1139-1150. doi: $10.1177 / 01461672952111002$

Dijksterhuis, A., and Bargh, J. A. (2001). The perception-behavior expressway: automatic effects of social perception on social behavior. Adv. Exp. Soc. Psychol. 33, 1-40. doi: 10.1016/S0065-2601(01)80003-4

Dovidio, J. F., and Gaertner, S. L. (2010). "Intergroup bias," in Handbook of Social Psychology, 5th Edn, Vol. 2, eds S. T. Fiske, D. Gilbert, and G. Lindzey (New York, NY: John Wiley \& Sons, Inc), 1084-1121.

Dolk, T., Hommel, B., Colzato, L. S., Schütz-Bosbach, S., Prinz, W., and Liepelt, R. (2011). How "social" is the social Simon effect? Front. Psychol. 2:84. doi: 10.3389/fpsyg.2011.00084

Fadiga, L., Craighero, L., and Olivier, E. (2005). Human motor cortex excitability during the perception of others' action. Curr. Opin. Neurobiol. 15, 213-218. doi: 10.1016/j.conb.2005.03.013

Fadiga, L., Fogassi, L., Pavesi, G., and Rizzolatti, G. (1995). Motor facilitation during action observation: a magnetic stimulation study. J. Neurophysiol. 73, 2608-2611.

Fiske, A. P. (1992). The four elementary forms of sociality: framework for a unified theory of social relations. Psychol. Rev. 99, 689-723. doi: 10.1037/0033295X.99.4.689

Fiske, S. T., Cuddy, A. J., and Glick, P. (2007). Universal dimensions of social cognition: warmth and competence. Trends Cogn. Sci. 11, 77-83. doi: 10.1016/j.tics.2006.11.005

Fox, E., Russo, R., and Dutton, K. (2002). Attentional bias for threat: evidence for delayed disengagement from emotional faces. Cogn. Emot. 16, 355-379. doi: $10.1080 / 02699930143000527$

Freeman, J. B., and Ambady, N. (2010). Mouse tracker: software for studying realtime mental processing using a computer mouse-tracking method. Behav. Res. Methods 42, 226-241. doi: 10.3758/BRM.42.1.226

Freeman, J. B., Ambady, N., Rule, N. O., and Johnson, K. L. (2008). Will a category cue attract you? Motor output reveals dynamic competition across person construal. J. Exp. Psychol. Gen. 137, 673. doi: 10.1037/a0013875
Freeman, J. B., Ma, Y., Han, S., and Ambady, N. (2013). Influences of culture and visual context on real-time social categorization. J. Exp. Soc. Psychol. 49, 206-210. doi: 10.1016/j.jesp.2012.10.015

Frischen, A., Bayliss, A. P., and Tipper, S. P. (2007). Gaze cueing of attention: visual attention, social cognition, and individual differences. Psychol. Bull. 133, 694-724. doi: 10.1037/0033-2909.133.4.694

Frith, C. D. (2007). The social brain? Philos. Trans. R. Soc. Lond. B Biol. Sci. 362, 671-678. doi: 10.1098/rstb.2006.2003

Fourkas, A. D., Avenanti, A., Urgesi, C., and Aglioti, S. M. (2006). Corticospinal facilitation during first and third person imagery. Exp. Brain Res. 168, 143-151. doi: 10.1007/s00221-005-0076-0

Gallese, V., Fadiga, L., Fogassi, L., and Rizzolatti, G. (1996). Action recognition in the premotor cortex. Brain 119, 593-609. doi: 10.1093/brain/119.2.593

Gutsell, J. N., and Inzlicht, M. (2010). Empathy constrained: prejudice predicts reduced mental simulation of actions during observation of outgroups. J. Exp. Soc. Psychol. 46, 841-845. doi: 10.1016/j.jesp.2010.03.011

Gutsell, J. N., and Inzlicht, M. (2013). "Using EEG mu-suppression to explore group biases in motor resonance," in Neuroscience of Prejudice and Intergroup Relations, eds B. Derks, D. Scheepers, and N. Ellemers (Milton Park: Psychology Press), 279-297.

Hayes, A. F. (2013). Introduction to Mediation, Moderation, and Conditional Process Analysis: A Regression-Based Approach. New York, NY: Guilford Press.

Hewstone, M., Rubin, M., and Willis, H. (2002). Intergroup bias. Annu. Rev. Psychol. 53, 575-604. doi: 10.1146/annurev.psych.53.100901.135109

Hilton, J. L., and von Hippel, W. (1996). Stereotypes. Annu. Rev. Psychol. 47, 237-271. doi: 10.1146/annurev.psych.47.1.237

Hurley, S. (2008). The shared circuits model (SCM): how control, mirroring, and simulation can enable imitation, deliberation, and mindreading. Behav. Brain Sci. 31, 1-2; discussion 22-58. doi: 10.1017/S0140525X07003123

Iacoboni, M. (2005). Neural mechanisms of imitation. Curr. Opin. Neurobiol. 15, 632-637. doi: 10.1016/j.conb.2005.10.010

Iacoboni, M. (2009). Imitation, empathy, and mirror neurons. Annu. Rev. Psychol. 60, 653-670. doi: 10.1146/annurev.psych.60.110707.163604

Iacoboni, M., Woods, R. P., Brass, M., Bekkering, H., Mazziotta, J. C., and Rizzolatti, G. (1999). Cortical mechanisms of human imitation. Science 286, 2526-2528. doi: 10.1126/science.286.5449.2526

Kaplan, J. T., Aziz-Zadeh, L., Uddin, L. Q., and Iacoboni, M. (2008). The self across the senses: an fMRI study of self-face and self-voice recognition. Soc. Cogn. Affect. Neurosci. 3, 218-223. doi: 10.1093/scan/nsn014

Kilner, J. M., Paulignan, Y., and Blakemore, S. J. (2003). An interference effect of observed biological movement on action. Curr. Biol. 13, 522-525. doi: $10.1016 /$ S0960-9822(03)00165-9

Knoblich, G., and Sebanz, N. (2006). The social nature of perception and action. Curr. Dir. Psychol. Sci. 15, 99-104. doi: 10.1111/j.0963-7214.2006. 00415. $\mathrm{x}$

Liew, S. L., Han, S., and Aziz-Zadeh, L. (2011). Familiarity modulates mirror neuron and mentalizing regions during intention understanding. Hum. Brain Mapp. 32, 1986-1997. doi: 10.1002/hbm.21164

Liuzza, M. T., Candidi, M., Sforza, A. L., and Aglioti, S. M. (2015). Harm avoiders suppress motor resonance to observed immoral actions. Soc. Cogn. Affect. Neurosci. 10, 72-77. doi: 10.1093/scan/nsu025

Losin, E. A. R., Iacoboni, M., Martin, A., Cross, K. A., and Dapretto, M. (2012). Race modulates neural activity during imitation. Neuroimage 59, 3594-3603. doi: 10.1016/j.neuroimage.2011.10.074

Mange, J., Chun, W. Y., Sharvit, K., and Belanger, J. J. (2012). Thinking about arabs and muslims makes americans shoot faster: effects of category accessibility on aggressive responses in a shooter paradigm. Eur. J. Soc. Psychol. 42, 552-556. doi: 10.1002/ejsp.1883

McConahay, J. B. (1986). "Modern racism, ambivalence, and the modern racism scale," in Prejudice, Discrimination, and Racism, eds J. F. Dovidio and S. L. Gaertner (San Diego, CA: Academic Press), 91-125.

Miles, L. K., Griffiths, J. L., Richardson, M. J., and Macrae, C. N. (2010). Too late to coordinate: contextual influences on behavioral synchrony. Eur. J. Soc. Psychol. 40, 52-60. doi: 10.1002/ejsp.721

Minio-Paluello, I., Baron-Cohen, S., Avenanti, A., Walsh, V., and Aglioti, S. M. (2009). Absence of embodied empathy during pain observation in Asperger syndrome. Biol. Psychiatry 65, 55-62. doi: 10.1016/j.biopsych.2008. 08.006 
Molnar-Szakacs, I., Wu, A. D., Robles, F. J., and Iacoboni, M. (2007). Do you see what I mean? Corticospinal excitability during observation of culture-specific gestures. PLoS ONE 2:e626. doi: 10.1371/journal.pone.0000626

Öhman, A., Flykt, A., and Esteves, F. (2001). Emotion drives attention: detecting the snake in the grass. J. Exp. Psychol. Gen. 130, 466-478. doi: 10.1037/00963445.130.3.466

Oswald, D. L. (2005). Understanding Anti-Arab Reactions Post-9/11: the role of threats, social categories, and personal ideologies1. J. Appl. Soc. Psychol. 35, 1775-1799. doi: 10.1111/j.1559-1816.2005.tb02195.x

Payne, B. K. (2001). Prejudice and perception: the role of automatic and controlled processes in misperceiving a weapon. J. Personal. Soc. Psychol. 81, 181-192. doi: 10.1037/0022-3514.81.2.181

Perani, D., Fazio, F., Borghese, N. A., Tettamanti, M., Ferrari, S., Decety, J., et al. (2001). Different brain correlates for watching real and virtual hand actions. Neuroimage 14, 749-758. doi: 10.1006/nimg.2001.0872

Pettigrew, T. F. (2008). Future directions for intergroup contact theory and research. Int. J. Intercult. Relat. 32, 187-199. doi: 10.1016/j.ijintrel.2007.12.002

Pettigrew, T. F., and Tropp, L. R. (2008). How does intergroup contact reduce prejudice? Meta-analytic tests of three mediators. Eur. J. Soc. Psychol. 38, 922-934. doi: 10.1002/ejsp.504

Plant, E. A., and Devine, P. G. (1998). Internal and external motivation to respond without prejudice. J. Personal. Soc. Psychol. 75, 811-832. doi: 10.1037/00223514.75.3.811

Posner, M. I. (1978). Chronometric Explorations of Mind. Lawrence: Erlbaum.

Posner, M. I. (1980). Orienting of attention. Q. J. Exp. Psychol. 32, 3-25. doi: 10.1080/00335558008248231

Press, C., Cook, J., Blakemore, S. J., and Kilner, J. (2011). Dynamic modulation of human motor activity when observing actions. J. Neurosci. 31, 2792-2800. doi: 10.1523/JNEUROSCI.1595-10.2011

Riek, B. M., Mania, E. W., and Gaertner, S. L. (2006). Intergroup threat and outgroup attitudes: a meta-analytic review. Personal. Soc. Psychol. Rev. 10, 336-353. doi: 10.1207/s15327957pspr1004_4

Righart, R., and De Gelder, B. (2008). Recognition of facial expressions is influenced by emotional scene gist. Cogn. Affect. Behav. Neurosci. 8, 264-272. doi: 10.3758/CABN.8.3.264

Rizzolatti, G., and Craighero, L. (2004). The mirror-neuron system. Annu. Rev. Neurosci. 27, 169-192. doi: 10.1146/annurev.neuro.27.070203.144230

Rizzolatti, G., Fogassi, L., and Gallese, V. (2001). Neurophysiological mechanisms underlying the understanding and imitation of action. Nat. Rev. Neurosci. 2, 661-670. doi: 10.1038/35090060

Rizzolatti, G., and Sinigaglia, C. (2010). The functional role of the parieto-frontal mirror circuit: interpretations and misinterpretations. Nat. Rev. Neurosci. 11, 264-274. doi: 10.1038/nrn2805

Roelofs, K., Hagenaars, M. A., and Stins, J. (2010). Facing freeze social threat induces bodily freeze in humans. Psychol. Sci. 21, 1575-1581. doi: $10.1177 / 0956797610384746$

Sacheli, L. M., Christensen, A., Giese, M. A., Taubert, N., Pavone, E. F., Aglioti, S. M., et al. (2015). Prejudiced interactions: implicit racial bias reduces predictive simulation during joint action with an out-group avatar. Sci. Rep. 5:8507. doi: 10.1038/srep08507

Sebanz, N., Knoblich, G., and Prinz, W. (2003). Representing others' actions: just like one's own? Cognition 88, B11-B21. doi: 10.1016/S0010-0277(03)00043-X
Stephan, W. G., and Stephan, C. W. (2000). "An integrated threat theory of prejudice," in Reducing Prejudice and Discrimination, ed. S. Oskamp (Hillsdale, NJ: Erlbaum), 23-46.

Stephan, W. G., Ybarra, O., and Bachman, G. (1999). Prejudice toward immigrants. J. Appl. Soc. Psychol. 29, 2221-2237. doi: 10.1111/j.1559-1816.1999.tb00107.x

Trawalter, S., Hoffman, K. M., and Waytz, A. (2012). Racial bias in perceptions of others' pain. PLoS ONE 7:e48546. doi: 10.1371/journal.pone.0048546

Trawalter, S., Todd, A. R., Baird, A. A., and Richeson, J. A. (2008). Attending to threat: race-based patterns of selective attention. J. Exp. Soc. Psychol. 44, 1322-1327. doi: 10.1016/j.jesp.2008.03.006

Todorov, A., Pakrashi, M., and Oosterhof, N. N. (2009). Evaluating faces on trustworthiness after minimal time exposure. Soc. Cogn. 27, 813-833. doi: 10.1521/soco.2009.27.6.813

Tsai, C. C., and Brass, M. (2007). Does the human motor system simulate Pinocchio's actions? Coacting with a human hand versus a wooden hand in a dyadic interaction. Psychol. Sci. 18, 1058-1062. doi: 10.1111/j.14679280.2007.02025.x

Uddin, L. Q., Molnar-Szakacs, I., Zaidel, E., and Iacoboni, M. (2006). rTMS to the right inferior parietal lobule disrupts self-other discrimination. Soc. Cogn. Affect. Neurosci. 1, 65-71. doi: 10.1093/scan/nsl003

Urgesi, C., Candidi, M., Fabbro, F., Romani, M., and Aglioti, S. M. (2006). Motor facilitation during action observation: topographic mapping of the target muscle and influence of the onlooker's posture. Eur. J. Neurosci. 23, 2522-2530. doi: 10.1111/j.1460-9568.2006.04772.x

Williams, J. M. G., Mathews, A., and MacLeod, C. (1996). The emotional Stroop task and psychopathology. Psychol. Bull. 120, 3-24. doi: 10.1037/00332909.120.1.3

Williams, J. M. G., Watts, F. N., MacLeod, C., and Mathews, A. (1988). Cognitive Psychology and Emotional Disorders. Hoboken, NJ: John Wiley \& Sons.

Wilson, M., and Knoblich, G. (2005). The case for motor involvement in perceiving conspecifics. Psychol. Bull. 131, 460-473. doi: 10.1037/0033-2909.131.3.460

Wojciszke, B. (2005). Morality and competence in person-and self-perception. Eur. Rev. Soc. Psychol. 16, 155-188. doi: 10.1080/10463280500229619

Wojciszke, B., Bazinska, R., and Jaworski, M. (1998). On the dominance of moral categories in impression formation. Pers. Soc. Psychol. Bull. 24, 1251-1263. doi: $10.1177 / 01461672982412001$

Conflict of Interest Statement: The authors declare that the research was conducted in the absence of any commercial or financial relationships that could be construed as a potential conflict of interest.

The reviewer FA and handling Editor declared their shared affiliation, and the handling Editor states that the process nevertheless met the standards of a fair and objective review.

Copyright () 2016 Capellini, Sacchi, Ricciardelli and Actis-Grosso. This is an openaccess article distributed under the terms of the Creative Commons Attribution License (CC BY). The use, distribution or reproduction in other forums is permitted, provided the original author(s) or licensor are credited and that the original publication in this journal is cited, in accordance with accepted academic practice. No use, distribution or reproduction is permitted which does not comply with these terms. 
OPEN ACCESS

Edited by:

Annalisa Setti,

University College Cork, Ireland

Reviewed by:

Rémy Versace,

Lumière University Lyon 2, France

Henrik Svensson,

University of Skövde, Sweden

${ }^{*}$ Correspondence:

Ruiming Wang

wangrm@scnu.edu.cn

Specialty section:

This article was submitted to

Cognition,

a section of the journal

Frontiers in Psychology

Received: 19 May 2016

Accepted: 22 August 2016 Published: 12 September 2016

Citation:

Xie J, Lu Z, Wang R and Cai ZG

(2016) Remember Hard But Think

Softly: Metaphorical Effects

of Hardness/Softness on Cognitive

Functions. Front. Psychol. 7:1343.

doi: 10.3389/fpsyg.2016.01343

\section{Remember Hard But Think Softly: Metaphorical Effects of Hardness/Softness on Cognitive Functions}

\author{
Jiushu Xie ${ }^{1,2}$, Zhi Lu' ${ }^{1,2}$, Ruiming Wang ${ }^{1,2 *}$ and Zhenguang G. Cai, ${ }^{3,4}$ \\ ${ }^{1}$ Center for Studies of Psychological Application, School of Psychology, South China Normal University, Guangzhou, China, \\ ${ }^{2}$ Guangdong Provincial Key Laboratory of Mental Health and Cognitive Science, South China Normal University, \\ Guangzhou, China, ${ }^{3}$ School of Psychology, University of East Anglia, Norwich, UK, ${ }^{4}$ Department of Experimental \\ Psychology, University College London, London, UK
}

Previous studies have found that bodily stimulation, such as hardness biases social judgment and evaluation via metaphorical association; however, it remains unclear whether bodily stimulation also affects cognitive functions, such as memory and creativity. The current study used metaphorical associations between "hard" and "rigid" and between "soft" and "flexible" in Chinese, to investigate whether the experience of hardness affects cognitive functions whose performance depends prospectively on rigidity (memory) and flexibility (creativity). In Experiment 1, we found that Chinesespeaking participants performed better at recalling previously memorized words while sitting on a hard-surface stool (the hard condition) than a cushioned one (the soft condition). In Experiment 2, participants sitting on a cushioned stool outperformed those sitting on a hard-surface stool on a Chinese riddle task, which required creative/flexible thinking, but not on an analogical reasoning task, which required both rigid and flexible thinking. The results suggest the hardness experience affects cognitive functions that are metaphorically associated with rigidity or flexibility. They support the embodiment proposition that cognitive functions and representations can be grounded in bodily states via metaphorical associations.

Keywords: embodied cognition, metaphor, tactile sensation, hardness, softness, memory, creativity, cognitive function

\section{INTRODUCTION}

Traditional theories of cognition take a dualist approach to the mind and the body. To use a computer metaphor, the mind and its cognitive functions are equated with the operational system and the algorithms whereas the body is assumed to function like the hardware (e.g., the keyboard and monitor) (Pylyshyn, 1984; Neisser, 2014). This approach implies that the mind is independent of the body, just as a software package is independent of a computer's physical settings. Such a dualist assumption has been challenged by a more recent embodied cognition approach, which assumes that the body plays a key role in shaping how the mind works (Lakoff and Johnson, 1980b; Kövecses, 2003; Barsalou, 2008; Landau et al., 2010; Meier et al., 2012). Over the past decade, research has converged to suggest that knowledge represented in our long-term memory consists of bodily or sensorimotor experiences acquired from interactions with the physical world (Mandler, 1992; Barsalou, 2003). From the beginning of human life, representations and understanding 
of the outside world are developed through interactions between sensorimotor systems and the environment (Smith, 2005). Thus, higher cognitive functions, such as memory, language, and reasoning, are grounded in perceptual, motor, and introspective contents instead of symbolic representations (Lakoff and Johnson, 1980b; Gallese, 2005, 2007; Barsalou, 2008; Barsalou, 2009, 2010; Gallese and Sinigaglia, 2011).

Cognitive functions can be embodied in two ways: sensorimotor embodiment and metaphorical association. First, when an event is experienced, the underlying sensorimotor states are partially stored. The representations underlying cognitive functions are simulations of past modal experiences rather than amodal symbols. When knowledge of the event is activated later, these sensorimotor states are partially stimulated. Thus, the compatibility of bodily states and cognitive states influences individuals' performance effectiveness (Barsalou, 1999; Barsalou et al., 2003). This perspective of simulations has been verified in a variety of studies demonstrating an interaction between sensorimotor information and cognitive processing. For instance, conceptual processing evokes multimodal perceptual information associated with a concept's referent (Solomon and Barsalou, 2001; Pecher et al., 2003, 2004; Solomon and Barsalou, 2004), and language comprehension makes use of sensorimotor simulation of the event that is being described in a sentence (Stanfield and Zwaan, 2001; Zwaan et al., 2002; Glenberg and Kaschak, 2003). A second way to achieve bodily grounding for cognitive functions is via metaphorical association, a mechanism that has been hypothesized to provide a "scaffold" for the acquisition and formation of new knowledge, especially that of abstract domains, such as time (Mandler, 1992; Lakoff and Johnson, 1999; Barsalou, 2003; Williams et al., 2009). For instance, time metaphorically recruits spatial representational vocabulary for its processing and representation (e.g., a time point can be before or after another, and duration can be long or short). Furthermore, the perception of time has been shown to be influenced by concurrent spatial information (Boroditsky, 2001; Casasanto and Boroditsky, 2008; Cai et al., 2013; cf. Cai and Connell, 2015).

One source of sensorimotor information that has been reported to provide bodily grounding for cognitive functions via metaphorical association is the sense of touch (Gallace and Spence, 2010). Among all the sensory modalities, the tactile sense is the first sense to develop and the last to fade (Gallace and Spence, 2010). Hence, during one's lifetime, tactile sensation accumulates enormous amounts of information to support cognitive functions. Specifically, many studies have revealed that tactile sensation can modulate higher-level cognitive functions (Brunye et al., 2012; Maister et al., 2013). The tactile sensation of heaviness, for instance, can provide grounding for abstract domains, such as value, importance, and confidence by way of a metaphorical association with "weight" (e.g., more important/valuable things carry more weight). In previous studies, people tended to judge a foreign currency as more valuable (Jostmann et al., 2009), to express more selfconfidence (Jostmann et al., 2009), and to rate job candidates as more suitable (Ackerman et al., 2010) when they were carrying a heavier physical load (e.g., holding a heavier clipboard).
These findings suggest that tactile sensation might modulate participants' social- and self-perception. The tactile sensation of heaviness has also been found to affect participants' cognitive function. Kaspar and Vennekotter (2015) found that participants performed worse in a riddle task (e.g., re-arranging "cctiat" into "tactic") if they concurrently held a heavier clipboard, presumably because a heavy load induced a "task-is-difficult" mindset, thereby hindering participants' performance. Indeed, tasks appear to be more difficult for people who are carrying a heavy load; e.g., people carrying a heavier backpack tend to judge a hill's slope to be steeper (Proffitt, 2006).

Further evidence concerning the effect of tactile sensations on social perception and cognitive function has been found in studies using the tactile sensation of hardness. Hardness is (in English at least) metaphorically associated with rigidity. A seminal study by Ackerman et al. (2010) tested whether the sensation of hardness would influence people's perception of these attributes in social situations. They showed that, when asked to evaluate the personality traits of a depicted employee, people in contact with a hard block of wood (thus exposed to a hard sensation) judged the employee to be more rigid than those in contact with a soft block of wood (thus exposed to a soft sensation). In addition, people who made their judgment while sitting on a hard-surface rather than on a cushioned stool judged the employee to be more stable and less emotional.

Although the above findings suggest that physical interactions with the tactile sensation of hardness influences participants' social impressions, it is less clear whether the sensation of hardness can have a similar effect on cognitive functions via metaphorical associations. Kim (2015) provided initial evidence that the sensation of hardness can shift people's rigidness in thinking. Using standard tests of creativity, Kim found that people were more likely to be divergent in their creativity (i.e., less rigid in their thinking) when they experienced a soft sensation (i.e., when squeezing a soft ball) but convergent in their creativity (i.e., more rigid in their thinking) when they experienced a hard sensation (i.e., squeezing a hard ball). Interesting as the results are, questionnaire-based standard tests are not ideal tools to explore whether and how cognitive functions can be influenced by the tactile sensation of hardness, because participants might understand each question in a questionnaire differently and avoid reporting some points that they did not want to report. Questionnaires also lack ecological validity and were not flexible. In addition, the manipulation of squeezing a soft vs. hard ball could have invited many confounds. For instance, it is possible that the observed effect could have been caused by other sensorimotor simulation, such as the strength applied to the ball (e.g., greater strength is needed to squeeze a hard ball) or the ease of squeezing a soft ball (e.g., a soft ball is more pliable, and therefore, easier to squeeze).

To explore more thoroughly whether the tactile sensation of hardness affects cognitive functions via metaphorical association, we used the metaphorical links between hardness/softness and rigidity/flexibility in Chinese. In Chinese, "hard" is associated with rigidity. For instance, rigid truths mean hard principles in Chinese (硬道理). In contrast, "soft" is associated with flexibility. For instance, the proverb “嘴巴是扁的, 舌头是软的” (lit., “the mouth 
is flat; the tongue is soft" means that people should be flexible in their communication). In addition, softness and flexibility are usually used together in Chinese as in 柔软灵活 (lit., “soft and flexible"). Hence, hardness is metaphorically associated with rigidity, whereas softness is metaphorically associated with flexibility.

As they are metaphorically associated with hardness and softness, rigidity and flexibility can also describe the manner in which certain cognitive functions are performed. A wellfunctioning memory system, for instance, encodes and retrieves information in a rigid manner when individuals require accurate memories in order to avoid the inconvenience of forgetting or false memories (Tanila et al., 1997). Of course, rigidness may be just one of mechanisms underlying the memory system. To update existing memories and avoid the memory loss, the system would also rely on the mechanisms of plasticity and reliability. Thus, while other types of memories, e.g., episodic memories and imagining possible future events, may mainly require flexibility and creativity mechanisms (Schacter and Addis, 2009; Spreng et al., 2009), remembering words for a subsequent memory test requires the rigid mechanism.

However, creativity requires people to think in a more flexible manner in order to view a problem with a new perspective, reveal hidden patterns, or generate innovative solutions (Zabelina and Robinson, 2010).

Of course, it is possible for a cognitive function to involve both manners of thinking. Analogical reasoning, for instance, requires people to have a rigid understanding of a problem (i.e., the source) and then to map that problem to a target using a flexible manner of thinking, so that is easier to understand (Sternberg, 1977; Sowa and Majumdar, 2003; Viskontas et al., 2004). The analogy between the structure of an atom and the solar system, for example, initially requires rigid thinking about the relationship between the nucleus and the electrons and then a more flexible search for a target (the solar system). Thus, if hardness experience affects cognitive function via a metaphorical association, we should expect hard and soft bodily stimulation to affect memory, creativity, and analogical reasoning in different ways. In particular, we would expect hard (rather than soft) bodily stimulation to promote explicit memory and soft (rather than hard) bodily stimulation to facilitate creativity. However, we would expect hardness stimulation to have little or no effect on analogical reasoning, as the rigidity and flexibility effects would cancel each other out, a hypothesis we tested in this study's two experiments.

In the experiments, we manipulated hardness bodily stimulation by having participants sit on either a hard-surface (hard condition) or a cushioned stool (soft condition) while they performed different cognitive tasks. We used three tasks that engage different cognitive functions: a memory-recall task (requiring a rigid thinking style for successful performance), a Chinese-riddle task (requiring a creative and flexible thinking style), and an analogical-reasoning task (requiring both rigid and creative thinking styles). In Experiment 1, participants were asked to perform a memory-recall task in which they memorized and recalled a list of words (León-Carrión et al., 2010). As the task mainly involved the cognitive function of memorization, we hypothesized hard bodily stimulation would be more likely to enhance task performance than soft bodily stimulation. Experiment 2 employed two tasks: a Chinese-riddle task, in which creative and flexible thinking was required for solutions, and an analogical-reasoning task that required participants to think in both a rigid and flexible manner (Kumar and Kumari, 1988; Beversdorf et al., 1999). We hypothesized that participants would perform better at solving the Chinese riddles if they sat on a cushioned rather than a hard-surface stool but would perform the analogical reasoning task at the same level, regardless of the stimulation condition.

\section{EXPERIMENT 1}

\section{Method \\ Participants}

Forty-five Mandarin-speaking students (29 women; mean age $=20.0, S D=2.2$ ) were recruited from South China Normal University, Guangzhou, China to participate in the experiment. None of them reported having a language disorder and all had normal or corrected-to-normal vision. Each participant was offered a small monetary reimbursement for participating in the study. All participants gave written informed consent in accordance with the Declaration of Helsinki. The study was approved by the Ethics Review Board of School of Psychology, South China Normal University.

\section{Materials}

We used two groups of stools to manipulate participants' sensations of hardness, which were identical except that one group had hard surfaces and the other had cushioned ones (see Supplementary Figure S1 for a sample of hard-surface stool).

The memory task consisted of 36 two-character Chinese words (see Supplementary Table 1). A pretest was administered to 25 participants who did not take part in the main experiment. Their ratings of the familiarity of the words on a 5-point scale ranging from 1 (extremely unfamiliar) to 5 (extremely familiar) showed that all of the words had a familiarity score greater than 4.5.

It is possible that these words may have a general tendency to be associated with the hard or soft sensation, and the hard-surface or cushioned stool may thus prime the recall of these words. To rule out this possible priming effect, we recruited another 20 participants to rate the association between each of the test words and hardness/softness on a 5-point Likert scale (1 indicated extremely soft while 5 indicated extremely hard). A one-sample $t$ test showed that the test words as a whole were not associated with either the hard or soft sensation $(M=3.07, S D=0.56$, $t(35)=0.76, p=0.454$, Cohen's $d=0.13)$.

\section{Procedure}

Participants were randomly assigned to one of the two hardness conditions and tested individually in a quiet cubicle. Depending on the condition, participants were seated on either hard-surface or cushioned stools naturally using their comfortable postures. They were then given a clipboard with a sheet of paper containing 36 words. The clipboard was placed on an office table in front 


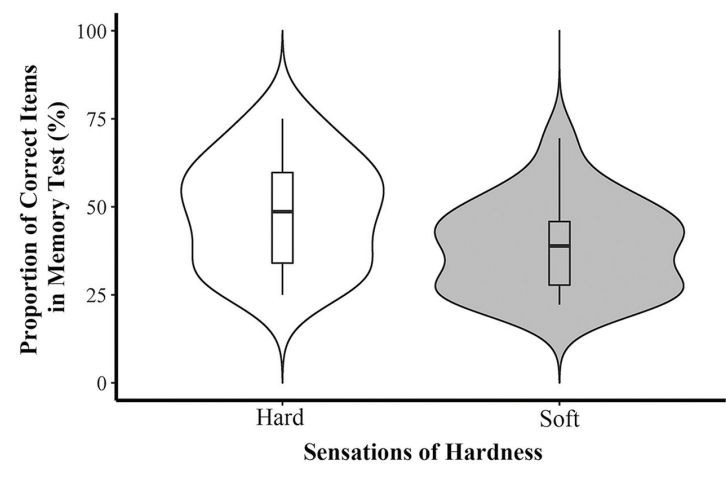

FIGURE 1 | Proportion of correct answers in recall task in Experiment 1. The white and gray areas display the data's probability density; the thick horizontal lines inlayed into the box correspond to medians; the lower and upper "hinges" of the box represent the first and third quartiles; and whiskers extends from the corresponding hinge to the highest/lowest value that is within $1.5 \times$ interquartile range of the hinge (subsequent figures also follow this layout).

of the participants. The participants were allowed 2 minutes to memorize the words. At the end of the memorization phase, the paper was collected and the participants took a 10-min break. Then, they recalled the words by writing them down on a new sheet of paper. No time limit was imposed on the recall phase. During the entirety of the experiment, the participants remained seated on the same stool. None of the participants reported any awareness of the hardness manipulation during the debriefing at the end of the experiment. In addition, previous studies have reported that body postures affected participants' cognitive performances (Stepper and Strack, 1993). However, we did not observe any systematic differences of body postures between hard and soft groups in this experiment.

\section{Results and Discussion}

We calculated the proportion of correctly recalled words out of the 36 test words. Given that none of the participants' scores fell beyond $\pm 3 S D s$, all participants' data were included in the analysis. An independent-samples $t$-test showed that participants sitting on the hard-surface stool correctly recalled more words ( $M=0.49, S D=0.15)$ than those who sat on the cushioned stool $(M=0.39, S D=0.13)(t(43)=2.35, p=0.023$, Cohen's $d=0.70$ ) (see Figure 1). These results suggest that sitting on a hard-surface stool, as compared to a soft one, facilitated participants' memorization. This finding suggests that hardness stimulation affects cognitive functions that underlie memory recall. In Experiment 2, we tested whether hardness stimulation affects creativity and analogical reasoning.

\section{EXPERIMENT 2}

\section{Method}

\section{Participants}

Another sample of 45 participants (32 women; mean age $=20.1$, $S D=2.4$ ) from the same population as those in Experiment 1 were paid to take part in the experiment. The study was approved by the Ethics Review Board of School of Psychology, South China Normal University.

\section{Materials}

The same stools from Experiment 1 were used. To construct the Chinese riddle test, we selected 10 riddles via a Chinese search engine (wenku.baidu.com). Each riddle contained orthographic and/or semantic cues to the answer (a Chinese character). For example, question: 上下合 (connect the upper [i.e., 上] and lower [下]); answer: 卡 (card) (see Supplementary Table 2). A pilot test with 8 participants (who did not take part in the main experiment) showed that on average, they correctly solved 5.9 out of the 10 riddles. The analogical reasoning test consisted of 28 questions. The stem of each question consisted of a Chinese word pair which exhibited a logical relationship (e.g., 自行车-公路 [bicycleroad]); the options were four word pairs, one of which expressed the same logical relationship as the one provided in the stem (e.g., 飞机-天空 [aircraft-sky]) (see Supplementary Table 3).

\section{Procedure}

As in Experiment 1, participants were randomly assigned to one of the two hardness conditions and were seated on the corresponding stool (hard-surface or cushioned) throughout the experiment. The two tests (Chinese riddles and analogical reasoning) were carried out sequentially, with their order counterbalanced across participants. For the Chinese riddle test, participants were given a paper-and-pencil questionnaire consisting of ten riddles, and allowed $5 \mathrm{~min}$ to solve as many riddles as possible. For the analogical reasoning test, participants were given a paper-and-pencil questionnaire consisting of 28 analogical reasoning questions and instructed to answer as many questions as possible within $5 \mathrm{~min}$. Participants were asked to remain seated until they had finished both tests. None of the participants reported an awareness of the hardness manipulation and showed any special body postures.

\section{Results and Discussion}

The proportion of correctly answered items out of all test items (i.e., 10 items in riddle test and 28 items in analogical reasoning test) on each test was analyzed by independent $t$-tests. One participant was excluded from the analysis of the Chinese riddle scores because his/her score exceeded 3 SDs. All participants were included in the analysis of the analogical reasoning scores ${ }^{1}$.

The results of the Chinese riddle test showed that participants' performance was better when they were seated on a soft stool $(M=60.45, S D=12.14)$ than when they were seated on a hardsurface stool $(M=48.57, S D=19.05)(t(41)=2.45, p=0.019$, Cohen's $d=0.74)$. The $t$-test of the scores on the analogical reasoning test showed that hardness did not affect participants' performance $\left(M_{\mathrm{soft}}=67.24, S D=12.26 ; M_{\text {hard }}=67.69\right.$, $S D=15.05 ; t(42)=-0.11, p=0.913$, Cohen's $d=-0.03)$ (see Figures 2 and 3 ).

\footnotetext{
${ }^{1}$ In another analysis of the analogical reasoning test, we also removed the participant who had been excluded from the riddle test. The result of the analogical reasoning test remained the same as reported above.
} 


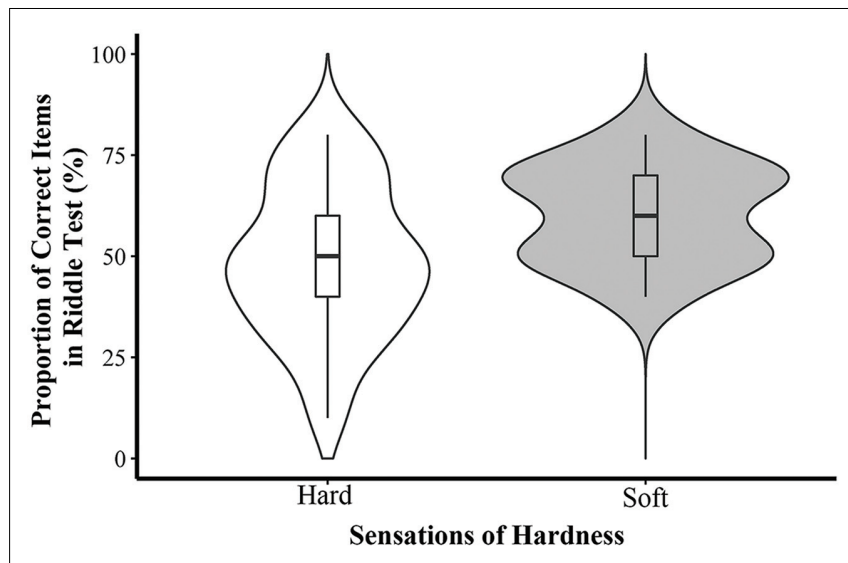

FIGURE 2 | Proportion of correct answers in Chinese riddle task in Experiment 2.

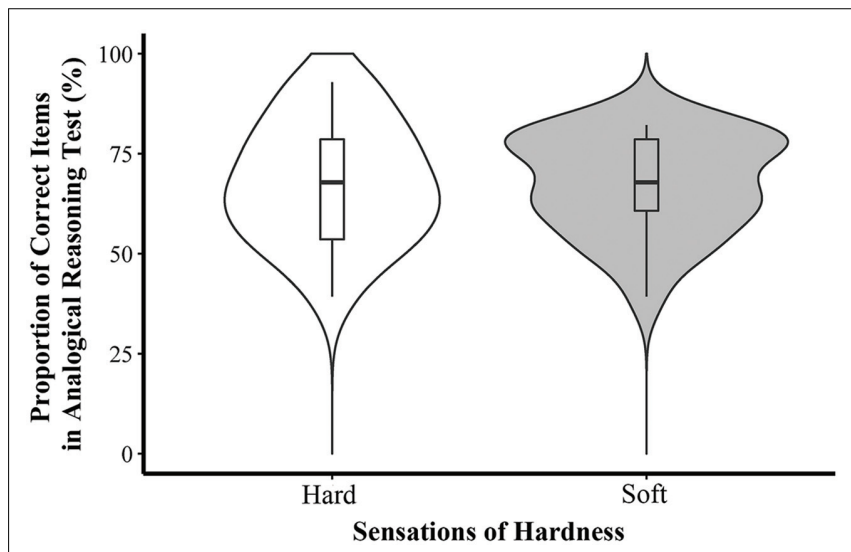

FIGURE 3 | Proportion of correct answers in analogical reasoning task in Experiment 2.

These results confirmed our hypothesis that soft bodily stimulation promotes creativity/flexibility during the Chinese riddle task via a metaphorical association between softness and flexibility. In the analogical reasoning task, soft stimulation might have improved participants' performance by promoting flexible thinking in the search for an analogical target; similarly, the hard stimulation might have improved performance through rigid thinking in relation to the source problem, thus cancelling out any possible effect by the soft simulation.

\section{GENERAL DISCUSSION}

In the current study, we tested whether the tactile sensation of hardness would affect participants' cognitive functions (i.e., memory, creativity, and analogical reasoning) via metaphorical associations. The results of Experiment 1 showed that hard bodily stimulation enhanced participants' memory: the participants who sat on a hard-surface stool accurately recalled more words in a memory test than those who sat on a cushioned stool. Experiment 2 revealed that soft bodily stimulation promoted participants' creativity: participants who sat on a soft stool correctly solved more Chinese riddle questions than those who sat on a hard stool. Hardness/softness stimulation, however, does not bias analogical reasoning, which presumably requires both rigid and flexible thinking. This, together with previous findings, confirms the embodied cognition proposition that the interplay between body and environment can affect how the mind works (Lakoff and Johnson, 1980b; Barsalou, 2008; Landau et al., 2010).

Many studies have reported that performance on memory tests is enhanced when the testing is conducted in the same setting as the learning (Godden and Baddeley, 1975; Smith et al., 1978; Smith, 1979). The current study extends this finding by suggesting that memory is improved when the learning and testing settings lead to a bodily state that is metaphorically congruent with the memory task (e.g., a hard stimulation leading to rigid thinking in the performance of a memory task). Similarly, our research also suggests that an environment that creates "soft" feelings, which is metaphorically congruent with the Chinese riddle task, can lead to better performance on creativity tasks (Kim, 2015). Thus, these results support our hypotheses that hardness would affect participants' cognitive functions via metaphorical associations.

The effects of hardness/softness with respect to cognitive functions occur via metaphorical association. As mentioned previously, people associate hardness with rigidness and softness with flexibility. For example, we use the term "hard science" to refer to the natural sciences, which require rigid adherence to a set of rules and methods in their quantitative research studies, and "soft science" to refer to the social sciences and humanities, which tend to rely more on argumentation and qualitative research methods. These metaphorical uses of hard and soft imply metaphorical links between the tactile experience of hardness and softness and the extent of rigidity in thinking. In the current study, hard feelings primed rigidity and its activation further facilitated the participants' performance of a memory task than did soft feelings, because memory is metaphorically associated with rigidity. Similarity, soft feelings primed flexibility that facilitated the participants' performance on the Chinese riddle task.

The current findings further extend the role of metaphor in creativity. Philosophers note that metaphors can create novel features of an object or situation, known as the creativity of metaphor. Take the metaphor, "There are some days the happy ocean lies// Like an unfingered harp, below the land." for instance. This metaphor emphasizes the sun's reflection on the ripples of a peaceful ocean and provides us a new view of the ocean (Indurkhya, 1999). The results of the Experiment 2 extend the premise that hardness affects creativity in cognitive function, indicating that metaphors not only create novel features directly, but may also create special metaphorical associations to improve people's creativity.

Thus, these findings suggest that bodily stimulation plays an important role in cognitive function, specifically, the manner in which people acquire new information or search for and retrieve existent information for problem solving (Messick, 1976; See Sternberg and Grigorenko, 1997; Kozhevnikov, 2007 for reviews). Prior research has suggested that cognitive function 
may be affected by external sensory experience. Mehta and Zhu (2009) presented evidence that a red and a blue visual background exerted different influences on the way people performed cognitive tasks. A red background biased people's attention towards details (e.g., leading to better word recall), whereas a blue background enhanced people's creativity (e.g., leading to more creative use of an object). These results are parallel with our findings that hard bodily stimulation enhanced memory performance, whereas soft bodily stimulation promoted creativity. Mehta and Zhu (2009) argued that these color effects on cognitive functions are caused by associations: the red color is associated with danger and mistakes, and therefore raises people's vigilance, which in turn, leads to better comprehension of details; the blue color, in contrast, is associated with openness, peace, and tranquility, which in turn, facilitates creativity in problem solving.

Although these associations may be common, it is unclear how they explain our results. Our daily experience offers a multitude of direct (i.e., sensorimotor perception-cognitive function) and indirect (sensorimotor perception-metaphorical associationcognitive function) associations between sensorimotor perceptions and conceptual meanings. For example, the association between warmth and prosocial feelings is mainly direct. Warmth is necessary for survival and it can make people feel safe. Hence, people generally associate warmth with prosocial feelings. When participants encountered social exclusion in one study, they preferred warm food and drink to reduce their bad feelings, in comparison to those who did not experience such exclusion (Zhong and Leonardelli, 2008).

Nevertheless, the effects of hardness on cognitive functions are likely to be based on indirect associations. In our daily lives, hardness stimulation does not have any direct functions or obvious meanings in memorization or creativity. Rarely do people explicitly experience better memory or a spur of creativity by having a particular tactile experience. Then, what are mechanisms underlying the observed associations? One interesting possibility is linguistic/metaphorical association, i.e., indirect association (Lakoff and Johnson, 1980a,b; Gibbs, 1994). Metaphor is the fundament of the conceptual system that is used to understand abstract concepts through superficially dissimilar concrete concepts. The concrete concepts, e.g., hardness and softness, come from individuals interactions with the environment. Conceptual metaphors help individuals adopt these concrete concepts as analogical framework for supporting their understanding of abstract concepts, e.g., memory and creativity (Landau et al., 2011). For instance, as the word "hard" implies rigidity (in Chinese at least), sitting on a hard-surface stool may instill some feeling of rigidness, which may provide metaphorical support for the memory system, and prime the formation of more rigid memories that are less susceptible to interference or forgetting. Similarly, as the word "soft" implies flexibility, a cushioned stool might leave the impression of physical flexibility, which metaphorically facilitates flexibility in thinking, thereby leading to increased creativity in the Chinese riddle task. Such a linguistically mediated metaphorical association is in line with recent research and highlights the importance of linguistic associations in cognitive processing (e.g., Andrews et al., 2009; Connell and Lynott, 2013).
Another potential account in line with indirect metaphorical account is social embodiment, which holds that individuals' performance is modulated by the compatibility of bodily states and cognitive states (Barsalou et al., 2003; Niedenthal, 2007; Gallese and Sinigaglia, 2011). In the current experiments, when participants sat on a hard-surface stool to learn a list of words, their tactile sensations (i.e., hardness) were compatible with their cognitive states (i.e., encoding words rigidly). Then, participants sitting on a hard-surface stool had better performance in the recalling test than those sitting on a soft stool. Similarly, when participants sat on a soft stool to solve the Chinese riddle question, their tactile sensation (i.e., softness) was compatible with their cognitive states (i.e., adopting flexible and creative thinking). As a result, the soft stool facilitated participants' performance in the Chinese riddle test than the hard one. Given that the current study did not intend to distinguish the underlying mechanisms of the observed effects but mainly focused on verifying the existence of such effects, future studies may distinguish the metaphorical and embodied accounts of the observed effects.

The results of the current experiments also verify the relationship between embodiment and metaphor. Barsalou (1999) proposed that perceptual symbol systems represent knowledge for cognitive processing. Gibbs and Berg (1999) further distinguished embodiment (e.g., bodily experience) and metaphor in these symbols of knowledge representation. They hold that knowledge is represented as perceptual symbols that are inherently structured by metaphor for the most part. Hence, metaphor may organize perceptual symbol systems. In the current study, memory and creativity were also grounded in perceptual experience and bodily states, such as tactile sensation. Metaphors, e.g., hardness as rigidity whereas softness as flexibility, help to ground less concrete concepts such as rigidity and flexibility in tactile sensation. When perceptual symbols are activated (e.g., by sitting on a cushioned/noncushioned stool), related metaphors are activated, which in turn lead to the associated abstract concepts.

Several embodied processes may interact with a cognitive function, such as analogical reasoning in the current study. Analogical reasoning requires people to understand a problem using rigid thinking, and then to use flexible thinking to transfer information of the problem to a target (Sternberg, 1977; Sowa and Majumdar, 2003; Viskontas et al., 2004). Hence, hard and soft stools affected the participants' performance in different ways, resulting in the absence of a metaphorical effect in the analogical reasoning test. However, this interpretation is merely our hypothesis. Follow-up studies should adopt different types of analogical reasoning tasks that emphasize hard and soft metaphorical associations differently to test our interpretation. In addition, further studies should examine whether such multiple embodied processes also interact with other cognitive functions, such as decision-making and moral judgment.

Whether comfort of stools and participants' mood modulate the observed findings is not clear. First, soft seats usually produce more even pressure distributions than hard ones, which makes soft seats more comfortable than hard ones. However, 
this effect is also modulated by individuals' adipose tissue. Lean individuals would also produce high-pressure peaks on very soft seats (Kolich, 2008). However, as we did not measure the participants' rating on the comfort of hard and soft stools, we do not know whether the observed effects are modulated or even induced by the comfort of stools. A second potential mechanism underlying the observed effect may be the participants' mood. Mood affects individuals' memory, such that a positive mood facilitated participants' learning for new brand names than a neural one (Lee and Sternthal, 1999). Unfortunately, we did not measure the participants' mood when they sat on hard or soft stools and thus the potential influence of mood for the observed effect is not clear. Nevertheless, these two factors are not confounding variables and may be, actually, mechanisms underlying the observed effects in this study. The tactile sensation of hardness may modulate participants' comfort levels and/or their mood, which then in turn induces the observed effects. Given that we have found the hardness of stools modulated the participants' memory and creativity, comfort and mood may be mediators between hardness and cognitive functions. Follow-up studies should use experimental manipulation or structural equation modeling to test these hypotheses.

Future research should also investigate whether the mechanism of metaphorical association works bi-directionally. Several studies have failed to observe a metaphorical effect of an abstract domain on a concrete domain (e.g., importance of physical weight on psychological significance) (Zhang and Li, 2012). Lee and Schwarz (2012) found that social suspicion and the perception of a fishy smell have a reciprocal effect on each other. As exposure to a fishy smell increases suspicion and undermines cooperation among people, an increase in social suspicion can similarly heighten people's sensitivity to a fishy smell. Given this observation, it would be interesting to see whether performance in memory and creativity tasks have a reciprocal effect on the perception of hardness.

In sum, the current study found that cognitive functions, such as memory and creativity might be modulated by the bodily experience of hardness, thereby lending support to the

\section{REFERENCES}

Ackerman, J. M., Nocera, C. C., and Bargh, J. A. (2010). Incidental haptic sensations influence social judgments and decisions. Science 328, 1712-1715. doi: 10.1126/science.1189993

Andrews, M., Vigliocco, G., and Vinson, D. (2009). Integrating experiential and distributional data to learn semantic representations. Psychol. Rev. 116, 463498. doi: $10.1037 / \mathrm{a} 0016261$

Barsalou, L. W. (1999). Perceptual symbol systems. Behav. Brain Sci. 22, 577-660. doi: 10.1017/S0140525X99532147

Barsalou, L. W. (2003). Situated simulation in the human conceptual system. Lang. Cogn. Process. 18, 513-562. doi: 10.1080/016909603440 00026

Barsalou, L. W. (2008). Grounded cognition. Annu. Rev. Psychol. 59, 617-645. doi: 10.1146/annurev.psych.59.103006.093639

Barsalou, L. W. (2009). Simulation, situated conceptualization, and prediction. Philos. Trans. R. Soc. Lond. B Biol. Sci. 364, 1281-1289. doi: 10.1098/ rstb.2008.0319 embodiment claim that cognition employs bodily states. These findings support the embodied account and have implications for the debates between embodiment and disembodiment (Clark, 1999; Tversky and Hard, 2009; Maglio and Trope, 2012; Pavlenko, 2012; Pulvermüller, 2013; Pulvermüller and Garagnani, 2014).

\section{AUTHOR CONTRIBUTIONS}

RW, ZL, and JX designed the experiment and analyzed the data. ZL collected the data and prepared the dataset for analyses. JX analyzed the data and wrote the first draft of the manuscript. ZGC, RW, and JX interpreted the data and revised the draft critically.

\section{FUNDING}

This research was funded by the National Natural Science Foundation of China (31571142), the National Excellent Doctoral Dissertation Foundation of China (201204), and by a UK Economic and Social Research Council grant (ES/L010224/1).

\section{ACKNOWLEDGMENTS}

We thank Dr. Yanli Huang for her valuable suggestions for the early version of this manuscript, Ms. Donggui Chen for her assistance in translating the experimental materials from Chinese to English in Supplementary Materials, Ms. Huihui Liu for her support in data collection, and Mr. Rubin Shi for his help in preparing supplementary figures.

\section{SUPPLEMENTARY MATERIAL}

The Supplementary Material for this article can be found online at: http://journal.frontiersin.org/article/10.3389/fpsyg. 2016.01343

Barsalou, L. W. (2010). Grounded cognition: past, present, and future. Top. Cogn. Sci. 2, 716-724. doi: 10.1111/j.1756-8765.2010.01115.x

Barsalou, L. W., Niedenthal, P. M., Barbey, A. K., and Ruppert, J. A. (2003). “Social embodiment," in The Psychology of Learning and Motivation, ed. B. H. Ross (San Diego, CA: Academic Press), 43-92.

Beversdorf, D. Q., Hughes, J. D., Steinberg, B. A., Lewis, L. D., and Heilman, K. M. (1999). Noradrenergic modulation of cognitive flexibility in problem solving. Neuroreport 10, 2763-2767. doi: 10.1097/00001756-199909090-00012

Boroditsky, L. (2001). Does language shape thought?: Mandarin and English speakers' conceptions of time. Cogn. Psychol. 43, 1-22. doi: 10.1006/ cogp.2001.0748

Brunye, T. T., Walters, E. K., Ditman, T., Gagnon, S. A., Mahoney, C. R., and Taylor, H. A. (2012). The fabric of thought: priming tactile properties during reading influences direct tactile perception. Cogn. Sci. 36, 1449-1467. doi: 10.1111/j.1551-6709.2012.01268.x

Cai, Z. G., and Connell, L. (2015). Space-time interdependence: evidence against asymmetric mapping between time and space. Cognition 136, 268-281. doi: 10.1016/j.cognition.2014.11.039 
Cai, Z. G., Connell, L., and Holler, J. (2013). Time does not flow without language: spatial distance affects temporal duration regardless of movement or direction. Psychon. Bull. Rev. 20, 973-980. doi: 10.3758/s13423-013-0414-3

Casasanto, D., and Boroditsky, L. (2008). Time in the mind: using space to think about time. Cognition 106, 579-593. doi: 10.1016/j.cognition.2007. 03.004

Clark, A. (1999). An embodied cognitive science? Trends Cogn. Sci. 3, 345-351. doi: 10.1016/S1364-6613(99)01361-3

Connell, L., and Lynott, D. (2013). Flexible and fast: linguistic shortcut affects both shallow and deep conceptual processing. Psychon. Bull. Rev. 20, 542-550. doi: 10.3758/s13423-012-0368-x

Gallace, A., and Spence, C. (2010). The science of interpersonal touch: an overview. Neurosci. Biobehav. Rev. 34, 246-259. doi: 10.1016/j.neubiorev.2008. 10.004

Gallese, V. (2005). Embodied simulation: from neurons to phenomenal experience. Phenomenol. Cogn. Sci. 4, 23-48. doi: 10.1007/s11097-005-4737-z

Gallese, V. (2007). Before and below 'theory of mind': embodied simulation and the neural correlates of social cognition. Philos. Trans. R. Soc. Lond. B Biol. Sci. 362, 659-669. doi: 10.1098/rstb.2006.2002

Gallese, V., and Sinigaglia, C. (2011). What is so special about embodied simulation? Trends Cogn. Sci. 15, 512-519. doi: 10.1016/j.tics.2011. 09.003

Gibbs, R. W. (1994). The Poetics of Mind: Figurative Thought, Language, and Understanding. Cambridge: Cambridge University Press.

Gibbs, R. W., and Berg, E. A. (1999). Embodied metaphor in perceptual symbols. Behav. Brain Sci. 22, 617-618. doi: 10.1017/S0140525X99312140

Glenberg, A. M., and Kaschak, M. P. (2003). “The body's contribution to language," in Psychology of Learning and Motivation, ed. B. H. Ross (San Diego, CA: Academic Press), 93-126.

Godden, D. R., and Baddeley, A. D. (1975). Context-dependent memory in two natural environments: on land and underwater. Br. J. Psychol. 66, 325-331. doi: 10.1111/j.2044-8295.1975.tb01468.x

Indurkhya, B. (1999). Creativity of metaphor in perceptual symbol systems. Behav. Brain Sci. 22, 621-622. doi: 10.1017/S0140525X99352146

Jostmann, N. B., Lakens, D., and Schubert, T. W. (2009). Weight as an embodiment of importance. Psychol. Sci. 20, 1169-1174. doi: 10.1111/j.14679280.2009.02426.x

Kaspar, K., and Vennekotter, A. (2015). Embodied information in cognitive tasks: haptic weight sensations affect task performance and processing style. $A d v$. Cogn. Psychol. 11, 64-76. doi: 10.5709/acp-0172-0

Kim, J. (2015). Physical activity benefits creativity: squeezing a ball for enhancing creativity. Creat. Res. J. 27, 328-333. doi: 10.1080/10400419.2015. 1087258

Kolich, M. (2008). A conceptual framework proposed to formalize the scientific investigation of automobile seat comfort. Appl. Ergon. 39, 15-27. doi: 10.1016/j.apergo.2007.01.003

Kövecses, Z. (2003). Metaphor and Emotion: Language, Culture, and Body in Human Feeling. Cambridge: Cambridge University Press.

Kozhevnikov, M. (2007). Cognitive styles in the context of modern psychology: toward an integrated framework of cognitive style. Psychol. Bull. 133, 464-481. doi: 10.1037/0033-2909.133.3.464

Kumar, D., and Kumari, S. (1988). Problem solving as a function of creativity and personality. Psychol. Stud. 33, 157-161.

Lakoff, G., and Johnson, M. (1980a). The metaphorical structure of the human conceptual system. Cogn. Sci. 4, 195-208. doi: 10.1016/S0364-0213(80)8 0017-6

Lakoff, G., and Johnson, M. (1980b). Metaphors We Live By. Chicago, IL: University of Chicago press.

Lakoff, G., and Johnson, M. (1999). Philosophy in the Flesh: The Embodied Mind and its Challenge to Western Thought. New York, NY: Basic Books (AZ).

Landau, M. J., Meier, B. P., and Keefer, L. A. (2010). A Metaphorenriched social cognition. Psychol. Bull. 136, 1045-1067. doi: 10.1037/a00 20970

Landau, M. J., Vess, M., Arndt, J., Rothschild, Z. K., Sullivan, D., and Atchley, R. A. (2011). Embodied metaphor and the "true" self: priming entity expansion and protection influences intrinsic self-expressions in selfperceptions and interpersonal behavior. J. Exp. Soc. Psychol. 47, 79-87. doi: 10.1016/j.jesp.2010.08.012
Lee, A. Y., and Sternthal, B. (1999). The effects of positive mood on memory. J. Consum. Res. 26, 115-127. doi: 10.1086/209554

Lee, S. W. S., and Schwarz, N. (2012). Bidirectionality, mediation, and moderation of metaphorical effects: the embodiment of social suspicion and fishy smells. J. Pers. Soc. Psychol. 103, 737-749. doi: 10.1037/a0029708

León-Carrión, J., Izzetoglu, M., Izzetoglu, K., Martín-Rodríguez, J. F., DamasLópez, J., Martin, J. M. B. Y., et al. (2010). Efficient learning produces spontaneous neural repetition suppression in prefrontal cortex. Behav. Brain Res. 208, 502-508. doi: 10.1016/j.bbr.2009.12.026

Maglio, S. J., and Trope, Y. (2012). Disembodiment: abstract construal attenuates the influence of contextual bodily state in judgment. J. Exp. Psychol. Gen. 141, 211-216. doi: 10.1037/a0024520

Maister, L., Tsiakkas, E., and Tsakiris, M. (2013). I feel your fear: shared touch between faces facilitates recognition of fearful facial expressions. Emotion 13, 7-13. doi: 10.1037/a0030884

Mandler, J. M. (1992). How to build a baby: II. Conceptual primitives. Psychol. Rev. 99, 587-604. doi: 10.1037/0033-295X.99.4.587

Mehta, R., and Zhu, R. J. (2009). Blue or red? Exploring the effect of color on cognitive task performances. Science 323, 1226-1229. doi: 10.1126/ science. 1169144

Meier, B. P., Schnall, S., Schwarz, N., and Bargh, J. A. (2012). Embodiment in social psychology. Top. Cogn. Sci. 4, 705-716. doi: 10.1111/j.1756-8765.2012. 01212.x

Messick, S. (1976). "Personality consistencies in cognition and creativity," in Individuality in Learning, ed. S. Messick (San Francisco, CA: Jossey-Bass), 4-22.

Neisser, U. (2014). Cognitive Psychology. New York, NY: Psychology Press.

Niedenthal, P. M. (2007). Embodying emotion. Science 316, 1002-1005. doi: $10.1126 /$ science. 1136930

Pavlenko, A. (2012). Affective processing in bilingual speakers: disembodied cognition? Int. J. Psychol. 47, 405-428. doi: 10.1080/00207594.2012. 743665

Pecher, D., Zeelenberg, R., and Barsalou, L. W. (2003). Verifying different-modality properties for concepts produces switching costs. Psychol. Sci. 14, 119-124. doi: 10.1111/1467-9280.t01-1-01429

Pecher, D., Zeelenberg, R., and Barsalou, L. W. (2004). Sensorimotor simulations underlie conceptual representations modality-specific effects of prior activation. Psychon. Bull. Rev. 11, 164-167. doi: 10.3758/BF03206477

Proffitt, D. R. (2006). Embodied perception and the economy of action. Perspect. Psychol. Sci. 1, 110-122. doi: 10.1111/j.1745-6916.2006.00008.x

Pulvermüller, F. (2013). Semantic embodiment, disembodiment or misembodiment? In search of meaning in modules and neuron circuits. Brain Lang. 127, 86-103. doi: 10.1016/j.bandl.2013.05.015

Pulvermüller, F., and Garagnani, M. (2014). From sensorimotor learning to memory cells in prefrontal and temporal association cortex: a neurocomputational study of disembodiment. Cortex 57, 1-21. doi: 10.1016/ j.cortex.2014.02.015

Pylyshyn, Z. W. (1984). Computation and Cognition. Cambridge, MA: MIT Press.

Schacter, D. L., and Addis, D. R. (2009). On the nature of medial temporal lobe contributions to the constructive simulation of future events. Philos. Trans. $R$. Soc. Lond. B Biol. Sci. 364, 1245-1253. doi: 10.1098/rstb.2008.0308

Smith, L. B. (2005). Cognition as a dynamic system: principles from embodiment. Dev. Rev. 25, 278-298. doi: 10.1016/J.Dr.2005.11.001

Smith, S. M. (1979). Remembering in and out of context. J. Exp. Psychol. Hum. Learn. Mem. 5, 460-471. doi: 10.1037/0278-7393.5.5.460

Smith, S. M., Glenberg, A. M., and Bjork, R. A. (1978). Environmental context and human memory. Mem. Cogn. 6, 342-353. doi: 10.3758/BF031 97465

Solomon, K. O., and Barsalou, L. W. (2001). Representing properties locally. Cogn. Psychol. 43, 129-169. doi: 10.1006/cogp.2001.0754

Solomon, K. O., and Barsalou, L. W. (2004). Perceptual simulation in property verification. Mem. Cogn. 32, 244-259. doi: 10.3758/BF03196856

Sowa, J., and Majumdar, A. (2003). "Analogical reasoning," in Conceptual Structures for Knowledge Creation and Communication, eds B. Ganter, A. de Moor, and W. Lex (Berlin: Springer), 16-36.

Spreng, R. N., Mar, R. A., and Kim, A. S. N. (2009). The common neural basis of autobiographical memory, prospection, navigation, theory of mind, and the default mode: a quantitative meta-analysis. J. Cogn. Neurosci. 21, 489-510. doi: 10.1162/jocn.2008.21029 
Stanfield, R. A., and Zwaan, R. A. (2001). The effect of implied orientation derived from verbal context on picture recognition. Psychol. Sci. 12, 153-156. doi: $10.1111 / 1467-9280.00326$

Stepper, S., and Strack, F. (1993). Proprioceptive determinants of emotional and nonemotional feelings. J. Pers. Soc. Psychol. 64, 211-220. doi: 10.1037/00223514.64.2.211

Sternberg, R. J. (1977). Component processes in analogical reasoning. Psychol. Rev. 84, 353-378. doi: 10.1037/0033-295X.84.4.353

Sternberg, R. J., and Grigorenko, E. L. (1997). Are cognitive styles still in style? Am. Psychol. 52, 700-712. doi: 10.1037/0003-066X.52.7.700

Tanila, H., Shapiro, M., Gallagher, M., and Eichenbaum, H. (1997). Brain aging: changes in the nature of information coding by the Hippocampus. J. Neurosci. 17, 5155-5166.

Tversky, B., and Hard, B. M. (2009). Embodied and disembodied cognition: spatial perspective-taking. Cognition 110, 124-129. doi: 10.1016/ j.cognition.2008.10.008

Viskontas, I. V., Morrison, R. G., Holyoak, K. J., Hummel, J. E., and Knowlton, B. J. (2004). Relational integration, inhibition, and analogical reasoning in older adults. Psychol. Aging 19, 581-591. doi: 10.1037/0882-7974.19.4.581

Williams, L. E., Huang, J. Y., and Bargh, J. A. (2009). The scaffolded mind: higher mental processes are grounded in early experience of the physical world. Eur. J. Soc. Psychol. 39, 1257-1267. doi: 10.1002/ejsp.665
Zabelina, D. L., and Robinson, M. D. (2010). Creativity as flexible cognitive control. Psychol. Aesthet. Creat. Arts 4, 136-143. doi: 10.1037/a0017379

Zhang, M., and Li, X. (2012). From physical weight to psychological significance: the contribution of semantic activations. J. Consum. Res. 38, 1063-1075. doi: $10.1086 / 661768$

Zhong, C. B., and Leonardelli, G. J. (2008). Cold and lonely: does social exclusion literally feel cold? Psychol. Sci. 19, 838-842. doi: 10.1111/j.14679280.2008.02165.x

Zwaan, R. A., Stanfield, R. A., and Yaxley, R. H. (2002). Language comprehenders mentally represent the shapes of objects. Psychol. Sci. 13, 168-171. doi: $10.1111 / 1467-9280.00430$

Conflict of Interest Statement: The authors declare that the research was conducted in the absence of any commercial or financial relationships that could be construed as a potential conflict of interest.

Copyright (c) $2016 \mathrm{Xie}, \mathrm{Lu}$, Wang and Cai. This is an open-access article distributed under the terms of the Creative Commons Attribution License (CC BY). The use, distribution or reproduction in other forums is permitted, provided the original author(s) or licensor are credited and that the original publication in this journal is cited, in accordance with accepted academic practice. No use, distribution or reproduction is permitted which does not comply with these terms. 


\title{
Virtual Reality as an Embodied Tool to Enhance Episodic Memory in Elderly
}

\author{
Claudia Repetto $^{1 *}$, Silvia Serino ${ }^{1,2}$, Manuela Macedonia ${ }^{3,4}$ and Giuseppe Riva ${ }^{1,2}$ \\ ${ }^{1}$ Catholic University of the Sacred Heart, Department of Psychology, Milan, Italy, ${ }^{2}$ Istituto Auxologico Italiano, Applied \\ Technology for Neuro-Psychology Lab, Milan, Italy, ${ }^{3}$ Johannes Kepler University, Information Engineering, Linz, Austria, ${ }^{4}$ Max \\ Planck Institute for Human Cognitive and Brain Sciences, Leipzig, Germany
}

Keywords: embodied cognition, episodic memory, virtual reality, memory training, embodied memory

OPEN ACCESS

Edited by:

Annalisa Setti,

University College Cork, Ireland

Reviewed by:

Nadia Pantidi,

University College Cork, Ireland

*Correspondence:

Claudia Repetto claudia.repetto@unicatt.it

Specialty section:

This article was submitted to

Cognition,

a section of the journal

Frontiers in Psychology

Received: 14 June 2016 Accepted: 07 November 2016 Published: 17 November 2016

Citation:

Repetto C, Serino S, Macedonia M and Riva G (2016) Virtual Reality as an Embodied Tool to Enhance Episodic Memory in Elderly.

Front. Psychol. 7:1839. doi: 10.3389/fpsyg.2016.01839
In the last decade, embodiment has dramatically influenced our conception of cognition. In this new frame, episodic memory, and particularly memory decline have been reinterpreted. Interventions supporting memory in the aging population address the connection between mind and body. Here, we discuss the use of Virtual Reality (VR) as an innovative tool to support episodic memory in older adults.

\section{THE EMBODIED NATURE OF EPISODIC MEMORY AND ITS IMPLICATIONS FOR AGING}

Traditional cognitive models of human mind describe it as processing abstract symbols and following predefined formal rules (Fodor, 1975, 1983).

An alternative view, the embodied cognition approach, claims however that the mind is inherently embodied: this is to say that perceptual and motor systems influence the way we construct concepts, make inferences, and use language (Barsalou, 2008; Shapiro, 2011). Empirical evidence supports this claim in different cognitive domains: in language comprehension (Hauk et al., 2004; Tettamanti et al., 2005; Repetto et al., 2013, 2015b; Repetto, 2014), second language learning (Macedonia et al., 2011; Repetto et al., 2015a; Macedonia and Repetto, 2016), and also in memory (Pezzulo et al., 2010; van Dam et al., 2013; Downing-Doucet and Guérard, 2014; Bochynska and Laeng, 2015; Lagacé and Guérard, 2015). Specifically, it has been argued that the ability to encode and retrieve information is constrained by the actions we can perform upon it and by the limitations/opportunities provided by our bodies (Glenberg, 1997). The embodied nature of memory appears particularly evident for episodic memories. They consist of past events with reference to the individuals themselves as participants of those events (Tulving, 2001, 2002) and linked to a place and a particular time. According to Tulving (2001), the essence of episodic retrieval is the "autonoetic consciousness," namely the subjective and conscious experience of mentally reliving a personal past event. This includes "all the attendant visual, kinesthetic, and spatial impressions" (Wilson, 2002 p.633).

It has been argued that the ontogeny of episodic memory is strictly connected with the onset of locomotion during infancy, and related to the maturation of the hippocampus (Glenberg and Hayes, 2016). This brain structure, together with the entorhinal cortex, contains different kinds of cells dedicated to different tasks. Hippocampal place cells code location (Ekstrom et al., 2003). Head-direction cells provide a viewing direction on the retrieved contents (Taube, 1998) and allow the generation of an egocentrically coherent representation in medial parietal areas. Grid cells in the entorhinal cortex support the process of updating a viewpoint in relation to self-motion signals (Boccara et al., 2010). Glenberg and Hayes (2016) specifically proposed that the alignment of hippocampal place cells and grid cells related to environmental cues triggers the encoding of space in which personal events are experienced. Embodied navigation and memory are thus strictly connected (Leutgeb et al., 2005; Buzsáki and Moser, 2013). 
Miller et al. (2013) developed an hybrid memory test assessing both spatial and episodic memory for a group of epileptic patients implanted with depth electrodes. The test was implemented in a virtual reality environment. In the encoding phase, patients were asked to drive in a virtual city and to deliver different items to stores; in the retrieval phase, patients were prompted to verbally recall the items delivered during navigation. Miller and colleagues found that place cells that fired at a specific location during virtual navigation were also activated during subsequent recall of the item associated to that location. This indicates that activity was reinstated during retrieval in episodic memory.

The embodied approach of memory also accounts for the decline of episodic memory described during aging (Koen and Yonelinas, 2014). Here, the critical element is the reduction of locomotion observed in older individuals. The decrease of locomotion results in the reduction of the opportunities for the hippocampal place cells and grid cells to be tuned with the environment (Glenberg and Hayes, 2016). It stands to reason that, in order to enhance episodic memory in older adults, trainings procedures should target self-locomotion and navigation tasks. To this extent, Virtual Reality systems (VR) could be employed as training tool.

\section{VIRTUAL REALITY AS A TOOL TO IMPROVE EPISODIC MEMORY IN THE AGING POPULATION}

Virtual reality allows users to create, explore and interact within environments that are perceived as near to reality. Typically, users entering a VR lab feel as being a part of this world and behave as if they were in the real world (Riva and Mantovani, 2012; Riva et al., 2014). Specifically, even if users do not always move their bodies in the real space, users have the subjective perception of being "in action." The effect of a virtual action on cognitive processing has been demonstrated by Repetto et al. (2015b) who have found that performing a virtual movement with a limb (i.e., virtual run-performed with the legs/feet) speeds up the comprehension of verbs that describe actions performed with the same limb (to kick, performed with legs/feet). On this base, VR can be used to support episodic memory, in particular in elderly (Morganti et al., 2013).

Three main features that VR implements may have an impact on episodic memory in elderly:

First VR allows to experience from an egocentric point of view. This feature places VR in an intermediate position between mere action observation (such as in a video) and real action execution (Serino et al., 2015), with an important rebound on brain activity (Serino et al., 2014). In a fMRI study, Strafella and Paus (2000) have demonstrated that cortical excitability is modified by the observation of movements performed by others. Futhermore, this modulation can be enhanced if the orientation of the movement is egocentric (Maeda et al., 2002). In a recent experiment, Bergouignan et al. (2014) tested the egocentric point of view in relation to encoding and retrieving real-life events. Participants seating in front of a "professor" had a social interaction with him. They saw a real scene filmed and projected through a head-mounted display (HMD) connected to cameras in different locations. The first camera was located exactly over the participants' head, so that participants looked at the scene from the same perspective as without the HMDs. The second camera was located $2 \mathrm{~m}$ in front of the participants and rotated $180^{\circ}$ to face them. This experimental manipulation yielded two different subjective frames of reference. In the former, the sense of bodily self was located inside of the physical body (in-body condition). In the latter the sense of bodily self was located outside the physical body (out-of-body condition). To induce the illusion of being in one of these two locations, they received a visuo-tactile stimulation on their chest with a rod, synchronous with the scene projected to HMD. After a week, participants' episodic memory of these life events was assessed. Results revealed a poorer recollection for life events encoded in the out-of-body condition. Furthermore, findings indicated that the retrieval of these life events was associated with activity changes in the hippocampus. Considering these findings, VR allowing an egocentric encoding and retrieval, could be a valid training tool for episodic memory.

Second, VR allows active navigation while the user actively explores the environment by manipulating keyboards, joysticks, or controllers. Within a virtual world, the user can choose directions and get the impression of walking or running simply by regulating the motion speed. Being the self-locomotion the base of embodiment and grounding consequently episodic memory (Glenberg and Hayes, 2016) virtual promenades could compensate for the decrease of the spontaneous motion in elderly. Jebara et al. (2014) investigated the effect of different types of virtual navigation on episodic memory in young and older adults. In a virtual environment designed as a city to be explored from inside a car, participants were asked to retrieve the events occurred with questions on "what," "where," and "when." Participants were assigned to four experimental conditions: (1) in the passive condition participants were the passengers, with no possibility to interact with the environment; (2) in the itinerary control, participants chose the road but did not drive the car; (3) in the low navigation control, participants displayed the car on rails they could control with pedals without choosing the directions; (4) in the high navigation control, participants drove as in real life choosing also directions. As expected, the low navigation and the itinerary control conditions enhanced episodic memory in both young and older adults. A study by Sauzéon et al. (2016) report similar findings and suggest that active navigation in episodic memory by means of VR benefits memory. In this study, younger and older adults explored two versions ( $A$ and $B$ ) of a virtual apartment either actively by choosing directions or passively (through a computer-guided tour of the apartment). The participants were instructed to memorize all the objects located in the different rooms for future recall. Performance was measured with different memory tests. The results underlined that active navigation increased object recognition in both groups, but did not influence other memory tasks (free recall, proactive interference, and semantic clustering). The authors discussed this finding as analogy between active navigation and the enactment effect (Engelkamp et al., 1994). Thereafter recall and recognition of items of verbal items are 
enhanced if participants previously execute semantically related actions to the words. The similarity between active virtual navigation and enactment is given because both procedures selectively impact item-specific processing as recognition, but not relational processing (for example semantic clustering of concepts). Following this perspective, active navigation in VR, can tap into item-specific processing (Pedroli et al., 2015), known to be more defective in older adults compared to relational processing (Dennis et al., 2007). Third, VR provides environmental enrichment by using flexible scenarios. They can in turn be implemented with different degrees of complexity. From bi-dimensional (2D) to tri-dimensional perspectives (3D), the amount of spatial information can increase the degree of enrichment. A beneficial effect of environmental enrichment toward hippocampal function in mice (in terms of neurogenesis and hippocampus-dependent learning and memory tasks) has been reported by Kempermann et al. (1997). Specifically, it has been proven that VR place cells can be activated by means of virtual navigation (Harvey et al., 2009). In humans, Clemenson and Stark (2015) have demonstrated that 3D in virtual environments improves memory in tasks known to be related to hippocampal activity. In their study, the authors trained naïve video gamers for 2 weeks on two different videogames. One was based on simple $2 \mathrm{D}$ graphics, and one was based on $3 \mathrm{D}$ complex graphics. The control group received no training. Visual and memory performance were tested: visual accuracy and visual processing speed in an enumeration task, memory discrimination between highly similar lure items from repeated items, and a spatial memory score. Participants trained with the $3 \mathrm{D}$ videogame outperformed both, the $2 \mathrm{D}$ gamers and the control group in the discrimination task and in the spatial memory task, but not in the visual task. Being the discrimination task associated with the activity of the hippocampus (Lacy et al., 2010), authors inferred that 3D enriched VR presumably impacts

\section{REFERENCES}

Barsalou, L. W. (2008). Grounded Cognition. Ann. Rev. Psychol. 59, 617-645. doi: 10.1146/annurev.psych.59.103006.093639

Bergouignan, L., Nyberg, L., and Ehrsson, H. H. (2014). Out-of-body-induced hippocampal amnesia. Proc. Natl. Acad. Sci. U.S.A. 111, 4421-4426. doi: 10.1073/pnas.1318801111

Boccara, C. N., Sargolini, F., Thoresen, V. H., Solstad, T., Witter, M. P., Moser, E. I., et al. (2010). Grid cells in pre- and parasubiculum. Nat. Neurosci. 13, 987-994. doi: $10.1038 / \mathrm{nn} .2602$

Bochynska, A., and Laeng, B. (2015). Tracking down the path of memory: eye scanpaths facilitate retrieval of visuospatial information. Cogn. Process. 16, 159-163. doi: 10.1007/s10339-015-0690-0

Buzsáki, G., and Moser, E. I. (2013). Memory, navigation and theta rhythm in the hippocampal-entorhinal system. Nat. Neurosci. 16, 130-138. doi: 10.1038/nn.3304

Castilla, D., Garcia-Palacios, A., Bréton-López, J., Miralles, I., Baños, R. M., Farfallini, L., et al. (2013). Process of design and usability evaluation of a telepsychology web and virtual reality system for the elderly: Butler. Int. J. Hum. Comput. Stud. 71, 350-362. doi: 10.1016/j.ijhcs.2012.10.017

Clemenson, G. D., and Stark, C. E. (2015). Virtual environmental enrichment through video games improves hippocampal-associated memory. J. Neurosci. 35, 16116-16125. doi: 10.1523/JNEUROSCI.258015.2015 also the hippocampal activity. Taken together, these findings support the view that episodic memory can be enhanced in older adults by means of enriched 3D scenarios. They can stimulate hippocampal activity which in turn supports episodic memory.

However, the use of VR with elderly might have some limitations per se: the first affects the usability of different devices such as HMD, joypad or other controllers (Castilla et al., 2013). Elder persons are reluctant toward technology and not used to handle (Manera et al., 2016). The second limitation has to do with the cognitive load of the task. If the task is too demanding, this can have detrimental effects on the memory performance if attentional resources might split between complex motor tasks and memory task (Jebara et al., 2014).

Despite these caveats, these studies pave the way for a new concept of trainings for episodic memory: proposing VR as an effective tool for active navigation in $3 \mathrm{D}$ enriched worlds.

\section{AUTHOR CONTRIBUTIONS}

CR, GR conceived the paper. CR, SS wrote the paper. MM, GR revised the paper.

\section{ACKNOWLEDGMENTS}

This work was partially supported by the Italian funded project "High-End and Low-End Virtual Reality Systems for the Rehabilitation of Frailty in the Elderly" (PE-2013-02355948), by the research project Tecnologia Positiva e Healthy Aging (Positive Technology and Healthy Aging) (Grant D.3.2., 2014) and by the research project "Ageing and Healthy Living: A Human Centered Approach in Research and innovation as Source of Quality Life," funded by Fondazione Cariplo within the 2014.
Dennis, N. A., Hongkeun, K., and Cabeza, R. (2007). Effects of aging on true and false memory formation: an fmri study. Neuropsychologia 45, 3157-3166. doi: 10.1016/j.neuropsychologia.2007.07.003

Downing-Doucet, F., and Guérard, K. (2014). A motor similarity effect in object memory. Psychonomic Bull. Rev. 21, 1033-1040. doi: 10.3758/s13423-0130570-5

Ekstrom, A. D., Kahana, M. J., Caplan, J. B., Fields, T. A., Isham, E. A., Newman, E. L., et al. (2003). Cellular networks underlying human spatial navigation. Nature 425, 184-188. doi: 10.1038/nature01964

Engelkamp, J., Zimmer, H. D., Mohr, G., and Sellen, O. (1994). Memory of selfperformed tasks: self-performing during recognition. Mem. Cogn. 22, 34-39. doi: 10.3758/BF03202759

Fodor, J. A. (1975). The Language of Thought. Cambridge: Harvard University Press.

Fodor, J. A. (1983). The Modularity of Mind. Boston: MIT press.

Glenberg, A. M. (1997). What memory is for. Behav. Brain Sci. 20, 1-55.

Glenberg, A. M., and Hayes, J. (2016). Contribution of embodiment to solving the riddle of infantile amnesia. Front. Psychol. 7:10. doi: 10.3389/fpsyg.2016.00010

Harvey, C. D., Collman, F., Dombeck, D. A., and Tank, D. W. (2009). Intracellular dynamics of hippocampal place cells during virtual navigation. Nature 461, 941-946. doi: 10.1038/nature08499

Hauk, O., Johnsrude, I., and Pulvermüller, F. (2004). Somatotopic representation of action words in human motor and premotor cortex. Neuron 41, 301-307. doi: 10.1016/S0896-6273(03)00838-9 
Jebara, N., Orriols, E., Zaoui, M., Berthoz, A., and Piolino, P. (2014). Effects of enactment in episodic memory: a pilot virtual reality study with young and elderly adults. Front. Aging Neurosci. 6:338. doi: 10.3389/fnagi.2014. 00338

Kempermann, G., Kuhn, H. G., and Gage, F. H. (1997). More hippocampal neurons in adult mice living in an enriched environment. Nature 386, 493-495. doi: 10.1038/386493a0

Koen, J. D., and Yonelinas, A. P. (2014). The effects of healthy aging, amnestic mild cognitive impairment, and alzheimer's disease on recollection and familiarity: a meta-analytic review. Neuropsychol. Rev. 24, 332-354. doi: 10.1007/s11065014-9266-5

Lacy, J. W., Yassa, M. A., Stark, S. M., Muftuler, L. T., and Stark, C. E. L. (2010). Distinct pattern separation related transfer functions in human CA3/dentate and CA1 revealed using high-resolution fmri and variable mnemonic similarity. Learn. Mem. 18, 15-18. doi: 10.1101/lm.1971111

Lagacé, S., and Guérard, K. (2015). when motor congruency modulates immediate memory for objects. Acta Psychol. 157, 65-73. doi: 10.1016/j.actpsy.2015.02.009

Leutgeb, S., Leutgeb, J. K., Moser, M. B., and Moser, E. I. (2005). Place cells, spatial maps and the population code for memory. Curr. Opin. Neurobiol. 15, 738-746. doi: 10.1016/j.conb.2005.10.002

Macedonia, M., Müller, K., and Friederici, A. D. (2011). The impact of iconic gestures on foreign language word learning and its neural substrate. Hum. Brain Mapp. 32, 982-998. doi: 10.1002/hbm.21084

Macedonia, M., and Repetto, C. (2016). Brief multisensory training enhances second language vocabulary acquisition in both high and low performers. Int. J. Learn. Teach. Edu. Res. 15, 42-53.

Maeda, F., Kleiner-Fisman, G., and Pascual-Leone, A. (2002). Motor facilitation while observing hand actions: specificity of the effect and role of observer's orientation. J. Neurophysiol. 87, 1329-1335. doi: 10.1152/jn.00773.2000

Manera, V., Chapoulie, E., Bourgeois, J., Guerchouche, R., David, R., Ondrej, J., et al. (2016). A feasibility study with image-based rendered virtual reality in patients with mild cognitive impairment and dementia. PLoS ONE 11:e0151487. doi: 10.1371/journal.pone.0151487

Miller, J. F., Neufang, M., Solway, A., Brandt, A., Trippel, M., Mader, I., et al. (2013), Neural activity in human hippocampal formation reveals the spatial context of retrieved memories. Science 342, 1111-1114. doi: 10.1126/science. 12 44056

Morganti, F., Stefanini, S., and Riva, G. (2013). From allo-to egocentric spatial ability in early Alzheimer's disease: a study with virtual reality spatial tasks. Cogn. Neurosci. 4, 171-180. doi: 10.1080/17588928.2013.85476

Pedroli, E., Serino, S., Cipresso, P., Pallavicini, F., and Riva, G. (2015). Assessment and rehabilitation of neglect using virtual reality: a systematic review. Front. Behav. Neurosci. 9:226. doi: 10.3389/fnbeh.2015.00226

Pezzulo, G., Barca, L., Bocconi, A. L., and Borghi, A. M. (2010). When affordances climb into your mind: advantages of motor simulation in a memory task performed by novice and expert rock climbers. Brain Cogn. 73, 68-73. doi: 10.1016/j.bandc.2010.03.002

Repetto, C. (2014). The use of virtual reality for language investigation and Learning. Front. Psychol. 5:1280. doi: 10.3389/fpsyg.2014.01280

Repetto, C., Colombo, B., Cipresso, P., and Riva, G. (2013). The effects of rTMS over the primary motor cortex: the link between action and language. Neuropsychologia 51, 8-13. doi: 10.1016/j.neuropsychologia.2012. 11.001

Repetto, C., Colombo, B., and Riva, G. (2015a). Is motor simulation involved during foreign language learning? A virtual reality experiment. SAGE Open 5. doi: $10.1177 / 2158244015609964$
Repetto, C., Cipresso, P., and Riva, G. (2015b). Virtual action and real action have different impacts on comprehension of concrete verbs. Front. Psychol. 6:176. doi: 10.3389/fpsyg.2015.00176

Riva, G., and Mantovani, F. (2012). From the body to the tools and back: a general framework for presence in mediated interactions. Interact. Comput. 24, 203-210. doi: 10.1016/j.intcom.2012.04.007

Riva, G., Waterworth, J., and Murray, D. (eds.). (2014). Interacting with Presence: HCI and the Sense of Presence in Computer-mediated Environments. Berlin: Walter de Gruyter GmbH \& Co KG.

Sauzéon, H., N'Kaoua, B., Arvind Pala, P., Taillade, M., and Guitton, P. (2016). Age and active navigation effects on episodic memory: a virtual reality study. $\mathrm{Br}$. J. Psychol. 107, 72-94. doi: 10.1111/bjop.12123

Serino, S., Cipresso, P., Morganti, F., and Riva, G. (2014). The role of egocentric and allocentric abilities in Alzheimer's disease: a systematic review. Ageing Res. Rev. 16, 32-44. doi: 10.1016/j.arr.2014.04.004

Serino, S., Morganti, F., Di Stefano, F., and Riva, G. (2015). Detecting early egocentric and allocentric impairments deficits in Alzheimer's disease: an experimental study with virtual reality. Front. Aging Neurosci. 7:88. doi: 10.3389/fnagi.2015.00088

Shapiro, L. A. (2011). Embodied Cognition. New York: NY: Routledge.

Strafella, A. P., and Paus, T. (2000). Modulation of cortical excitability during action observation: a transcranial magnetic stimulation study. Neuroreport 11, 2289-2292.

Taube, J. S. (1998). Head direction cells and the neurophysiological basis for a sense of direction. Prog. in Neurobiol. 55, 225-256. doi: 10.1016/S03010082(98)00004-5

Tettamanti, M., Buccino, G., Saccuman, M. C., Gallese, V., Danna, M., Scifo, P., et al. (2005). Listening to action-related sentences activates fronto-parietal motor circuits. J. Cogn. Neurosci. 17, 273-281. doi: 10.1162/08989290531 24965

Tulving, E. (2001). Episodic memory and common sense: how far apart?" Philos. Trans. R. Soc. Lond. B Biol. Sci. 356, 1505-1515. doi: 10.1098/rstb. 2001.0937

Tulving, E. (2002). Episodic memory: from mind to brain. Ann. Rev. Psychol. 53, 1-25. doi: 10.1146/annurev.psych.53.100901.135114

van Dam, W. O., Rueschemeyer, S. A., Bekkering, H., Lindemann, O. (2013). Embodied grounding of memory: toward the effects of motor execution on memory consolidation. Q. J. Exp. Psychol. 66, 2310-2328. doi: $10.1080 / 17470218.2013 .777084$

Wilson, M. (2002). Six views of embodied cognition. Psychonomic Bull. Rev. 9 , 625-636. doi: 10.3758/BF03196322

Conflict of Interest Statement: The authors declare that the research was conducted in the absence of any commercial or financial relationships that could be construed as a potential conflict of interest.

The reviewer NP and the handling Editor declared their shared affiliation, and the handling Editor states that the process nevertheless met the standards of a fair and objective review.

Copyright (C) 2016 Repetto, Serino, Macedonia and Riva. This is an open-access article distributed under the terms of the Creative Commons Attribution License (CC $B Y)$. The use, distribution or reproduction in other forums is permitted, provided the original author(s) or licensor are credited and that the original publication in this journal is cited, in accordance with accepted academic practice. No use, distribution or reproduction is permitted which does not comply with these terms. 
OPEN ACCESS

Edited by:

Annalisa Setti,

University College Cork, Ireland

Reviewed by:

Alexander Skulmowski,

Technische Universität Chemnitz,

Germany

Conor Linehan

University College Cork, Ireland

*Correspondence:

Mina C. Johnson-Glenberg

mina.johnson@asu.edu

Specialty section:

This article was submitted to

Cognition,

a section of the journal

Frontiers in Psychology

Received: 07 June 2016 Accepted: 03 November 2016 Published: 25 November 2016

Citation:

Johnson-Glenberg MC,

Megowan-Romanowicz C, Birchfield DA and Savio-Ramos $C$ (2016) Effects of Embodied Learning and Digital Platform on the Retention of Physics Content: Centripetal Force.

Front. Psychol. 7:1819.

doi: 10.3389/fpsyg.2016.01819

\section{Effects of Embodied Learning and Digital Platform on the Retention of Physics Content: Centripetal Force}

\author{
Mina C. Johnson-Glenberg ${ }^{1,2 *}$, Colleen Megowan-Romanowicz ${ }^{3}$, David A. Birchfield ${ }^{4}$ \\ and Caroline Savio-Ramos ${ }^{5}$
}

\begin{abstract}
${ }^{1}$ Department of Psychology, Arizona State University, Tempe, AZ, USA, ${ }^{2}$ Behavioural Sciences Institute, Radboud University, Nijmegen, Netherlands, ${ }^{3}$ American Modeling Teachers Association, Sacramento, CA, USA, ${ }^{4}$ SMALLab Learning, LLC, North Hollywood, CA, USA, ${ }^{5}$ Mary Lou Fulton Teachers College, Arizona State University, Tempe, AZ, USA
\end{abstract}

Embodiment theory proposes that knowledge is grounded in sensorimotor systems, and that learning can be facilitated to the extent that lessons can be mapped to these systems. This study with 109 college-age participants addresses two overarching questions: (a) how are immediate and delayed learning gains affected by the degree to which a lesson is embodied, and (b) how do the affordances of three different educational platforms affect immediate and delayed learning? Six 50 min-long lessons on centripetal force were created. The first factor was the degree of embodiment with two levels: (1) low and (2) high. The second factor was platform with three levels: (1) a large scale "mixed reality" immersive environment containing both digital and hands-on components called SMALLab, (2) an interactive whiteboard system, and (3) a mouse-driven desktop computer. Pre-tests, post-tests, and 1-week follow-up (retention or delayed learning gains) tests were administered resulting in a $2 \times 3 \times 3$ design. Two knowledge subtests were analyzed, one that relied on more declarative knowledge and one that relied on more generative knowledge, e.g., hand-drawing vectors. Regardless of condition, participants made significant immediate learning gains from pre-test to post-test. There were no significant main effects or interactions due to platform or embodiment on immediate learning. However, from post-test to followup the level of embodiment interacted significantly with time, such that participants in the high embodiment conditions performed better on the subtest devoted to generative knowledge questions. We posit that better retention of certain types of knowledge can be seen over time when more embodiment is present during the encoding phase. This sort of retention may not appear on more traditional factual/declarative tests. Educational technology designers should consider using more sensorimotor feedback and gestural congruency when designing and opportunities for instructor professional development need to be provided as well.

Keywords: embodied learning, STEM, education, mixed reality, virtual reality, centripetal force, design principles 


\section{INTRODUCTION}

Embodiment theory proposes that knowledge is grounded in sensorimotor systems. An extension of that proposition would be that learning can be facilitated to the extent that lessons are created that map to and activate those systems. For this series on embodiment across the life span, we focus on learning in adolescence and early adulthood. A taxonomy on embodied is education is presented and a randomized controlled trial is included that assesses the effects of learning platform and amount of embodiment on the learning of physics content.

Physics concepts are an obvious choice for the study of embodied learning because physical interactions are part of experience from the moment the brain/body begins to experience the world. A thrown object moves through the air along a parabolic trajectory. An object swung in a circle, like a yo-yo spun overhead, must be pulled toward the center of the circle by the string to remain in curvilinear motion. However, the world is complex, and people will induce incorrect concepts. For example, although we feel a force in our arm and physical body when swinging a yo-yo, it is not as clear that the same force is acting on the yo-yo. If the string breaks and the yo-yo flies off, which path will the yo-yo follow, a straight or a curved one?

Naive beliefs about what causes observed behaviors of real objects in motion can be thought of as primitive "mental models" or phenomenological primitives (p-prims; DiSessa, 1988, 2000; Redish, 1994; Johnson-Laird, 1998; Hestenes, 2006), and these are frequently at odds with the expert models of physicists (Sengupta and Wilensky, 2009). These pieces of intuitive knowledge about how the world works are powerful and preserved, even in the face of exceptions (DiSessa, 2000). Since a p-prim is considered selfexplanatory it is rarely mentioned in an explanation, it remains implicit. It is this implicit knowledge structure into which new physics knowledge is assimilated by the novice.

Reiner et al. (2000) posit that it is our generalized knowledge of the properties of material substances and how they behave that is responsible for the collection of naive beliefs that we draw upon when trying to learn a new physics concept. Our knowledge of substances includes the following properties: substances are 'pushable', frictional, containable, consumable, locational, movable, stable, corpuscular (have surface area and volume), additive, inertial, and gravity-sensitive. Novice physics students tend to explain collisions as constrained by internal properties of moving objects such as a ball possessing a force (Halloun and Hestenes, 1985a), this would explain some misconceptions about objects in motion.

\section{The Choice of Centripetal Force}

In the usual course of science, a research question is posed and then content and tests are created to answer the question. In an inspirational flip, the content of this multimedia study was actually inspired by a test item. The overarching goal was to assess how the amount of embodiment designed in to a lesson affected learning and the choice to focus on centripetal force (CF) arose from a classic item from the Force Concept Inventory (Hestenes et al., 1992). Figure 1 shows the CF item that so readily lends itself to embodiment. Students often choose option A. When they pick a curved path after release, students are revealing an incorrect circular impetus notion of CF (McCloskey et al., 1980). We wanted to know whether a 50 min lesson on CF based on the principles of embodiment could help to overcome several misconceptions associated with CF, including the impetus model.

\section{Plain Folks Physics}

Natural philosophers, physics education researchers, and learning scientists have devoted a great deal of effort to examining and describing how "just plain folks" understand physics concepts like force and motion (Viennot, 1979; McCloskey et al., 1980; Halloun and Hestenes, 1985a; DiSessa, 1988, 1993; Arons, 1997; Lakoff and Johnson, 1999). Surprisingly, the fact that we are experimental physicists since birth interferes with learning formal basic physics concepts. We bring to the learning experience well-entrenched ideas based on our observations and interactions with the real world, but those experiences do not easily allow us to separate effects of multiple forces (e.g., gravity, friction, and CFs) and can produce naïve conceptions that are often in conflict with formal physical laws (Halloun and Hestenes, 1985b; Lakoff and Johnson, 1999). McCloskey et al. (1980) described students' intuitive physics knowledge as it relates to objects in circular motion, positing a sort of 'circular impetus' in that objects moving in a circle are endowed with an internal force that will keep them moving in a circular path. To address scientific misconceptions with new media and embodiment, we designed a study that varied along several physical and virtual dimensions. We chose fairly abstract content (CF) and focused on two common misconceptions: (a) that an object will continue in a circle when released from CF (impetus model), and (b) that the radius of distance effects the force in an additive monotonic manner, such that, the longer the yo-yo string, the greater the CF. This second misconception is sometimes called the "more is more" default. It may be based on Reif 's (1995) suggestion that students' reasoning in physics is based on retrieved plausible knowledge fragments, e.g., experiences or events, and the reasoning is tempered by the need for "cognitive efficiency." That is, the expenditure of the least possible cognitive resources to arrive at a solution.

It is incontrovertible that physics can be difficult to learn. After a semester of college physics, the mean score for freshman physics majors at Harvard on an inventory of basic force concepts was a modest 77\% (Hestenes et al., 1992). For many years, physics educators have merely presented formulae and proofs and assumed mastery would follow. For example, these instructors may believe that to learn about CF, a student need only be taught that $\mathrm{F}_{C}=\mathrm{mv}^{2} / \mathrm{r}$ and the definitions of those terms to do well on traditional fill-in-the-blank tests. This is akin to believing the human information processing system is isomorphic to a computer and a modal. Yet, when students merely memorize and practice symbol manipulation they are still not achieving consistently high scores and we do not see generalization.

\section{Embodied Education}

Suppose, however, that the human information processing system is a biological system that evolved in the service of action in the world (Wilson, 2003; Barsalou, 2008; Glenberg, 
A steel ball is attached to a string and is swung in a circular path in a horizontal plane as illustrated in the accompanying figure.

At the point $\mathrm{P}$ indicated in the figure, the string suddenly breaks near the ball.

If these events are observed from directly above as in the figure, which path would the ball most closely follow after the string breaks?

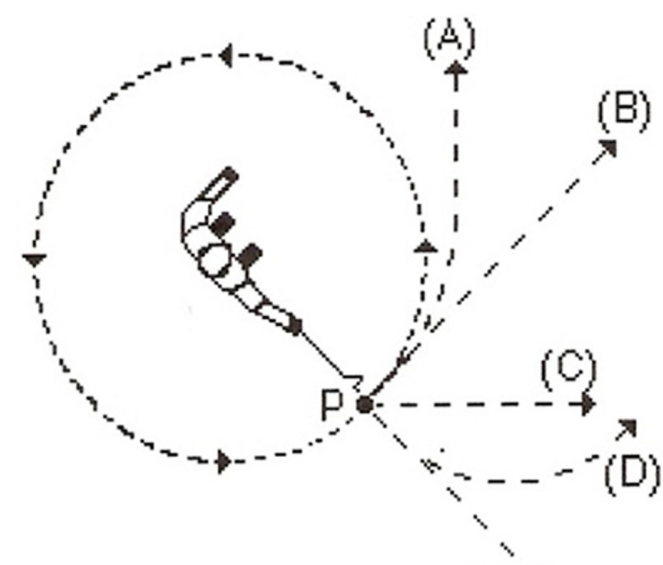

(E)

FIGURE 1 | Inspirational item from FCI test. (Item 7 from the Force Concept Inventory, Hestenes, Wells, and Swackhamer, 1992-reprinted with permission from The American Modeling Teachers Association.)

2008; Glenberg et al., 2013). In this case, learning may be more effective if it were based not so much on symbols and their manipulation, but on perceptual processes and the actions afforded in learning environments. In this case, learning about CF might be better facilitated by experiencing CF with the body. What if the variables in the equation above could be kinesthetically experienced? For example, what if learners could swing objects around their heads and feel the differences in the force when the mass of the object is altered, when the speed changes, and when the length of the tether is altered? Would that type of embodied learning result in a deeper knowledge structure? Would the new knowledge be retained longer? This new view of human information processing, that sensorimotor activation is also important, is the basis for the embodied cognition educational framework. This article assesses learning gains in three platforms and varies the amount to embodiment in each in an attempt to pull apart the most efficacious components. When we understand these components better, they can be folded into established classroom pedagogies from the learning sciences (e.g., situated learning) to create more powerful lessons.

The embodiment framework proposes that knowledge is highly dependent on sensorimotor activity. For education, when learners physiologically feel forces and exert agency over those forces during a lesson, they may more deeply comprehend forces in the world. Learning is primed by what we perceive, and what we expect in the world as we move about it, in addition to how we interact with the objects and situations discovered. In education, it may be that knowledge is not simply in "the extracted verbal or formal description of a situation, but rather in the perceptual interpretations and motoric interactions" in lessons (Goldstone et al., 2008). Such an approach would not seem to cover the learning of abstract materials in language, mathematics, or sciences. However, the notion of embodied simulation (Barsalou, 1999; Glenberg and Gallese, 2011) addresses this potential limitation. Learners may simulate mentally the constructs to be learned using perception and emotion as well.

The equation for $\mathrm{CF}, F_{c}=m v^{2} / r$, may at first appear to be a jumble of meaningless symbols. We propose that understanding the equation will be facilitated when learners map the symbols to sensorimotor experiences. Thus, a learner must map " $m$ " to prior or new experiences with objects of differing mass and must coordinate experiences of speed (the $v^{2}$ ), and radius (the $r$ ) to create a dynamic mental simulation of CF. The end product of this mental work is called 'comprehension,' it means that the learner has been able to create and apply appropriate mental simulations. Perceptual symbols may well underlie all cognition. But how are those first learned? We posit via interactions with the physical world. With this experiential basis established more complex and abstract thoughts can be created. One of our working theories is that reactivating some of the motor activities associated with a learned concept may aid in teaching new associated concepts. The hypothesis is that when the body uses meaningful (congruent) gestures and motoric actions to learn a new concept then the learning signal will be strengthened and primed to activate the knowledge upon recall. Our goal in this study was to create a lesson that would activate (or reactivate) many of the same sensorimotor and cognitive systems associated with CF while correcting several misconceptions.

\section{Two Centripetal Force Misconceptions}

A misconception that the majority of freshmen in physics still hold is that an object released from CF maintains a curvilinear trajectory after the point of release (Halloun and Hestenes, 1985b). This is called the impetus model. Second, students often come to the concept of CF with the misconception that a longer radius will always result more CF. The intervention was designed to give learners varying kinetic and visual experiences with different points of release and variable lengths of radius in a guided discovery 
manner in several mixed and augmented reality (AR) environments.

There are multiple non-mediated hands-on CF classroom experiences for students (Moore et al., 1981). A popular one involves a string, straw, and washers. Our lab believes that adding digital components to the hands-on experience can have powerful repercussions for learning. As Klahr et al. (2007) state, ... "inherent pragmatic advantages of virtual materials in science may make them the preferred instructional medium" (p. 183). Thus, many more trials can occur with a virtual mousetrap car compared to reconfiguring a new physical car for each handson trial. By adding digital components to hands-on components in a mixed or virtual reality (VR) platform, learners are able to easily pause during a lesson and reflect on what is happening in the instant. In our intervention learners were also able to observe the digital trail (called a ghost trail) left by the bob as it traveled after being released, thereby confirming whether the trajectory after release was straight or curved.

\section{Gesture and Embodiment}

Goldin-Meadow et al. (2001) propose that gesture and speech form an integrated, synergistic system in which effort expended in one modality can "lighten the load on the system as a whole," that is, gesturing may actually shift some of the load from verbal working memory to other cognitive systems. Much of the earlier work on gesture and embodiment was done with videotaping experiments followed by human coding, but with affordable motion capture becoming more ubiquitous, more research is being done on grosser body movements and learning. Gestures (what might also be labeled as "instrumented gestures") and full-body movements can be designed now to drive simulations. Digital simulations can be powerful learning aids when created with proper design heuristics (Mayer and Moreno, 2003). Especially larger display simulations can be engaging for leaners. Higher levels of engagement, and more positive attitudes toward science can be seen when whole body movement is integrated into large digitized science lessons (Lindgren et al., 2016). The medical education field has an ongoing history of researching the efficacy of simulations, immersive learning, and skill acquisition, as an example, surgeons perform better after training with the gesture-based Wii (Giannotti et al., 2013). In the next section we describe some differences between augmented and mixed reality (MR) platforms so the reader understands why certain design choices were made.

\section{Augmented and Mixed Reality}

If using the body aids in learning, and receiving immediate digital feedback on actions can facilitate learning and skill acquisition, then perhaps we should be designing for platforms that integrate the two: gesture and user-driven simulation. Platforms that mesh virtual (digital worlds) with physical (kinetic worlds) are called MR environments. Impressive early taxonomic work on MR was done by Milgram and Kishino (1994). They proposed a three dimensional taxonomy and a "virtuality continuum" (Milgram and Kishino, 1994). The real world was one end point and a fully virtual world another end point on the continuum.
In the middle was a "MR" world where physical and digital components would merge. It has become acceptable to use the term "AR" to also describe a segment of the middle space. We reserve the term "VR" to refer to fully enclosed, immersive spaces [e.g., head mounted displays (HMD) or four walled CAVEs].

Educational designers need guidance on how to design with gesture for embodied learning in AR/VR environments. Lindgren and Johnson-Glenberg (2013) recently published six precepts to follow while designing for embodied education and JohnsonGlenberg et al. (2014a) present an early version of an embodied taxonomy for education. In this article, the taxonomy is enhanced and includes a physics lesson and study done in SMALLab (Situated Multimedia Arts Learning Lab). SMALLab is a MR platform where learners hold trackable objects and can control and manipulate interactive digitized media. It is only very recently that guidelines for creating educational content for AR/VR and MR environments are starting to come out (JohnsonGlenberg, 2012; Wu et al., 2013). Thus, this is an emerging area.

\section{The Taxonomy for Educational Embodiment}

According to our proposed taxonomy, for content to be considered minimally embodied it should contain three constructs: (a) sensorimotoric engagement, (b) gestural congruency, that is, how well-mapped the evoked gesture is to the content to be learned, and (c) evoke a sense of immersion. If content is an animation on small screen or monitor offering no interactivity to the user, then it should be called a "simulation" and not be referred to with the terms "embodied, virtual, mixed, or augmented reality." The three axes or constructs were chosen to account for how the body might move and how "present" the learner might feel in the lesson. The taxonomy should be considered a work in progress. The three constructs of embodiment in education occur on three continuous axes, but are partitioned binarily as low and high in order to make it more tractable. The resulting eight sets are then binned along the one dimension of embodiment into four degrees. See Table 1. The 4th degree is the highest because the three constructs are highly present in the content; the 1st degree is the lowest because all constructs are low in the content. The 3 rd and 2 nd degrees each contain three sets with combinatorial mixtures of lows and highs. The edges between adjacent degrees can be considered fuzzy, but

TABLE 1 | Construct magnitude within degrees in the Embodied Education Taxonomy; H, High, L, Low.

\begin{tabular}{lllllll}
\hline Degree & 4th $3 r d$ 3rd 3rd 2nd 2nd 2nd 1st
\end{tabular}

Embodiment construct

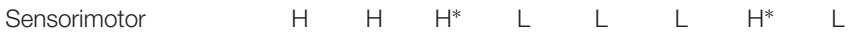

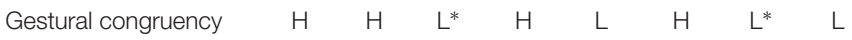

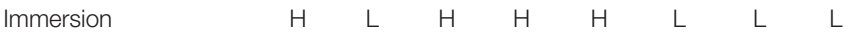

* It would be odd to require a large movement that was poorly mapped to the content to be learned. 
discernable differences are noticeable between degrees separated by more than one step (i.e., 4 th and 2 nd degree, or 1 st and $3 \mathrm{rd}$ degree). In mathematics this ordering is called a "weak ordering" because the highest class (first set termed the 4th degree) has no predecessor, and the last class (last set termed 1st degree) has no successor. We note that it would be odd to purposefully design content that is high on sensorimotor input but low on gestural congruency (e.g., Why make a user swipe a finger back and forth across an entire tablet three times to turn one virtual page?).

The degrees are:

4 th degree $=$ (a) Sensorimotor engagement - High. The system can map (via motion capture, etc.) the whole body, or any part of the body, which can act as the controller of the system. If locomotion is included then visual parallax is also engaged (Campos et al., 2000) and this further increases sensorimotor activation. (b) Gestural congruency - High. There are multiple instances of gestures that drive the system, and these are consistently designed to map to the content being learned. E.g., spinning the arm makes a virtual gear spin the same speed and direction on the screen. (c) Sense of immersionHigh. A very large display is used so the learner perceives environment as very immersive, or a HMD can be used that covers a very large percentage of the field of vision (FOV); borders are not readily apparent (e.g., a participant might stand in the middle of a floor projection with a 21 foot diagonal and when looking down the boarders are not apparent). Participant appears to be fully engaged and/or reports feeling "in the world."

3rd degree $=$ (a) Sensorimotor engagement - The whole body could be used as the controller, but the user remains in one place [e.g., standing at an Interactive Whiteboard (IWB)]. (b) Gestural congruency - The system should contain one or more instances of this. (c) Sense of immersion - A large screen display or floor projection should induce the learner to perceive the environment as immersive; however, borders are usually present in the peripheral. A participant may report they on occasion felt they were "in the world."

2nd degree $=$ (a) Sensorimotor engagement - Learner is generally seated, but there is some upper body movement of the arm or fingers. (b) Gestural congruency - Probably not a defining feature of the content, although there is always some interactivity (e.g., finger swipe to advance, spin mouse for a circle on screen), (c) Sense of immersion - The display covers less than $50 \%$ of FOV and borders are always present no matter the fixation point (e.g., a 16 inch monitor, or tablet-sized screen).

1st degree $=$ (a) Sensorimotor engagement - Low. Learner is generally seated, but there is some upper body movement, usually for a key press occurs. The learner is primarily observing a video/simulation. (b) Gestural congruency - Low. There is no learning-related mapping between gesture and content, the users' movements are elicited primarily for navigation (e.g., tap for next screen). (c) Sense of immersion - Low. The display covers far less than $50 \%$ of FOV and borders are always present (e.g., small form display generally tablet or smartphone screen).

\section{More on the three constructs}

In this section the three constructs of embodiment in education are further explicated.

\section{Sensorimotor engagement}

More muscular movement engages more sensorimotor systems and this translates to larger areas in the sensorimotor cortex being activated. We propose that using larger learning gestures (e.g., moving an arm, rather than a finger) may result in a stronger learning signal. That is, using the arm with the shoulder joint as the fulcrum to simulate a water pump handle may be more meaningful in terms of learning than using the index finger. The reader may ask whether this sort of distinction really matters for the adult brain. Perhaps for a child it may be important to map the meaning of words to actions, but in the adult brain, packed with overlearned words, how much importance would the body metaphor hold for learning new concepts? Brain imaging studies reveal intriguing results. Hauk et al. (2004) measured brain activity using functional magnetic resonance imaging (fMRI) while people listened to action verbs such as lick, pick, and kick; significantly more somatotopic activation of the premotor and motor cortical systems that specifically control the mouth, the hands, and the legs (respectively) was observed. If these overlearned words still activate specific motor areas in the adult brain, and if Goldin-Meadow et al.'s (2001) postulation is correct that gesturing helps to off-load cognition, then perhaps it makes sense to teach science content through the body. A compelling example comes from Kontra's lab (Kontra et al., 2015), students who physically held two bicycle wheels spinning on an axle learned more about angular momentum compared to students who observed a partner holding the wheels. In an extension of their lab study, Kontra et al. (2015) pushed further with an fMRI experiment that revealed that the action group did better than the observe group on tests, and that the level of the BOLD signal in the brain motor regions of interest (left M1/S1) significantly predicted test performance ( $r=0.58, p=<0.009$ ) for both groups. Kontra et al. (2015) tout this as a model that explains how physical experience, relative to observation, increases "activation of the sensorimotor systems important for representing dynamic physical concepts." (p. 6).

Surely emotion and the other senses play a large role in learning and have a place in an embodied theory for education. This article is primarily focused on gesture and kinetics. Nonetheless, it should be mentioned that emotions, and all of the senses, including proprioception, have a place in embodiment theory. If the learning platform and the content afford strong reasons for using more modalities, then those should be considered during design.

\section{Gestural congruency}

Segal et al. (2010) show that when students interacted in a more gesturally congruent manner (i.e., tapping out a number versus selecting a symbol for the number) on a tablet-based math game, the tapping students made fewer errors on a post-test. Koch et al. (2011) report that participants reacted faster in the condition that meshed Stroop choices with congruent gestures compared to 
those in the incongruent gesture group. Our lab's recent studies also support the superiority of gestural congruency for learning about vectors (Johnson-Glenberg et al., submitted).

These gestures are based on what Antle and others call "body metaphors." Antle designed her Sound Maker virtual reality system (Antle et al., 2009) using viable physical mappings for volume, tempo, pitch, and rhythm, e.g., tempo was associated with speed of movement through the room, pitch was associated with movement up and down in 3D space, or toward and away from in a $2 \mathrm{D}$ space, etc. She counsels for "interactional mappings that preserve structural isomorphisms between lived experience and the target domain." Thus, designers should strive for gestural congruency using movements that coincide with real life and cultural experiences, e.g., raising the hand upward usually signifies something going higher. There is a history of this research in cognitive psychology using other names like Self-Performed Tasks (SPT; Engelkamp and Zimmer, 1994; Engelkamp, 2001). A representative study from Engelkamp would compare three groups of participants: one that heard a list of unrelated action phrases ("lift the hat"), one that performed the action without the object, and one that performed the task with the object. The consistent finding was that the self-performing participants recalled more of the phrases than those who merely heard the phrases. When assessing for learning after actions have been performed we cite the encoding specificity hypothesis (Tulving and Tomson, 1973) which holds that content will be better recalled when the cues match the method with which the content was encoded. In our modern and new world of digitization, we are able to easily add the virtual objects to any lesson, what are the effects of that?

\section{Immersion}

Coomans and Timmermans (1997) state that immersion is “...the feeling of being deeply engaged... (in) a make believe world as if it was real." (p. 279). Immersion is subjective, difficult to precisely quantify, and results have been mixed on its effects on learning. In the medical research community the concept of immersion in a task is well-received. A meta-analysis by Miller and Bugnariu (2016) showed that for high-immersion virtual environments treatment response was overwhelmingly positive for those with autism spectrum disorder who were learning social skills. Gutiérrez et al. (2007) showed that students who learned how to treat a head trauma victim via a proprietary virtual HMD (they called this the "full immersion" condition) showed significantly better learning than students who learned on a laptop monitor (called "partial immersion" condition). In the paper it is not explained why those condition definitions were chosen. However, in a small $n$ correlational study run by Bailenson's group (Bailey et al., 2012), participants learned multiple environmental messages while in a VR shower. They then filled out a five item Physical Presence questionnaire and the higher the presence score, the significantly less content participants remembered on a cued recall task. This negative correlation had not been predicted. The authors speculate that after a highly vivid sensory experience, participants may have had limited cognitive resources left over to dedicate to the memory task. Thus, the idea that an immersive VR or MR environment will indiscriminately enhance learning has not been fully supported yet.

The field needs to rigorously define and operationalize immersion for the sake of learning, then we will be better able to weave instances of congruent gestures into effective and immersive educational environments. Slater and Wilbur (1997) proposed that the immersive capability of a virtual environment depends on the degree to which it is "inclusive, extensive, surrounding, vivid, and matching." Each of these components influences, but is not the sole determinant of the user's perceptual experience. Inclusive refers to whether signals pertaining to the physical world have been eliminated (e.g., joystick, weight of wearables, etc.). Extensive refers to the number of sensory modalities that are part of the experience. Surrounding refers to the visual presentation including FOV and the degree to which the physical world is shut out. Vivid refers to the fidelity and resolution of the simulation. Matching refers to whether the viewpoint of the environment is modified to match the user's perspective (e.g., in an HMD when the user moves left, the environment moves as well). The construct of immersion is complex with several measurable components, under the category of inclusive we should also place physiomarkers (i.e., heart rate, skin conductance, pupil dilation, etc). For our study, we focus primarily on the sense of surrounding for immersion. Thus, FOV served as our primary marker or determinant. Borders defined the edge of the learning platform and three very distinct FOV's existed in each platform, with SMALLab, the MR platform to be described in the next section, being the most immersive and extensive.

\section{Crossing Learning Platform with Amount of Embodiment}

The study was designed to use a mixture of platforms that are readily available in schools and at least one that is innovative and very immersive. The SMALLab platform provided the greatest opportunity for use of the whole body and locomotion (and consequently, the greatest range of sensorimotor experiences). These platforms are sometimes called EMRELEs for Embodied Mixed Reality Learning Environments and one might expect the greatest learning gains to be seen in the condition with more whole body activity (Johnson-Glenberg et al., 2014a). To ask the question of how learning gains are affected by platform crossed with embodiment, three different platforms were selected: SMALLab with a very large projected floor display [21 foot (252 inch) diagonal], an IWB (78 inch diagonal), and a traditional desktop with a monitor (16 inch diagonal). The hypothesis is that the high embodied, 4th degree SMALLab platform which encourages larger, stronger sensorimotor signals (by actually spinning objects), and a greater sense of immersion (defined by FOV) will result in better learning and greater delayed learning gains. If stronger memory traces can be practiced with more haptic and large-display visual feedback, then perhaps these traces will aid in bootstrapping new 
knowledge to the learner's existing knowledge structures. Six conditions that varied in amount of embodiment (low versus high) as afforded by the three platforms were created.

\section{Delayed Learning Gains}

The final factor in the experiment was time. As the work on memory consolidation continues (Walker and Stickgold, 2004; Stickgold and Walker, 2007, 2013), it seems critical to ask whether there were group differential effects on knowledge over time. A student may not be able to answer some questions based on more labile forms of memory, for example, a memorized verbal description of a rule or definition after a delay. However, if adding a strong sensorimotor trace increases memory via more complex connections, then a different slope (interaction) for content memory by condition may emerge. With time, group differences might emerge.

In sum, the primary question was whether levels of embodiment in mediated learning platforms affect immediate and delayed learning. We hypothesized that a congruency between learned content and assessment metric might be felicitous for those who learned in a more isomorphic manner, that is, those who used grosser gestures to and more sensorimotor engagement might perform better on a test that included movement via drawing.

Three hypotheses were tested and our predictions and rationales are listed after each.

(1) Platform should be predictive of both immediate gains and delayed learning gains. We predict that the platforms that afford more embodiment and haptic interaction will show greater comparative gains. That is, learners using larger gestures on a platform like an IWB should learn more than those on a computer using more constrained mousedriven movements; however, the greatest gains should be seen on the MR platform called SMALLab because it affords the most sensorimotor engagement as well as locomotion.

(2) Level of embodiment should be positively predictive of both immediate and delayed learning gains. This is hypothesized because a high degree of embodiment implies that the gestures used while encoding were congruent to the content to be learned. This congruency should strengthen the memory trace and facilitate recall of the newly learned content for both post-tests and delayed tests. The greater the amount of embodied gestures that are congruently mapped to the content, then the better the participants should perform on all post-intervention assessments.

(3) The SMALLab high embodied condition should be the one to demonstrate the greatest learning gains. It is the platform that affords the greatest amount of embodiment. If learners are encoding in a more embodied and immersive manner, they may be better at over-riding incorrect analogies about how spinning objects operate in the real world. We want to encourage novice learners' unstable and incorrect mental models to begin to resemble the experts' more veridical models. By using larger body gestures in immersive environments learners may be better able to rehearse and "cohere their pieces" of knowledge, or p-prims, into the correct structure for understanding complex physics concepts.

\section{MATERIALS AND METHODS}

\section{Participants}

From the introductory psychology research pool at a large university, 110 participants were recruited. This study was carried out under the auspices of the Internal Review Board at Arizona State University and with written informed consent from all participants. Because some computer-collected data were lost, some analyses have data from only 105 participants. In addition, data from one participant were eliminated because the participant's score on the post-test was more than four standard deviations below the mean. Of the remaining 109 participants, $32 \%$ were female, $85 \%$ were native English speakers, the median number of high school physics courses completed was 1.0 (with a reported range of $0-4$ or more), and the median number of college physics courses was 0 (range of $0-3$ ).

\section{Design}

Before starting to code the CF content, we held over a dozen design meetings focused on how to create the conditions. It should be stated again that the constructs in the taxonomy are not orthogonal: sensorimotor activation, gestural congruency, and immersion. In addition, we did not have the resources to run every variation, i.e., each construct varied by high versus low, by the three platforms. Indeed, as noted in Table 1, some conditions are not particularly ecologically valid for educational purposes (why create a non-sensical high sensorimotor/no gestural congruency condition, i.e., doing jumping jacks to learn about CF?). Some educational platforms afford various immutable properties. To simply turn off "immersion" in SMALLab is nonfelicitous because SMALLab is at its core a large projection, motion capture environment. Turning off the graphics with realtime feedback would have rendered it a human-tutored lesson with a spinning manipulable; that platform would not have addressed timely new media and design issues.

Working within the stated framework, we assumed low versus high embodiment (as conceptualized by the three constructs simultaneously) and crossed the two levels of embodiment with the three platforms (i.e., SMALLab, IWB, and desktop), this resulted in six conditions. We crossed platform with embodiment and not immersion because high-immersion is folded into the definition of high embodiment. Second, we are asking questions relevant to the current state of educational technology. The types of platforms (and their affordances) that could be placed in modern classrooms drove the study. At the time of the study only desktops and IWB's were in general use. There were few tablets and it would not be expeditious to research non-existent platforms (i.e., SMALLab without a floor projection).

The experiment was a mixed $2 \times 3 \times 3$ factorial design. The first two factors were manipulated between-subjects: (1) 
TABLE 2 | Salient differences between conditions.

\begin{tabular}{|c|c|c|c|c|}
\hline & & Desktop & Interactive whiteboard & SMALLab \\
\hline \multicolumn{5}{|l|}{ Platform } \\
\hline \multirow[t]{3}{*}{ Physical description } & Control device & Mouse & Trackable pen & Trackable manipulables \\
\hline & Body position & Seated & Standing & Standing, spinning \\
\hline & Display size & 16 inch diag. & 78 inch diag. & 252 inch diag. \\
\hline \multicolumn{5}{|c|}{ Embodiment conditions } \\
\hline \multirow[t]{3}{*}{ Low embodiment } & Sensorimotor & $\begin{array}{l}\text { Hand moves mouse to control } \\
\text { virtual bob }\end{array}$ & $\begin{array}{l}\text { Hand and arm hold pen to control } \\
\text { virtual bob }\end{array}$ & $\begin{array}{l}\text { Hand and arm hold trackable } \\
\text { wand to control virtual bob }\end{array}$ \\
\hline & Gestural Congruency & $\begin{array}{l}\text { Mouse controls speed slider on } \\
\text { screen, left to right }\end{array}$ & $\begin{array}{l}\text { Tracking pen controls speed } \\
\text { slider on screen left to right }\end{array}$ & $\begin{array}{l}\text { Hand controls speed slider } \\
\text { projected on floor, left or right }\end{array}$ \\
\hline & Immersiveness-primarily FOV & Low & Medium & High \\
\hline \multirow[t]{3}{*}{ High embodiment } & Sensorimotor & $\begin{array}{l}\text { Hand moves mouse to control } \\
\text { virtual bob }\end{array}$ & $\begin{array}{l}\text { Hand and arm hold pen to control } \\
\text { virtual bob }\end{array}$ & $\begin{array}{l}\text { Hand and arm hold physical } \\
\text { manipulables to control a physical } \\
\text { bob, and body spins in circle }\end{array}$ \\
\hline & Gestural Congruency & $\begin{array}{l}\text { Mouse moves in small circles, } \\
\text { maps to circular movement of } \\
\text { virtual bob }\end{array}$ & $\begin{array}{l}\text { Pen moves in large circles, maps } \\
\text { to circular movement of virtual } \\
\text { bob }\end{array}$ & $\begin{array}{l}\text { Swinging the physical bob } \\
\text { overhead. Also, the whole body } \\
\text { spins around to release the bob }\end{array}$ \\
\hline & Immersiveness-primarily FOV & Low & Medium & High \\
\hline
\end{tabular}

Except for key device nouns the experimenter script did not vary between conditions.

embodiment with two levels - low or high, and 2) platform with three levels - Desktop, IWB, or SMALLab. Table 2 gives examples of the salient differences between the six conditions. The within-subjects factor, test time, had three levels: pre-test (same day), post-test (immediately after instruction- same day), and follow-up (an average of 1 week after instruction).

\section{Materials and Procedure}

All conditions included a pre-test, watching a 3-min vocabulary animated video (wherein the four CF terms in the equation were explained), the instructional intervention (i.e., experimental manipulation), a post-test, and a delayed follow-up test. The instructional intervention - hereafter referred to as the lesson consisted of the participant interacting one-on-one with one of two experimenters to learn key concepts and the proportional relationships represented in the CF equation. Participants manipulated either a real or a simulated "bob" that spun around a fixed point.

\section{The Three Platforms and Lesson Variations}

A group of five high school physics teachers met for multiple sessions to co-design the content. A master script was written for the two experimenters to follow as they instructed participants. The first coded version of the lesson was for the extreme lesson, i.e., the "all bells and whistles" version, that was deemed to be 4 th degree with the most embodiment. The first lesson was high on all constructs: sensorimotor, gestural congruency, and immersion and that lesson was best afforded by the platform called SMALLab. The following lessons were then tailored down along the dimensions of gross body movements, congruency of gesture, and immersion as described in the taxonomy, until ending up with the desktop and mouse version with little interactivity. It is important to note that the instructions and question prompts from the experimenters remained the same throughout the six conditions, only short phrases were altered "spin the mouse" versus "sin the bob." All lessons were equated for time on task.

In the extreme lesson, i.e., high embodied SMALLab, the three variables in the CF equation were instantiated in a multimodal manner. For example, (1) radius corresponded directly to the length of a physical string attached to a bob that was spun overhead, (2) mass corresponded directly to number of weight packets placed inside the spinning bob, and (3) velocity was controlled directly by the participant spinning the tangible bob overhead with his/her hand. Thus, the participant received realtime sensorimotor/haptic feedback, as well as aural and virtual visual feedback (e.g., increase in pitch as the bob swung faster, bar charts corresponded to bob's speed). The pitch increase (aural) and bar charts (visual) feedback were given in all six lessons.

In the low embodied condition the participants had control over the bob, but in an indirect manner - through a virtual slider. The media-rich dynamic graphics in the animations were visually engaging and as the speed of the spinning bob increased pitch always increased accordingly. Thus, we make the distinction between high and low embodiment, and we avoid the descriptor of "no embodiment." One of the greatest differences between the low and high conditions that might not be readily apparent in Table $\mathbf{1}$ is that controlling the bob was not "well mapped" in the low embodied conditions. Participants in the low embodied SMALLab condition used a tracking wand to move a slider to increase speed of the bob. Whereas, in the high embodied conditions, the physical act of spinning the mouse or pen, or swinging the bob overhead actually corresponded directly to and drove the speed of the virtual bob. In juxtaposition, in the low embodied conditions, the speed of the bob was driven by lateral placement on a virtual slider. Thus, location of a point along a short horizontal slider drove speed and not a congruent action. There was some agency involved in selecting that speed, but there was not a one-to-one, direct gesture-driven mapping associated 


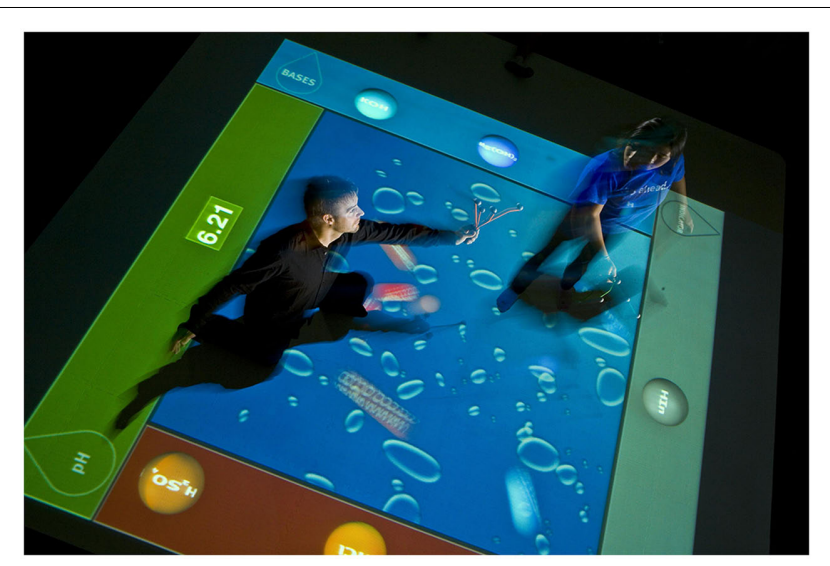

FIGURE 2 | Example of SMALLab floor projection with marker-based motion capture.

with the speed of the bob. This is what is meant by "not well mapped." To see a video to help conceptualize, please visit www.embodied-games.com/games/all/centripetal-force or https: //www.youtube.com/watch? $\mathrm{v}=\mathrm{oFiXtcXRpVE}$.

\section{Condition 1 - High Embodied SMALLab}

The Situated Multimedia Arts Learning Lab (SMALLab) is a $15 \times 15 \times 15$ feet interactive space designed to engage multiple sensory systems including vision, audition, and kinesthetics. See Figure 2 for an example of the how the floor projection works.

The MR environment includes both digital components (projected graphics on the floor) and tangible, physical components (motion tracking wands and manipulable handheld objects). The platform provides an engaging mesh of the real and the virtual. The system uses 12 infrared NaturalPoint Optitrack cameras for real-time motion tracking of handheld rigid-body objects. Participants manipulate projected images on the floor with the handhelds. The wands and tracked spinning bobs are tracked in $X, Y, Z$ coordinates with millimeter precision. One of the notable differences between this platform and a traditional desktop or IWB platform is that participants can locomote through the immersive environment thus providing multiple opportunities for congruency between dimensions of action and dimensions of the content being learned, as well as parallax (Johnson-Glenberg et al., 2014a) and Campos (Campos et al., 2000) for reasons why locomotion may represent a special case of embodied learning from a developmental perspective.

\section{New tangibles}

Two new tangible objects were constructed for this study - a 'swinger' and a 'flinger.' Figure 3 shows the swinger, it is a tracked bob or ball on a string of varying length. The mass inside the ball can be varied during the lesson by inserting taped weight packets inside the bob, and the radius can be adjusted by switching between two lengths of string.

Figure 4 shows a student using the swinger in the high embodiment condition. The adjustable string was created to

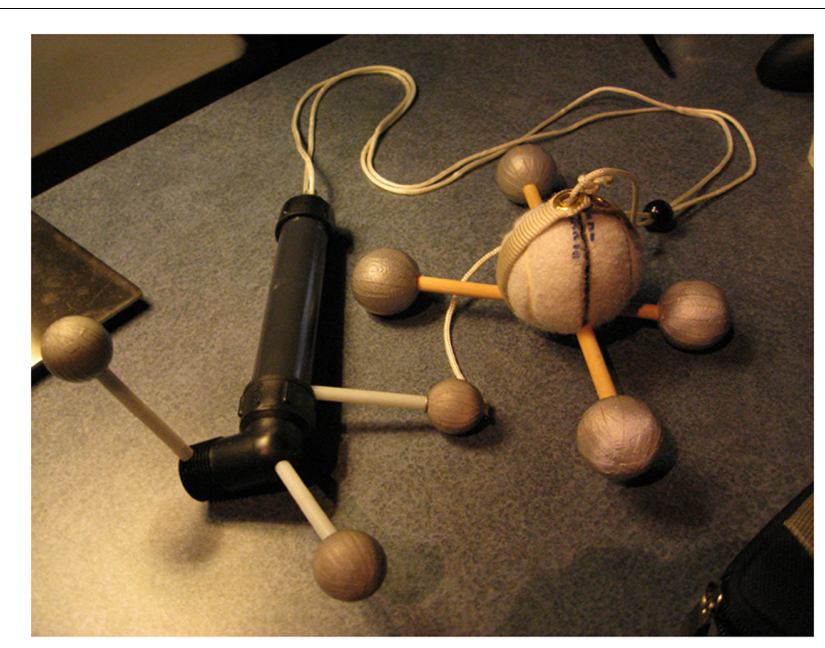

FIGURE 3 | The "Swinger" used in the high embodied SMALLab condition.

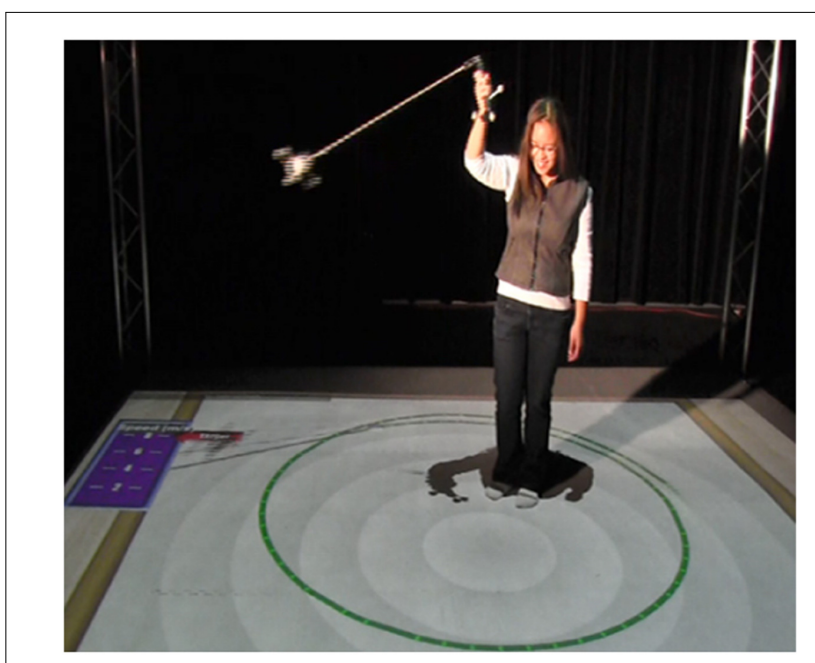

FIGURE 4 | The "Swinger" in action. Note dynamic simulations projected on the floor giving real-time feedback.

address the radius misconception that more is more. The swinger was always swung overhead.

The second tangible was the flinger. See Figure 5. This dualcomponent wand was used to assess the learning of trajectory at point of release. The brass lever on the top of the handle serves as the release mechanism. Participants physically spun or rotated their entire bodies around holding the flinger in front so they could watch it at all times. They then released the bob at a time of their choosing to hit a target on the floor. That is, the tennis ball (the 'bob' part) of the unit would disengage and fly from the tracked handle when the brass lever was depressed. The smaller tracked spheres on both components are covered with retroreflective tape and this allows the IR cameras to map the positions of both the handle and the moving bob. Thus, when the bob is released, both the handle in the hand and the released bob moving 

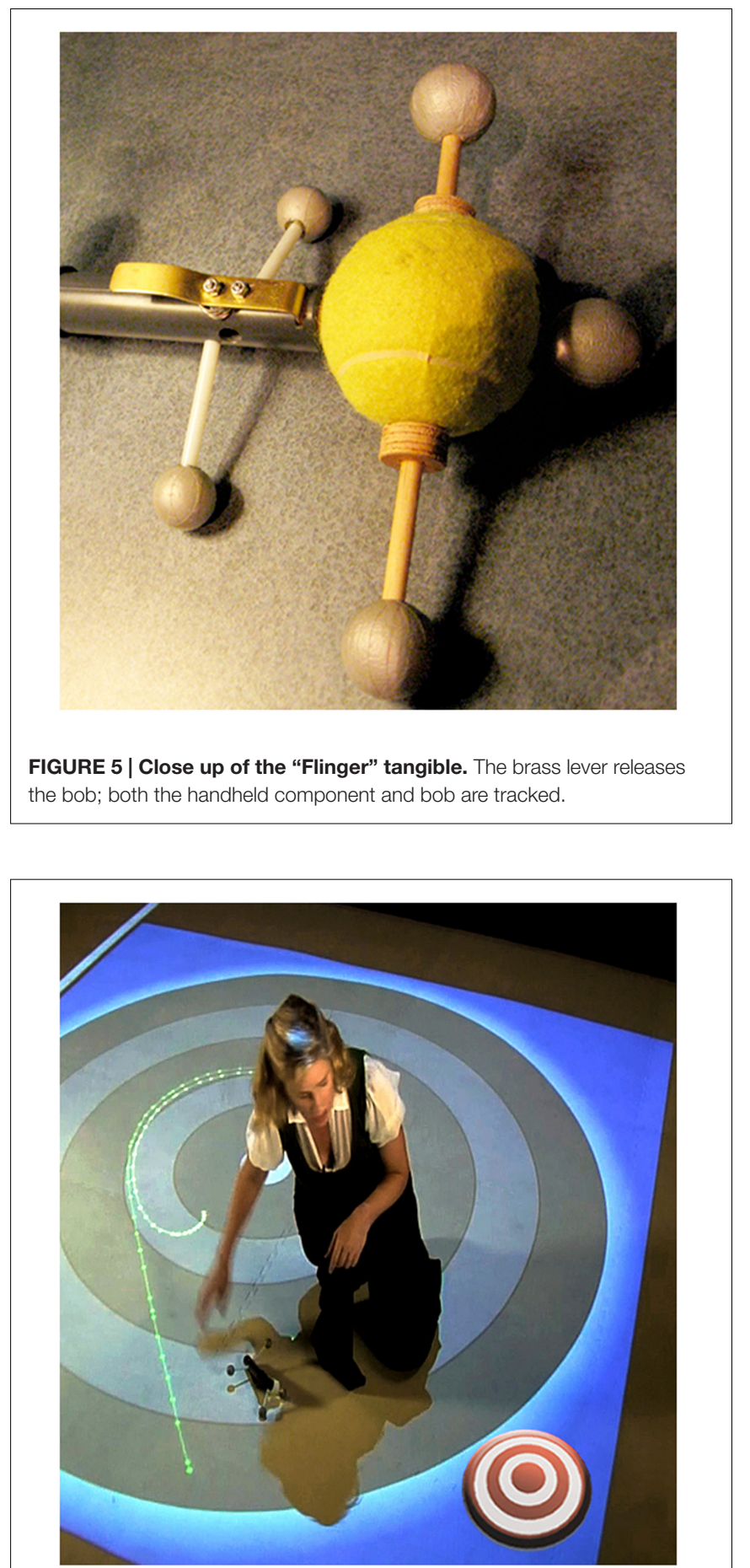

FIGURE 6 | The Target Game. There are two "ghost trails" on the floor. The line that continues to curve represents the handheld component. The line that straightens represents the bob's tangent at point of release.

through the air can be tracked for several seconds. Participants are able to observe how the bob flies at the point of release and begin to address the impetus misconception regarding trajectory at point of release.

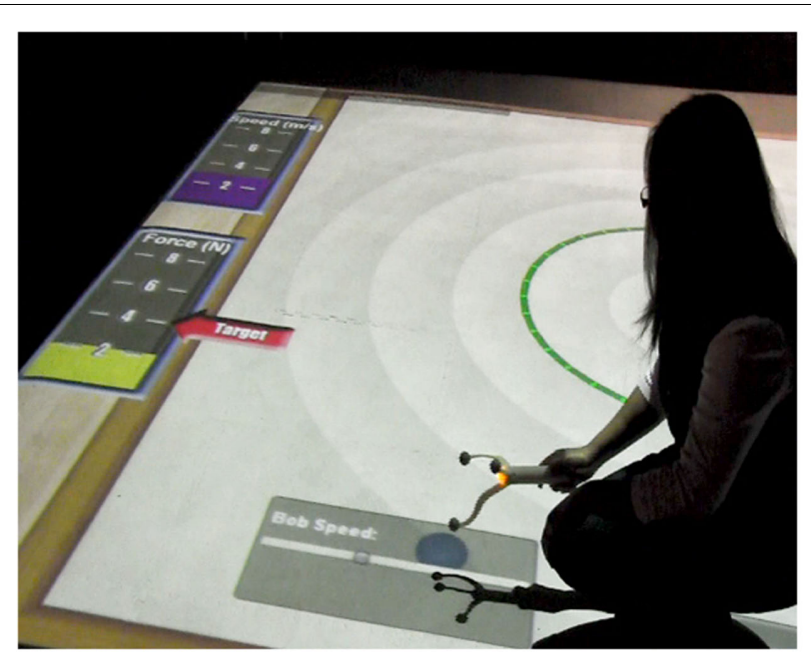

FIGURE 7 | Student in low embodied condition using trackable wand to change Speed. (example of low gestural congruency).

Figure 6 shows a user who has released the bob trying to hit the virtual bulls-eye target on the ground during the Target Game. It also shows the mediated ghost trail feedback on the floor projection. It is important that both sets of tangible objects are tracked. Again, users receive visual feedback on placement of both the handle (in Figure 6 the curling line) and the traveling bob (the straight line) from user-created movements. The visual feedback provides irrefutable evidence that the bob travels in a straight line after release.

Given the full-body kinesthetic experience in SMALLab, this lesson is considered highly embodied. The experimenters followed a memorized script. The script did not vary substantively between conditions, only a noun or two might change. Experimenter effects were controlled for in this manner. Each element in the CF equation was introduced one at a time using a "guided discovery" method so that participants would not be overwhelmed with the physics concepts.

\section{Condition 2 - Low Embodied SMALLab}

In the low embodied SMALLab condition, participants used a different rigid-body trackable object. This was an extant 3Dprinted plastic wand that has been used for other studies. It has a unique configuration of retro-reflective spheres and performs much like a "wireless mouse." Participants in this condition used the plastic wand to control the virtual slider and signal a release from spin via $X, Y, Z$ placement of the wand.

In all three low embodied conditions, the participant controlled speed of spin with a horizontal virtual slider. In this SMALLab low embodied condition the slider graphic can be seen in the bottom of Figure 7. Again, the participants had a small amount of agency over the speed of the bob, but their gestures or actions were not highly congruent (as in the previous condition). The digital slider action is left-to-right linear and does not afford the physical, circular kinesthetics of swinging an object overhead. In addition, speed of moving the wand did not correspond to 


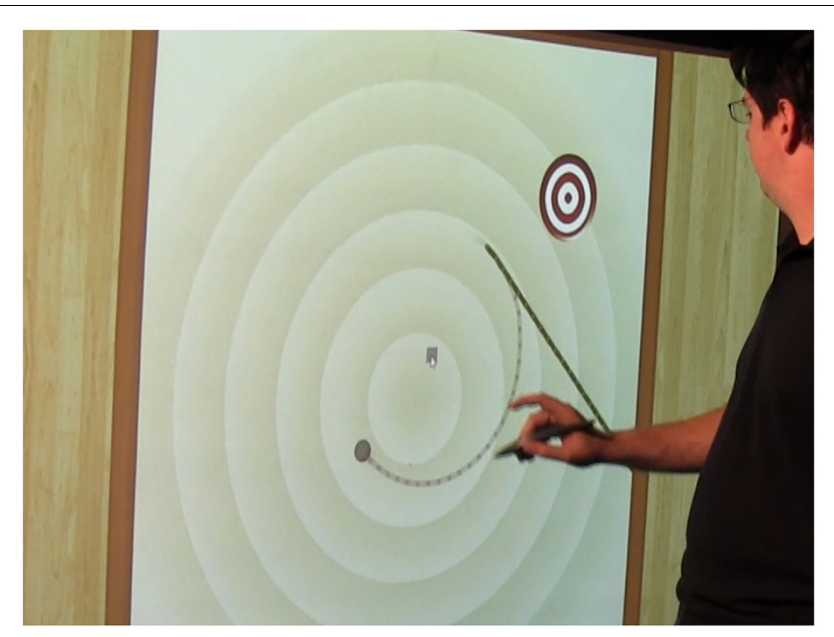

FIGURE 8 | Participant at the whiteboard in the high embodied Interactive Whiteboard (IWB) condition using the tracking pen.

speed of the bob spinning, only placement of a virtual marker on the slider altered speed.

Figure 7 shows a student adjusting the simulated bob speed using the wand over the slider. Note the bar graph on the upper left that shows immediate feedback as the participant attempts to match a target speed, similar to the high embodied condition. When the participant wished to release the bob from its virtually tethered spin and hit the target during the Target Game, s/he merely raised the wand up (in the $Z$ axis) and the virtual bob was released. The virtual bob left the same type of ghost trail on the floor as the physical bob.

\section{Condition 3 - High Embodied Interactive Whiteboard}

In the IWB platform, the participants used the tracking pen provided with the Promethean ACTIVboard. In the high embodied condition, participants started the bob spinning by directly moving the tracking pen in a circular motion on the large vertical board. The velocity of the hand movement directly controlled the velocity of the bob, i.e., the virtual bob is linked to the pen's tip. This would be considered highly gesturally congruent. For the Target Game or "trajectory at release" phase of the lesson, participants lifted their index finger from a triggerbutton on the pen to release the bob.

Figure 8 shows a student who missed hitting the target on his first try. It should be noted that in all six conditions during the Target Game the bob's path was always tracked and presented via a ghost trail.

\section{Condition 4 - Low Embodied IWB}

In the low embodied condition on the IWB participants again interacted with the left-to-right virtual slider at the bottom of the IWB screen (similar to the gesture used in all other low embodied conditions). Moving the pen along the slider increased or decreased the spin, but not in a direct one to one manner, what mattered was placement of a marker on the horizontal slider.
To release the bob from the spin during the Target Game in the low embodied condition, the student watched the bob spin and then tapped a virtual "release" button on the IWB screen at the desired moment of release. Thus, there was some agency associated with the task, but it would still be considered to have low levels of gestural congruency. In all three low embodied conditions for the Target Game, participants were always able to choose the release points.

\section{Condition 5 - High Embodied Desktop}

In the desktop platform, the participants viewed a 16 inch monitor and used a Windows 7 tower machine on the floor. Participants sat at a desk and used the mouse on a table to start the bob spinning in a circular motion. The direction and velocity of the hand controlled and directly mapped to the direction and velocity of the virtual bob, so there was gesturally congruent feedback. For trajectory at release during the Target Game, the participant lifted the index finger off the left mouse button to release the bob.

\section{Condition 6 - Low Embodied Desktop}

In the low embodied condition, participants were presented with the same virtual left to right slider as was developed for the two other low embodied conditions. They used the mouse to click and drag the marker on the virtual slider to affect bob speed. In all low AND high embodied conditions participants generated their own real-time data for the spinning sections. It should be noted, in all low embodied conditions they did not merely watch animations, but had varying degrees of agency over all simulations. Participants saw graphs and arrows and feedback related to velocities that were self-generated, albeit in a low embodied manner via the slider. For the Target Game participants clicked on an on screen button to control the moment of the bob's release.

\section{Assessment Measures}

A 20 item test was designed in conjunction with two high school physics teachers. See Appendix A. All three tests were invariant, except that the post-test and follow-up tests included one final item (item 21 an open-ended question) that was not used in these analyses as it was not included in the pre-test. Two distinct subtests were designed, a computerized declarative subtest and a hand-drawn generative subtest.

\section{Declarative Knowledge Computerized Subtest}

The first, on-line subtest (items 1-13) was administered using a computer. It was designed to primarily tap memory for information explicitly presented during the lessons. The first five items in this section were open-ended questions querying definitions of CF terms. The definitions were scored on a $0-2$ scale. Items 6-13 described or showed images of circular motion events and answer choices were displayed in a four item multiple choice format. Of these eight image-based items, four of them ended with the prompt, "Explain why you chose that answer." Participants typed in responses and these were scored on a scale of $0-2$, often these resulted in one word responses. 
A

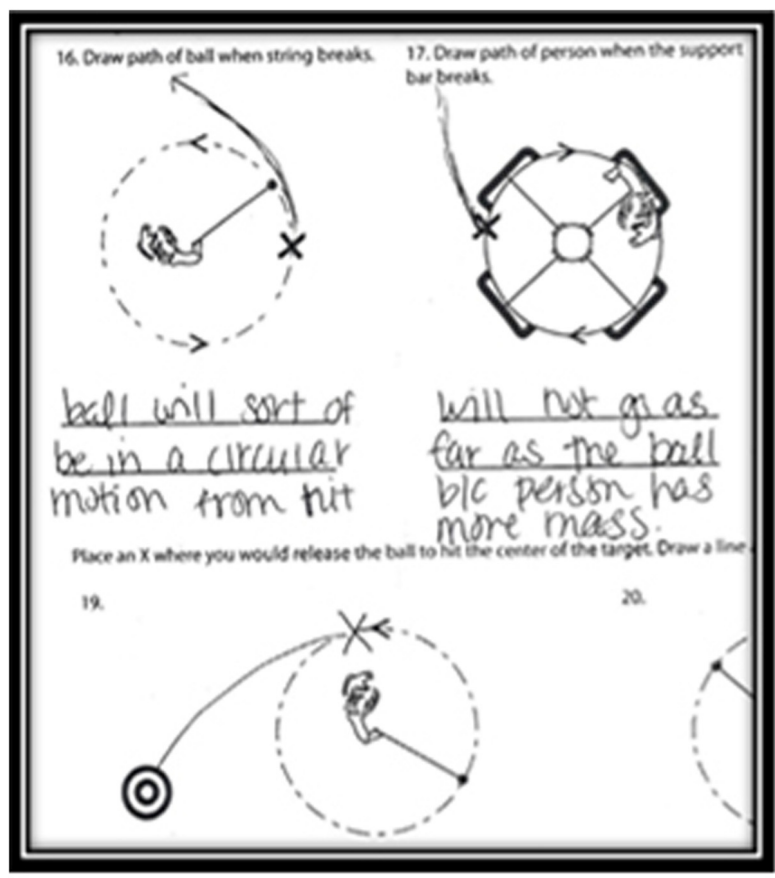

B

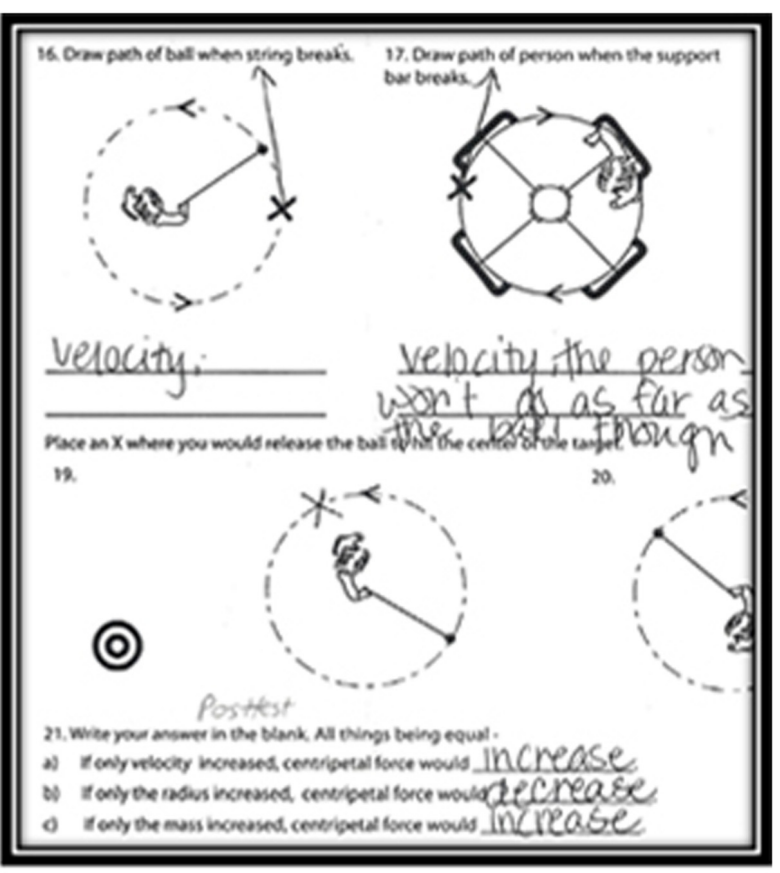

FIGURE 9 | Example of pre-test and post-test answers from same participant showing that the impetus model has been corrected.

\section{Generative Knowledge Subtest}

Items 14 through 21 were completed using a paper-based generative (off-line) subtest. The first seven items were designed to allow the student to create, with few constraints, answers to questions about several key CF concepts. The first two items displayed images of a bob swinging around a pivot (a human or a tetherball pole) and the participant was prompted to draw an arrow representing the "force on the ball," (i.e., CF). The first five items also ended with the prompt, "Explain how you decided to draw the arrow the way you did." These were scored 0-2. The next three items displayed bird's eye views of CF events and queried the participant to draw arrows that represented the path that the ball (or person) would take when released from CF at a point on the circle labeled with an X. Explanations were requested as well. The final two items were near-transfer items and required the student place an $\mathrm{X}$ on the dotted circular path to indicate where to release the ball so that it would hit the target. The left panel in Figure 9 shows the same participant's answer in the pre-test, on the left and post-test, on the right. That participant held the incorrect impetus model at pre-test, but drew a correct straight trajectory for the ball after release at post-test, correcting the misconception.

\section{Reliability}

Two scoring techniques and reliabilities are described here: consensus tangent scoring and inter-rater correlations. For the seven off-line generative subtest items that required a tangent to be drawn (items 14 through 20). The instructions requested that the participant draw either "arrows or lines" to show the path of a released object. The veracity of the tangents was assessed by two blind scorers who needed to come to consensus. The subject ID with condition was blocked out on the top of each page. Both scorers sat together and needed to agree when there was a dispute. First, an expert overlay sheet (created by the first author) was made with the correct tangent drawn. Each participantconstructed tangent received two sub-scores. The first sub-score addressed whether the tangent appeared straight, range $0-2$. The second sub-score was more objective and included the degrees of deviation from the expert angle at the circle. Theta, $\theta$, was measured with a protractor. A zero score was awarded for any angle greater than $39^{\circ}$ from the expert-generated angle, one point for any angle between 20 and $39^{\circ}$, and two points for any angle less than $20^{\circ}$ from the expert-generated angle. The two sub-scores where then summed and divided by two, thus the mean score of the two agreed-upon sub-scores was entered as the one score for that item (range 0-2). This was a way to take into account both global curve and degree of curve at start point; it penalized the occasional drawing that started out straight with an exact angle match at the beginning, but ended with an anomalous curved tail.

The other test items were comprised of multiple choice and short responses to open-ended prompts. A rubric for the prompt answers was created by a physics teacher and scores ranged from $0-2$. Half of the tests were randomly selected for blind review. A first pass on scoring was made by a graduate student blind to condition. A second pass was made by a research assistant blind to condition on a random subset of half the tests. This research assistant was trained on the rubric and scored all items except the line drawings. Inter-rater reliability was high on the pre-test, 
Pearson $r(54)=0.98, p<0.001$, and acceptable on the post-test, $r(54)=0.81, p<0.001$.

\section{Procedure}

After the pre-tests and a 3-min introductory video on vocabulary terms, the experimental intervention began and lasted approximately $50 \mathrm{~min}$. The two experimenters each followed a script based on the Tasks subsection below in "Tasks. The Same Procedure with Eight Tasks Was Followed in Each Condition." All lessons were advanced with a remote control held by the experimenter. Participants then took the immediate post-test. Five to six days later, they were reminded via email to return to the laboratory for the follow-up test (mode of return days for follow-up = seven). Follow-ups could occur between day 7 and 10 post-intervention. One research credit and 10 dollars were offered as further incentive to return (69 of the original 105 participants returned for follow-up).

Participants were randomly assigned to one of six conditions. The intervention or lesson included eight tasks that focused on the relationships between CF and (a) speed, (b) radius, and (c) mass - the variables in the equation. Although the tasks and learning goals were the same across the six conditions, the specific implementations varied by platform affordances and the degree of embodiment.

\section{Tasks}

The Same Procedure with Eight Tasks Was Followed in Each Condition.

\section{Speed}

The participant was instructed to vary the speed of the bob and was prompted to talk aloud about observations. There were three subtasks in this section. In the first subtask, the participant manipulated the speed of the bob (by congruent or noncongruent gestures in the high and low embodied conditions, respectively), and the speed was indicated by the dots in the green arc projection (Figure 2). The participants were prompted to explain why the dots in the arc trail spread out with an increase in speed, and to describe how the audio (the pitch) was affected by speed of the bob. If incorrect three times a row the experimenter supplies the answer. In the second subtask, a digital arrow perpendicular to the length of the string and tangent to the arc at bob location was added. The arrow became elongated with increased speed. The participant then varied the speed of the bob and observed changes in the length of the arrow. The participant was then asked if s/he knew the difference between velocity and speed. If the participant's answer was incorrect on the third prompt the correct answer was supplied. (The corrective procedure was the same across all conditions, the correct answer was supplied after three incorrect attempts). Third, a purple bar graph that indicated speed was projected, and the participant was asked to match the speed of the bob to a target level shown on the purple graph. The participant was asked to explain the relationship between the speed of the bob and the bar graph. Throughout the intervention, at the end of each of the eight tasks the participant was asked to summarize how the graphical representations related to the manipulation of the bob.

\section{Trajectory at release}

The participant was instructed to imagine him/herself as the bob and asked to either trace (if on the IWB or desktop conditions) or walk (if in the SMALLab high condition) the path that would be traveled by the bob if the string were to break at a point designated by a red "X" projected onto the bob's circular path. Participants were then asked why they created that path at the point of release. If the answer was incorrect, e.g., was curvilinear, s/he was prompted to try again. The participant was asked to explicitly state that objects travel in a straight path to the circle when released from circular motion. Once the idea of a straight line was established, the red " $\mathrm{X}$ " was moved to a different position along the circular path and the participant was asked to repeat the exercise for a total of four times predicting the traveled pathway.

\section{Target Game}

The participant was then advanced to the third task, the Target Game. A projected red bull's eye target was placed in one of four locations. In the low embodied conditions, participants released the virtual bob with various methods: press a button on either the IWB pen or mouse, or use an upward "swiping" motion with the wand in SMALLab. In the three high embodied conditions the participants physically started the bob swinging with the input device associated with platform, e.g., in the SMALLab platform participants spun their bodies around in a circle and released the flinger with the brass lever; in the IWB platform participants moved the pen in a circle and then released; in the desktop platform participants spun the mouse in a circle on the table before releasing the mouse button. Thus, the high embodied participants received four trials of physical practice spinning and releasing. The low embodied participants stopped and started four simulations of the release. In all conditions, when the target was hit, audio feedback of clapping was played. The target moved location for each trial.

\section{Centripetal force vector}

The fourth task involved exploring the relationship between speed and CF. The participant was asked to start spinning the bob in a circle and notice that a new, yellow arrow had been added to the graphic display. Participants were encouraged to adjust the speed of the bob to see what happened to the yellow arrow. The tail of the yellow arrow was placed in the center of the bob and the arrow pointed toward the pivot running down the length of the string (tether). The arrow represented the pulling force exerted by the string (CF). The participant was then introduced to a realtime tracked yellow bar graph and asked what it represented. If incorrect a third time the answer was supplied. Alongside the graph, a target marker was placed on either the number 2, 4, 6 , or 8 and the participant was asked to swing the bob so its force would match the target value. When the value was matched, the participant received audio feedback in the form of clapping and cheering. Upon completion of the two trials, the second, purple bar graph was shown below the yellow graph and labeled "meters/second (m/s)." The yellow bar graph for force was labeled in Newtons (N), the SI unit of force. The participant was asked to spin the bob at $2 \mathrm{~m} / \mathrm{s}$. A marker was placed at the corresponding value for $1 \mathrm{~N}$ on the force bar graph. The participant was asked 
to predict what would happen to CF if the speed of the bob were to double. After the guess, the participant was asked to double the speed to $4 \mathrm{~m} / \mathrm{s}$ and verify the answer. Then, s/he was asked to double the speed, again, to $8 \mathrm{~m} / \mathrm{s}$. The goal was for participants to discover that force is proportional to the square of the speed rather than linearly related to speed. The participant was asked to state this relationship out loud.

\section{Radius}

In the fifth task added radios to the conceptual framework. The length of the string was varied to explore the relation between radius and $\mathrm{CF}$. The participant was asked to predict what would happen to the force on the string if speed were kept constant and the length of the string increased. The majority responded that he force would increase if the length increased. The participant was also asked why a change in force might happen. The participant was then made to try the different string lengths to verify the prediction made.

In the desktop, IWB, and the SMALLab low embodied conditions, the length of the string was changed by clicking a button labeled "shorter" or "longer." In the SMALLab high embodied condition, the original $0.5 \mathrm{~m}$ string was physically replaced with a $1.0 \mathrm{~m}$ string. As the high embodied participants swung the longer "swinger" manipulable over their heads, they often spontaneously noted how different the sensation felt and that it was "easier" to spin the longer one. Very few participants could articulate why this might be so.

\section{Mini-lesson on varying radii}

The sixth task consisted of a graphical and verbal explanation as to why the CF is greater when the radius is smaller. This was the second misconception we wanted to address. For the lesson, two concentric circles of different radii were shown on the screen (or floor for SMALLab). This graphic can be seen on the left in Appendix B (also called Figure 11). There was a brief explanation of what the vectors represented, and then the experimenter would click a button on the remote and the translated vectors (on the right) were projected on the floor or screen.

The vectors were translated so that the tail of the vector at time $=\mathrm{t}$ was aligned with the tail of the vector at time $=\mathrm{t}+1$. Thus, the translated gray vectors in the figure show the change in direction needed to keep the bob on a circular path at two successive time points. It was explained to the participant that the greater change in direction associated with the shorter radius (string) required a greater force. That is, the angular change in the "short radius" vectors' directions required a greater pull on the string to keep the bob on the circular path at the same speed. When the experimenters talked through these graphics, the explanations often elicited comprehending "ohhhs" from participants. This was a real aha moment for many in the experiment.

\section{Mass}

The seventh task required the participant to vary the mass of the bob while holding the speed constant. (To help participants hold the bob's speed constant there was both the digital representation of the bob's speed and variable pitch sonic feedback.) First, the participants were asked to predict what would happen if speed were held constant and mass were doubled. After responding, the experimenter encouraged the participant to add more mass to the bob and see what happened to the force. At the conclusion of this task, participants were asked to describe the relation between mass and force. In the SMALLab highembodied condition a packet weighing $100 \mathrm{~g}$ was added to the hollow bob to double its mass (there was already $100 \mathrm{~g}$ in the center of the bob). This allowed participants to feel that an increase in force was needed to keep spinning the bob at the target speed. In the other five conditions (without that manipulable bob) an increase in mass was simulated by the projected bob increasing in size with the click of a button.

\section{Applying all three variables}

In the eighth and final task, the participants were asked to name the three variables that affected the CF between the string and the bob (speed, radius, and mass). In a simulation, using two of the three variables, with the third variable held constant, the participants were asked to manipulate the two variables to match a target force. They practiced this until correct. After successfully matching this target force on a bar graph, the experimenter described a situation in which someone was swinging a bucket around in a circle overhead as fast as possible. The participants were asked which two factors could be changed to increase the force between the person and the bucket.

\section{Experimenter Fidelity}

The two experimenters moved the participants through the sections with remote clickers (SMALLab, IWB) or hitting the appropriate advance keys on the keyboard (desktop condition). Because the experimenters actively queried at the end of each section and also answered participants' questions; we refer to this a "guided inquiry" lesson. Both experimenters memorized a script they helped to write. At the end of each section the experimenter would inquire about the relationship between all the elements in the task. Lessons varied only slightly between condition and almost every response from an experimenter was scripted. The experimenters clicked to advance to preordered sections after the participant answered queries correctly or the experimenter had supplied the correct answer after three attempts. There was little room for experimenter variability. The experiment lasted two semesters ( $\sim 5$ months) with the same two experimenters. The first author observed each experimenter twice in the first month of the experiment. The only feedback given and this was given to both the experimenters - was to be certain to make sure that if a participant supplied an incorrect answer three times in a row, only then should the experimenter gave the correct answer (e.g., one experimenter gave an answer after two attempts on one task, and the other experimenter gave an answer after four attempts on a task).

\section{RESULTS}

Invariant tests were administered at three time points: pre-test, post-test, and a delayed test. There were no significant differences 
on pre-tests between conditions, $F<2.0$. A two factor factorial ANOVA that included the between-subject factors of platform and level of embodiment was used to analyze (a) the total pre-test score, (b) the on-line (declarative recall) subtest, and (c) the offline (generative) subtest. There were no significant main effects or interactions in any of the pre-intervention analyses.

\section{Analyses on Whole Test}

An ANOVA was run on the whole test (both subsections) analyzing the difference between pre-test to post-test (withinsubjects) in addition to platform and level of embodiment. The overall immediate learning increase from pre-test to post-test was significant, $F_{(1,99)}=459.89, p<0.001$. However, all of the groups improved similarly, with no other main effects or interactions reaching significance $(F$ 's $<2.0)$. Of the 109 students who completed the immediate post-test, 69 also completed the delayed test. The attrition rate did not differ across the six conditions, $\mathrm{X}_{(5)}^{2}=2.32, p=0.80$. An ANOVA was conducted using post-test to follow-up as the within-subjects variable, i.e., on delayed learning gains, and there were no significant group differences. In Appendix $\mathrm{C}$ are the tables for the descriptives and effects sizes for total test scores (the sum of both subtests).

\section{Analyses on the Subtests}

Because we had reason to suspect that the high embodied groups might perform differently on the more generative subtest that were more sensitive to embodied learning, we analyzed those separately. There were no significant pre-test differences on the subtests $(F<2.0)$. Tables 3 and 4 list the Means and SDs for the on-line (declarative) and the off-line (generative) subtests.

The two subtests varied in important ways. First, the on-line declarative subtest was taken on the computer, and its scores reflected more of the ability to retrieve knowledge rather than apply knowledge and demonstrate it in an unconstrained manner (e.g., no multiple choice items are on the generative subtest).

TABLE 3 | Descriptives for on-line declarative subtest.

\begin{tabular}{lrcc}
\hline Condition & $\begin{array}{c}\text { Pre-test } \\
\boldsymbol{M}(\boldsymbol{S D})\end{array}$ & $\begin{array}{c}\text { Post-test } \\
\boldsymbol{M}(\boldsymbol{S D})\end{array}$ & $\begin{array}{c}\text { Follow-up } \\
\boldsymbol{M} \text { (SD) }\end{array}$ \\
\hline SMALLab low embodied & $10.39(5.63)$ & $21.64(4.42)$ & $22.07(2.89)$ \\
SMALLab high embodied & $9.22(4.47)$ & $20.43(4.83)$ & $21.25(3.68)$ \\
IWB low embodied & $9.28(4.45)$ & $20.47(3.57)$ & $20.78(3.46)$ \\
IWB high embodied & $11.38(4.92)$ & $21.16(3.86)$ & $21.29(4.11)$ \\
Desktop low embodied & $9.69(4.80)$ & $20.81(5.19)$ & $20.18(6.66)$ \\
Desktop high embodied & $9.92(3.13)$ & $21.66(2.00)$ & $20.58(4.50)$
\end{tabular}

TABLE 4 | Descriptives for off-line generative subtest.

\begin{tabular}{lccc}
\hline Condition & $\begin{array}{c}\text { Pre-test } \\
\boldsymbol{M}(\boldsymbol{S D})\end{array}$ & $\begin{array}{c}\text { Post-test } \\
\boldsymbol{M} \text { (SD) }\end{array}$ & $\begin{array}{c}\text { Follow-up } \\
\boldsymbol{M} \text { (SD) }\end{array}$ \\
\hline SMALLab low embodied & $16.86(8.18)$ & $25.86(5.28)$ & $25.81(6.04)$ \\
SMALLab high embodied & $16.48(7.66)$ & $24.10(7.31)$ & $25.60(6.45)$ \\
IWB low embodied & $13.34(8.84)$ & $25.12(6.37)$ & $21.89(8.91)$ \\
IWB high embodied & $15.44(8.70)$ & $26.59(3.57)$ & $27.25(2.60)$ \\
Desktop low embodied & $11.88(8.95)$ & $23.09(6.62)$ & $25.14(6.44)$
\end{tabular}

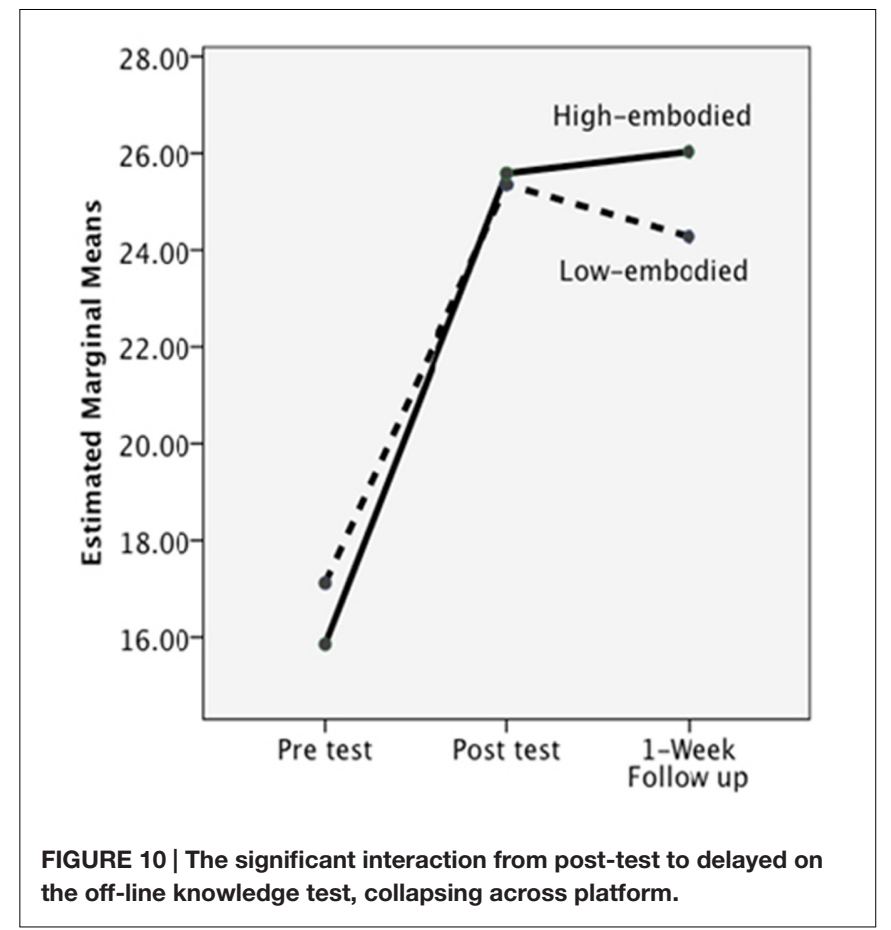

Second, the majority of the items on the off-line generative subtest items required a type of gestural congruency to produce the answer. Participants needed to draw or generate trajectories to show the path that the bob would take when released. It was hypothesized that the congruency between learned content (more sensorimotor activity) and assessment metric might be felicitous for those who learned in a more isomorphic manner, i.e., those in the high embodied groups who practiced releasing the bob with more gestural congruency.

\section{Delayed Effects}

In Table 3, the gains between groups are almost "lock step similar." ANOVA analyses on group differences resulted in $F^{\prime}$ s $<2.00$, and all analyses on time resulted in $F^{\prime}$ s $<2.00$. However, for the generative subtest, there was a significant interaction between level of embodiment and post-test to followup (i.e., delayed learning gains), $F_{(1,62)}=4.83, p=0.03$. As depicted in Figure 10, at the post-test, the low and high embodied groups performed similarly, but, with the passage of 1 week, the low embodied group did not retain as much of their new understanding of CF compared to the high embodied group. The largest decrease in retention was seen in the low embodied IWB group. The figure presents the results collapsed across platform.

\section{Aptitude by Treatment Effect}

We also performed an analysis to determine if the interaction was modified by an aptitude by treatment effect. These analyses used multi-level modeling (MLM) for several reasons. First, the measure of aptitude, the pre-test score (which correlated significantly with number of semesters of high school physics, number of semesters of college physics, and GPA), was continuous. Second, this technique obviates concerns regarding 
sphericity. In addition, because this method uses maximum likelihood estimation, missing data are optimally handled. In the analyses, two degrees of freedom for platform were used to represent SMALLab versus the desktop and IWB versus desktop. The following variables were entered into the model and all variables were centered at the grand mean: the pre-test score, a variable representing delayed testing (delta of post-test to delayed follow-up), and the two effects-coded variables representing platform, and degree of embodiment. In addition, we entered interactions of pre-test by embodiment, pre-test by delayed, embodiment by delayed, and the three-way interaction of pretest by delayed by embodiment. There was a significant main effect of the pre-test, $t_{(106.35)}=5.05, p<0.001$ and a significant two-way interaction of embodiment by delayed learning gains, $t_{(69.97)}=2.52, p=0.014$. Both of these replicate effects reported above. No other effects were significant (all $p$ 's $>0.17$ ), indicating no further aptitude by treatment interactions.

\section{DISCUSSION}

The study began with three predictions, that: (1) platform would be predictive of both immediate and delayed learning gains; (2) level of embodiment would be predictive of both immediate and delayed learning gains; and (3) the individual condition with the greatest changes would be the MR SMALLab high embodied one because it afforded the greatest amount of gestural congruency, sensorimotor feedback, and largest FOV. The first prediction was not supported, platform did not affect immediate learning gains. When the two levels of embodiment were collapsed, the three platform groups did not perform significantly different on post-test, nor did they perform differentially on the delayed tests. Thus, type of platform used to deliver a well-designed multimedia lesson is not a significant predictor of differential learning.

The second prediction regarding embodiment was partially supported. Although there were no differences for immediate learning, significant differences were seen on the delayed gains on the generative subtest. There was a significant effect for embodiment on the non-computerized, generative subtest, that is, from post-test to the 1 week follow-up, those who learned the content in a more embodied manner showed an advantage on recall of generative physics information. This result was primarily driven by the decrease in scores on the IWB low embodied condition and increases in scores for all high embodied conditions. This result can also be discussed using 'levels of processing' terminology. Those in the low embodied conditions primarily received visual and auditory feedback and used a minimum of action while learning, those in the high embodied conditions were able to use instrumented gestures with more sensorimotor feedback, while also receiving the visual and auditory feedback. Adding the motor trace is another level of processing and may strengthen the encoding signal. It may also prime the pathways that learners activated when first encountering force and it became a perceptual symbol. In addition, adding the motoric gestures may have been "disruptive" during the time of processing. Disruptive is a positive term because often being exposed to disruptive or difficult events (e.g., testing) during processing can lead to better retention and delayed learning gains (Bjork, 1994). Thus, there may be several long term advantages to including physical embodiment in the design of lessons on topics that deal with forces.

The third prediction that the SMALLab high embodied cell would demonstrate the greatest gains was only descriptively supported via an increase in comparative effect sizes (see Appendix C); however, inferential statistics did not reveal a statistically significant increase. The SMALLab high embodied condition still demonstrated the highest effect size from post-test to delayed follow-up, Cohen's $d=0.22$. This is non-trivial given that no physics training occurred in the interim, and it is twice the size of the next highest delayed gain (also seen in a high embodied condition).

\section{The Unexpected Immediate Effects}

The entire group of participants displayed similar gains from pre-test to post-test regardless of condition and this was somewhat unexpected. In retrospect, there may be several reasons why this occurred. First, all six conditions were designed to engage participants and promote the development of a robust CF mental model. That is, all six lessons contained optimal inquiry-based science pedagogy (Hestenes, 1996; Megowan-Romanowicz, 2010) such as using high-quality simulations, diagrams, and active user-control of key aspects in the simulations. Even in the low embodied conditions the participants experienced more agency in navigating the pace of the simulation or animation than is offered by many of the popular science education "gizmos" (simulations or learning objects) currently available for science education. The control conditions should be considered very state-of-theart.

From the point of view of embodiment theory, these highquality visual simulations made all of the conditions partially embodied. That is, in all of the conditions, there were multiple opportunities to ground abstract CF concepts such as force, velocity, mass, and radius in components of the simulations. The conditions differed primarily in the amount of kinesthetics and gestural congruency. Cook and Goldin-Meadow (2006) report that "gesturing makes learning last" in the domain of learning a new mathematical concept. We saw that the condition with the most gesturing and movement via whole body (high embodied SMALLab) was indeed the condition in which the learning persevered more robustly.

Second, a decision was made early on that we would not consciously allow students to leave the study with incorrect mental models. That would have felt somewhat unethical. Thus, when participants answered a prompt incorrectly (e.g., replying that "a longer string would result in more CF"), participants were asked to run through the task again and to answer the question again. If they made the same incorrect conclusion three times in a row, the experimenter explicitly supplied the correct answer. This corrective guidance assured that the knowledge needed to show competency on the post-test was voiced at least one time either by the participant or experimenter. It is still worth noting that no one scored $100 \%$ on the post-test. 


\section{Delayed Gains Seen on Appropriate Subtest}

On average the participants in the three high embodied conditions demonstrated a significant delayed gain on the generative subtest. This may be due to the multiple instances of gestural congruency during encoding and because the high embodied condition elicited more sensorimotor activity. A greater amount of physical movement should activate complex motor neuron patterns and these will be associated with the learning signal. Cook and Goldin-Meadow (2006) hypothesize that their significant delay test results seen in the gesture and gesture/speech groups may be because, “...expressing information in gesture may produce stronger and more robust memory traces than expressing information in speech because of the larger motor movement." In addition, motor planning, though unconscious, recruits resources that have downstream effects on attention and may affect delayed learning gains. We have seen similar delayed results on nutrition knowledge tests when comparing low and high embodied learning conditions in an exergame, i.e., greater retention effects were seen in the delayed knowledge tests for the active, more embodied group (Johnson-Glenberg et al., 2014b). In this current study, the generative subtest was composed of several items that required participants create answers with movements that either mimicked the movement of the bob in flight, or were generated from recall and not recognition. The act of drawing may have also gesturally reified the meaning of the encoded content.

\section{Gains in the SMALLab High Embodied Cell}

Although the greatest delayed learning gains were seen in the SMALLab high embodied cell, the difference was not statistically significant. There are some power concerns with the analyses due to delayed test attrition, but we will also mention two issues exclusive to SMALLab: novelty and technology problems. First, the novelty of the immersive SMALLab experience can distract from learning at first. When SMALLab is used in schools, participants have several days to acclimate to the technology and use the motion tracking wands. They are also able to observe peers perform. In contrast, during the experiment, there was no extended formal period of adaptation. Participants walk immediately into a very techy-looking truss system with multiple draping wires and are simply told, "Above are infrared cameras that track the motion of certain handheld objects." In this individual experiment experience, the participant is active from start to finish with no chance to observe. Yes, it is novel and that can be engaging (see a sample dialog from a participant in Appendix D to get a sense of the flow and how engaging the platform can be). But, SMALLab also requires a tremendous amount of sensory integration in a very short time span when used in a non-collaborative, one shot experimental situation. In addition, there were the requisite technology woes. All 12 infrared cameras must be tightly calibrated and synced for the system to work correctly, otherwise jitter is introduced into the floor projections. The experimenters reported that four sessions seemed to have arrows that "jumped around" a bit. Consequently, error variability was introduced into some of the high embodied SMALLab sessions that was never present in the other five conditions. Nonetheless, the effect size in the SMALLab high embodied condition was twice that seen in the next highest delayed learning gains condition. This lends some support to the theory that grosser body movements and the ability to directly manipulate and feel CF effects may contribute to greater delayed learning gains. For example, in the SMALLab high embodied condition they were able to insert weighted mass packets into the "swinger" bob, and then swing the heavier bob overhead and directly experience greater exertion in their core. This experiential "feeling" of the effects of greater mass is probably very clarifying. It may be more effective than merely showing the bob getting larger in a graphical manner (as was done in the five other swingerless conditions). In sum, there were both negative and positive consequences associated with the SMALLab high embodied condition.

\section{Creating Embodied Content}

It may be the case that certain topics may lend themselves more readily to being taught in an embodied manner. Perhaps it is more straightforward to teach about forces with the body than to teach about justice. We can assert with some assurance that the amount of embodiment in a lesson is important for delayed learning gains in the context of this CF experiment. Although we were surprised that platform did not have an effect, it may also be the case that being freed from platform dependency is a very good thing for education. Creating an optimal lesson might not be highly dependent on the exact technology used. Optimal lessons, those that encourage retention of knowledge, may rely more on the extent of gestural congruency and/or sense of immersion designed into the lesson and less on the technology. A recent example of an embodied lesson delivered via a simple "instruction animation" on a computer monitor comes from Pouw et al. (2016). The topic was levers and a seesaw analogy was provided to the middle school students. They were encouraged to stretch their arms out and think of their torsos as the fulcrum. In the study virtual weights appear on an avatar's outstretched arms and the students must decide which weight is heavier. This is a succinct and elegant meshing of body metaphor with applied science, and it does not require expensive technology to deliver the message.

If it is the amount of embodiment designed into the lesson that is crucial, and teachers do not necessarily need large truss systems, etc., to activate embodiment, then by creatively designing for mouse and extant tracking pads, we should be able to produce highly embodied content. Instructional designers should strive to create lessons that are highly embodied, generative, and include gestural congruencies that are well-mapped to the learning goals. In addition, new cost-effective motion tracking technologies are rapidly entering the market, including VR units that track and respond to hand position. We are optimistic these technologies will come down in price and make their way into classrooms. When that happens, we stress that teachers needed to be properly trained to use new media techniques. Nathan and Alibali (Nathan et al., 2014) found a relationship between action and cognition and experimenter's language (prompts and 
hints) as participants learned geometry proofs. This suggests that providing guided scripts for instructors is important to get the most out of a technology-supported, mediated embodied lesson.

One way to think about the undifferentiated immediate gains in this study is to note that the "instructors" were highly trained (the experimenters actually helped to write the physics script and were experts). The participants learned via one-on-one guidance with the correct answer eventually supplied, thus, we may have created an artificial plateau for gains. Perhaps the novice learners could learn no more due to the platforms' influence because the human instruction quality was so high? The constructs to be varied in a future study could deal with levels of embodiment as well as quality of the instructor. Personally, we are interested in designing technology to aid teachers, not supplant them. We do not always insert guiding avatars into our systems. Thus, we highly recommend that designers focus on professional development and lesson plans as well. Interestingly, Harris and Sass (2007) found that teacher pre-service training generally had little influence on student productivity. One exception was that content-focused teacher professional development was positively associated with productivity in middle and high school math though. Designers of mediated STEM lessons need to also take time to create lesson plans for teachers. Not only plans, but teachers should receive specific training on mediated, embodied lessons multiple times immediately preceding use of the specific technology. We delve more into professional development.

\section{How Does the Embodiment Taxonomy Relate to These Physics Lessons?}

Finally, the field needs to use the term "embodied" in a more codified manner. Which is why we continue to refine the Educational Embodiment Taxonomy (Johnson-Glenberg et al., 2014a). We have left an explication of the six lessons to the end, should the readers wish to make their own mappings before we put forth ours. Again the three constructs were: (a) amount of sensorimotoric engagement, (b) gestural congruency, and (c) immersion. Using the taxonomy, we suggest the six lessons be classified thusly:

4th degree - SMALLab high embodied.

3rd degree - Both SMALLab low embodied and IWB high embodied conditions because many of the gestures were congruent (although these conditions were not as haptic and kinetic as SMALLab high embodied), and both contained larger FOVs.

2nd Degree - Both IWB low embodied and desktop high embodied conditions because the first had a larger FOV, and the second allowed for gesturally congruency - albeit via smaller circular mouse movements.

1st degree - Desktop low embodied because the learner primarily stopped and started the simulations so there was no gestural congruency, and it contained a smaller FOV.

What would a truly non-embodied lesson look like? Such a lesson might be text only, it would never makes reference to anthropomorphization ("flip your hand to transpose a table"). The non-embodied text could include symbols, but no pictures (images), no animations, nor auditory cues (e.g., pitch increase with speed). It would not be multimodal. On the other hand, there is no guarantee that a learner would not spontaneously create a rich visual mental model from text alone; if perceptual symbols are unconsciously activated, then can any content ever be truly "unembodied"?

\section{Future Directions}

This is one of the first attempts to use highly embodied methods to teach physics in a rich multimedia MR environment. Important lessons in design for content with congruent gestures were learned. The ultimate goal is to design lessons for classrooms, to make sure the best pedagogies have a broad reach. We were also able to pilot the CF lesson in an 11th grade science classroom with SMALLab. We have observed SMALLab in this high school setting for several years. We have consistently seen that interest from the observing students (only 1-4 students can be active in the center at one time) is always high the first half of a class session, but attention begins to wane after (a) a student has been active, or (b) they have observed up to four rounds. One way to keep the observing students engaged is to assign tasks to small groups (a strong technique borrowed from Reciprocal Teaching by Palincsar and Brown (1984) and Rosenshine and Meister (1994). Groups of three to four students ring the space around SMALLab and each group holds a small whiteboard. The groups write down their predictions, for example, if speed is increased will CF increase linearly? Yes or no, and why. Each round would be evaluated post-performance and the prediction shared with the entire class; discussion was encouraged as to why a prediction was correct or incorrect. This sort of student-centered teaching with technology may not be something all teachers are familiar with.

It is our job as designers to think through how the content will be used in real classrooms and to also guide teachers in how to be most effective. We encourage designers of educational technology to think through the non-tech parts of the lesson as well. Lesson plans should be available and within each lesson designers need to build in time for student reflection and discourse. There have been instances where we allowed teachers to use our embodied systems after only one webinar worth of training, in general those have resulted in less successful lessons. We give space to the topic of professional development here because even if a designer creates a seamless lesson that sublimely meshes technology and embodiment, when a teacher does not know how to use it, it is wasted experience for all. Teachers' practices are crucial, and many teachers are interested in integrating more technology into their lessons. Recent research supports that subject areas are key predictors in the success of technology integration; however, the effect of subject area on technology integration is not well understood (Howard et al., 2015), however, it seems in the area of STEM technology is more readily integrated. The model for professional development-at-a-distance is changing and we need to proactively support teachers who are use cutting edge technologies with multiple training sessions and access to videotaped real world lessons.

We predict that results from this study will generalize beyond CF. Indeed, the team has created embodied content for, and 
researched in, a range of topics including: gears (JohnsonGlenberg et al., 2015), disease transmission (Johnson-Glenberg et al., 2014a), geology (Birchfield and Johnson-Glenberg, 2010), and metaphor comprehension using other motion capture techniques and SMALLab (Hatton et al., 2010). The MR sessions that have been most successful are the ones where the teachers had very active roles in co-designing the content and/or they received more than two sessions of on-line training.

More research is clearly needed on emerging learning technologies and how various technologies can afford more or less embodiment. AR with tablets or smart devices is poised for large scale dissemination in classrooms. Designers need to be creative about adding gestures to learning with small form devices and mapping gestural congruency. The accelerometer should be integrated into lessons where add value is predicted (e.g., force in physics). Little is known about how the observing students are affected by learning in highly mediated high embodiment lessons (although see Kontra et al., 2015). Less is known about how gestures affect a learner's sense of agency or further enjoyment and engagement while learning. We are encouraged by those who create immersive classrooms (Lui et al., 2014) and use MR platforms in educational and informal learning spaces (Lindgren and Moshell, 2011; Tscholl and Lindgren, 2014) and the gains reported in learning. We do not see technology receding from the modern classroom.

\section{Lessons for Designers}

One foremost tenet for designers is to design for interactivity. The learner should be able to create content as s/he learns. Learners also need immediate, yet non-disruptive, feedback. In the field of STEM, how many high-quality, interactive science objects are available for free? The PHET simulations are outstanding examples, but there is currently not one for CF. The first 25 objects in a Google search on CF (date of retrieval September 2016) were observational videos or worksheets. Not one hit in the first few pages was an interactive multimedia object. We should fill this void (a void in STEM overall) by creating browser-enabled objects that encourage learners to use a mouse or tablet surface as a gesture-based interaction tool. For example, for CF, the learners should be able to spin a bob and release it with user-generated actions. There should be multiple chances for exploration and failure as the learners try to hit a stable target. Learners should be able to draw predictive paths and vectors and then assess the veracity of their actions. They should spin and release a bob and try to hit stable, then moving targets as they "leveled up" and reify concepts. The action should match the learning goal, e.g., if speed of spin is important, then the speed at which the finger circles on the screen needs to part of the displayed information. That is a strong example of gestural mapping and congruency. A weaker example would be a lesson where the learner types in a number for speed and the bob automatically spins to a facsimile of that speed. That is not gesturally congruent. When new media instructional designers understand the limits of the technology and how to map gestures to the key content to be learned (e.g., don't use a hand "push" motion with a Kinect to set a gear train spinning), use a hand "spin" motion, (Johnson-Glenberg et al.,
2015), then larger and more sustained learning gains may be seen.

Creating quality educational content also encompasses the concepts of ecological validity and transferability. This is one of the problems pointed out by Shute et al.'s (2015) analysis of a well-designed videogame compared to a popular computerized brain training regime. What is being taught should promote generalization and learning transfer beyond the "actual tasks" performed in the intervention. Although we believe that embodiment principles generalize to other topics, we cannot say if the specific content learned in our lesson will transfer. This current study focused on delayed gains and did not assess transfer to other far domains (e.g., how is the $r$ in the CF equation denominator similar to the $r$ in the equation for Coulomb's law?). We hypothesize that when transfer does occur it may be because that lesson created a situation where the learner's p-prims no longer contain misconceptions and the knowledge pieces have been well-integrated into the previous knowledge structure so the new pieces can now be applied to other scientific constructs. In this study, the majority of participants who once held the impetus model had corrected it by post-test.

\section{New Assessments}

New types of assessments should be included with new interactive learning objects. Not only does this satisfy some constraints associated with encoding specificity (i.e., Tulving and Thomson, 1973), but the field should continue to research whether knowledge is indeed retained longer when learned in a more embodied manner. Testing is itself a method for increasing learning (Roedinger and Karpicke, 2006) and new embodied methods for testing should continue to be developed. It is now easy to gather in-process data while the participants are in the act of learning (Plass et al., 2013) and creative assessments should also take advantage of what motion capture data can reveal. Affordable new technologies (e.g., Microsoft Kinect, Intel RealSense) provide rapid sampling of rich data streams and reveal how learners move through 3D space and make decisions during learning. The trick is to design the content to elicit meaningful gestural actions at key decision points during the lesson. Recently our lab ran a study using randomized control trials on the topic of electric fields and vectors. In a traditional post-intervention declarative style test, the participants in the high embodied groups did not show a difference in learning compared to low embodied. However, when post-intervention knowledge was assessed with a large Intuous Wacom tablet that afforded and measured acceleration of hand-drawn vectors, then the high embodied conditions demonstrated significant knowledge differences (Johnson-Glenberg and Megowan-Romanowicz, submitted). Instrument sensitivity may play a role in this current study as well, it appears that allowing participants to generate and draw freehand on paper (the off-line subtest) revealed knowledge that could not be gathered on the less sensitive keyboard-based task (the on-line, declarative subtest).

\section{Sleep and Consolidation}

Finally, the delayed test results raise issues regarding sensorimotor, gestural signals, and delayed effects. What is 
driving the delayed effect, and can it be enhanced with more or less delay? Memory consolidation is surely occurring; skill acquisition and procedural knowledge are known to be affected by sleep (Walker and Stickgold, 2004; Stickgold and Walker, 2007), but little is known about how type of learning (embodied or not) interacts with optimal length of sleep for memory consolidation in humans. Multiple follow-up test points may address this.

\section{CONCLUSION}

The experiment examined the effects of embodiment and learning platform on immediate and delayed learning. All participants made impressive significant gains on the immediate post-test. Prior to data collection, we hypothesized that students in the highly embodied conditions would outperform those in the low embodied conditions. We did not see this result at immediate post-test; however, we did see significant gains for the high embodied group on the 1 week delayed subtest that allowed the participants to be more generative. Participants retained more generative physics knowledge after learning in the highly embodied lessons regardless of the learning platform. This may be because adding an extra motor trace during encoding of new knowledge strengthens or "coheres" new knowledge to old knowledge structures in a more felicitous manner.

Based on these results, two general conclusions are drawn. First, principles of embodiment can be applied effectively for the enhanced delayed learning gains of certain STEM material and for overcoming incorrect mental models. Second, we suggest that instructional designers create lessons that contain more embodiment when possible, this means considering the amount of sensorimotor engagement, gestural congruency, and immersion when designing. It appears that the effects of high embodiment may be more robustly revealed on delayed tests. Designers who only have access to mouse and keyboard technology can still be creative about how to make the content gesturally congruent, e.g., spinning the mouse in a circle for a CF lesson. Given that education is about retaining material for future application, it is important that assessment measures be given several times after an intervention. The assessment measures should be sensitive to the instructional methodologies, e.g., including hand-drawn objects may reveal differences in

\section{REFERENCES}

Antle, A. A., Corness, G., and Droumeva, M. (2009). What the body knows: Exploring the benefits of embodied metaphors in hybrid physical digital environments. J. Interact. Comput. 21, 66-75. doi: 10.1016/j.intcom.2008. 10.005

Arons, A. (1997). Teaching Introductory Physics. New York: John Wiley \& Sons.

Bailey, J., Bailenson, J. N., Won, A. S., Flora, J., and Armel, K. C. (2012). Presence and memory: immersive virtual reality effects on cued recall. Paper presented at the Proceedings of the International Society for Presence Research Annual Conference, Philadelphia, PA.

Barsalou, L. W. (1999). Perceptual symbol systems. Behav. Brain Sci. 22, 577-660. doi: 10.1017/S0140525X99532147

Barsalou, L. W. (2008). Grounded cognition. Annu. Rev. Psychol. 59, 617-645. doi: 10.1146/annurev.psych.59.103006.093639 learning. Finally, we are hopeful that the emergence of costeffective forms of motion tracking technology herald a new age for the inclusion of more embodied content into classrooms, and that teachers will be more effectively trained in how to use these new methodologies.

\section{AUTHOR CONTRIBUTIONS}

MJ-G co-designed project, oversaw study, analyzed results, and wrote majority of the manuscript. CM-R co-designed study, acted as physics expert, created tests, wrote much of the introduction. DB co-designed study, coded some of the content, co-created the mixed reality platform called SMALLab. He then left ASU and had no input in the data analyses, results, or discussion sections. $\mathrm{He}$ is an unbiased co-author. CS-R was the lead experimenter as a graduate student and helped to co-design the tests.

\section{FUNDING}

This material is based upon work supported by the National Science Foundation under Grant No1020367. Any opinions, findings, and conclusions or recommendations expressed in this material are those of the author(s) and do not necessarily reflect the views of the National Science Foundation.

\section{ACKNOWLEDGMENTS}

Special thanks go to Arthur Glenberg as well as the SMALLab group at School of Arts, Media and Engineering at ASU and in particular Kelly Phillips. Gratitude is also expressed to Benjamin Luke, Christopher Dean, Elizabeth Marsh, James Gee, Grisha Colemen, David Tinapple, Brian Kim, Aisling Keliher, Wilhelmina Savenye, Barbara Kinach, and Lisa Tolentino.

\section{SUPPLEMENTARY MATERIAL}

The Supplementary Material for this article can be found online at: http://journal.frontiersin.org/article/10.3389/fpsyg. 2016.01819/full\#supplementary-material

Birchfield, D., and Johnson-Glenberg, M. C. (2010). A next gen interface for embodied learning: SMALLab and the geological layer cake. Int. J. Gaming Comput. Mediat. Simul. 2, 49-58. doi: 10.4018/jgcms.2010010105

Bjork, R. A. (1994). Memory and Metamemory Considerations in the Training of Human Beings. Cambridge, MA: MIT Press.

Campos, J. J., Anderson, D. I., Barbu-Roth, M. A., Hubbard, E. M., Hertenstein, M. J., and Witherington, D. (2000). Travel broadens the mind. Infancy 1, 149-219. doi: 10.1207/S15327078IN0102\_1

Cook, S., and Goldin-Meadow, S. (2006). The role of gesture in learning: do children use their hands to change their minds. J. Cogn. Dev. 7, 211-232. doi: $10.1207 /$ s15327647jcd0702\_4

Coomans, M. K. D., and Timmermans, H. J. P. (1997). "Towards a taxonomy of virtual reality user interfaces," Proceedings of the IEEE Computer Society 17th International Conference on Information Visualisation, Washington, DC, 279. doi: 10.1109/IV.1997.626531 
DiSessa, A. A. (1988). "Knowlege in pieces," in Constructivism in the Computer Age, eds G. Forman and P. Pufall (Hillsdale, NJ: Erlbaum), 49-70.

DiSessa, A. A. (1993). Toward an epistemology of physics. Cogn. Instr. 10, 105-225. doi: 10.1080/07370008.1985.9649008

DiSessa, A. A. (2000). Changing Minds: Computers, Learning, and Literacy. Cambridge, MA: MIT Press.

Engelkamp, J. (2001). “Action memory: a systems-oriented approach,” in Memory for Action: A Distinct Form of Episodic Memory? eds H. D. Zimmer, R. L. Cohen, M. J. Guynn, J. Engelkamp, R. Kormi-Nouri, and M. A. Foley (New York: Oxford University Press), 49-96.

Engelkamp, J., and Zimmer, H. D. (1994). The Human Memory: A Multimodal Approach. Seattle, WA: Hogrefe.

Giannotti, D., Patrizi, G., Di Rocco, G., Vestri, A. R., Semproni, C. P., Fiengo, L., et al. (2013). Play to become a surgeon: impact of nintendo WII training on laparoscopic skills. PLoS ONE 8:e57372. doi: 10.1371/journal.pone.00 57372

Glenberg, A. M. (2008). "Embodiment for education," in Handbook of Cognitive Science: An Embodied Approach, eds P. Calvo and A. Gomila (Amsterdam: Elsevier), 355-372.

Glenberg, A. M., and Gallese, V. (2011). Action-based language: a theory of language acquisition,comprehension, and production. Cortex 48, 905-922. doi: 10.1016/j.cortex.2011.04.010

Glenberg, A. M., Witt, J. K., and Metcalfe, J. (2013). From the revolution to embodiment: 25 years of cognitive psychology. Pers. Psychol. Sci. 8, 573-585. doi: $10.1177 / 1745691613498098$

Goldin-Meadow, S., Nusbaum, H., Kelly, S. D., and Wagner, S. (2001). Explaining math: gesturing lightens the load. Psychol. Sci. 12, 516-522. doi: 10.1111/14679280.00395

Goldstone, R., Landy, D., and Son, J. Y. (2008). "A well-grounded education: The role of perception in science and mathematics," in Symbols, Embodiement and Meaning: A Debate, eds M. DeVega, A. M. Glenberg, and A. C. Graesser (Cambridge: Oxford University Press).

Gutiérrez, F., Pierce, J., Vergara, V. M., Coulter, R., Saland, L., Caudell, T. P., et al. (2007). The effect of degree of immersion upon learning performance in virtual reality simulations for medical education. Stud. Health Technol. Inform. 125, 155-160.

Halloun, I. A., and Hestenes, D. (1985a). Common sense concepts about motion. Am. J. Phys. 53, 1056-1065. doi: 10.1119/1.14031

Halloun, I. A., and Hestenes, D. (1985b). The initial knowledge state of college physics students. Am. J. Phys. 53, 1043-1055. doi: 10.1119/1.14030

Harris, D. N. H., and Sass, T. R. (2007). Teacher Training, Teacher Quality and Student Achievement Calder Working Papers. Washington, DC: The Urban Institute.

Hatton, S., Birchfield, D., and Megowan-Romanowicz, C. M. (2010). Learning Metaphor Through Mixed-Reality Game Design and Game Play. Available at: http://docplayer.net/18963434-Learning-metaphor-through-mixed-realitygame-design-and-game-play.html

Hauk, O., Johnsrude, I., and Pulvermüller, F. (2004). Somatotopic representation of action words in human motor and premotor cortex. Neuron 41, 301-307. doi: 10.1016/S0896-6273(03)00838-9

Hestenes, D. (1996). Modeling methodology for physics teachers. Paper Presented at the International Conference on Undergraduate Physics, College Park, MD.

Hestenes, D. (2006). Notes for a modeling theory of science cognition and instruction. Paper Presented at the GIREP Conrerence, Modeling and Physics and Physics Education, Amsterdam.

Hestenes, D., Wells, M., and Swackhamer, G. (1992). Force concept inventory. Phys. Teach. 30, 141-158. doi: 10.1119/1.2343497

Howard, S. K., Chan, A., Mozejko, A., and Caputi, P. (2015). Technology practices: confirmatory factor analysis and exploration of teachers' technology integration in subject areas. Comput. Educ. 90, 24-35. doi: 10.1016/j.compedu.2015. 09.008

Johnson-Glenberg, M. (2012). What is learning in a mixed reality environment and what does an "Embodied Lesson" mean. Paper Presented at the ARVELSig, Special group of AERA. Tempe, AZ: Arizona State University.

Johnson-Glenberg, M. C., Birchfield, D., Koziupa, T., and Tolentino, L. (2014a). Collaborative embodied learning in mixed reality motion-capture environments: two science studies. J. Educ. Psychol. 106, 86-104. doi: 10.1037/ a0034008

Johnson-Glenberg, M. C., Savio-Ramos, C., and Henry, H. (2014b). “Alien Health”: a nutrition instruction exergame using the kinect sensor. Games Health J. 3, 241-251. doi: 10.1089/g4h.2013.0094

Johnson-Glenberg, M. C., Birchfield, D., Megowan-Romanowicz, M. C., and Snow, E. L. (2015). If the gear fits, spin it! Embodied education and in-game assessments. Int. J. Gaming Comput. Based Simul. 7, 40-65.

Johnson-Laird, P. N. (1998). Computer and the Mind: An Introduction to Cognitive Science. Cambridge: Harvard University Press.

Klahr, D., Triona, L. M., and Williams, C. (2007). Hands on what? The relative effectiveness of physical versus virtual materials in an engineering design project by middle school children. J. Res. Sci. Teach. 44, 183-203. doi: 10.1002/ tea. 20152

Koch, S. C., Glawe, S., and Holt, D. V. (2011). Up and down, front and back: movement and meaning in the vertical and sagittal axes. Soc. Psychol. 42, 214-224. doi: 10.1027/1864-9335/a000065

Kontra, C., Lyons, D., Fischer, S., and Beilock, S. L. (2015). Physical experience enhances science learning. Psychol. Sci. 26, 737-749. doi: 10.1177/ 0956797615569355

Lakoff, G., and Johnson, M. (1999). Philosophy in the Flesh: The Embodied Mind and its Challenge to Western Thought. New York, NY: Basic Books.

Lindgren, R., and Johnson-Glenberg, M. C. (2013). Emboldened by embodiment: six precepts regarding the future of embodied learning and mixed reality technologies. Educ. Res. 42, 445-452. doi: 10.3102/0013189X135 11661

Lindgren, R., and Moshell, J. (2011). Supporting children's learning with bodybased metaphors in a mixed reality environment. Paper Presented at the Proceedings of the 10th International Conference on Interaction Design and Children, Ann Arbor, MI.

Lindgren, R., Tscholl, M., Wang, S., and Johnson, E. (2016). Enhancing learning and engagement through embodied interaction within a mixed reality simulation. Comput. Educ. 95, 174-183. doi: 10.1016/j.compedu.2016.01.001

Lui, M., Kuhn, A., Acosta, A., Niño-Soto, M. I., Quintana, C., and Slotta, J. D. (2014). Using mobile tools in immersive environments to support science inquiry. CHI '14 Ext. Abstr. Hum. Factors in Computing Syst. 978, 403-406. doi: $10.1145 / 2559206.2574796$

Mayer, R., and Moreno, R. (2003). Nine ways to reduce cognitive load in multimedia learning. Educ. Psychol. 38, 43-52. doi: 10.1207/ S15326985EP3801 \_6

McCloskey, M., Caramazza, A., and Green, B. (1980). Curvilinear motion in the absence of external forces: naive beliefs about the motion of objects. Sci. Educ. 210, 1139-1141.

Megowan-Romanowicz, C. (2010). Modeling Instruction: how to do it! Paper Presented at the Chinese Association of Physics Education Research, Beijing.

Milgram, P., and Kishino, A. F. (1994). Taxonomy of mixed reality visual displays. IEICE Trans. Inform. Syst E77-D, 1321-1329.

Miller, H. L., and Bugnariu, N. L. (2016). Level of immersion in virtual environments impacts the ability to assess and teach social skills in autism spectrum disorder. Cyberpsychol. Behav. Soc. Netw. 19, 246-256. doi: 10.1089/ cyber.2014.0682

Moore, W., Cormier, R., and Smith Phelan, L. (1981). Project Physics Handbook. New York, NY: Holt Rinehart \& Winston.

Nathan, M. J., Walkington, C., Boncoddo, R., Pier, E. L., Williams, C. C., and Alibali, M. W. (2014). Actions speak louder with words: The roles of action and pedagogical language for grounding mathematical reasoning. Learn. Instr. 33, 182-193. doi: 10.1016/j.learninstruc.2014.07.001

Palincsar, A., and Brown, A. (1984). Reciprocal teaching of comprehensionfostering and comprehension monitoring activities. Cogn. Instr. 2, $117-175$.

Plass, J. L., Frye, J., Isbister, K., Perlin, K., Homer, B. D., Kaczetow, W., et al. (2013). "Metrics in simulations and games for learning," in Game Analytics: Maximizing the Value of Player Data, eds A. Drachen, M. S. El-Nasr, and A. Canossa (London: Springer-Verlag), 697-730.

Pouw, W. T., Gog, T., Zwaan, R., and Paas, F. (2016). Augmenting instructional animations with a body analogy to help children learn about physical systems. Front. Psychol. 7:860. doi: 10.3389/fpsyg.2016.00860 
Redish, E. F. (1994). Implications of cognitive studies for teaching physics. Am. J. Phys. 62, 796-803. doi: 10.1119/1.17461

Reif, F. (1995). Millikan lecture 1994: understanding and teaching important scientific thought processes. Am. J. Phys. 63, 17-34. doi: 10.1119/ 1.17764

Reiner, M., Slotta, J. D., Chi, M. T. H., and Resnick, L. B. (2000). Naive physics reasoning: a commitment to substance-based conceptions. Cogn. Instr. 18, 1-35. doi: 10.1207/S1532690XCI1801\_01

Roedinger, H. L., and Karpicke, J. D. (2006). The power of testing memory: basic research and implications for educational practice. Perspect. Psychol. Sci. 1, 181-210. doi: 10.1111/j.1745-6916.2006.00012

Rosenshine, B., and Meister, C. (1994). Reciprocal teaching: a review of the research. Rev. Educ. Res. 64, 479-530. doi: 10.3102/00346543064004479

Segal, A., Black, J., and Tversky, B. (2010). Do gestural interfaces promotoe learning? congruent gestures promote performance in math. Paper Presented at the 51st Meeting of the Psychonomic Society Conference, St. Louis, MO.

Sengupta, P., and Wilensky, U. (2009). Learning electricity with NIELS: thinking with electrons and thinking in levels. Int. J. Comput. Math. Learn. 14, 21-50. doi: 10.1007/s10758-009-9144-z

Shute, V. J., Ventura, M., and Ke, F. (2015). The power of play: the effects of Portal 2 and Lumosity on cognitive and noncognitive skills. Comput. Educ. 80, 58-67. doi: 10.1016/j.compedu.2014.08.013

Slater, M., and Wilbur, S. (1997). A framework for immersive virtual environments (FIVE): speculations on the role of presence in virtual environments. Presence 6, 603-616. doi: 10.1162/pres.1997.6.6.603

Stickgold, R., and Walker, M. (2007). Sleep-dependent memory consolidation and reconsolidation. Sleep Med. 8, 331-343. doi: 10.1016/j.sleep.2007.03.011

Stickgold, R., and Walker, M. P. (2013). Sleep-dependent memory triage: evolving generalization through selective processing. Nat. Neurosci. 16, 139-145. doi: 10.1038/nn.3303

Tscholl, M., and Lindgren, R. (2014). Empowering digital interactions with real world conversation. Tech. Trends 58, 56-63. doi: 10.1007/s11528-013-0721-6

Tulving, E., and Thomson, D. M. (1973). Encoding specificity and retrieval processes in episodic memory. Psychol. Rev. 80, 352-373. doi: 10.1037/h0020071

Viennot, L. (1979). Spontaneous reasoning in elementary dynamics. Eur. J. Sci. Educ. 1, 205-211. doi: 10.1080/0140528790010209
Walker, M. P., and Stickgold, R. (2004). Sleep-dependent learning and memory consolidation. Neuron 44, 121-133. doi: 10.1016/j.neuron.2004.08.031

Wilson, M. (2003). Six views of embodied cognition. Psychon. Bull. Rev. 9, 625-636. doi: 10.3758/BF03196322

Wu, H., Lee, S. W., Chang, H., and Liang, J. (2013). Current status, opportunities and challenges of augmented reality in education. Comput. Educ. 62, 41-49. doi: $10.1016 /$ j.compedu.2012.10.024

Conflict of Interest Statement: DB is listed as a co-author because he helped to write the NSF grant that secured the funding for the project and he contributed in the first year to co-design the study and program some of the conditions. In year two of the grant when participants were recruited, Dr. Birchfield decided to leave academia and the PI-ship was handed over to the first author. DB moved to California to run the company called SMALLab Learning, LLC. www.smallableanring.com. The site is dedicated to embodied learning, but there is no content for sale on that site that corresponds to this work or the topic of centripetal force. The scenario described in this manuscript is not a commercial product. DB had no input into the project after the creation of the six conditions. He never worked with the data nor wrote sections in this article. Nonetheless, he deserves co-authorship because it would not have come to pass without him. All the other authors declare that the research was conducted in the absence of any commercial or financial relationships that could be construed as a potential conflict of interest.

The reviewer CL and handling Editor declared their shared affiliation, and the handling Editor states that the process nevertheless met the standards of a fair and objective review.

Copyright (C) 2016 Johnson-Glenberg, Megowan-Romanowicz, Birchfield and Savio-Ramos. This is an open-access article distributed under the terms of the Creative Commons Attribution License (CC BY). The use, distribution or reproduction in other forums is permitted, provided the original author(s) or licensor are credited and that the original publication in this journal is cited, in accordance with accepted academic practice. No use, distribution or reproduction is permitted which does not comply with these terms. 


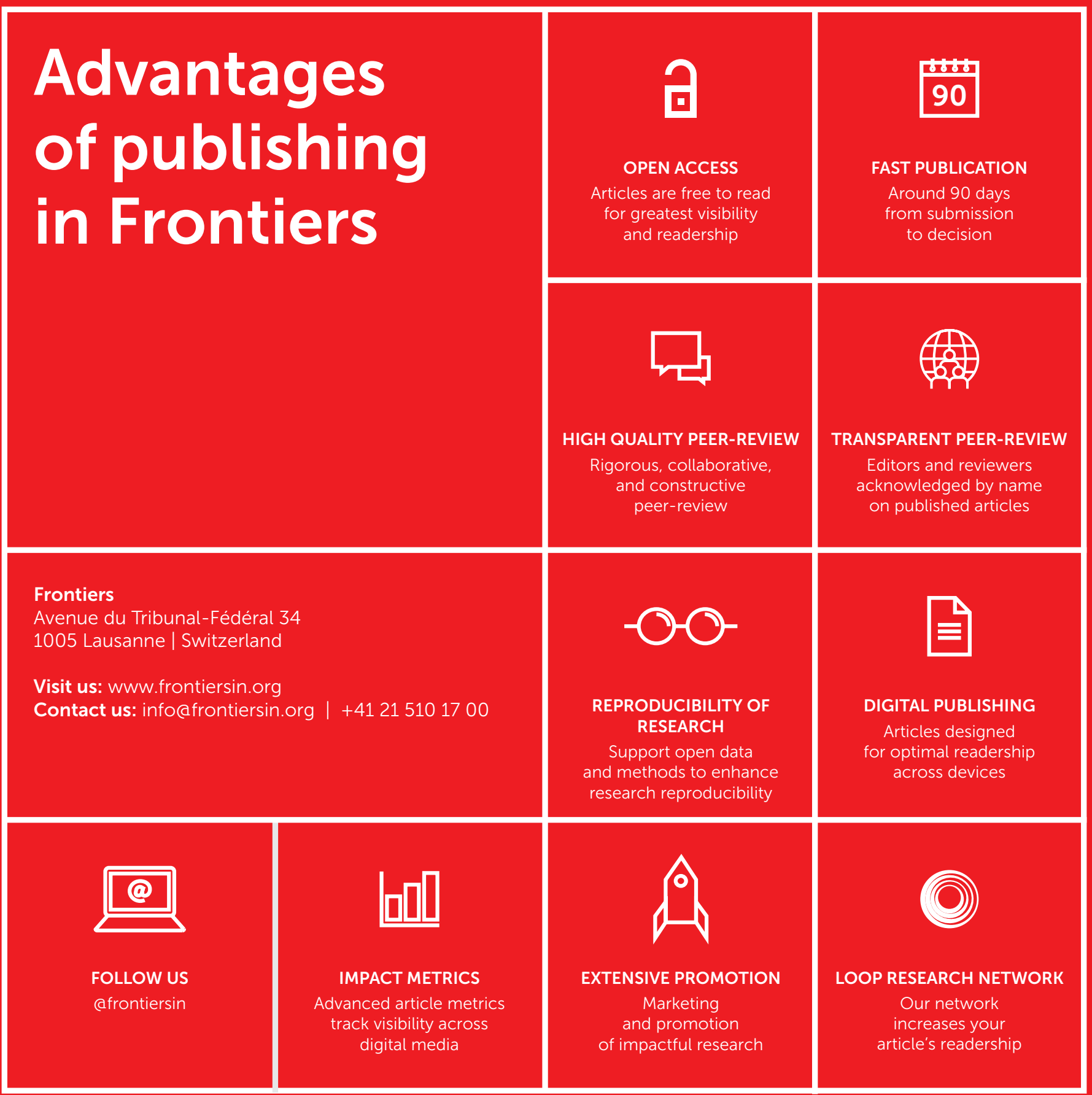

